\title{
Materials Safety Data Sheets The Basis for Control of Toxic Chemicals Volume 3
}

MASTER

E. E. Ketchen

W. E. Porter 


\section{DISCLAIMER}

This report was prepared as an account of work sponsored by an agency of the United States Government. Neither the United States Government nor any agency Thereof, nor any of their employees, makes any warranty, express or implied, or assumes any legal liability or responsibility for the accuracy, completeness, or usefulness of any information, apparatus, product, or process disclosed, or represents that its use would not infringe privately owned rights. Reference herein to any specific commercial product, process, or service by trade name, trademark, manufacturer, or otherwise does not necessarily constitute or imply its endorsement, recommendation, or favoring by the United States Government or any agency thereof. The views and opinions of authors expressed herein do not necessarily state or reflect those of the United States Government or any agency thereof. 


\section{DISCLAIMER}

Portions of this document may be illegible in electronic image products. Images are produced from the best available original document. 


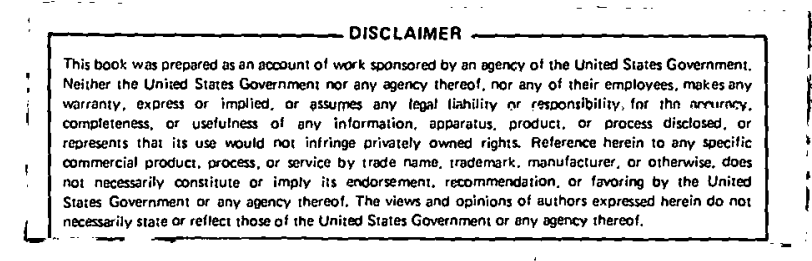

(Rev. Dec. 1979)

ORNL/TM-6981/V3

Contract No. W-7405-eng-26

HEALTH DIVISION

Industrial Hygiene Department

MATERIAL SAFETY DATA SHEETS

THE BASIS FOR CONIROL OF TOXIC CHEMICALS

VOLUME 3

E. E. Ketchen

W. E. Porter

Date Published: September 1979

OAK RIDGE NATIONAL LABORATORY

Oak Ridge, Tennessee 37830

operated by

UNION CARBIDE CORPORATION

for the

Department of Energy 

FOREWORD

The Material Safety Data Sheets contained in this volume are the basis for the Toxic Chemical Control Program developed by the Industrial Hygiene Department, Health Division, ORNL. The three volumes are the update and expansion of ORNL/TM-5721 and ORNL/TM-5722 "Material Safety Data Sheets The Basis for Control of Toxic Chemicals", Volume I and Volume II. As such, they are a valuable adjunct to the data cards issued with specific chemicals. The chemicals are identified by name, stores catalog number where appropriate, and sequence numbers from the NIOSH Registry of Toxic Effects of Chemical Substances, 1977 Edition, if available.

The data sheets were developed and compiled to aid in apprising the employees of hazards peculiar to the handling and/or use of specific toxic chemicals. Space limitation necessitate the use of descriptive medical terms and toxicological abbreviations. A glossary and an abbreviation list were developed to define some of those sometimes unfamiliar terms and abbreviations. Questions concerning these data sheets should be referred to the Industrial Hygiene Department.

The three volumes are being printed in a looseleaf form to accommodate additional data sheets and updated sheets without the re-issue of new volumes. The page numbers are keyed to the catalog number in the chemical stores at ORNL. 


\section{THIS PAGE}

\section{WAS INTENTIONALLY \\ LEFT BLANK}


(Rev. Dec. 1979)

\section{TABLE OF CONTENTS}

FOREWORD

1. Glossary of Terms

2. Abbreviations

3. Material Safety Data Sheets

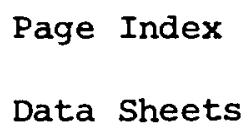

4. Appendix

Chemical Abstracts Service Registry Numbers (Continuation of ORNL/TM-6981/V2) 


\section{PAGES $1-1$ to $1-2$ \\ WERE INTENTIONALLY \\ LEFT BLANK}




\begin{tabular}{|c|c|}
\hline Albumin & A water-soluble protein excreted in urine \\
\hline Anemia & $\begin{array}{l}\text { A condition in which the blood is } \\
\text { deficient in hemoglobin and/or numbers } \\
\text { of red cells compared with normal blood }\end{array}$ \\
\hline Ane'sthesia & $\begin{array}{l}\text { Loss of feeling or sensation, especialiy } \\
\text { the loss of tactile sensibility }\end{array}$ \\
\hline Anorexia & Lack or loss of the appetite for food \\
\hline Anosmia & Absence of the sense of smell \\
\hline Anuria & $\begin{array}{l}\text { Total suppression of urinary output by } \\
\text { the kidney }\end{array}$ \\
\hline Apathy & Lack of feeling or emotion, indifference \\
\hline Arthralgia & Neuralgia or pain in a joint. \\
\hline Asbestosis & $\begin{array}{l}\text { Lung disease caused by prolonged inhaling } \\
\text { of asbestos }\end{array}$ \\
\hline Asphyxia & Suffocation \\
\hline Bronchiolitis & Capillary bronchitis \\
\hline Bronchitis & Inflammation of the bronchial tubes \\
\hline Bronchopneumonia & $\begin{array}{l}\text { Inflammation of the lungs following in-. } \\
\text { fection of the upper respiratory tract, } \\
\text { infectious fevers, or debilitating diseases }\end{array}$ \\
\hline Bronchospasms & Bronchial spasm \\
\hline Carcinolld & $\begin{array}{l}\Lambda \text { malignant new growth made up of ept- } \\
\text { thelial cells, which give rise to } \\
\text { metastases }\end{array}$ \\
\hline Cataract & $\begin{array}{l}\text { An opacity of the crystalline eye lens } \\
\text { or its capsule }\end{array}$ \\
\hline Cirrhosis & $\begin{array}{l}\text { A disease of the liver marked by the } \\
\text { progressive destruction of liver cells }\end{array}$ \\
\hline Conjunctivitis & $\begin{array}{l}\text { Inflammation of the conjunctiva, the } \\
\text { delicate membrane that lines the eyelids } \\
\text { and covers the eyeballs }\end{array}$ \\
\hline
\end{tabular}




$$
\bar{I}-4
$$

Convulsion

Cornea

Cyanosis

Delirium

Dermatitis

Dyspena

Emphysema

Epigastric

Erosion

Euphoria

Gangrene

Fastronteritis

Goiters

Hallucinations

Hematemess is

Hematuria

Hemoglobinuria

Hemolytic Anemia

Hepatitis

Hyperactivity
A violent contraction of the voluntary muscles

Transparent structure of the external layer of the eyeball

Blueness of the skin caused by insufficient oxygenation of the blood

A mental disturbance marked by illusion, hallucinations, physical restlessness, and incoherence

Inflammation of the skin

Difficult or labored breathing

À swelling or inflation due to presence of air in the interstices of the connective tissues of the lungs

Pertaining to the belly or (epigastrium)

An eating or gnawing away

Exaggerated sense of well-being

Anemic necrosis of tissue

Inflammation of the slundich and intestines

Enlargement of the thyroid gland

A sense perception not founded upon objective reality

Vnmiting of blood

Discharge of biood in the urine

Presence of hemoglubin in the urine

Anemia due to active destruction of red cells

Inflammation of the liver

Overactivity 
Hyperemia

Hyperpnea

Hypotension

Hypothermia

Inebriation

Ingestion

Insomnia

Intoxication

Lacrimation

Laryngitis

Lassitude

Lethargy

Leukopenia

Malaise

Malignant tumors

Mottling

Myalgia

Narcosis

Narcotic
Excess of blood in any part of the body Abnormal exaggeration of the respiratory movements

Abnormally low blood pressure

Abnormally low temperature

The condition of being drunk

The act of taking food, medicines, etc. into the body by mouth

Inability to sleep

The condition produced by excessive use of alcohol; also, the state of being poisoned

The secretion and discharge of tears

Sore throat, hoarseness, and cough due to inflammation of the larynx

Weakness, exhaustion

A condition of drowsiness of mental origin

Reduction of white cells in the blood

Uneasiness, discomfort, and distress

Tumors that tend to become progressively worse and result in death

A condition of a tissue spotted with patches of color

Pain in a muscle or muscles

A state of profound unconsciousness produced by a drug

Having the effect of producing sleep or stupor and at the same time relieving pain $(\operatorname{adj}$. 
Nausea

Necrosis

Nephritis

Neuritis

Neurotoxic

Uligurıa

Upacity

Opaque

Pallor

Palpitation

Paill edema

Paralysis

Petechida

Pharyngitis

Pneumonitis

Polyneuritis

Prostration

Paychic disturbances

Pil monary edema

Pulmonary fibrosis

Pyorrhed

Retching

Salivation
Tending to vomit, feeling of sickness at the stomach

Death of a circumscribed portion of tissue

Inflammation of the kidney (hemmorrhagic)

Inflammation of a nerve

Poisonous or destructive to nerve tissue

Reduced daily output of urine

The condition of heing npaque

Impervious to lighll rays

Páleness

Unduly rapid beating of the heart

Edema of the optic papilla

Loss or impairment due to lesion of muscular mechanism

A small area of discoloration formed by the effusion of blood

InC. 1.mullidiun uf the pharynx

Localized inflammation of the lung

Inflammation of many nerves at once

Extreme exhaustion or powerlessness

Menlal disturbances

Fluid in the lungs

Fibrous tissues in the lungs

A discharge of pus, particularly from the gums

A strong involuntary effort to vomit

An excessive discharge of saliva 
Somnolence

Stomatitis

Stupor

Thiocyanate Intoxication

Tinnitus

Ulceration

Vertigo
Sleepiness

Inflammation of the mouth

Partial or nearly complete unconsciousness

Characterized by central nervous system stimulation and depression, and muscular incoordination

Ringing in the ears

The formation of an ulcer

A sensation as if the external world were revolving around the patient or if he were revolving in space; the term is sometimes erroneously used as a synonym for dizziness 
2. KEY TO ABBREVIA'TIONS 


\section{THIS PAGE}

\section{WAS INTENTIONALLY LEFT BLANK}


KEY TO ABBREVIATIONS

$\begin{array}{ll}\text { cC } & \text { cubic centimeter } \\ \text { CNS } & \text { central nervous system effects } \\ \text { gm } & \text { gram } \\ \text { H } & \text { hour } \\ \text { IRR } & \text { irritant effects } \\ \text { kg } & \text { kilogram (one thousand grams) } \\ \text { LC50 } & \text { lethal concentration } 50 \text { percent kill } \\ \text { LCLo } & \text { lowest published lethal concentration } \\ \text { LD50 } & \text { lethal dose 50 percent kill } \\ \text { LDLo } & \text { lowest published lethal dose } \\ \text { C.M. } & \text { cubic meter } \\ \text { mg } & \text { milligram (one thousandth of a gram; } 10^{-3} \text { gm) } \\ \text { ml } & \text { milliliter } \\ \text { Ppm } & \text { parts per million ( } / v \text { ) } \\ \text { TCLo } & \text { lowest published toxic concentration } \\ \text { TDLo } & \text { lowest published toxic dose } \\ \text { TLV } & \text { threshold limit value } \\ \text { TWA } & \text { time-weighted average } \\ \text { W } & \text { week } \\ \text { Y } & \text { year }\end{array}$


3. MATERIAL SAFETY DATA SHEETS 


\section{$\underline{\text { PAGE INDEX }}$}

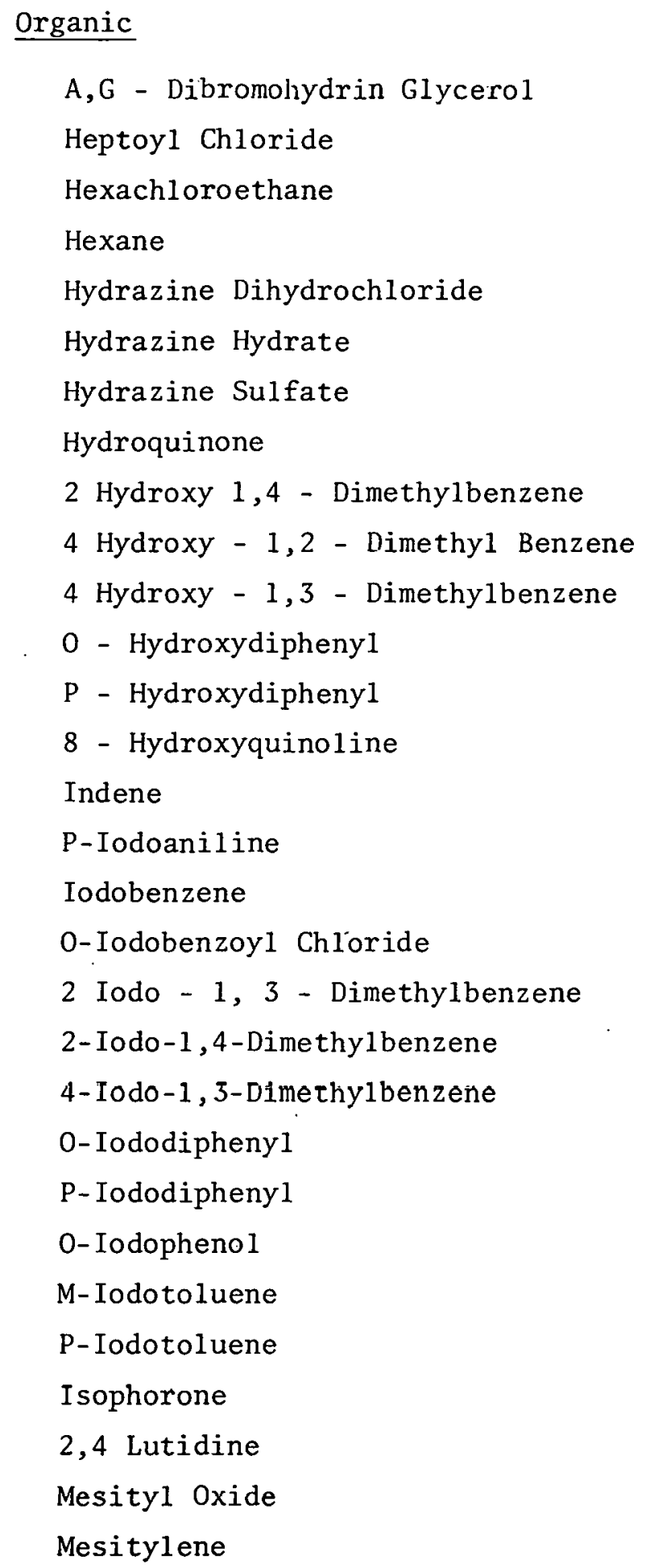

Page No.

263-0606

267-1707

267-2407

267-3207

267-5507

267-5787

267-5947

267-6737

267-8207

267-8307

267-8407

267-8647

267-8677

267-9857

278-0308

278-1308

278-1808

278-2308

278-2708

278-2808

278-2908

278-3308

278-3408

278-4308

278-4708

278-4908

278-5808

278-2919

305-2601

305-2701 
Methyl Acetate

$305-5631$

Methy1 Acrylate

305-6101

Methylamine

305-7601

Methylamine Hydrochloride

305-7701

Methyl N-Amy1 Ketone

305-9601

$\mathrm{N}$-Methyl aniline

306-0202

Methyl Bromoacetate

306-2702

Methyl B-Bromopropionate

306-3702

Methyl Iso-Buty1 Kotono

$306-4702$

Methyl Carbonate

306-6202

P-Methyl cyclohexanul

3ن்7-U3U3

M-Methylcyclohexanone

307-0703

P-Methylcyclohexanone

$307-0903$

Methyl A, B-Dibromopropionate

307-1303

Methylene Bromide

$307-2103$

Methylene Chloride

307-2203

Methylene Iodide

$307-2303$

Methylethylamine Hydrochloride

307-2803

Methylethylaniline

307-3303

Mothyr Ethyrl Ketone

$307-3813$

Methylhydrazinc Sulfate

307-5803

Mcthyl Iodide

307-7803

A-Methylnaphthalene

308-0404

B-Methylnaph thal ene

$308-0474$

4 Methy1-2-Pentanol

$308-4904$

Methyl-P-Phenylenediamine Dihydrochloride

308-5904

A-Methy 1-A-Pheny1 hydraz ine

308-6404

Methyl-Iso-Propyl carbinol

$308-8404$

Methyl Iso-Propyl Ketone

308-8904

Methyl Sulfate

$309-1605$

Methyl Thiocyanate

309-2105

Methyl-Iso-Thiocyanate

309-2205 
Methy1-M-Toluidine

309-3605

Methyl-0-Toluidine

309-3705

Methyl $P$ Toluidine

309-3805

Morpholine

309-8205

Myristoyl Chloride

309-9205

Amsco Diluent 125-82

314-0286

Naphthalene

314-0706

A-Naphthaleneacetonitrile

$314-1206$

A-Naphthol

314-2706

B-Naphthol

314-2806

A-Naphthonitrile

314-3706

A-Naphthylamine

314-5306

B - Naphthylamine Hydrochloride

314-5906

A-Naphthylhydrazine Hydrochloride

314-6306

1-Naphthyl Isocyanate

314-6806

B-Naphthy1 Isocyanate

314-6906

3 Nitro-4 Acetylaminotoluene

315-0907

5 Nitro-2-Aminoanisole

315-1907

b Nitro-4-Amino-1,

3-Dimethyl Benzene

315-2407

3-Nitro-4 Aminophenetole

315-2907

3-Nitro-4-Aminotoluene

315-3407

4-Nitro-2-Aminotoluene

315-3507

M-Nitroanil ine

$315-3807$

O-Nitroaniline

315-3907

P-Nitrobenzal Bromide

315-5407

Nitrobenzene

$315-6857$

P-Nitrobenzoyl Chloride

316-0208

P-Nitrobenzyl Bromide

316-0608

P-Nitrobenzyl Chloride

316-0808

$\mathrm{M}$-Nitrobromobenzene

316-1108

O-Nitrobromobenzene

316-1208

P-Nitrobromobenzene

316-1308 
M-Nitrochlorobenzene

316-1708

O-Nitrochlorobenzene

316-1808

P-Nitrochlorobenzene

316-1908

2-Nitro-P-Cymene

216-2608

2 Nitro-1,4 Diaminobenzene

316-3108

4-Nitro-1,2-Diaminobenzene

316-3308

O-Nitrodiethylaniline

316-3708

M-Nitrodimethylaniline

$316-4108$

2-Nitro, 1,4-Dimethylbenzene

$316-4608$

3-Nitro-1, 2-Dimethylbenzene

$316-4808$

p-Nitrotluorobenzene

$316-7108$

M-Nitroiodobenzene

316-8108

O-Nitroiodobenzene

316-8208

P-Nitroiodobenzene

316-8308

Nitromesitylene

316-8608

Nitromethane

316-9108

P-Nitromethylaniline

316-9608

5 Nitro-1-Naphthylamine

317-1209

M-Nitrophenol

$317-3239$

$P$ Nitrophonol

$317-3439$

O-Nitrophenol Sodium Salt

317-3709

P-Nitrophenylhydrazine

$317-4709$

P-Nitrophenylhydrazine Hydrochloride

$317-4909$

I-Nitropropane

$317-6209$

2-Nitropropane

317-6309

2-Nitroresorcinol

3.1 7-72.n9

P-Nitrosodietlyylaniline

317-8009

P-Nitrosodimethylaniline

317-8209

2-Nitroso 1-Napthol

31. 7-8809

Nitrosopiperidene

317-9409

Nitrosoresorcinol

Sodium Salt

317-9609

$\mathrm{M}$-Nitrotoluene

317-9709 
O-Nitrotoluene

317-9809

P-Nitrotoluene

317-9909

Oxalyl Chloride

$321-0301$

Palmitoyl Chloride

326-0402

Pentamethylene Bromide

326-3402

M-Phenetidine

326-5902

O-Phenetidine

$326-6002$

P-Phenetidine

326-6102

Phenol

326-6904

G-Phenoxybutyronitrile

326-7902

Phenylacetyl Chloride

327-0603

M-Phenylenediamine

$327-2603$

P-Phenylene Diamine

$327-2813$

M-Phenylenediamine Dihydrochloride

$327-2903$

B-Phenylethyl Bromide

327-3603

Phenylhydrazine

327-5103

Phenylhydrazine Hydrochloride

327-5203

Phenyl Isocyanate

327-5603

Phenyl Isothiocyanate

327-6103

P-Phenylphenacyl Bromide

$327-7603$

B-Phenylpropionyl Chloride

327-8103

1-Pheny1semicarbazide

328-0704

4 Phenylthiosemicarbazide

328-1704

Phenyl thiourea

328-2204

Picoline

328-5704

B-Picoline

328-5904

G-Picoline

328-5904

Picramide

328-6204

Picryl Chloride

$328-6404$

Propionitrile

328-9504

Propionyl Chloride

329-0305

Iso-Propyl Acetate

329-1305

Iso-Propyl amine

329-1805

$\mathrm{N}$-Propyl amine

329-1905 
$\mathrm{N}$-Propylaniline

$329-2305$

$\mathrm{N}$-Propylben zene

329-3305

N-Propy1 Chlorocarbonate

329-4405

Propylene Bromide

$329-4805$

Propylene Bromohydrin

329-4905

Propylene Chloride

329-5105

Propylene Chlorohydrin

329-5205

Propylene Glycol

329-5605

Propylene Oxide

$32.9-5805$

Isopropyl lodide

$32.9-6.305$

Quinone

341-1906

Resorcinol

348-0607

Semicarbazide Hydrochloride

357-1708

Strychnine Sulfate

$357-2708$

Succinonitrile

357-3908

Succinyl Chloride

$357-4108$

Sulfanil amide

357-5009

Tetra bromoethane

(acetylene tetrabromide)

365-0839

Tetrabromophthalic Anyhydride

365-1809

Totra-N.Butylammonium Iodide

$365-2309$

$1,1,2,2$-l'etrachlorocthane

365-2809

Tetrachlorocthylene

(Perchloroethylene)

$365-3309$

Tetrachlorohydroquinone

365-3809

Tetrachorophtha1ic Anhydride

365-4309

Tetrahydrofurañ

$365-4809$

Tetramethylammonium Bromide

365-5809

Tetramethylammonium Chloridc

$365-5909$

Tetramethyl ammonium Hydroxide

365-6009

Tetramethylammonium Iodide

365-6109

Tetramethylene Chloride

365-7309

Thioacetamide

365-8509

Thionyl Chloride

$365-9309$ 
Thiophenol

365-9709

Thiosemicabazide

366-0401

Thiourea

366-0901

o-Tolidine Dihydrochloride

366-1501

Toluene

366-2401

Toluhydroquinone

366-3401

M-Toluidine

366-3901

0 -Toluidine

366-4001

$\mathrm{P}$-Toluidine

$366-4101$

P-Toluidine Hydrochloride

366-4401

2, 4,6-Tribromoanil ine

366-7401

1,3,5 Tribromobenzene

366-7901

1,2,3 Tribromopropane

366-8401

2,4,6 - Trichloroaniline

366-9401

1,2,4-richlorobenzene

366-9611

1,1,2 Trichloroethane

367-0084

Trichloroethylene

367-0102

2,4,6 Trichlorophenol

367-0602

1,2,3 Trichloropropane

:67-1102

Triethylamine

$367-2102$

Trimethylamine

367-3102

Trimethylene Bromide

367-3502

Trimethylene Glycol

367-3902

2,4,6 - Trinitroresorcinol

367-5602

Tri-N-Propylamine

367-8102

Tribromoethylene

367-8602

Xylene

387-1425

M-Xylene

387-1505

$0-X y l$ ene

307-1605

P-Xylene

387-1705

o-Xylylene Bromide

387-2905

M-Xyly1 Bromide

387-3405

0-Xyly1 Bromide

387-3505 
Materials

Camphor Gum

619-3032

Cleaning Mixture \#49

623-5020

Fungicide

649-3001

Pyridine

731-8305

Organic Gases

Methyl Chloride

$967-6070$ 


PRODUCT DESIGNATION
$03-263-0606$
$A, G-$ Dibromohydrin Glycerol

\section{MATERIAL SAFETY DATA SHEET}

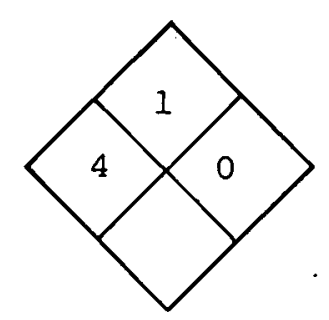

\section{SECTION I SOURCE AND NOMENCLATURE}

MANUFACTURER'S NAME

EMERGENCY TELEPHONE NO.

ADDRESS (NUMBER, STREET, CITY, STATE, ZIP CODE)

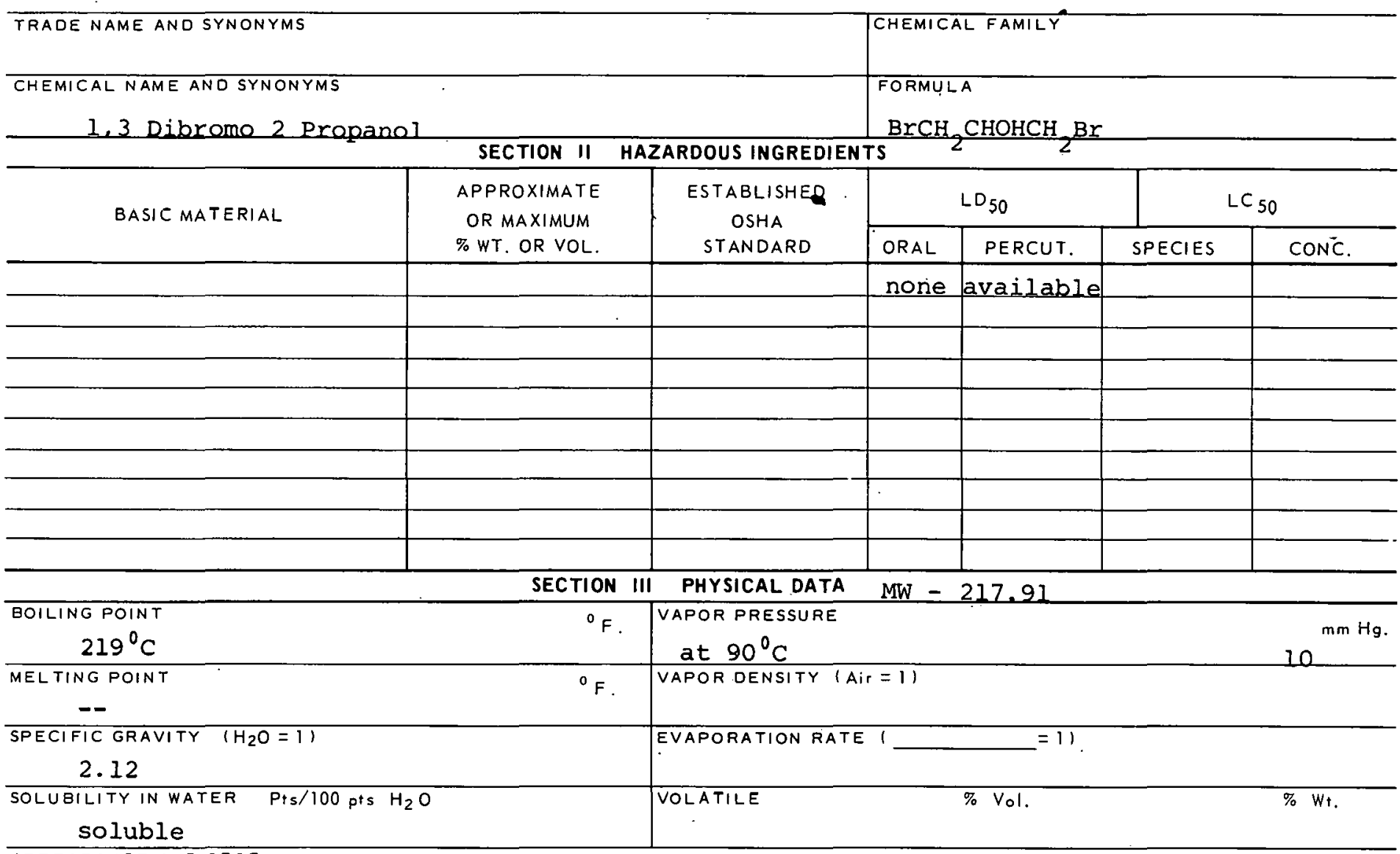

APPEARANCE AND ODOR

Yellow liquid

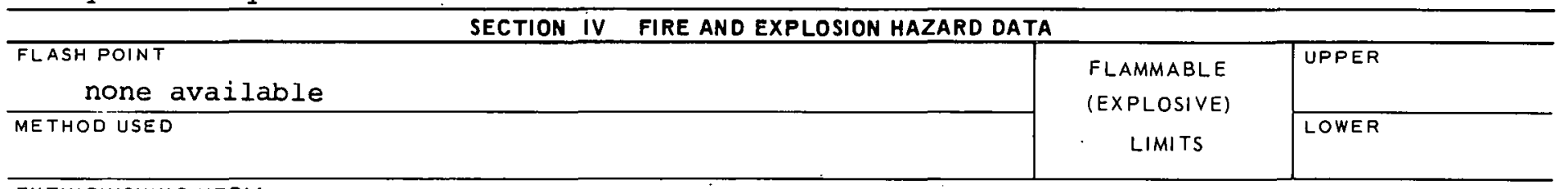

EXTINGUISHING MEDIA

alcohol foam, carbon dioxide, dry chemical

SPECIAL FIRE FIGHTING PROCEDURES

UNUSUAL FIRE ANDEXPLOSION HAZARDS 
$A, G$ - Dibromohydrin Glycerol

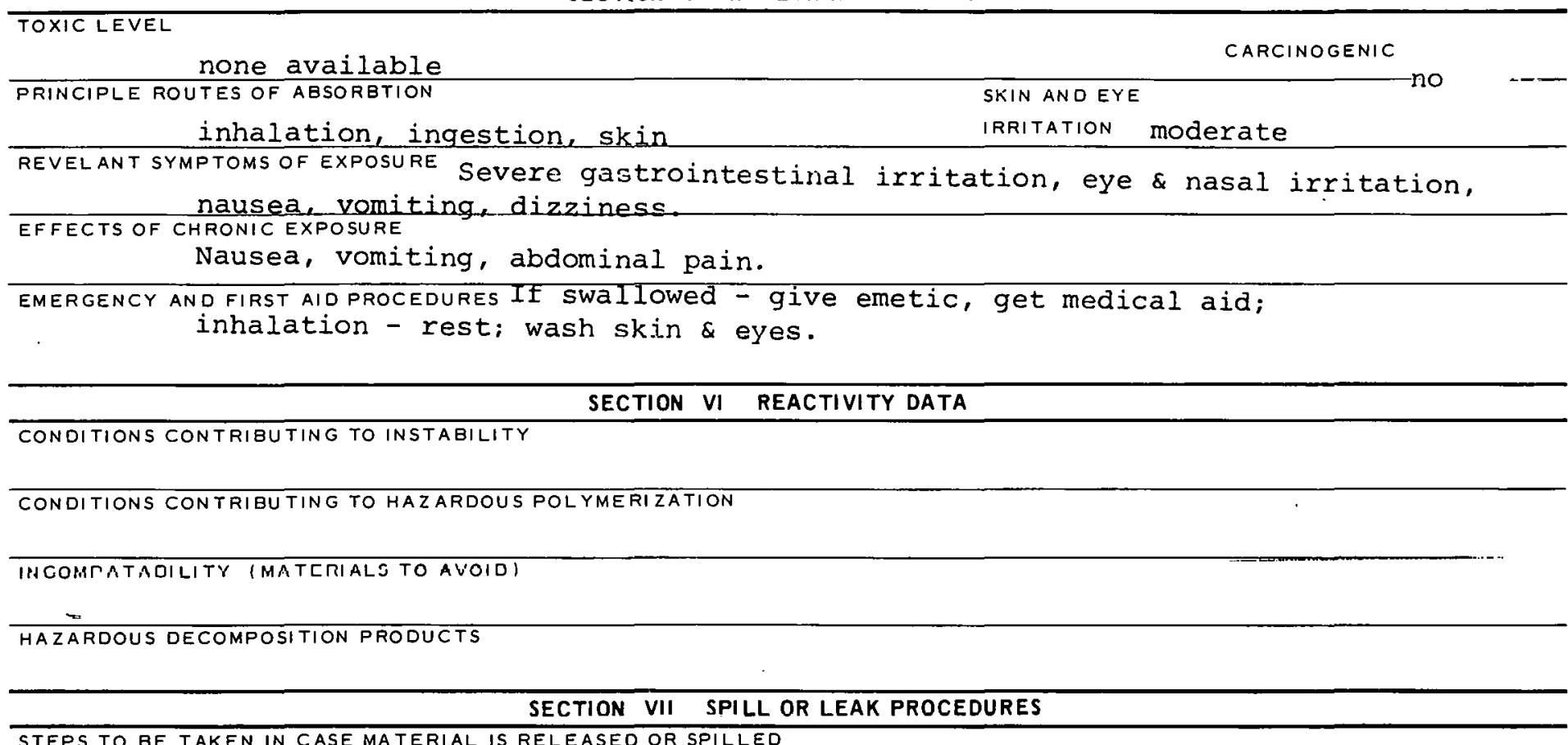

STEPS TO BE TAKEN IN CASE MATERIAL IS RELEASED OR SPILLED

WASTE DISPOSAL METHOD

SECTION VIII SPECIAL PROTECTION INFORMATION

\begin{tabular}{l|l}
\hline VENTILATION REQUIREMENTS LOCAL EXHAUST & PROTECTIVE EQUIPMENT (SPECIFY TYPES) EYE
\end{tabular} Guggles

MECHANICAL (GENERAL)

GLOVES

Rubber

SPECIAL

RESPIRATOR

OTHER PROTECTIVE EQUIPMENT

With proper filter.

\section{SECTION IX SPECIAL PRECAUTIONS}

PRECAUTIONS TO BE TAKEN IN HANDLING AND STORAGE

OTHER PRECAUTIONS

SIGNATURE

DATE

263-0606B 
ADORESS (NUMBER, STREET, CITY, STATE, ZIP CODE)

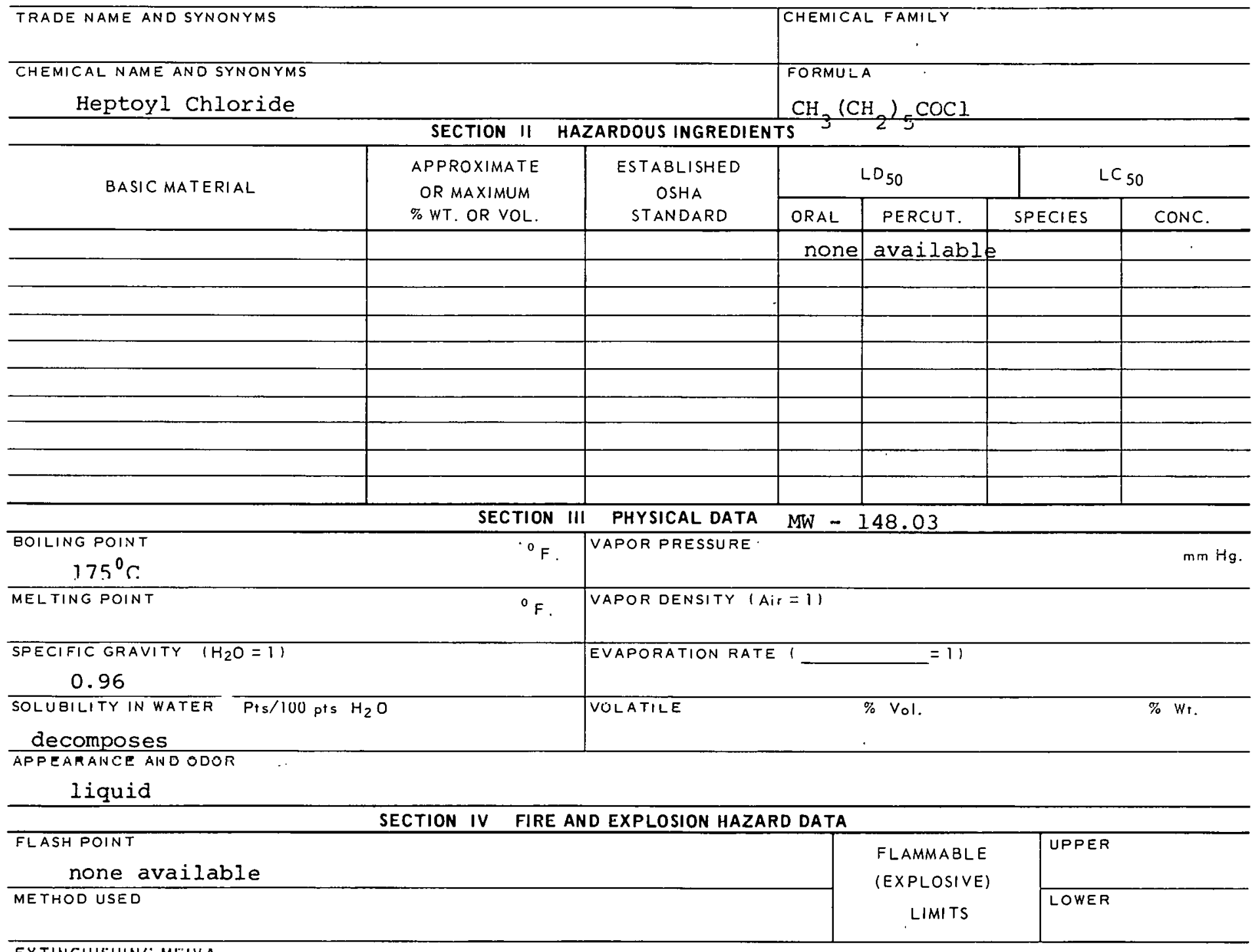

EXTINGUISHING MEUUIA

alcohol foam, carbon dioxide, dry chemical

SPECIAL FIRE FIGHTING PROCEDURES

UNUSUAL FIRE AND EXPLOSION HAZARDS

Reacts with steam or water to produce $\mathrm{HCl}$. 


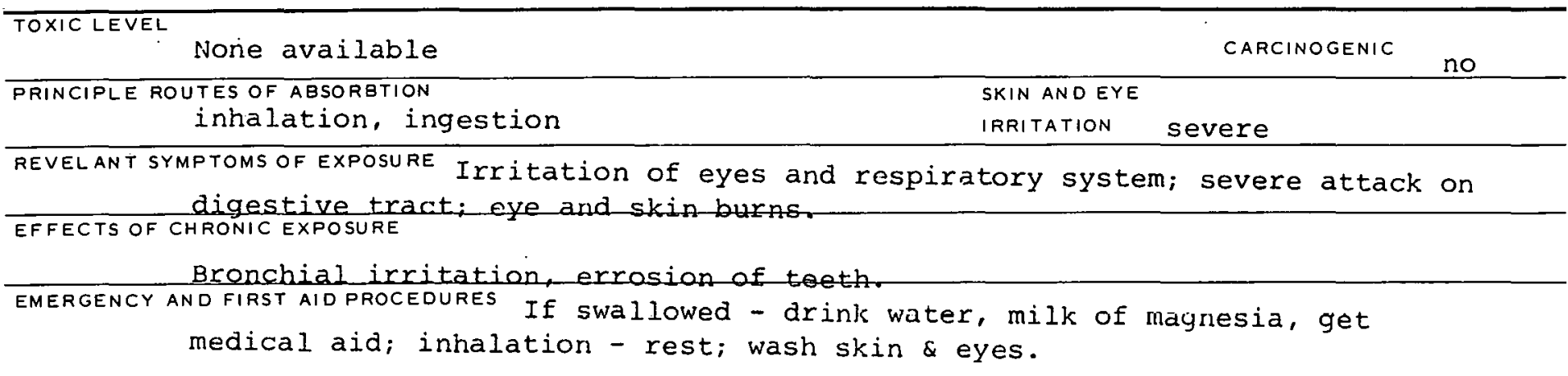

\section{SECTION VI REACTIVITY DATA}

CONDITIONS CONTRIBUTING TO INSTABILITY

CONDITIONS CONTRIBU TING TO HAZARDOUS POLYMERIZATION

IPICOMSATAOILITY (AAATEDIALSTR AVRIN)

Reacts violently with water or alcohol.

HAZARDQUS DECOMPOSITION PRODUCYS

SECTION VII SPILL OR LEAK PROCEDURES

STEPS TO EE TAKEN IN CASE MATERIAL IS RELEASED OR SPILLED

WASTE DISPOSAL METHOD

SECTION VIII SPECIAL PROTECTION INFORMATION

\begin{tabular}{|c|c|}
\hline VENTILATION REQUIREMENTS LOCAL EXHAUST & $\begin{array}{l}\text { PROTECTIVE EQUIPMENT (SPECIFY TYPES) EYE } \\
\text { Goggles }\end{array}$ \\
\hline MECHANICAL (GENERAL) & $\begin{array}{l}\text { Ruboves } \\
\text { Rubber }\end{array}$ \\
\hline SPECIAL & $\begin{array}{l}\text { RESPIRATOR } \\
\text { With proper filter }\end{array}$ \\
\hline
\end{tabular}

OTHER PROTECTIVE EQUIPMENT

rubber apron

SECTION IX SPECIAL PRECAUTIONS

PRECAUTIONS TO BE TAKEN IN HANDLING AND STORAGE

OTHER PRECAUTIONS

SIGNATURE

DATE

$267-1707 B$ 


\section{PRODUCT DESIGNATION}

03-267-2407

Hexachloroethane
MATERIAL SAFETY

DATA SHEET

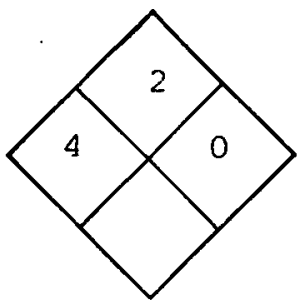

\section{SECTION I SOURCE AND NOMENCLATURE}

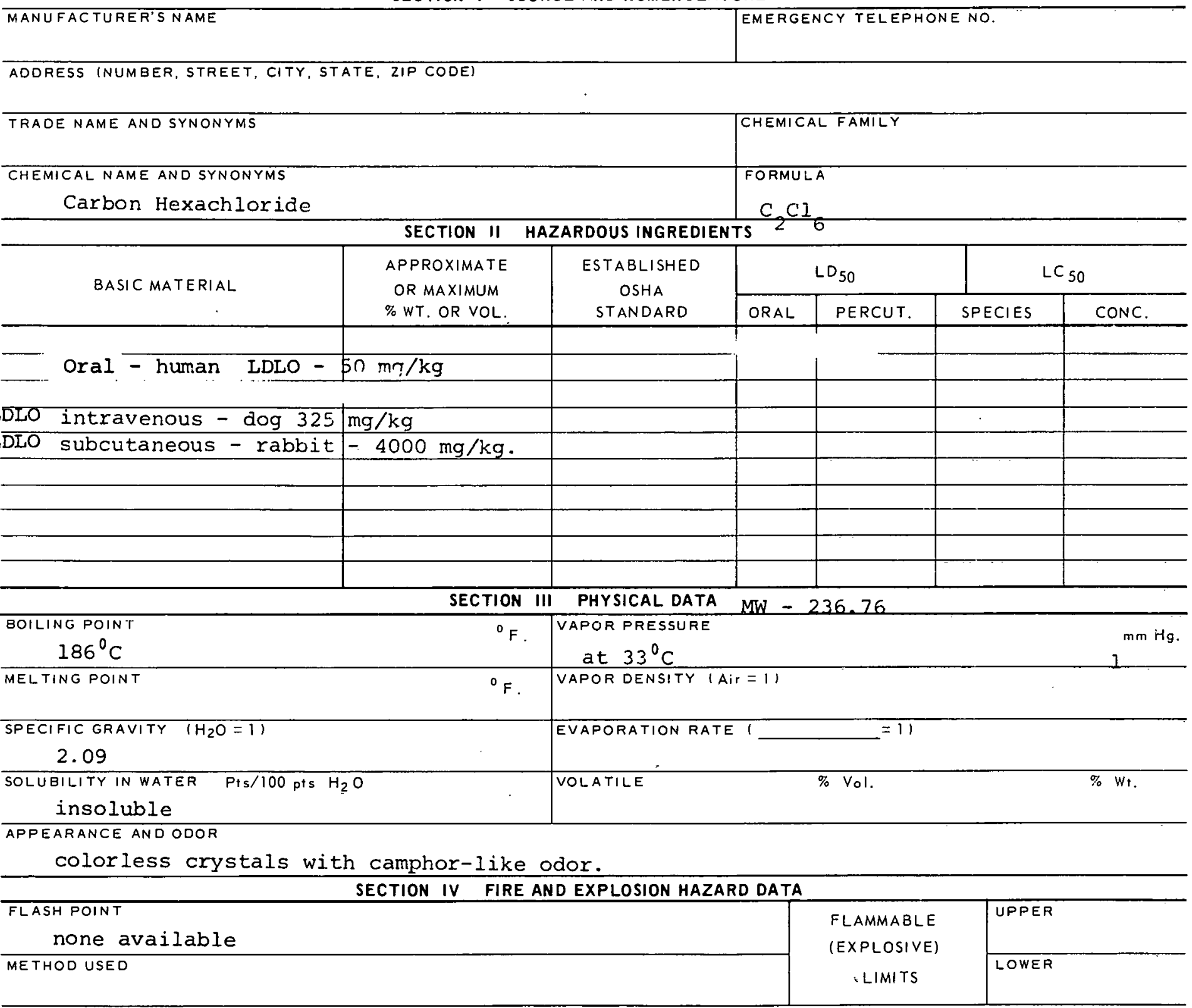

EXTINGUISHING MEDIA

Alcohol foam, carbon dioxide, dry chemical.

SPECIAL FIRE FIGHTING PROCEDURES

UNUSUAL FIRE AND EXPLOSION HAZARDS 
TOXIC LEVEL

TLV - 1 PPM, $10 \mathrm{mg} / \mathrm{C} . \mathrm{M}$. skin

CARCINOGENIC

PRINCIPLE ROUTES OF ABSORBTION
Inhalation, ingestion SKIN AND EYE

Inhalation, ingestion IRRITATION

REVELANT SYMPTOMS OF EXPOSURE

Eyes, skin, mucous membrane and lung irritation;

narcotic in high concentrations.

EFFECTS OF CHRONIC EXPOSURE

Liver injury

EMERGENCY AND FIRST AIDPROEEDURES If swallowed - give emetic, get medical aid;

inhalation - rest; wash from skin \& eyes.

\section{SECTION VI REACTIVITY DATA}

CONDITIONS CONTRIBUTING TO INSTABILITY

CONDITIONS CONTRIBUTING TO HAZARDOUS POLYMERIZATION

INR.NMPATARII.I TY IMATERIAI.S TR AVחIn)

Avoid alkalies and metals.

HAZARDOUS DECOMPOSITION PRODUCTS

SECTION VII SPILL OR LEAK PROCEDURES

STEPS TO BE TAKEN IN CASE MATERIAL IS RELEASED OR SPILLED

WASTE DISPOSAL METHOD

SECTION VIII SPECIAL PROTECTION INFORMATION

\begin{tabular}{l|l}
\hline VENTILATION REQUIREMENTS LOCAL EXHAUST & $\begin{array}{l}\text { PROTECTIVE EQUIPMENT (SPECIFY TYPES) EYE } \\
\text { gOggles }\end{array}$ \\
\hline MECHANICAL (GENERAL) & $\begin{array}{l}\text { GLOVES } \\
\text { rubber }\end{array}$ \\
\hline SPECIAL & $\begin{array}{l}\text { RESPIRATOR } \\
\text { with proper filter }\end{array}$ \\
\hline
\end{tabular}

OTHER PROTECTIVE EQUIPMENT

rubber apron

SECTION IX SPECIAL PRECAUTIONS

PRECAUTIONS TO BE TAKEN IN HANDLING AND STORAGE

OTHER PRECAUTIONS

\begin{tabular}{l|l}
\hline SIGNATURE & DATE \\
\hline
\end{tabular}


$03-267-3207$

\section{PRODUCT DESIGNATION}

03-267-3255

03-267-3257

Hexare
MATERIAL SAFETY DATA SHEET

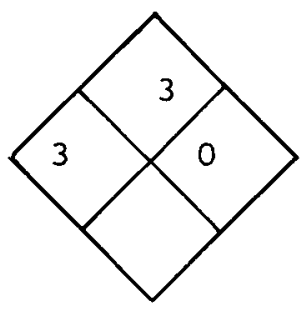

\section{SECTION I SOURCE AND NOMENCLATURE}

\begin{tabular}{l|l}
\hline MANUFACTURER'S NAME & EMERGENCY TELEPHONE NO. \\
\hline ADDRESS (NUMBER, STREET, CITY, STATE, ZIP CODE) & \\
\hline
\end{tabular}

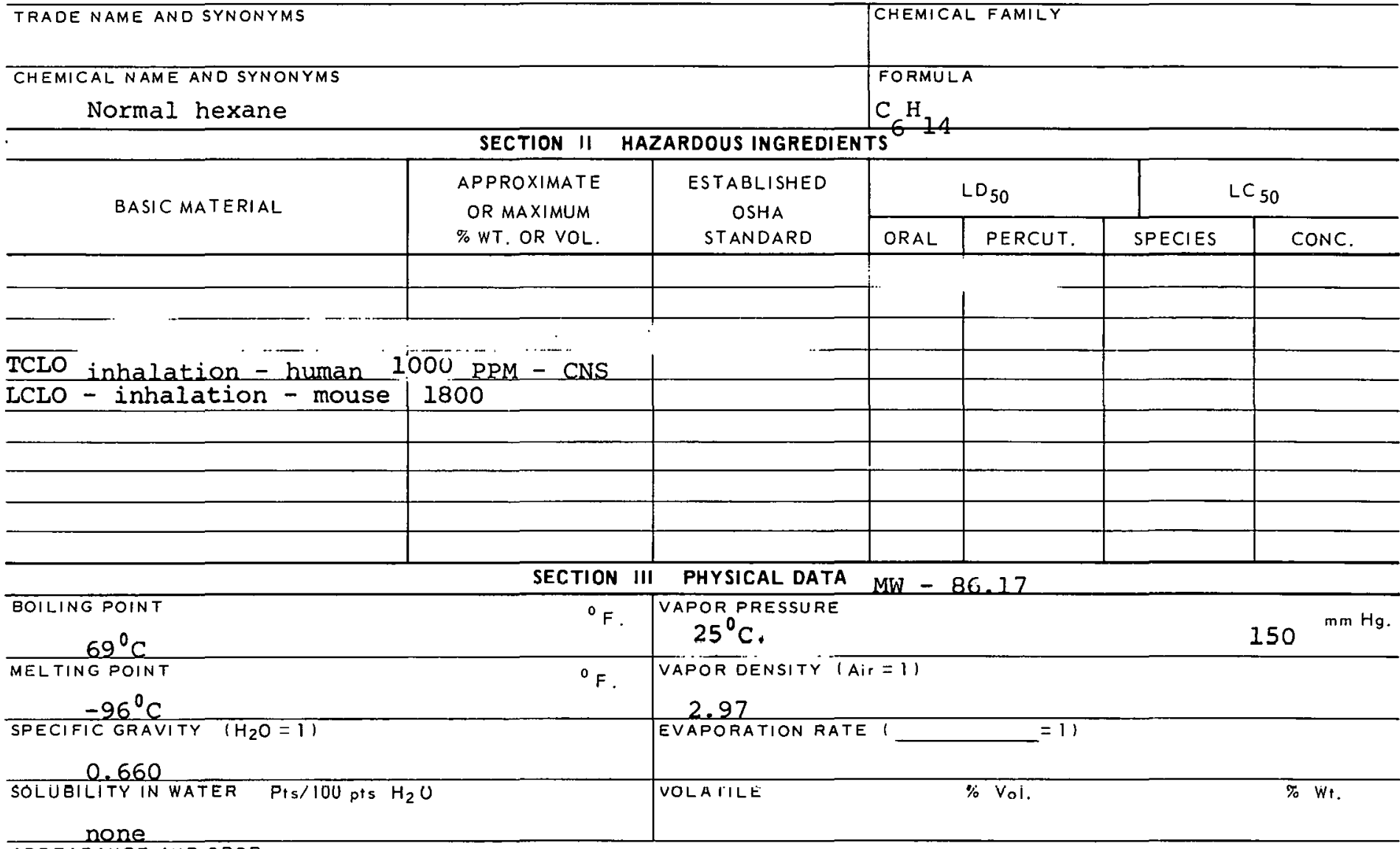

APPEARANCE AND ODOR

colorless liquid

\section{FLASH POINT}

$$
-7^{\circ} \mathrm{F}
$$

METHOO USEO

EXTINGUISHING MEDIA

\section{SECTION IV FIRE AND EXPLOSION HAZARD DATA}

\begin{tabular}{c|c} 
FLAMMABLE & UPPER \\
(EXPLOSIVE) & $7.5 \%$ \\
LIMITS & LOWER \\
& $1.1 \%$
\end{tabular}

carbon dioxide, dry chemical

SPECIAL FIRE FIGHTING PROCEDURES 
REVELANT SYMPTOMS OF EXPOSURE EFFECTS OF CHRGNIC and skin ir intation.

Dizziness, convulsions, coma, chemical pneumonia,

Dermatitis.

EMERGENCY AND FIRST AID PROCEDURES Inhalation - rest, give oxygen as needed; if swallowed get medical aid; wash from skin \& eyes.

\section{SECTION VI REACTIVITY DATA}

CONDITIONS CONTRIBUTING TO INSTABILITY

CONDITIONS CONTRIBUTING TO HAZARDOUS POLYMERIZATION

INCOMPATABILITY (MATERIALS TO AVOIO)

HAZAROQUS DECOMPOSITION PRODUCTE

SECTION VII SPILL OR LEAK PROCEDURES

STEPS TO BE TAKEN IN CASE MATERIAL IS RELEASED OR SPILLED

Absorb on paper towel, evaporate in hood.

WASTE DISPOSAL METHOD

\section{SECTION VIII SPECIAL PROTECTION INFORMATION}

\begin{tabular}{l|l}
\hline VENTILATION REQUIREMENTS LOCAL EXHAUST & $\begin{array}{c}\text { PROTECTIVE EQUIPMENT (SPECIFY TYPES) EYE } \\
\text { safetY glaSSES }\end{array}$ \\
\hline MECHANICAL IGENERAL) & $\begin{array}{c}\text { GLOVES } \\
\text { rubber }\end{array}$ \\
\hline SPECIAL & $\begin{array}{c}\text { RCJFIRATOR } \\
\text { with chemical filter }\end{array}$ \\
\hline
\end{tabular}

OTHER PROTECTIVE EQUIPMENT

Coveralls

SECTION IX SPECIAL PRECAUTIONS

PRECAUTIONS TO BE TAKEN IN HANDLING AND STORAGE

store in cool, well-ventilated area.

OTHER PRECAUTIONS 
PRODUCT DESIGNATION

03-267-5507

Hydrazine Dihydrochloride
MATERIAL SAFETY

DATA SHEET

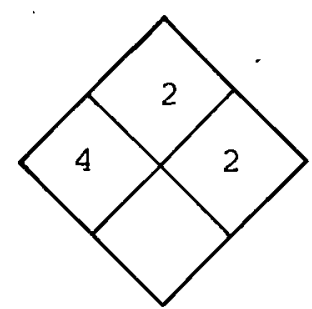

\section{SECTION I SOURCE AND NOMENCLATURE}

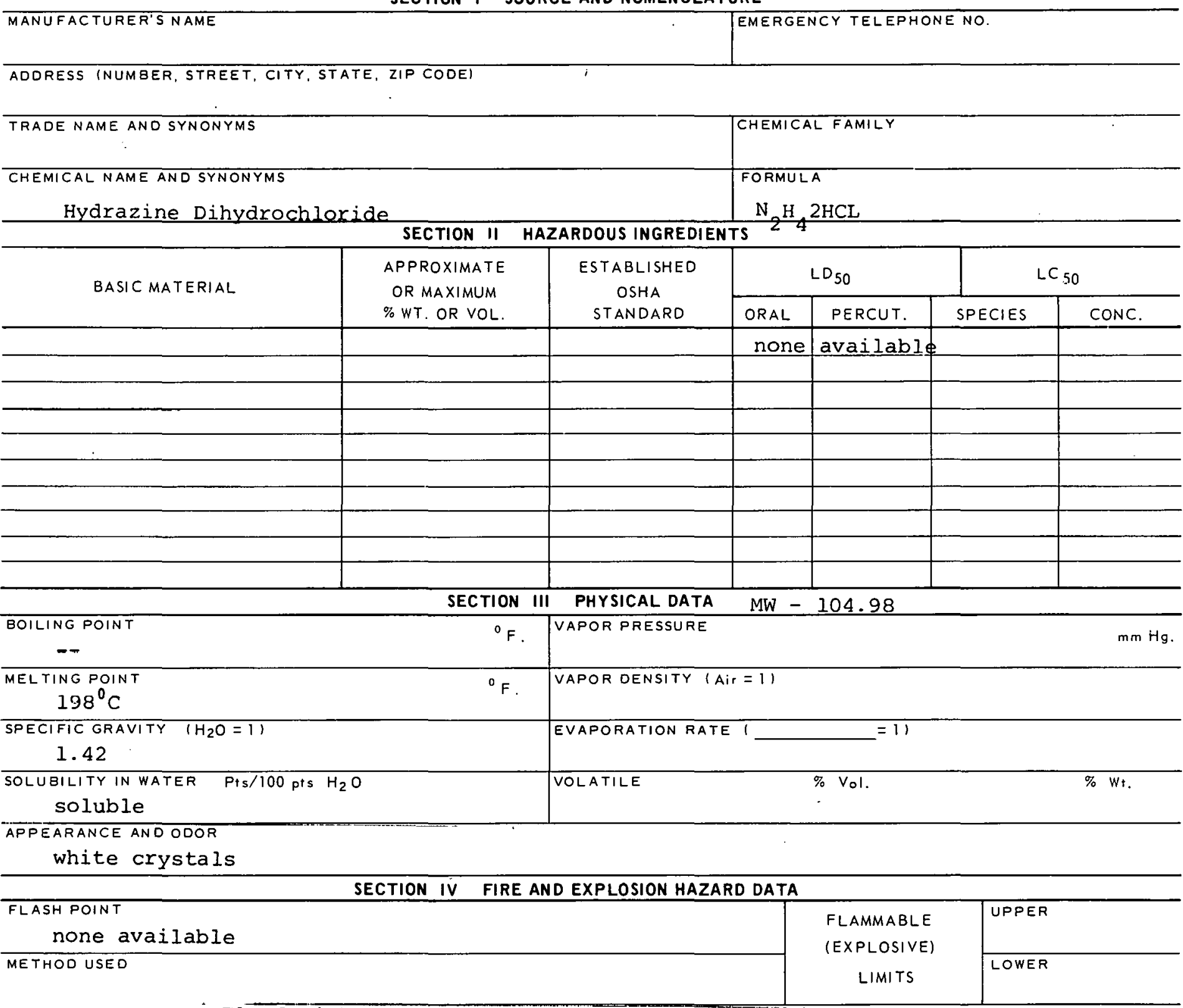

extinguishing media Flood with water; dry chemical and carbon dioxide

may be used to extinguish fire.

SPECIAL FIRE FIGHTING PROCEDURES

Wear full protective clothing.

UNUSUAL FIRE AND EXPLOSION HAZARDS 
Hydrazine Dihydrochloride

\section{SECTION $V$ HEALTH HAZARD DATA}

\section{TOXIC LEVEL}

None available.

PRINCIPLE ROUTES OF ABSORBTION

Inhalation, ingestion, skin

CARCINOGENIC no

REVELANT SYMPTOMS OF EXPOSURE Ingestion - vomiting and convulsions; inhalation -

vomiting, respiratory distress, convulsions, eye damage.

EFFECTS OF CHRONIC EXPOSURE Liver damage, hemolytic anemia and convulsive seizures, lung and kidney damage, allergic reaction.

EMERGENCY AND FIRST AID PROCEDURES If swallowed - drink water, get medical aid; inhalation -

artificial respiration, oxygen; wash skin \& eyes.

CONDITIONS CONTRIBUTING TO INSTABILITY

CONDITIONS CONTRIQUTING TO HAZARDOUS POLYMERIZATION

IACOMFATABILITY (WATERIALJ TO AVOID)

avoid oxidizing agents.

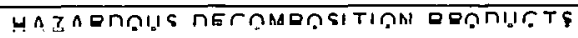

\section{SECTION VI REACTIVITY DATA}

SECTION VII SPILL OR LEAK PROCEDURES

STEPS TO BE TAKEN IN CASE MATERIAL IS RELEASED OR SPILLED

WASTE DISPOSAL METHOD

SECTION VIII SPECIAL PROTECTION INFORMATION

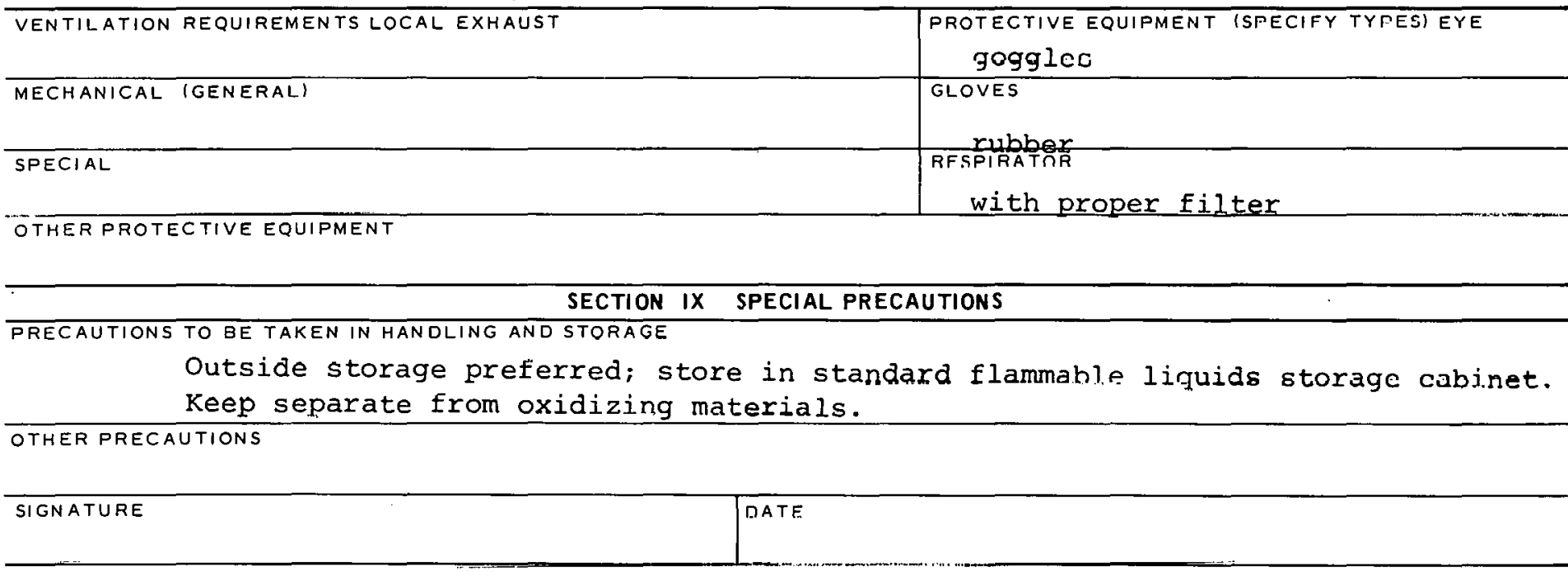




\section{PRODUCT DESIGNATION}

Hydrazine hydrate

03-267-5787
MATERIAL SAFETY

DATA SHEET

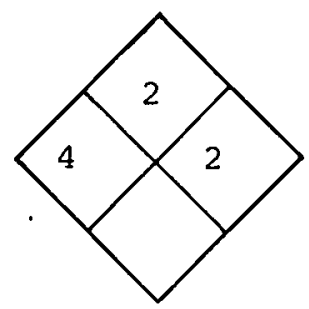

\section{SECTION I SOURCE AND NOMENCLATURE}

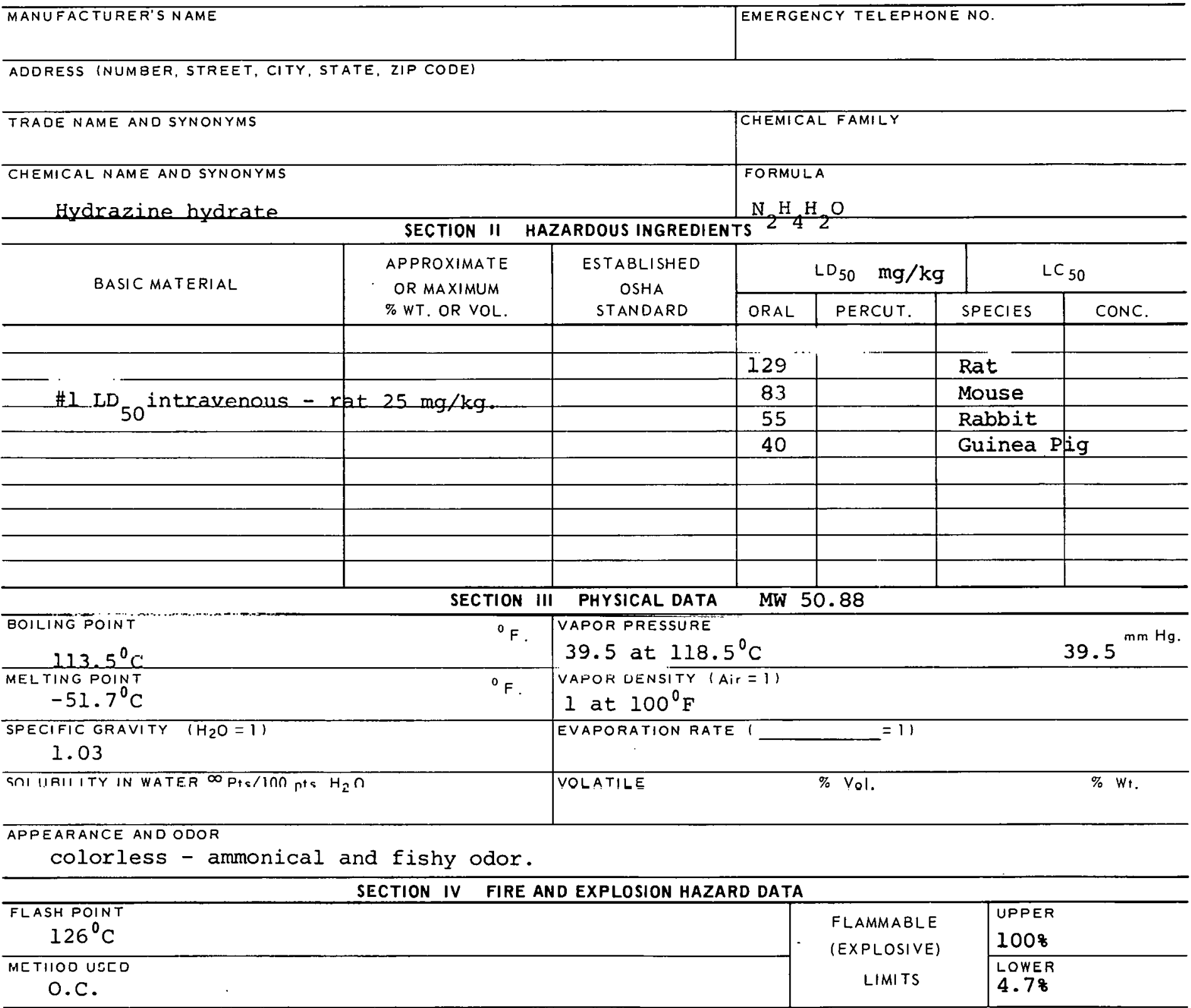

EXTINGUISHING MEDIA

Water, dry chemicals and $\mathrm{CO}_{2}$, alcohol foam -

SPECIAL FIRE FIGHTING PROCEDURES

Water spray to disperse vapors. Wear full protective clothing.

UNUSUAL FIRE AND EXPLOSION HAZAROS

Spontaneous ignition with oxidants such as nitric acid and hydrogen peroxide. 
TOXIC LEVEL

TLV - I PPM, $1.3 \mathrm{mg} / \mathrm{C.M}$.

CARCINOGENIC yes

PRINCIPLE ROU'TES UF ABSORBIION

Ingestion, inhalation, skin absorption SKIN AND EYE

IRRITATION Severe on liquid contact REVELANT SYMPTOMS OF EXPOSURE Inhalation - vomiting, respiratory distress and convulsions;

direct skin contact - burns; ingestion - vomiting and convulsions, eye damage. EFFECTS OF CHRONIC EXPOSURE Lung and kidney damage, allergic reaction,

Liver damage, hemolytic anemia and convulsive seizures.

EMERGENCY AND FIRST AID PROCEDURES

\section{SECTION VI REACTIVITY DATA}

CONDITIONS CONTRIBUTING TO INSTABILITY

Heat and strong oxidizing agents.

CONDITIONS CONTRIBUTING TO HAZARDOUS POLYMERIZATION

INCOMPATABILITY (MATERIALS TO AVOID)

Spontaneous ignition with strong oxidants such as nitric acid or $\mathrm{H}_{2} \mathrm{O}_{2}$.

HAIARDOUS DECOMPOSITION PRODUCTS

SECTION VII SPILL OR LEAK PROCEDURES

STEPS TO BE TAKEN IN CASE MATERIAL IS RELEASED OR SPILLED

Use water spray to disperse vapors.

WASTE DISPOSAL METHOD

Dilute to non-inflammable mixture.

SECTION VIII SPECIAL PROTECTION INFORMATION

\begin{tabular}{l|l}
\hline VENTILATION REQUIREMENTS LOCAL EXHAUST. & PROTECTIVE EQUIPMENT (SPECIFY TYPES) EYE
\end{tabular}

Use in hood

MECHANICAL (GENERAL)

SPECISL

OTHER PROTECTIVE EQUIPMENT

Full protective clothing recommended.

\section{SECTION IX SPECIAL PRECAUTIONS}

PRECAUTIONS TO BE TAKEN IN HANDLING AND STORAGE

Maintain under nitrogen atmosphere.

OTHER PRECAUTIONS

Keep away from strong oxidizing agents.

SIGN ATURE

DATE

267-5787B 


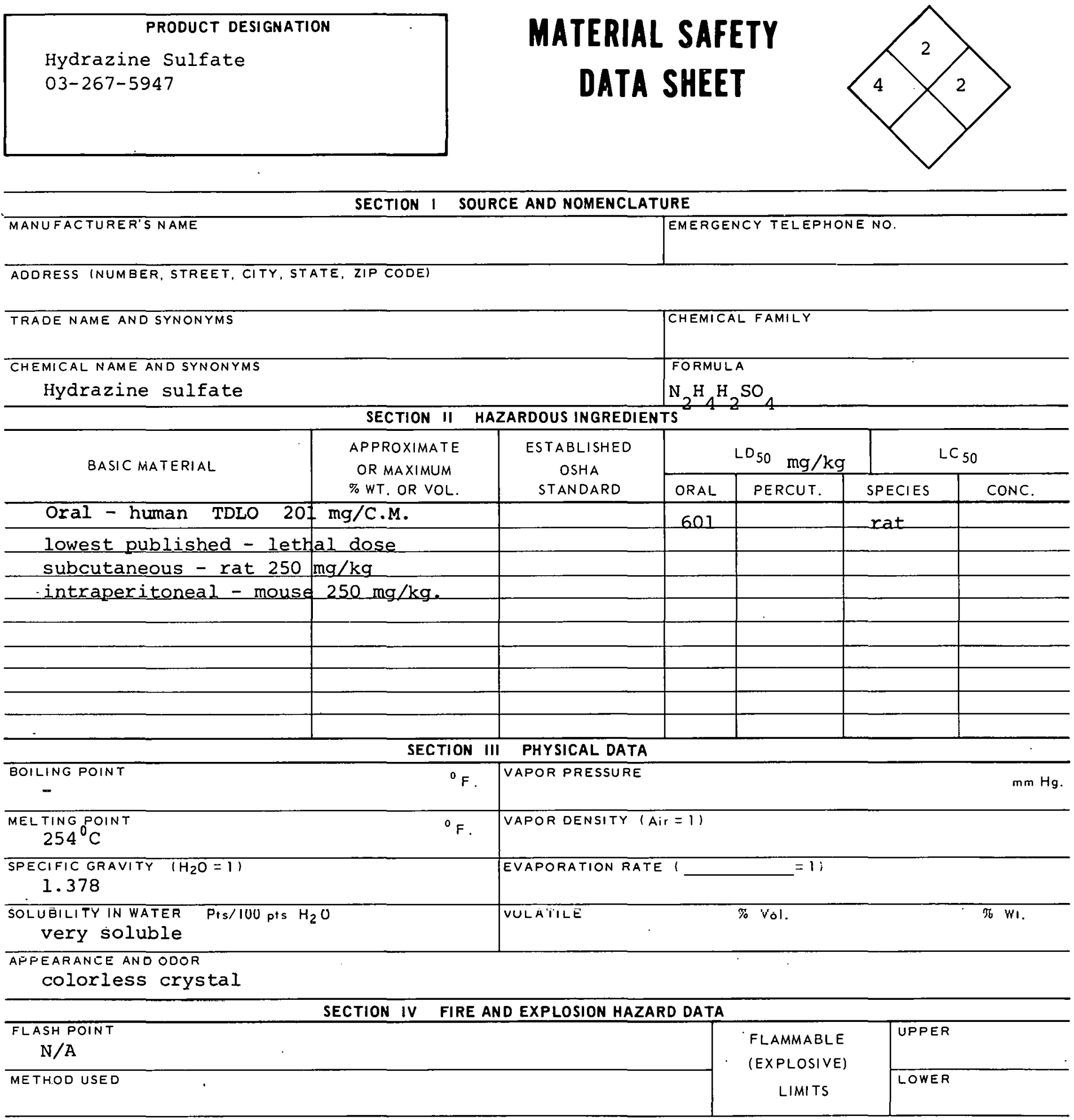

EXTINGUISHING MEDIA

Water, dry chemical and $\mathrm{CO}_{2}$

SPECIAL HIRE FIGHTING PROCEOURES water spray to disperse hydrazine vapor - wear full protective clothing.

UNUSUAL FIRE AND EXPLOSION HAZARDS

May react vigorously with strong oxidizing agents. 


\section{PRODUCT DESIGNATION \\ Hydrazine sulfate}

SECTION $V$ HEALTH HAZARD DATA

TOXIC LEVEL

none available

PRINCIPLE ROUTES OF ABSORETION

ingestion, skin

REVELANT SYMPTOMS OF EXPOSURE

urns - from dixect skin contact.

Ingestion - vomiting and convulsions, eye damage, respiratory distress. EFFECTS OF CHRONIC EXPOSURE Lung and kidney damage, allergic reaction

Liver damage, hemolytic, anemia and convulsive seizures.

EMERGENCY AND FIRST AID PROCEDURES Skin contact - drench with water; if swallowed - wash mouth, give water to drink, get medical aid; inhalation - oxygen or artificial respiration as necessary.

\section{SECTION VI REACTIVITY DATA}

CONDITIONS CONTRIBUTING TO INSTABILITY

Heat and strong oxidizing agents.

CONDITIONS CONTRIBUTING TO HAZARDOUS POLYMERIZATION

INCOMPATABILITY IMATFRIAISTR AVOIII!

Reacts vigorously with strong oxidizing agents. HAZARDOUS DECOMPOSITION PRODUCTS

SECTION VII SPILL OR LEAK PROCEDURES

STEPS TO BE TAKEN IN CASE MATERIAL. IS RELEASED OR SPILLED

flush with water

WASTE DISPOSAL METHOD

SECTION VIII SPECIAL PROTECTION INFORMATION

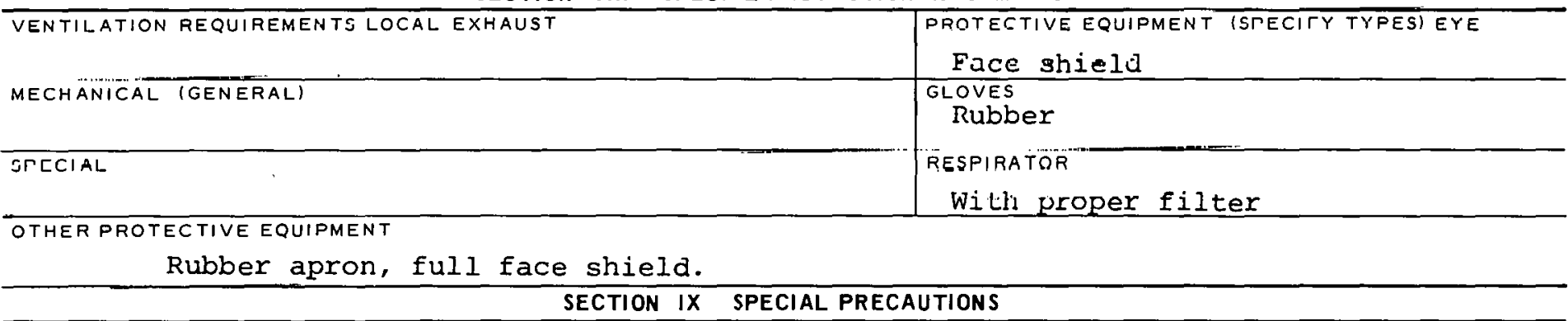

PRECAUTIONS TO BE TAKEN IN HANDLING AND STORAGE

OTHER PRECAUTIONS

Keep away from strong oxidizing agents

SIGNATURE

DATE

$267-5947 B$ 


\section{PRODUCT DESIGNATION}

$03-267-6737$

Hydroquinone
MATERIAL SAFETY

DATA SHEET

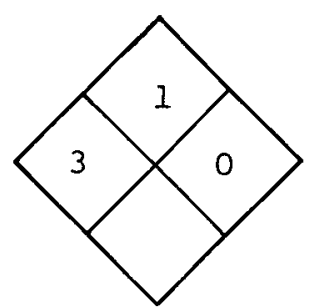

SECTION I SOURCE AND NOMENCLATURE

AODRESS (NUMBER, STREET, CITY, STATE, ZIP CODE)

\section{TRADE NAME AND SYNONYMS \\ P dihydroxy benzene \\ CHEMICAL NAME AND SYNONYMS \\ 1, 4 Benzene diol}

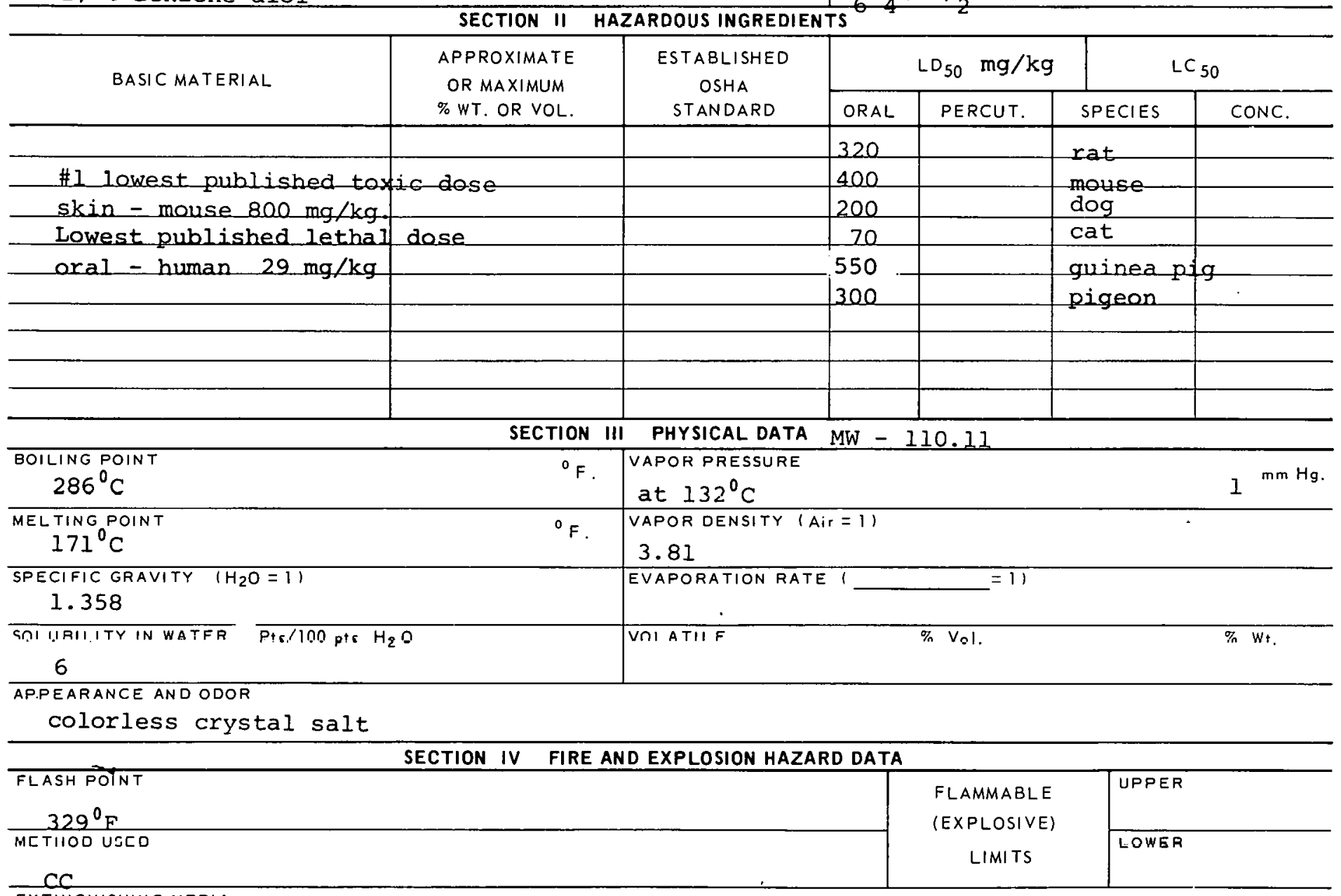

EXTINGUISHING MEDIA

water, carbon dioxide, ary chemical

SPECIAL FIRE FIGHTING PROCEOURES

UNUSUAL FIRE AND EXPLOSION HAZARDS 


\section{TOXIC LEVEL}

TLV $2 \mathrm{mg} / \mathrm{C} . \mathrm{M}$.

PRINCIPLE ROUTES OF ABSORGTION

Ingestion, skin, inhalation of dust

SKIN AND EYE

IRRITATION SEvere

REVELANT SYMPTOMS OF EXPosuRE Nausea, dizziness, increased respiration, headache, cyanosis, delirium and collapse, depigmentation of eyes and skin.

EFFECTS OF CHRONIC EXPOSURE

Eye damage, dermatitis.

EMERGENCY AND FIRST AID PROCEDURES Inhalation - rest; if swallowed - induce vomiting, gastric

lavage, medical aid; wash from eyes and skin.

\section{SECTION VI REACTIVITY DATA}

CONOITIONS CONTRIBUTING TO INSTABILITY

CONOITIONS CONTRIBUTING TO HAZARDOUS POLYMERIZATION

INCOMPATABILITY (MATERIALS TO AVOID)

IIAZARDOUE DEGOMROEITIOH RRODUGTE

SECTION VII SPILL OR LEAK PROCEDURES

STEPS TO GE TAKEN IN CASE MATERIAL IS RELEASED OR SPILLED

Brush onto paper, burn paper on iron pan in hood.

WASTE DISPOSAL METHOD

\section{SECTION VIII SPECIAL PROTECTION INFORMATION}

\begin{tabular}{|c|c|}
\hline VENTILATION REQUIREMENTS LOCAL EXHAUST & $\begin{array}{l}\text { PROTECTIVE EQUIPMENT (SPECIFY TYPES) EYE } \\
\text { safety goggles }\end{array}$ \\
\hline MECHANICAL (GENERAL) & $\begin{array}{l}\text { GLOVES } \\
\text { rubber }\end{array}$ \\
\hline SPECIAL & 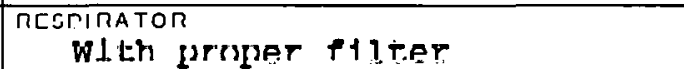 \\
\hline
\end{tabular}

OTHER PROTECTIVE EQỤIPMENT

\section{SECTION IX SPECIAL PRECAUTIONS}

PRECAUTIONS TO BE TAKEN IN HANDLING AND STORAGE

Keep tightly closed, store in place away from sunlight. 


\section{PRODUCT DESIGNATION}

03-267-8207

2 Hydroxy 1,4 - Dimethylbenzene
MATERIAL SAFETY

DATA SHEET

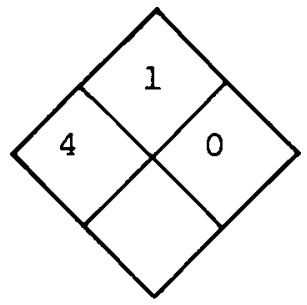

SECTION I SOURCE AND NOMENCLATURE

\begin{tabular}{l|l}
\hline MANUFACTURER'S NAME & EMERGENCY TELEPHONE NO. \\
\hline ADDRESS (NUMOER, STREET, CITY, STATE, ZIP CODE) & \\
\hline
\end{tabular}

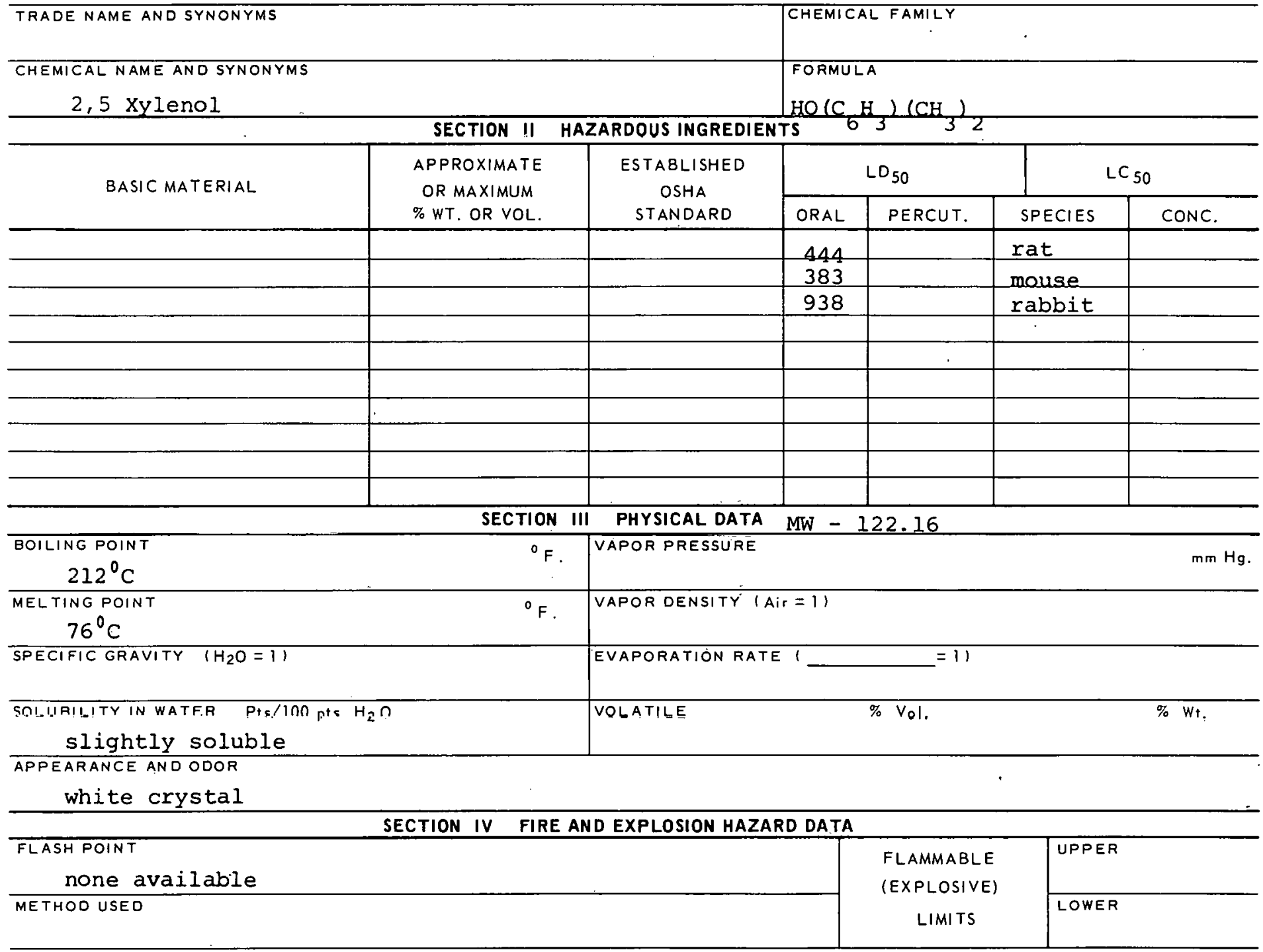

EXTINGUISHING MEDIA

Foam, carbon dioxide, dry chemical

SPECIAL FIRE FIGHTING PROCEDURES

Wear self-contained breathing apparatus.

UNUSUAL FIRE AND EXPLOSION HAZAROS 
2 Hydroxy 1,4 Dimethylbenzene

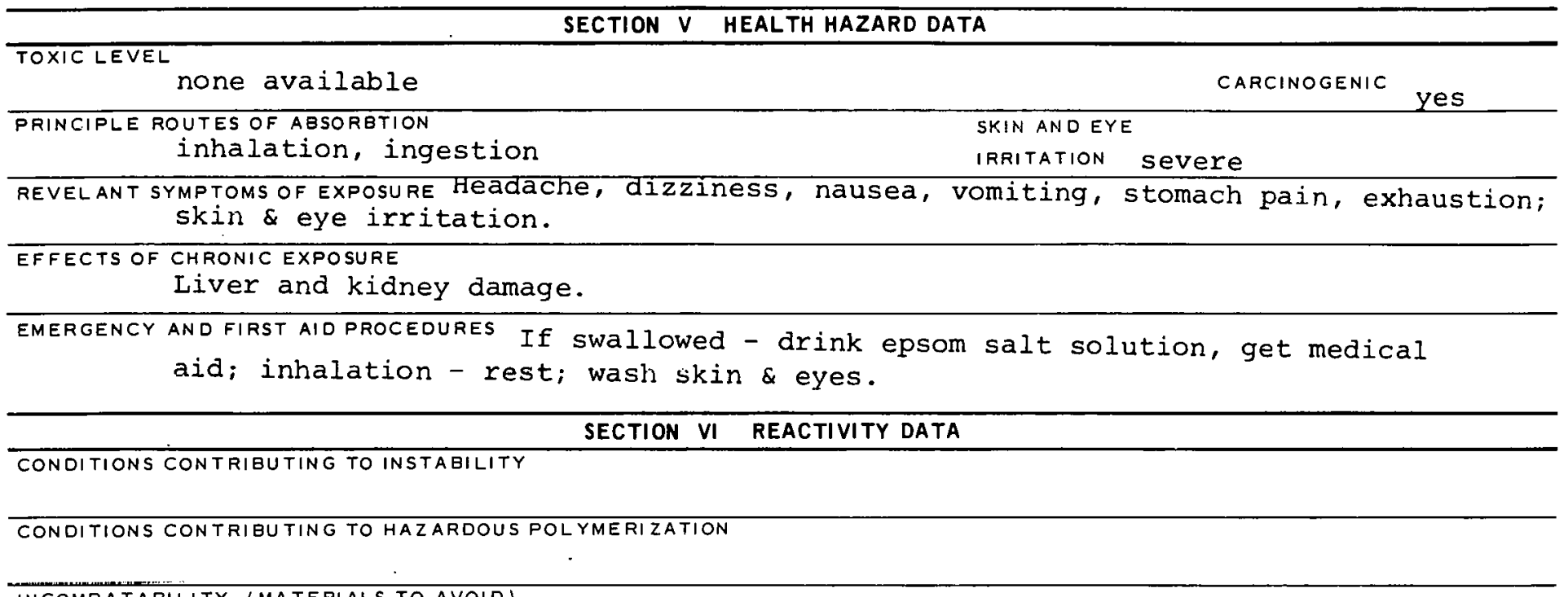

INCIOMPATARI I.I TY (MATERIAI,.S TO AVRIN)

HAZARDOUS DECOMPOSITION PRODUCTS

SECTION VII SPILL OR LEAK PROCEDURES

STEPS TO BE TAKEN IN CASE MA TERIAL IS RELEASED OR SPILLED

WASTE DISPOSAL METHOD

\section{SECTION VIII SPECIAL PROTECTION INFORMATION}

\begin{tabular}{|c|c|}
\hline VENTILATION REQUIREMENTS LOCAL EXHAUST & $\begin{array}{l}\text { PROTECTIVE EQUIPMENT (SPECIFY TYPES) EYE } \\
\text { goggles }\end{array}$ \\
\hline MECHANICAL (GENERAL) & $\begin{array}{l}\text { GLOVES } \\
\text { rubber }\end{array}$ \\
\hline SPECIAL & $\begin{array}{l}\text { RESPIRATOR } \\
\text { seTf-ront.. ined }\end{array}$ \\
\hline
\end{tabular}

OTHER PROTECTIVE EQUIPMENT

\section{SECTION IX SPECIAL PRECAUTIONS}

PRECAUTIONS TO BE TAKEN IN HANDLING AND STORAGE

UTHER RRECAUTIONS

SIGNATURE

DATE 
CAS $: 000095658$

\begin{tabular}{|c|}
\hline PRODUCT DESIGNATION \\
$43-2.67-8307$ \\
4 Hydroxy - 1,2 - Dimethyl benzene
\end{tabular}

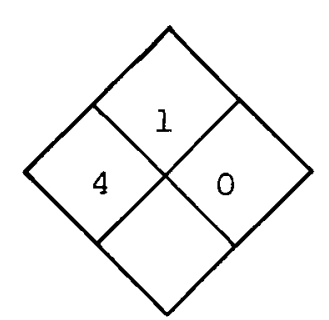

\section{SECTION I SOURCE AND NOMENCLATURE}

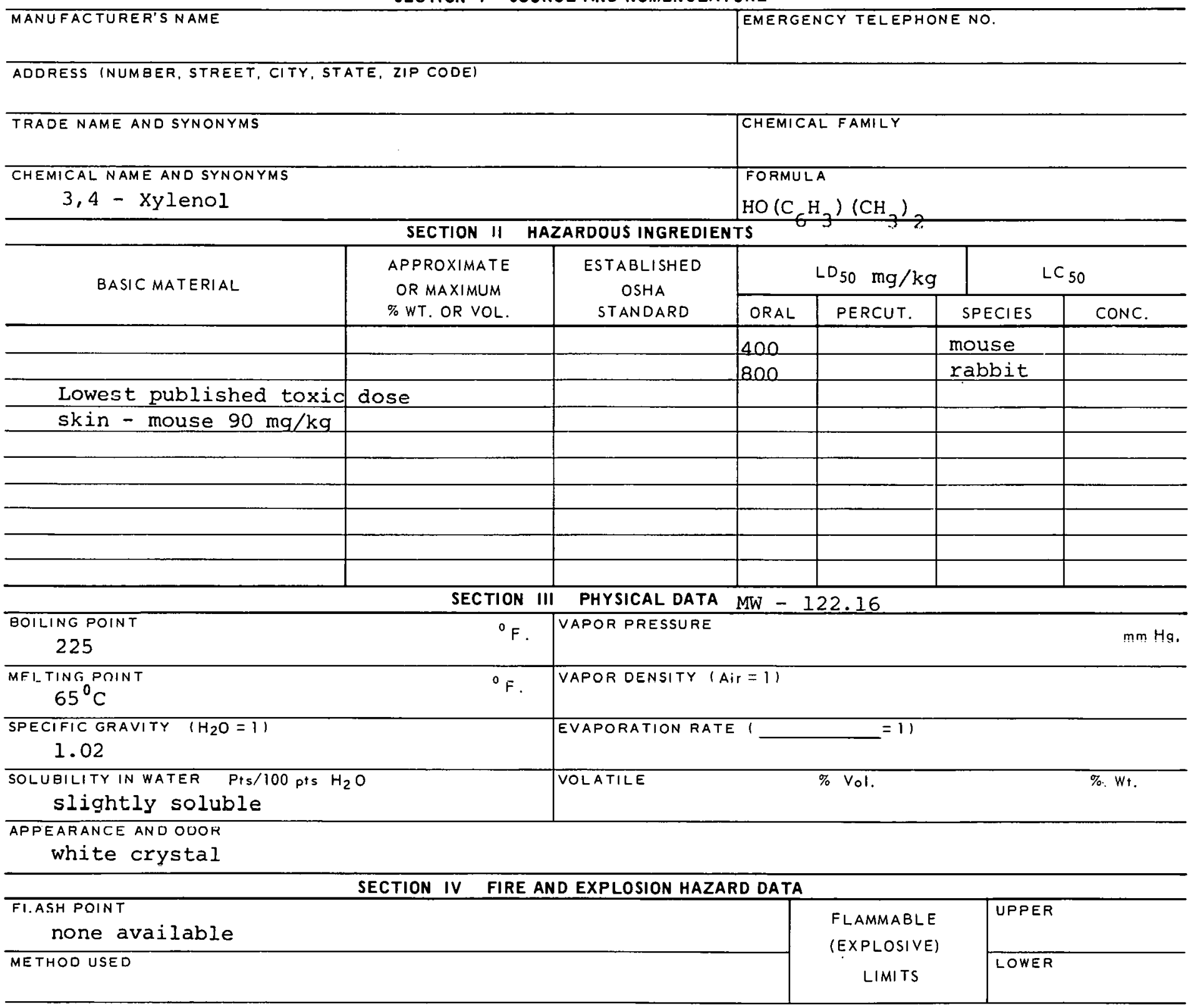

EXTINGUISHING MEDIA

foam. carbon dioxide, dry chemiral

SPECIAL FIRE FIGHTING PROCEDURES

wear self-contained breathing apparatus. 
4 Hydrcxy - 1,2 - Dime thylbenzene

SECTION $V$ HEALTH HAZARD DATA

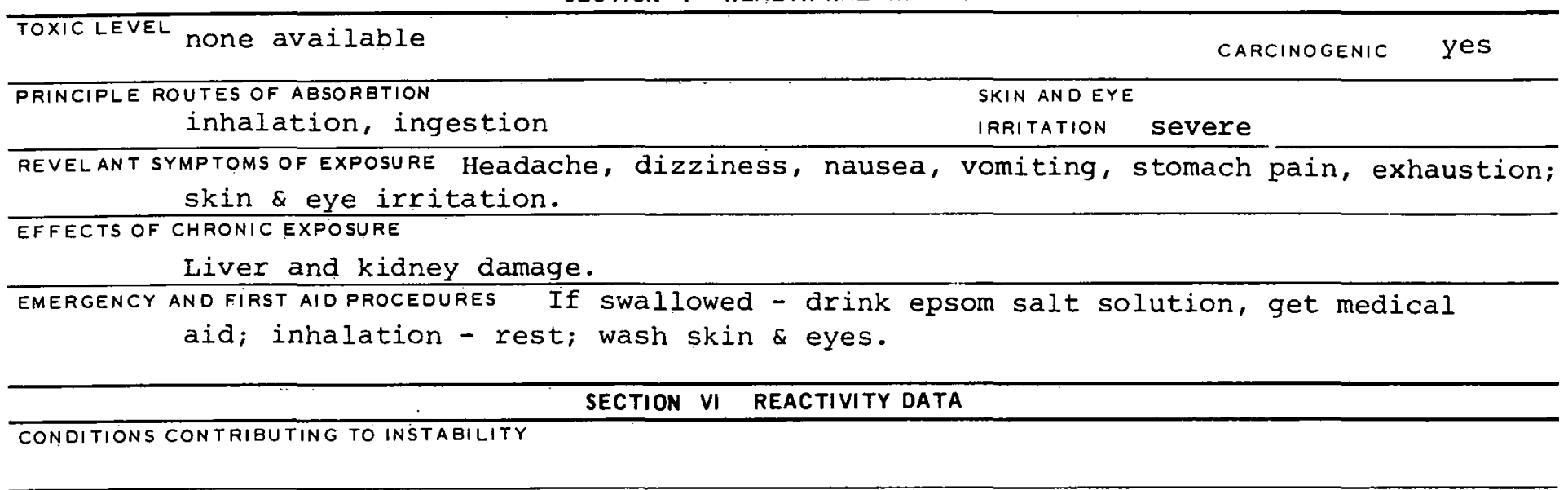

CONDITIONS CONTRIBUTING TO HAZARDOUS POLYMERIZATION

INCOMPATABILITY (MATERIALS TO AVOID)

HAZARDOUS DECOMPOSITION PRODUCTS

SECTION VII SPILL OR LEAK PROCEDURES

STEPS TO BE TAKEN IN CASE MATERIAL IS RELEASED OR SPILLED

\begin{tabular}{|c|c|}
\hline SECTION VIII & SPECIAL PROTECTION INFORMATION \\
\hline VENTILATION REQUIREMENTS LOCAL EXHAUST & \begin{tabular}{|l|l} 
& $\begin{array}{l}\text { PROTECTIVE EQUIPMENT (SPECIFY TYPES) EYE } \\
\text { goggles }\end{array}$ \\
\end{tabular} \\
\hline MECHANICAL (GENERAL) & $\begin{array}{l}\text { GLOVES } \\
\text { rubber }\end{array}$ \\
\hline SFCCIAL & $\begin{array}{l}\text { RCERIRATOR } \\
\text { Self-conläined }\end{array}$ \\
\hline \multicolumn{2}{|l|}{ OTHER PROTECTIVE EQUIPMENT } \\
\hline \multicolumn{2}{|c|}{$\begin{array}{l}\text { SECTION IX SPECIAL PRECAUTIONS } \\
\end{array}$} \\
\hline \multicolumn{2}{|c|}{ PRECAUTIONS TO BE TAKEN IN HANDLING AND STORAGE } \\
\hline \multicolumn{2}{|l|}{ OTHER PRECAUTIONS } \\
\hline SIGNATURE & DATE \\
\hline
\end{tabular}




\section{PRODUCT DESIGNATION}

03-267-8407

4 Hydroxy - 1,3 - Dimethy]benzene
MATERIAL SAFETY

DATA SHEET

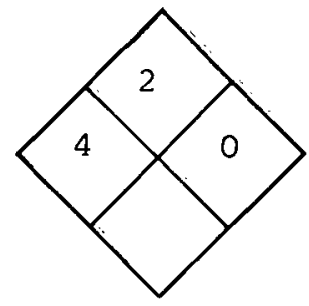

\section{SECTION I SOURCE AND NOMENCLATURE}

\section{MANUFACTURER'SNAME \\ TRADE NAME AND SYNONYMS \\ CHEMICAL NAME AND SYNONYMS \\ 2,4 - XYlenol}

ADDRESS INUMBER, STREET, CITY, STATE, ZIP CODE
BASIC MATERIAL
SECTION II APPROXIMATE

OR MAXIMUM

\% WT. OR VOL.
HAZARDOUS INGREDIENTS

$\left.\mathrm{HO}\left(\mathrm{C}_{6} \mathrm{H}_{3}\right) \mathrm{CH}_{3}\right)_{2}$

\begin{tabular}{c}
$\begin{array}{c}\text { ESTABLISHED } \\
\text { OSHA } \\
\text { STANDARD }\end{array}$ \\
\hline \\
\hline \\
\hline \\
\hline \\
\hline
\end{tabular}

SECTION III

PHYSICAL DATA

\begin{tabular}{|l|c|l|l|l|}
\hline \multicolumn{2}{|c|}{ LD $_{50} \mathrm{mg} / \mathrm{kg}$} & \multicolumn{2}{c}{ LC $_{50}$} \\
\hline ORAL & PERCUT. & SPECIES & CONC. \\
\hline
\end{tabular}

BOILING POINT

$$
21.2^{\circ} \mathrm{C}
$$

MELTING POINT $26^{\circ} \mathrm{C}$

SPECIFIC GRAVITY $\left(\mathrm{H}_{2} \mathrm{O}=1\right)$

1.04

SOLUDILITY IPI WATER $\mathrm{P}+\mathrm{g}, 100 \mathrm{pts} \mathrm{H}_{2} \mathrm{O}$ slightly soluble

APPEARANCE AND ODOR yellow-brown liquid
EMERGENCY TELEPHONE NO. 


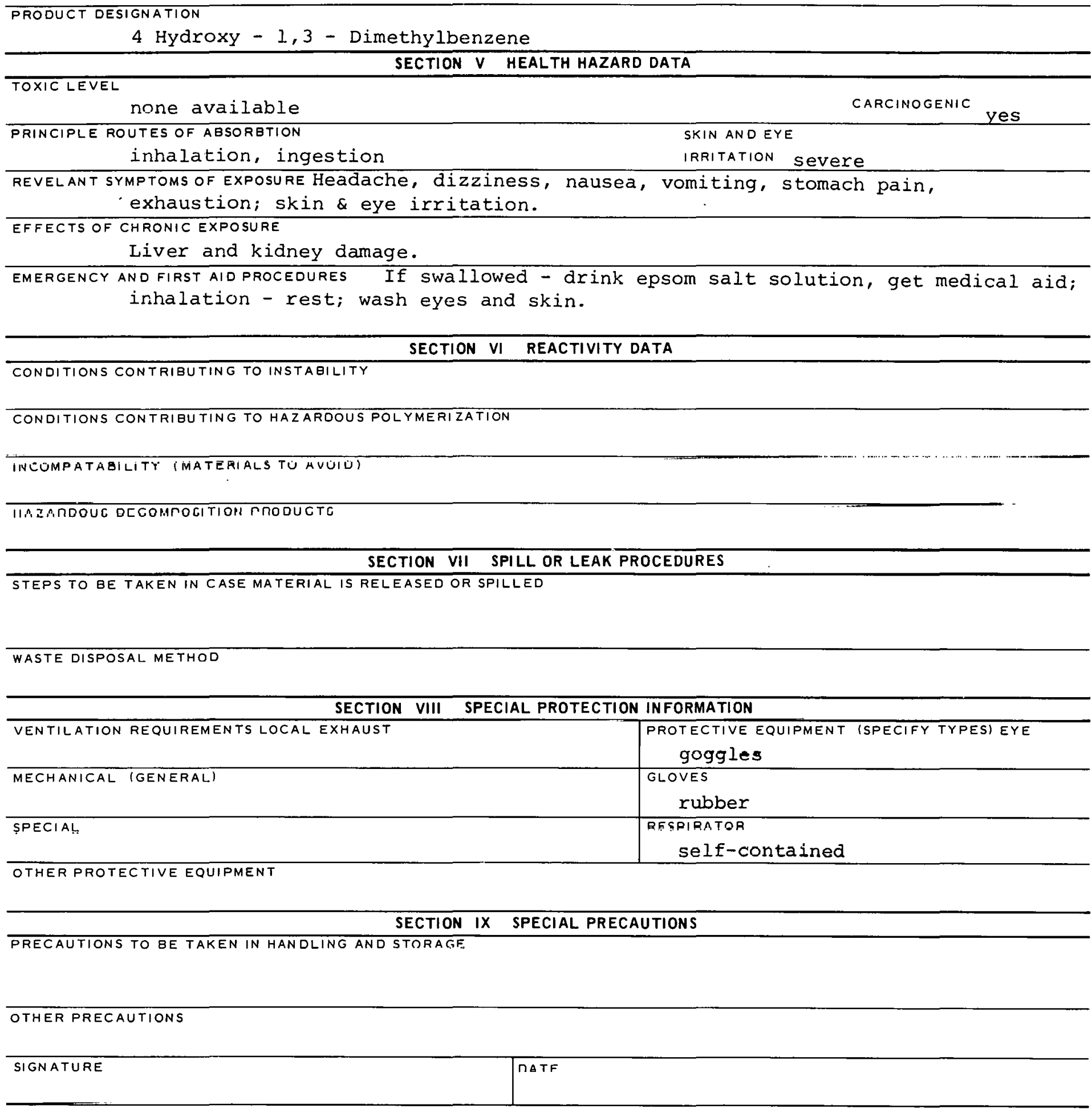




\begin{tabular}{|c|} 
PRODUCT DESIGNATION \\
$03-267-8647$ \\
$0-$ HydroxydiphenYl
\end{tabular}

\section{MATERIAL SAFETY DATA SHEET}

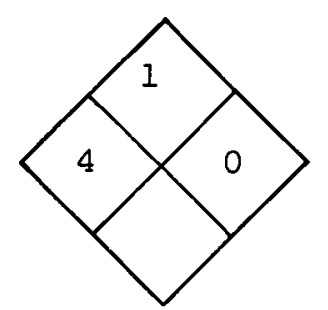

\section{SECTION I SOURCE AND NOMENCLATURE}

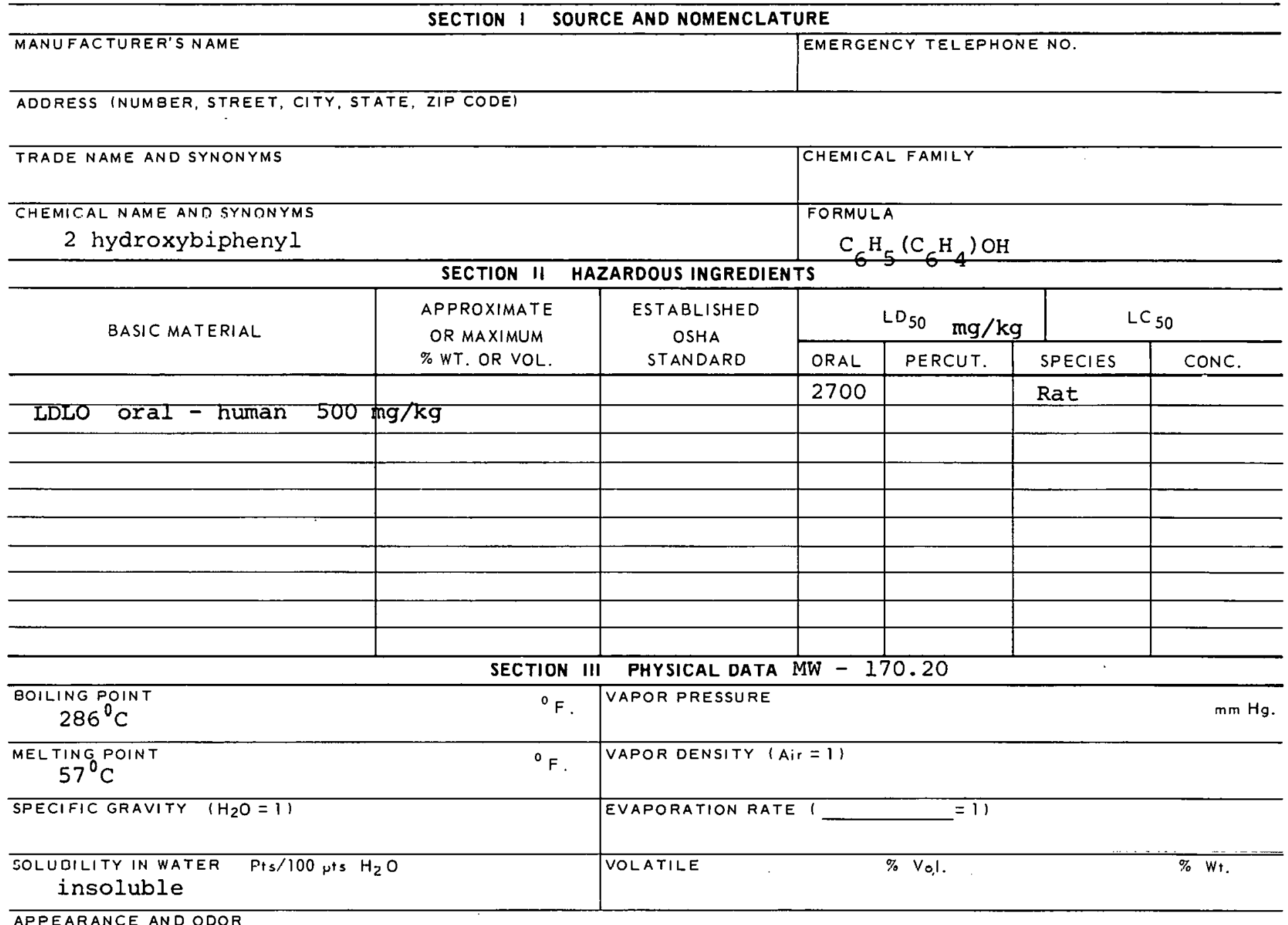

APPEARANCE AND ODOR

white solid

\begin{tabular}{l|c|c}
\hline \multicolumn{2}{c}{ SECTION IV FIRE AND EXPLOSION HAZARD DATA } \\
\hline $\begin{array}{l}\text { FLASH POINT } \\
\text { nONe available }\end{array}$ & $\begin{array}{c}\text { FLAMMABLE } \\
\text { (EXPLOSIVE) }\end{array}$ \\
\hline METHOD USED & LIMITS & LOWER \\
\hline
\end{tabular}

EXTINGUISHING MEDIA

carbon dioxide or dry chemical

SPECIAL FIRE FIGHTING PROCEDURES

water may cause frothing.

UNUSUAL FIRE AND FXPILOSION HAZARDS 


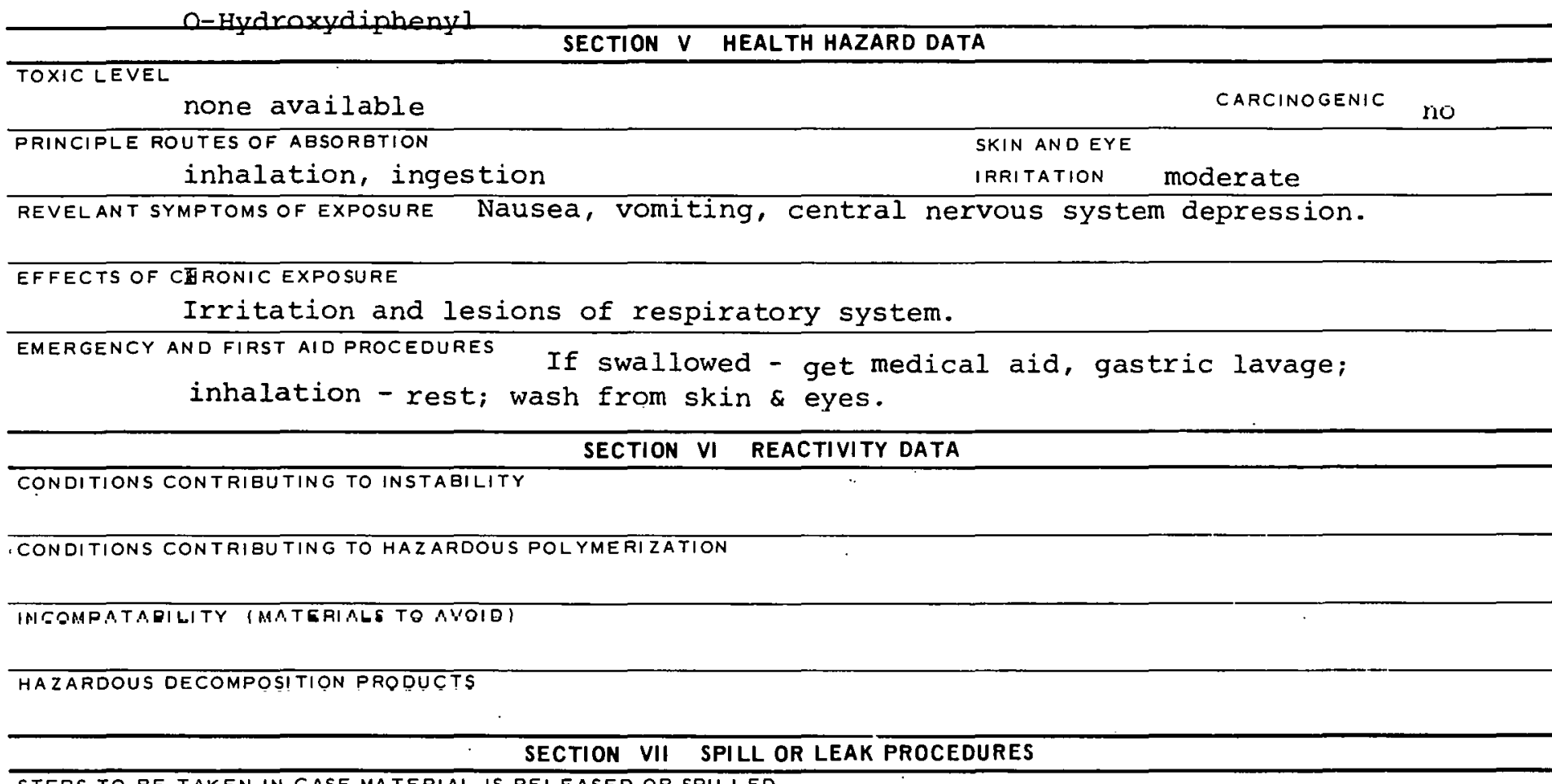

STEPS TO BE TAKEN IN CASE MATERIAL IS RELEASED OR SPILLEO

WASTE DISPOSAL METHOD

SECTION VIII SPECIAL PROTECTION INFORMATION

\begin{tabular}{l|l|l}
\hline VENTILATION REQUIREMENTS LOCAL EXHAUST & PROTFC.TIVE EQUIPMENT ISPECIFY TYPESI EYE
\end{tabular}

MECHANICAL (GENERAL)

Goggles

GLOVES

SPECIAL

Polyzinyl alcohol

RESPIRÁTOR

OTHER FROTECTIVE EQUIPMENT

with proper filter

SECTION IX SPECIAL PRECAUTIONS

PRECAUTIONS TO BE TAKEN IN HANDLING AND STORAGE

OTHER PRECAUTIONS 
PRODUCT DESIGNATION

03-267-8677

P - Hydroxydiphenyl
MATERIAL SAFETY

DATA SHEET

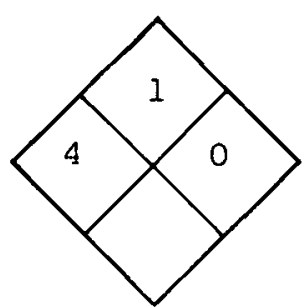

\section{SECTION 1 SOURCE AND NOMENCLATURE}

\section{MANUFACTURER'S NAME}

EMERGENCY TELEPHONE NO.

ADDRESS (NUMBER, STREET, CITY, STATE, ZIP CODE)

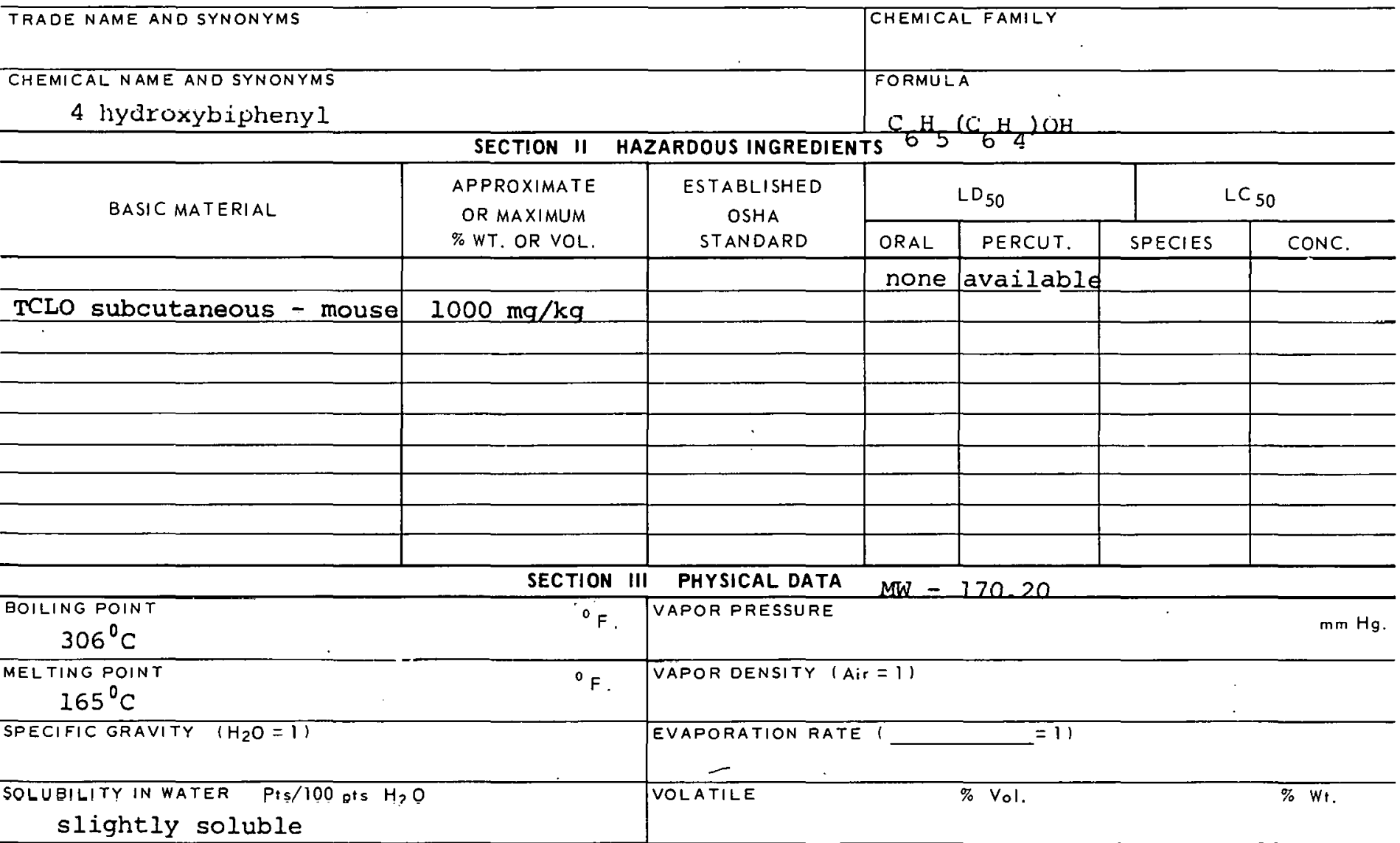
APPEARANCE AND ODOR

white solid

\section{SECTION IV FIRE AND EXPLOSION HAZARD DATA}

FLASH POINT
none available

EXTINGUISHING MEQIA

carbon dioxide or dry chemical

SPECIAL FIRE FIGHTING PROCEOURES

water may cause frothing.

UNUSUAL FIRE AND EXPLOSION HAZARDS

FLAMMABLE

(EXPLOSIVE)

LIMITS

\section{UPPER}

LOWER 


\begin{tabular}{ccc}
\hline & SECTION $V$ & HEALTH HAZARD DATA \\
\hline TOXIC LEVEL none available & CARCINOGENIC & neOPLAStiC \\
\hline PRINCIPLE ROUTES OF ABSORBTION & SKIN ANDEYE \\
inhalation, ingestion & IRRITATION & moderatc \\
\hline
\end{tabular}

Irritation and lesions of respiratory system. EMERGENCY AND FIRST AID PROCEOURES If swallowed - get medical aid, gastric lavage; inhalation rest; wash from skin \& eyes.

\section{SECTION VI REACTIVITY DATA}

CONDITIONS CONTRIBUTING TO INSTABILITY

CONDITIONS CONTRIBUTING TO HAZARDOUS POLYMERIZATION

INCOMPATABILITY (MATERIALS TO AVOID)

HALARUUUS LEG.UMRUSI IISIV PRULUL. 1 S

SECTION VII SPILL OR LEAK PROCEDURES

STEPS TO BE TAKEN IN CASE MATERIAL IS RELEASED OR SPILLED

WASTE DISPOSAL METHOD

SECTION VIII SPECIAL PROTECTION INFORMATION

\begin{tabular}{|c|c|}
\hline VENTILATION REQUIREMENTS LOCAL EXHAUST & $\begin{array}{l}\text { PROTECTIVE EQUIPMENT (SPECIFY TYPES) EYE } \\
\text { GOggleS }\end{array}$ \\
\hline MECHANICAL (GENERAL) & $\begin{array}{l}\text { GLOVES } \\
\text { polyVinyl alcohol }\end{array}$ \\
\hline SPERIAI. & $\begin{array}{l}\text { nCERInATOR } \\
\text { With proper filter }\end{array}$ \\
\hline \multicolumn{2}{|l|}{ OTHER PROTECTIVE EQUIPMENT } \\
\hline \multicolumn{2}{|c|}{ SECTION IX SPECIAL PRECAUTIONS } \\
\hline \multicolumn{2}{|c|}{ PRECAUTIONS TO BE TAKEN IN HANDLING ANO STORAGE } \\
\hline \multicolumn{2}{|l|}{ OTHER PRECAUTIONS } \\
\hline SIGNATURE & DATE \\
\hline
\end{tabular}


CA-000148243

VC42000

PRODUCT DESIGNATION

03-267-9857

03-267-9837

8-Hydroxyquinoline

MATERIAL SAFETY

DATA SHEET

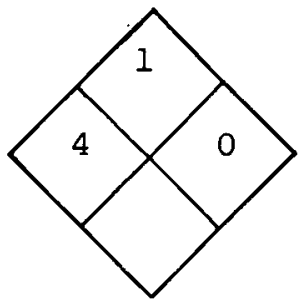

SECTION I SOURCE AND NOMENCLATURE

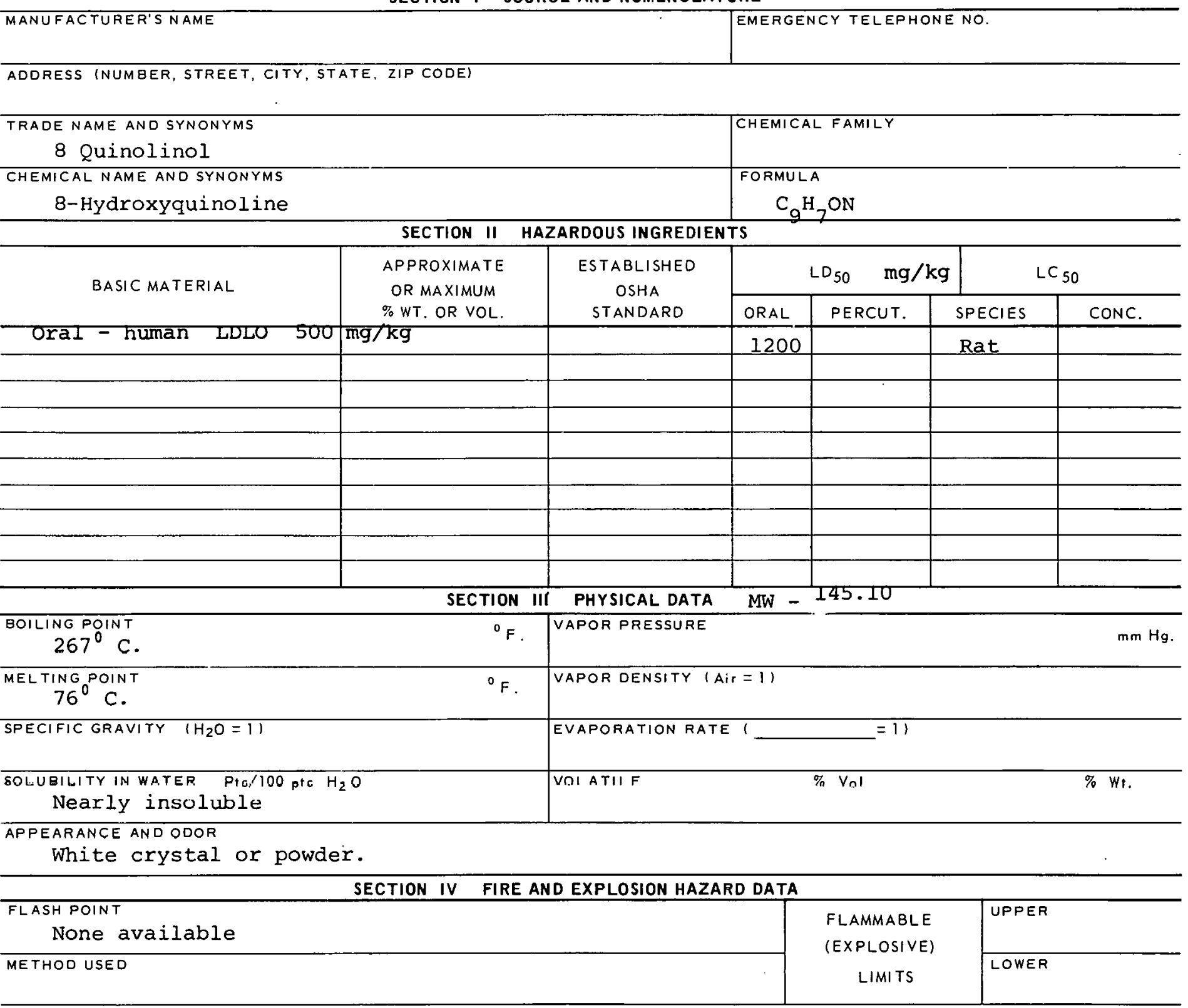

EXTINCUIEHIHC MEDIA

Alcohol foam, carbon dioxide, dry chemical.

SPECIAL FIRE FIGHTING PROCEDURES

UNUSUAL FIRE ANO EXPLOSION HAZARDS

When heated to decomposition, it emits highly toxic fumes of oxides of nitrogen. 


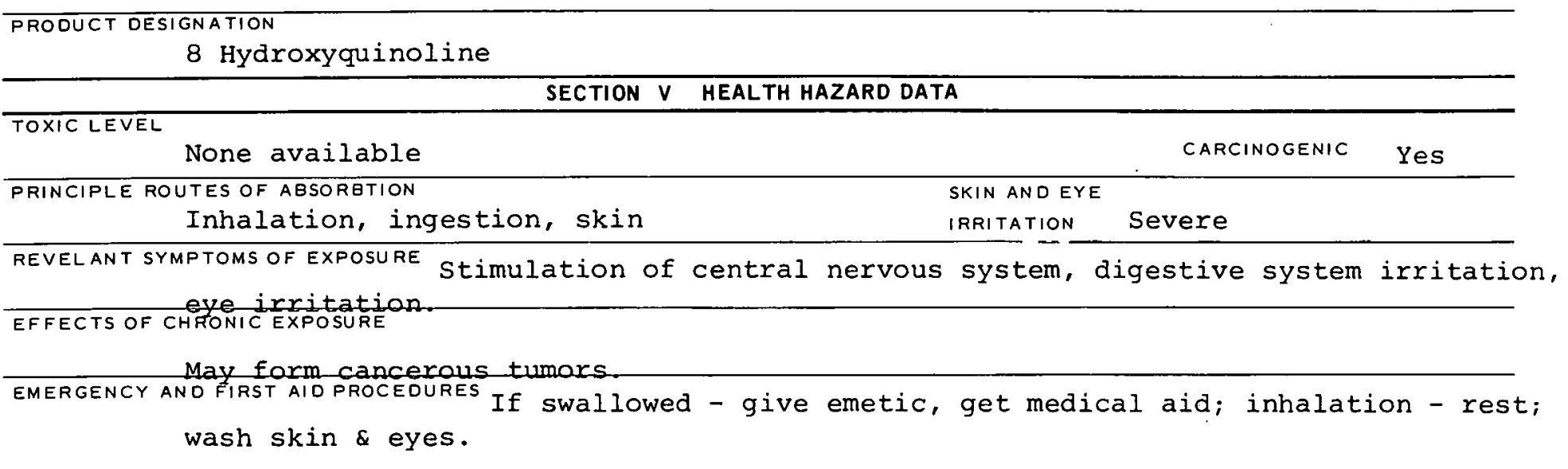

SECTION VI REACTIVITY DATA

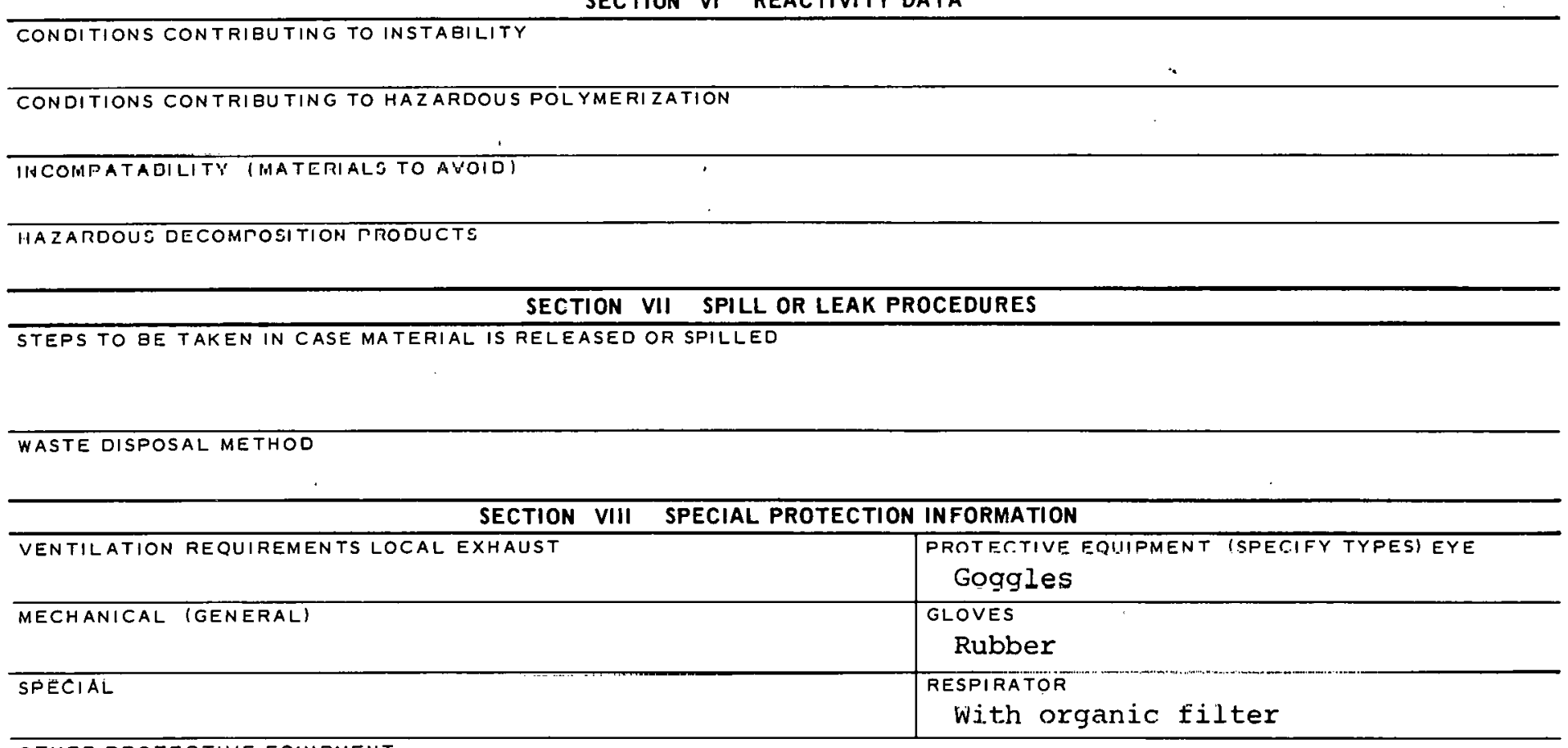

OTHER PROTECTIVE EQUIFMENT

SECTION IX SPECIAL PRECAUTIONS

PRECAUTIONS TO BE TAKEN IN HANDLING AND STORAGE

OTHER PRECAUTIONS

SIGNATURE

OATE

267-9857B 


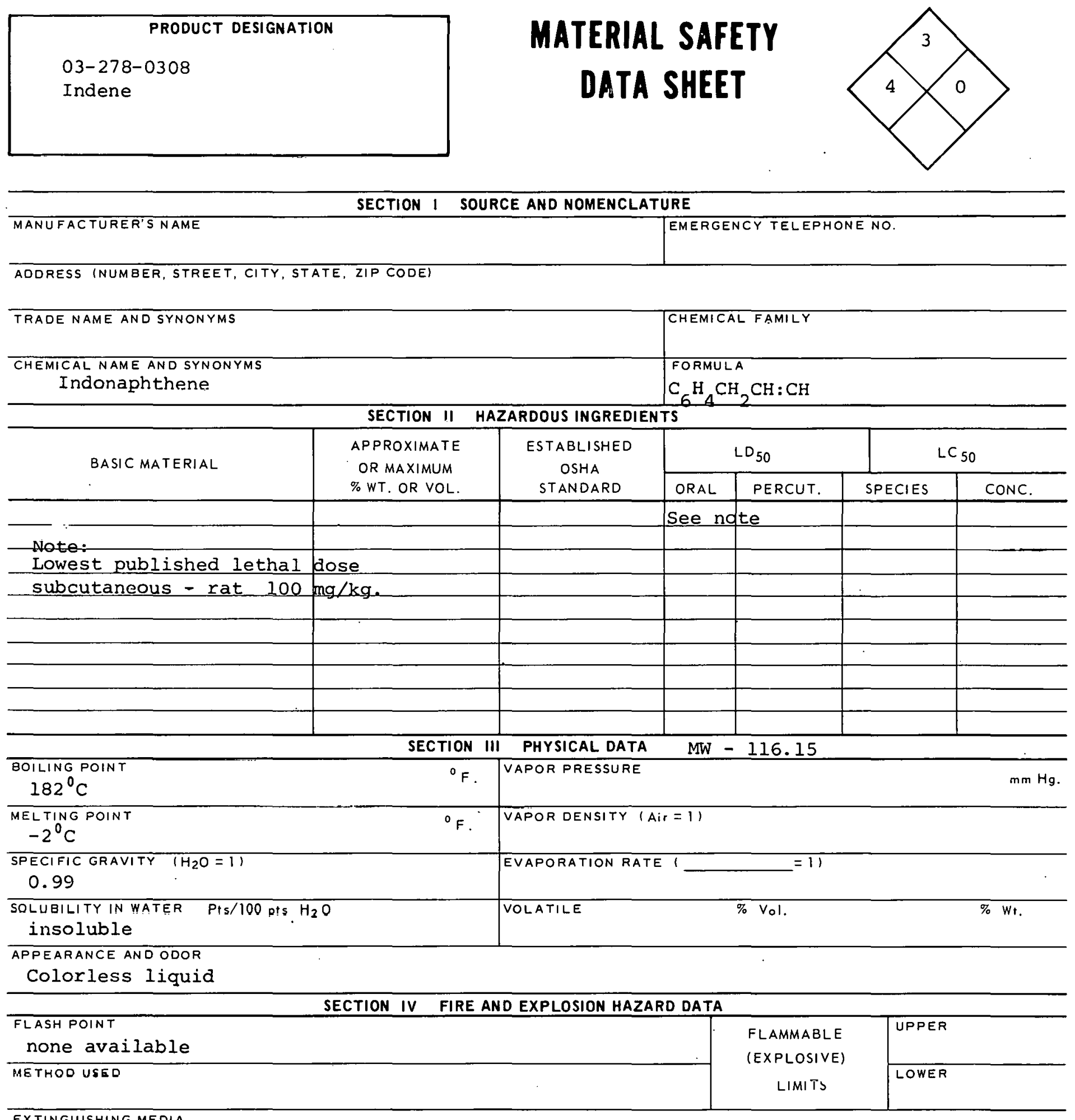

F.XTINGIIISHING MEDIA

Water spray carbon dioxide, dry chemical or alcohol foam.

SPECIAL FIRE FIGHTING PROCEOURES

Wear self-contained breathing apparatus.

UNUSUAL FIRE AND EXPLOSION HAZAROS 


\section{TOXIC LEVEL}

TLV - $10 \mathrm{PPM}, 45 \mathrm{mg} / \mathrm{C.M}$.

CARCINOGENIC

PRINCIPLE ROUTES OF ABSORBTION
Inhalation, ingestion, skin

Inhalation, ingestion, skin IRRITATION slight

REVELANT SYMPTOMS OF EXPOSURE Irxitation of mucous membranes and lungs,

pulmonary edema.

EFFECTS OF CHRONIC EXPOSURE

Dermatitis, liver and kidney damage.

EMERGENCY AND FIRST AID PROCEDURES If swallowed - give emetic, get medical aid; inhalation rest; wash from skin \& eyes.

\begin{tabular}{lll}
\hline SONDITIONS CONTRIBUTING TO INSTABILITY & SECTION VI REACTIVITY DATA \\
\hline
\end{tabular}

CONDITIONS CONTRIEUTING TO HAZARDOUS POLYMERIZATION

IICOMCATADILITY (MATERIALJ TO AVOIO)

HAZARDOUS DECOMPOSITION PRODUCTS

SECTION VII SPILL OR LEAK PROCEDURES

STEPS TO BE TAKEN IN CASE MATERIAL IS RELEASEO OR SPILLED

WASTE DISPOSAL METHOD

SECTION VIII SPECIAL PROTECTION INFORMATION

\begin{tabular}{l|l}
\hline VENTILATION REQUIREMENTS LOCAL EXHAUST & $\begin{array}{l}\text { PROTECTIVE EQUIPMENT (SPECIFY TYPES) EYE } \\
\text { GoggleS }\end{array}$ \\
\hline MECHANIGAL (GENERAL) & $\begin{array}{l}\text { SLUVES } \\
\text { Rubber }\end{array}$ \\
\hline SPECIAL & RESPIRATOR \\
With proper filter
\end{tabular}

OTHER PROTECTIVE EQUIPMENT

SECTION IX SPECIAL PRECAUTIONS

PRECAUTIONS TO BE TAKEN IN HANOLING AND STORAGE

OTHER PRECAUTIONS

SIGNATURE

DATE

278-0308B 
CAS-000540374

PRODUCT DESIGNATION

$03-278-1308$

P-Iodoaniline
BY38500

MATERIAL SAFETY

DATA SHEET

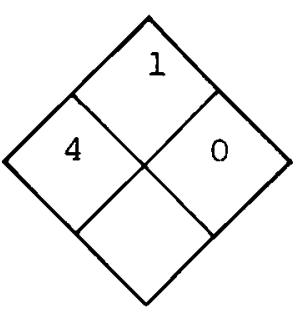

\section{SECTION I SOURCE AND NOMENCLATURE}

MANUFACTURER'S NAME

EMERGENCY TELEPHONE NO.

AODRESS (NUMBER, STREET, CITY, STATE, ZIP CODE)

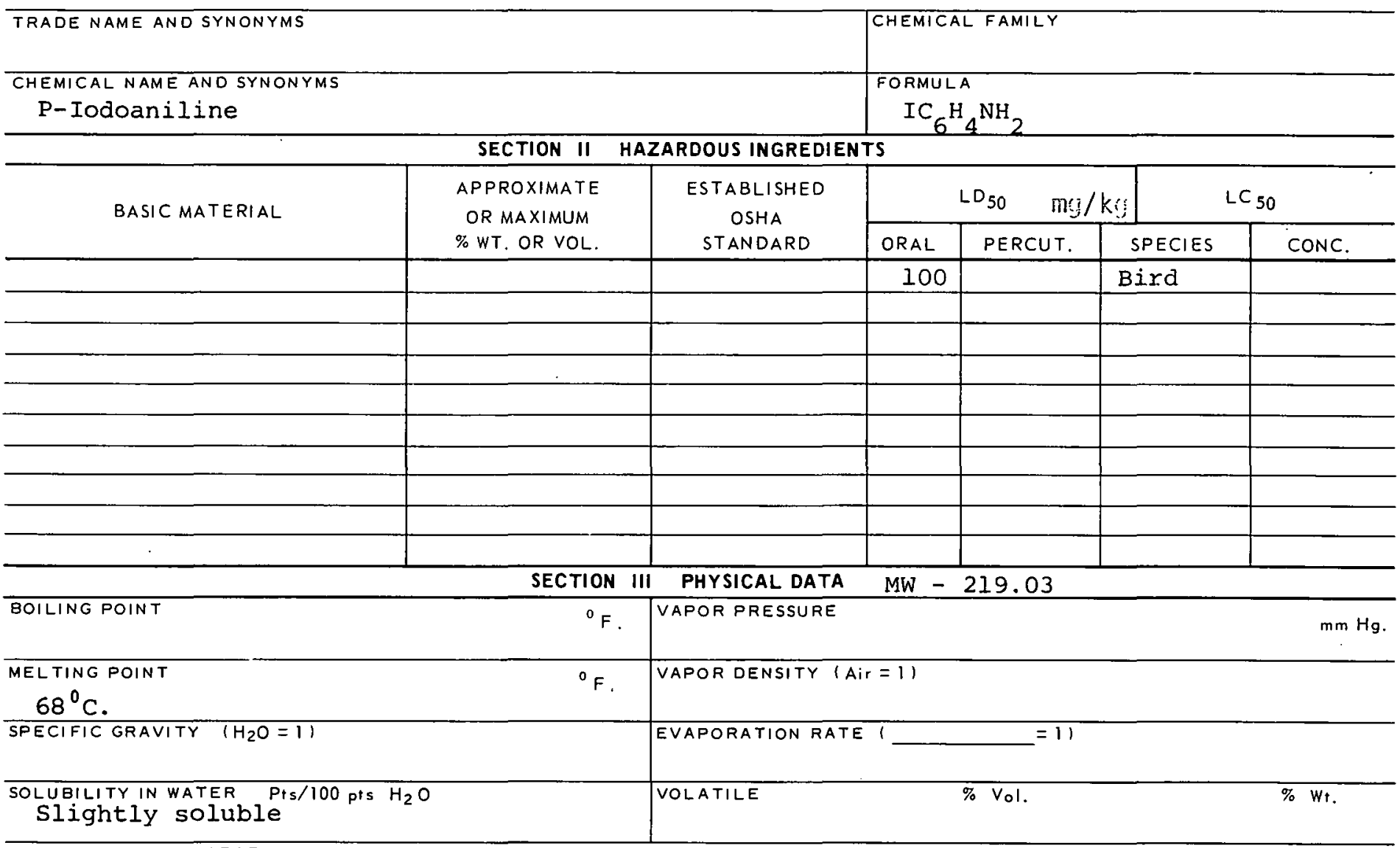

APPEARANCE AND ODOR

Yellow crystal

SECTION IV FIRE AND EXPLOSION HAZARD DATA

FLASH POINT
None available
METHOO USED

EXTINGUISHING MEDIA

Water spray, carbon dioxide, alcohol foam.

SPECIAL FIRE FIGHTING PROCEDURES

Self-contained breathing apparatus.

UNUSUAL FIRE ANO EXPLOSION HATARDS

Vapor may form explosive mixture with air.

FLAMMABLE (EXPLOS!VE) LIMITS
UPPER

LOWER 


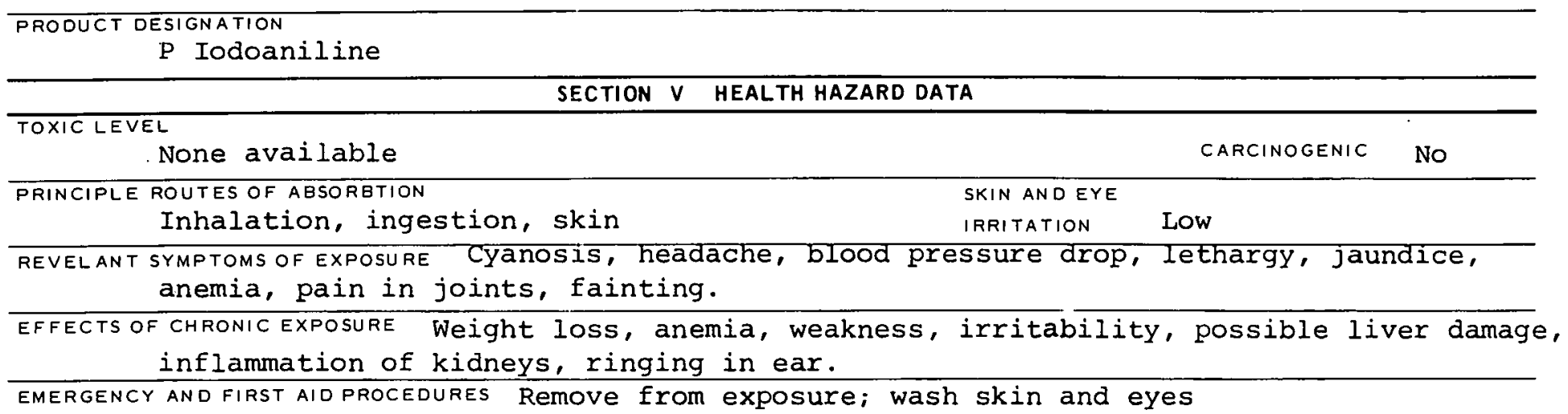

\section{SECTION VI REACTIVITY DATA}

CONDITIONS CONTRIBUTING TO INSTABILITY

CONDITIONS CONTRIBUTING TO HAZARDOUS POLYMERIZATION

INCOMPATABILITY (MATERINLS TO AVOIO)

Sodium and potassium peroxides, ozone, perchloric acid.

HA7ARONIIS DFCOMPOSITION PRODIIS.TS

\section{SECTION VII SPILL OR LEAK PROCEDURES}

STEPS TO BE TAKEN IN CASE MATERIAL IS RELEASED OR SPILLED

Wear protective clothing during clean-up.

WASTE DISPOSAL METHOD

SECTION VIII SPECIAL PROTECTION INFORMATION

\begin{tabular}{|c|c|}
\hline VENTILATION REQUIREMENTS LOCAL EXHAUST & $\begin{array}{l}\text { PROTECTIVE EOUIPMENT (SPECIFY TYPESIEYE } \\
\text { GOggles }\end{array}$ \\
\hline MELCHANILAL IGENERAL) & $\begin{array}{l}\text { RLOVES } \\
\text { Rubber }\end{array}$ \\
\hline SPECIAL & $\begin{array}{l}\text { RFSPIRATOR } \\
\text { Selt-contained }\end{array}$ \\
\hline
\end{tabular}

OTHER PROTECTIVE EQUIPMENT

Protective clothing

\section{SECTION IX SPECIAL PRECAUTIONS}

PRECAUTIONS TO BE TAKEN IN HANDLING AND STORAGE

Store in flammable liquids cabinet. 


\begin{tabular}{|c|}
\hline PRODUCT DESIGNATION \\
$03-278-1808$ \\
Iodobenzene
\end{tabular}

\section{MATERIAL SAFETY DATA SHEET}

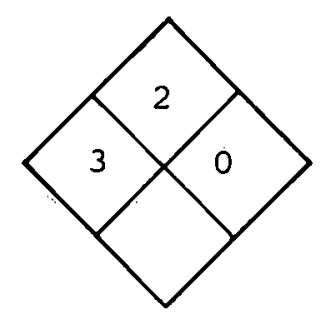

SECTION I SOURCE AND NOMENCLATURE

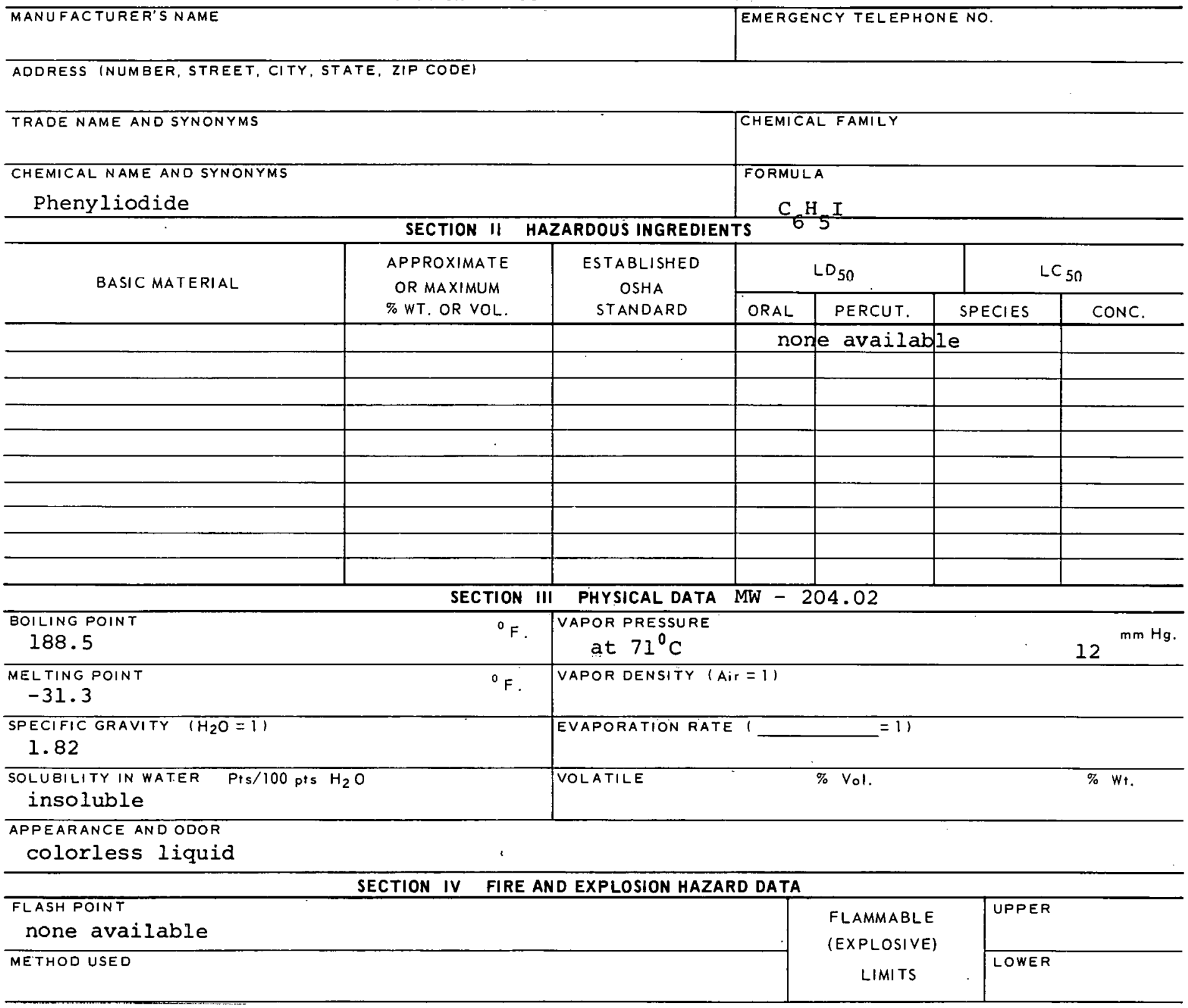

EXTINGUISHING MEDIA

water, foam, carbon dioxide, dry chemical.

SPECIAL FIRE FIGHTING PROCEDURES

Wear goggles and self-contained breathing apparatus. 
TOXIC LEVEL

none available

PRINCIPLE ROUTES OF ABSORETION

inhalation, ingestion, skin

REVELANT SYMPTOMS OF EXPOSURE CYAnOSis

drowsiness, unconsciousness.

EFFECTS OF CHRONIC EXPOSURE

Lung, liver and kidney damage.

EMERGENCY AND FIRST AIDPROCEDURES Inhalation - rest; if swallowed - give emetic, get medical aid; wash from skin \& eyes.

SECTION VI REACTIVITY DATA
CARCINOGENIC SKIN AND EYE

IRRITATION

severe

\section{severe}

deep and rapid respiration, irregular pulse,
CONDITIONS CONTRIBUTING TO HAZARDOUS POLYMERIZATION

INCOMPATAEILITY (MATERIALS TO AVOIB)

HAZARDOUS DECOMPOSITION PRODUCTS

SECTION VII SPILL OR LEAK PROCEDURES

STEPS TO BE TAKEN IN CASE MATERIAL IS RELEASED OR SPILLED

WASTE DISPOSAL METHOD

SECTION VIII SPECIAL PROTECTION INFORMATION

\begin{tabular}{|c|c|}
\hline SECTION VIII & SPECIAL PROTECTION INFORMATION \\
\hline VENTILATION REQUIREMENTS LOCAL EXHAUST & $\begin{array}{l}\text { PROTECTIVE EQUIPMENT (SPECIFY TYPES) EYE } \\
\text { Goggles }\end{array}$ \\
\hline MECHANICAL (GENERAL) & $\begin{array}{l}\text { GLOVES } \\
\text { Polyvinyl alcohol }\end{array}$ \\
\hline SPECIAL & $\begin{array}{l}\text { RFGPIRATRR } \\
\text { EcIf-contained breathing appardlus. }\end{array}$ \\
\hline
\end{tabular}

OTHER PROTECTIVE EQUIPMENT

\section{SECTION IX SPECIAL PRECAUTIONS}

PRECAUTIONS TO BE TAKEN IN HANDLING AND STORAGE

\section{OTHER PRECAUTIONS}

SIGNATURE

DATE

$278-1808 B$ 


PRODUCT DESIGNATION
03-278-2308
O-Iodobenzoyl Chloride

\section{MATERIAL SAFETY \\ DATA SHEET}

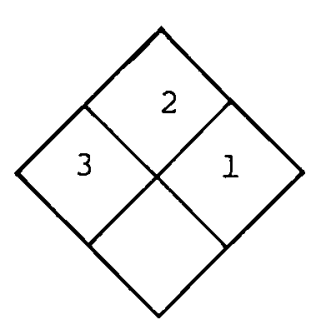

\section{SECTION I SOURCE AND NOMENCLATURE}

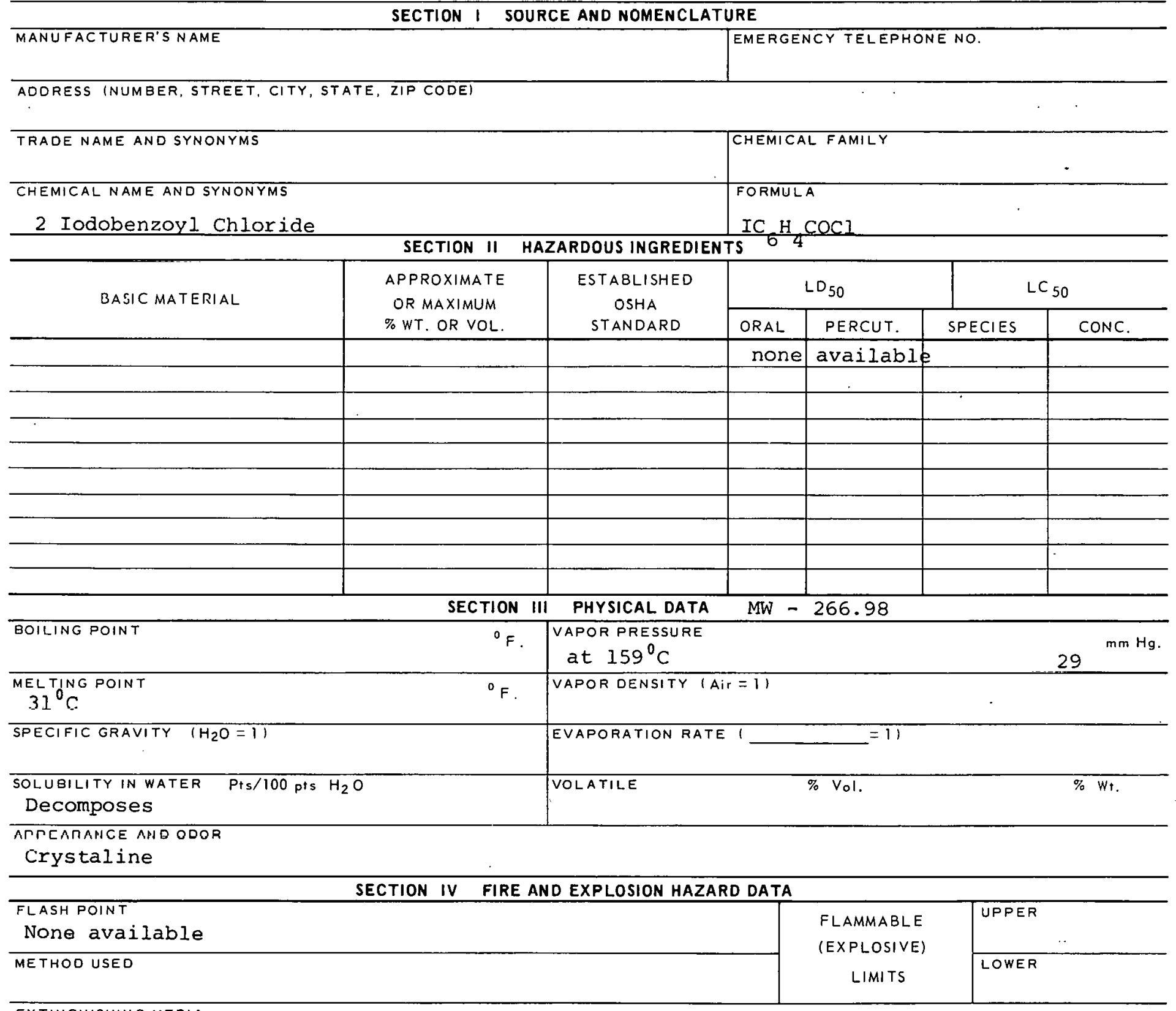

EXTINGUISHING MEDIA

Water spray, foam, carbon dioxide or dry chemical.

SPECIAL FIRE FIGHTING PROCEOURES

Full protective clothing.

UNUSUAL FIRE AND EXPLOSION HAZARDS 


\section{TOXIC LEVEL}

None available

PRINCIPLE ROUTES OF AESORETION

Inhalation, ingestion
CARCINOGENIC

SKIN AND EYE

IRRITATION no

severe

REVELANT SYMPTOMS OF EXPOSURE Irritation of respiratory system, skin \& eye burns, mouth and digestive tract burns.

EFFECTS OF CHRONIC EXPOSURE

EMERGENCY AND FIRST AID PROCEDURES If swallowed - drink water, milk of magnesia, get medical aid; inhalation - rest, oxygen, artificial respiration; wash skin \& eyes.

\section{SECTION VI REACTIVITY DATA}

CONDITIONS CONTRIBUTING TO INSTABILITY

CONDITIONS CONTRIBU TING TO HAZARDOUS POLYMERIZATION

IACOMPATARI!.ITY (MATFRIA!STOAVIII)

May react with oxidizing materials. HAZARDOUS DECOMPOSITION PRODUCTS

When heated, phosgene is produced.

SECTION VII SPILL OR LEAK PROCEDURES

STEPS TO BE TAKEN IN CASE MATERIAL IS RELEASED OR SPILLED

WASTE DISPOSAL METHOD

SECTION VIII SPECIAL PROTECTION INFORMATION

\begin{tabular}{|c|c|}
\hline VENTILATION REQUIREMENTS LOCAL EXHAUST & $\begin{array}{l}\text { PROTECTIVE EQUIPMENT (SPECIFY TYPES) EYE } \\
\text { GOggles }\end{array}$ \\
\hline MECHANICAL (GENERAL) & $\begin{array}{l}\text { GLOVES } \\
\text { Ruthher. }\end{array}$ \\
\hline SWELIAL & $\begin{array}{l}\text { RESPIRATOR } \\
\text { With proper filter }\end{array}$ \\
\hline
\end{tabular}

OTHER PROTECTIVE EQUIPMENT

\section{SECTION IX SPECIAL PRECAUTIONS}

PRECAUTIONS TO BE TAKEN IN HANDLING AND STORAGE

Store in cool, dry, well-ventilated location. OTHER PRECAUTIONS 

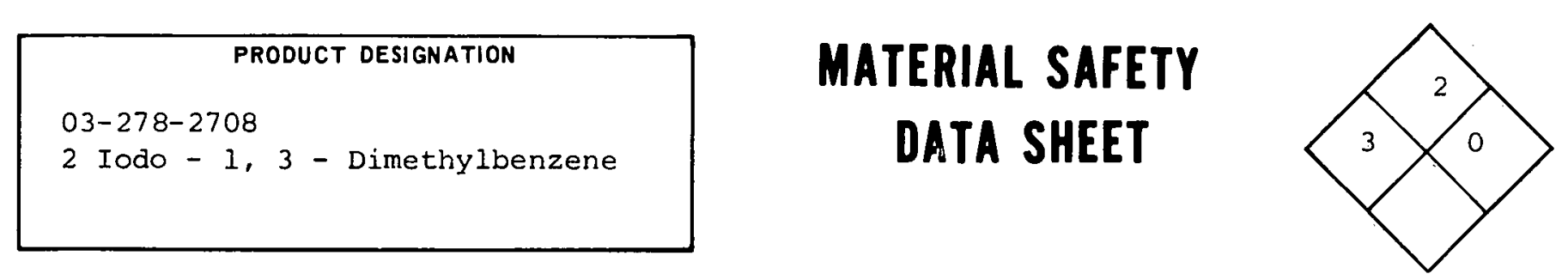

SECTION I SOURCE AND NOMENCLATURE

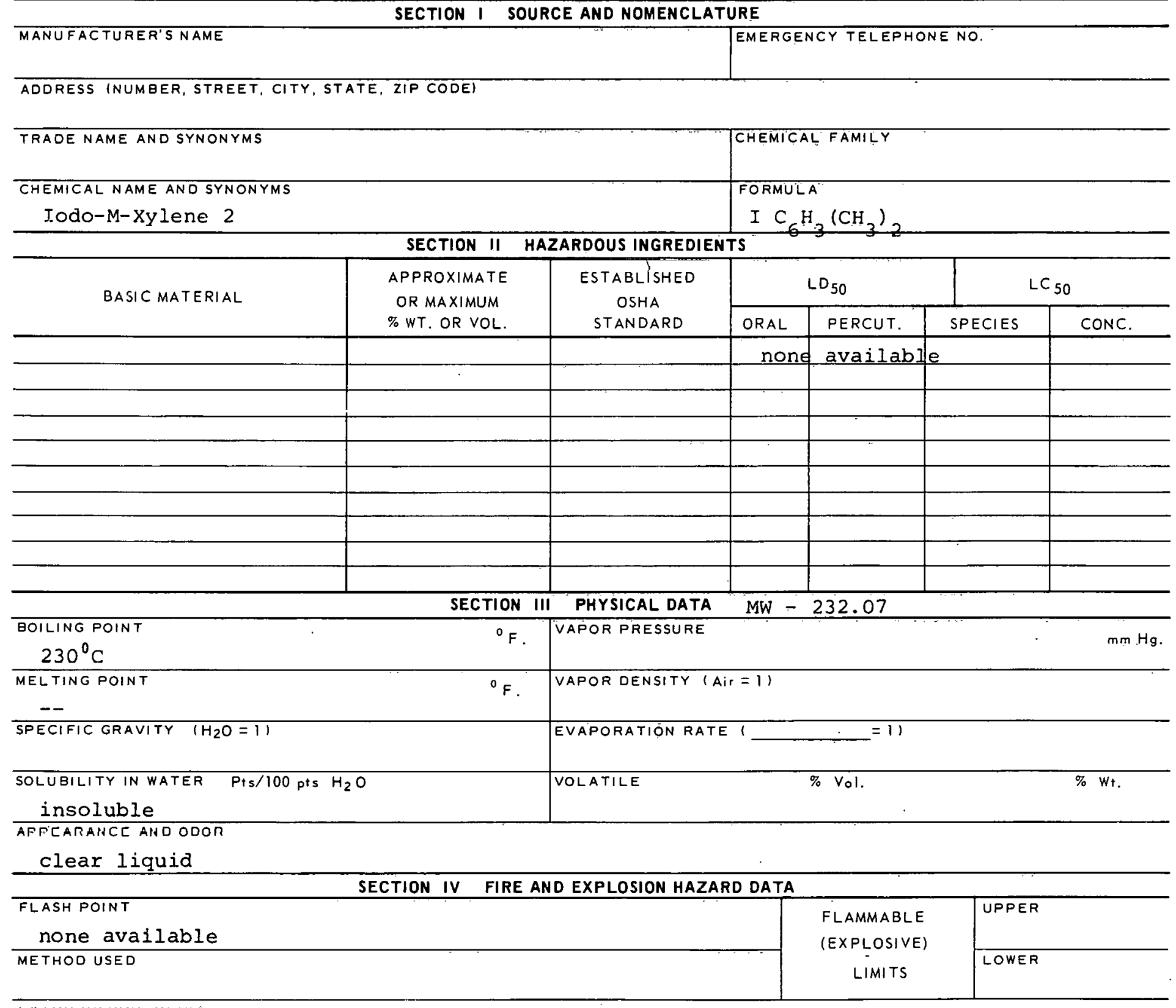

EXIINGUISHING MEUIA

Foam, carbon dioxide, dry chemical. Water spray may be used to reduce rate of burning.

SPECIAL FIRE FIGHTING PROCEDURES

Wear self-contained breathing apparatus.

UNUSUAL FIRE AND EXPLOSION HAZARDS 
2 Iodo - 1,3 - Dimethylbenzene

SECTION $V$ HEALTH HAZARD DATA

TOXICLEVEL

none available

PRINCIPLE ROUTES OF AESORBTION

inhalation, ingestion

SKIN AND EYE

CARCINOGENIC no

REVELANT SYMPTOMS OF EXPOSURE Irritation of respiratory tract, disturbance of vision,
dizziness, nausea, collapse, coma; burning of skin and eyes.

REVELANT SYMPTOMS OF EXPOSURE Irritation of respiratory tract, disturbance
dizziness, nausea, collapse, coma; burning of skin and eyes.

EFFECTS OF CHRONIC EXPOSURE

Dermatitis, blood and liver damage.

EMERGENCY AND FIRST AID PROCEDURES Inhalation - rest, get medical aid; ingestion - give saline cathartics and demulcents, get medical aid; wash skin \& eyes.

\section{SECTION VI REACTIVITY DATA}

CONDITIONS CONTRIBUTING TO INSTABILITY

CONDITIONS CONTRIBUTING TO HAZARDOUS POLYMERIZATION

INCOMPA IABILIIY IMATERIALS IU AVUIUI

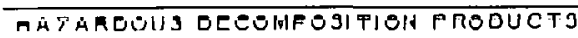

SECTION VII SPILL OR LEAK PROCEDURES

STEPS TO BE TAKEN IN CASE MATERIAL IS RELEASED OR SPILLED

WASTE OISPOSAL METHOD

SECTION VIII SPECIAL PROTECTION INFORMATION

\begin{tabular}{|c|c|}
\hline VENTILATION REQUIREMENTS LOCAL EXHAUST & $\begin{array}{l}\text { PROTECTIVE EQUIPMENT (SPECIFY TYPES) EYL } \\
\text { GOggleS }\end{array}$ \\
\hline MECHANICAL (GENERAL) & $\begin{array}{l}\text { GLOVES } \\
\text { Rubber }\end{array}$ \\
\hline SPFC.IAI & $\begin{array}{l}\text { RFSPIRATAR } \\
\text { Self-contained }\end{array}$ \\
\hline
\end{tabular}

OTHER PROTECTIVE EQUIPMENT

\section{SECTION IX SPECIAL PRECAUTIONS}

PRECAUTIONS TO BE TAKEN IN HANDLING AND STORAGE

Outside storage preterred; inside storage flammable liquids storage cabinet.

OTHER PRECAUTIONS

SIGNATURE

DATE

$278-2708 B$ 

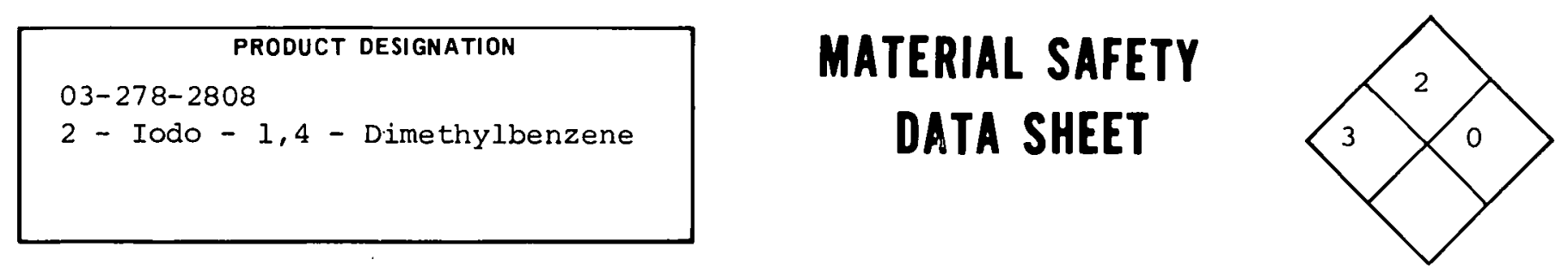

SECTION I SOURCE AND NOMENCLATURE

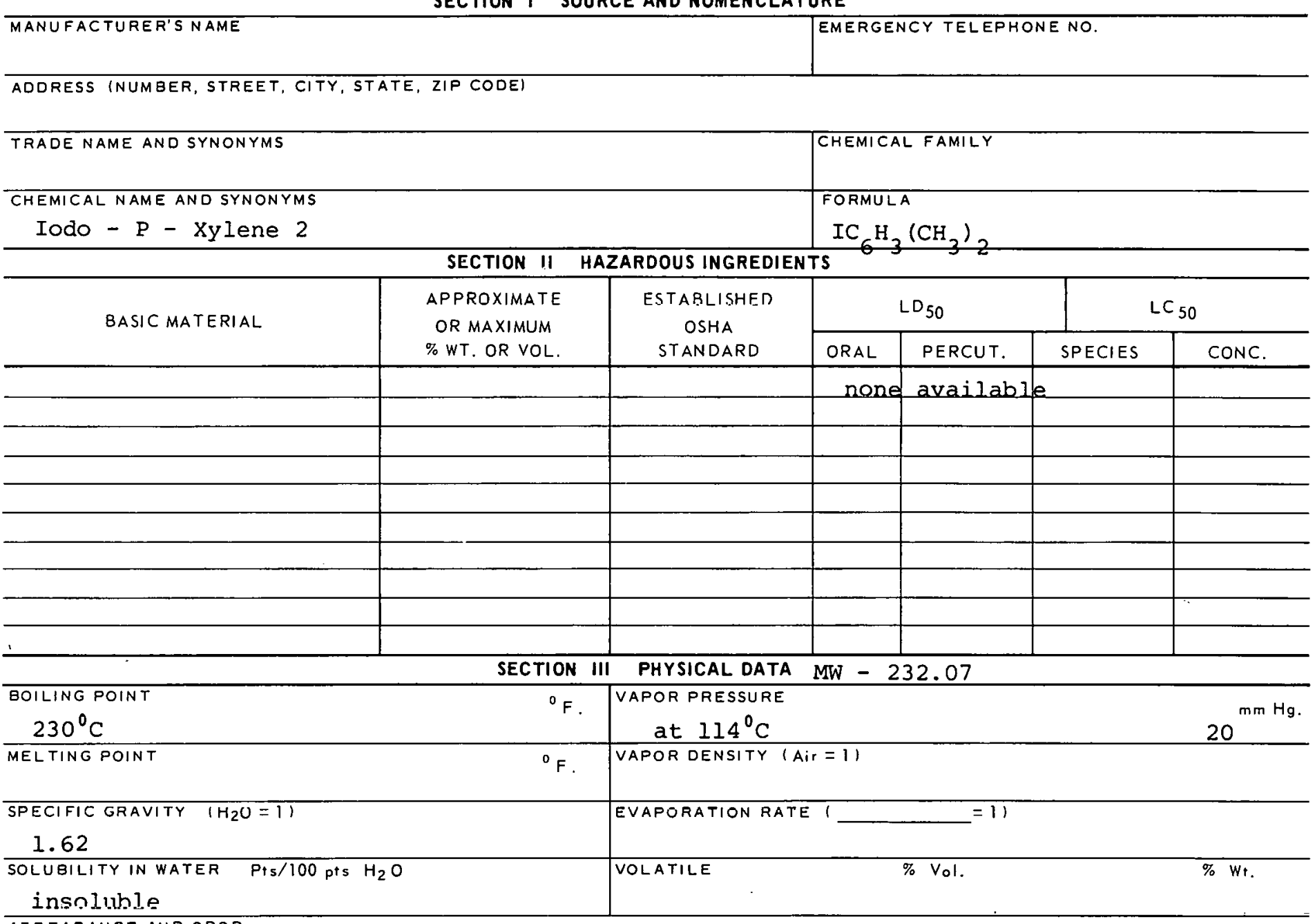

APPEARANCE AND ODOR

clear liquid

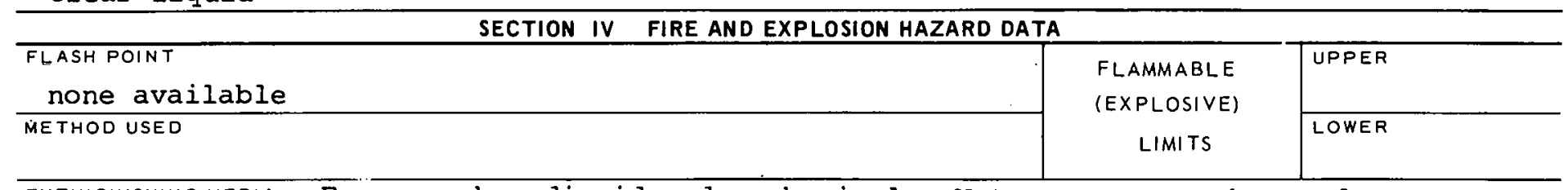

EXTINGUISHING MEDIA Foam, carbon dioxide, dry chemical. Water spray may be used to redure ratee of hurning.

SPECIAL FIRE FIGHTING PROCEDURES

Wear self-contained breathing apparatus.

UNUSUAL FIRE ANDEXPLOSION HAZARUS 
2 Iodo - 1,4 - Dimethylbenzene

SECTION $\checkmark$ HEALTH HAZARD DATA

TOXIC LEVEL

None available

PRINCIPLE ROUTES OF ABSORETION

CARCINOGENIC

Inhalation, ingestion SKIN ANO EYE

IRRITATION severe

REVELANT SYMPTOMS OF EXPOSURE Irritation of respiratory tract, disturbance of vision, dizziness, nausea, collapse, coma; burning of skin \& eyes.

EFFECTS OF CHRONIC EXPOSURE

Dermatitis, blood and liver damage.

EMERGENCY AND FIRST AID PROCEDURES Inhalation - rest, get medical aid; ingestion - give saline water cathartics and demuclcents, get medical aid; wash skin \& eyes.

SECTION VI REACTIVITY DATA

CONDITIONS CONTRIBUTING TO INSTABILITY

CONDITIONS CONTRIBUTING TO HAZARDOUS POLYMERIZATION

IHCONHATADILITY (MATERIALE TO AVOID)

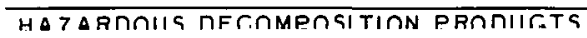

SECTION VII SPILL OR LEAK PROCEDURES

STEPS TO BE TAKEN IN CASE MATERIAL IS RELEASED OR SPILLED

WASTE DISPOSAL METHOD

SECTION VIII SPECIAL PROTECTION INFORMATION

\begin{tabular}{l|l}
\hline VENTILATION REQUIREMENTS LOCAL EXHAUST & $\begin{array}{l}\text { PROTECTIVE EQUIPMENT (SPECIFY TYPES) EYE } \\
\text { GOggles }\end{array}$ \\
\hline MECHANICAL IGENERAL) & GLOVES \\
\hline SPECIAL & RUbSPIRATOR \\
& Self-COntained \\
\hline
\end{tabular}

OTHER FFOTECTIVE EQUIPMENT

\section{SECTION IX SPECIAL PRECAUTIONS}

PRECAUTIONS TO $8 E$ TAKEN IN HANDLING AND STORAGE

Outside storage preferred; inside storage flammable liquids storage cabinet.

OTHER PRECAUTIONS

\begin{tabular}{|l|l|}
\hline SIGNATURE & DATE \\
\hline
\end{tabular} 


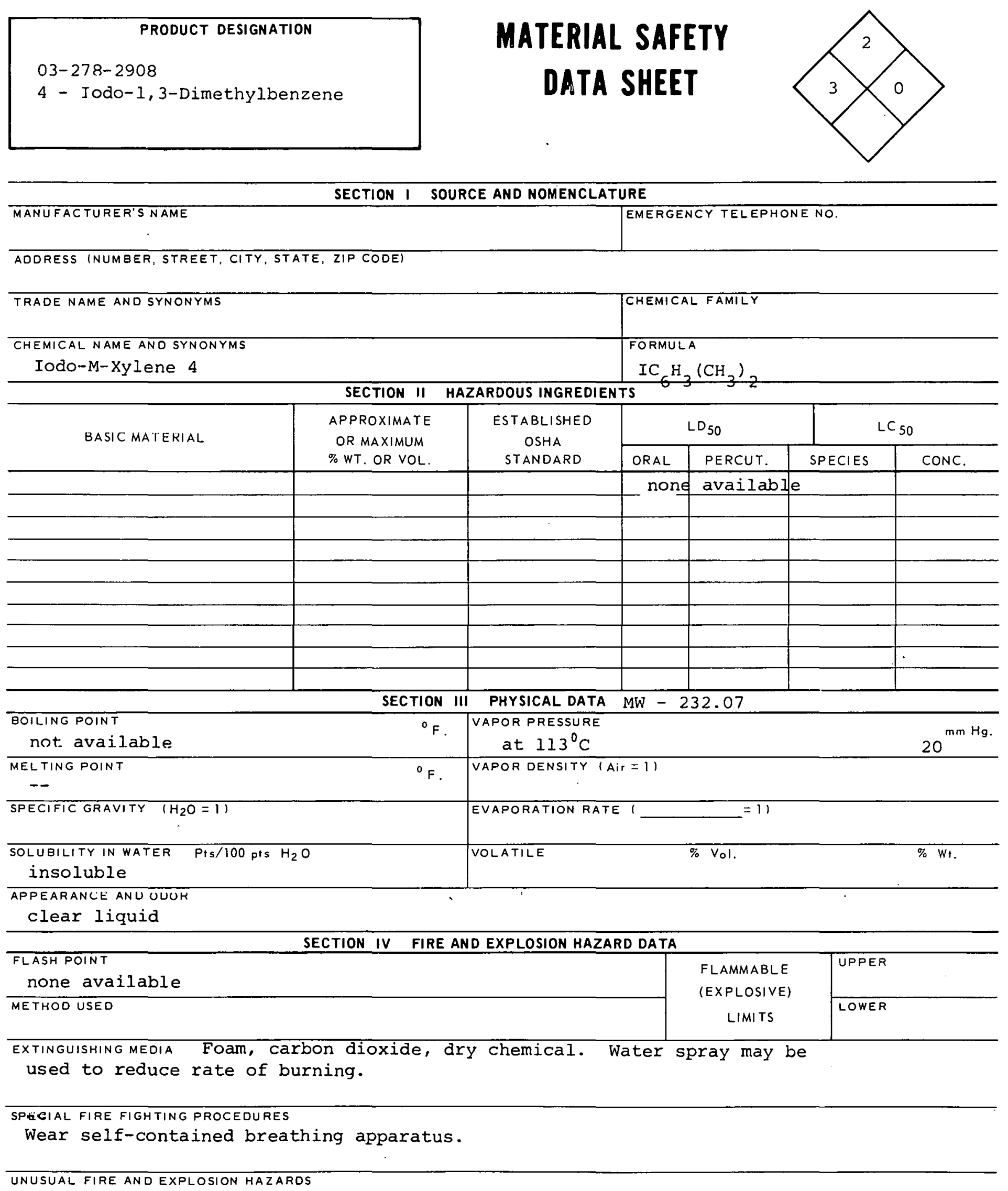


4-Iodo-1,3-Dimethylbenzene

\section{SECTION $V$ HEALTH HAZARD DATA}

TOXICLEVEL

None available

PRINCIPLE ROUTES OF ABSORBTION

Inhalation, ingestion

CARCINOCENIC

no

IRRITATION severe

REVELANT SYMPTOMS OF EXPOSURE Irritation of respiratory tract; disturbance of vision, dizziness, nausea, collapse, coma; burning of skin \& eyes.

EFFECTS OF CHRONIC EXPOSURE

Dermatitis, blood \& liver damage.

EMERGENCY AND FIRST AID PROCEDURES Inhalation - rest, get medical aid; if swallowed give saline water cathartics and demulcents, get medical aid; wash skin and eyes.

\section{SECTION VI REACTIVITY DATA}

CONDITIONS CONTRIBUTING TO INSTABILITY

CONDITIONS CONTRIBUTING TO HAZARDOUS POLYMERIZATION

IICOMPATADILITY IMATERIAL3 TO AVOIOI

HATARMNIIS REROMADSS!T!OM! PROQUET\&

SECTION VII SPILL OR LEAK PROCEDURES

STEPS TO BE TAKEN IN CASE MATERIAL IS RELEASED OR SPILLED

WASTE DISPOSAL METHOD

SECTION VIII SPECIAL PROTECTION INFORMATION

\begin{tabular}{l|l}
\hline VENTILATION REQUIREMENTS LOCAL EXHAIIST & $\begin{array}{l}\text { PROTECTIVE EQUIPMENT (SPECIFY TYPESI EYE } \\
\text { GOGgleS }\end{array}$ \\
\hline MECHANICAL IGENERAL) & GLOVES \\
& Rubber \\
\hline SPECIAL & RESPIAATOR \\
& Self-COntained
\end{tabular}

OTHER PROTECTIVE EQUIPMENT

SECTION IX SPECIAL PRECAUTIONS

PRECAUTIONS TO BE TAKEN IN HANDLING AND STORAGE Outside storage preferred; inside storage flammable Iiquids storage cabinet.

OTHER PRECAUTIONS

SIGNATURE

DATE

$278-2908 B$ 


\begin{tabular}{|c|}
\multicolumn{1}{|c|}{ PROdUCT DESIGNATION } \\
$\begin{array}{l}\text { 03-278-3308 } \\
\text { O-Iododiphenyl }\end{array}$ \\
\end{tabular}

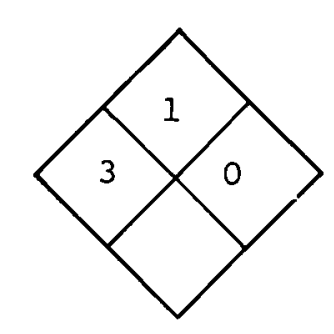

SECTION I SOURCE AND NOMENCLATURE

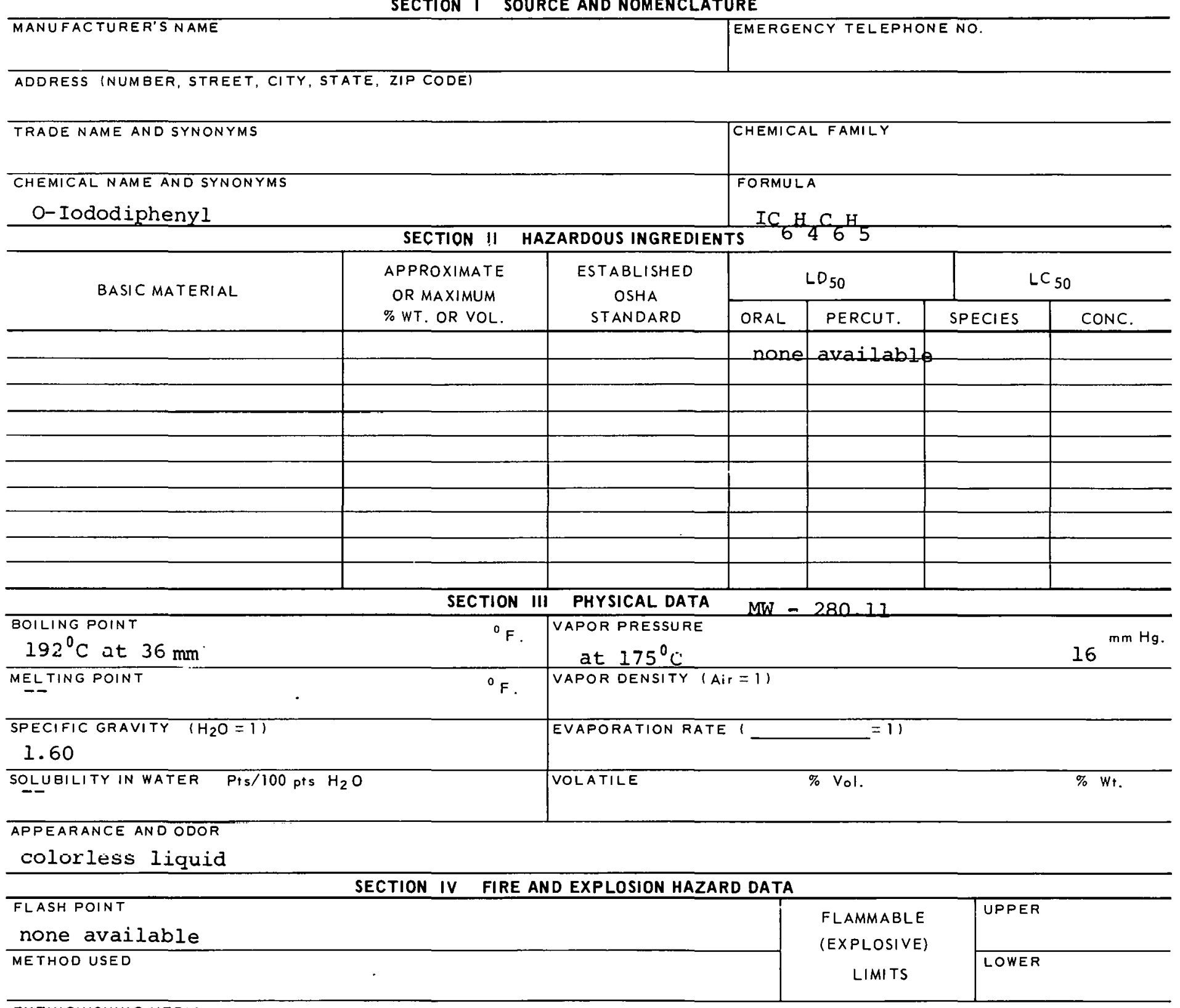

EXTINGUISHING MEDIA

water, foam, carbon dioxide, dry chemical

SPECIAL FIRE FIGHTING PROCEDURES

UNUSUAL FIRE AND EXPLOSION HAZARDS 


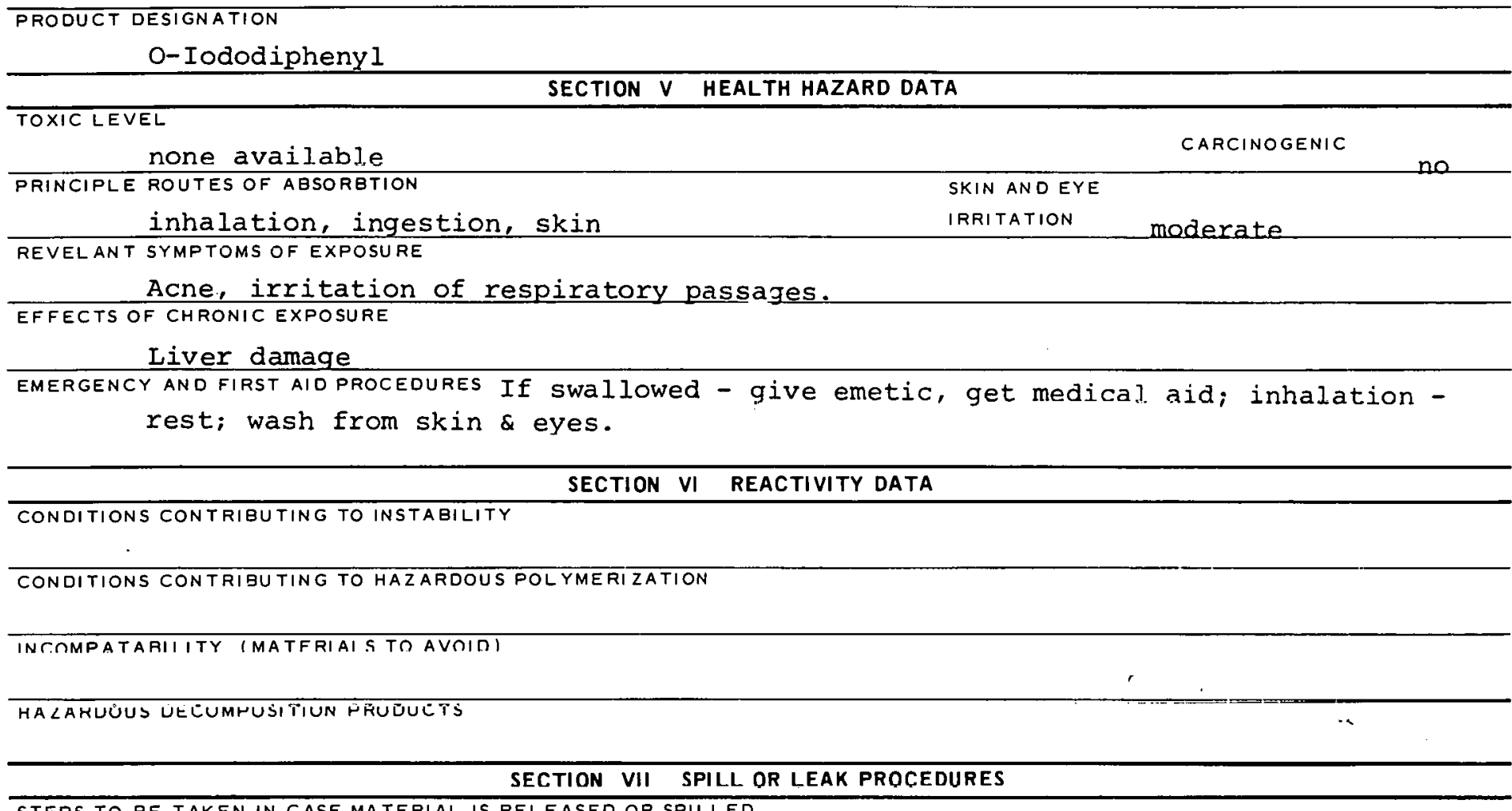

STEPS TO BE TAKEN IN CASE MATERIAL IS RELEASED OR SPILLED

WASTE DISPOSAL METHOD

SECTION VIII SPECIAL PROTECTION INFORMATION

\begin{tabular}{|c|c|}
\hline $\begin{array}{l}\text { VENTILATION REQUIREMENTS LOCAL EXHAUST } \\
\text {. }\end{array}$ & $\begin{array}{l}\text { PROTECTIVE EQUIPMENT (SPECIFY TYPES) EYE } \\
\text { GOgg } 1 \text { PSS }\end{array}$ \\
\hline MECHANICAL IGENERAL) & $\begin{array}{l}\text { GLOVES } \\
\text { Rubber }\end{array}$ \\
\hline SPEEIAL & $\begin{array}{l}\text { YESHIKAIUH } \\
\text { Śelf-contained }\end{array}$ \\
\hline
\end{tabular}

OTHER PROTECTIVE EQUIPMENT

SECTION IX SPECIAL PRECAUTIONS

PRECAUTIONS TO BE TAKEN IN HANDLING AND STORAGE

OTHER PRECAUTIONS

SIGNATURE

DATE

278-3308B 


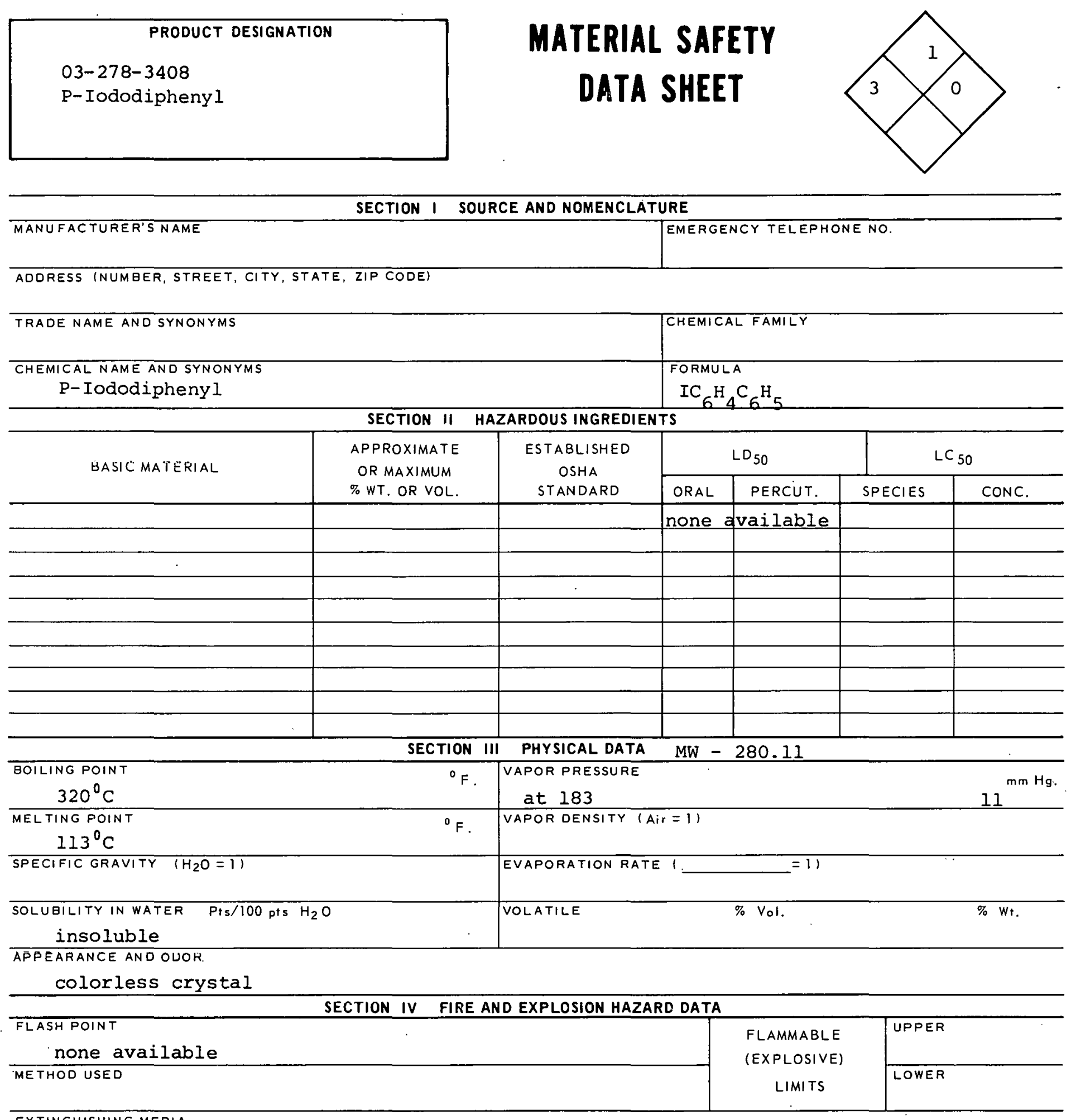

EXTINGUISHING MEDIA

water, foam, carbon dioxide, dry chemical.

SPECIAL FIRE FIGHTING PROCEDURES

UNUSUAL FIRE AND EXPLOSION HAZARDS 


\section{TOXIC LEVEL}

none available

PRINCIPLE ROUIES OF ABSORBTION

inhalation, ingestion, skin

CARCINOGENIC SKIN ANOEYE IRRITATION moderate

REVELANT SYMPTOMS OF EXPOSURE

Acne, irritation of respiratory passages.

EFFECTS OF CHRONIC EXPOSURE

Liver damage

EMERGENCY AND FIRST AID PROCEDURES

rest; wash skin \& eyes.

If swallowed - give emetic, get medical aid; inhalation SECTION VI REACTIVITY DATA

CONDITIONS CONTRIBUTING TO INSTABILITY

CONDITIONS CONTRIBUTING TO HAZARDOUS POLYMERIZATION

INCOMPATABILITY (MATERIALS TO AVOID)

HAZARDOUS DECOMPOSITION PRODUCTS

SECTION VII SPILL OR LEAK PROCEDURES

STEPS TO BE TAKEN IN CASE MATERIAL IS RELEASED OR SPILLED

WASTE DISPOSAL METHOD

SECTION VIII SPECIAL PROTECTION INFORMATION

\begin{tabular}{l|l}
\hline VENTILATION REQUIREMENTS LOCAL EXHAUST & $\begin{array}{l}\text { PROTECTIVE EQUIPMENT (SPECIFY TYPES) EYE } \\
\text { gOggles }\end{array}$ \\
\hline MECHANICAL IGENERAL) & $\begin{array}{l}\text { GLOVES } \\
\text { rubber }\end{array}$ \\
\hline SPFCIAI & RESFIRÁÜR \\
SElP-COntained
\end{tabular}

SECTION IX SPECIAL PRECAUTIONS

PRECAUTIONS TO BE TAKEN IN HANDLING AND STORAGE

OTHER PRECAUTIONS

SIGNATURE

DATE

$278-3408 B$ 


\section{SL -55000 \\ MATERIAL SAFETY \\ DATA SHEET}

PRODUCT DESIGNATION

$03-278-4308$

o- Iodophenol

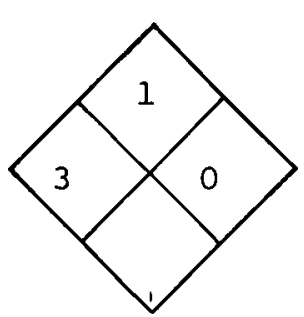

SECTION I SOURCE AND NOMENCLATURE

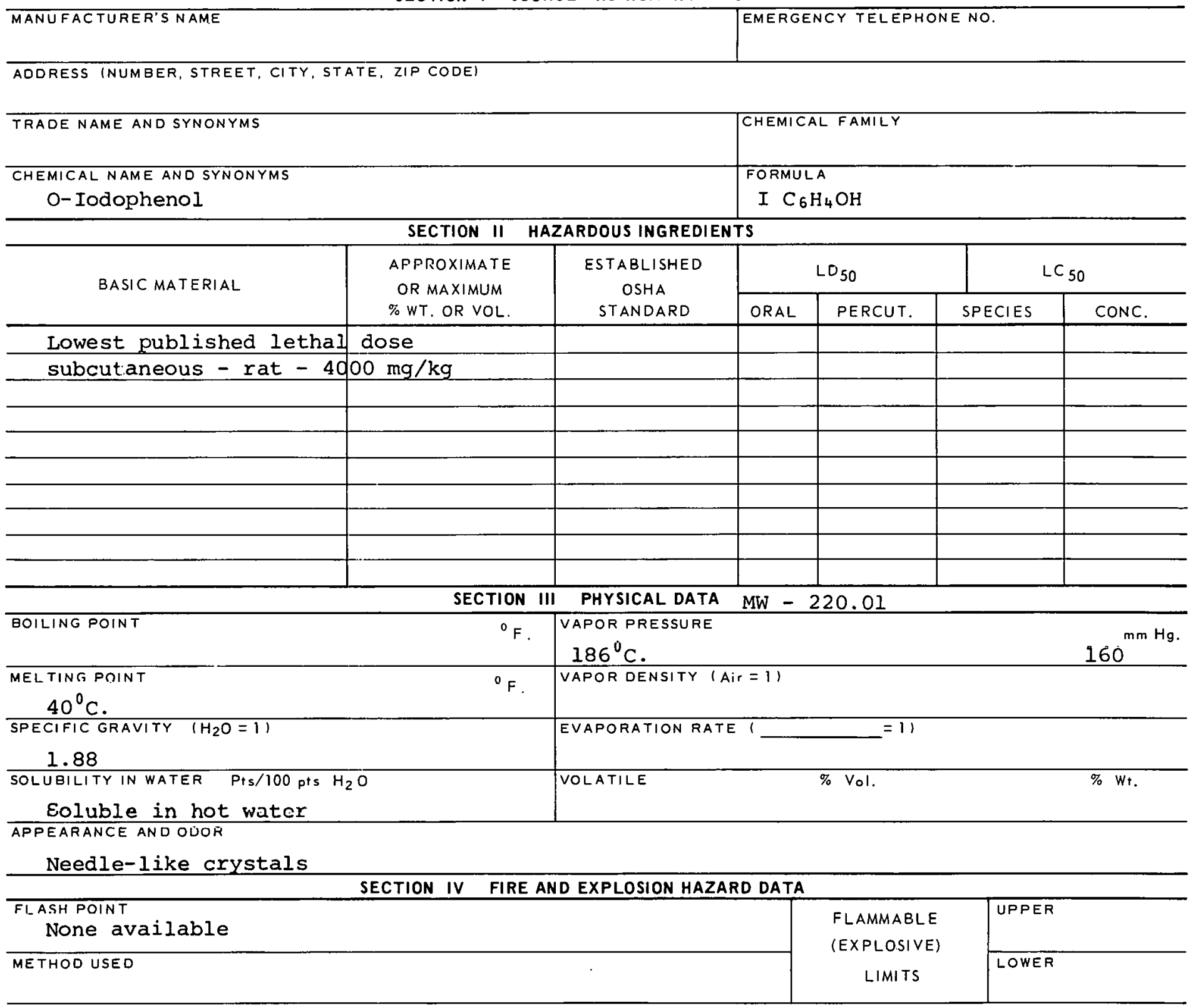

EXTINGUISHING MEDIA

Dry Elemical, alcohol foam, aarbon dioxide

SPECIAL FIRE FIGHTING PROCEDURES

Self-contained

UNUSUAL FIRE AND EXPLOSION HAZARDS

May liberate indine fumes on heating. 


\section{SECTION $V$ HEALTH HAZARD DATA}

\section{TOXIC LEVEL}

None available

CARCINOGENIC NO

PRINCIPLE ROUTES OF ABSORBTION

Inhalation, ingestion, skin

SKIN AND EYE

IRRITATION Severe

REVELANT SYMPTOMS OF EXPOSURE EYe, skin and mucous membrane irritation, dermatitis, cyanosis.

EFFECTS OF CHRONIC EXPOSURE

Liver damage

EMERGENCY AND FIRST AID PROCEOURES Inhalation - rest; wash skin \& eyes; if swallowed give emetic, get medical aid.

SECTION VI REACTIVITY DATA

CONDITIONS CONTRIBUTING TO INSTABILITY

CONDITIONS CONTRIBUTING TO HAZARDOUS POLYMERIZATION

INRIMPPATARII ITY IMATFRIAIS TR AVOIRI

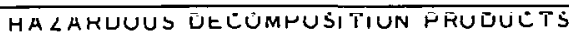

SECTION VII SPILL OR LEAK PROCEDURES

- STEPS TO BE TAKEN IN CASE MATERIAL IS RELEASED OR SPILLED

Pour sand to soda ash 9:1 mixture on spill and sweep into box for disposal.

WASTE DISPOSAL METHOD

\section{SECTION VIII SPECIAL PROTECTION INFORMATION}

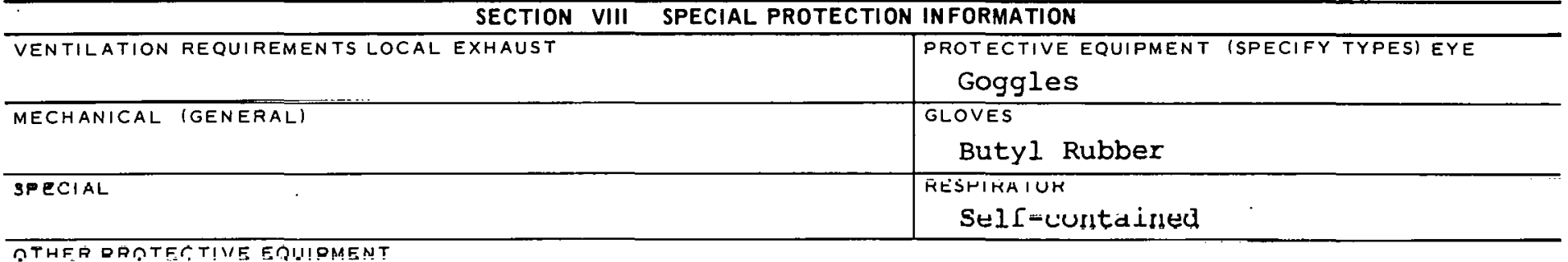

Protective clothing

SECTION IX SPECIAL PRECAUTIONS

PRECAUTIONS TO BE TAKEN IN HANDLING AND STORAGE

store in cool, dry place.

OTHER PRECAUTIONS 

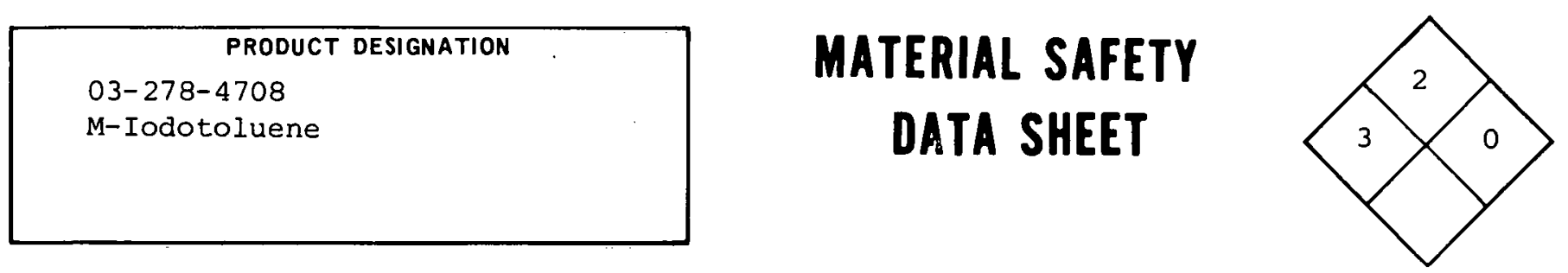

SECTION I SOURCE AND NOMENCLATURE

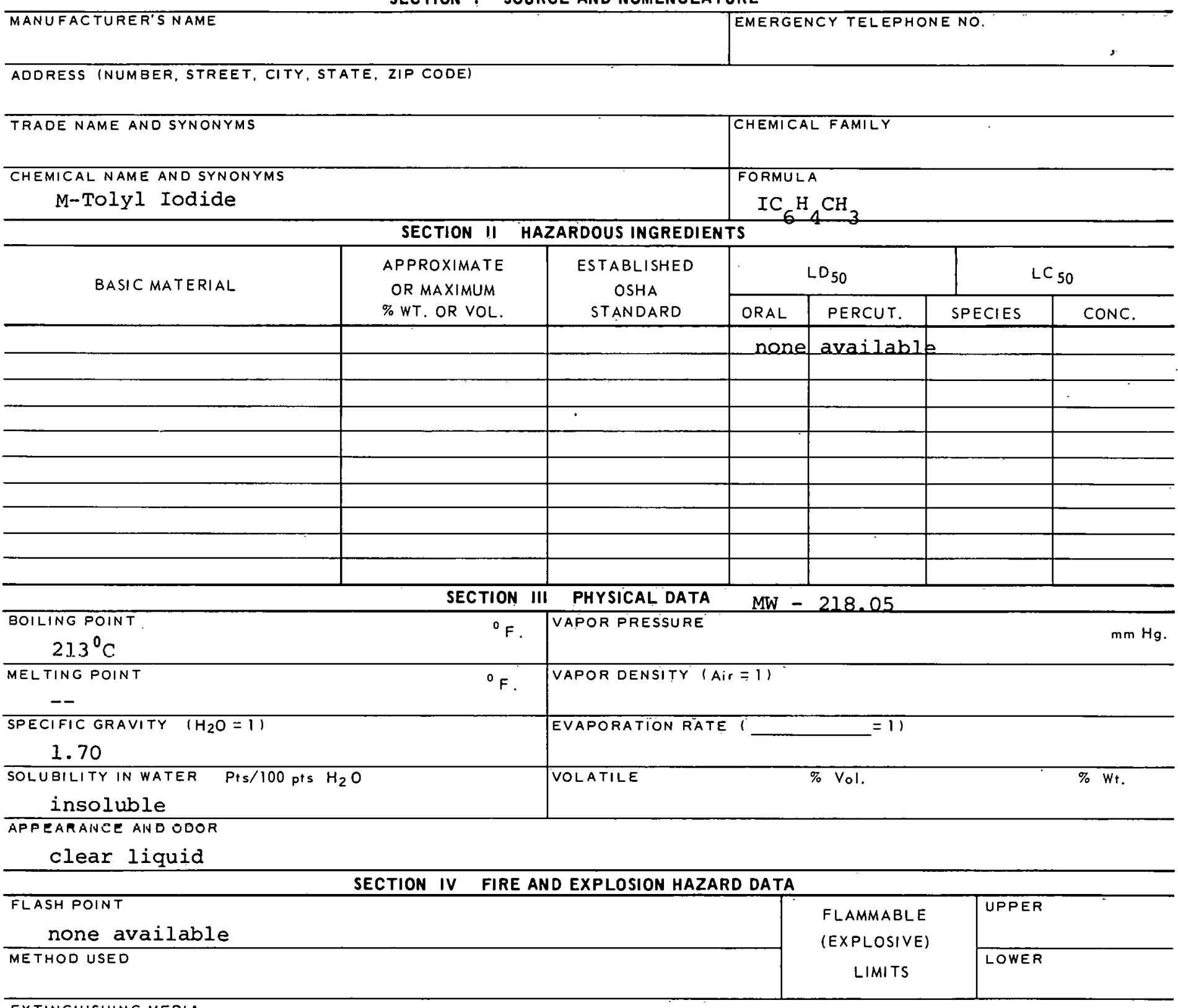

EXTINGUISHING MEDIA

alcohol foam, carbon dioxide, dry chemical. SPECIAL FIRE FIGHTING PROCEDURES

UNUSUAL FIRE AND EXPLOSION HAZARDS 


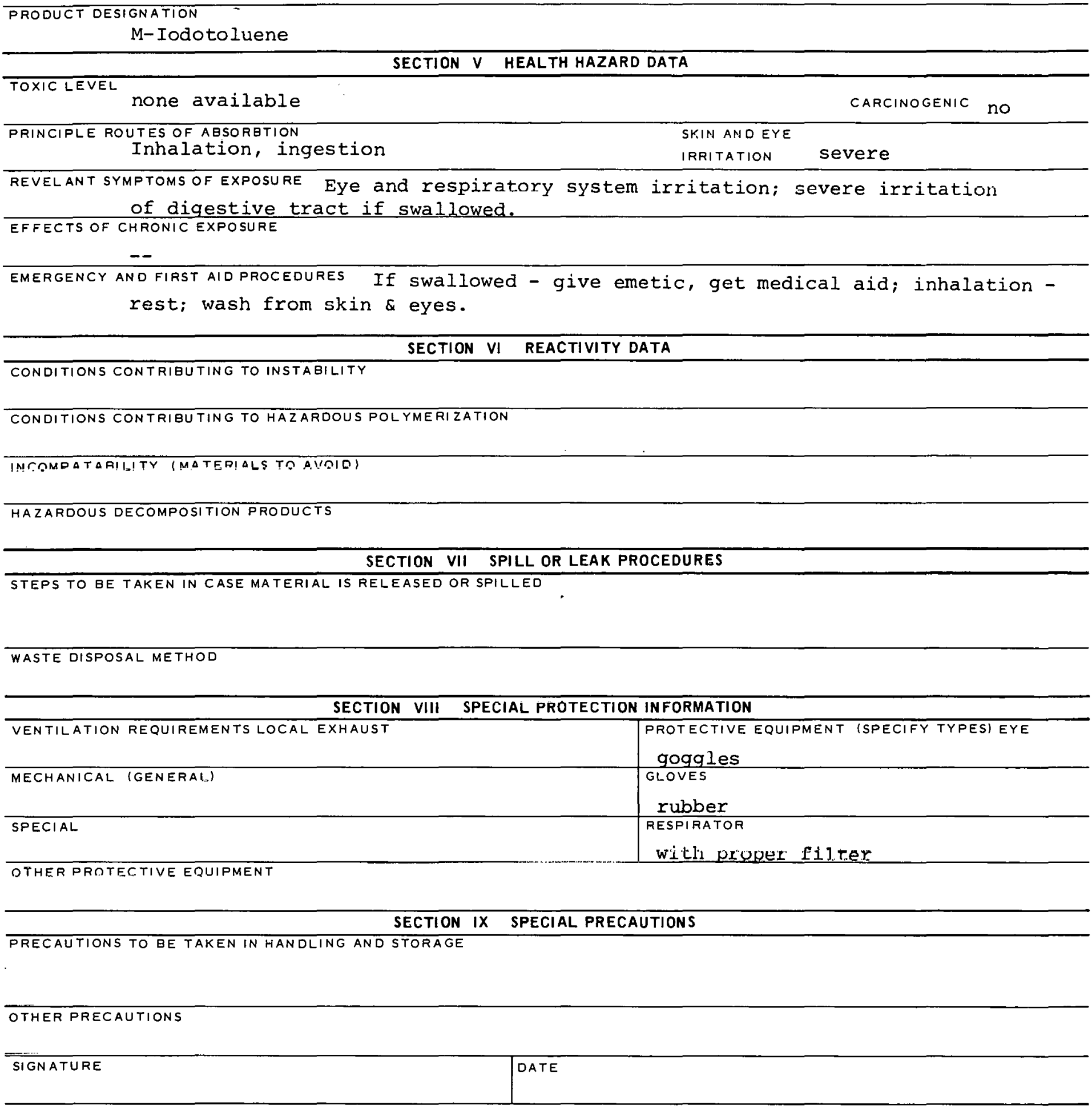



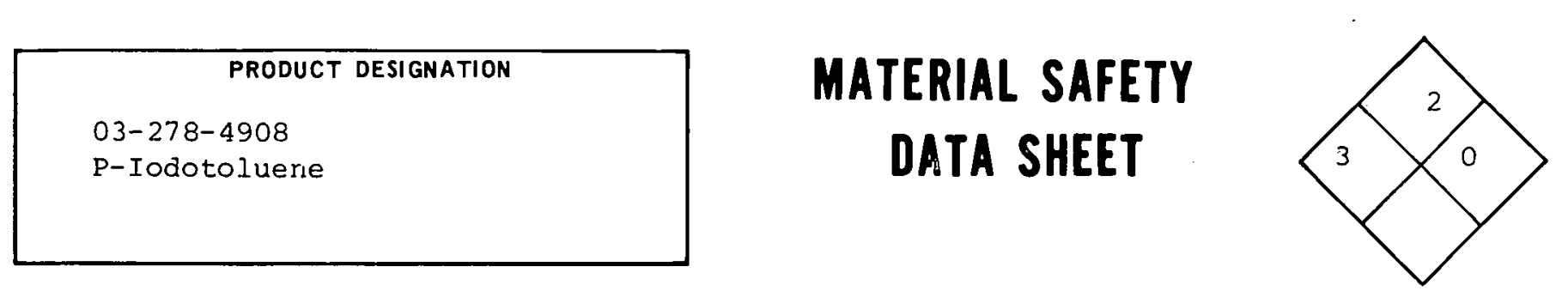

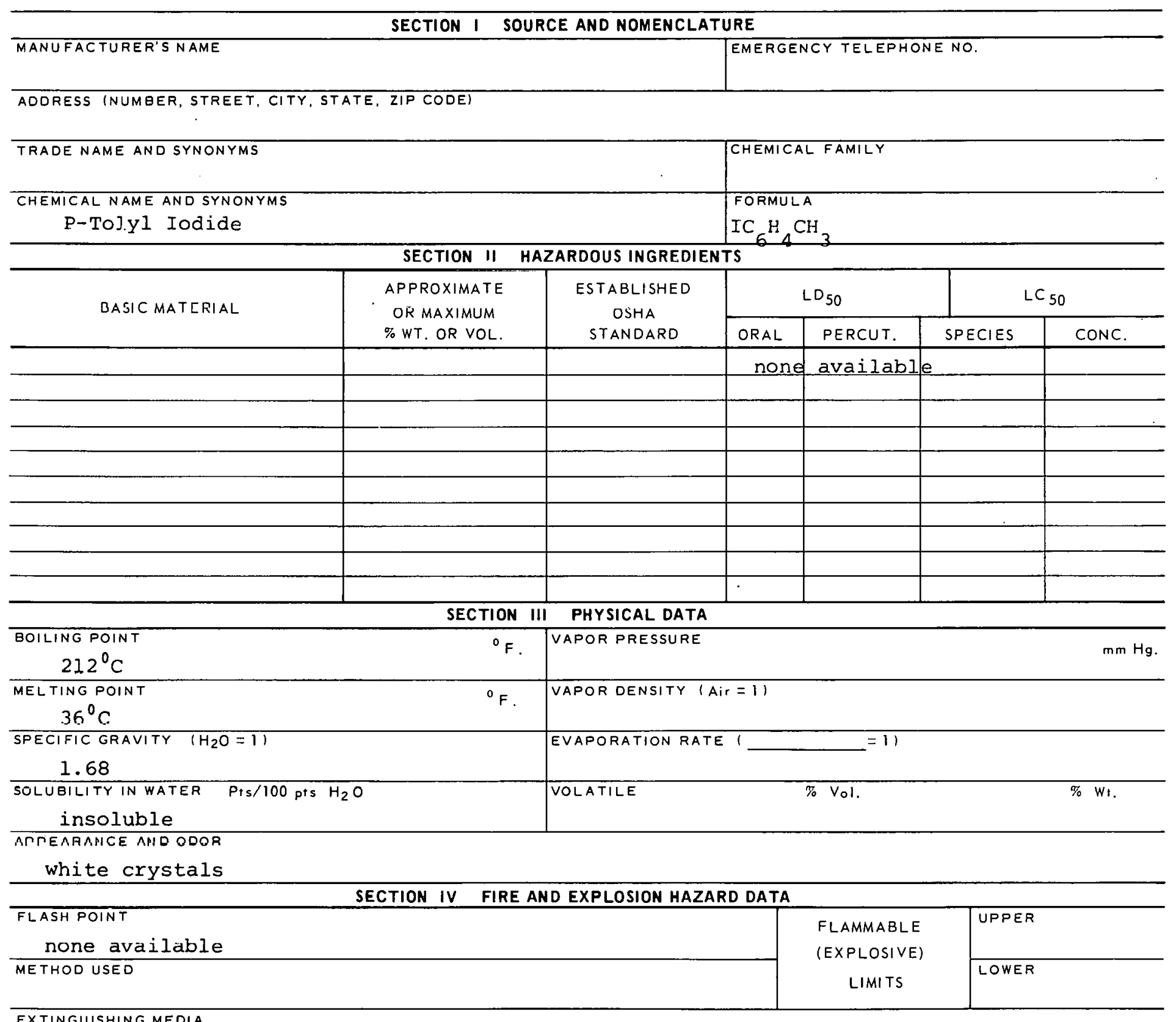

EXTINGUISHING MEDIA

alcohol foam, carbon dioxide, dry chemiçal

SPECIAL FIRE FIGHTING PROCEDURES

UNUSUAL FIRE AND EXPLOSION HAZARDS 


\section{TOXIC LEVEL}

None available

PRINCIPLE ROUTES OF ABSORBTION

Inhalation, ingestion

CARCINOGENIC

REVELANT SYMPTOMS OF EXPOSURE EYe and respiratory system irritation, severe irritation of digestive tract if swallowed.

EFFECTS OF CHRONIC EXPOSURE

$--$

EMERGENCY AND FIRST AID PROCEDURES If swallowed - give emetic, get medical aid; inhalation rest; wash from skin \& eyes.

\section{SECTION VI REACTIVITY DATA}

CONDITIONS CONTRIBUTING TO INSTABILITY

CONDITIONS CONTRIBUTING TO HAZARDOUS POLYMERIZATION

IIdCOMFATADILITY (MATERIALE TO AYOID)

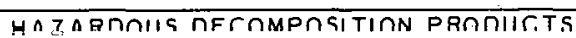

SECTION VII SPILL OR LEAK PROCEDURES

STE'PS TO BE TAKEN IN CASE MATERIAL IS RELEASED OR SPILLED

WASTE DISPOSAL METHOD

SECTION VIII SPECIAL PROTECTION INFORMATION

\begin{tabular}{l|l}
\hline VENTILATION REQUIREMENTS LOCAL EXHAUST & $\begin{array}{l}\text { PROTECTIVE EQUIPMENT (SPECIFY TYPES) EYE } \\
\text { GOGgles }\end{array}$ \\
\hline MECHANICAL (GENERAL) & $\begin{array}{l}\text { GLOVES } \\
\text { Rubber }\end{array}$ \\
\hline SPECIAL & $\begin{array}{l}\text { RESPIRATOR } \\
\text { With proper filter }\end{array}$ \\
\hline
\end{tabular}

OTHER PROTECTIVE EQUIPMENT

SECTION IX SPECIAL PRECAUTIONS

PRECAUTIONS TO BE TAKEN IN HANDLING AND STORAGE

OTHER PRECAUTIONS 
CAS : 000078591

GW77000

\begin{tabular}{|l|}
\hline \multicolumn{1}{|c|}{ PRODUCT DESIGNATION } \\
03-278-5808 \\
Isophorone \\
\hline
\end{tabular}

\section{MATERIAL SAFETY DATA SHEET}

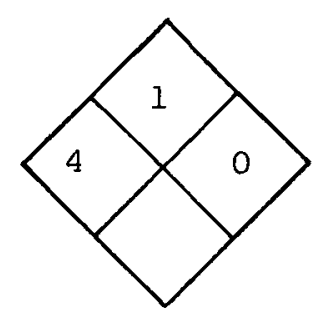

SECTION I SOURCE AND NOMENCLATURE

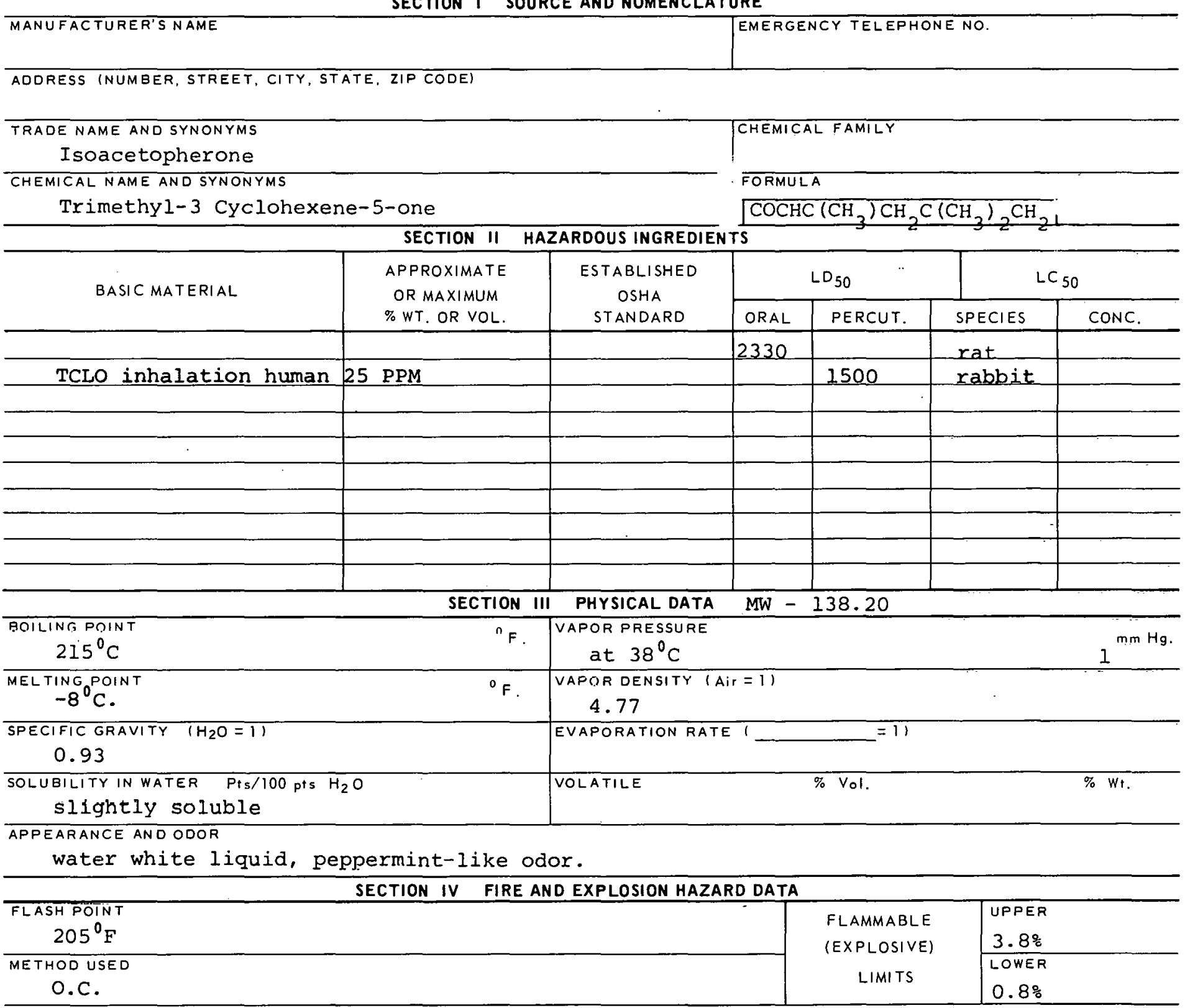

EXTINGUISHING MEDIA

Foam; carbon dioxide, dry chentical

SPECIAL FIRE FIGHTING PROCEDURES

UNUSUAL FIRE AND EXPLOSION HAZARDS 
Isophorone

\section{SECTION V HEALTH HAZARD DATA}

TOXIC LEVEL

$\mathrm{TLV}-5 \mathrm{PPM}, 25 \mathrm{mg} / \mathrm{C.M}$.

CARCINOGENIC

PRINCIPLE ROUTES OF ABSORBTION

Ingestion, inhalation

SKIN AND EYE

IRRITATION moderate to severe

REVELANT SYMPTOMS OF EXPOSURE EYe, nose and throat irritation, nausea, headache,

dizziness, faintness, inebriation.

EFFECTS OF CHRONIC EXPOSURE

Kidney poison.

EMERGENCY AND FIRST AID PROCEDURES

If swallowed - give emetic, get medical aid;

inhalation - give oxygen as needed; wash skin \& eyes.

\section{SECTION VI REACTIVITY DATA}

CONDITIONS CONTRIBUTING TO INSTABILITY

CONDITIONS CONTRIBUTING TO HAZARDOUS POLYMERIZATION

INCOMトAT"ÄBILIT' (MATERIALS TU AVUID)

Can react with oxidizing materials.

IIRZARDOUG DEGOMTOGITIOR RMOQUETC

SECTION VII SPILL OR LEAK PROCEDURES

STEPS TO BE TAKEN IN CASE MATERIAL IS RELEASED OR SPILLED

$A b s o r b$ on paper towel; evaporate in hood.

WASTE DISPOSAL METHOD

SECTION VIII SPECIAL PROTECTION INFORMATION

\begin{tabular}{|c|c|}
\hline VENTILATION REQUIREMENTS LOCAL EXHAUST & $\begin{array}{l}\text { PROTECTIVE EQUIPMENT (SPECIFY TYPES) EYE } \\
\text { SOgglCG }\end{array}$ \\
\hline MECHANICAL IGENERAL) & $\begin{array}{l}\text { GLOVES } \\
\text { Rubber }\end{array}$ \\
\hline SPECIAL & $\begin{array}{l}\text { RF.SPIRATOR } \\
\text { With propex Filtex }\end{array}$ \\
\hline
\end{tabular}

UTHER HRUTECTIVE EQUIFMENT

Coveralls

SECTION IX SPECIAL PRECAUTIONS

PRECAUTIONS TO BE TAKEN IN HANDLING AND STORAGE

OTHER PRECAUTIONS

SIGNATURE

DATE

$278-5808 B$ 
03-296-2919

2,4 Lutidine

MATERIAL SAFETY

DATA SHEET

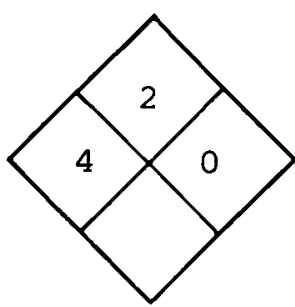

\section{SECTION I SOURCE AND NOMENCLATURE}

MANUFACTURER'S NAME

TEMERGENCY TELEPHONE NO.

ADDRESS INUMBER, STREET, CITY, STATE, ZIP CODE)

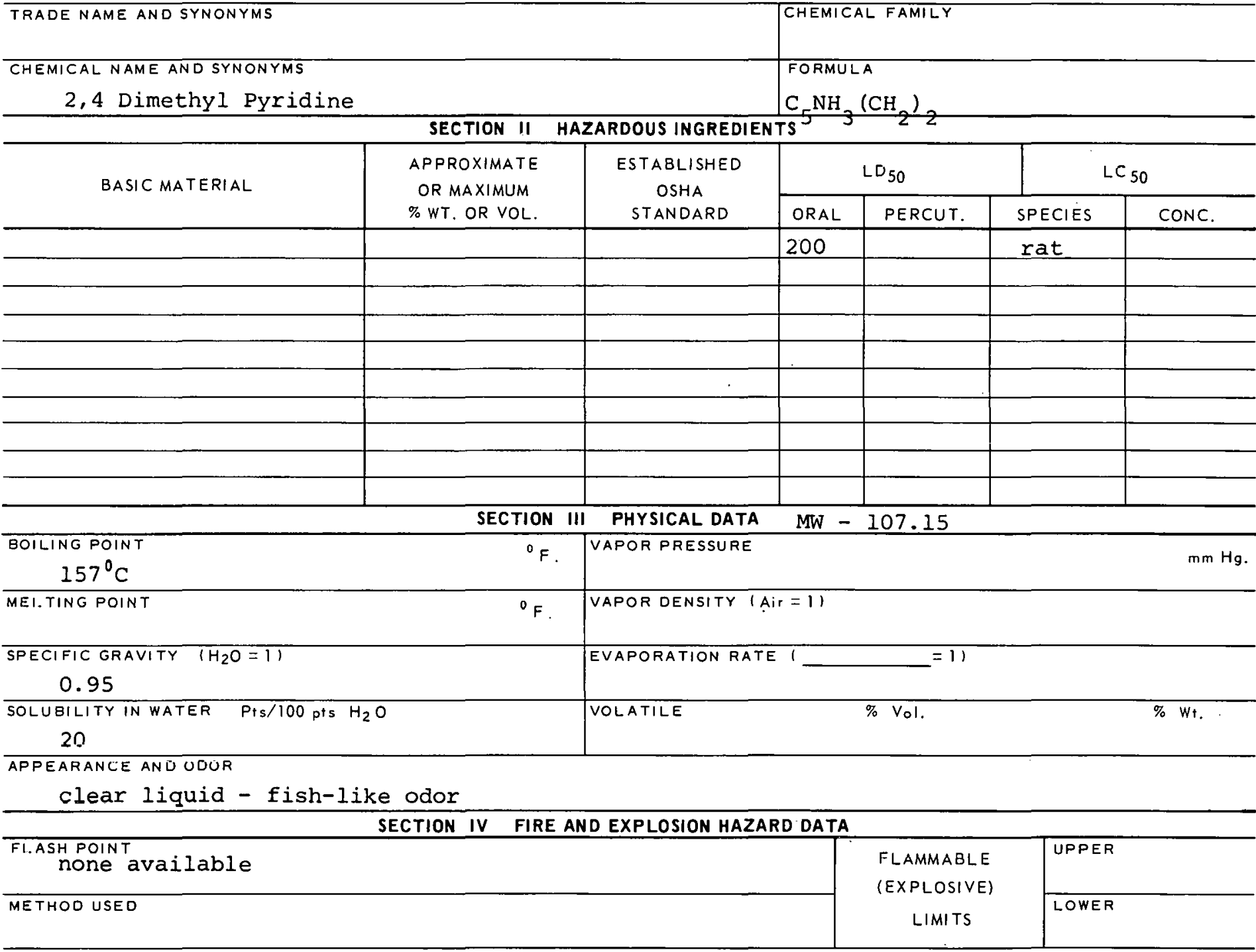

EXTINGUISHING MEDIA

alcohol foam, carbon dioxide, dry chemical

SPECIAL FIRE FIGHTING PROCEDURES

Wear goggles and self-contained breathing apparatus. UNUSUAL FIRE AND EXPLOSION HAZAROS 


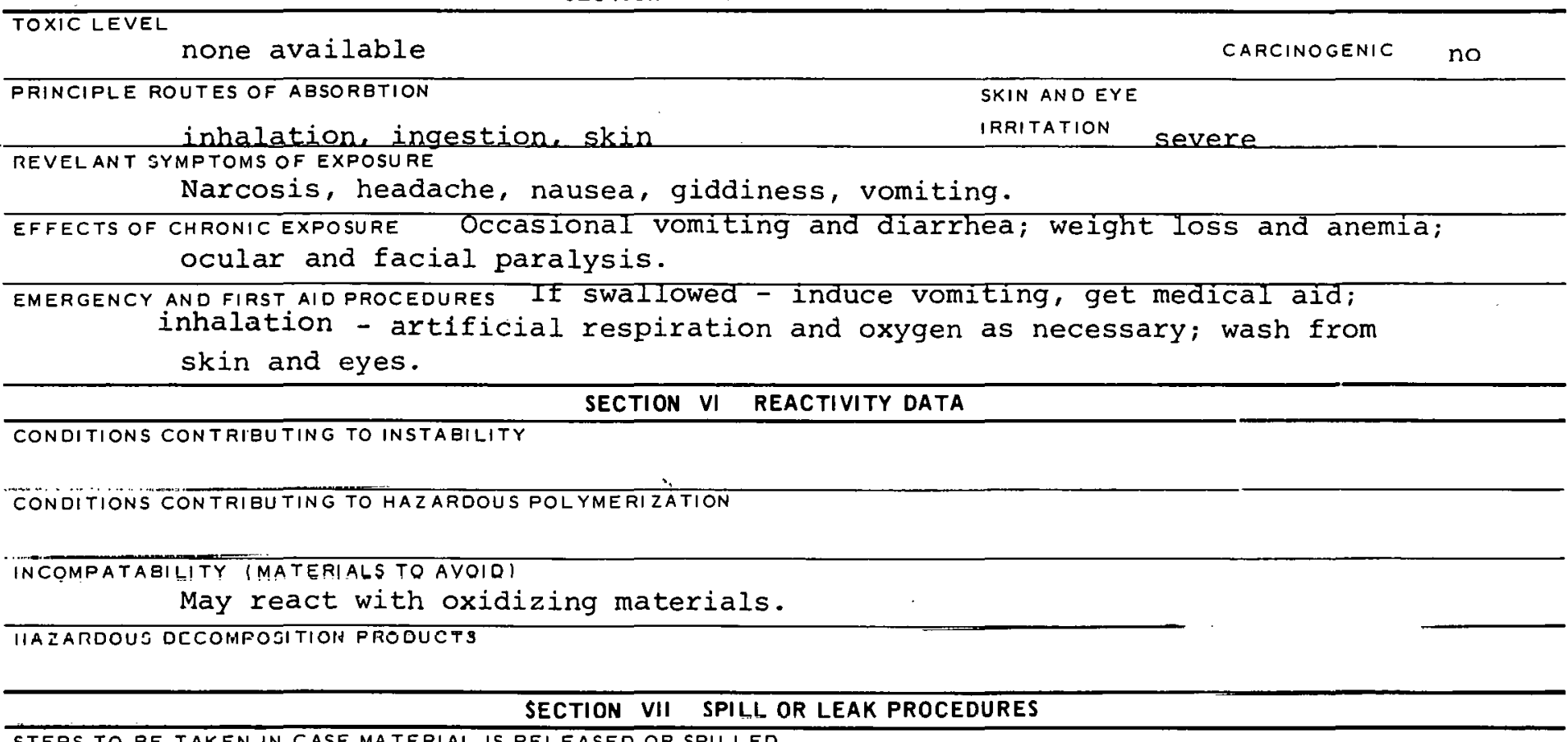

STEPS TO BE TAKEN IN CASE MA TERIAL IS RELEASED OR SPILLED

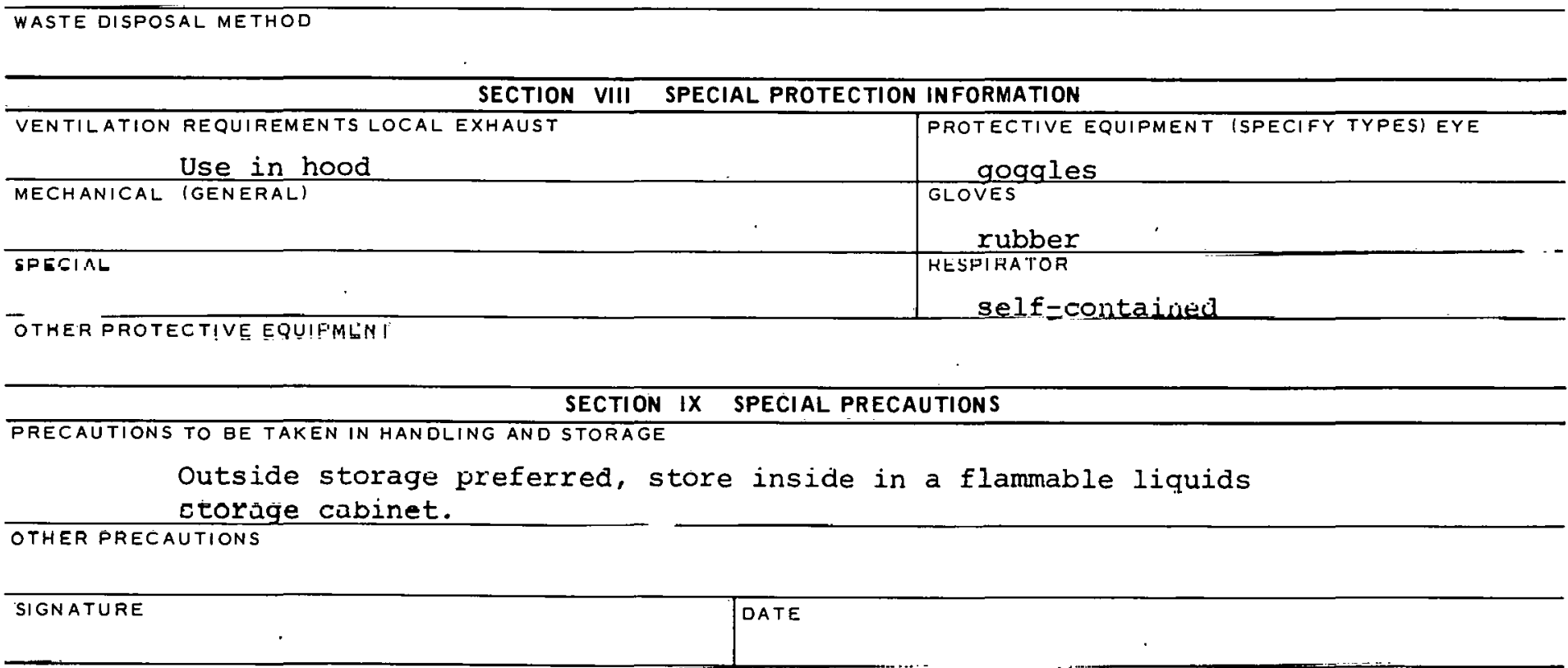




\section{PRODUCT DESIGNATION}

03-305-2601

Mesityl oxide
MATERIAL SAFETY

DATA SHEET

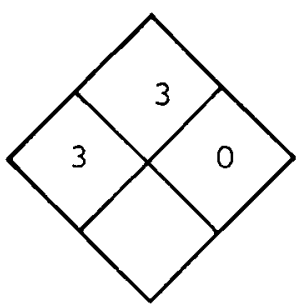

\section{SECTION I SOURCE AND NOMENCLATURE}

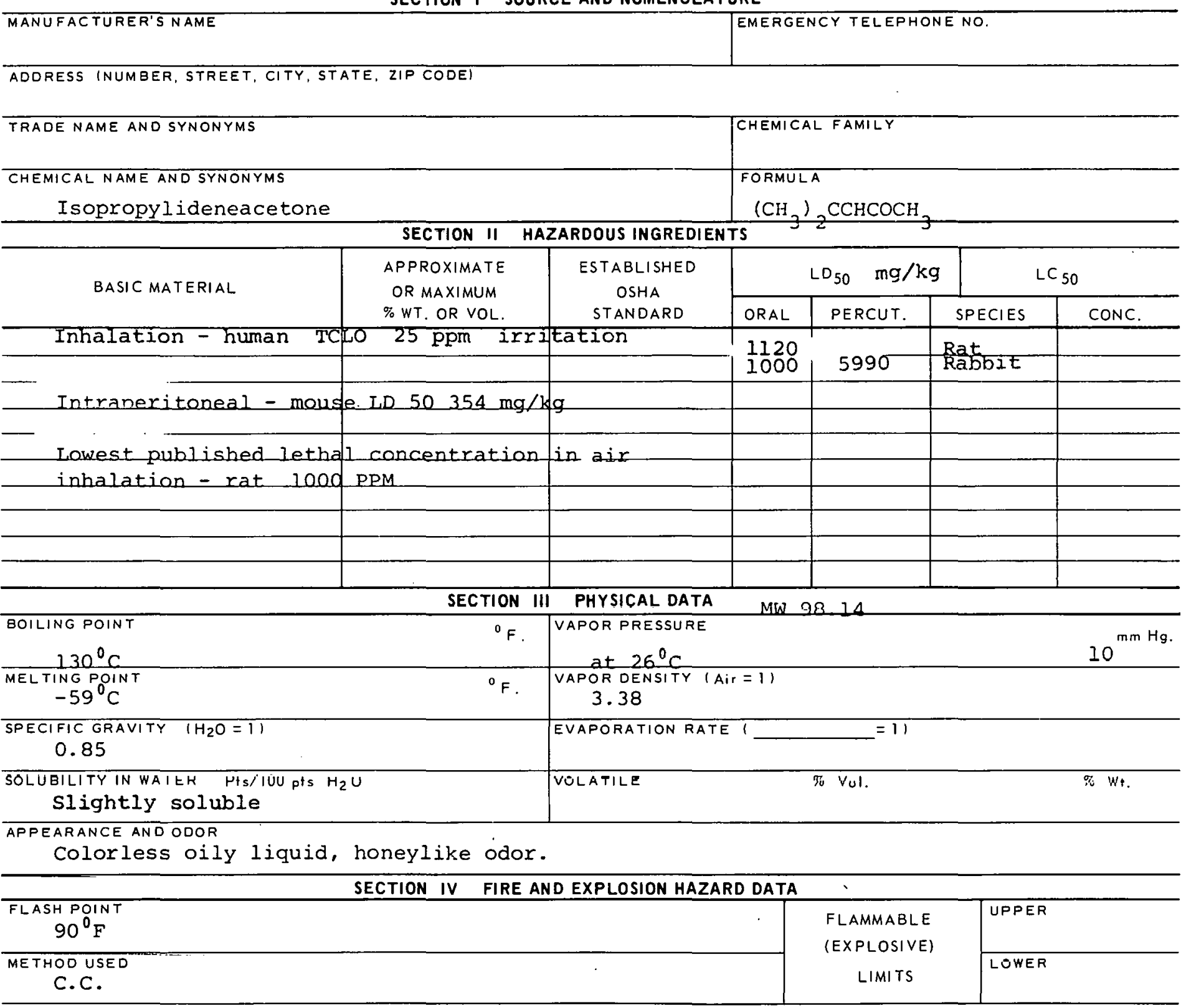

EXTINGUISHING MEDIA

Alcohol foam, carbon dioxide, dry chemical. Water may be ineffective.

SPECIAL FIRE FIGHTING PROCEDURES

Wear full protective clothing.

UHUGUAL TIRE AND EXRLOEION HAZARDS 
Mesityl oxide

TOXIC LEVEL

TLV - 25 PPM, $100 \mathrm{mg} / \mathrm{C} . \mathrm{M}$. PRINCIPLE ROUTES OF ABSORBTION

Inhalation, ingestion, skin IRRITATION severe

CARCINOCENIC

REVELANT SYMPTOMSOF EXPOSURE Dermatitis, corneal injury, respiratory irritation,

lowering of respiratory and heart rate, loss of reflexes, coma.

EFFECTS OF CHRONIC EXPOSURE

Kidney, liver and lung damage.

EMERGENCY AND FIRST AID PROCEDURES Inhalation - give oxygen as needed; if swallowed -

give emetic, get medical aid; wash from skin \& eyes.

\section{SECTION VI REACTIVITY DATA}

CONDITIONS CONTRIBUTING TO INSTABILITY

CONDITIONS CONTRIBUTING TO HAZARDOUS POLYMERIZATION

IACOMFATAELLIT'I (MATERIALS TO AVUIO)

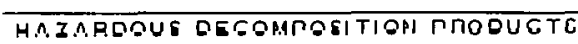

SECTION VII SPILL OR LEAK PROCEDURES

STEPS TO BE TAKEN IN CASE MATERIAL IS RELEASED OR SPILLED

Absorb on paper towel; evaporate in hood.

WASTE DISPOSAL METHOD

SECTION VIII SPECIAL PROTECTION INFORMATION

\begin{tabular}{l|l}
\hline \multicolumn{2}{c}{ SECTION VIII SPECIAL PROTECTION INFORMATION } \\
\hline VENTILATION REQUIREMENTS LOCAL EXHAUST & $\begin{array}{c}\text { PROTECTIVE EQUIPMENT ISPECIFY TYPESI EYE } \\
\text { GOGgles }\end{array}$ \\
\hline MECHANICAL IGENERAL) & GLOVES \\
Rubber & PESPIRATOR \\
WPECIAL & With proper filter \\
\hline
\end{tabular}

OTHER PROTECTIVE EQUIPMENT

SECTION IX SPECIAL PRECAUTIONS

PRECAUTIONS TO BE TAKEN IN HANDLING AND STORAGE

Store in well ventilated area. Isolate from oxidizing materials.

OTHER PRECAUTIONS

SIGNATURE

RATF

$305-2601 B$ 
CAS : 000108678

\section{PRODUCT DESIGNATION}

03-305-2701

Mesitylene
MATERIAL SAFETY

DATA SHEET

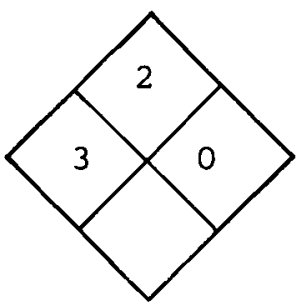

SECTION I SOURCE AND NOMENCLATURE

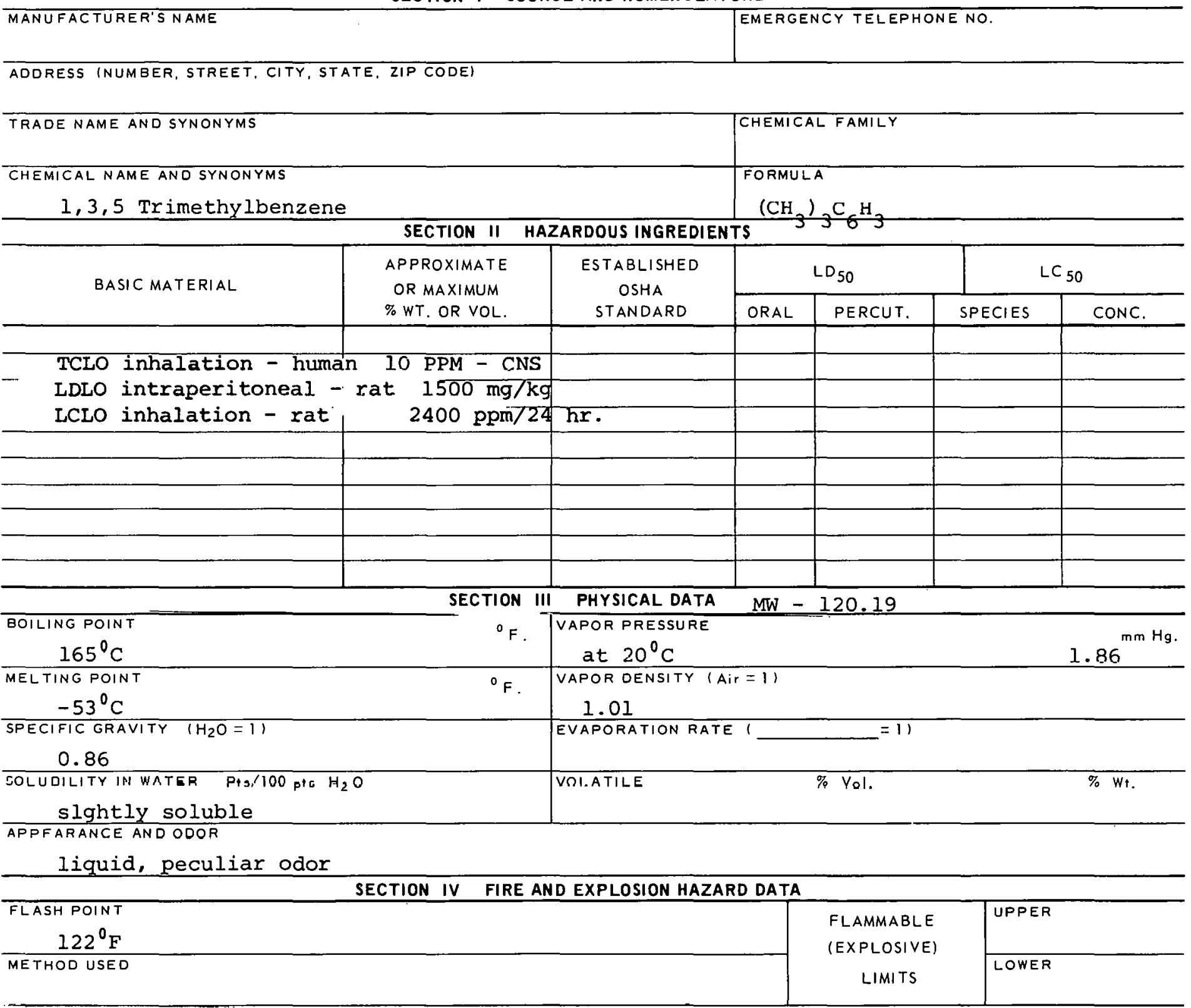

EXTINGUIGIIING MCDIA

Alcohol foam, carbon dioxide, dry chemical

SPECIAL FIRF. FIGHTING PROCEDURES

Wear full protective clothing.

IINUSIIAL FIRE AND EXPLOSI ON HAZARDS 


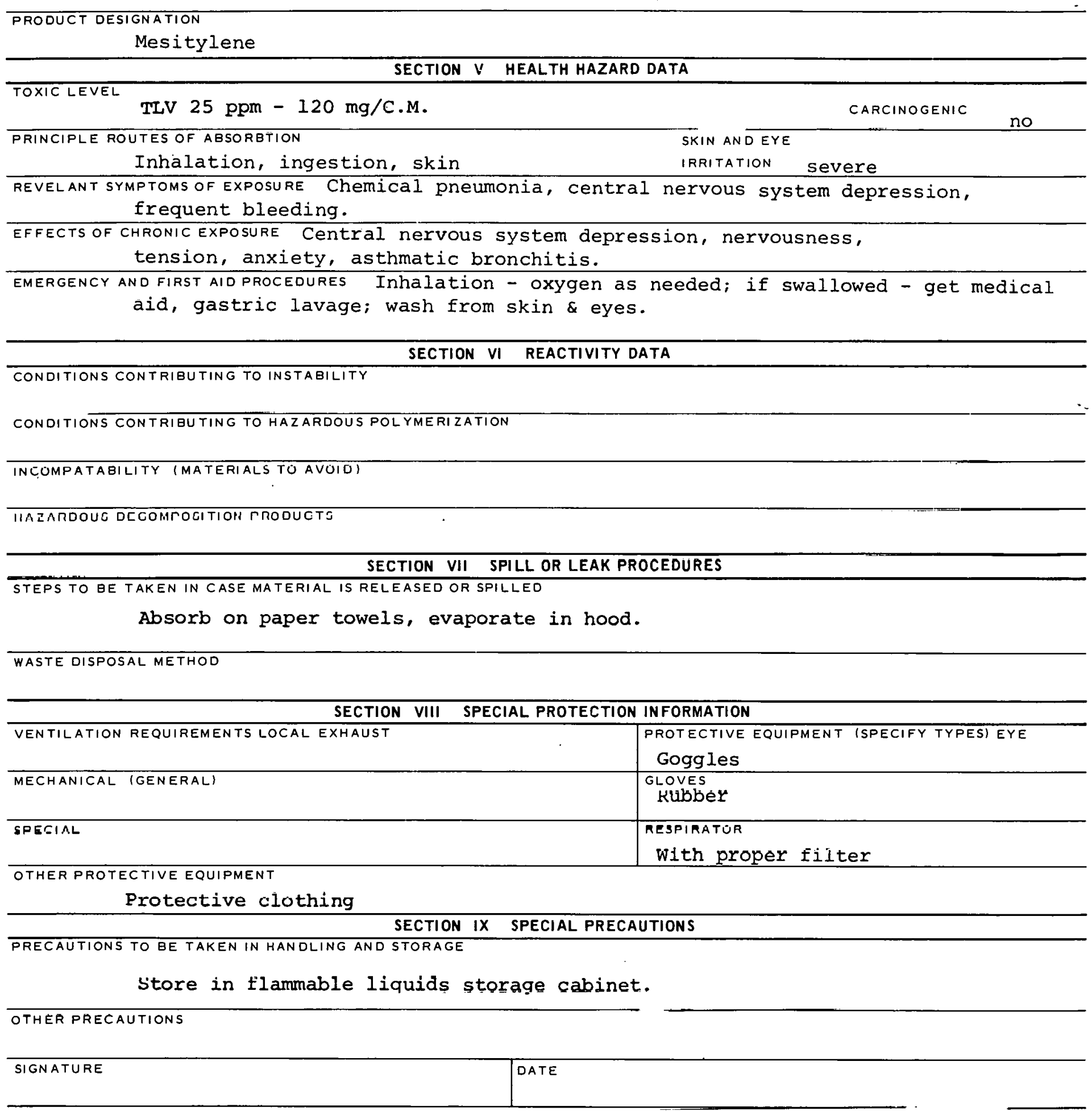


CAS : 000079209

\begin{tabular}{|c|}
\hline PRODUCT DESIGNATION \\
$03-305-5631$ \\
$03-305-5671$ \\
Methyl Acetate \\
\hline
\end{tabular}

nastoon

MATERIAL SAFETY

DATA SHEET

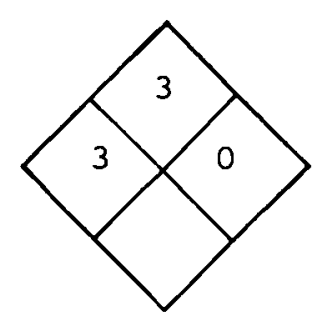

SECTION I SOURCE AND NOMENCLATURE

\begin{tabular}{l|l|l}
\hline MANUFAC TURER'S NAME & EMERGENCY TELEPHONE NO. \\
\hline ADDRESS (NUMBER, STREET, CITY, STATE, ZIP CODE)
\end{tabular}

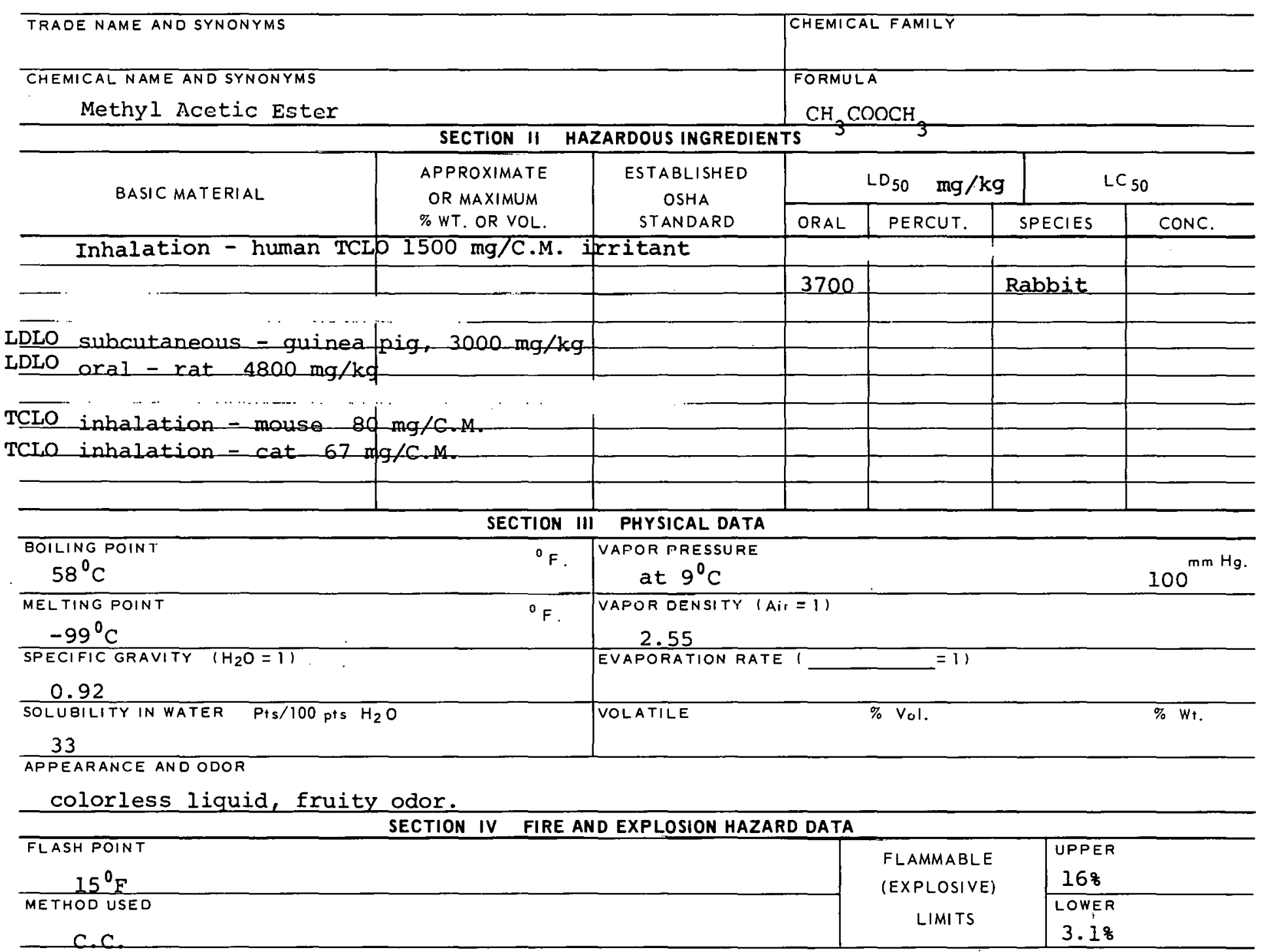

EXTINGUISHING MEDIA

Alcohol foam, carbon dioxide, dry chemical

SPECIAL FIRE FIGHTING PROCEDURES

UNUSUAL FIRE AND EXPLOSION HAZARDS 


\section{TOXIC LEVEL}

200 PPM, $610 \mathrm{mg} / \mathrm{C} . \mathrm{M}$.

PRINCIPI.F ROUTES OF AESORBTION

Ingestion, inhalation, skin SKIN AND EYE

CARCINOGENIC IRRITATION

REVELANT SYMPTOMS OF EXPOSURE Irritation of eyes and upper respiratory system, dizziness and depression, palpitation of heart.

EFFECTS OF CHRONIC EXPOSURE

$--$

EMERGENCY AND FIRST AID PROCEDURES -inhalation - rest; wash eyes and skin.

\section{SECTION VI REACTIVITY DATA}

CONDITIONS CONTRIBUTING TO INSTABILITY

CONDITIONS CONTRIBUTING TO HAZARDOUS POLYMERIZATION

INEOMPATABILITI (MATEAIALSTU AVUIU)

HAZARDOUS DECOMPOSITION PRODUCTS

SECTION VII SPILL OR LEAK PROCEDURES

STEHS TO BE IAKEN IN CASE MATERIAL IS RELEASED OR SPILLEO

Absorb on paper towels; evaporate in hood.

WASTE DISPOSAL METHOD

SECTION VIII SPECIAL PROTECTION INFORMATION

\begin{tabular}{l|l}
\hline VENTILATION REQUIREMENTS LOCAL EXHAUST & PROTECTIVE EQUIPMENT (SPECIFY TYPES) EYE
\end{tabular}

MECHANICAL (GENERAL)

$\overline{\text { SPECIAL }}$

Goggles

GLOVES

Neoprene

OTHER PROTECTIVE EQUIPMENT

RESPIRATOR

Self-sontained

SECTION IX SPECIAL PRECAUTIONS

PRECAUTIONS TO BE TAKEN IN HANDLING AND STORAGE

Store in closed container in well-ventilated area.

OTHER PRECAIITIONS

SIGNATURE

DATE

305-5631B 


\section{PRODUCT DESIGNATION}

03-305-6101

Methyl Acrylate

\section{MATERIAL SAFETY \\ DATA SHEET}

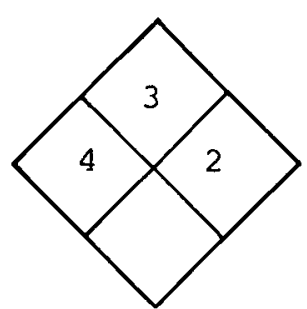

\section{SECTION I SOURCE AND NOMENCLATURE}

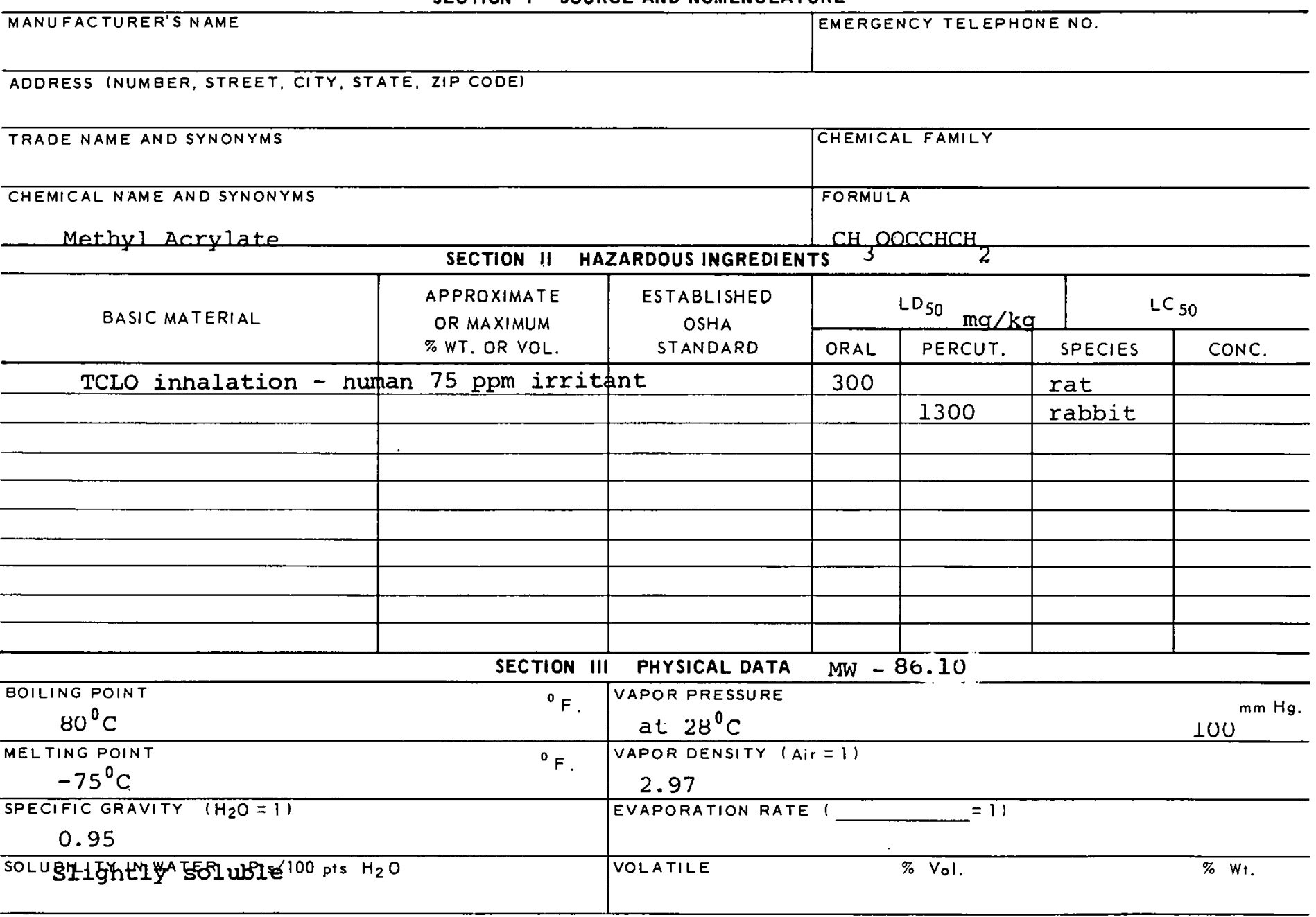

APFEARANCE AND ODOR

Colorless liquid, acrici odor.

SECTION IV FIRE AND EXPLOSION HAZARD DATA

\begin{tabular}{l|c|c}
\hline FLASH POINT & FLAMMABLE. & UPPER \\
$27^{\circ} \mathrm{C}$ & (EXPLOSIVE) & LOWER \\
\cline { 2 - 3 } $\begin{array}{l}\text { METHOD USED } \\
\text { O.C. }\end{array}$ & LIMITS & n \\
\hline
\end{tabular}

EXTINGUISHING MEDIA

Carbon dioxide, dry chemical, foam.

SPECIAL FIRE FIGHTING PROCEDURES

Water spray may be ineffective. Wear self-contained breathing apparatus.

UNUSUAL FIRE AND EXPLOSION HAZARDS

Heat may cause polymerization and rapid expansion. 
PRINCIPLE ROUTES OF ABSORATION

Inhalation, ingestion

SKIN AND EYE

IRRITATION

No

REVELANT SYMPTOMS OF EXPOSURE

Eyes, nose \& throat irritation, headache, skin eruptions.

EFFECTS OF CHRONIC EXPOSURE

Lung, liver and kidney damage.

EMERGENCY ANO FIRST AID PROCEDURES If swallowed - give emetic; get medical aid;

inhalation - artificial respiration and oxygen as needed. Wash from skin

\& eyes.

SECTION VI REACTIVITY DATA

CONDITIONS CONTRIBUTING TO INSTABILITY

CONDITIONS CONTRIBUTING TO HAZARDOUS POLYMERIZATION

Hedt

INCOMPATABILITY (MATERIALS TO AVOID)

HAZARDOUJ DECOMFOBITIOIA PRODUET 3

SECTION VII SPILL OR LEAK PROCEDURES

STEPS TO BE TAKEN IN CASE MATERIAL IS RELEASED OR SPILLED

Absorb on paper towels; evaporate in hood.

WASTE DISPOSAL METHOD

SECTION VIII SPECIAL PROTECTION IN FORMATION

\begin{tabular}{l|l}
\hline VENTILATION REQUIREMENTS LOCAL EXHAUST & $\begin{array}{l}\text { PROTECTIVE EQUIPMENT (SPECIFY TYPES) EYE } \\
\text { GOggles }\end{array}$ \\
\hline MECHANICAL IGENERAL) & GLOVES \\
\hline STEGIAL & Neoprene rubber \\
& SeLt-contained \\
\hline
\end{tabular}

OTHER PROTECTIVE EQUIPMENT

\section{SECTION IX SPECIAL PRECAUTIONS}

PRECAUTIONS TO BE TAKEN IN HANDLING AND STORAGE

Outside or detached storage preferred; inside - standard flammable Iijuils cabinet.

OTHER PRECAUTIONS 
Methylamine

( $25 \%$ in $\mathrm{H}_{2} \mathrm{O}$ )

DATA SHEET

03-305-7601

SECTION I SOURCE AND NOMENCLATURE

MANUFACTURER'S NAME
ADDRESS INUMBER, STREET, CI
TRADE NAME AND SYNONYMS
CHEMICAL NAME AND SYNONYMS
ME ThYl AMINE

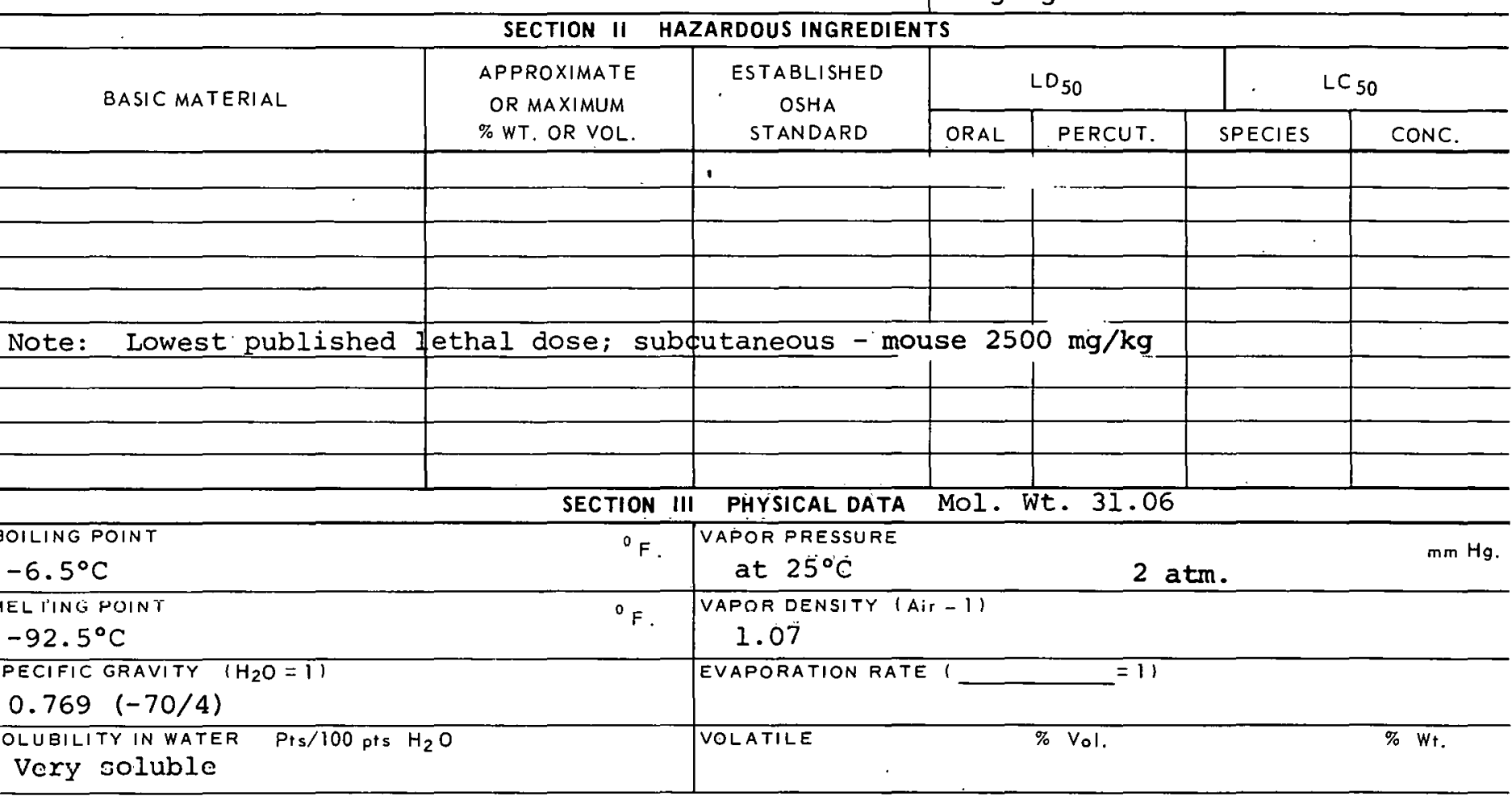

APPEARANCE AND ODOR

Colorless Iiquid with strong ammoniacal odor.

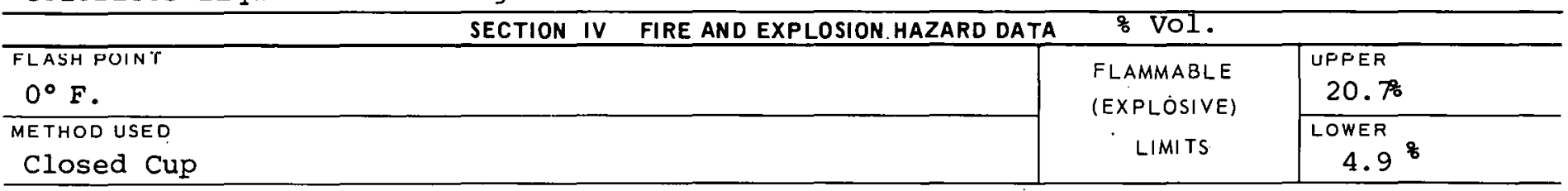

\section{EXTINGUISHING MEDIA}

water spray, carbon dloxide, dry chemical and "alculiul". fuam.

SPECIAL FIRE FIGHTING PROCEDURES

Wear full protective clothing.

UNUSUAL FIRE ANDEXPLOSION HAZAROS

Contact with mcrcury can produce explosion. May flash back. 


\section{TOXIC LEVEL}

TLV $10 \mathrm{ppm}, 12 \mathrm{mg} / \mathrm{C} . \mathrm{M}$. PRINCIPLE ROUTES OF ABSORBTION Inhalation of vapor

SKIN AND EYE

IRRITATION Moderate to severe

REVELANT SYMPTOMS OF EXPOSURE Vapor is an irritant to respiratory system and eyes, may affect central nervous system, coughing, choking. EFFECTS OF CHRONIC EXPOSURE

EMERGENCY AND FIRST AID PROCEDURES Remove patient from exposure; if on skin or eyes, drench or irrigate thoroughly with water, rest, keep warm, apply artificial respiration if necessary; obtain medical attention.

\section{SECTION VI REACTIVITY DATA}

CONDITIONS CONTRIBUTING TO INSTABILITY

CONDITIONS CONTRIBUTING TO HAZARDOUS POLYMERIZATION

INCOMPATARII ITY (MATFRIAI STO AVDID)

Mereury

HAZARDOUS DECOMPOSITION PRODUCTS

SF.CTION VII SPILL OR LEAK PROCEDURES

STEPS TO BE TAKEN IN CASE MATERIAL IS RELEASED OR SPILLED

Spread sodium bisulfate on spill and sprinkle with water. Drain into sewer.

WASTE DISPOSAL METHOD

\section{SECTION VIII SPECIAL PROTECTION INFORMATION}

\begin{tabular}{|c|c|c|}
\hline VENTILATION REQUIREMENTS LOCAL EXHAUST & & $\begin{array}{l}\text { PROTECTIVE EQUIPMENT (SPECIFY TYPES) EYE } \\
\text { Goggles or safety glasses }\end{array}$ \\
\hline MECHANICAL (GENERAL) & & $\begin{array}{l}\text { GLOVES } \\
\text { Rubber (butyl) }\end{array}$ \\
\hline SPECIAL & & $\begin{array}{l}\text { RESPIRATOR } \\
\text { w//aPPYOpriato filter }\end{array}$ \\
\hline \multicolumn{3}{|l|}{ Full-face shield, protective clothing. } \\
\hline \multicolumn{3}{|c|}{ SECTION IX SPECIAL PRECAUTIONS } \\
\hline \multicolumn{3}{|c|}{$\begin{array}{l}\text { PRECAUTIONS TO BE TAKEN IN HANDLING AND STORAGE } \\
\text { Should be stored in standard flammable liquids } \\
\text { moroury. }\end{array}$} \\
\hline \multicolumn{3}{|l|}{ OTHER PRECAUTIONS } \\
\hline SIGNATURE & DATE & \\
\hline
\end{tabular}



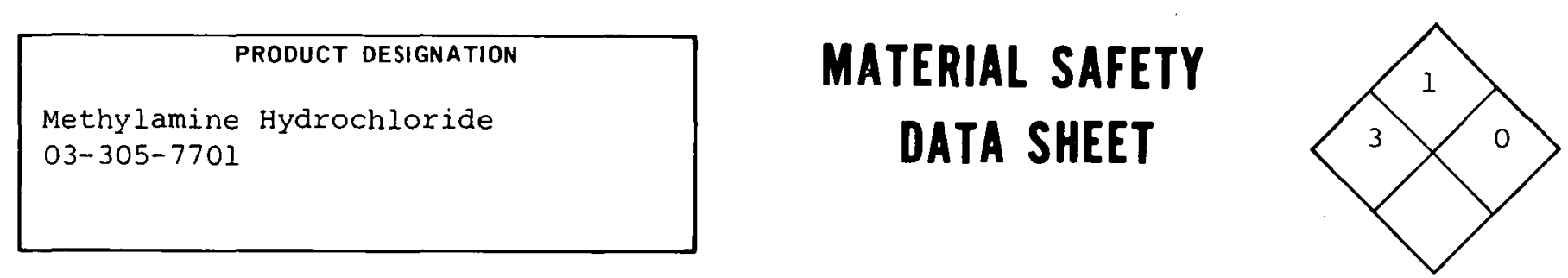

\section{SECTION I SOURCE AND NOMENCLATURE}

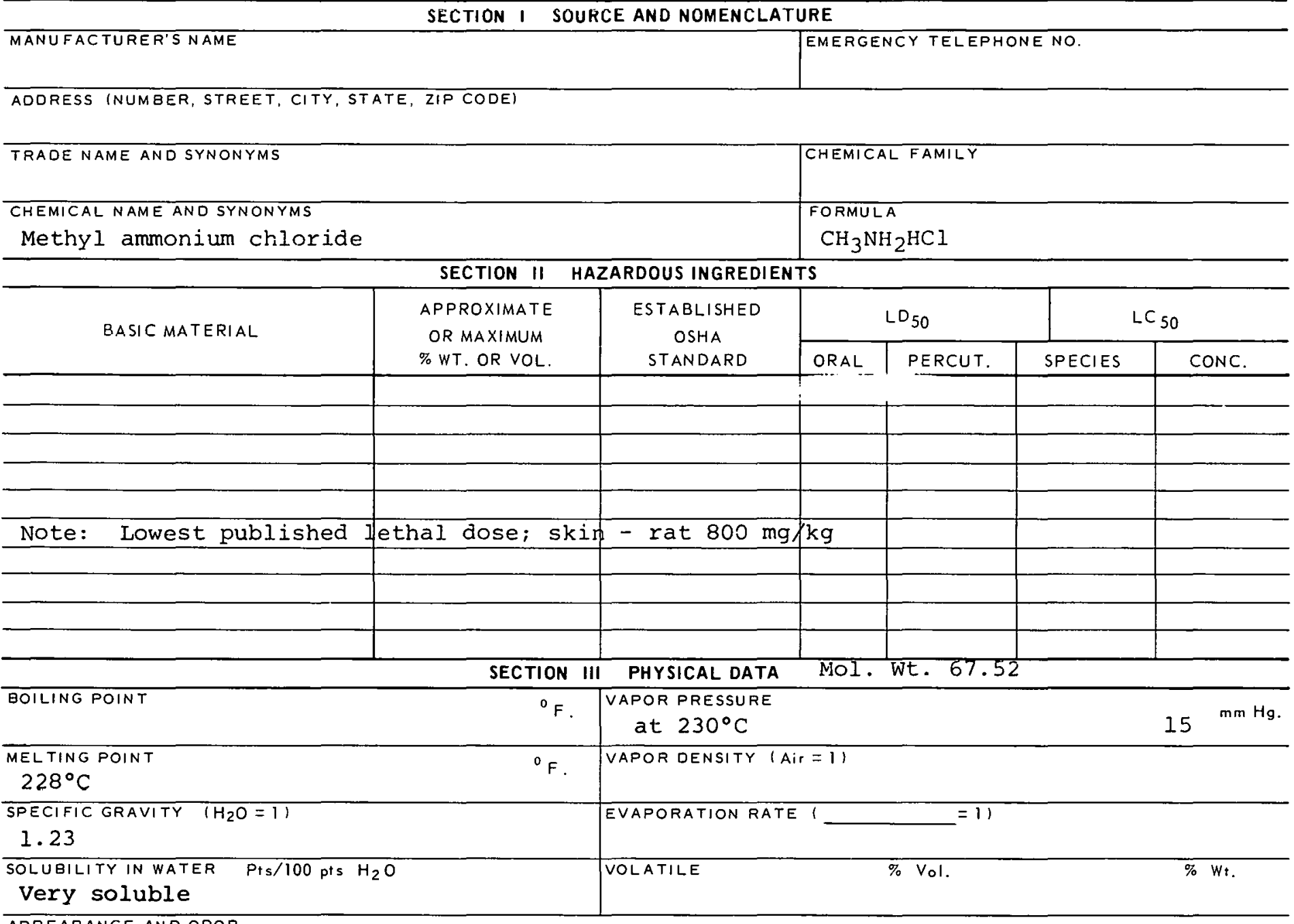

APPEARANCE AND ODQR

SECTION IV FIRE AND EXPLOSION HAZARD DATA

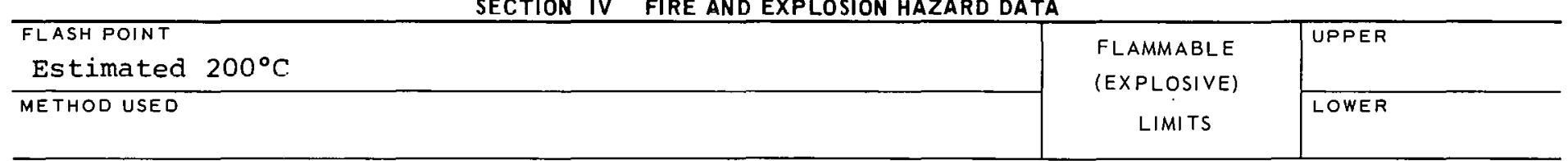

EXTINGUISHING MEDIA

water spray, alcohol foam, $\mathrm{CO}_{2}$, dry chemical.

SPECIAL FIRE FIGHTING PROCEDURES

Wear full protective clothing

UNUSUAL FIRE AND EXPLOSION HAZAROS 


\section{TOXIC LEVEL}

None available

PRINCIPLE ROUTES OF ABSORBTION

Ingestion, inhalation of dust REVELANT SYMPTOMS OF EXPOSURE

faintness and anxiety.

EFFECTS OF CHRONIC EXPOSURE

EMERGENCY AND FIRST AID PROCEDURES Inhalation - rest, keep warm, get medical attention; if swallowed - wash mouth with water, give plenty of water to drink; wash skin and eyes with water.

\begin{tabular}{ll}
\hline & SECTION VI REACTIVITY DATA \\
\hline CONDITIONS CONTRIBUTING TO INSTABILITY &
\end{tabular}

CONDITIONS CONTRIBUTING TO HAZARDOUS POLYMERIZATION

INCOMPATABILITY IMATERIALS TO AVOID

HAZARDOUS DECOMPOSITION PRODUCTS

SECTION VII SPILL OR LEAK PROCEDURES

STEPS TO BE TAKEN IN CASE MATERIAL IS RELEASED OR SPILLED

\section{SECTION VIII SPECIAL PROTECTION INFORMATION}

\begin{tabular}{|c|c|}
\hline VENTILATION REQUIREMENTS LOCAL EXHAUST & $\begin{array}{l}\text { PROTECTIVE EQUIPMENT (SPECIFY TYPES) EYE } \\
\text { Safety glasses }\end{array}$ \\
\hline MECHANICAL (GENERAL) & $\begin{array}{l}\text { GLOVES } \\
\text { Rubber }\end{array}$ \\
\hline SГEGIML & $\begin{array}{l}\text { RESFIRATUR } \\
\text { W/approprlate filter }\end{array}$ \\
\hline
\end{tabular}

OTHER PROTECTIVE EQUIPMENT

Face shield

SECTION IX SPECIAL PRECAUTIONS

PRECAUTIONS TO BE TAKEN IN HANDLING AND STORAGE

OTHER PRECAUTIONS

SIGNATURE

DATE

$305-7701 B$ 
CAS : 000110430

PRODUCT DESIGNATION

03-305-9601

Methyl N-Amyl Ketone
MJ50750

MATERIAL SAFETY

DATA SHEET

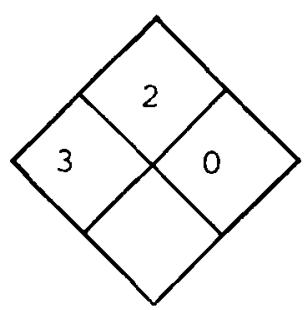

\section{SECTION I SOURCE AND NOMENCLATURE}

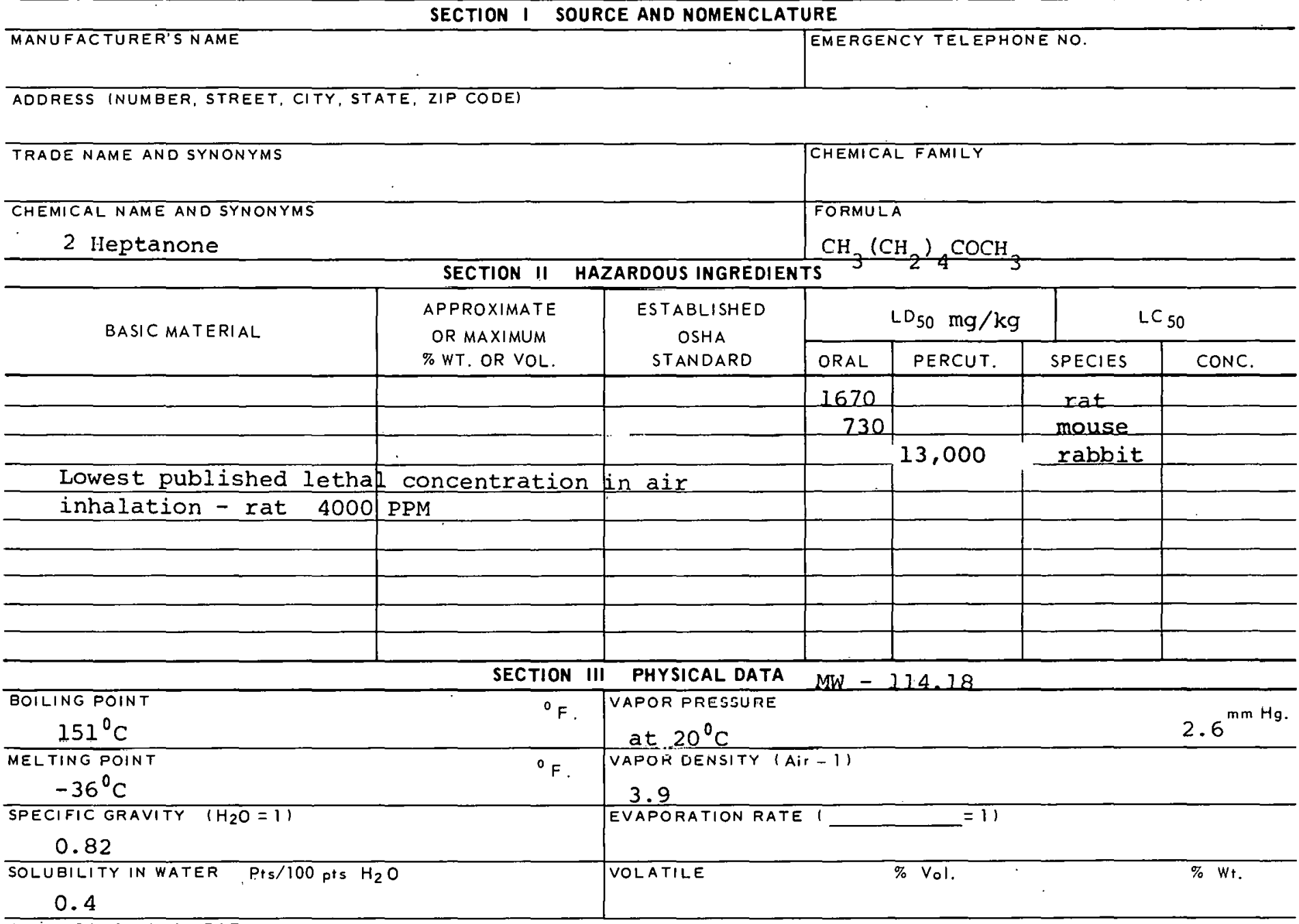

APPEARANCE AND ODOR

Water white liquid

SECTION IV FIRE AND EXPLOSION HAZARD DATA

\begin{tabular}{|c|c|c|}
\hline $\begin{array}{c}\text { FLASH POINT } \\
120^{\circ} \mathrm{F}\end{array}$ & $\begin{array}{l}\text { FLAMMABLE } \\
\text { (EXPLOSIVE) }\end{array}$ & UPPER \\
\hline $\begin{array}{c}\text { METHOD USED } \\
\text { O.C. }\end{array}$ & LIMITS & LOWER \\
\hline
\end{tabular}

EXT!NGUISHING MEDIA

Foam, carbon dioxide, dry chemical

SPECIAL FIRE FIGHTING PROCEDURES

UNUSUAL FIRE AND EXPLOSION HAZARDS 
Methyl N-Amyl Ketone SECTION $V$ HEALTH HAZARD DATA

TOXIC LEVEL

$100 \mathrm{PPM}, 465 \mathrm{mg} / \mathrm{kg}$.

PRINCIPLE ROUTES OF AESORBTION

Inhalation, ingestion

CARCINOGENIC

SKIN AND EYE

IRRITATION moderate

REVELANT SYMPTOMS OF EXPOSURE

Irritation of mucous membranes, narcosis, eye irritation.

EFFECTS OF CHRONIC EXPOSURE

$--$

EMERGENCY AND FIRST AID PROCEDURES

rest; wash eyes and skin.

If swallowed - give emetic, get medical aid; inhalation -

SECTION VI REACTIVITY DATA

CONOITIONS CONTRIBUTING TO INSTABILITY

CONDITIONS CONTRIBUTING TO HAZARDOUS POLYMERIZATION

INCOMPATABILITY (MATERIALS TO AVOIO)

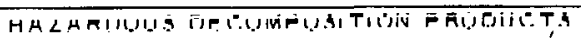

SECTION VII SPILL OR LEAK PROCEDURES

STEPS TO BE TAKEN IN CASE MATERIAL IS RELEASED OR SPILLED

WASTE DISPOSAL METHOD

SECTION VIII SPECIAL PROTECTION INFORMATION

\begin{tabular}{|c|c|}
\hline VENTILATION REQUIREMENTS LOCAL EXHAUST & $\begin{array}{l}\text { PROTECTIVE EQUIPMENT (SPECIFY TYPES) EYE } \\
\text { GOggleS }\end{array}$ \\
\hline MEGIIAPHIGAL (GENERAL) & $\begin{array}{l}\text { OLUVEJ } \\
\text { Neoprene }\end{array}$ \\
\hline SPFC,IAI & $\begin{array}{l}\text { REETIAATOR } \\
\text { With proper filter }\end{array}$ \\
\hline
\end{tabular}

OTHER PROTECTIVE EOUIPMENT

SECTION IX SPECIAL PRECAUTIONS

PRECAUTIONS TO BE TAKEN IN HANDLING ANO STORAGE

OTHER PRECAUTIONS

SIGNATURE

DATE

DATE

$305-9601 B$ 
CAS : 000100618

PRODUCT DESIGNATION

03-306-0202

$\mathrm{N}$-Methylaniline
BY45500

MATERIAL SAFETY

DATA SHEET

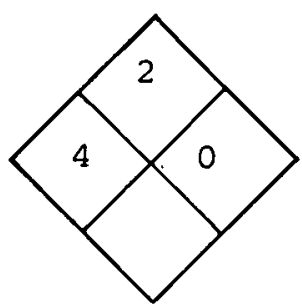

\section{SECTION I SOURCE AND NOMENCLATURE}

MANUFACTURER'S NAME

ADDRESS (NUMBER, STREET, CITY, STATE, ZIP CODE)

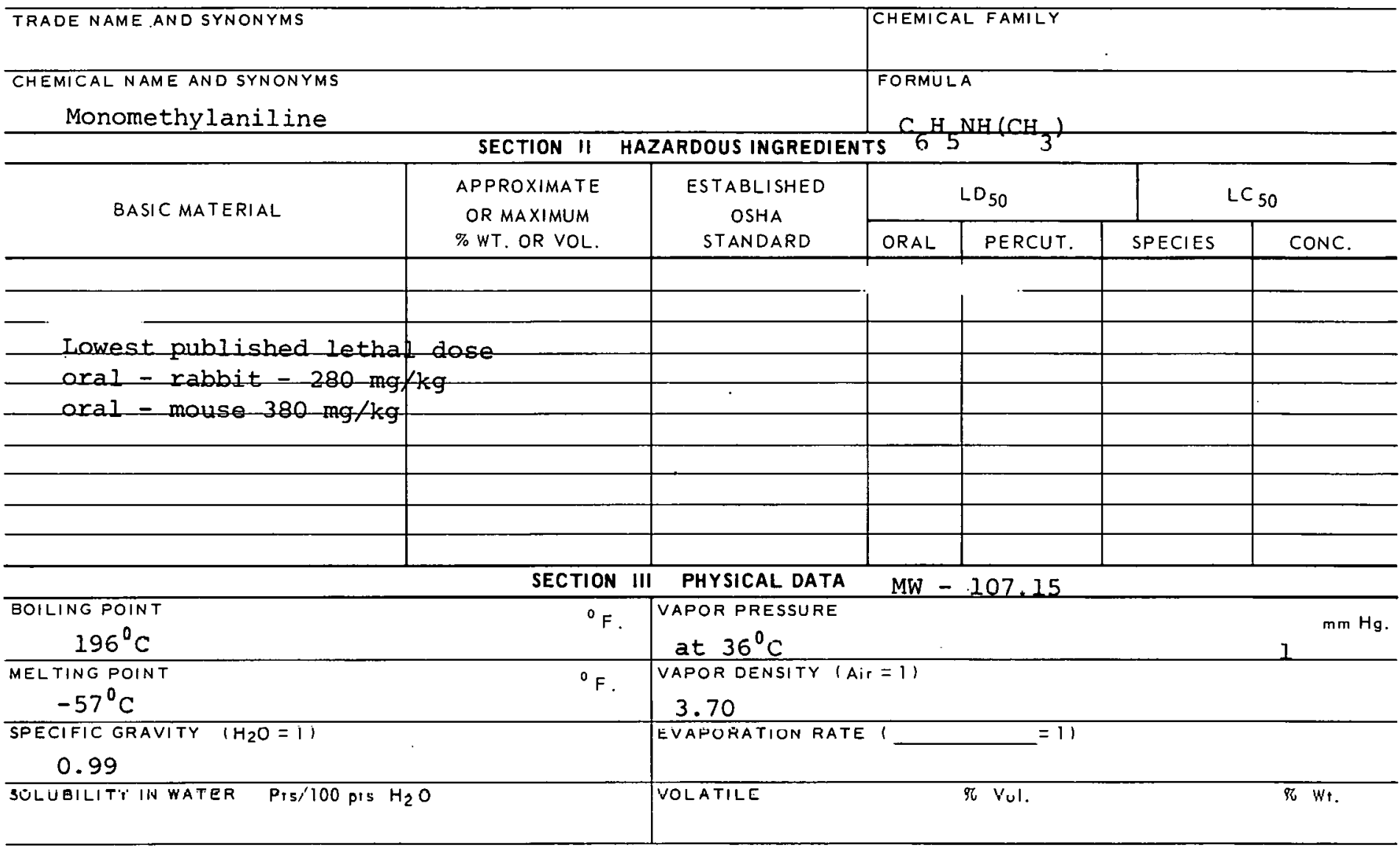

APPEARANCE AND ODOR

Reddish brown, oily liquid.

SECTION IV FIRE AND EXPLOSION HAZARD DATA

\begin{tabular}{|c|c|c|}
\hline $\begin{array}{l}\text { FLASH POINT } \\
\text { None available }\end{array}$ & FLAMMABLE & UPPER \\
\hline METHOD USED & & LOWER \\
\hline
\end{tabular}

EXIINGUISHING MEUIA

Alcohol foam, carbon dioxide, dry chemical

SFECIAL FIRE FIGHTING PROCEDURES

UNUSUAL FIRE AND EXPLDS!ON HATARIS

hen heated to decomposition, emits aniline fumes. 


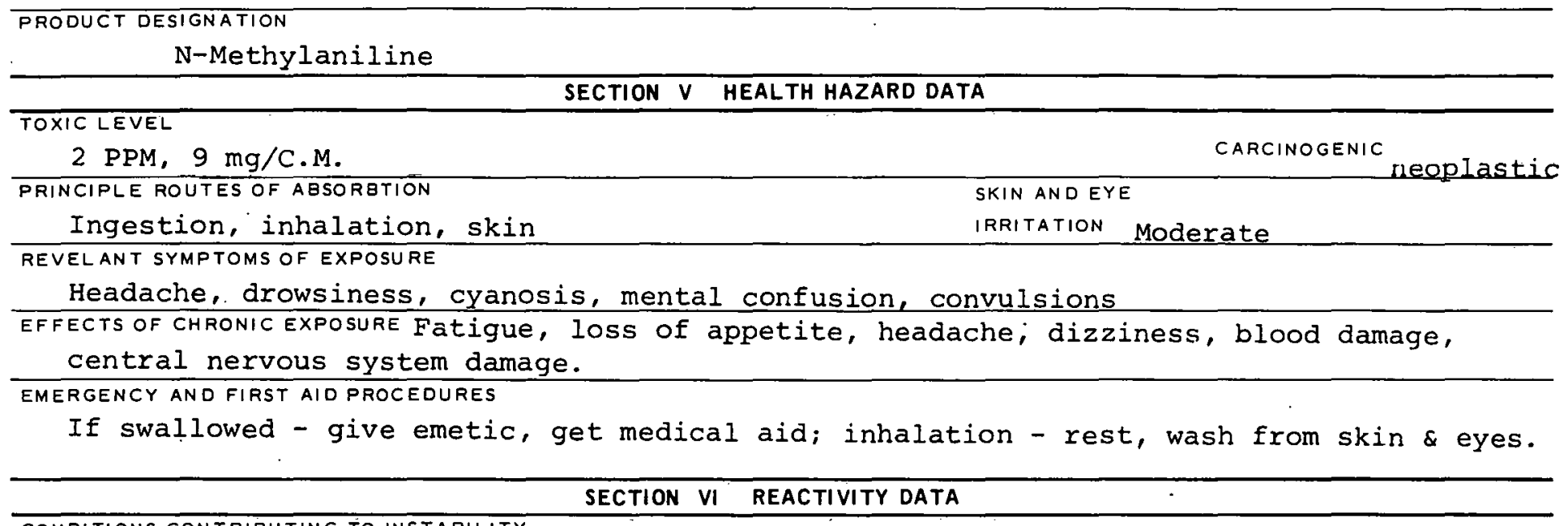




\begin{tabular}{|l|l|l|l|}
\hline PRODUCT DESIGNATION \\
$03-306-2702$ \\
Methyl Bromoacetate
\end{tabular}

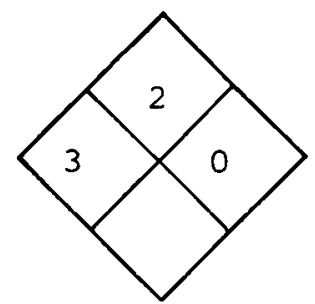

SECTION I SOURCE AND NOMENCLATURE

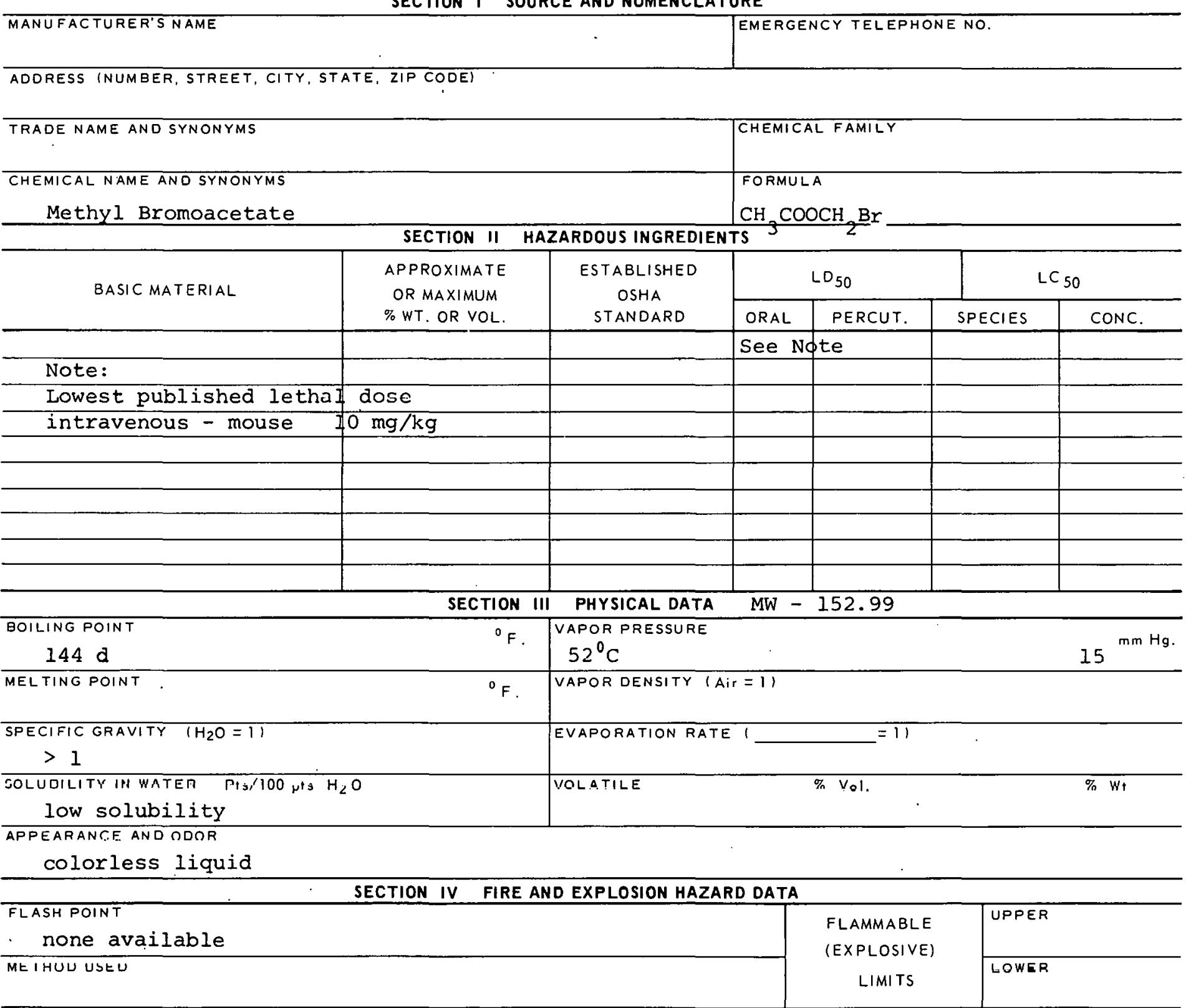

EXTINOUISHING MEDIA

Alcohol foam, carbon dioxide, dry chemical and water.

SPECIAL FIRE FIGHTING PROCEDURES 
Methyl Bromoacetate

\section{TOXIC LEVEL}

None available

PRINCIPLE ROUTES OF ABSORBTION

Inhalation, ingestion, skin SIN AND EYE

IRRITATION

CARCINOCENIC

REVELANT SYMPTOMS OF EXPOSURE Irritation of eyes and upper respiratory system, dizziness and depression, palpitation of heart.

EFFECTS OF CHRONIC EXPOSURE

$--$

EMERGENCY AND FIRST AIDPROCEDURES If swallowed - give emetic, get medical aid;

inhalation - rest; wash from skin \& eyes.

SECTION VI REACTIVITY DATA

CONDITIONS CONTRIBUTING TO INSTABILITY

CONDITIONS CONTRIBUTING TO HAZARDOUSS POLYMEER! ZAT!QN

INCOMPATABILITY (MATERIALS TO AVOID)

HAZARDOUS DECOMPOSITION PRODUCTS

SECTION VII SPILL OR LEAK PROCEDURES

STEPS TO BE TAKEN IN CASE MATERIAL IS RELEASED OR SPILLED

SECTION VIII SPECIAL PROTECTION INFORMATION

\begin{tabular}{|c|c|}
\hline VENTILATION REQUIREMENTS LOCAL EXHAUST & $\begin{array}{l}\text { PROTECTIVE EQUIPMENT (SPECIFY TYPES) EYE } \\
\text { GOggles }\end{array}$ \\
\hline MECHANICAL (GENERAL) & $\begin{array}{l}\text { GLOVES } \\
\text { Rubber }\end{array}$ \\
\hline SFEこIAL & $\begin{array}{l}\text { RFSPIRATh } \\
\text { With proper filter }\end{array}$ \\
\hline
\end{tabular}

OTHER FROTECTIVE CQUIPMENT

SECTION IX SPECIAL PRECAUTIONS

PRECAUTIONS TO.BE TAKEN IN HANDLING AND STORAGE

Store in standard flammable liquids cabinet. OTHER PRECAUTIONS

JIGNATURE

OATE

$306-2702 B$ 


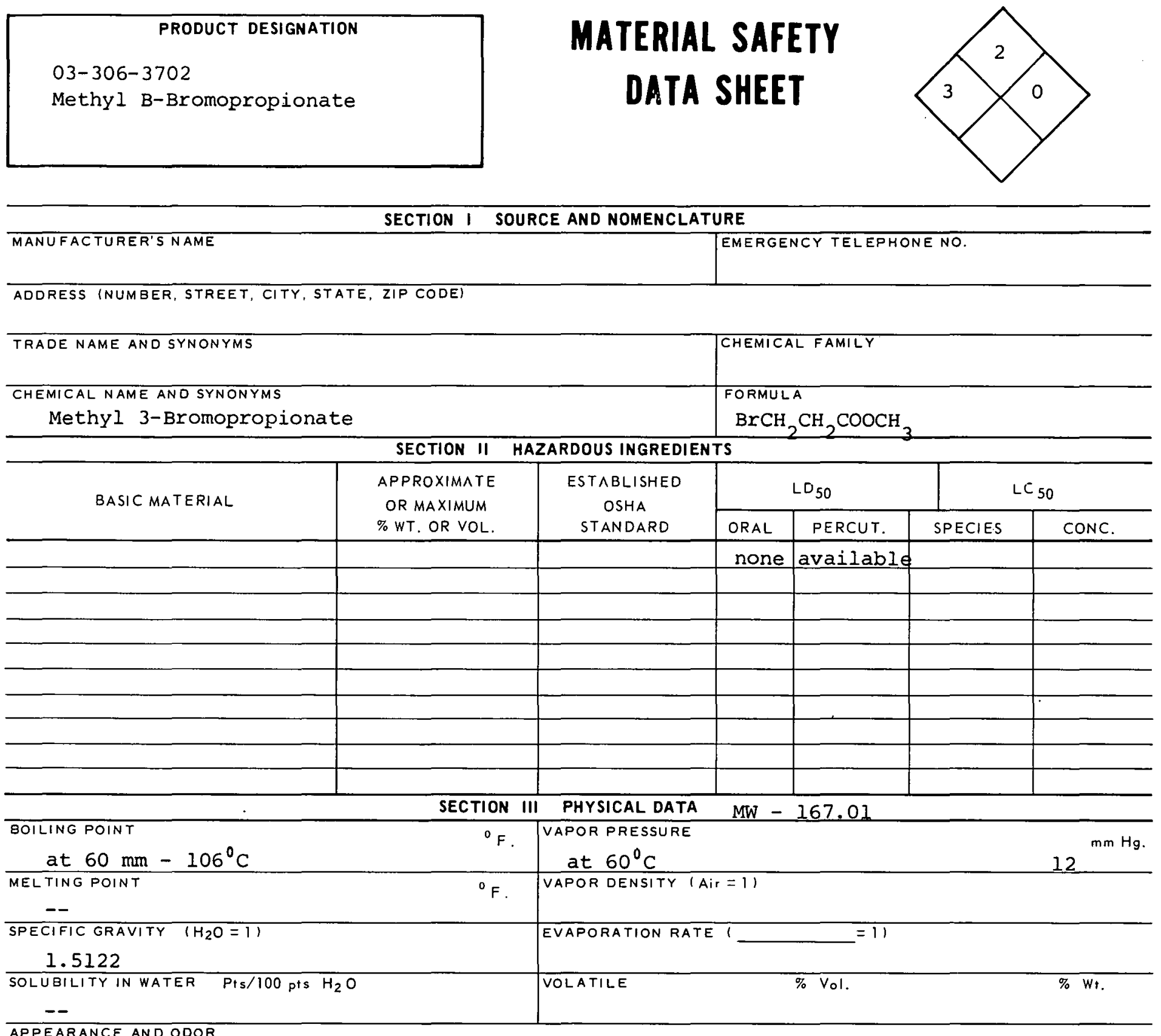

APPEARANCE AND ODOR

Colorless liquid

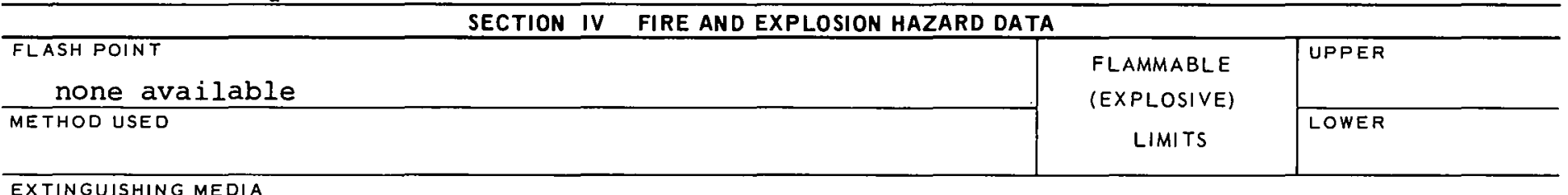

Foam, carbon dioxide, dry chemical.

SPECIAL FIRE FIGHTING PROCEDURES

UNUSUAL FIRE AND EXPLOSION HAZARDS 
Methyl B-Bromopropionate

SECTION $V$ HEALTH HAZARD DATA

TOXIC LEVEL

none available

PRINCIPLE ROUTES OF ABSORBTION

inhalation, ingestion, skin

CARCINOGENIC

SKIN ANDEYE CARCINOGENIC no

REVELANT SYMPTOMSOF EXPOSURE Irxitation of eyes and upper respiratory syste, dizzinesS and depression, palpitation of heart. EFFECTS OF CHRONIC EXPOSURE

$--$

EMERGENCY AND FIRST AID PROCEDURES If swallowed - give emetic, get medical aid;

inhalation - rest; wash from skin \& eyes.

SECTION VI REACTIVITY DATA

CONDITIONS CONTRIBUTING TO INSTABILITY

CONDITIONS CONTRIBUTING TO HAZARDOUS POLYMERIZATION

IACOMPATAEILITY (MATERIALE TO AVOID)

HA ZARDOIIS DFR.MMPOSITION PROMIIT.TS

SECTION VII SPILL OR LEAK PROCEDURES

STEPS TO BE TAKEN IN CASE MATERIAL IS RELEASED OR SPILLED

WASTE DISPOSAL METHOD

SECTION VIII SPECIAL PROTECTION INFORMATION

\begin{tabular}{l|l}
\hline \multicolumn{2}{c}{ SECTION VIII SPECIAL PROTECTION INFORMATION } \\
\hline VENTILATION REQUIREMENTS LOCAL EXHAUST & $\begin{array}{c}\text { PROTECTIVE EQUIPMENT (SPECIFY TYPES) EYE } \\
\text { GOggles }\end{array}$ \\
\hline MECHANICAL IGENERAL) & $\begin{array}{c}\text { GLOVES } \\
\text { Rubber }\end{array}$ \\
\hline SEECIAL & $\begin{array}{c}\text { RESPIRATOR } \\
\text { With proper filter. }\end{array}$ \\
\hline
\end{tabular}

OTHER PROTECTIVE EQUIPMENT

\section{SECTION IX SPECIAL PRECAUTIONS}

PRECAUTIONS TO BE TAKEN IN HANDLING AND STORAGE

Store in standard flammable liquids cabinet.

OTHER PRECAUTIONS

\begin{tabular}{l|l|l}
\hline SIGNATURE & DATE \\
\hline
\end{tabular}




\begin{tabular}{|c|} 
PRODUCT DESIGNATION \\
03-306-4702 \\
Methyl Iso-Butyl Ketone
\end{tabular}

\section{MATERIAL SAFETY DATA SHEET}

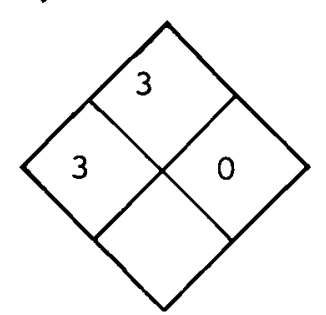

SECTION I SOURCE AND NOMENCLATURE

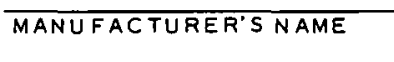

(1)

.

EMERGENCY TELEPHONE NO.

ADDRESS (NUMBER, STREET, CITY, STATE, ZIP CODE)

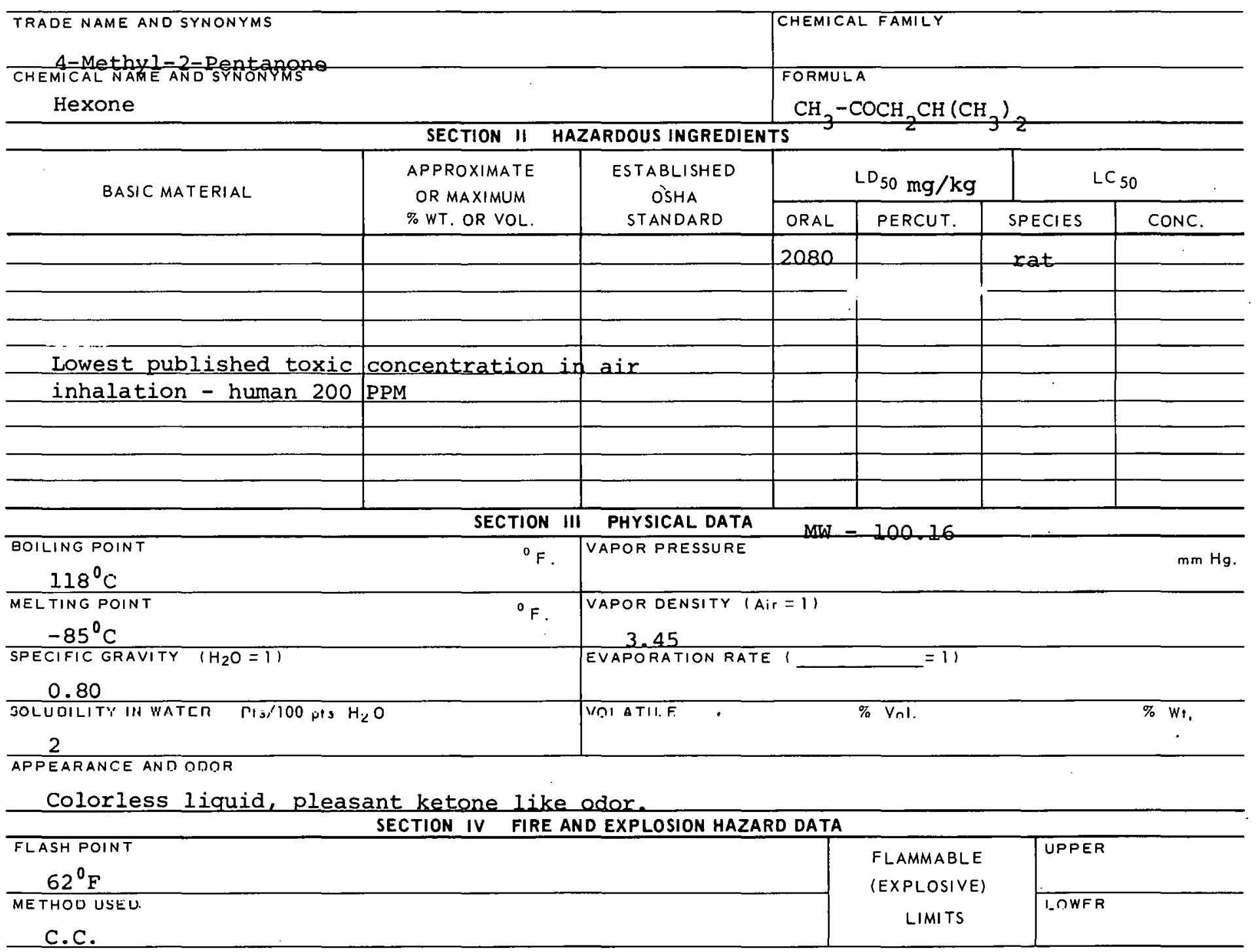

EXTINGUISHING MEOIA

Foam, carbon dioxide, dry chemical. Water may be ineffective.

SPECIAL FIRE FIGHTING PROCEDIIRES

Wear goggles \& self-contained breathing apparatus. 


\section{TOXIC LEVEL}

$100 \mathrm{PPM}, 410 \mathrm{mg} / \mathrm{C} . \dot{\mathrm{M}}$.

PRINCIPLE ROUTES OF ABSOROTION

Inhalation, ingestion
CARCINOGENIC

SKIN AND EYE

IRRITATION

moderate

REVELANT SYMPTOMS OF EXPOSURE Weakness, loss of appetite, stomach ache, nausea, vomiting,

eye and nasal irritation, narcosis, headache, nausea, lowering of body temperature EFFECTS OF CHRONIC EXPOSURE

$--$

EMERGENCY AND FIRST AID PROCEDURES If swallowed-give emetic, get medical aid, gastric lavage;

inhalation - rest; oxygen as needed; wash skin \& eyes.

\section{SECTION VI REACTIVITY DATA}

CONDITIONS CONTRIBUTING TO INSTABILITY

CONDITIONS CONTRIBU TING TO HAZARDOUS POLYMERIZATION

IBISOMPATABILITY (MATEMIALE TO AVOID)

HAZARDOUS DECOMPOSITION PRODUCTS

SECTION VII SPILL OR LEAK PROCEDURCS

STEPS TO BE TAKEN IN CASE MATERIAL IS RELEASED OR SPILLED

Absorb on paper towels; evaporate in hood.

WASTE DISPOSAL METHOD

SECTION VIII SPECIAL PROTECTION INFORMATION

\begin{tabular}{l|l|l}
\hline VENTILATION REQUIREMENTS LOCAL EXHAUST & PROTECTIVE EQUIPMENT (SPECIFY TYPES) EYE
\end{tabular}

MECHANICAL (GENERAL)

Goggles

GLOVES

SPECIAI.

RESPIRATUH

SeI Cuntained

OTHER PROTECTIVE EQUIPMENT

SECTION IX SPECIAL PRECAUTIONS

PRECAUTIONS TO BE TAKEN IN HANDLING AND STORAGE

Outside storage preferable, inside storage in a standard flammable

liquid cabinet.

OTHER PRECAUTIONS

SIGNATURE

DATE

$306-4702 B$ 
CAS : 000616386

\section{PRODUCT DESIGNATION}

03-306-6202

Methyl Carbonate

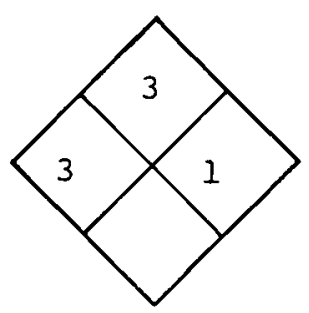

\section{SECTION I SOURCE AND NOMENCLATURE}

\section{MANUFACTURER'S NAME \\ TRADE NAME AND SYNONYMS \\ CHEMICAL NAME ANO SYNONYMS \\ dimethyl carbonate}

ADDRESS (NUMBER, STREET, CITY, STATE, ZIP CODE)

BASIC MATERIAL

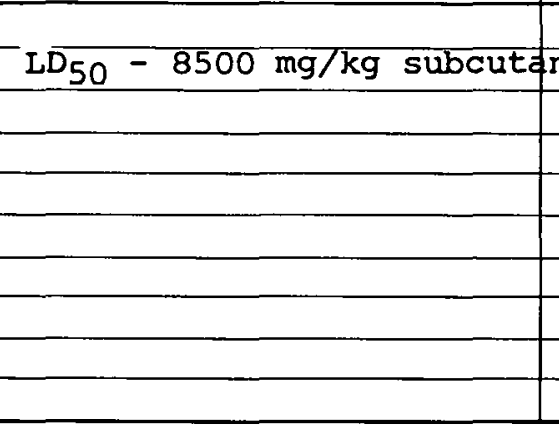

EUILING HOINI
$90^{\circ} \mathrm{C}$

MEL TING POINT

$1^{\circ} \mathrm{C}$

SPECIFIC GRAVITY $\left(\mathrm{H}_{2} \mathrm{O}=\prod\right.$

$$
1.07
$$

SOLUBILITY IN WATER Pts/100 pts $\mathrm{H}_{2} \mathrm{O}$ insoluble

APPEARANCE AND ODOR

colorless liquid, pleasant odor.

SECTION IV FIRE AND EXPLOSION HAZARD DATA

FLASH POINT

$$
66^{\circ} \mathrm{F}
$$

METHOD USED

O.C.

EXTINGUISHING MEDIA Alcohol foam, carbon dioxide, dry chemical. Water may be ineffective.

SPECIAL FIRE FIGHTING PROCEDURES

Wear goggles and self-contained breathing apparatus.
EMERGENCY TELEPHONENO.

\section{SECTION II HAZARDOUS INGREDIENTS CO $\left.\mathrm{COCH}_{3}\right)_{2}$}

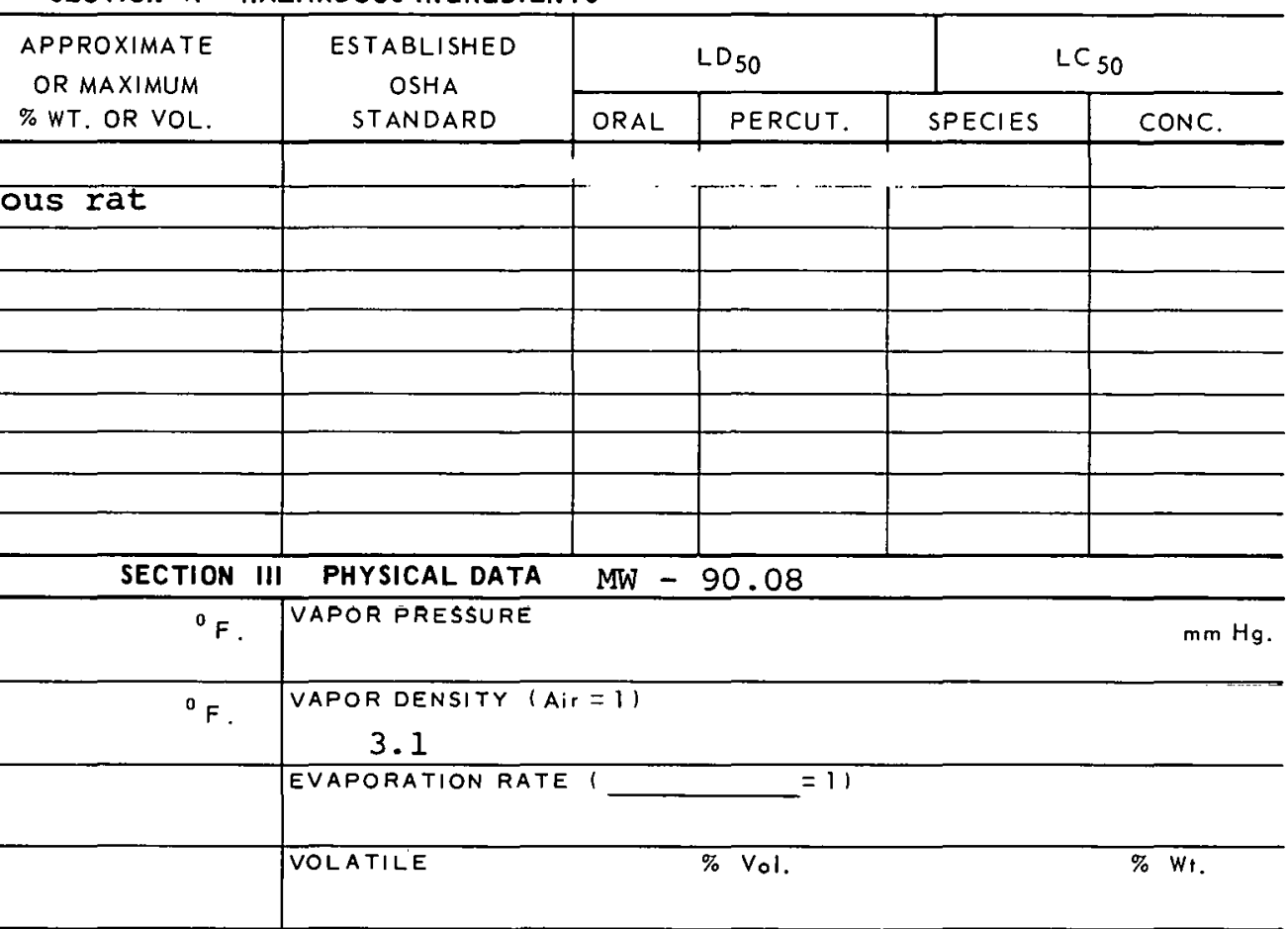

UNUSUAL FIRE AND EXPLOSION HAZAROS

FLAMMABLE

(EXPLOSIVE)

LIMITS 


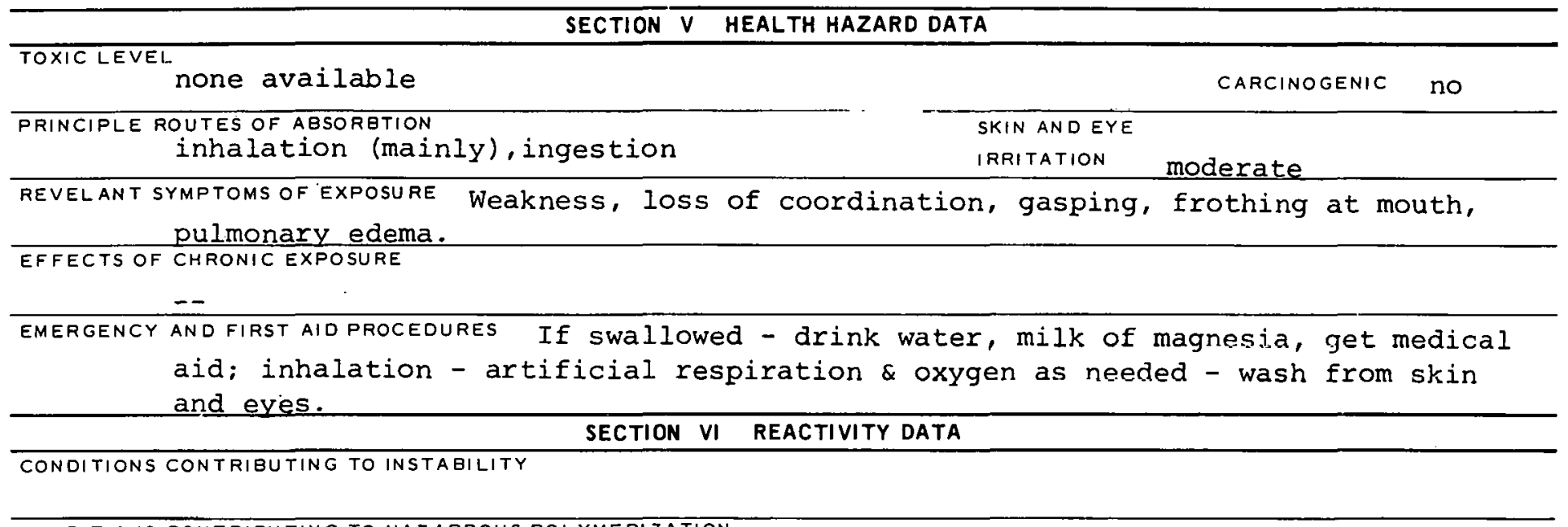

CONDITIONS CONTRIBU TING TO HAZARDOUS POLYMERIZATION

INCOMPATABILITY (MATERIALS TO AVQIQ)

IIAZARDOUJ OLCOMFOSITIOA PRODUCT3

SECTION VII SPILL OR LEAK PROCEDURES

STEPS TO BE TAKEN IN CASE MATERIAL IS RELEASED OR SPILLEO

WASTE DISPOSAL METHOD

SECTION VIII SPECIAL PROTECTION INFORMATION

\begin{tabular}{l|l}
\hline VENTILATION REQUIREMENTS LOCAL EXHAUST & $\begin{array}{l}\text { PROTECTIVE EQUIPMENT (SPECIFY TYPES) EYE } \\
\text { GOggles }\end{array}$ \\
\hline MECHANICAL (GENERAL) & $\begin{array}{c}\text { GLOVES } \\
\text { Rubber }\end{array}$ \\
\hline STCCIAL & KESHIRAIUH \\
& Self-contained \\
\hline
\end{tabular}

OTHER PROTECTIVE EQUIPMENT

SECTION IX SPECIAL PRECAUTIONS

PRECAUTIONS TO BE TAKEN IN HANDLING AND STORAGE
Outside storage preferred, store inside flammable liquids storage cabinet.

OTHER PRECAUTIONS

SIGNATURE

DATE

$306-6202 B$ 
03-307-0303

PRODUCT DESIGNATION

P-Methylcyclohexanol
MATERIAL SAFETY

DATA SHEET

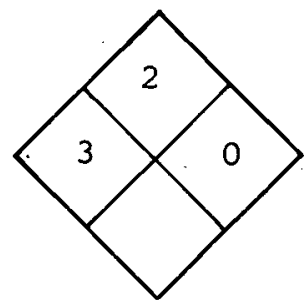

SECTION I SOURCE AND NOMENCLATURE

MANUFACTURER'S NAME
ADORESS INUMBER, STREET, CITY, STAT
TRADE NAME AND SYNONYMS
HeXahYdrome thYl PhEnol
CHEMICAL NAME AND SYNONYMS
HeXahydrocresol

\begin{tabular}{|c|c|c|c|c|c|c|}
\hline \multirow{3}{*}{ BASIC MATERIAL } & SECTION !I & AZARDOUS INGREDIENTS & & & & \\
\hline & \multirow{2}{*}{$\begin{array}{l}\text { APPROXIMATE } \\
\text { OR MAXIMUM } \\
\% \text { WT. OR VOL. }\end{array}$} & \multirow{2}{*}{$\begin{array}{c}\text { ESTABLISHED } \\
\text { OSHA } \\
\text { STANDARD }\end{array}$} & \multicolumn{2}{|r|}{$\mathrm{LD}_{50} \mathrm{mg} / \mathrm{kg}$} & \multicolumn{2}{|c|}{$\mathrm{LC}_{50}$} \\
\hline & & & \multirow{2}{*}{$\frac{\text { ORAL }}{1660}$} & \multirow[t]{2}{*}{ PERCUT. } & SPECIES & \multirow[t]{2}{*}{ CONC. } \\
\hline Inhalation - human & TCLO 500 PPM & & & & Rat & \\
\hline Lowest published lethal & $d \Omega=0$ & & & & & \\
\hline \multicolumn{7}{|c|}{ oral - rabbit $1750 \mathrm{mg} / \mathrm{kg}$} \\
\hline & & & & & & \\
\hline & & & & & & \\
\hline & & & & & & \\
\hline & & & & & & \\
\hline & & & & & & \\
\hline & & & & & & \\
\hline \multicolumn{7}{|c|}{ SECTION III PHYSICAL DATA MW - 114.18} \\
\hline BOILING POINT & ${ }^{0} \mathrm{~F}$ & VAPQR PRESSURE & & & & $\mathrm{mm} \mathrm{Ha}$ \\
\hline $174^{\circ} \mathrm{C}$ & & at $30^{\circ} \mathrm{C}$ & & & & 1.5 \\
\hline MELTING POINT & ${ }^{\circ} \mathrm{F}$. & VAPOR DENSITY IAIT & $=11$ & & & \\
\hline$-50^{\circ} \mathrm{C}$ & & 3.93 & & & & \\
\hline SPECIFIC GRAVITY $\left(\mathrm{H}_{2} \mathrm{O}=1\right)$ & & EVAPORATION RATE & 1 & $=11$ & & \\
\hline \multicolumn{7}{|l|}{0.92} \\
\hline SOI UIRIIITY IN WATER Pts/INOR R.ts $\mathrm{H}_{2}$ & & VOLATILE & & $\% V_{n} l$. & & $\% \mathrm{Wt}$. \\
\hline \multicolumn{7}{|l|}{ very slightly soluble } \\
\hline \multicolumn{7}{|c|}{ APPEARANCE AND ODOR } \\
\hline \multicolumn{7}{|c|}{ colorless, viscous liguid, aromatic, menthol odor. } \\
\hline \multicolumn{7}{|c|}{ SECTION IV FIRE AND EXPLOSION HAZARD DATA } \\
\hline $\begin{array}{c}\text { FLASH POINT } \\
154^{\circ} \mathrm{F}\end{array}$ & & & & \multirow{2}{*}{$\begin{array}{c}\text { FLAMMABLE } \\
\text { (EXPLOSIVE) } \\
\text { LIMITS }\end{array}$} & \multicolumn{2}{|l|}{ UPPER } \\
\hline $\begin{array}{l}\text { METHOD USED } \\
\text { C.C. }\end{array}$ & & & & & LOWER & \\
\hline
\end{tabular}

EXTINGUISHING MEDIA

Foam, carbon dioxide or dry chemicals.

SPECIAL FIRE FIGHTING PROCEDURES

UNUSUAL FIRE ANO EXPLOSION HAZARDS 


\section{TOXIC LEVEL}

$50 \mathrm{PPM}, 235 \mathrm{mg} / \mathrm{C} . \mathrm{M}$.

CARCINOGENIC

PRINCIPLE ROUTES OF ABSORETION

Inhalation, ingestion, skin

SKIN ANO EYE

IRRITATION severe

REVELANT SYMPTOMS OF EXPOSURE Salivation, eye and mucous membrane irritation, tremors,

narcosis, headache, gross hemorrhage.

EFFECTS OF CHRONIC EXPOSURE

Liver and kidney damage

EMERGENCY AND FIRST AID PROCEDURES If swallowed - give emetic, get medical aid, gastric lavage; inhalation - rest, oxygen as needed; wash from eyes \& skin.

SECTION VI REACTIVITY DATA

CONDITIONS CONTRIBUTING TO INSTABILITY

CONDITIONS CONTRIBUTING TO HAZARDOUS POLYMERIZATION

INCOMRATADILITY (MATCRIALJ TO A'VOIO)

can react with oxidizing materials.

HAZARDOUS DECOMPOSITION PRODUCTS

SECTION VII SPILL OR LEAK PROCEDURES

STEPS TO BE TAKEN IN CASE MATERIAL IS RELEASED OR SPILLED

WASTE DISPOSAL METHOD

SECTION VIII SPECIAL PROTECTION INFORMATION

\begin{tabular}{l|l}
\hline VENTILATION REQUIREMENTS LOCAL EXHAUST & $\begin{array}{l}\text { PROTECTIVE EQUIPMENT (SPECIFY TYPES) EYE } \\
\text { gOggles }\end{array}$ \\
\hline MECHANICAL IGENERAL) & $\begin{array}{l}\text { GLOVES } \\
\text { rubber }\end{array}$ \\
\hline SPECIAL & $\begin{array}{l}\text { RESPIRATOR } \\
\text { with proper filter }\end{array}$ \\
\hline
\end{tabular}

OTHER PROTECTIVE EQUIPMENT

SECTION IX SPECIAL PRECAUTIONS

P'RECAUTIONS TO BE TAKEN IN HANDLING AND STORAGE

OTHER PRECAUTIONS 


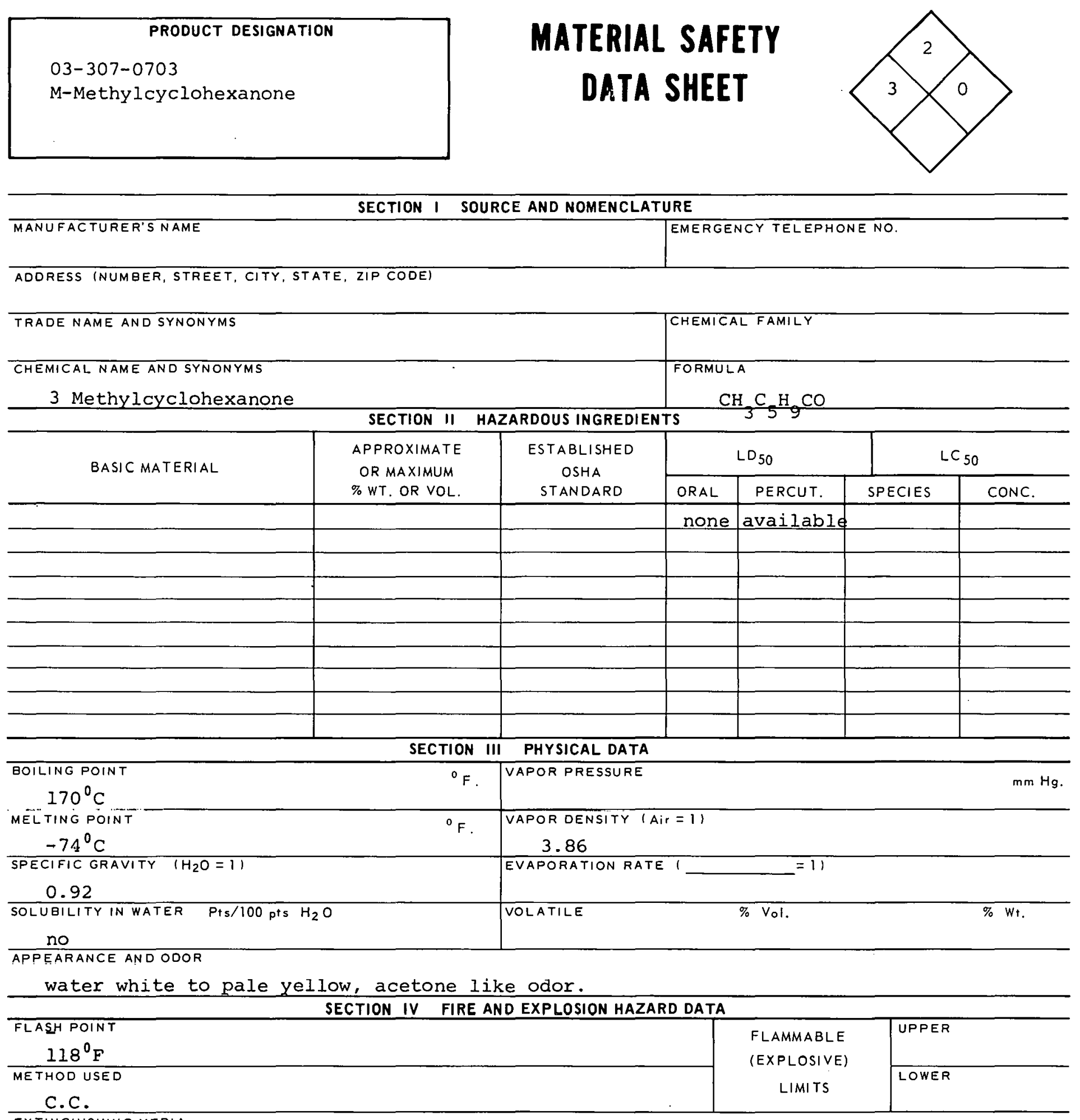

EXTINGUISHING MEDIA

Foam, carbon dioxide, dry chemical

SPECIAL FIRE FIGHTING PROCEDURES

UNUSUAL FIRE AND EXPLOSION HAZARDS 


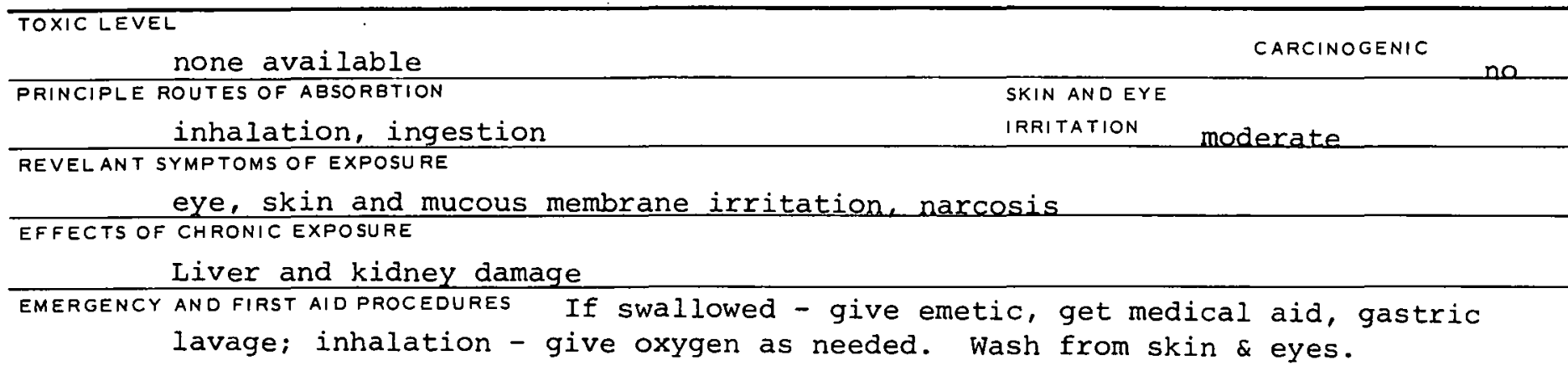

\begin{tabular}{l}
\hline SECTION VI REACTIVITY.DATA \\
\hline CONDITIONS CONTRIBUTING TO INSTABILITY \\
\hline CONDITIONS CONTRIBUTING TO HAZARDOUS POLYMERIZATION \\
\hline HAZARDOUS DECOMPOSITION PRODUETS \\
\hline STEPS TO BE TAKEN IN CASE MATERIAL IS RELEASED OR SPILLED
\end{tabular}

STEPS TO BE TAKEN IN CASE MATERIAL IS RELEASED OR SPILLED

\section{WASTE DISPOSAL METHOD}

\section{SECTION VIII SPECIAL PROTECTION INFORMATION}

\begin{tabular}{l|l} 
SECTION VIII SPECIAL PROTECTION INFORMATION \\
\hline VENTILATION REQUIREMENTS LOCAL EXHAUST & $\begin{array}{c}\text { PROTECTIVE EQUIPMENT (SPECIFY TYPESI EYE } \\
\text { Safety gOggles }\end{array}$ \\
\hline MECHANICAL (GENERAL) & $\begin{array}{c}\text { GLOVES } \\
\text { Rubber }\end{array}$ \\
\hline STCCIAL. & RESHIRAIOR \\
With proper fiIter
\end{tabular}

OTHER PROTECTIVE EQUIPMENT

SECTION IX SPECIAL PRECAUTIONS

PRECAUTIONS TO BE TAKEN IN HANDLING AND STORAGE

OTHER PRECAUTIONS

SIGNATURE 


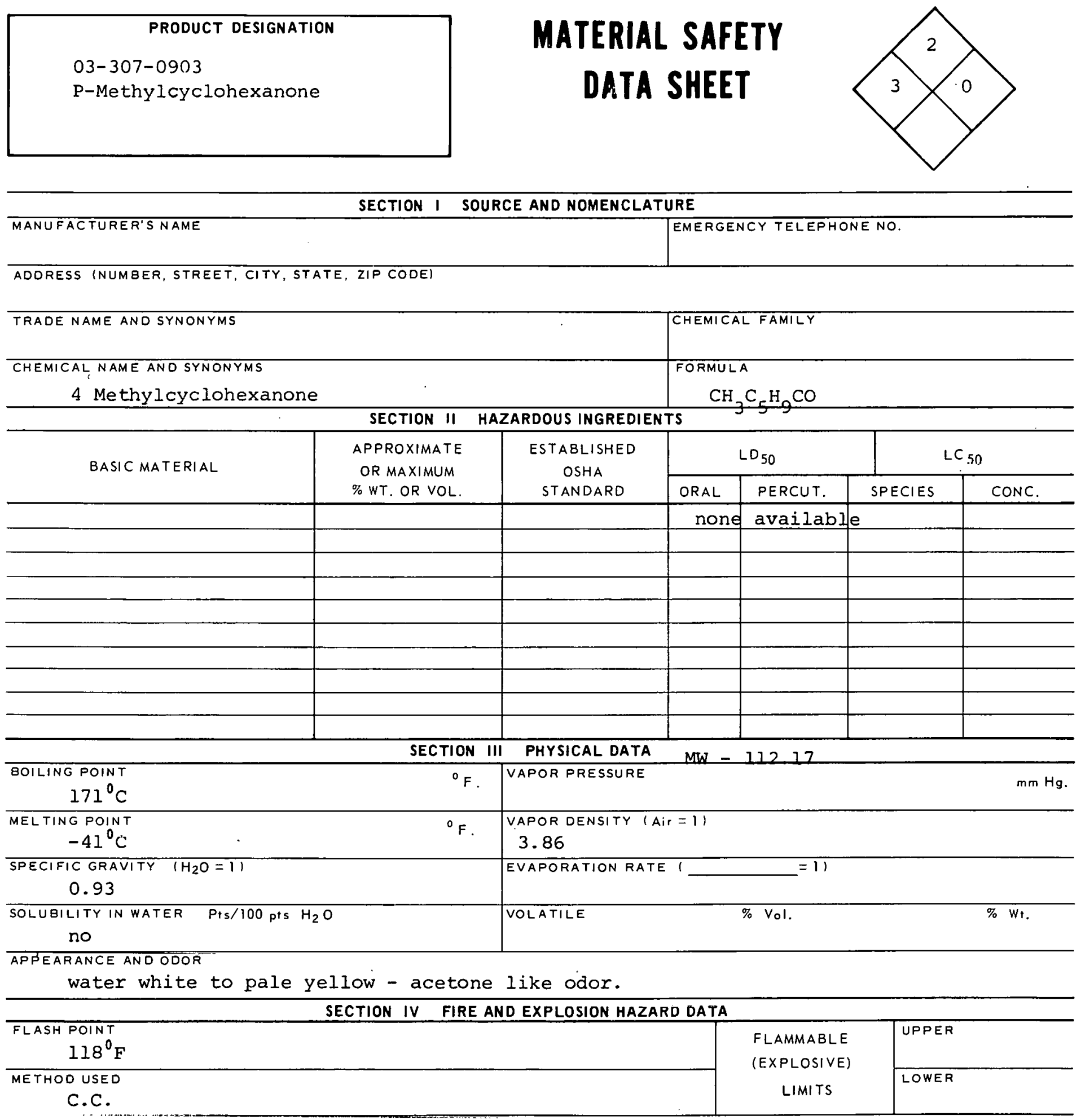

EXTINGUISHING MEDIA

Foam, carbon dioxide, dry chemical

SPECIAL FIRE FIGHTING PROCEOURES

UNUSUAL FIRE AND EXPLOSION HAZARDS 
P-Methylcyclohexanone

SECTION $V$ HEALTH HAZARD DATA

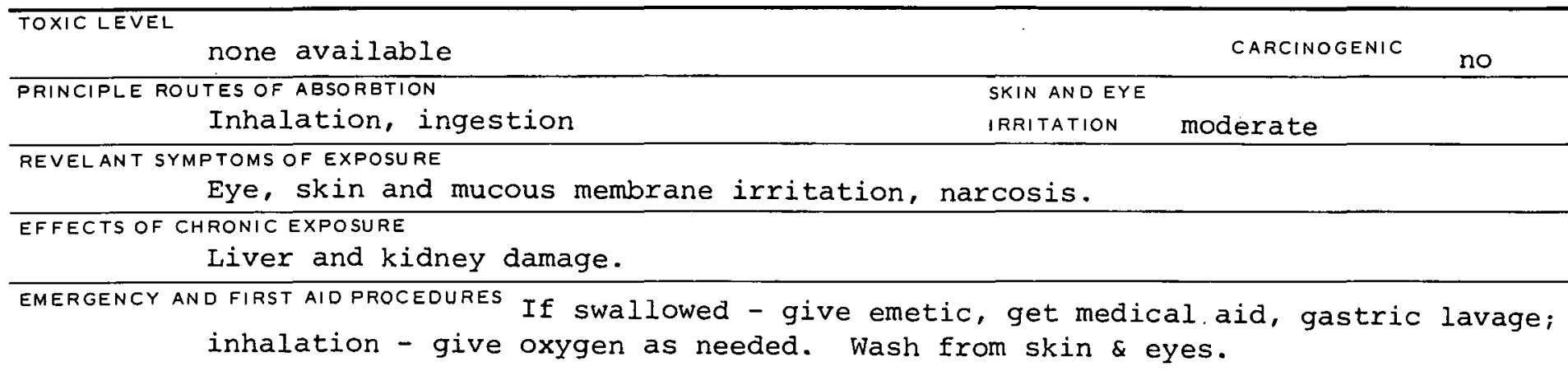

\section{SECTION VI REACTIVITY DATA}

CONDITIONS CONTRIBUTING TO INSTABILITY

CONDITIONS CONTRIBUTING TO HAZARDOUS POLYMERIZATION

INCOMHATABILITY IMATERHIALS TO AVOIOI

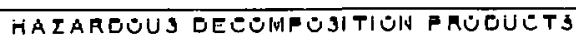

SECTION VII SPILL OR LEAK PROCEDURES

STEPS TO BE TAKEN IN CASE MATERIAL IS RELEASED OR SPILLED

\section{WASTE DISPOSAL METHOD}

\section{SECTION VIII SPECIAL PROTECTION INFORMATION}

\begin{tabular}{|c|c|}
\hline VENTILATION REQUIREMENTS LOCAL EXHAUST & $\begin{array}{l}\text { PROTECTIVE EQUIPMENT (SPECIFY TYPES) EYE } \\
\text { Safety goggles }\end{array}$ \\
\hline MECHANICAL (GENERAL) & $\begin{array}{l}\text { GLOVES } \\
\text { Rubber }\end{array}$ \\
\hline SPFC.IAI & $\begin{array}{l}\text { REGTIRATOR } \\
\text { With proper filter }\end{array}$ \\
\hline
\end{tabular}

\section{OTHER PROTECTIVE EQUIPMENT}

\section{SECTION IX SPECIAL PRECAUTIONS}

PRECAUTIONS TO BE TAKEN IN HANDLING AND STORAGE

OTHER PRECAUTIONS 


\section{PRODUCT DESIGNATION}

03-307-1303

Methyl A, B-Dibromopropionate
MATERIAL SAFETY

DATA SHEET

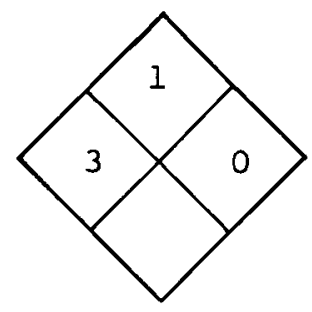

SECTION I SOURCE AND NOMENCLATURE

MANU FACTURER'S NAME

EMERGENCY TELEPHONE NO.

ADDRESS (NUMBER, STREET, CITY, STATE, ZIP CODE)

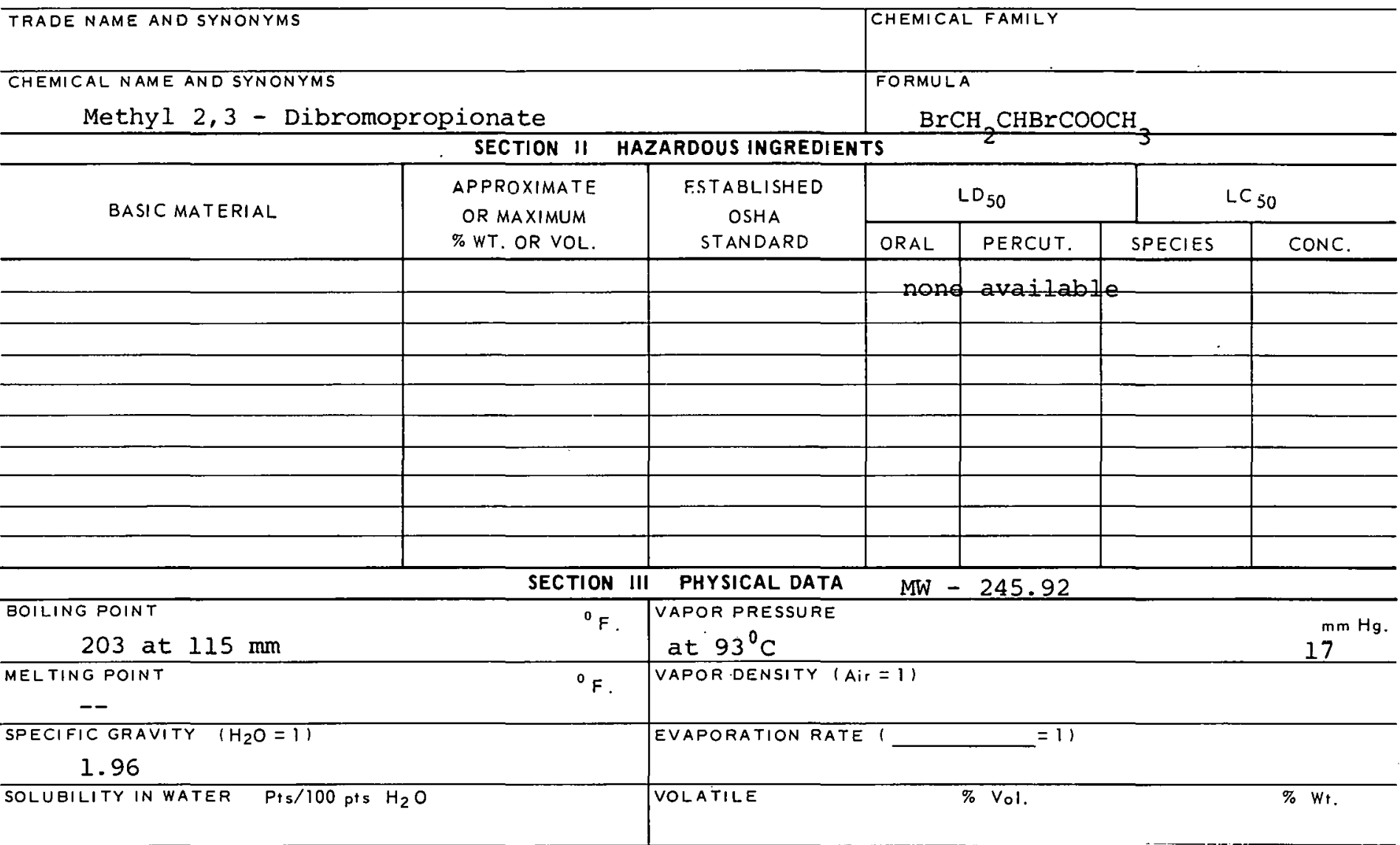

APPEARANCE AND ODOR

clear liquid

\begin{tabular}{|c|c|c|c|c|}
\hline & SECTION IV & FIRE AND EXPLOSION HAZARD DATA & & \\
\hline $\begin{array}{l}\text { FLASH POINT } \\
\text { none available }\end{array}$ & & & \multirow{2}{*}{$\begin{array}{c}\text { FLAMMABLE } \\
\text { (EXPLOSIVE) } \\
\text { LIMITS }\end{array}$} & UPPER \\
\hline METHOD USED & & & & LOWER \\
\hline
\end{tabular}

EXTINGUISHING MEDIA

Foam, carbon dioxide, dry chemical, wațer.

SPECIAL FIRE FIGHTING PROCEDURES

UNUSUAL FIRE ANU EXPLOSION HAZARDS 


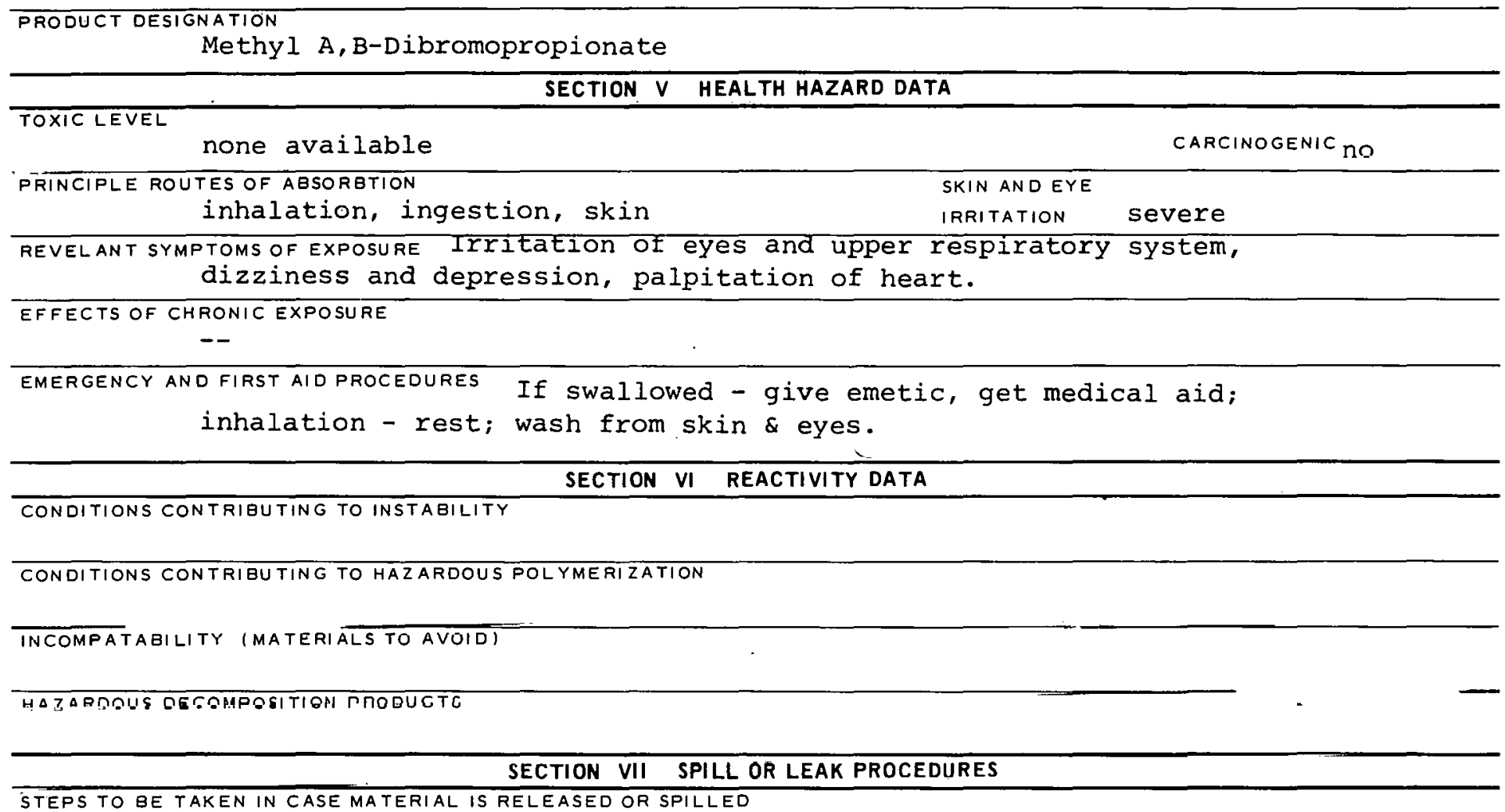

\begin{tabular}{|c|c|c|}
\hline SECTION VIII & \multicolumn{2}{|c|}{ SPECIAL PROTECTION INFORMATION } \\
\hline \multicolumn{2}{|l|}{ VENTILATION REQUIREMENTS LOCAL EXHAUST } & $\begin{array}{l}\text { PROTECTIVE EQUIPMENT (SPECIFY TYPES) EYE } \\
\text { gOggles }\end{array}$ \\
\hline \multicolumn{2}{|l|}{ MECHANICAL (GENERAL) } & $\begin{array}{l}\text { GLOVES } \\
\text { rubber }\end{array}$ \\
\hline \multicolumn{2}{|l|}{ SPECIAL } & $\begin{array}{l}\text { RESPIRATUK } \\
\text { with proper filter }\end{array}$ \\
\hline \multicolumn{3}{|c|}{$\begin{array}{l}\text { SECTION IX SPECIAL PRECAUTIONS } \\
\text { SP }\end{array}$} \\
\hline \multicolumn{3}{|c|}{$\begin{array}{l}\text { PRECAUTIONS TO BE TAKEN IN HANDLING AND STORAGE } \\
\text { Store in standard flammable liquids cabinet. }\end{array}$} \\
\hline \multicolumn{3}{|l|}{ OTHER PRECAUTIUNS } \\
\hline SIGNATURE & DATE & \\
\hline
\end{tabular}




\begin{tabular}{|l|}
\hline \multicolumn{1}{|c|}{ PRODUCT DESIGNATION } \\
03-307-2103 \\
Methylene Bromide \\
\hline
\end{tabular}

\section{SECTION I SOURCE AND NOMENCLATURE}

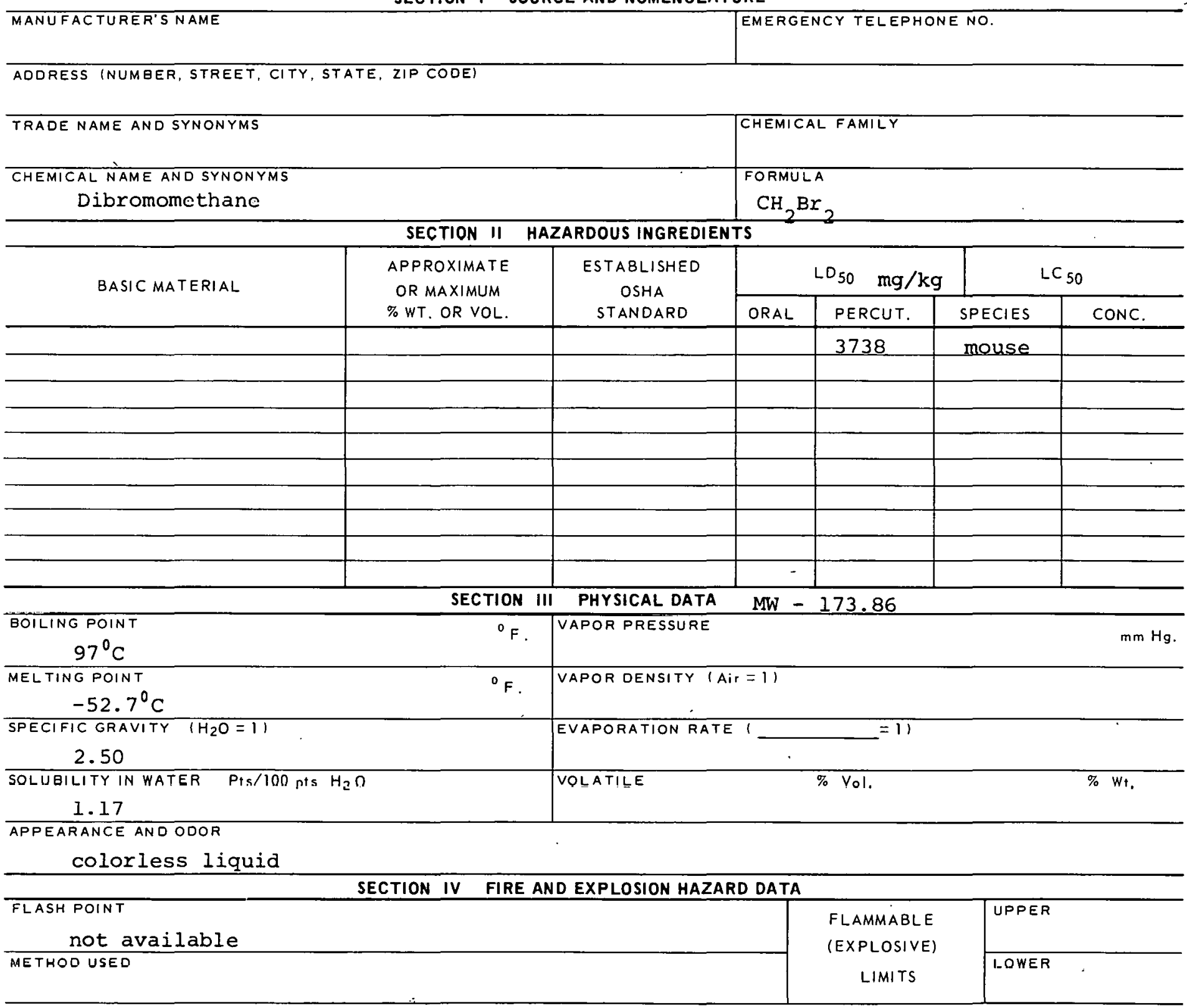

FXTINGIIISHING MFDIA

Probably inflammable like $\mathrm{CH}_{2} \mathrm{Cl}_{2}$

SPECIAL FIRE FIGHTING PROCEDURES

UNUSUAL FIRE AND EXPLOSION HAZARDS

May generate $\mathrm{COBr}_{2}$ when in contact with flame or very hot metal surfaces. 
Methylene Bromide

SECTION $V$ HEALTH HAZARD DATA

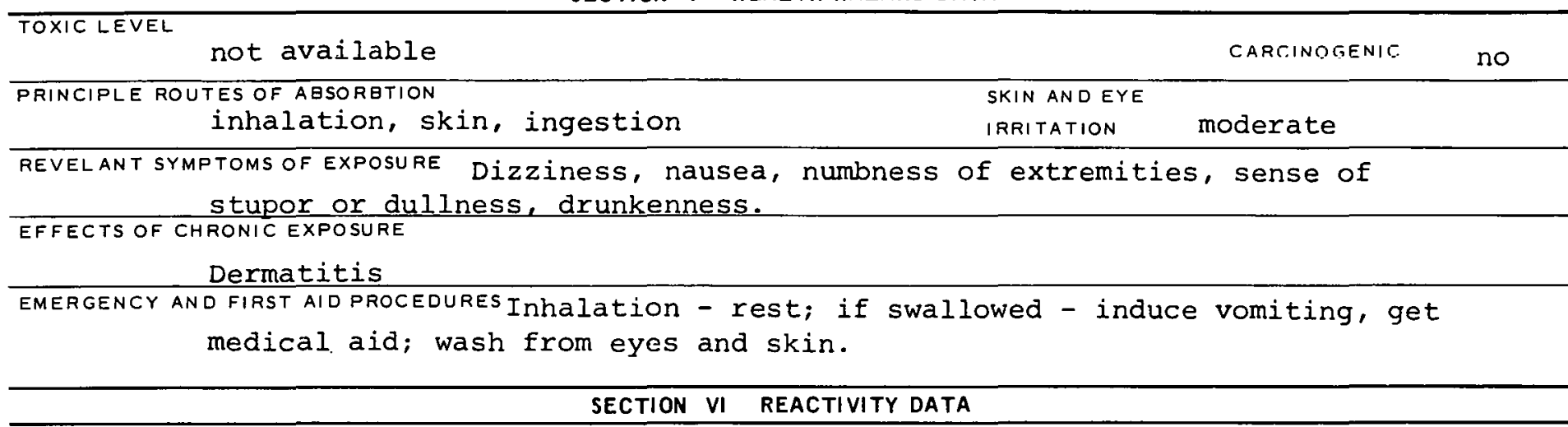

CONDITIONS CONTRIBUTING TO INSTABILITY

CONDITIONS CONTRIBUTING TO HAZARDOUS POLYMERIZATION

INCOMPATABILITY (MATERIALS TO AVOID)

Alkali metals

HALARUUUS UECUMHUSI IIUN HHUUUC' IS

May generate toxic COBr? on contact with flame or very hot metal ourfaceg. SECTION VII SPILL OR LEAK PROCEDURES

STEPS TO BE TAKEN IN CASE MATERIAL IS RELEASED OR SPILLED

WASTE DISPOSAL METHOD

\begin{tabular}{|c|c|c|}
\hline SECTION & SPECIAL PROTECTION & INFORMATION \\
\hline $\begin{array}{l}\text { VENTILATION REQUIREMENTS LOCAL EXHAUST } \\
\text {. }\end{array}$ & & $\begin{array}{l}\text { PROTECTIVE EQUIPMENT (SPECIFY TYPES) EYE } \\
\text { gOggles }\end{array}$ \\
\hline MECIIANICAL (GENERAL) & & GLUVES \\
\hline EחEGIML & & AESPIRATUN \\
\hline
\end{tabular}

OTHER PROTEC.TIVE EQUIPMENT

SECTION IX SPECIAL PRECAUTIONS

PRECAUTIONS TO BE TAKEN IN HANDLING AND STORAGE

\section{OTIICR PRECAUTIONS}

\begin{tabular}{l|l|l|}
\hline SIGNATIIAF & UATE \\
\hline
\end{tabular}




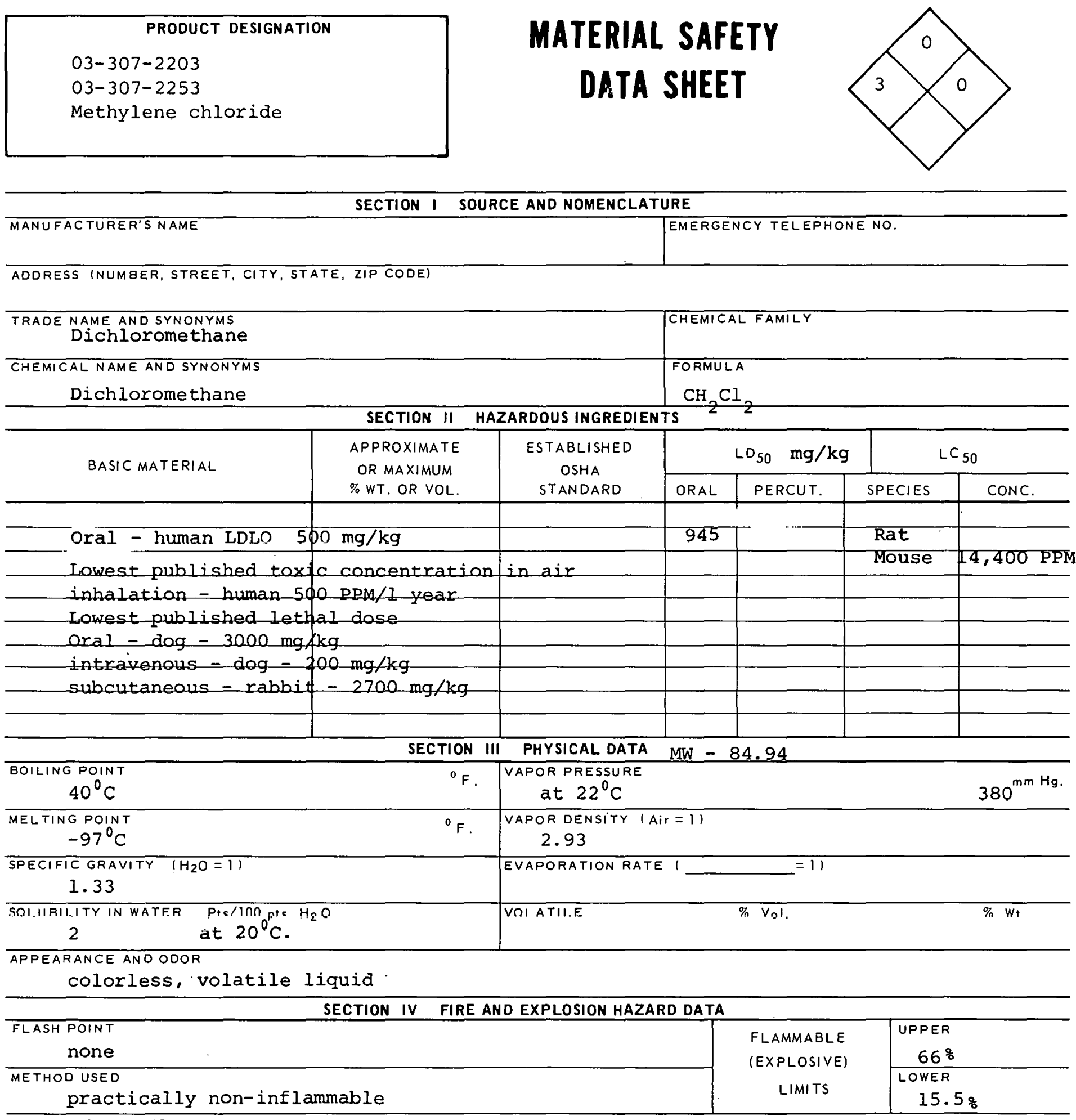

EXTINGUISHING MEDIA

SPECIAL FIRE FIGHTING PROCEDURES

UNUSUAL FIRE ANO EXPLOSION HAZARDS

May generate phosgene on contact with flame or very hot metal surfaces. 


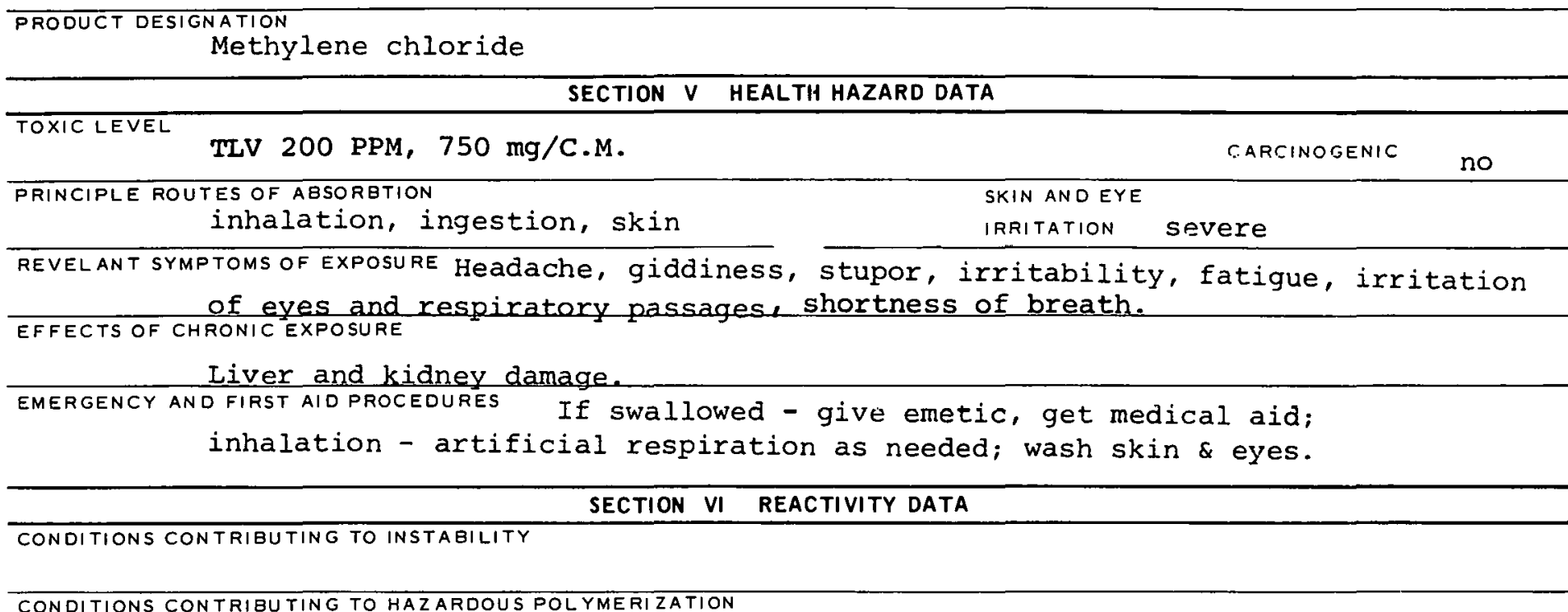

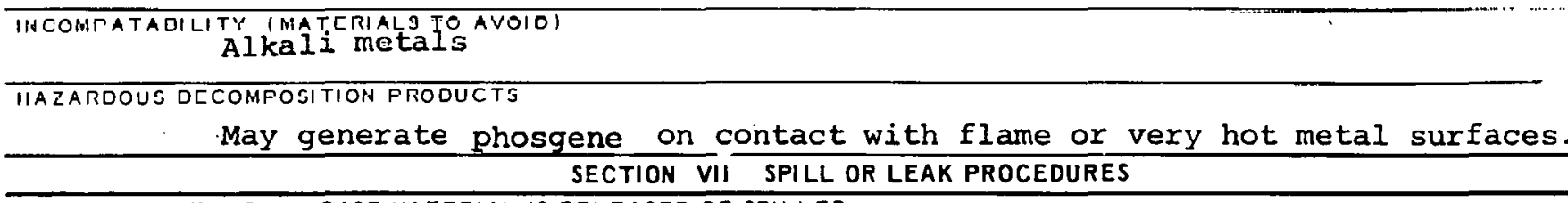

STEPS TO BE TAKEN IN CASE MATERIAL IS RELEASED OR SPILLED

WASTE DISPOSAL METHOD

SECTION VIII SPECIAL PROTECTION INFORMATION

\begin{tabular}{|c|c|c|c|}
\hline \multicolumn{4}{|c|}{ SECTION VIII SPECIAL PROTECTION INFORMATION } \\
\hline \multicolumn{3}{|c|}{ VENTILATION REQUIREMENTS LOCAL EXHAUST } & $\begin{array}{l}\text { PROTECTIVE CQUIPMENT (SPECIFY TYPES) EYE } \\
\text { satety goggles }\end{array}$ \\
\hline \multicolumn{3}{|l|}{ MECHANICAL (GENERAL) } & $\begin{array}{l}\text { CLOVES } \\
\text { rubber }\end{array}$ \\
\hline \multicolumn{3}{|l|}{ SPECIAL } & $\begin{array}{l}\text { RFSPIAATOR } \\
\text { self-contained }\end{array}$ \\
\hline \multicolumn{4}{|c|}{$\begin{array}{l}\text { OTHEA PROTECTIVE EQUIPMENI } \\
\text { Rubber, protective clothing. }\end{array}$} \\
\hline \multicolumn{4}{|c|}{ SPECIAL PRECAUTIONS } \\
\hline
\end{tabular}

store in cool, dry,well-ventilated area away from fire hazarde. OTHER PRECAUTIONS

SIGNATURE กATE 
PRODUCT DESIGNATION

03-307-2303

Methylene Iodide
MATERIAL SAFETY

DATA SHEET

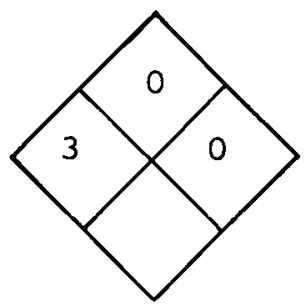

SECTION I SOURCE AND NOMENCLATURE

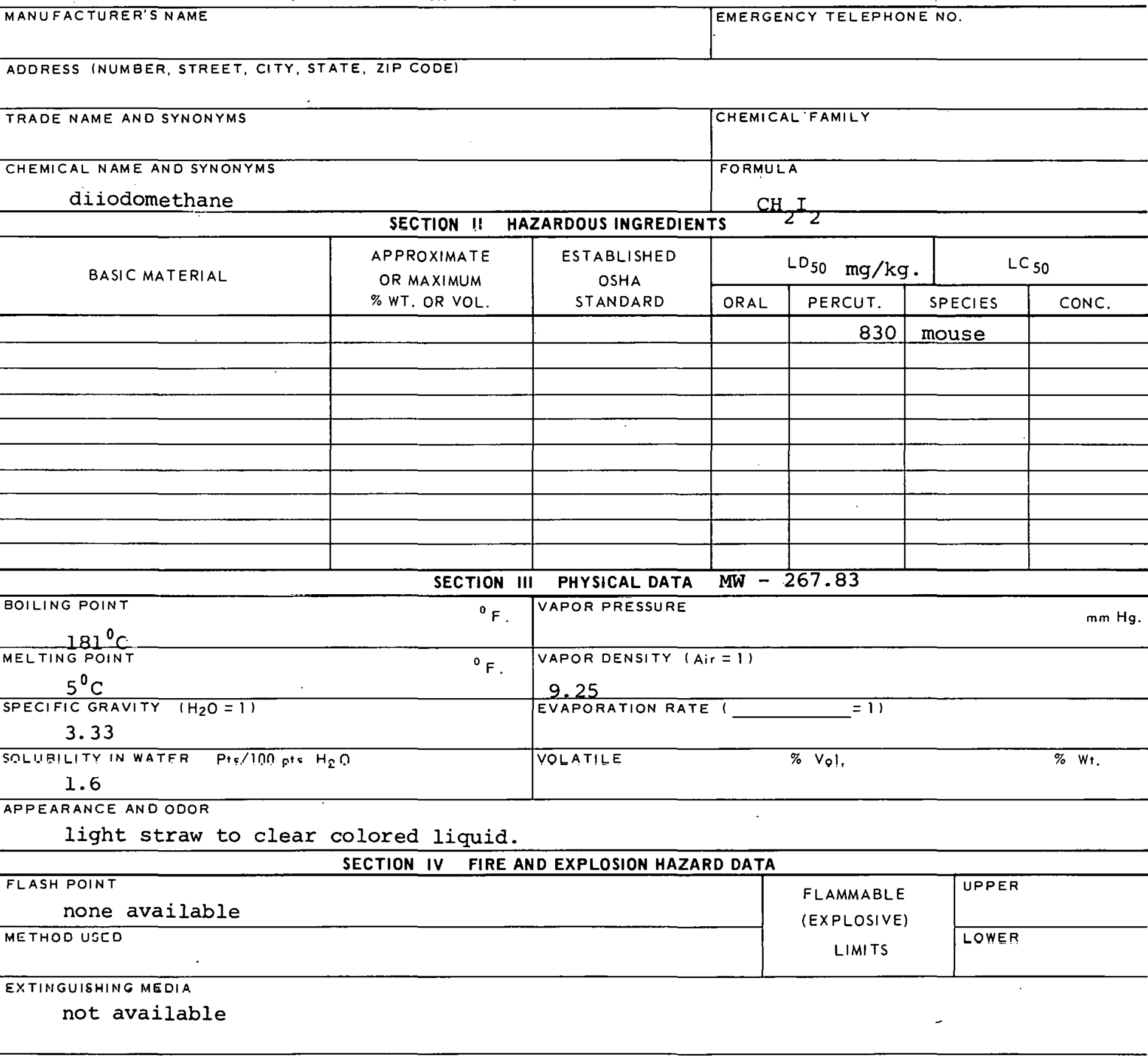

SPECIAL FIRE FIGHTING PROCEDURES

$--$

UNUSUAL FIRE AND EXPLOSION HAZARDS

When heated to decomposition point - emits toxic fumes of iodides. 


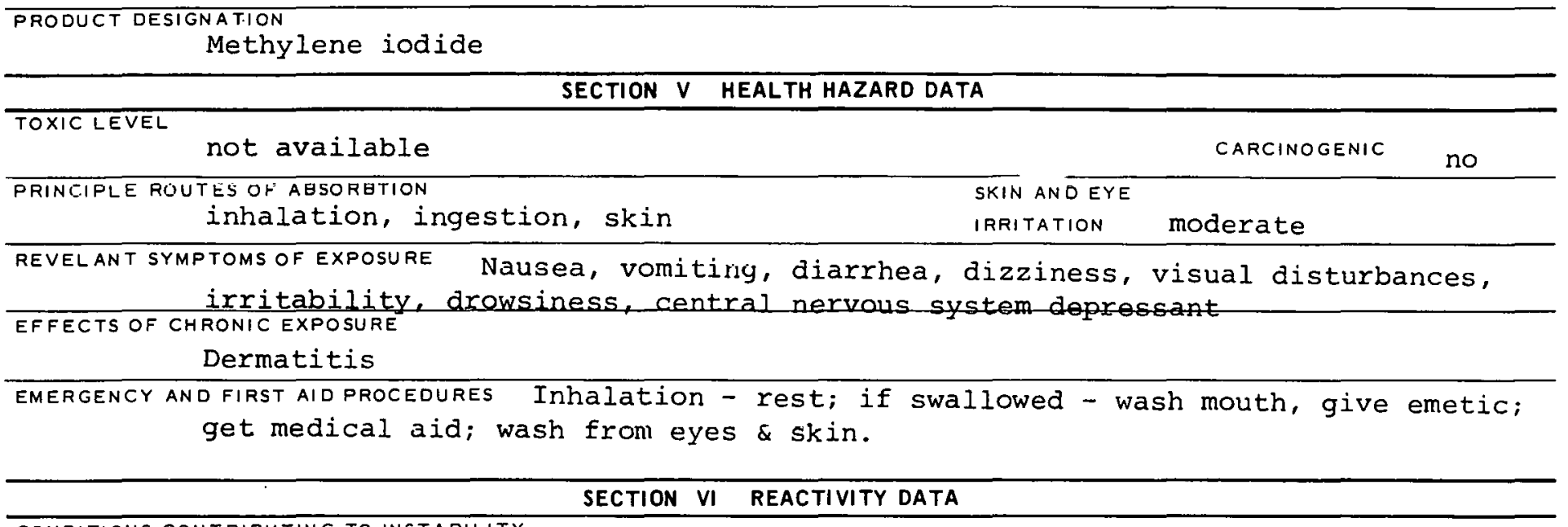

CONDITIONS CONTRIBUTING TO INSTABILITY

CONDITIONS CONTRIBUTING TO HAZARDOUS POLYMERIZATION

INCOMPATHEILITY IMATERIALS TO AVOIOI

HAZARDOUS DECOMPOSITION PRODUCTS

SECTION VII SPILL OR LEAK PROCEDURES

STEPS TO BE TAKEN IN CASE MATERIAL IS RELEASED OR SPILLED

WASTE DISPOSAL METHOO

SECTION VIII SPECIAL PROTECTION INFORMATION

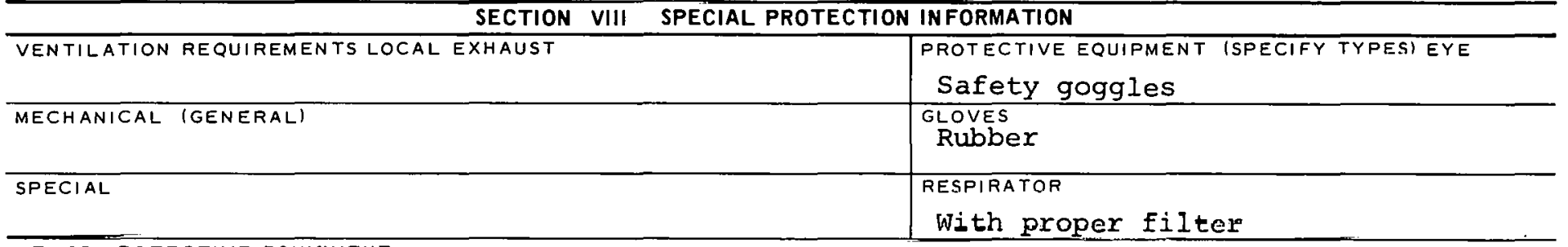

OTHER PROTECTIVE EQUIPMENT

SECTION IX SPECIAL PRECAUTIONS

PRECAUTIONS TO BE TAKEN IN HANDLING AND STORAGE

OTHER PRECAUTIONS

\begin{tabular}{l|l}
\hline SIGNATURE & DATE
\end{tabular} 
03-307-2803

Methylethylamine

Hydrochloride

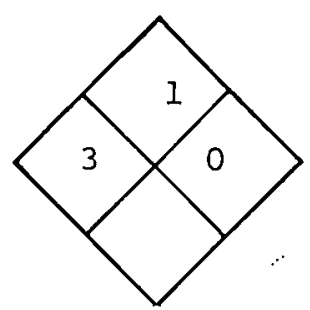

SECTION I SOURCE AND NOMENCLATURE

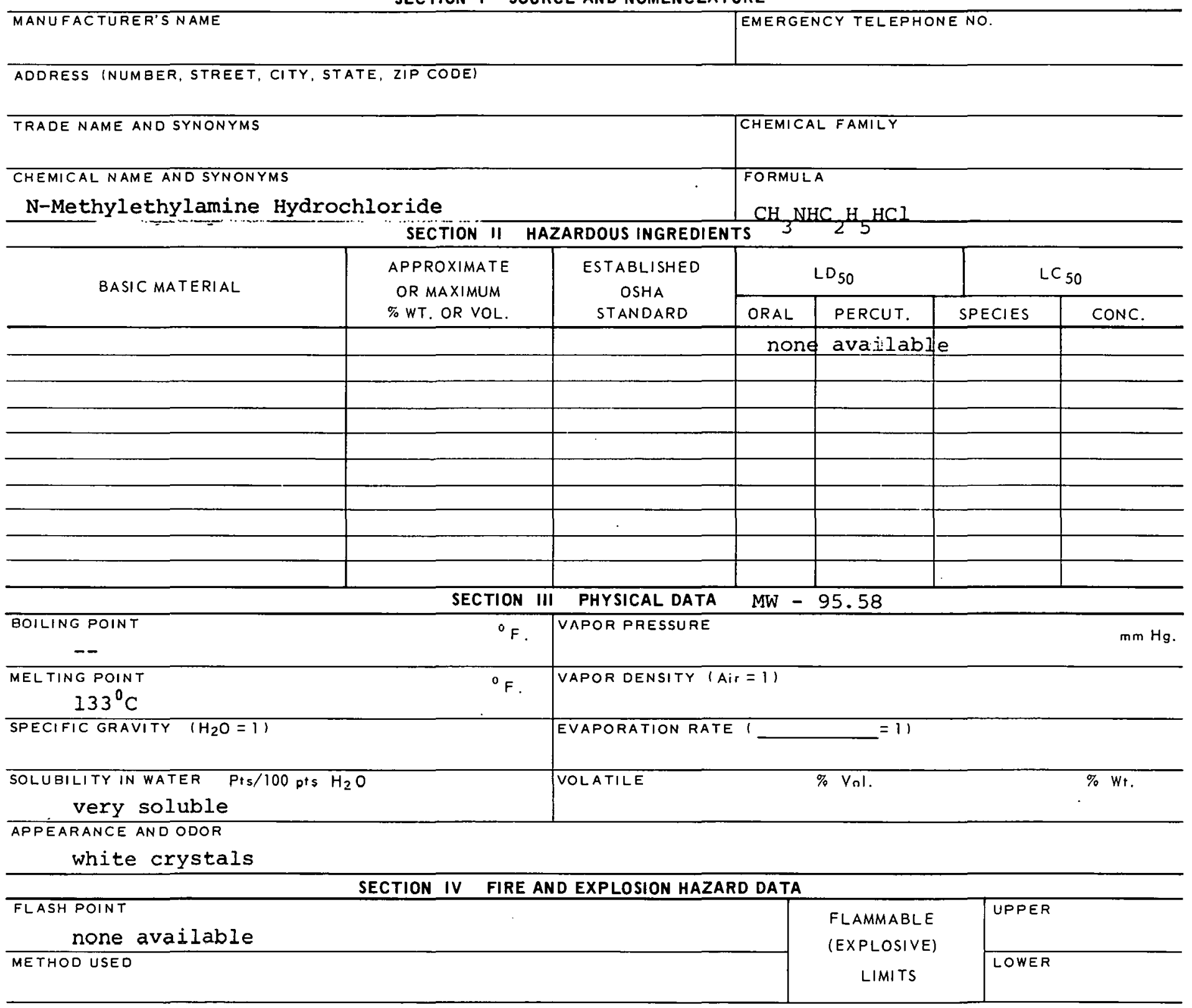

EXTINGUISHING MEDIA

Alcohol foam, carbon dioxide, dry chemical

SPECIAL FIRE FIGHTING PROCEOURES

Wear self-contained breathing apparatus.

UNUSUAL FIRE AND EXPLOSION HAZARDS 
Methylethylamine Hydrochloride SECTION $V$ HEALTH HAZARD DATA

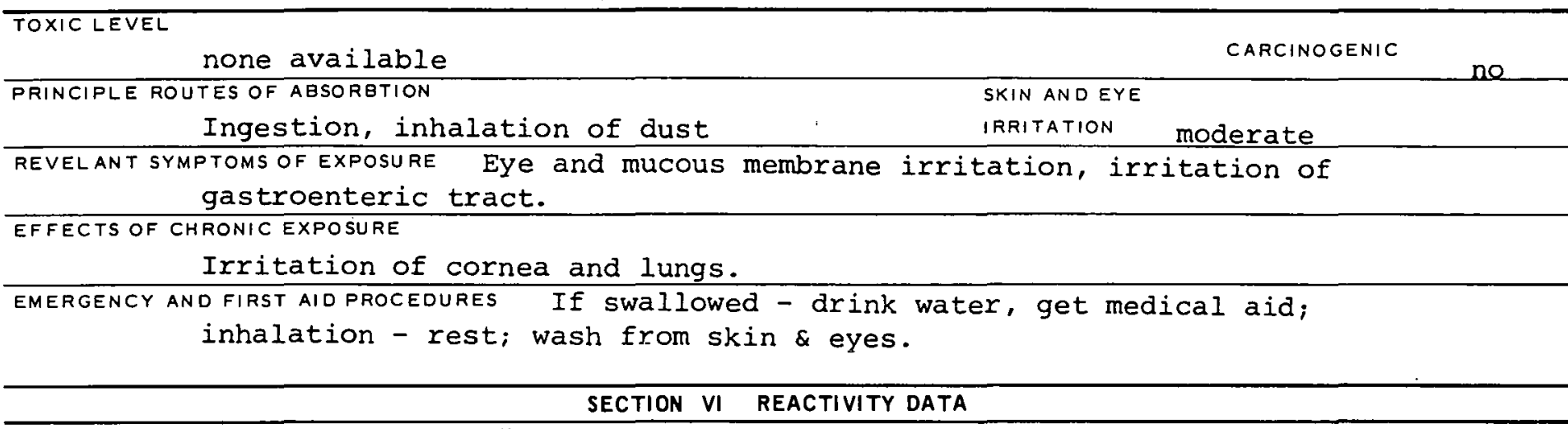

CONDITIONS CONTRIBUTING TO INSTABILITY

CONDITIONS CONTRIBUTING TO HAZARDOUS POLYMERIZATION

INSOMPATARII.ITY (MATFRIAI S TR AVOID)

HAZARDOUS DECOMPOSITION PRODUCTS

SECTION VII SPILL OR LEAK PROCEDURES

STEPS TO BE TAKEN IN CASE MATERIAL IS RELEASED OR SPILLED

WASTE DISPOSAL METHOD

SECTION VIII SPECIAL PROTECTION INFORMATION

\begin{tabular}{|c|c|}
\hline VENTILATION REQUIREMENTS LOCAL EXHAUST & $\begin{array}{l}\text { PROTECTIVE EQUIPMENT (SPECIFY TYPES) EYE } \\
\text { GOgglies }\end{array}$ \\
\hline MECHANICAL (GENERAL) & $\begin{array}{l}\text { GLOVES } \\
\text { Rubber }\end{array}$ \\
\hline SPECIAL & $\begin{array}{l}\text { RESPIRATOR } \\
\text { Selt-contained }\end{array}$ \\
\hline
\end{tabular}

OTHER PROTECTIVE EQUIPMENT

SECTION IX SPECIAL PRECAUTIONS

PRECAUTIONS TO BE TAKEN IN HANDLING AND STORAGE

OTHER PRECAUTIONS

SIGNATURE

DATE

$307-2803 B$ 


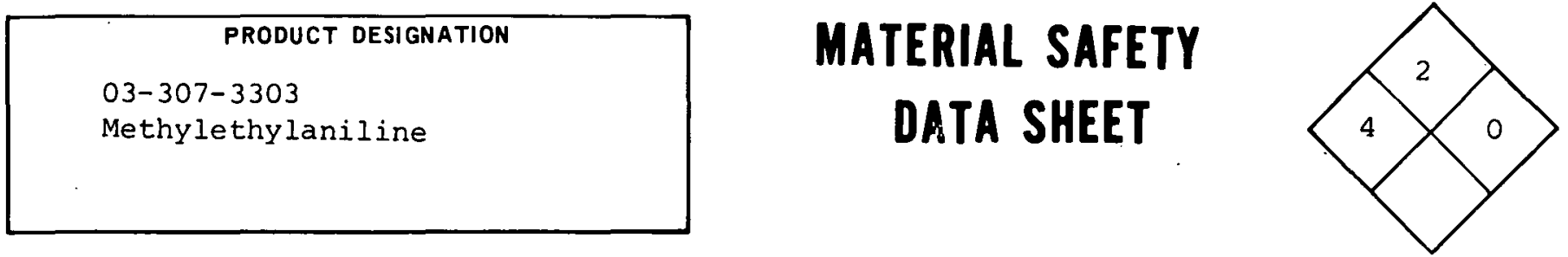

\section{SECTION I SOURCE AND NOMENCLATURE}

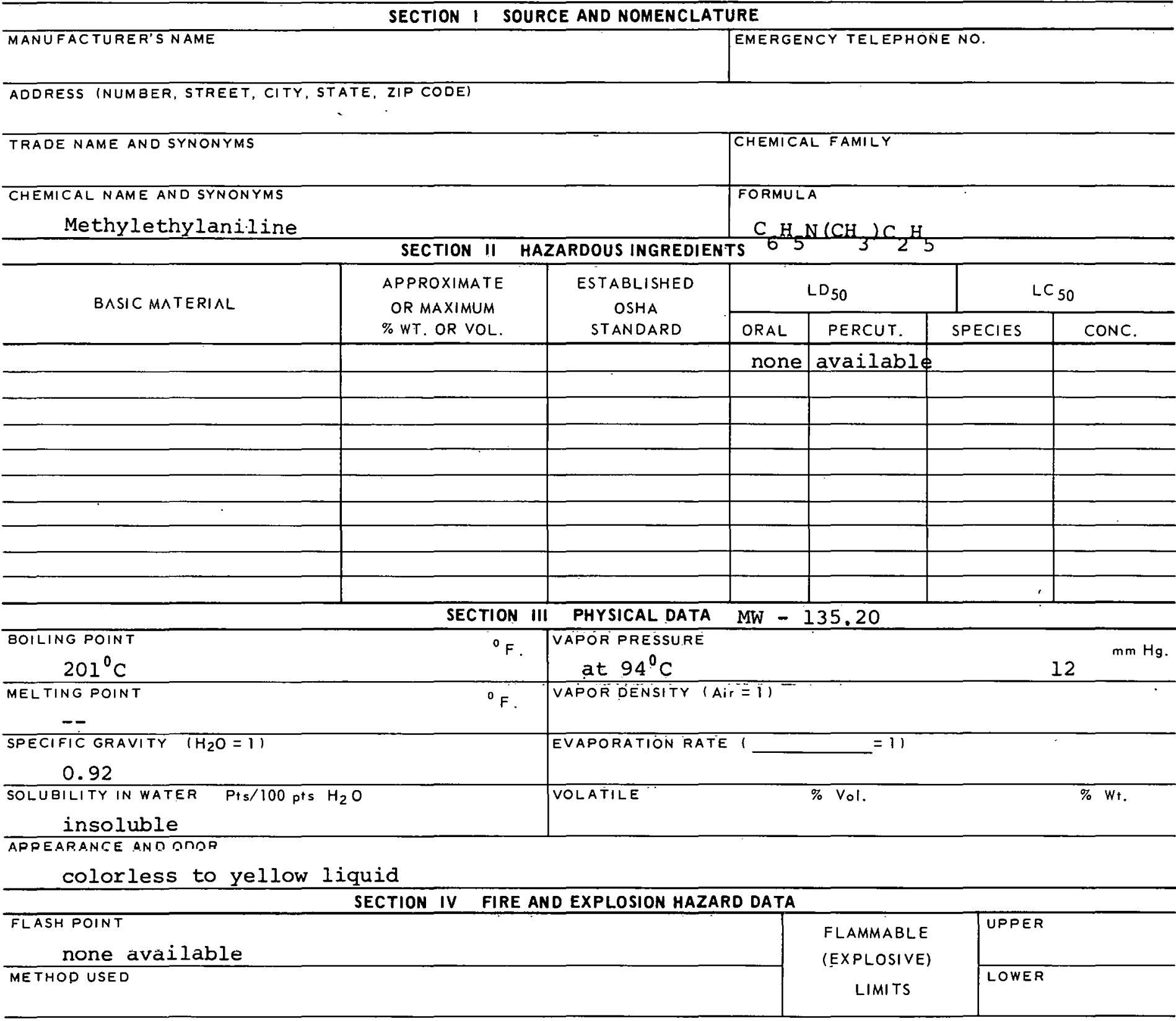

EXTINGUISHING MEDIA

Alcohol foam, water spray, carbon dioxide, dry chemical

SPECIAL FIRE FIGHTING PROCEDURES

UNUSUAL FIRE AND EXPLOSION HAZARDS 


\section{TOXIC LEVEL}

PRINCIPLE ROUTESOF ABSORETPle

inhalation, ingestion, skin

REVELANT SYMPTOMS OF EXPOSURE Weakness, tremors, cyanosis, drowsiness, central nervous

system depression.

EFFECTS OF CHRONIC EXPOSURE

Fatigue, loss of appetite, headache, dizziness

EMERGENCY AND FIRST AID PROCEDURES If swallowed - give emetic, get medical aid; inhalation rest; wash from skin \& eyes.

CONDITIONS CONTRIBUTING TO INSTABILITY

CONDITIONS CONTRIBUTING TO HAZARDOUS POLYMERIZATION

INCOMSATABILITY (MATLRIALS TO AVOID)

HAZARDOUS DECOMPOSITION PRODUCTS

SECTION VII SPILL OR LEAK PROCEDURES

STEPS TO BE TAKEN IN CASE MATERIAL IS RELEASED OR SPILLED

WASTE DISPOSAL METHOD

SECTION VIII SPECIAL PROTECTION INFORMATION

\begin{tabular}{l|l|l}
\hline VENTILATION REQUIREMENTS LOCAL EXHAUST & PROTECTIVE EQUIPMENT (SPECIFY TYPES) EYE
\end{tabular}

MECHANISAI (RENERAL)

Goggles

GLOVES

Polyvinyl gloves

SPECIAL

RESPIRATOR

OTHER PROTECTIVE EQUIPMENT

Whth proper I1Lter

\section{SECTION IX SPECIAL PRECAUTIONS}

PRECAUTIONS TO BE TAKEN IN HANDLING AND STORAGE

\section{OTHER PRECAUTIONS}


PRODUCT DESIGNATION

$03-307-3843$

$03-307-3853$

Methyl Ethyl Ketone
MATERIAL SAFETY

DATA SHEET

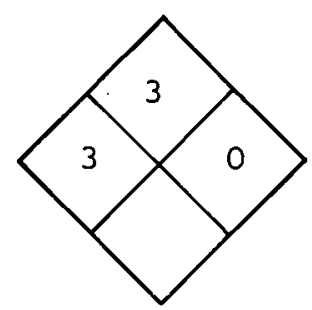

\section{SECTION I SOURCE AND NOMENCLATURE}

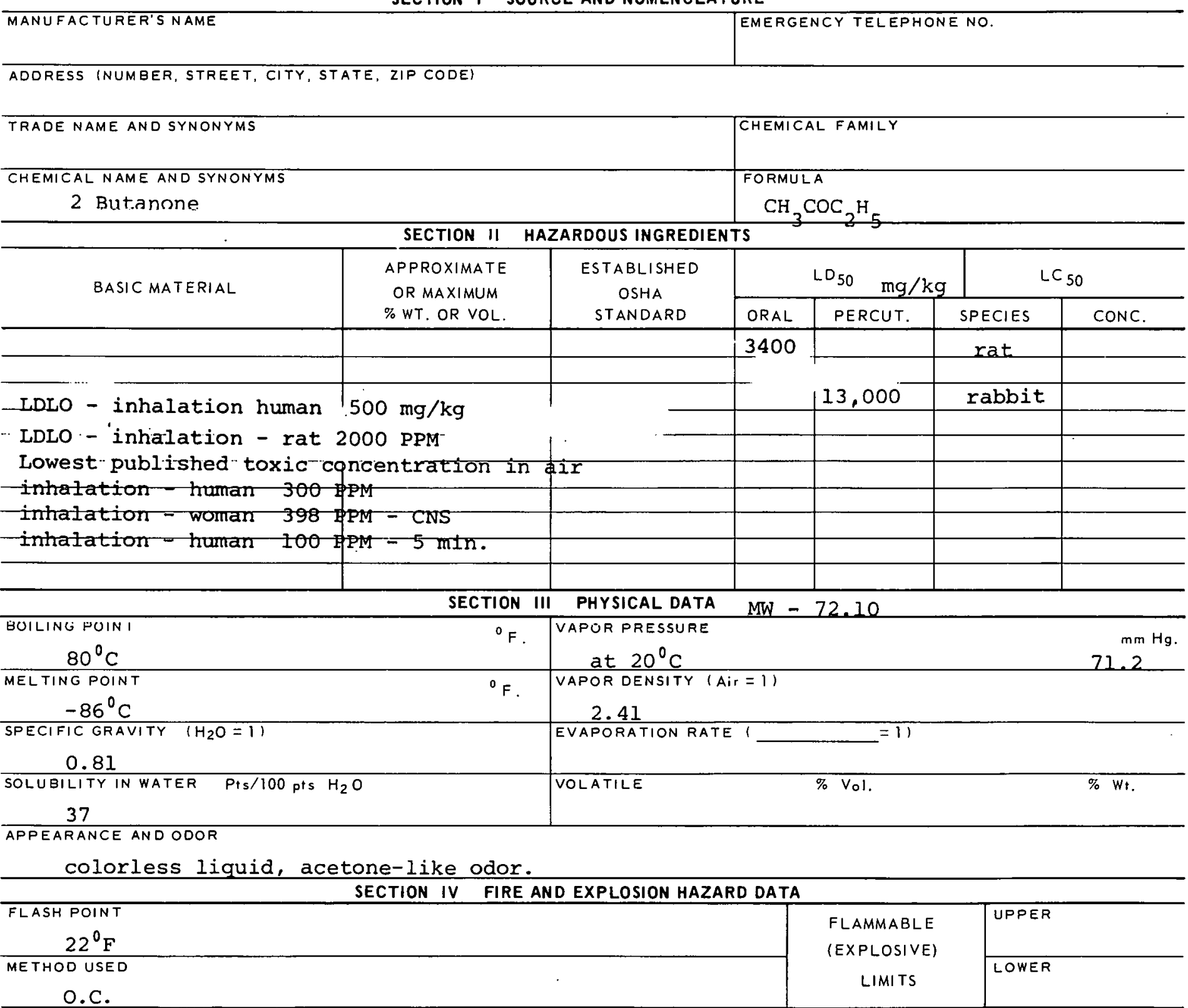

EXTINGUISHING MEDIA

Alcohol foam, carbon dioxide, dry chemical. Water may be ineffective.

SPECIAL FIRE FIGHTING PROCEDURES

UNUSUAL FIRE AND EXPLOSION HAZAROS

Moderate explosion hazard when exposed to flame. 
TOXIC LEVEL

$200 \mathrm{PPM}, 590 \mathrm{mg} / \mathrm{C} . \mathrm{M}$.

CARCINOGENIC severe PRINCIPLE ROUTES OF ABSORBTION

Inhalation, ingestion REVELANT SYMPTOMS OF EXPOSURE

Eye and nasal passage irritation, narcotic symptoms, drying \& irritation of skin. EFFECTS OF CHRONIC EXXPOSURE

--

EMERGENCY AND FIRST AID PROCEDURES If swallowed - give emetic, get medical aid -

inhalation - rest; wash from skin \& eyes.

SECTION VI REACTIVITY DATA

CONDITIONS CONTRIBUTING TO INSTABILITY

CONDITIONS CONTRIBUTING TO HAZARDOUS POLYMERIZATION

INCOMPATABILITY (MATERIALS TO AVOID)

TIAZARDOUS DLCOMTOEITIOH TRODUCTE

SECTION VII SPILL OR LEAK PROCEDURES

STEPS TO BE TAKEN IN CASE MATERIAL IS RELEASED OR SPILLED

Absorb on paper towels; evaporate in hood.

SKIN AND EYE

IRRITATION

severe

WASTE DISPOSAL METHOD

SECTION VIII SPECIAL PROTECTION INFORMATION

VENTILATION REQUIREMENTS LOCAL EXHAUST

PROTECTIVE EQUIPMENT (SPECIFY TYPES) EYE

MECHANICAL IGENERAL)

Goggles

GLOVES

Rubber

SPECIAL

RLSTIRATOR

With proper filler

OTHER PROTECTIVE EQUIPMENT

Protective clothing

\section{SECTION IX SPECIAL PRECAUTIONS}

PRECAUTIONS TO BE TAKEN IN HANDLING AND STORAGE

Store in standard safety cabinet.

\section{OTHER PRECAUTIONS}

SIGNATURE 


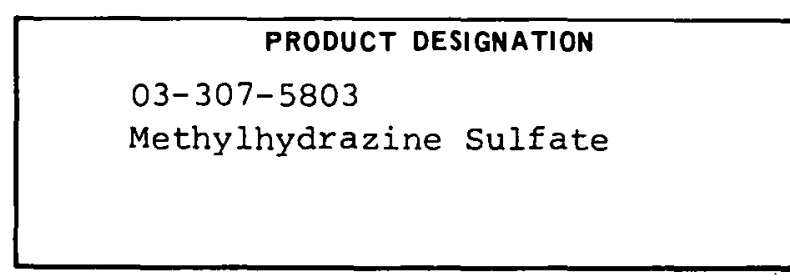

\section{MATERIAL SAFETY DATA SHEET}

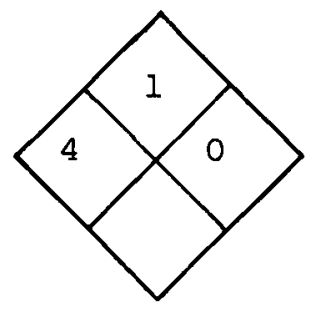

SECTION I SOURCE AND NOMENCLATURE

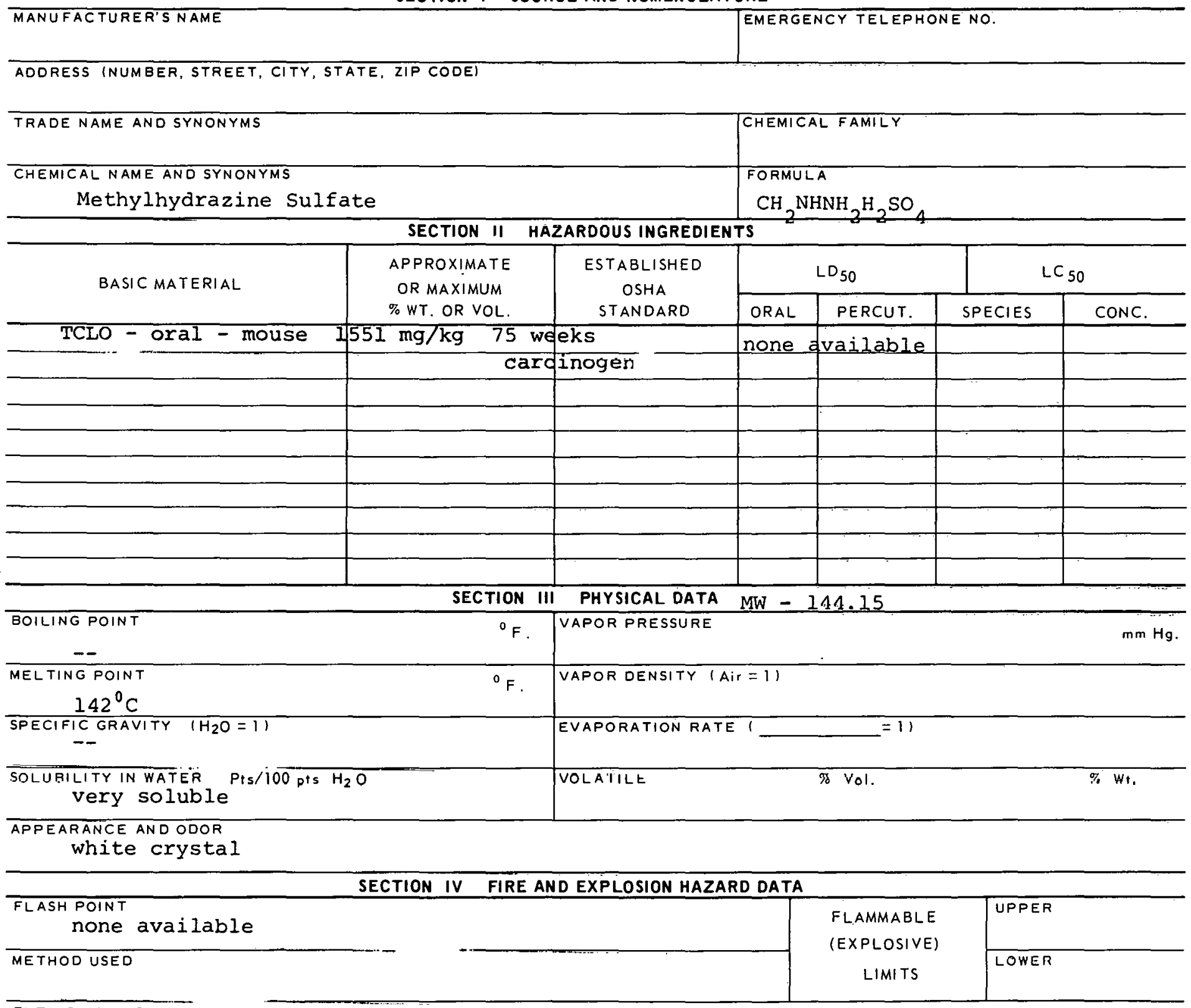

EXTINGUISHING MEDIA

Alcohol foam, carbon dioxide, dry chemical.

SPECIAL FIRE FIGHTING PROCEOURES

UNUSUAL FIRE AND EXPLOSION HALARDS 


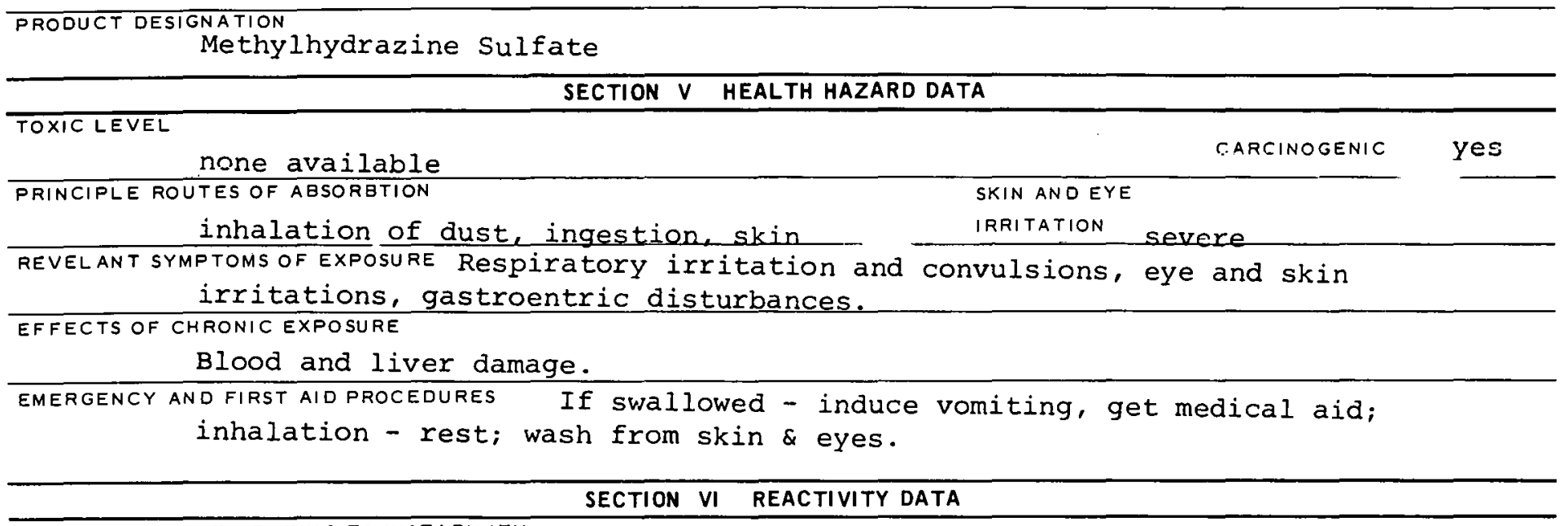

CONDITIONS CONTRIBUTING TO INSTABILITY

CONDITIONS CONTRIBUTING TO HAZARDOUS POLYMERIZATION

IMEOAPATARILITY TAATEAIALE TO AVOIDI

HAZARDOUS DECOMPOS!TIQN PRODUCTS

SECTION VII SPILL OR LEAK PROCEDURES

STEPS TO BE TAKEN IN CASE MATERIAL IS RELEASED OR SPILLED

WASTE DISPOSAL METHOD

SECTION VIII SPECIAL PROTECTION INFORMATION

\begin{tabular}{l|l}
\hline \multicolumn{1}{c}{ SECTION VIII SPECIAL PROTECTION INFORMATION } \\
\hline VENTILATION REQUIREMENTS LOCAL EXHAUST & $\begin{array}{l}\text { PROTECTIVE CQUIPMENT (SPECIFY TYPES) EYE } \\
\text { GOGGLES }\end{array}$ \\
\hline MECHANICAL IGENERAL) & GLOVEE \\
Rubber \\
\hline SPECIAL & RFSPIRATNR \\
\hline OTHER PROTECTIVE EQUIPMENT & With proper fiIter \\
\hline
\end{tabular}

PRECAUTIONS TO BE TAKEN IN HANDLING AND STORAGE

OTHER PRECAUTIONS

SIGNATURE

חATF

$307-5803 B$ 


PRODUCT DESIGNATION
$03-307-7803$
Methyl Iodide

\section{MATERIAL SAFETY DATA SHEET}

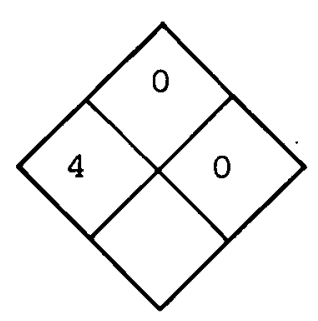

\section{SECTION I SOURCE AND NOMENCLATURE}

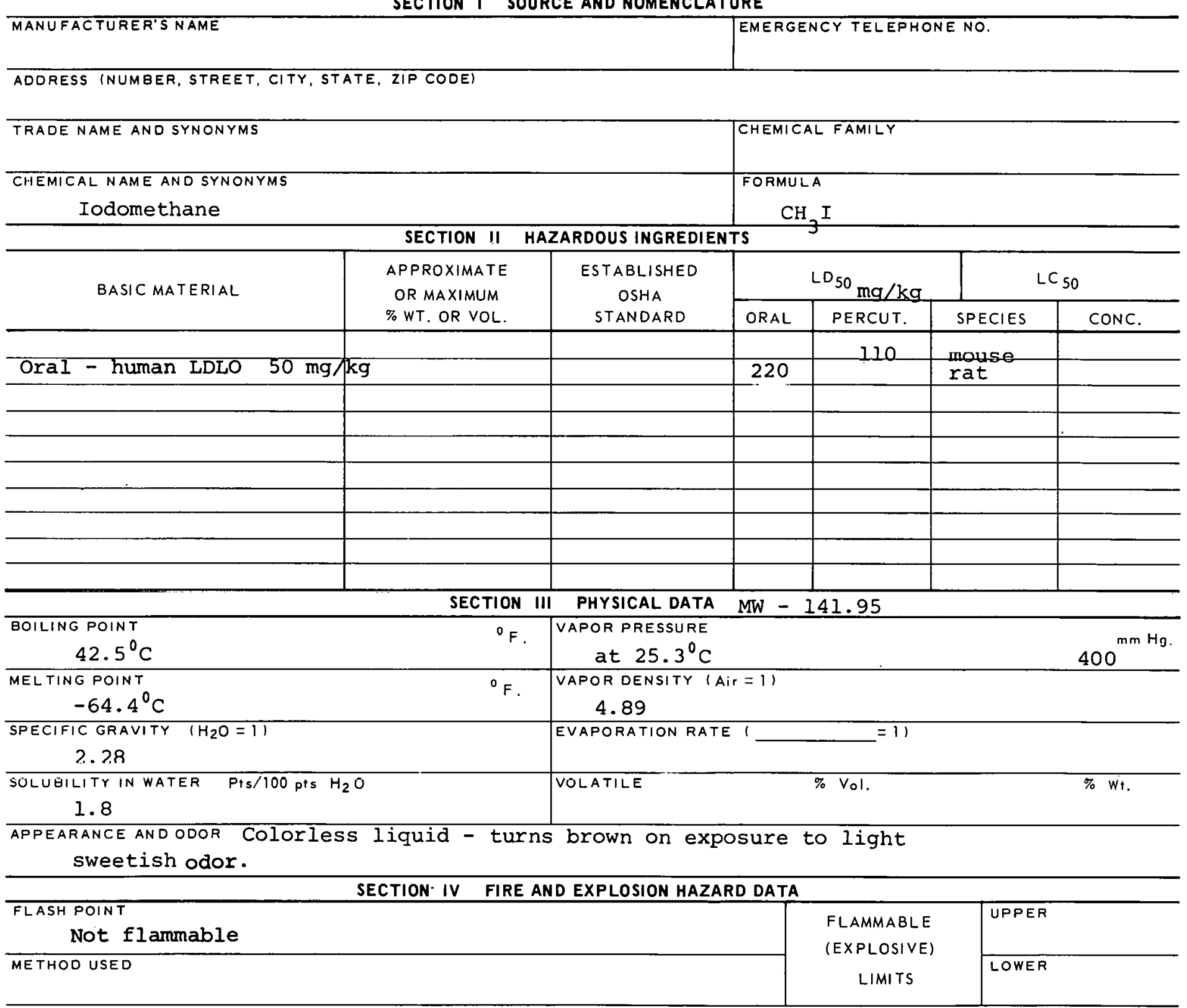

EXTINGUISHING MEDIA

not available

SPECIAL FIRE FIGHTING PROCEDURES

--

UNUSUAL FIRE AND EXPLOSION HAZAROS

When heated to decomposition point - emits toxic fumes of iodides. 
TOXIC LEVEL

$T L V$ - $5 \mathrm{PPM}, 28 \mathrm{mg} / \mathrm{CM}$.

CARCINOGENIC

PRINCIPLE ROUTES OF ABSORBTION
inhalation, ingestion, skin SKIN AND EYE

IRRITATION

severe

REVELANT SYMPTOMS of EXPOSURE Nausea, vomiting, diarrhea, dizziness, visual disturbances, irritability, drowsiness, central nervous system depressant.

EFFECTS OF CHRONIC EXPOSURE

Dermatitis

EMERGENCY AND FIRST AID PROCEDURES Inhalation - rest; if swallowed - wash mouth, give emetic, get medical aid; wash from eyes \& skin.

\section{SECTION VI REACTIVITY DATA}

CONDITIONS CONTRIBUTING TO INSTABILITY

CONDITIONS CONTRIBUTING TO HAZARDOUS POLYMERIZATION

INCOMPATAEILITY (MATERIALS TQ AVOID)

IIAZARDOUS DECOMFOSITION PRODUCTS

SECTION VII SPILL OR LEAK PROCEOURES

STEPS TO BE TAKEN IN CASE MATERIAL IS RELEASED OR SPILLED

Absorb with paper towels; evaporate in hood.

WASTTE DISPOSAL METHOD

SECTION VIII SPECIAL PROTECTION INFORMATION

\begin{tabular}{l|l}
\hline VENTILATION REQUIREMENTS LOCAL EXHAUST & PROTECTIVE EQUIPMENT (SPECIFY TYPES) EYE
\end{tabular}

MECHANICAL (GENERAL) safety goggles

GLOVES

rubber

SPECIA.L

RESPIRATUR

with proper. filter

OTHER PROTECTIVE EQUIPMENT

SECTION IX SPECIAL PRECAUTIONS

PRECAUTIONS TO BE TAKEN IN HANDLING AND STORAGE

Store in tightly closed containers in dark place.

OTHER PRECAUTIONS

SIGNATURE

DATE

$307-7803 B$ 


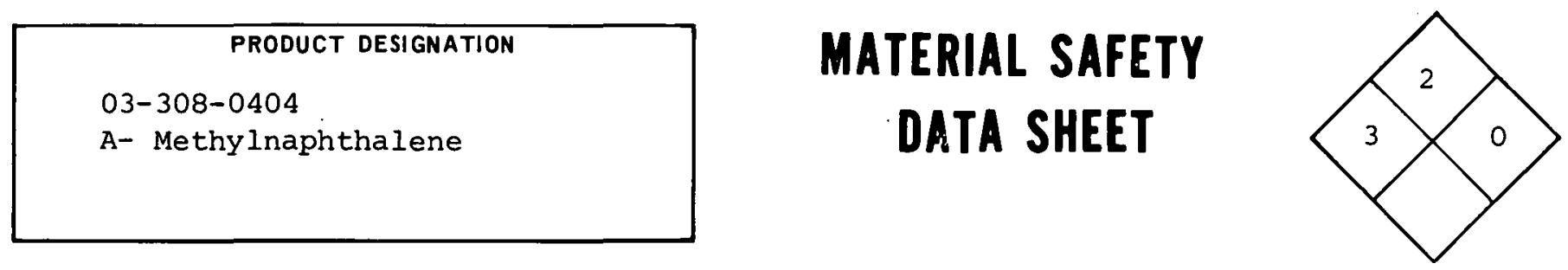

SECTION I SOURCE AND NOMENCLATURE

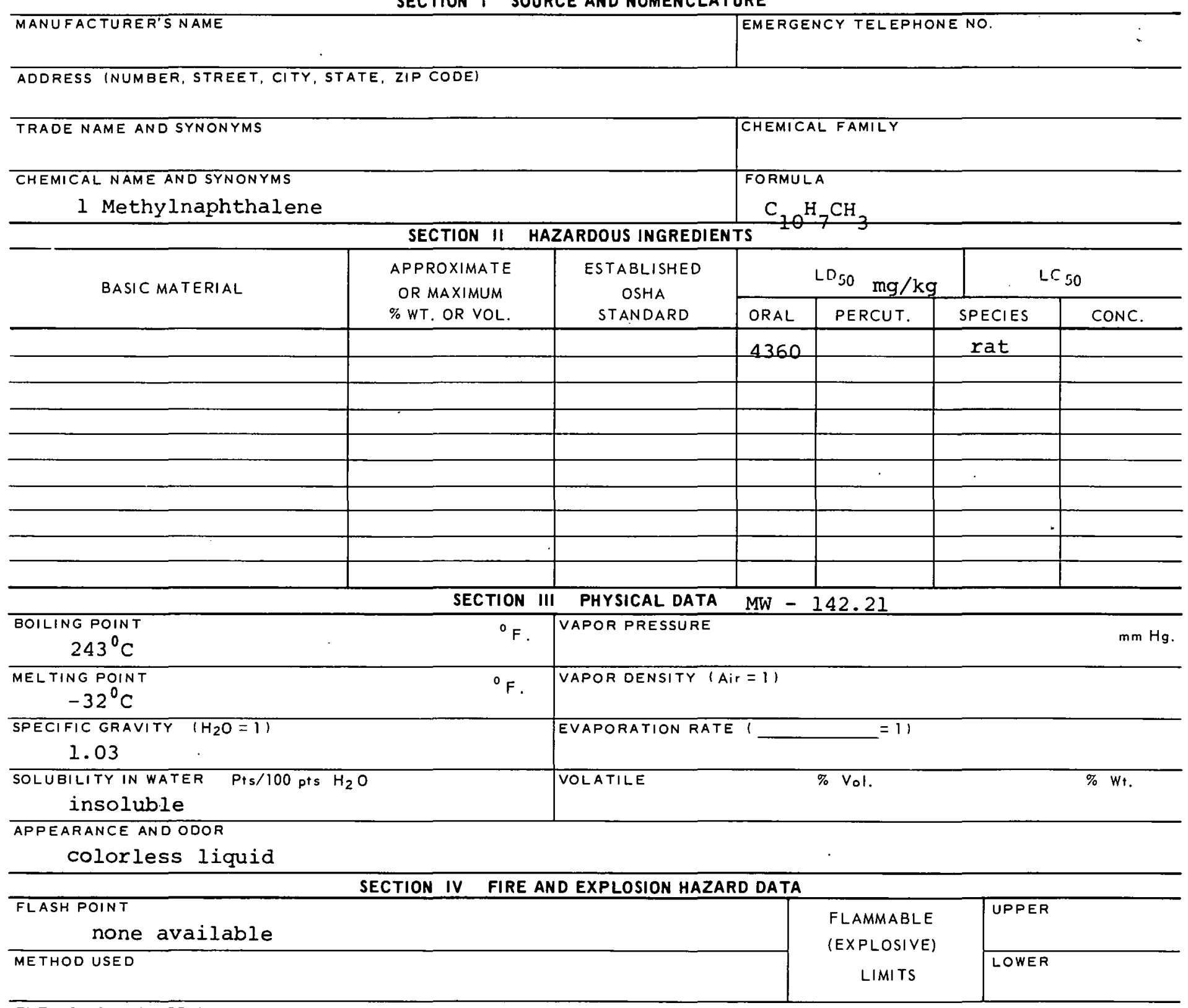

EXTINGUISHING MEDIA

Alcohol foam, carbon dioxide, dry chemical

SPECIAL FIRE FIGHTING PROCEDURES

Self-contained breathing apparatus.

UNUSUAL FIRE AND EXPLOSION HAZARDS 
A-Methylnaphthalene

SECTION $V$ HEALTH HAZARD DATA

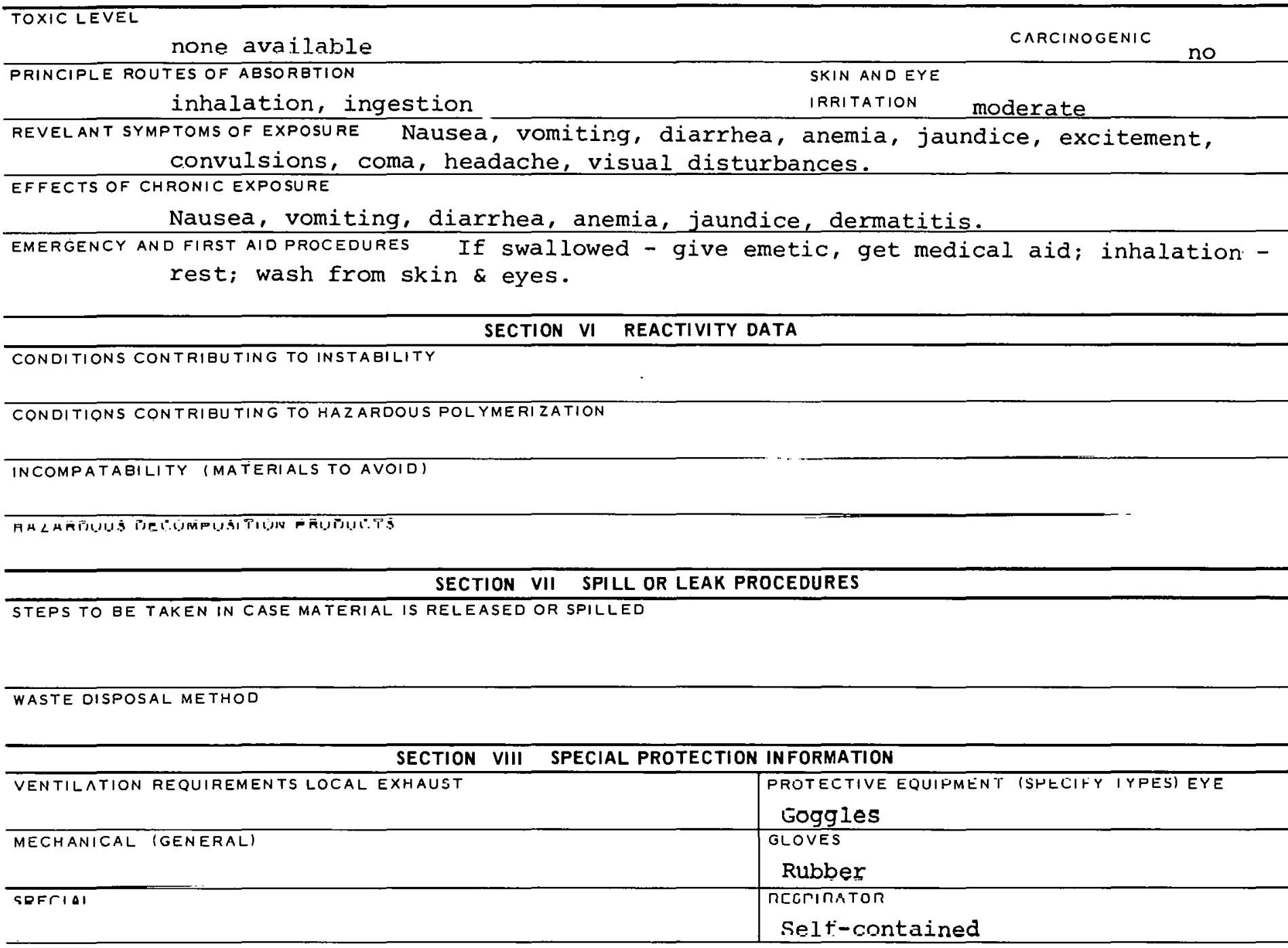

OTHER PROTECTIVE EOUIPMENT

SECTION IX SPECIAL PRECAUTIONS

PRECAUTIONS TO BE TAKEN IN HANDLING AND STORAGE

OTHEF TAECAUTIOHE

SIGNATURE

DATE

$308-0404 B$ 


\begin{tabular}{|c|}
\hline PRODUCT DESIGNATION \\
03-308-0474 \\
B-Methylnaphthalene
\end{tabular}$\quad$ MATERIAL SAFETY

\section{SECTION I SOURCE AND NOMENCLATURE}

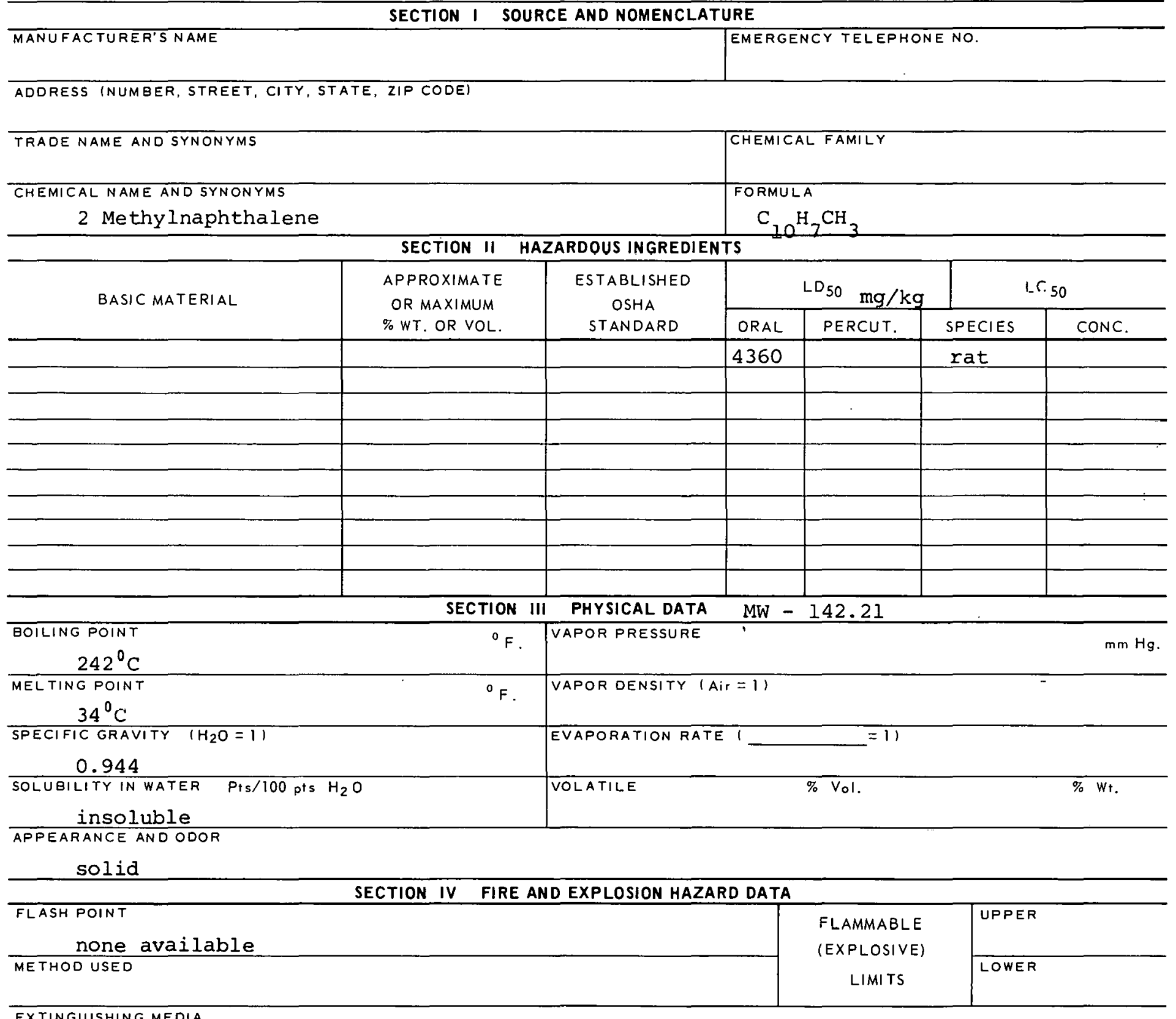

EXTINGUISHING MEDIA

alcohol foam, carbon dioxide, dry chemical

SPECIAL FIRE FIGHTING PROCEDURES

self-contained breathing apparatus.

UNUSUAL FIRE AND EXPLOSION HAZARDS 
B-Methylnaphthalene

\section{SECTION $V$ HEALTH HAZARD DATA}

TOXIC LEVEL

none available

PRINCIPLE ROUTES OF ABSORATION

Inhalation, ingestion

CARCINOGENIC

CARCINOGEN
SKIN ANDEYE
IRRITATION

REVELANT SYMPTOMS OF EXPOSURE

convulsions,

Nausea, vomiting, diarrhea, anemia, jaundice, excitement, coma, headache, visual disturbances.

EFFECTS OF CHRONIC EXPOSURE Nausea, vomiting, diarrhea, anemia, jaundice, dermatitis.

EMERGENCY AND FIRST AID PROCEDURES Ingestion - give emetic, get medical aid; inhalation rest; wash skin \& eyes.

SECTION VI REACTIVITY DATA

CONDITIONS CONTRIBUTING TO INSTABILITY

CONDITIONS CONTRIBUTING TO HAZARDOUS POLYMERIZATION

IIGOMPATABI WITY (MATERIALE TO AVQID)

HAZARDOUS DECOMPQS! TI ON PRQDUCTS

SECTION VII SPILL OR LEAK PROCEDURES

STEPS TO BE TAKEN IN CASE MATERIAL IS RELEASED OR SPILLED

WASTE DISPOSAL METHOD

SECTION VIII SPECIAL PROTECTION INFORMATION

\begin{tabular}{|c|c|}
\hline VENTILATION REQUIREMENTS LOCAL EXHAUST & $\begin{array}{l}\text { PROTECTIVE EQUIPMENT (SPECIFY TYPES) EYE } \\
\text { GOgglOS }\end{array}$ \\
\hline MECHANICAL (GENERAL) & $\begin{array}{l}\text { GLOVES } \\
\text { Rubber }\end{array}$ \\
\hline SPECIAL & $\begin{array}{l}\text { RESPIRATOR } \\
\text { Self-contained }\end{array}$ \\
\hline
\end{tabular}

OTHER PROTECTIVE EQUIPMENT

SECTION IX SPECIAL PRECAUTIONS

PRECAUTIONS TO BE TAKEN IN HANDLING AND STORAGE

OTHER PRECAUTIONS

SIGNATURE

DATE

$308-0474 B$ 


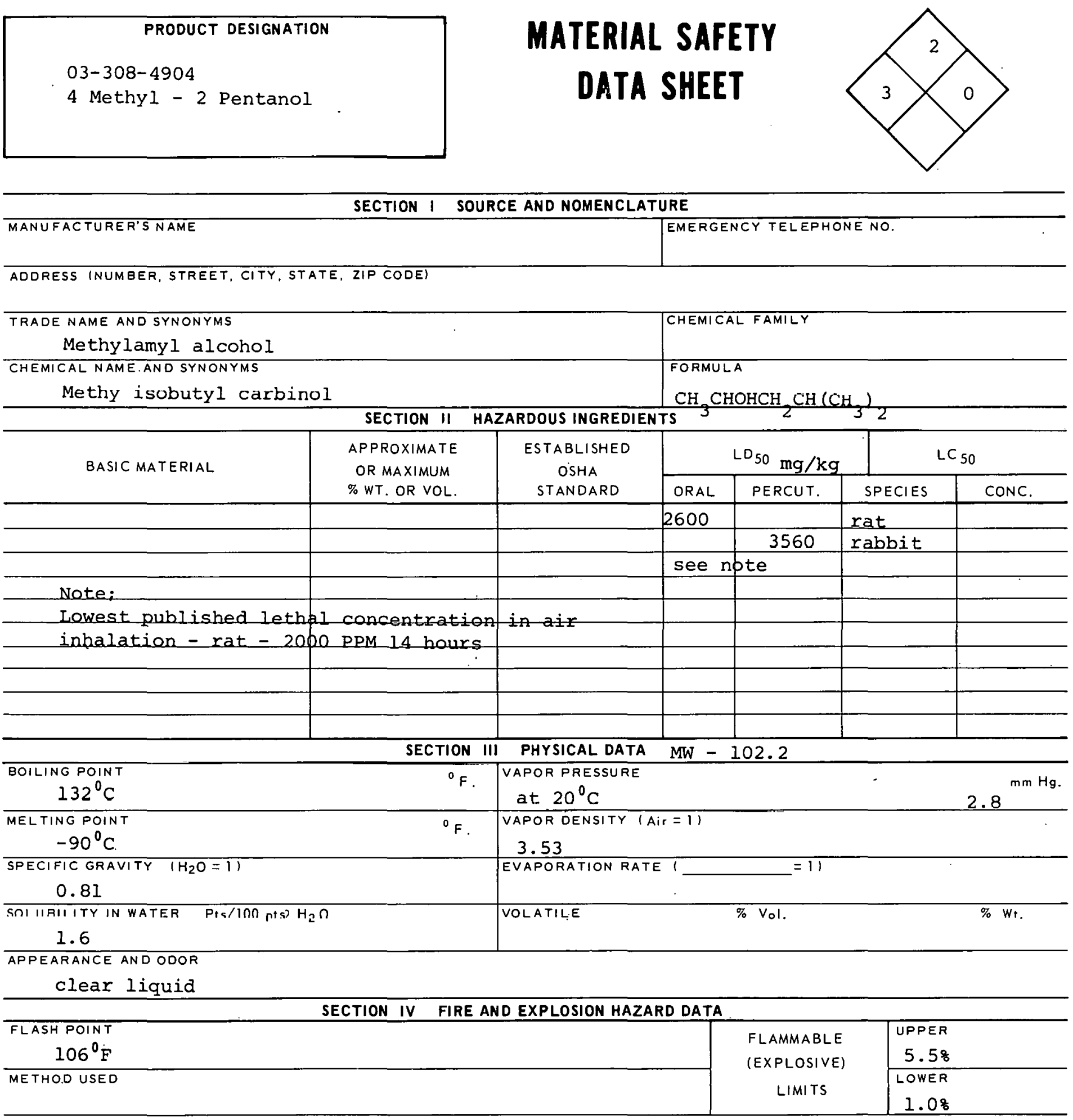

EXTINGUISHING MEDIA

Alcohol foam, carbon dioxide, dry chemical

SPECIAL FIRE FIGHTING PROCEDURES

UNUSUAL FIRE AND EXPLOSION HAZARDS 
4 Methyl 2 Pentanol

SECTION V HEALTH HAZARD DATA

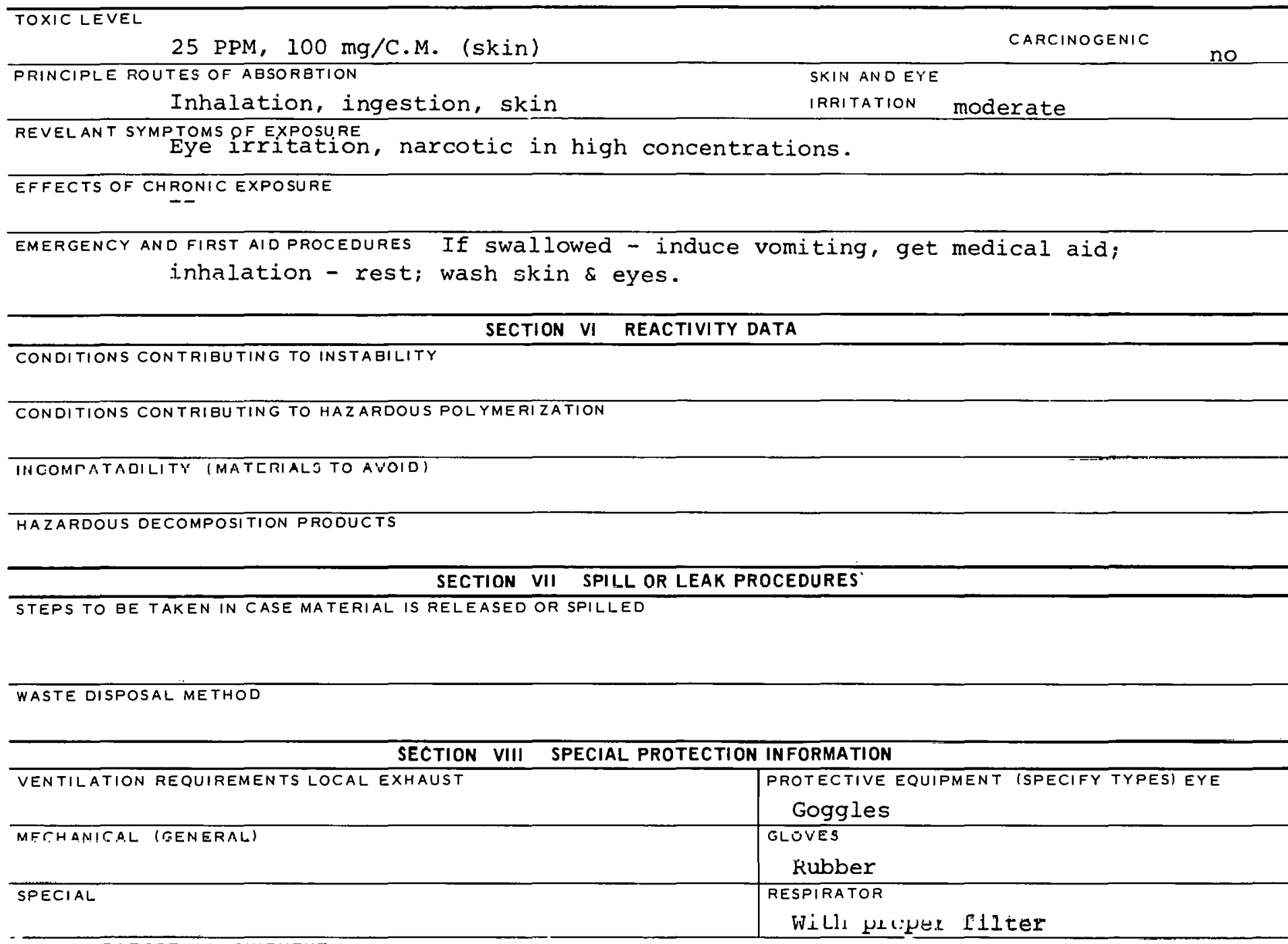

OTHER PROTECTIVE LQUIPMENT

SECTION IX SPECIAL PRECAUTIONS

PRECAUTIONS TO BE TAKEN IN HANDLING AND STORAGE

OTHER PRECAUTIONS

SIGNATURE

DATE

$308-4904 B$ 


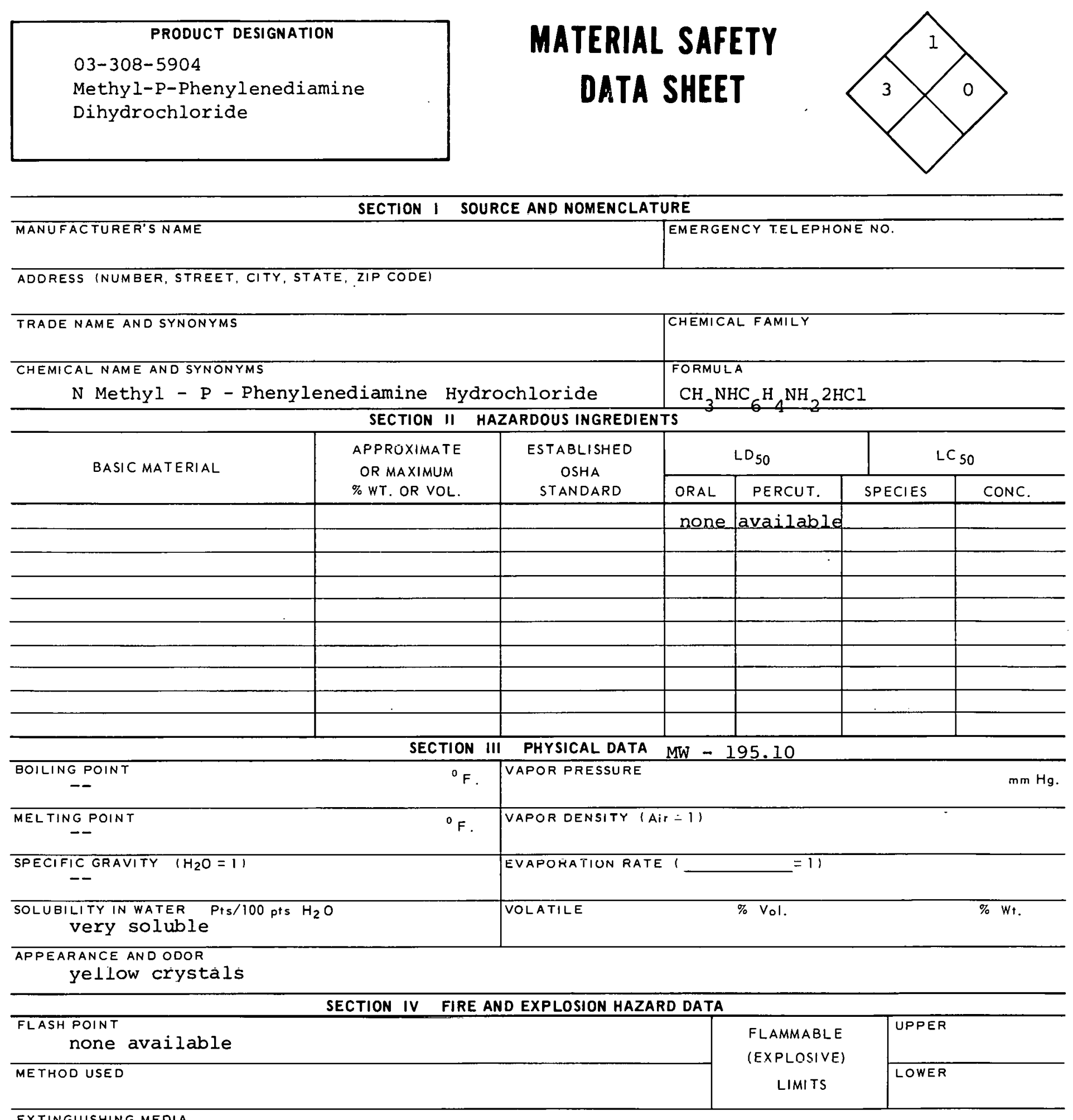

EXTINGUISHING MEOIA

Alcohol foam, carbon dioxide, dry chemical SPECIAL FIRE FIGHTING PROCEDURES 


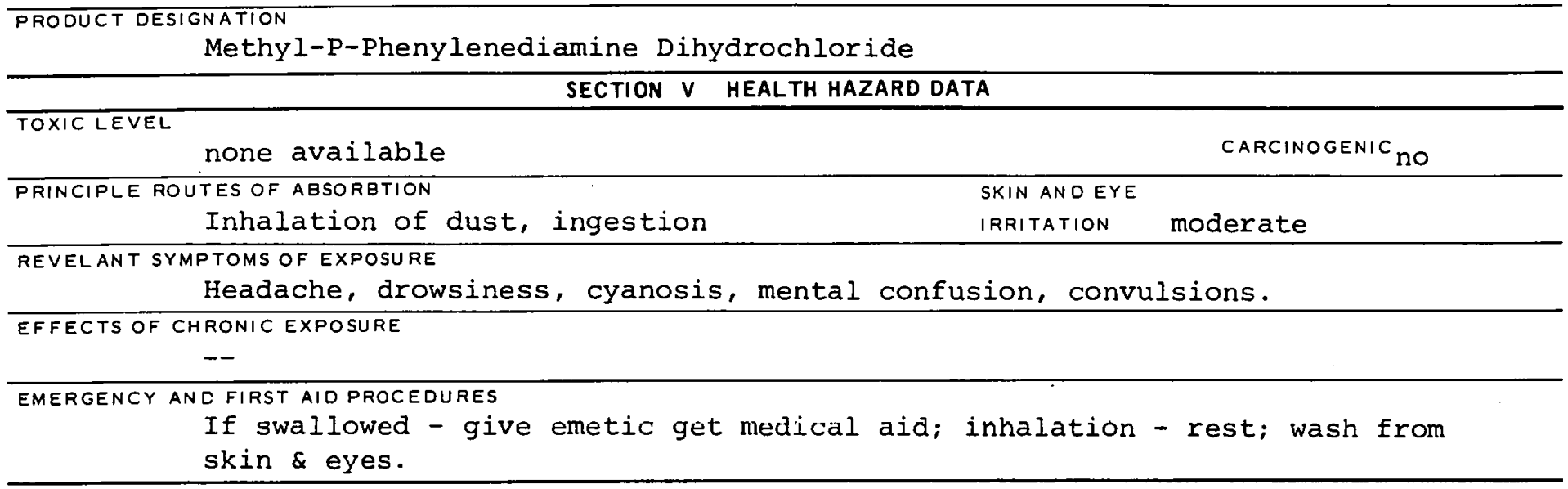

SECTION VI REACTIVITY DATA

CONDITIONS CONTRIBUTING TO INSTABILITY

CONDITIONS CONTRIBUTING TO HAZARDOUS POLYMERIZATION

INCOMPATABILITY (MATERIALS TO AVOID)

HAZARDOUS DESOMROSITION PRODUCTS

SECTION VII SPILL OR LEAK PROCEDURES

STEPS TO BE TAKEN IN CASE MATERIAL IS RELEASED OR SPILLED

WASTE DISPOSAL METHOD

SECTION VIII SPECIAL PROTECTION INFORMATION

\begin{tabular}{l|l}
\hline VENTILATION REQUIREMENTS LOCAL EXHAUST & $\begin{array}{l}\text { PROTECTIVE EQUIPMENT (SPECIFY TYPESI EYE } \\
\text { gOggleS }\end{array}$ \\
\hline MECHANICAL IGENERAL) & GLOVES \\
Rubber
\end{tabular}

OTHER PROTECTIVE EQUIPMENT

SECTION IX SPECIAL PRECAUTIONS

PRECAUTIONS TO BE TAKEN IN HANDLING AND STORAGE

OTHER PRECAUTIONS

SIGNATURE

DATE

$308-5904 B$ 


\begin{tabular}{|c|}
\hline PRODUCT DESIGNATION \\
03-308-6404 \\
A-Methyl-A-Phenylhydrazine
\end{tabular}

\section{MATERIAL SAFETY \\ DATA SHEET}

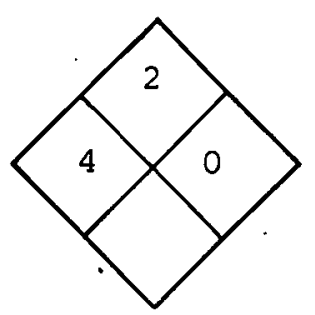

SECTION I SOURCE AND NOMENCLATURE

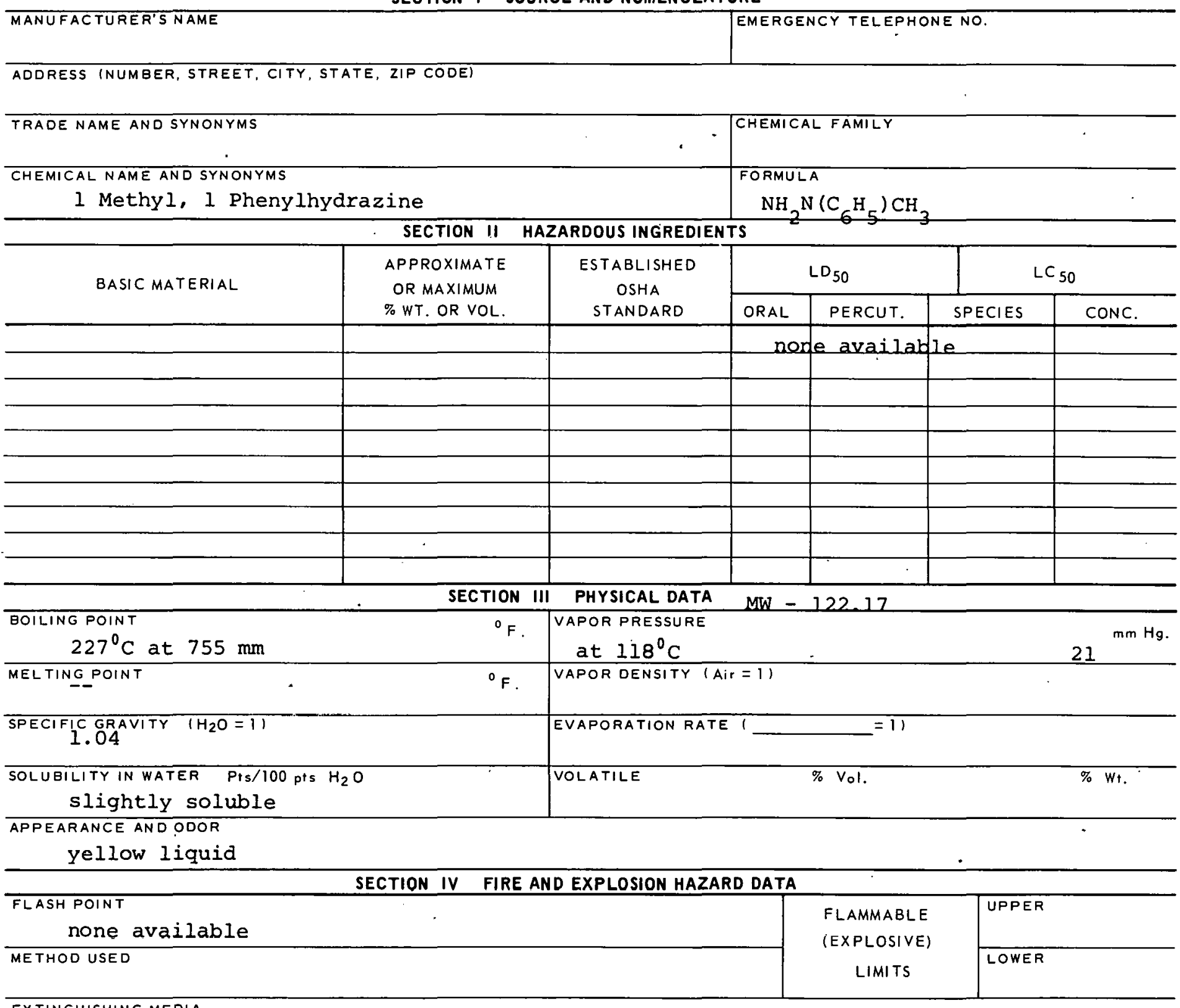

EXTINGUISHING MEDIA

alcohol foam, water, carbon dioxide

SPECIAL FIRE FIGHTING PROCEDURES

UNUSUAL FIRE AND EXPLOSION HAZARDS When heated to decomposition point, it gives off fumes

of nitrogen compounds. 


\section{TOXIC LEVEL}

none available

CARCINOGENIC

PRINCIPLE ROUTES OF ABSORBTION

Inhalation, ingestion, skin

REVELANT SYMPTOMS OF EXPOSURE Irritation of skin, eyes and mucous membranes. gastroentric disturbances.

EFFECTS OF CHRONIC EXPOSURE

Blood and liver damage, loss of appetite.

EMERGENCY AND FIRST AID PROCEDURES Inhalation - rest; if swallowed - induce vomiting, get medical aid; wash from skin \& eyes.

SECTION VI REACTIVITY DATA

CONDITIONS CONTRIBUTING TO INSTAEILITY

CONDITIONS CONTRIBUTING TO HAZARDOUS POLYMERIZATION

IHGOMPATAEILITY (MATEP!ALSTO AVO!RI

Avoia lead dioxide

HAZARDOUS DECOMPOSITION PRODUCTS

SECTION VII SPILL OR LEAK PROCEDURES

STEPS TO BE TAKEN IN CASE MATERIAL IS RELEASED OR SPILLED

WASTE DISPOSAL METHOD

SECTION VIII SPECIAL PROTECTION INFORMATION

\begin{tabular}{l|l}
\hline VENTILATION REQUIREMENTS LOCAL EXHAUST & $\begin{array}{l}\text { PROTECTIVE EQUIPMENT (SPECIFY TYPESI EYE } \\
\text { GOggleS }\end{array}$ \\
\hline MECHANICAL. IGENERALI & $\begin{array}{l}\text { GLOVES } \\
\text { Rubber }\end{array}$ \\
\hline SPECIAL & $\begin{array}{l}\text { RESPIRATOR } \\
\text { SeIf-COntained }\end{array}$ \\
\hline
\end{tabular}

OTHER PROTECTIVE EQUIPMENT

SECTION IX SPECIAL PRECAUTIONS

PRECAUTIONS TO BE TAKEN IN HANDLING AND STORAGE

OTHER PRECAUTIONS

SIGNATIIRE

O.A.TE

$308-6404 B$ 


\begin{tabular}{|l|}
\hline PRODUCT DESIGNATION \\
03-308-8404 \\
Methyl-Iso-propylcarbinol
\end{tabular}

\section{SECTION I SOURCE AND NOMENCLATURE}

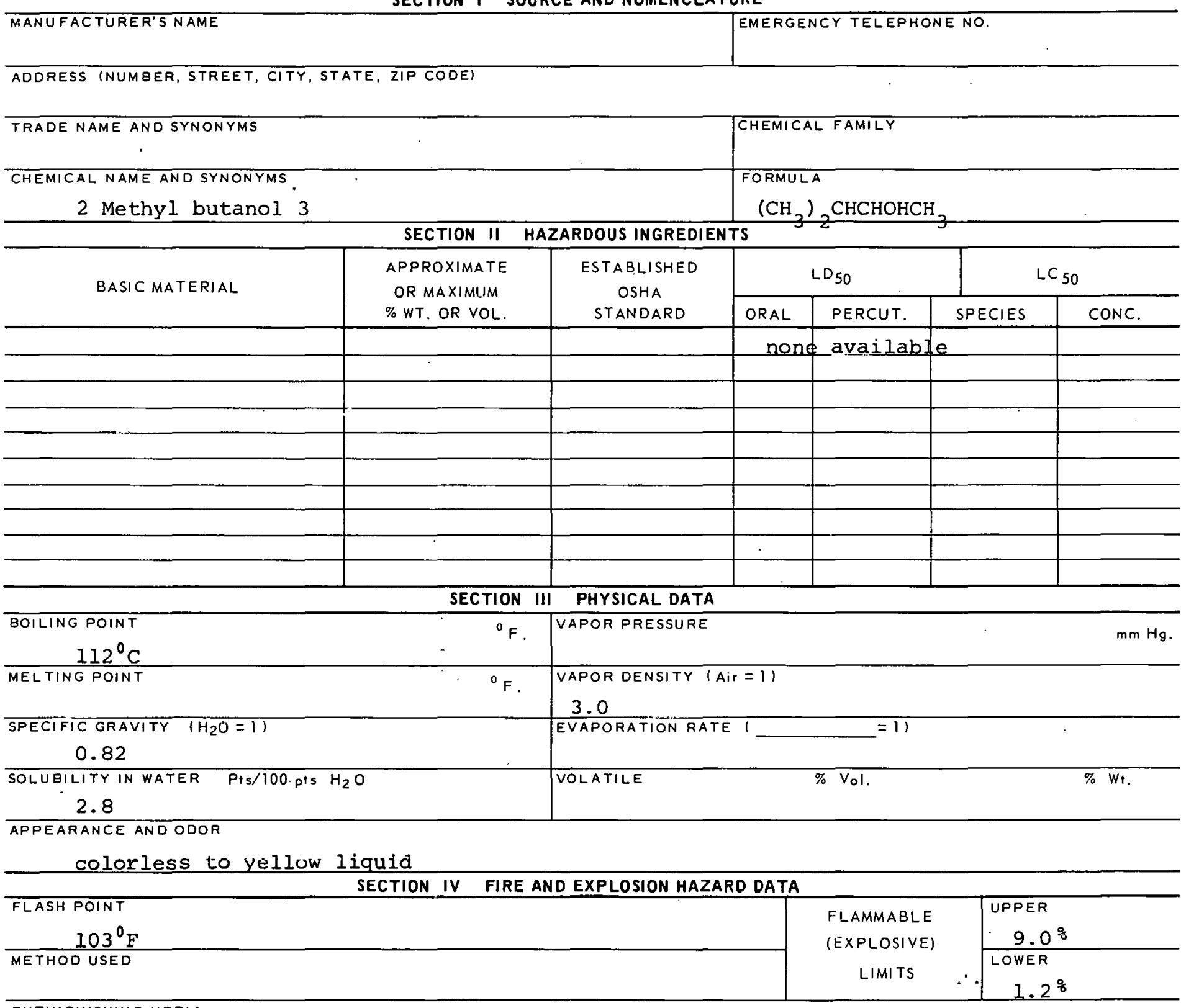

EXTINGUISHING MEDIA

alcohol foam, carbon dioxide, dry chemical.

SPECIAL FIRE FIGHTING PROCEDURES

UNUSUAL FIRE AND EXPLOSION HAZARDS 
Methyl - Iso - Propylcarbinol

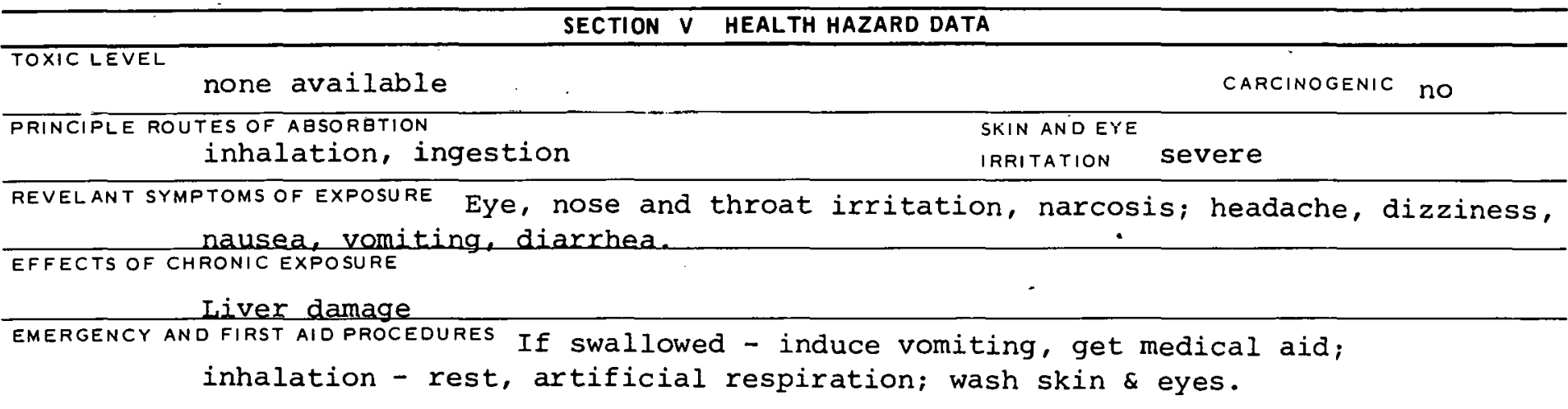

SECTION VI REACTIVITY DATA

CONDITIONS CONTRIBUTING TO INSTABILITY

CONDITIONS CONTRIBUTING TO HAZARDOUS POL YMERIZATION

INCAMPATABILITY (MATERIALS TO AVOIO)

HALAHUUUS DECOMPOSITIOH RRODUCTS

SECTION VII SPILL OR LEAK PROCEDURES

STEPS TO BE TAKEN IN CASE MATERIAL IS RELEASED OR SPILLED

\section{WASTE DISPOSAL METHOD}

\section{SECTION VIII SPECIAL PROTECTION INFORMATION}

\begin{tabular}{l|l}
\hline VENTILATION REQUIREMENTS LOCAL EXHAUST & PROTECTIVE EQUIPMENT (SPECIFY TYPES) EYE \\
\hline MECHANICAL IGENERAL) & GLOVIES \\
\hline SPECIAL & Rubber \\
& RESPIRATOR \\
\hline OTHER PROTECTIVE EQUIPMENT & With proper filtcir
\end{tabular}

OTHER PROTECTIVE EQUIPMENT

SECTION IX SPECIAL PRECAUTIONS

PRECAUTIONS TO BE TAKEN IN HANDLING AND STORAGE

OTHER PRECAUTIONS

SIGNATURE

DATE

$308-8404 B$ 


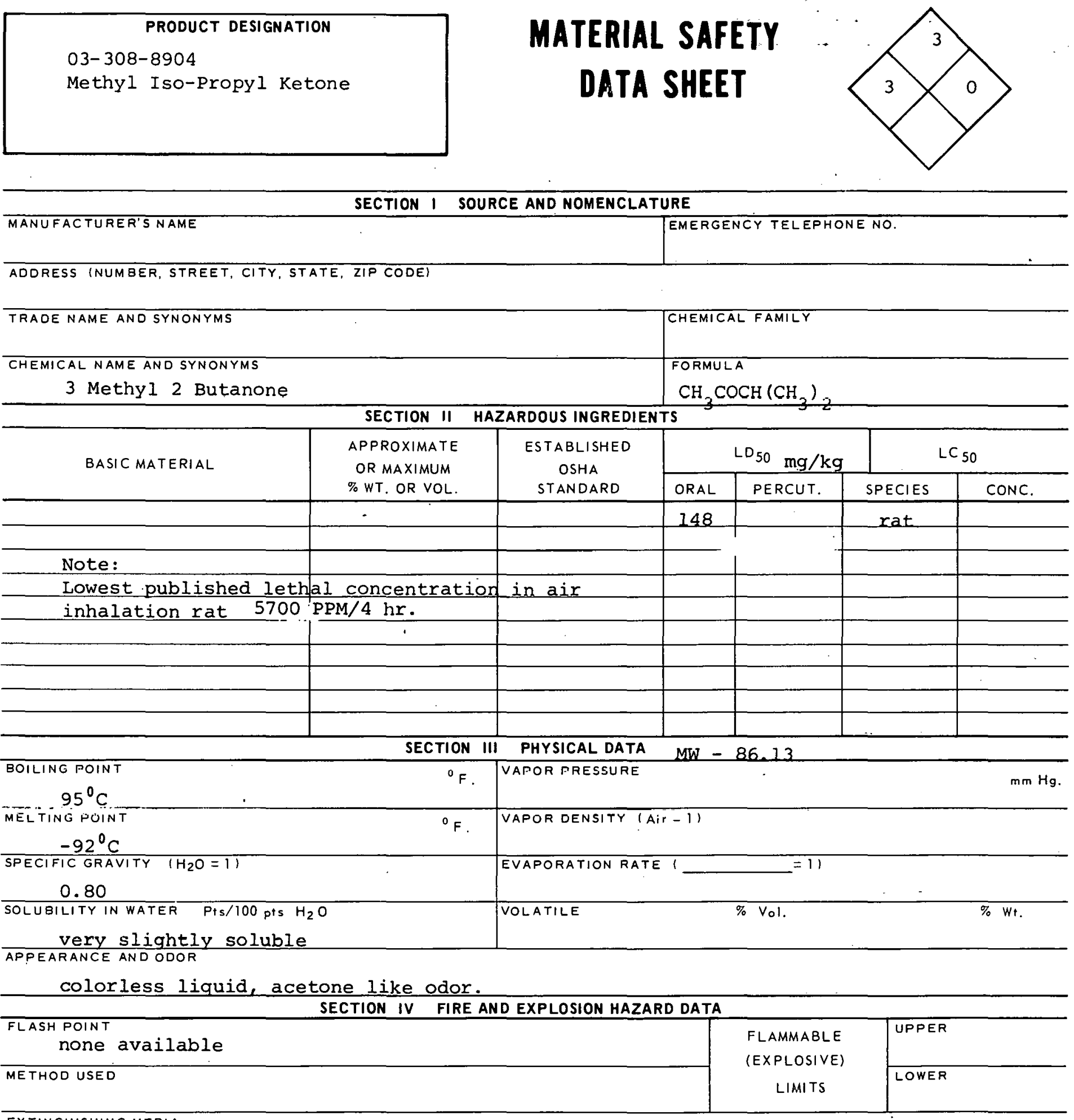

EXTINGUISHING MEDIA alcühul füan̆, cárbún diúxide, dry cileinical.

SPECIAL FIRE FIGHTING PROCEDURES 
Methyl Isopropyl Ketone

SECTION $V$ HEALTH HAZARD DATA

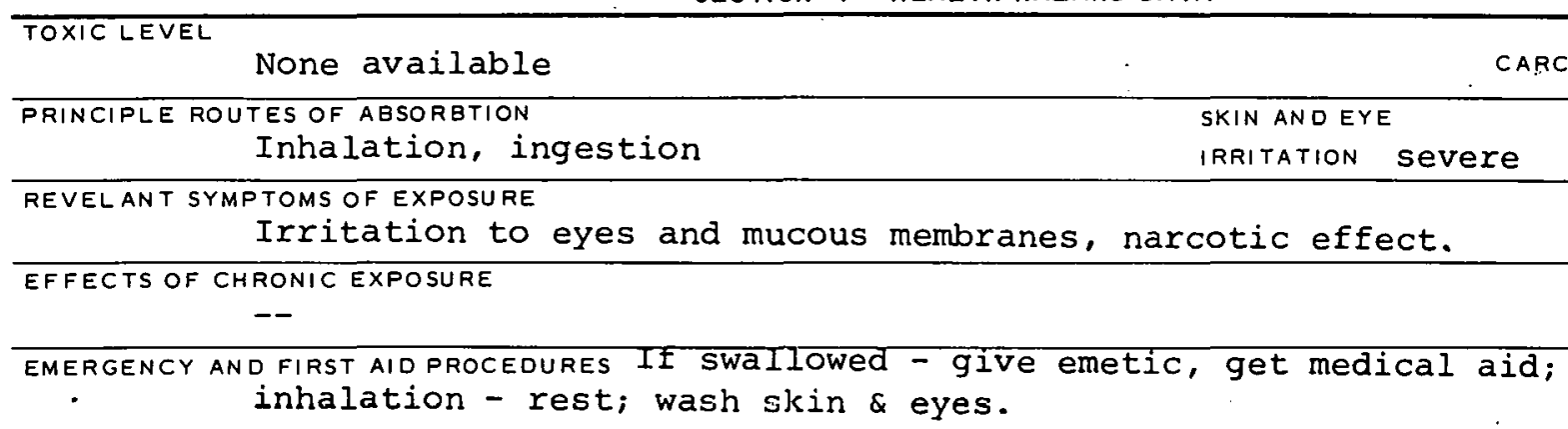

\section{SECTION VI REACTIVITY DATA}

CONDITIONS CONTRIBUTING TO INSTABILITY

CONOITIONS CONTRIBUTING TO HAZARDOUS POLYMERIZATION

INCOMPATABILITY (MATERIALS TO AVOID)

HA 7ARMNIIS RFGOMPRSITION PRIDUCTS

SECTION VII SPILL OR LEAK PROCEDURES

STEPS TO BE TAKEN IN CASE MATERIAL IS RELEASED OR SPILLED

WASTE OISPOSAL METHOD

- SECTION VIII SPECIAL PROTECTION INFORMATION

\begin{tabular}{|c|c|}
\hline VENTILATION REQUIREMENTS LOCAL EXHAUST & $\begin{array}{l}\text { PROTECTIVE EQUIPMENT (SPECIFY TYPES) EYE } \\
\text { Goggles }\end{array}$ \\
\hline MECHANICAL IGENERALI & $\begin{array}{l}\text { GLOVES } \\
\text { Rubber }\end{array}$ \\
\hline SPECIAL & $\begin{array}{l}\text { RESPIRATOR } \\
\text { Witl plopex lilter }\end{array}$ \\
\hline
\end{tabular}

OTHER PROTECTIVE EQUIPMENT

\section{SECTION IX SPECIAL PRECAUTIONS}

PRECAUTIONS TO BE TAKEN IN HANDLING AND STORAGE

\section{OTHER PRECAUTIONS}

SIGNATURE 


\section{MATERIAL SAFETY DATA SHEET}

Methyl Sulfate

03-309-1605

SECTION I SOURCE AND NOMENCLATURE

\section{MANUFACTURER'S NAME}

ADDRESS (NUMBER, STREET, CITY, STATE, ZIP CODE)

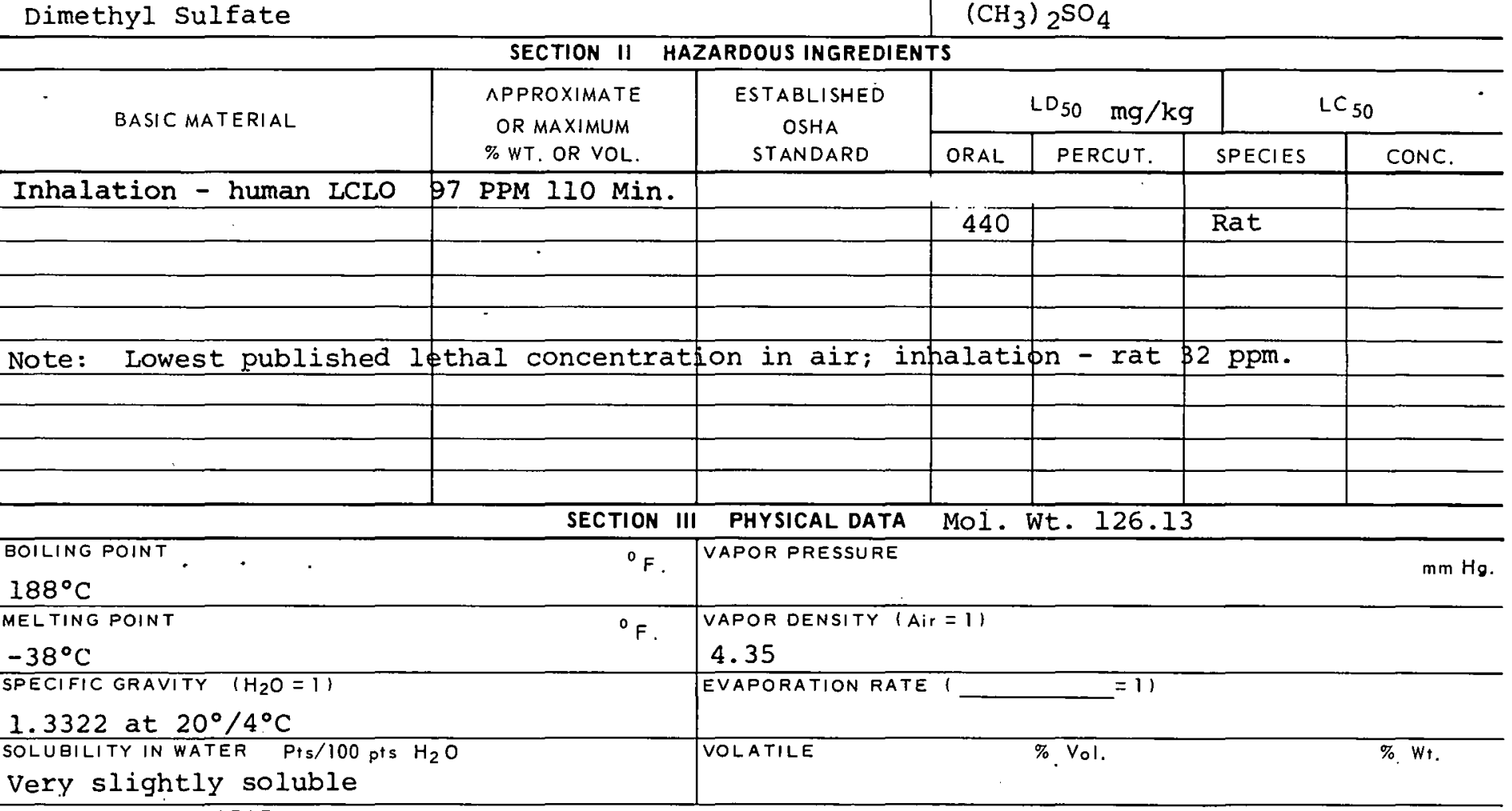

APPEARANCE AND ODOR

Colorless liquid

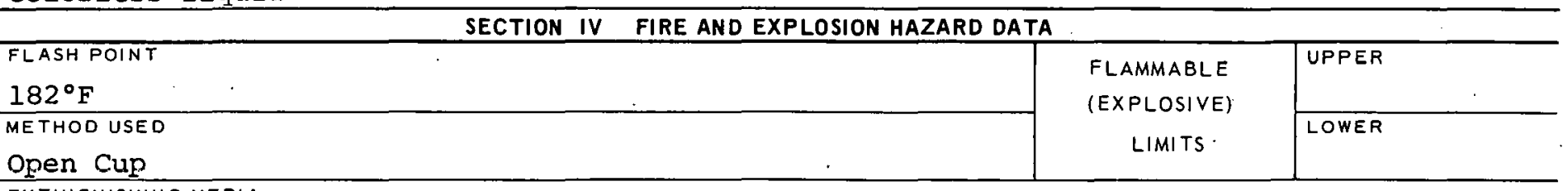

EXTINGUISHING MEDIA

Water spray, dry chemical, foam, or carbon dioxide.

SPECIAL FIRE FIGHTING PROCEDURES

Wear full protective clothing.

UNUSUAL FIRE ANDEXPLOSION HAZARDS 


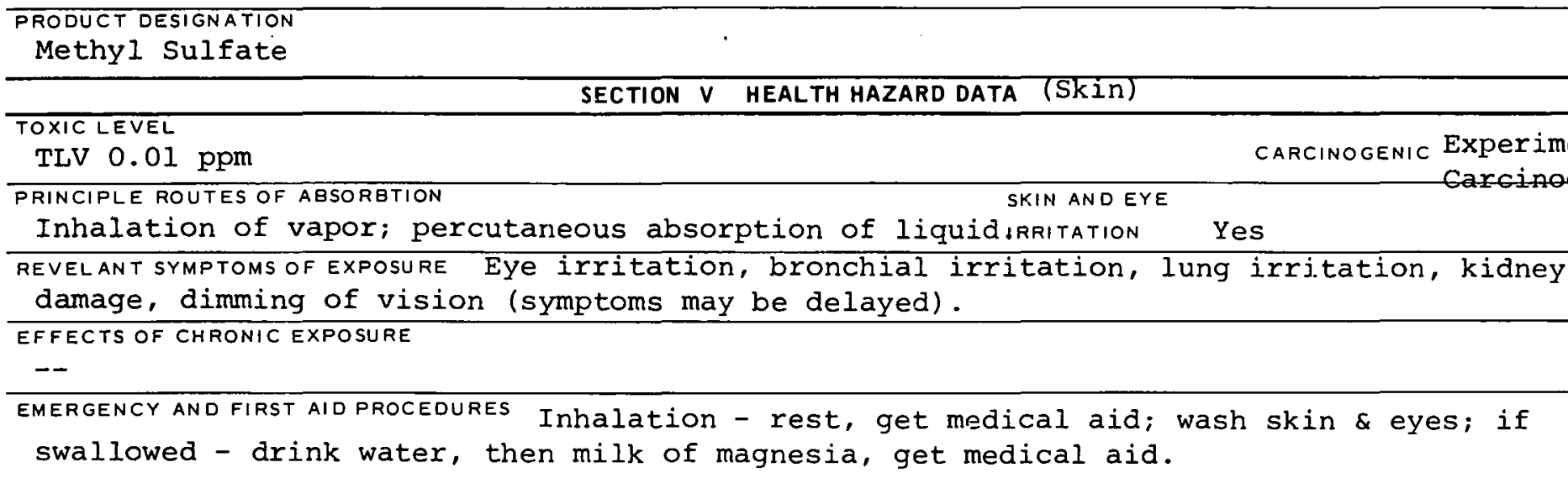


$C A-000556649$

XIL15750

\section{PRODUCT DESIGNATION}

03-309-2105

Methyl Thiocyanate

MATERIAL SAFETY

DATA SHEET

\section{SECTION I SOURCE AND NOMENCLATURE}

\section{MANUFACTURER'S NAME \\ TRADE NAME AND SYNONYMS \\ CHEMICAL NAME AND SYNONYMS \\ Methyl Sulfocyanate}

ADDRESS (NUMBER, STREET, CITY, STATE, ZIP CODE)

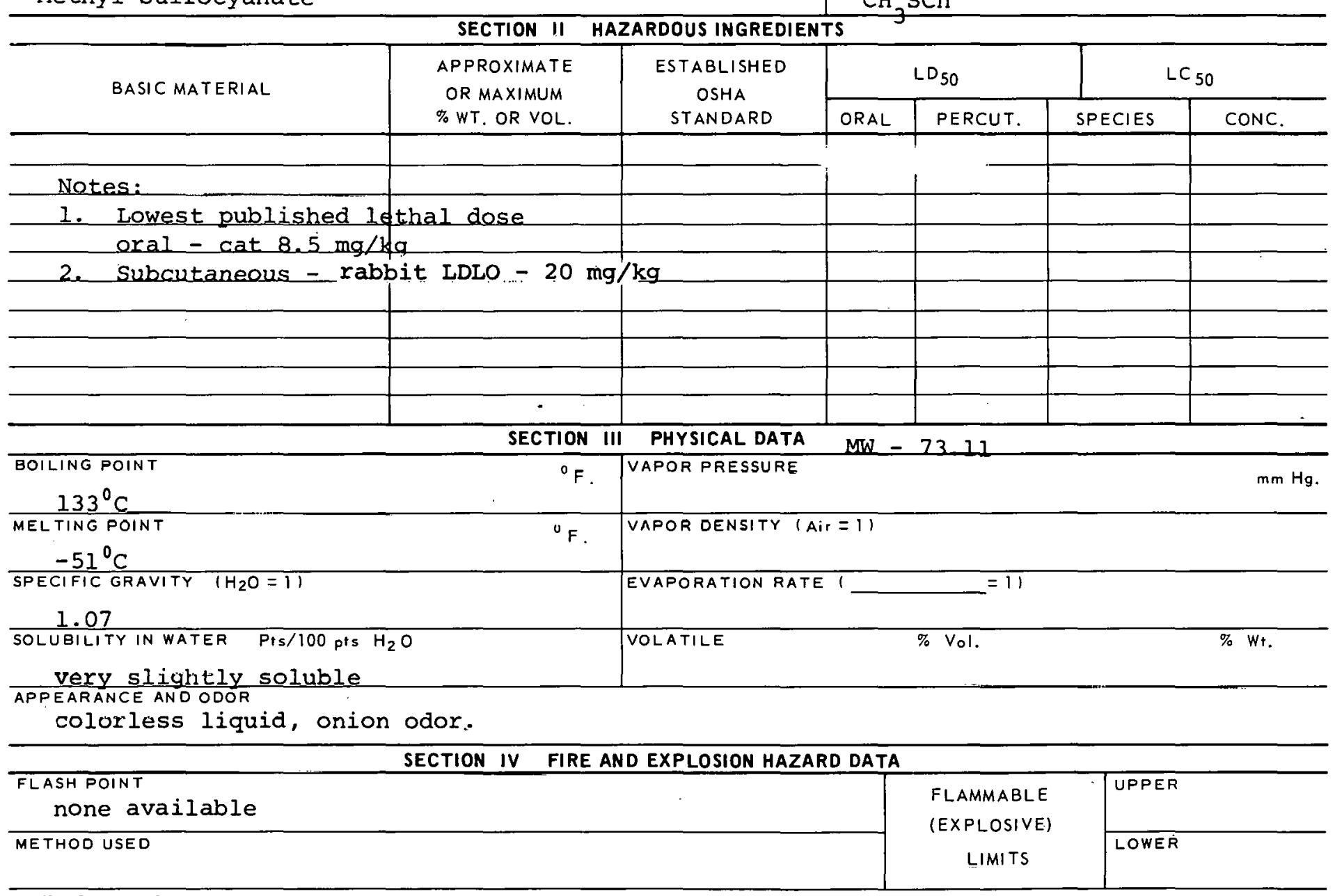

EXTINGUISHING MEDIA

water, alcohol foam, carbon dloxide, dry chemical.

SPECIAL FIRE FIGHTING PROCEDURES

Wear self-contained breathing apparatus and goggles.

UNUSUAL FIRE AND EXPLOSION HAZAROS 
Methyl Thiocyanate

\section{SECTION $V$ HEALTH HAZARD DATA}

TOXIC LEVEL

none available

PRINCIPLE ROUTES OF ABSORBTION

ingestion, inhalation

CARCINOGENIC

CARCINOGENIC no
SKIN ANDEYE
IRRITATION moderate

REVELANT SYMPTOMS OF EXPOSURE

eye and mucous membrane irritation. Thiocyanate intoxication. EFFECTS OF CHRONIC EXPOSURE

Goiters.

EMERGENCY AND FIRST AID PROCEDURES If swallowed - give emetic, get medical aid, gastric

lavage; inhalation - rest, give oxygen as needed. Wash from skin \& eyes.

\section{SECTION VI REACTIVITY DATA}

CONDITIONS CONTRIBUTING TO INSTABILITY

CONDITIONS CONTRIBU TING TO HAZAROQUS POLYMERIZATION

INCOMPATABILITY (MAT.ERIALS TO AVQIO)

HAZARDOUS DECOMPOSITION PRODUCTS

SECTION VII SPILL OR LEAK PROCEDURES

STEPS TO BE TAKEN IN CASE MATERIAL IS RELEASED OR SPILLED

WASTE DISPOSAL METHOD

SECTION VIII SPECIAL PROTECTION INFORMATION

\begin{tabular}{l|l}
\hline \multicolumn{2}{c}{ SECTION VIII SPECIALPROTECTION INFORMATION } \\
\hline MECHANICAL (GENERAL) & $\begin{array}{c}\text { PROTECTIVE EQUIPMENT (SPECIFY TYPESI EYE } \\
\text { GOggles }\end{array}$ \\
\hline SPECIAL & GLOVES \\
& Rubber \\
\hline
\end{tabular}

OTHER PROTECTIVE EQUIPMENT

SECTION IX SPECIAL PRECAUTIONS

PRECAUTIONS TO BE TAKEN IN HANDLING AND STORAGE

OTHER PRECAUTIONS

SIGNATURE

DATE

309-2105B 
CAS : 000109046

\begin{tabular}{|l|}
\hline \multicolumn{1}{|c|}{ PRODUCT DESIGNATION } \\
03-221-9100 \\
2-Bromopyridine \\
\end{tabular}

MATERIAL SAFETY DATA SHEET

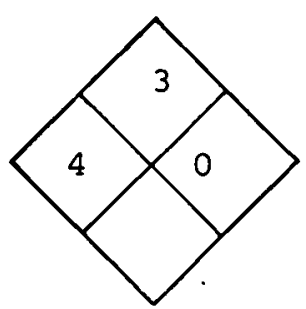

\section{SECTION I SOURCE AND NOMENCLATURE}

\section{MANUFACTURER'S NAME}

ADDRESS (NUMEER, STREET, CITY, STATE, ZIP CODE)

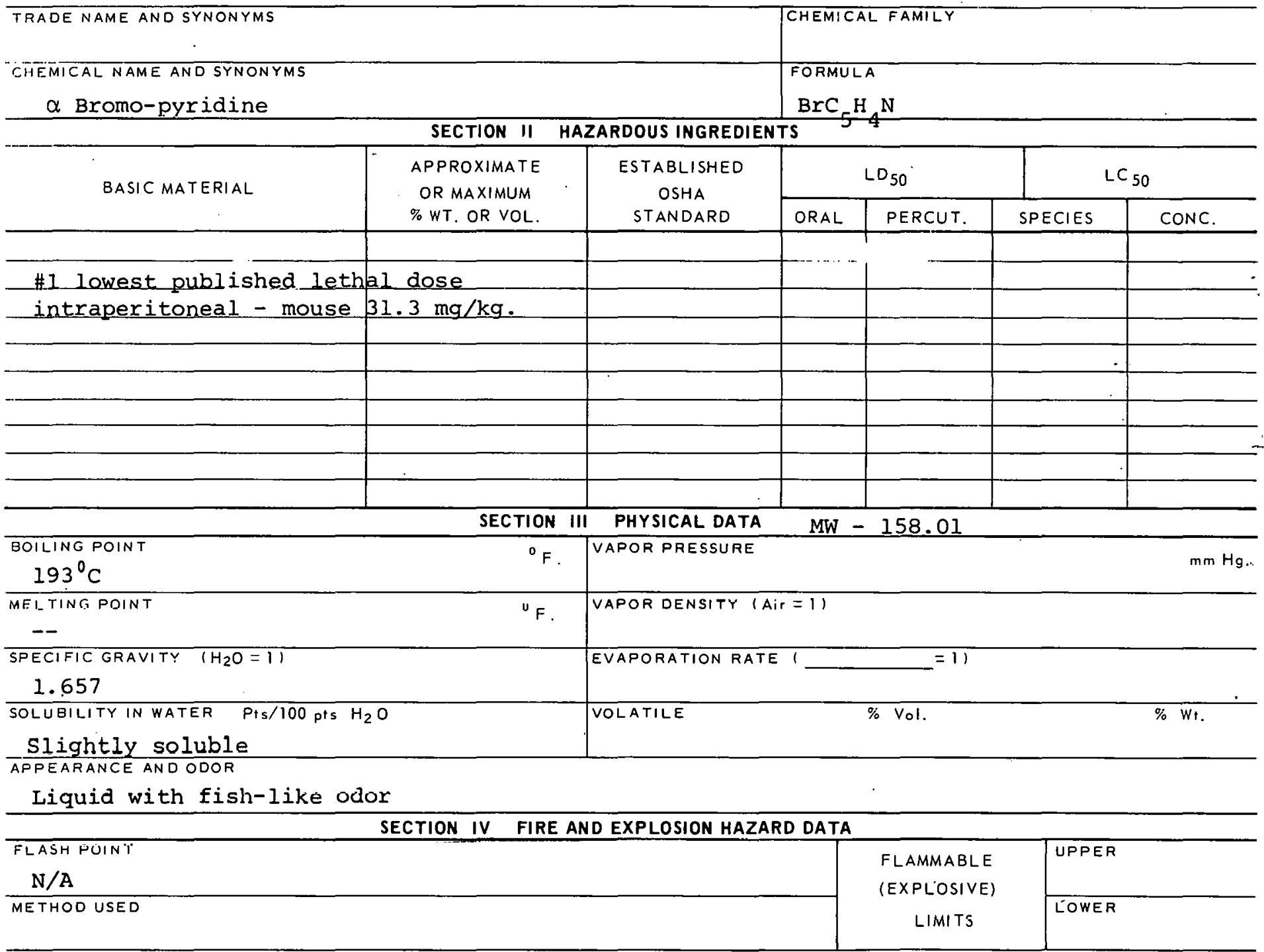

EXTINGUISHING MEDIA

Alcohol foam, carbon dioxide, dry chemical

SPECIAL FIRE FIGHTING PROCEDURES

Wear goggles and self-contained breathing apparatus. 


\section{TOXIC LEVEL}

None available

CARCINOGENIC

No

PRINCIPLE ROUTES OF ABSORBTION

Inhalation, ingestion, skin

SKIN AND LYE

REVELANT SYMPTOMS of EXPosuRE Narcosis, headache, nausea, giddiness, vomiting;

oral - liver \& kidney damage.

CTS OF CHRONIC EXPOSURE OCCasional

ocular and facial paralysis.

EMERGENCY AND FIRST AID PROCEOURES Inhalation - rest; if swallowed - induce vomiting, then gastric lavage; wash skin \& eyes.

\section{SECTION VI REACTIVITY DATA}

CONOITIONS CONTRIBUTING TO INSTABILITY

CONDITIONS CONTRIBU TING TO HAZARDOUS POLYMERIZATION

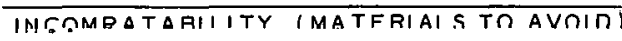

HAZARDOUS DECOMPOSITION PRODUCTS

SECTION VII SPILL OR LEAK PROCEDURES

STEPS TO BE TAKEN IN CASE MATERIAL IS RELEASED OR SPILLED

WASTE DISPOSAL METHOD

SECTION VIII SPECIAL PROTECTION INFORMATION

VENTILATION REQUIREMENTS LOCAL EXHAUST

Use in hood

MECHANICAL (GENERAL)

Goggles

GLOVES

Rubber

SPECIAL

RESPIRATOR

OTHER PROTECTIVE EOUIPMENT

Self-contained breathing apparatus

SECTION IX SPECIAL PRECAUTIONS

PRECAUTIONS TO BE TAKEN IN HANDLING AND STORAGE

OTHER PRECAUTIONS 


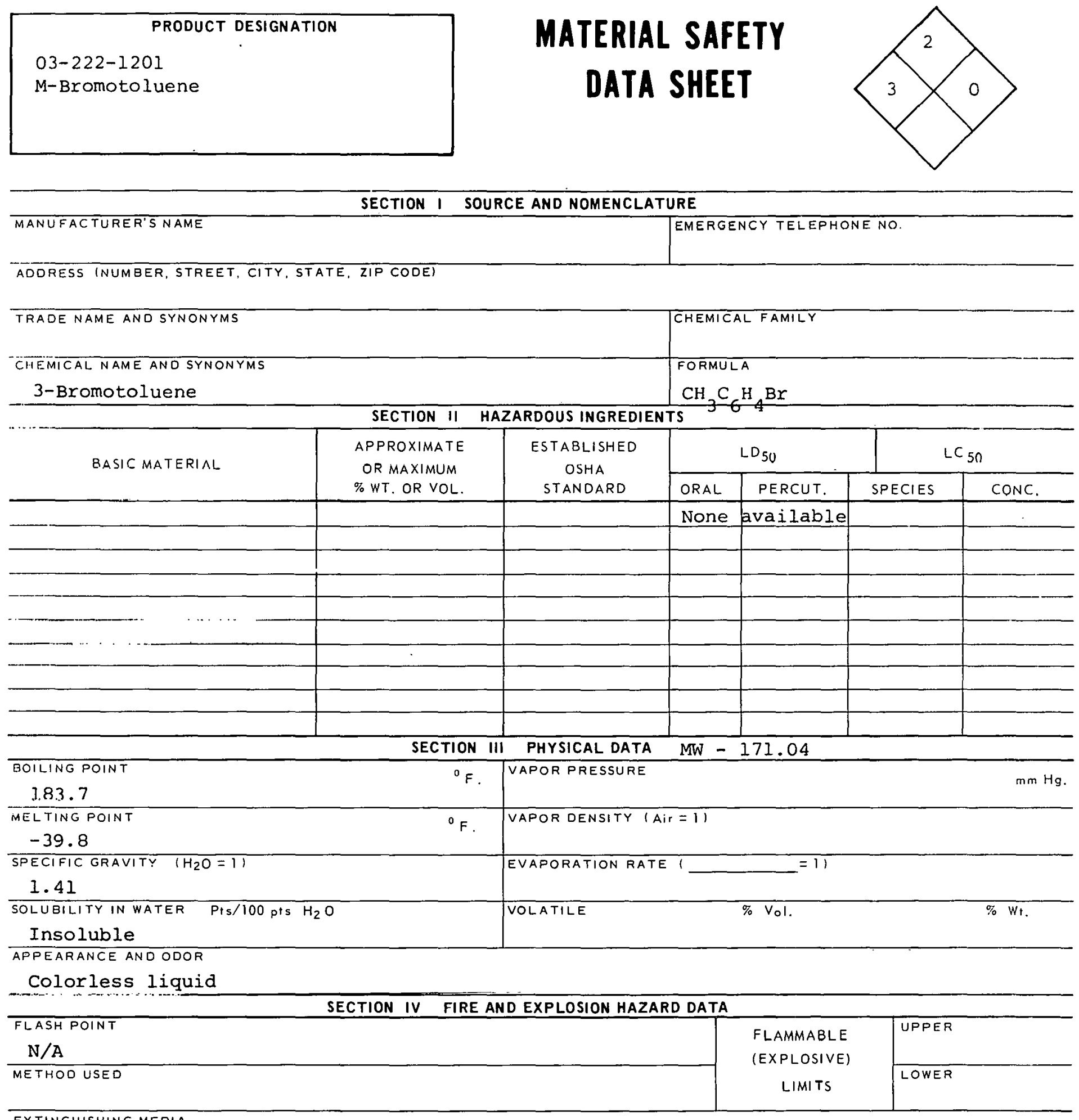

EXTINGUISHING MEOIA

Alcohol foam, carbon dioxide, dry chemical

SPECIAL FIRE FIGHTING PROCEDURES

UNUSUAL FIRE ANO EXPLOSION HAZARDS 


\section{TOXIC LEVEL \\ None available}

PRINCIPLE ROUTES OF ABSORBTION

Inhalation, ingestion internal irritation of digestive tract.

SKIN AND EYE IRRITATION Severe

REVELANT SYMPTOMS OF EXPOSURE EYe \& respiratory system irritation, severe

EFFECTS OF CHRONIC EXPOSURE

$--$

EMERGENCY AND FIRST AID PROCEDURES

Inhalation - rest; if swallowed - give emetic, get medical aid; wash eyes \& skin.

SECTION VI REACTIVITY DATA

CONDITIONS CONTRIBUTING TO INSTABILITY

CONDITIONS CONTRIBUTING TO HAZARDOUS POLYMERIZATION

INCOMPATABILITY (MATERIALS TO AVOIU)

HAZARDOUS DECOMPOSITION PRODUCTS

SECTION VII SPILL OR LEAK PROCEDURES

STEPS TO BE TAKEN IN CASE MATERIAL IS RELEASED OR SPILLED

SECTION VIII SPECIAL PROTECTION INFORMATION

\begin{tabular}{l|l}
\hline VENTILATION REQUIREMENTS LOCAL EXHAUST & $\begin{array}{c}\text { PROTECTIVE EQUIPMENT (SPECIFY TYPES) EYE } \\
\text { GOggles }\end{array}$ \\
\hline MECHANICAL IGENERAL) & $\begin{array}{c}\text { RLOVES } \\
\text { Rubber }\end{array}$ \\
\hline SPECIAL & RESPIRATOR \\
With propcr filtcr
\end{tabular}

OTIIER TROTLCTIVE EQUIPMEMT

With proper filtcr

\section{SECTION IX SPECIAL PRECAUTIONS}

PRECAUTIONS TO BE TAKEN IN HANDLING AND STORAGE

OTHER PKEGAUIIGNS 

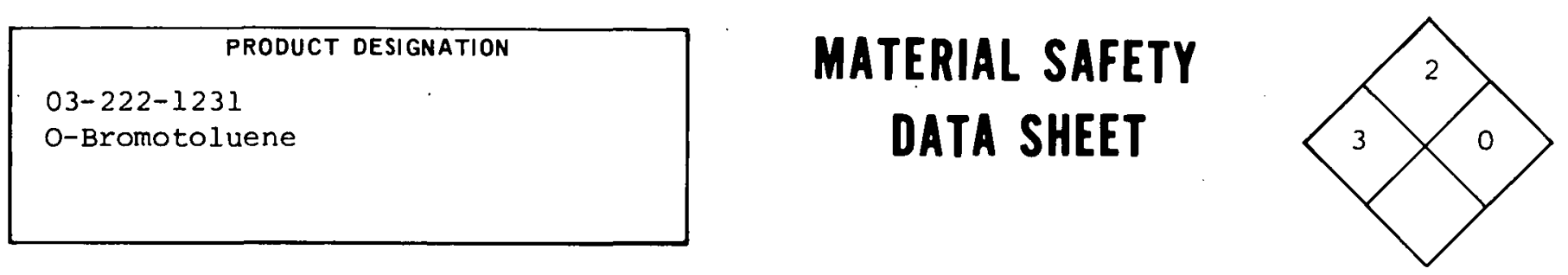

\begin{tabular}{l|l|l}
\hline \multicolumn{2}{|c|}{ SECTION I SOURCE AND NOMENCLATURE } \\
\hline MANU FACTURER'S NAME & EMERGENCY TELEPHONE NO. \\
\hline ADORESS (NUMBER, STREET, CITY, STATE, ZIP CODE)
\end{tabular}

ADORESS (NUMBER, STREET, CITY, STATE, ZIP CODE)

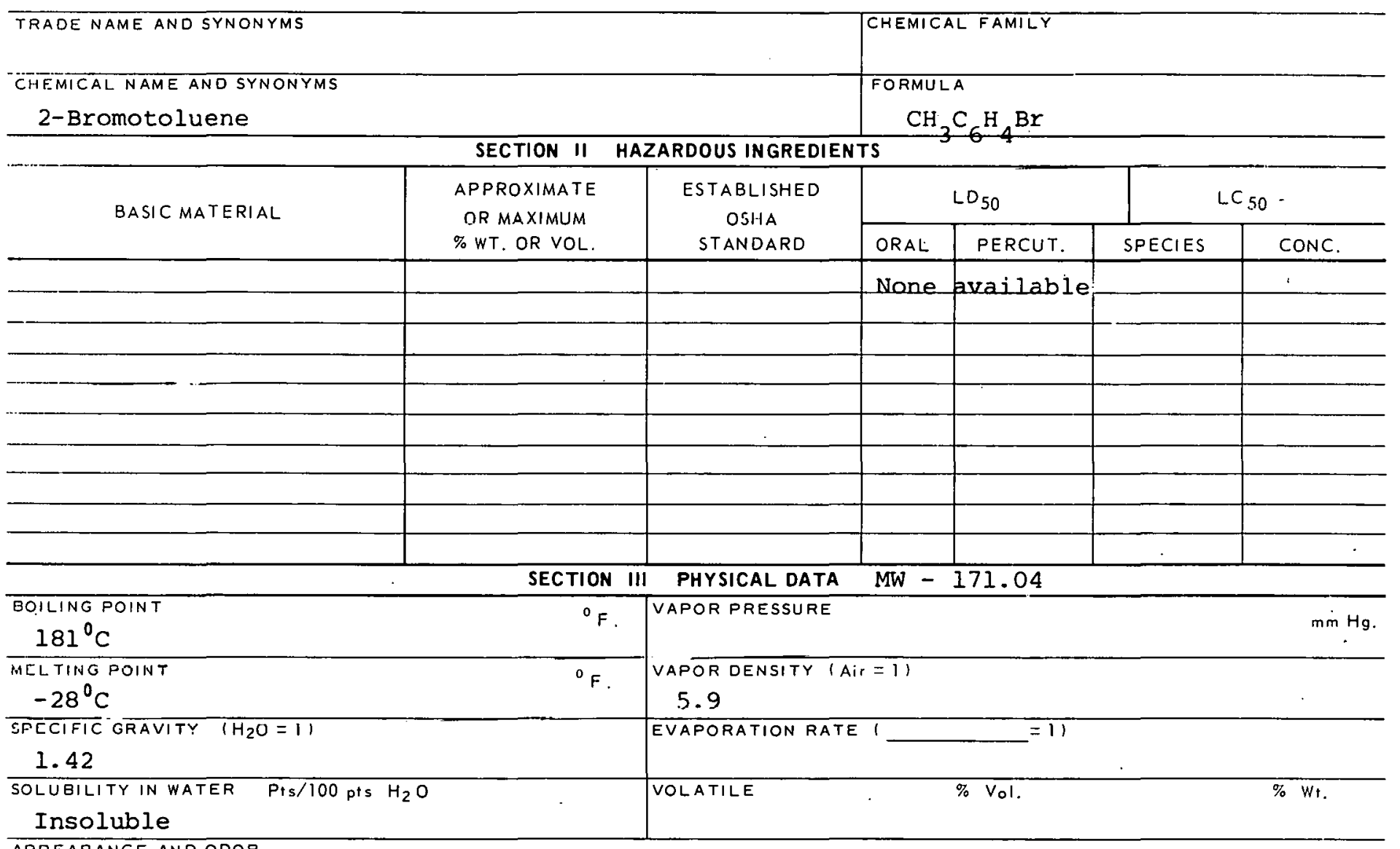

APPEARANCE AND ODOR

Colorless Iiquid

\begin{tabular}{|c|c|c|c|c|}
\hline & SECTION IV & FIRE AND EXPLOSION HAZARD DATA & & \\
\hline $\begin{array}{c}\text { FLASH POINT } \\
175^{\circ} \mathrm{F}\end{array}$ & & & \multirow{2}{*}{$\begin{array}{c}\text { FLAMMABLE } \\
\text { (EXPLOSIVE) } \\
\text { LIMITS }\end{array}$} & UPPER \\
\hline METHOD USED & & & & LOWER \\
\hline
\end{tabular}

EXTINGUISHING MEDIA

Alcohol foam, carbon dioxide, dry chemical

SPECIAL FIRE FIGHTING PROCEDURES

UNUSUAL FIRE AND EXPLOSION HAZARDS 


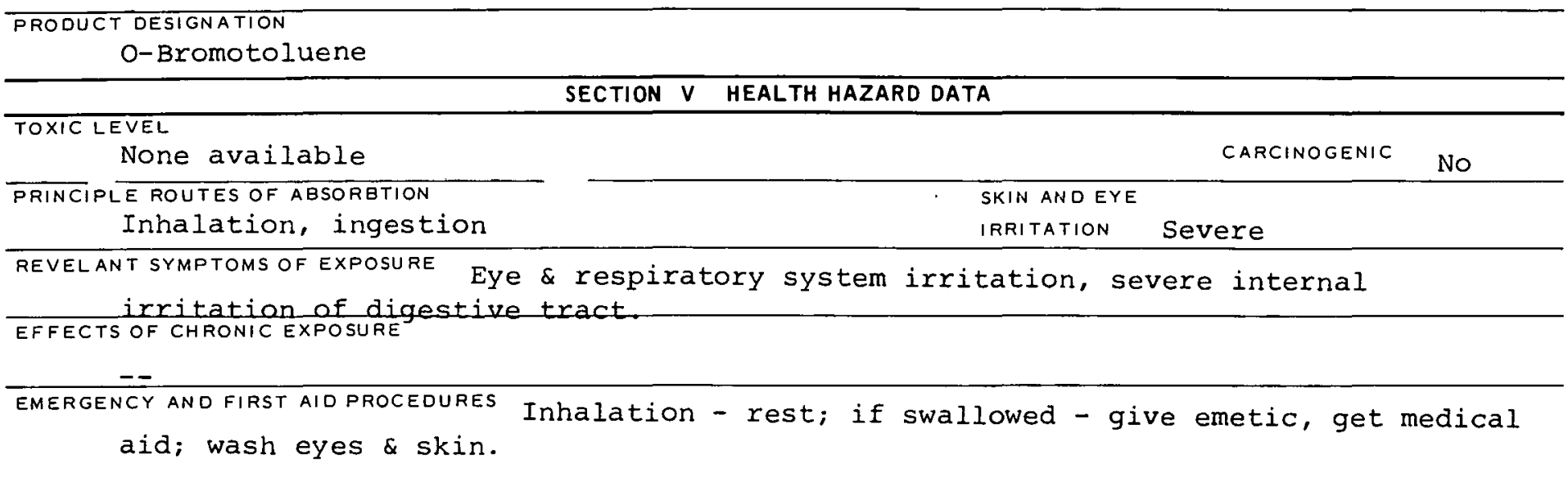

\begin{tabular}{l} 
SECTION VI REACTIVITY DATA \\
\hline CONDITIONS CONTRIBUTING TO INSTABILITY \\
\hline CONDITIONS CONTRIBUTING TO HAZARDOUS POL YMERIZATION
\end{tabular}

CONDITIONS CONTRIBUTING TO HAZARDOUS POLYMERIZATION

INCOMPATABILITY (MATERIALS TO AVOIO)

HAZARDOUS DECOMPOSITION PRODUCTS

SECTION VII SPILL OR LEAK PROCEDURES

STEPS TO BE TAKEN IN CASE MATERIAL IS RELEASED OR SPILLED

WASTE DISPOSAL METHOD

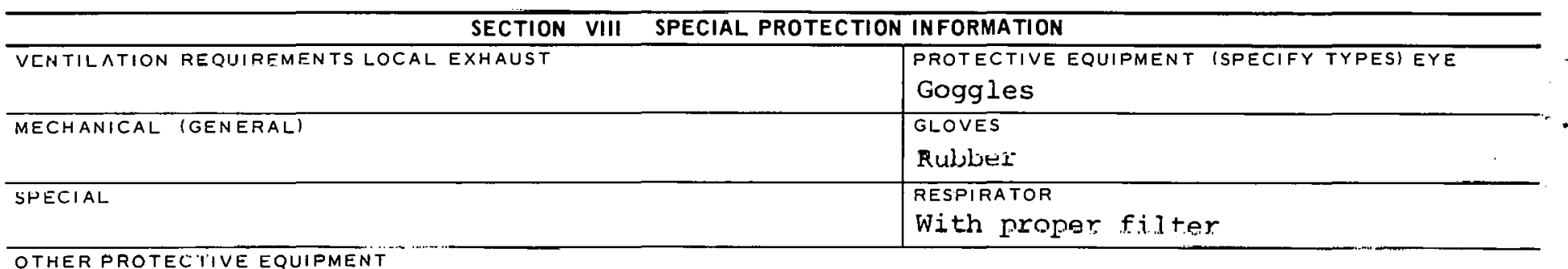

SECTION IX SPECIAL PRECAUTIONS

PRECAUTIONS TO BE TAKEN IN HANDLING AND STORAGE

OTHER PRECAUTIONS

SIGNATURE

DATE

$222-1231 B$ 
03-222-1261

P-Bromotoluene
MATERIAL SAFETY

DATA SHEET

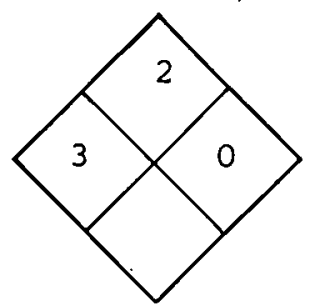

SECTION I SOURCE AND NOMENCLATURE

MANUFACTURER'S NAME

EMERGENCY TELEPHONE NO.

ADDRESS (NUMBER, STREET, CITY, STATE, ZIP CODE)

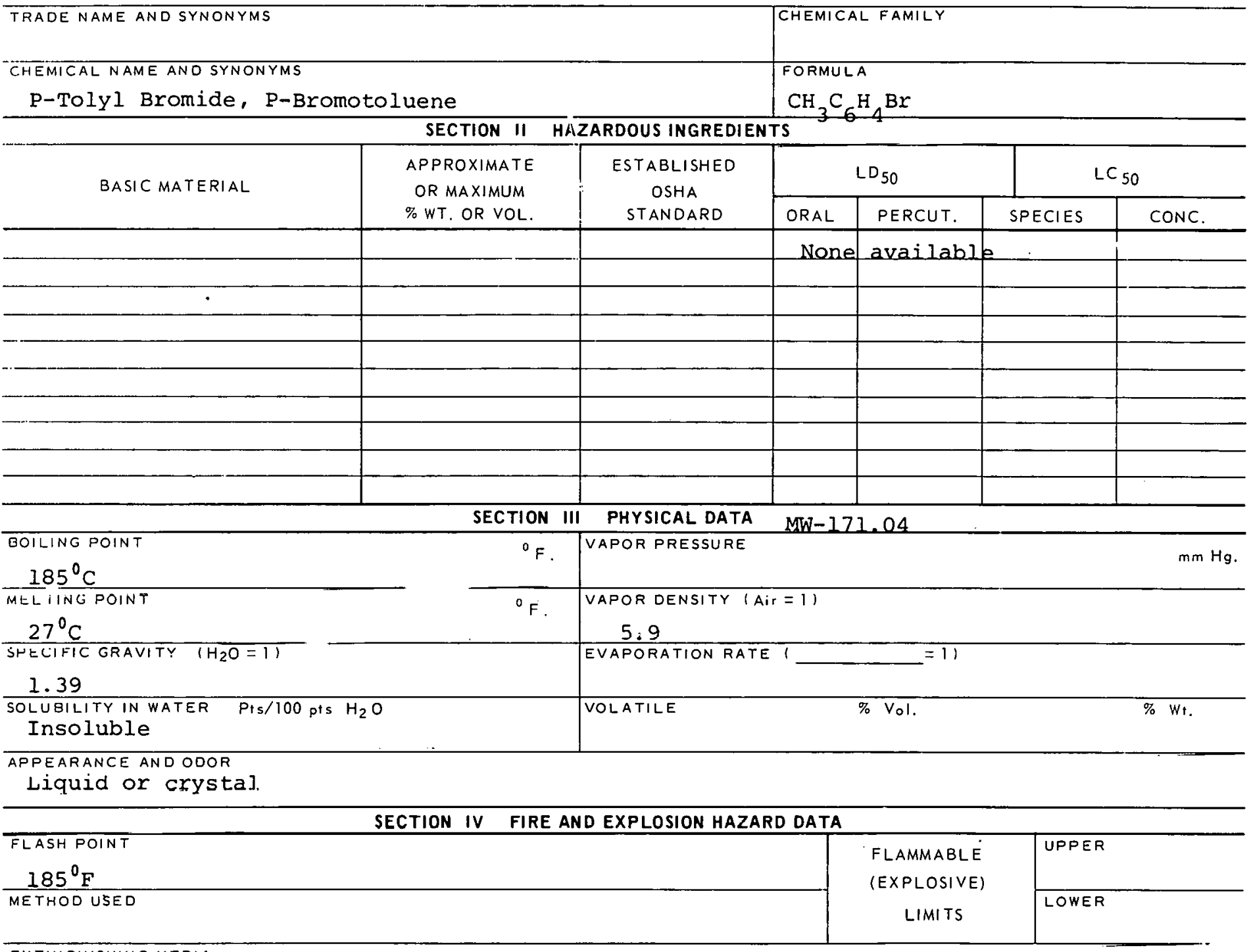

EXTINGUISHING MEDIA

Alcohol foam, carbon dioxide, dry chemical

SPECIAL FIRE FIGHYING PROCEDURES

UNUSUAL FIRE ANDEXPLOSION HAZARDS 


\section{TOXICLEVEL \\ None available}

PRINCIPLE ROUTES OF ARSORBTION

Inhalation, ingestion

REVELANT SYMPTOMS OF EXPOSURE 'EY irritation of digestive tract.

EFFECTS OF CHRONIC EXPOSURE

$--$

EMERGENCY AND FIRST AID PROCEDURES Inhalation - rest; if swallowed - give emetic, get medical aid; wash eyes \& skin.

SKIN AND EYE

IRRITATION Severe

\section{SECTION VI REACTIVITY DATA}

CONDITIONS CONTRIBUTING TO INSTABILITY

CONDITIONS CONTRIBUTING TO HAZARDOUS POLYMERIZATION

INCOMEATARII I TY IMATERIALS TO AVOIDI

HAZARDOUS DECOMPOSITION PRODUCTS

SECTION VII SPILL OR LEAK PROCEDURES

STEPS TO BE TAKEN IN CASE MATERIAL IS RELEASED OR SPILLED

SECTION VIII SPECIAL PROTECTION INFORMATION

\begin{tabular}{l|l}
\hline VENTILATION REQUIREMENTS LOCAL EXHAUST & $\begin{array}{l}\text { PROTECTIVE EQUIPMENT ISPECIFY TYPESI EYE } \\
\text { GoggleS }\end{array}$ \\
\hline MECHANICAL IGENERAL) & $\begin{array}{l}\text { GLOVES } \\
\text { Rubber }\end{array}$ \\
\hline SPEGIAL. & $\begin{array}{l}\text { RESPIRATOR } \\
\text { W1th proper f1lter }\end{array}$ \\
\hline
\end{tabular}

OTHER PROTECTIVE EQUIPMEN I

CARCINOGENIC No

\section{WASTE DISPOSAL METHOD}

\section{SECTION IX SPECIAL PRECAUTIONS}

PRECAUTIONS TO BE TAKEN IN HANDLING AND STORAGE

OIHER PMECAUTIONS 
03-222-2701
03-222-2721
$03-222-2751$
03-222-2761
N-Butyl Acetate

\section{MATERIAL SAFETY DATA SHEET}

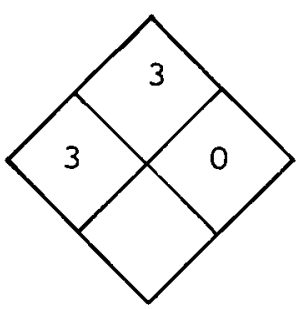

\section{SECTION I SOURCE AND NOMENCLATURE}

\section{MANUFACTURER'S NAME \\ $\overline{T R A D E} \overline{\text { NAME AND SYNONYMS }}$ \\ CHEMICAL NAME AND SYNONYMS \\ Butyl Acetate}

ADDRESS (NUMBER, STREET, CITY, STATE, ZIP CODE)

BASIC MATERIAL

LDLO oral - human $500 \mathrm{mg} / \mathrm{kg}$

Lowest published toxic concentration in air

\section{Inhalation - human 200 SPM}

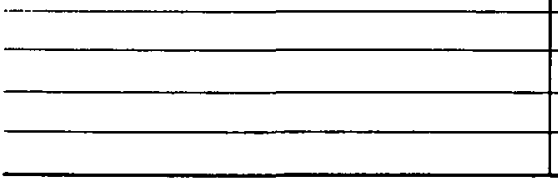

BOILING POINT

$$
126^{\circ} \mathrm{C}
$$

MELTING POINT

$$
-74^{\circ} \mathrm{C}
$$

SPECIFIC GRAVITY $\left(\mathrm{H}_{2} \mathrm{O}=1\right)$

\subsection{8}

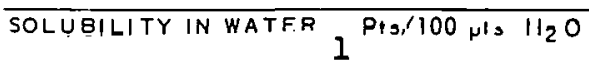

APPEARANCE AND OOOR

Colorless liquid

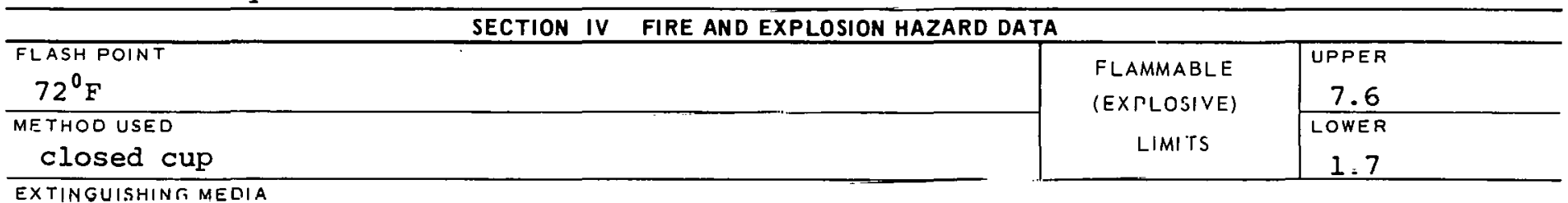

EXTINGUISHINF MEDIA

Alcohol foam, carbon dioxide, dry chemical
SECTION II HAZARDOUS INGREDIENTS

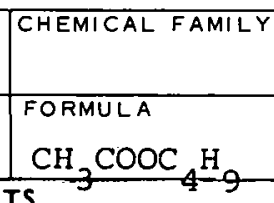

\begin{tabular}{|c|c|c|c|}
\hline \multicolumn{2}{|c|}{$L D_{50}$} & $\mathrm{mg} / \mathrm{kg}$ & $\operatorname{LC}_{50}$ \\
\hline ORAL & PERCUT. & SPECIES & CONC. \\
\hline 7056 & & mouse & \\
\hline See & Note & & \\
\hline 4000 & & rat & \\
\hline & & & \\
\hline & & & \\
\hline & & & \\
\hline & & & \\
\hline & & & \\
\hline & & & \\
\hline & & & \\
\hline
\end{tabular}

SECTION III PHYSICAL DATA MW-116.16

${ }^{\circ} \mathrm{F}$. VAPOR PRESSURE

at $25^{\circ} \mathrm{C}$

${ }^{\circ} \mathrm{F}$.

VAPOR OENSITY (Air $=1$ )

4.0

EVAPORATION RATE

VOLA TILE

$\% \mathrm{Vol}$ $=11$

$\% W_{t}$

SPECIAL FIRE FIGHTING PROCEDIIRES

Water may be ineffective.

UNUSUAL FIRE AND EXPLOSION HAZARDS 


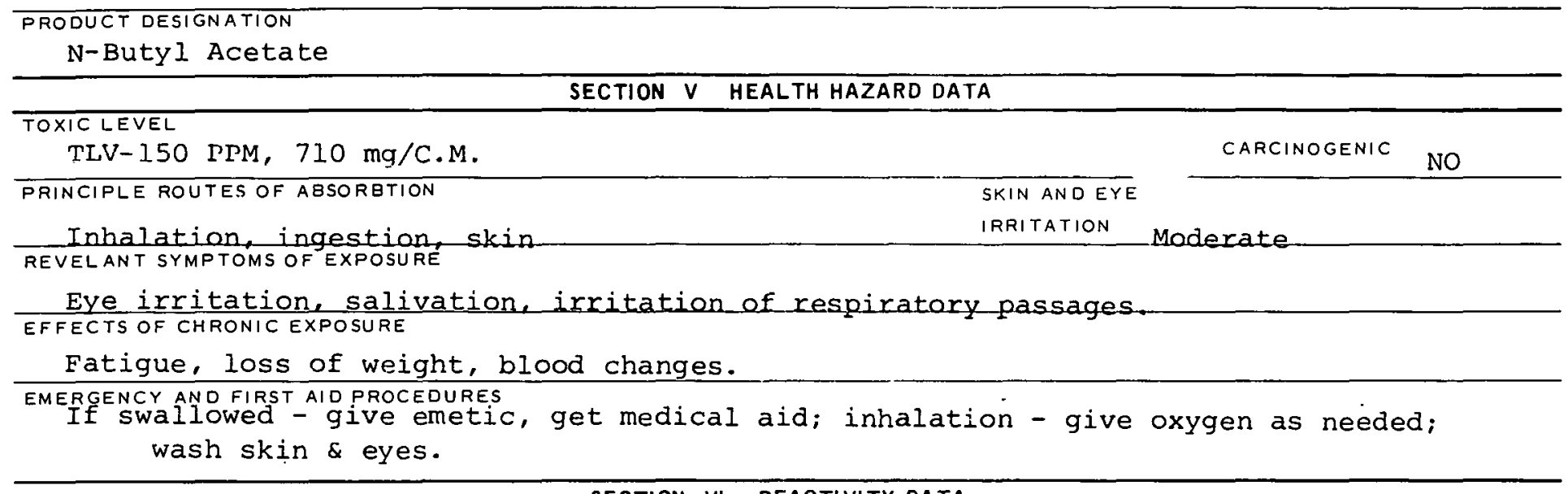

SECTION VI REACTIVITY DATA

CONDITIONS CONTRIBUTING TO INSTABILITY

CONDITIONS GONTRIBIJTING TO HAZARDOUS POLYMERIZATION

INCOMPATABILITY (MATERIALS 10 AVUIU)

Can react with oxidizing materials

HAZARDOÚS DELUMNUSIIIUN PR̃IUUET3

SECTION VII SPILL OR LEAK PROCEDURES

STEPS TO BE TAKEN IN GASE MATERIAL IS RELEASED OR SPILLEO

Absorb on paper, evaporate in hood, burn paper.

WASIE DISPOSAL METIIOD

SECTION VIII SPECIAL PROTECTION INFORMATION

\begin{tabular}{l|l}
\hline VEN IILATION REQUIREMENTS LOCAL EXHAUST & $\begin{array}{l}\text { PROTECTIVE EQUIPMENT (SPECIFY TYPES) EYE } \\
\text { GNgg].eS }\end{array}$ \\
\hline MECHANICAL IGENERAL) & $\begin{array}{l}\text { GLOVES } \\
\text { Rubber }\end{array}$ \\
\hline SFECIAL & $\begin{array}{l}\text { RESPIRATOR } \\
\text { With propex fi.1.ter }\end{array}$ \\
\hline
\end{tabular}

OTHER PROTECTIVE EQUIPMENT

Rubber protective clothing

\section{SECTION IX SPECIAL PRECAUTIONS}

PRECAUTIONS TO BE TAKEN IN HANDLING AND STORAGE

store in cool, well-ventilated place away from source of ignition. 


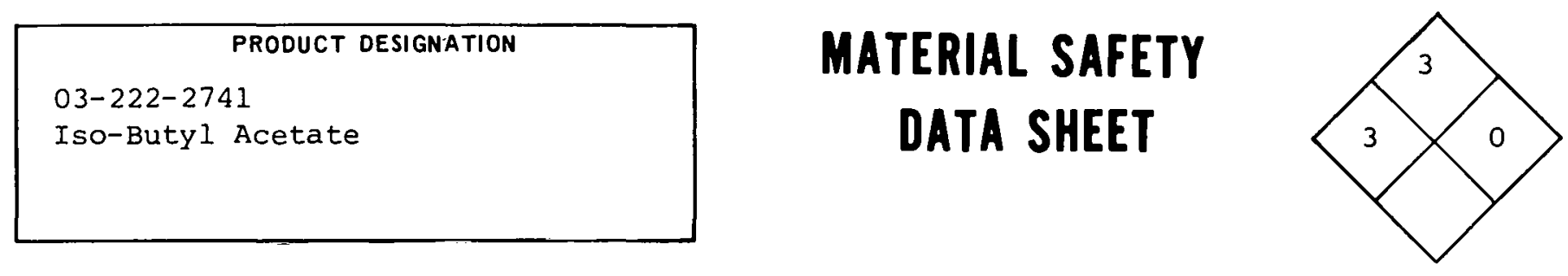

SECTION I SOURCE AND NOMENCLATURE

MANUFACTURER'S NAME

ME

TE, ZIPCODEY

EMERGENCY TELEPHONE NO.

AODRESS (NUMBER, STREET, CITY, STATE, ZIP CODE)

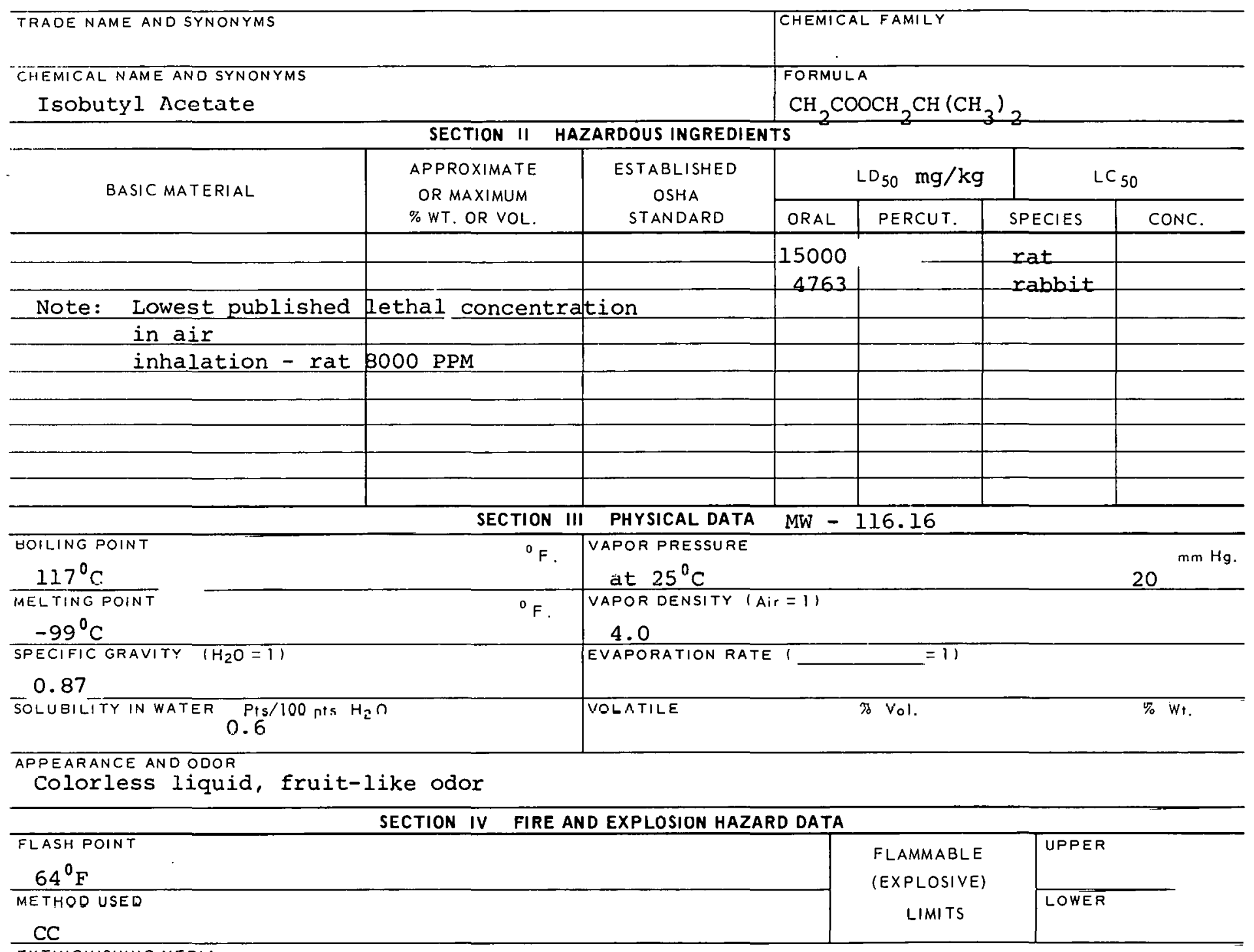

EXTINGUISHING MEDIA

Alcohol foam, carbon dioxide, dry chemical

SPECIAL FIRE FIGHTING PROCEDURES

Water may be ineffective.

UNUSUAL FIRE AND EXPLOSION HAZARDS 
Iso-Butyl Acetate

SECTION $V$ HEALTH HAZARD DATA

TOXIC LEVEL

TLV-150 PPM, $700 \mathrm{mg} / \mathrm{C} . \mathrm{M}$.

PRINCIPLE ROUTES OF ABSORBTION SKIN AND EYE

CARCINOGENIC

Inhalation, ingestion

IRRITATION Moderate

REVELANT SYMPTOMS OF EXPOSURE Irritation of eyes \& respiratory passages, salivation.

EFFECTS OF CHRONIC EXPOSURE

Fatigue, loss of weight, blood changes.

EMERGENCY AND FIRST AID PROCEDURES If swallowed - give emetic, get medical aid; inhalation give oxygen as needed; wash from skin \& eyes.

CONDITIONS CONTRIBUTING TO INSTABILITY

SECTION VI REACTIVITY DATA

CONDITIONS CONTRIBUTING TO HAZARDOUS POLYMERIZATION

INCOMPATABILI IY IMATERIAL3 TO AVOID)

Can react with oxidizing materials

HALAẼLUIJ OECOMTOCITIOH RAOQUCTS

SECTION VII SPILL OR LEAK PROCEDURES

STFPS TO BE TAKEN IN CASE MATERIAL IS RELEASED OR SPILLED

Absorb on paper: evaporate in hood.

$\therefore$

WASTE DISPOSAL METHOD

SECTION VIII SPECIAL PROTECTION INFORMATION

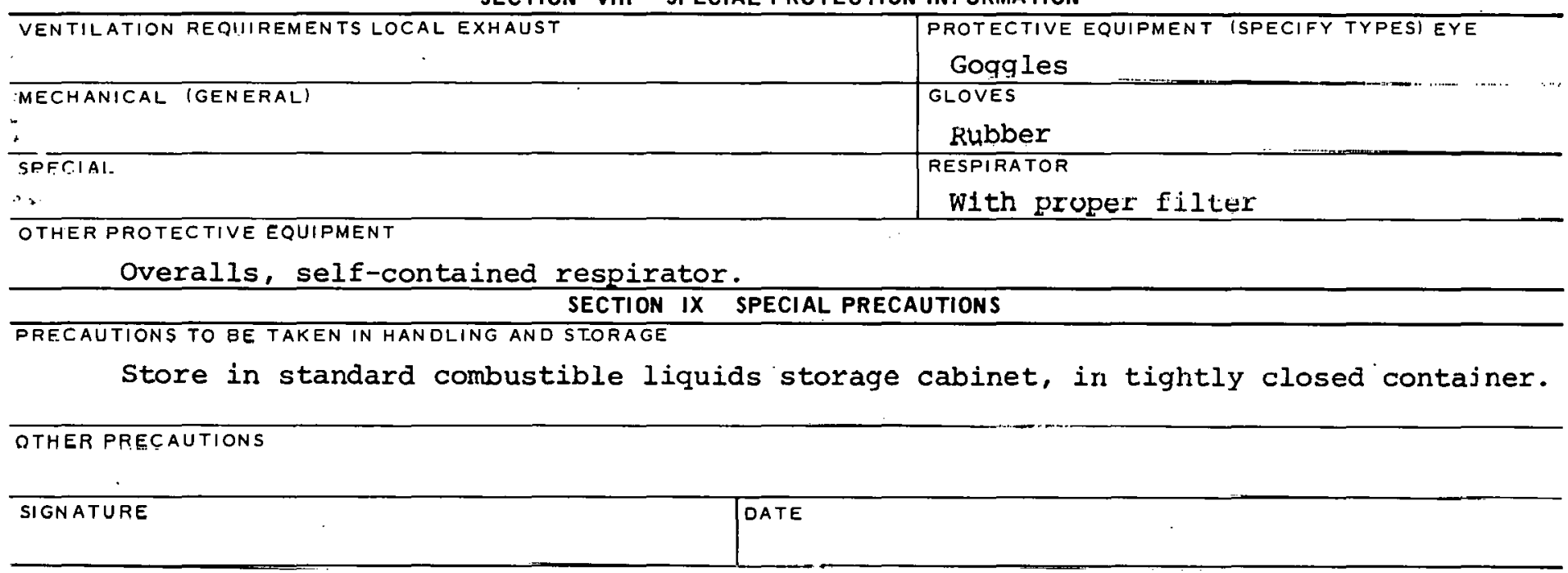


PRODUCT DESIGNATION

03-222-2781

Sec-Butyl Acetate
MATERIAL SAFETY

DATA SHEET

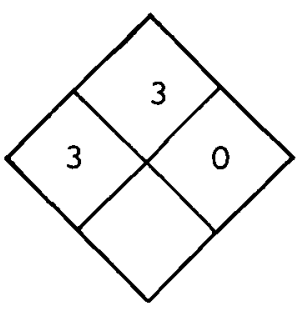

\section{SECTION I SOURCE AND NOMENCLATURE}

MANUFACTURER'S NAME

|EMERGENCY TELEPHONE NO

ADDRESS (NUMBER, STREET, CITY, STATE, ZIP CODE)

TRADE NAME AND SYNONYMS

CHEMICAL NAME AND SYNONYMS

Sec - Butyl Acetate

BASIC MATERIAL

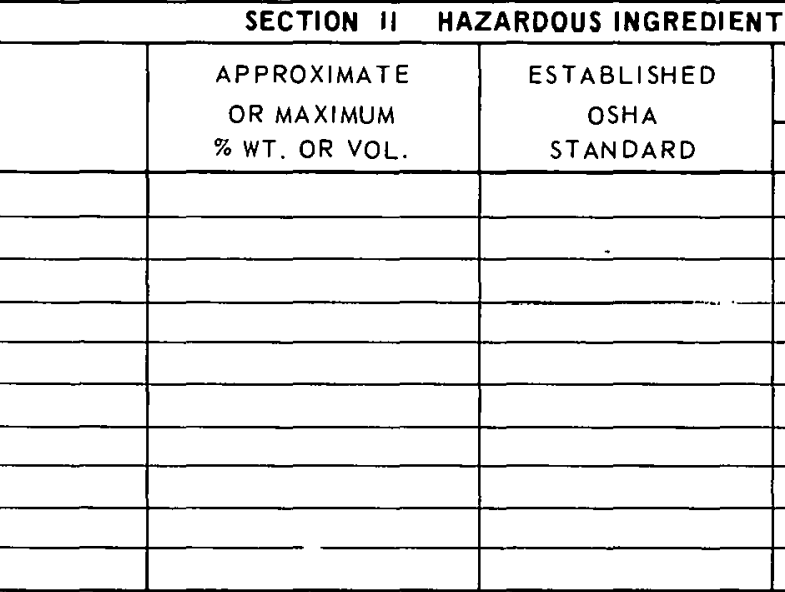

SECTION III PHYSICAL DATA

BOILING POINT

$113^{\circ} \mathrm{C}$

MELTING POINT

-

SPECIFIC GRAVITY $\left(\mathrm{H}_{2} \mathrm{O}=1\right)$

0.87

SOLUBILITY IN WATER $3^{\text {Pis } / 100 \text { pts } \mathrm{H}_{2} \mathrm{O}}$

${ }^{0} \mathrm{~F}$.

VAPOR PRESSURE

at $25^{\circ} \mathrm{C}$

${ }^{\circ} \mathrm{F}$ VAPOR DENSITY (Air $=1$ )

4.0

EVAPORATION RATE

VOLATILE

$\% \mathrm{Vol}$

CHEMICAL FAMILY
FORMULA
$\mathrm{CH}_{3} \mathrm{COOCH}\left(\mathrm{CH}_{3}\right) \mathrm{C}_{2} \mathrm{H}_{5}$

\begin{tabular}{l|l|l|l}
\multicolumn{2}{c|}{ LD $_{50}$} & \multicolumn{2}{c}{ LC $_{50}$} \\
\hline ORAL & PERCUT. & SPECIES & CONC. \\
\hline None & available & & $\therefore$ \\
\hline & & & $\ddots$ \\
\hline & & & $\ddots$ \\
\hline & & & $\ddots$ \\
\hline
\end{tabular}

MiN-116.16

$\mathrm{mm} \mathrm{Hg}$

APPEARANEE AND UUUH

Colorless liquid, mild odor

SECTION IV FIRE AND EXPLOSION HAZARD DATA

\begin{tabular}{|c|c|c|}
\hline $\begin{array}{l}\text { FLASH POINT } \\
88^{0} \mathrm{~F}\end{array}$ & \multirow{2}{*}{$\begin{array}{c}\text { FLAMM } \\
\text { (EXPLOSIVE) } \\
\text { LIMITS }\end{array}$} & UPPER \\
\hline $\begin{array}{l}\text { METHOD USED } \\
\text { O.C. }\end{array}$ & & LOWER \\
\hline
\end{tabular}

EXTINGUISHING MEDIA

Alcohol foam, carbon dioxide, dry chemical

SPECIAL FIRE FIGHTING PROCEDURES

water may be ineffective

UNUSUAL FIRE AND EXPLOSION HAZAROS 


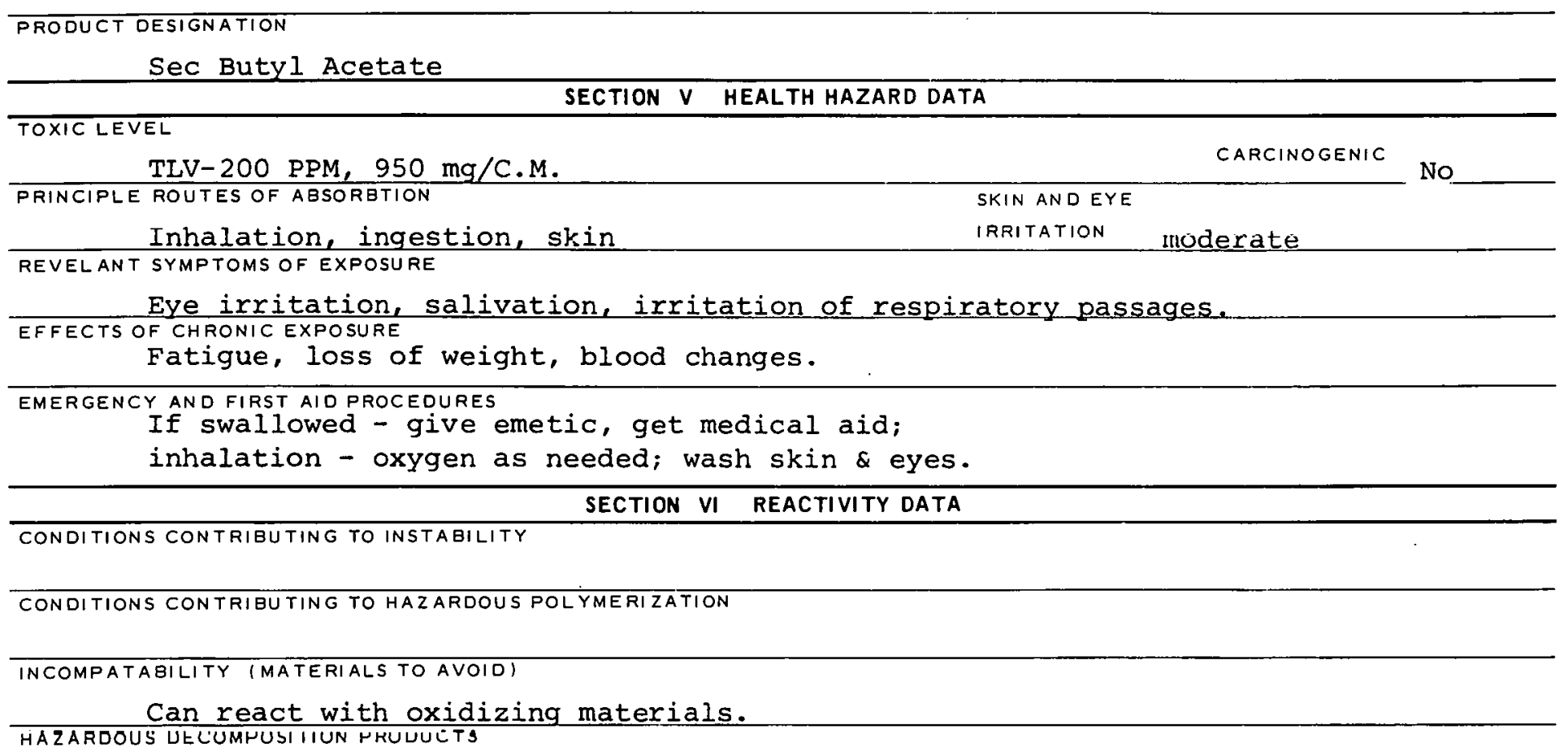

SECTION VII SPILL OR LEAK PROCLDURES

STEPS TO BE TAKEN IN CASE MATERIAL IS RELEASED OR SPILLED

WASTE DISPOSAL METHOD

\begin{tabular}{l|l}
\hline \multicolumn{2}{c}{ SECTION VIII SPECIAL PROTECTION INFORMATION } \\
\hline VEN IILATION REQUIREMENTS LOCAL EXHAUST & $\begin{array}{c}\text { FROTECTIVE EQUIPMENT (SPECIFY TYPES) EYE } \\
\text { Goggles }\end{array}$ \\
\hline MECHANICAL IGENERALI & $\begin{array}{c}\text { GLOVES } \\
\text { Rubber }\end{array}$ \\
\hline SPECIAL & RESPIRATOR \\
& With proper filter \\
\hline
\end{tabular}

\section{SECTION IX SPECIAL PRECAUTIONS}

PRECAUTIONS TO BE TAKEN IN HANDLING AND STORAGE

\section{OTHER PRECAUTIONS}

SIGNATURE

DATE

$222-2781 B$ 
CAS: 000513495

\begin{tabular}{|l|}
\hline PRODUCT DESIGNATION \\
03-222-3701 \\
Sec Butylamine \\
\end{tabular}

EO- 32250

\section{MATERIAL SAFETY DATA SHEET}

SECTION I SOURCE AND NOMENCLATURE
EMERGENCY TELEPHONE NO.

\begin{tabular}{l} 
MANUFACTURER'S NAME \\
ADDRESS INUMEER, STREET. CI \\
TRADE NAME AND SYNONYMS \\
CHEMICAL NAME ANO SYNONYMS \\
2-aMinObutanE \\
\hline
\end{tabular}

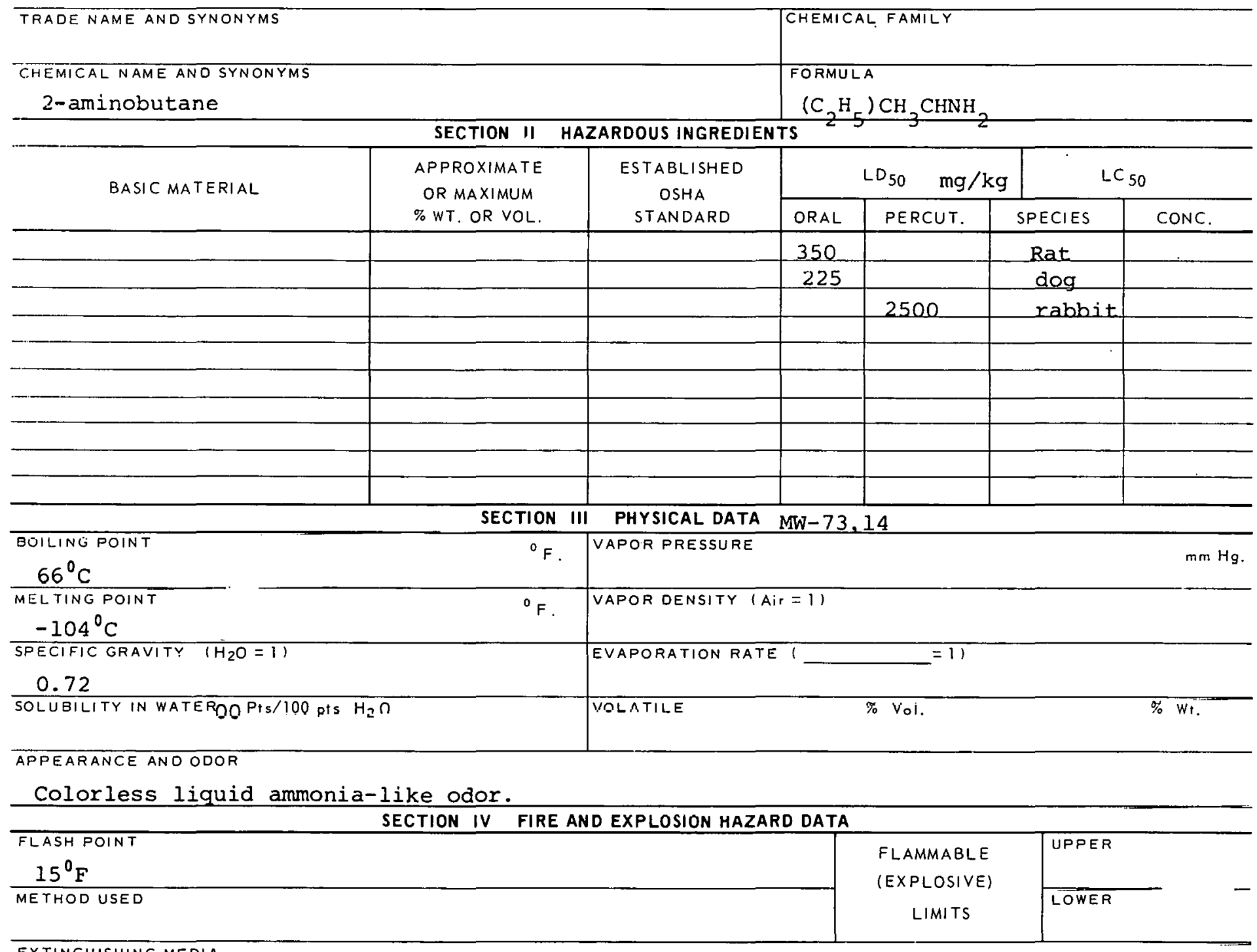

EXTINGUISHING MEDIA

Alcohol foam, carbon dioxide, dry chemical

(P CODE)

\begin{tabular}{l|l} 
SECTION I SOURCE AND NOMENCLATURE \\
EMERGENCY TELEPHONE NO.
\end{tabular}

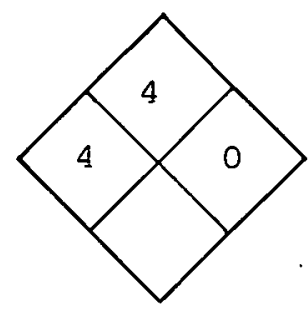

SPECIAL FIRE FIGHTING PROCEDURES

Wear goggles \& self contained breathing apparatus.

UNUSUAL FIRE AND EXPLOSION HAZARDS 


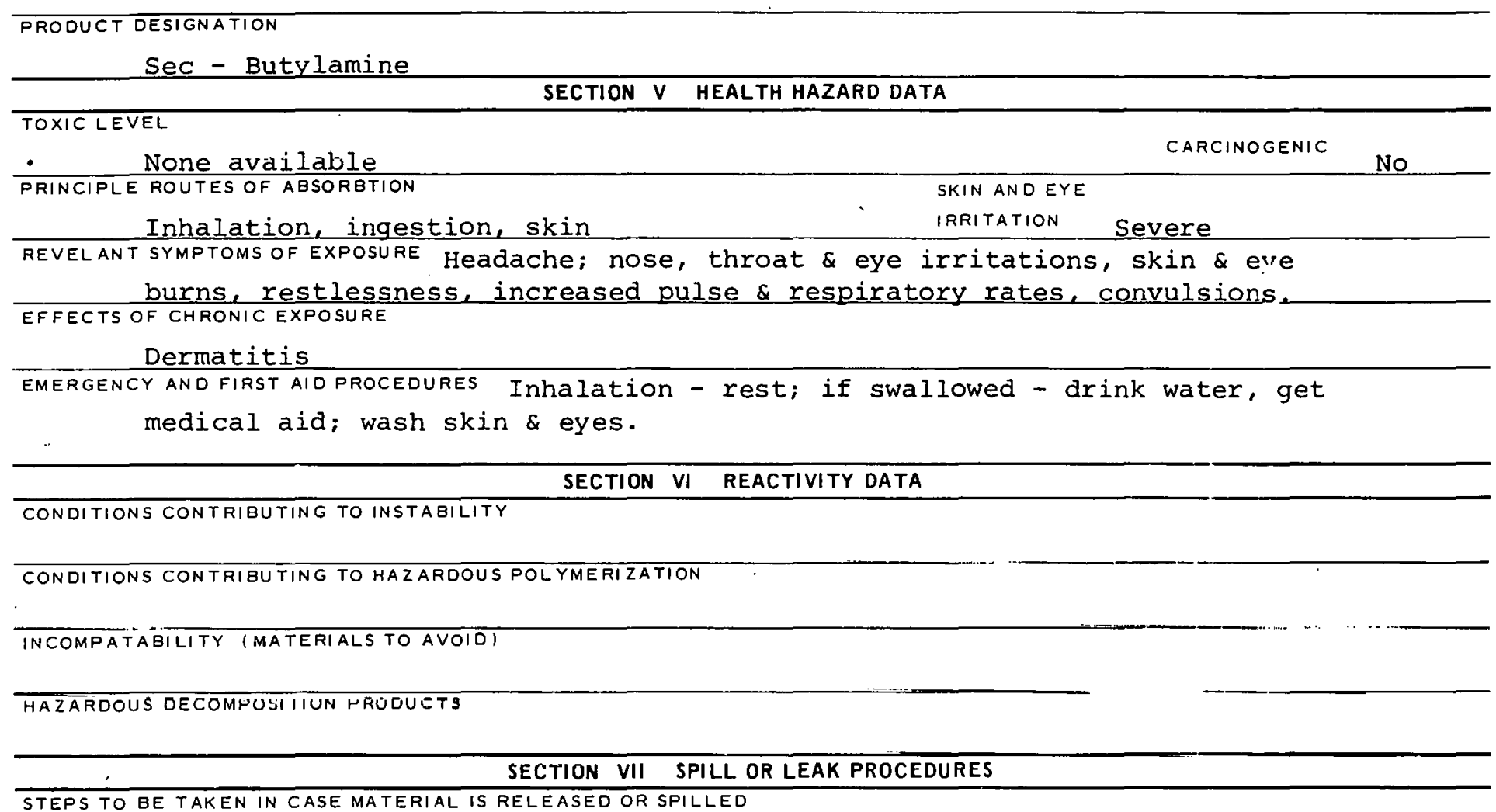

STEPS TO BE TAKEN IN CASE MATERIAL IS RELEASED OR SPILLED

WASTE DISPOSAL METHOD

SECTION VIII SPECIAL PROTECTION INFORMATION

\begin{tabular}{l|l|l}
\multicolumn{2}{c}{ SECTION VIII SPECIAL PROTECTION INFORMATION } \\
\hline VENTILATION REQUIREMENTS LOCAL EXIIAUST & $\begin{array}{c}\text { PROTEC.TIVE EQUIPMENT (SPECIFY TYPES) EYE } \\
\text { Goggles }\end{array}$ \\
\hline MECHANICAL IGENERAL) & $\begin{array}{c}\text { GLOVES } \\
\text { Rubber }\end{array}$ \\
\hline SPECIAL & $\begin{array}{c}\text { RESPIRATOR } \\
\text { Self contained breathing apparatus }\end{array}$ \\
\hline
\end{tabular}

OTHER PROTECTIVE EQUIPMENT

SECTION IX SPECIAL PRECAUTIONS

PRECAUTIONS TO BE TAKEN IN HANDLING AND STORAGE

OTHER FRECAUTIONC

3IGNATURL

NATF

$222-3701 B$ 


\section{MATERIAL SAFETY DATA SHEET}

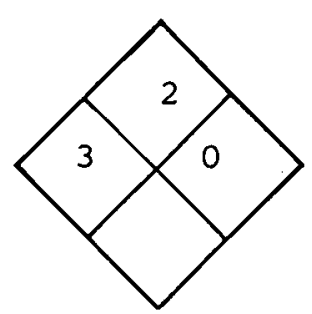

SECTION I SOURCE AND NOMENCLATURE

\begin{tabular}{l|l}
\hline MANU FAC TURER'S NAME & EMERGENCY TELEPHONE NO. \\
\hline ADDRESS (NUMBER, STREET, CITY, STATE, ZIP CODE)
\end{tabular}

\section{TRADE NAME AND SYNONYMS \\ CHEMICAL NAME AND SYNONYMS \\ 1-Phenylbutane}

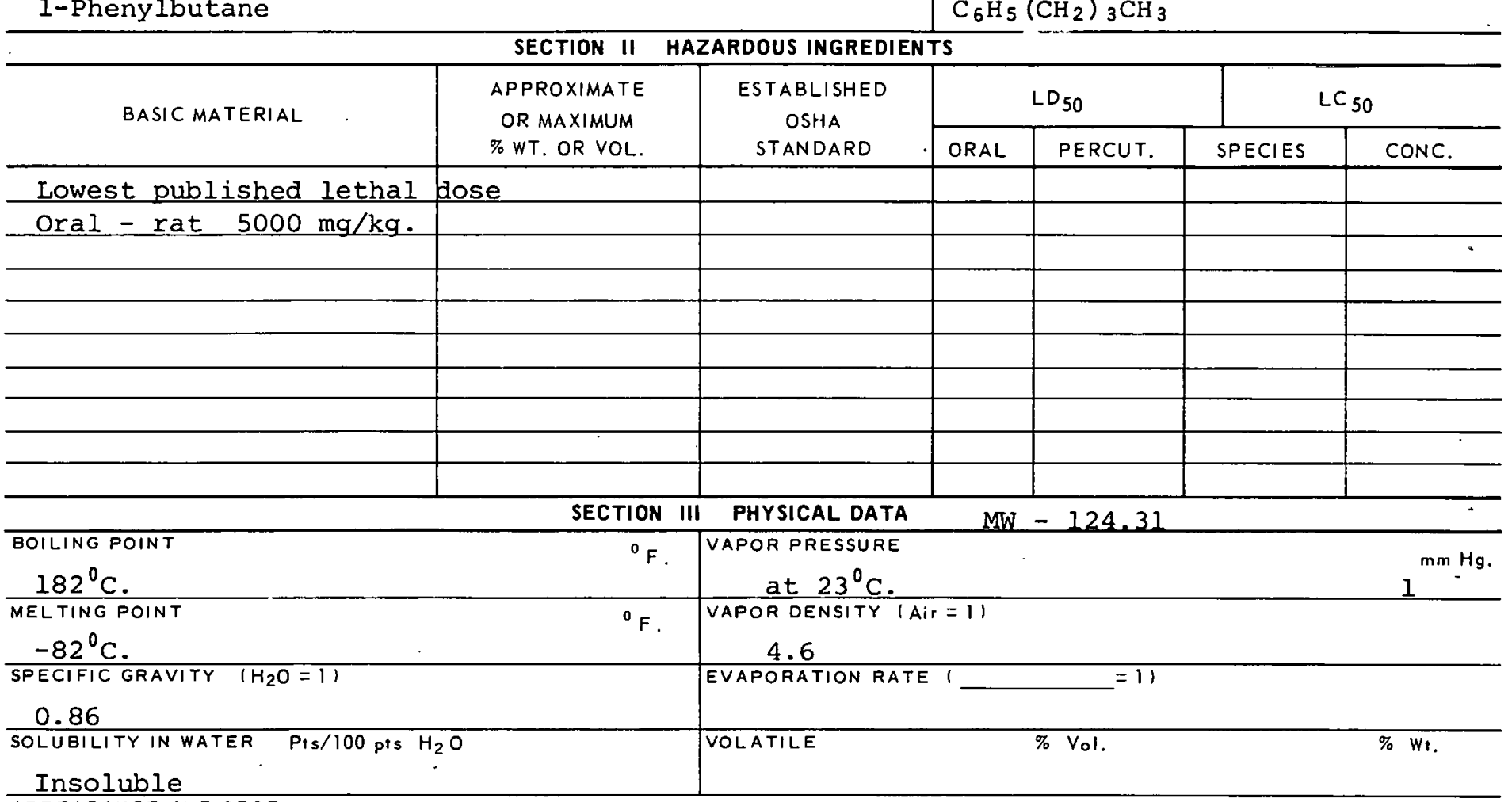

Insoluble

APPEARANCE AND ODOR

\section{Colorless liquid}

\begin{tabular}{l}
\hline \\
\hline FLASH POINT \\
$160^{\circ} \mathrm{F}$. \\
METHOD USED \\
OC \\
EXTINGUISHING MEDIA
\end{tabular}

\section{CHEMICAL FAMILY \\ FORMULA}

TS

OR MAXIMUM OSHA

$\%$ WT. OR VOL.
PRESSURE

$$
4.6
$$

OLATILE $\%$ Vol. $\% W_{\text {t }}$

\begin{tabular}{c|r} 
FLAMMABLE & UPPER \\
(EXPLOSIVE) & $5.8 \%$ \\
\cline { 2 - 2 } LIMITS & LOWER \\
& $0.8 \%$
\end{tabular}

Foam, carbon dioxide, dry chemical

SPECIAL FIRE FIGHTING PROCEDURES 


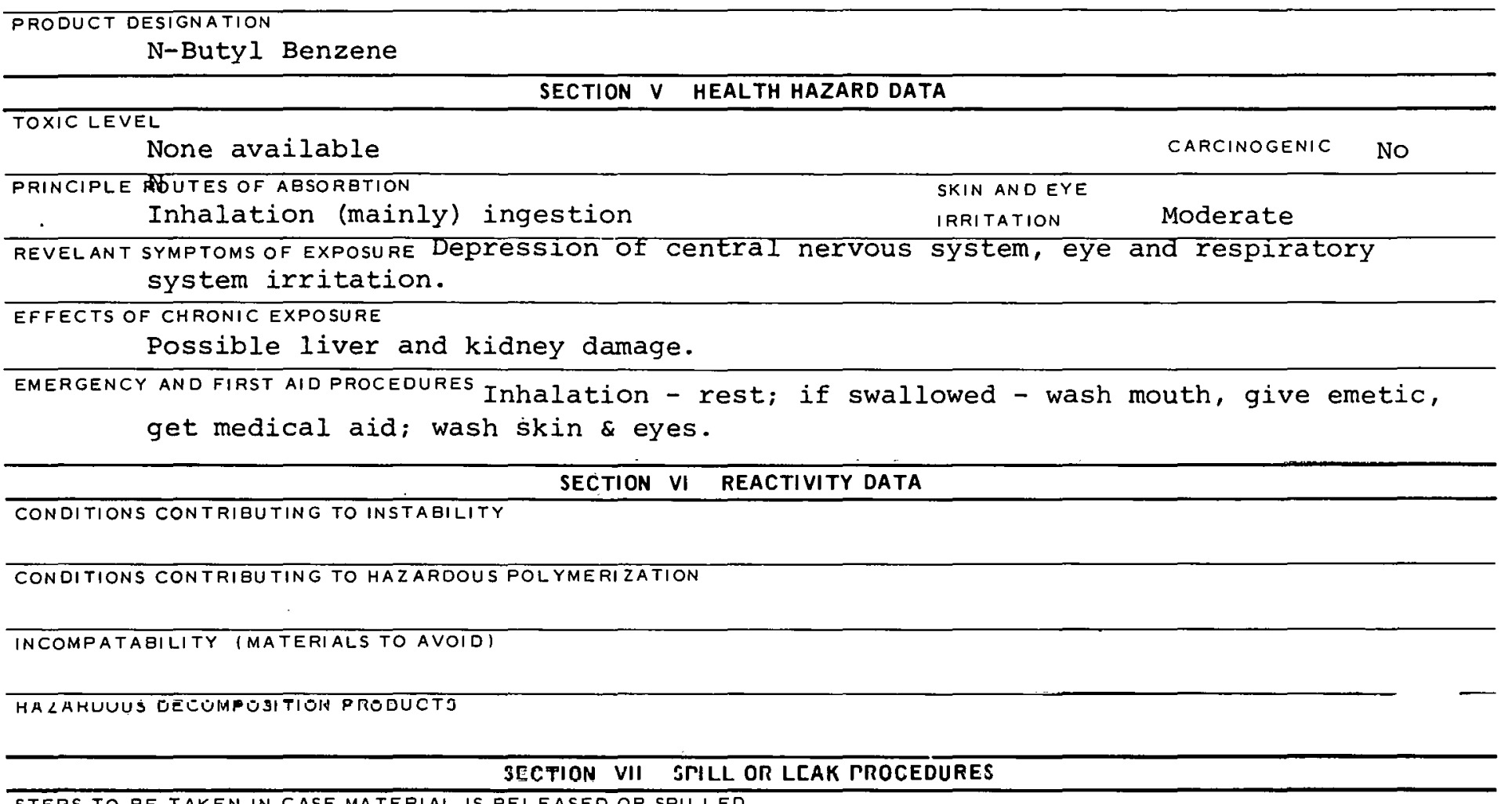

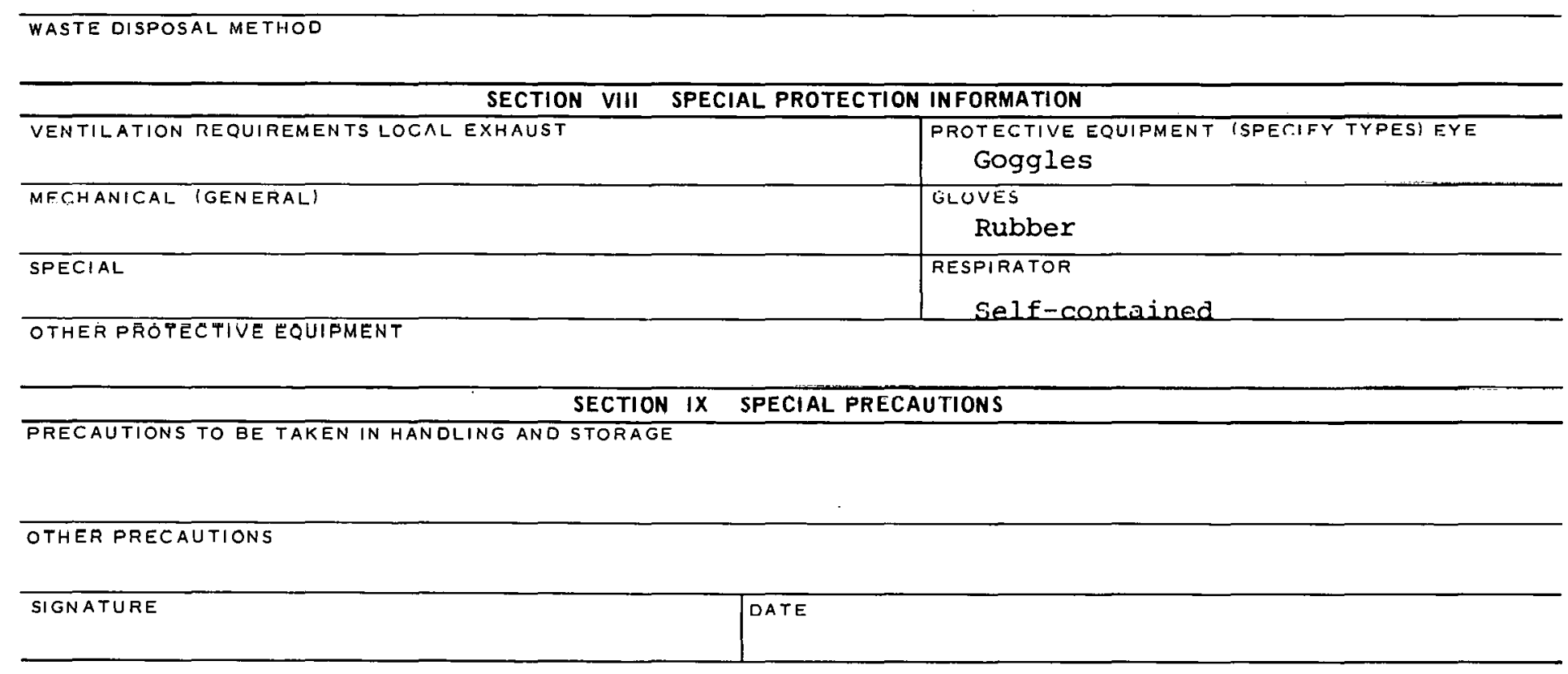


CAS : 000135988

\section{PRODUCT DESIGNATION}

03-222-4731

Sec-Butyl Benzene

\section{MATERIAL SAFETY \\ DATA SHEET}

\section{SECTION I SOURCE AND NOMENCLATURE}

MANUFACTURER'S NAME

ADDRESS (NUMBER, STREET, CITY, STATE, ZIP CODE)

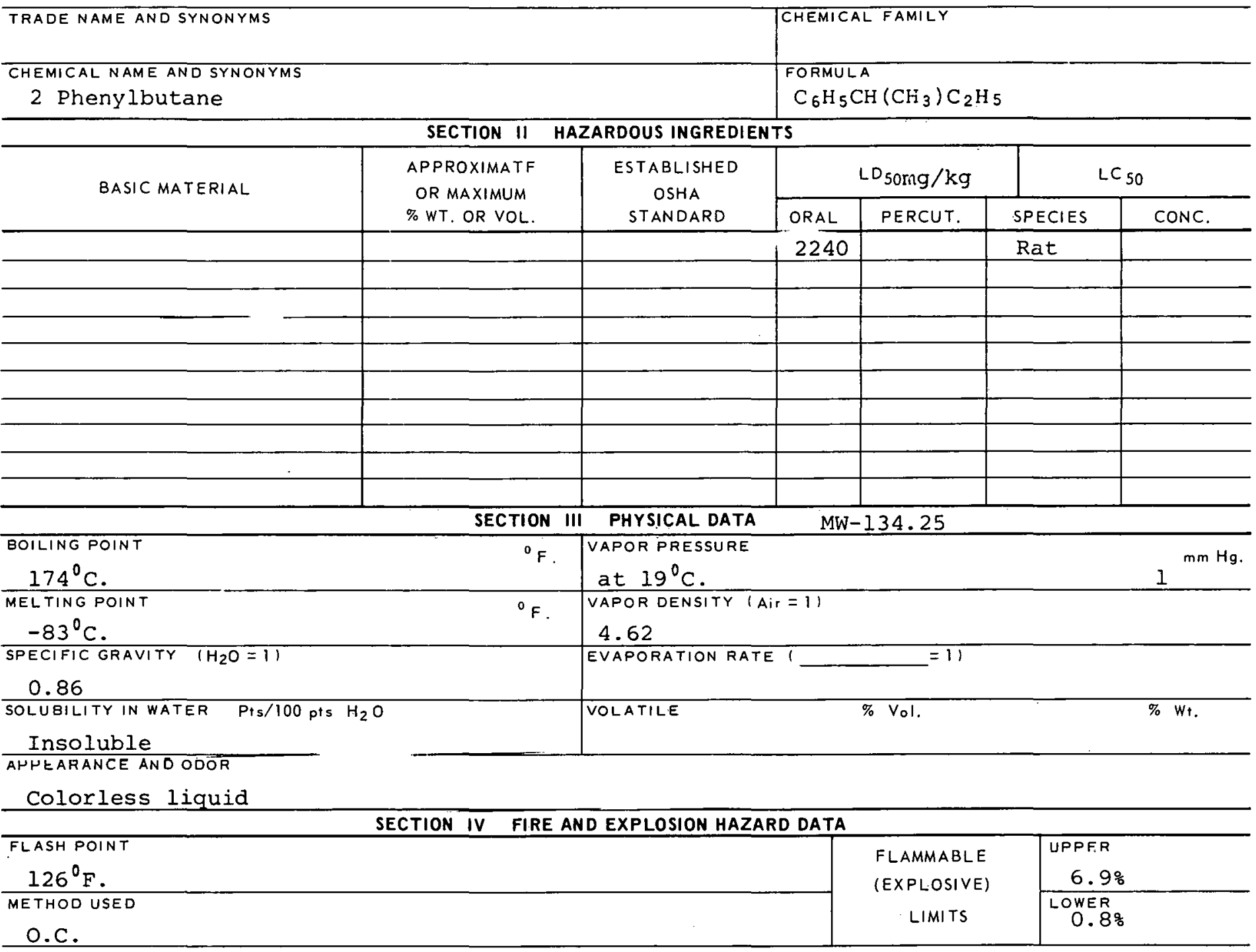

EXTINGUISHING MEDIA

Foam, carbon dioxide, dry cliemicd.

SPECIAL FIRE FIGHTING PROCEDURES

UNUSUAL FIRE AND EXPLOSION HAZARDS 
TOXIC LEVEL

None Available

PRINCIPLE ROUTES OF ABSORBTION

Inhalation (mainly) ingestion

REVELANT SYMPTOMS OF EXPOSURE Depression of central nervous system, system irritation.

EFFECTS OF CHRONIC EXPOSURE

Possible liver and kidney damage.

EMERGENCY AND FIRST AIDPROCEDURES Inhalation - rest; if swallowed - give emetic, get

medical aid; wash from skin and eyes.

\section{SECTION VI REACTIVITY DATA}

CONDITIONS CONTRIBUTING TO INSTABILITY

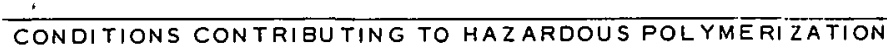

INCOMPÁTABILIIY (MAIERIAL3 TO AYOID

HALARUUUS PEESMPOSITIOH RRONITTS

SECTION VII SPILL OR LEAK PROCEDURES

STEPS TO BE TAKEN IN C.ASE MATERIAL IS RELEASED OR SPILLED

WASTE DISPOSAL METHOD

SECTION VIII SPECIAL PROTECTION INFORMATION

\begin{tabular}{|c|c|}
\hline VENTILATION REQUIRFMENTS LOCAL EXHAUST & $\begin{array}{l}\text { PROTECTIVE EQUIPMENT (SPECIFY TYPES) EYE } \\
\text { GOggleS }\end{array}$ \\
\hline MECHANICAL (GENERAL) & $\begin{array}{l}\text { GLOVES } \\
\text { Rubber }\end{array}$ \\
\hline EmErial & 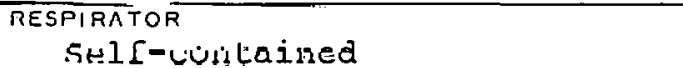 \\
\hline
\end{tabular}

OTHER PROTECTIVE EQUIFMENT

SECTION IX SPECIAL PRECAUTIONS

PRECAIITIONS TO BE TAKEN IN HANDLING AND STORAGE

ITHFR PRECAUTIONS

SIGNATURE

DATE

$222-4731 B$
CARCINOGENIC NO

Moderate

eye and respiratory

SKIN ANDEYE

IRRITATION 
CAS : 000098066

\section{PRODUCT DESIGNATION}

03-222-4761

Tert-Butyl benzene

\section{MATERIAL SAFETY DATA SHEET}

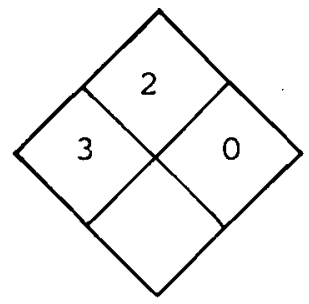

\section{SECTION I SOURCE AND NOMENCLATURE}

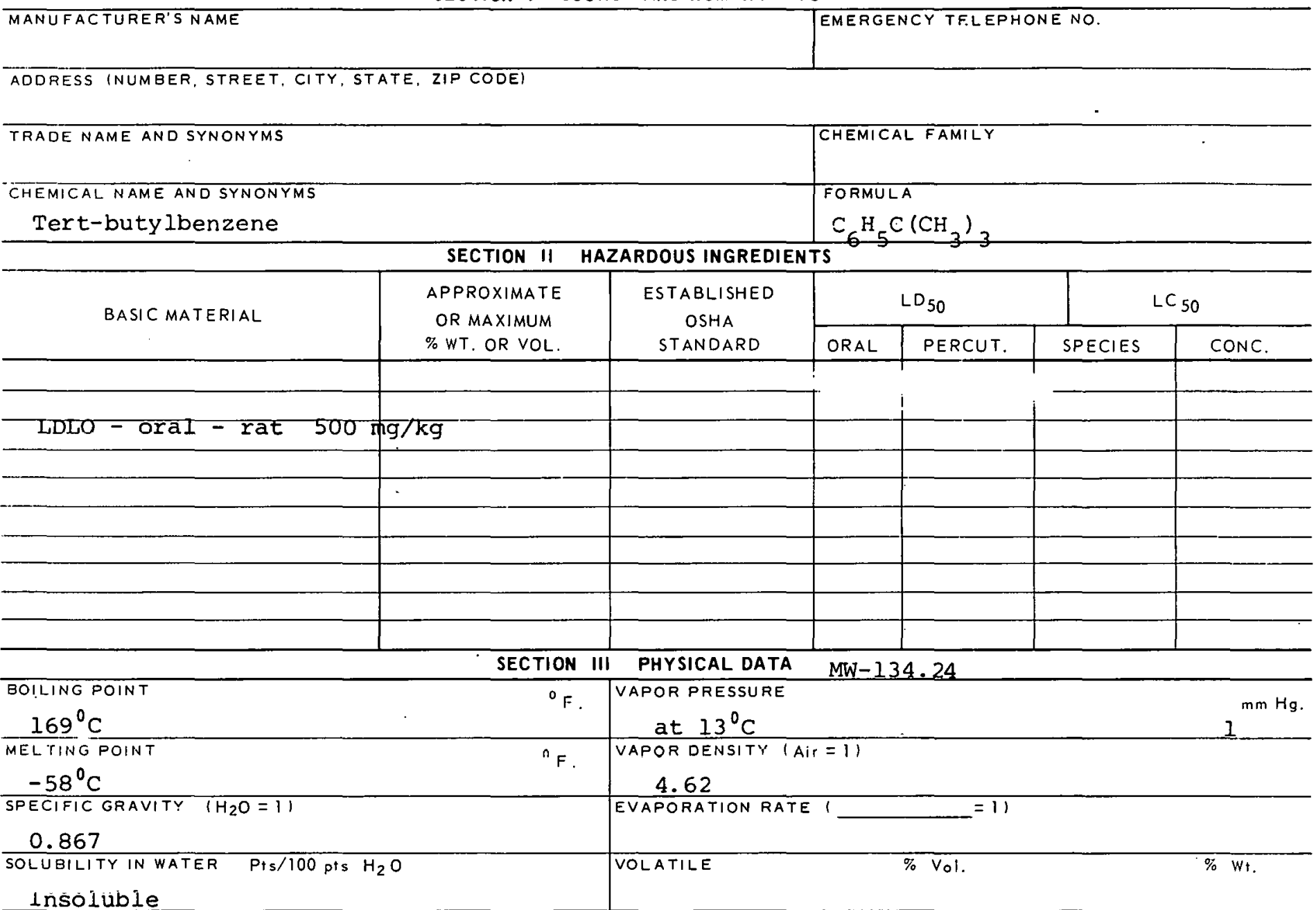

APPEARANCE AND ODOR

Colorless liquid

\begin{tabular}{l|c|c}
\hline \multicolumn{1}{c}{ SECTION IV FIRE AND EXPLOSION HAZARD DATA } & \\
\hline FLASH POINT & FLAMMABLE & UPPER \\
$160^{\circ} \mathrm{F}$ & 5.8 & (EXPLOSIVE) \\
\cline { 2 - 3 } METHOD USED & LOWER \\
C.C. & 0.8 & LIMITS \\
\hline
\end{tabular}

EXTINGUISHING MEDIA

Foam, carbon dioxide, dry chemical

SPECIAL FIRE FIGHTING PROCEDURES

Wear goggles and self contained breathing apparatus.

UNUSUAL FIRE AND EXPLOSION HAZAROS 
TOXIC LEVEL

Not available

PRINCIPLE ROUTES OF ABSORETION

Inhalation (mainly) ingestion

CARCINOGENIC No REVELANT SYMPTOMS OF EXPOSURE

Depression of central nervous system, eye \& respiratory system irritation. EFFECTS OF CHRONIC EXPOSURE

Possible liver \& kidney damage.

EMERGENCY AND FIRST AID PROCEDURES Inhalation - rest; if swallowed - wash mouth, give emetic, get medical aid; wash skin \& eyes.

\begin{tabular}{l}
\hline CONDITIONS CONTRIBUTING TO INSTABILITY \\
\hline CONDITIONS CONTRIBUTING TO HAZARDOUS POLYMERIZATION VI REACTIVITY DATA \\
\hline HIVEOMIFATADILITY IMATERIALE TO AVIOIOI \\
\hline HAZARDOUS DECOMPOSITION PRODUCTS \\
\hline STEPS TO BE TAKEN IN CASE MATERIAL IS RELEASED OR SPILLED
\end{tabular}
STEPS TO BE TAKEN IN CASE MATERIAL IS RELEASED OR SPILLED

WASTE DISPOSAL METHOD

SECTION VIII SPECIAL PROTECTION INFORMATION

\begin{tabular}{l|l}
\hline \multicolumn{1}{c}{ SECTION VIII SPECIAL PROTECTION INFORMATION } \\
\hline VENTILATION REQUIREMENTS LOCAL FXHAIST & $\begin{array}{c}\text { PROTECTIVE EQUIPMENT (SPECIFY TYPES) EYE } \\
\text { Goggles }\end{array}$ \\
\hline MECHANICAL (GENERAL) & $\begin{array}{c}\text { GLOVES } \\
\text { Rubber }\end{array}$ \\
\hline SPECIAL & $\begin{array}{c}\text { RESPIRATOR } \\
\text { SeIf COntained }\end{array}$ \\
\hline OTHER PROTECTIVE EQUIPMENT & \\
\hline
\end{tabular}

PRECAUTIONS TO BE TAKEN IN HANDLING AND STORAGE

OTHER PRECAUTIONS

SIGNATURE

DATE 


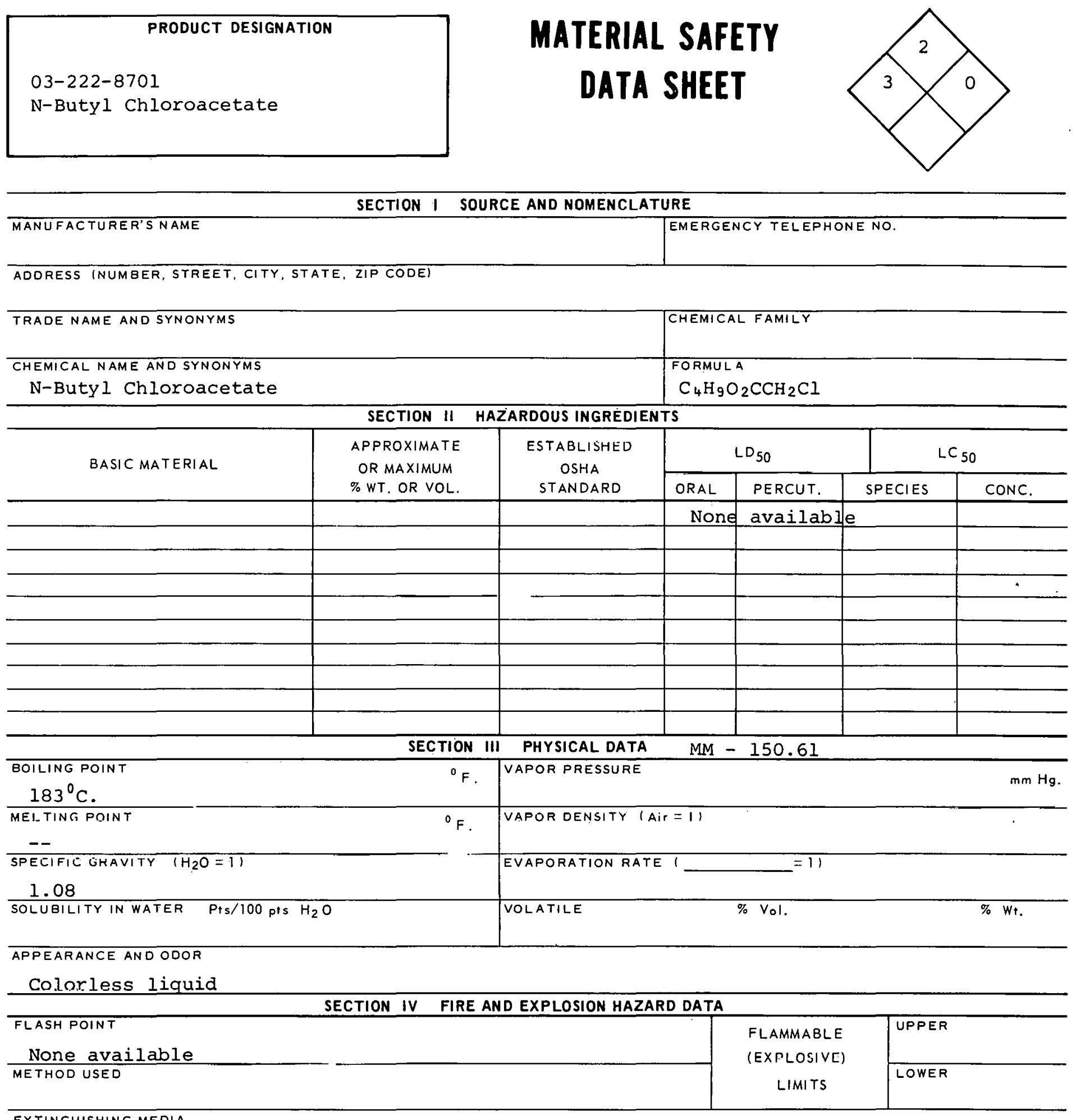

EXTINGUISHING MEDIA

Alcohol foam, carbon dioxide, dry chemical.

SPECIAL FIRE FIGHTING PROCEDURES

Water may be ineffective.

UNUSUAL FIRE AND EXPLOSION HAZARDS 


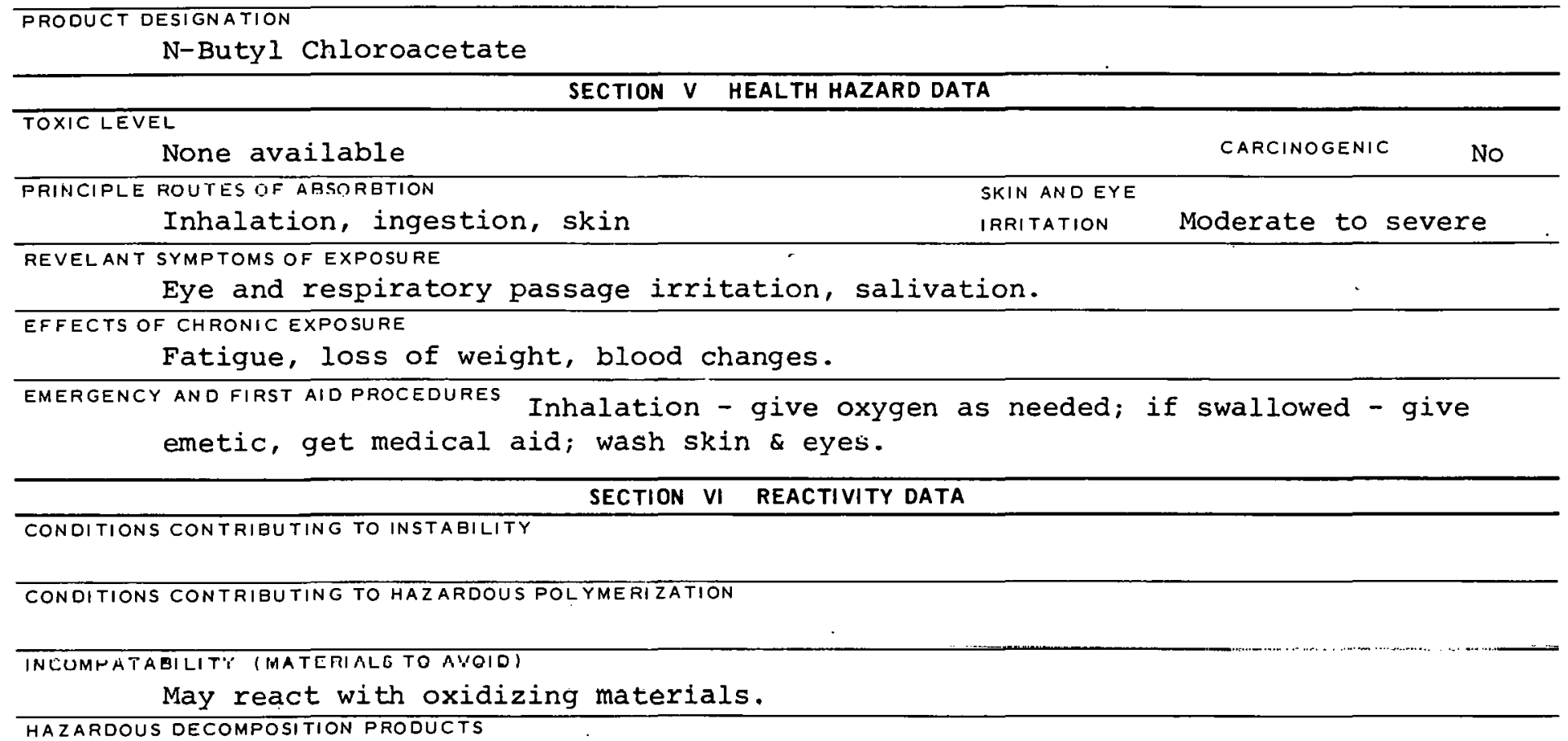

SECTION VII SPILL OR LEAK PROCEDURES

STEPS TO BE TAKEN IN CASE MATERIAL IS RELEASED OR SPILLED

WASTE DISPOSAL METHOD

\begin{tabular}{l|l}
\hline \multicolumn{1}{c}{ SECTION VIII SPECIAL PROTECTION INFORMATION } \\
\hline VENTILATION REQUIREMENTS LOCAL EXHAUST & $\begin{array}{c}\text { PROTECTIVE EQUIPMENT ISPECIFY TYPESIEYE } \\
\text { GOggles }\end{array}$ \\
\hline MECIHAPIICAL (GENERAL) & $\begin{array}{c}\text { GLOVES } \\
\text { Rubber }\end{array}$ \\
\hline SPECIAL & $\begin{array}{c}\text { RESPIRATOR } \\
\text { With proper filter }\end{array}$ \\
\hline
\end{tabular}

OTHER PROTECTIVE EQUIPMENT

SECTION IX SPECIAL PRECAUTIONS

PRECAUTIONS TO BE TAKEN IN HANDLING AND STORAGE

OTHER PRECAUTIONS

EICHATURE

NATF

$222-8701 B$ 
PRODUCT DESI.GNATION

033-222-9211

$\alpha$-Butylene Bromide
MATERIAL SAFETY

DATA SHEET

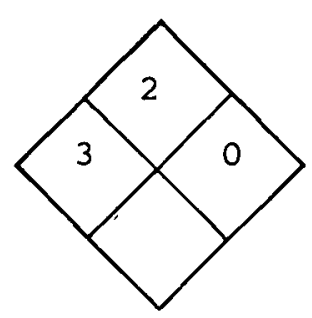

SECTION I SOURCE AND NOMENCLATURE

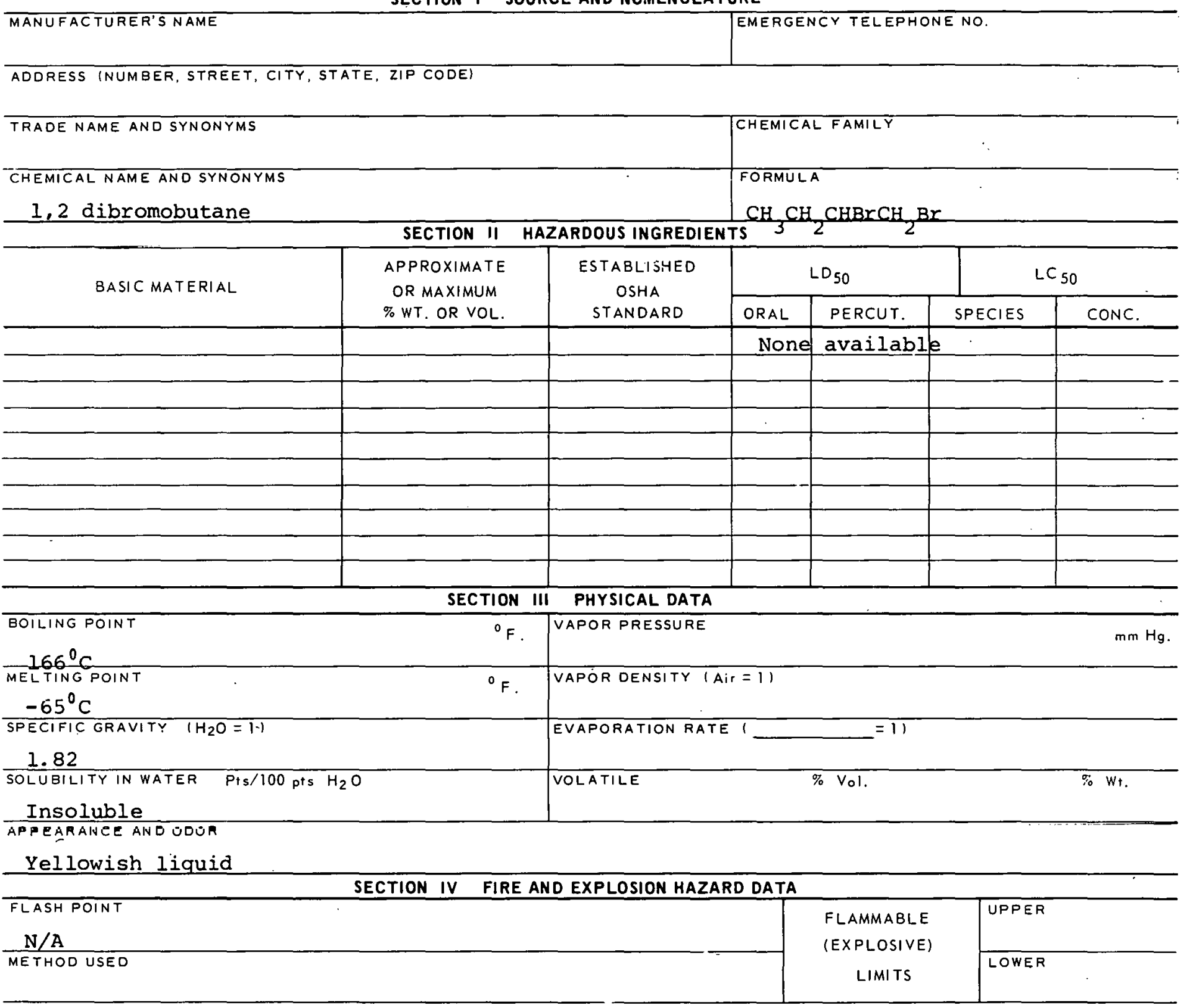

EXTINGUISHING MEDIA

Water, foam, carbon dioxide, dry chemical

SPECIAL FIRE FIGHTING PROCEDURES

Water may be ineffective.

UNUSUAL FIRE AND EXPLOSION HAZARDS 
TOXICLEVEL

None available

PRINCIPLE ROUTES OF ABSORBTION

Inhalation, ingestion

REVELANT SYMPTOMS OF EXPOSURE

Eye irritation, headache, vomiting, drowsiness, narcosis EFFECTS OF CHRONIC EXPOSURE

Dermatitis, liver \& kidney damage

EMERGENCY AND FIRST AID PROCEDURES Inhalation - rest; if swallowed - wash mouth, give emetic, get medical aid; wash from skin \& eyes.

SECTION VI REACTIVITY DATA
SKIN AND EYE

IRRITATION

Moderate

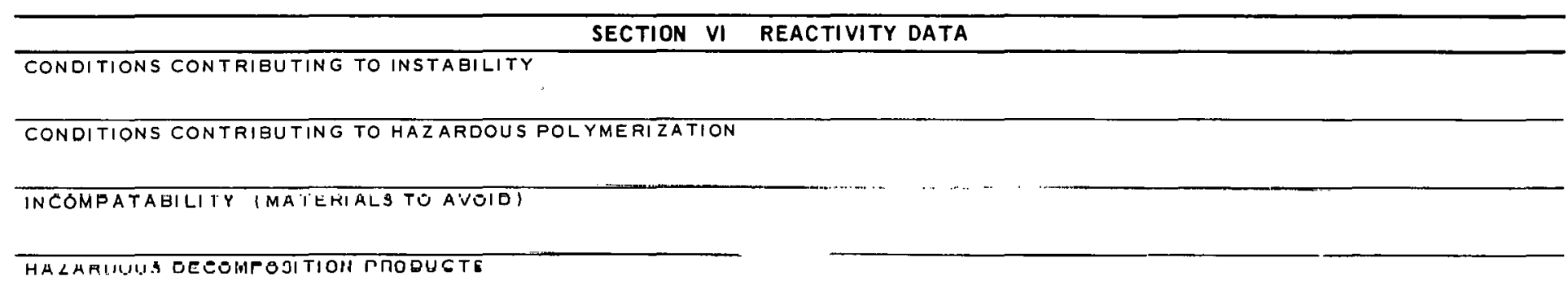

SECTION VII SPILL OR LEAK PROCEDURES

STEPS TO BE TAKEN IN CASE MATERIAL IS RELEASED OR SPILLED

Wear self contained breathing apparatus

WASTE DISPOSAL METHOD

SECTION VIII SPECIAL PROTECTION INFORMATION

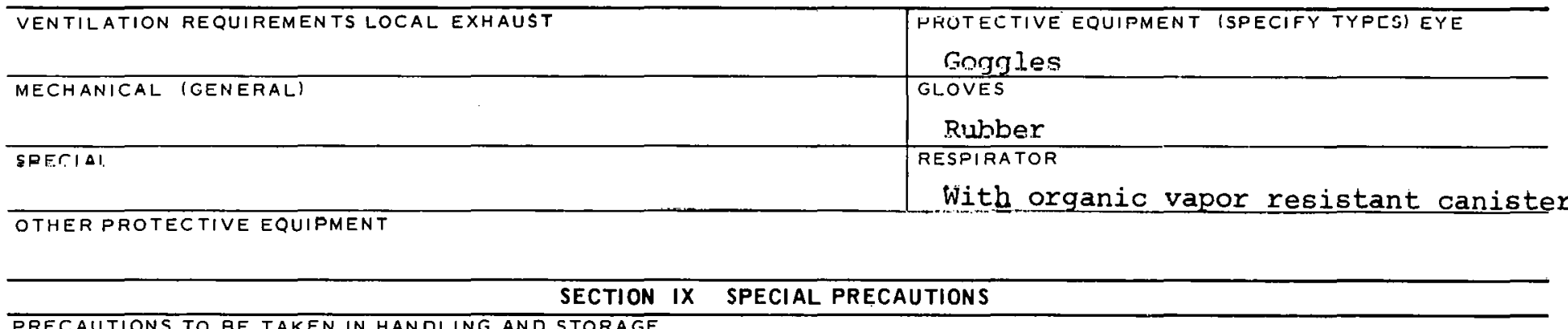

OTHER PREGAUTIONS

SIGNATURE

DATE

$222-9211 B$ 

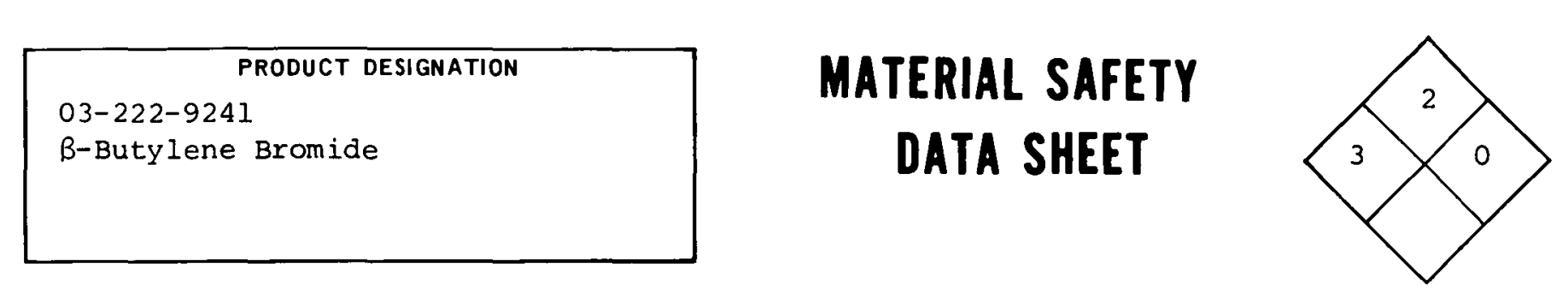

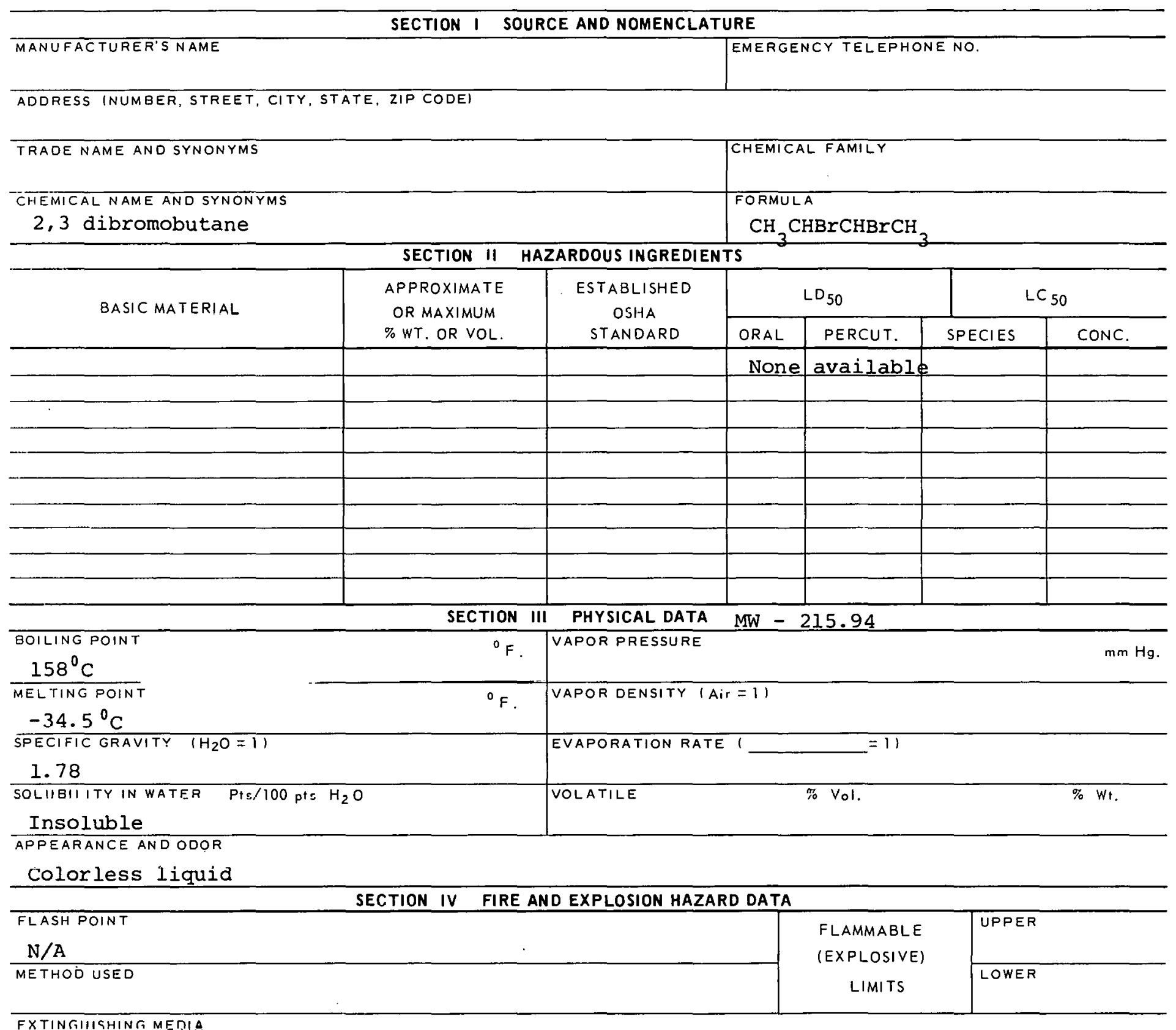

FXTINGIIISHING MENIA

water, foam, carbon dioxide, dry chemical

SPECIAL FIRE FIGHTING PROCEDURES

Water may be ineffective

UNUSUAL FIRE AND EXPLOSION HAZARDS 
B-butylene bromide

SECTION V HEALTH HAZARD DATA

TOXIC LEVEL

None available

CARCINOGENIC

SKIN AND EYE

IRRITATION Moderate

IRRITATION Moderate

Inhalation, ingestion

REVELANT SYMPTOMS OF EXPOSURE Eye irritation, headache, vomiting, drowsiness,

narcosis, liver \& kidney damage.

EFFECTS OF CHRONIC EXPOSURE

Dermatitis, liver \& kidney damage

EMERGENCY AND FIRST AID PROCEDURES Inhalation - rest; if swallowed - wash mouth, give

emetic; get medical aid; wash from skin \& eyes.

\section{SECTION VI REACTIVITY DATA}

CONDITIONS CONTRIBUTING TO INSTABILITY

CONDITIONS CONTRIBUTING TO HAZARDOUS POLYMERIZATION

INCOMPATABILITY (MATERIALS TO AVOIU)

IIAZARDOUS DECOMPOSITION PRODUCTS

SECTION VII SPILL OR LEAK PROC.EDIIRES

STEPS TO BE TAKEN IN CASE MATERIAL IS RELEASED OR SPILLED

Wear self contained breathing apparatus.

WASTE DISPOSAL METHOD

SECTION VIII SPECIAL PROTECTION INFORMATION

VENTILATION REQUIREMENTS LOCAL EXHAUST

$\therefore$

MECHANICAL IGENERAL)

.

SPECIAI

.

OTHER.PROTECTIVE EQUIPMENT

PROTECTIVE EQUIPMENT (SPECIFY TYPES) EYE

Gogyles

GLOVEE

Rubber

RESPIRATOR

With organic vapor resistant canister.

SECTION IX SPECIAL PRECAUTIONS

PRECAUTIONS TO BE TAKEN IN HANDLING AND STORAGE

OTHER PRECAUTIONS

SIGNATURE

UATE

222-924IB 


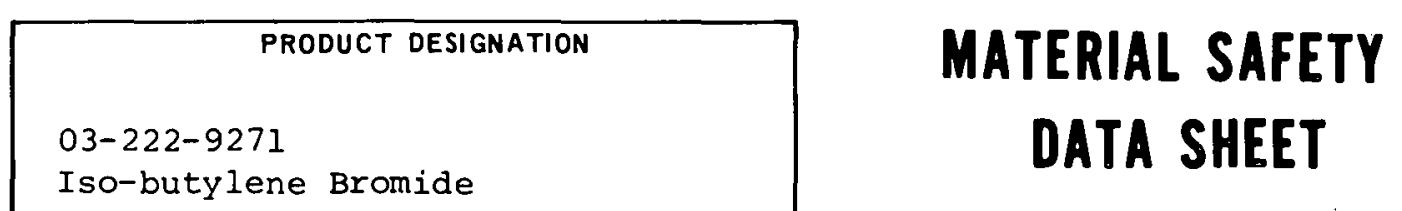

SECTION I SOURCE AND NOMENCLATURE

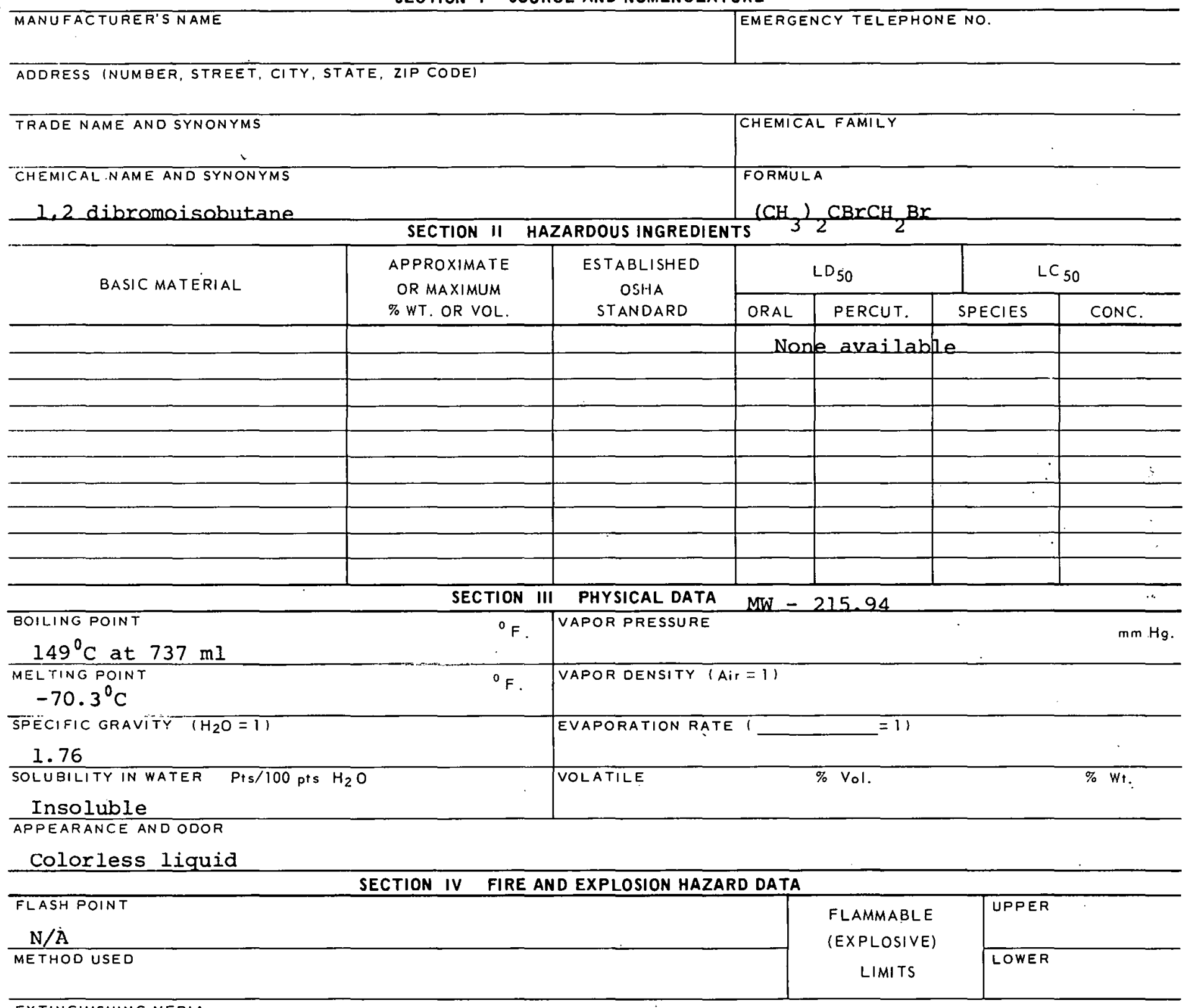

EXTINGUISHING MEDIA

Water, foam, carbon dioxide, dry chemical

SPECIAL FIRE.FIGHTING PROCEDURES

Water may be ineffective

UNUSUAL FIRE AND EXPLOSION HAZARDS 


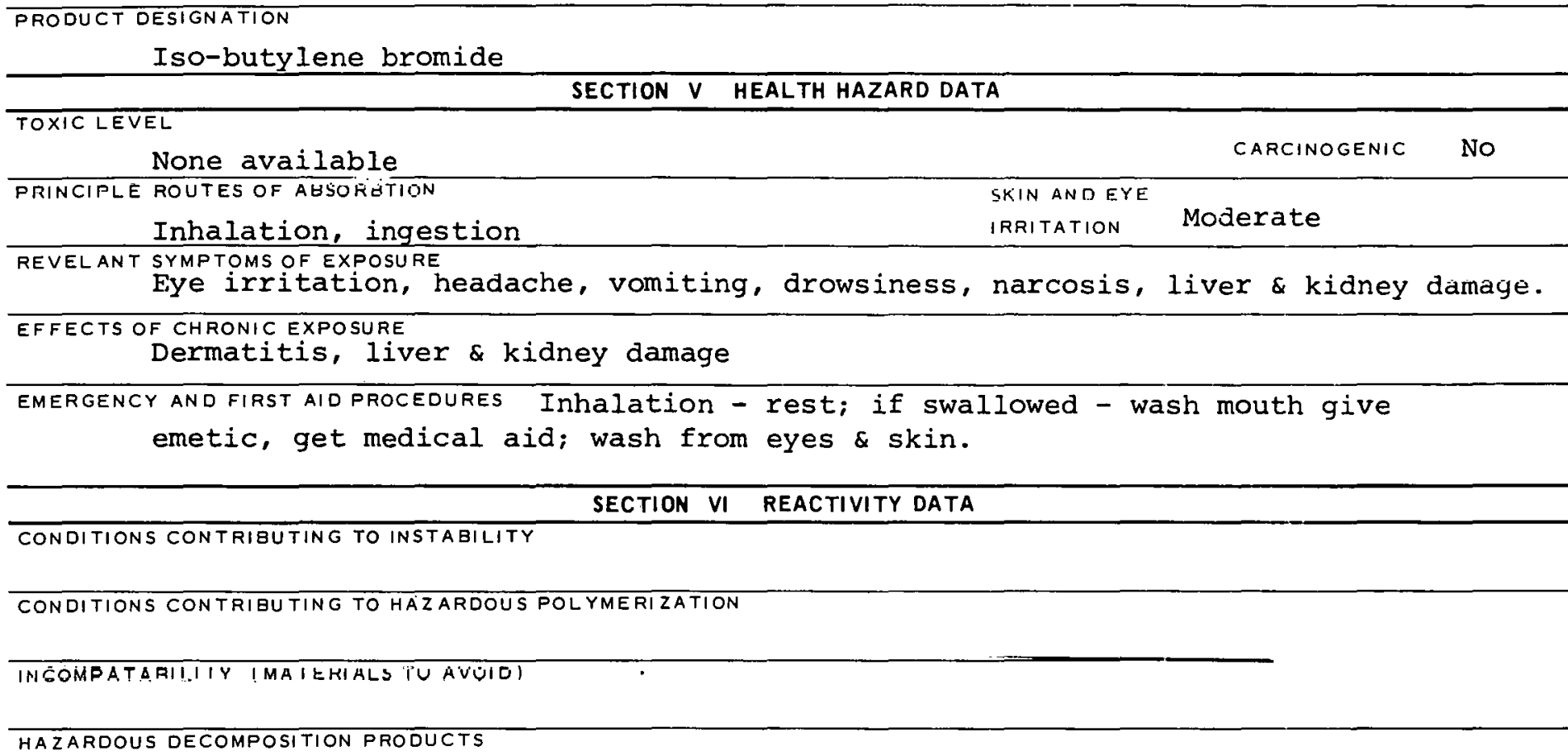

SECTION VII SPILL OR LEAK PROCEDURES

STEPS TO BE TAKEN IN CASE MATERIAL IS RELEASEU UR SPILLED

Wear self contained breathing apparatus.

WASTE DISPOSAL METHOD

SECTION VIII SPECIAL PROTECTION INFORMATION

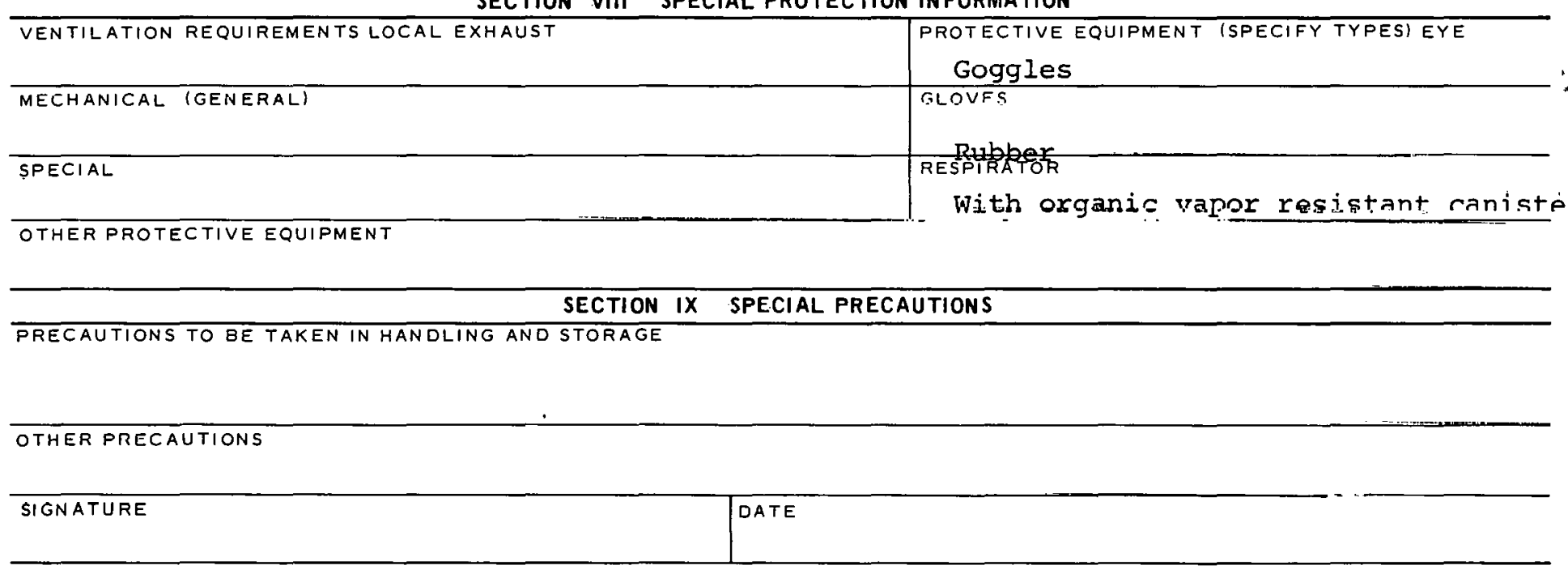


CAS : 000592847

LQ55000

\begin{tabular}{|c|}
\hline PRODUCT DESIGNATION \\
03-223-0302 \\
N-Butyl Formate \\
\end{tabular}

MATERIAL SAFETY

DATA SHEET

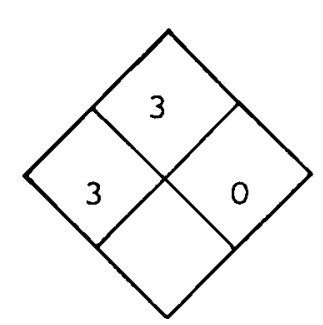

\section{SECTION I SOURCE AND NOMENCLATURE}

\begin{tabular}{l|l}
\hline \multicolumn{2}{c}{ SECTION I SOURCE AND NOMENCLATURE } \\
\hline MANUFAC TURER'S NAME & EMERGENCY TELEPHONE NO. \\
\hline AODRESS (NUMBER, STREET, CITY, STATE, ZIP CODE)
\end{tabular}

ADDRESS (NUMBER, STREET, CITY, STATE, ZIP CODE)

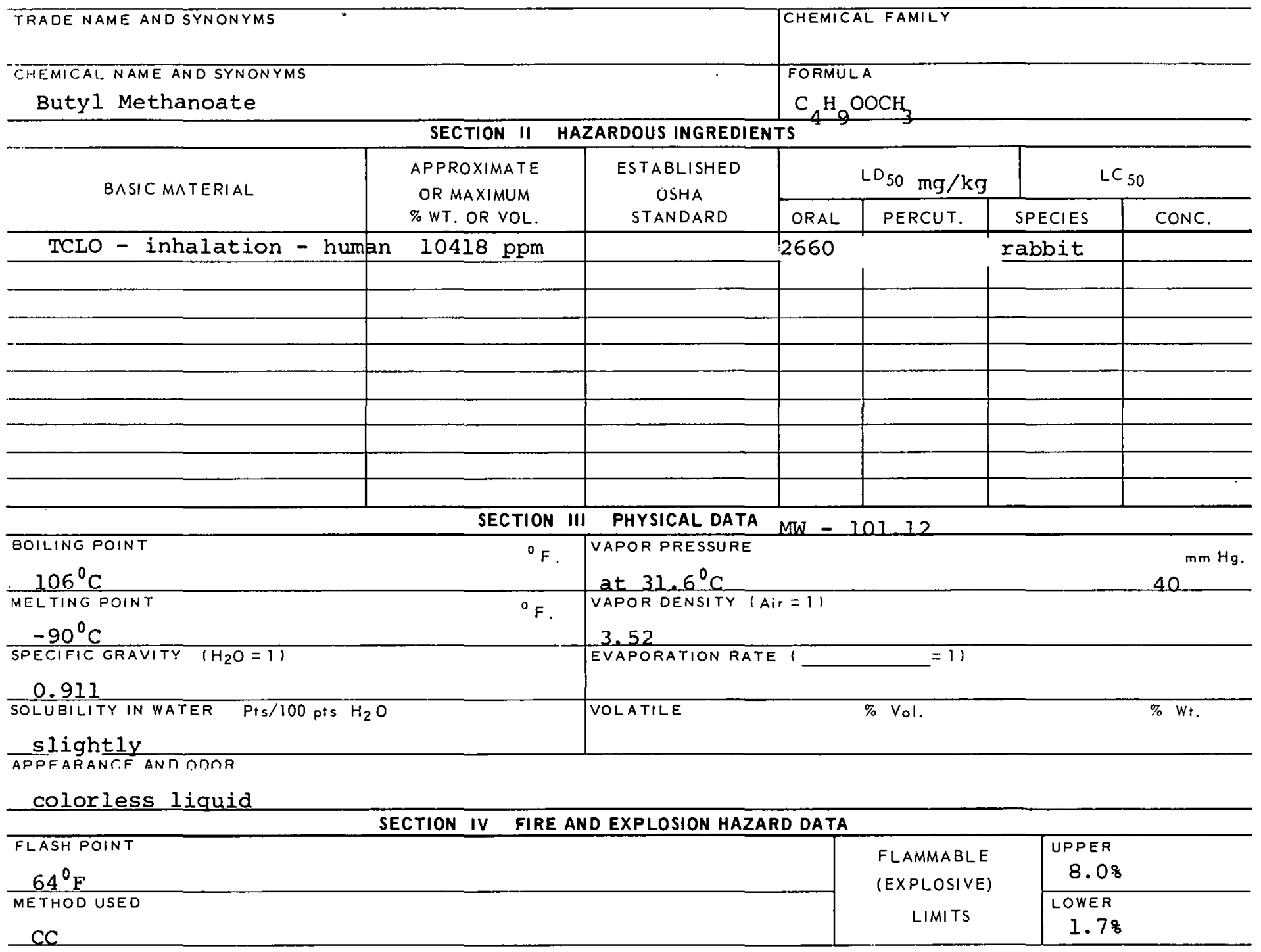

EXTINGUISHING MEDIA

Alcohol foam, carbon dioxide, dry chemical

SPECIAL FIRE FIGHTING PROCEDURES

Water may be ineffective

UNUSUAL FIRE AND EXPLOSION HAZAROS 


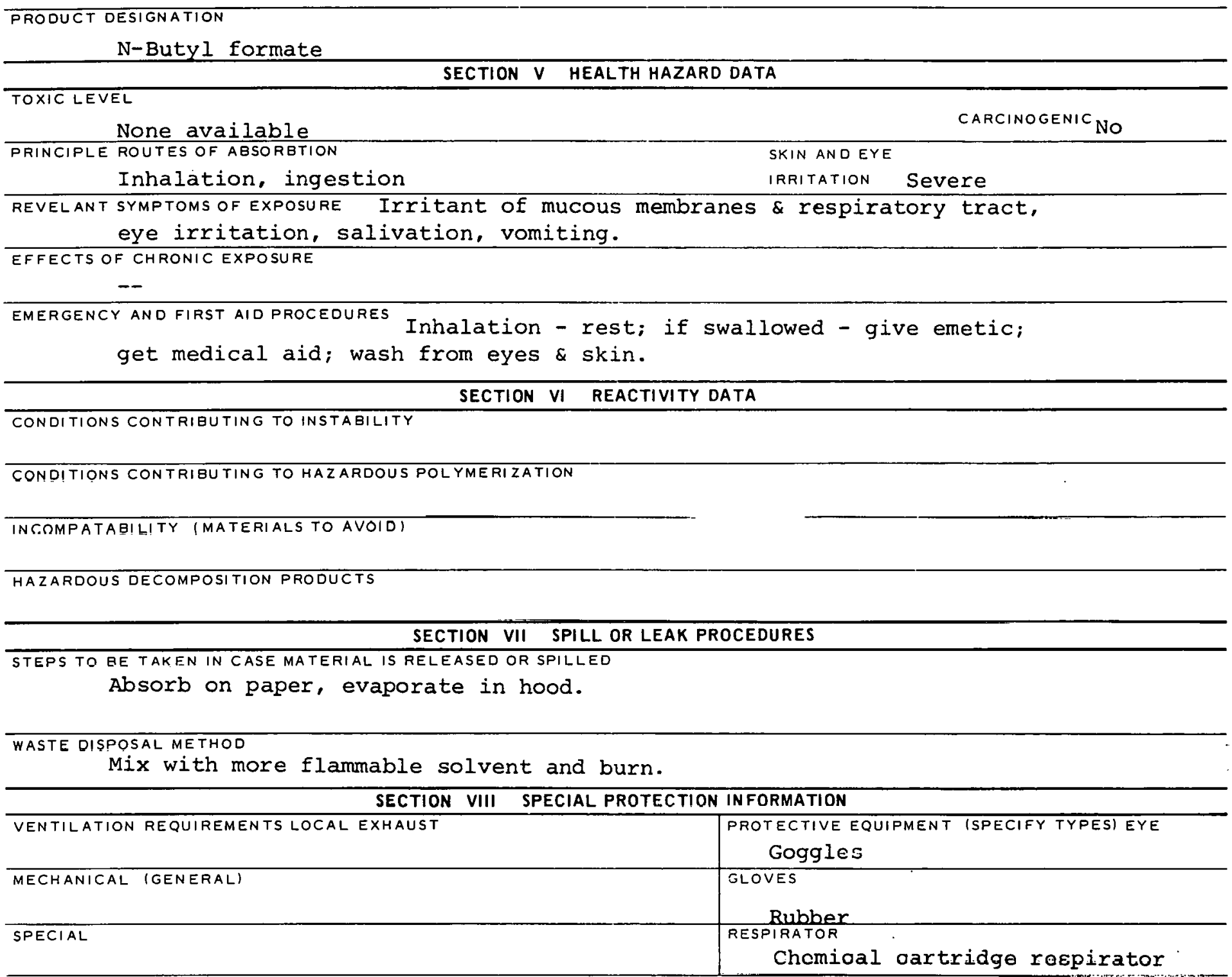

OTHER PROTECTIVE EUUIHMEN I

overalls

SECTION IX SPECIAL PRECAUTIONS

PRECAUTIONS TO BE TAKEN IN HANDLING AND STORAGE

Keep away from sources of ignition.

OI HER PRECAUTIONS

SIGNATURE

DATE

223-0302B 


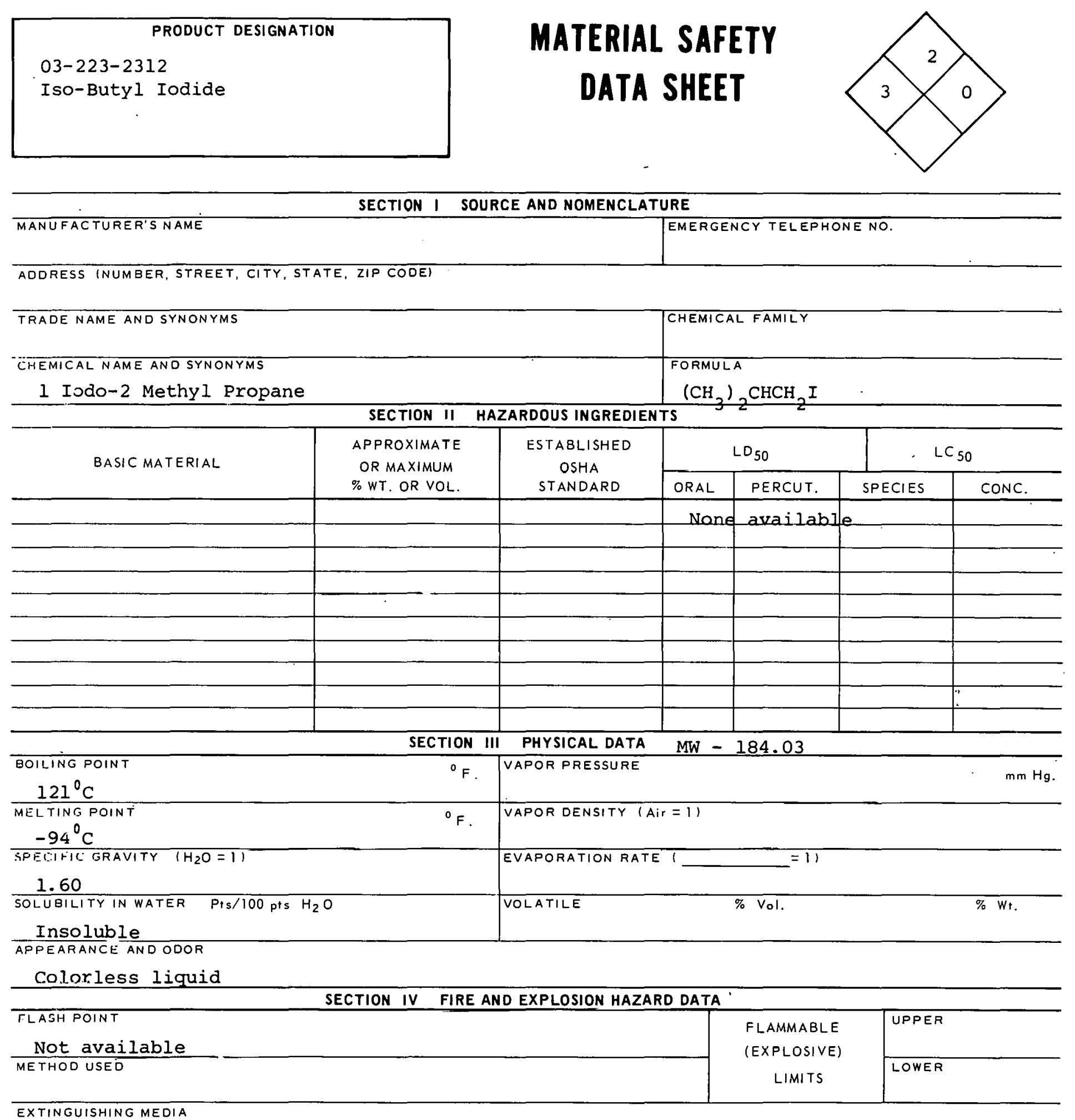

water, carbon dioxide, dry chemical

SPECIAL FIRE FIGHTING PROCEDURES

UNUSUAL FIRE AND EXPLOSION HAZARDS

When heated to decomposition point emits toxic fumes of iodates. 


\section{TOXIC LEVEL}

Not available

PRINCIPLE ROUTES OF ABSORBTION

Inhalation, ingestion, skin

REVELANT SYMPTOMS OF EXPOSURE Nausea, vomiting, diarrhea, dizziness, visual disturbances,

irritability, drowsiness, central nervous system depression.

Dermatitis

EMERGENCY AND'FIRST AID PROCEDURES Inhalation - rest; if swallowed - wash mouth, give emetic, get medical aid; wash skin \& eyes.

\section{SECTION VI REACTIVITY DATA}

CARCINOGENIC

SKIN AND EYE No

IRRITATION

sight EFFECTS OF CHRONIC EXPOSURE

CONDITIONS CONTRIBUTING TO HAZARDOUS POLYMERIZATION

IAGOMPATA.BI!.ITY (MATF.RIAL.S TO AVOID)

HAZAROOUS DECOMPOSITION PRODUCTS

SECTION VII SPILL OR LEAK PROCEDURES

STEPS TO BE TAKEN IN CASE MATERIAL IS RELEASED OR SPILLED

WASTE OISPOSAL METHOD

SECTION VIII SPECIAL PROTECTION INFORMATION

\begin{tabular}{l|l}
\hline VENTILATION REQUIREMENTS LOCAL EXHAUST & $\begin{array}{l}\text { PROTECTIVE EQUIPMENT (SPECIFY TYPESI EYE } \\
\text { GOGgleS }\end{array}$ \\
\hline MECHANICAL IGENERAL) & GLOVES \\
\hline SPECIAL & RESPbIRATOR \\
& With proper tilter
\end{tabular}

SECTION IX SPECIAL PRECAUTIONS

PRECAUTIONS TO BE TAKEN IN HANDLING AND STORAGE

OTHER PRECAUTIONS

SIGNATURE

DATE

$223-2312 B$ 


\begin{tabular}{|l} 
PRODUCT DESIGNATION \\
03-223-2332 \\
N-Butyl Iodide
\end{tabular}

\section{MATERIAL SAFETY \\ DATA SHEET}

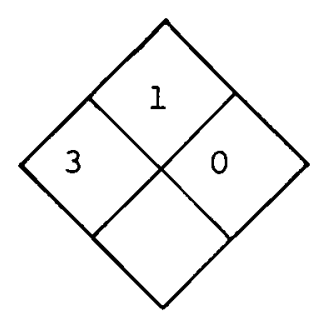

SECTION I SOURCE AND NOMENCLATURE

\section{MANUFACTURER'S NAME \\ TRADE NAME AND SYNONYMS \\ CHEMICAL NAME AND SYNONYMS \\ 1-Iodobutane}

ADDRESS (NUMBER, STREET, CITY, STATE, ZIP CODE)

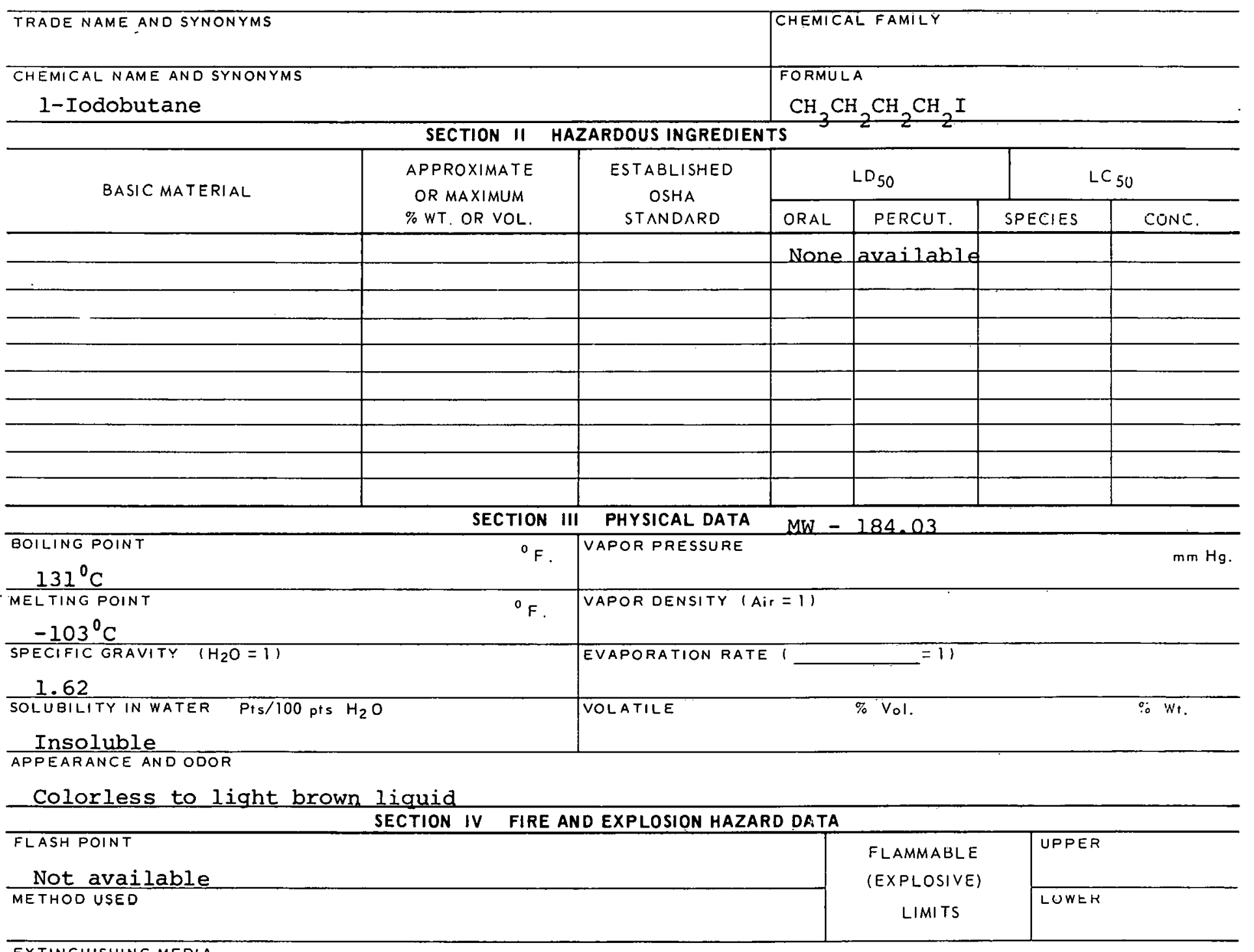

EXTINGUISHING MEDIA

Water, carbun diuxtde, ary chemical

SPECIAL FIRE FIGHTING PROCIFIIRES

--

UNUSUAL FIRE AND EXPLOSION HAZARDS

When heated to decomposition point emits toxic fumes of iodates. 


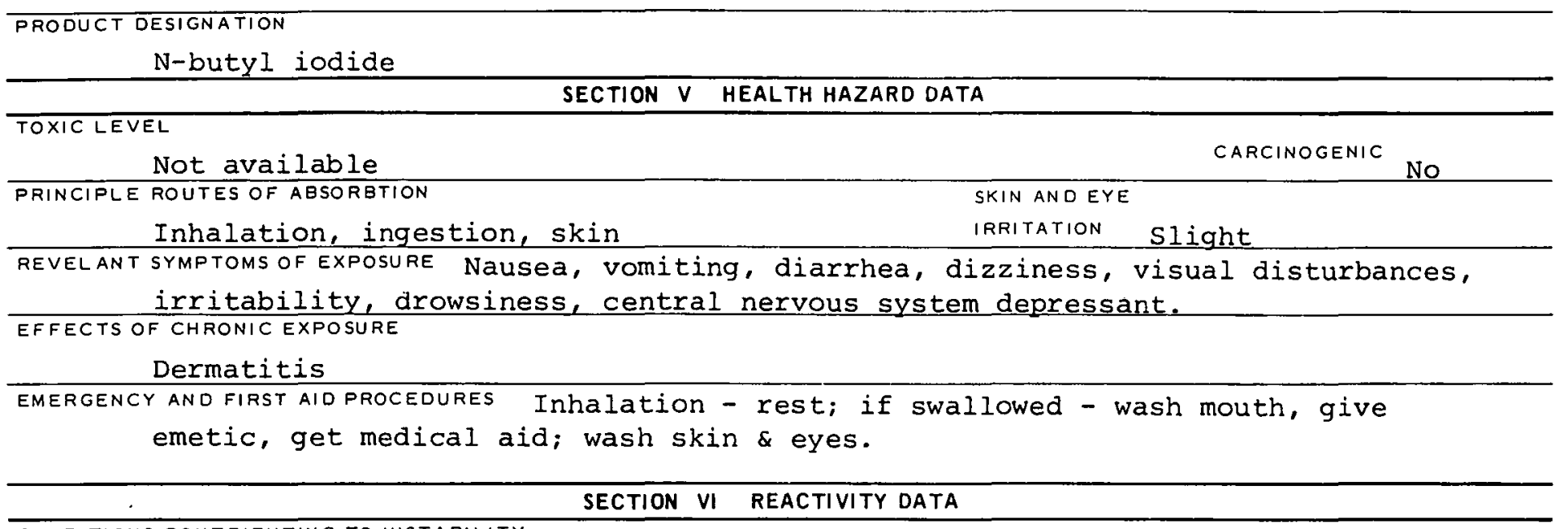

CONDITIONS CONTRIBUTING TO INSTABILITY

CONDITIONS CONTRIBUTING TO HAZARDOUS POLYMERIZATION

INCOMPATABILIIY (MA I ERIALS TS AVUIO)

HAZARDOUS DECOMPOSITION PROUUCTS

SECTION VII SPILL OR LEAK PROCEDURES

STEPS TO BE TAKEN IN CASE MA TERIAL IS RELEASEO OR SPILLED

WASTE DISPOSAL METHOD

\section{SECTION VIII SPECIAL PROTECTION INFORMATION}

\begin{tabular}{|c|c|}
\hline VENTILATION REQUIREMENTS LOCAL EXHAUST & $\begin{array}{l}\text { PROTECTIVE EQUIPMENT ISPECIFY TYPESI EYE } \\
\text { Safety goggles }\end{array}$ \\
\hline MECHANICAL (GENERAL) & $\begin{array}{l}\text { GLOVES } \\
\text { Rubber }\end{array}$ \\
\hline SPECIAL & $\begin{array}{l}\text { RESPIRATOR } \\
\text { Witli proper filter }\end{array}$ \\
\hline
\end{tabular}

SECTION IX SPECIAL PRECAUTIONS

PRECAUTIONS TO BE TAKEN IN HANDLING AND STORAGE

OTHER PRECAUTIOOHS 


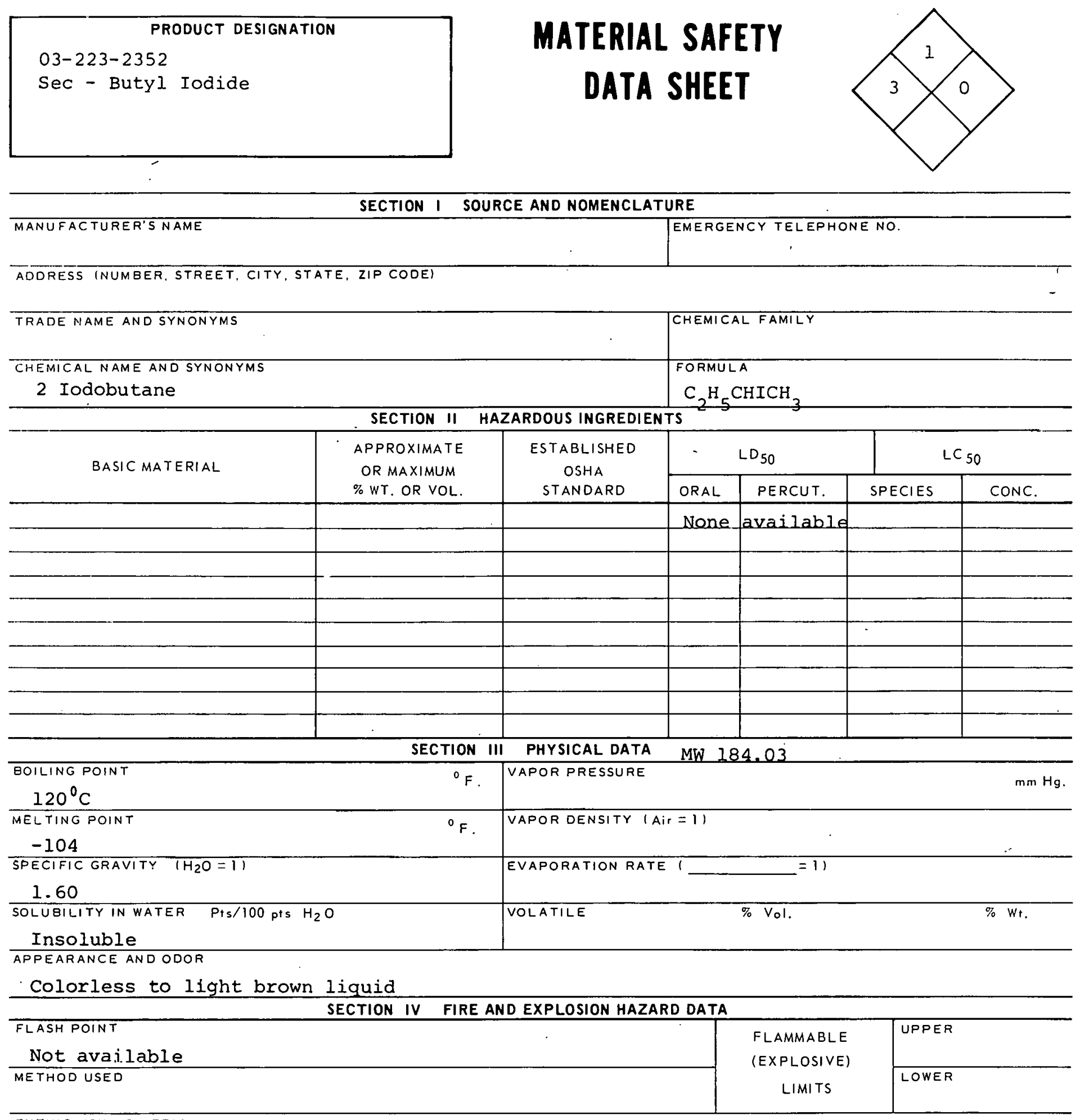

EXTINGUISHING MEOIA

Water, carbon dioxide, dry chemical

SPEGIAL FIRE FIGHTING PROCEOURES

$--$

UNUSUAL FIRE AND EXPLOSION.HAZARDS

When heated to decomposition point emits toxic fumes of iodates. 
Sec-butyl iodide

SECTION $V$ HEALTH HAZARD DATA

TOXICLEVEL

Not available

PRINCIPLE ROUTES OF ABSORBTION

Inhalation, ingestion, skin

CARCINOGENIC

No

SKIN AND EYE

IRRITATION Slight

REVELANT SYMPTOMS OF EXPOSURE Nausea, vomiting, diarrhea, dizziness, visual disturbances, irritability, drowsiness, central nervous system depressant

EFFECTS OF CHRONIC EXPOSURE

Dermatitis

EMERGENCY AND FIRST AID PROCEDURES

get medical aid; wash skin \& eyes.

SECTION VI REACTIVITY DATA

CONDITIONS CONTRIBUTING TO INSTABILITY

CONDITIONS CONTRIBUTING TO HAZARDOUS POLYMERIZATION

TINCOMTATADILITY MATERIAI TO AVRIOT

HAZARDOUS DECOMPOSITION PRODUCTS

SECTION VII SPILL OR LEAK PROCEDURES

STEPS TO BE TAKEN IN CASE MATERIAL IS RELEASED OR SPILLED

WASTE DISPOSAL METHOD

SECTION VIII SPECIAL PROTECTION INFORMATION

\begin{tabular}{|c|c|}
\hline VENTILATION REQUIREMENTS LOCAL EXHAUST & $\begin{array}{l}\text { PROTECTIVE EQUIPMENT (SPECIFY TYPES) EYE } \\
\text { GOggleS................... }\end{array}$ \\
\hline MECHANICAL (GENERAL) & $\begin{array}{l}\text { GLOVES } \\
\text { Rubber }\end{array}$ \\
\hline SPECIAL & $\begin{array}{l}\text { REEPIRATOR } \\
\text { WITh proper filter: }\end{array}$ \\
\hline
\end{tabular}

OTHER PROTECTIVE EQUIPMENT

SECTION IX SPECIAL PRECAUTIONS

PRECAUTIONS TO BE TAKEN IN HANDLING AND STORAGE

OTHER PRECAUTIONS

\begin{tabular}{l|l}
\hline SIGNATURE & DATE \\
\hline
\end{tabular}

\begin{tabular}{l|l}
\hline SIGNATURE & DATE \\
\hline
\end{tabular}

$223-2352 A$ 


\section{PRODUCT DESIGNATION}

03-223-2372

Tert-Butyl Iodide
MATERIAL SAFETY

DATA SHEET

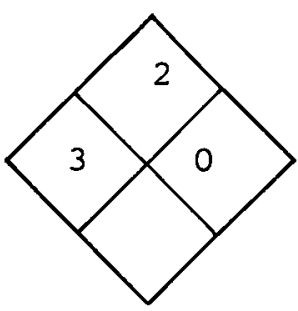

\section{SECTION I SOURCE AND NOMENCLATURE}

MANUFACTURER'S NAME

EMERGENCY TELEPHONE NO.

ADDRESS (NUMBER, STREET, CITY, STATE, ZIP CODE)

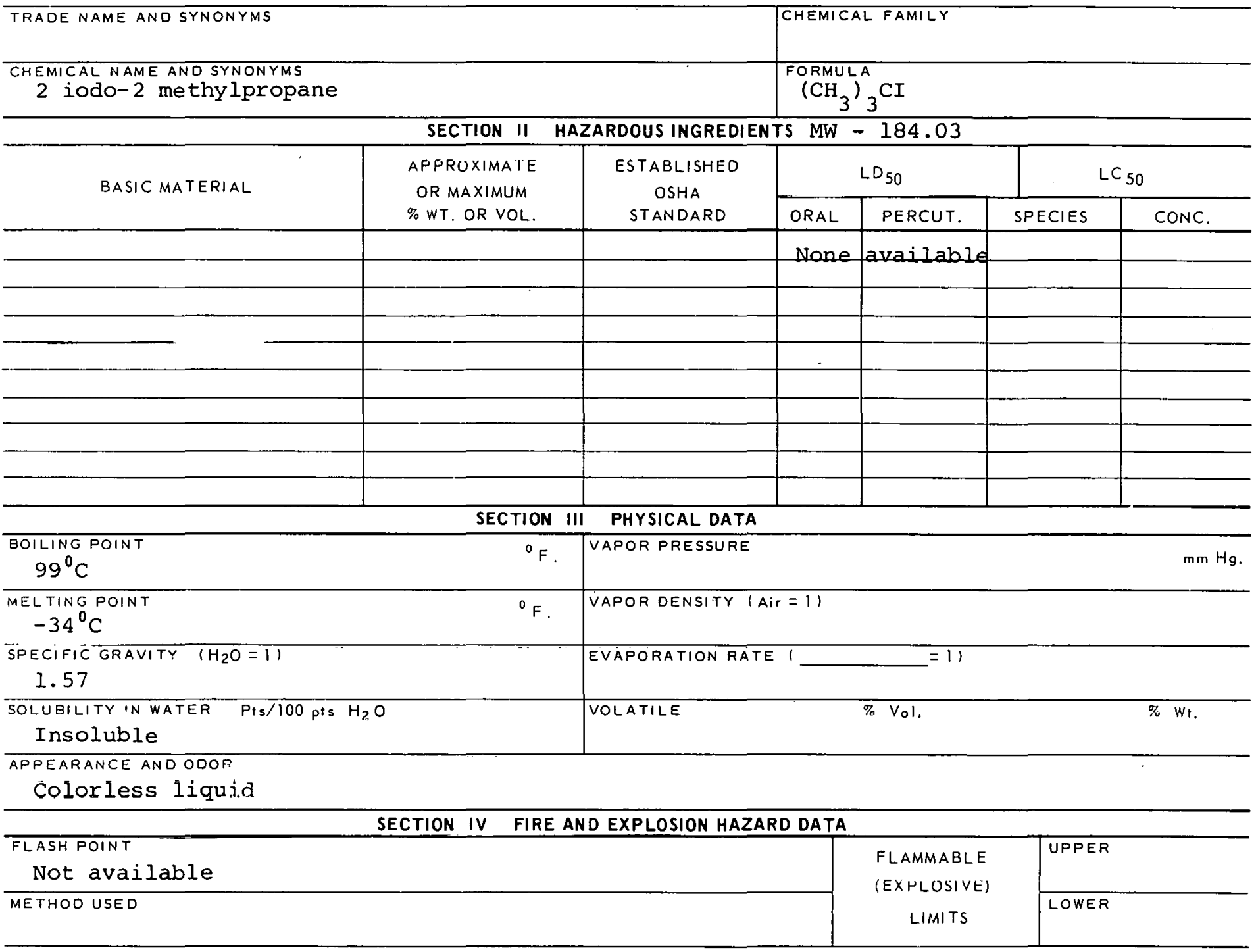

EXTINGUISHING MEDIA

Water, carbon dioxide, dry chemical

SPECIAL FIRE FIGHTING PROCEDURES

$-$

UNUSUAL FIRE AND EXPLOSION HAZARDS

When heated to decomposition point emits toxic fumes of iodates. 
TOXIC LEVEL

Not available

CARCINOGENIC

PRINCIPI,E ROUTES OF ABSORBTION

SKIN AND EYE

Inhalation, ingestion, skin

IRRITATION slight

REVELANT SYMPTOMS OF EXPOSURE Nausea, vomiting, diarrhea, dizziness, visual disturbances, irritability, drowsiness, central nervous system depression.

EFFECTS OF CHRONIC EXPOSURE

Dermatitis

EMERGENCY AND FIRST AID PROCEDURES Inhalation - rest; if swallowed - wash mouth, give emetic, get medical aid; wash from skin \& eyes.

\section{SECTION VI REACTIVITY DATA}

CONDITIONS CONTRIBUTING TO INSTABILITY

CONDITIONS CONTRIBUTING TO HAZARDOUS POLYMERIZATION

INTIMPATARII.ITY (MATERIALS TO AVOID)

HAZARDOUS DECOMPOSITION PRODUCTS

SECTION VII SPILL OR LEAK PROCEDURES

STEPS TO BE TAKEN IN CASE MATERIAL IS RELEASED OR SPILLED

WASTE DISPOSAL METHOD

SECTION VIII SPECIAL PROTECTION INFORMATION

\begin{tabular}{l|l}
\hline VENTILATION REQUIREMENTS LOCAL EXHAUST & $\begin{array}{c}\text { PROTECTIVE EQUIPMENT (SPECIFY TYPES) EYE } \\
\text { GOggles }\end{array}$ \\
\hline MECHANICAL IGENERAL) & $\begin{array}{c}\text { GLOVES } \\
\text { Rubber }\end{array}$ \\
\hline SPECIAL & $\begin{array}{c}\text { RESPIRATOR } \\
\text { with proper filter }\end{array}$ \\
\hline
\end{tabular}

OTHER PROTECTIVE EQUIPMENT

With proper filter

\section{SECTION IX SPECIAL PRECAUTIONS}

PRECAUTIONS TO BE TAKEN IN HANDLING AND STORAGE

OIHEK HKELAUTIUNS

SIGNATURE

DATE

$223-2372 B$ 
CAS - 000109740

PRODUCT DESIGNATION

03-225-6403

N-Butyronitrile
ET875000

MATERIAL SAFETY

DATA SHEET

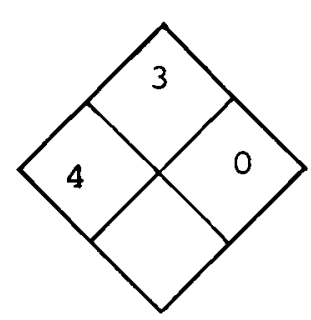

SECTION I SOURCE AND NOMENCLATURE

MANUFACTURER'S NAME

EMERGENCY TELEPHONE NO.

AODRESS (NUMBER, STREET, CITY, STATE, ZIP CODE)

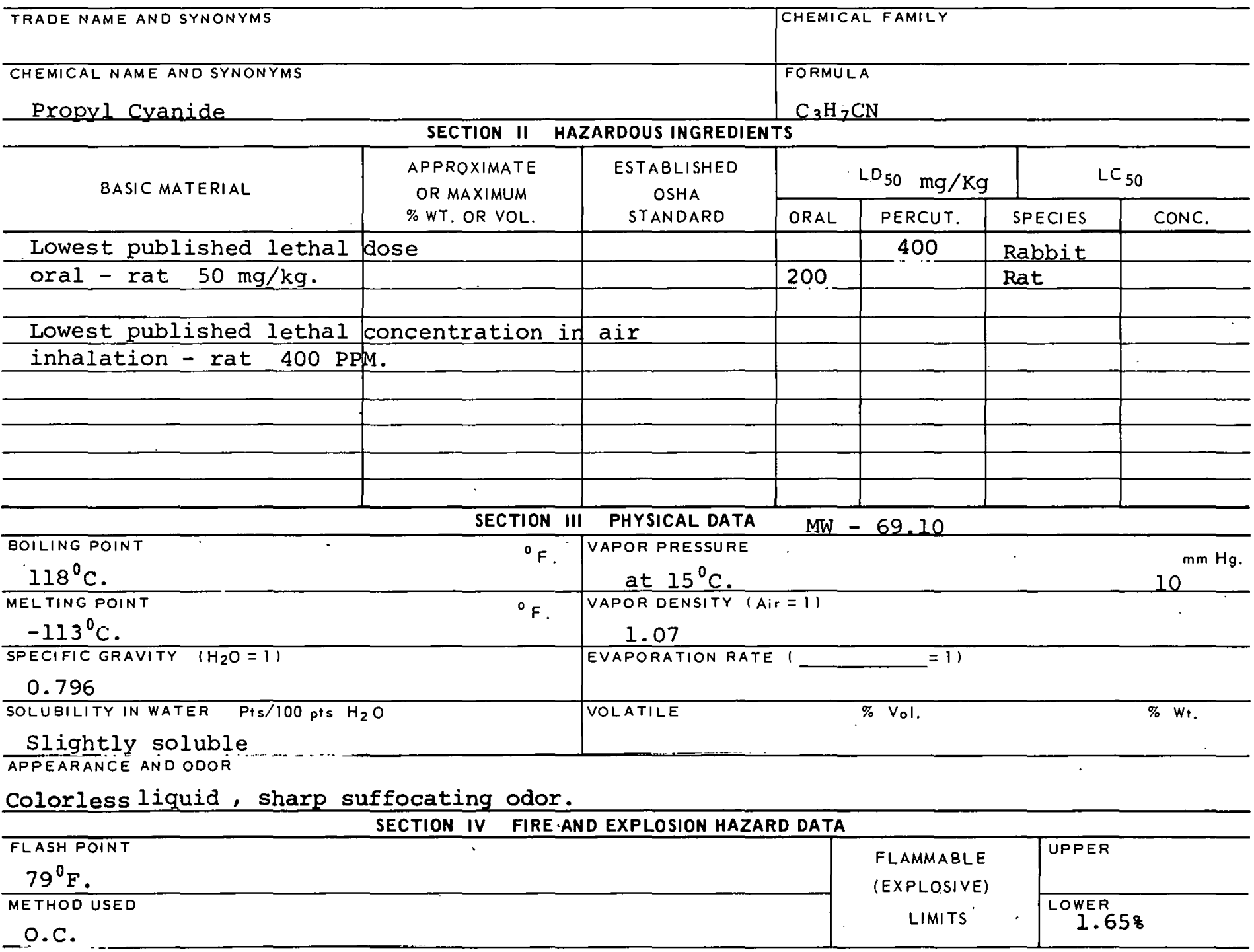

EXTINGUISHING MEDIA

Dry chemical, alcohol foam or carbon dioxide.

SPECIAL FIRE FIGHTING PROCEDURES

Protective clothing, self-contained breathing apparatus.

UNUSUAL FIRE AND EXPI_OSION HAZARDS 
TOXIC LEVEL

TWA-TLV-8 PPM, $22 \mathrm{mg} / \mathrm{C} . \mathrm{M}$. (NIOSH Value)

PRINCIPLE ROUTES OF ABSOFBTION

Inhalation, ingestion, skin

CARCINOGENIC

CARCINOGENIC NO

SKIN AND EYE

IRRITATION Moderate

REVELANT SYMPTOMS OF EXPOSURE Dizziness, rapid respiration, headache, drowsiness, drop in blood pressure and pulse, delayed symptoms.

EFFECTS OF CHRONIC EXPOSURE Eye irritation, loss of appetite, weight loss, mental deterioration.

EMERGENCY AND FIRST AID PROCEDURES If swallowed - inhalation of amyl nitrite, induce vomiting, or gastric lavage, wash skin \& eyes; inhalation, rest.

\section{SECTION VI REACTIVITY DATA}

CONDITIONS CONTRIBUTING TO INSTABILITY

CONDITIONS CONTRIBUTING TO HAZARDOUS POLYMERIZATION

INCOMPATABILITY (MATERIALS TO AVOID)

HAZAKUOUS DECOMPOSITION PRODUCTS

When heated to decomposition omits highly toxic cyanide fumes.

SECTION VII SPILL OR LEAK PROCEDURES

STEPS TO BE TAKEN IN CASE MATERIAL IS RELEASED OR SPILLED

Absorb with paper towels - evaporate in hood.

WASTE OISPOSAL METHOD Add excess alcoholic sodium hydroxide, after one hour, evaporate

alcohol, then add calcium hypochlorite SECTION VIII SPECIAL PROTECTION INFORMATION

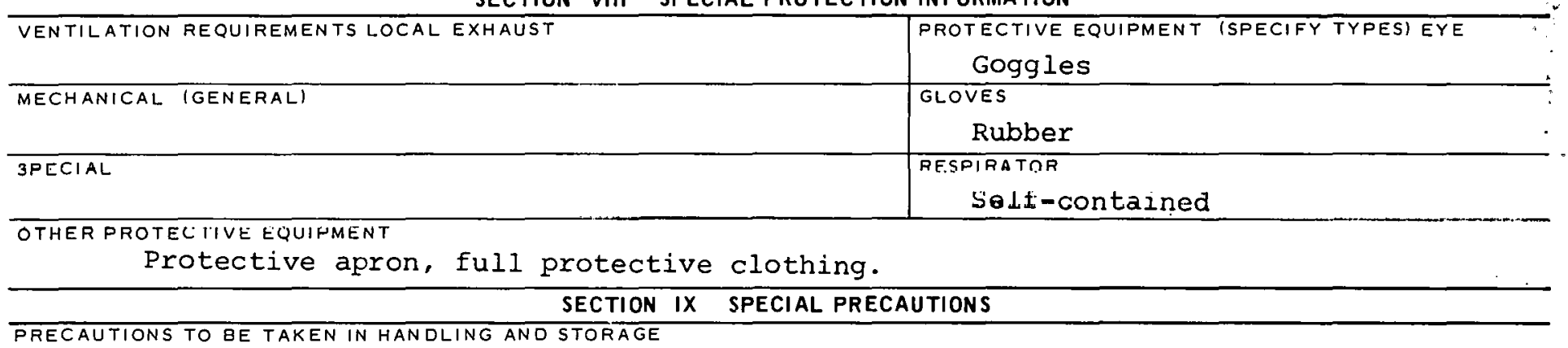

PRECAUTIONS TO BE TAKEN IN HANDLING AND STORAGE

OTHER PRECAUTIONS 


\begin{tabular}{|l|}
\multicolumn{1}{|c|}{ PRODUCT DESIGNATION } \\
Carbon Disulfide \\
$03-227-4004$
\end{tabular}

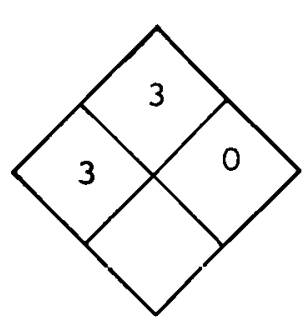

SECTION I SOURCE AND NOMENCLATURE

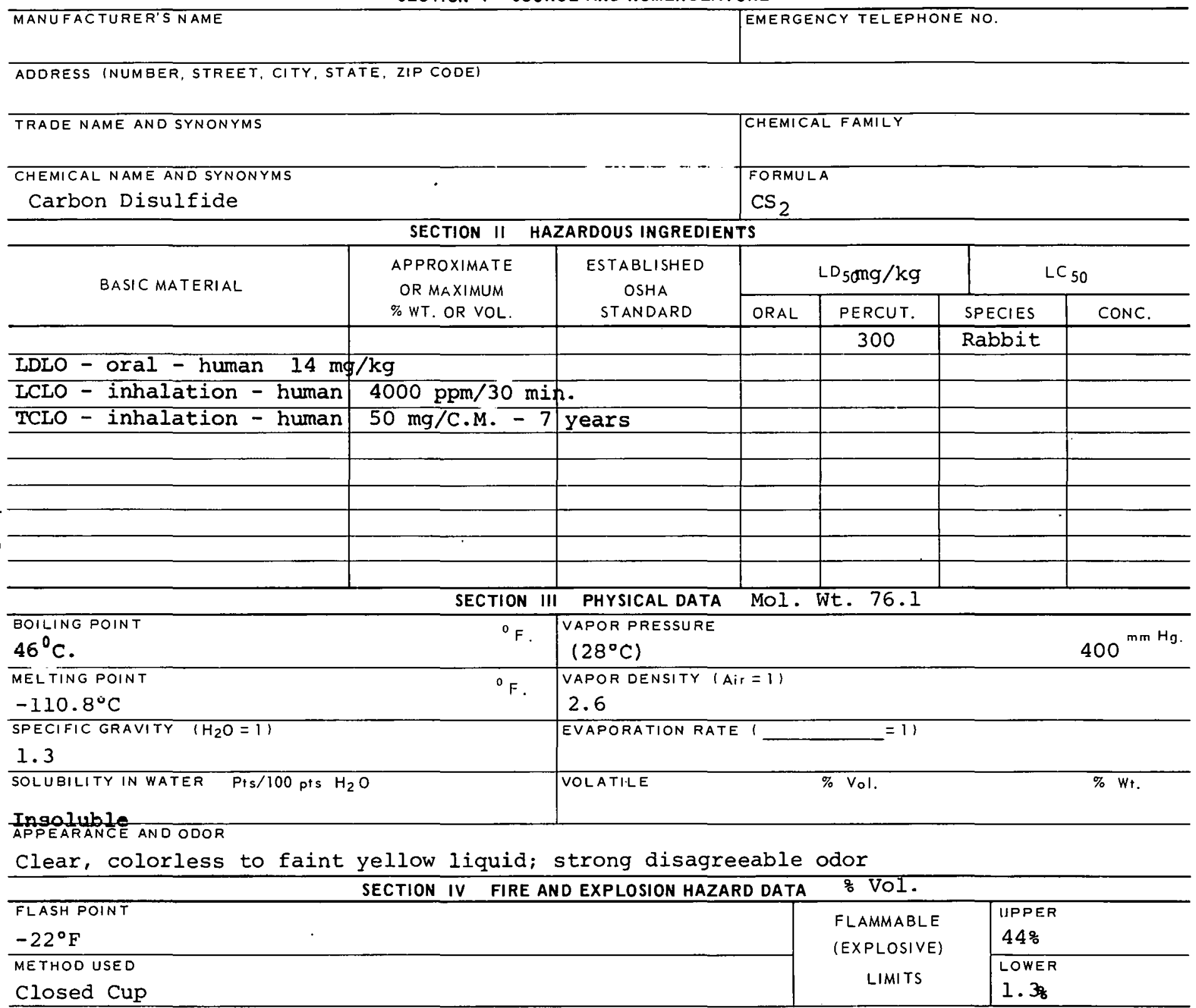

\section{EXTINGUISHING MEDIA}

Foam ineffective; use dry chemical, carbon dioxide, or other inert gas.

SPECIAL FIRE FIGHTING PROCEDURES

Wear self-contained breathing apparatus.

UNUSUAL FIRE ANDEXPLOSION HAZARDS

Gives off flammable vapors at low temperatures over a wide range; forms explosive mixtures in air; flash back. 
TOXIC LEVEL

TLV $20 \mathrm{ppm}, 60 \mathrm{mg} / \mathrm{C} . \mathrm{M}$.

PRINCIPLE ROUTES OF ABSORETION

Inhalation and prolonged skin contact

REVELANT SYMPTOMS OF EXPOSURE

Restlessness, blurred vision, nausea, vomiting, headache

EFFECTS OF CHRONIC EXPOSURE Bizarre sensations in extremities, sensory loss, muscular weakness,

memory loss, irritability, blurred vision, insomnia, mental depression, partial blindness, EMERGENCY AND FIRST AID PROCEDURES

parkinsonian tremor, heart, liver, kidney damage.

Remove from exposure ; apply artificial respiration; give amyl nitrite or sodium nitrite;

rest, keep warm; obtain medical attention.

SECTION VI REACTIVITY DATA

CONDITIONS CONTRIBUTING TO INSTABILITY

CONDITIONS CONTRIBUTING TO HAZARDOUS POL YMERIZATION

INCOMPAIAGILITY (MATERIALS TOAVOID) Azides, chlorine, chlorine monoxide, fluorine, nitric oxide, nitrogen dioxide, (permanganates and sulfuric acid), potassium and zinc

HAZARDOUS DECOMPOSITION PRODUCTS

SECTION VII SPILL OR LEAK PROCEDURES

STEPS TO BE TAKEN IN CASE MATERIAL IS RELEASED OR SPILLED

WASTE DIGPOSAL METHOD

Provide safe place for burning it. (Do not pour on ground.)

SECTION VIII SPECIAL PROTECTION INFORMATION

VENTILATION REQUIREMENTS LOCAL EXHAUST

MLCIIANICAL (CENCRAL)

SPECIAL

(20)

DIII

PROTECTIVE EQUIPMENT (SPECIFY TYPES) EYE
Goggles, face shield

GLOVES

Rubber

OTHER PROTECTIVE EQUIPMENT

Self-contained breathing apparatus. SECTION IX SPECIAL PRECAUTIONS

PRECAUTIONS TO BE TAKEN IN HANDLING AND STORAGE

Keep away from sources of ignition, good ventilation. Protect against lightening or static electricity:

OTHER PRECAUTIONS 
CAS : 000558134

\begin{tabular}{|c|}
\hline PRODUCT DESIGNATION \\
$03-227-4504$ \\
Carbon Tetrabromide \\
\hline
\end{tabular}

\section{FG47250 \\ MATERIAL SAFETY \\ DATA SHEET}

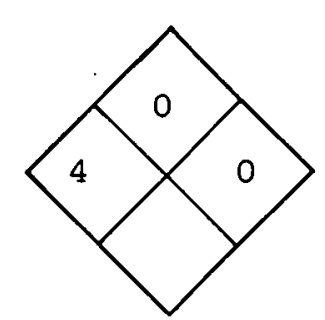

\section{SECTION I SOURCE AND NOMENCLATURE}

\begin{tabular}{|c|c|c|c|c|c|c|}
\hline \multicolumn{7}{|c|}{ SECTION I SOURCE AND NOMENCLATURE } \\
\hline \multicolumn{3}{|c|}{ MANUFACTURER'S NAME } & \multicolumn{4}{|c|}{ EMERGENCY TELEPHONE NO. } \\
\hline \multicolumn{7}{|c|}{ ADDRESS (NUMEER, STREET, CITY, STATE, ZIP CODE) } \\
\hline \multicolumn{3}{|l|}{ TRADE NAME AND SYNONYMS } & \multicolumn{4}{|c|}{ CHEMICAL FAMILY } \\
\hline \multicolumn{3}{|l|}{ CHEMICAL NAME AND SYNONYMS } & \multicolumn{4}{|c|}{ FORMULA } \\
\hline \multicolumn{3}{|l|}{ Tetrabromomethane } & \multicolumn{4}{|c|}{$\mathrm{C} \mathrm{Br}$} \\
\hline \multicolumn{7}{|c|}{ SECTION II HAZARDOUS INGREDIENTS } \\
\hline \multirow{2}{*}{ BASIC MATERIAL } & \multirow{2}{*}{$\begin{array}{l}\text { APPROXIMATE } \\
\text { OR MAXIMUM } \\
\% \text { WT. OR VOL. }\end{array}$} & \multirow{2}{*}{$\begin{array}{c}\text { ESTABLISHED } \\
\text { OSHA } \\
\text { STANDARD } \\
\end{array}$} & \multicolumn{2}{|c|}{$\mathrm{LD}_{50}$} & \multicolumn{2}{|c|}{$L_{C} C_{0}$} \\
\hline & & & ORAL & PERCUT. & SPECIES & CONC. \\
\hline \multicolumn{7}{|c|}{ Lowest Published letblaldose } \\
\hline \multirow{2}{*}{\multicolumn{7}{|c|}{$\begin{array}{l}\text { oral - rat } 1000 \mathrm{mg} / \mathrm{kg} . \\
\text { LD50 - subcutaneous - mpuse } 298 \mathrm{mg} / \mathrm{kg}\end{array}$}} \\
\hline & & & & & & 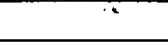 \\
\hline & & & & & & $\ldots$ \\
\hline & & & & & 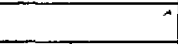 & \\
\hline & & & & & $\because$ & $\therefore$ \\
\hline & & & & & - & 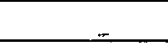 \\
\hline & & & & & & $\dot{3}$ \\
\hline \multicolumn{7}{|c|}{ SECTION III PHYSICAL DATA $\quad$ MW -331.67} \\
\hline $\begin{array}{l}\text { BOILING POINT } \\
190^{\circ} \mathrm{C}\end{array}$ & ${ }^{\circ} \mathrm{F}$ & VAPOR PRESSURE & & & 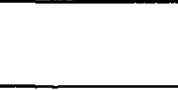 & mm' $\mathrm{Hg}$. \\
\hline $\begin{array}{l}\text { MEL TING POINT } \\
\alpha-48{ }^{\circ} \mathrm{C}, \beta 90^{\circ} \mathrm{C}\end{array}$ & ${ }^{0} \mathrm{~F}$ & $\begin{array}{l}\text { VAPOR DENSITY IA } \\
\quad 11.4\end{array}$ & $=11$ & & $\therefore$ & . \\
\hline $\begin{array}{l}\text { SPECIFIC GRAVITY }\left(\mathrm{H}_{2} \mathrm{O}=1\right) \\
\quad 3.42\end{array}$ & & EVAPORATION RAT & 1 & $=11$ & & (2) \\
\hline $\begin{array}{c}\text { SOLUBILITY IN WATER Pts/100 pts } \mathrm{H}_{2} \\
0.024\end{array}$ & & VOLATILE & 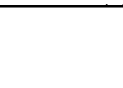 & $\%$ vol. & & $\begin{array}{ll}\% & \text { Wt. }\end{array}$ \\
\hline \multicolumn{7}{|l|}{ APPEARANCE AND ODOR } \\
\hline \multicolumn{7}{|l|}{ Colorless solid } \\
\hline \multicolumn{7}{|c|}{ SECTION IV FIRE AND EXPLOSION HAZARD DATA } \\
\hline \multicolumn{4}{|l|}{$\begin{array}{l}\text { FLASH POINT } \\
\text { Nonflammable }\end{array}$} & \multirow{2}{*}{$\begin{array}{c}\text { FLAMIIABLE } \\
\text { (EXPLOSIVE) } \\
\text { LIMITS }\end{array}$} & \multicolumn{2}{|l|}{ UPPER } \\
\hline \multicolumn{4}{|l|}{ METHOD USED } & & \multicolumn{2}{|l|}{ LOWER } \\
\hline EXTINGUISHING MEDIA & & & & & & \\
\hline $\begin{array}{l}\text { SPECIAL FIRE FIGHTING PROCEDURES } \\
\text { Self contained respirator }\end{array}$ & if heated in a & fire. & . & & & \\
\hline
\end{tabular}

UNUSUAL FIRE AND EXPLOSION HAZARDS 
TOXIC LEVEL

TLV 0.1 PPM, $1.4 \mathrm{mg} / \mathrm{C} . \mathrm{M}$.

CARCINOGENIC

PRINCIFLE ROUTES OF ABSORBTION SKIN AND EYE

Inhalation, ingestion, skin IRRITATION Severe

REVELANT SYMPTOMS OF EXPOSURE EYe and upper respiratory irritation, lung, liver \& kidney damage, damage to cornea.

EFFECTS OF CHRONIC EXPOSURE

Liver damage

EMERGENCY AND FIRST AID PROCEDURES Inhalation \& rest, get medical aid; if swallowed -

give emetic, get medical aid; wash from eyes and skin.

\section{SECTION VI REACTIVITY DATA}

CONDITIONS CONTRIBUTING TO INSTABILITY

CONDITIONS CONTRIBUTING TO HAZARDOUS POLYMERIZATION

INCOMFATADILITY (MATERIALS TO AVOID)

HAZARDOUS DECOMPOSITION PRODUCTS

SECTION VII SPILL OR LEAK PROCEDURES

STEPS TO BE TAKEN IN CASE MATERIAL IS RELEASED OR SPILLED

Collect on paper, evaporate on dish in hood.

WASTE DISFOSAL METHOD

SECTION VIII SPECIAL PROTECTION INFORMATION

\begin{tabular}{|c|c|}
\hline VENTILATION REQUIREMENTS LOCAL EXHAUST & $\begin{array}{l}\text { PROTECTIVE EQUIPMENT (SPECIFY TYPES) EYE } \\
\text { Safety goggles }\end{array}$ \\
\hline MECHANICAL (GENFRAI) & $\begin{array}{l}\text { GLOVES } \\
\text { Rubbcr }\end{array}$ \\
\hline SPECIAL & $\begin{array}{l}\text { RESPIRATOR } \\
\text { SPlf rnntained }\end{array}$ \\
\hline
\end{tabular}

OTHER PROTECTIVE EQUIPMENT

SECTION IX SPECIAL PRECAUTIONS

PRECAUTIONS TO BE TAKEN IN HANDLING AND STORAGE

OTHER PRECAUTIONS

SIGNATURE 
Carbon Tetrachloride

03-227-4604

$03-227-4624$

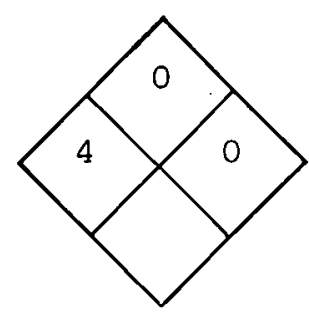

SECTION I SOURCE AND NOMENCLATURE

\begin{tabular}{l|l}
\hline MANUFACTURER'S NAME & EMERGENCY TELEPHONE NO. \\
\hline ADDRESS (NUMBER, STREET, CITY, STATE, ZIP CODE)
\end{tabular}

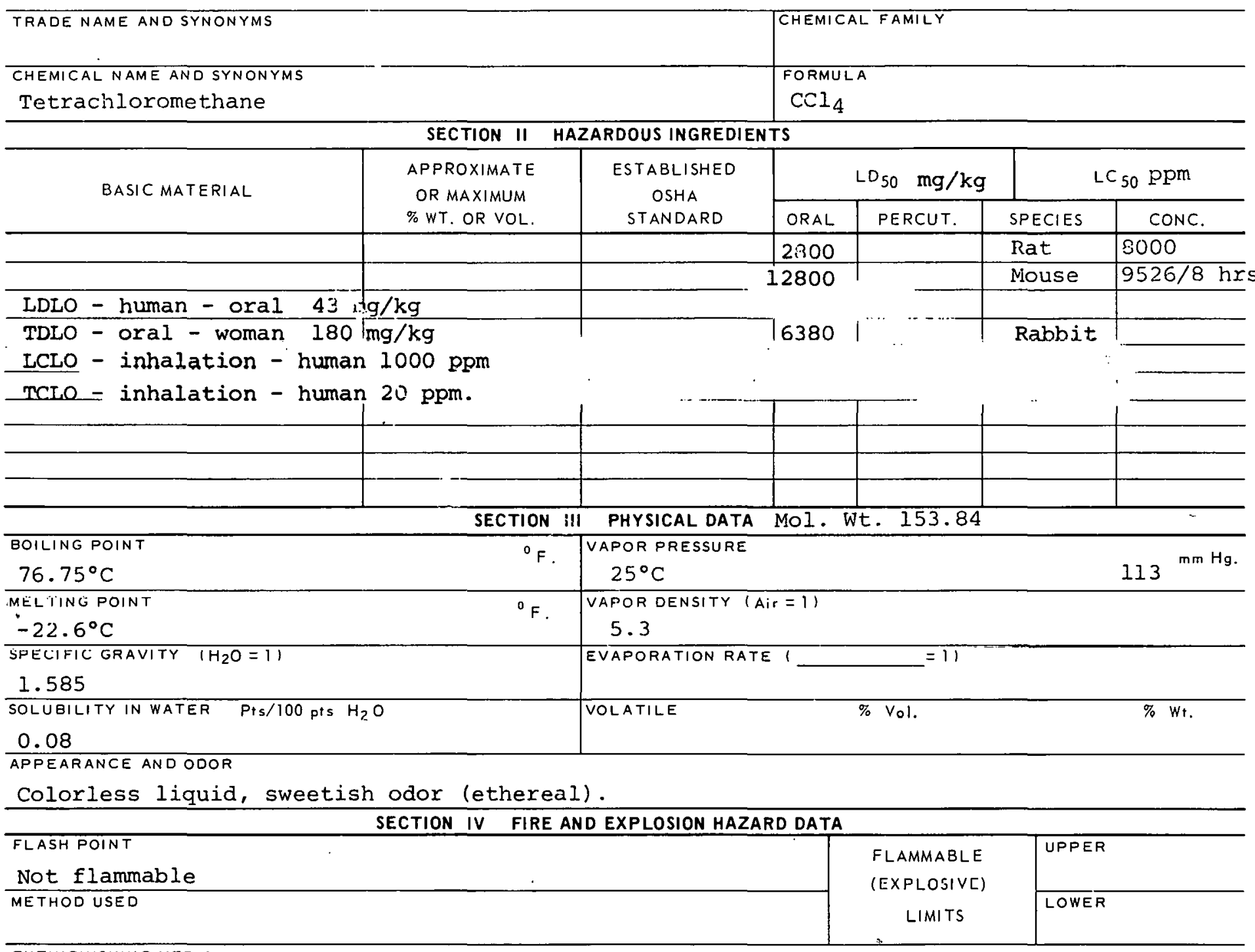

EXTINGUISHING MEDIA

Water

SPECIAL FIRE FIGHTING PROCEDURES

Wear self-contained breathing apparatus.

UNUSUAL FIRE AND EXPLOSION HAZARDS

Emits toxic fumes. 


\section{SECTION $V$ HEALTH HAZARD DATA}

TOXIC LEVEL

TLV $10 \mathrm{ppm}, 25 \mathrm{mg} / \mathrm{C} . \mathrm{M}$.

PRINCIPLE ROUTES OF ABSORETION

Inhalation of vapor, ingestion, sometimes skin absorptiBRITATION Skin

REVELANT SYMPTOMS OF EXPOSURE Abdominal pain, nausea, vomiting, dizziness, loss of blood pressure, respiratory slowing, and fall of blood pressure. Delayed symptoms of liver or kidney EFFECTS OF CHRONIC EXPOSURE damage.

Liver, kidney damage, anemia, loss of appetite and weight, dermatitis, loss of memory, EMERGENCY AND FIRST AID PROCEDURES anOreXia.

Remove from exposure, rest, keep warm; if necessary, qive artificial respiration. Do not give stimulants. Obtain medical aid.

SECTION VI REACTIVITY DATA

CONDITIONS CONTRIBUTING TO INSTABILITY

CONDITIONS CONTRIBUTING TO HAZARDOUS POLYMERIZATION

INCOMPATABILITY (MATERIALS TO AVOIDI Allyl alcohol, aluminum, barium, beryllium, disilane,

ethylene, tluorlne, alkalies, magnesium, LOX, plutonium, tetrasilane, trisilane, uranium, HAZARDOUS DECOMPOSITION PRODUCTS waX, zirCONium.

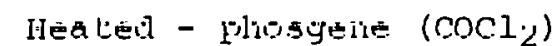

SECTION VII SPILL OR LEAK PROCEDURES

STEPS TO BE TAKEN IN CASE MATERIAL IS RELEASED OR SPILLED

WASTF MIGPRSAI MFTHOR

SECTION VIII SPECIAL PROTECTION INFORMATION

\begin{tabular}{l|l}
\hline VENTILATION REQUIREMENTS LOCAL EXHAUST & PROTECTIVE EQUIPMENT (SPECIFY TYPES) EYE
\end{tabular} Goggles

MESHANICAL. IGENERAL) GLOVES

Polyrinyl gloves

SPECIAL.

RESPIRATOR

Organic tilter

OTHER PROTECTIVE EQUIPMENT

Self-contained breathing apparatus

SECTION IX SPECIAL PRECAUTIONS

PRECAUTIONS TO BE TAKEN IN HANDLING AND STORAGE

Protect from physical damage. Store in cool, dry, well-ventilated location away from any area where the fire hazard may be acute.

OTHER PRECAUTIONS

SIGNATURE.

DATE

$227-4604 B$ 
UXI0500

PRODUCT DESIGNATION
$03-227-5004$
Catechol

\section{MATERIAL SAFETY DATA SHEET}

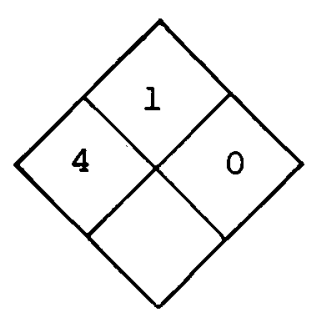

SECTION I SOURCE AND NOMENCLATURE

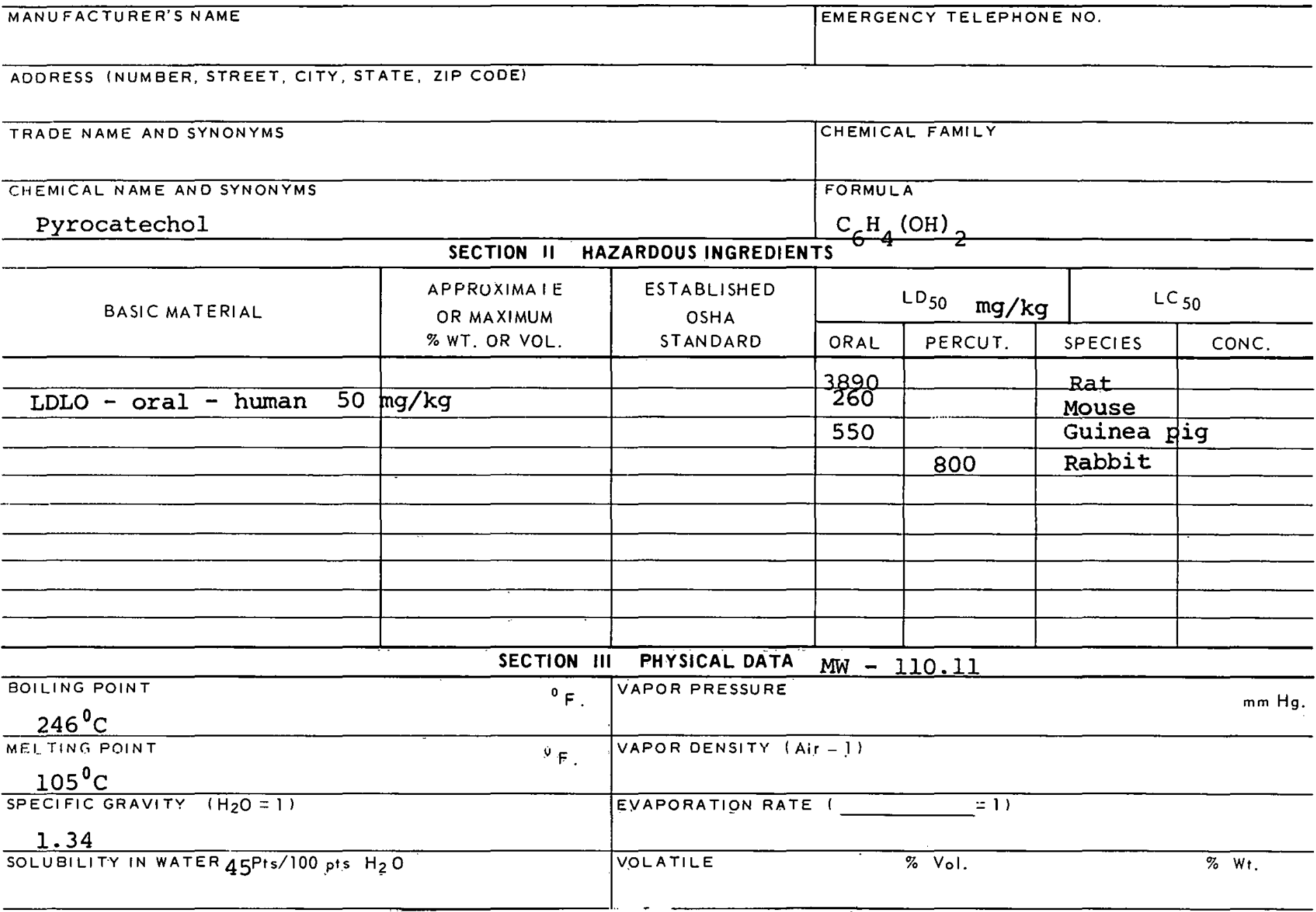

APPEARANCE AND ODOR

Colorless crystal salt

\begin{tabular}{|c|c|c|c|c|}
\hline & SECTION IV & FIRE AND EXPLOSION HAZARD DATA & & \\
\hline $\begin{array}{c}\text { FLASH POINT } \\
261^{\circ} \mathrm{F}\end{array}$ & & & \multirow{2}{*}{$\begin{array}{c}\text { FLAMMABLE } \\
\text { (EXPLOSIVE) } \\
\text { LIMITS }\end{array}$} & UPPER \\
\hline $\begin{array}{l}\text { METHOD USED } \\
\text { CC }\end{array}$ & & & & LOWER \\
\hline
\end{tabular}

EXTINGUISHING MEDIA

Water, carbon aloxiae, dry chemical

SPECIAL FIRE FIGHTINC FROCEOURES

UNUSUAL FIRE AND EXPLOSION HAZARDS 


\section{TOXIC LEVEL}

TLV $5 \mathrm{ppm}, 20 \mathrm{mg} / \mathrm{C} . \mathrm{M}$.

PRINCIPLE ROUTES OF ABSORETION

Skin, ingestion

REVELANT SYMPTOMS OF EXPOSURE

Central nervous
CARCINOGENIC No

SKIN AND EYE

IRRITATION VErY severe

\section{EFFECTS OF CHRONIC EXPOSURE}

Blood damage, dermatitis

EMERGENCY AND FIRST AID PROCEOURES Wash eyes and skin; if swallowed - drink water, then

2 tsp. epsom salts in water; get medical aid.

\section{SECTION VI REACTIVITY DATA}

CONDITIONS CONTRIBUTING TO INSTABILITY

CONDITIONS CONTRIBU TING TO HAZARDOUS POLYMERIZATION

INCOMPATABILITY (MATEREIALS TO AVOIOI

HAZARDOUS DECOMPOSITION PRODUCTS

SECTION VII SPILL OR LEAK PROCEDURES

STEPS TO BE TAKEN IN CASE MATERIAL IS RELEASED OR SPILLED

WASTE DISPOSAL METHOD

SECTION VIII SPECIAL PROTECTION INFORMATION

\begin{tabular}{l|l}
\hline \multicolumn{2}{c}{ SECTION VIII SPECIAL PROTECTION INFORMATION } \\
\hline $\begin{array}{l}\text { VENTILATION REQUIREMENTS LOCAL EXHAUST } \\
\text { MECHANICAL IGENERALI }\end{array}$ & $\begin{array}{c}\text { PROTECTIVE EQUIPMENT (SPECIFY TYPESI EYE } \\
\text { Safety glasSes }\end{array}$ \\
\hline SPECIAL & $\begin{array}{c}\text { GLOVES } \\
\text { Rubber }\end{array}$ \\
\hline
\end{tabular}

UTHERPROTECTIVE EQUIFMENT

\section{SECTION IX SPECIAL PRECAUTIONS}

PRECAUTIONS TO BE TAKEN IN HANDLING AND STORAGE 
CAS : 000118752

\begin{tabular}{|l|}
\hline \multicolumn{1}{|c|}{ PRODUCT DESIGNATION } \\
03-227-7504 \\
Chloranil \\
\end{tabular}

\section{MATERIAL SAFETY DATA SHEET}

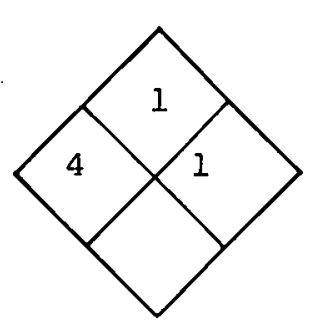

SECTION I SOURCE AND NOMENCLATURE

MANUFACTURER'S NAME \begin{tabular}{l|l}
- & EMERGENCY TELEPHONE NO.
\end{tabular}

ADORESS (NUMBER, STREET, CITY, STATE, ZIP CODE)

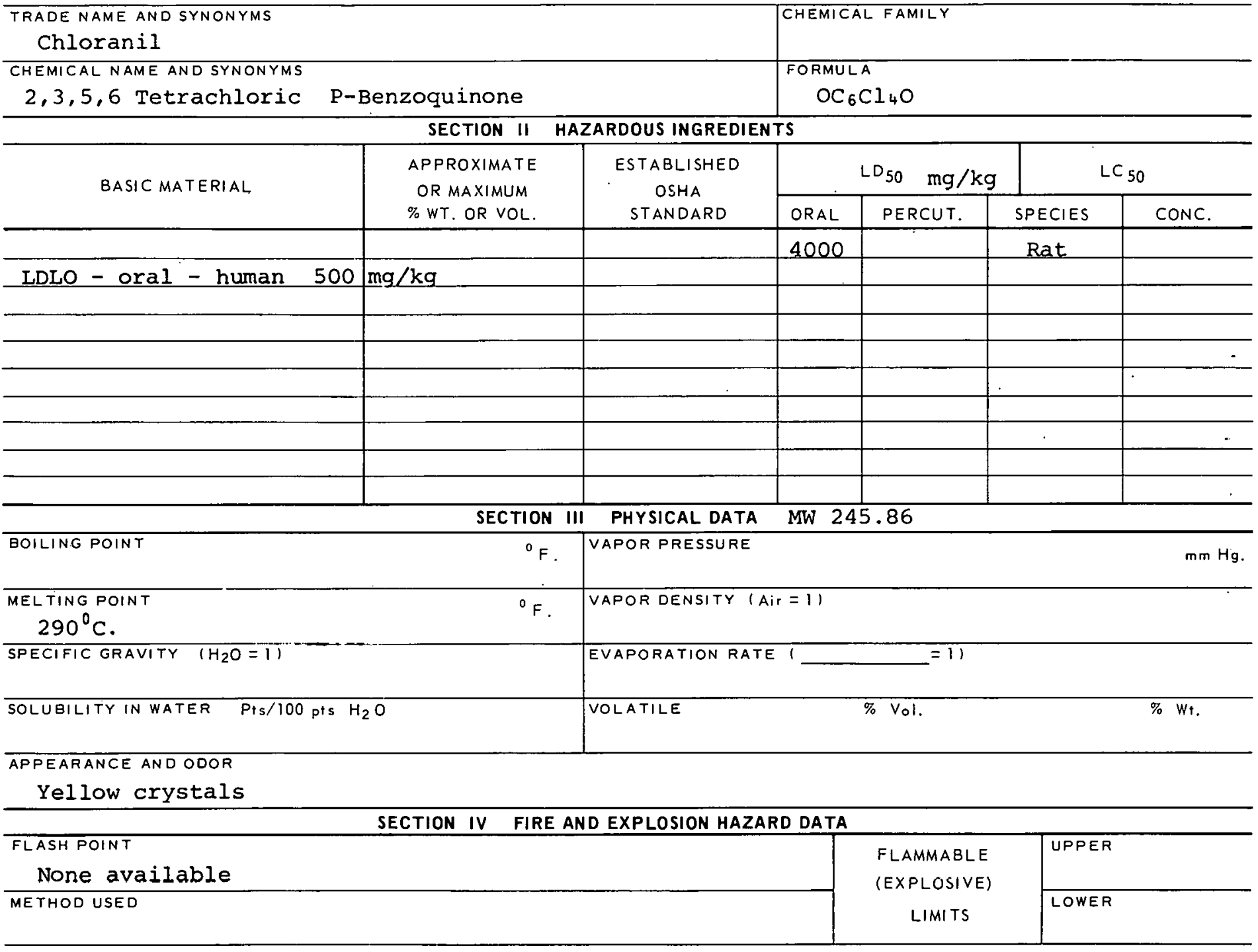

EXTINGUISHING MEDIA

Dry chemical, carbon dioxide, alcohol foam. SPECIAL FIRE FIGHTING PROCEDURES

UNUSUAL FIRE AND EXPLOSION HAZAROS 


\section{TOXIC LEVEL \\ None available}

Inhalation, ingestion
PRINCIPLE ROUTES OF ABSORBTION

CARCINOGENIC

Yes

SKIN AND EYE

IRRITATION SEvere

REVELANT SYMPTOMS OF EXPOSURE Irritation to skin, eye and mucous membrane,

headache, dizziness.

EFFECTS OF CHRONIC EXPOSURE

May cause cancer of liver or kidneys.

EMERGENCY AND FIRST AIDPROCEDURES Wash skin \& eyes; if swallowed - gastric lavage, get

medical aid; inhalation - rest.

SECTION VI REACTIVITY DATA

CONDITIONS CONTRIBUTING TO INSTABILITY

CONDITIONS CONTRIBUTING TO HAZARDOUS POLYMERIZATION

TNCUMPATABILITY (MATERIALS TO AVOID)

HAZARDOUS DECOMPOSITION PRODUETS

SECTION VII SPILL OR LEAK PROCEDURES

STEPS TO BE TAKEN IN CASE MATERIAL IS RELEASED OR SPILLED

WASTE DISPOSAL METHOD

SECTION VIII SPECIAL PROTECTION INFORMATION

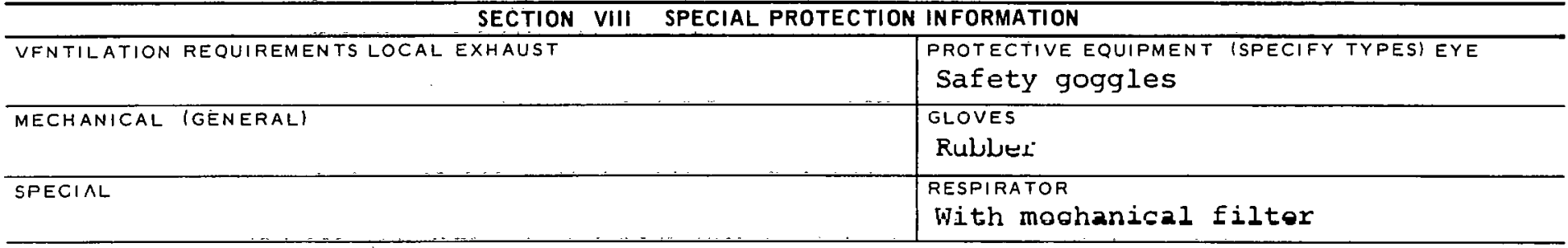

OTHER PROTECTIVE EQUIPMENT

\section{SECTION IX SPECIAL PRECAUTIONS}

PRECAUTIONS TO BE TAKEN IN HANDLING AND STORAGE

OTHER PRECIAU IIONS

SIGNATURE

DATE

$227-7504 B$ 
CAS : 000078955

PRODUCT DESIGNATION

03-228-0105

Chloroacetone
UCO 7000

MATERIAL SAFETY

DATA SHEET

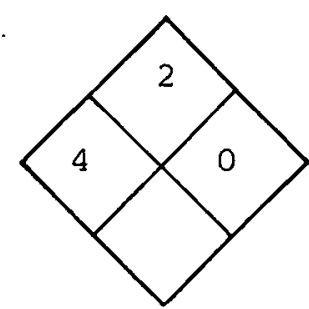

SECTION I SOURCE AND NOMENCLATURE

\begin{tabular}{l} 
MANUFACTURER'S NAME \\
AODRESS INUMBER, STREET, CITY \\
\hline TRADE NAME AND SYNONYMS \\
CHEMICAL NAME AND SYNONYMS \\
1 ChIOYO 2 PrOpanONE
\end{tabular}

SECTION II HAZARDOUS INGREDIENTS

BASIC MATERIAL

\begin{tabular}{l|l} 
APPROXIMATE & ESTABLISHED
\end{tabular}

OR MAXIMUM $\quad$ OSHA

$\%$ WT. OR VOL.

STANDARD

Lowest published lethal concentration in air Inhalation - human - $60 \$$ PPM/l0 minutes.

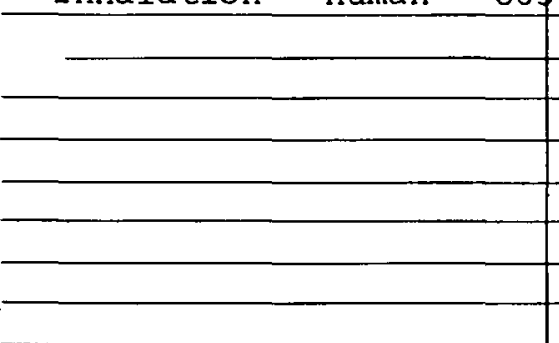

BOILING POINT

$121^{\circ} \mathrm{C}$.

MELTING POINT

$-44.5^{\circ}$

SPECIFIC GRAVITY $\left(\mathrm{H}_{2} \mathrm{O}=1\right)$

1.16

SOLUBILITY IN WATER Pts/100 pts $\mathrm{H}_{2} \mathrm{O}$

$\infty$

APPEARANCE AND ODOR

Colorless liquid, pungent odor.

SECTION IV FIRE AND EXPLOSION HAZARD DATA

FLASH POINT

None available

METHOD USED

SECTION III PHYSICAL DATA MW - 95.53

${ }^{\circ} \mathrm{F}$. VAPOR PRESSURE

ORMULA

$\mathrm{CH}_{3} \mathrm{COCH}_{2} \mathrm{Cl}$

\begin{tabular}{|l|l|l|l}
\hline \multicolumn{2}{|c|}{ LD $_{50}$} & \multicolumn{2}{c}{ LC SO $^{2}$} \\
\hline ORAL & PERCUT. & SPECIES & CONC. \\
\hline & & & \\
\hline & & & \\
\hline & & & \\
\hline & & & \\
\hline & & & \\
\hline & & & \\
\hline MW -95.53 & & \\
\hline
\end{tabular}

EXTINGUISHING MEDIA

${ }^{\circ} \mathrm{F} . \quad$ VAPOR DENSITY (Air $=11$

EVAPORATION RATE

VOLATILE

\% Vol.

EXTINGUISHING MEDIA

Alcohol foam

Water may be ineffective.

SPECIAL FIRE FIGHTING PROCEDURES

UNUSUAL FIRE AND FXPI..OSION HATARDS

Emits phosgene on heating to decomposition point. 


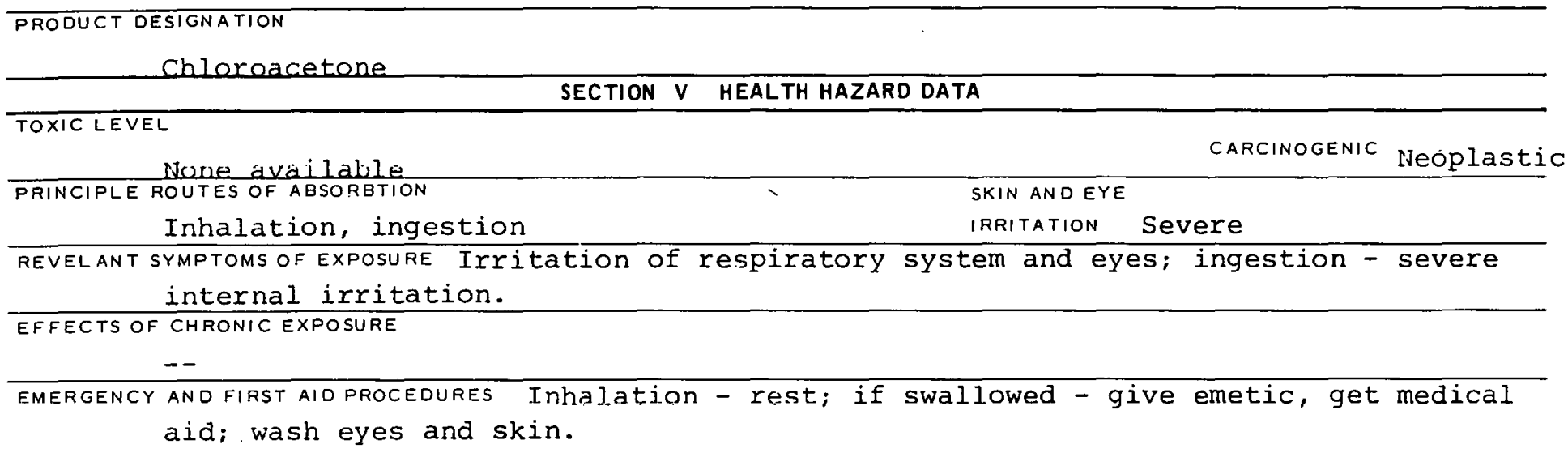

\section{SECTION VI REACTIVITY DATA}

CONDITIONS CONTRIBUTING TO INSTABILITY

CONDITIONS CONTRIBUTING TO HAZARDOUS POLYMERIZATION

INCOMPA I ABILI IY (MA I ERIALS IU AVUIOU)

MATARDOUS DECOMPOSITION PRODUETS

SECTION VII SPILL OR LEAK PROCEDURES

STEPS TO BE TAKEN IN CASE MATERIAL IS RELEASEO OR SPILLED

WASTE DISPOSAL METHOD

SECTION VIII SPECIAL PROTECTION INFORMATION

\begin{tabular}{l|l}
\hline VENTILATION REQUIREMENTS LOCAL EXHAUST & $\begin{array}{c}\text { PROTECTIVE EQUIPMENT (SPECIFY TYPES) EYE } \\
\text { GOGglPS }\end{array}$ \\
\hline MECHANICAL (GENERAL) & $\begin{array}{c}\text { GLOVES } \\
\text { Rưbber }\end{array}$ \\
\hline GPFCIAI & $\begin{array}{c}\text { RESPIRATOR } \\
\text { Self-contained }\end{array}$ \\
\hline
\end{tabular}

OTHER PROTECTIVE EQUIPMENT

SECTION IX SPECIAL PRECAUTIONS

PRECAUTIONS TO $8 E$ TAKEN IN HANDLING AND STORAGE 


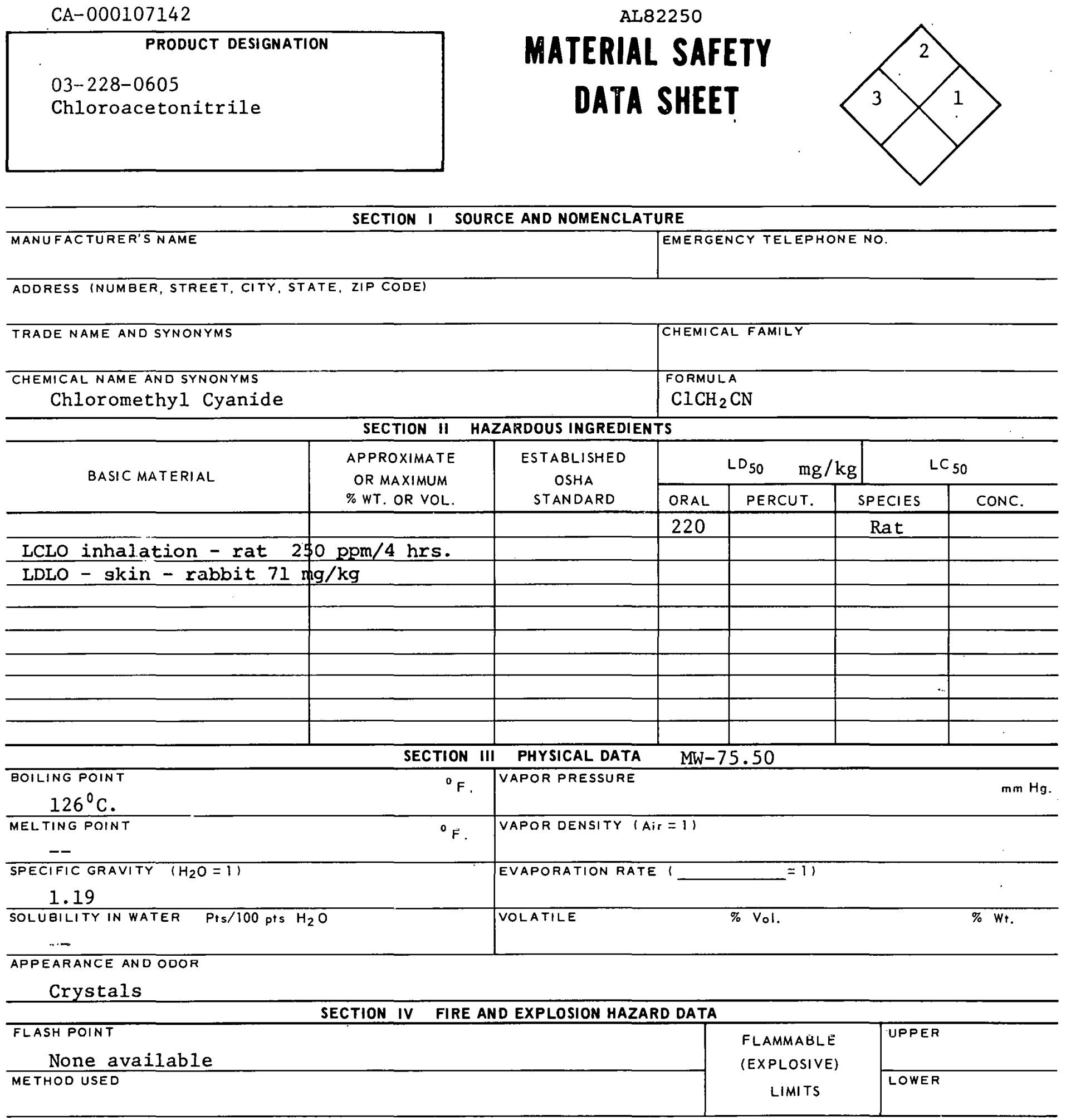

EXTINGUISHING MEDIA

Alcohol foam, dry chemical, carbon dioxide, watcr may bc incffcctive. SPECIAL FIRE FIGHTING PROCEOURES

Wear self,-contained breathing apparatus. 


\section{TOXIC LEVEL \\ None available}

PRINCIPLE ROUTES OF ABSORBTION Inhalation, ingestion, skin
CARCINOGENIC

No

SKIN AND EYE

IRRITATION Mild to moderate

REVELANT SYMPTOMS OF EXPOSURE Dizziness, rapid respiration, headache, drowsiness, drop in blood pressure, rapid pulse, unconsciousness.

EFFECTS OF CHRONIC EXPOSURE Dizziness, weakness, lung congestion, eye irritation, loss of appetite and weight, mental deterioration.

EMERGENCY AND FIRST AID PROCEDURES Remove from area, give amyl nitrite inhalation every 5 minutes, artificial respiration with oxygen, wash skin \& eyes, get medical aid.

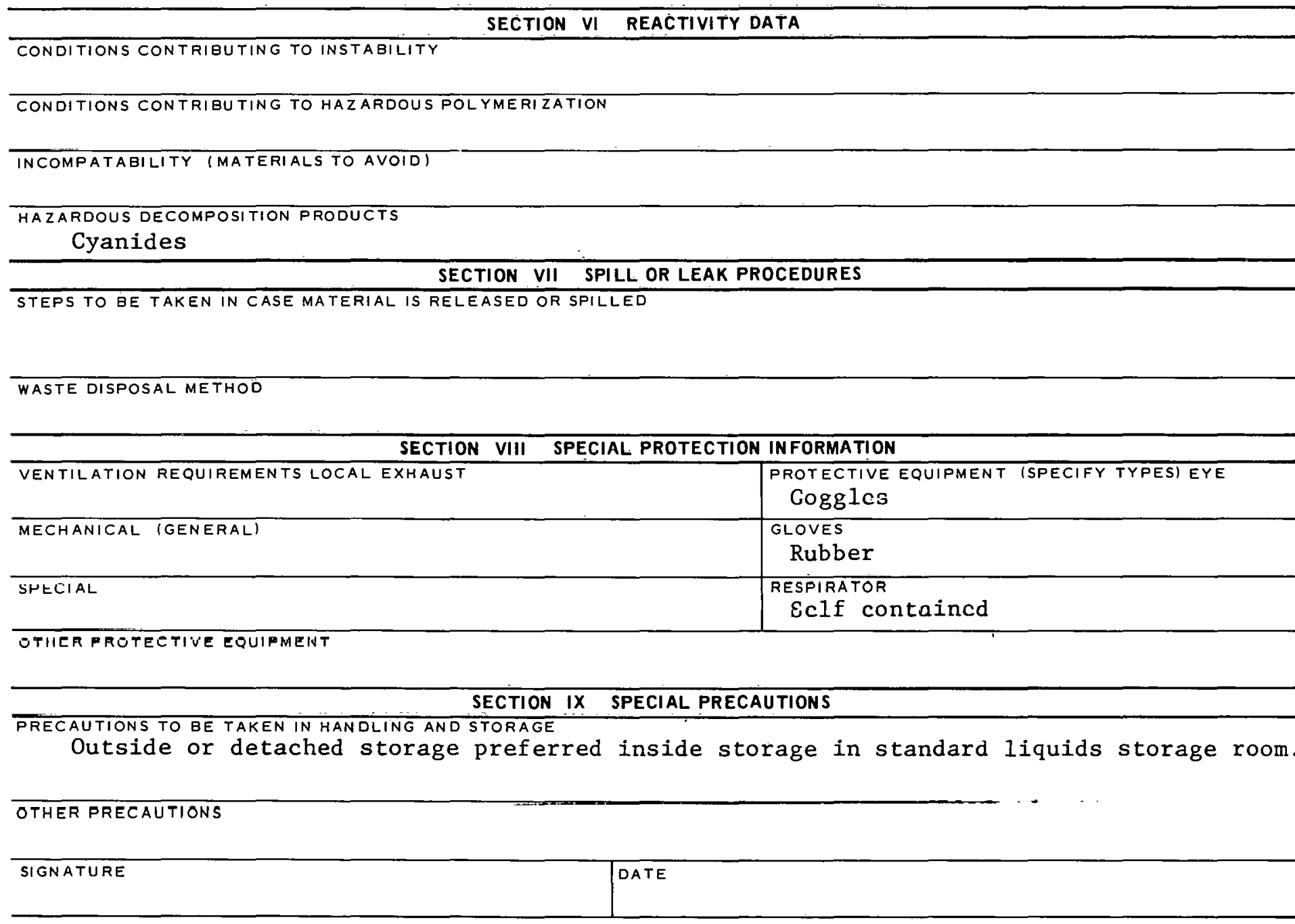




\section{PRODUCT DESIGNATION}

03-228-1100

$\alpha$-chloro-acetophenone

\section{MATERIAL SAFETY DATA SHEET}

\section{SECTION I SOURCE AND NOMENCLATURE}

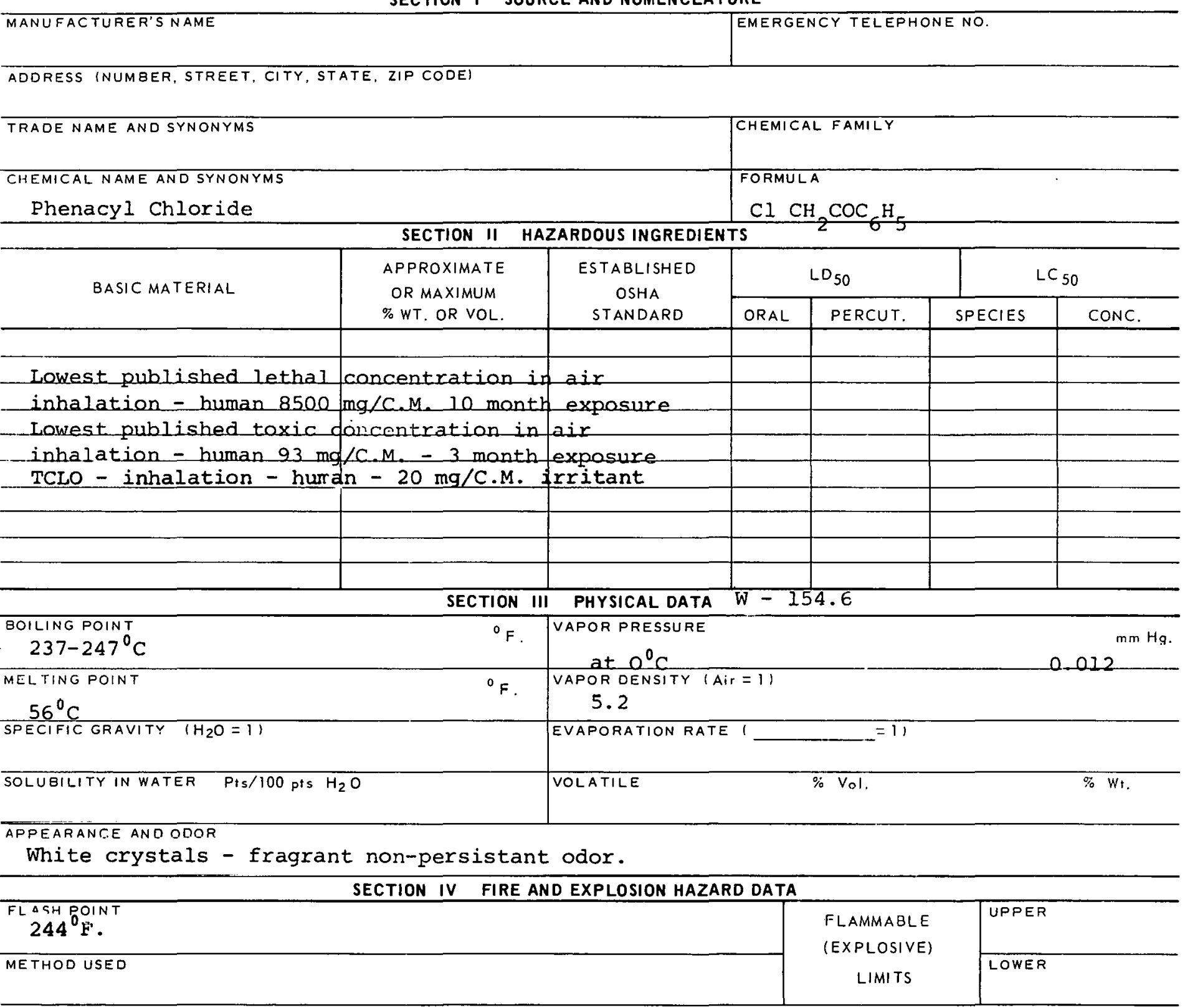

EXTINGUISHING MEDIA

Carbon dioxide, dry chemical

CDER,IAL FIRE FIGHTING PROCEDURES

UNUSUAL FIRE AND EXRLOSION HAZARDS

May react with water or steam to produce toxic and corrosive fumes. 


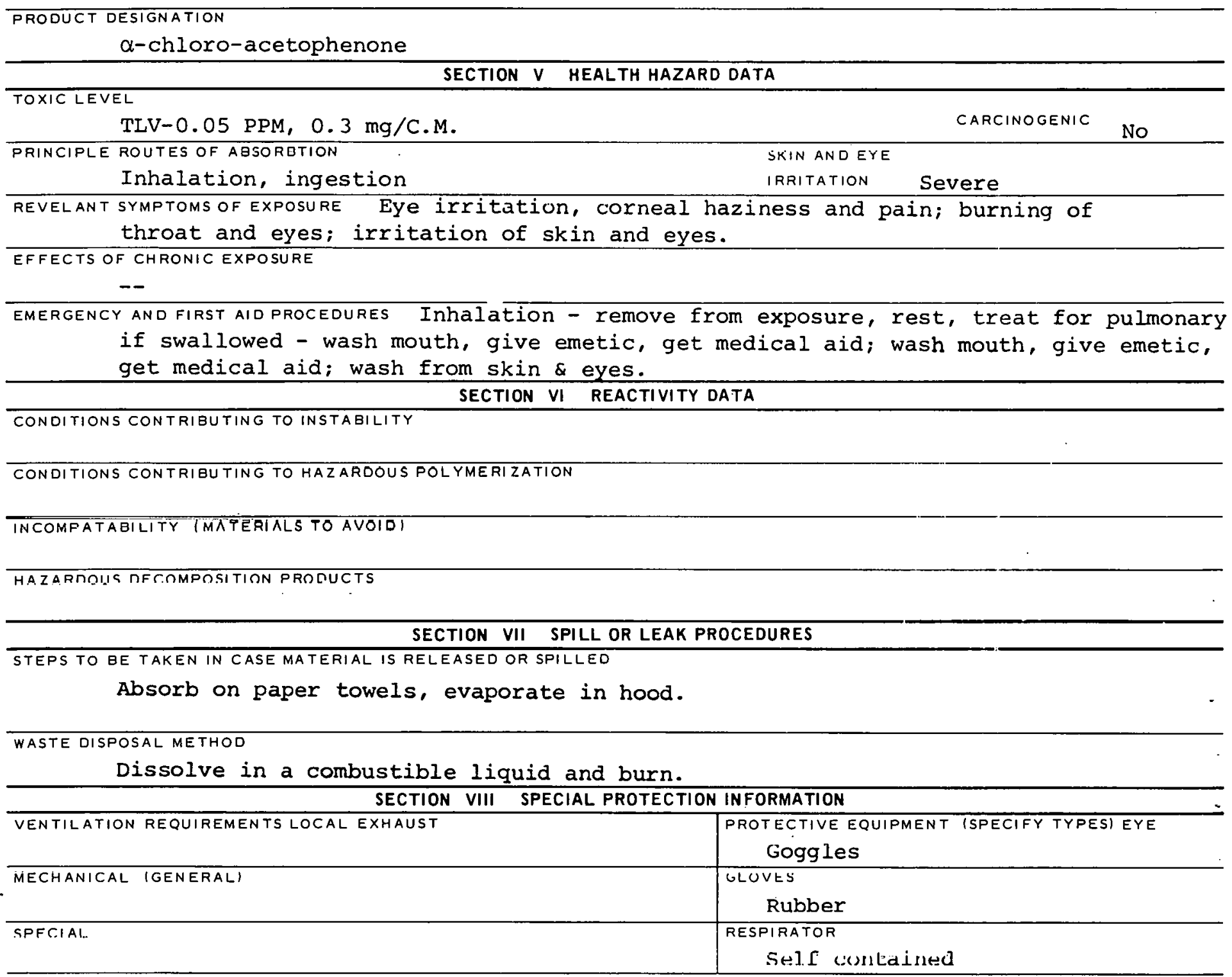

OTHER PROTECTIVE EQUIPMENT

Protecting clothing

SECTION IX SPECIAL PRECAUTIONS

PRECAUTIONS TO BE TAKEN IN HANDLING AND STORAGE

OTHER PRECAUTIONS

SIGNATURE

DATE

$228-1100 B$ 


\section{PRODUCT DESIGNATION}

02-228-1605

Chloroacetyl chloride
MATERIAL SAFETY

DATA SHEET

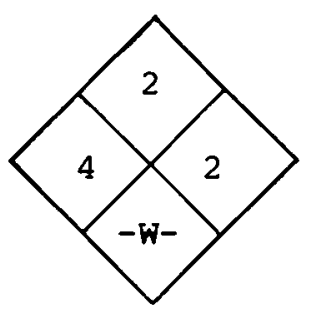

SECTION I SOURCE AND NOMENCLATURE

MANUFACTURER'S NAME

EMERGENCY TELEPHONE NO

ADDRESS (NUMBER, STREET, CITY, STATE, ZIP CODE)

\section{TRADE NAME AND SYNONYMS \\ CHEMICAL NAME AND SYNONYMS \\ $\alpha$ - chloroacetyl chloride}

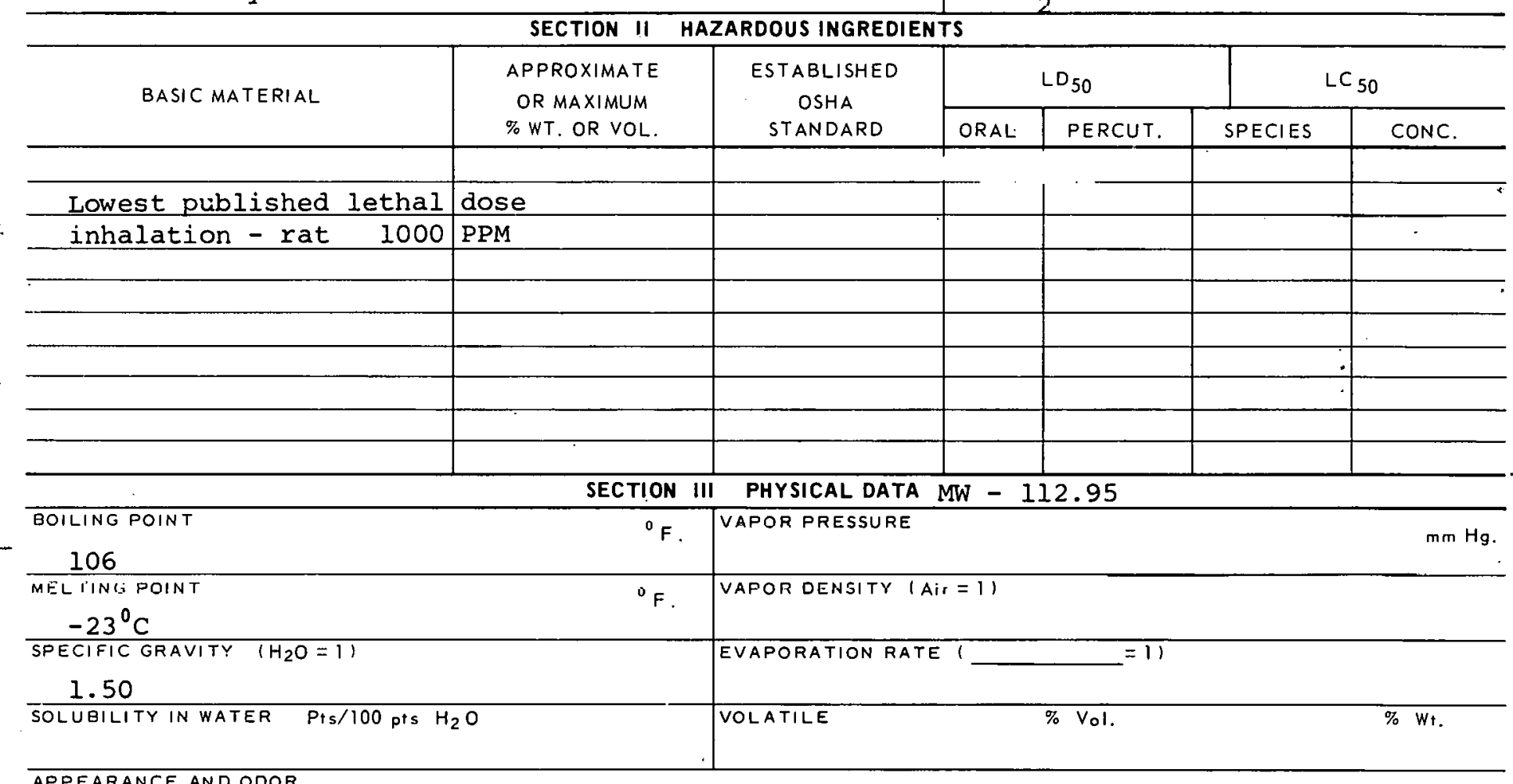

APPEARANCE AND ODOR

Water white or slightly yellow liqui.d

\begin{tabular}{l|c|c}
\hline \multicolumn{1}{c}{ SECTION IV FIRE AND EXPLOSION HAZARD DATA } \\
\hline $\begin{array}{l}\text { FLASH POINT } \\
\text { N/A }\end{array}$ & $\begin{array}{c}\text { FLAMMABLE } \\
\text { (EXPLOSIVE) } \\
\text { LIMITS }\end{array}$ \\
\hline METHOD'USED & LOWER \\
\hline $\begin{array}{l}\text { EXTINGUISHING MEDIA } \\
\text { DO not uEe water or foam } \\
\text { use carbon dioXide or dry chemical }\end{array}$ \\
\hline SPECIAL FIRE FIGHTING PROCEDURES
\end{tabular}

UNUSUAL FIRE AND EXPLOSION HAZAROS

When heated to decomposition point gives off

toxic chloride fumes 
Chloroacetyl Chloride

SECTION $V$ HEALTH HAZARD DATA

TOXIC LEVEL

$50 \mathrm{PPB}$

PRINCIFLE ROUTES OF ABSORBIION

Inhalation, ingestion

REVELANT SYMPTOMS OF EXPOSURE Irritation of eyes and respiratory system; severe

internal damage if swallowed; eye \& skin burns.

EFFECTS OF CHRONIC EXPOSURE

Bronchial irritation, errosion of teeth. EMERGENCY AND FIRST AID PROCEDURES

Inhalation - rest, get medical aid; if swallowed -

drink water, milk of magnesia, get medical aid; wash eyes \& skin.

CONDITIONS CONTRIBUTING TO INSTABILITY

SECTION VI REACTIVITY DATA

CONDITIONS CONTRIBUTING TO HAZARDOUS POLYMERIZATION

INCOMPATABILITY (MAIEHIALSTU AVUIU)

Reacts with water to produce H Cl alcohol \& dimethyl sulfoxide HATARIOIIS DECOMPOSITION PRODUCTS

SECTION VII SPILL OR LEAK PROCEDURES

STEPS TO BE TAKEN IN CASE MATERIAL IS RELEASED OR SPILLED

Cover spill with sodium bicarbonate

WASTE DISPOSAL METHOD

SECTION VIII SPECIAL PROTECTION INFORMATION

\begin{tabular}{l|l}
\hline VENTILATION REQUIREMENTS LOCAL EXHAUST & $\begin{array}{l}\text { PROTECTIVE EQUIPMENT ISPECIFY TYPESIEYE } \\
\text { GoggleS }\end{array}$ \\
\hline MECHANILAL IGENEHALI & GLOVES \\
Subber \\
\hline SPECIAL & RESPIRATOR \\
& Self contained \\
\hline
\end{tabular}

OTHER. PROTECTIVE EQUIPMENT

Rubber apron

SECTION IX SPECIAL PRECAUTIONS

PRECAUTIONS TO BE TAKEN IN HANDLING AND STORAGE

OTHER PRECAUTIONS

SIGNATURE

DATE

$228-1605 B$ 
CAS : 000095512

\section{PRODUCT DESIGNATION}

03-228-2635

o-Chloroaniline
MATERIAL SAFETY

DATA SHEET

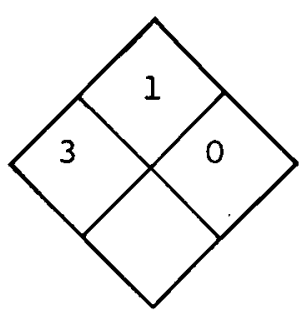

SECTION I SOURCE AND NOMENCLATURE

\section{MANUFACTURER'S NAME \\ TRADE NAME AND SYNONYMS \\ Fast Yellow GC Base CHEMICAL NAME AND SYNONYMS O-Chloroaniline}

ADDRESS (NUMBER, STREET, CITY, STATE, ZIP CODE)

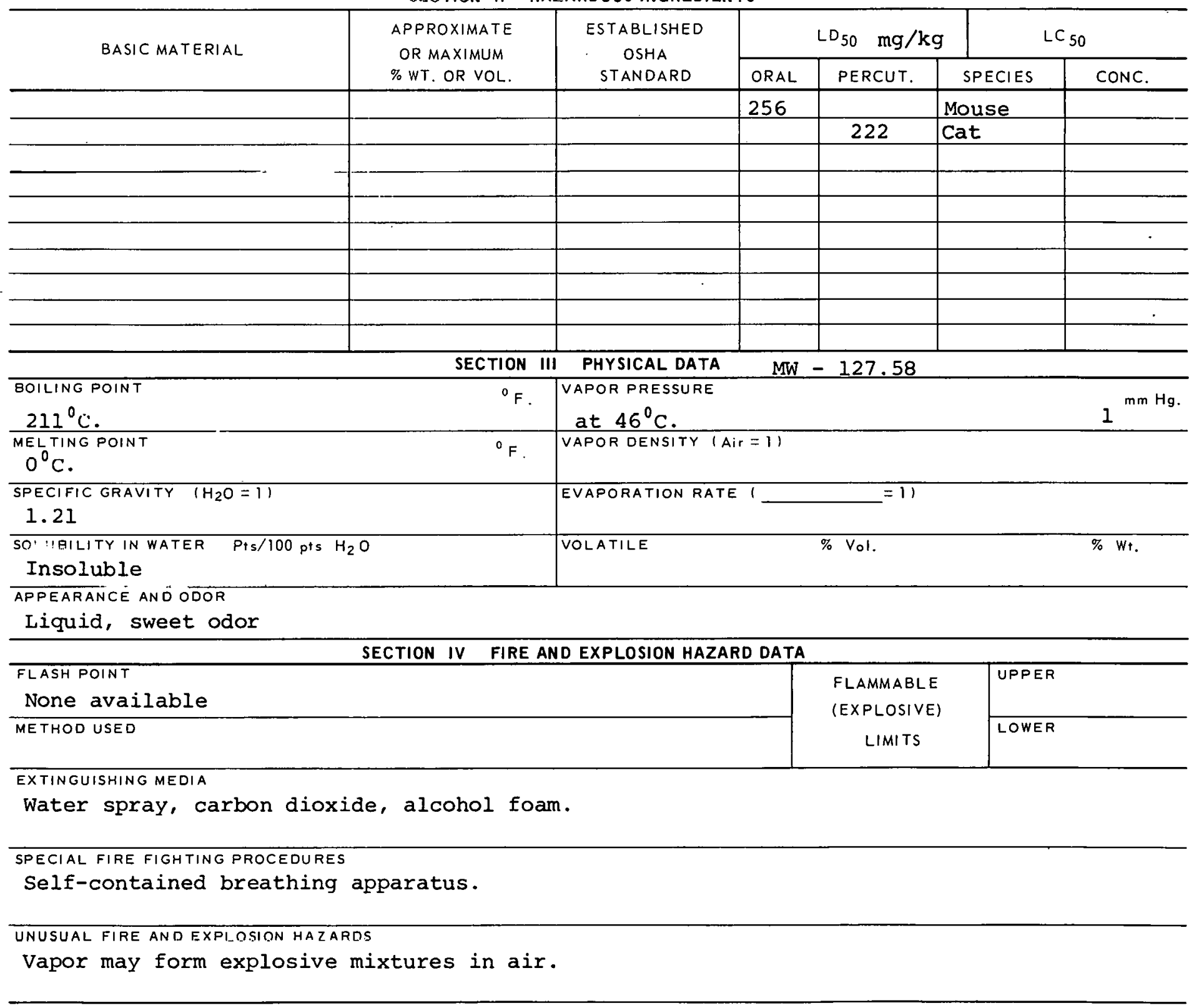




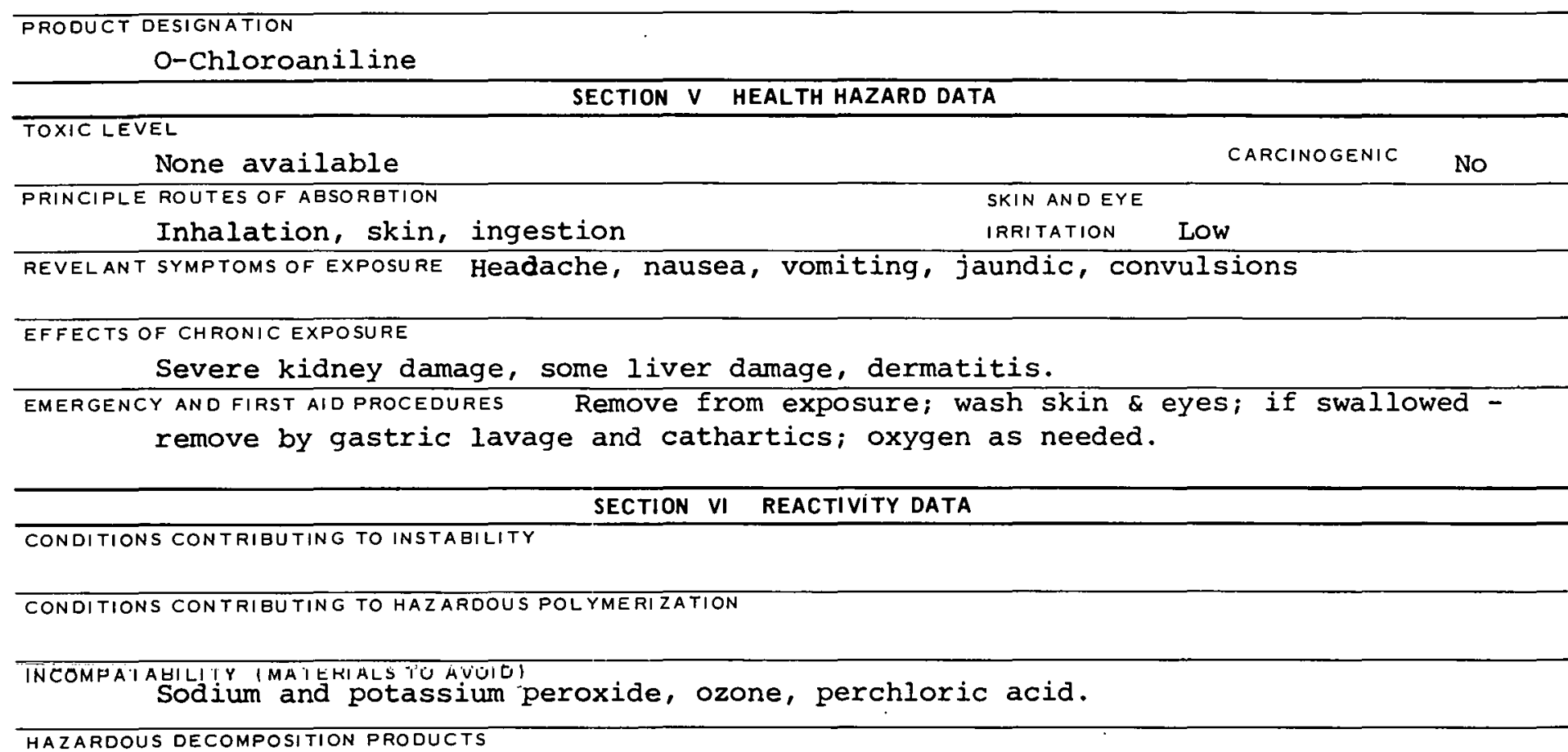

HAZARDOUS DECOMPOSITION PRODUCTS

SECTION VII SPILL OR LEAK PROCEDURES

STEPS TO BE TAKEN IN CASE MATERIAL IS RELEASED OR SPILLED

Wear protective clothing during cleanup.

WASTE DISPOSAL METHOD

SECTION VIII SPECIAL PROTECTION INFORMATION

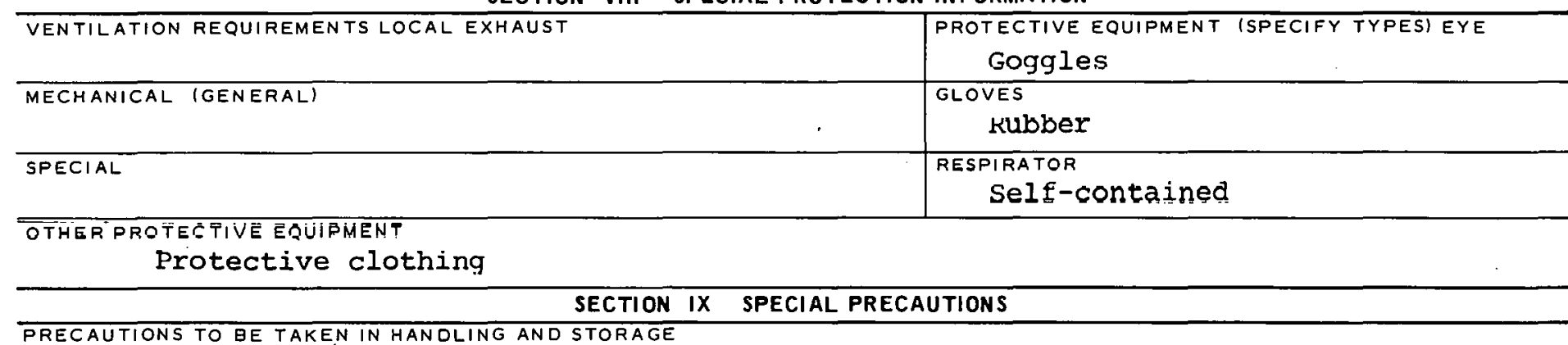

PRECAUTIONS TO BE TAKEN IN HANDLING AND STORAGE

Store in flammable liquids cabinets.

UTHER PRECAUTION3

SIGNATURE

DATE

$228-2635 B$ 


\begin{tabular}{|l|}
\hline PRODUCT DESIGNATION \\
03-228-2665 \\
P-Chloroaniline \\
\end{tabular}

\section{MATERIAL SAFETY DATA SHEET}

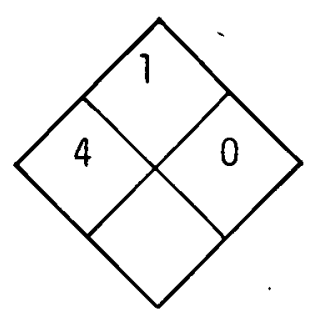

SECTION I SOURCE AND NOMENCLATURE

AODRESS (NUMBER, STREET, CITY, STATE, ZIP CODE)

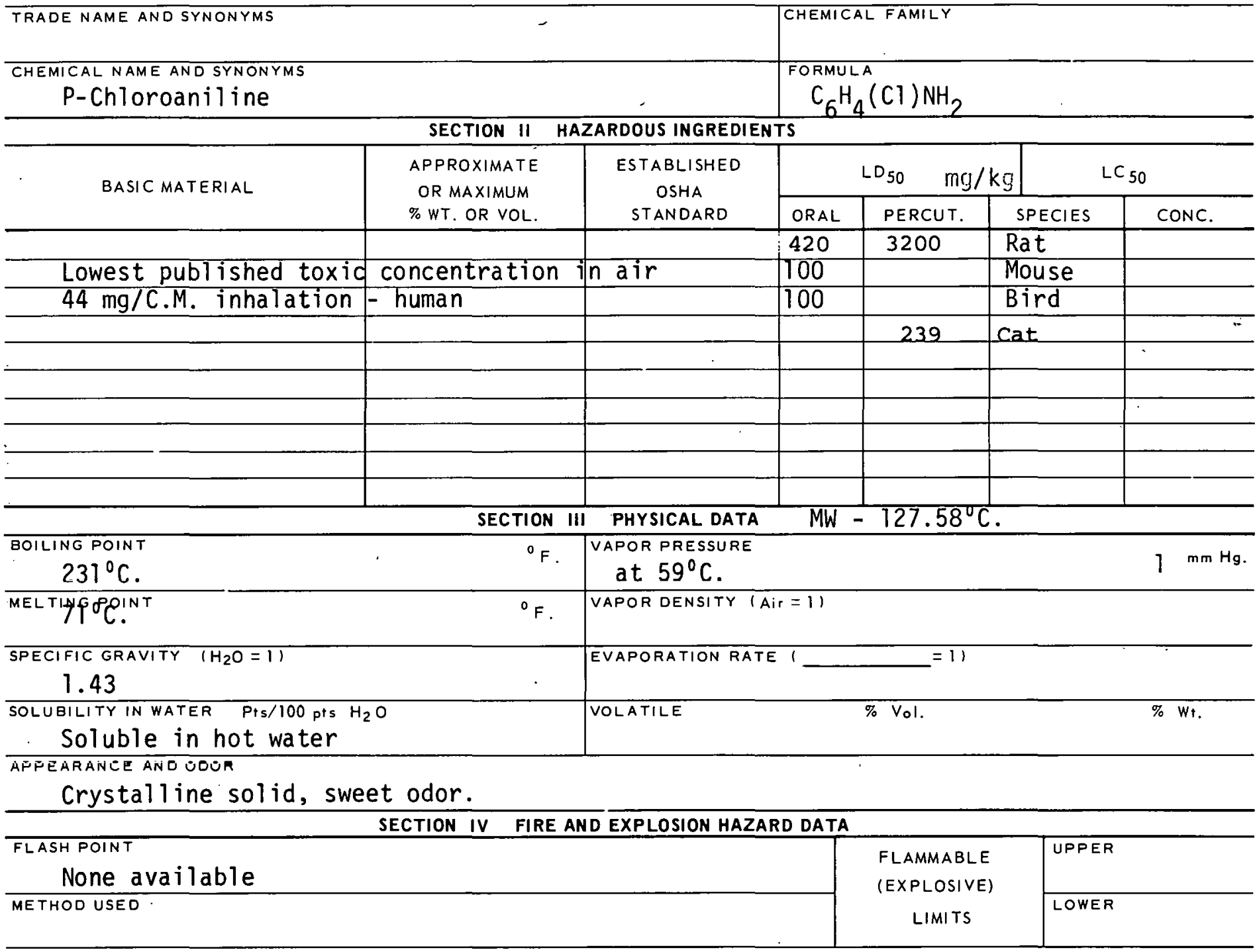

EXTINGUISHING MEDIA

Water spray, carbon dioxide, alcohol foam.

SPECIAL FIRE FIGHTING PROCEDURES

Self-contained breathing apparatus.

UNUSUAL FIRE ANO EXPLOSION HAZARDS

Vapor may form explosive mixtures in air. 


\section{TOXIC LEVEL}

None Avajlable

PRINCIPLE ROUTES OF ABSORATION

Inhalation, skin, ingestion

CARCINOGENIC NO
ANT SYMPTOMS OF EXPOSURE Cyanosis, headache, blood pressure drop, lethargy, jaundice
REVELANT SYMPTOMS OF EXPOSURE Cyanosis, he
$\&$ anemia, pain in joints, fainting.

EFFECTS OF CHRONiC EXPOSURE Weight loss, anemia, weakness and irritability, possible liver

damage, inflammation of kidney, ringing in ears.

EMERGENCY AND FIRST AID PROCEDURES Remove from exposure; wash from skin \& eyes; if

swallowed - remove by gastric lavage and catharsis; give oxygen as needed.

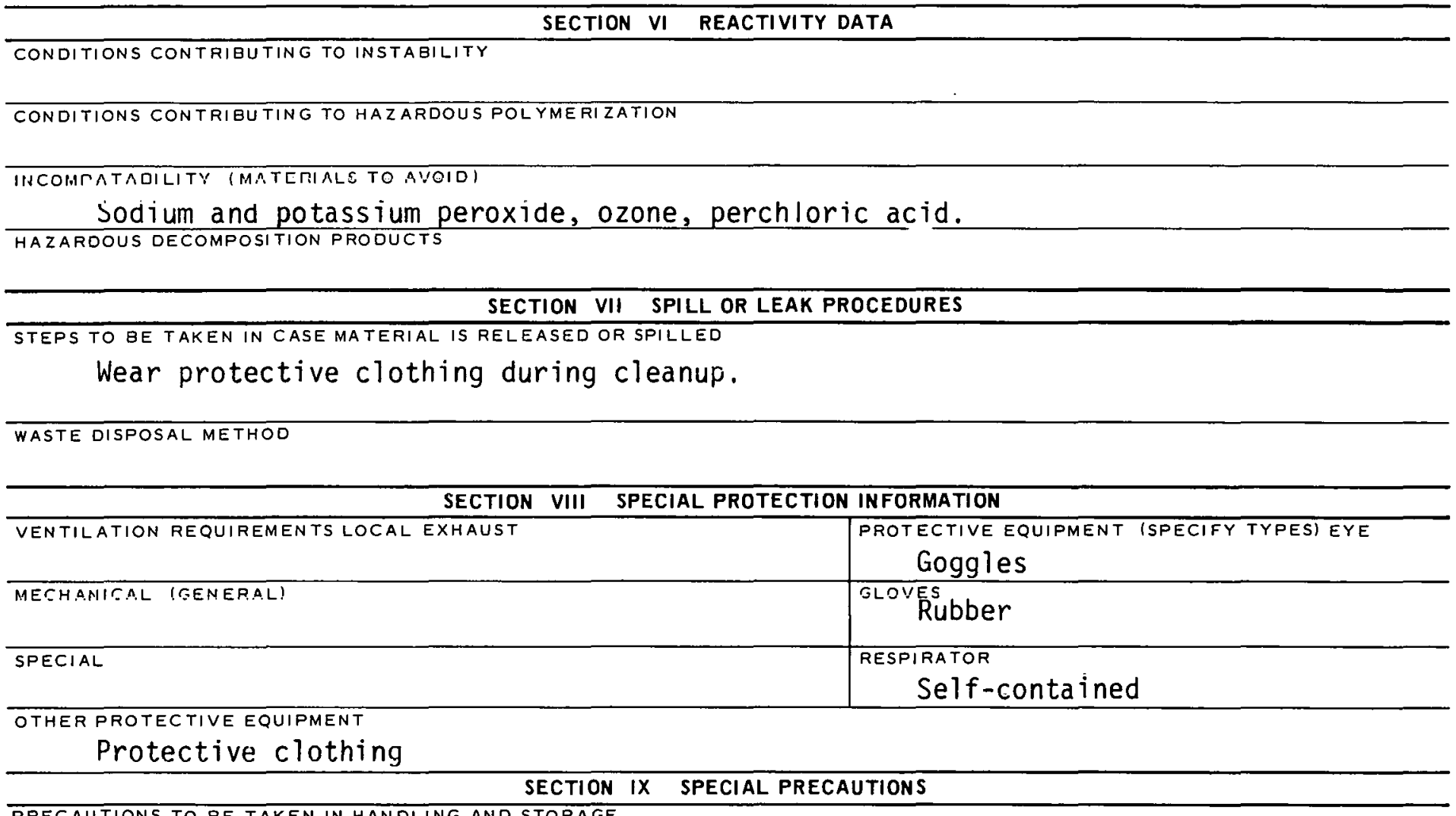

PRECAUTIONS TO BE TAKEN IN HANDLING AND STORAGE

Store in flammable liquids cabinet. 
CAS : 000089985

\begin{tabular}{|l|}
\hline PRODUCT DESIGNATION \\
03-228-3635 \\
O-chlorobenzaldehyde \\
\end{tabular}

CU50750

\section{MATERIAL SAFETY \\ DATA SHEET}

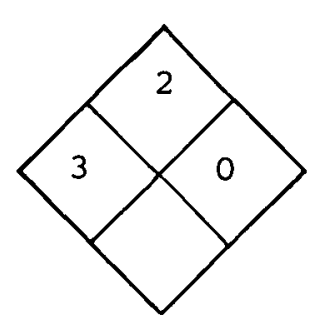

\section{SECTION I SOURCE AND NOMENCLATURE}

MANUFACTURER'S NAME

ADORESS (NUMBER, STREET, CITY, STATE, ZIP CODE)

TRADE NAME ANO SYNONYMS

CHEMICAL FAMILY

CHEMICAL NAME ANO SYNONYMS

2-chlorobenzaldehyde

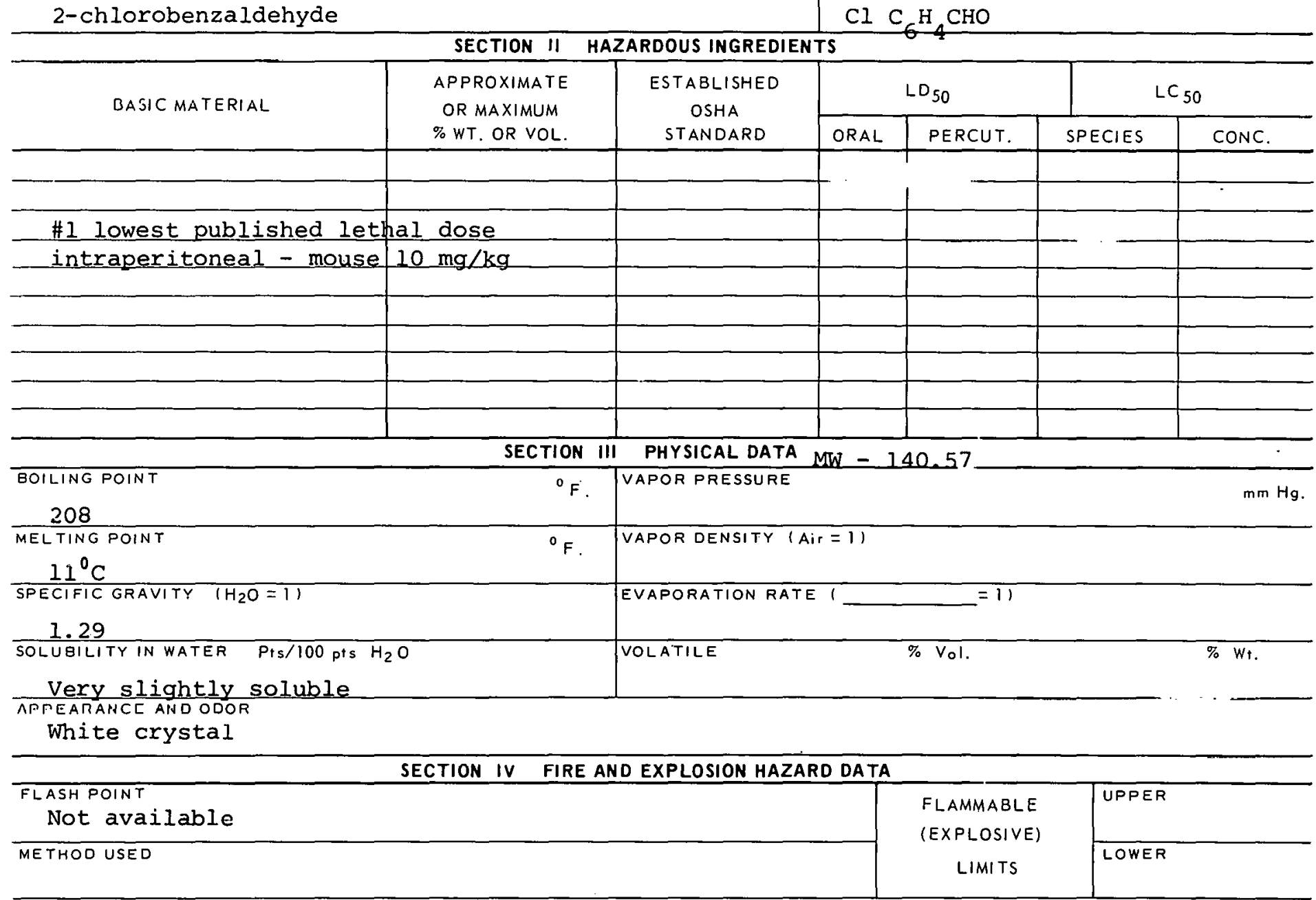

EXTINGUISHING MEDIA

Foam, carbon dioxide, dry chemical

SPECIAL FIRE FIGHTING PROCEDURES

UNUSUAL FIRE AND EXPLOSION HAZARDS 


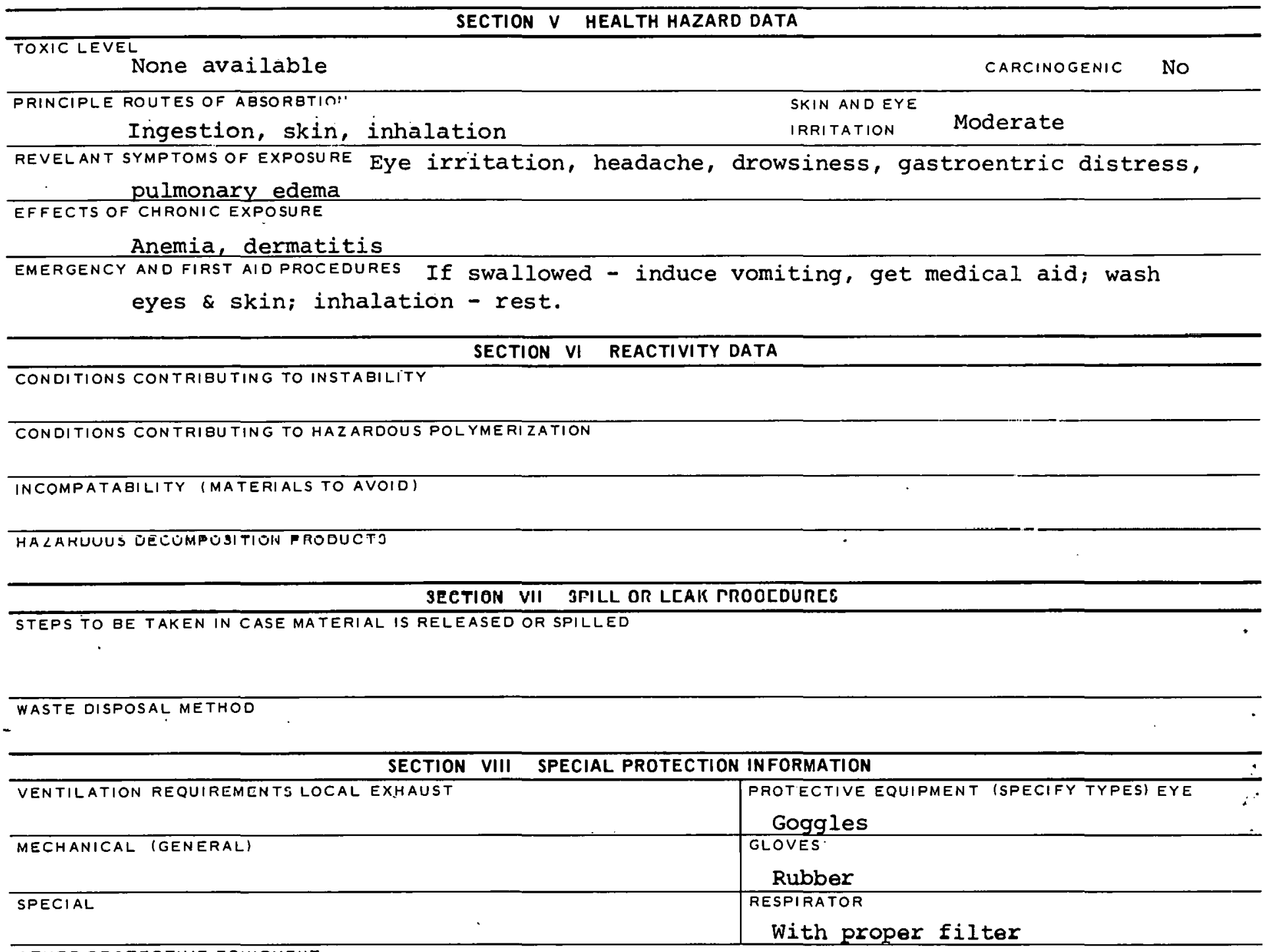

OTHER PROTECTIVE EQUIPMENT

\section{SECTION IX SPECIAL PRECAUTIONS}

PRECAUTIONS TO BE TAKEN IN HANDLING AND STORAGE

\section{OTHER PRECAUTIONS}

SIGNATIIRE

DATE

$228 \cdots 3635 B$ 


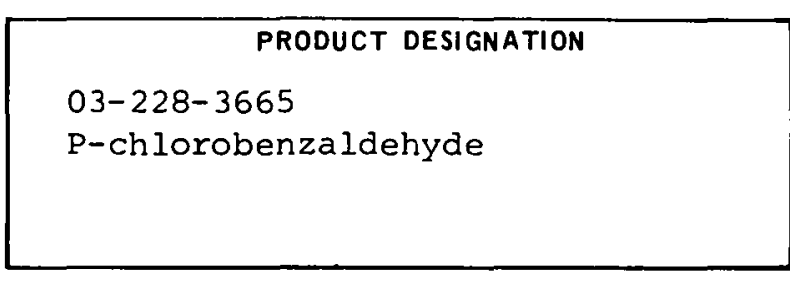

\section{MATERIAL SAFETY DATA SHEET}

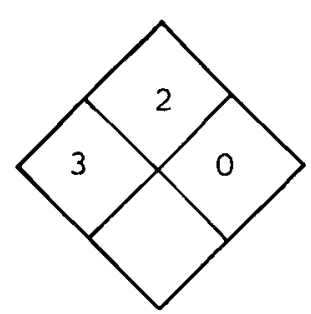

\section{SECTION I SOURCE AND NOMENCLATURE}

\begin{tabular}{l|l}
\hline MANUFACTURER'S NAME & EMERGENCY TELEPHONE NO. \\
\hline ADDRESS (NUMBER, STREET, CITY, STATE, ZIP CODE)
\end{tabular}

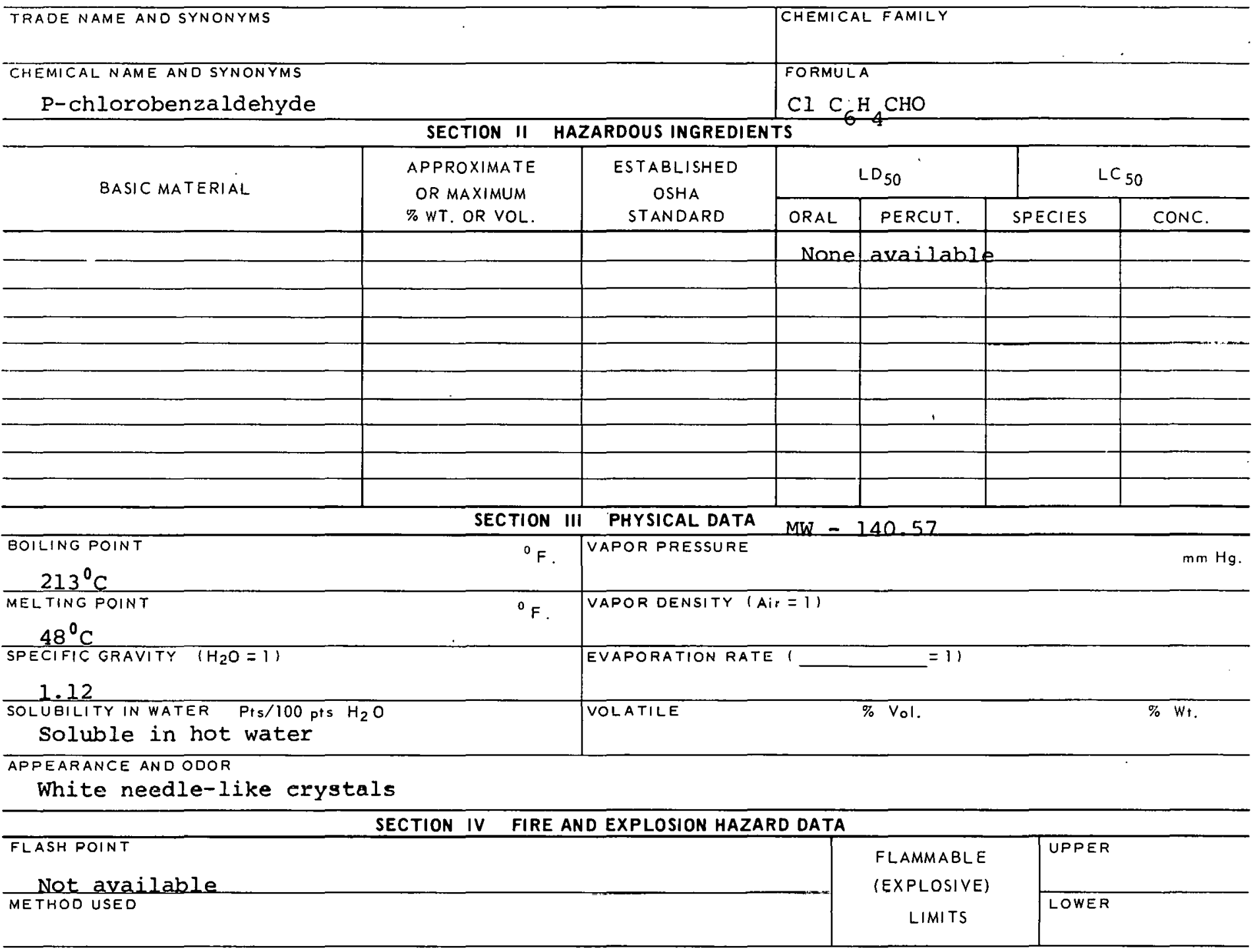

EXTINGUISHING MEOIA

Foam, carbon dioxide, dry chemical

SPECIAL FIRE FIGHTING PROCEDURES

UNUSUAL FIRE AND EXPLOSION HAZARDS 


\section{SECTION $V$ HEALTH HAZARD DATA}

\section{TOXIC LEVEL}

Not available

PRINCIPLE ROUTES OF ABSORATION

Ingestion, skin, inhalation of dust

CARCINOGENIC

No

SKIN AND EYF

IRRITATION moderate

REVELANT SYMPTOMS OF EXPOSURE
EYe irritation, headache, drowsiness, gastroentric distress, pulmonary edema

EFFECTS OF CHRONIC EXPOSURE

Anemia, dermatitis

EMERGENCY AND FIRST AID PROCEDURES

eyes \& skin; inhalation - rest.

SECTION VI REACTIVITY DATA

CONDITIONS CONTRIBUTING TO INSTABILITY

CONDITIONS CONTRIBUTING TO HAZARDOUS POLYMERIZATION

INCOMMA I AHILIIY IMATERIALS TE AV́VIDI

HAZARDOUS OECOMPOSITION PRODUCTS

SECTION VII SPILL OR LEAK PROCEDURES

STEPS TO BE TAKEN IN CASE MATERIAL IS RELEASED OR SPILLED

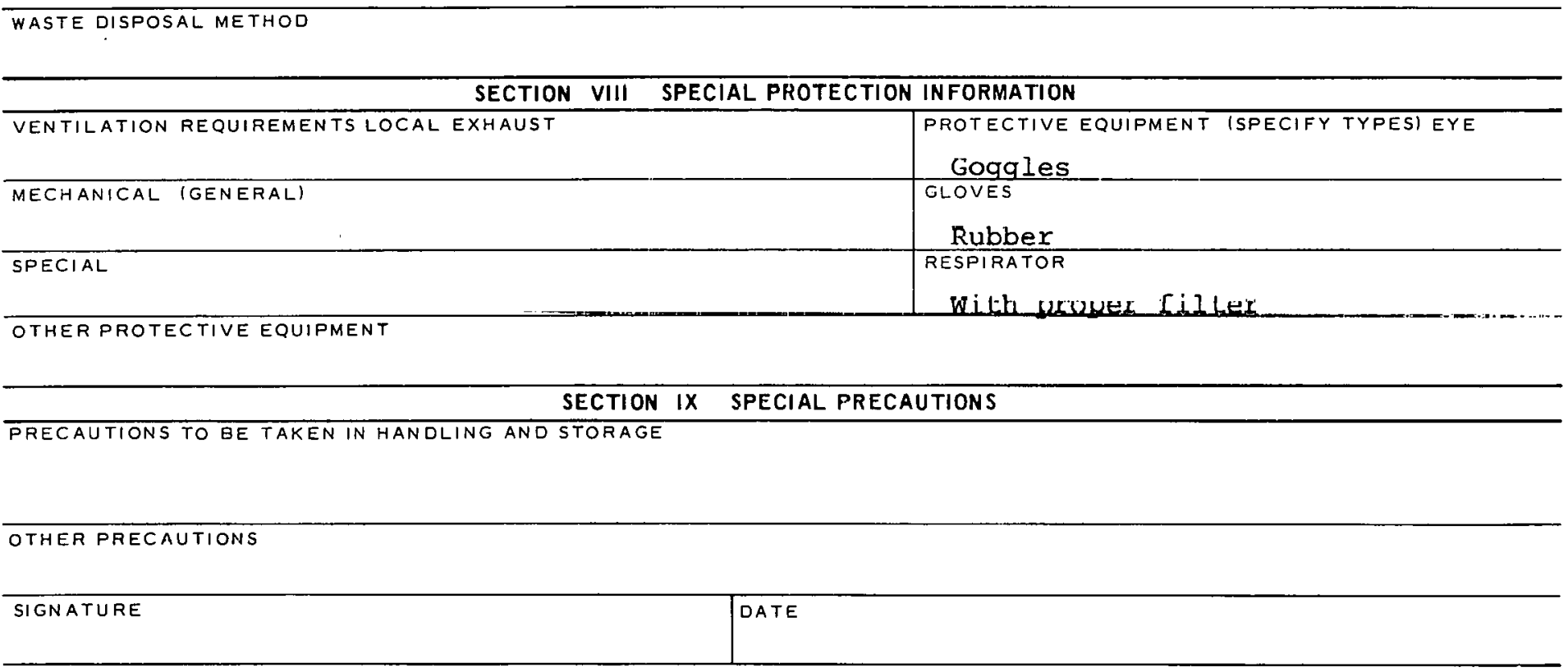


CAS : 000108907

PRODUCT DESIGNATION
03-228-4105
Chlorobenzene

\section{SECTION I SOURCE AND NOMENCLATURE}

MANUFACTURER'S NAME

MATERIAL SAFETY

DATA SHEET

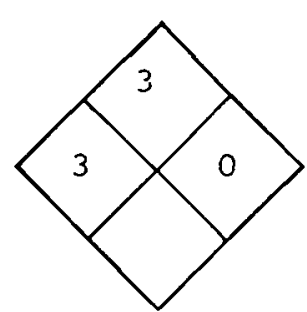

ADDRESS (NUMBER, STREET, CITY, STATE, ZIP CODE)

TRADE NAME AND SYNONYMS

Chlorobenzol

CHEMICAL NAME AND SYNONYMS

PhenYl chloride

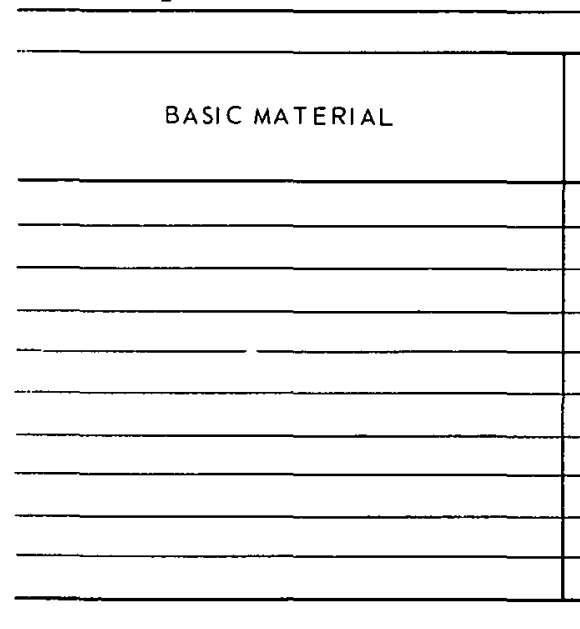

BOILING POINT

$131.7^{\circ} \mathrm{C}$

MELTING POINT

$-55.6^{\circ} \mathrm{C}$

SPECIFIC GRAVITY $\left(\mathrm{H}_{2} \mathrm{O}=11\right.$

1.113

SOLUBILITY IN WATER Pis/100 pis $\mathrm{H}_{2} \mathrm{O}$

APPEARANCE AND ODOR

Clear colorless liquid with aromatic odor.

SECTION IV FIRE AND EXPLOSION HAZARD DATA

FLASH POINT

$85^{\circ} \mathrm{F}$

METHOD USED

Closcd Cup

EXTINGUISHING MEDIA

Carbon dioxide; dry chemical, foam, water spray

SPECIAL FIRE FIGHTING PROCEDURES

Wear goggles and self contained breathing apparatus. SECTION $\|$ HAZARDOUS INGREDIENTS ${ }_{6}{ }_{5} \mathrm{Cl}$
EMERGENCY TELEPHONE NO. \begin{tabular}{l} 
CHEMICAL FAMILY \\
FORMULA \\
$\mathrm{C}_{6} \mathrm{H}_{5} \mathrm{Cl}$ \\
\hline
\end{tabular}

\begin{tabular}{|c|l|l|l}
\hline \multicolumn{3}{|c|}{ DD $_{50} \mathrm{mg} / \mathrm{kg}$} & \multicolumn{2}{c}{ LC $_{50}$} \\
\hline ORAL & PERCUT. & SPECIES & CONC. \\
\hline 2910 & & Rat & \\
\hline 2830 & & Rabbit & \\
\hline & & & \\
\hline & & & \\
\hline & & & \\
\hline & & & \\
\hline & & & \\
\hline
\end{tabular}

SECTION III PHYSICAL DATA

${ }^{0} \mathrm{~F}$.

at $22.2^{\circ} \mathrm{C}$

$10^{\mathrm{mm} \mathrm{Hg}}$

${ }^{0} \mathrm{~F}$.

3.88

EVAPORATION RATE I

$=11$
FLAMMABLE

(EXPLOSIVE)

LIMITS
UPPER

7.18

LOWER

1. 38

NUSUAL FIRE AND EXPLOSION HAZARDS 
TOXIC LEVEL

TLU - 75 PPM, $350 \mathrm{mg} / \mathrm{C} . \mathrm{M}$.

CARCINOGENIC No

PRINCIPLE ROUTES OF ABSORBTION

Inhalation, ingestion, skin

SKIN AND EYE

IRRITATION YES

REVELANT SYMPTOMS OF EXPOSURE Cyanosis, deep \& rapid respiration, irregular pulse, drowsiness, unconsciousness

EFFECTS OF CHRONIC EXPOSURE

Lung, liver and kidney damage.

EMERGENCY AND FIRST AID PROCEDURES Inhalation - remove from exposure, rest; if swallowed wash mouth, give emetic, get medical aid; wash eyes and skin.

SECTION VI REACTIVITY DATA

CONDITIONS CONTRIBUTING TO INSTABILITY

CONDITIONS CONTRIBUTING TO HAZARDOUS POLYMERIZATION

TIVEOMPATADILITY IMATERIALS TO AVOIOI

HAZAROOUS DECOMPOSITION PRODUCTS

SECTION VII SPILL OR LEAK PROCEDURES

STEPS TO BE TAKEN IN CASE MATERIAL IS RELEASED OR SPILLED

Absorb spill with paper towels and evaporate in hood.

WASTE DISPOSAL METHOD

SECTION VIII SPECIAL PROTECTION INFORMATION

\begin{tabular}{|c|c|}
\hline VENTILATION REQUIREMENTS LOCAL EXHAUST & $\begin{array}{l}\text { PROTECTIVE EQUIPMENT (SPECIFY TYPES) EYE } \\
\text { GOggles }\end{array}$ \\
\hline MECHAHICAL (GEHERAL) & $\begin{array}{l}\text { GLOVEJ } \\
\text { Polyvinyl alcohol }\end{array}$ \\
\hline SPECIAL & $\begin{array}{l}\text { RESPIRATOR } \\
\text { Belf contained breatlinily apparalus }\end{array}$ \\
\hline
\end{tabular}

OTHER PROTECTIVE EQUIPMENT

SECTION IX SPECIAL PRECAUTIONS

PRECAUTIONS TO BE TAKEN IN HANDLING AND STORAGE

Store in tightly closed containers and protect against physical damage.

OTHER PRECAUTIONS

SIGNATURE

DATE

$228-4105 B$ 
CAS- 000623030

\section{MATERIAL SAFETY DATA SHEET}

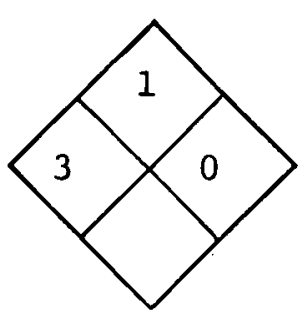

SECTION I SOURCE AND NOMENCLATURE

MANUFACTURER'S NAME

ADDRESS (NUMBER, STREET, CITY, STATE, ZIP CODE)

\section{TRADE NAME AND SYNONYMS \\ CHEMICAL NAME AND SYNONYMS \\ P-Chlorobenzonitrile}

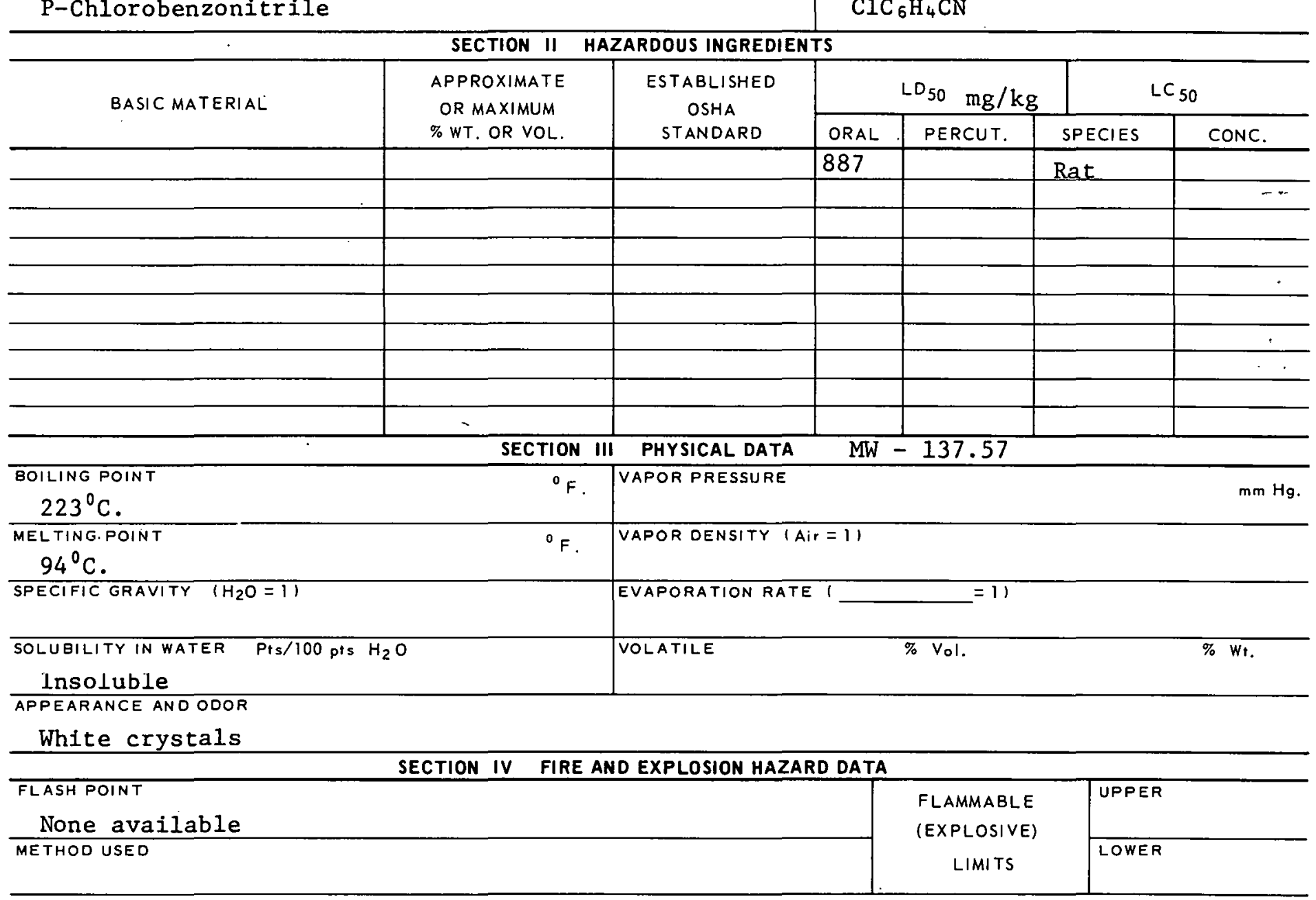

EXTINGUISHING MEDIA

Alcohol foam, carbon dioxide, dry chemical.

SPECIAL FIRE FIGHTING PROCEDURES

Wear self-contained breathing apparatus.

UNUSUAL FIRE AND EXPLOSION HAZARDS 


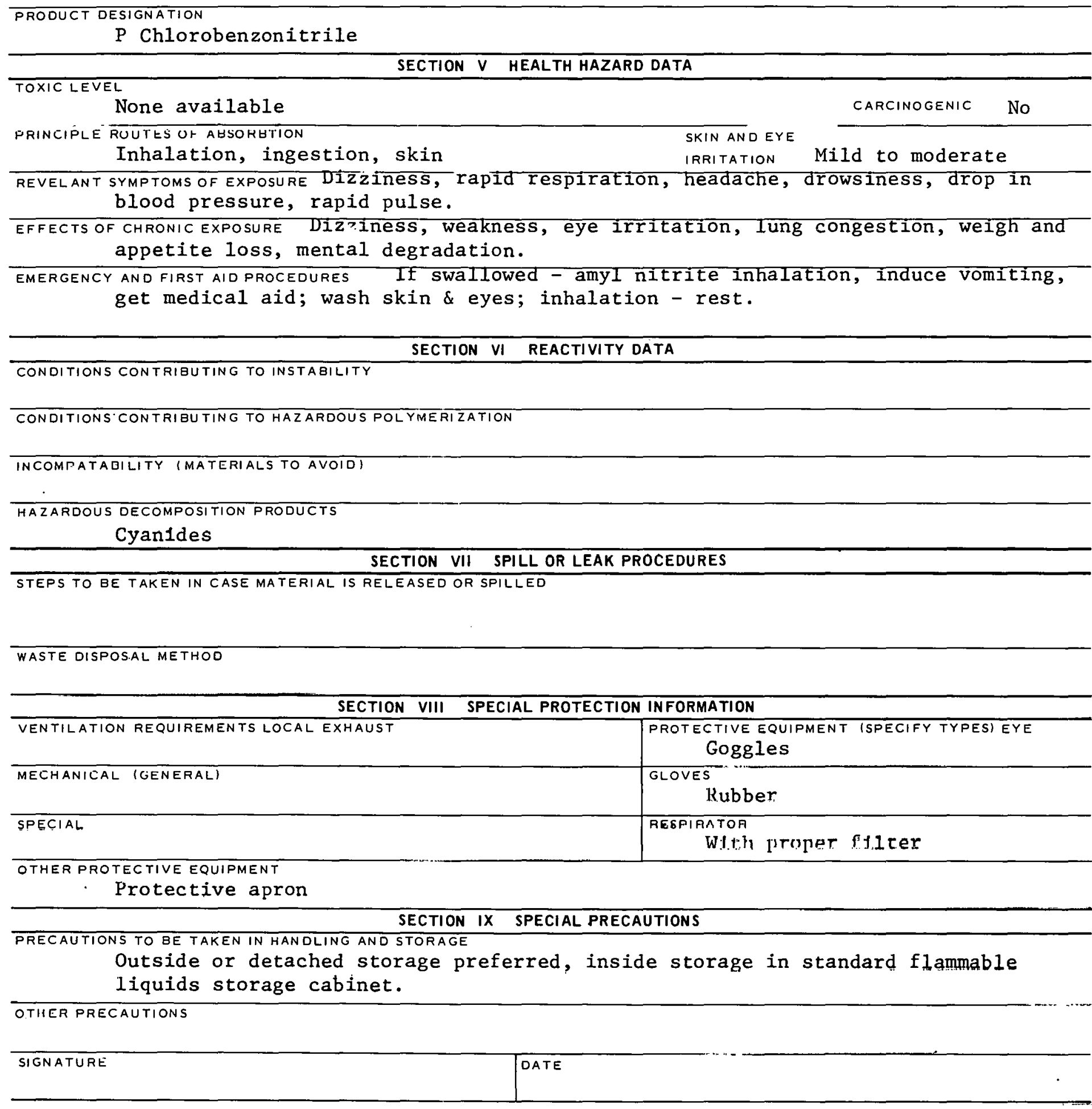




\begin{tabular}{|l|}
\hline PRODUCT DESIGNATION \\
03-228-5635 \\
O-chlorobenzoyl chloride \\
\end{tabular}

\section{MATERIAL SAFETY DATA SHEET}

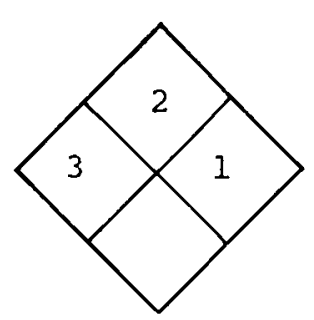

\section{SECTION I SOURCE AND NOMENCLATURE}

MANUFACTURER'S NAME
ADDRESS INUMBER, STREET, CITY, STATE
TRADE NAME AND SYNONYMS
CHEMICAL NAME ANO SYNONYMS
1,2 ChlOYObENZOYl chloride

EMERGENCY TELEPHONE NO.

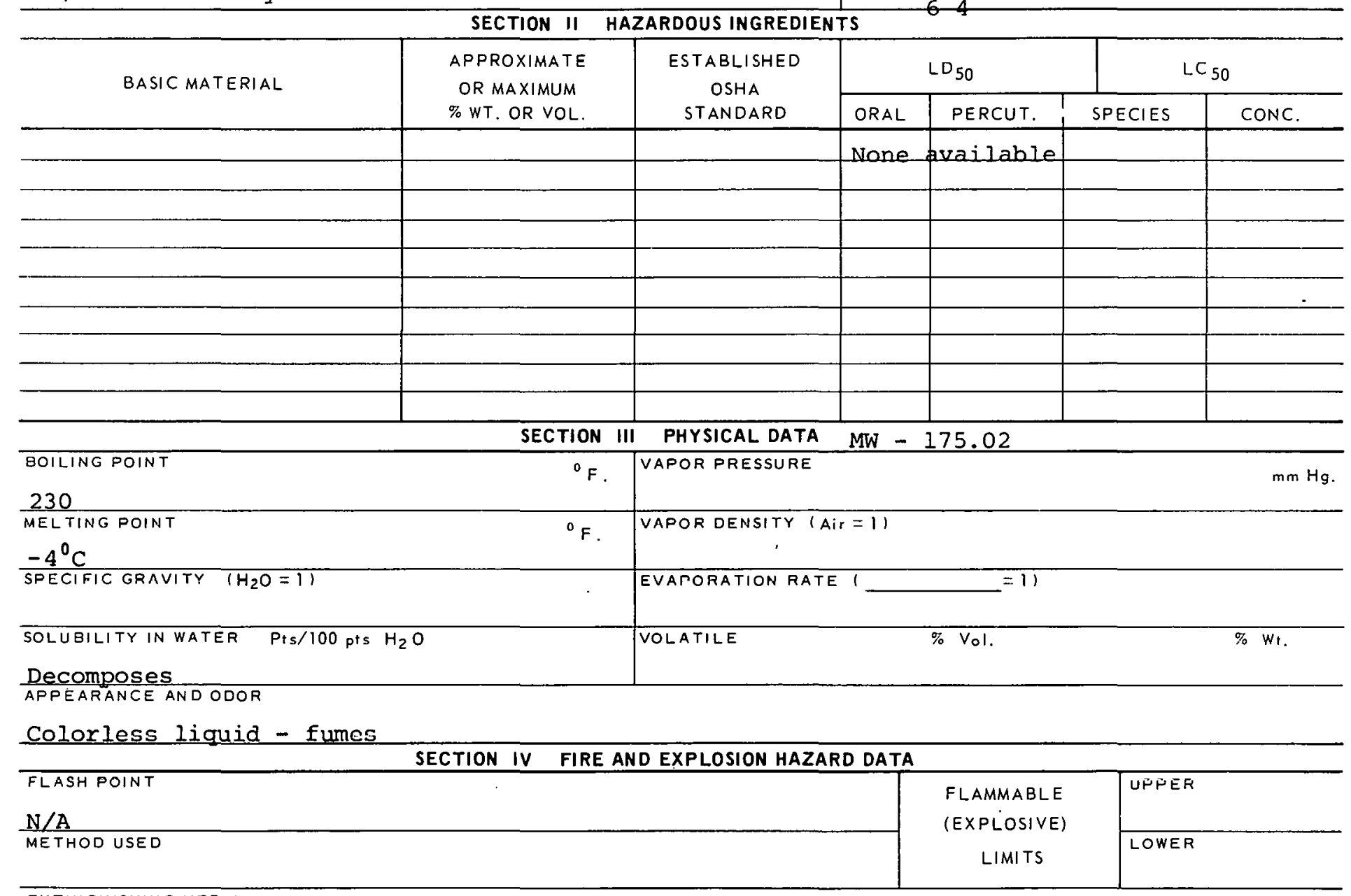

EXTINGUISHING MEDIA

\begin{tabular}{l|l} 
& CHEMICAL FAMILY \\
& $\begin{array}{l}\text { FORMULA } \\
\text { SECTION II HAZARDOUS INGREDIENTS }\end{array}{ }_{6}{ }_{4}{ }_{4} \mathrm{COCl}$ \\
\hline
\end{tabular}

Water spray, foam, carbon dioxide or dry chemical

SPECIAL FIRE FIGHTING PROCEDURES

Full protective clothing

UNUSUAL FIRE ANDEXPLOSION HAZARDS 
PRODUCT DESIGNATION

O-Chlorobenzoyl Chloride

SECTION $V$ HEALTH HAZARD DATA

\section{TOXIC LEVEL}

None available

PRINCIPLE ROUTES OF ABSORETION

CARCINOGENIC

Inhalation, ingestion

REVELANT SYMPTOMS OF EXPOSURE

mouth and digestive tract burns.

EFFECTS OF CHRONIC EXPOSURE

$--$

EMERGENCY AND FIRST AID PROCEDURES Inhalation - rest, artificial respiration if needed;

if swallowed - drink water, milk of magnesia, get medical aid; wash skin \& eyes.

\section{SECTION VI REACTIVITY DATA}

CONDITIONS CONTRIBUTING TO INSTABILITY

CONDITIONS CONTRIBUTING TO HAZARDOUS POLYMERIZATION

INCOMPATAEILITY IMATERIALS TO AVOIDI

dimethyl sulfoxide

HAZARDOUS DECOMPOSITION PRODUCTS

When heated, phosgene in produced

SECTION VII SPILL OR LEAK PROCEDURES

STEPS TO BE TAKEN IN CASE MATERIAL IS RELEASED OR SPILLED

WASTE DISPOSAL ME I'HOU

SECTION VIII SPECIAL PROTECTION INFORMATION

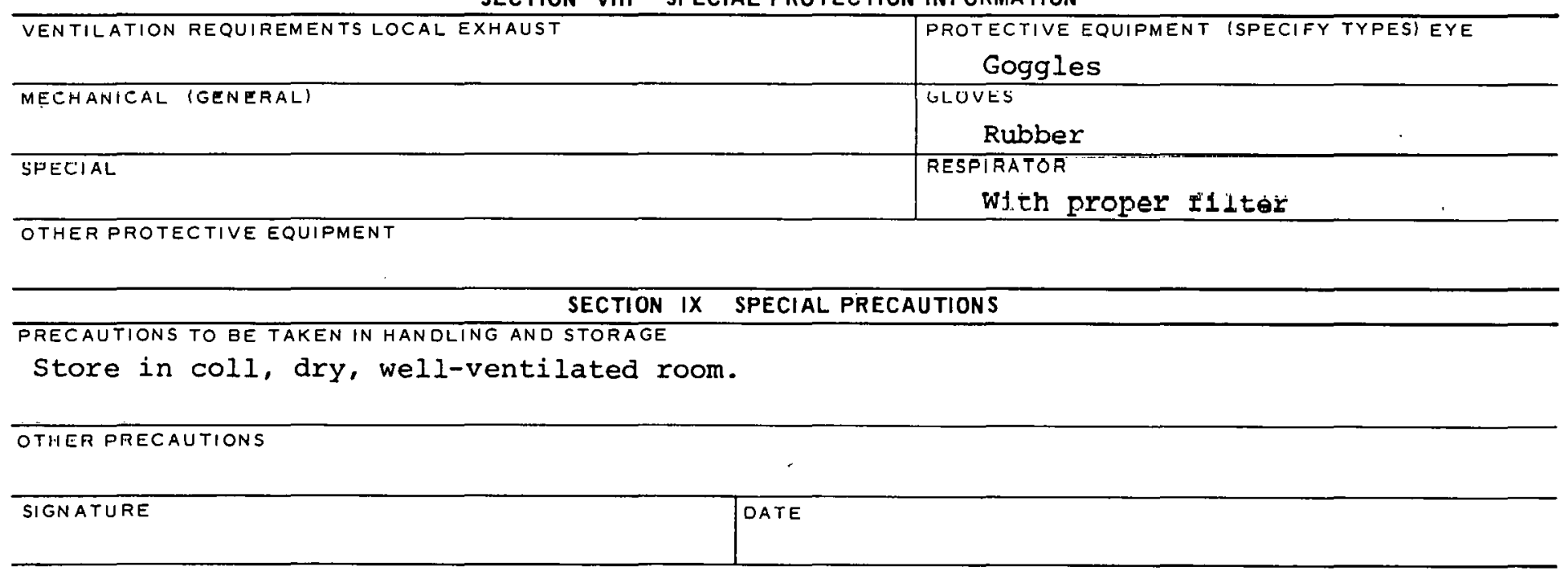




\section{3-228-5665 \\ P-Chlorobenzoyl Chloride}

MATERIAL SAFETY

DATA SHEET

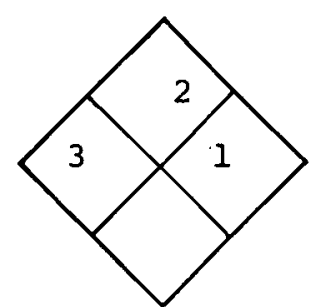

\section{SECTION I SOURCE AND NOMENCLATURE}

MANU FACTURER'S NAME

ADORESS (NUMBER, STREET, CITY, STATE, ZIP CODE)

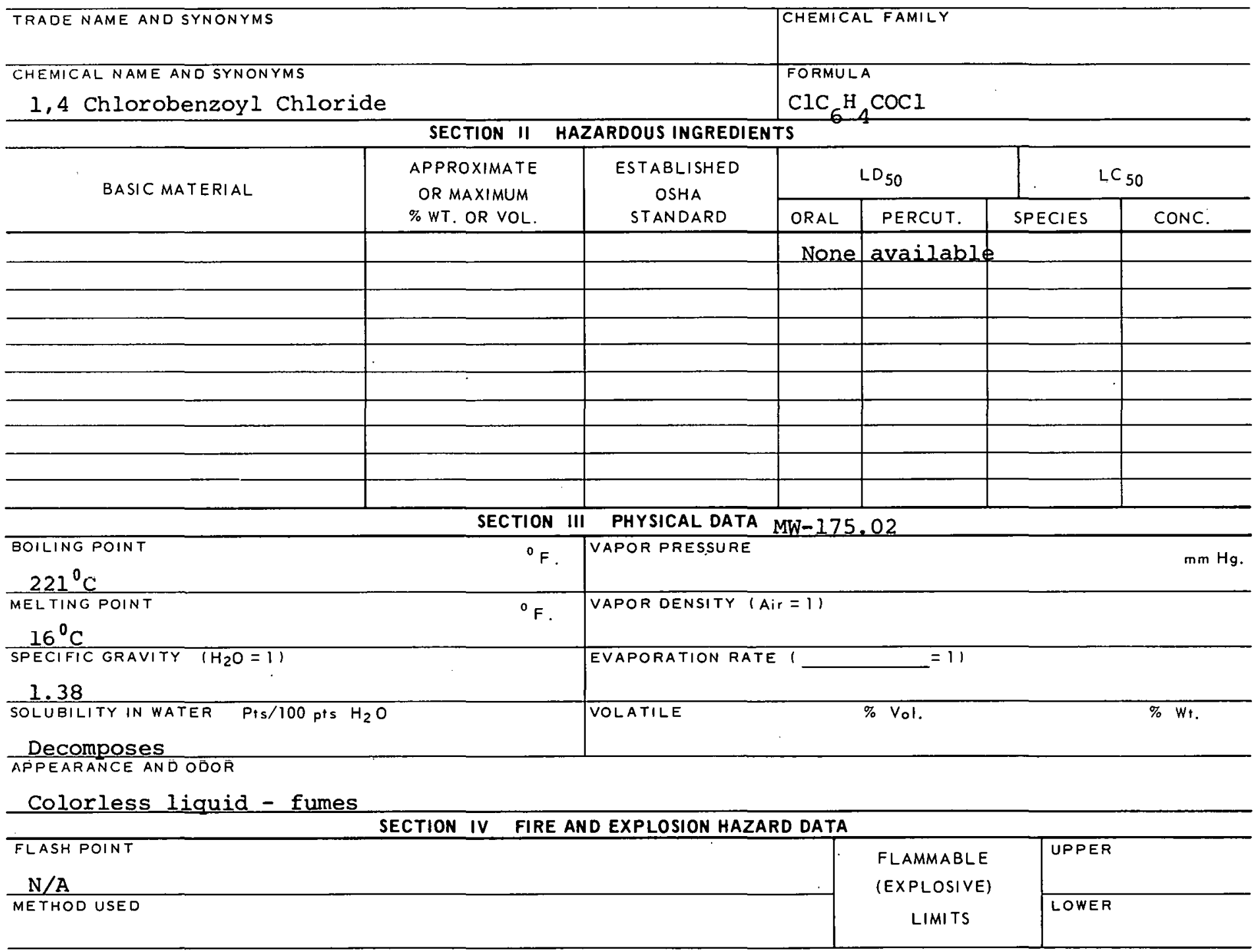

EXTINGUISHING MEDIA

Water spray, foam, carbon dioxide or dry chemical.

SPECIAL FIRE FIGHTING PROCEDURES

Full protective clothing.

UNUSUAL FIRE AND EXPLOSION HAZARDS 
TOXIC LEVEL

None available

PRINCIPLE ROUTES OF ABSORBTION

Inhalation, ingestion REVELANT SYMPTOMS OF EXPOSURE

mouth and digestive tract burns.

EFFECTS OF CHRONIC EXPOSURE

$--$

EMERGENCY AND FIRST AID PROCEDURES Inhalation - rest, artificial respiration as needed;

ingestion-drink water, milk of magnesia, get medical aid; wash eyes \& skin.

\section{SECTION VI REACTIVITY DATA}

CONDITIONS CONTRIBUTING TO INSTABILITY

CONDITIONS CONTRIBUTING TO HAZARDOUS POLYMERIZATION

INCOMPATABILITY (MATERI.ALS TO AVOID)

Dimethyl sulfoxide

HAZARDOUS DECOMPOSITION PRODUCTS

When heated, phosgene is produced

SECTION VII SPILL OR LEAK PROCEDURES

STEPS TO BE TAKEN IN CASE MATERIAL IS RELEASED OR SPILLED

WASTE DISPOSAL METHOD

SECTION VIII SPECIAL PROTECTION INFORMATION

\begin{tabular}{l|l}
\hline VENTILATION REQUIREMENTS LOCAL EXHAUST & $\begin{array}{l}\text { PROTECTIVE EQUIPMENT ISPECIFY TYPESIEYE } \\
\text { Goggles }\end{array}$ \\
\hline MECHANICAL IGENERAL) & GLOVES \\
Rubber \\
\hline SPECIAL & RESPIRATOR \\
With proper filter
\end{tabular}

OTHER'PROTECTIVE EQUIPMENT

SECTION IX SPECIAL PRECAUTIONS

PRECAUTIONS TO BE TAKEN IN HANDLING AND STORAGE

Store in cool, dry, well-ventilated location.

OTHER PRECAUTIONS

SIGNATURE

OATE

$228-5665 B$ 


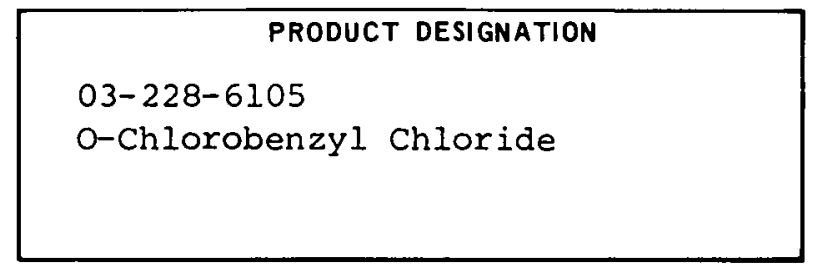

\section{MATERIAL SAFETY \\ DATA SHEET}

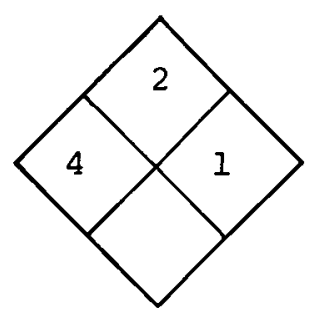

SECTION I SOURCE AND NOMENCLATURE

\begin{tabular}{l|l}
\hline MANUFACTURER'S NAME & EMERGENCY TELEPHONE NO. \\
\hline ADDRESS (NUMBER, STREET, CITY, STATE, ZIP CODE)
\end{tabular}

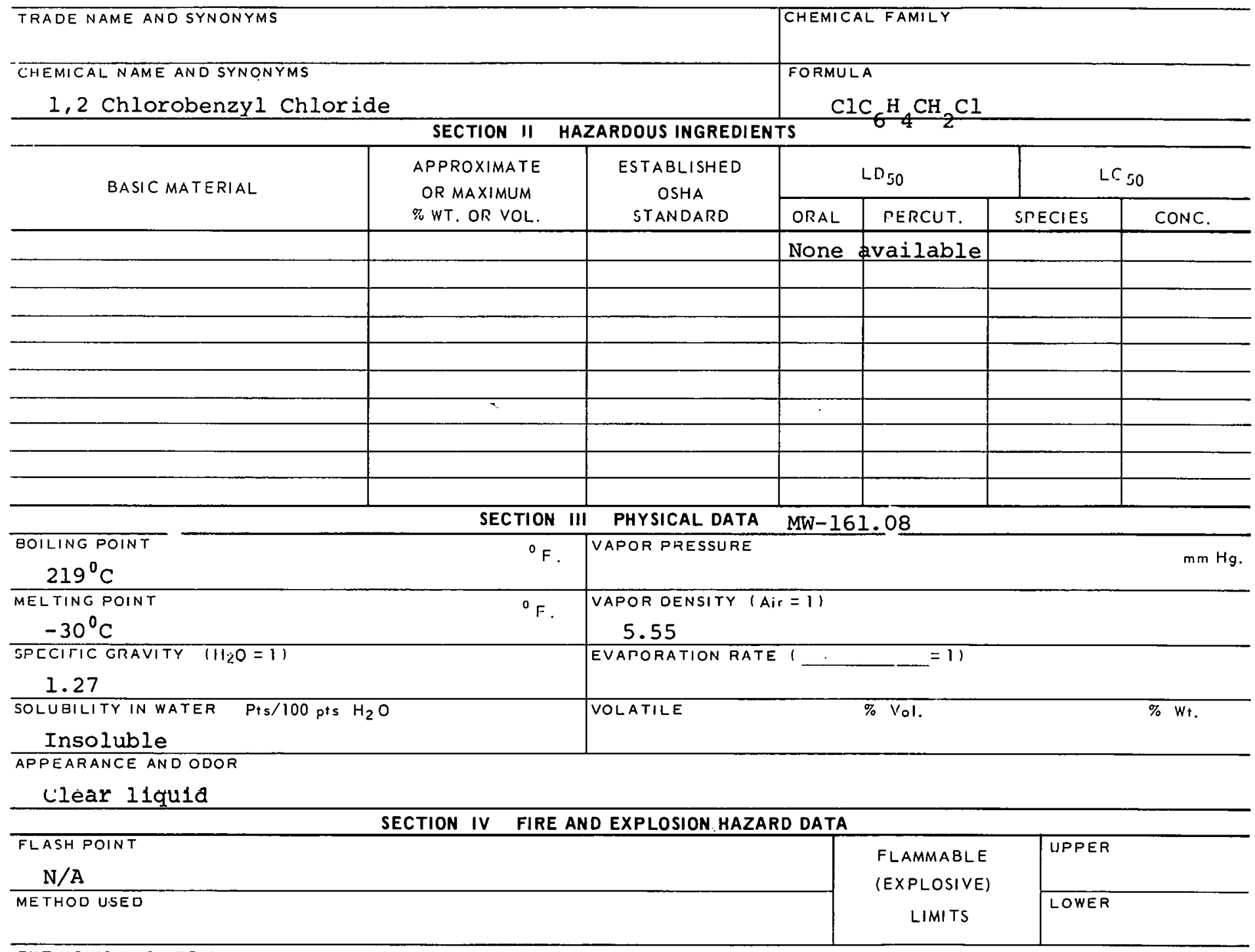

EXTINGUISHING MEDIA

Water spray, dry chemical, carbon dioxide

SPECIAL HIHE FIGHIING NHULEUUKES

UNUSUAL FIRE AND EXPLOSION HAZAROS 


\section{PRODUCT DESIGNATION \\ O-Chlorobenzyl Chloride}

SECTION $V$ HEALTH HAZARD DATA

TOXIC LEVEL

None available

PRINCIPLE ROUTES OF ABSORBTION

Inhalation, ingestion

CARCINOGENIC

SKIN AND EYE

IRRITATION

High

REVELANT SYMPTOMS OF EXPOSURE EYe, nose \& throat irritation; severe burns to mouth and digestive tract if swallowed.

EFFECTS OF CHRONIC EXPOSURE

--

EMERGENCY AND FIRST AIDPROCEDURES If inhaled - rest; if swallowed - give emetic, get medical aid; wash skin \& eyes.

\begin{tabular}{l} 
SECTION VI REACTIVITY DATA \\
\hline CONDITIONS CONTRIBUTING TO INSTABILITY \\
CONDITIONS CONTRIBUTING TO HAZARDOUS POLYMERIZATION May undergo exothermic condensatj.on in \\
pYesence of COpper aluminum, iron, zinc, magnesium, tin and other metals.
\end{tabular}
INCOMPATABILITY (MATERIALS TO AVO! D!

HAZARDOUS DECOMPOSITION PRODUCTS

SECTION VII SPILL OR LEAK PROCEDURES

STEPS TO BE TAKEN IN CASE MATERIAL IS RELEASED OR SPILLEO

Flush spills with large quantities of water and ventilate area to remove vapors. WASTE DISPOSAL METHOD

\begin{tabular}{l|l}
\hline \multicolumn{1}{c}{ SECTION VIII SPECIAL PROTECTION INFORMATION } \\
\hline VENTILATION REQUIREMENTS LOCAL EXHAUST & $\begin{array}{c}\text { PROTECTIVE EQUIPMENT (SPECIFY TYPES) EYE } \\
\text { Goggles }\end{array}$ \\
\hline MECHANICAL IGENERAL) & $\begin{array}{c}\text { GLOVES } \\
\text { Rubber }\end{array}$ \\
\hline SPECIAL & $\begin{array}{c}\text { RESPIRATOR } \\
\text { With proper filter }\end{array}$ \\
\hline
\end{tabular}

OTIIEAPROTECTIVE EQUIFMEINT

\section{SECTION IX SPECIAL PRECAUTIONS}

PRECAUTIONS TO BE TAKEN IN HANDLING AND STORAGE

OTHER PRECAUTIONS

SIGNATURE

DATE

$228-6105 B$ 

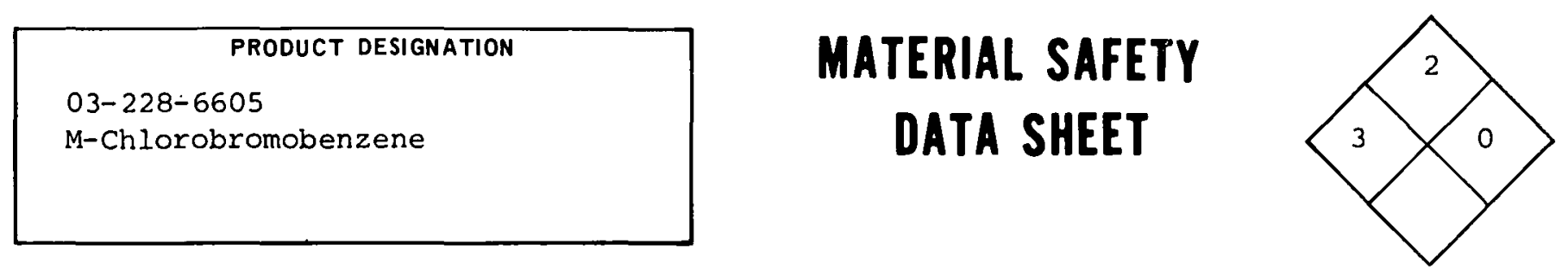

SECTION I SOURCE AND NOMENCLATURE

\begin{tabular}{l|l}
\hline MANUFACTURER'S NAME & EMERGENCY TELEPHONE NO. \\
\hline ADDRESS INUMBER, STREET, CITY, STATE, ZIP CODE) &
\end{tabular}

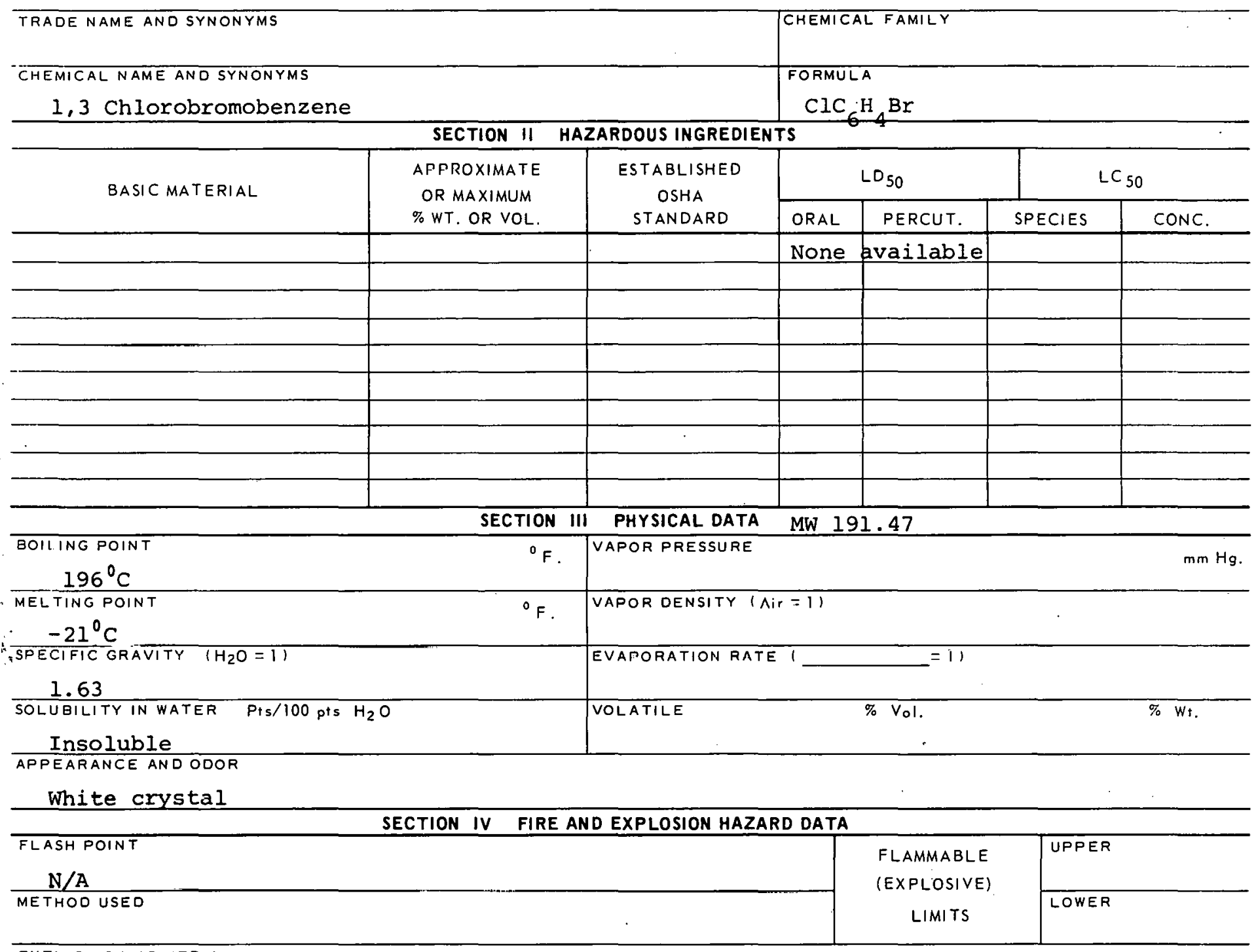

EXTINGUISHING MEDIA

Water spray, carbon dioxide, foam, dry chemical

- SPECIAL FIRE FIGHTING PROCEDURES

Wear goggles and self-contained breathing apparatus.

UNUSUAL FIRE AND EXPLOSION HAZAROS 
TOXIC LEVEL

Not available

PRINCIPLE ROUTES OF ABSORBTION

Inhalation, ingestion, skin

REVELANT SYMPTOMS OF EXPOSURE CYAROSis

drowsiness, unconsciousness.

EFFECTS OF CHRONIC EXPOSURE

Lung, liver \& kidney damage

EMERGENCY ANO FIRST AID PROCEDURES

Inhalation - rest; if swallowed - give emetic; get medical aid; wash eves and skin.

\section{SECTION VI REACTIVITY DATA}

CARCINOGENIC SKIN AND EYE IRRITATION Severe

\section{CONOITIONS CONTRIBUTING TO INSTABILITY}

CONDITIONS CONTRIBUTING TO HAZARDOUS POLYMERIZATION

INCOMPATABILITY (MATERIALS TO ÁVOTDI

HAZARDOUS DECOMPOSITION PROOUCTS

SEC̈IIUUN VII SHILL UK LLAK PRUCEDURES

STEPS TO BE TAKEN IN CASE MATERIAL IS RELEASED OR SPILLED

WASTE DISPOSAL METHOD

\section{SECTION VIII SPECIAL PROTECTION INFORMATION}

\begin{tabular}{|c|c|}
\hline VENTILATION REQUIREMENTS LOCAL EXHAUST & $\begin{array}{l}\text { PROTFCTIVE EQUIPMENT (SPECIFY TYPES) EYE } \\
\text { GOggleS }\end{array}$ \\
\hline MELHANILAL IGENERALI & $\begin{array}{l}\text { GLOVES } \\
\text { POlyvinyl alcohol }\end{array}$ \\
\hline SPECIAL & $\begin{array}{l}\text { RESPIRATOR } \\
\text { With proper tilleri }\end{array}$ \\
\hline
\end{tabular}

OTHER PROTECTIVE EQUIPMENT

\section{SECTION IX SPECIAL PRECAUTIONS}

PRECAUTIONS TO BE TAKEN IN HANDLING AND STORAGE

OTHER PRECAUTIONS 
03-228-6665

4 Chlorobromobenzene

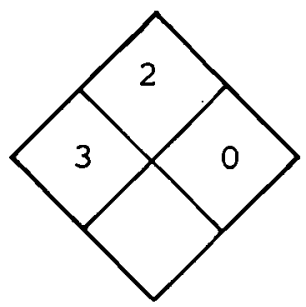

SECTION I SOURCE AND NOMENCLATURE

\section{MANU FACTURER'S NAME \\ 1, 4 Chlorobromobenzene \\ CHEMICAL NAME AND SYNONYMS \\ P Chlorobromobenzene}

ADDRESS (NUMBER, STREET, CITY, STATE, ZIP CODE)

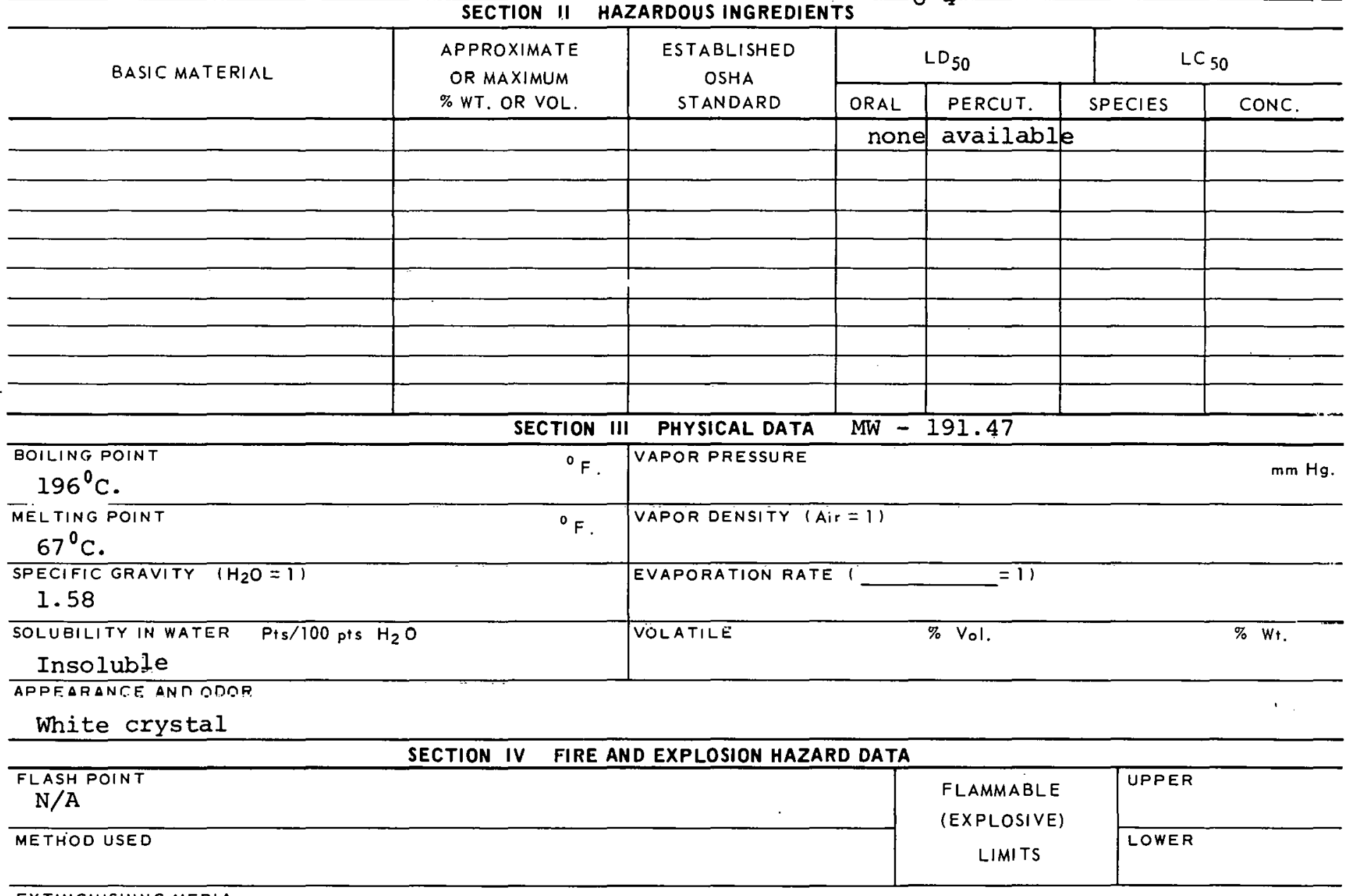

\section{EXTINCUISIINA MEDIA}

water spray, carbon dioxide, foam, dry chemical.

SPECIAL FIRE FIGHTING PROCEOURES

Wear goggles and self-contained breathing apparatus. 


\section{Chlorobromobenzene} SECTION $V$ HEALTH HAZARD DATA

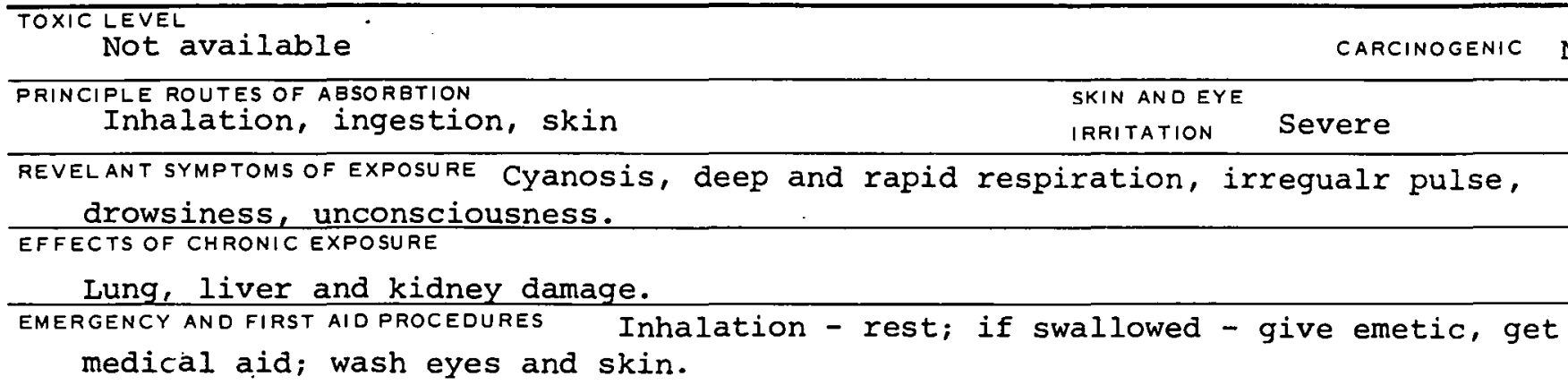




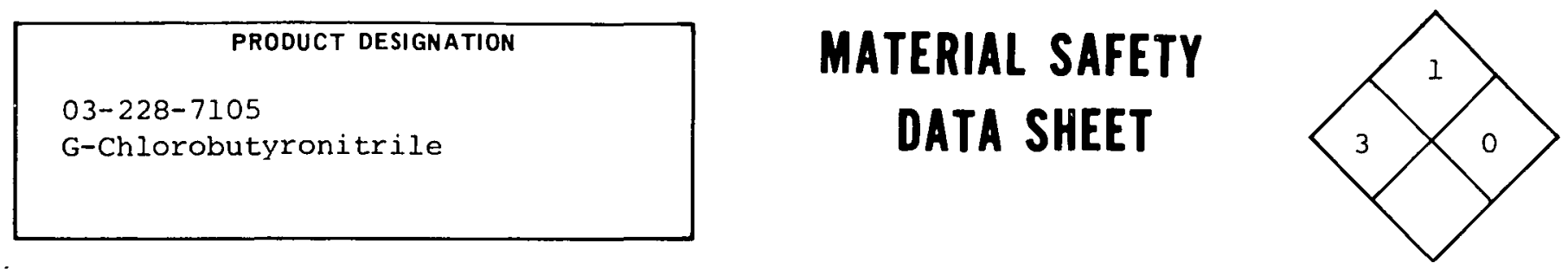

SECTION I- SOURCE AND NOMENCLATURE

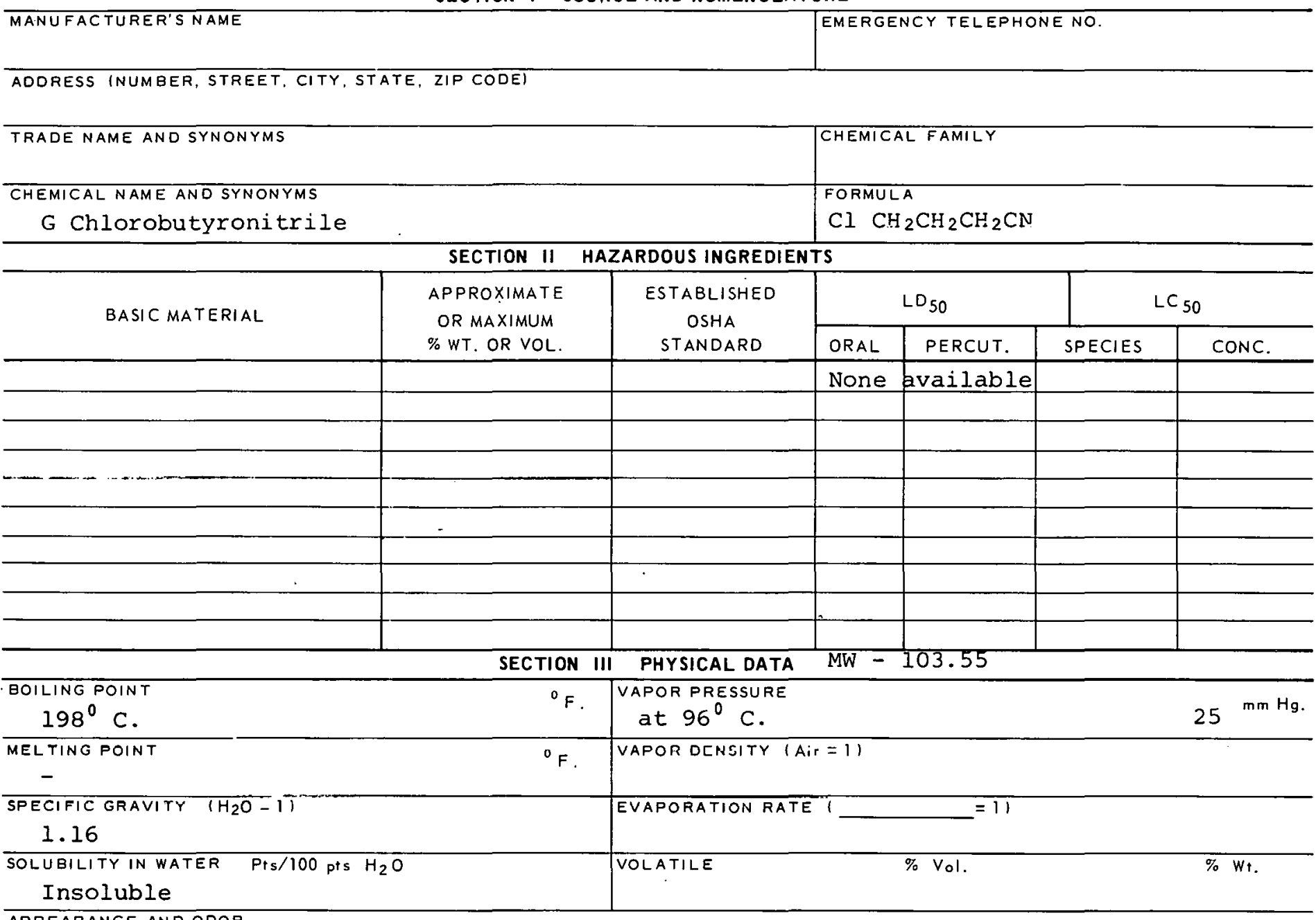

APPEARANCE AND ODOR

Tu.iquid

SECTION IV FIRE AND EXPLOSION HAZARD DATA

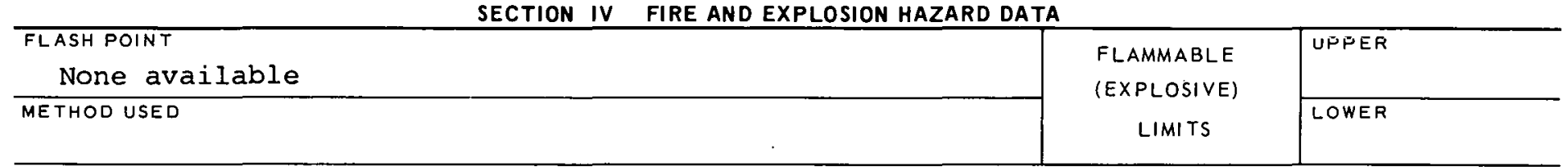

EXTINGUISHING MEDIA

Alcohol foam, carbon dioxide, dry chemical.

SPECIAL FIRE FIGHTING PROCEDURES

Self-contained breathing apparatus.

UNUSUAL FIRE ANDEXPLOSION HALARUS 
TOXIC LEVEL

None available

PRINCIPLE ROUTES OF ADSOROTION

Inhalation, ingestion, skin

REVELANT SYMPTOMS OF EXPOSURE Dizziness: rapid respiration, headache, drowsiness, drop in blood

pressure, pulse, delayed symptoms.

EFFECTS OF CHRONIC EXPOSURE

Eve irxitation, loss of appetite and weight, mental deterioration.

EMERGENCY AND FIRST AIDPROCEDURES If swallowed - inhalation of amyl nitrite, induce

vomiting or gastric lavage; wash skin \& eyes; inhalation - rest.

\section{SECTION VI REACTIVITY DATA}

CONOITIONS CONTRIBUTING TO INSTABILITY

CONDITIONS CONTRIBUTING TO HAZARDOUS POLYMERIZATION

INCOMPATABILITY (MATERIALS TO AVOID)

HAZARDOUS DECOMPOSITION PRODUCTS

When heated to decomposition emits highly toxic cyanide fumes

SECTION VII SPILL OR LEAK PROCEDURES

STEPS TO BE TAKEN IN CASE MATERIAL IS RELEASED OR SPILLED

Absorb with paper towels - evaporatc in hood.

WASTE DISPOSAL METHOD Add excess alcoholic sodium hydroxide, after one hour, evaporate alcohol, then add calcium hypochloride.

SECTION VIII SPECIAL PROTECTION INFORMATION

\begin{tabular}{l|l}
\hline VENTILATION REQUIREMENTS LOCAL EXHAUST & $\begin{array}{l}\text { PROTECTIVE EQUIPMENT ISPECIFY TYPES) EYF } \\
\text { GOgGlES }\end{array}$ \\
\hline MECHANICAL IGENERAL) & GLOVES \\
Rubber \\
\hline SPECIAL & RESPIRATRR \\
& ECIf eontQined \\
\hline
\end{tabular}

DTHER PROTECTIVE EQUIPMENT

Protective apron.

PRECAUTIONS TO BE TAKEN IN HANDLING AND STORAGE

SECTION IX SPECIAL PRECAUTIONS

OTHER TRECAUTIONS

SIGNATURE

DATE

$228-7105 B$ 


\begin{tabular}{|l|}
\hline PRODUCT DESIGNATION \\
Chloroform (trichloromethane) \\
$03-228-9135$ \\
$03-228-9215$
\end{tabular}

\section{MATERIAL SAFETY DATA SHEET}

SECTION I SOURCE AND NOMENCLATURE

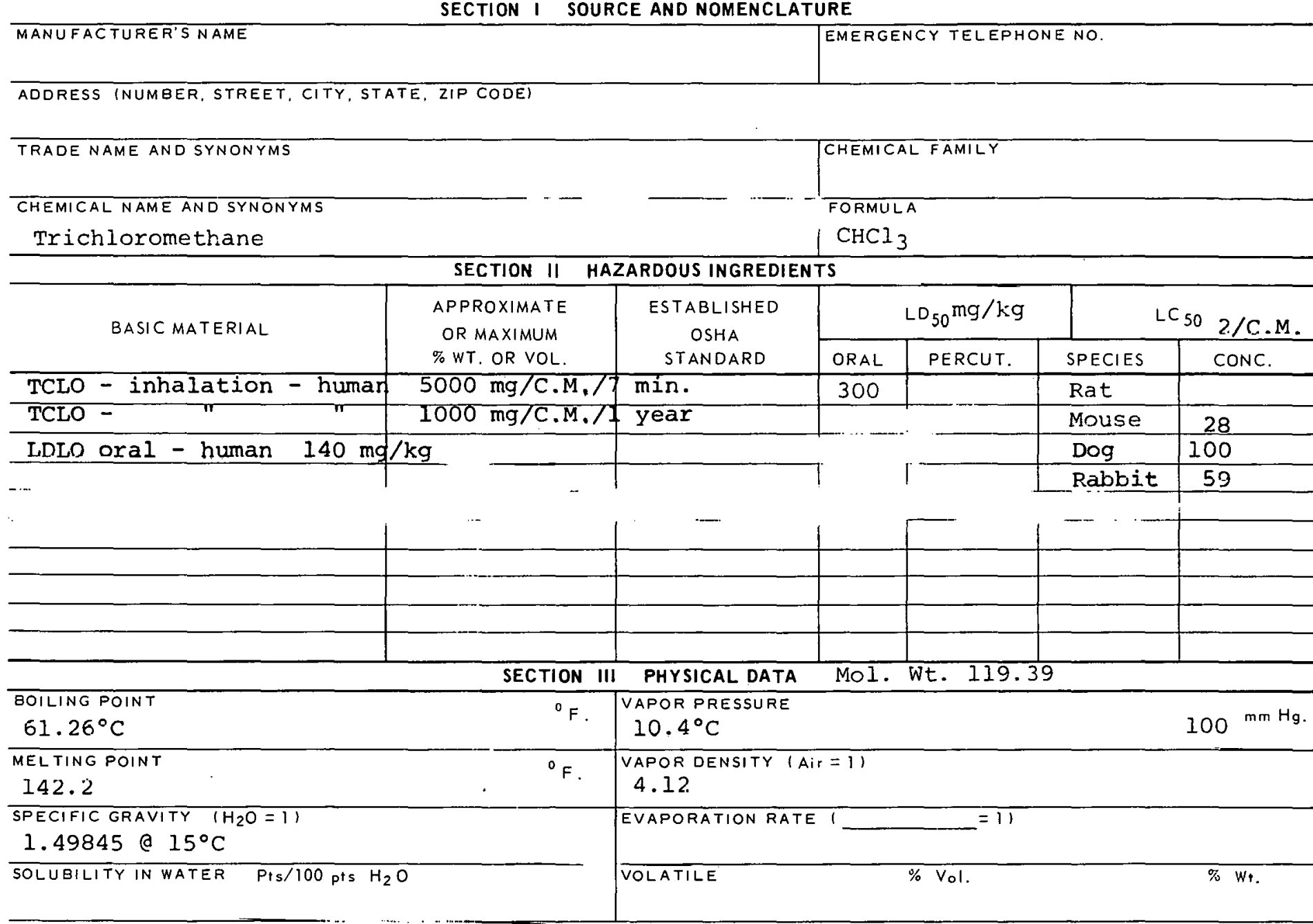

AFTEARANCE AND ODOR

Heavy, water-white, volatile, sweet-tasting liquid with characteristic odor. SECTION IV FIRE AND EXPLOSION HAZARD DATA

FLASH POINT
Not flammable

METHOD USED

EXTINGUISHING MEDIA

Water, foam, $\mathrm{CO}_{2}$, ctc.

SPECIAL FIRE FIGHTING PROCEDURES

Wear self-contained breathing apparatus.

UNUSUAL FIRE AND EXPLOSION HAZARRS

Emits toxic and irritating fumes.
FLAMMABLE

(EXPLOSIVE)

LIMITS

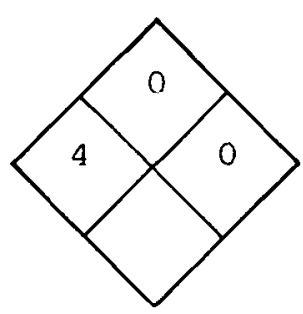


PRODUCT DESIGNATION

Cnloroform

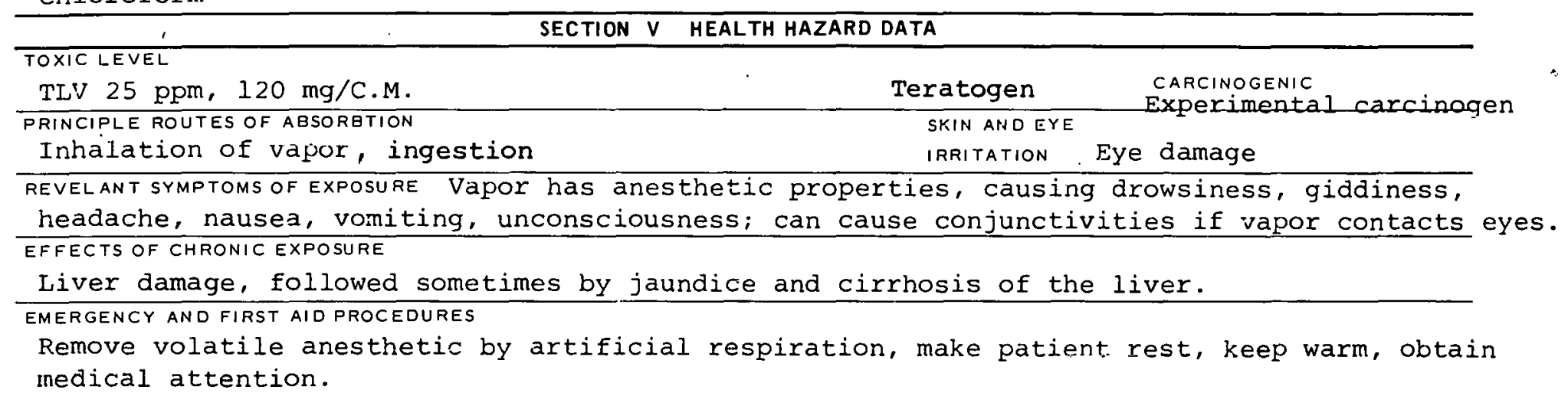

\begin{tabular}{l}
\hline SECTION VI REACTIVITY DATA \\
\hline CONDITIONS CONTRIBUTING TO INSTABILITY \\
\hline CONDITIONS CONTRIBUTING TO HAZARDOUS POLYMERIZATION
\end{tabular}

CONDITIONS CONTRIBUTING TO HAZARDOUS POLYMERIZATION

INCOMPATABILITY (MATERIALS TO AVOIO) Alkali metals, aretone, aluminum, disilane, llagnesium, nitroyen tetroxide, sodium methylate. HAZARDOUS DECOMPOSITION PRODUCTS

SECTION VII SPILL OR LEAK PROCEDURES

STEPS TO BE TAKEN IN CASE MATERIAL IS RELEASED OR SPILLED

WASTE OISPOSAL METHOD

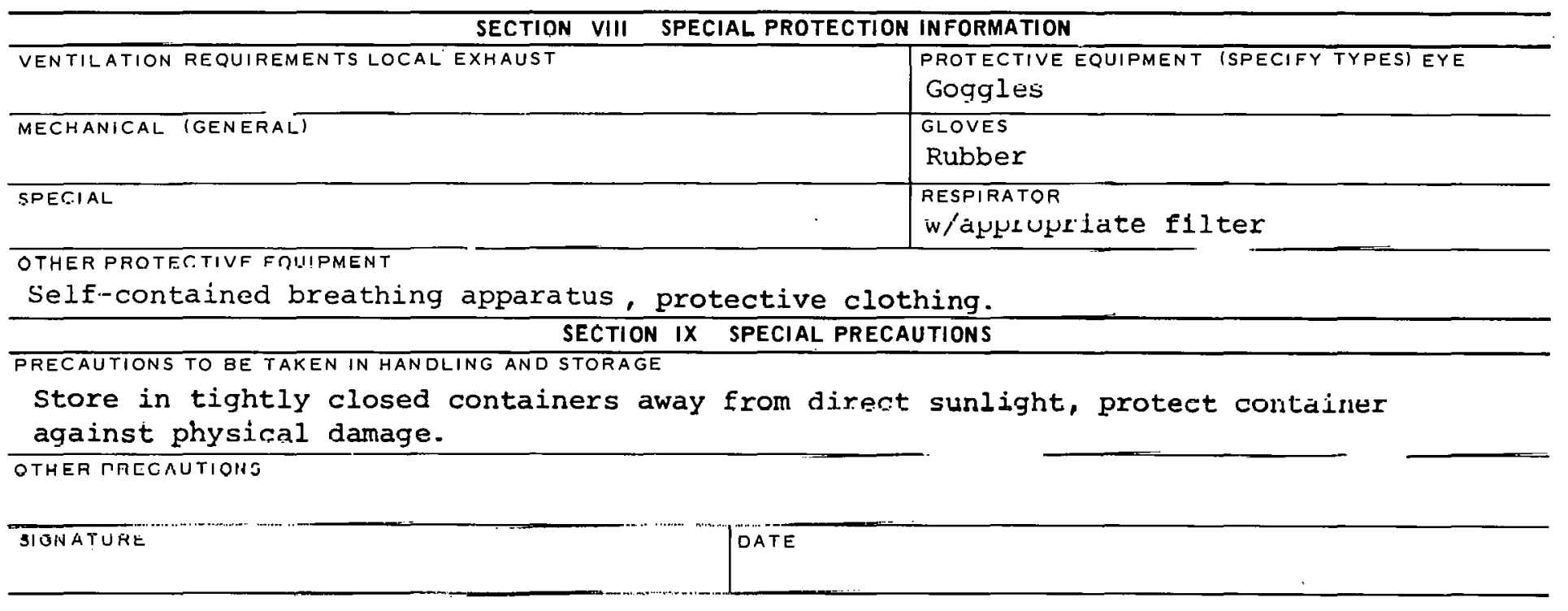


03-228-9605

PRODUCT DESIGNATION

2 Chloro-5-Hydroxytoluene
MATERIAL SAFETY

DATA SHEET

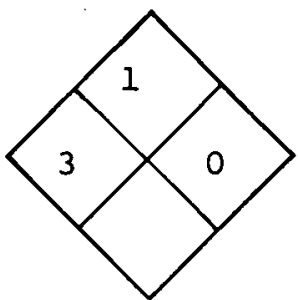

SECTION I SOURCE AND NOMENCLATURE

MANUFACTURER'S NAME

\begin{tabular}{|l|l} 
& EMERGENCY TELEPHONE NO. \\
\hline
\end{tabular}

ADORESS (NUMBER, STREET, CITY, STATE, ZIP CODE)

TRADE NAME AND SYNONYMS

CHEMICAL FAMILY

CHEMICAL NAME AND SYNONYMS

FORMULA

6 Chloro-M-Cresol

$\mathrm{Cl}\left(\mathrm{C}_{6} \mathrm{H}_{3}\right)(\mathrm{OH}) \mathrm{CH}_{3}$

\begin{tabular}{|c|c|}
\hline Chloro-M-Cresol \\
\hline BASIC MATERIAL \\
\hline \\
\hline \\
\hline
\end{tabular}
SECTION II HAZARDOUS INGREDIENTS

\begin{tabular}{l} 
SECTION II HAZARDOUS INGREDIENTS \\
\hline \multirow{2}{*}{$\begin{array}{c}\text { APPROXIMATE } \\
\text { OR MAXIMUM } \\
\% \text { WT. OR VOL. }\end{array}$}
\end{tabular}

BOILING POINT

$235^{\circ} \mathrm{C}$.

MELTING POINT

$66^{\circ} \mathrm{C}$.

SPECIFIC GRAVITY $\left(\mathrm{H}_{2} \mathrm{O}=.1\right)$

SOLUBILITY IN WATER P\$s/100 pts $\mathrm{H}_{2} \mathrm{O}$

38

APPEARANCE AND ODOR

White crystals

SECTION III PHYSICAL DATA

${ }^{\circ} \mathrm{F}$.

$M W-142.59$

${ }^{\mathrm{o}} \mathrm{F}$. VAPOR DENSITY (Air $=1)$

EVAPORATION RATE I

$=11$

VOLATILE

\% Vol.

$\% W_{t}$.

\begin{tabular}{l|c|c} 
White crystals & SECTION IV FIRE AND EXPLOSION HAZARD DATA \\
\hline Fi_ASH POINT & FLAMMABLE & UPPER \\
NOne available & (EXPLOSIVE) & LIMITS \\
\cline { 2 - 3 } METHOD USEO & LOWER & \\
\hline
\end{tabular}

EXTINGUISHING MEDIA

Alcuhul fuam, carbon dioxide, dry chemical.

SPECIAL FIRE FIGHTING PROCEDURES

UNUSUAL FIRE ANDEXPLOSION HAZARDS 


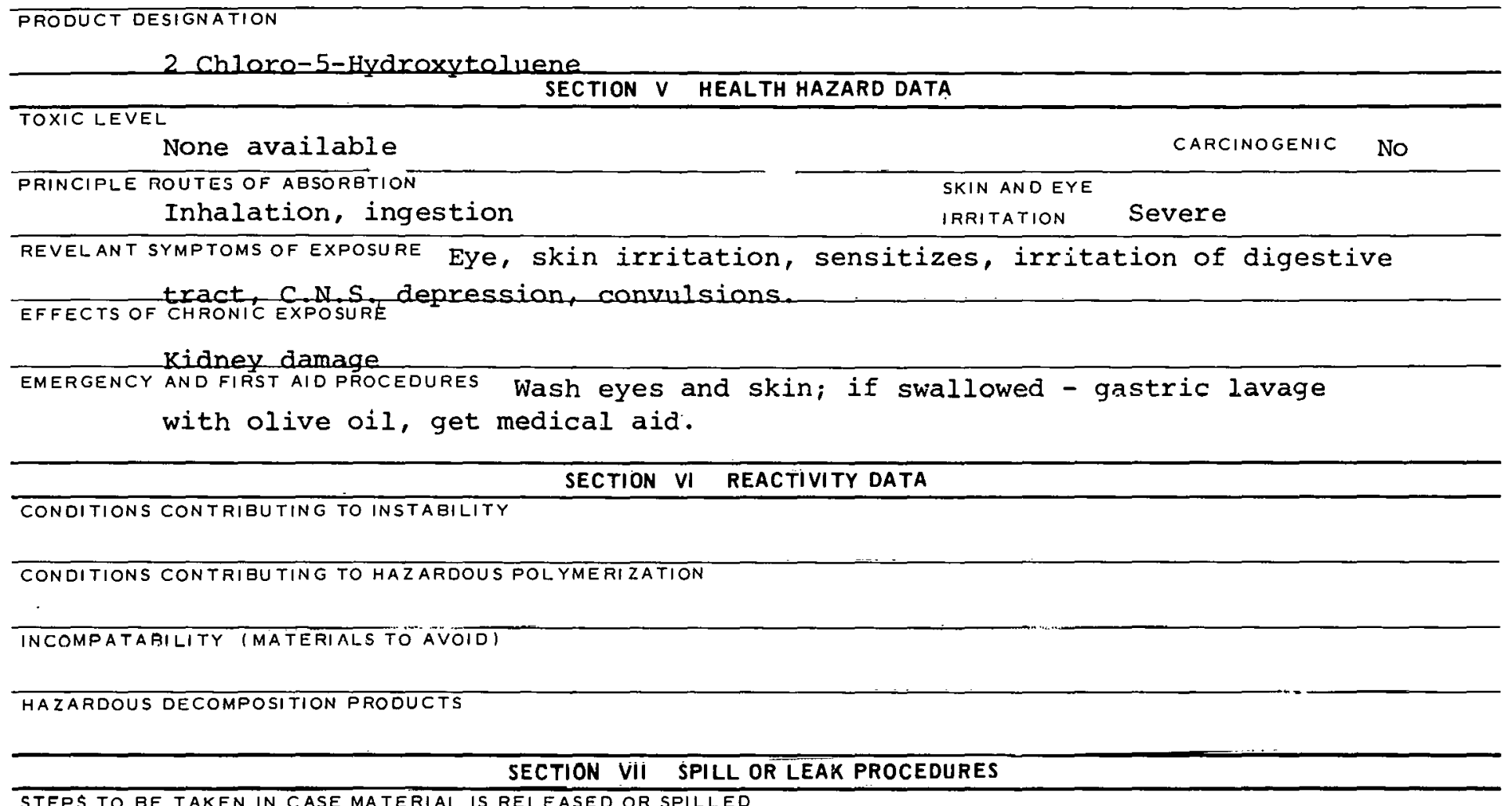
STEPS TO BE TAKEN IN CASE MATERIAL IS RELEASED OR SPILLED

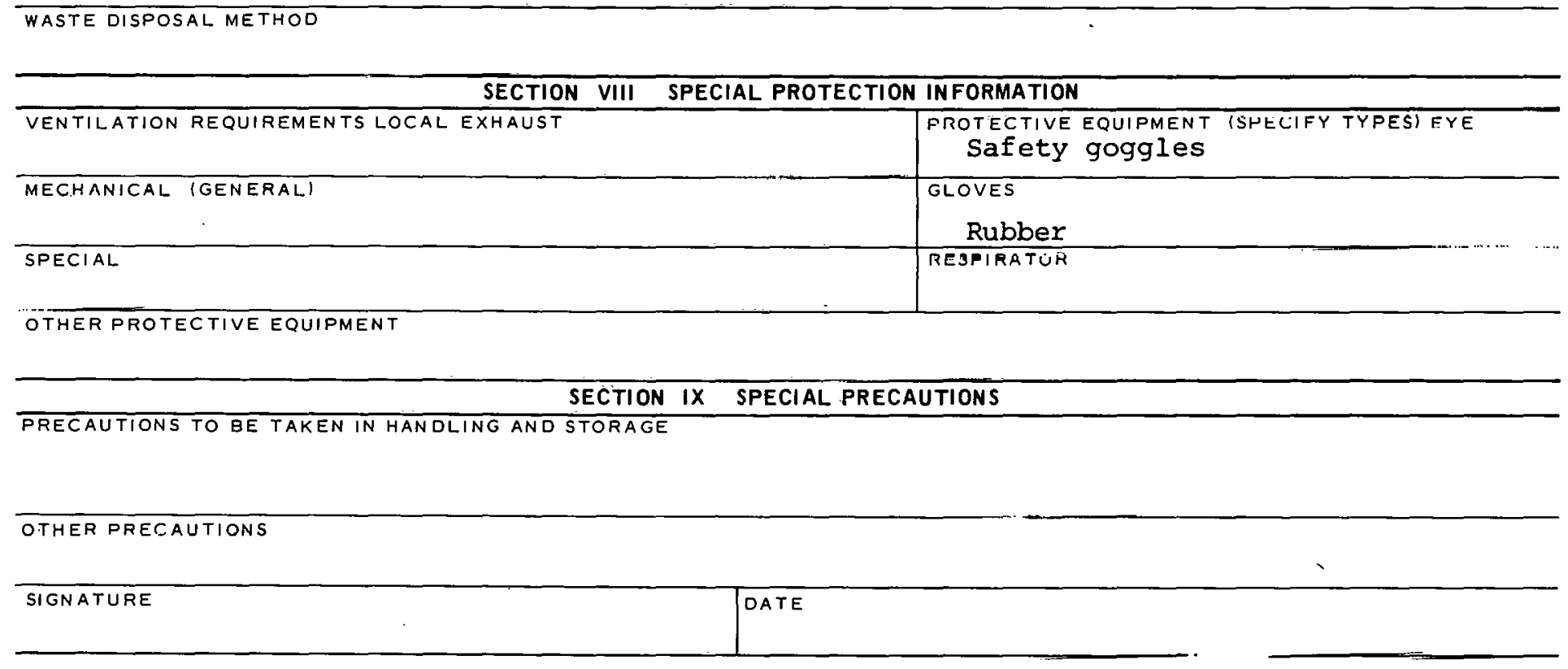




$\left.\begin{array}{|l|l|l|l|l|}\hline \text { PRODUCT DESIGNATION } \\ \text { 03-229-0206 } \\ \text { M-Chloroiodobenzene }\end{array}\right]$

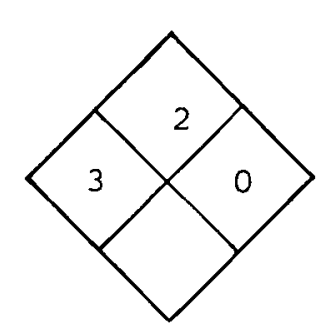

\section{SECTION I SOURCE AND NOMENCLATURE}

MANUFACTURER'S NAME
ADDRESS (NUMBER, STREET, CITY, STATE, ZIP CODE)

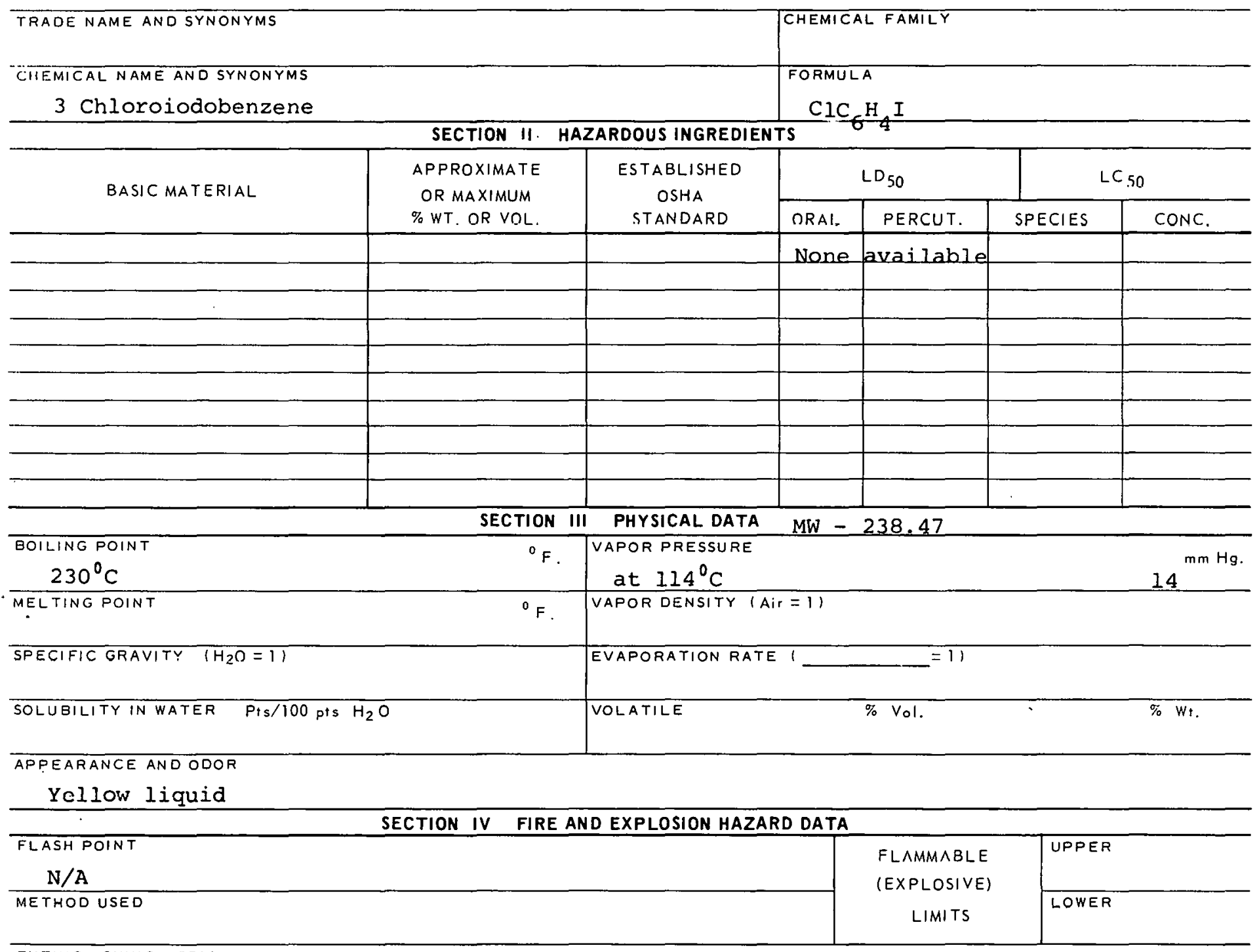

EXTINGUISHING MEDIA

Water spray, carbon dioxide, foam dry chemical

SPEGIAL FIRE FIGHTING PROCEOIJRES

Wear goggles and self-contained breathing apparatus.

UNUSUAL FIRE AND EXPLOSION HAZARDS 


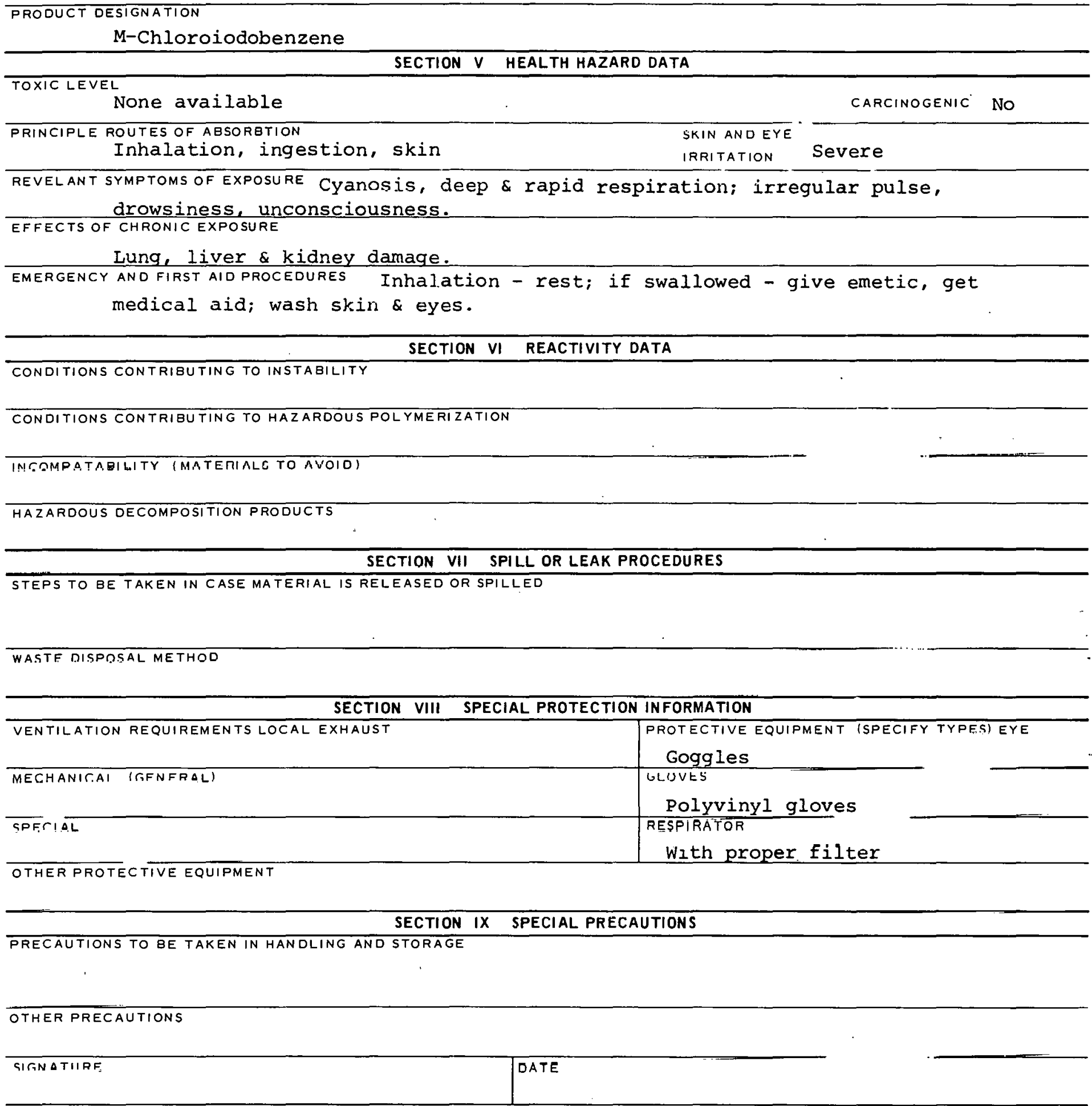




PRODUCT DESIGNATION
$03-229-0236$
O-Chloroiodobenzene

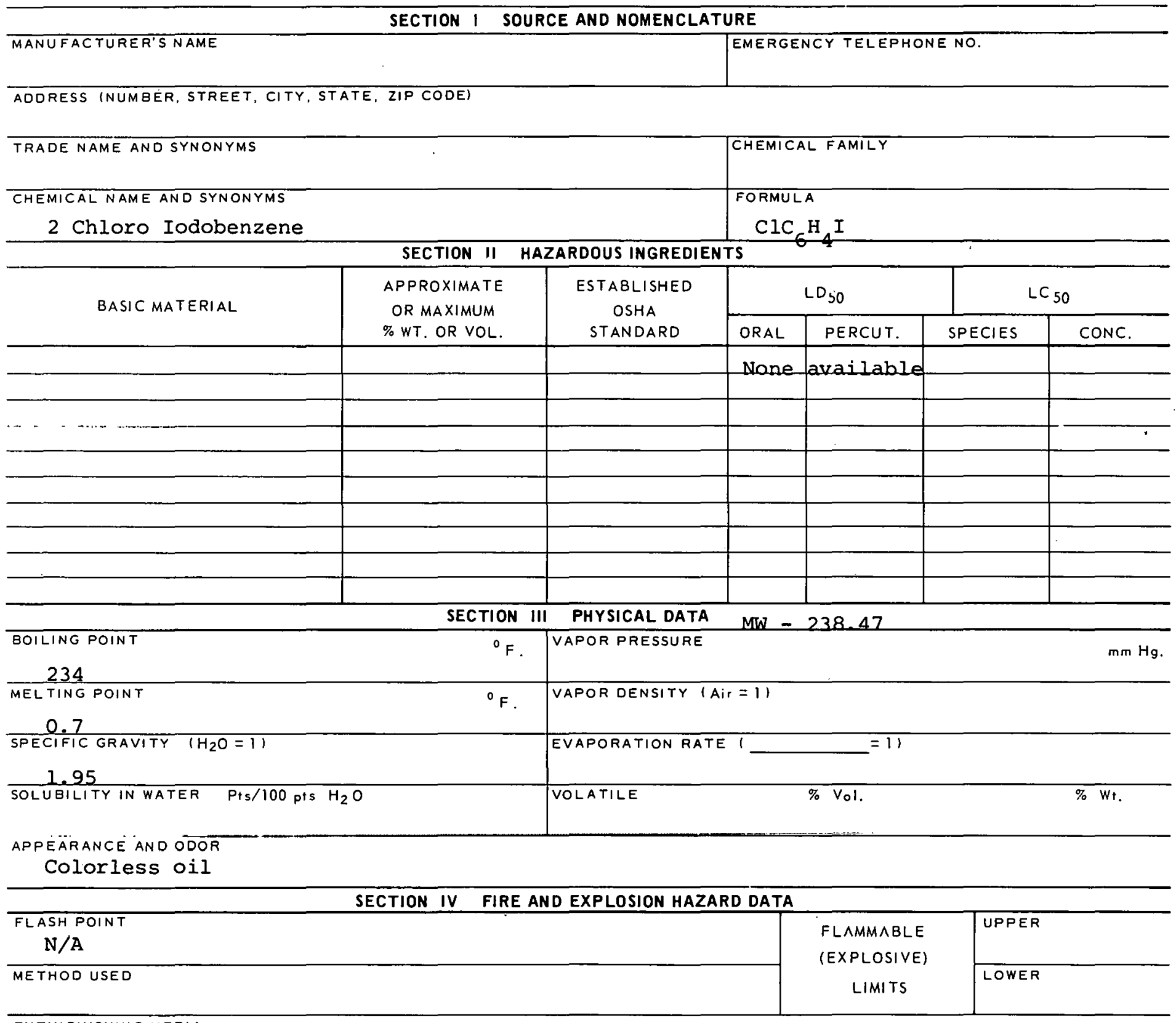

EXTINGUISHING MEDIA

Water spray, carbon dioxide, foam, dry chemical

SPECIAL FIRE FIGHTING PROCEDURES

Wear goggles and self contained breathing apparatus.

UNUSUAL FIRE AND EXPLOSION HAZARDS 
O-Chloroiodobenzene

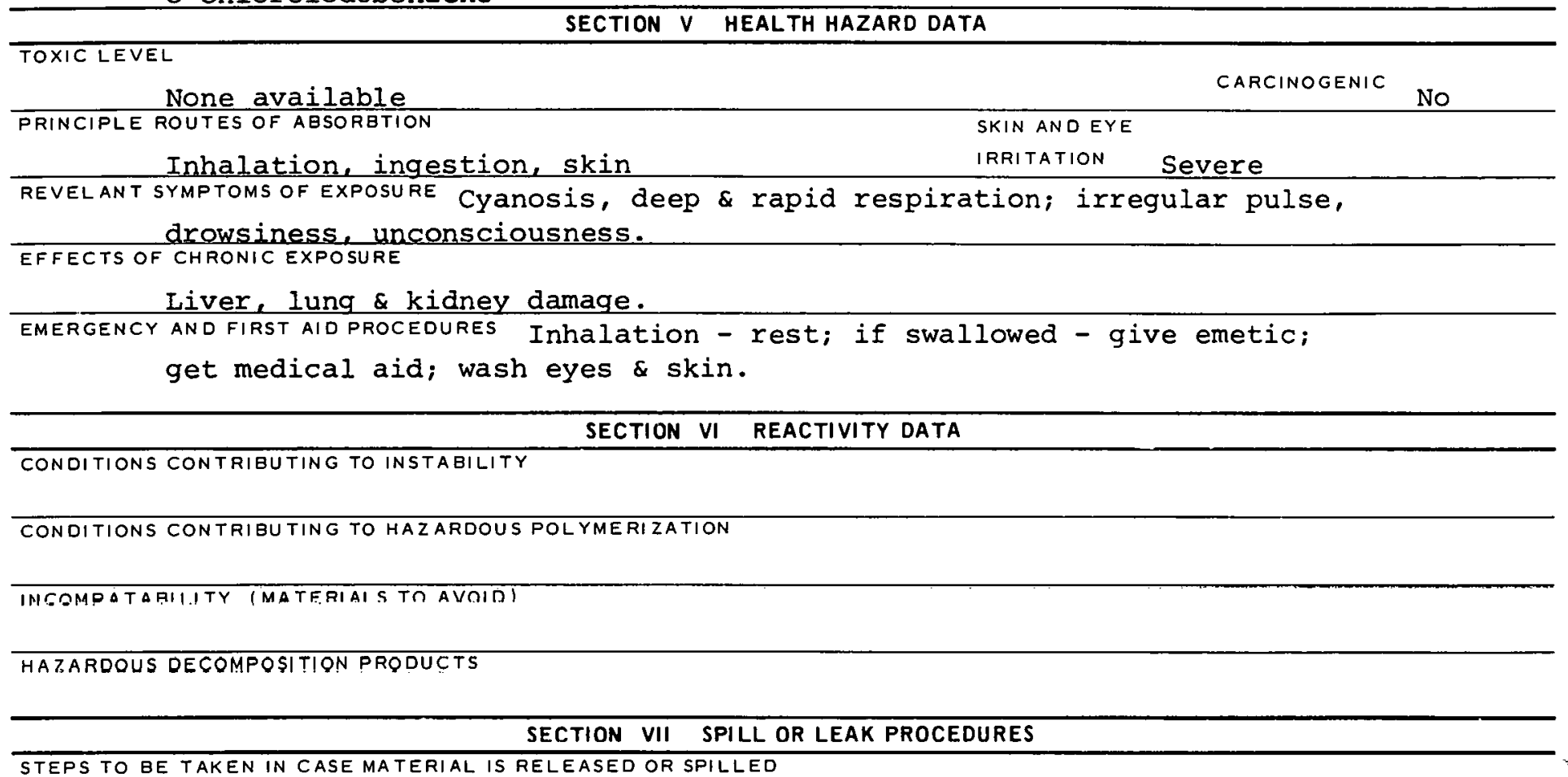

WASTE DISPOSAL METHOD

SECTION VIII SPECIAL PROTECTION INFORMATION

\begin{tabular}{|c|c|}
\hline SECTION VIII & SPECIAL PROTECTION INFORMATION \\
\hline VENTILATION REQUIREMENTS LOCAL EXHAUST & \begin{tabular}{l|l} 
& PROTECTIVE EQUIPMENT (SPECIFY TYPES)EYE \\
GOQgles
\end{tabular} \\
\hline MECHANICAL (GENERAL) & $\begin{array}{l}\text { GLOVES } \\
\text { Polyviny } 1 \text { alcohol }\end{array}$ \\
\hline SPECIAL & $\begin{array}{l}\text { RESPIRATOR } \\
\text { With proper fllter }\end{array}$ \\
\hline
\end{tabular}

\section{SECTION IX SPECIAL PRECAUTIONS}

PRECAUTIONS TO BE TAKEN IN HANDLING AND STORAGE

OTHER PRECAUTIONS

SIGNATURE

DATE

SIGNATURE

DATE

$229-0236 B$ 


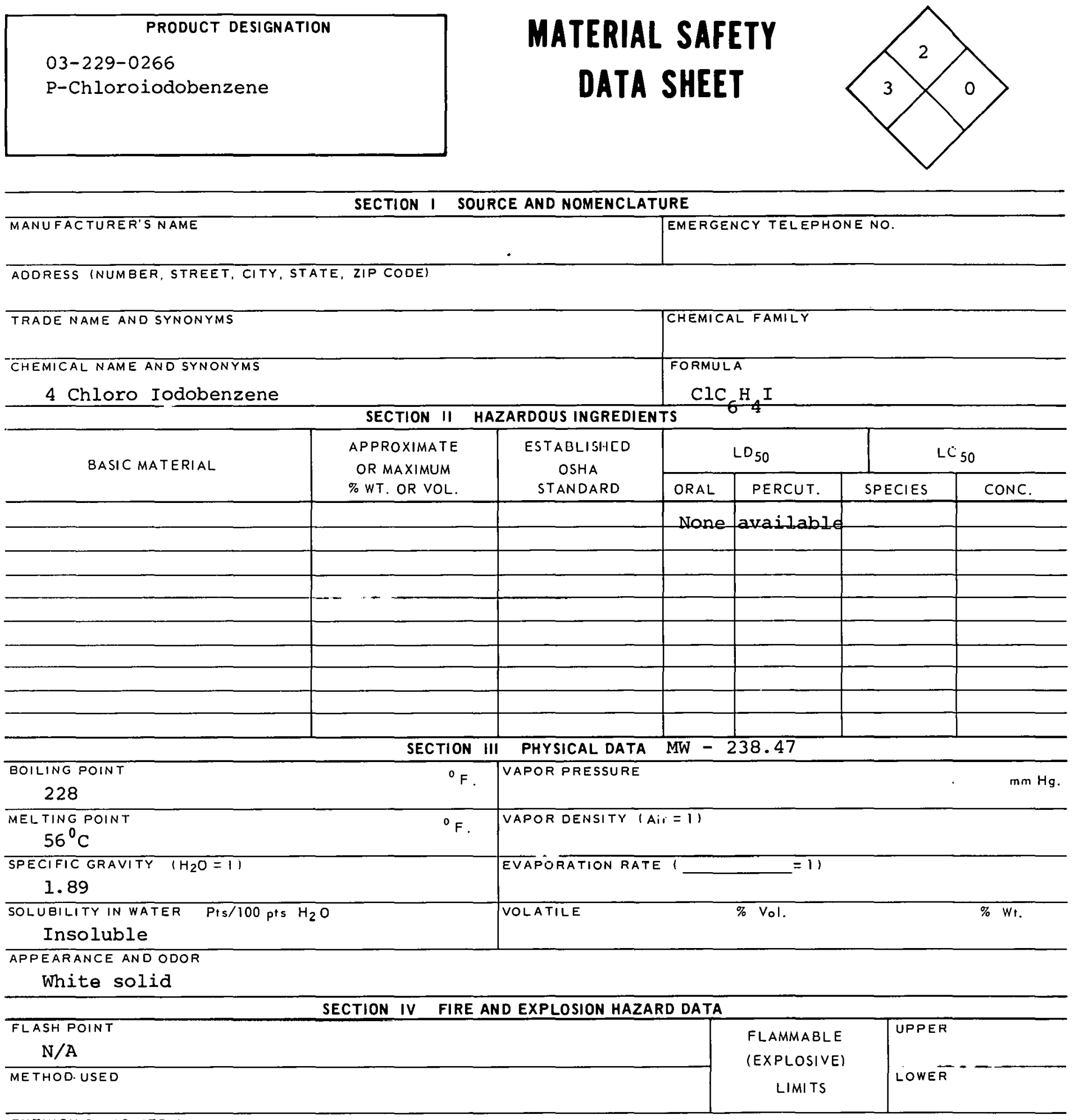

EXTINGUISHING MEDIA

Water spray, carbon dioxide, foam, dry chemical

SPECIAL FIRE FIGHTING PROCEDURES

Wear goggles and self-contained breathing apparatus.

UNUSUAL FIRE AND EXPLOSION HAZARDS 


\title{
P-Chloroiodobenzene
} SECTION $V$ HEALTH HAZARD DATA

\section{TOXIC LEVEL}

None available

PRINCIPLE ROUTES OF ABSORBTION

Inhalation, ingestion, skin

CARCINOGENIC SKIN ANDEYE IRRITATION

\section{REVELANT SYMPTOMS OF EXPOSURE Cyanosis, deep and rapid respiration, irregular pulse,}

drowsiness, unconsciousness.

EFFECTS OF CHRONIC EXPOSURE

Lung. liver \& kidney damage.

EMERGENCY AND FIRST AIO PROCEDURES Inhalation - rest; if swallowed - give emetic, get medical aid; wash eyes \& skin.

\section{SECTION VI REACTIVITY DATA}

\begin{abstract}
CONDITIONS CONTRIBUTING TO INSTABILITY
CONDITIONS CONTRIBUTING TO HA.ZARDOUS POLYMERIZATION

INCOMPATABILITY (MATERIALS TO AVOIO)

HAZARDOUS DECOMPOSITION PRODUCTS

SECTION VII SPILL OR LEAK PROCEDURES

STEPS TO BE TAKEN IN CASE MATERIAL IS RELEASED OR SPILLED
\end{abstract}

WASTE DISPOSAL METHOD

SECTION VIII SPECIAL PROTECTION INFORMATION

\begin{tabular}{l|l}
\hline VENTILATION REQUIREMENTS LOCAL EXHAUST & $\begin{array}{l}\text { PROTECTIVE EQUIPMENT (SPECIFY TYPES) EYE } \\
\text { Goggles }\end{array}$ \\
\hline MECHANICAL IGENERAL) & $\begin{array}{c}\text { GLOVES } \\
\text { POlYVinYl gloves }\end{array}$ \\
\hline GPECIAL & RESPIRATOR \\
With proper filter
\end{tabular}

OTHER PROTECTIVE EQUIPMENT

SECTION IX SPECIAL PRECAUTIONS

PRECAUTIONS TO BE TAKEN IN HANDLING AND STORAGE 


\section{PRODUCT DESIGNATION}

03-229-3216

1 Chloro-1-Nitropropane
MATERIAL SAFETY

DATA SHEET

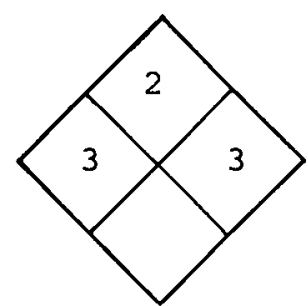

SECTION I SOURCE AND NOMENCLATURE

ADDRESS (NUMBER, STREET, CITY, STATE, ZIP CODE)

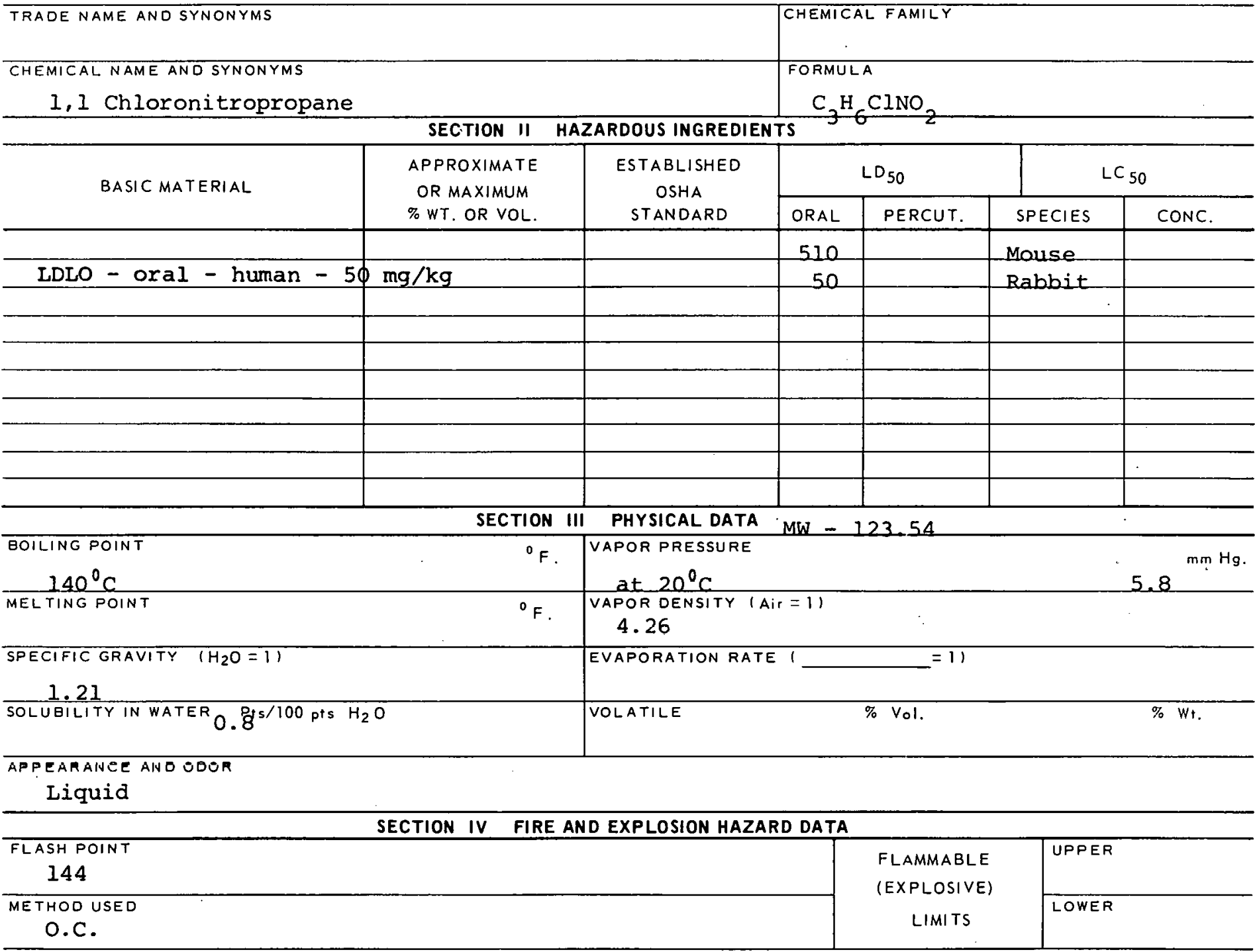

EXTINGUISHING MEDIA

Alcohol foam, water, carbon dioxide, dry chemical

SPECIAL FIRE FIGHTING PROCEOURES

UNUSUAL FIRE AN $\cap$ EXPLOSION HAZARDS

May explode when heated 


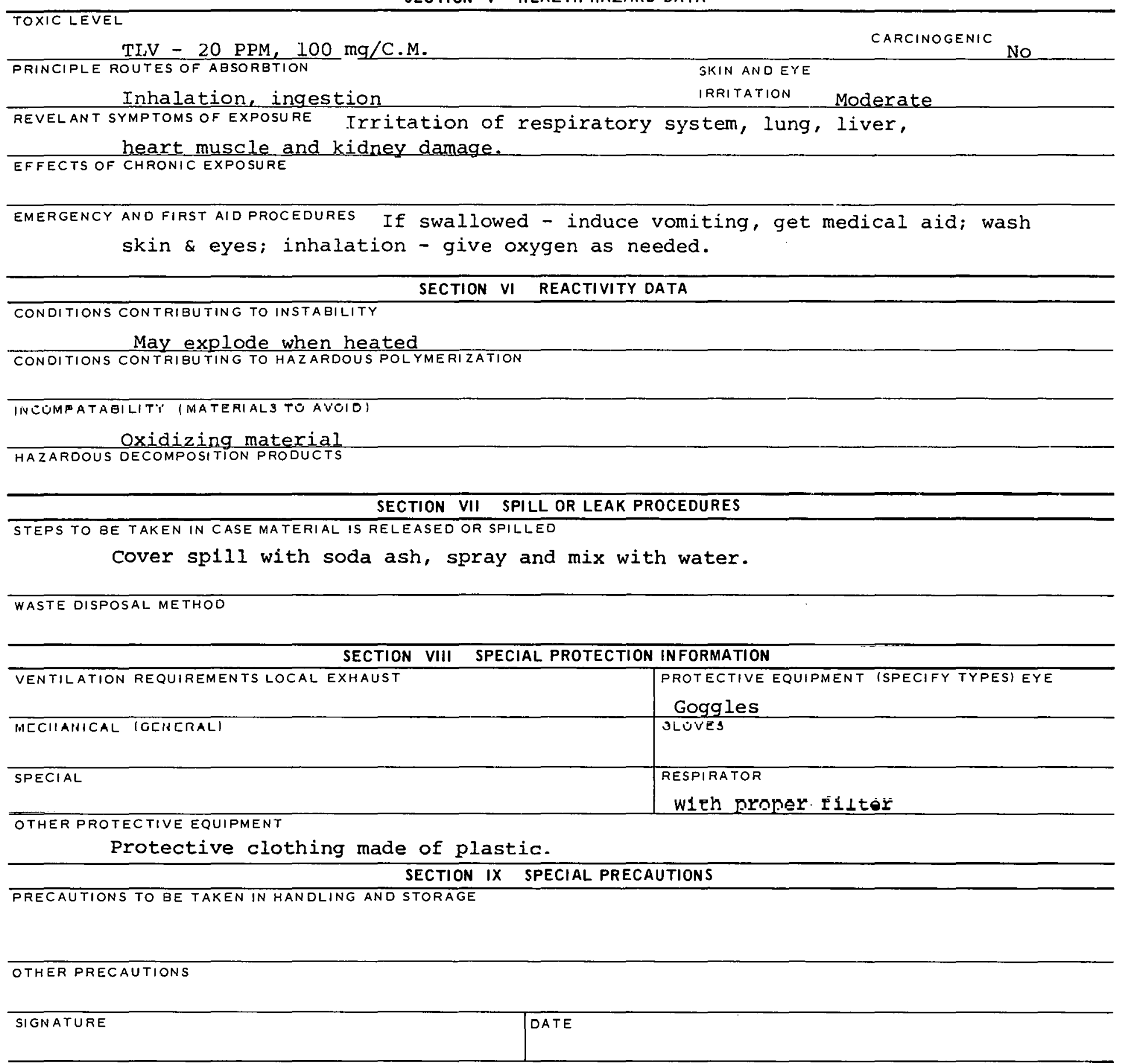


03-229-4216

M-Chlorophenol
MATERIAL SAFETY

DATA SHEET

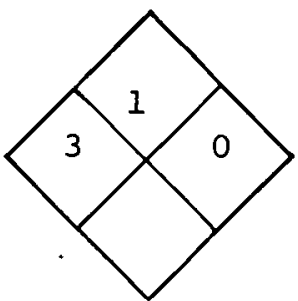

\section{SECTION I SOURCE AND NOMENCLATURE}

MANUFACTURER'S NAME

ET, CITY STATE, ZIP CODE)

EMERGENCY TELEPHONE NO.

ADDRESS (NUMBER, STREET, CITY, STATE, ZIP CODE)

TRADE NAME AND SYNONYMS
3 ChIORO- 1 hYdrOXYbenzene
CHEMICAL NAME AND SYNONYMS
M ChlorOphenOl

\begin{tabular}{l|l} 
& CHEMICAL FAMILY \\
& $\begin{array}{r}\text { FORMULA } \\
\mathrm{Cl} \mathrm{C}_{6} \mathrm{H}_{4} \mathrm{OH}\end{array}$ \\
\hline
\end{tabular}

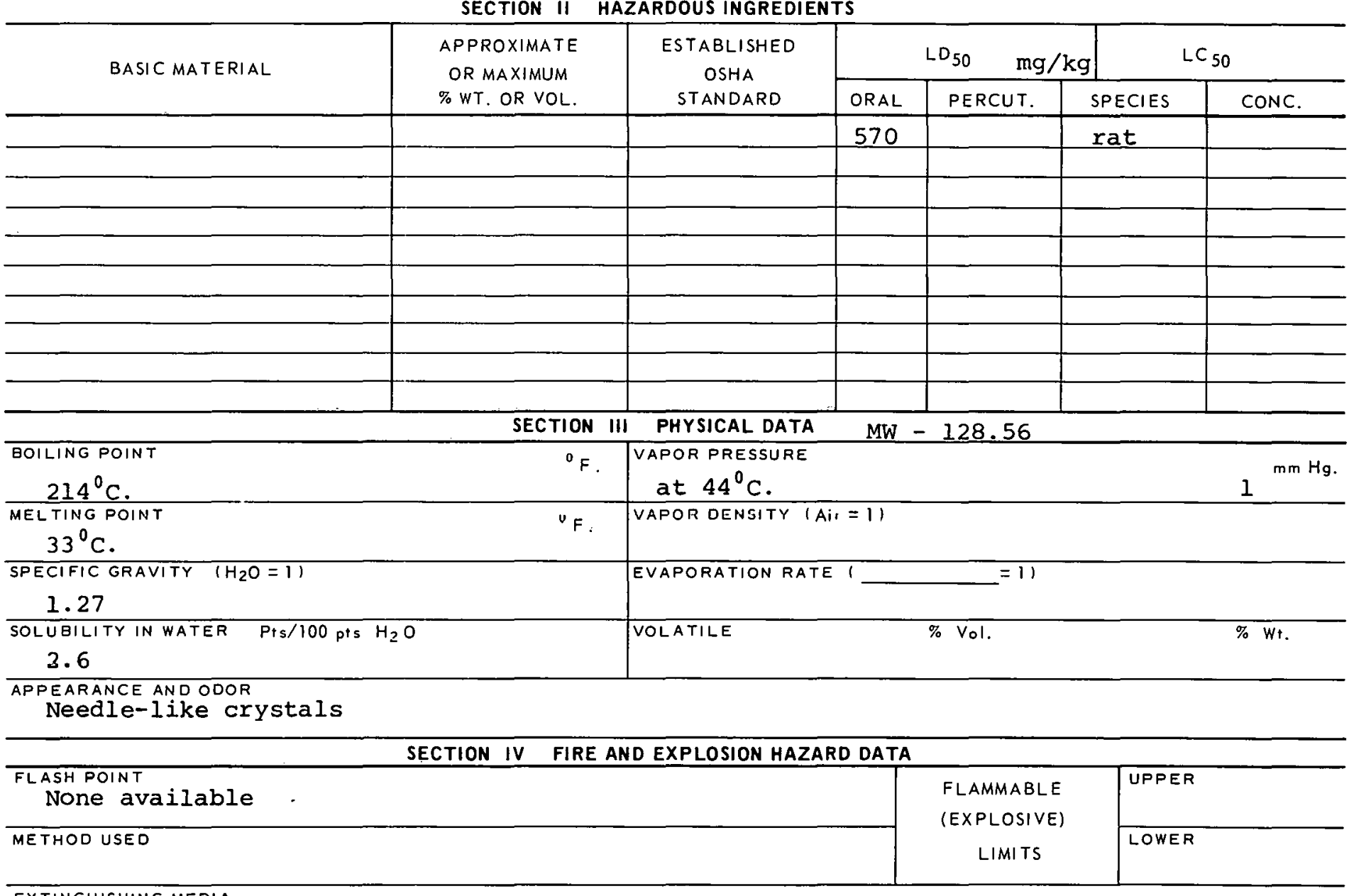

EXTINGUISHING MEDIA

Alcohol foam, carbon dioxide, dry chemical.

SPECIAL FIRE FIGHTING PROCEDURES

UNUSUAL FIRE AND EXPLOSION HAZARDS 


\section{TOXIC LEVEL None available}

PRINCIPLE ROUTES OF ABSORBTION

Inhalation, ingestion, skin SKIN AND EYE

IRRITATION

\section{Severe}

REVELANT SYMPTOMS OF EXPOSURE EYe skin and mucous membrane irritation,

dermatitis, cyanosis.

EFFECTS OF CHRONIC EXPOSURE

Liver damage.

EMERGENCY AND FIRST AID PROCEDURES Inhalation - rest; wash from skin \& eyes; if swallowed give emetic, get medical aid.

SECTION VI REACTIVITY DATA

CONDITIONS CONTRIBUTING TO INSTABILITY

CONDITIONS CONTRIBUTING TO HAZARDOUS POLYMERIZATION

INGOMRATAEIGITY (MATERIALS TQ AVO!R)

HAZARDOUS DECOMPOSITION PRODUCTS

SECTION VII SPILL OR LEAK PROCEDURES

STEPS TO BE TAKEN IN CASE MATERIAL IS RELEASED OR SPILLED

Put a mixture of sand 9 and 1 soda ash. Put intc box for disposal.

WASTE DISPOSAL. METHOD

SECTION VIII SPECIAL PROTECTION INFORMATION

\begin{tabular}{l|l}
\hline VENTILATION REQUIREMENTS LOCAL EXHAUST & $\begin{array}{l}\text { PROTECTIVE EQUIPMENT (SPECIFY TYPES) EYE } \\
\text { GOgGIES }\end{array}$ \\
\hline MECHANICAL IGENERAL) & $\begin{array}{c}\text { GLOVES ButYl Rubber } \\
\text { But }\end{array}$ \\
\hline SPECIAL & $\begin{array}{c}\text { RESPIRATOR } \\
\text { Self-COntained }\end{array}$ \\
\hline
\end{tabular}

OTHER PROTECTIVE EQUIPMENT

Protective clothing

SECTION IX SPECIAL PRECAUTIONS

PRECAUTIONS TO BE TAKEN IN HANDLING AND STORAGE

store in cool, dry place.

OTHER PRECAUTIONS 


PRODUCT DESIGNATION
03-229-4246
O-Ch1orophenol

MATERIAL SAFETY DATA SHEET

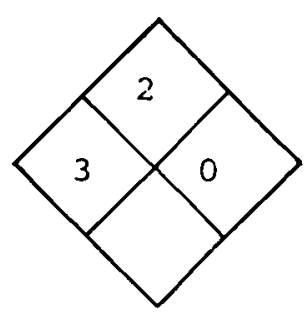

SECTION I SOURCE AND NOMENCLATURE

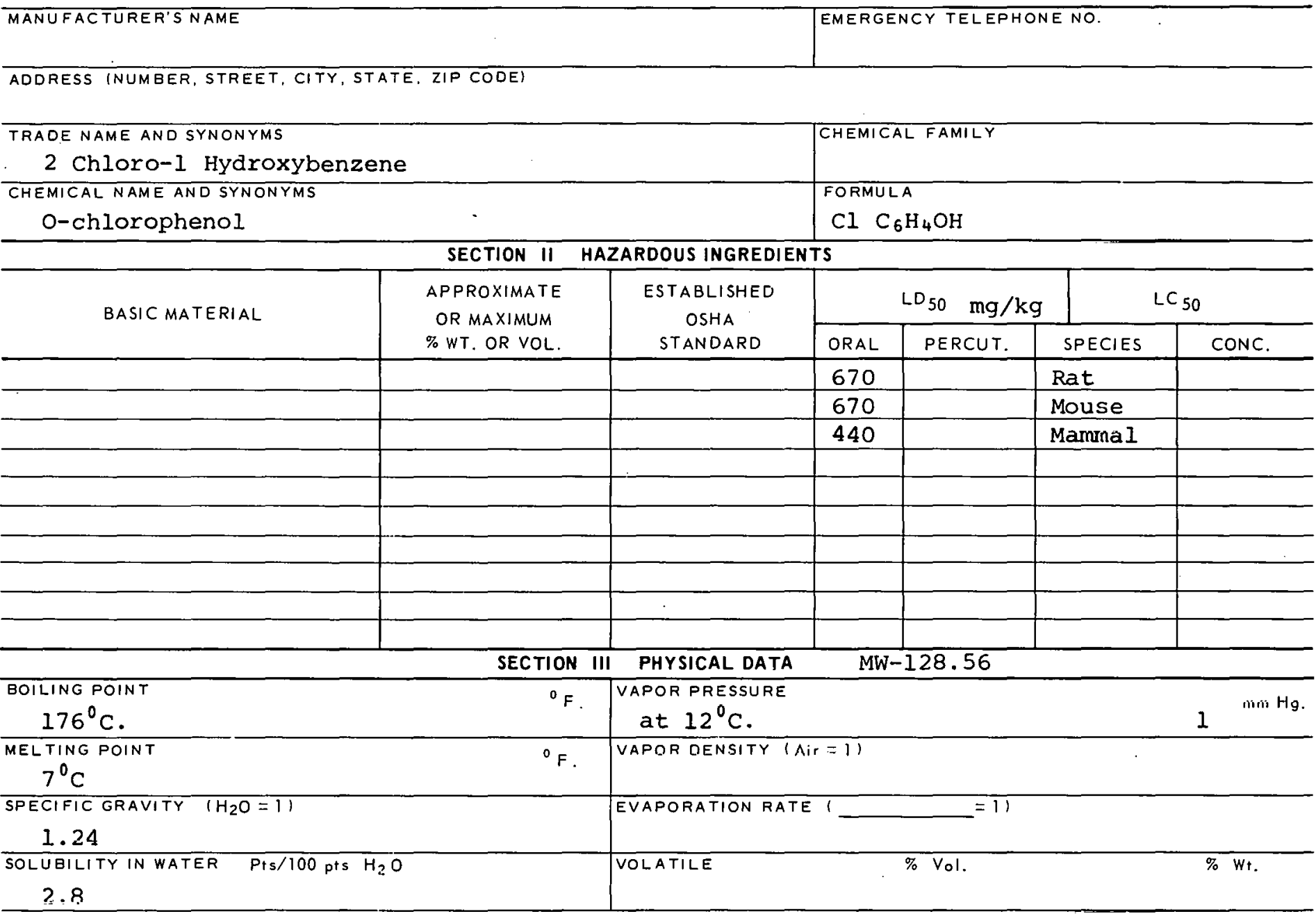
APPEARANCE AND ODOR

Colorless liquid

\begin{tabular}{|c|c|c|c|c|}
\hline & SECTION IV & FIRE AND EXPLOSION HAZARD DATA & & \\
\hline $\begin{array}{c}\text { FLASH POINT } \\
147^{\circ} \mathrm{F} .\end{array}$ & & & \multirow{2}{*}{$\begin{array}{c}\text { FLAMMABLE } \\
\text { (EXPLOSIVE) } \\
\text { LIMITS }\end{array}$} & UPFER \\
\hline METHOD USED & & & & LOWER \\
\hline
\end{tabular}

EXTINGUISHING MEDIA-

Alcohol fnam, carbon dioxide - dry chemical.

SPECIAL FIRE FIGHTING PROCEDURES

UNUSUAL FIRE AND EXPLOSION HAZARDS 


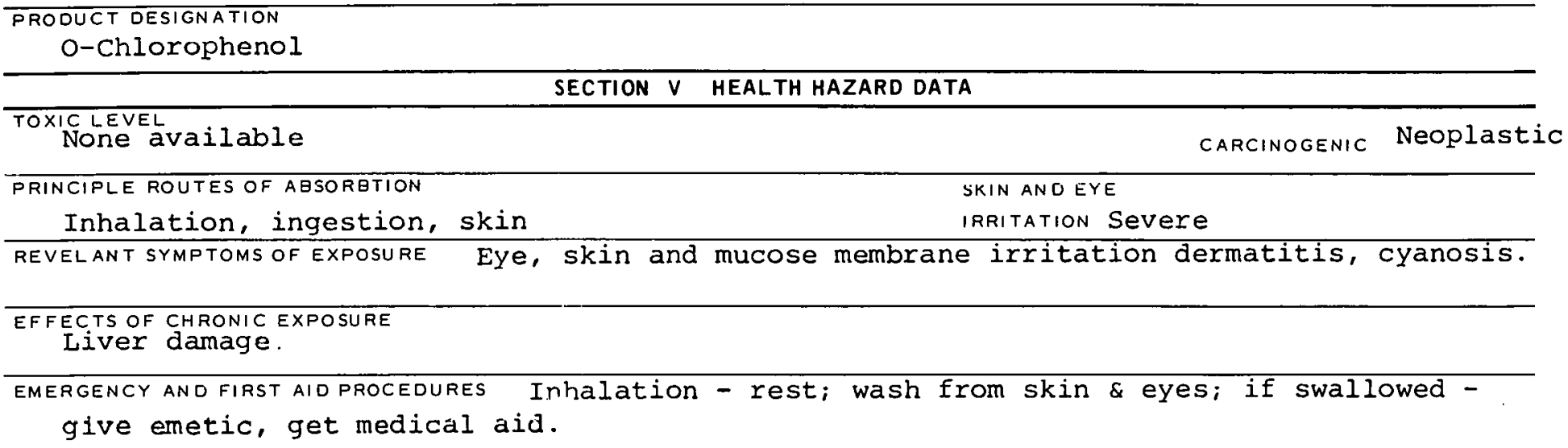


CAS : 000106489

PRODUCT DESIGNATION

03-229-4276

P-Chlorophenol
MATERIAL SAFETY

DATA SHEET

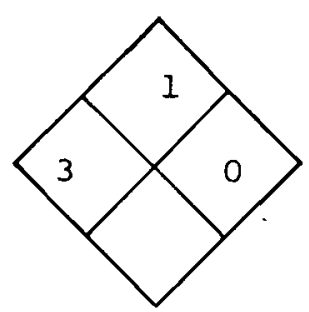

\section{SECTION I SOURCE AND NOMENCLATURE}

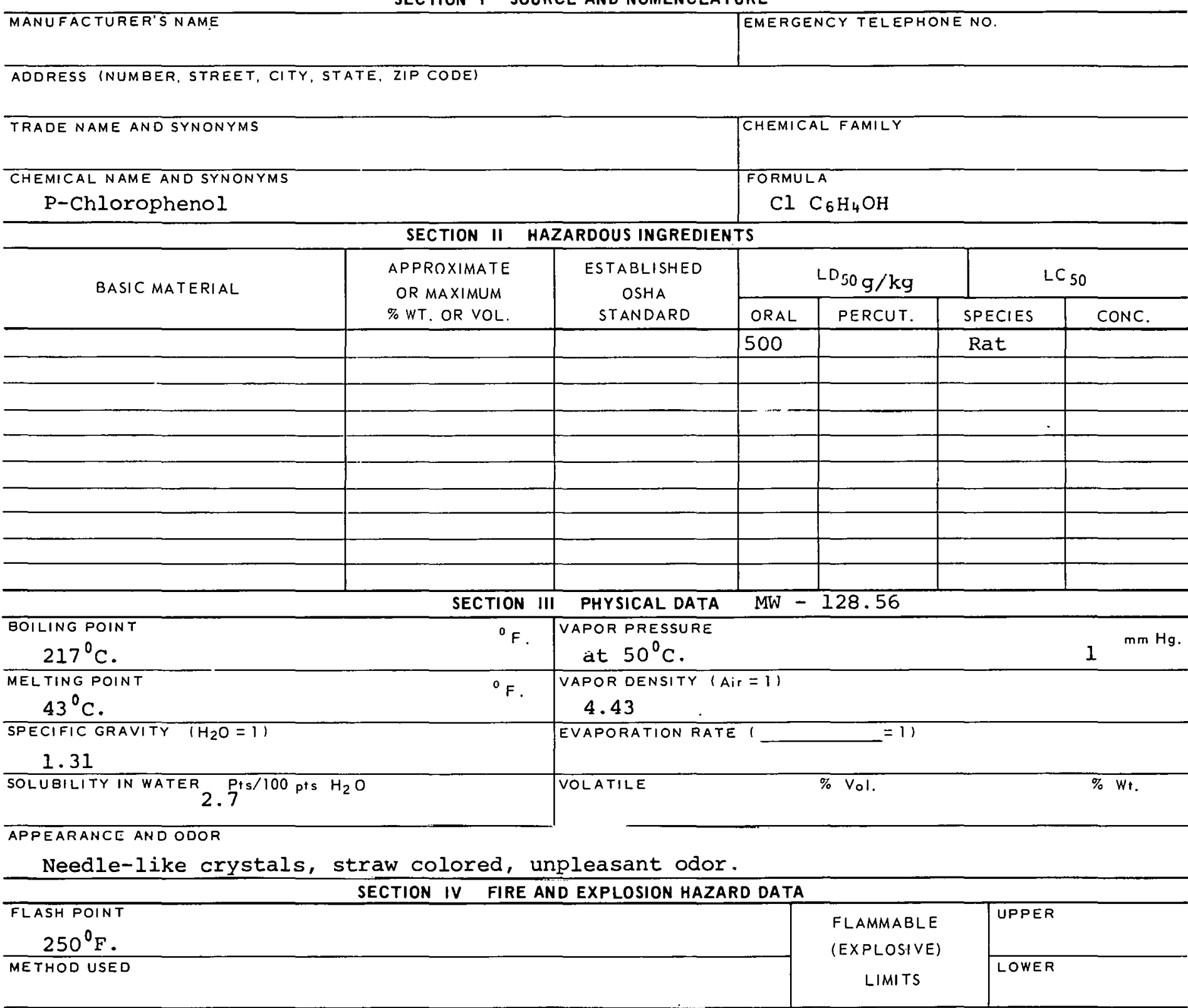

EXTINGUISHING MEDIA

Alcohnl foam, dry chemical, carbon dioxide.

SPECIAL FIRE FIGHTING PROCEOURES

UNUSUAL FIRE AND EXPLOSION IIAZARDS 


\begin{tabular}{|c|c|c|c|}
\hline TOXIC LEVEL & & & \\
\hline None available & & CARCINOGENIC & No \\
\hline PRINGIPLE FOUTES OF ABSORBTION & SKIN AND EYE & & \\
\hline Inhalation, ingestion, skin & IRRITATION & Severe & \\
\hline
\end{tabular}

REVELANT SYMPTOMS OF EXPOSURE EYe, skin and mucous membrane irritation dermatitis, cyanosis.

EFFECTS OF CHRONIC EXPOSURE

Liver damage.

EMERGENCY AND FIRST AID PROCEDURES Inhalation - rest; wash from skin \& eyes. If swallowed give emetic, get medical aid.

SECTION VI REACTIVITY DATA

CONDITIONS CONTRIBUTING TO INSTABILITY

CONDITIONS CONTRIBUTING TO HAZARDOUS POLYMERIZATION

IICOHFATAOILIT'i (MATERIALS TU AVUIO'

HAZARDOUS DECOMPOSITION PRODUCTS

SECTION VII SPILL OR LEAK PROCEDURES

STEPS TO BE TAKEN IN CASE MATERIAL IS RELEASED OR SPILLED

Pour a mixture of sand to soda ash ( $9: 1)$ on spill and sweep into

box for disposal.

WASTE OISPOSAL METHOD

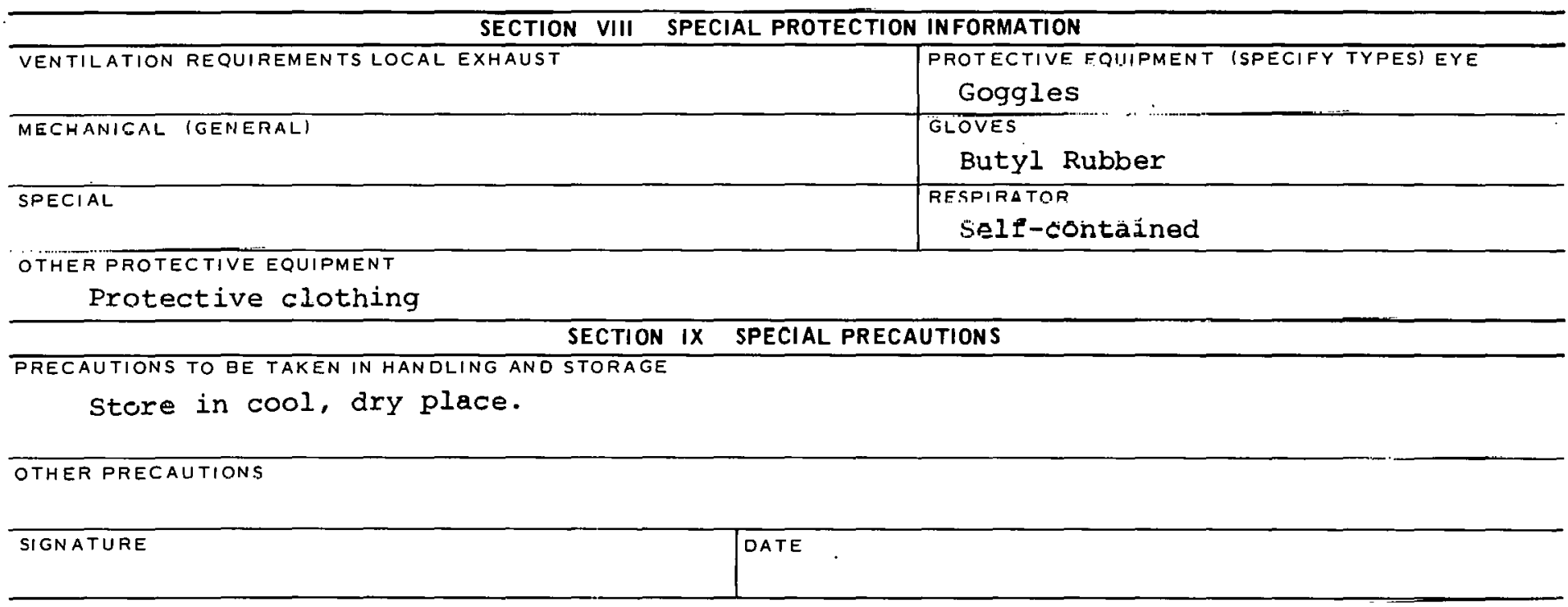




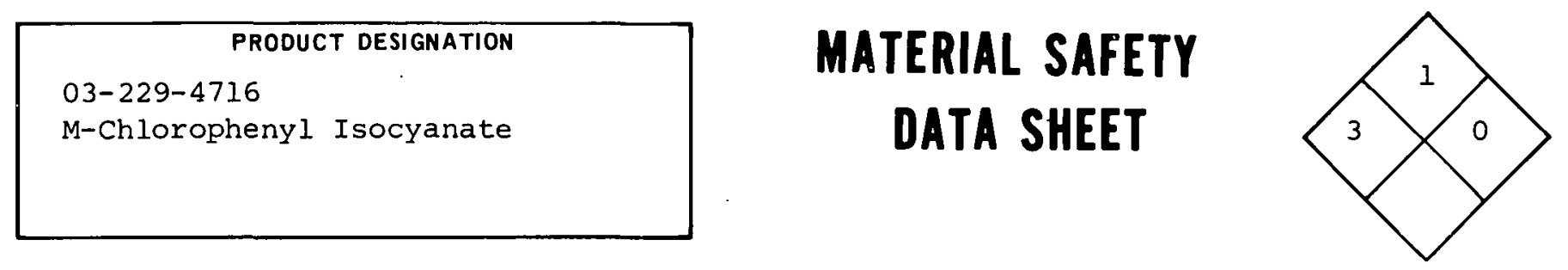

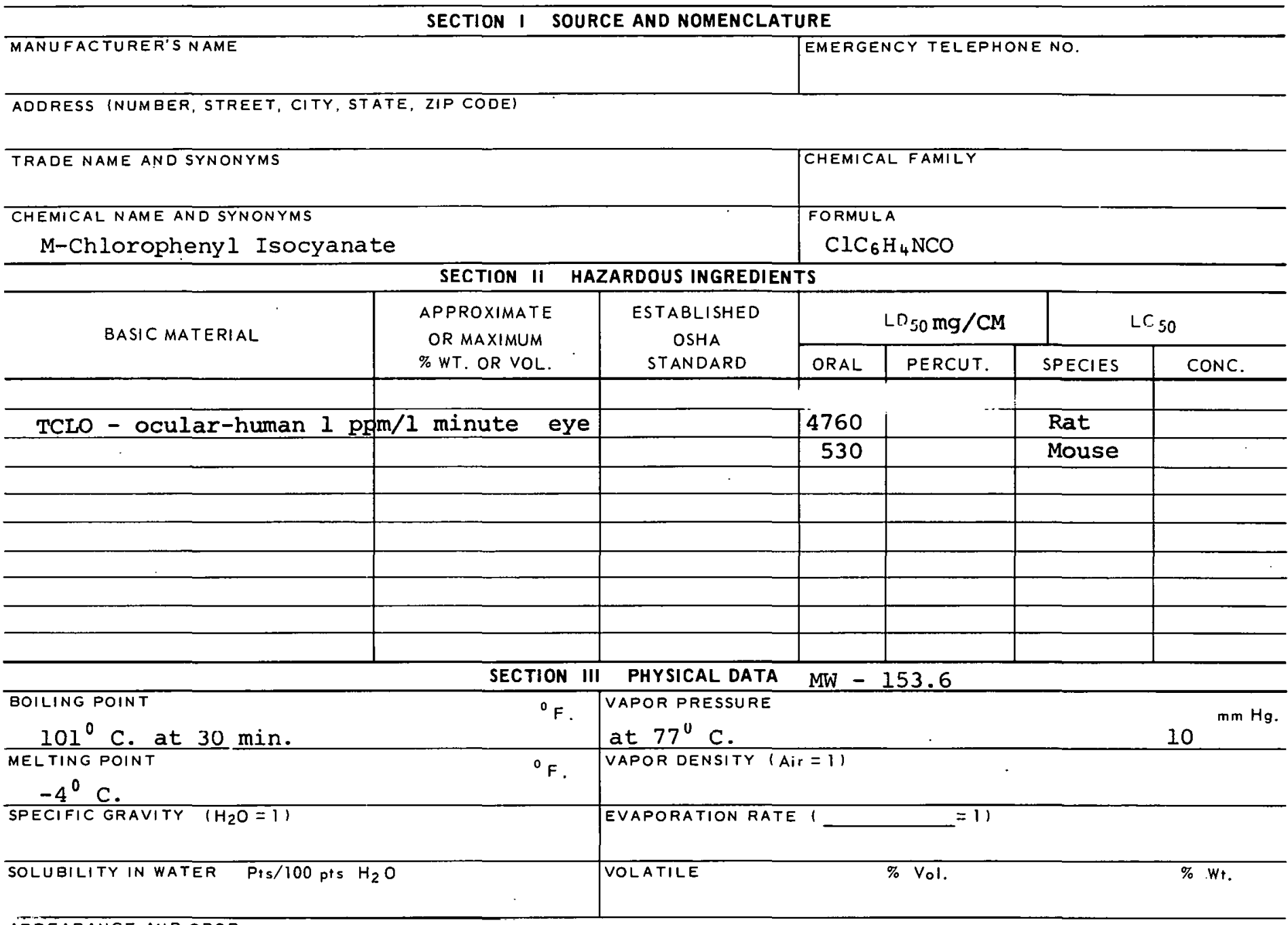

APPEARANCE AND ODOR

Water white liquid.

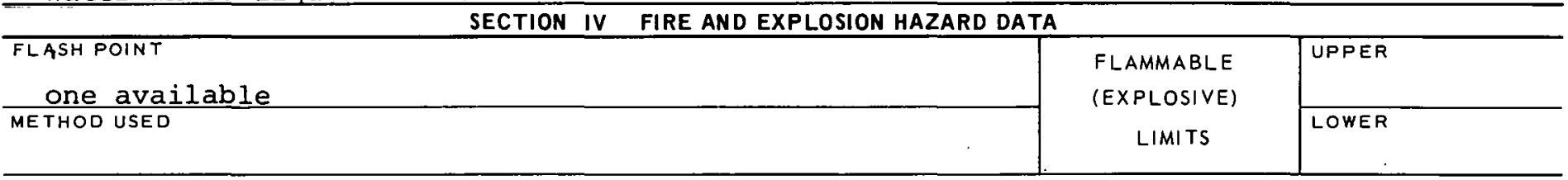

EXTINGUISH!NG MEDIA

Alcohol foam, carbon dioxide, ary chemical.

SPECIAL FIRE FIGHTING PROCEDURES

Wear self-contained breathing apparatus.

UNUSUAL FIRE AND EXPI OSION HAZARDS

Powerful lachrymater. 


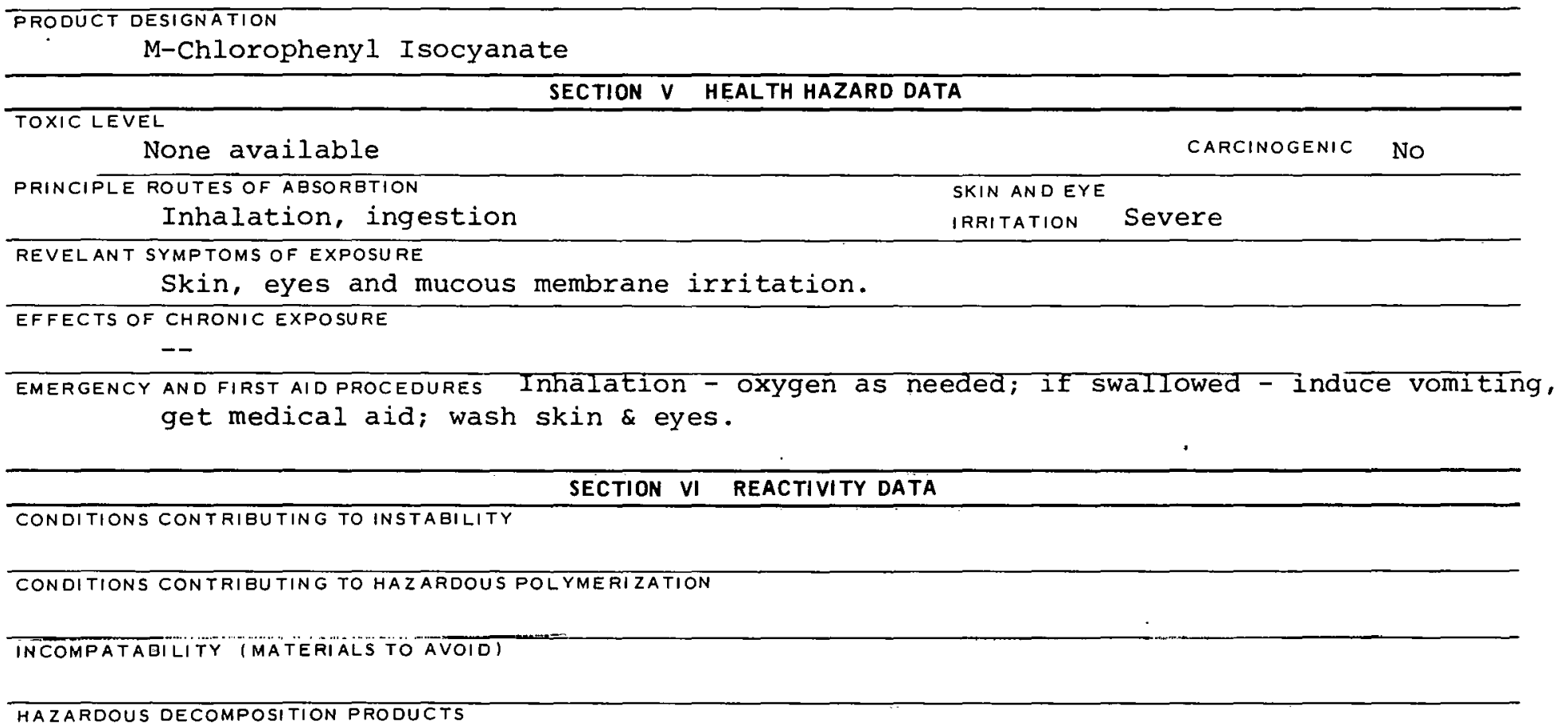

SECTION VII SPILL OR LEAK PROCEDURES

STEPS TO BE TAKEN IN CASE MATERIAL IS RELEASED OR SPILLED

WASTE DISPOSAL METHOD

SECTION VIII SPECIAL PROTECTION INFORMATION

\begin{tabular}{l|l}
\hline VENTILATION REQUIREMENTS LOCAL EXHAUST & $\begin{array}{l}\text { FROTECTIVE EQUIPMENT (SPECIFY TYPESI EYE } \\
\text { GOgGleS }\end{array}$ \\
\hline MECHANICAL IGENERAL) & $\begin{array}{c}\text { GLOVES } \\
\text { Rubber }\end{array}$ \\
\hline SPECIAL & $\begin{array}{c}\text { RESPIRATOR } \\
\text { Self-containCd }\end{array}$ \\
\hline
\end{tabular}

OTHER PROTECTIVE EQUIPMENT

\section{SECTION IX SPECIAL PRECAUTIONS}

PRECAUTIONS TO BE TAKEN IN HANDLING AND STORAGE

\begin{tabular}{l|l|l|}
\hline OTHER PRECAUTIONS & DATE \\
\hline SIGNATURE & \\
\hline
\end{tabular}




\begin{tabular}{|l|}
\hline \multicolumn{1}{|c|}{ PRODUCT DESIGNATION } \\
03-229-4746 \\
O-Ch1orophenyl Isocyanate
\end{tabular}

\section{MATERIAL SAFETY \\ DATA SHEET}

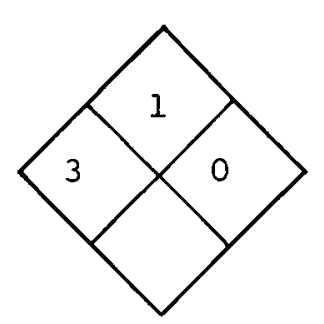

\section{SECTION I SOURCE AND NOMENCLATURE}

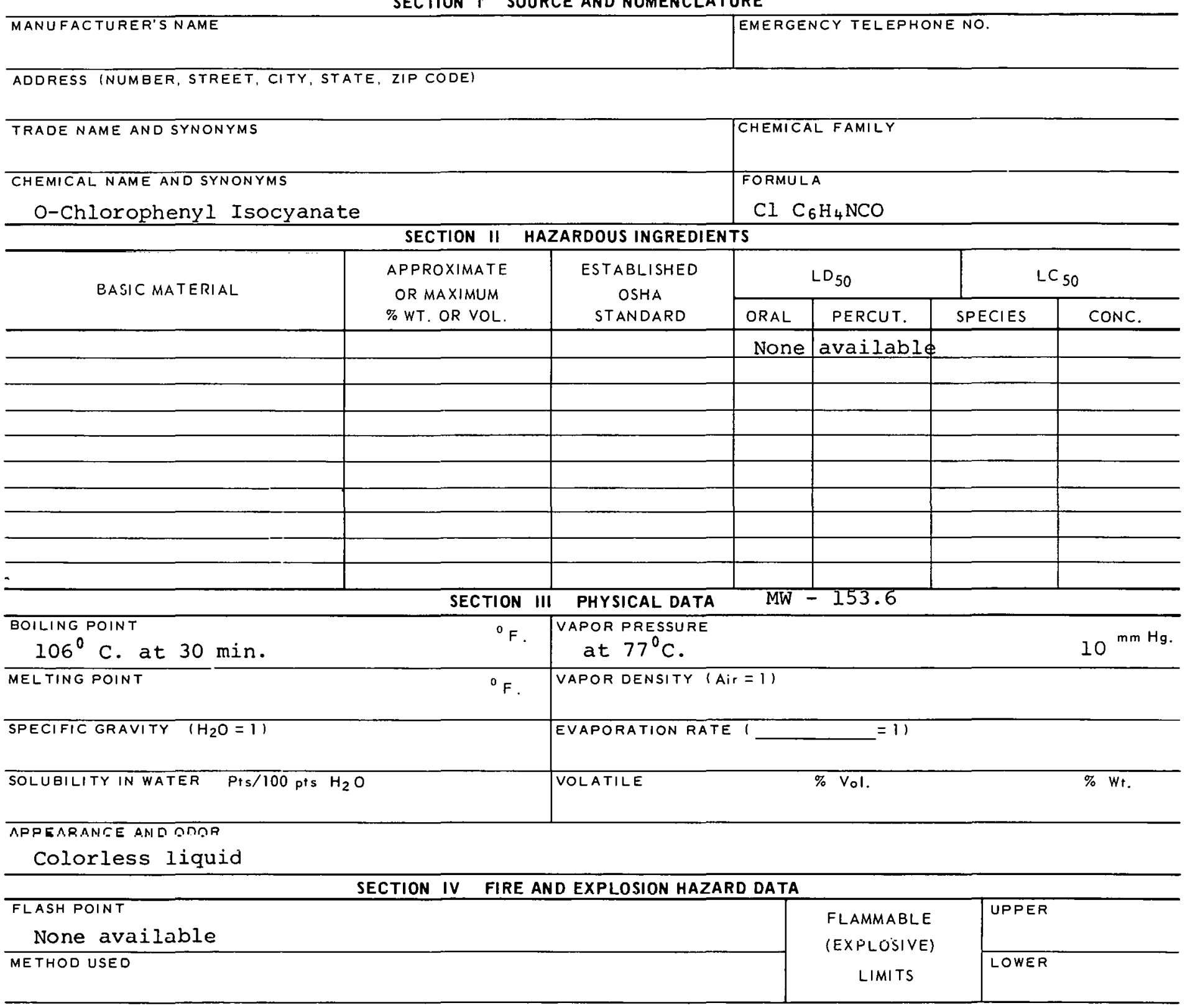

EXTINGUISHING MEDIA

Alcohol foam, carbon dioxide, dry chemical.

SPECIAL FIRE FIGHTING PROCEDURES

Wear self-contained breathing apparatus.

UNUSUAL FIRE AND EXPLOSION HAZARDS

Powerful lachrymater 


\section{TOXIC LEVEL}

None available PRINCIPLE ROUTES OF ABSORBTION

Inhalation, ingestion

REVELANT SYMPTOMS OF EXPOSURE

Irritation of skin, eyes and mucous membrane.

EFFECTS OF CHRONIC EXPOSURE

-.

EMERGENCY AND FIRST AID PROCEDURES Inhaltion - oxygen as needed; if swallowed - induCe

vomiting, get medical aid; wash skin \& eyes.

\section{SECTION VI REACTIVITY DATA}

CONDITIONS CONTRIBUTING TO INSTABILITY

CONDITIONS CONTRIBUTING TO HAZARDOUS POLYMERIZATION

INCOMTATADILITY (MATERIALS TO AVOID)

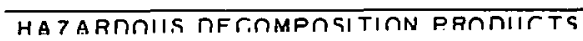

SECTION VII SPILL OR LEAK PROCEDURES

STEPS TO BE TAKEN IN CASE MATERIAL IS RELEASED OR SPILLED

WASTE DISPOSAL METHOD

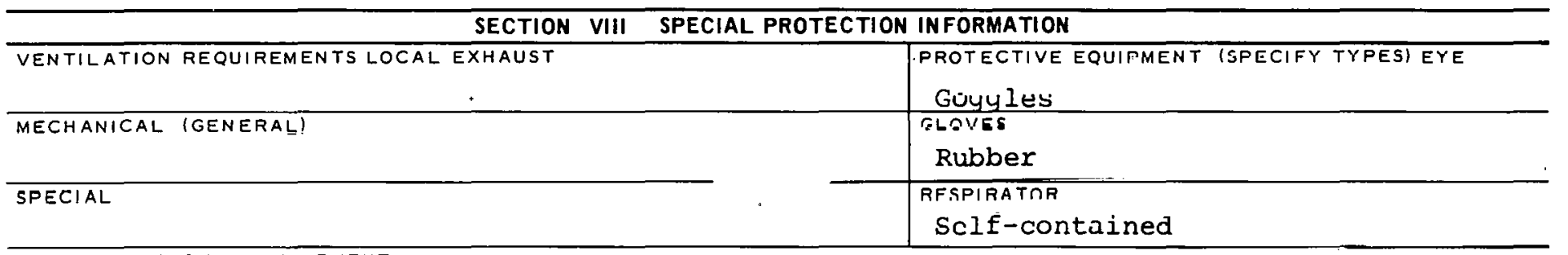

OTHER FROTLCTIVE EQUIPMENT

SECTION IX SPECIAL PRECAUTIONS

PRECAUTIONS TO BE TAKEN IN HANDLING AND STORAGE

OTHER PRECAUTIONS

SIGNATURE

DATE

$229-4746 B$ 


PRODUCT DESIGNATION
03-229-6466
3-Chloro, 1,2 Propanediol

\section{MATERIAL SAFETY DATA SHEET}

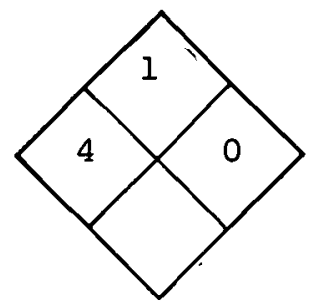

SECTION I SOURCE AND NOMENCLATURE

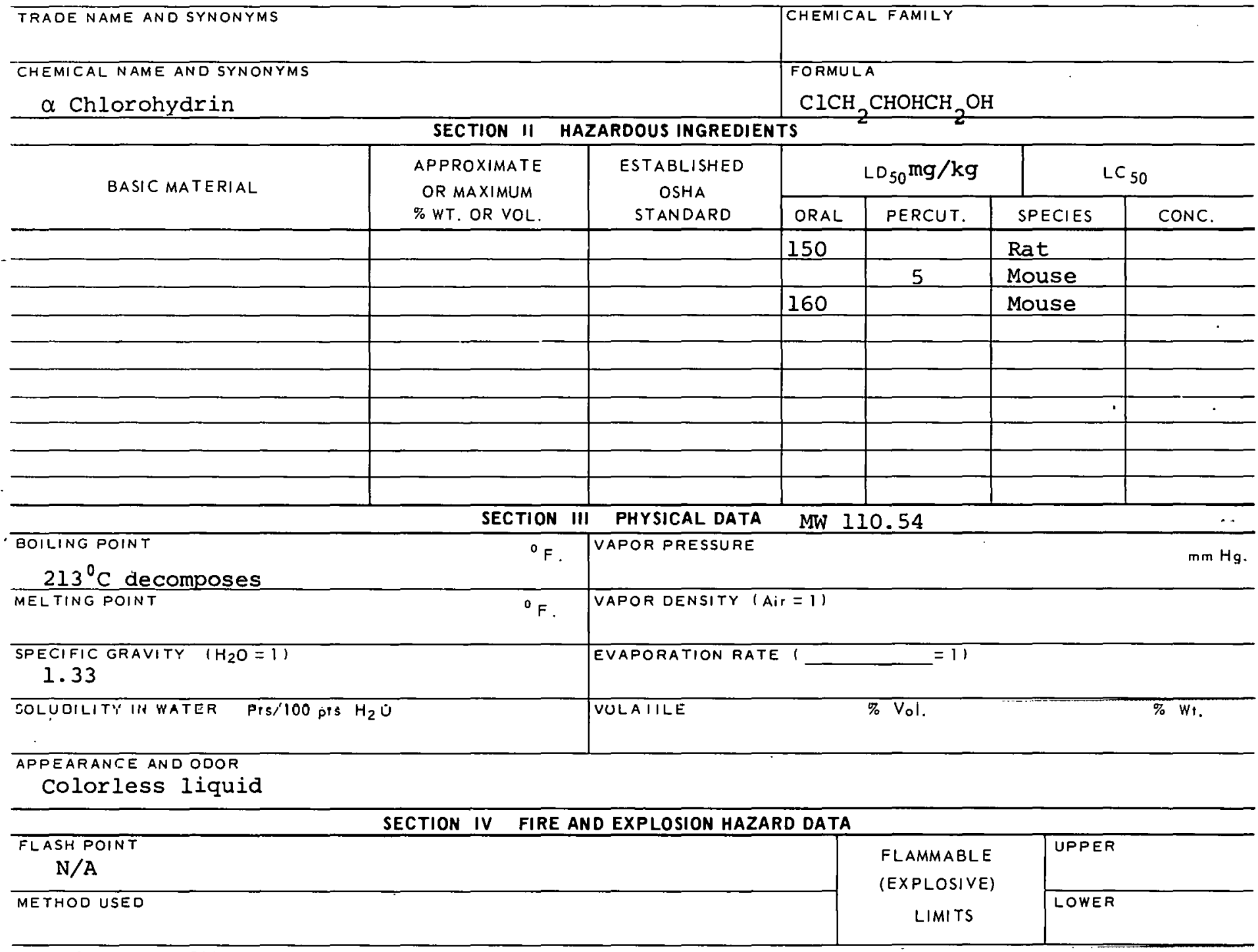

EXTINGUISHINE MEOIA

Alcohol foam, carbon dioxide, dry chemical SPECIAL FIRE FIGHTING PROCEDURES 
3 Chloro - 1,2 Propanediol

\section{SECTION $V$ HEALTH HAZARD DATA}

TOXIC LEVEL

None available

CARCINOGENIC No

PRINCIPLE ROUTES OF ABSORBTION

Inhalation, ingestion, skin

SKIN AND EYE

IRRITATION MOderate

REVELANT SYMPTOMS OF EXPOSURE eye and nasal irritation, nausea, vomiting, giddiness, dizziness, incoordination, coma

EFFECTS OF CHRONIC EXPOSURE

Nausea, vomiting, abdominal pain

EMERGENCY AND FIRST AID PROCEDURES Wash eyes and skin; if swallowed - give emetic; get

medical aid; inhalation - rest, give oxygen as needed.

$$
\text { SECTION VI REACTIVITY DATA }
$$

CONDITIONS CONTRIBUTING TO INSTABILITY

CONDITIONS CONTRIBUTING TO HAZARDOUS POLYMERIZATION

INCOMPATABILITY (MATERIALSTO AVQIO)

HAZARDOUS DECOMPOSITION PRODUCTS

SECTION VII SPILL OR LEAK PROCEDURES

STEPS TO BE TAKEN IN CASE MATERIAL IS RELEASED OR SPILLED

WASTE DISPOSAL METHOD

SECTION VIII SPECIAL PROTECTION INFORMATION

\begin{tabular}{l|c}
\hline VENTILATION REQUIREMENTS LOCAL EXHAUST & $\begin{array}{c}\text { GOTOTECTIVE EQUIPMENT (SPECIFY TYPESI EYE } \\
\text { MECHANICAL IGENERAL) }\end{array}$ \\
\hline SPECIAL & $\begin{array}{c}\text { GLOVES } \\
\text { Rubber }\end{array}$ \\
\hline OTHER PROTECTIVE EQUIPMENT & $\begin{array}{c}\text { REgPIRATOR } \\
\text { WIth proper filter }\end{array}$ \\
\hline
\end{tabular}

OTHER PROTECTIVE EQUIPMENT

SECTION IX SPECIAL PRECAUTIONS

PRECAUTIONS TO BE TAKEN IN HANDLING AND STORAGE

OTHER PRECAUTIONS

SIGNATURE

DATE

$229-6466 B$ 


\section{PRODUCT DESIGNATION}

03-229-7716

B-Chloropropionyl Chloride
MATERIAL SAFETY

DATA SHEET

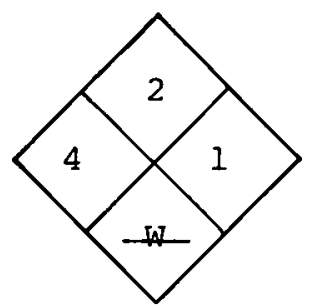

SECTION I SOURCE AND NOMENCLATURE

MANUFACTURER'S NAME

ADDRESS (NUMBER, STREET, CITY, STATE, ZIP CODE)

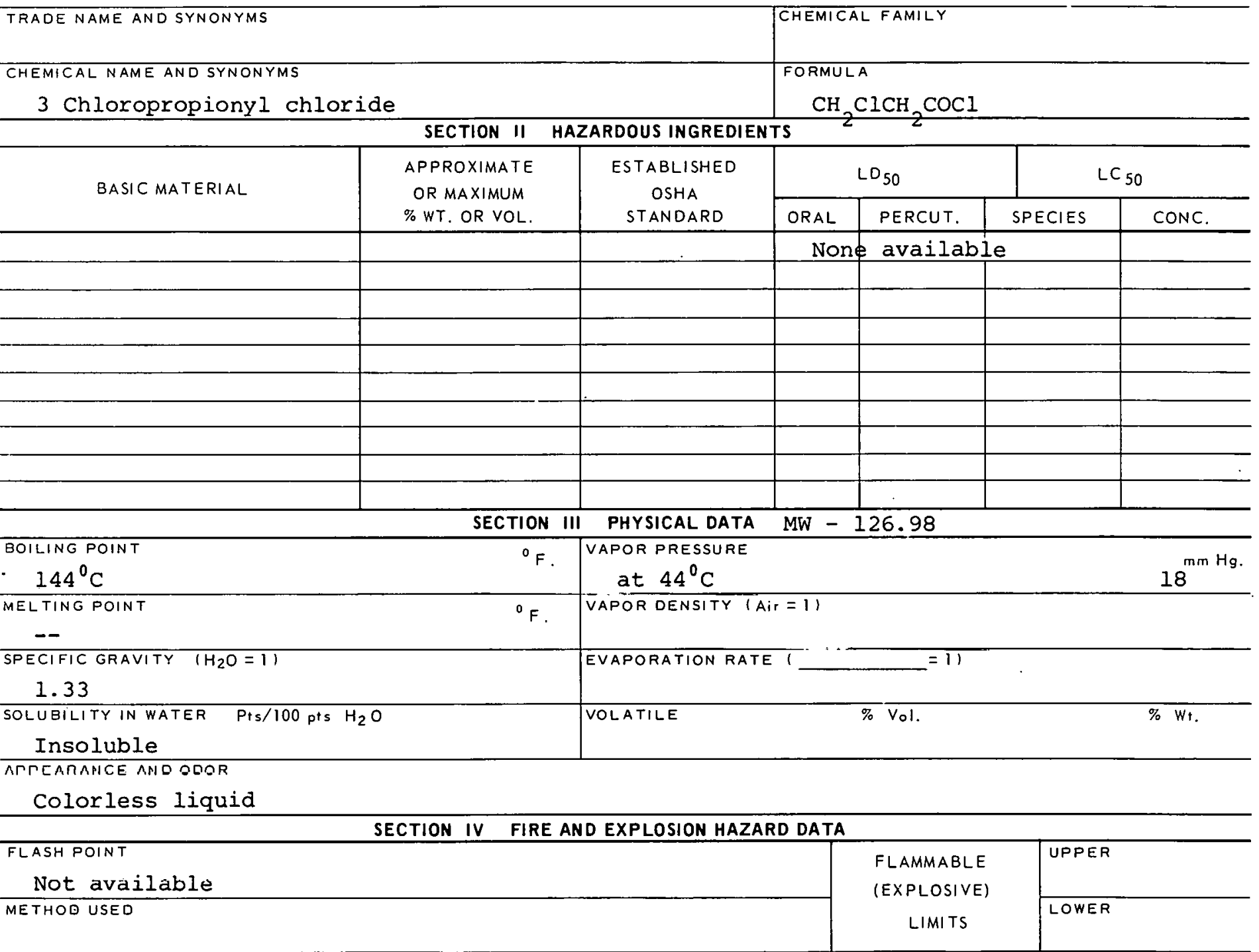

EXTINGUISHING MEDIA

Carbon dioxide, dry chemical

SPECIAL FIRE FIGHTING PROCEOURES

UNUSUAL FIRE AND EXPLOSION HAZARDS 


\section{TOXIC L'EVEL}

None available

PRINCIPLE ROIITES OF ABSORBTION

CARCINOGENIC

Inhalation, ingestion

SKIN AND EYE

IRRITATION Severe

REVELANT SYMPTOMS OF EXPOSURE Cyanosis, coughing, headache, pulmonary edema,

ulceration of skin, dizziness.

EFFECTS OF CHRONIC EXPOSURE Erosion of teeth, bronchial irritation and chronic cough.

EMERGENCY AND FIRST AID PROCEDURES Ingestion - drink water, milk of magnesia, get medical

aid; inhalation - artificial respiration as needed; wash eyes \& skin.

\section{SECTION VI REACTIVITY DATA}

\section{CONDITIONS CONTRIBUTING TO INSTABILITY}

CONDITIONS CONTRIBUTING TO HAZARDOUS POLYMERIZATION

INCOMPATABILITY (MATERIALS TO AVOIDI May react violently with water, alonhol, and almethyl sulfoxide.

HAZARDOUS DECOMPOSITION PRODUCTS

\section{SECTION VII SPILL OR LEAK PROCEDURES}

STEPS TO BE TAKEN IN CASE MATERIAL IS RELEASED OR SPILLED

WASTE DISPOSAL METHOD

SECTION VIII SPECIAL PROTECTION INFORMATION

\begin{tabular}{|c|c|}
\hline VENTILATION REQUIREMENTS LOCAL EXHAUST & $\begin{array}{l}\text { PROTECTIVE EQUIPMENT (SPECIFY TYPES) EYE } \\
\text { Goggles }\end{array}$ \\
\hline MECHANICAL IGENERAL) & $\begin{array}{l}\text { GLuves } \\
\text { Rubber }\end{array}$ \\
\hline SPEC!AL & $\begin{array}{l}\text { RESPIRATOR } \\
\text { Se } 1 t \text {-Contained }\end{array}$ \\
\hline
\end{tabular}

OTHER PROTECTIVE EQUIPMENT

PRECAUTIONS TO BE TAKEN IN HANDLING AND STORAGE

OTHER PRECAUTIONS

SIGNATURE

UAIE

$229-7716 B$ 
CAS : 000109091

US59500

\begin{tabular}{|l} 
PRODUCT DESIGNATION \\
03-229-9216 \\
2 Chloropyridine
\end{tabular}

\section{MATERIAL SAFETY \\ DATA SHEET}

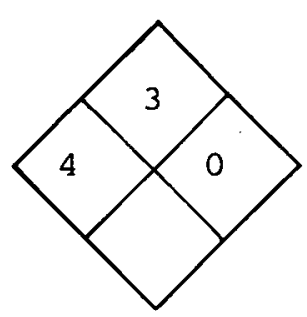

SECTION I SOURCE AND NOMENCLATURE

MANUFACTURER'S NAME

ADDRESS (NUMBER, STREET, CITY, STATE, ZIP CODE)

TRADE NAME AND SYNONYMS

$\alpha$ chloropyridine

CHEMICAL NAME ANO SYNONYMS

2- chloropyridine

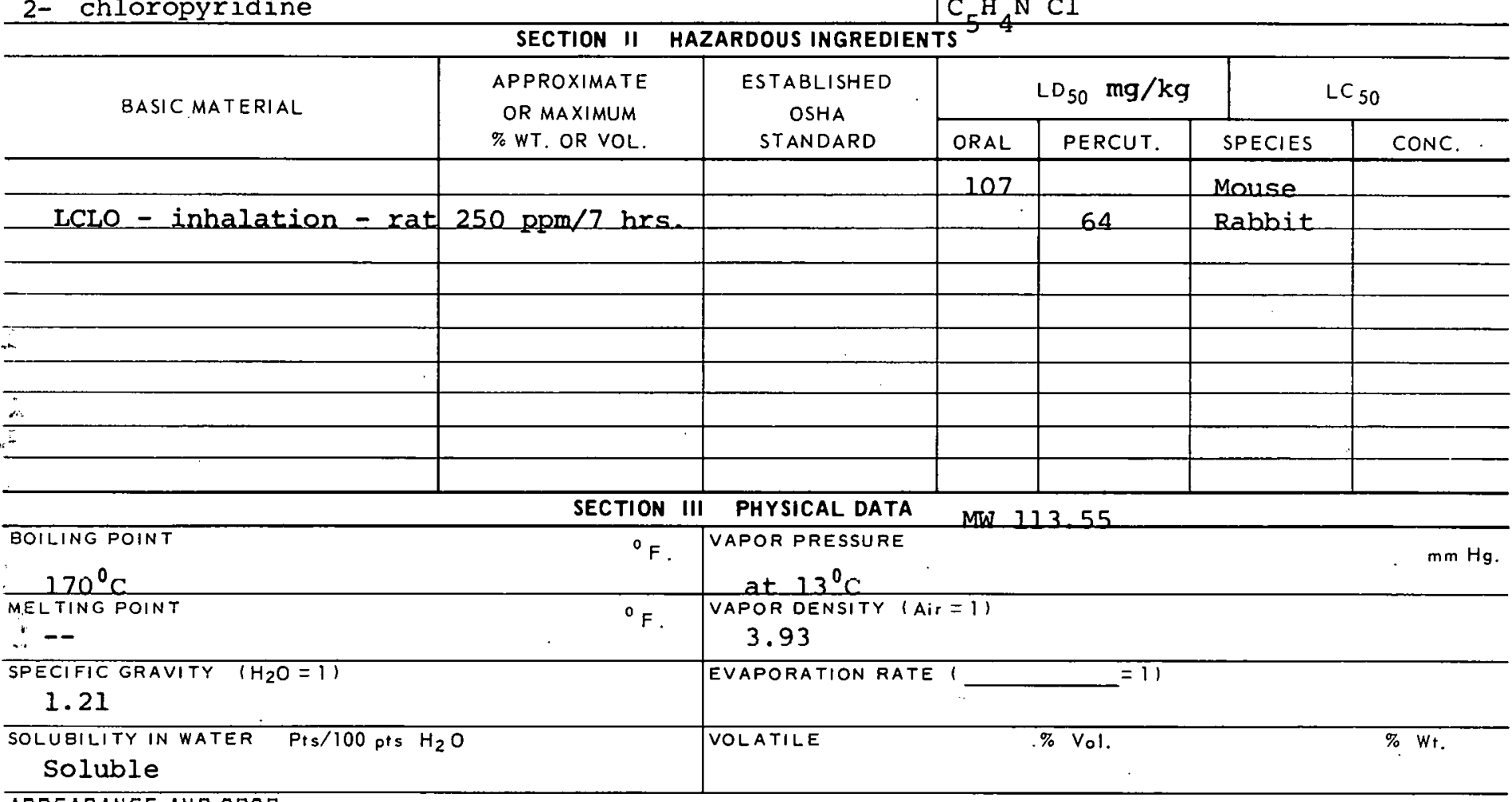

APPEARANCE AND ODOR

Oil with fish-like odor.

SECTION IV FIRE AND EXPLOSION HAZARD DATA

\begin{tabular}{l|c|c}
\hline \multicolumn{2}{c}{ SECTION IV FIRE AND EXPLOSION HAZARD DATA } \\
\hline $\begin{array}{l}\text { FLASH POINT } \\
\text { N/A }\end{array}$ & $\begin{array}{c}\text { FLAMMABLE } \\
\text { (EXPLOSIVE) }\end{array}$ \\
\hline METHOD USED & LIMITS & LOWER \\
\hline
\end{tabular}

EXTINGUISHING MEDIA

Alcohol foam, carbon dioxide, dry chemical.

SPECIAL FIRE FIGHTING PROCEDURES Wear goggles and self-contained breathing apparatus.

UNUSUAL FIRE AND EXPLOSION HAZARDS 
TOXIC LEVEL

None available

PRINCIPLE ROUTES OF ABSORBTION

CARCINOGENIC

Inhalation, ingestion, skin SKIN ANDEYE IRRITATION

REVELANT SYMPTOMS OF EXPOSURE Narcosis, headache, nausea, giddiness, vomiting,

oral - liver \& kidney damage.

EFFECTS OF CHRONIC EXPOSURE Occasional vomiting and diarrhea; weight loss \& anemia;

ocular and facial paralysis.

EMERGENCY AND FIRST AID PROCEDURES Inhalation - rest; if swallowed - induce vomiting,

then gastric lavage; wash skin \& eyes.

\section{SECTION VI REACTIVITY DATA}

CONDITIONS CONTRIBUTING TO INSTABILITY

CONDITIONS CONTRIBUTING TO HAZARDOUS POLYMERIZATION

INCOWPATADILITY (MATERIALJ TO AVOID)

May react with oxidizing materials

HAZARDOUS DECOMPOSITION PROOUCTS

SECTION VII SPILL OR LEAK PROCEDURES

STEPS TO BE TAKEN IN CASE MATERIAL IS RELEASED OR SPILLED

WASTE DISPOSAL METHOD

SECTION VIII SPECIAL PROTECTION INFORMATION

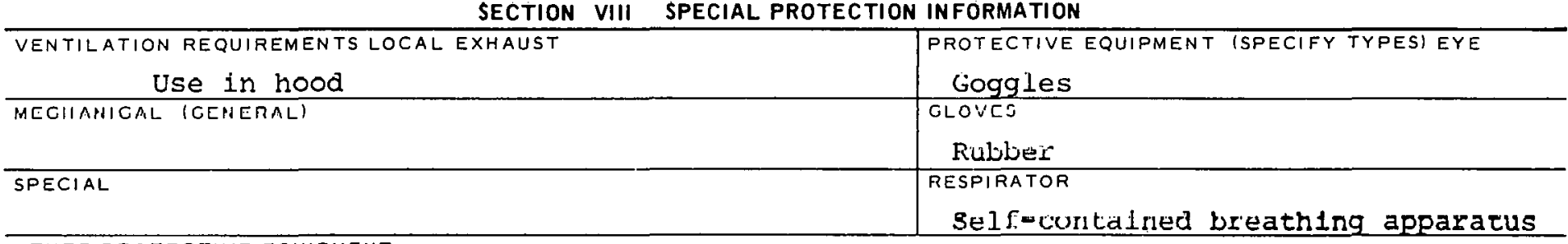

OTHER PROTECTIVE EQUIPMENT

SECTION IX SPECIAL PRECAUTIONS

PRECAUTIONS TO BE TAKEN IN HANDLING AND STORAGE

OTHER PRECAUTIONS

\begin{tabular}{l|l|l}
\hline SIGNATURE & DATE \\
\hline
\end{tabular} 

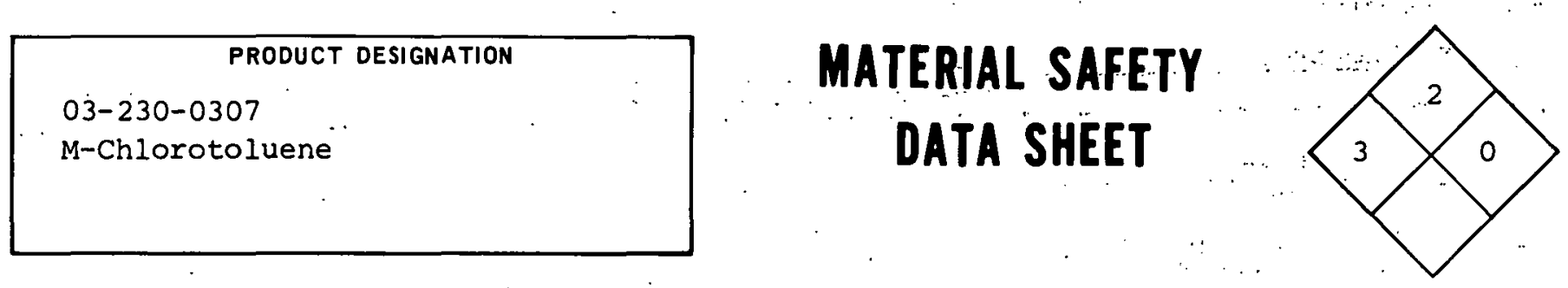

SECTION I SOURCE AND NOMENCLATURE

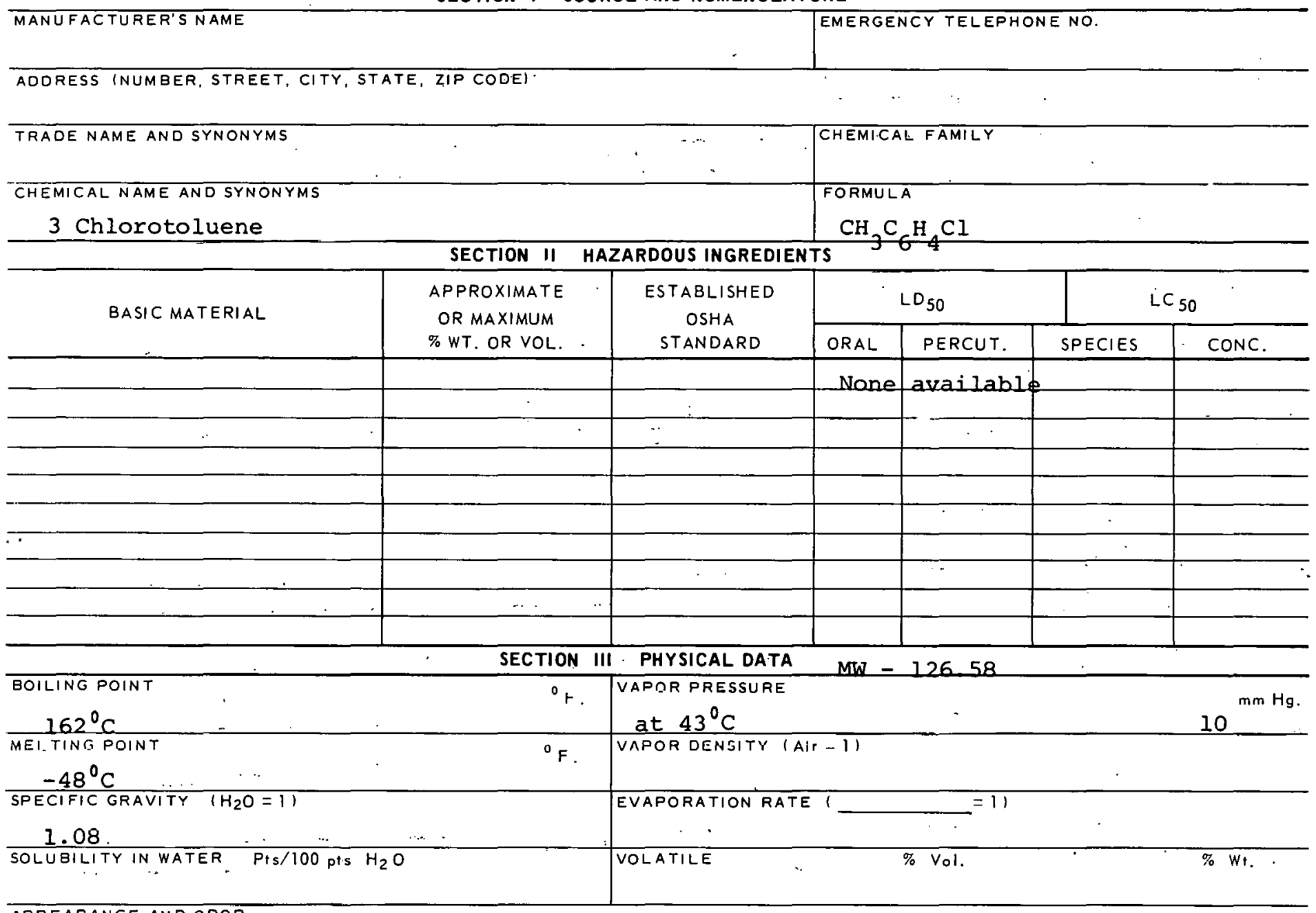

APPEARANCE AND ODOR

Colorless liguid

\begin{tabular}{l|c|c}
\hline \multicolumn{3}{c}{ SECTION IV FIRE AND EXPLOSION HAZARD DATA } \\
\hline $\begin{array}{l}\text { FLASH POINT } \\
\text { NOt available }\end{array}$ & $\begin{array}{c}\text { FLAMMABLE } \\
\text { (EXPLOSIVE) } \\
\text { LIMITS }\end{array}$ & $\begin{array}{l}\text { LOWER } \\
\text { LIMOD USED }\end{array}$ \\
\hline
\end{tabular}

EXTINGUISHING MEOIA

Alcohol fuäli, carbon dioxide, dry chemical

SPECIAL FIRE FIGHTING PROCEDURES

UNUSUAL FIRE AND EXPLOSION HAZARDS 
PRODUCT DESIGNATION

M-Chlorotoluene

TOXICLEVEL SECTION V MEALTH HAZARD OATA

None available

PRINCIPLE ROUTES OF ABSORBTION

Inhalation, ingestion

CARCINOGENIC No SKIN AND EYE

IRAITATION

Severe

REVELANT SYMPTOMS OF EXPOSURE EYe \& respiratory system irritation, severe internal

damage, narcotic effect

EFFECTS OF CHRONIC EXPOSURE

$--$

EMERGENCY AND FIRST AID PROCEOURES Inhalation - rest; if swallowed - give emetic,

get medical aid; wash skin \& eyes.

SECTION VI REACTIVITY DATA

CONDITIONS CONTRIBUTING TO INSTABILITY

CONDITIONS CON.TRIBUTING TO HAZARDOUS POLYMEAIZATION

INCOMPATABILITY (MATERIALS TO AVOID)

HAZARDOUS DECOMPOSITION PRODUCTS

SECTION VII SPILL OR LEAK PROCEDURES

STEPS TO BE TAKEN IN CASE MA TERIAL IS RELEASED OR SPILLED

WASTE DISPOSAL METHOD

SECTION VIII SPECIAL PROTECTION IAFORMATION

\begin{tabular}{|c|c|}
\hline VENTILATION REQUIREMENTS LOCAL EXHAUST & $\begin{array}{l}\text { PROTECTIVE EQUIPMENT ISPECIFY TYPESI EYE } \\
\text { Goggles. }\end{array}$ \\
\hline MECHANICAL IGENEAALI) & GLOVES \\
\hline SPECIAL & $\begin{array}{l}\text { RESPIRATOR } \\
\text { With proper filter }\end{array}$ \\
\hline
\end{tabular}

OTHER PROTECTIVE EOUIPMENT

\section{SECTION IX SPECIAL PRECAUTIONS}

PAECAUTIONS TO BE TAKEN IN HANDLINO ANO STORAGE

\section{OTHER PRECAUTIONS}

SIGNATURE 


\section{RECENED BY ThS SEP 31981}



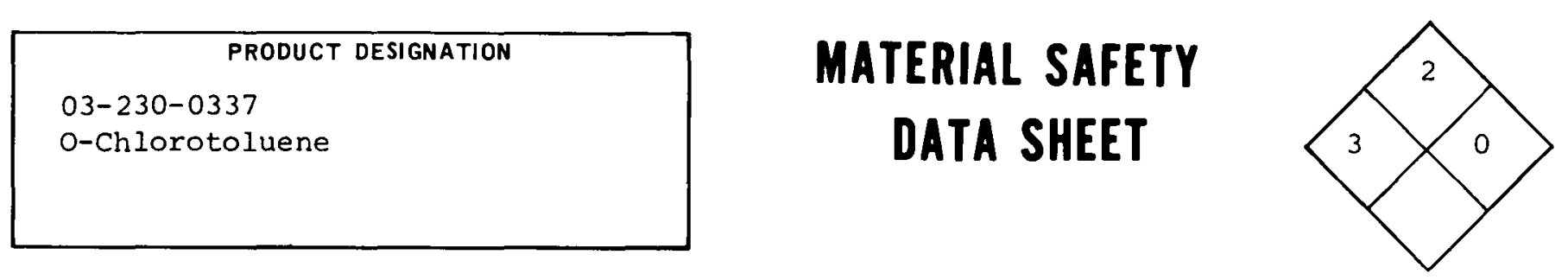

SECTION I SOURCE AND NOMENCLATURE

MANUFACTURER'S NAME
ADDRESS INUMBER, STREET, CITY,
TRADE NAME AND SYNONYMS
CHEMICAL NAME AND SYNONYMS
2 ChlOYOTOIUENE

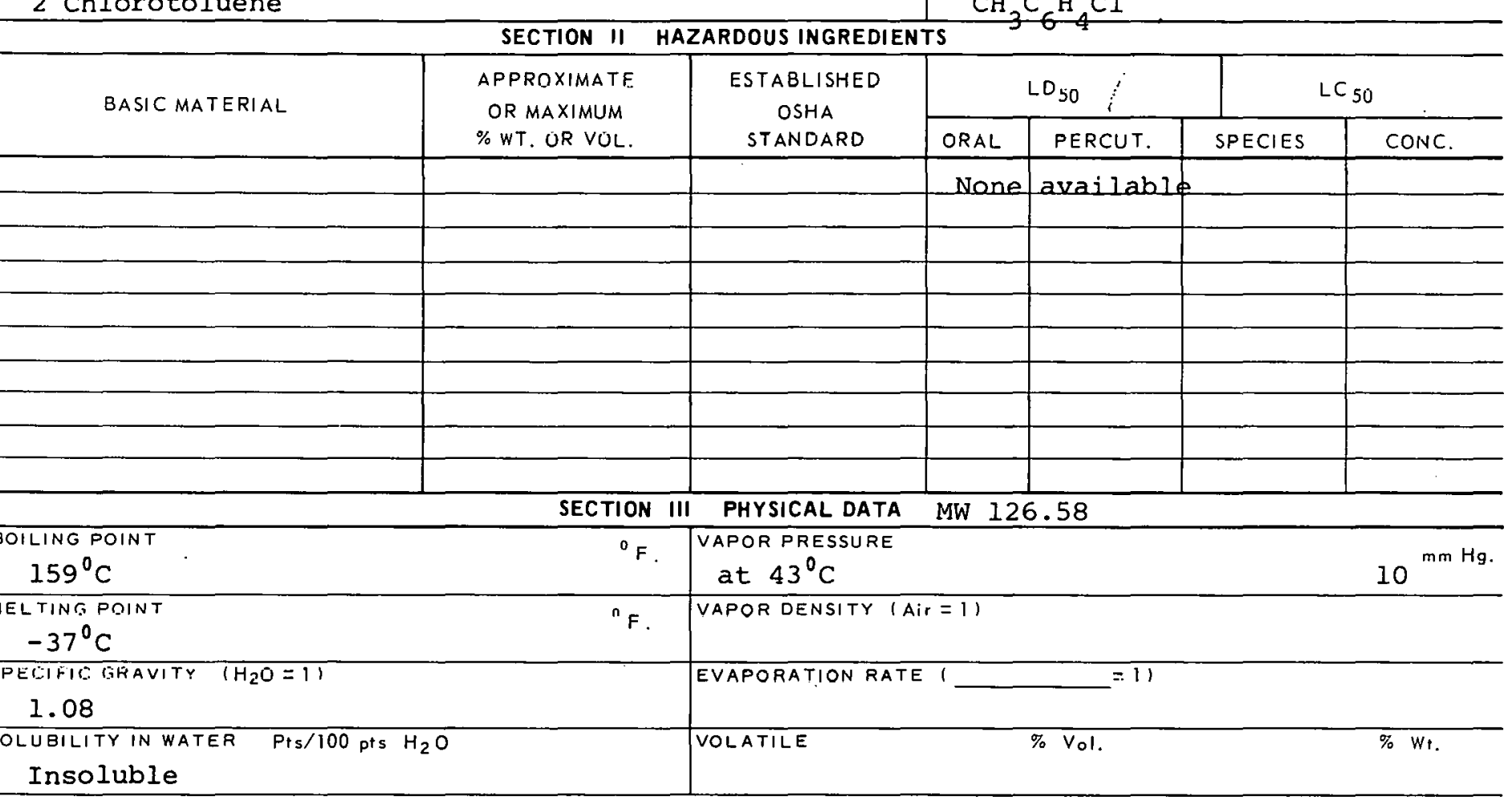
APPEARANCE AND ODOR

\section{Coloriless liquid}

\section{SECTION IV FIRE AND EXPLOSION HAZARD DATA}

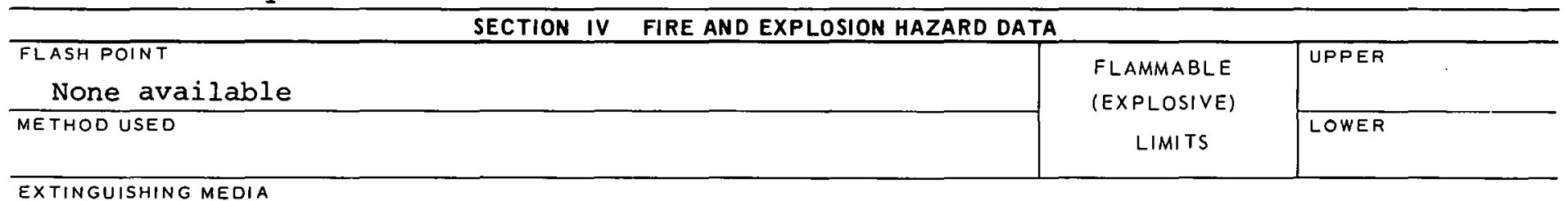

EXTINGUISHING MEDIA

Alcohol foam, carbon dioxide, dry chemical

SPECIAL FIRE FIGHTING PROCEDURES

$-$

UNUSUAL FIRE AND EXPLOSION HAZARDS

-- 


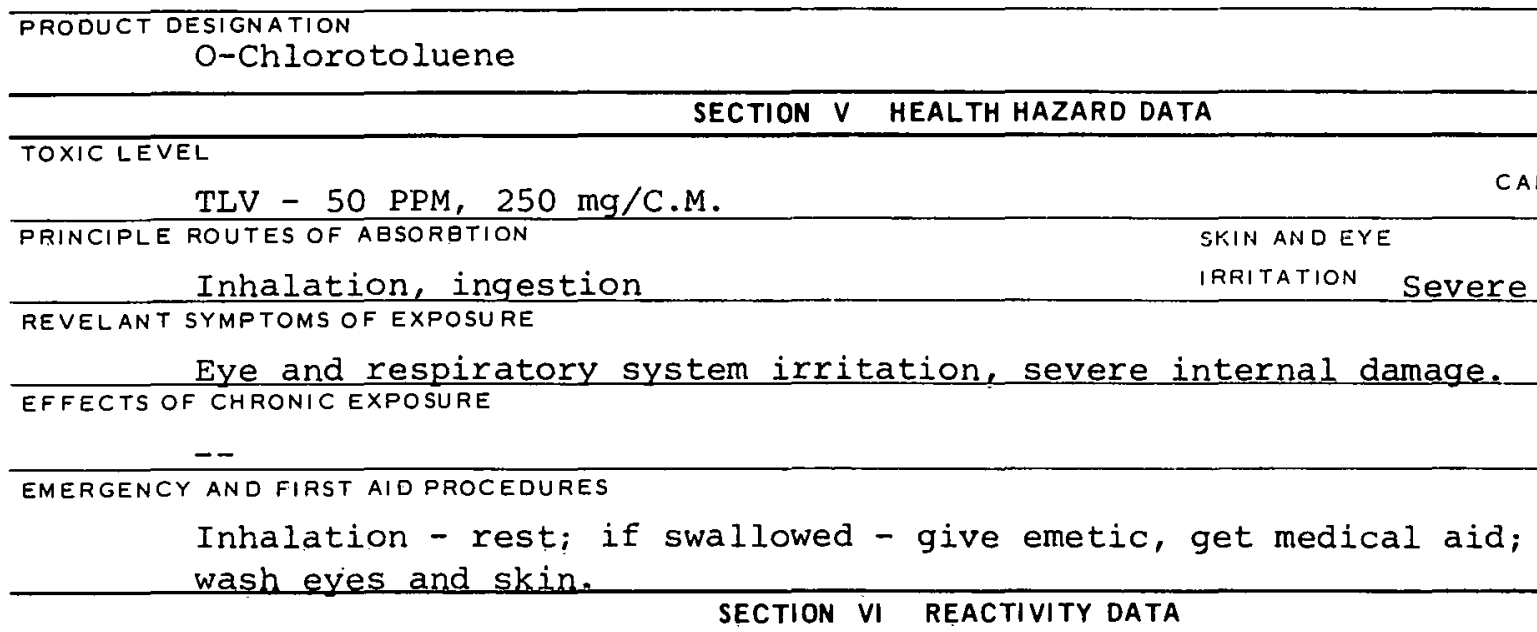




PRODUCT DESIGNATION
03-230-0367
P-Chlorotoluene

\section{MATERIAL SAFETY DATA SHEET}

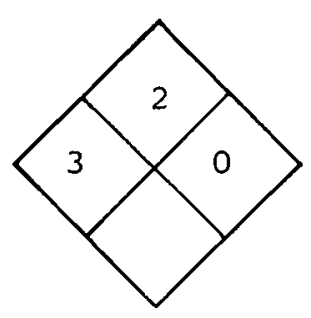

\section{SECTION I SOURCE AND NOMENCLATURE}

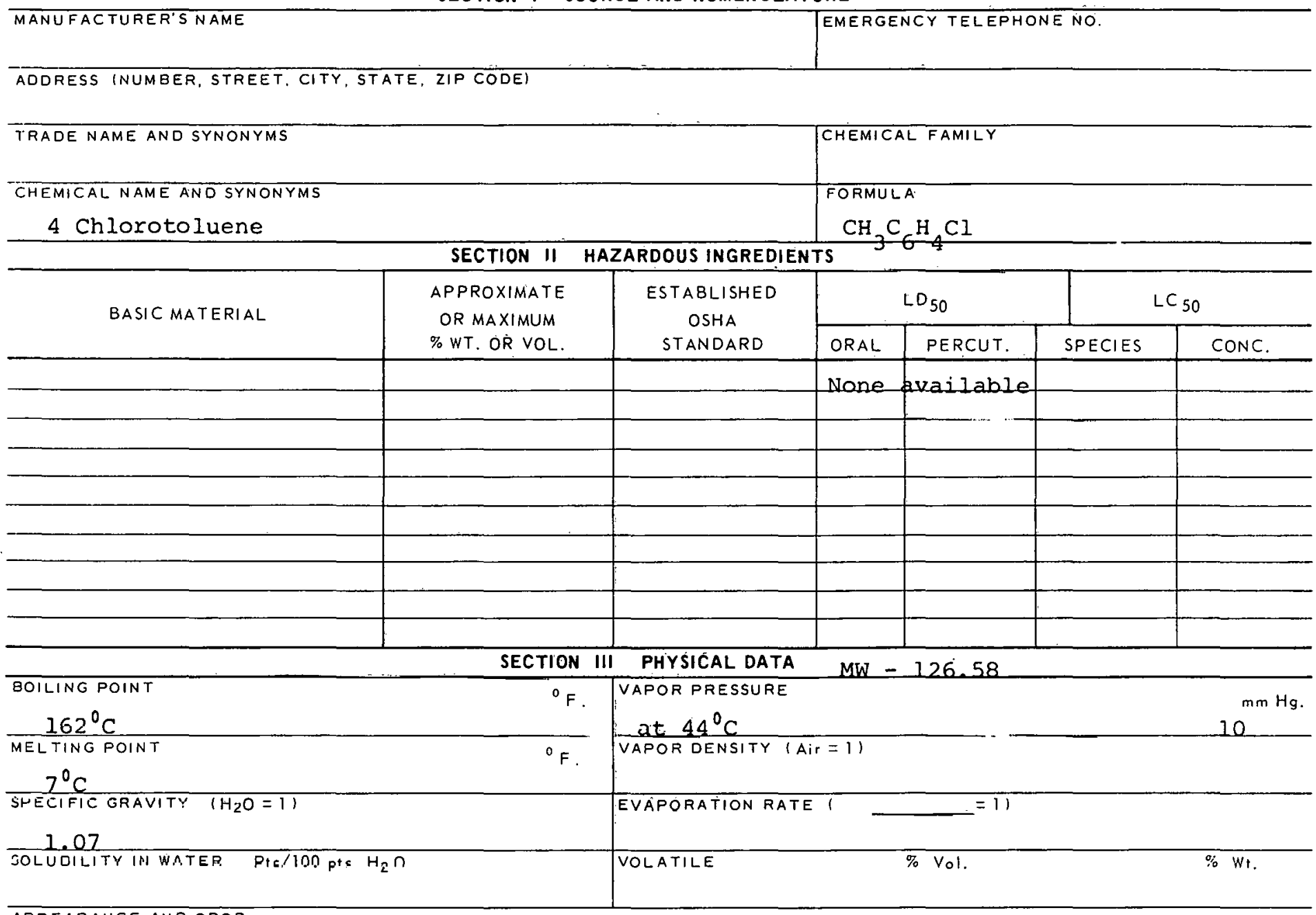

APPEARANCE AND ODOR

Colorless liquid

\begin{tabular}{l|c|c}
\hline \multicolumn{2}{c}{ SECTION IV FIRE AND EXPLOSION HAZARD DATA } & \\
\hline FLASH POINT & FLAMMABLE & UPPER \\
\hline ME I HUU USED & (EXPLOSIVE) \\
\cline { 2 - 4 } & LIMITS & LOWER \\
\hline
\end{tabular}

EXTINGUIGHING MLDIA

Alcohol foam, carbon dioxide, dry chemical

SPECIAL FIRE FIGHTING PROCEDURES

IINUSUAL FIRE AND EXPLOSION HAZARDS 


\section{TOXIC LEVEL}

None available

PRINCIPLE ROUTES OF ABSORBTION

Inhalation, ingestion
CARCINOGENIC No IRRITATION Severe

REVELANT SYMPTOMS OF EXPOSURE EYe \& respiratory system irritation, severe internal damage if swallowed.

EFFECTS OF CHRONIC EXPOSURE

$--$

EMERGENCY AND FIRST AID PROCEDURES Inhalation - rest; if swallowed - give emetic, get medical aid; wash skin \& eyes.

\section{SECTION VI REACTIVITY DATA}

CONDITIONS CONTRIBUTING TO INSTABILITY

CONDITIONS CONTRIBUTING TO HAZARDOUS POLYMERIZATION

INCOMPATABILITY (MATERIALS TO AVOID)

HAZARDOUS DECOMPOSITION PRODUCTS

SECTION VII SPILL OR LEAK PROCEDURES

STEPS TO BE TAKEN IN CASE MATERIAL IS RELEASED OR SPILLED

WASTE DISPOSAL METHOD

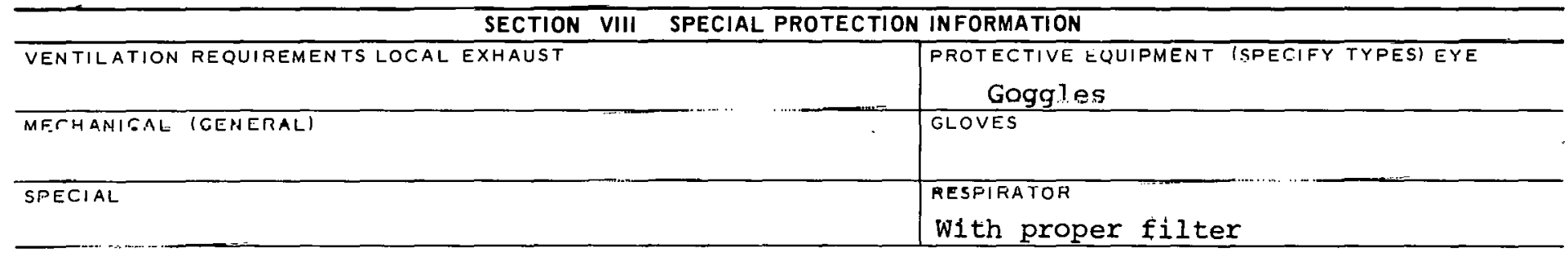

OTHER PROTECTIVE EQUIPMENT

PRECAUTIONS TO BE TAKEN IN HANDLING AND STORAGE

OTHER PRECAUTIONS

SIGNATURE

DATE

SIGNATURE 


\section{PRODUCT DESIGNATION}

03-309-2205

Methyl-Iso-Thiocyanate
MATERIAL SAFETY

DATA SHEET

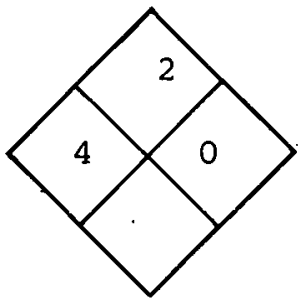

SECTION I SOURCE AND NOMENCLATURE

MANUFACTURER'S NAME

\section{E}

CITY, STATE, ZIP CODEI

ADDRESS (NUMBER, STREET, CITY, STATE, ZIP CODE)

\section{TRADE NAME AND SYNONYMS \\ Methyl Mustard Oil CHEMICAL NAME AND SYNONYMS \\ Methyl Isothiocyanate}

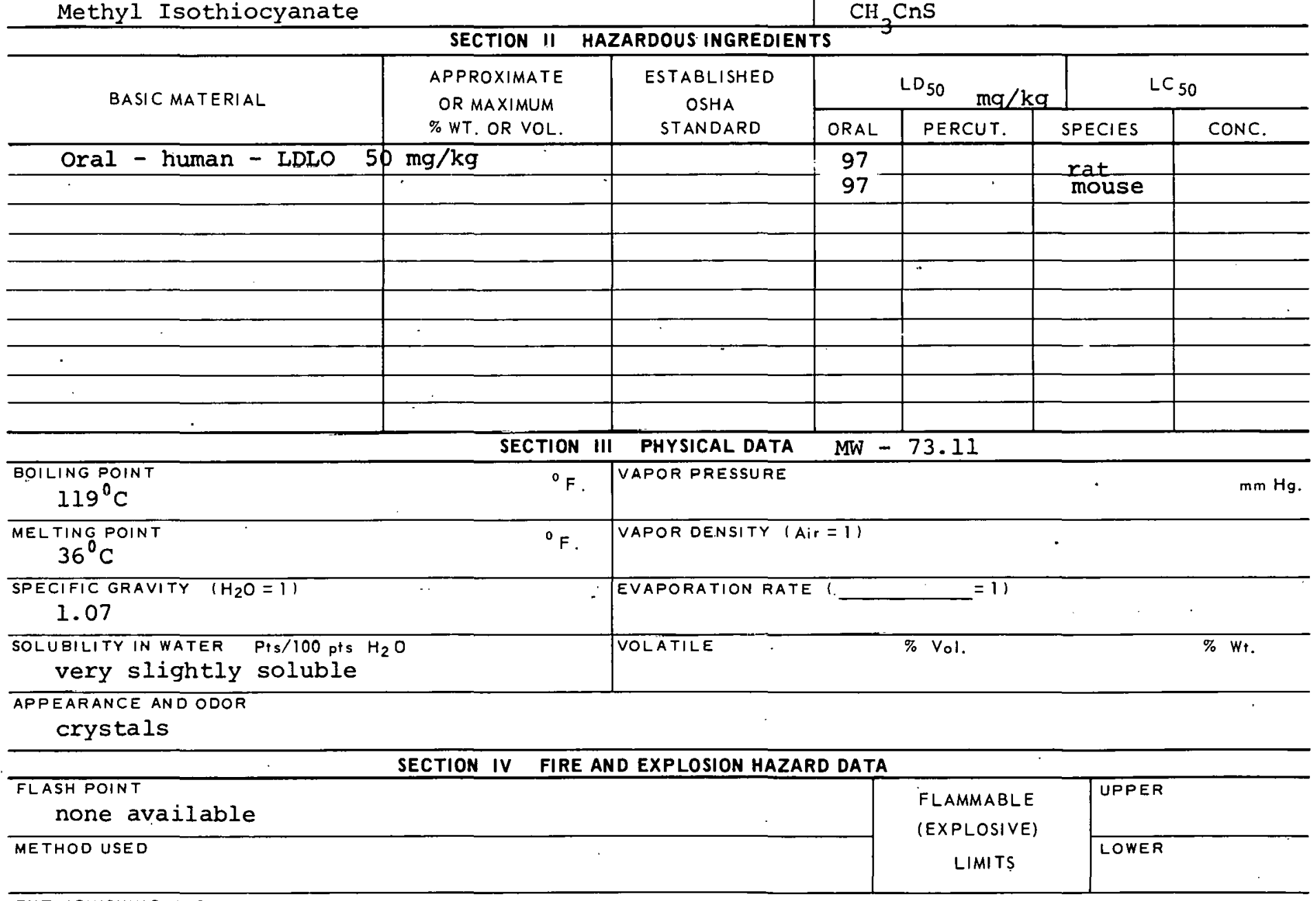

EXTINGUISHING MEDIA

water, alcohol foam, carbon dioxide, dry chemical.
EMERGENCY TELEPHONE NO. 


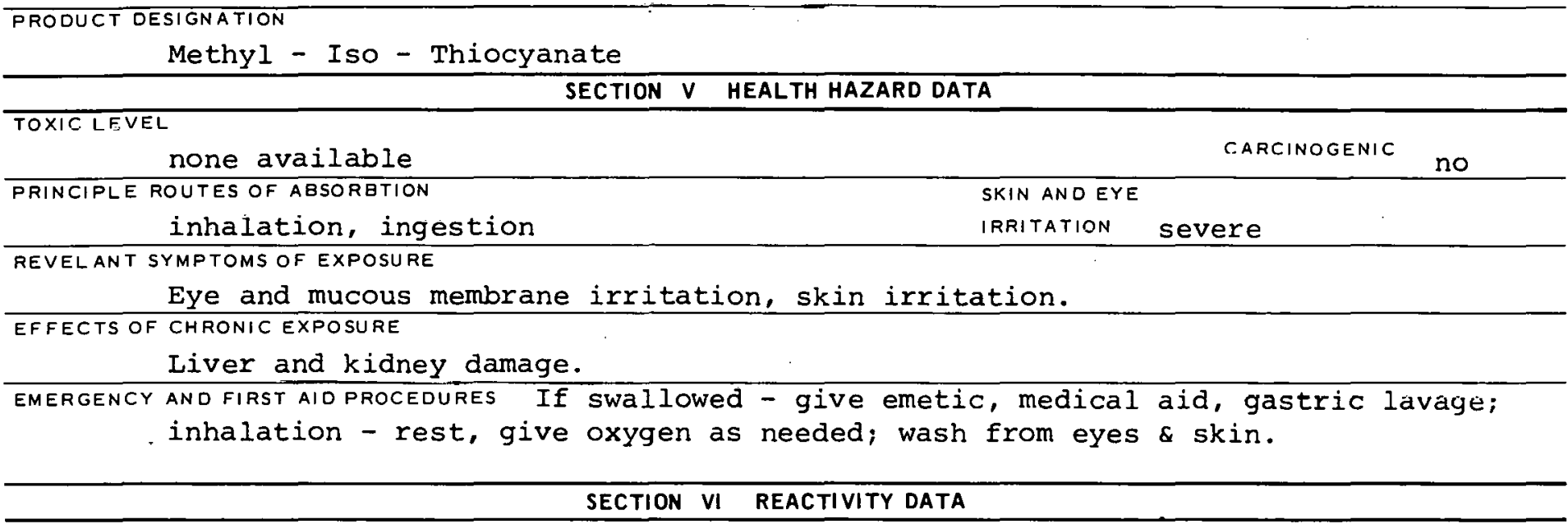

CONDITIONS COONTRIBUTING TO INSTABILITY

T.NNIITIONS CONTRIBUTING TO HAZARDOUS POLYMERIZATION

INCOMPATABILITY (MATERIALS TO AVOID)

HAZARDOUS DECOMPOSITION PRODUCTS

SECTION VII SPILL OR LEAK PROCEDURES

STEPS TO BE TAKEN IN CASE MATERIAL IS RELEASED OR SPILLEO

$A b s o r b$ on paper towels; evaporate in hood.

WASTE DISPOSAL METHOD

SECTION VIII SPECIAL PROTECTION INFORMATION

\begin{tabular}{l|l}
\hline VENTILATION REQUIREMENTS LOCAL EXHAUST & $\begin{array}{l}\text { PROTECTIVE EQUIPMENT (SPECIFY TYPES) EYE } \\
\text { Goggles }\end{array}$ \\
\hline MECHANICAL IGENERAL) & $\begin{array}{l}\text { fi.oVES } \\
\text { Ruhber (butyl.) }\end{array}$ \\
\hline SPECIAL & $\begin{array}{l}\text { RESPIRATOR } \\
\text { Self-contained }\end{array}$ \\
\hline
\end{tabular}

OTHER PROTECTIVE EQUIPMENT

Coveralis

SECTION IX SPECIAL PRECAUTIONS

PRECAUTIONS TO BE TAKEN IN HANDLING AND STORAGE

OTHER PRECAUTIONS

SIGNATURE

DATE 


\begin{tabular}{|l|}
\hline PRODUCT DESIGNATION \\
03-309-3605 \\
Methyl-M-Toluidine \\
\end{tabular}

\section{MATERIAL SAFETY \\ DATA SHEET}

\section{SECTION I SOURCE AND NOMENCLATURE}

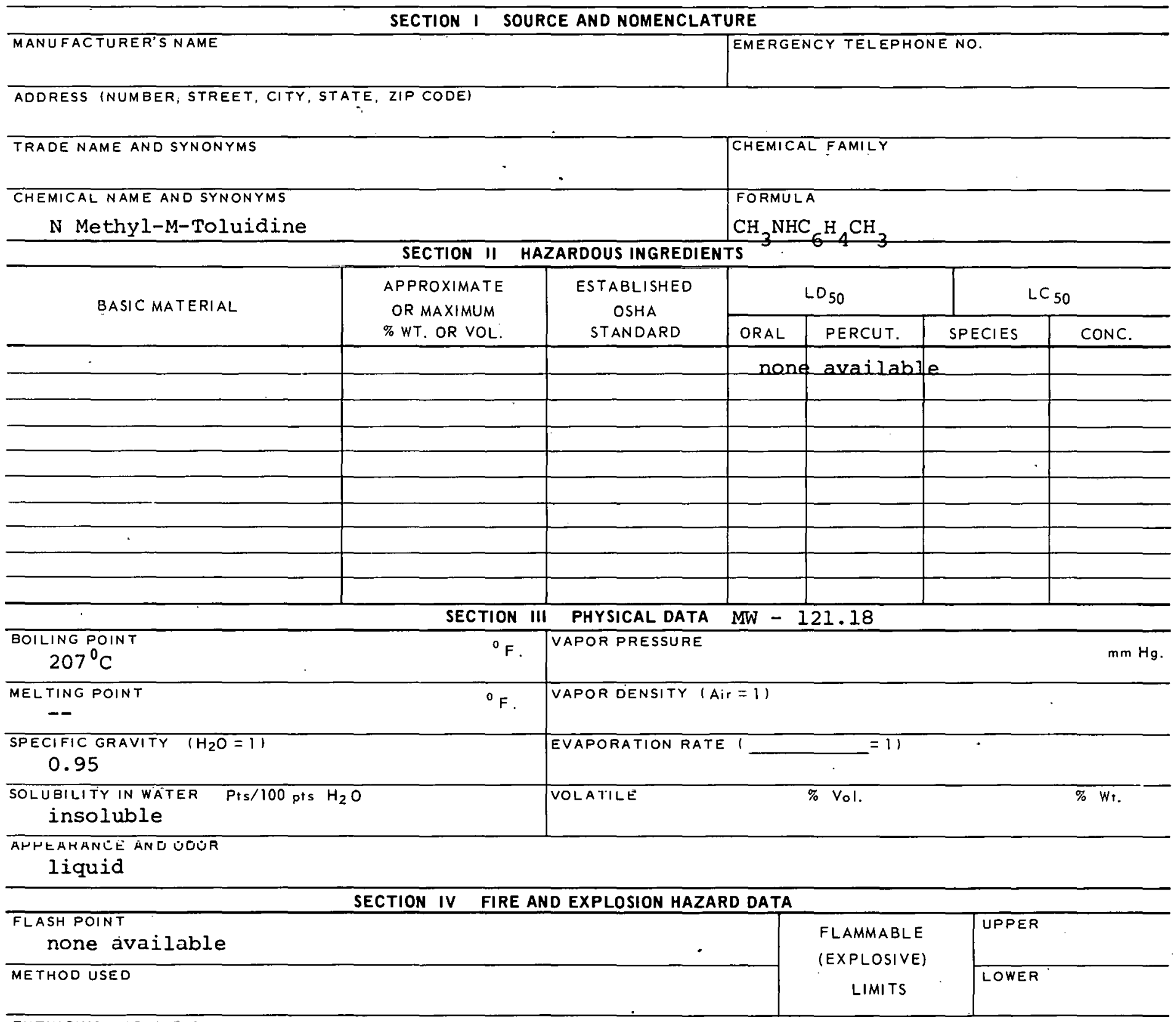

EXTINGUISHING MEDIA

Alcohol foam, carbon dioxide, ary chemical.

SPECIAL FIRE FIGHTING PROCEDURES

Wear full protective clothing.

UNUSUAL FIRE AND EXPLOSION HAZARDS 


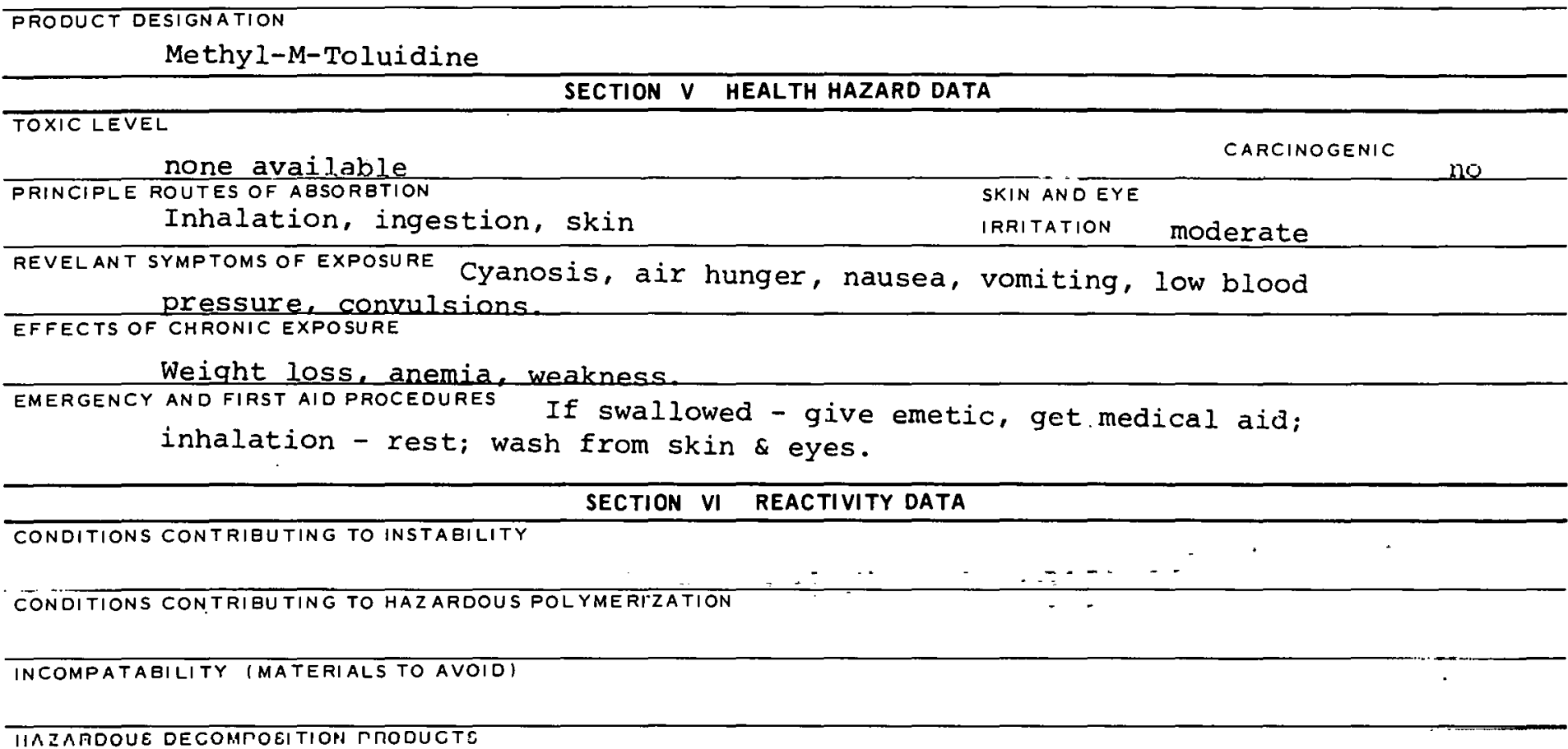

IIAZARDOUE DECOMTOEITION RRODUCTE

SECTION VII SPILL OR LEAK PROCEDURES

STEPS TO BE TAKEN IN CASE MATERIAL IS RELEASED OR SPILLED

WASTE DISPOSAL METHOD

SECTION VIII SPECIAL PROTECTION IN FORMATION

\begin{tabular}{l|l}
\hline VENTILATION REQUIREMENTS LOCAL EXHAUST & $\begin{array}{l}\text { PROTECTIVE EQUIPMENT (SPECIFY TYPES) EYE } \\
\text { Goggles }\end{array}$ \\
\hline MECHANICAL IGENERAL) & $\begin{array}{l}\text { GLOVES } \\
\text { Rubber }\end{array}$ \\
\hline SPECIAL & $\begin{array}{l}\text { REGPIRATOR } \\
\text { With proper filter }\end{array}$ \\
\hline
\end{tabular}

OTHER PROTECTIVE EQUIPMENT

SECTION IX SPECIAL PRECAUTIONS

PRECAUTIONS TO BE TAKEN IN HANDLING AND STORAGE

Outside storage preferred. Inside storage in a standard flammable liquids

storage cabinet.

OTHER PRECAUTIONS

SIGNATURE

DATE

309-3605B 


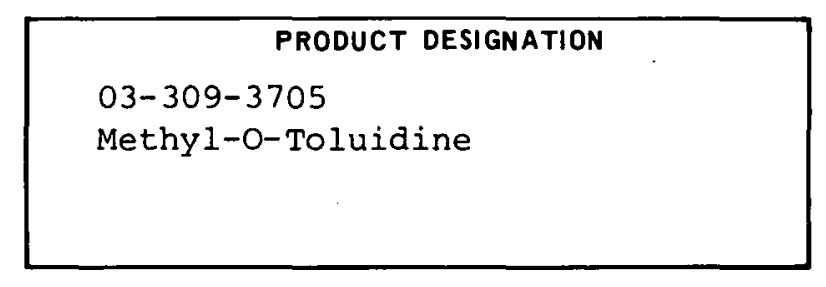

\section{MATERIAL SAFETY \\ DATA SHEET}

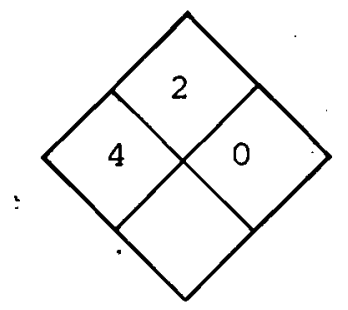

MANUFACTURER'S NAME
ADDRESS INUMBER, STREET, CITY,
TRADE NAME AND SYNONYMS
CHEMICAL NAME AND SYNONYMS
N MEthYl-O-TOluidine

\section{SECTION I SOURCE AND NOMENCLATURE}

EMERGENCY TELEPHONE NO.

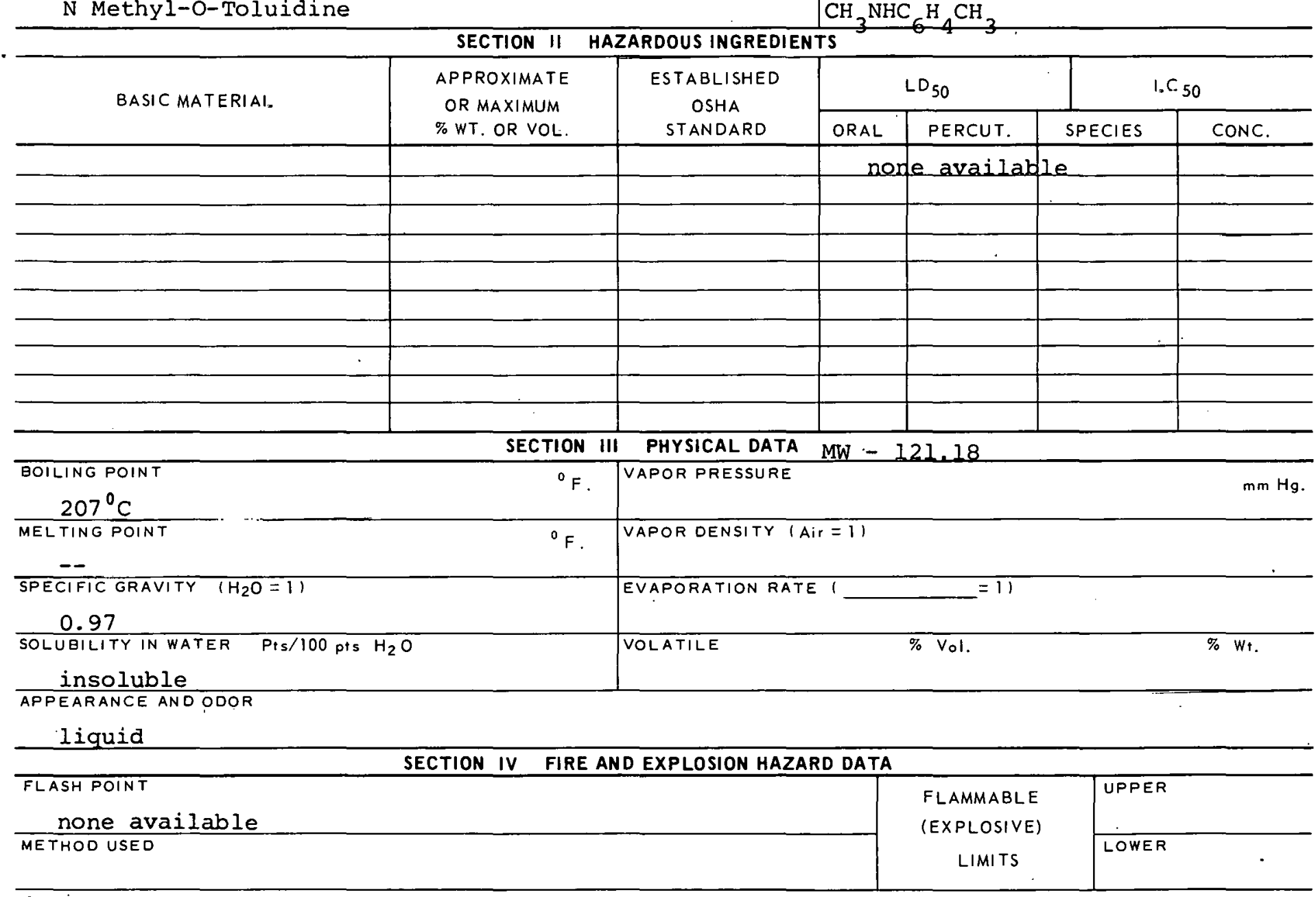

EXTINGUISHING MEDIA

Alcohol foam, carbon dioxide, dry chemical

SPECIAL FIRE FIGHTING PROCEDURES

Wear full protective clothing.

UNUSUAL FIRE AND EXPLOSION HAZARDS 
TOXIC LEVEL none available

PRINCIPLE ROUTES OF ABSORBTION

Inhalation, ingestion, skin

SKIN AND EYE

IRRITATION moderate

REVELANT SYMPTOMS OF EXPOSURE Cyanosis, air hunger, nausea, vomiting, low blood pressure, convulsions.

EFFECTS OF CHRONIC EXPOSURE

Weight loss, anemia, weakness

EMERGENCY AND FIRST AID PROCEDURES If swallowed - give emetic, get medical aid;

inhalation - rest; wash from skin \& eyes.

\begin{tabular}{l} 
SECTION VI REACTIVITY DATA \\
\hline CONDITIONS CONTRIBUTING TO INSTABILITY \\
\hline CONOITIONS CONTRIBUTING TO HATARDOUS POL YMERIZATION
\end{tabular}

CONOITIONS CONTRIBUTING TO HAZAROOUS POLYMERIZATION

INCOMPATABILITY (MATERIALS TO AVOIOI)

HALARDÖUS DECOMPOSTTION PKUUUL IS

SECTION VII SPILL OR LEAK PROCEDURES

STEPS TO BE TAKEN IN CASE MATERIAL IS RELEASED OR SPILLED

\section{WASTE DISPOSAL METHOD}

SECTION VIII SPECIAL PROTECTION INFORMATION

\begin{tabular}{|c|c|}
\hline VENTILATION REQUIREMENTS LOCAL EXHAUST & $\begin{array}{l}\text { PROTECTIVE EQUIPMENT (SPECIFY TYPES) EYE } \\
\text { ringglPS }\end{array}$ \\
\hline MECHANICAL (GENERAL) & $\begin{array}{l}\text { GLOVES } \\
\text { Rubber }\end{array}$ \\
\hline SHELIAL & $\begin{array}{l}\text { RESPIRATOR } \\
\text { With proper filer }\end{array}$ \\
\hline
\end{tabular}

SECTION IX SPECIAL PRECAUTIONS

PRECAUTIONS TO BE TAKEN IN HANDLING AND STORAGE

Outside storage preferred. Inside storage in a standard flammable liquids

storage cabinet.

UTHER FRECAUTIOHS 


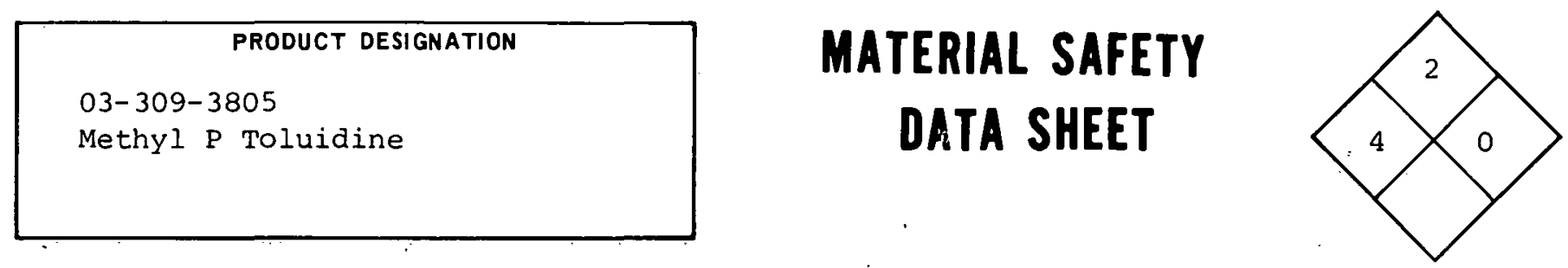

SECTION I SOURCE AND NOMENCLATURE

MANUFACTURER'S NAME

|EMERGENCY TELEPHONE NO.

ADDRESS INUMBER, STREET, CITY, STATE, ZIP CODE)

TRADE NAME ANO SYNONYMS

CHEMICAL FAMILY

CHEMICAL NAME ANO SYNONYMS

$N$ Methyl P Toluidine

FORMULA

$\mathrm{CH}_{3} \mathrm{NHC}_{6} \mathrm{H}_{4} \mathrm{CH}_{3}$

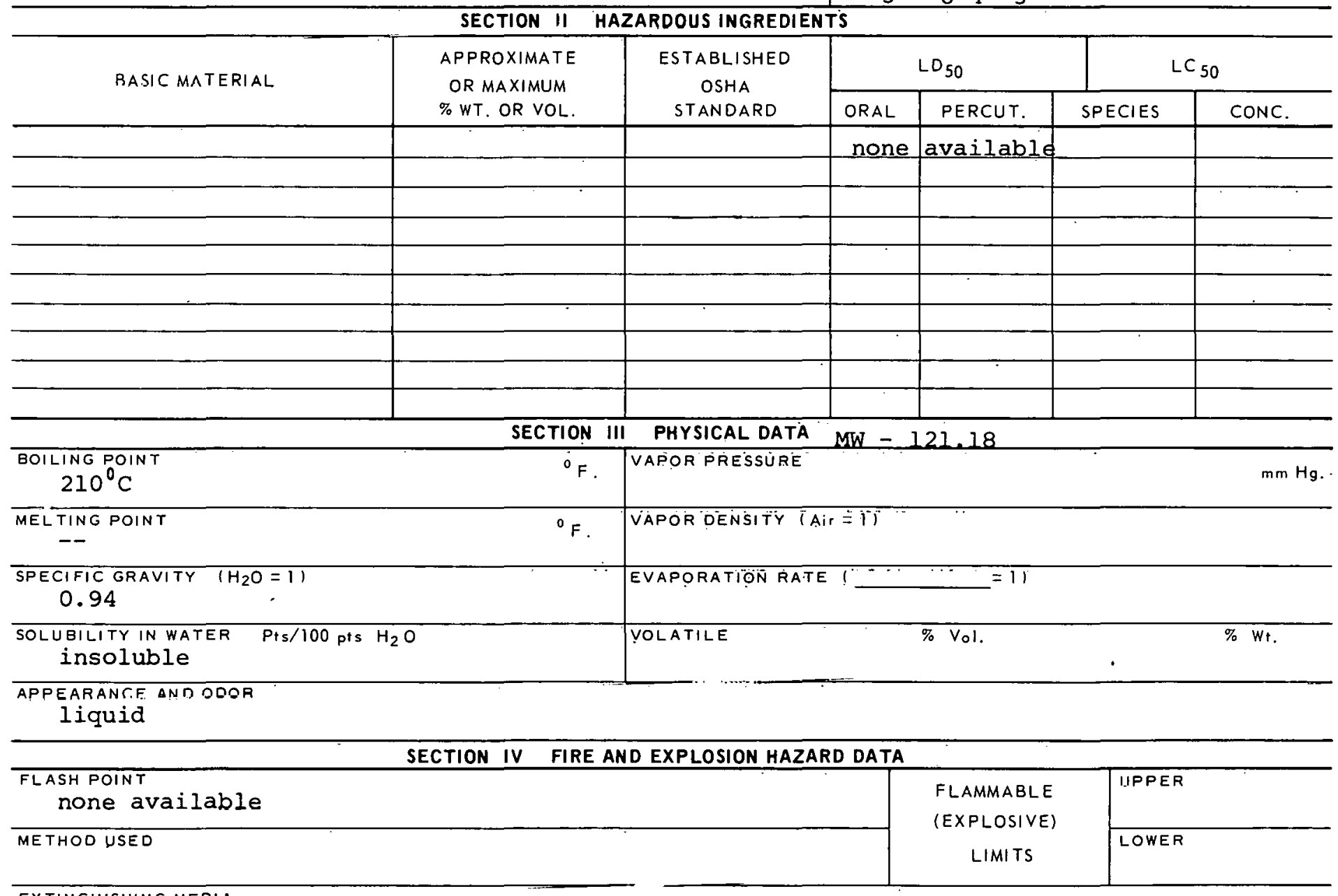

EXTINGUISHINC MEDIA

alcohol foam, carbnn dioxide, dry chemical

SPECIAL FIRE FIGHTING PROCEDURES

wear full protective clothing

UNUSUAL FIRE AND EXPLOSION HAZARDS 


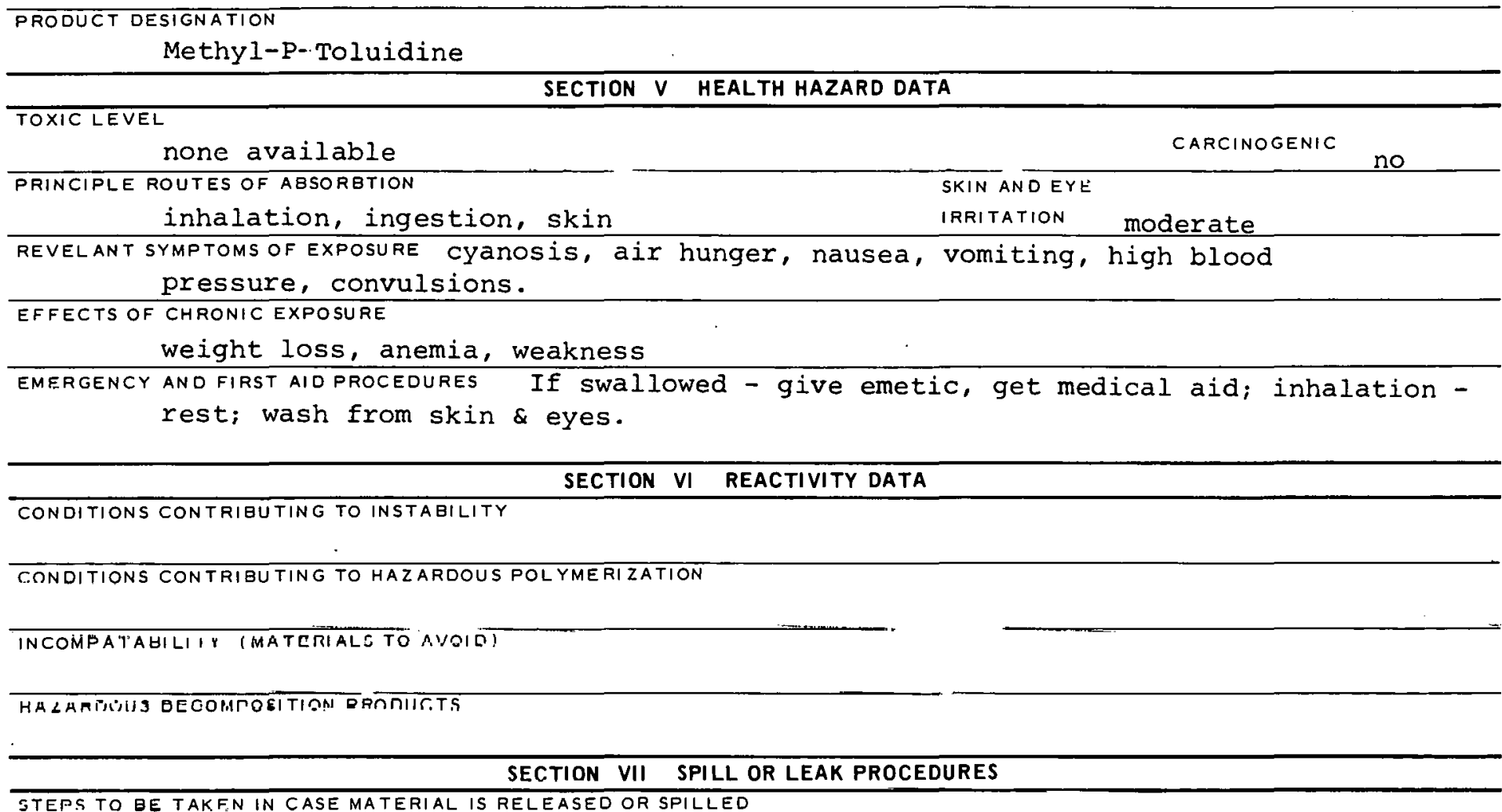

STEPS TO BE TAKFN IN CASE MATERIAL IS RELEASED OR SPILLED

WASTE DISPOSAL METHOD

SECTION VIII SPECIAL PROTECTION INFORMATION

\begin{tabular}{l|l}
\hline VENTILATION RFQUIREMENTS LOCAL EXHAUST & $\begin{array}{c}\text { PROTECTIVE EQUIPMENT (SPECIFY TYPESIEYE } \\
\text { gOggles }\end{array}$ \\
\hline MECHANICAL IGENERALI & $\begin{array}{c}\text { GLOVES } \\
\text { rubber }\end{array}$ \\
\hline SRECIAL. & $\begin{array}{c}\text { RESPIRATOR } \\
\text { with proper filter }\end{array}$ \\
\hline
\end{tabular}

OTHER PROTECTIVE EQUIPMENT

\section{SECTION IX SPECIAL PRECAUTIONS}

PRECAUTIONS TO BE TAKEN IN HANDLING AND STORAGE

Outside storage preferred. Inside storage in a standard flammable liquids storage cabinet.

OTHER PRECAUTIONS 


PRODUCT DESIGNATION
03-309-8205
03-309-8305
Morpholine

\section{MATERIAL SAFETY \\ DATA SHEET}

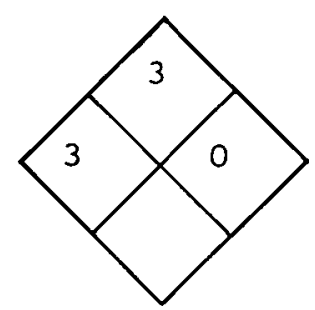

\section{SECTION I SOURCE AND NOMENCLATURE}

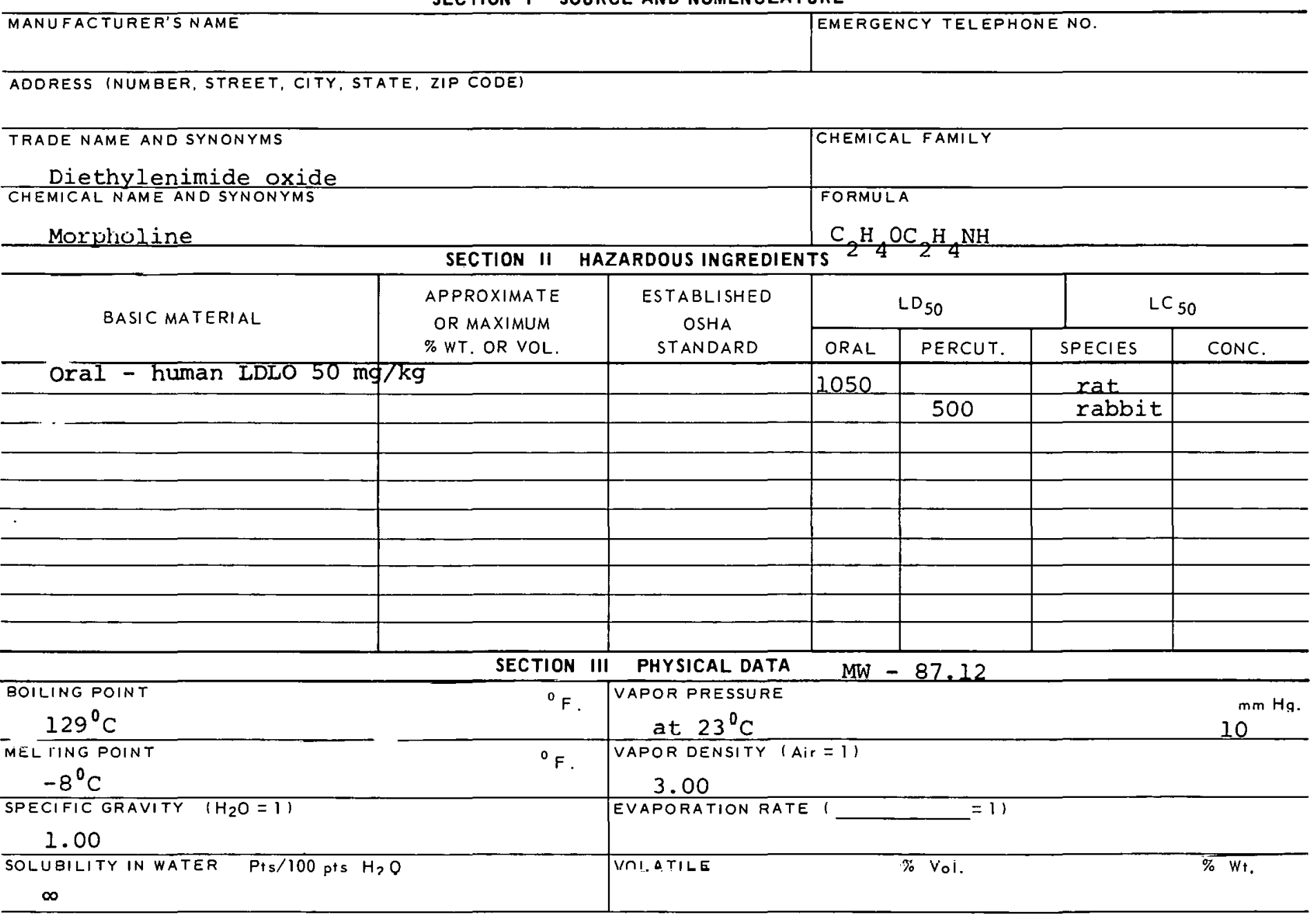

AP.PEARANCE AND ODOR

Colorless, hygroscopic oil, pepper-like odor.

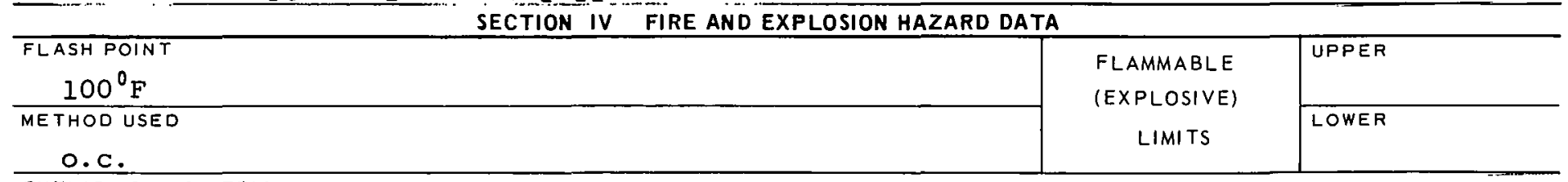

EXTINGUISHING MEDIA

Alcohol foam, carbon dioxide, dry chemical.

SPECIAL FIRE FIGHTING PROCEDURES

UNUSUAL FIRE AND EXPLOSION HAZARDS 


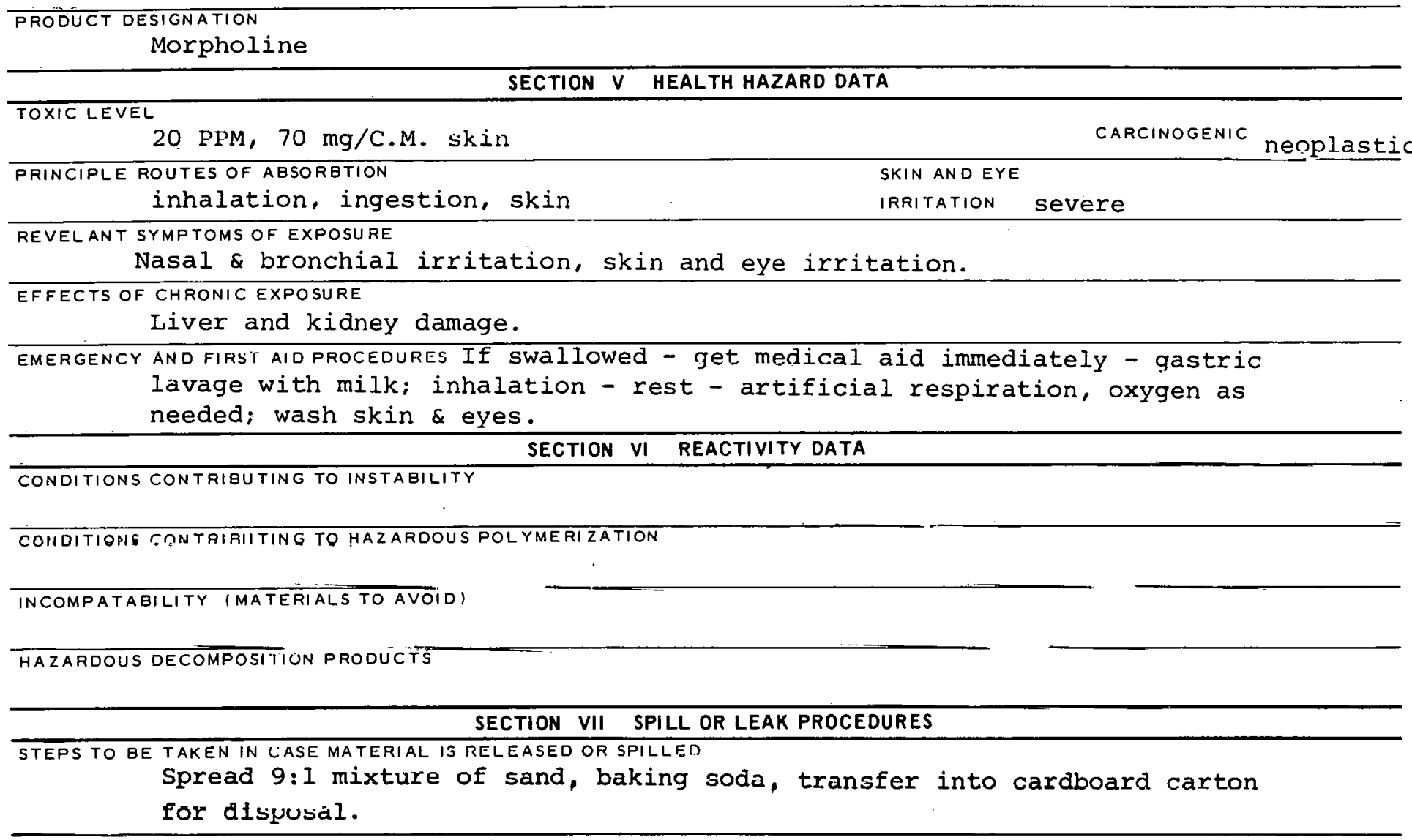

WASTE DISPOSAL METHOD

SECTION VIII SPECIAL PROTECTION INFORMATION

\begin{tabular}{|c|c|c|}
\hline VENTILATION & REQUIREMENTS LOCAL EXHAUST & $\begin{array}{l}\text { PROTECTIVE EQUIPMENT (SPECIFY TYPES) EYE } \\
\text { gחgglPS }\end{array}$ \\
\hline MECHANICAL & (GENERAL) & $\begin{array}{l}\text { GLOVES } \\
\text { rubber (butyl) }\end{array}$ \\
\hline$S F C \overline{C I \wedge L}$ & & $\begin{array}{l}\text { RESPIRATOR } \\
\text { with proper filter }\end{array}$ \\
\hline
\end{tabular}

OTHER FROTECTIVE EQUIPMENT

Plastic coveralls

SECTION IX SPECIAL PRECAUTIONS

PRECAUTIONS TO BE TAKEN IN HANDLING AND STOPRAGE

store tightly closed bottle in a dark, cool place.

OTHER PKECAUTIONS

SIGNATURE

OATF

309-8205B 


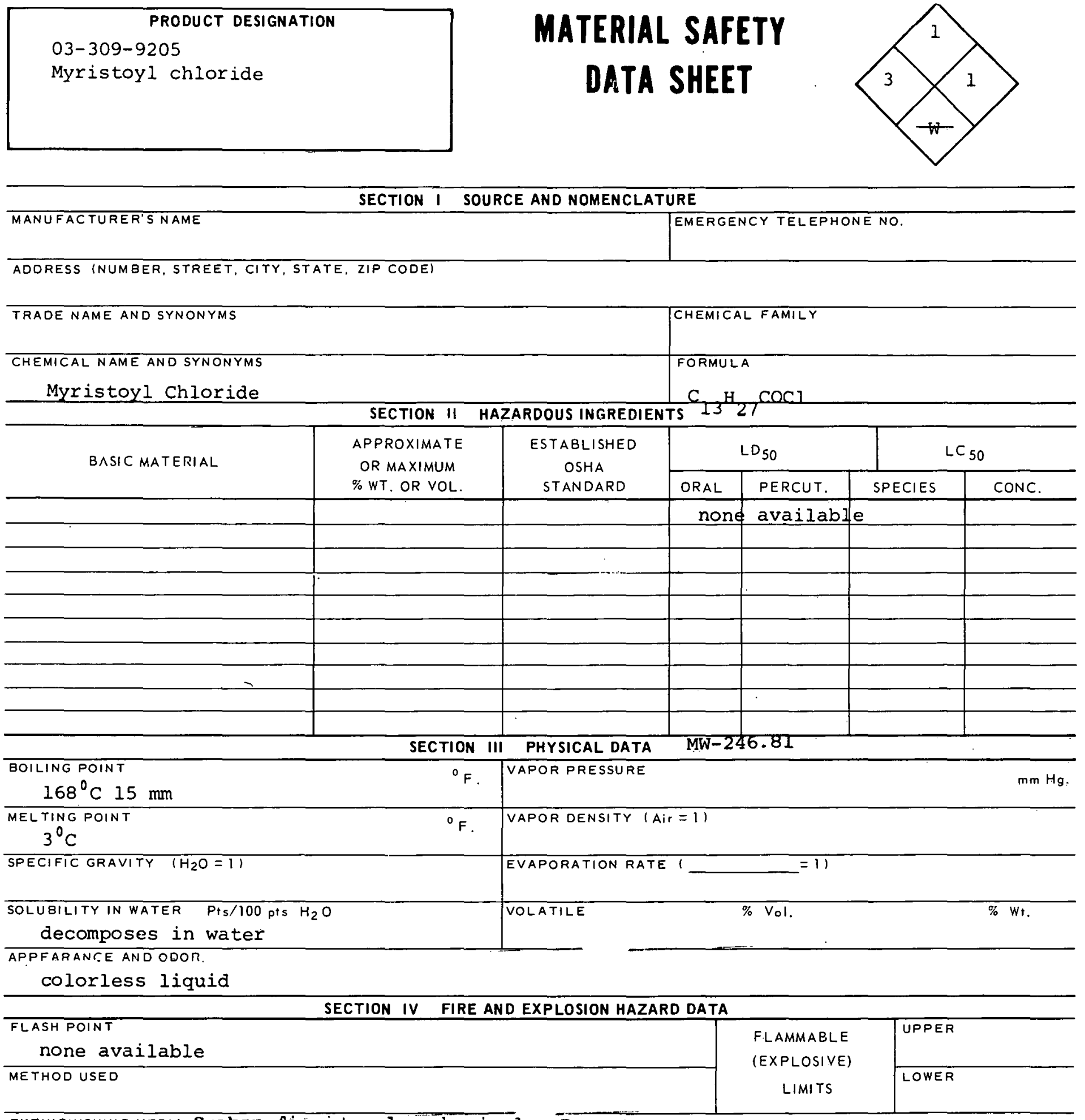

Extincuishlis medin Carbon dioxide, dry chemical. Do not use water.

SPECIAL FIRE FIGHTING PROCEDURES

Full protective clothing, self-contained breathing apparatus.

UNUSUAL FIRE AND EXPLOSION HAZARDS

When heated to decomposition point, gives off toxic $\mathrm{HCl}$. 


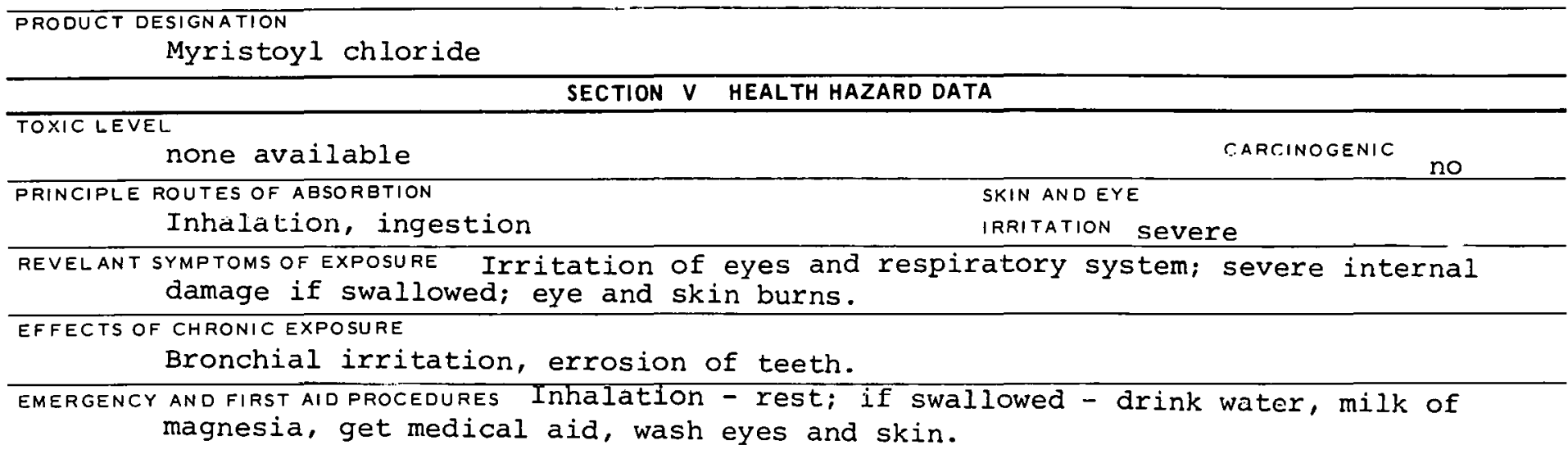

\begin{tabular}{|c|c|c|c|}
\hline SECTION & REACTIVITY DATA & & \\
\hline \multicolumn{4}{|l|}{ CONDITIONS CONTRIBUTING TO INSTABILITY } \\
\hline \multicolumn{4}{|l|}{ SONDITINNS COONTRIBUTING TO HAZARDOUS POLYMERIZA IIUN } \\
\hline \multicolumn{4}{|l|}{ INCOMPATABILITY (MATERIALS TO AVOID) } \\
\hline \multicolumn{4}{|l|}{ Reacts with water or alcohol } \\
\hline \multicolumn{4}{|l|}{ HAZARDOUS DECOMPOSITION PRODUCYS } \\
\hline SECTION VII & SPILL OR LEAK PROCEDURES & & . \\
\hline
\end{tabular}

STEPS TO BE TAKEN IN CASE MATERIAL IS RELEASED OR SPILLED

Flush spills with large quantities of water and ventilate area to remove vapor. WASTE DISPOSAL METHOD

\section{SECTION VIII SPECIAL PROTECTION INFORMATION}

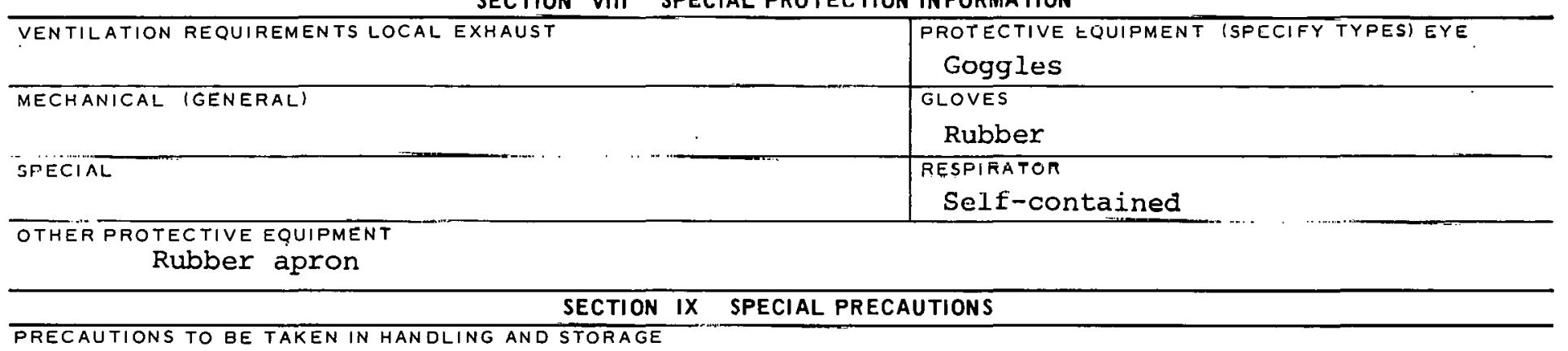

PRECAUTIONS TO BE TAKEN IN HANDLING AND STORAGE

OTHER PRECAUTIONS

SIGNATURE

DATE

$309-9205 B$ 


\section{PRODUCT DESIGNATION}

03-314-0286

Amsco Diluent 125-82
MATERIAL SAFETY

DATA SHEET

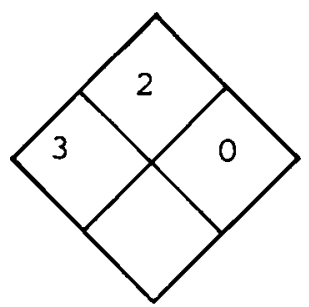

\section{SECTION I SOURCE AND NOMENCLATURE}

\section{MANUFACTURER'S NAME}

Amsco Division-Union Oil

ADORESS (NUMBER, STREET, CITY, STATE, ZIP CODE)

\section{TRADE NAME AND SYNONYMS \\ Amsco odorless mineral spirits \\ CHEMICAL NAME AND SYNONYMS \\ Amsco Diluent 125-82}

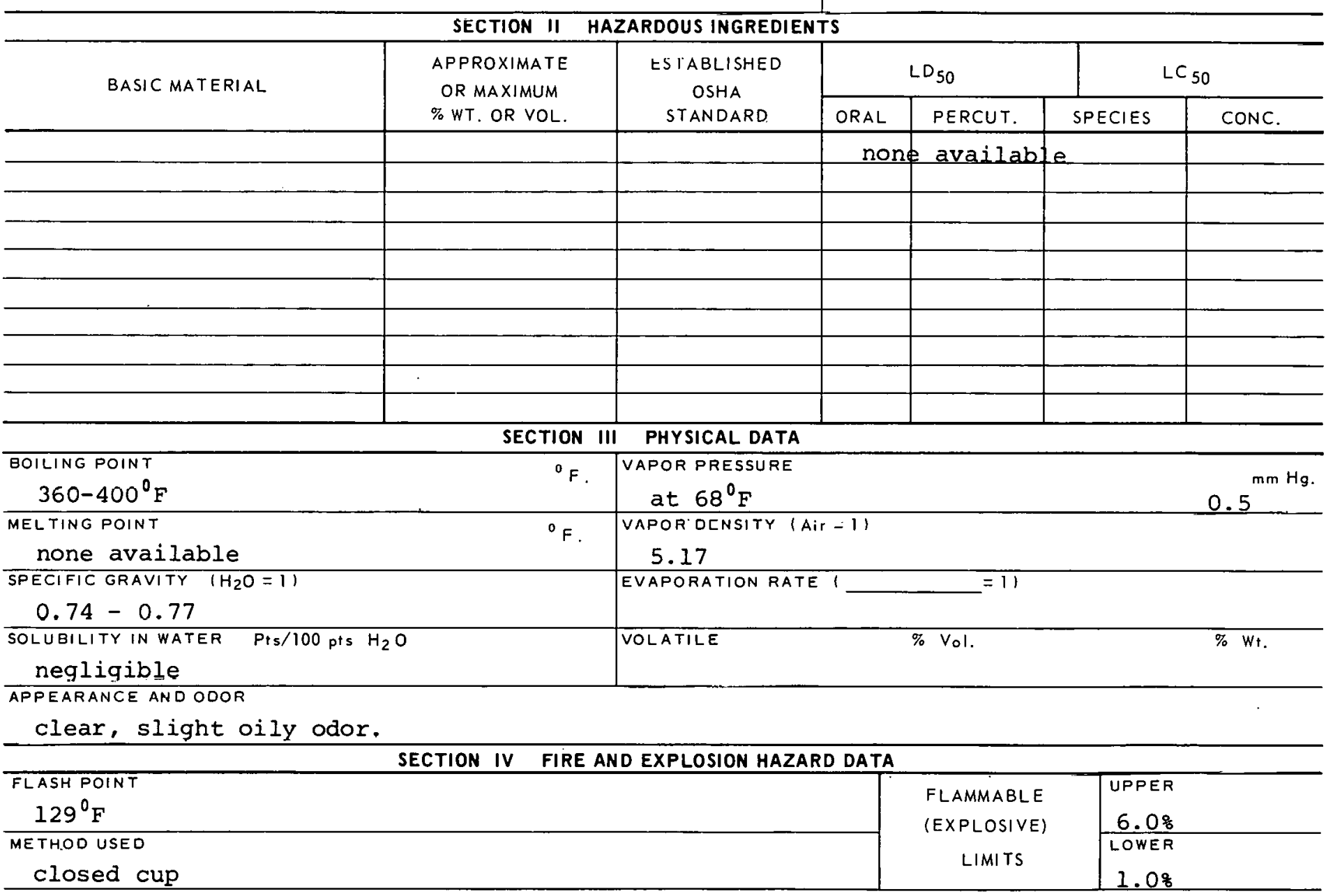

EXTINGUISHING MEDIA

water spray, $\mathrm{CO}_{2}$, foam, dry chemical

SPECIAL FIRE FIGHTING PROCEOURES

none

UNUSUAL FIRE AND EXPLOSION HAZARDS

none

CHEMICAL FAMILY

Petroleum hydrocarbon family Pormula 


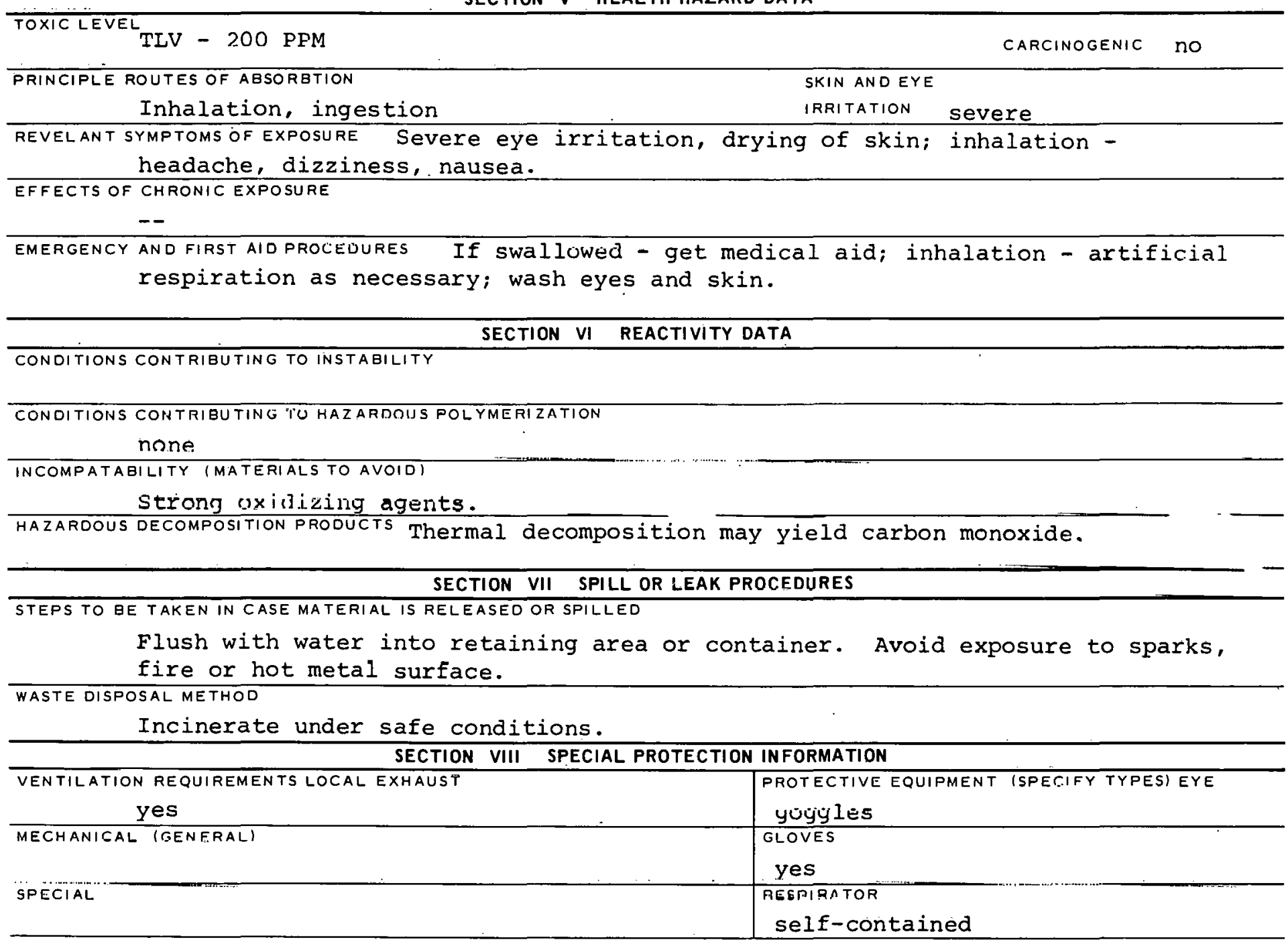

OTHER PROTECTIVE EQUIPMENT

Eye bath and safety shower.

SECTION IX SPECIAL PRECAUTIONS

PRECAUTIONS TO BE TAKEN IN HANDLING AND STORAGE

Keep away from heat, sparks and open flame. Use with adequate ventilation.

OTHER PRECAUTIONS

\begin{tabular}{l|l|l}
\hline SIGNATURE & OATE \\
\hline
\end{tabular}


CAS : 000091203

\section{PRODUCT DESIGNATION}

03-314-0706

Naphthalene
MATERIAL SAFETY

DATA SHEET

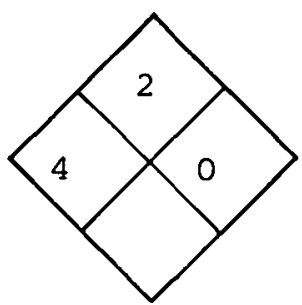

\section{SECTION I SOURCE AND NOMENCLATURE}

\begin{tabular}{l|l|l}
\hline SECTION I SOURCE AND NOMENCLATURE \\
\hline MANUFACTURER'S NAME & EMERGENCY TELEPHONE NO. \\
\hline ADDRESS (NUMBER, STREET, CITY, STATE, ZIP CODE)
\end{tabular}

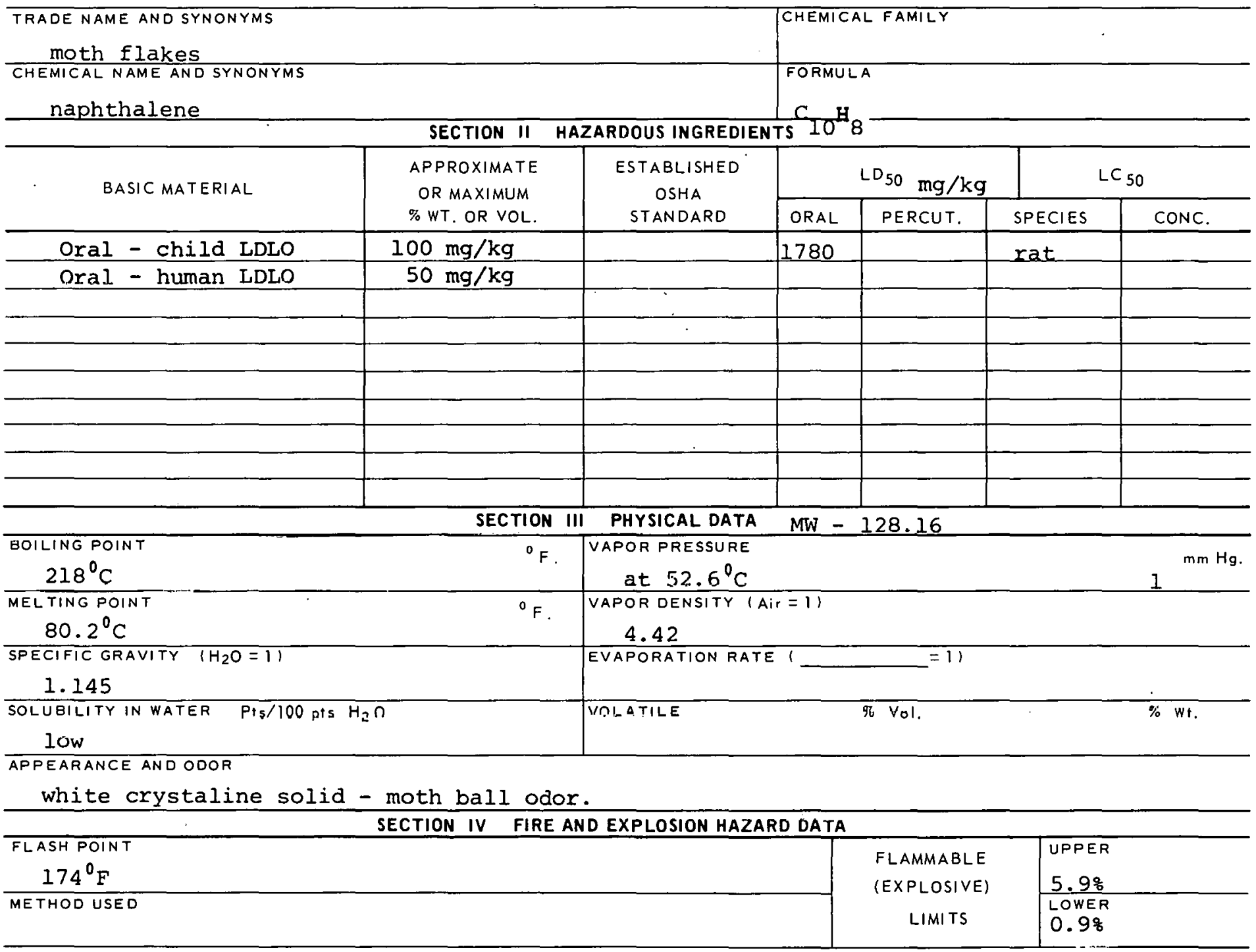

EXTINGUISHING MEDIA

Water, carbon dioxide, dry chemical or foam.

\section{SPECIAL FIRE FIGHTING PROCEDURES}

Wear goggles and self-contained breathing apparatus. UNUSUAL FIRE AND EXPLOSION HAZARDS 
TOXICLEVEL

TLV - $10 \mathrm{PPM}, 50 \mathrm{mg} / \mathrm{C} . \mathrm{M}$.

PRINCIPLE ROUTES OF ABSORBTION SKIN AND EYE

inhalation, ingestion

IRRITATION yes

REVELANT SYMPTOMS OF EXPOSURE Gastroenteric distress, tremors, convulsions, fever, respiratory failure, headache, nausea, vomiting.

EFFECTS OF CHRONIC EXPOSURE

clouding of lens.

EMERGENCY AND FIRST AID PROCEDURES Inhalation - rest; if swallowed - gastric lavage,

blood transfusion, oxygen.

SECTION VI REACTIVITY DATA

CONDITIONS CONTRIBUTING TO INSTABILITY

CONDITIONS CONTRIBUTING TO HAZARDOUS POL YMERI ZATION

INCOMPATABILITY (MATERIALS TO AVOID)

HAZARDOUS DECOMPOSITION PRODUCTS

SECTION VII SPILL OR LEAK PROCEDURES

STEPS TO AF. TAKEN IN CASE MATERIAL IS RELEASED OR SPILLED

WASTE DISPQSAL METHOD

\section{SECTION VIII SPECIAL PROTECTION INFORMATION}

\begin{tabular}{l|l}
\hline VENTILATION REQUIREMENTS LOCAL EXHAUST & \\
\hline MECHANICAL IGENERAL) & GLOVES \\
\hline SPECIAL & FEEPIRATOR \\
\hline
\end{tabular}

OTHER PROTECTIVE EQUIPMENT

SECTION IX SPECIAL PRECAUTIONS

PRECAUTIONS TO BE TAKEN IN HANDLING AND STORAGE

OTHER PRECAUTIONS

SIGNATURE

DATE

314-0706B 


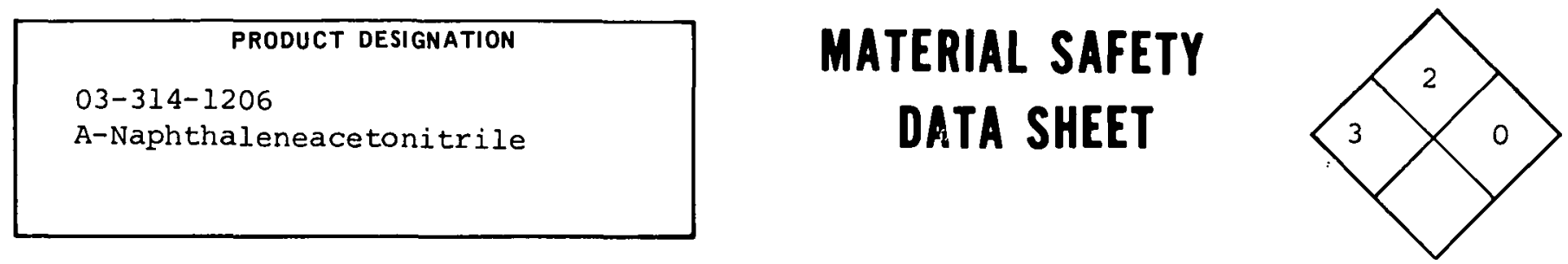

\section{SECTION I SOURCE AND NOMENCLATURE}

MANUFACTURER'S NAME

IEMERGENCY TELEPHONE NO.

ADDRESS (NUMGER, STREET, CITY, STATE, ZIP CODE)

TRADE NAME AND SYNONYMS

1 Naphthaleneacetonitrile

CHEMICAL NAME AND SYNONYMS

$\alpha$ naphthaleneacetonitrile

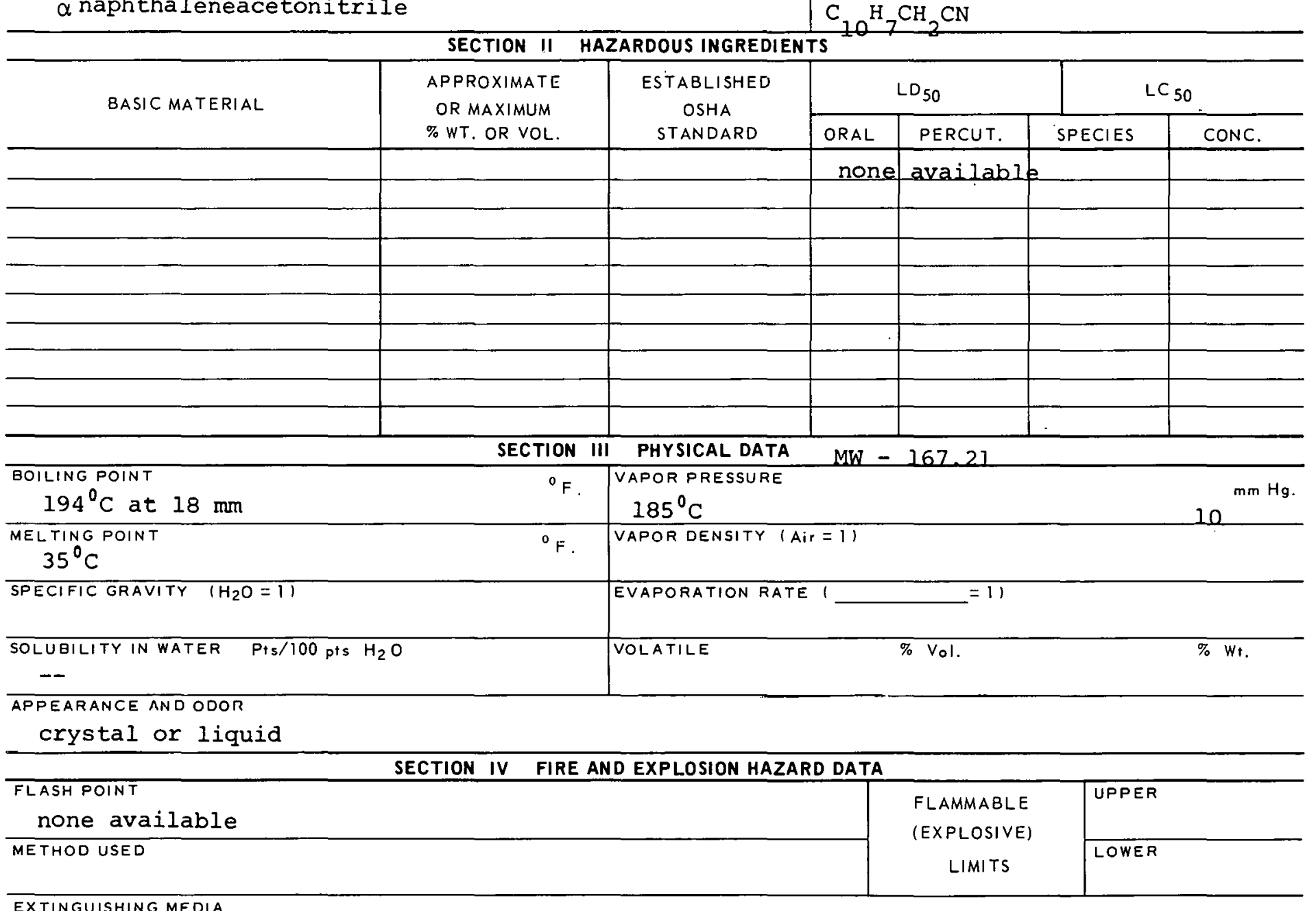

EXTINGUISHING MEDIA

alcohol foam, carbon dioxide, dry chemical

SPECIAL FIRE FIGHTING PROCEDURES

UNUSUAL FIRE ANO EXFLOSION HAZARDS 
SECTION $V$ HEALTH HAZARD DATA

TOXIC LEVEL

PRINCIPLE ROUTE OF OA

CARCINOGENIC

inhalation, ingestion (mainly)

SKIN AND EYE

IRRITATION moderate

REVELANT SYMPTOMS OF EXPOSURE Dizziness, rapid respiration, headache, drowsiness, drop in blood pressure, rapid pulse.

EFFECTS OF CHRONIC EXPOSURE Dizziness, weakness, hoarseness, lung congestion, eye irritation, loss of weight and appetite.

EMERGENCY AND FIRST AID PROCEDURES If swallowed - cyanide antidote; inhalation - inhale amyl -

nitrite artificial respiration; get medical aid; wash skin \& eyes.

\section{SECTION VI REACTIVITY DATA}

CONDITIONS CONTRIBUTING TO INSTABILITY

CONDITIONS CONTRIBUTING TO HAZARDOUS POLYMERIZATION

INCOMPATABILITY (MATERIALS TO AVOID)

HAZARDOUS DECOMPOSITION PRODUCTS

SECTION VII SPILL OR LEAK PROCEDURES

STEPS TO BE TAKEN IN CASE MATERIAL IS RELEAGED OR SPILLED

WASTE DISPOSAL METHOD

SECTION VIII SPECIAL PROTECTION INFORMATION

VENTILATION REQUIREMENTS LOCAL EXHAUST

MECHANICAL (GENERAL)

Safety yoyyles

GLOVES

Rubber

SPECIAL

OTIER PROTECTIVE EQUIPMENT

PESPIBATOR

With proper filter

\section{SECTION IX SPECIAL PRECAUTIONS}

PRECAUTIONS TO BE TAKEN IN HANDLING AND STORAGE

OTHËR PRECAUTIONS

SIGNATURE

DATE

$314-1206 B$ 
CAS-000090153

PRODUCT DESIGNATION

03-314-2706

A-Naphthol
QL28000

MATERIAL SAFETY

DATA SHEET

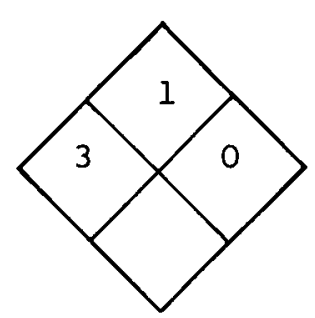

\section{SECTION I SOURCE AND NOMENCLATURE}

ADDRESS (NUMBER, STREET, CITY, STATE, ZIP CODE)

TRADE NAME AND SYNONYMS

A-Hydroxynaph thalene

CHEMICAL NAME AND SYNONYMS

A-Naphthol

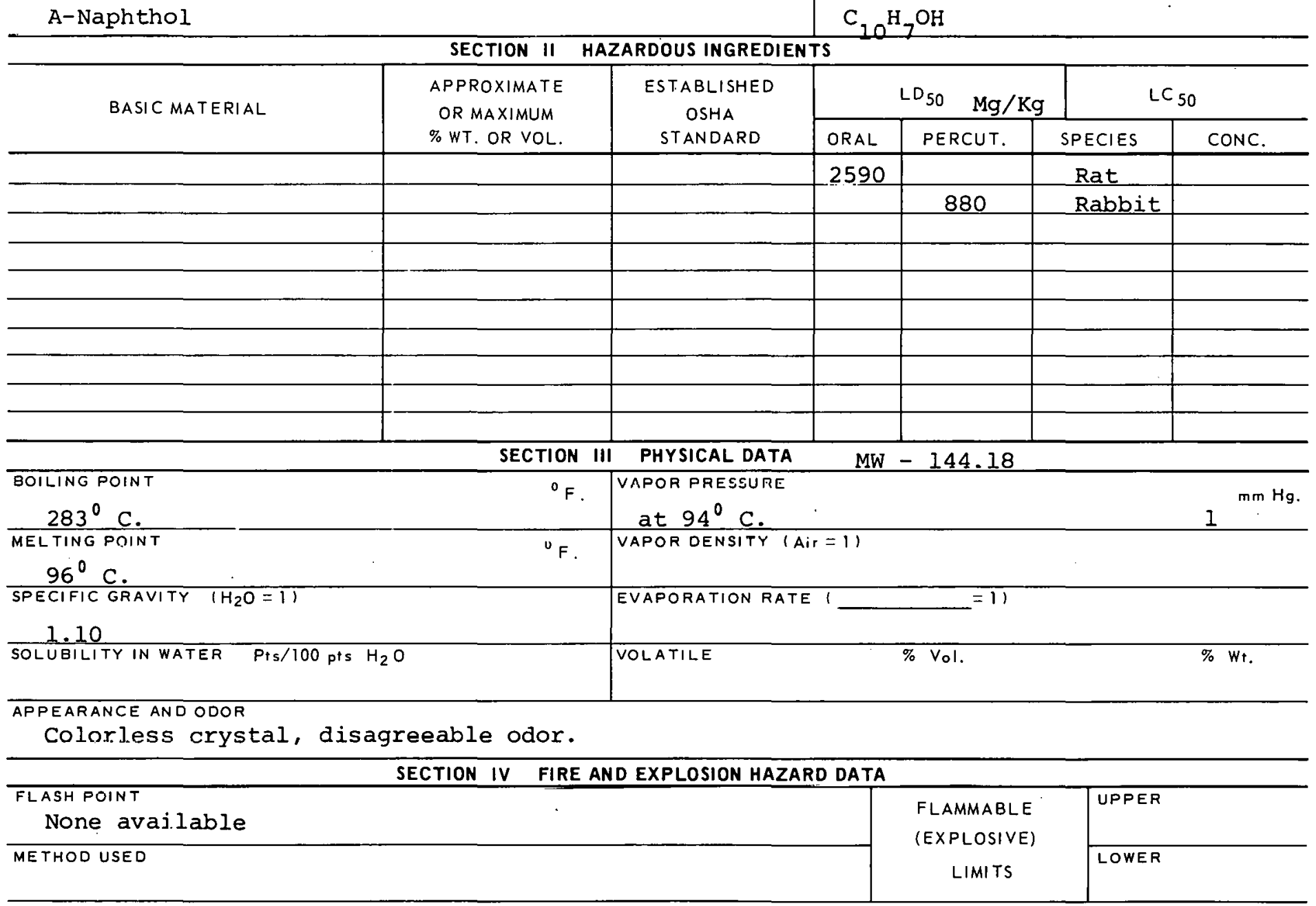

EXTINGUISHING MEDIA

Carbon dioxide, dry chemical.

SPECIAL FIRE FIGHTING PROCEDURES

UNUSUAL FIRE AND EXPLOSION HAZARDS 


\section{A-Naphthol}

SECTION $V$ HEALTH HAZARD DATA

\section{TOXICLEVEL}

None available

CARCINOGENIC

No

PRINCIPLE ROUTES OF ABSORBTION

SKIN AND EYE

Inhalation, ingestion \& skin

IRRITATION

REVELANT SYMPTOMS OF EXPOSURE

Skin and mucous membrane irritation, eye damage.

EFFECTS OF CHRONIC EXPOSURE

Kidney damage, dermatitis.

EMERGENCY AND FIRST AID PROCEDURES Inhalation - rest; if swallowed - drink water, epsom salt solution, get medical aid; wash skin \& eyes.

CONDITIONS CONTRIBUTING TO INSTABILITY

SECTION VI REACTIVITY DATA

CONOITIONS CONTRIBUTING TO HAZARDOUS POLYMERIZATION

INCOMCATAEI GITY (AAATERIAI STO AVOID)

HAZARDOUS DECOMPOSITION PRODUCTS

SECTION VII SPILL OR LEAK PROCEDURES

STEPS TO BE TAKEN IN CASE MATERIAL IS RELEASED OR SPILLED

WASTE DISPOSAL METHOD

SECTION VIII SPECIAL PROTECTION INFORMATION

\begin{tabular}{|c|c|}
\hline VENTILATION REQUIREMENTS LOCAL EXHAUST & $\begin{array}{l}\text { PROTECTIVE EQUIPMENT (SPECIFY TYPES) EYE } \\
\text { Safety goggles }\end{array}$ \\
\hline MECHANICAL (GENERAL) & $\begin{array}{l}\text { GLoves } \\
\text { Rubber }\end{array}$ \\
\hline SPECIAL & $\begin{array}{l}\text { REEPIRATOR } \\
\text { With proper Filter }\end{array}$ \\
\hline
\end{tabular}

OTHER PROTECTIVE EQUIPMENT

SECTION IX SPECIAL PRECAUTIONS

PRECAUTIONS TO BE TAKEN IN HANDLING AND STORAGE

OTHER PRECAUTIONS

SIGNATURE

DATE

$314-2706 B$ 
CAS -000135193

QL29750

PRODUCT DESIGNATION

MATERIAL SAFETY

DATA SHEET

$03 \quad 314-2806$

B-Naphthol

SECTION I SOURCE AND NOMENCLATURE

MANUFACTURER'S NAME

EMERGENCY TELEPHONE NO

ADORESS (NUMBER, STREET, CITY, STATE, ZIP CODE)

TRADE NAME AND SYNONYMS

B-Hyd roxynaphthalene

CHEMICAL NAME AND SYNONYMS

B-Naphthol

SECTION II HAZARDOUS INGREDIENTS

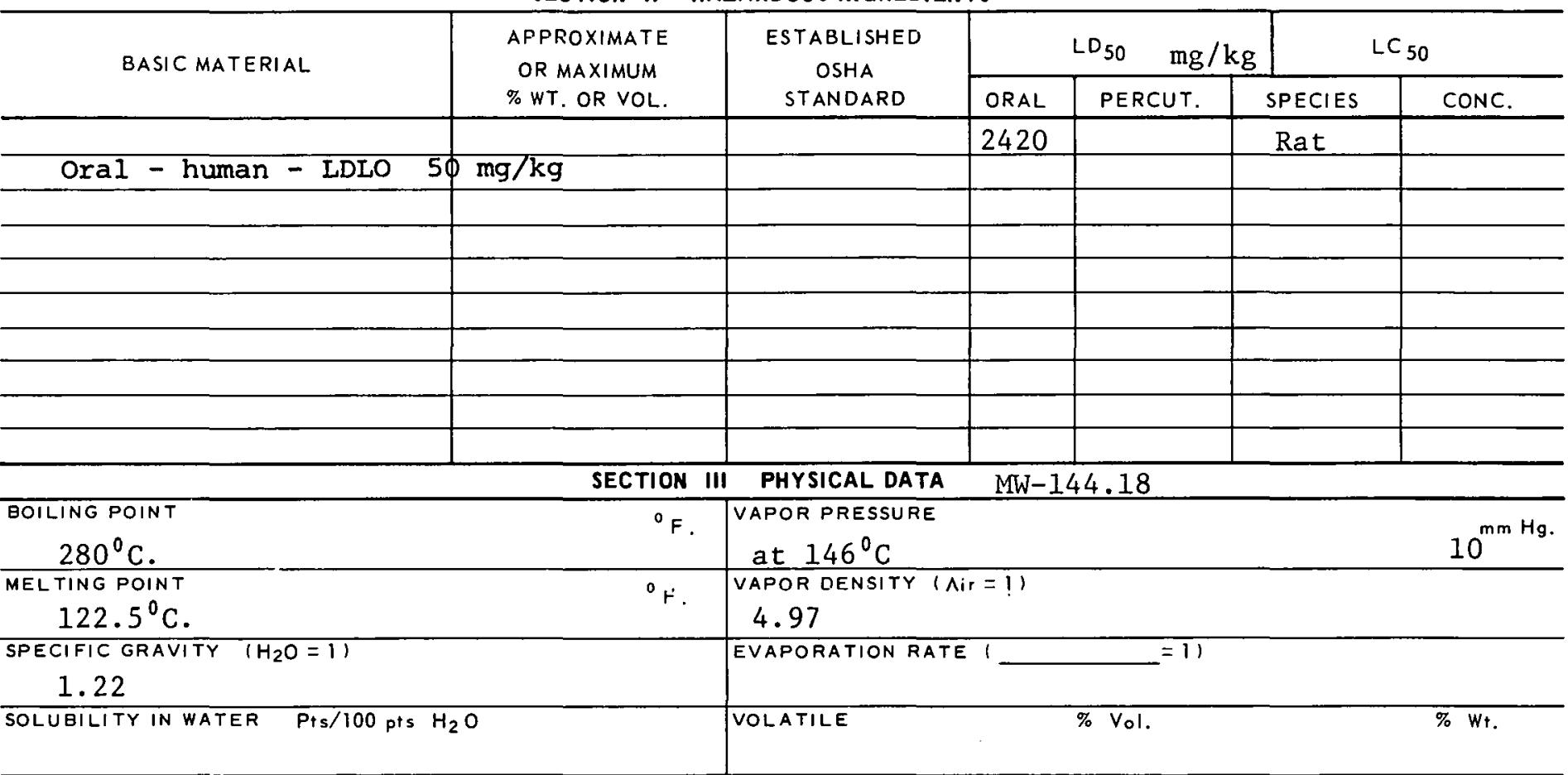

APPEARANCE AND ODOR

White to yellow white crystal, p henolic odor.

SECTION IV FIRE AND EXPLOSION HAZARD DATA

\begin{tabular}{l|c|c}
\hline FLASH POINT & SECTION IV FIRE AND EXPLOSION HAZARD DATA & FLAMMABLE \\
322 & UPPER & (EXPLOSIVE) \\
\cline { 1 - 2 } & LIMITS & LOWER \\
\hline
\end{tabular}

EXTINGUISHING MEDIA

Carbon dloxide, dry chemical.

SPECIAL FIRE FIGHTING PROCEDURES

UNUSUAL FIRE AND EXPLOSION HAZARDS 


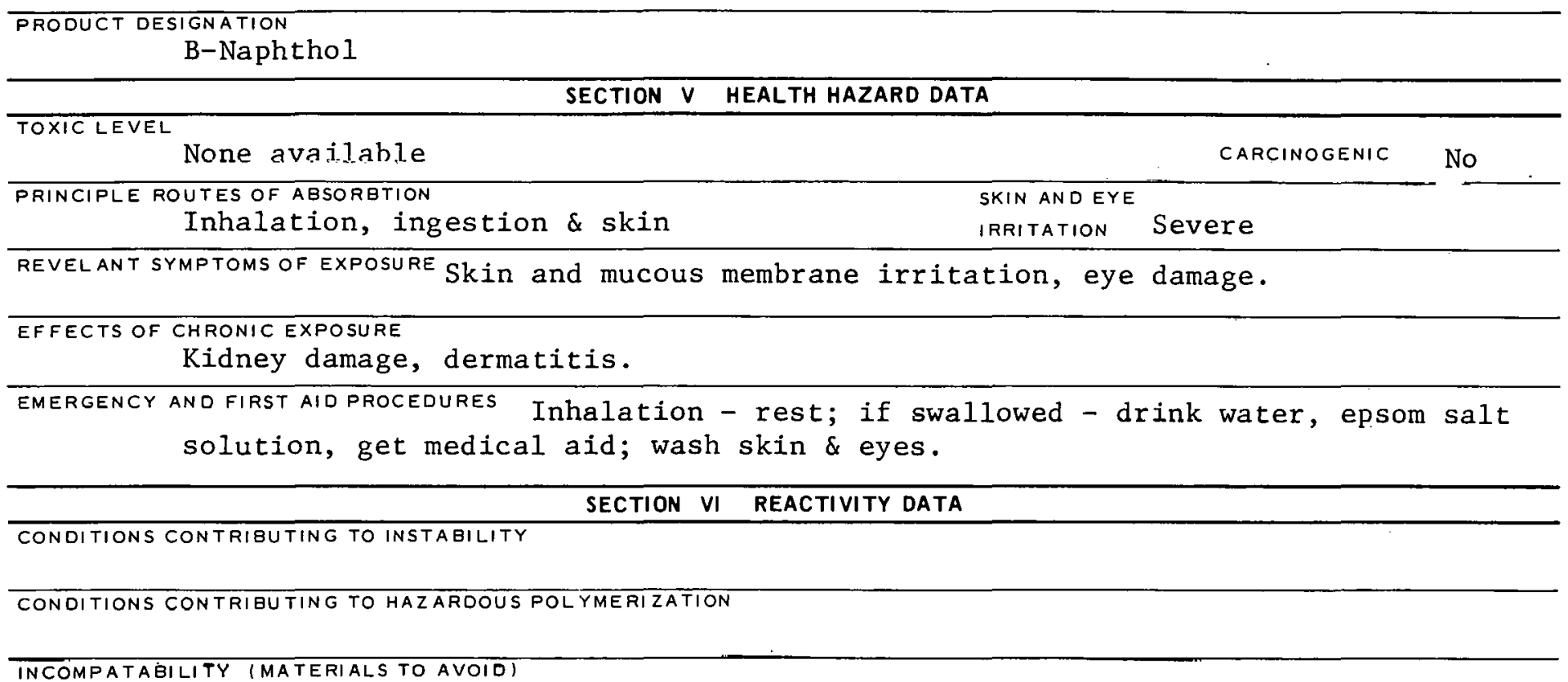

HAZAHUUUS UELUNFEMITIGIA FRODUCTC
STEPS TO BE TAKEN IN CASE MATERIAL IS RELEASED OR SPILLED
SECTION VII SPILL OR LEAK PROCEDURES

STEPS TO BE TAKEN IN CASE MATERIAL IS RELEASED OR SPILLED

\begin{tabular}{l|l}
\hline WASTE DISPOSAL METHOD \\
\hline \multicolumn{1}{c}{ SECTION VIII SPECIAL PROTECTION INFORMATION } \\
\hline VENTILATION REQUIREMEN TS LOCAL EXHAUST & $\begin{array}{c}\text { PROTECTIVE EQUIPMENT (SPECIFY TYPESIEYE } \\
\text { SafetY gOggles }\end{array}$ \\
\hline MECHANICAL IGENERAL) & $\begin{array}{l}\text { GLOVES } \\
\text { Rubber }\end{array}$ \\
\hline GPECIAL & $\begin{array}{l}\text { RESPIRATOR } \\
\text { With Proper filter }\end{array}$ \\
\hline
\end{tabular}

OTHER PROTECTIVE EQUIPMENT

SECTION IX SPECIAL PRECAUTIONS

PRECAUTIONS TO BE TAKEN IN HANDLING AND STORAGE

OTHER PRECAUTIONS

\begin{tabular}{l|l|l|}
\hline SIGNATURE & DATE \\
\hline
\end{tabular}




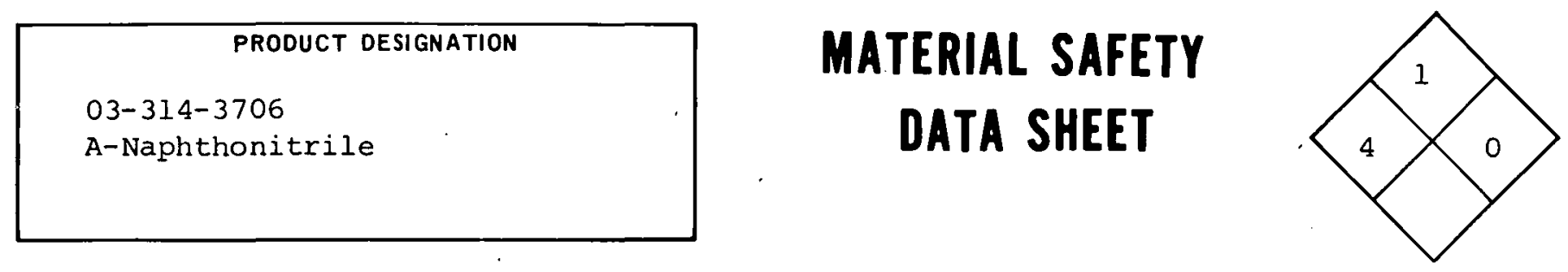

SECTION I SOURCE AND NOMENCLATURE

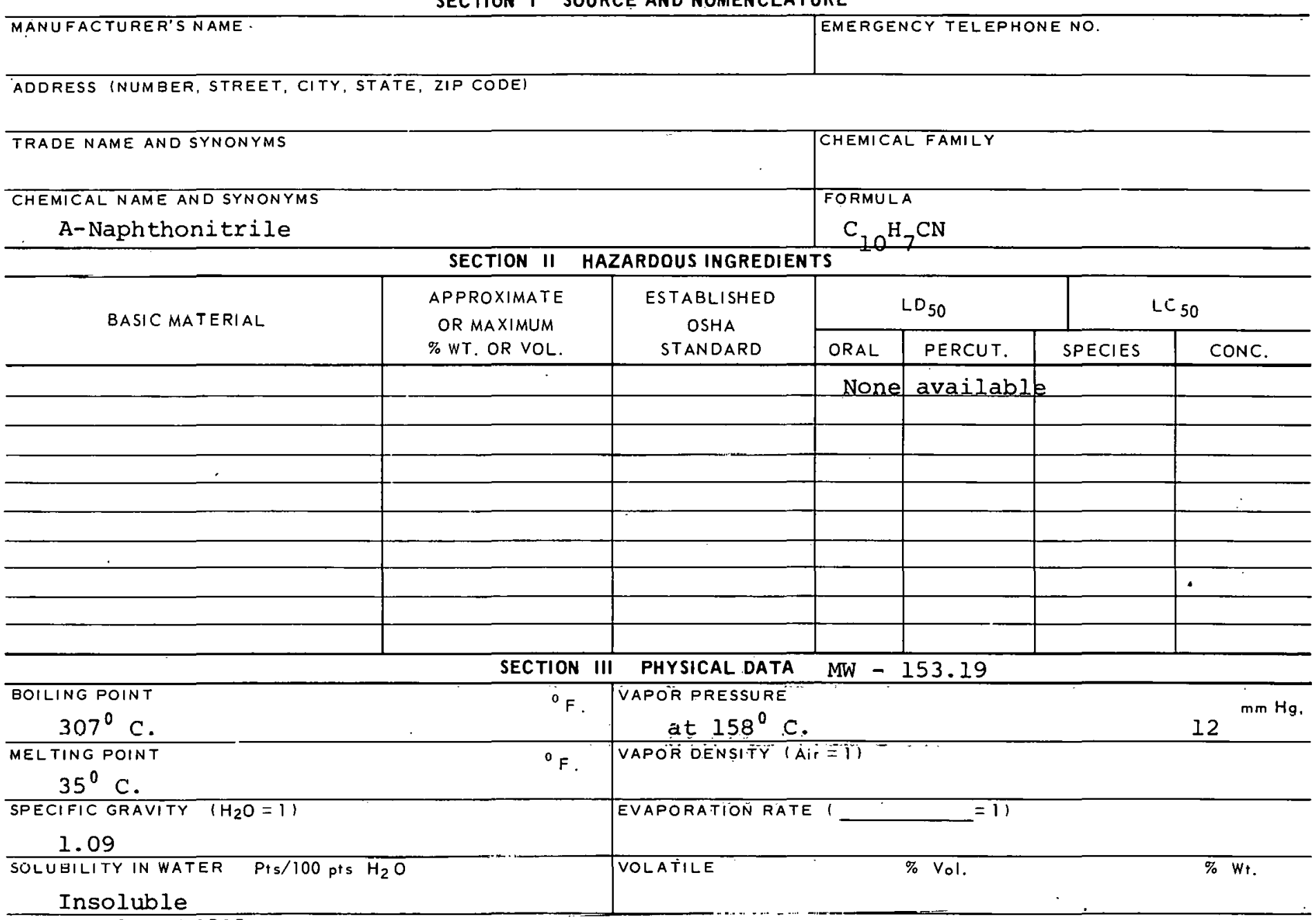

ATTEARAHCE ANO OOUR

Leaf-like crystals

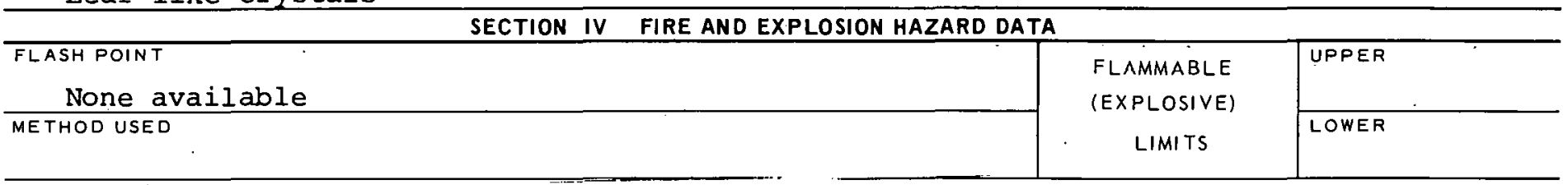

EXTINGUISHING MEDIA

Alcohol foam, carbon dioxide, dry chemical.

SPECIAL FIRE FIGHTING PROCEDURES

UNUSUAL FIRE AND EXPLOSION HAZARDS 


\section{TOXIC LEVEL}

None available

PRINCIPLE ROUTES OF ABSORBTION

Inhalation, ingestion, skin REVELANT SYMPTOMS OF EXPOSURE Headache, nausea, vomiting, abdominal cramping, unconsciousness, convulsions. EFFECTS OF CHRONIC EXPOSURE

Flushing of face, nausea, jaundice. EMERGENCY AND FIRST AID PROCEDURES Wash skin \& eyes; inhalation - inhale amyl nitrite vapor; if swallowed - give emetic, get medical aid.

\section{SECTION VI REACTIVITY DATA}

CARCINOGENIC No SKIN AND EYE IRRITATION Moderate

CONDITIONS CONTRIBUTING TO INSTABILITY

CONDITIONS CONTRIBU TING TO HAZAROOUS POLYMERIZATION

INCOMFATADILITY (MATFR.IAISTO AVOID)

HA TARNAIIS RFR.NMPQSITION PRQDUCTS

$+$

SECTION VII SPILL OR LEAK PROCEDURES

STEPS TO BE TAKEN IN CASE MATERIAL IS RELEASED OR SPILLED

\section{WASTE DISPOSAL METHOD}

\section{SECTION VIII SPECIAL PROTECTION INFORMATION}

\begin{tabular}{l|l}
\hline VENTILATION REQUIREMENTS LOCAL EXHAUST & $\begin{array}{c}\text { PROTECTIVE EQUIPMENT (SPECIFY TYPES) EYE } \\
\text { GOggles }\end{array}$ \\
\hline MECHANICAL IGENERAL) & $\begin{array}{c}\text { GLOVES } \\
\text { Rubber }\end{array}$ \\
\hline SPECIAL & $\begin{array}{c}\text { RESPIRATOR } \\
\text { With organio filtor }\end{array}$ \\
\hline
\end{tabular}

OTIER FROTECTIVE EQUIPMENT

SECTION IX SPECIAL PRECAUTIONS

PRECAUTIONS TO BE TAKEN IN HANOLING AND STORAGE

OTHER PRECAUTIONS

SIGNATURE

DATE

$314-3706 B$ 
CAS -000134327

\begin{tabular}{|c|} 
PRODUCT DESIGNATION \\
$03-314-5306$ \\
A-Naphthylamine \\
\end{tabular}

\section{MATERIAL SAFETY \\ DATA SHEET}

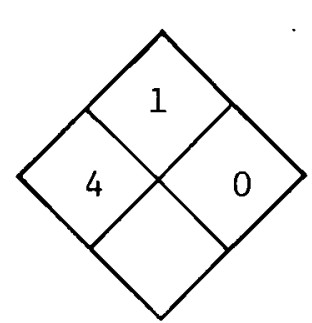

SECTION I SOURCE AND NOMENCLATURE

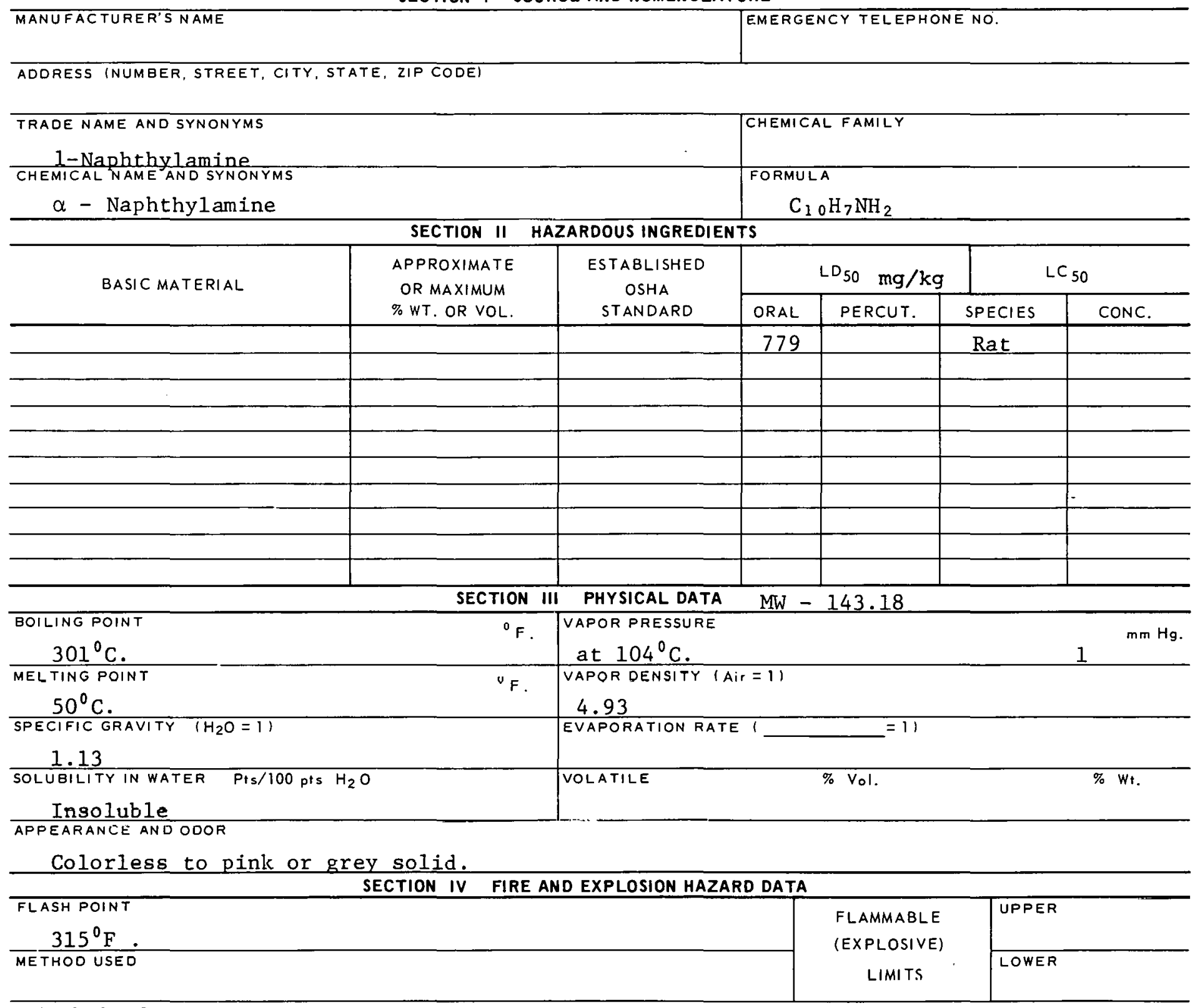

EXTINGUISHING MEDIA

Watcr or foam may caust fruthing. Carbon dioxide, dry chemical.

SPECIAL FIRE FIGHTING PROCEDURES

UNUSUAL FIRE AND EXPLOSION HAZARRS

Can react with oxidizing materiale. 
A-Naphthylamine SECTION $V$ HEALTH HAZARD DATA

TOXIC LEVEL

None available

CARCINOGENIC Yes PRINCIPLE ROUTES OF ABSORBTION SKIN AND EYE

Inhalation, ingestion, skin IRRITATION Moderate

REVELANT SYMPTOMS OF EXPOSURE

Eye irritation

EFFECTS OF CHRONIC EXPOSURE

Bladder tumors.

EMERGENCY AND FIRST AID PROCEDURES

aid; wash skin and eyes.

Inhalation - rest; if swallowed - emetic, get medical

SECTION VI REACTIVITY DATA

CONDITIONS CONTRIBUTING TO INSTABILITY

COHAITIONS CONTRIBUTING TO HAZARDOUS POL YMERIZATION

INCOMPATABILITY (MATERIALS TO ÁVOID)

HA ZAROOUS DECOMROSITIUN RKUUU.TS

SECTION VII SPILL OR LEAK PROCEDURES

STEPS TO BE TAKEN IN CASE MATERIAL IS RELEASED OR SPILLED

WASTE OISPOSAL METHOD

\section{SECTION VIII SPECIAL PROTECTION INFORMATION}

VENIILATION REQUIREMENTS LOCAL EXHAUST

MECHANICAL (GLNERAL) Safety gnggles GLOVES

Rubber

SFEEIAL

RESPIRATOR Filter type respirator for dust, mist and fumes.

OTHER PROTECTIVE EQUIPMENT

Full protective clothing. SECTION IX SPECIAL PRECAUTIONS

PRECAUTIONS TO BE TAKEN IN HANDLING AND STORAGE

Store in sealed container.

OTIIER RAESAUTIOML 


\begin{tabular}{|c|}
\hline PRODUCT DESIGNATION \\
93-314-5906 \\
$\beta-$ Naphthylamine Hydrochloride
\end{tabular}

\section{SECTION I SOURCE AND NOMENCLATURE}

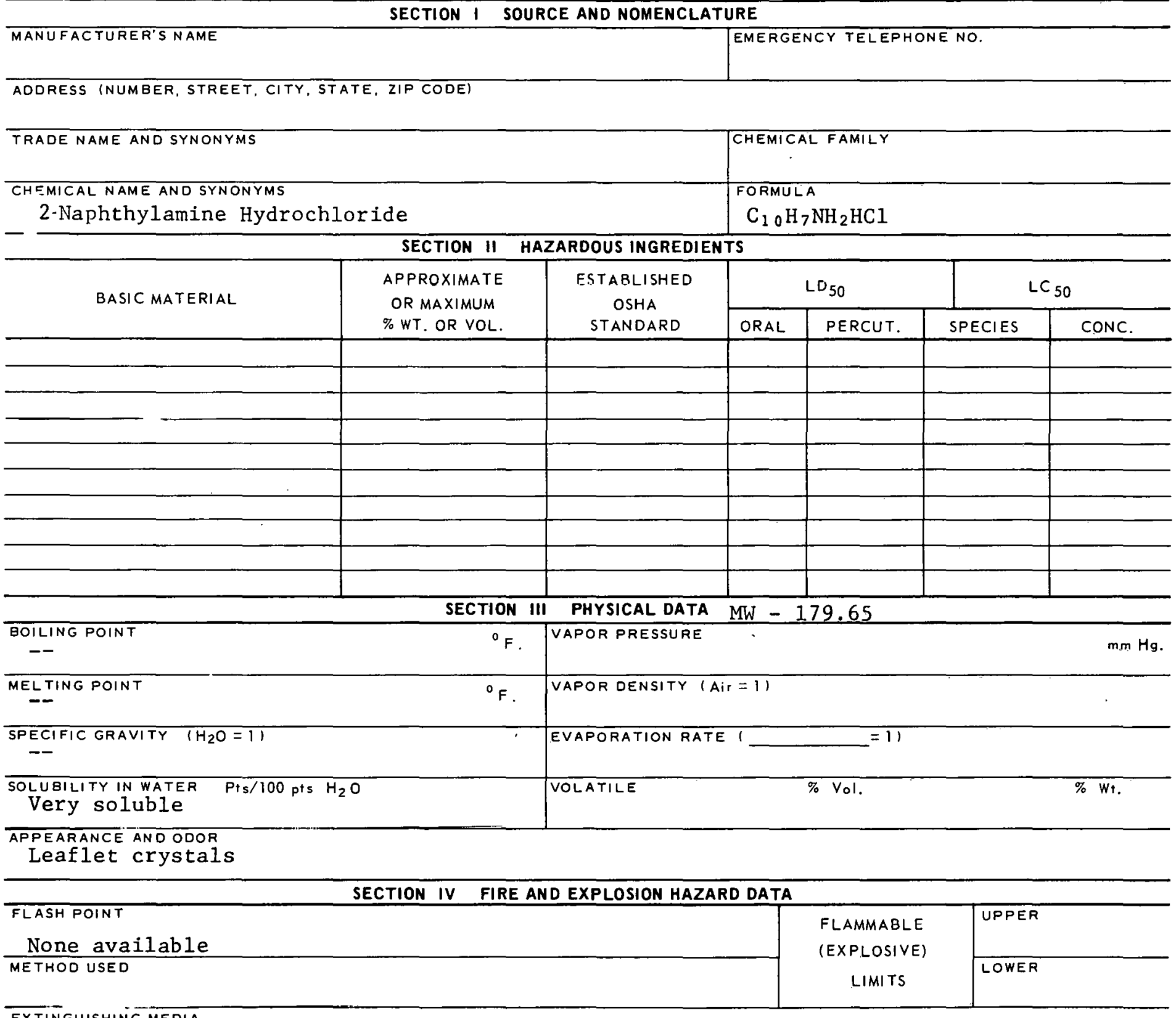

EXTINGUISHING MEDIA

Carbon dioxide, dry chemical.

SPECIAL FIRE FIGHTING PROCEDURES

UNUSUAL FIRE ANOEXPIOSION HAZARDS 
B-Naphthylamine Hydrochloride

SECTION $V$ HEALTH HAZARD DATA

\begin{tabular}{l}
$\begin{array}{l}\text { TOXICLEVEL } \\
\text { NOne available }\end{array}$ \\
$\begin{array}{c}\text { CARCINOGENIC } \\
\text { PRINCIPLE ROUTES OF ABSORBTION } \\
\text { Inhalation, ingestion, skin }\end{array}$ \\
$\begin{array}{l}\text { REVELANT SYMPTOMS OF EXPOSURE } \\
\text { EYe irritation }\end{array}$ \\
$\begin{array}{l}\text { EFFECTS OF CHRONIC EXPOSURE } \\
\text { Bladder tumors }\end{array}$ \\
\hline EMERGENCY AND FIRST AID PROCEDURES If swallowed - give emetic, get medical aid; inhalation -
\end{tabular}

SECTION VI REACTIVITY DATA

CONDITIONS CONTRIBUTING TO INSTABILITY

CONDITIONS CONTRIBUTING TO HAZARDOUS POLYMERIZATION

INCOMPATABILITY (MATERIALS TO AVOID)

HAZARDOUS DECOMPOSITION PRODUCTS

SECTION VII SPILL OR LEAK PROCEDURES

STEPS TO BE TAKEN IN CASE MATERIAL IS RELE ASED OR SPILLED

WASTE DISPOSAL METHOD

SECTION VIII SPECIAL PROTECTION INFORMATION

VENTILATION REQUIREMENTS LOCAL EXHAUST

MECIIANICAL (GENERAL)

SPECIAL

OTHER PROTECTIVE EQUIPMENT

Ful1 protective clothing.

PRECAIITIONS TO GE TAKEN IN HANDLING AND STORAGE
PROTECTIVE EQUIPMENT (SPECIFY TYPES) EYE

Safety goggles

GLOVES

Rubber

RESPIRATOR

F1lter type for dust, mist and funes

OTHER PHECAUIIUNS

SIGNATURE

UAIE

314-5906B 
03-314-6306

A-Naph thylhydrazine

DATA SHEET

Hydrochloride

\section{SECTION I SOURCE AND NOMENCLATURE}

MANUFACTURER'S NAME

EMERGENCY TELEPHONE NO.

ADDRESS (NUMBER, STREET, GITY, STATE, ZIP CODE)

TRADE NAME AND SYNONYMS

CHEMICAL FAMILY

CHEMICAL NAME ANO SYNONYMS

a Naphthylhydrazine hydrochloride

FORMULA

$\mathrm{C}_{20} \mathrm{H}_{7} \mathrm{NHNH}_{2} \mathrm{HCl}$ SECTION II HAZARDOUS INGREDIENTS

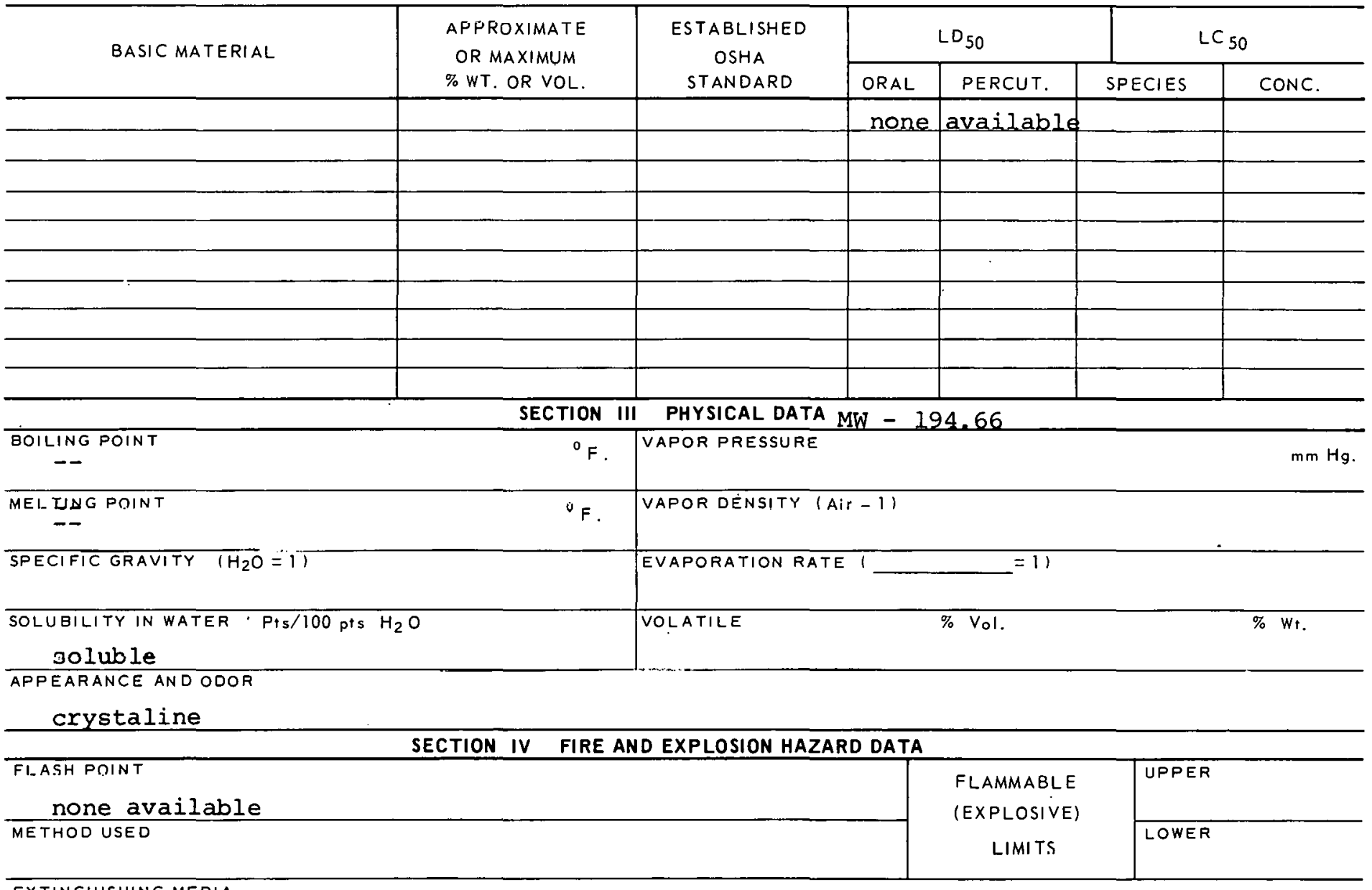

EXTINGUISHING MEDIA

SPECIAL FIRE FIGHTING PROS.EDURES

UNUSUAL FIRE AND EXPLOSION HAZARDS 
A-Naphthylhydrazine Hydrochloride SECTION $V$ HEALTH HAZARD DATA

\section{TOXIC LEVEL}

none available

CARGINOGENIC. PRINCIPLE ROUTES OF ABSORBTION inhalation of dust, ingestion, skin SKIN AND EYE IRRITATION

CARCINOGENIC: no
SKINANDEYE
IRRITATION SEVere

REVELANT SYMPTOMS OF EXPOSURE Irritation of skin, eyes and mucous membranes - gastroentric disturbances, anemia.

EFFECTS OF CHRONIC EXPOSURE

Blood and liver damage, loss of appetite.

EMERGENCY AND FIRST AID PROCEDURES Inhalation - rest; if swallowed - induce vomiting, get medical aid; wash from skin \& eyes.

\section{SECTION VI REACTIVITY DATA}

CONDITIONS CONTRIBUTING TO INSTABILITY

CONDITIONS CONTRIBUTING TO HAZARDOUS POLYMERIZATION

INCOMPATABILITY IMATERIALS TO AVOID)

HAZARDOUS DECOMPOSITION PRODUCTS

SECTION VII SPILL OR LEAK PROCEDURES

STEPS TO BE TAKEN IN CASE MATERIAL IS RELEASED OR SPILLED

WASTE DISPOSAL METHOD

SECTION VIII SPECIAL PROTECTION INFORMATION

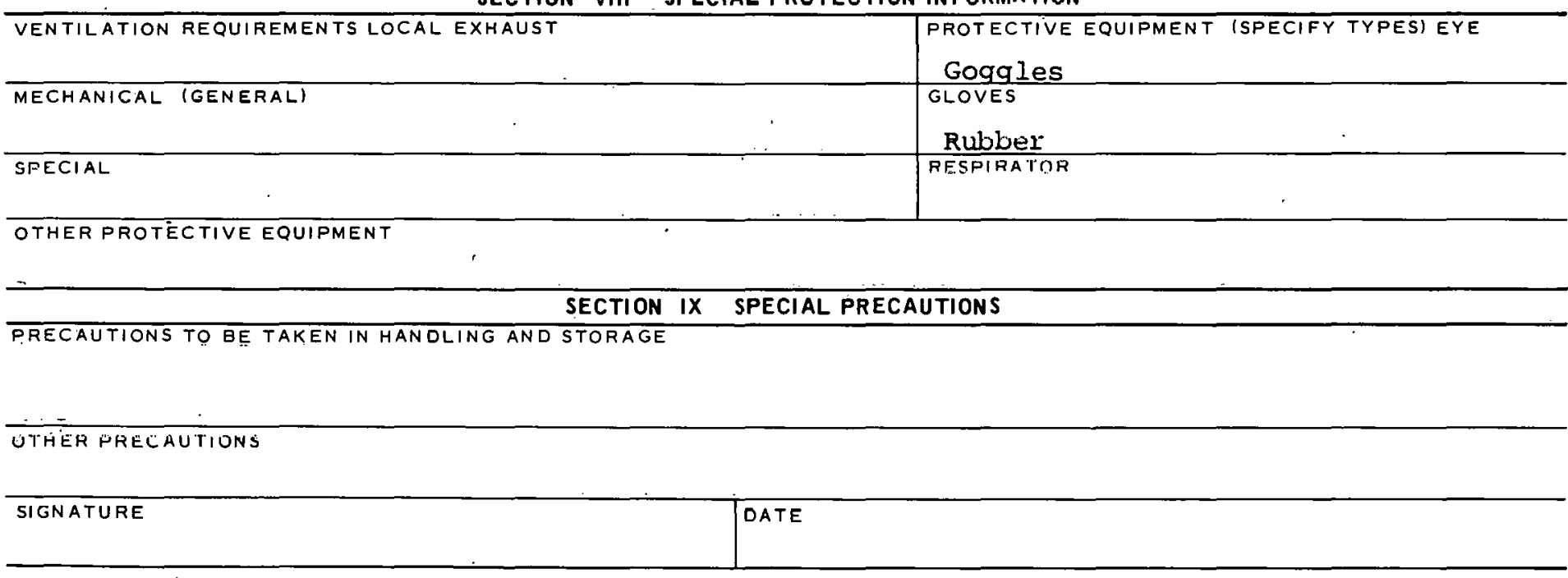


EAS! OOOS5

\begin{tabular}{l|}
\multicolumn{1}{|c|}{ PRODUCT DESIGNATION } \\
03-314-6806 MATERIAL SAFETY \\
l-NaphthyI Isocyanate
\end{tabular}

SECTION I SOURCE AND NOMENCLATURE

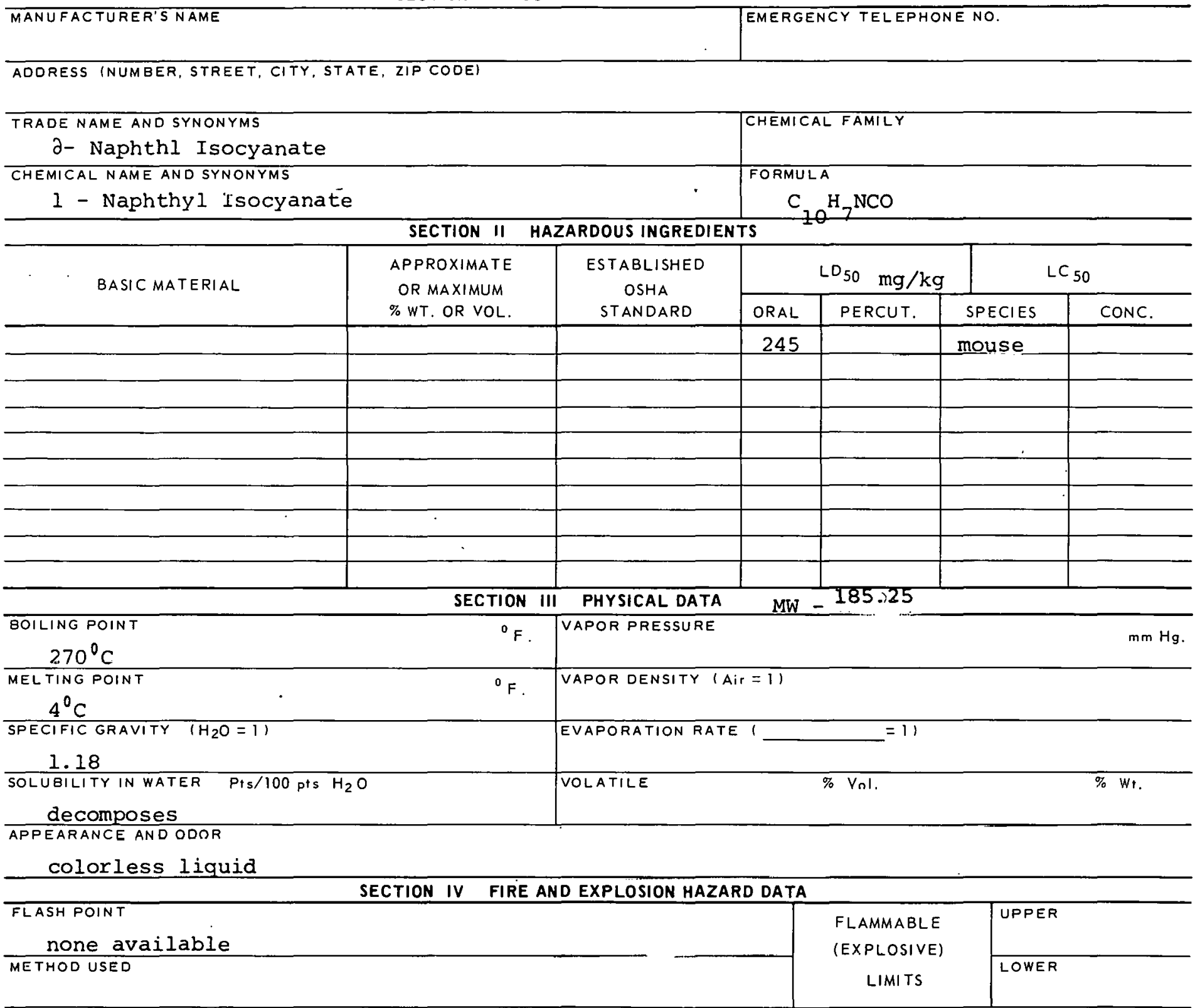

EXTINGUISHING MEDIA

alcohol foam, carbon dioxide, dry chemical

SPECIAL FIRE FIGHTING PROCEDURES

UNUSUAL FIRE AND EXPLOSION HATARIS 
TOXIC LEVEL

none available

CARCINOGENIC

PRINCIPLE ROUTES OF ABSORBTION

inhalation, ingestion, skin

SKIN AND EYE

IRRITATION

severe

REVELANT SYMPTOMS OF EXPOSURE

skin, eye and respiratory tract irritation.

EFFECTS OF CHRONIC EXPOSURE

allergic sensitization of respiratory tract.

EMERGENCY AND FIRST AID PROCEDURES

if swallowed - induce vomiting, get medical aid; inhalation - rest;

wash from skin \& eyes.

SECTION VI REACTIVITY DATA

CONDITIONS CONTRIBUTING TO INSTABILITY
CONDITIONS CONTRIBIITING TO HAZARDOUS POLYMERIZATION
INCOMPATABILITY (MATERIALS TO AVOID)
HAZARDOUS DECOMPOSITION PRODUCTS
SECTION VII SPILL OR LEAK PROCEDURES

STEPS TO BE TAKEN IN CASE MATERIAL IS RELEASED OR SPILLED

WASTE DISPOSAL METHOD

SECTION VIII SPECIAL PROTECTION INFORMATION

\begin{tabular}{l|l}
\hline VENTILATION REQUIREMENTS LOCAL EXHAUST & $\begin{array}{c}\text { PROTECTIVE EQUIPMENT (SPECIFY TYPES) EYE } \\
\text { gOggles }\end{array}$ \\
\hline MECHANICAL IGENERAL) & $\begin{array}{c}\text { rubber } \\
\text { SPECIAL }\end{array}$ \\
\hline
\end{tabular}

OTHER PROTECTIVE EQUIPMENT

with proper filter

\section{SECTION IX SPECIAL PRECAUTIONS}

PRECAUTIONS TO BE TAKEN IN HANDLING AND STORAGE

OTHER PRECAUTIONS

SIGNATURE

DATE

314-6806B 

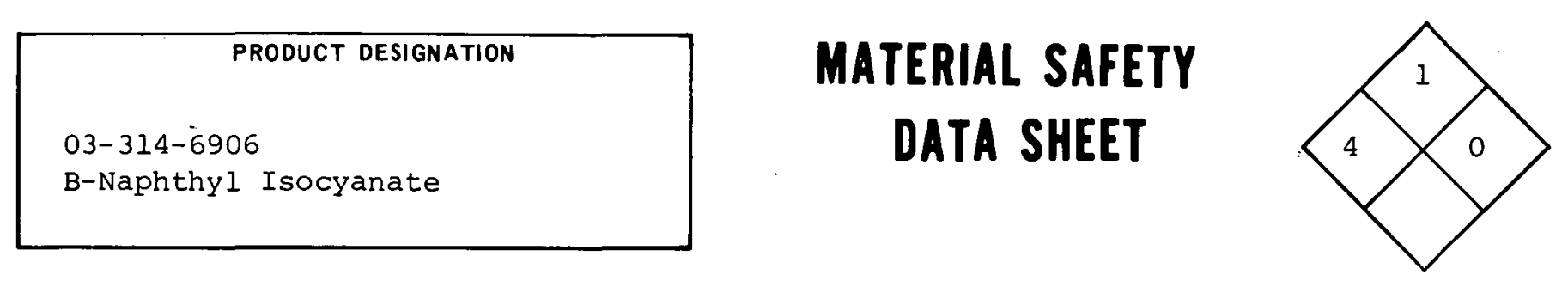

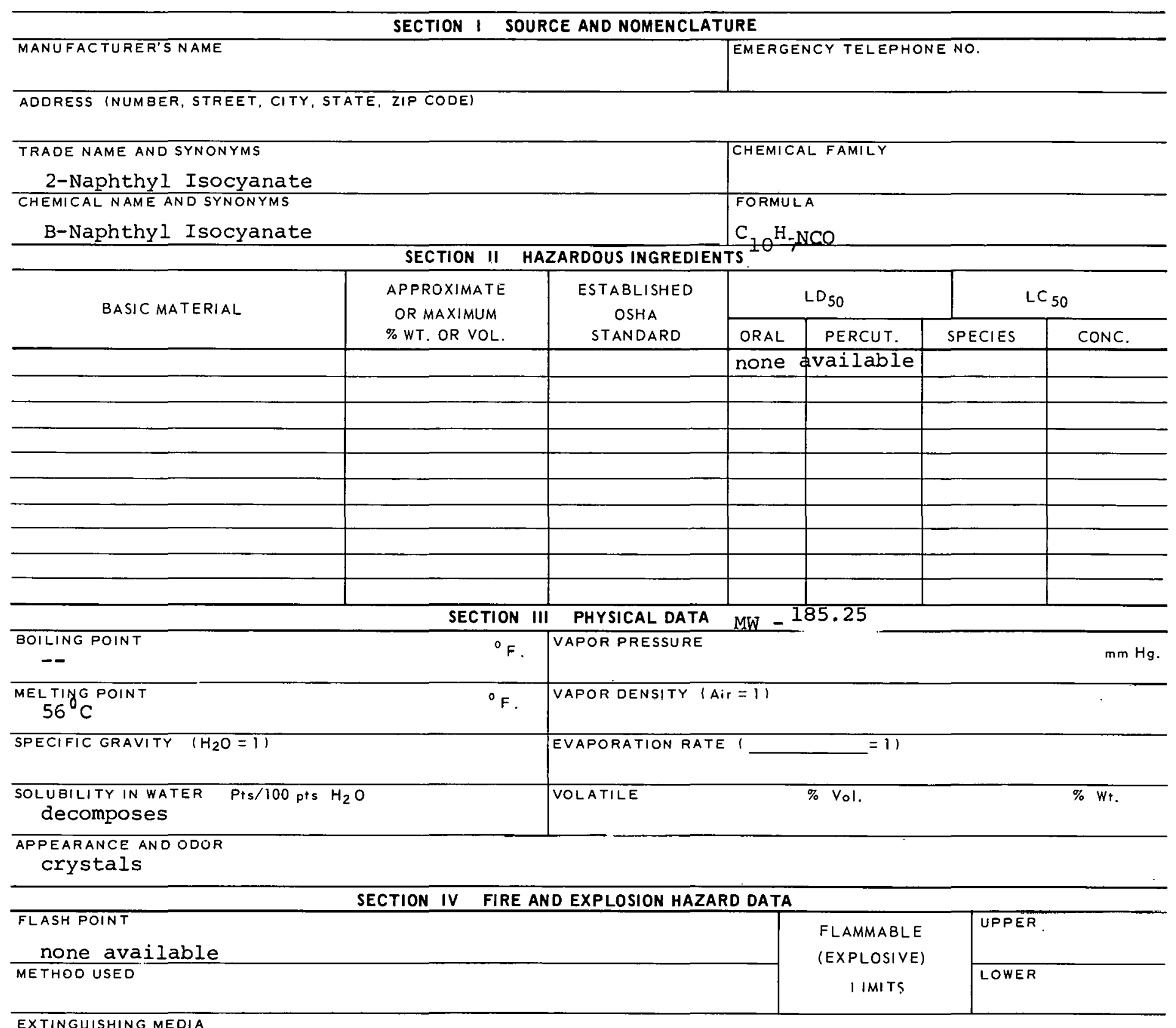

alcohol foam, carbon dioxide, dry chemical.

SPECIAL FIRE FIGHTING PROCEDURES

UNUSUAL FIRE AND EXPLOSION HAZARDS 


\section{TOXIC LEVEL}

none available

PRINCIPLE ROUTES OF ABSORATION

Inhalation, ingestion, skin

CARCINOGENIC IRRITATION SEVEre

\section{REVELANT SYMPTOMS OF EXPOSURE}

Skin, eye and respiratory tract irritation.

EFFECTS OF CHRONIC EXPOSURE

Sensitization of respiratory tract.

EMERGENCY AND FIRST AID PROCEDURES If swallowed - induce vomiting, get medical aid;

inhalation - rest; wash from skin \& eyes.

SECTION VI REACTIVITY DATA

CONDITIONS CONTRIBUTING TO INSTABILITY

CONDITIONS GONTRIBUTING TO HAZARDOUS POLYMERIZATION

INCOMPATABILITY (MATERIALS TO AVOID)

HAZARDOUS DECOMPOSITION PRODUCTS

SECTION VII SPILL OR LEAK PROCEDURES

STEPS TO BE TAKEN IN CASE MATERIAL IS RELEASED OR SPILLED

WASTE DISPOSAL METHOD

SECTION VIII SPECIAL PROTECTION INFORMATION

\begin{tabular}{|c|c|}
\hline VENTILATION REQUIREMENTS LOCAL EXHAUST & $\begin{array}{l}\text { PROTECTIVE EQUIPMENT (SFECIFY TYPESI EYE } \\
\text { gOggles }\end{array}$ \\
\hline MECHANICAL (GENERAL) & $\begin{array}{l}\text { GLOVES } \\
\text { rubber }\end{array}$ \\
\hline SPECIAL & $\begin{array}{l}\text { RESPIRATOR } \\
\text { with proper filter }\end{array}$ \\
\hline
\end{tabular}

OTHER PROTECTIVE EOUIPMENT

\section{SECTION IX SPECIAL PRECAUTIONS}

PRECAUTIONS TO BE TAKEN IN HANDLING AND STORAGE

OTHER PRECAUTIONS

SGNATURE

DATE

$314-6906 \mathrm{~B}$ 
PRODUCT DESIGNATION

03-315-0907

3 Nitro-4 Acetylaminotoluene
MATERIAL SAFETY

DATA SHEET

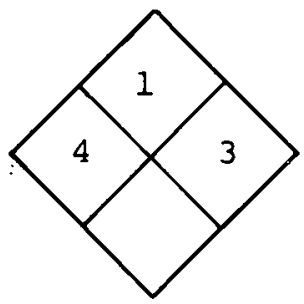

SECTION I SOURCE AND NOMENCLATURE

MANUFAC TURER'S NAME
ADORESS INUMBER, STREET, CITY, STATE, ZIP COD
TRADE NAME AND SYNONYMS
2 NitrO-P-ACEtOtoluidide
CHEMICAL NAME AND SYNONYMS
3 Nitro 4 ACetYlamino Toluene

SECTION II HAZARDOUS INGREDIENTS

CHEMICAL FAMILY
FORMULA
$\mathrm{NO}_{2} \mathrm{C}_{6} \mathrm{H}_{3}\left(\mathrm{CH}_{3}\right)\left(\mathrm{NHCOCH}_{3}\right)$

BASIC MATERIAL

\begin{tabular}{|c|c|c|}
\hline $\begin{array}{c}\text { APPROXIMATE } \\
\text { OR MAXIMUM } \\
\% \text { WT. OR VOL. }\end{array}$ & $\begin{array}{c}\text { ESTABLISHED } \\
\text { OSHA } \\
\text { STANDARD }\end{array}$ & \\
${ } &{ } \\
{\hline} &{ } &{ } \\
{\hline} &{ } &{ } \\
{\hline} &{ } &{ } \\
{\hline} &{ } &{ } \\
{\hline} &{ } &{ } \\
{\hline}$
\end{tabular}

BOILING POINT

$--$

MEL TING POINT

$93^{\circ} \mathrm{C}$

SPECIFIC GRAVITY $\left(\mathrm{H}_{2} \mathrm{O}=1\right)$

$--$

SOLUBILITY IN WATER Pis/100 pts $\mathrm{H}_{2} \mathrm{O}$

Soluble

APPEARANCF AND ODOR

yellow crystals

\section{FLASH POINT}

none available

METHOD USED

EXTINGUISHING MEDIA

SECTION III PHYSICAL DATA

\begin{tabular}{|l|l}
${ }^{0} \mathrm{~F}$ & VAPOR PRESSURE
\end{tabular}

MW - 194.19

\begin{tabular}{l|l|l|l|l}
\multicolumn{2}{c|}{ LD $_{50}$} & \multicolumn{2}{c}{ LC $_{50}$} \\
\hline ORAL & PERCUT. & SPECIES & CONC. \\
\hline none & available & & \\
\hline & & & \\
\hline & & & \\
\hline & & & \\
\hline & & & & \\
\hline & & & \\
\hline & & & \\
\hline
\end{tabular}

${ }^{\circ} \mathrm{F}$.

VAPOR DENSITY ( Air $=1)$

EVAPORATION RATE 1 $=11$

VOLATILE

$\%$ vol.

\% Wt.

SECTION IV FIRE AND EXPLOSION HAZARD DATA.
FLAMMABLE

(EXPLOSIVE)

LIMITS
UPPER

LOWER

carbon dioxide, dry chemiral

SPECIAL FIRE FIGHTING PROCEOURES

UNUSUAL FIRE ANO EXPLOSION HAZARDS 
3 Nitro-4 Acetylaminotoluene

SECTION $V$ HEALTH HAZARD DATA

TOXIC LEVEL

none available

PRINCIPLE ROUTES OF ABSORBTION

inhalation, ingestion, skin

CARCINOGENIC no

REVELANT SYMPTOMS OF EXPOSURE SKIN AND EYE

IRRITATION slight

difficulty in breathing, cyanosis, vomiting, headache

EFFECTS OF CHRONIC EXPOSURE

blood damage

EMERGENCY AND FIRST AID PROCEDURES If swallowed - give emetic, get medical aid;

inhalation - rest; wash from skin \& eyes.

\section{SECTION VI REACTIVITY DATA}

CONDITIONS CONTRIBUTING TO INSTABILITY

CONDITIONS CONTRIBUTING TO HAZARDOUS POLYMERIZATION

INCOMPA IABILI Y Y IMA IERTIALSTO AVOIOI

May explode when heated with sulfuric acid.

חATAROOUS BECOMTOCITIOH RTOOUETS

SECTION VII SPILL OR LEAK PROCEDURES

STEPS TO BE TAKEN IN CASE MATERIAL IS RELEASED OR SPILLED

WASTE DISPOSAL METHOD

SECTION VIII SPECIAL PROTECTION INFORMATION

\begin{tabular}{|c|c|}
\hline SECTION VIII & SPECIAL PROTECTION INFORMATION \\
\hline VENTILATION REQUIREMENTS LOCAL EXHAUST & \begin{tabular}{l|l} 
& PROILCIIVE EQUIPMENT (SPECIFY TYPES) EYE \\
gOggles
\end{tabular} \\
\hline BAESHANIGAL ICEFNFRAII & $\begin{array}{l}\text { GLOVES } \\
\text { rubber }\end{array}$ \\
\hline SPFr.IAI & $\begin{array}{l}\text { RESPIRATOR } \\
\text { self-contained }\end{array}$ \\
\hline
\end{tabular}

OTHĒ PROTECTIVE EQUIPMEN I

SECTION IX SPECIAL PRECAUTIONS

PRECAUTIONS TO BE TAKEN IN HANDLING AND STORAGE

OTHER PREC.AUTIONS

SIGNATURE

DATE

315-0907B 


\section{PRODUCT DESIGNATION}

03-315-1907

5 Nitro-2-Aminoanisole
MATERIAL SAFETY DATA SHEET

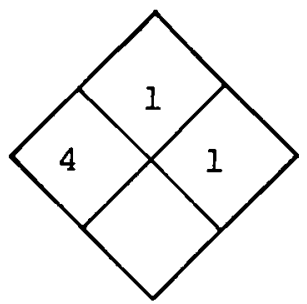

\section{SECTION I SOURCE AND NOMENCLATURE}

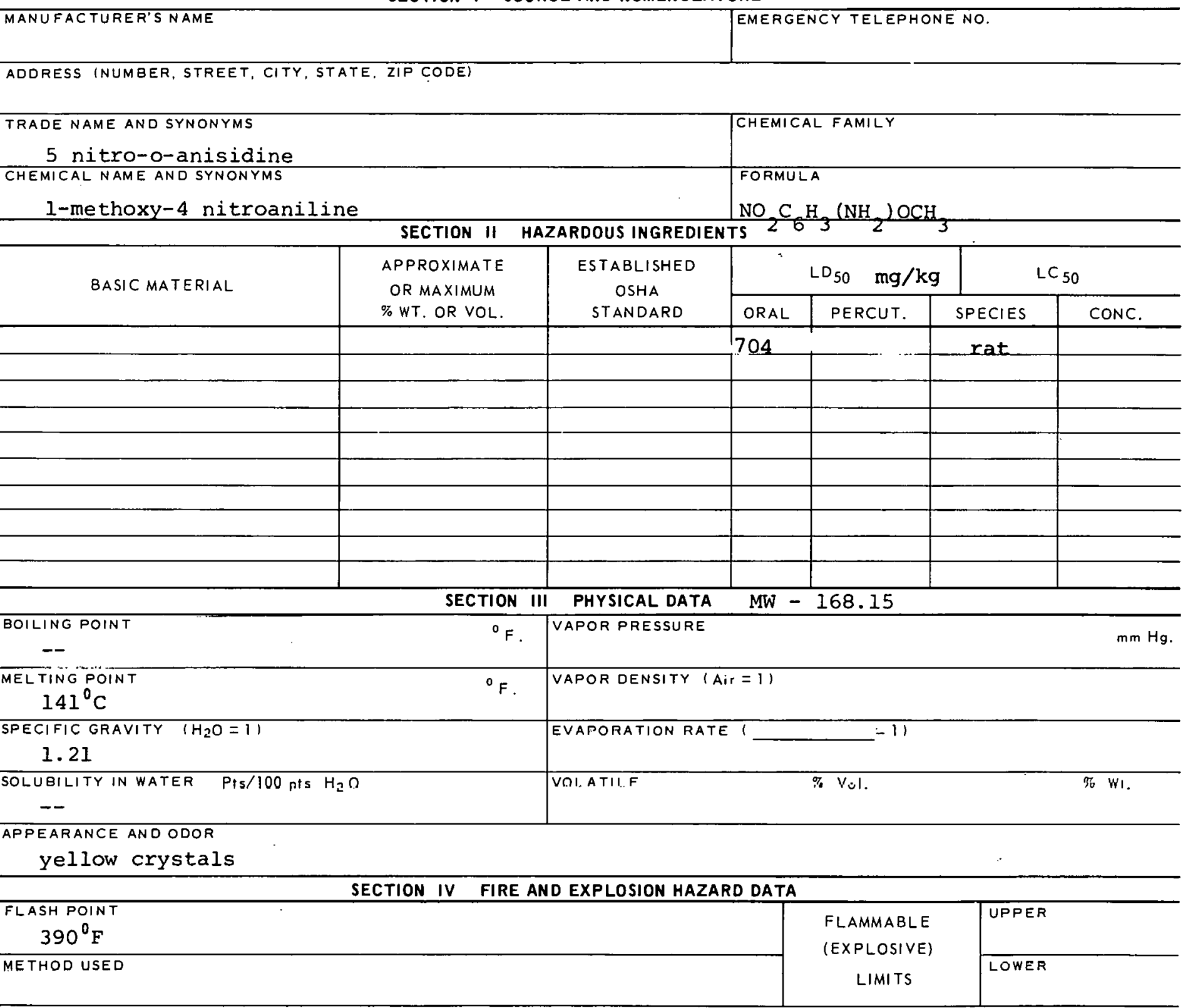

EXTINGUISHING MEDIA

water spray, carbon dioxide, dry chemical, foam

SPECIAL FIRE FIGHTING PROCEDURES

wear full protective clothing.

UNUSUAL FIRE AND EXPLOSION HAZARDS 
SECTION $\checkmark$ HEALTH HAZARD DATA

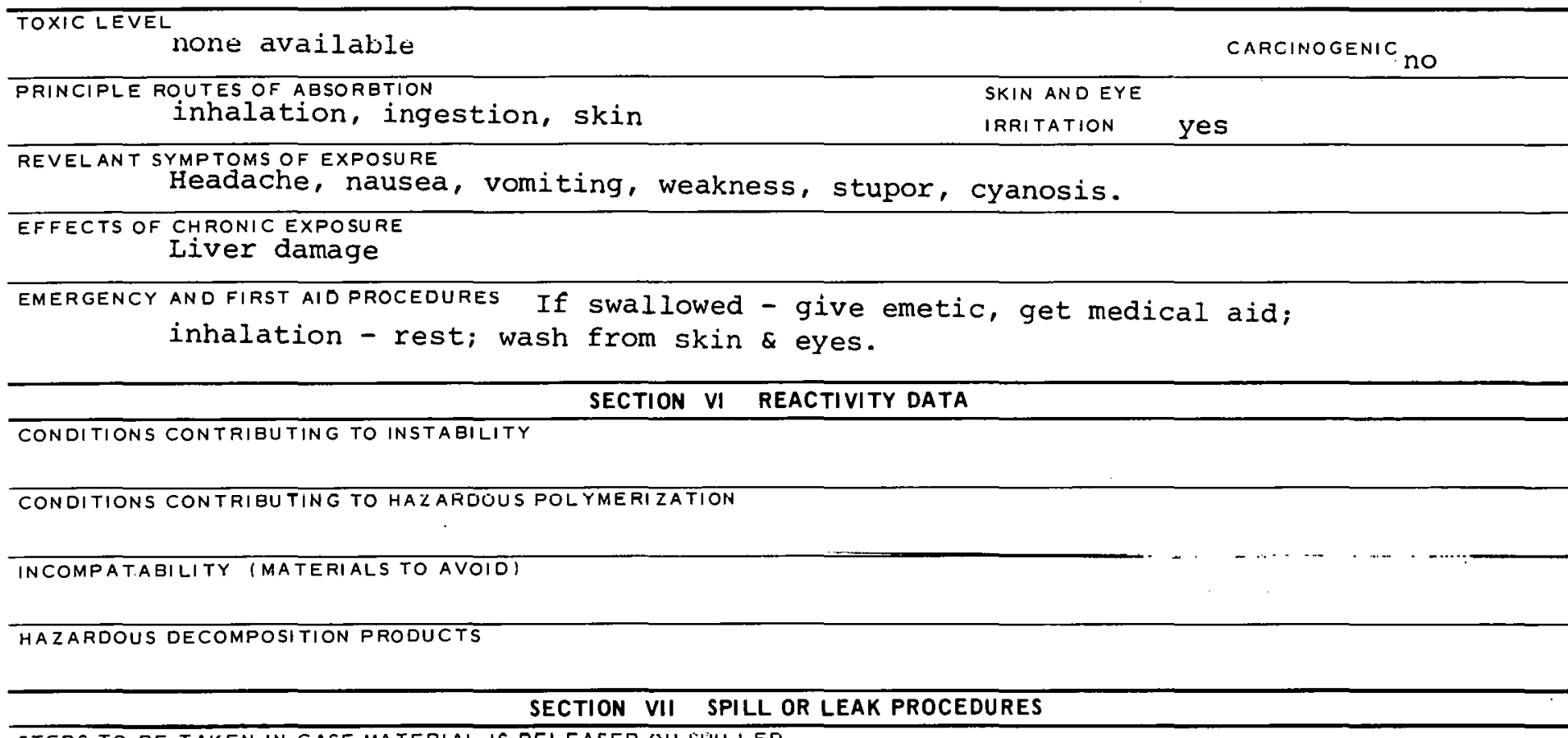

STEPS TO BE TAKEN IN CASE MATERIAL IS RELEASED OK SHILLED

WASTE DISPOSAL METHOD

SECTION VIII SPECIAL PROTECTION INFORMATION

\begin{tabular}{|c|c|}
\hline VENTILATION REQUIREMENTS LOCAL EXHAUST & $\begin{array}{l}\text { PROTECTIVE EQUIPMENT (SPECIFY TYPESI EYE } \\
\text { gOggles }\end{array}$ \\
\hline MECHANICAL (GENERAL) & $\begin{array}{l}\text { GLOVES } \\
\text { rubber }\end{array}$ \\
\hline$\overline{S P E C I A L}$ & $\begin{array}{l}\text { RESPIRATOR } \\
\text { with proper filter }\end{array}$ \\
\hline
\end{tabular}

OTHER PROTECTIVE EQUIPMENT

SECTION IX SPECIAL PRECAUTIONS

PRECAUTIONS TO BE TAKEN IN HANDLING AND STORAGE

OTHER PRECAUTIONS

SIGNATURE

OATE

$315-1907 \mathrm{~B}$ 


PRODUCT DESIGNATION
03-315-2407
5 Nitro-4-Amino-1,
3-Dimethyl Benzene

\section{SECTION I SOURCE AND NOMENCLATURE}

MANUFACTURER'S NAME

EMERGENCY TELEPHONE NO.

ADDRESS (NUMBER, STREET, CITY, STATE, ZIP CODE)

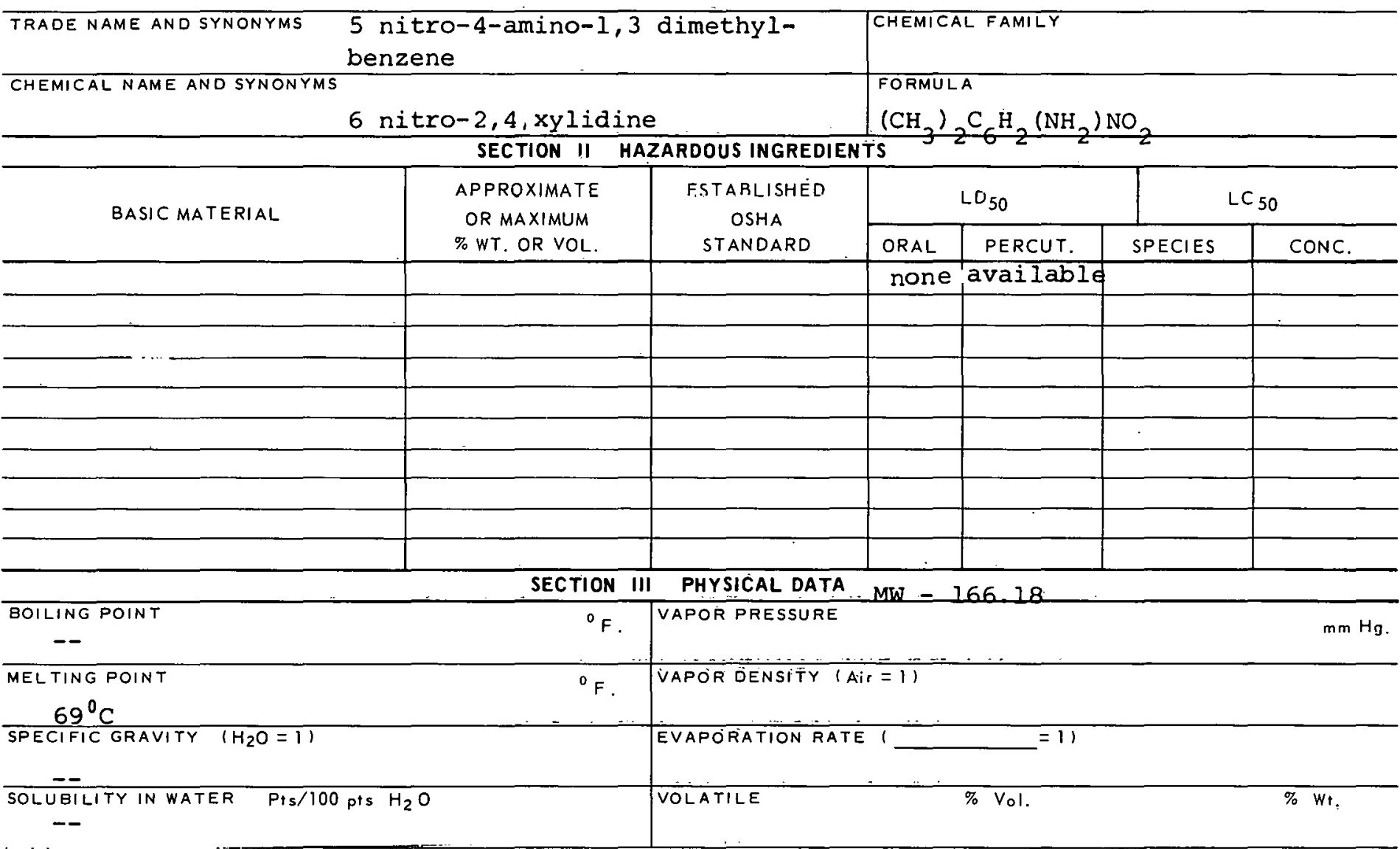

APPEARANCE AND ODOR

crystaline

\begin{tabular}{l|c|l}
\hline \multicolumn{2}{|c|}{ SECTION IV FIRE AND EXPLOSION HAZARD DATA } \\
\hline $\begin{array}{l}\text { FLASH POINT } \\
\text { nOne available }\end{array}$ & FLAMMABLE & UPPER \\
\hline METHOD USED & LIMITS & LOWER \\
\hline EXTINGUISHING MEDIA & & \\
\hline
\end{tabular}

EXTINGUISHING MEDIA

water spray, carbon dioxide, dry chemical

SPECIAL FIRE FIGHTING PROCEDURES

UNUSUAL FIRE AND EXPLOSION HAZARDS 
5 Nitro-4-amino-1,3-Dimethy 1-Benzene

SECTION $V$ HEALTH HAZARD DATA

TOXIC LEVEL

none available

PRINCIPLE ROUTES OF ABSORBTION

inhalation, ingestion, skin

CARCINOGENIC no

REVELANT SYMPTOMS OF EXPOSURE

SKIN AND EYE

IRRITATION YeS

Cyanosis, headache, nausea, vomiting, weakness, stupor.

EFFECTS OF CHRONIC EXPOSURE

Liver damage

EMERGENCY AND FIRST AID PROCEDURES If swallowed - give emetic, get medical aid;

inhalation - rest; wash from skin \& eyes.

\section{SECTION VI REACTIVITY DATA}

CONDITIONS CONTRIBUTING TO INSTABILITY

CONDITIONS CONTRIBUTING TO HAZARDOUS POLYMERIZATION

IN COMPATABILITY (MATERIALS TO AVOIO)

HALARÜUS EEEOMPOMTION FRODUCTS

SECTION VII SPILL OR LEAK PROCEDURES

STEPS TO BE TAKEN IN CASE MATERIAL IS RELEASED OR SPILLED

WASTE DISPOSAL METHOD

SECTION VIII SPECIAL PROTECTION INFORMATION

\begin{tabular}{l|l}
\hline \multicolumn{2}{c}{ SECTION VIII SPECIAL PROTECTION INFORMATION } \\
\hline VENTILATION REQUIREMENTS LOCAL EXHAUST & $\begin{array}{l}\text { PROTECTIVE EQUIPMENT (SPECIFY TYPES) EYE } \\
\text { gOggIES }\end{array}$ \\
\hline MECHANICAL IGENERAL) & $\begin{array}{c}\text { GLOVES } \\
\text { rubber }\end{array}$ \\
\hline SPECIAL & $\begin{array}{c}\text { RESPIRATQR } \\
\text { with proper filter }\end{array}$ \\
\hline
\end{tabular}

OTHER PROTECTIVE EQUIPMEN'T

\section{SECTION IX SPECIAL PRECAUTIONS}

PRECAUTIONS TO BE TAKEN IN HANDLING AND STORAGE

\section{OTHER PRECAUTIONS}

SIGNATURE

DATE

$315-2407 B$ 


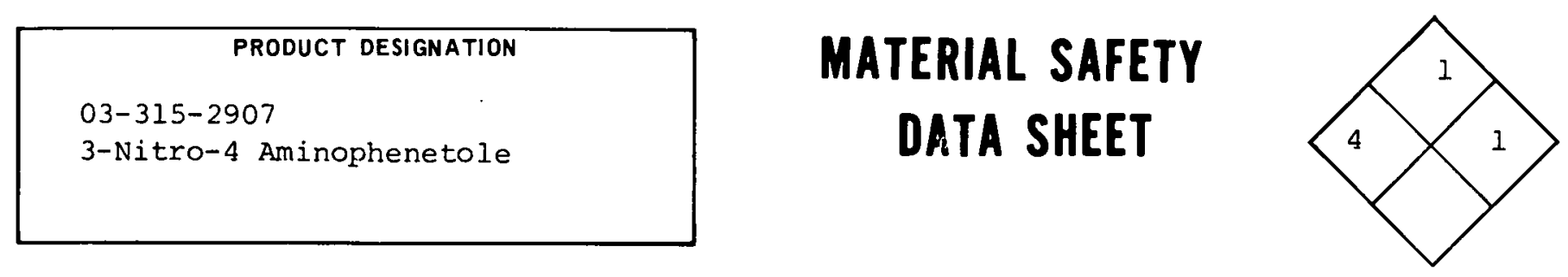

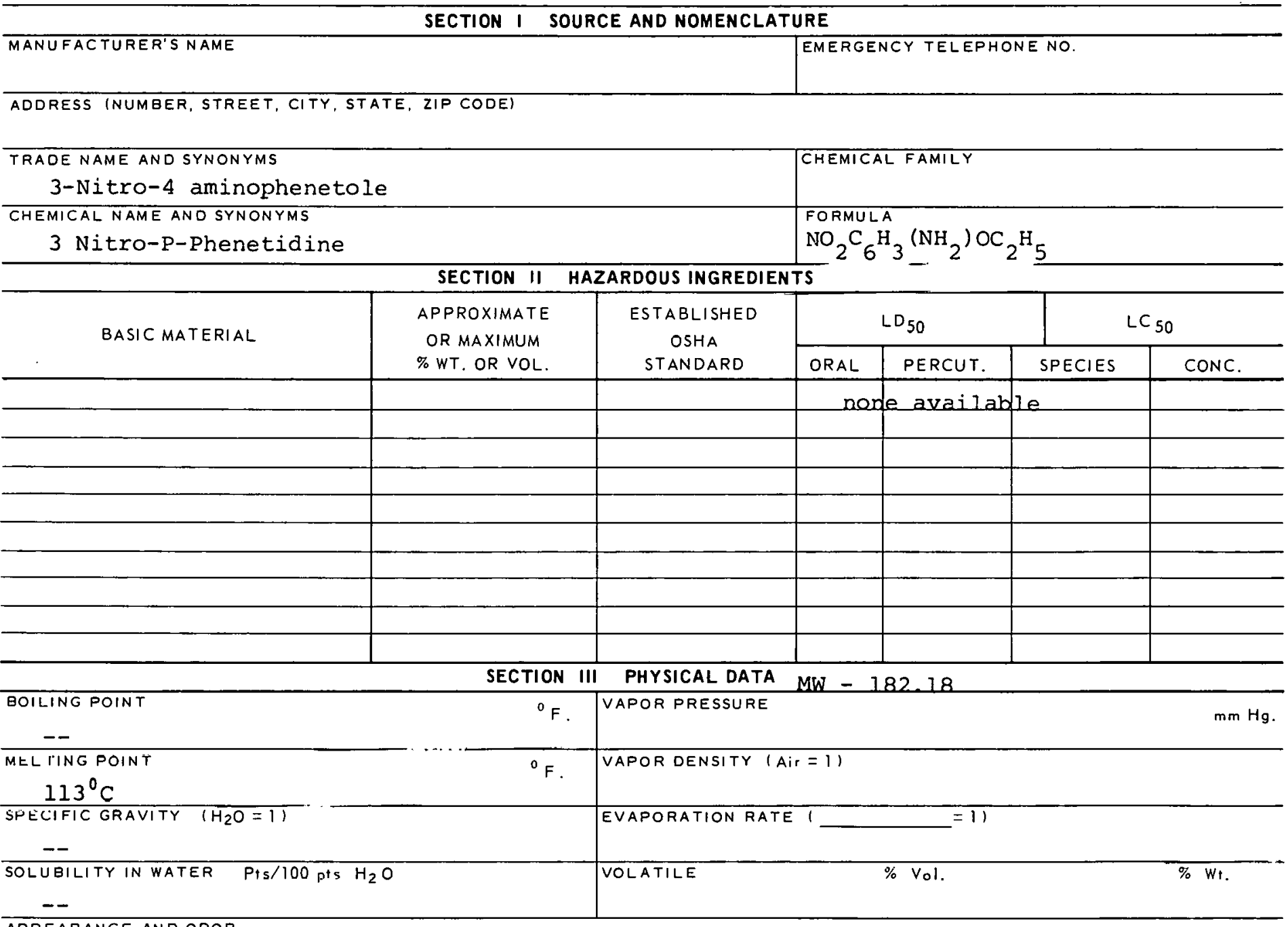

APPEARANCE AND ODOR

red crystals

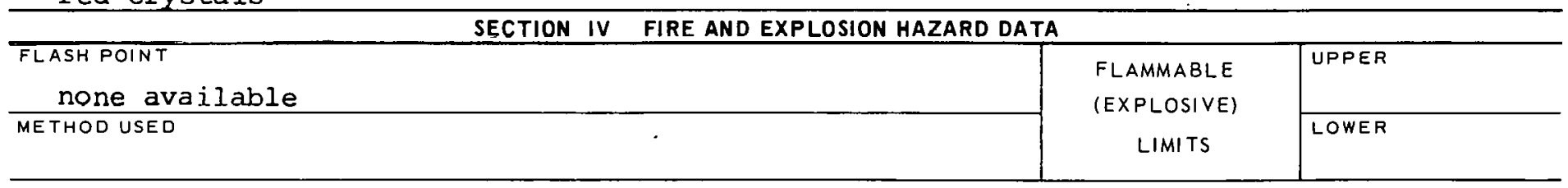

EXTINGUISHING MEDIA

water spray, carbon dioxide, dry chemical

SPECIAL FIRE FIGHTING PROCEDURES

UNUSUAL FIRE AND $\overline{\text { EXPLOSION HAZARDS }}$ 


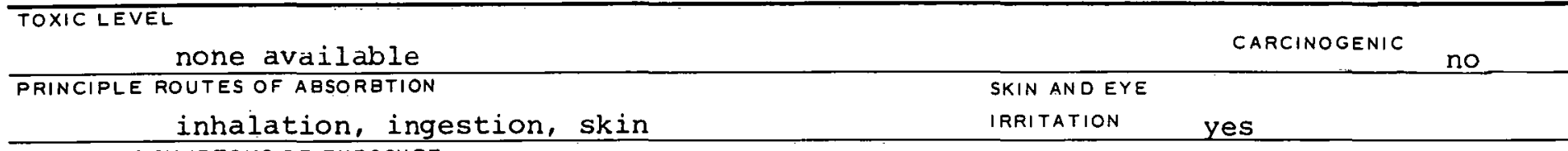

REVELANT SYMPTOMS OF EXPOSURE

Cyanosis, headache, nausea, vomiting, weakness, stupor. EFFECTS OF CHRONIC EXPOSURE

Liver damage

EMERGENCY AND FIRST AID PROCEDURES If swallowed - give emetic, get medical aid;

inhalation - rest; wash from skin \& eyes.

\begin{tabular}{l} 
SECTION VI REACTIVITY DATA \\
\hline CONDITIONS CONTRIBUTING TO INSTABILITY \\
\hline CONDITIONS CONTRIBUTING TO HAZARDOUS POLYMERIZATION
\end{tabular}

INCOMPATABILITY (MATERIALS TO AVOID)

HAZARDOUS DECOMPOSITION PRODUCTS

SECTION VII SPILL OR LEAK PROCEDURES

STEPS TO BE TAKEN IN CASE MATERIAL IS RELEASED OR SPILLED

\section{WASTE DISPOSAL METHOD}

\section{SECTION VIII SPECIAL PROTECTION INFORMATION}

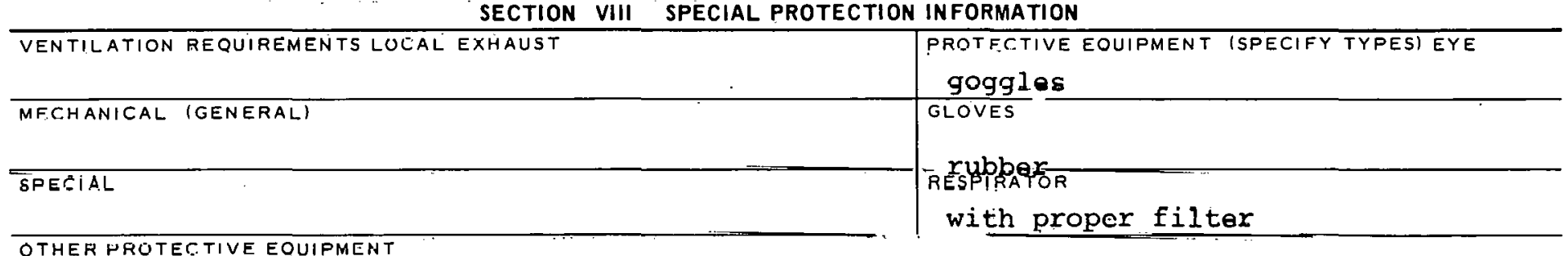

OTHER HROTECTIVE EOUIPMENT

SECTION IX SPECIAL PRECAUTIONS

PRECAUTIONS TO BE TAKEN IN HANDLING AND STORAGE

\section{UTHER PRECAUTIOONS}


CAิS:000119324e

\begin{tabular}{|c|}
\hline PRODUCT DESIGNATION \\
$03-315-3407$ \\
$3-N i t r o-4-$ Aminotoluene \\
\hline
\end{tabular}

\section{MATERIAL SAFETY DATA SHEET}

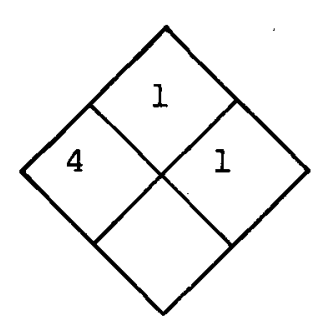

SECTION I SOURCE AND NOMENCLATURE

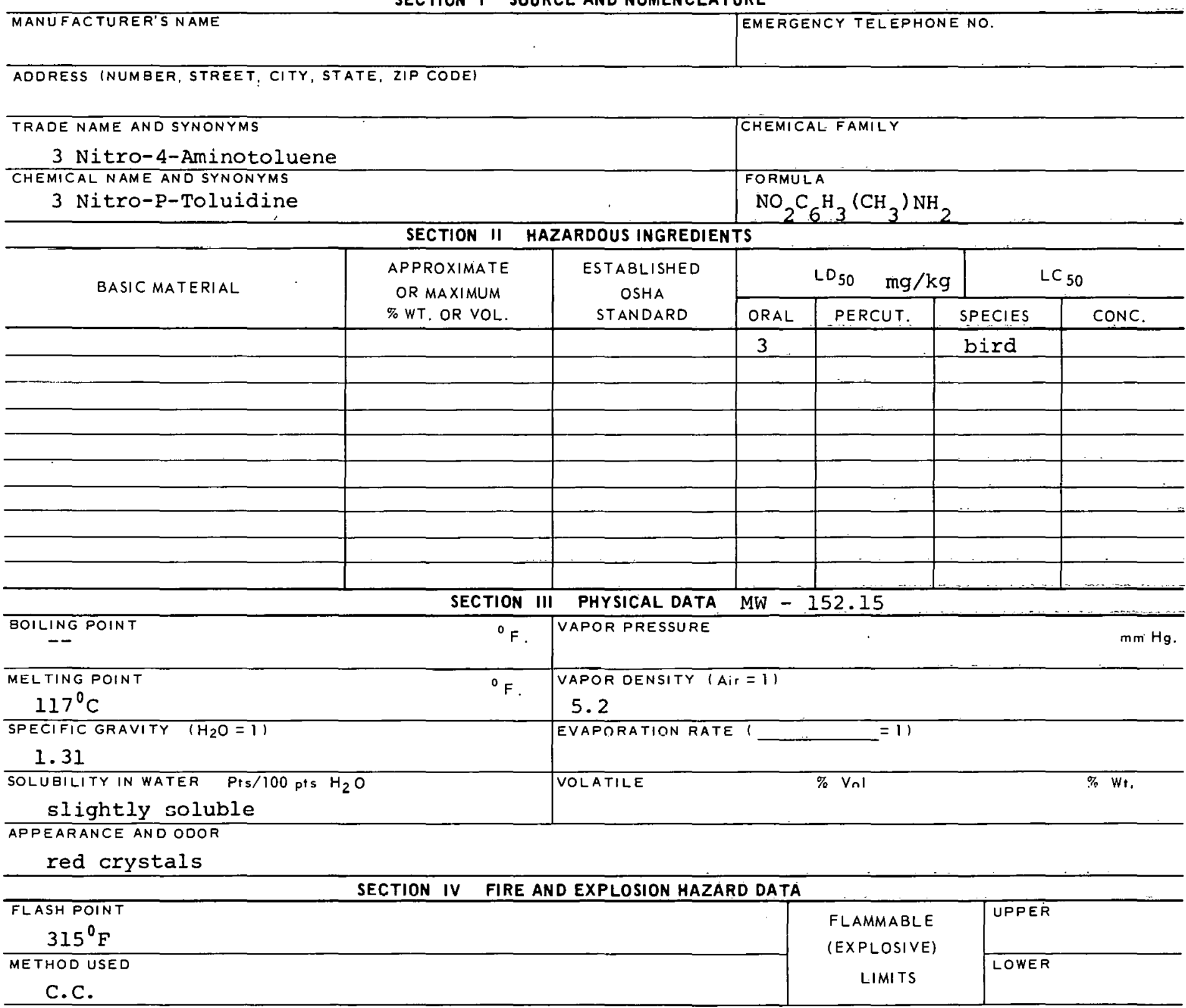

EXTINGUISHING MEDIA

Water spray, carbon dioxide, dry chemical

SPECIAL FIRE FIGHTING PROCEDURES

Wear full protective clothing.

UNUSUAL FIRE AND EXPLOSION HAZAROS 


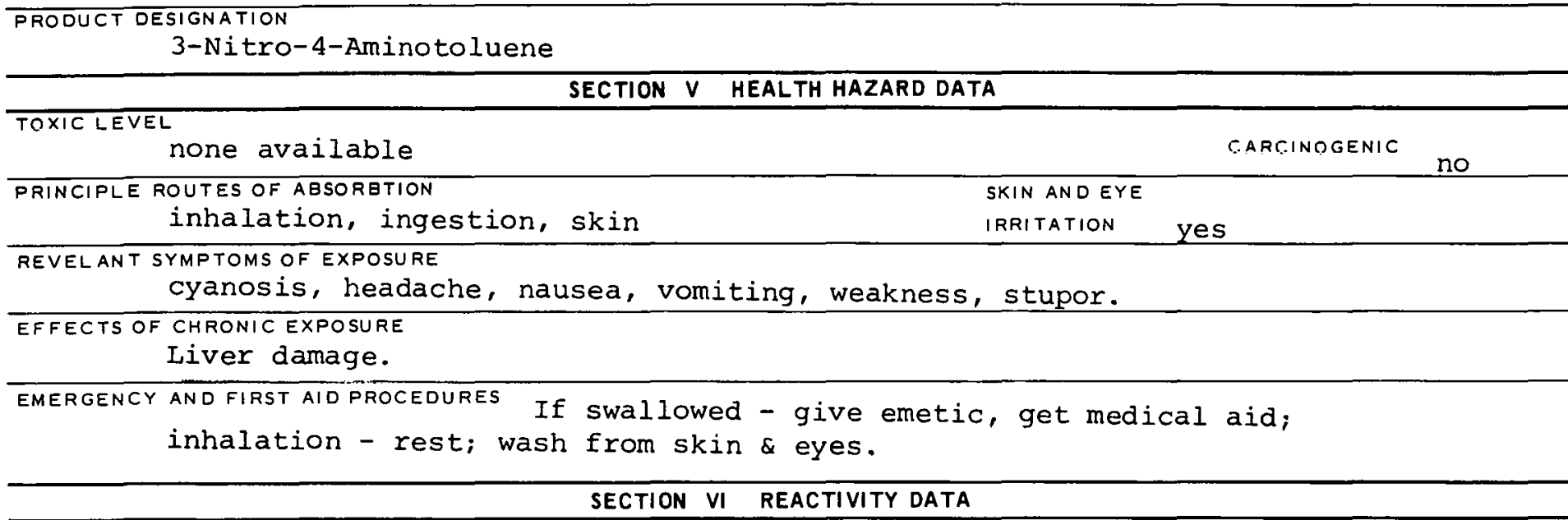

CONDITIONS CONTRIBUTING TO INSTABILITY

CONDITIONS CONTRIBUTING TO HAZARDOU S POLYMERIZATION

INCOMPATABILITY (MATERIALS TO AVOID)

HAZARDOUS DECOMPOSITION PRODUCTS

SECTION VII SPILL OR LEAK PROCEDURES

STEPS TO BE TAKEN IN CASE MATERIAL IS RELEASED OR SPILLED

WASTE DISPOSAL METHOD

\section{SECTION VIII SPECIAL PROTECTION INFORMATION}

\begin{tabular}{l|l}
\hline VENTILATION REQUIREMENTS LOCAL EXHAUST & PROTECTIVE EQUIPMENT (SPECIFY TYPESI EYE
\end{tabular} goggles

MECHANICAL (GENERAL)

SPECIAL

OTHER PROTECTIVE EQUIPMENT

SECTION IX SPECIAL PRECAUTIONS

PRECAUTIONS TO BE TAKEN IN HANDLING AND STORAGE

OTHER PRECAUTTONS

SIGNATURE

DATE

$315-3407 B$ 


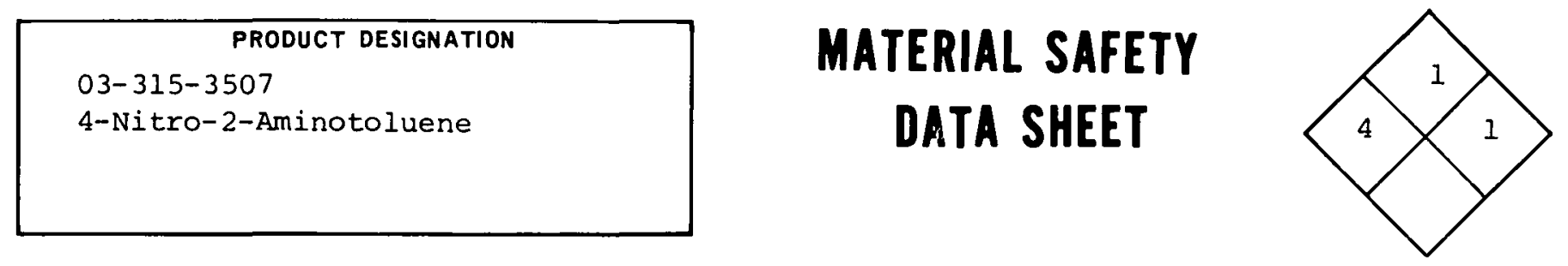

SECTION I SOURCE AND NOMENCLATURE

MANUFACTURER'S NAME

TEMERGENCY TELEPHONE NO.

ADDRESS (NUMBER, STREET, CITY, STATE, ZIP CODE)

TRADE NAME AND SYNONYMS

4 nitro-2-aminotoluene

CHEMICAL NAME AND SYNONYMS

4 nitro-o-toluidine

SECTION \| HAZARDOUS INGREDIENTS

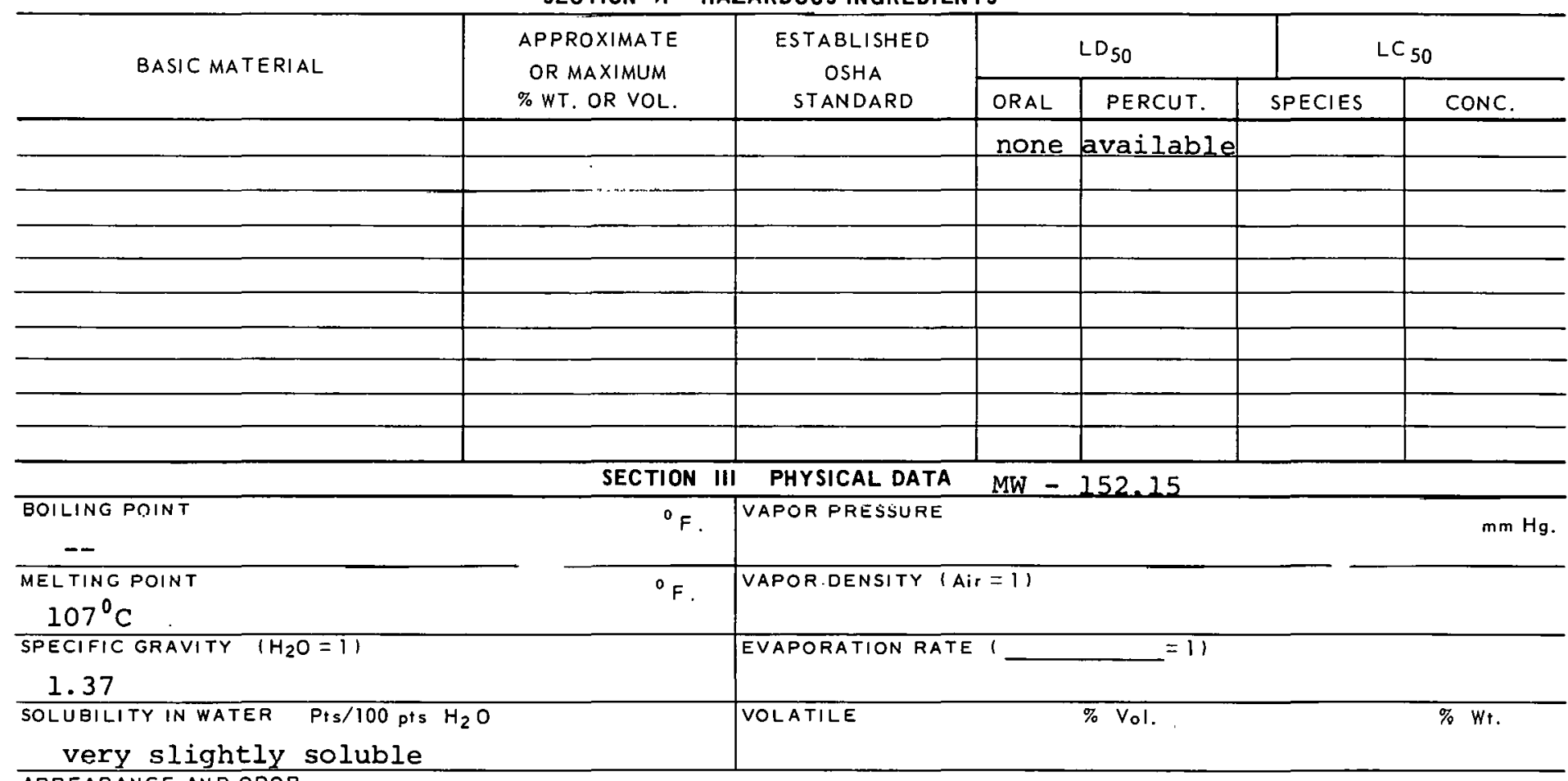

APPEARANCE AND ODOR

yellow crystals

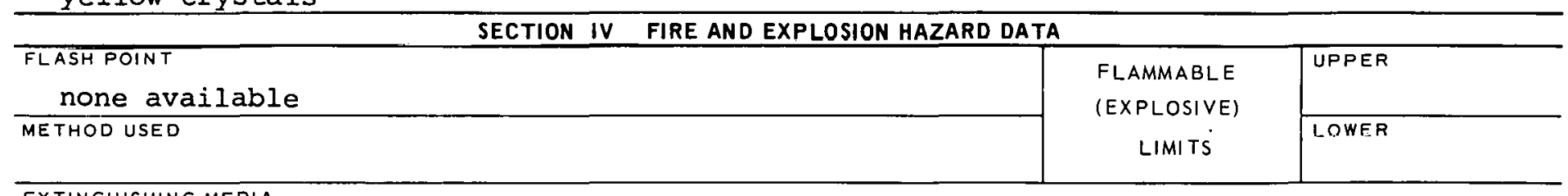

EXTINGUISHING MEDIA

water spray, carbon dioxide, dry chemical

SPECIAL FIRE FIGHTING PROCEDURES

wear full protective clothing.

UNUSUAL FIRE AND EXPLOSION HAZARDS 


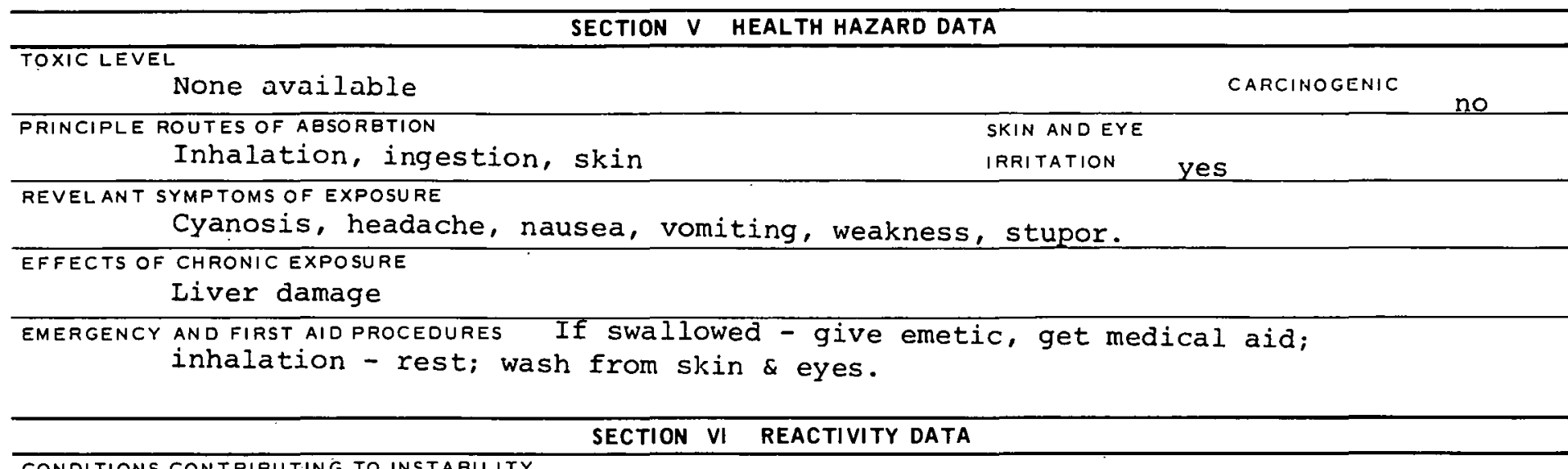

CONOITIONS CONTRIBUTING TO INSTABILITY

CONDITIONS CONTRIBU TING TO HAZARDOUS POLYMERIZAT!ON

INCOMPATABILITY (MATERIALS TO AVOIO)

HAZARDOUS DECOMPOSITION PRODUCTS

SECTION VII SPILL OR LEAK PROCEDURES

STEPS TO BE TAKEN IN CASE MATERIAL IS RELEASEL UR SFILLED

WASTE DISPOSAL METHOD

SECTION VIII SPECIAL PROTECTION INFORMATION

\begin{tabular}{|c|c|}
\hline VENTILATION REQUIREMENTS LOCAL EXHAUST & $\begin{array}{l}\text { PROTECTIVE EQUIPMEN'1 (SPECIFY TYPES) EYE } \\
\text { GOggleS }\end{array}$ \\
\hline MECHANICAL (GENERAL) & $\begin{array}{l}\text { GLOVES } \\
\text { Rubber }\end{array}$ \\
\hline SPECIAL & FESPIRATOR \\
\hline
\end{tabular}

SECTION IX SPECIAL PRECAUTIONS

PRECAUTIONS TO BE TAKEN IN HANDLING AND STORAGE

OTHER PRECAUTIONS

SIGNATURE

DATE

$315-3507 B$ 
CAS : 00099092

BY 68250

\begin{tabular}{|c|}
\hline PRODUCT DESIGNATION \\
03-315-3807 \\
M-Nitroaniline \\
\hline
\end{tabular}

MATERIAL SAFETY

DATA SHEET

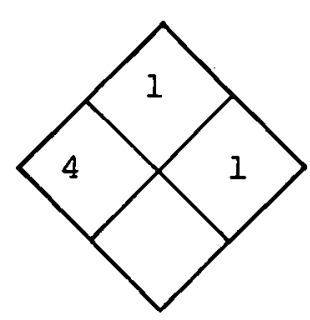

\section{SECTION I SOURCE AND NOMENCLATURE}

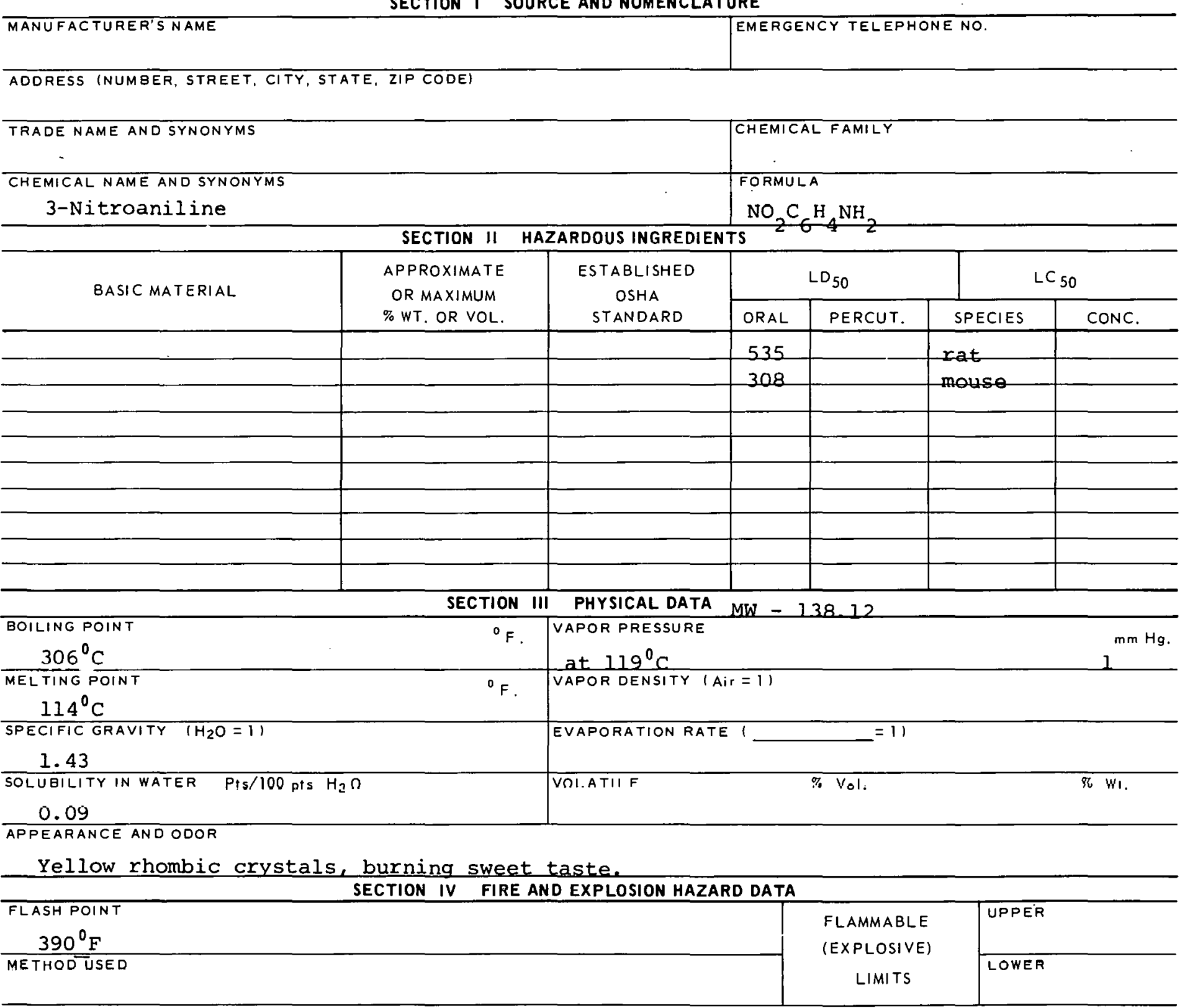

EXTINGUISHING MEOIA

Water spray, carbon dioxide, dry chemical

SPECIAL FIRE FIGHTING PROCEDURES

Wear full protective clothing. 


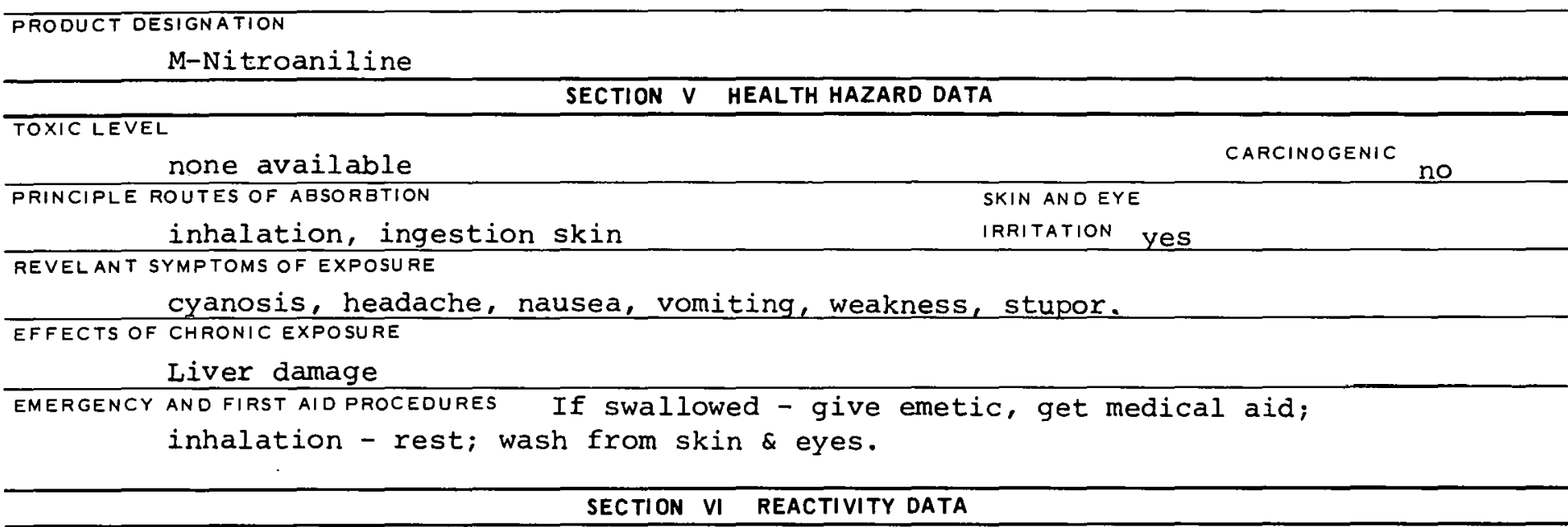

CONDITIONS CONTRIBUTING TO INSTABILITY

CONDITIONS CONTRIBUTING TO HAZARDOUS POLYMERIZATION

INCOMPATABILITY (MATERIALS TO AVOID)

HAZARDOUS DECOMPOSITION PRODUCTS

SECTION VII SPILL OR LEAK PROCEDURES

STEPS TO BE TAKEN IN CASE MATERIAL IS RELEASED OR SPILLED

WASTE DISPOSAL METHOD

SECTION VIII SPECIAL PROTECTION INFORMATION

\begin{tabular}{l|l}
\hline VENTILATION REQUIREMENTS LOCAL EXHAUST & $\begin{array}{l}\text { gROTECTIVE EQUIPMENT (SPECIFY TYPES) EYE } \\
\text { MECHANICAL IGENERAL) }\end{array}$ \\
\hline SPECIAL & $\begin{array}{l}\text { GLOVES } \\
\text { rubber (butyl) }\end{array}$ \\
\hline
\end{tabular}

OTHER PROTECTIVEEQUIPMENT
Protective wOrk clothes

SECTION IX SPECIAL PRECAUTIONS

PRECAUTIONS TO BE TAKEN IN HANDLING AND STORAGE

Protect against moisture and physical damage.

OTHER PRECAUTIONS

SIGNATURE

DATE

$315-3807 B$ 


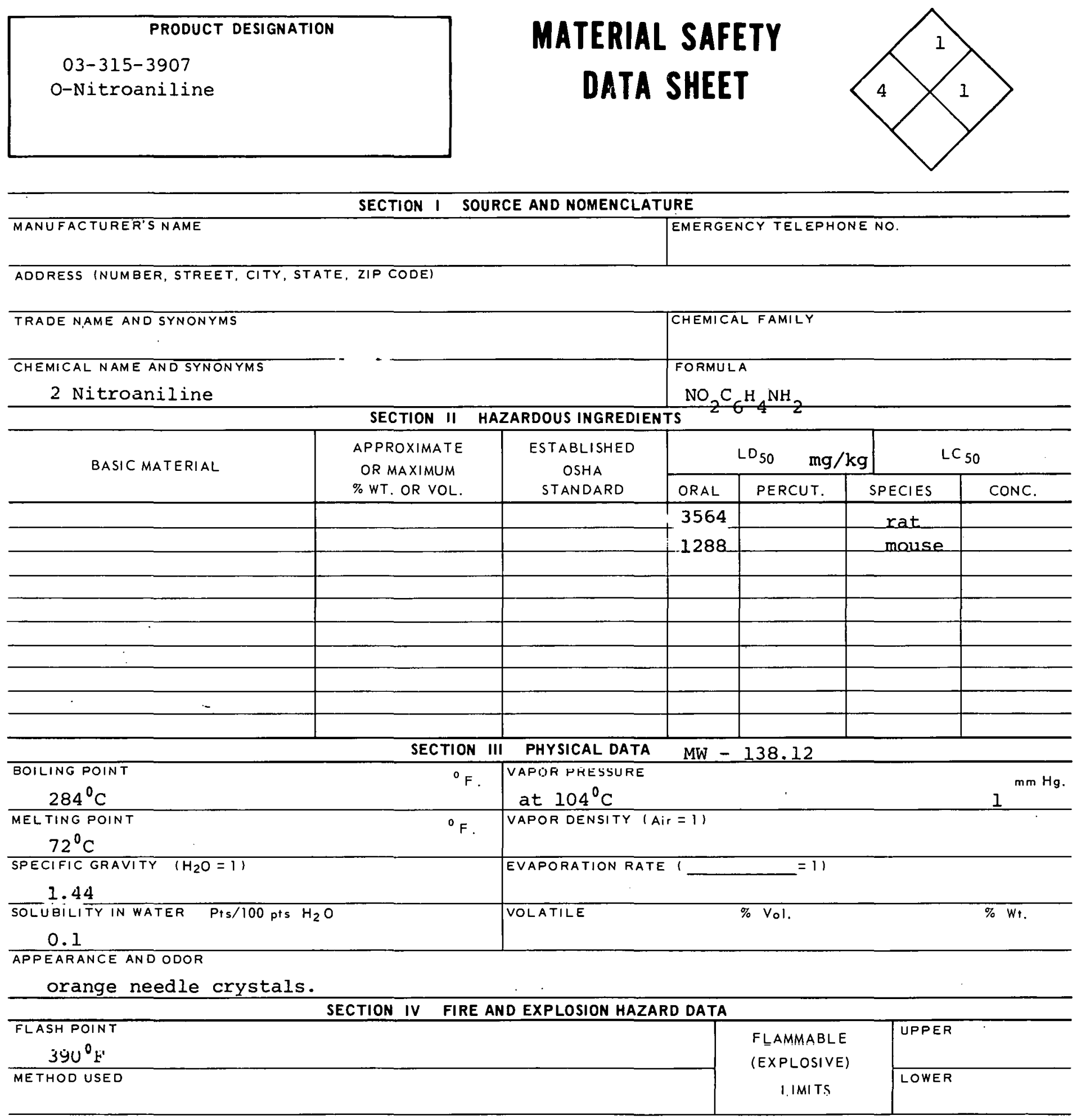

EXTINGUISHING MEDIA

water spray, carbon dioxide, dry chemical

SPECIAL FIRE FIGHTING PROCEDURES

Wear full protective clothing.

UNUSUAL FIRE AND EXPLOSION HAZARDS 


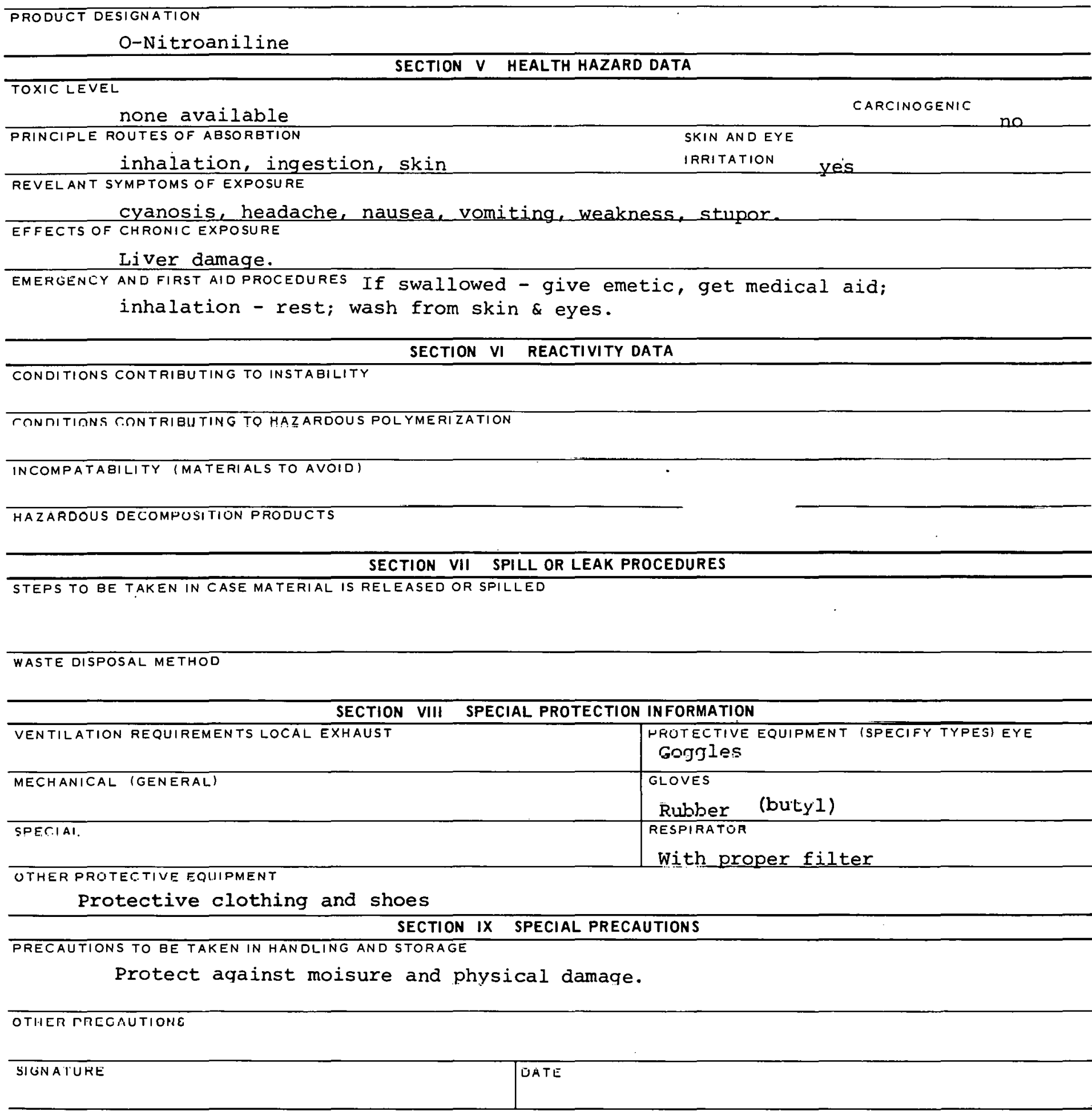




\begin{tabular}{|c|}
\hline PRODUCT DESIGNATION \\
03-315-5407 \\
P-Nitrobenzal Bromide \\
\hline
\end{tabular}

\section{SECTION I SOURCE AND NOMENCLATURE}

\section{MANUFACTURER'S NAME \\ ADDRESS (NUMBER, STREET, CITY, STATE, ZIP CODE) \\ TRADE NAME AND SYNONYMS \\ $\alpha \alpha$-Dibromo-P-Nitrotoluene \\ CHEMICAL NAME AND SYNONYMS \\ P-Nitrobenzal Bromide}

BASIC MATERIAL

\begin{tabular}{l|l} 
& CHEMICAL FAMILY \\
& $\begin{array}{l}\text { FORMULA } \\
\text { SECTION II HAZARDOUS INGREDIENTS }\end{array}{ }_{2} \mathrm{C}_{6} \mathrm{H}_{4} \mathrm{CHBr}_{2}$
\end{tabular}
SECTION II HAZARDOUS INGREDIENTS \begin{tabular}{l|l} 
APPROXIMATE & ESTABLISHED
\end{tabular} OR MAXIMUM OSHA $\%$ WT. OR VOL.

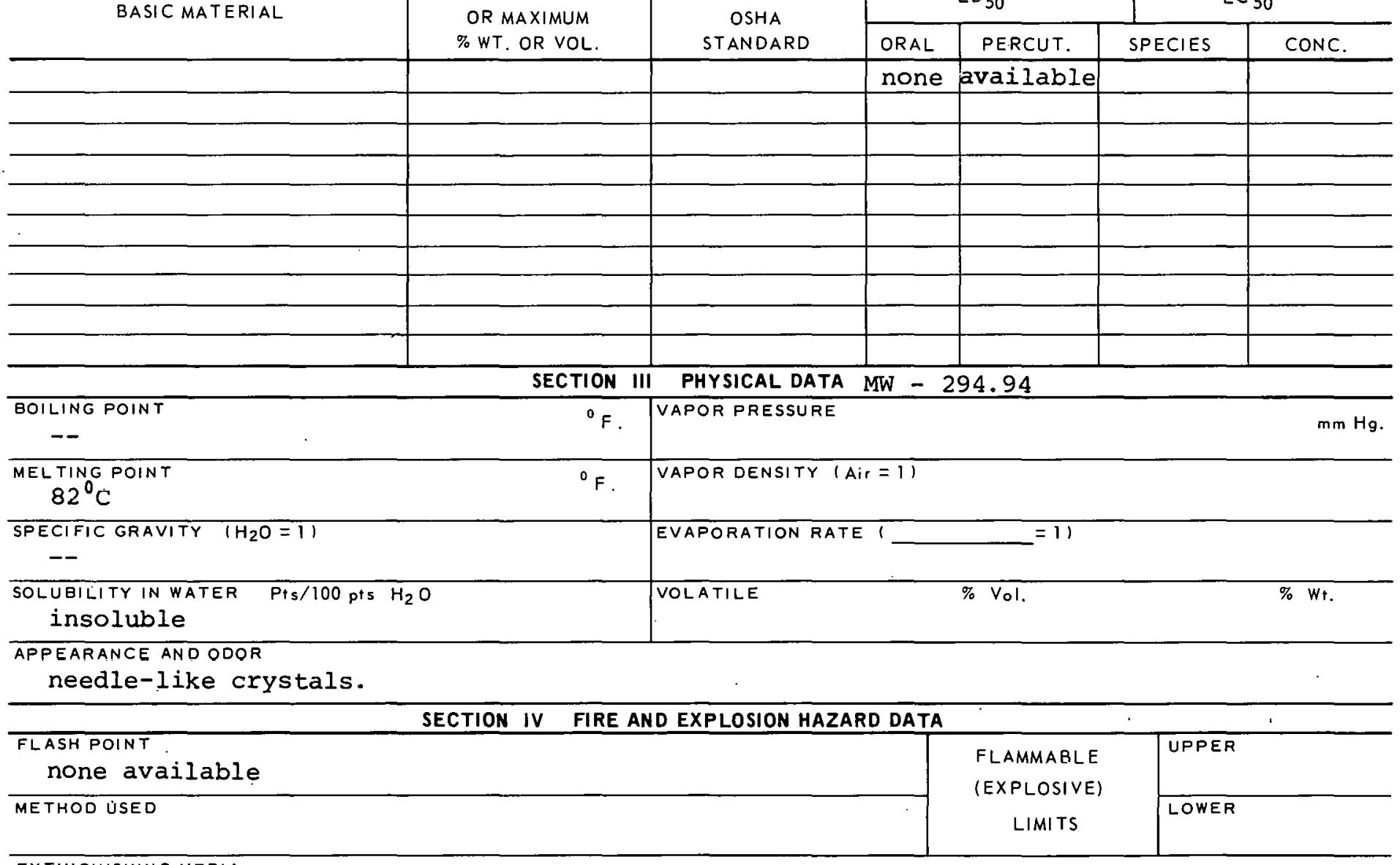

\section{EXTINGUISHING MEDIA}

water spray, carbon dioxide, dry chemical.

SPECIAL FIRE FIGHTING PROCEDURES 
TOXIC LEVEL

$$
\text { none available }
$$

CARCINOGENIC nO

PRINCIPLE ROUTES OF ABSORETION Inhalation, ingestion, skin SKIN ANO EYE IRRITATION SIIght

REVELANT SYMPTOMS OF EXPOSURE

Difficulty in breathing, cyanosis, vomiting, headache

EFFECTS OF CHRONIC EXPOSURE

Blood damage

EMERGENCY AND FIRST AID PROCEDURES If swallowed - give emetic, get medical aid;

inhalation - rest; wash from skin \& eyes.

CONDITIONS CONTRIBUTING TO INSTABILITY

SECTION VI REACTIVITY DATA

CONDITIONS CONTRIBU TING TO HAZARDOUS POLYMERIZATION

INCOMPATABILITY (MATERIALS TO AVOID)

May explode when heated with sulfuric acid. HAZARDOUS DECOMPOSITION PRODUCTS

SECTION VII SPILL OR LEAK PROCEDURES

STEPS TO BE TAKEN IN CASE MATERIAL IS RELEASED OR SPILLED

WASTE DISPOSAL METHOD

SECTION VIII SPECIAL PROTECTION INFORMATION

VENTILATION REQUIREMENTS LOCAL EXHAUST

MECHANICAL (GENERAL)

Goggles

GLOVES

Rubber

SFECIAL

REBPIRATOR

Self-contained

OTHER PROTECTIVE EQUIPMENT

SECTION IX SPECIAL PRECAUTIONS

PRECAUTIONS TO BE TAKEN IN HANDLING AND STORAGE

Outside or detached storage preferred; inside storage in standard flammable liquids cabinct:

OTHER PRECAUTIONS

SIGNATURE

DATE

$315-5407 B$ 


\begin{tabular}{|c|}
\hline PRODUCT DESIGNATION \\
$03-315-6857$ \\
Nitrobenzene \\
\hline
\end{tabular}

\section{MATERIAL SAFETY DATA SHEET}

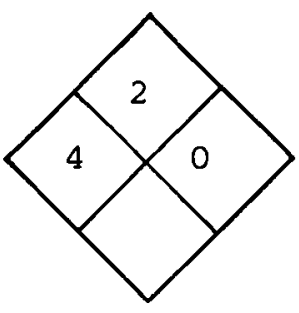

SECTION I SOURCE AND NOMENCLATURE

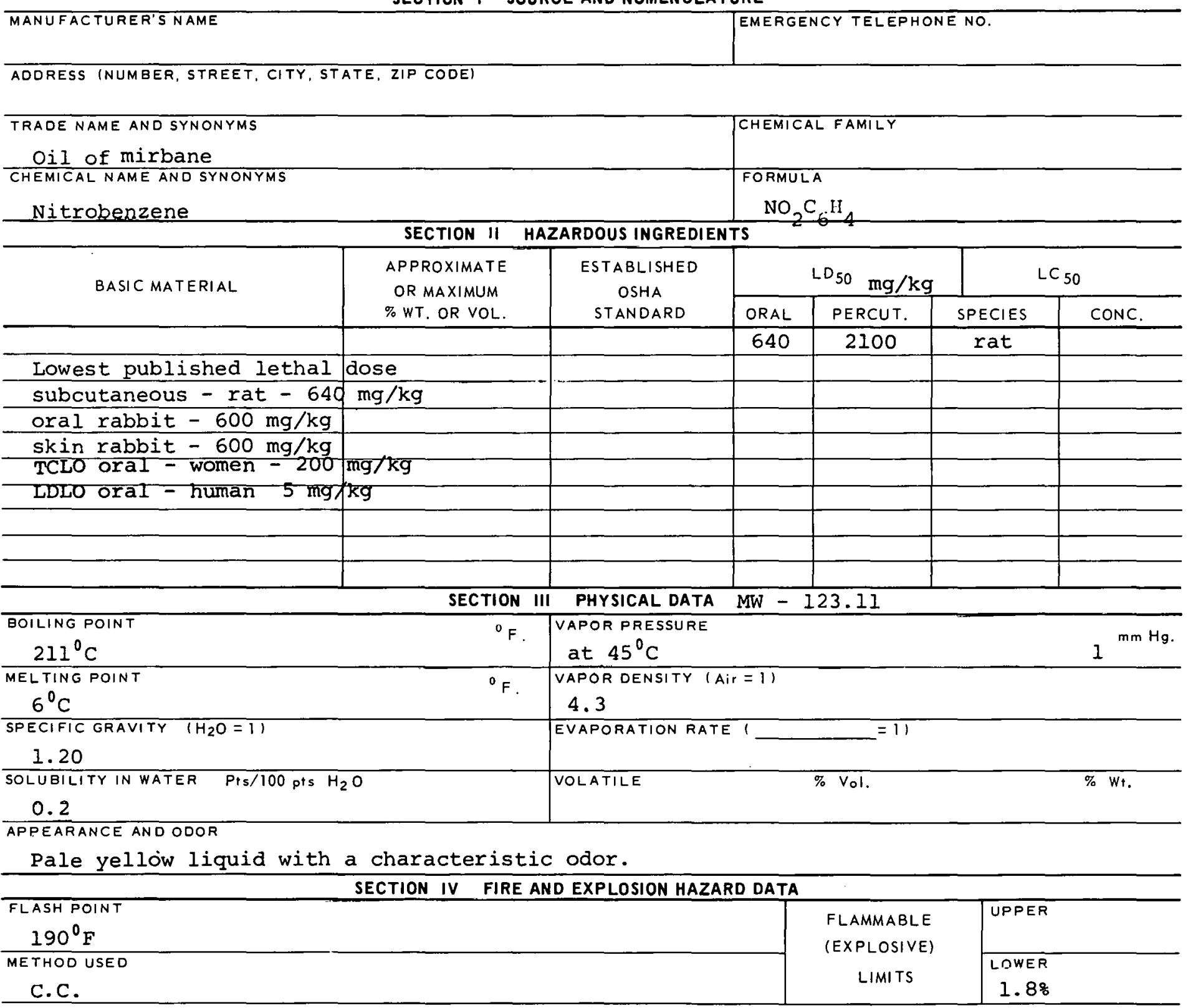

EXTINGUISHING MEDIA

water spray, carbon dioxide, dry chemical or foam.

SPECIAL FIRE FIGHTING PROCEDURES

Wear complete protective clothing.

UNUSUAL FIRE AND EXPLOSION HAZARDS

Store separate froin uxidizing materials, heat or sources of ignition. 
TOXIC LEVEL

TLV - I PPM, $5 \mathrm{mg} / \mathrm{C} . \mathrm{M}$. skin PRINCIPLE ROUTES OF ABSORBTION

Inhalation, ingestion, skin
Teratogen CARCINOGENIC SKIN AND EYE IRRITATION slight

REVELANT SYMPTOMS OF EXPOSURE Cyanosis, excitement, weakness, difficult breathing, shock and circulatory failure.

EFFECTS OF CHRONIC EXPOSURE

Headache, vertigo, blood damage.

EMERGENCY AND FIRST AIO PROCEOURES If swallowed - give emetic, get medical aid; inhalation rest; wash from skin \& eyes.

\section{SECTION VI REACTIVITY DATA}

CONDITIONS CONTRIBUTING TO INSTABILITY

CRNDITIONS CONTRIBU TING TO HAZARDOUS POLYMERIZATION

"'INCOMPATABILITY (MATERIALS TO AVUID)

Avoid, nitric acid, aniline with glycerine, and aluminum chloride with phenol. HAZARDOUS UELUMHUSI IIIN RUOUCTO

SECTION VII SPILL OR LEAK PROCEDURES

STEPS TO BE TAKEN IN CASF: MATERIAL IS RELEASED OR SPILLED

WASTE DISPOSAL METHOD

\section{SECTION VIII SPECIAL PROTECTION INFORMATION}

\begin{tabular}{|c|c|}
\hline VENTILATION REQUIREMENTS LOCAL EXHAUST & $\begin{array}{l}\text { PROTECTIVE EQUIPMENT (SPECIFY TYPESI EYE } \\
\text { gOggleS }\end{array}$ \\
\hline MECHANICAL (GENERAL) & $\begin{array}{l}\text { GLOVES } \\
\text { rubber }\end{array}$ \\
\hline SPECIAL & $\begin{array}{l}\text { RESPIRATOR } \\
\text { with proper filter }\end{array}$ \\
\hline
\end{tabular}

OTHER PROTECTIVE EQUIPMENT

\section{SECTION IX SPECIAL PRECAUTIONS}

PRECAUTIONS TO BE TAKEN IN HANDLING AND STORAGE

OTHER PAEGAUTIONS

SIGIATURE

DATE

$315-6857 \mathrm{~B}$ 


\begin{tabular}{|c|}
\hline PRODUCT DESIGNATION \\
03-316-0208 \\
P-Nitrobenzoyl chloride \\
.
\end{tabular}

\section{MATERIAL SAFETY DATA SHEET}

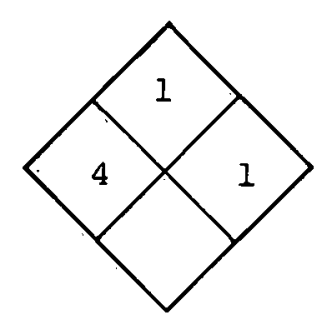

\section{SECTION I SOURCE AND NOMENCLATURE}

\begin{tabular}{l|l|l}
\hline MANUFACTURER'S NAME & EMERGENCY TELEPHONE NO. \\
\hline ADDRESS (NUMBER, STREET, CITY, STATE, ZIP CODE)
\end{tabular}

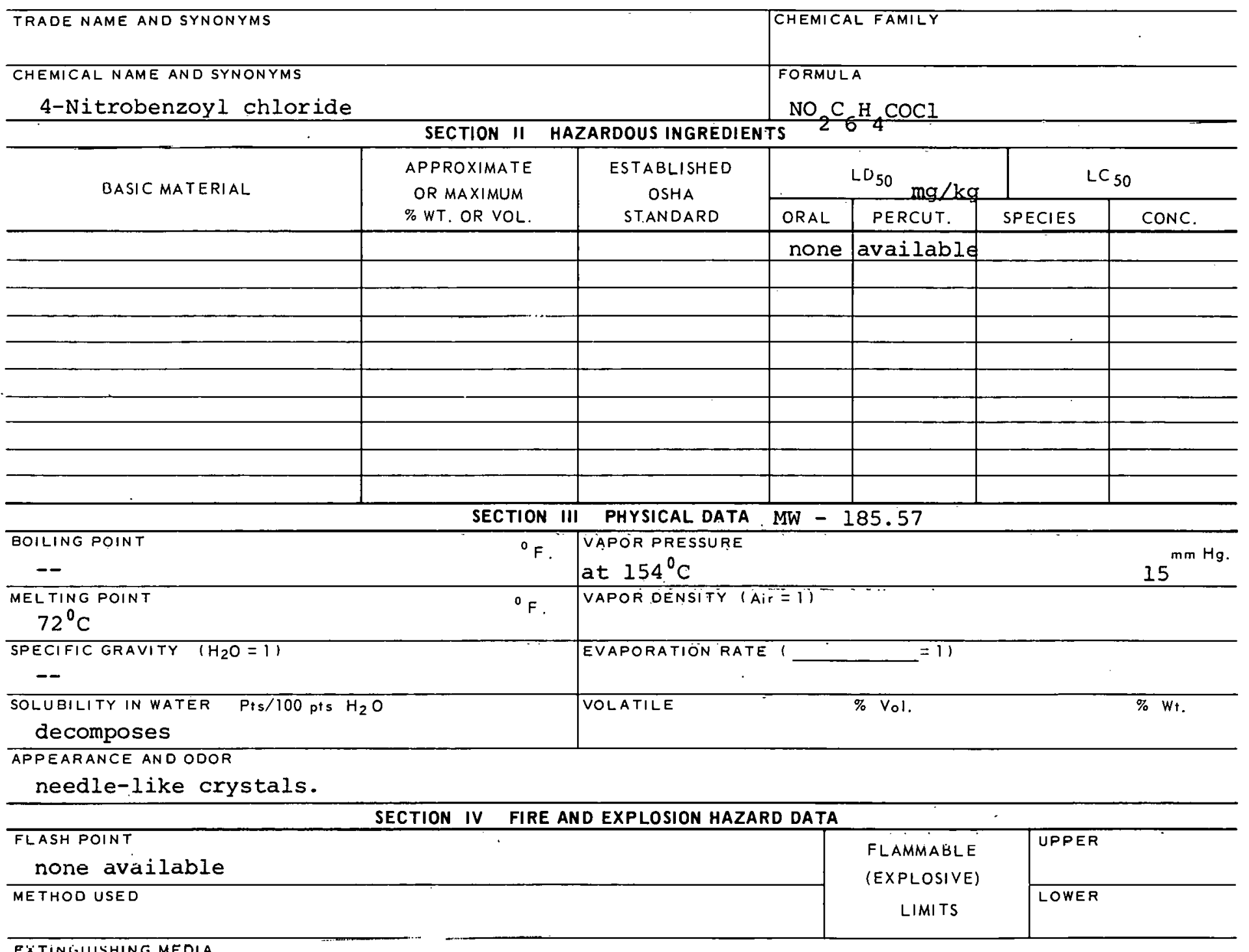

EXTINGUISHING MEDIA

carbon dioxide, dry chemical

SPECIAL FIRE FIGHTING PROCEDURES

UNUSUAL FIRE AND EXPLOSION HAZARDS 


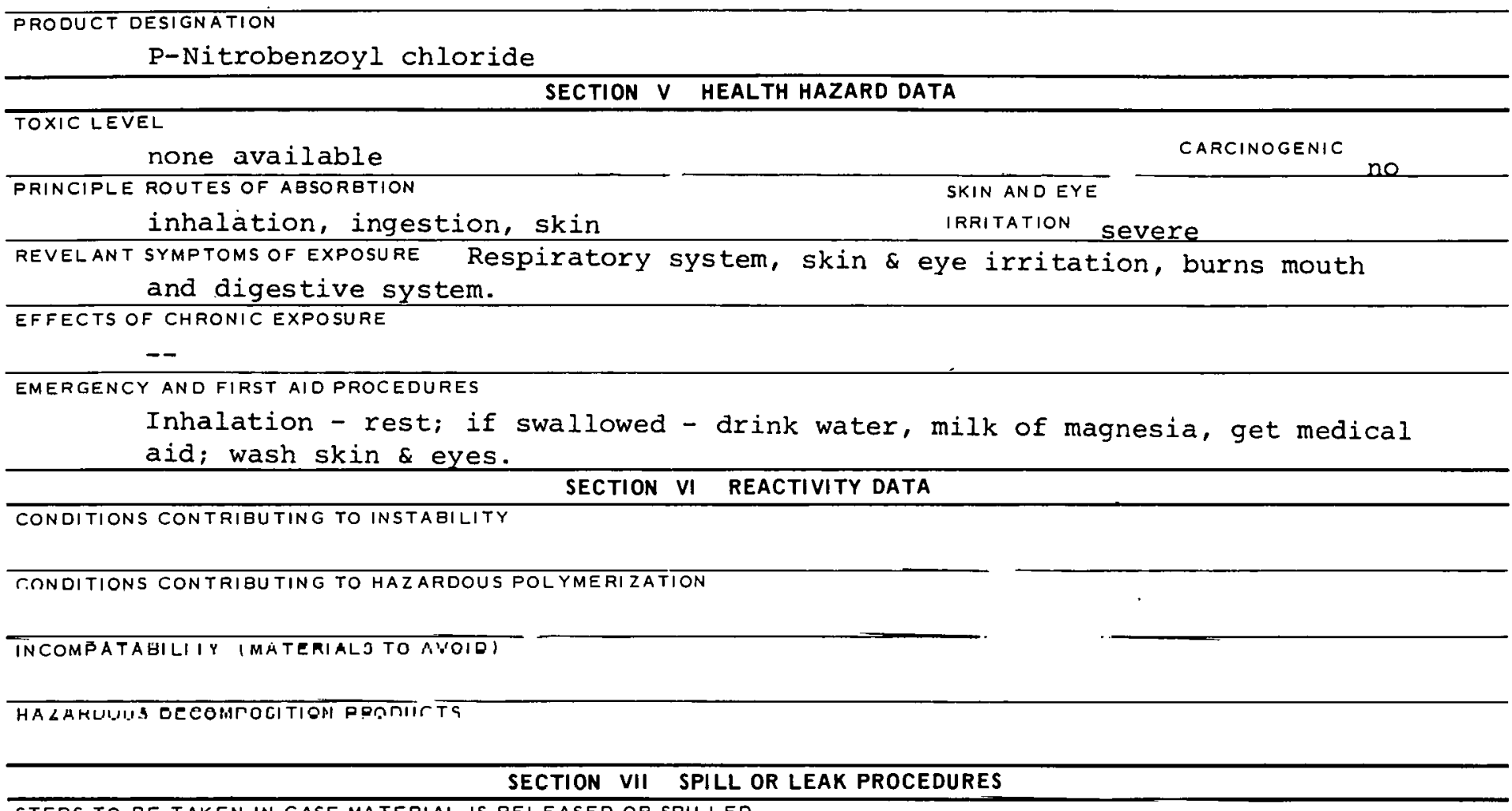

STLFS TO BE TAKEN IN CASE MATERIAL IS RELEASED OR SPILLED

WASTE DISPOSAL MF.THOD

SECTION VIII SPECIAL PROTECTION INFORMATION

\begin{tabular}{l|l}
\hline VENTILATION REQUIREMENTS LOCAL EXHAUST & PROTECTIVE EQUIPMENT (SPECIFY TYPES) EYE
\end{tabular}

MECHANICAL (GENERAL)

Goggles

GLOVES

Rubber

qPFrIAI

RESPIRATOR

OTHER PROTECTIVE EQUIPMENT

With proper filtor

SECTION IX SPECIAL PRECAUTIONS

PRECAIITIONS TO BE TAKEN IN HANDLING AND STORAGE

OTHER PRECAUTIONS

SIGNATURE

\begin{tabular}{l|l} 
OATE \\
\hline
\end{tabular}

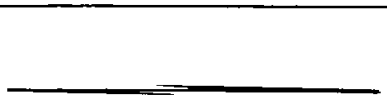

316-0208B 

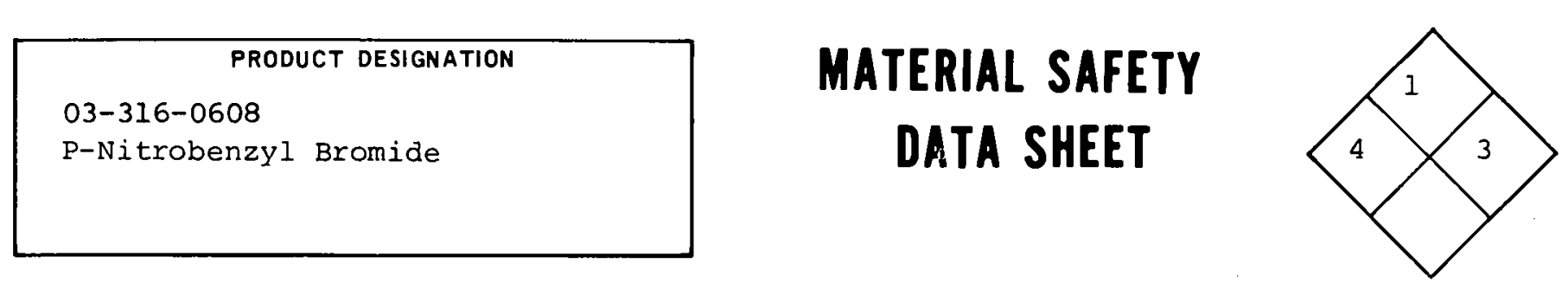

SECTION I SOURCE AND NOMENCLATURE

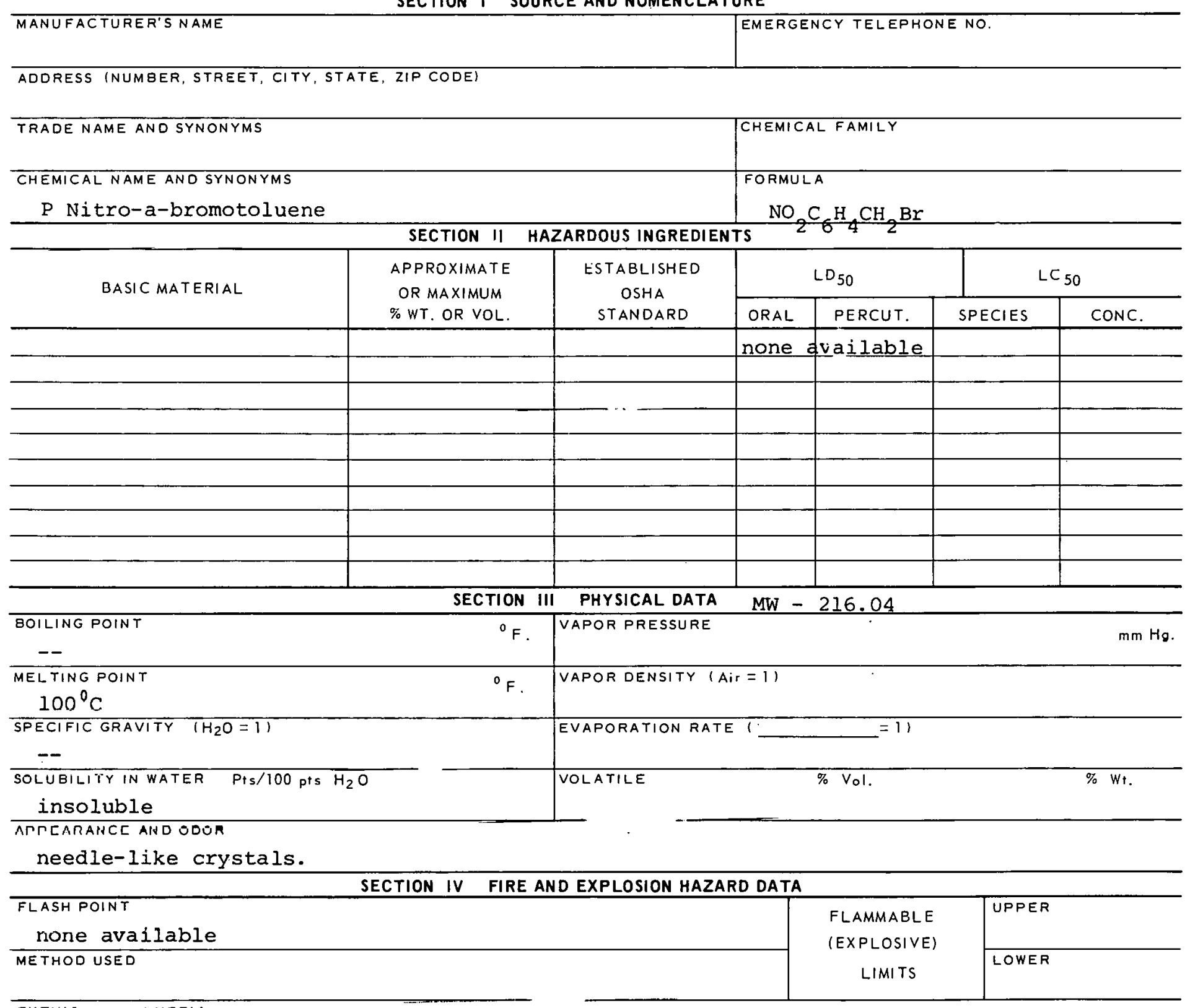

EXTINGUISHING MEDIA

water, carbon dioxide, dry chemicals.

SPECIAL FIRE FIGHTING PROCEDURES

UNUSUAL FIRE AND EXPLOSION HAZARDS 
TOXIC LEVEL

none available

CARCINOGENIC no

PRINCIPLE ROUTES OF ABSORBTION

inhalation, ingestion, skin

SKIN AND EYE

IRRITATION SIIght

REVELANT SYMPTOMS OF EXPOSURE

Difficulty in breathing, cyanosis, vomiting, headache.

EFFECTS OF CHRONIC EXPOSURE

Blood damage.

EMERGENCY AND FIRST AID PROCEDURES If SWALlowed - give emetic, get medical aid; inhalation rest; wash from skin \& eyes.

SECTION VI REACTIVITY DATA

CONDITIONS CONTRIBUTING TO INSTABILITY

EOHOITIOHE SOMTRIRIITING TO HAZARDOUS POLYMERIZATION

INCOMPATABILITY (MATERIALS TO AVOID)

May explode when heated with sulfuric acid.

HAZARDOUS DECOMPOSITION HROOUCTS

SECTION VII SPILL OR LEAK PROCEDURES

STEPS TO BE TAKEN IN CASE MATERIAL IS RELEASED OR SPILLED

WASTE DISPOSAL METHOD

SECTION VIII SPECIAL PROTECTION INFORMATION

\begin{tabular}{l|l}
\hline VENTILATION REQUIREMENTS LOCAL EXHAUST & $\begin{array}{l}\text { PROTECTIVE EQUIPMENT (SPECIFY TYPES) EYE } \\
\text { gNGglPS }\end{array}$ \\
\hline MECHANICAL IGENERAL) & $\begin{array}{l}\text { GLOVES } \\
\text { rubber }\end{array}$ \\
SPCCIAL & $\begin{array}{l}\text { RFSPIRATRR } \\
\text { self-contained }\end{array}$ \\
\hline
\end{tabular}

OTHER PROTECTIVE EQUIPMENT

SECTION IX SPECIAL PRECAUTIONS

PREÇAUTIONS TO BE TAKEN IN HANDLING AND STORAGE

OTHER PRECAUTIONS 

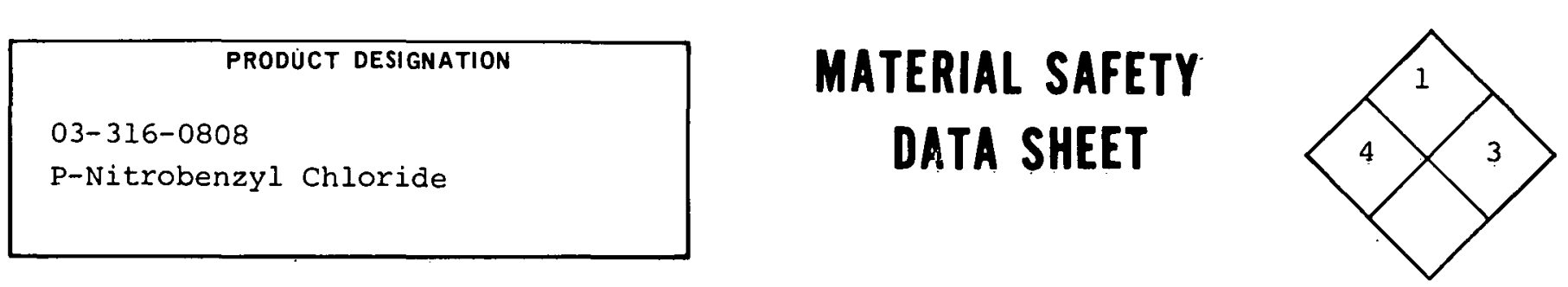

\section{SECTION I SOURCE AND NOMENCLATURE}

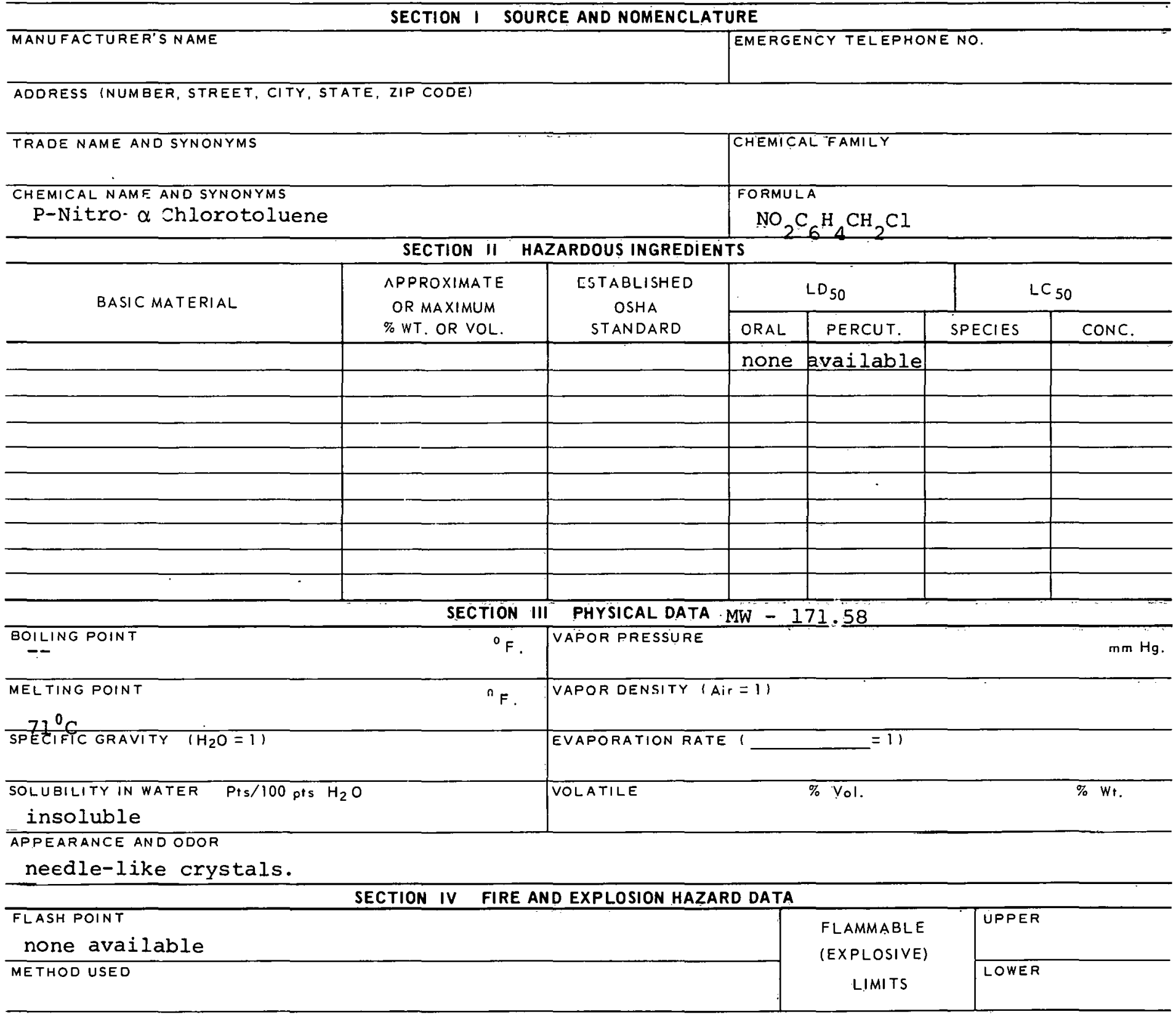

EXTINGUISHING MEDIA

water, carbon dioxide, dry chemical.

SPECIAL FIRE FIGHTING PROCEDURES

UNUSUAL FIRE AND EXPLOSION HAZARDS 


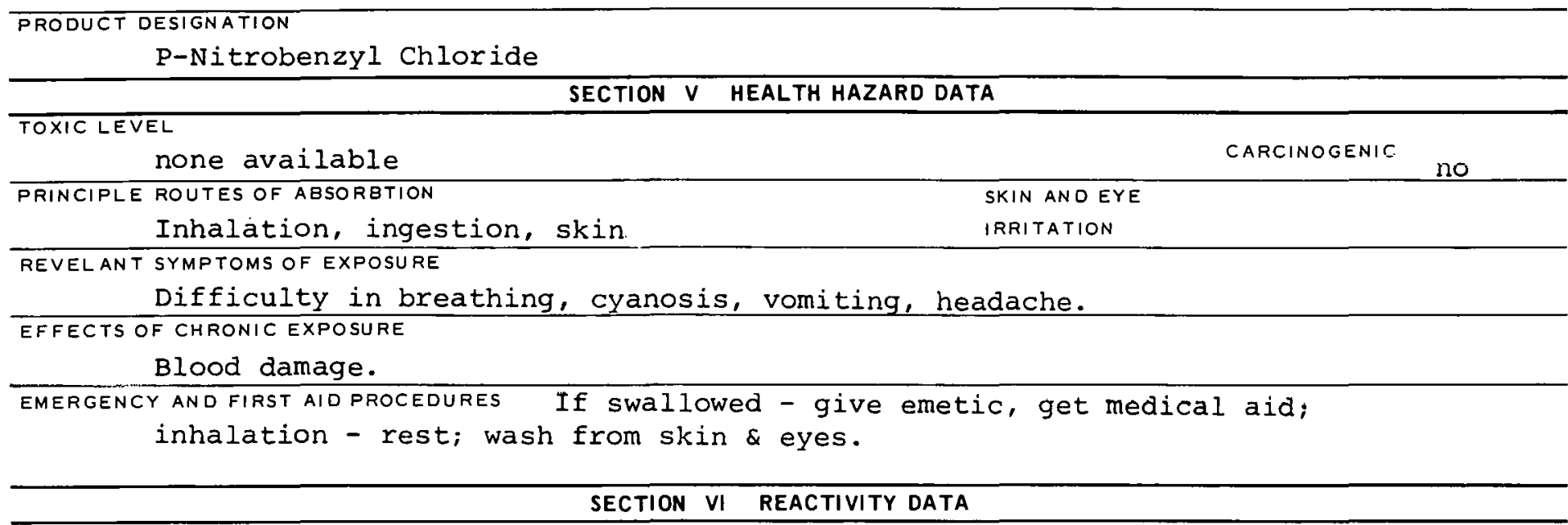

CONDITIONS CONTRIBUTING TO INSTABILITY

CONDITIONS CONTRIBUTING TO HAZARDOUS POLYMERIZATION

INCOMPATABILITY (MATERIALS TO AVOID)

May explode when heated with sulfuric acid. HAZARDOUS DECOMPOSITION PRODUCTS

SECTION VII SPILL OR LEAK PROCEDURES

STEPS TO BE TAKEN IN CASE MATERIAL IS RELEASEO OR SPILLED

WASTE DISPOSAL METHOD

SECTION VIII SPECIAL PROTECTION INFORMATION

\begin{tabular}{l|l}
\hline VENTILATION REQUIREMENTS LOCAL EXHAUST & $\begin{array}{l}\text { PROTECTIVE EQUIPMENT (SPECIFY TYPES) EYE } \\
\text { GOgg LES }\end{array}$ \\
\hline MECHANICAL IGENERAL) & $\begin{array}{l}\text { GLOVES } \\
\text { Rubher }\end{array}$ \\
\hline SPECIAL & $\begin{array}{l}\text { KESHIRATUR } \\
\text { Self-contained }\end{array}$ \\
\hline
\end{tabular}

OTHER PROTECTIVE EQUIPMENT

PRECAUTIONS TO BE TAKEN IN HANDLING AND STORAGE

UTHER PRECAUTIONS

SIGNATURE DATE 


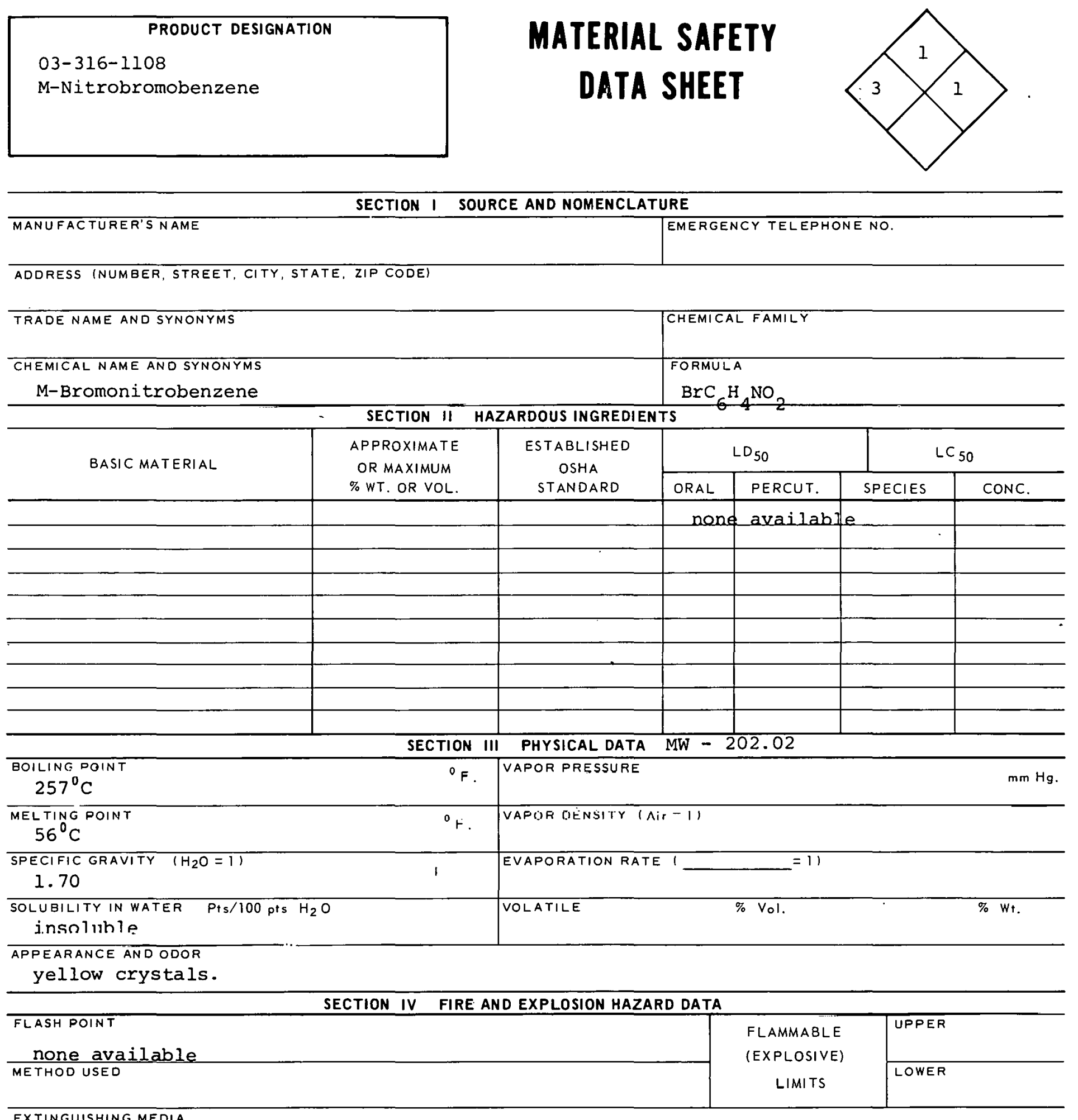

water spray, carbon dioxide, dry chemical, foam.

SPECIAL FIRE FIGHTING PROCEDURES

Wear self-contained breathing apparatus.

UNUSUAL FIRE AND EXPLOSION HAZARDS 


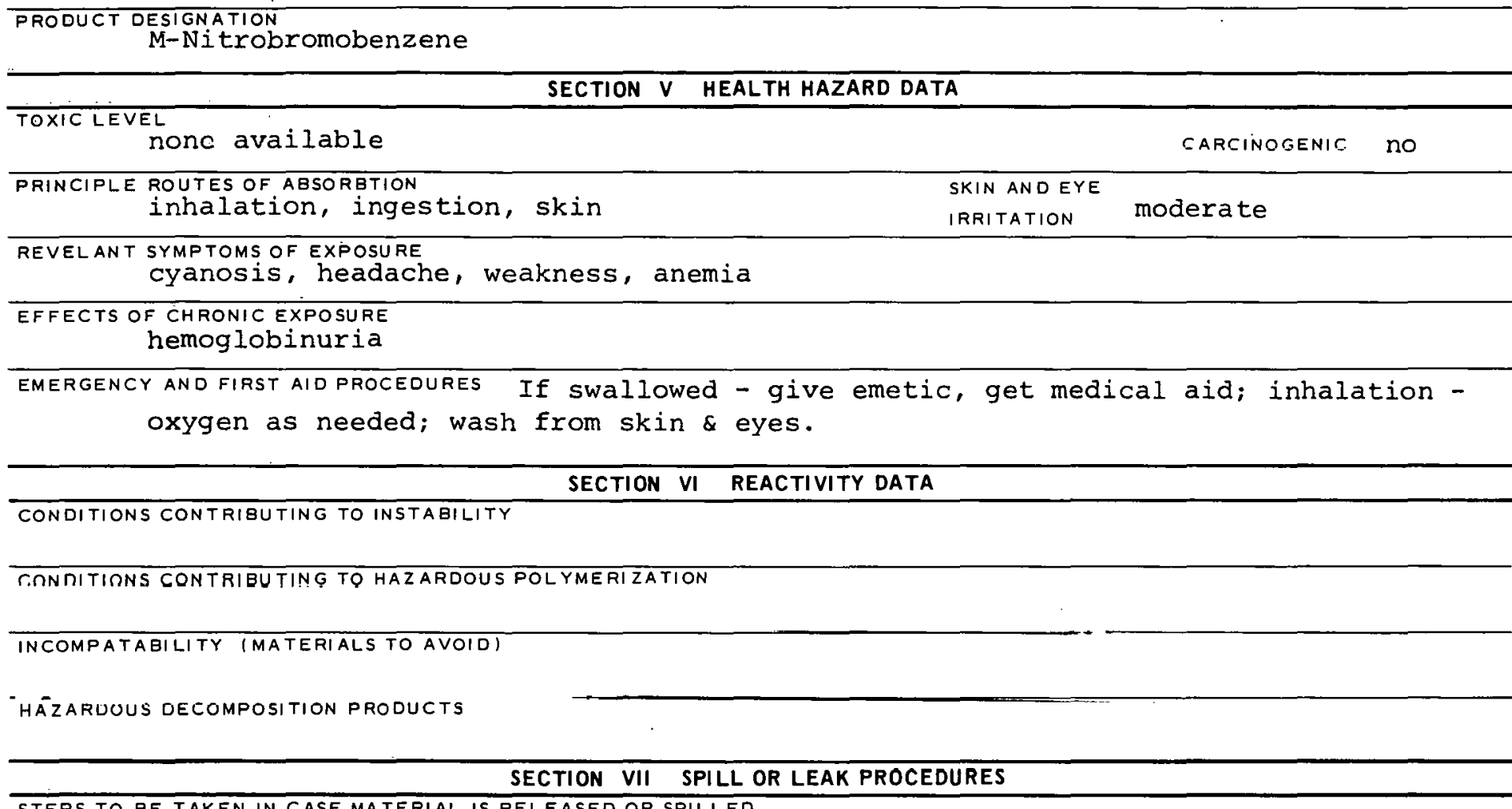

STEPS TO BE TAKEN IN CASE MATERIAL IS RELEASED OR SPILLED

WASTE DISPOSAL METHOD

SECTION VIII SPECIAL PROTECTION INFORMATION

\begin{tabular}{l|l}
\hline VENTILATION REQUIREMENTS LOCAL EXHAUST & $\begin{array}{c}\text { PROTECTIVE EQUIPMENT (SPECIFY TYPES) EYE } \\
\text { GOggleS }\end{array}$ \\
\hline MECHANICAL IGENERAL) & GLOVES \\
Rubber
\end{tabular}

OTHER PROTECTIVE EQUIPMENT

SECTION IX SPECIAL PRECAUTIONS

PRECAUTIONS TO BE TAKEN IN HANDLING AND STORAGE

Store away from oxidizing materials and sources of heat.

OTHER FREEAUTIONB

SIENATUAE

DATE

$316-1108 B$ 


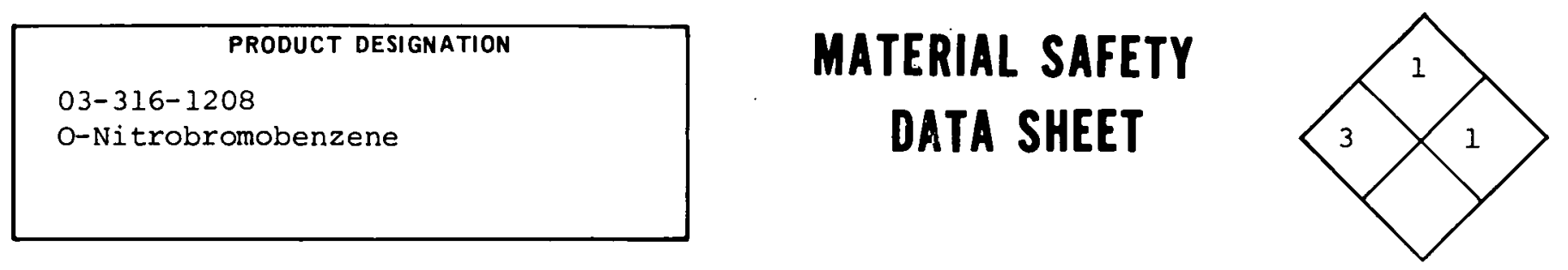

\section{SECTION I SOURCE AND NOMENCLATURE}

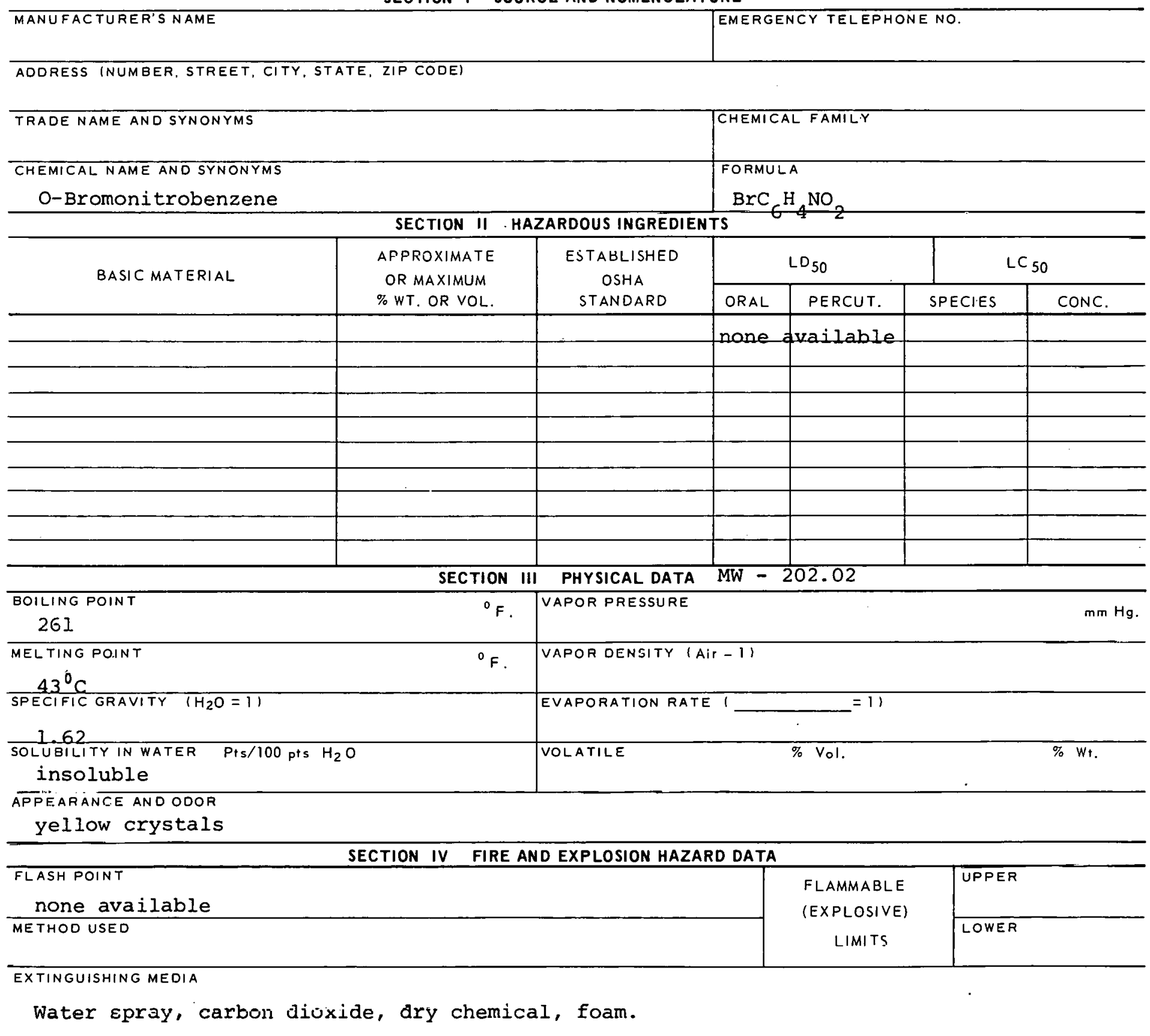

SPECIAL FIRE FIGHTING PROCEDURES

Wear self-contained breathing apparatus.

UNUSUAL FIRE AND EXPLOSION HAZARDS 


\section{TOXICLEVEL} none available

PRINCIPLE ROUTES OF ABSORBTION inhalation, ingestion, skin

REVELANT SYMPTOMS OF EXPOSURE cyanosis, headache, weakness, anemia.

EFFECTS OF CHRONIC EXPOSURE hemoglobinuria

EMERGENCY AND FIRST AID PROCEDURES If swallowed - give emetic, get medical aid; inhalation oxygen if needed; wash from skin \& eyes.

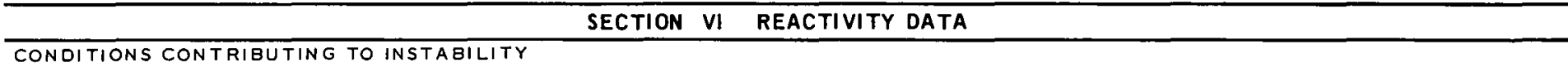

GONDIT!ONG CONTRIBUTING TO HAZARDOUS POLYMERI ZATION

INCOMPATABILITY (MATERIALS TO AVOID)

HAZARDOUS DECOMPOSITION PRODUCTS

SECTION VII SPILL OR LEAK PROCEDURES

STLFS TO BE TAKEN IN CASE MATERIAL IS RELEASED OR SPILLED

\section{WASTE DISPOSAL METHOD}

\section{SECTION VIII SPECIAL PROTECTION INFORMATION}

\begin{tabular}{l|l}
\hline VENTILATION REQUIREMENTS LOCAL EXHAUST & $\begin{array}{l}\text { PROTECTIVE EQUIPMENT (SPECIFY TYPES) EYE } \\
\text { GOggles }\end{array}$ \\
\hline MECHANICAL IGENERALI & $\begin{array}{l}\text { GLOVES } \\
\text { Kubber }\end{array}$ \\
\hline SPECIAL & $\begin{array}{l}\text { EESPIRATDR } \\
\text { Self-contained }\end{array}$ \\
\hline OTHER PROTECTIVE EQUIPMENT
\end{tabular}

OTHER PROTECTIVE EQUIPMENT

SECTION IX SPECIAL PRECAUTIONS

PRECAUTIONS TO BE TAKEN IN HANDLING AND STORAGE

Store away from oxidizing materials and sources of heat.

OTHER PRECAUTIONS

SIGNATURE

D̈ATE 


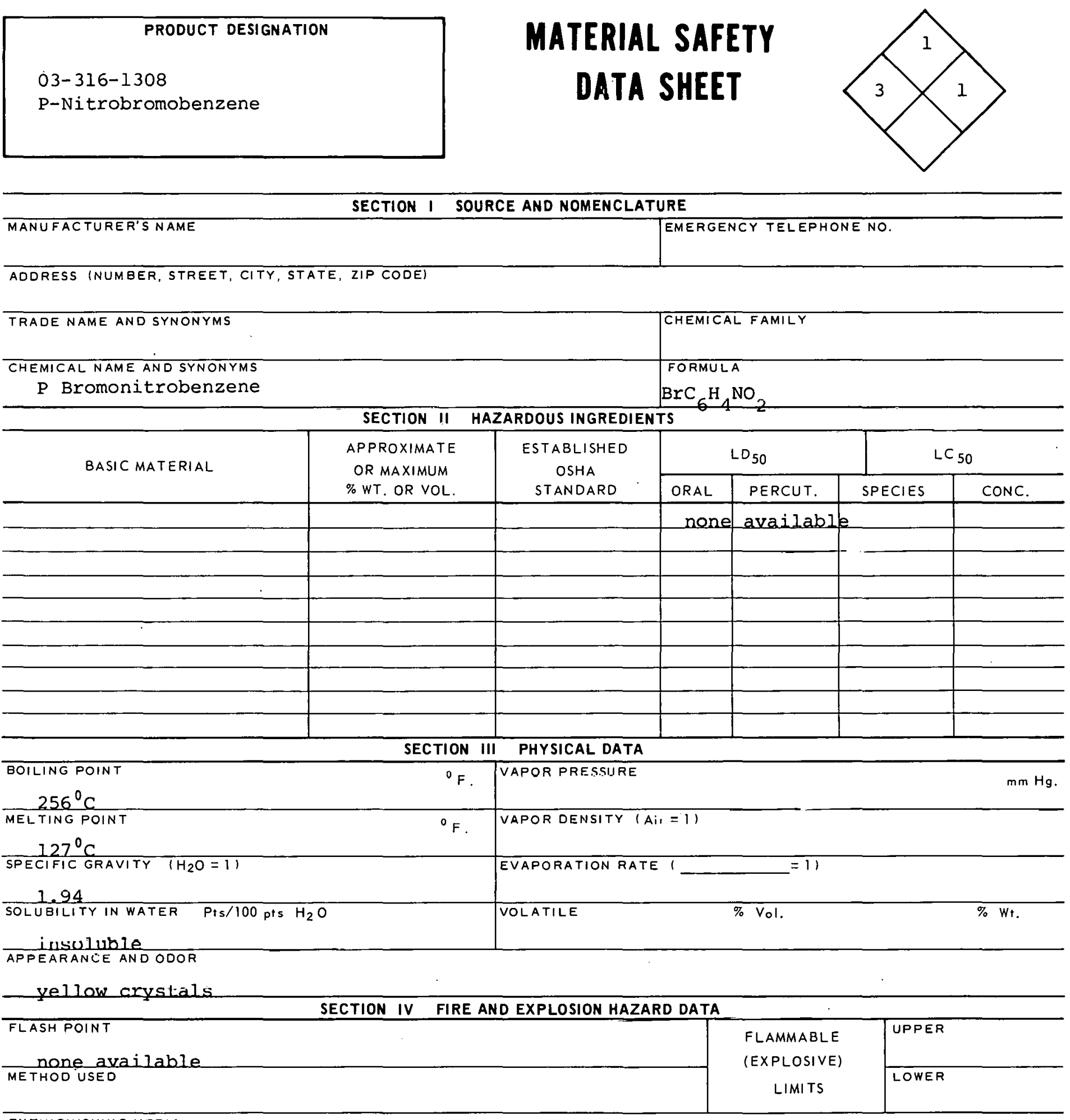

EXTINGUISHING MEDIA

water spray, carbon dioxide, dry chemical, foam.

SPECIAL FIRE FIGHTING PROCEDURES

Wear self-contained breathing apparatus.

UNUSUAL FIRE AND EXPLOSION HAZARDS 


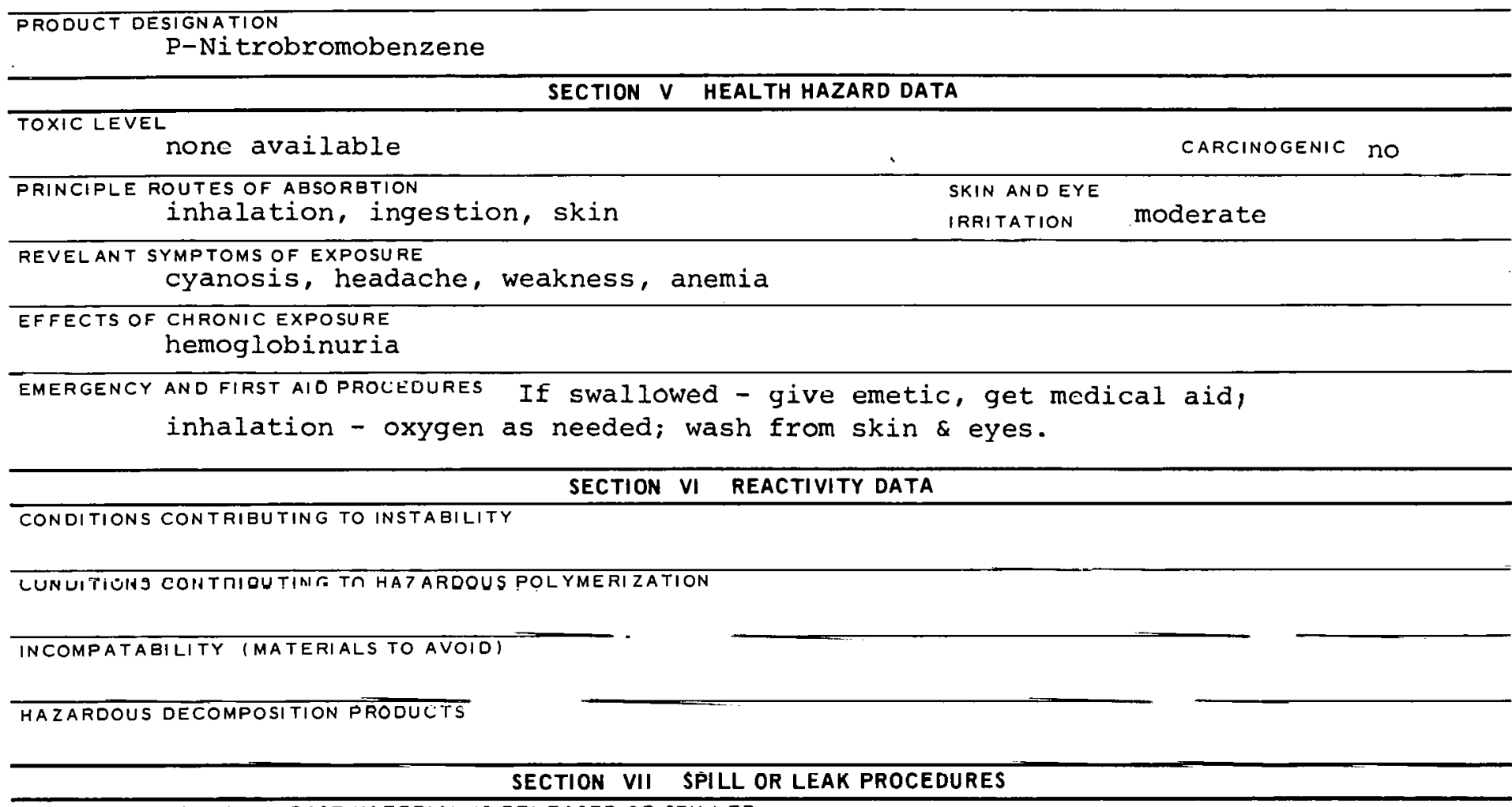

STEPS TO BE TAKEN IN CASE MATERIAL IS RELEASED OR SPILLED

WASTE DISPOSAL METHOD

SECTION VIII SPECIAL PROTECTION INFORMATION

\begin{tabular}{l|l}
\hline VENTILATION REQUIREMENTS LOCAL EXHAUST & $\begin{array}{l}\text { PROTECTIVE EQUIPMENT (SPECIFY TYPES) EYE } \\
\text { GOgGles }\end{array}$ \\
\hline MECHANICAL IGENERAL) & $\begin{array}{l}\text { GLOVES } \\
\text { RUbber }\end{array}$ \\
\hline SHECIAL & $\begin{array}{l}\text { RESPIRATOR } \\
\text { Self-contained }\end{array}$ \\
\hline
\end{tabular}

OTHER PROTECTIVE EQUIPMENT

SECTION IX SPECIAL PRECAUTIONS

PRECAUTIONS TO BE TAKEN IN HANDLING AND STORAGE

store away from oxidizing materials and sources of heat.

OTHER PRECAUTIONS

SIGNATURE

DATE

$316-1308 B$ 


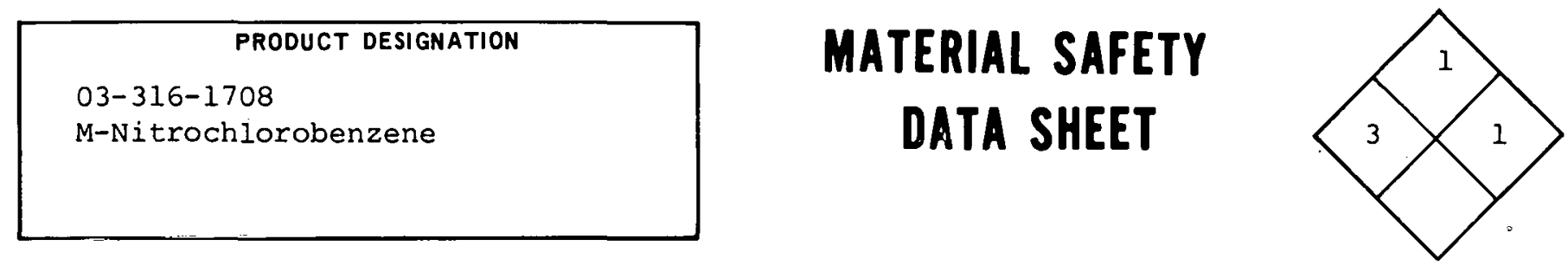

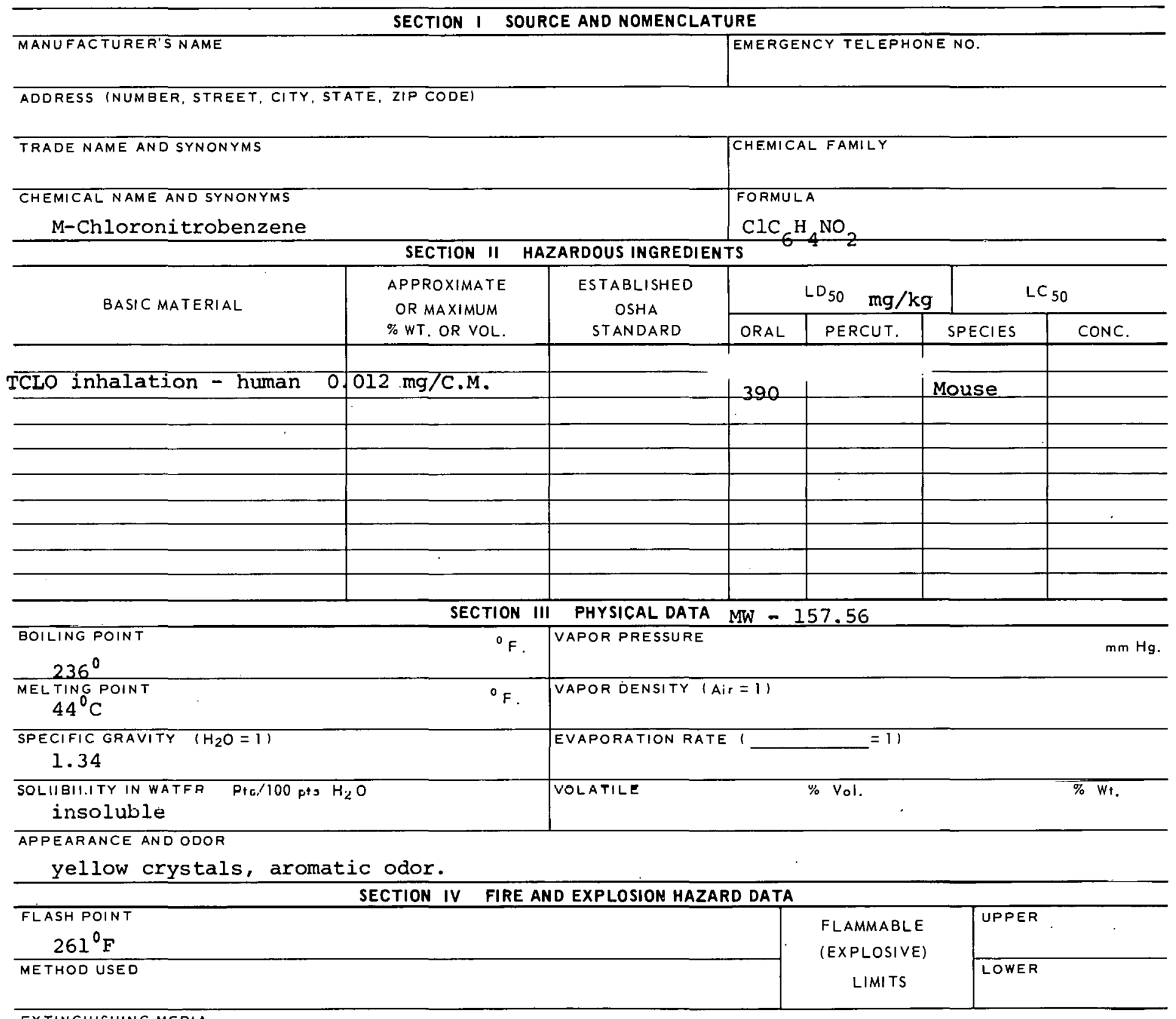

EXTINGUISHING MEDIA

water spray, carbon dioxide, dry chemical, foam

SPECIAL FIRE FIGHTING PROCEDURES

wear self-contained breathing apparatus.

UNUSUAL FIRE AND EXPLOSION HAZARDS 


\section{TOXIC LEVEL}

none available

PRINCIPLE ROUTES OF ABSORBTION

inhalation, ingestion, skin

REVELANT SYMPTOMS OF EXPOSURE

cyanosis, headache, weakness, anemia.

EFFECTS OF CHRONIC EXPOSURE

Hemoglobinuria

EMERGENCY AND FIRST AID PROCEDURES If swallowed - give emetic, get medical aid; inhalation oxygen if needed; wash from skin \& eyes.

\section{SECTION VI REACTIVITY DATA}

CONDITIONS CONTRIBUTING TO INSTABILITY

GONMITIONS CONTRIBU TING TO HAZARDOUS POLYMERIZATION

INCOMPATABILITY (MAIERIALS IUÁVUIO)

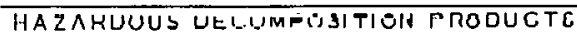

SECTION VII SPILL OR LEAK PROCEDURES

STEPS TO BE TAKFN IN CASE MATERIAL IS RELEASED OR SPILLED

SECTION VIII SPECIAL PROTECTION INFORMATION

\begin{tabular}{l|l}
\hline VENTILATION REQUIREMENTS LOCAL EXHAUST & $\begin{array}{l}\text { PROTECTIVE EQUIPMENT (SPECIFY TYPESIEYE } \\
\text { GOQqleS }\end{array}$ \\
\hline MECHANICAL (GENERAL) & $\begin{array}{l}\text { GLOVES } \\
\text { RUbber }\end{array}$ \\
\hline CMEGIAL & RESPIRATOR \\
& SeIf-COntained \\
\hline
\end{tabular}

OTHFR PROTECTIVE EQUIPMENT

\section{SECTION IX SPECIAL PRECAUTIONS}

PRECAUTIONS TO BE TAKFN IN HANDLING AND STORAGE

Store away from oxidizing materials and sources of heat. OTHER PRECAUTIONS

\begin{tabular}{l|l|l}
\hline SIGNATIJRE & DATE \\
\hline
\end{tabular}

$316-1708 B$ 
CAS : 000088733

CZ08750

\section{PRODUCT DESIGNATION}

03-316-1808

o-nitrochlorobenzene
MATERIAL SAFETY

DATA SHEET

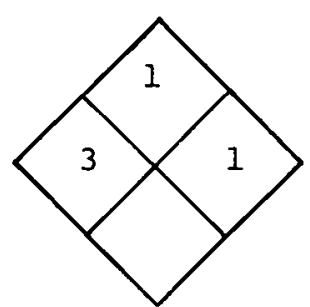

SECTION I SOURCE AND NOMENCLATURE

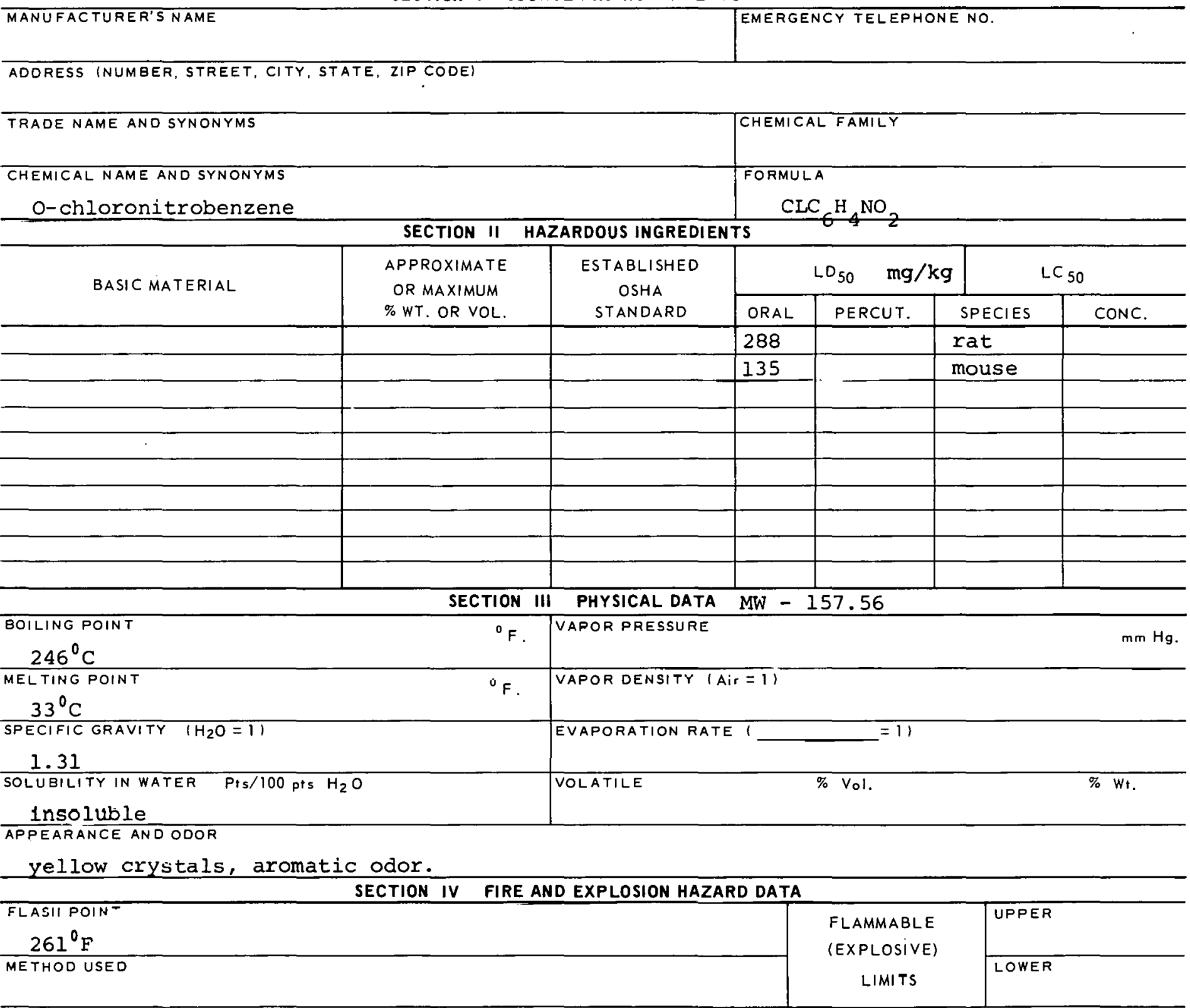

EXTINGUISHING MEDIA

water spray, carbon dioxide, dry chemical, foam.

SPECIAL FIRE FIGHTING PROCEDURES

Wear self-contained breathing apparatus.

UNUSUAL FIRE AND EXPLOSION HAZARDS 


\begin{tabular}{ccc}
\hline & SECTION $V$ HEALTH HAZARD DATA & \\
\hline $\begin{array}{c}\text { TOXIC LEVEL } \\
\text { none available }\end{array}$ & CARCINOGENIC nO \\
\hline $\begin{array}{c}\text { PRINCIPLE ROUTES OF ABSORBTION } \\
\text { inhalation, ingestion, skin }\end{array}$ & SKIN ANDEYE moderate \\
\hline
\end{tabular}

REVELANT SYMPTOMS OF EXPOSURE

Cyanosis, headache, weakness, anemia

EFFECTS OF CHRONIC EXPOSURE

hemoglobinuria

EMERGENCY AND FIRST AID PROCEDURES If swallowed - give emetic, get medical aid; inhalation oxygen if needed; wash from skin \& eyes.

\section{SECTION VI REACTIVITY DATA}

CONDITIONS CONTRIBUTING TO INSTABILITY

IONMITIONS CONTRIEUTING TO HAZARIOUS POLYMERI ZATION

INCOMPATABILITY (MATERIALS TO AVOIDI

HAZARDOUS DECOMPOSTTION FROOLULIS

SECTION VII SPILL OR LEAK PROCEDURES

STEPS TO BE TAKEN IN CASE MATERIAL IS RELEASED OR SPILLED

WASTE DISPOSAL METHOD

SECTION VIII SPECIAL PROTECTION INFORMATION

\begin{tabular}{l|l}
\hline VENTILATION REQUIREMENTS LOCAL EXHAUST & PROTECTIVE EOUIPMENT (SPECIFY TYPES) EYE \\
\hline MECHANICAL IGENERAL) & GLOVES \\
& rubber \\
\hline SPFCIAL. & RESPIRATOR \\
& self-contained \\
\hline
\end{tabular}

OTHER PROTECTIVE EQUIFMENT

SECTION IX SPECIAL PRECAUTIONS

PRECAUTIONS TO BE TAKEN IN HANDLING AND STORAGE

Store away from oxidizing materials and sources of heat.

OTHER PRECAUTIONS

SIENATUKL

DATE

$316-1808 \mathrm{~B}$ 
CAS : 000100005

CZ10500

PRODUCT DESIGNATION

03-316-1908

$\mathrm{P}-\mathrm{Nitrochlorobenzene}$

MATERIAL SAFETY

DATA SHEET

SECTION I SOURCE AND NOMENCLATURE

MANUFACTURER'S NAME

IEMERGENCY TELEPHONENO.

ADDRESS (NUMBER, STREET, CITY, STATE, ZIP CODE)

TRAOE NAME AND SYNONYMS

CHEMICAL FAMILY

CHEMICAL NAME AND SYNONYMS

$P$ Chloronitrobenzene

FORMULA

$\mathrm{ClC}_{6} \mathrm{H}_{4} \mathrm{NO}_{2}$

SECTION II HAZARDOUS INGREDIENTS

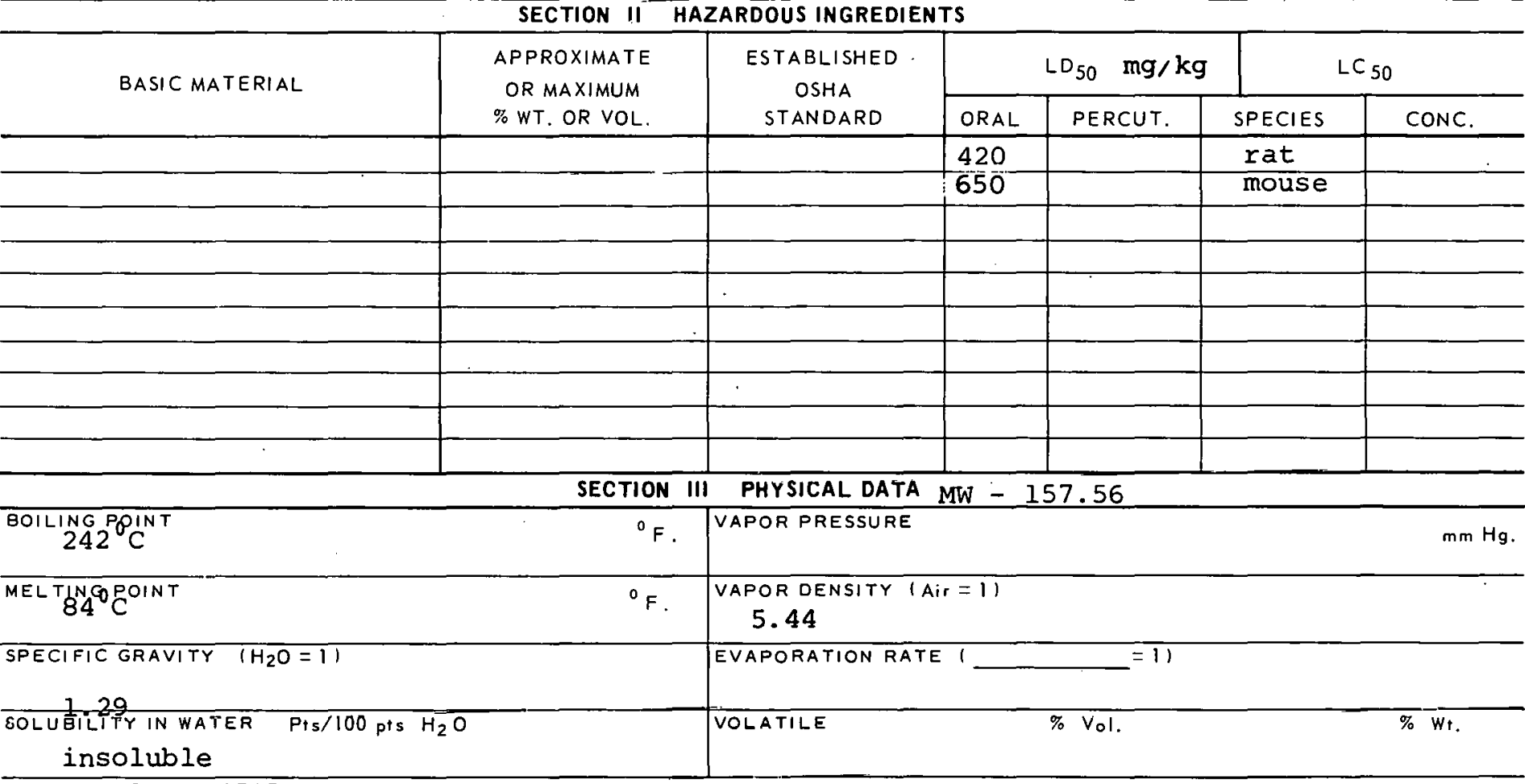

APPEARANCE AND ODOR

yellow crystals, aromatic odor.

SECTION IV FIRE AND EXPLOSION HAZARD DATA

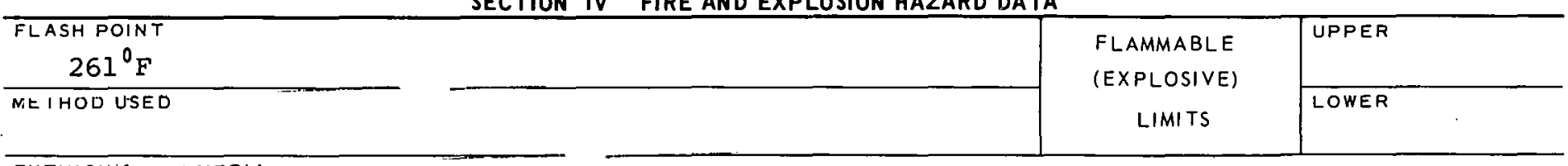

EXTINGLISHING MEOIA

water spray, carbon dioxide, dry chemical, foam

SPECIAL FIRE FIGHTING PROCEDURES

wear self-contained breathing apparatus.

UNUSIIAL FIRE AND EXRLOGIOIN HAZARUS 


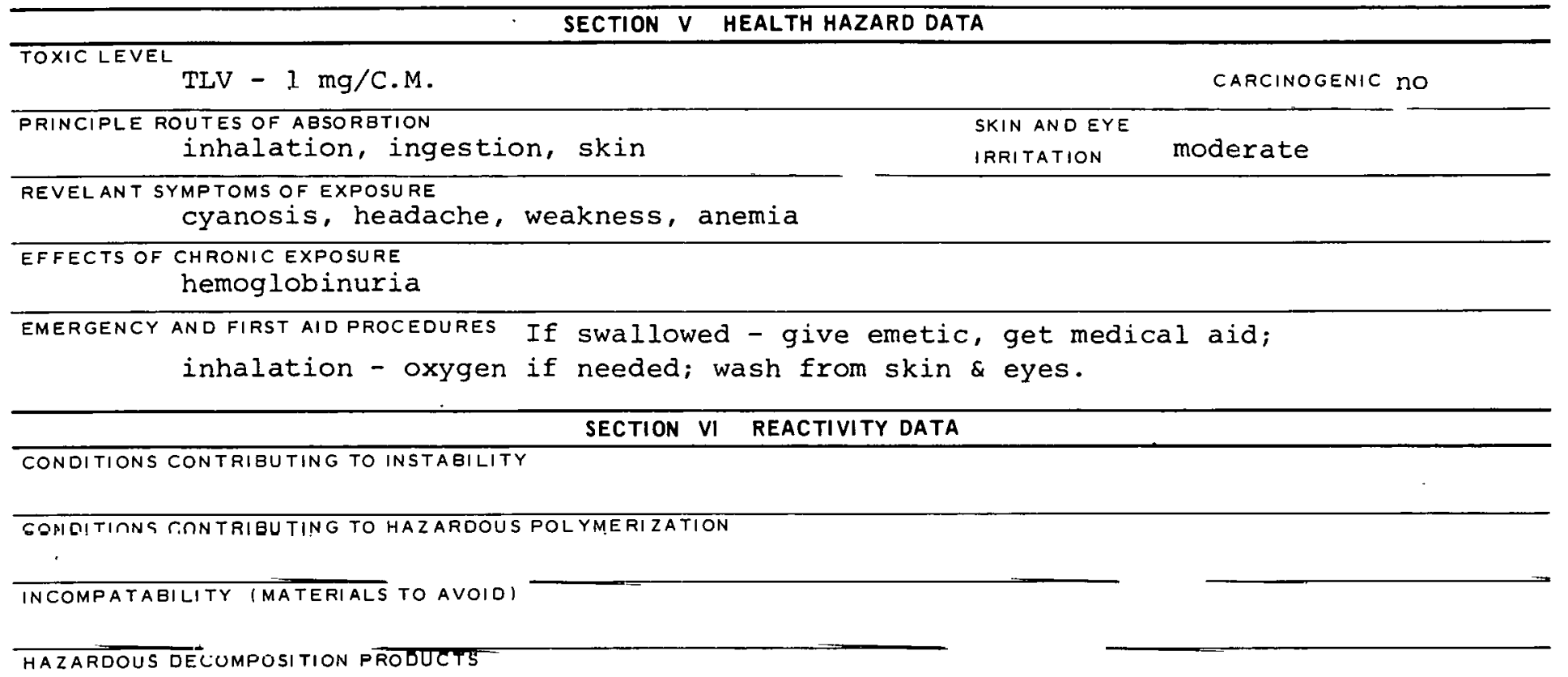

SECTION VII SPILL OR LEAK PROCEDURES

STEPS TO BE TAKEN IN CASE MATERIAL IS RELEASED OR SPILLED

WASTE DISPOSAL METHOD

SECTION VIII SPECIAL PROTECTION INFORMATION

\begin{tabular}{|c|c|}
\hline VENTILATION REQUIREMEN'TS LOCAL EXHAUST & $\begin{array}{l}\text { PROTECTIVE EQUIPMENT (SPECIFY TYPES) EYE } \\
\text { gOggleS }\end{array}$ \\
\hline MECHANICAL (GENERAL) & $\begin{array}{l}\text { rI RVES } \\
\text { rubber }\end{array}$ \\
\hline$S P E C|\Delta|$ & $\begin{array}{l}\text { RESPIRATOR } \\
\text { self-contained }\end{array}$ \\
\hline
\end{tabular}

OTHEH PROTFCTIVE EOUIFMENT

SECTION IX SPECIAL PRECAUTIONS

PRECAUTIONS TO BE TAKEN IN HANDLING AND STORAGE

Store away from oxidizing materials and sources of hear.

OTIILR RRECAUTIOMS

SIGNATURE 


\begin{tabular}{|l|}
\hline PRODUCT DESIGNATION \\
03-316-2608 \\
2-Nitro- P-Cymene
\end{tabular}

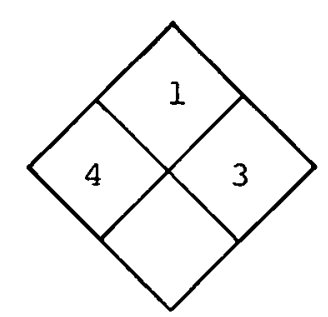

SECTION I SOURCE AND NOMENCLATURE

MANUFACTURER'S NAME
ADDRESS INUMBER, STREET, CITY,
TRADE NAME ANO SYNONYMS
CHEMICAL NAME AND SYNONYMS
2 NitYO-P-CYMENE

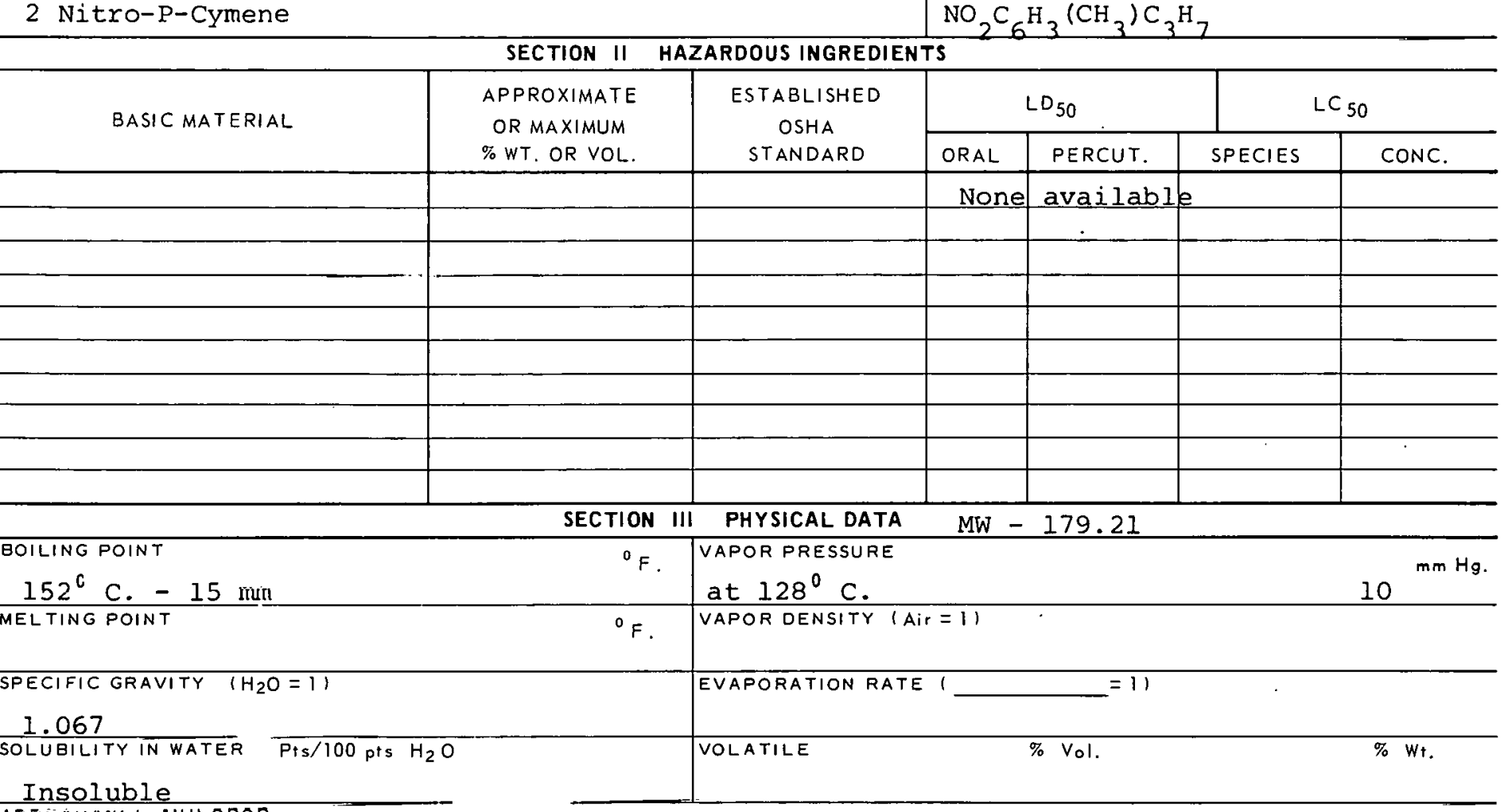

APFEARANLE ANU ODOR

Oil

\begin{tabular}{|c|c|c|c|c|}
\hline & SECTION IV & FIRE AND EXPLOSION HAZARD DATA & & \\
\hline $\begin{array}{l}\text { FLASH POINT } \\
\text { None available }\end{array}$ & & & \multirow{2}{*}{$\begin{array}{c}\text { FLAMMABLE } \\
\text { (EXPLOSIVE) } \\
\text { LIMITS }\end{array}$} & UPPER \\
\hline METHOD USED & & & & LOWER \\
\hline
\end{tabular}

\section{EXTINGUISHING MEDIA}

Foam, carbon dioxide, dry chemical.

SPECIAL FIRE FIGHTING PROCEDURES

UNUSUAL FIRE AND EXPLOSION HAZARDS 
TOXIC LEVEL

None available

PRINCIPLE ROUTES OF ABSORBTION

Inhalation, ingestion, skin
CARCINOGENIC No SKIN AND EYE

IRRITATION Slight

REVELANT SYMPTOMS OF EXPOSURE

Difficulty in breathing, cyanosis, vomiting, headache.

EFFECTS OF CHRONIC EXPOSURE

$--$

EMERGENCY AND FIRST AID PROCEDURES rest; wash skin and eyes.

If swallowed - yive emetic, get madical aid; inhalation SECTION VI REACTIVITY DATA

\section{CONDITIONS CONTRIBUTING TO INSTABILITY \\ CONDITIONS CONTRIBU TING TO HAZARDOUS POLYMERIZATION \\ INCOMPATABILITY (MATERIALS TO AVOID) \\ May explode when heated with sulfuric acid. HAZARDOUS DECOMPOSITION PRODUCTS}

SECTION VII SPILL OR LEAK PROCEDURES

STEPS TO BE TAKEN IN CASE MATERIAL IS RELEASED OR SPILLED

WASTE DISPOSAL METHOD

SECTION VIII SPECIAL PROTECTION INFORMATION

\begin{tabular}{l|l}
\hline VENTILATION REQUIREMENTS LOCAL EXHAÚST & $\begin{array}{c}\text { PHOUTECTIVE EQUIPMENT (SPECIFY TYPES) EYE } \\
\text { GUYYlES }\end{array}$ \\
\hline MECHANICAL IGENERAL) & $\begin{array}{c}\text { GLOVES } \\
\text { Rubber }\end{array}$ \\
\hline SPECIAL & $\begin{array}{c}\text { RE3PIRATOR } \\
\text { Self contained }\end{array}$ \\
\hline
\end{tabular}

OTHER PROTECTIVE EQUIPMENT

\section{SECTION IX SPECIAL PRECAUTIONS}

PRECAUTIONS TO BE TAKEN IN HANDLING AND STORAGE

Outside or detached storage preferred; inside storage in standard flammable

Iiquids cabinet.

OTHER PRECAUTIONS

SIGNATURE UATE 


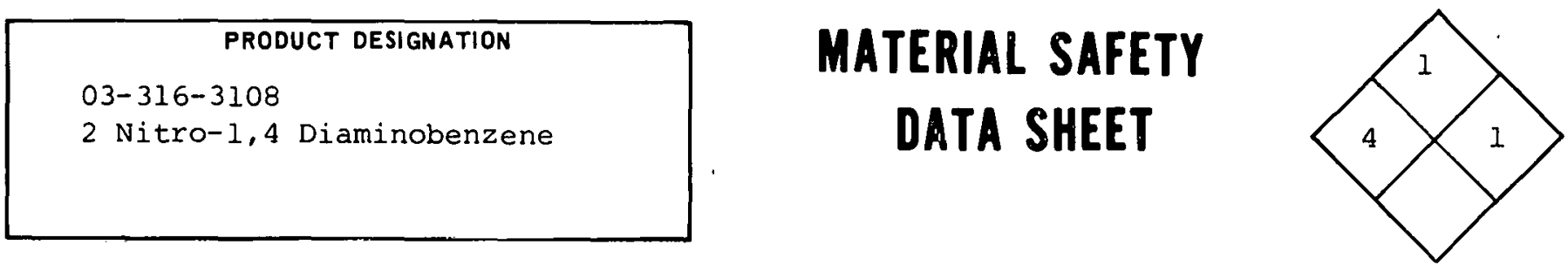

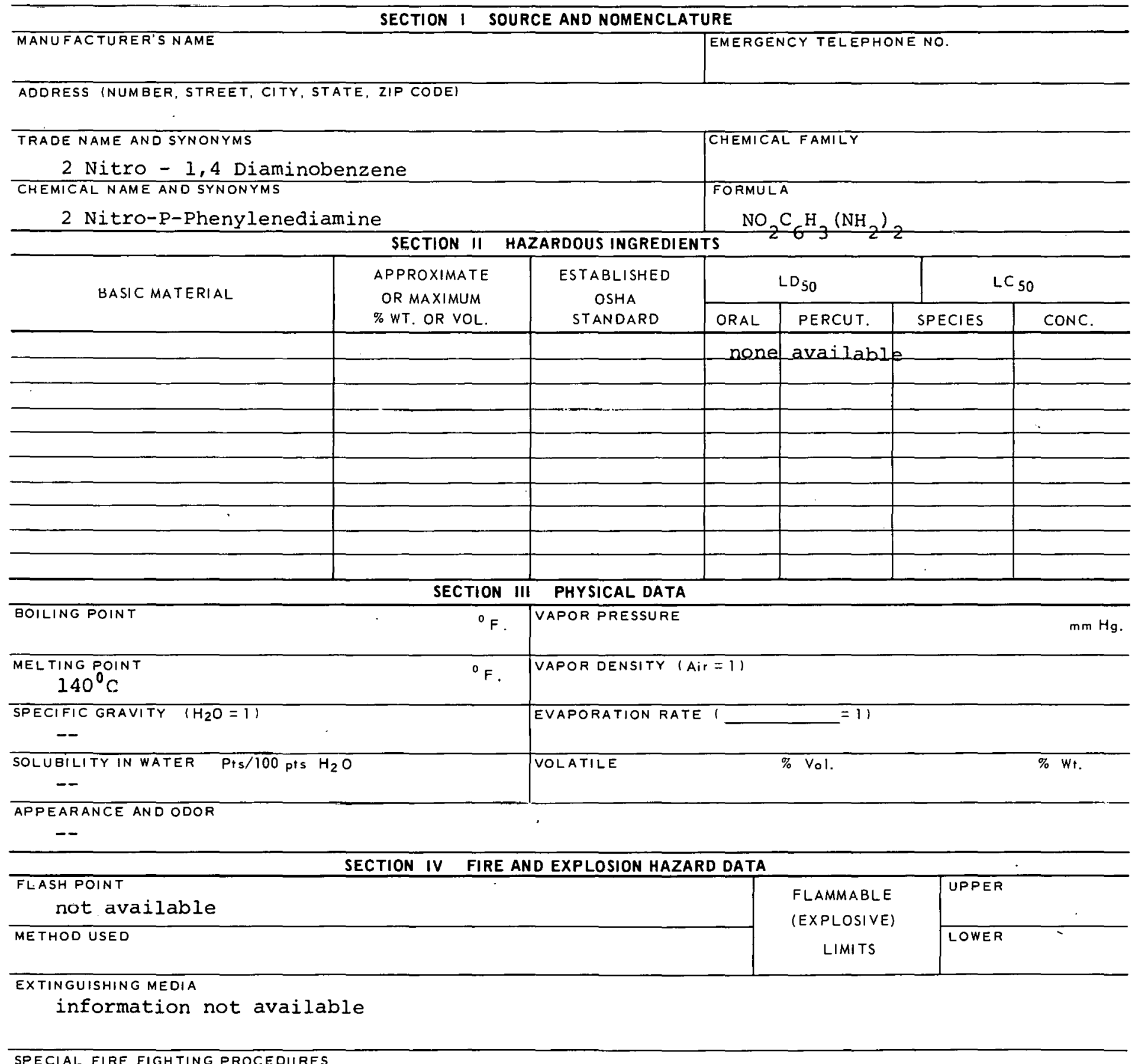

SPECIAL FIRE FIGHTING PROCEDURES

UNUSUAL FIRE AND EXPLOSION HAZARDS 


\section{SECTION $V$ HEALTH HAZARD DATA}

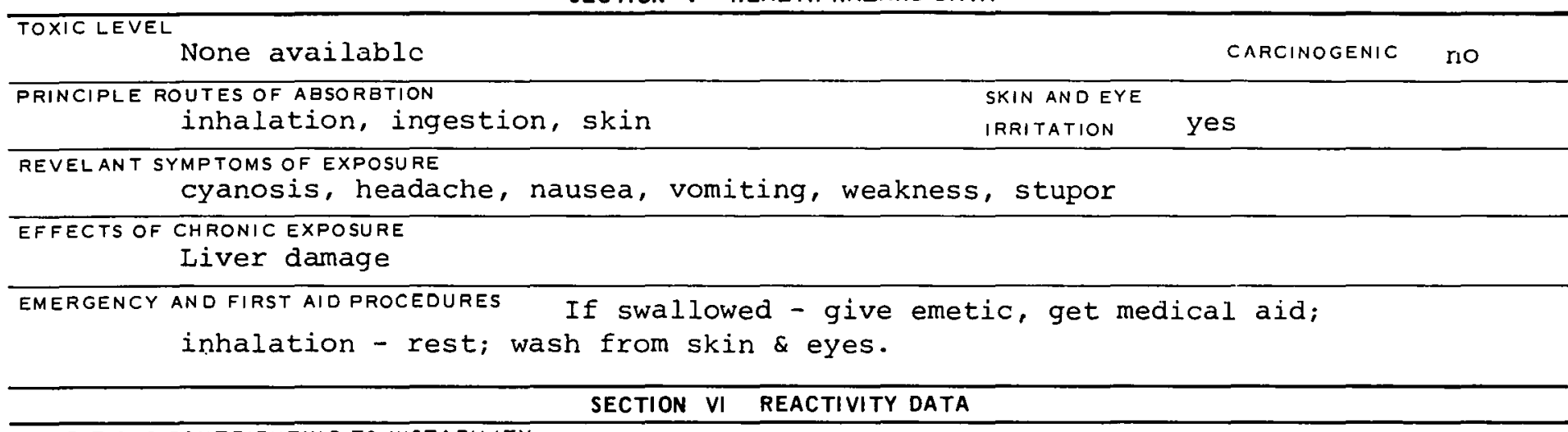

CONDITIONS CONTRIBUTING TO INSTABILITY

CONDITIONS CONTRIBUTING TO HAZAKUUUS POLYMERIZATION

INCOMPATABILITY (MATERIALS TO AVOIO)

$\overline{\text { HAZARDOUS DECOMPOSITION PRODUCTS }}$

SECTION VII SPILL OR LEAK PROCEDURES

STEPS TO BE TAKEN IN CASE MATERIAL IS RELEASED OH SPILLED

SECTION VIII SPECIAL PROTECTION INFORMATION

\begin{tabular}{l|l}
\hline VENTILATION REQUIREMENTS LOCAL EXHAUST & $\begin{array}{l}\text { PROTECTIVE EQUIPMENT (SPECIFY TYPES) EYE } \\
\text { gOggles }\end{array}$ \\
\hline MECHANICAL IGENERAL) & $\begin{array}{l}\text { GLOVES } \\
\text { rubber }\end{array}$ \\
\hline SPECIAL & RESPIRATOR \\
with proper filter
\end{tabular}

OTHER PROTECTIVE EQUIPMENT

\section{SECTION IX SPECIAL PRECAUTIONS}

PREGALTIONS TO BE TAKEN IN HANDLING AND STORAGE

information not available

OTHER PRECAUTIONS

SIGNATURE

OATE

$316-3108 \mathrm{~B}$ 


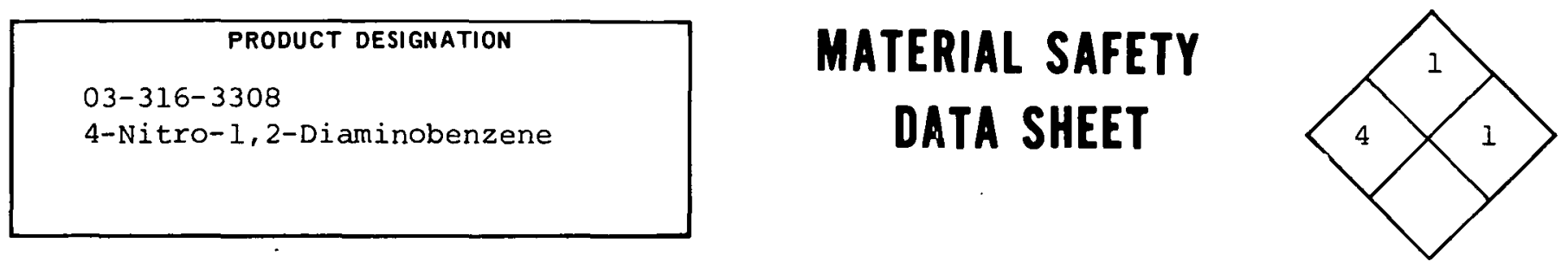

\section{SECTION I SOURCE AND NOMENCLATURE}

MANUFACTURER'S NAME

EMERGENCY TELEPHONE NO.

ADDRESS (NUMBER, STREET, CITY, STATE, ZIP CODE)

TRADE NAME AND SYNONYMS

4 Nitro-1,2-Diaminobenzene CHEMICAL NAME AND SYNONYMS

4 Nitro-P-Phenylenediamine

\begin{tabular}{l|l} 
& CHEMICAL FAMILY \\
& $\begin{array}{l}\text { FORMULA } \\
\mathrm{NO}_{2} \mathrm{C}_{6} \mathrm{H}_{3}\left(\mathrm{CH}_{3}\right)_{2}\end{array}$ \\
\hline SECTION II HAZARDOUS INGREDIENTS &
\end{tabular}

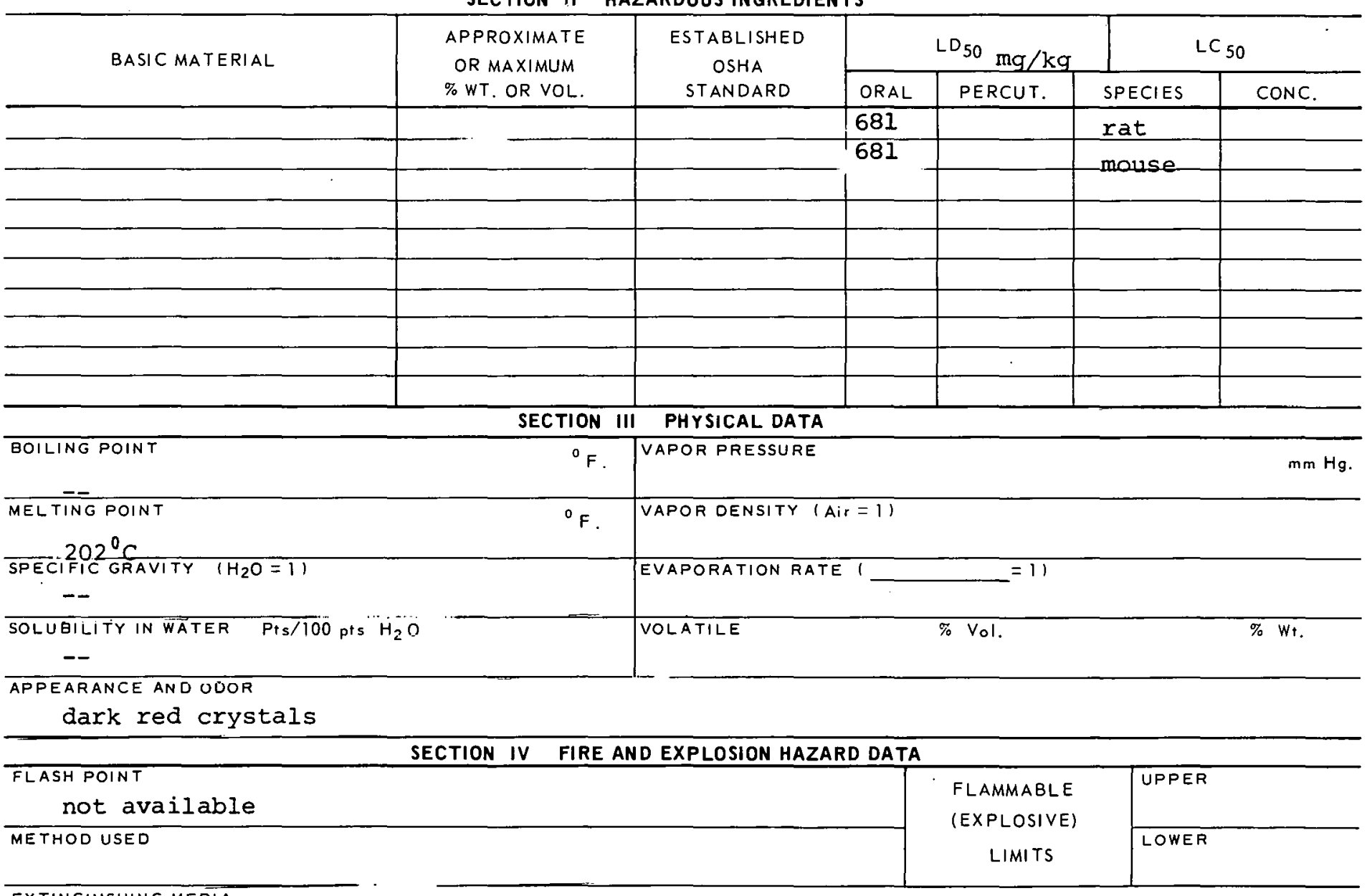

EXTINGUISHING MEDIA

information not available

SPECIAL FIHE FIGHTING PROCEDURES

UNUSUAL PIRE AND EXFLUSIUN HAZARDS 


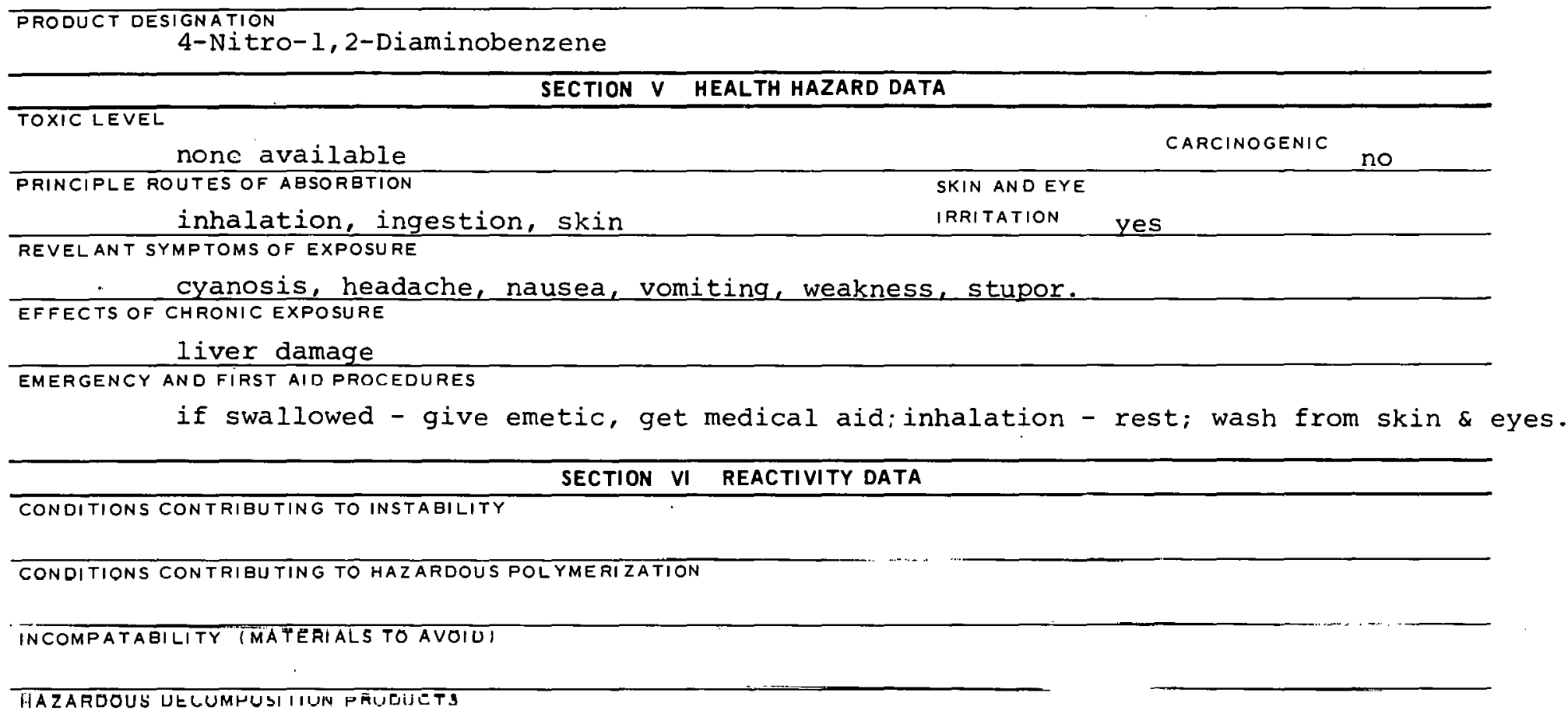

\begin{tabular}{l|l}
\hline VENTILATION REQUIREMENTS LOCAL EXHAUST & $\begin{array}{l}\text { PROTECTIVE EOUIPMENT (SPECIFY TYPES) EYE } \\
\text { GOggIES }\end{array}$ \\
\hline MECHANICAL IGENERAL) & $\begin{array}{c}\text { GLOVES } \\
\text { Rubber }\end{array}$ \\
\hline SPEGIAL & $\begin{array}{c}\text { RESTPIRATOR } \\
\text { With proper filter }\end{array}$ \\
\hline
\end{tabular}

\section{SECTION IX SPECIAL PRECAUTIONS}

PRECAUTIONS TO BE TAKEN IN HANDLING AND STORAGE

Information not available.

\section{OTHER PRECAIUTIONS}

SIGNATIIRF-

DATE

$316-3308 B$ 


PRODUCT DESIGNATION
03-316-3708
O-Nitrodiethylaniline

SECTION I SOURCE AND NOMENCLATURE

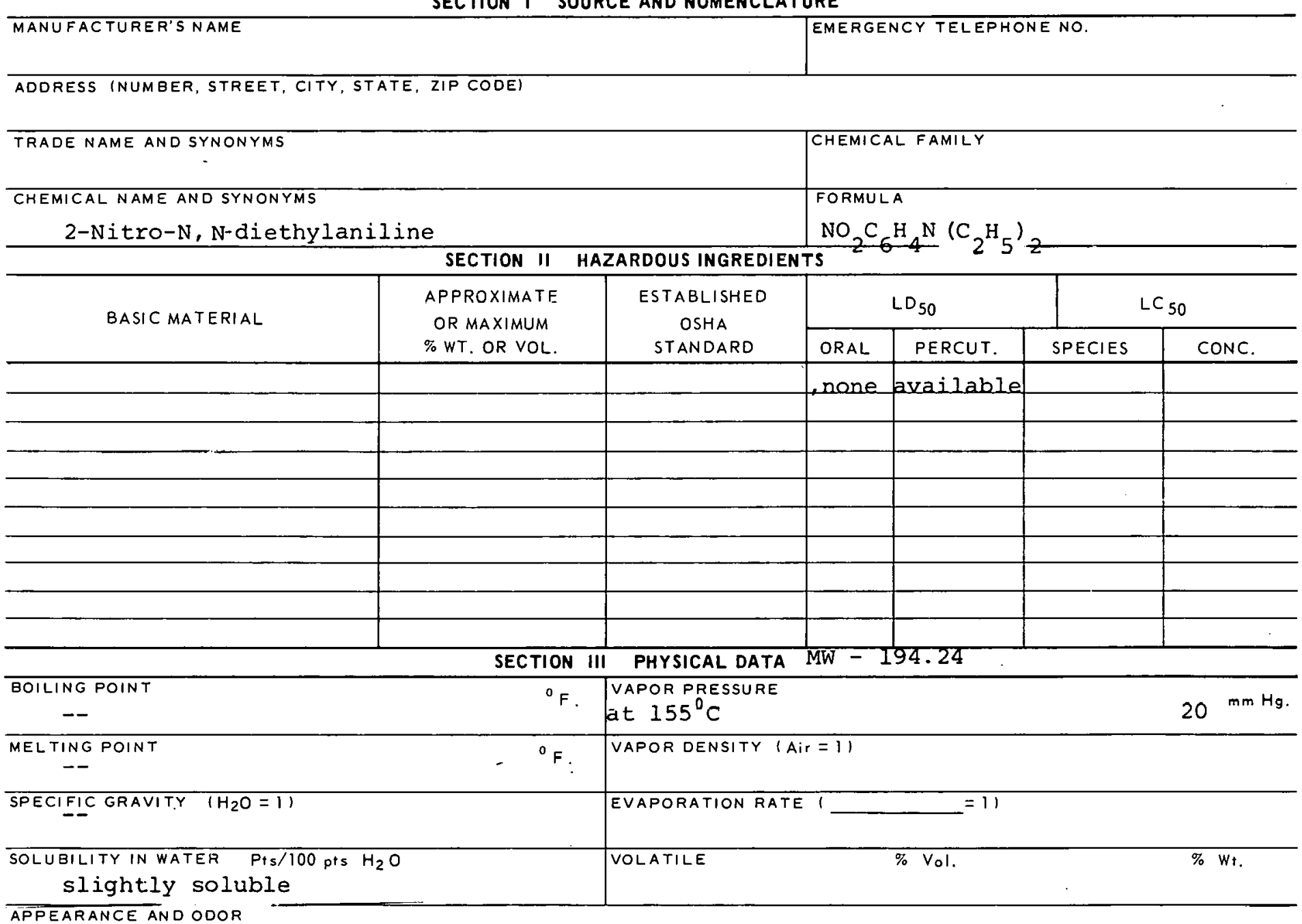

APPEARANCE AND ODOR

yellow liquid

\begin{tabular}{l|c|l}
\hline \multicolumn{2}{c}{ SECTION IV FIRE AND EXPLOSION HAZARD DATA } \\
\hline $\begin{array}{l}\text { FLASH POINT } \\
\text { nOne available }\end{array}$ & FLAMMABLE & UPPER \\
\hline METHOD USEO & LIMITS & LOWER \\
\hline
\end{tabular}

EXTINGUISHING MEDIA

information not available

SPECIAL FIRE FIGHTING PROCEDURES

UNUSUAL FIRE AND EXPLOSION HAZAROS 


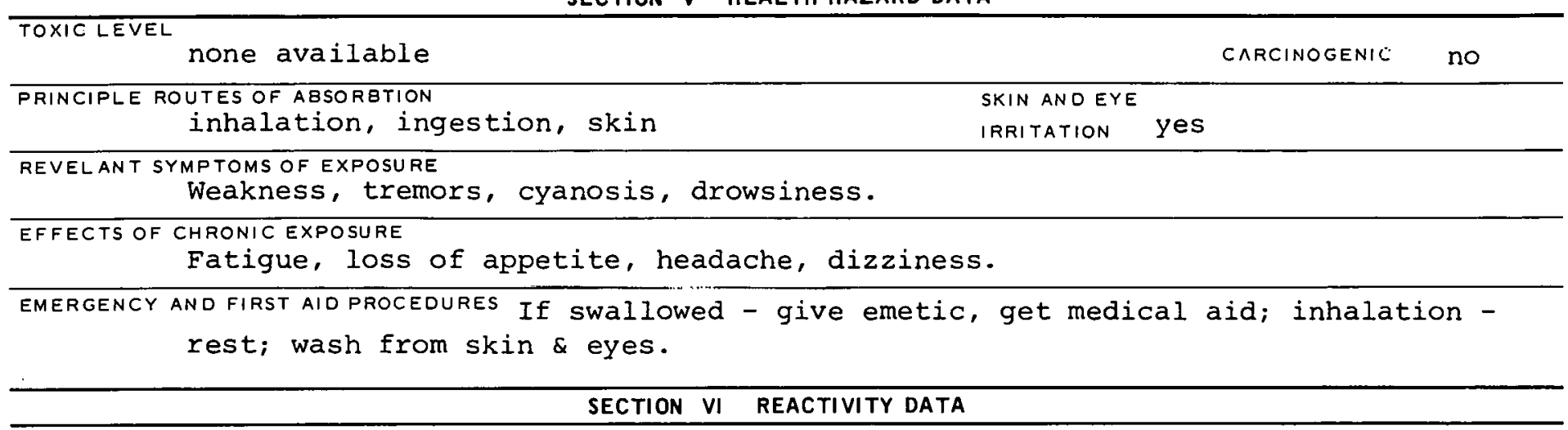

CONDITIONS CONTRIBUTING TO INSTABILITY

GONDITIOMS CONTRIRIITING TO HAZARDOUS POLYMERIZATION

INCOMPATABILITY (MATERIALS TO AVOID)

HAZARDOUS DECOMPOSITION PRODUCTS

SECTION VII SPILL OR LEAK PROCEDURES

STEPS TO BE TAKEN IN CASE MATERIAL IS RELEASED OR SPILLED

WASTC OISPOSAL METHOD

SECTION VIII SPECIAL PROTECTION INFORMATION

\begin{tabular}{|c|c|}
\hline VFNTILATION REQUIREMENTS LOCAL EXHAUST & $\begin{array}{l}\text { PROTECTIVE EQUIPMENT (SPECIFY TYPES) EYE } \\
\text { GUGgleS }\end{array}$ \\
\hline MECHANICAL (GENERAL) & $\begin{array}{l}\text { GLOVES } \\
\text { Rubber }\end{array}$ \\
\hline SPECIAL & $\begin{array}{l}\text { RESPIRATOR } \\
\text { With proper filter }\end{array}$ \\
\hline
\end{tabular}

OTHER PROTECTIVE EQUIPMENT

SECTION IX SPECIAL PRECAUTIONS

PRECAUTIONS TO BE TAKEN IN HANDLING AND STORAGE

\section{OTHER PRECAUTIONS}




\begin{tabular}{c} 
PRODUCT DESIGNATION \\
03-316-4108 \\
M-Nitrodimethylaniline \\
$r$ \\
\hline
\end{tabular}

\section{SECTION I SOURCE AND NOMENCLATURE}

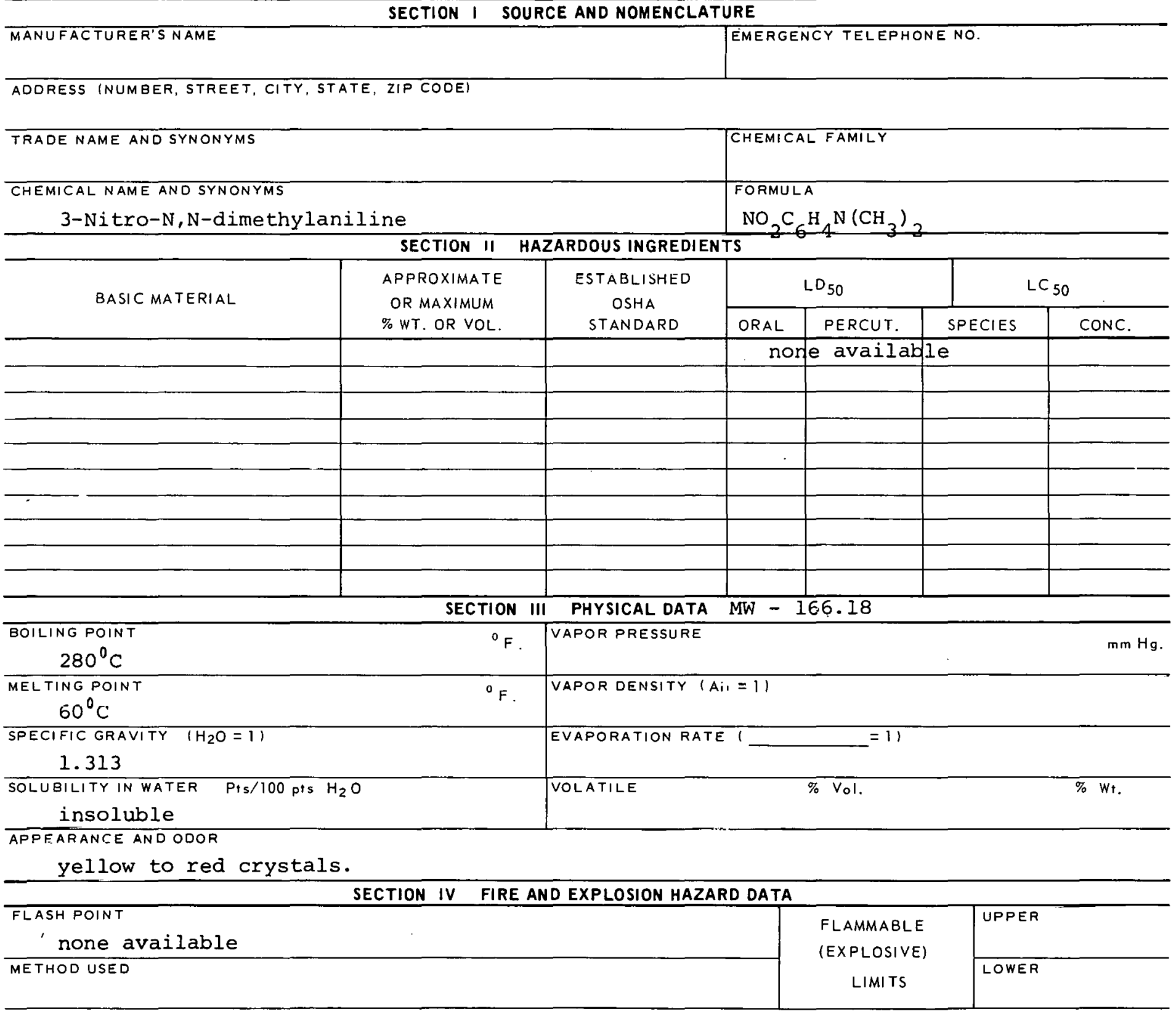

EXTINGUISHING MEDIA

Foam, carbon dioxide, dry chemical.

SPECIAL FIRE FIGHTING PROCEDURES

UNUSUAL FIRE ANDEXPLOSION HAZARDS 
$\mathrm{M}-\mathrm{Ni}$ trodime thylaniline

\section{SECTION $V$ HEALTH HAZARD DATA}

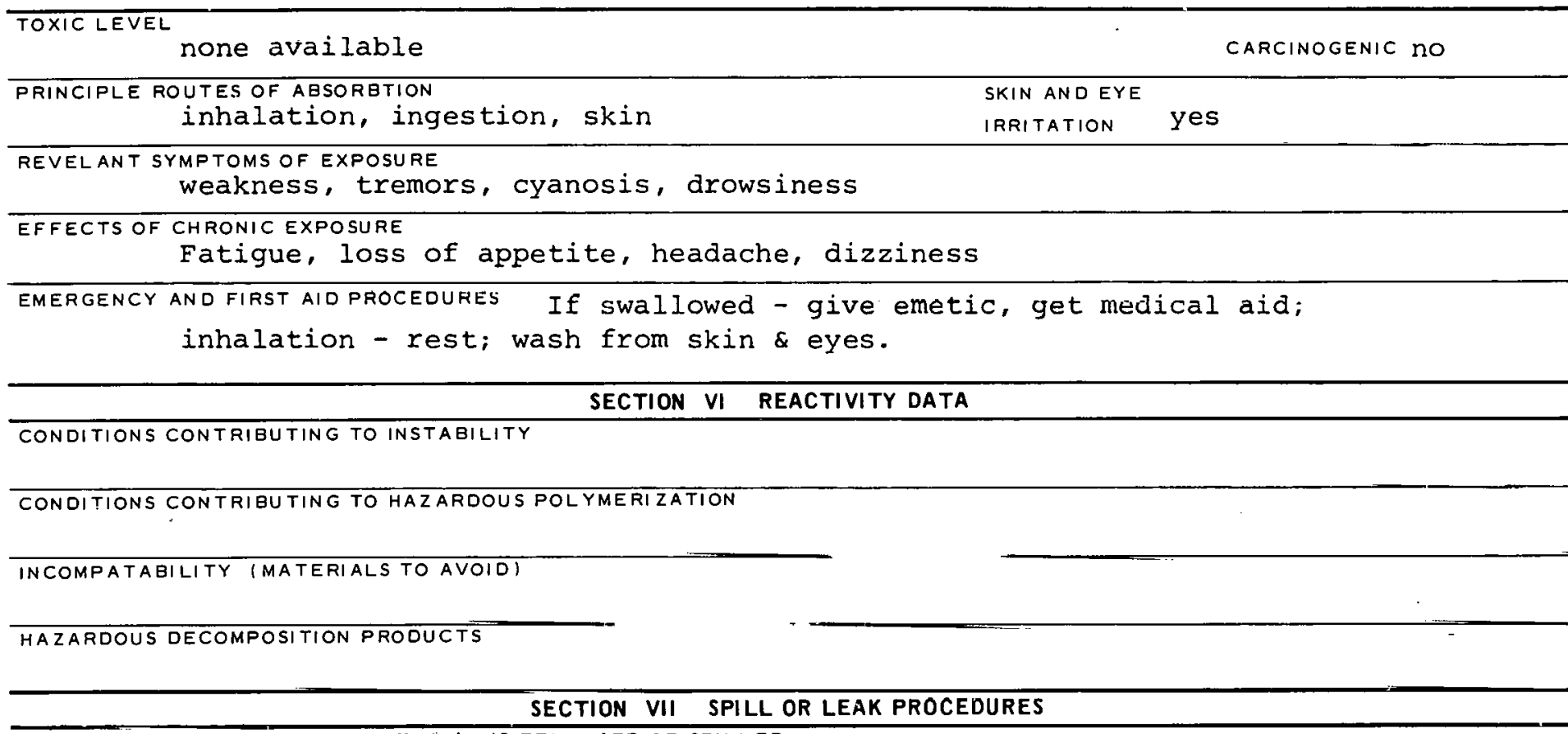

STEPS TO BE TAKEN IN CASE MATERIAL IS RELEASED OR SPILLED

WASTE DISPOSAL METHOD

SECTION VIII SPECIAL PROTECTION INFORMATION

\begin{tabular}{|c|c|}
\hline VENTILATION REQUIREMENTS LOCAL EXHAUST & $\begin{array}{l}\text { PROTECTIVE EQUIPMENT (SPECIFY TYPES) EYE } \\
\text { yUggleS }\end{array}$ \\
\hline MECHANICAL (GENERAL) & $\begin{array}{l}\text { GLOVES } \\
\text { rubber }\end{array}$ \\
\hline$\triangle \overline{P E C I A L}$ & $\begin{array}{l}\text { RCGCIMATQA } \\
\text { with proper filter }\end{array}$ \\
\hline
\end{tabular}

OTHER PROTECTIVE EQUIFMENT

SECTION IX SPECIAL PRECAUTIONS

PRFC.AIITIONS TO BE TAKEN IN HANDLING AND STORAGE

OTHER PRECAUTIONS

SIGNATURE

DATE

$316-41088$ 


\begin{tabular}{|c|}
\hline PRODUCT DESIGNATION \\
03-316-4608 \\
2-Nitro, 1,4-Dimethylbenzene \\
\hline
\end{tabular}

\section{MATERIAL SAFETY DATA SHEET}

\section{SECTION I SOURCE AND NOMENCLATURE}

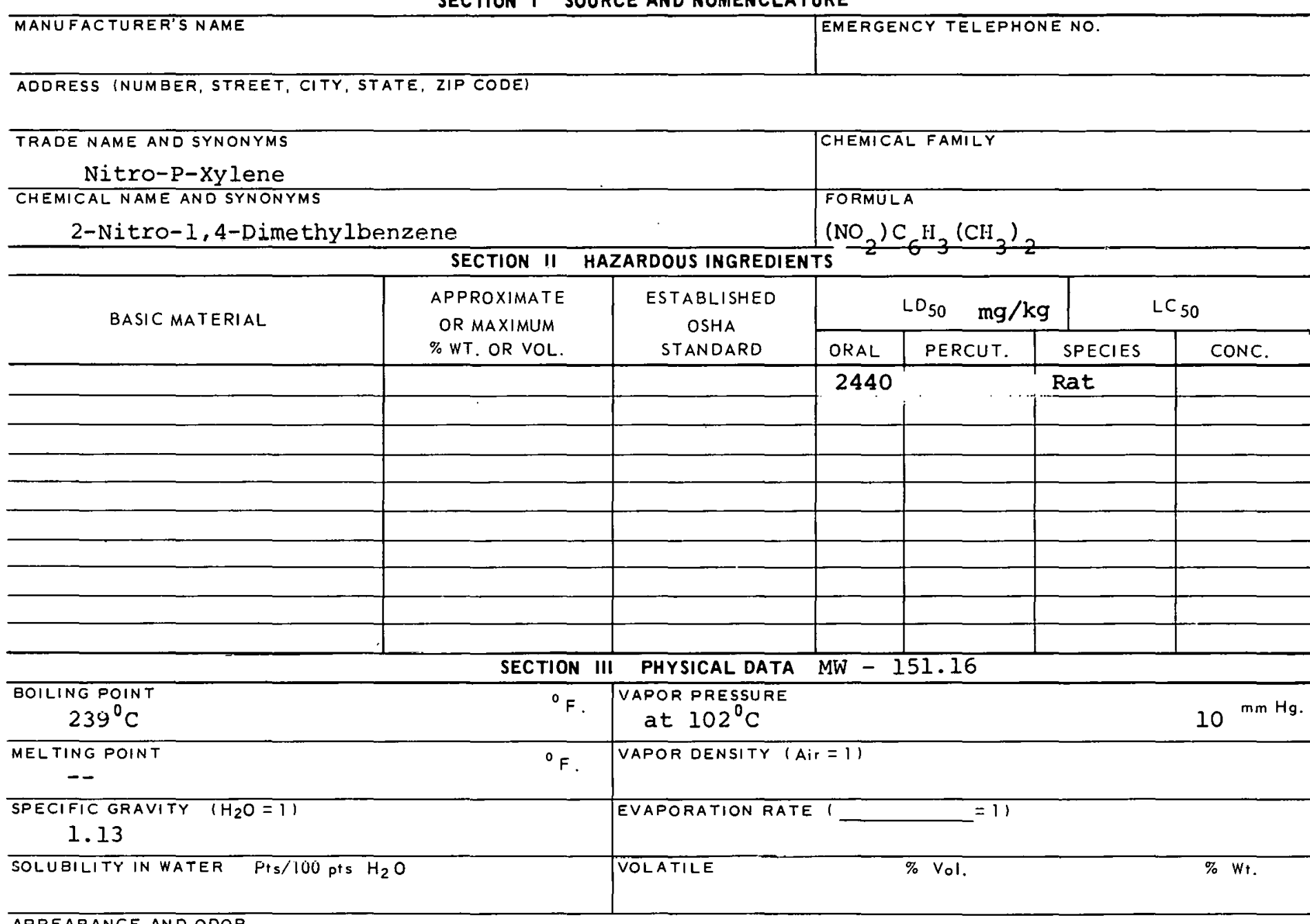

APPEARANCE AND ODOR

yellow Iiquid

\begin{tabular}{|c|c|c|c|c|}
\hline & SECTION IV & FIRE AND EXPLOSION HAZARD DATA. & & \\
\hline $\begin{array}{l}\text { FLASH POINT } \\
\text { none available }\end{array}$ & & & \multirow{2}{*}{$\begin{array}{c}\text { FLAMMABLE } \\
\text { (EXPLOSIVE) } \\
\text { LIMITS }\end{array}$} & UPPER \\
\hline METHOD USED & & & & LOWER \\
\hline
\end{tabular}

EXTINGUISHING MEDIA

Information - not available.

SPECIAL FIRE FIGHTING PROCEDURES

UNUSUAL FIRE AND EXPLOSION HAZARDS 
TOXIC LEVEL

none available

PRINCIPLE ROUTES OF ABSORBTION

inhalation, ingestion, skin

CARCINOGENIC

REVELANT SYMPTOMS OF EXPOSURE

SKIN AND EYE

IRRITATION

difficulty in breathing, cyanosis, vomiting, headache.

EFFECTS OF CHRONIC EXPOSURE

blood damage.

EMERGENCY AND FIRST AID PROCEDURES If swallowed - give emetic, get medical aid;

inhalation - rest; wash from skin \& eyes.

\section{SECTION VI REACTIVITY DATA}

CONDITIONS CONTRIBUTING TO INSTABILITY

CONDITIONS CONTRIBU TING TO HAZARDOUS POLYMERIZATION

INCOMPATABILITY (MATERIALS IU AVUIU)

May explode when heated with sulfuric acid HAZARUUUS UELUMHUSITIÜS ROBUCTO

SECTION VII SPILL OR LEAK PROCEDURES

STEPS TO BE TAKEN IN CASE MA TERIAL IS RELEASED OR SPILLED

WASTE DISPOSAL METHOD

SECTION VIII SPECIAL PROTECTION INFORMATION

\begin{tabular}{l|l}
\hline VENTILATION REQUIREMENTS LOCAL EXHAUST & $\begin{array}{l}\text { PROTECTIVE EQUIPMENT (SPECIFY TYPES) EYE } \\
\text { gRgG TPS }\end{array}$ \\
\hline MECHAINICAL (CLNLRAL) & GLOVES \\
rubber \\
\hline GRCGIAL & RESPIRATOR \\
& selt-Contained \\
\hline
\end{tabular}

OTHER PROTECTIVE EOUIPMENT

\section{SECTION IX SPECIAL PRECAUTIONS}

PRECAUTIONS TO BE TAKEN IN HANDLING AND STORAGE

Information not available.

OTHER PRECAUTIONS

SITSATIIRF

DATE

$316-4608 B$ 


\begin{tabular}{|c|}
\hline PRODUCT DESIGNATION \\
03-316-4808 \\
3-Nitro-1,2-Dimethylbenzene \\
\end{tabular}

\section{MATERIAL SAFETY \\ DATA SHEET}

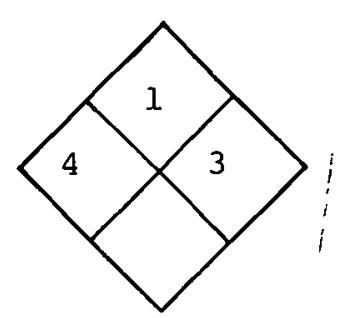

\section{SECTION I SOURCE AND NOMENCLATURE}

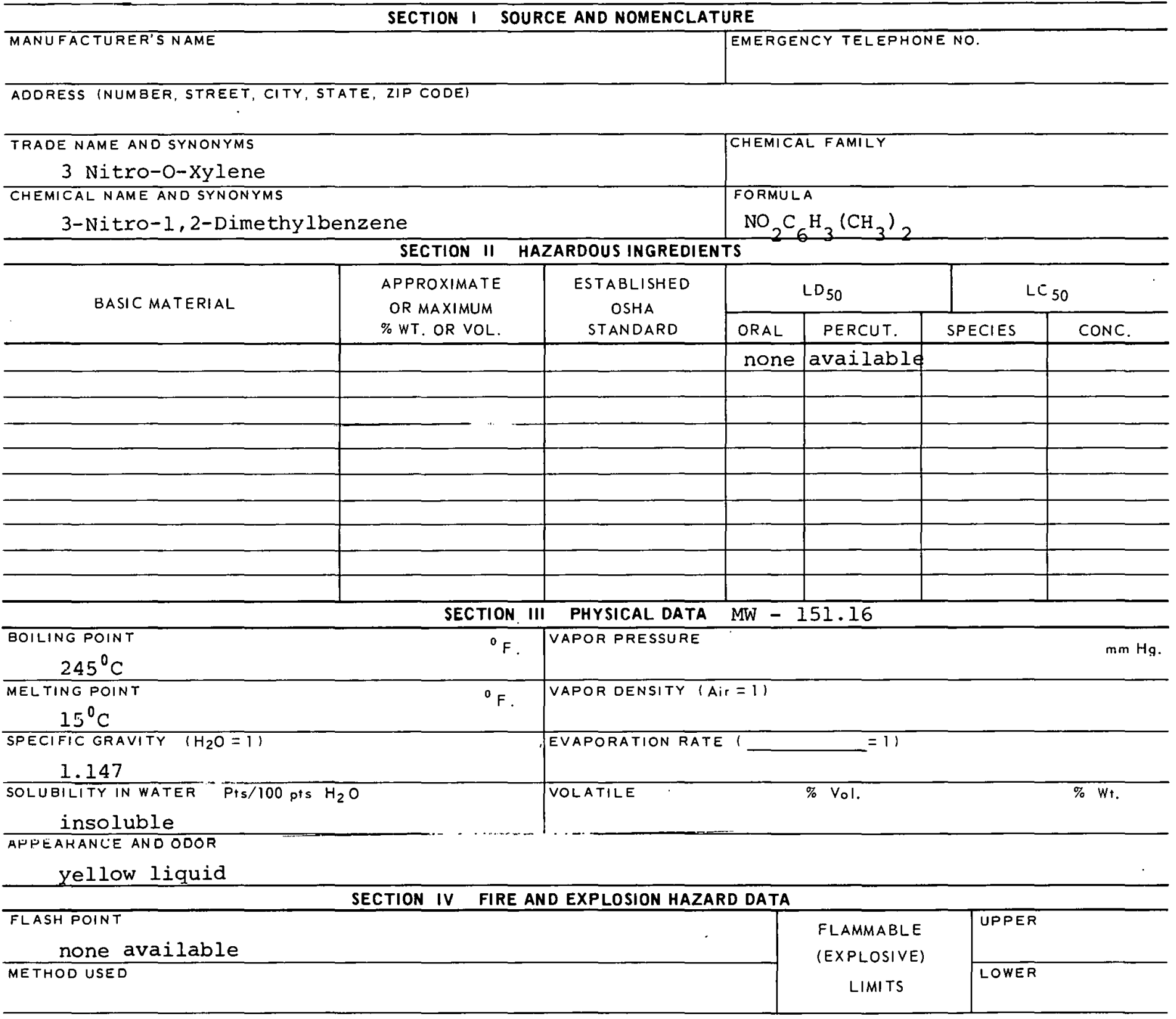

EXTINGUISHING MEDIA

information not available

SPECIAL FIRE FIGHTING PROCEDURES

UNUSUAL FIRE AND EXPLOSION HAZAROS 
3-Nitro-1,2-Dimethylbenzene

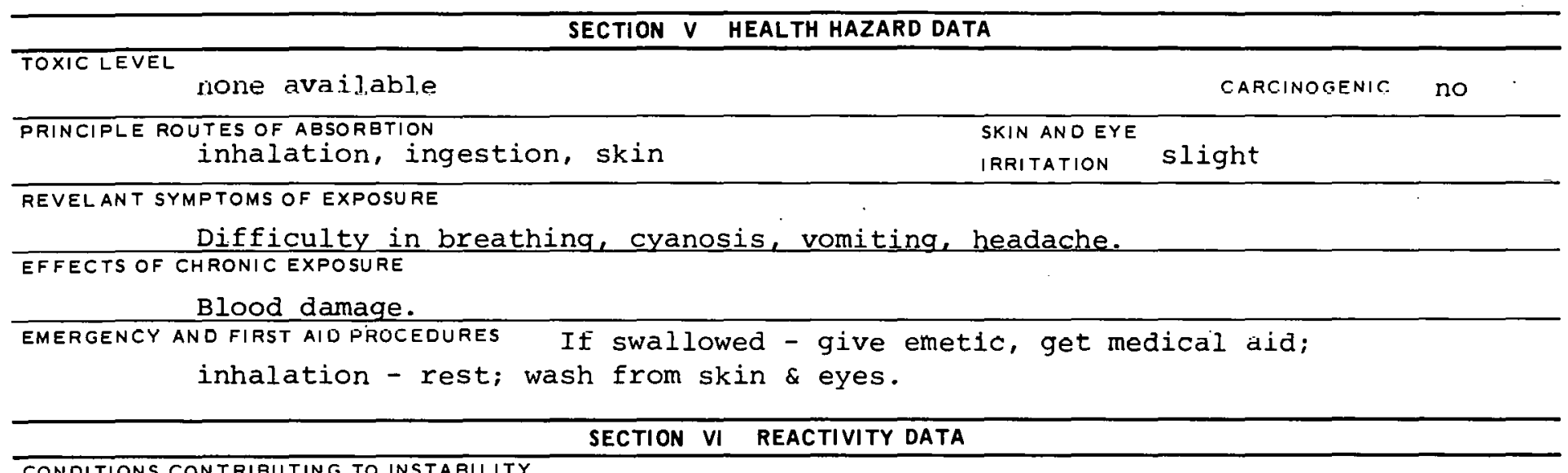

CONOITIONS CONTRIBUTING TO INSTABILITY

CONDITIONS CON TRIBUTING TO HAZARDOUS POLYMERIZATION

INCOMPATABILITY (MATERIALS TO AVOID)

May explode when heated with sulfuric acid.

HAZARDOUS DECOMPOSITION PRODUCTS

SECTION VII SPILL OR LEAK PROCEDURES

STEPS TO BE TAKEN IN CASE MATEHIAL IS RELEASED OR SPILLED

WASTE DISPOSAL METHOD

SECTION VIII SPECIAL PROTECTION INFORMATION

\begin{tabular}{|c|c|}
\hline VENTILATION REQUIREMENTS LOCAL EXHAUST & $\begin{array}{l}\text { PROTECTIVE EQUIPMENT ISPECIFY TYPESIEYE } \\
\text { GOgg LES }\end{array}$ \\
\hline MECHANIGAL (GENERAL) & $\begin{array}{l}\text { GLOVES } \\
\text { Kubber }\end{array}$ \\
\hline SRECIAL & $\begin{array}{l}\text { REBPlRATOR } \\
\text { Self-contained }\end{array}$ \\
\hline
\end{tabular}

OTHER PROTECTIVE EQUIPMENT

\section{SECTION IX SPECIAL PRECAUTIONS}

PRECAUTIONS TO BE TAKEN IN HANDLING AND STORAGE

Information not avallable.

OTHËR PRECAUTIONS 


\section{PRODUCT DESIGNATION}

03-316-7108

P-Nitrofluorobenzene
MATERIAL SAFETY

DATA SHEET

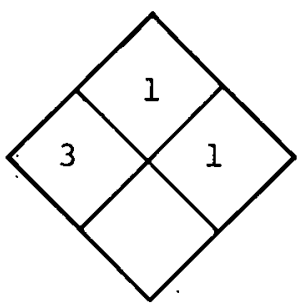

SECTION I SOURCE AND NOMENCLATURE

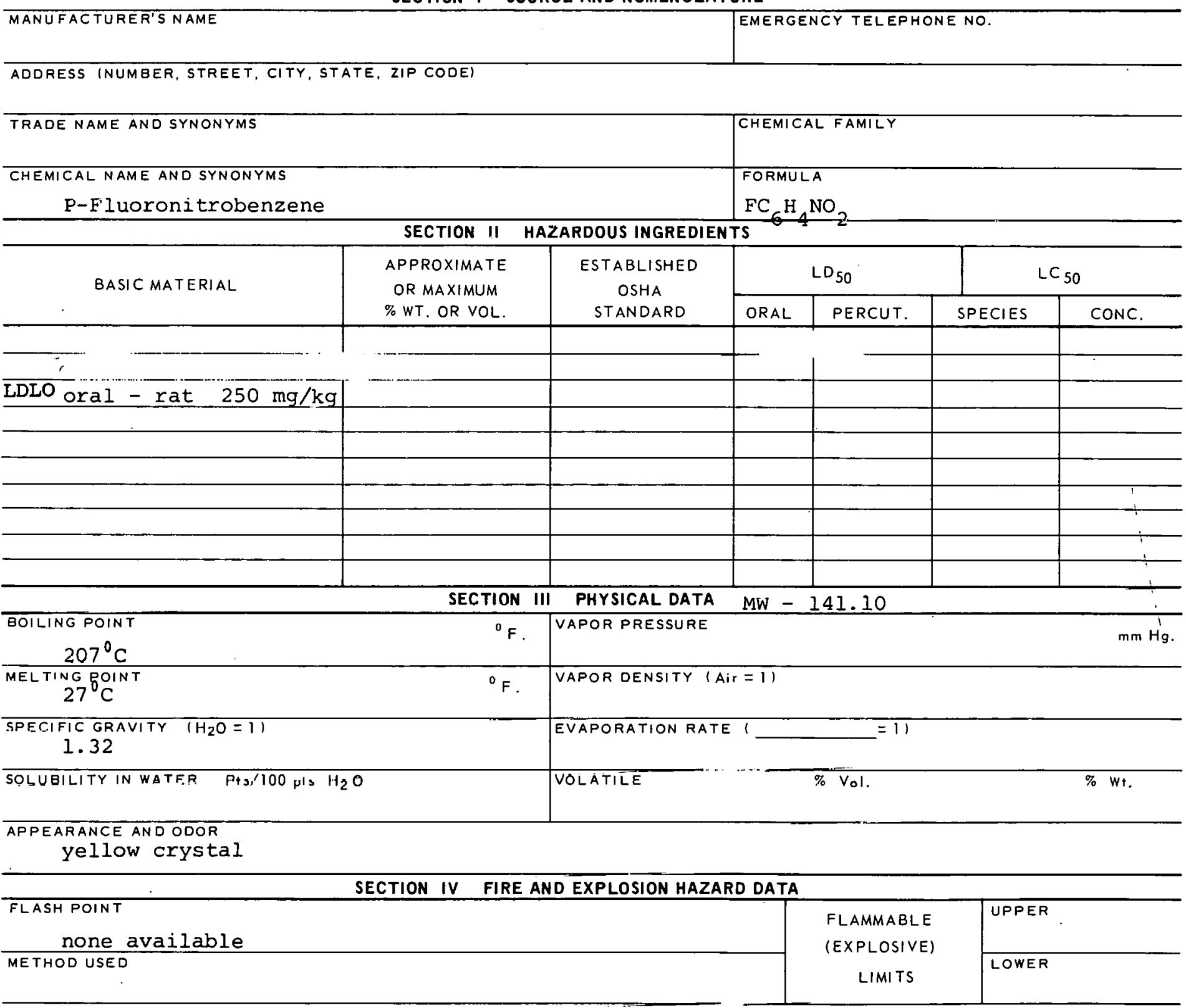

EXTINGIIISHING MEDIA

water spray, carbon dioxide, dry chemical, foam.

SPECIAL FIRE FIGHTING PROC.EDURES

wear self-contained breathing apparatus.

UNUSUAL FIRE AND EXPLOSION HAZARIS 


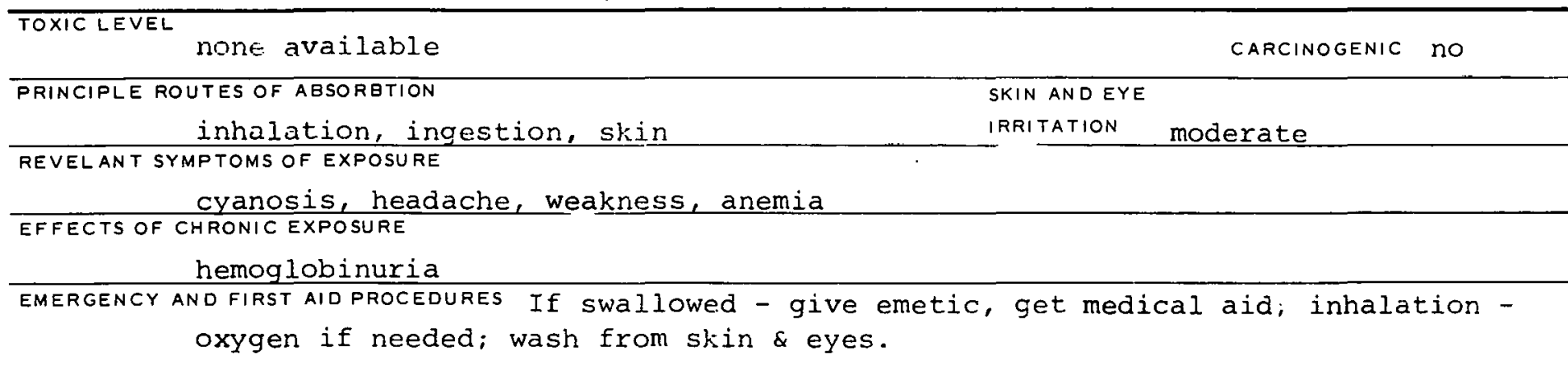

SECTION VI REACTIVITY DATA

CONDITIONS CONTRIBUTING TO INSTABILITY

GOHQITIONS RONTRIRIITING TO HAZARDOUS POLYMERIZATION

INCOMPATABILITY (MATERIALS TO AVOIDI)

HAZARDOUS DECOMPOSI TION PRODUCTS

SECTION VII SPILL OR LEAK PROCEDURES

STEPS TO BE TAKEN IN CASE MATERIAL IS RELEASED OR SPILLED

WASTE DISPOSAL METHOD

SECTION VIII SPECIAL PROTECTION INFORMATION

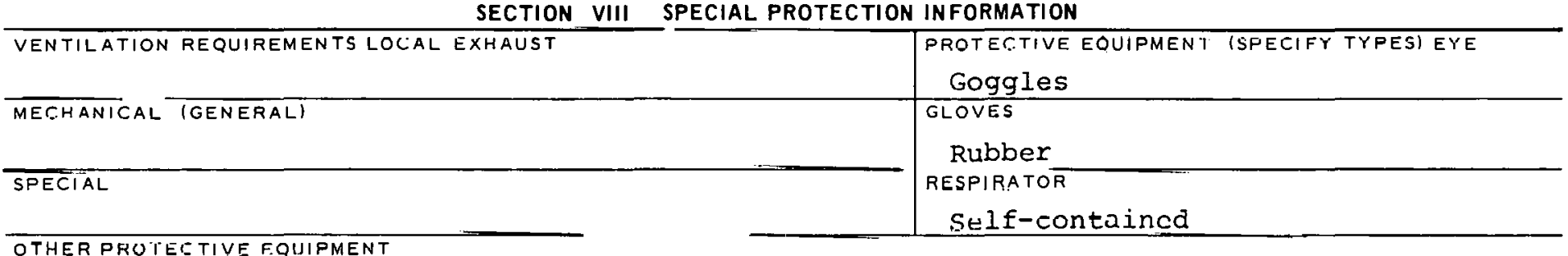

OTHER PROTECTIVE F.QUIPMENT

SECTION IX SPECIAL PRECAUTIONS

PRECAUTIONS TO BE TAKEN IN HANOLING AND STORAGE

Store away from oxidizing materials and sources of heat.

OTHER FRCCAUTIOHE

SIGNATURE

DATE

$316-7108 \mathrm{~B}$ 
03-316-8108

M-Nitroiodobenzene
MATERIAL SAFETY

DATA SHEET

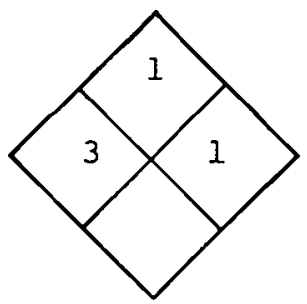

\section{SECTION I SOURCE AND NOMENCLATURE}

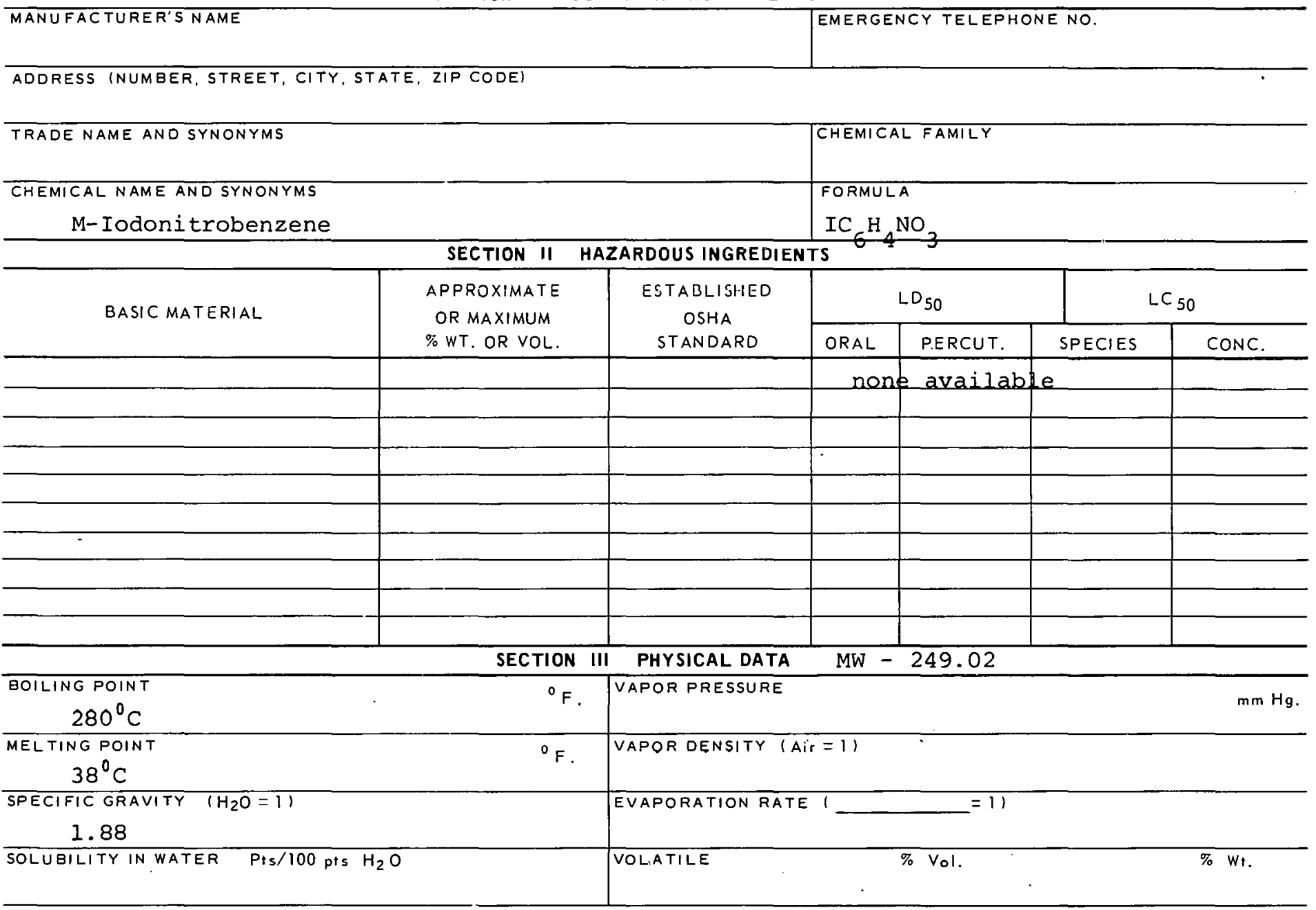

APPEARANCE AND ODOR

crystaline

SECTION IV FIRE AND EXPLOSION HAZARD DATA

FLASH POIN T

none available

METHOD USED

EXTINGUISHING MEDIA

water spray, carbon dioxide, dry chemical, fnam

SPECIAL FIRE FIGHTING PROCEDURES

Wear self-contained breathing apparatus.

UNUSUAL FIRE AND EXPLOSION HAZARDS

\begin{tabular}{l|l}
$\begin{array}{c}\text { FLAMMABLE } \\
\text { (EXPLOSIVE) } \\
\text { LIMITS }\end{array}$ & UPPER \\
\cline { 2 - 2 } & LOWER \\
\hline
\end{tabular}




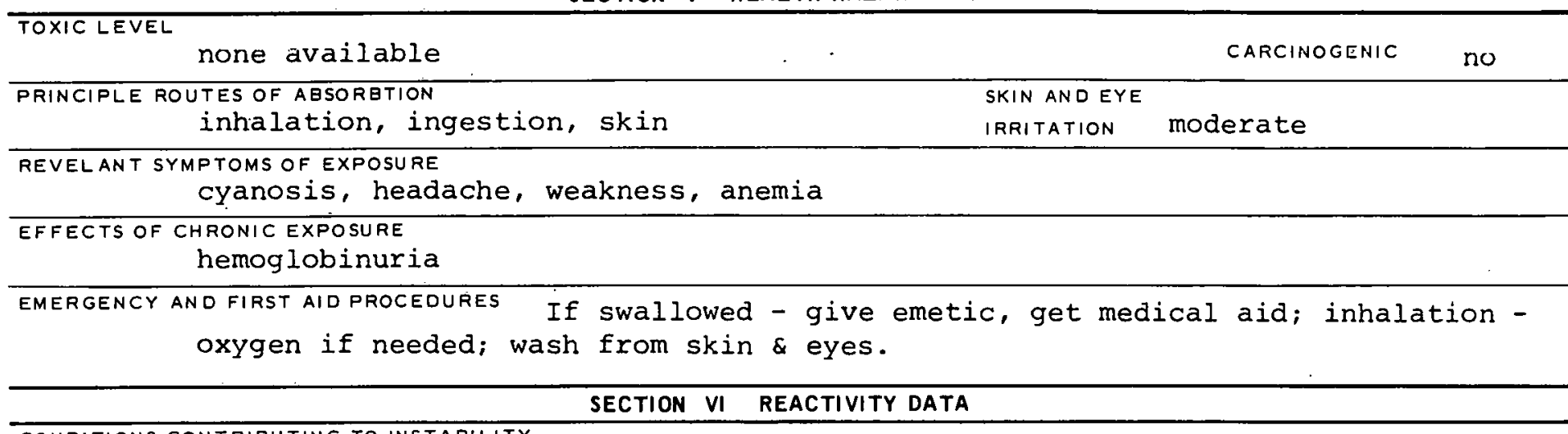

CONDITIONS CONTRIBUTING TO INSTABILITY

CONDITIONS CONTRIBUTING TO HAZARDOUS POLYMERIZATION

INCOMPATABILITY (MATÉRIALS TO AVOIO)

HAZARDOUS DECOMPOSITION PRODUCTS

SECTION VII SPILL OR LEAK PROCEDURES

STEPS TO BE TAKEN IN CASE MATERIAL IS RELEASED OR SPILLED

WASTE DISPOSAL METHOD

SECTION VIII SPECIAL PROTECTION INFORMATION

\begin{tabular}{l|l}
\hline VENTILATION REQUIREMENTS LOCAL EXHAUST & $\begin{array}{c}\text { PROTECTIVE EQUIPMENT (SPECIFY TYPESIEYE } \\
\text { GOgGlE3 }\end{array}$ \\
\hline MECHANICAL IGENERALI & $\begin{array}{c}\text { GLOVES } \\
\text { Rubber }\end{array}$ \\
\hline SPECIAL & RESPIRATOR \\
Self-contained
\end{tabular}

OTHER PROTECTIVE EQUIPMENT

SECTION IX SPECIAL PRECAUTIONS

PRECAUTIONS TO BE TAKEN IN HANOLING AND STORAGE

Store away from oxidizing materials and sources of heat.

OTHER PRECAUTIONS

SIGNATURE

DATE

$316-8108 B$ 


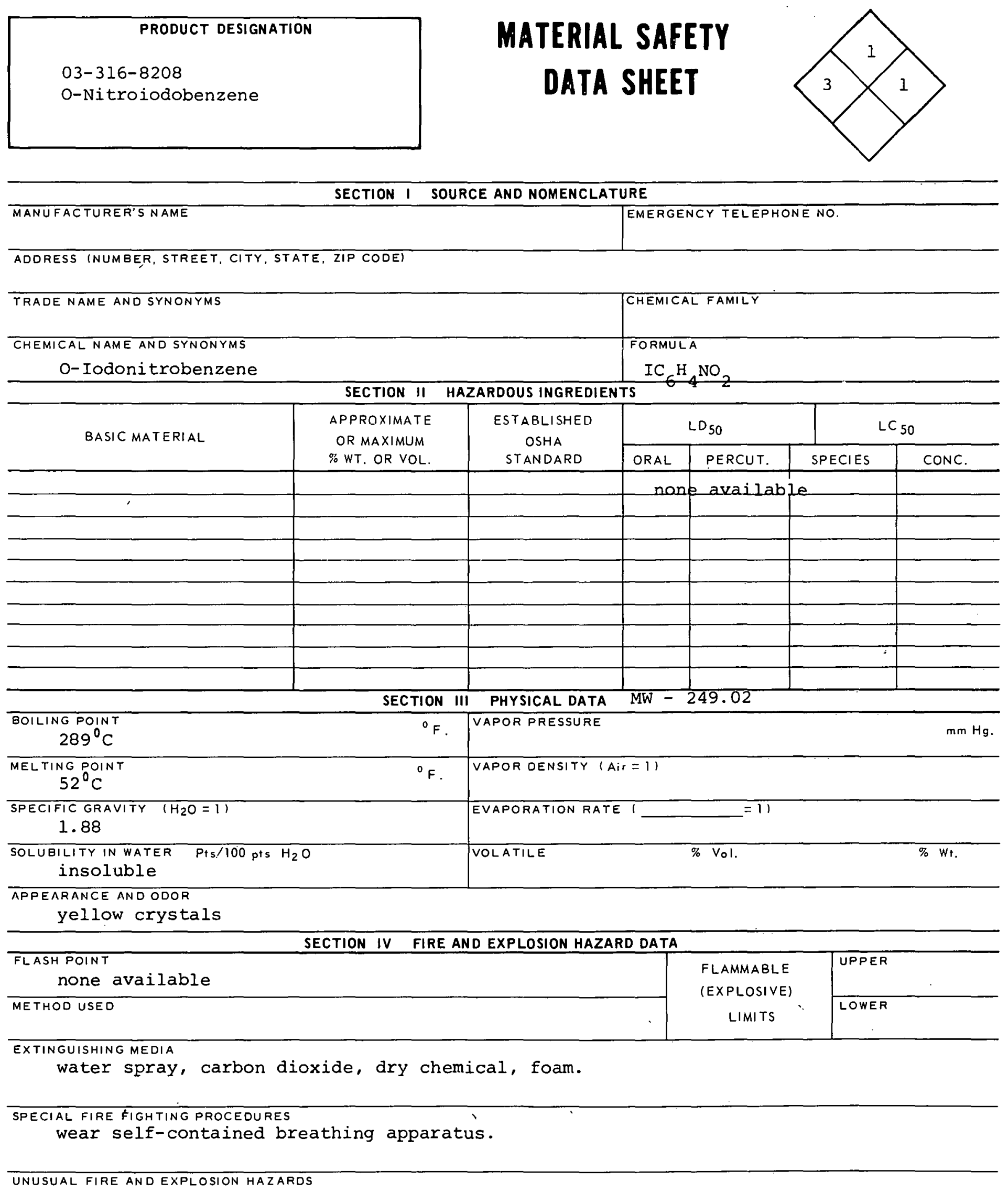




\begin{tabular}{l|l}
\hline VENTILATION REQUIREMENTS LOCAL EXHAUST & $\begin{array}{l}\text { PROTECTIVE EQUIPMENT ISPECIFY TYPESIEYE } \\
\text { GOggleS }\end{array}$ \\
\hline MECIIANICAL ICENERAL) & GLOVES \\
\hline SPEGIAL & HESPIHATUA \\
Self-contained
\end{tabular}

OTHER PROTECTIVE EQUIPMENT

\section{SECTION IX SPECIAL PRECAUTIONS}

PRECAUTIONS TO BE TAKEN IN HANDLING AND STORAGE

Store away from oxidizing materials and sources of heat.

OTIIER RREGAUTIOHC

SIOINATURE

UAIE 


PRODUCT DESIGNATION
O3-316-8308
P-Nitroiodobenzene

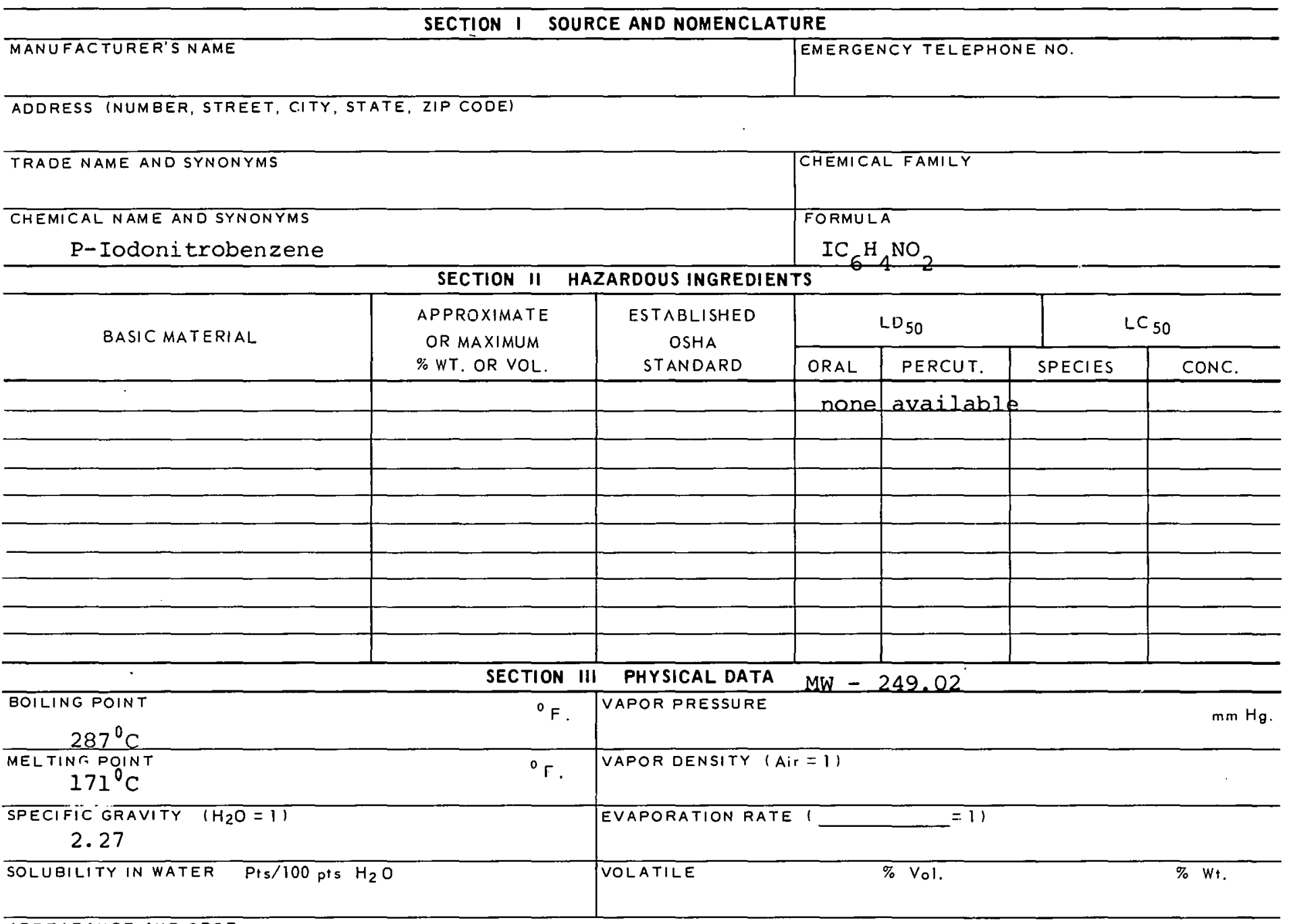

APPEARANCE AND ODOR

yellow crystals

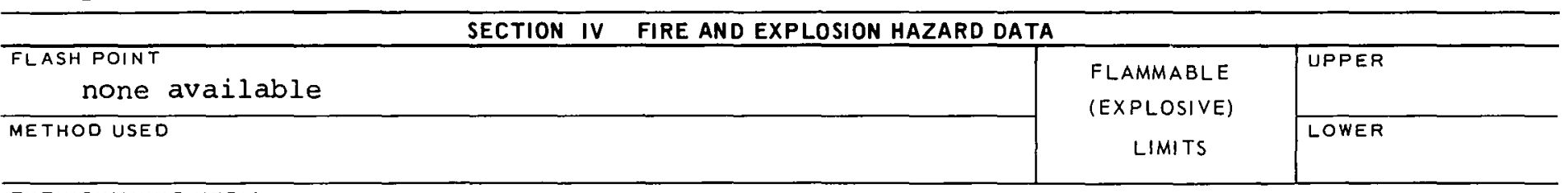

EXTINGUISHING MEDIA

water spray, carbon dioxide, dry chemical, foam.

SPECIAL FIRE FIGHTING PROCEDURES

Wear self-contained breathing apparatus.

UNUSUAL FIRE AND EXPLOSION HAZARDS 


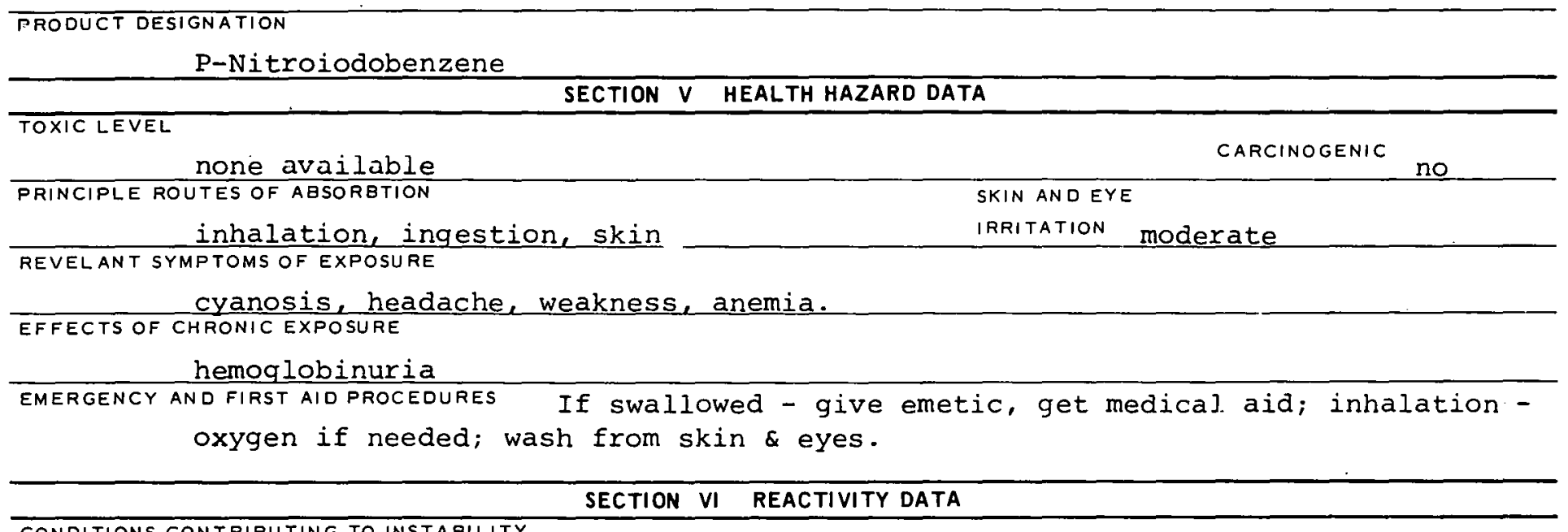

CONDITIONS CONTRIBUTING TO INSTABILITY

CONDITIONS CONTRIBUTING TO HAZARDOUS POLYMERIZATION

INCOMPA TABILITY (MATERIALS TO AVOID)

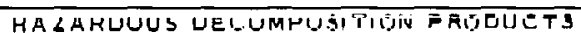

SECTION VII SPILL OR LEAK PROCEDURES

STEPS TO BE TAKEN IN CASE MATERIAL IS RELEASED OR SPILLED

WASTE DISPOSAL METHOD

SECTION VIII SPECIAL PROTECTION INFORMATION

\begin{tabular}{l|l}
\hline \multicolumn{2}{c}{ SECTION VIII SPECIAL PROTECTION INFORMATION } \\
\hline VENTILATION REQUIREMENTS LOCAL EXHAUST & $\begin{array}{c}\text { PROTECTIVE EQUIPMENT (SPECIFY TYPES) EYE } \\
\text { GOGgles }\end{array}$ \\
\hline MECHANICAL IGENERAL) & $\begin{array}{c}\text { GLOVES } \\
\text { Rubber }\end{array}$ \\
\hline SPECIAI. & $\begin{array}{l}\text { RF.SPIRATOR } \\
\text { With propex fj.lter }\end{array}$ \\
\hline
\end{tabular}

OTHER PROTECTIVE EQUIPMENT

\section{SECTION IX SPECIAL PRECAUTIONS}

PRECAUTIONS TO BE TAKEN IN HANDLING AND STORAGE

Store away from oxidizing materials and sources of heat.

OTHER PRECALTIONS

SIGNATURE

DATF

$316-8308 B$ 


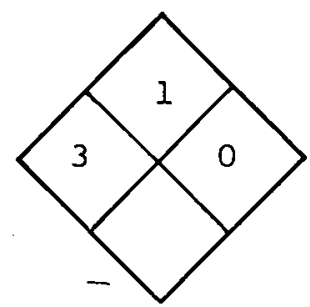

\section{SECTION I SOURCE AND NOMENCLATURE}

MANUFACTURER'S NAME
ADDRESS INUMBER, STREET, CITY,
TRADE NAME AND SYNONYMS
CHEMICAL NAME AND SYNONYMS
2 NitromeSitYlene

\begin{tabular}{l|l} 
& EMERGENCY TELEPHONE NO.
\end{tabular}

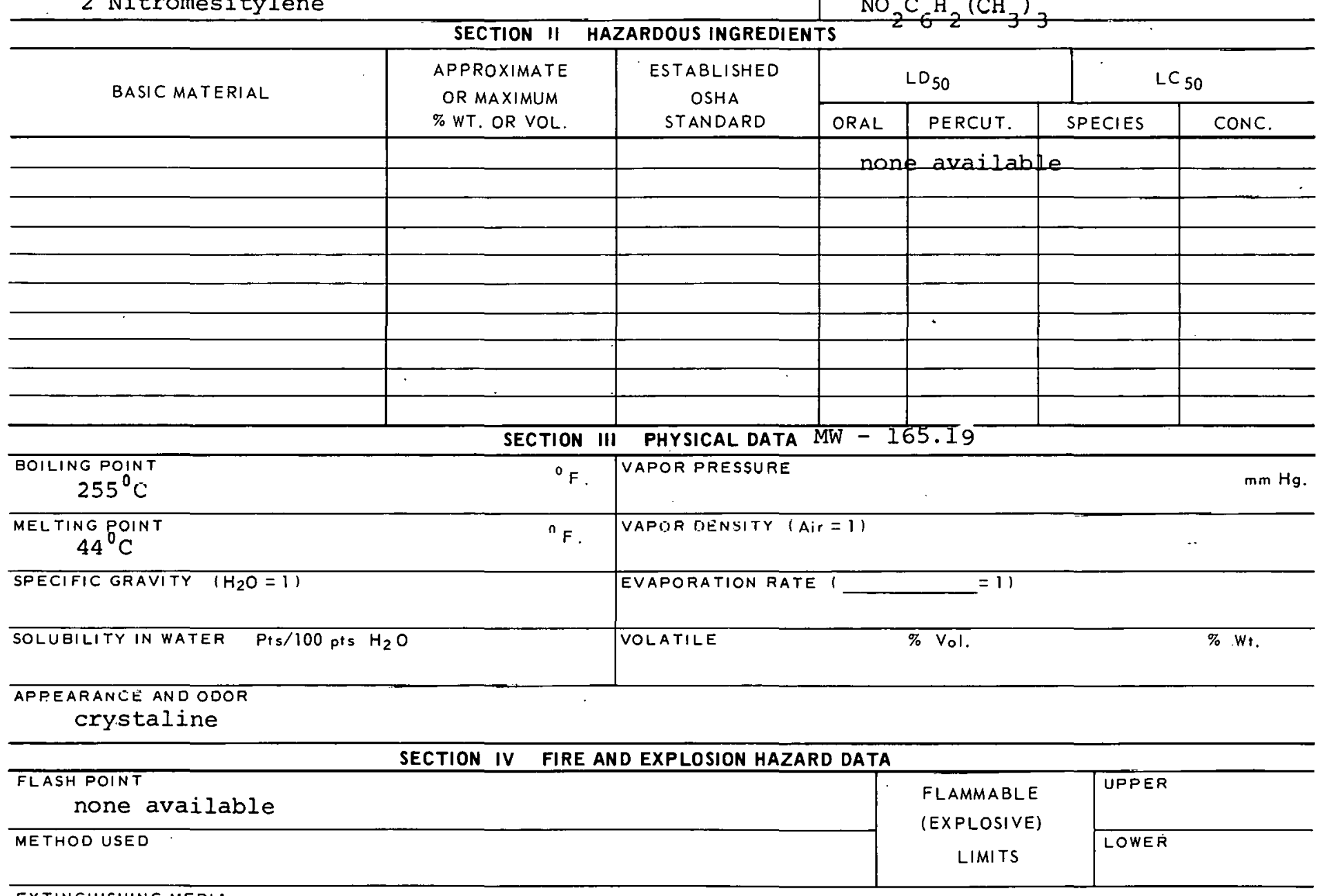

EXTINGUISHING MEDIA

information not available.

SPECIAL FIRE FIGHTING PROCEDURES

UNUSUAL FIRE ANDEXPLOSION HAZARDS 
TOXICLEVEL none available

PRINCIPLE ROUTES OF ABSORBTION inhalation, ingestion, skin

SKIN AND EYE

IRRITATION

severe

REVELANT SYMPTOMS OF EXPOSURE chemical pneumonia, central nervous system depression, frequent bleeding.

EFFECTS OF CHRONIC EXPOSURE

Nervous tension, anxiety, asthmatic bronchitis.

EMERGENCY AND FIRST AID PROCEDURES Inhalation - oxygen as needed; if swallowed - get medical aid, gastric lavage; wash from skin \& eyes.

\title{
SECTION VI REACTIVITY DATA
}

\begin{abstract}
C.ONDITIONS CONTRIBUTING TO INSTABILITY
CONOITIONS CONTRIBUTING TO HAZARDOUS POLYMERIZAIION

INCOMPATABILITY (MATERIALS TO AVOID)

HAZARDOUS DECOMPOSITION PRODUCTS

SECTION VII SPILL OR LEAK PROCEDURES
\end{abstract}

STEPS TO BE TAKEN IN CASE MATERIAL IS RELEASED OR SPILLED

SECTION VIII SPECIAL PROTECTION INFORMATION

\begin{tabular}{l|l}
\hline $\begin{array}{l}\text { VENTILATION REQUIREMENTS LOCAL EXHAUST } \\
\text { MECHANICAL IGENERAL) }\end{array}$ & $\begin{array}{c}\text { PROTECTIVE EQUIPMENT (SPECIFY TYFES) EYE } \\
\text { Gogg] }\end{array}$ \\
\hline SPECIAI & $\begin{array}{c}\text { GLOVES } \\
\text { Rubber }\end{array}$ \\
\hline OTHER PROTECTIVE EQUIPMENT & $\begin{array}{c}\text { RESHIRATOA } \\
\text { With proper filter }\end{array}$ \\
\hline
\end{tabular}

OTHER PRECAUUTIONS

$\overline{\text { SIGNATURE }}$

DATE

SIONATURE

$\overline{0 A}$

$316-8608 B$ 


\section{PRODUCT DESIGNATION}

03-316-9108

Nitromethane
MATERIAL SAFETY

DATA SHEET

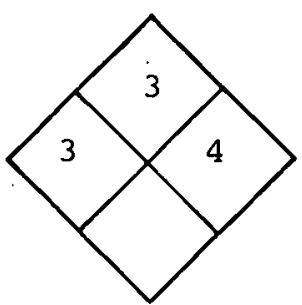

SECTION I SOURCE AND NOMENCLATURE

MANUFACTURER'S NAME

ADORESS (NUMBER, STREET, CITY, STATE, ZIP CODE)

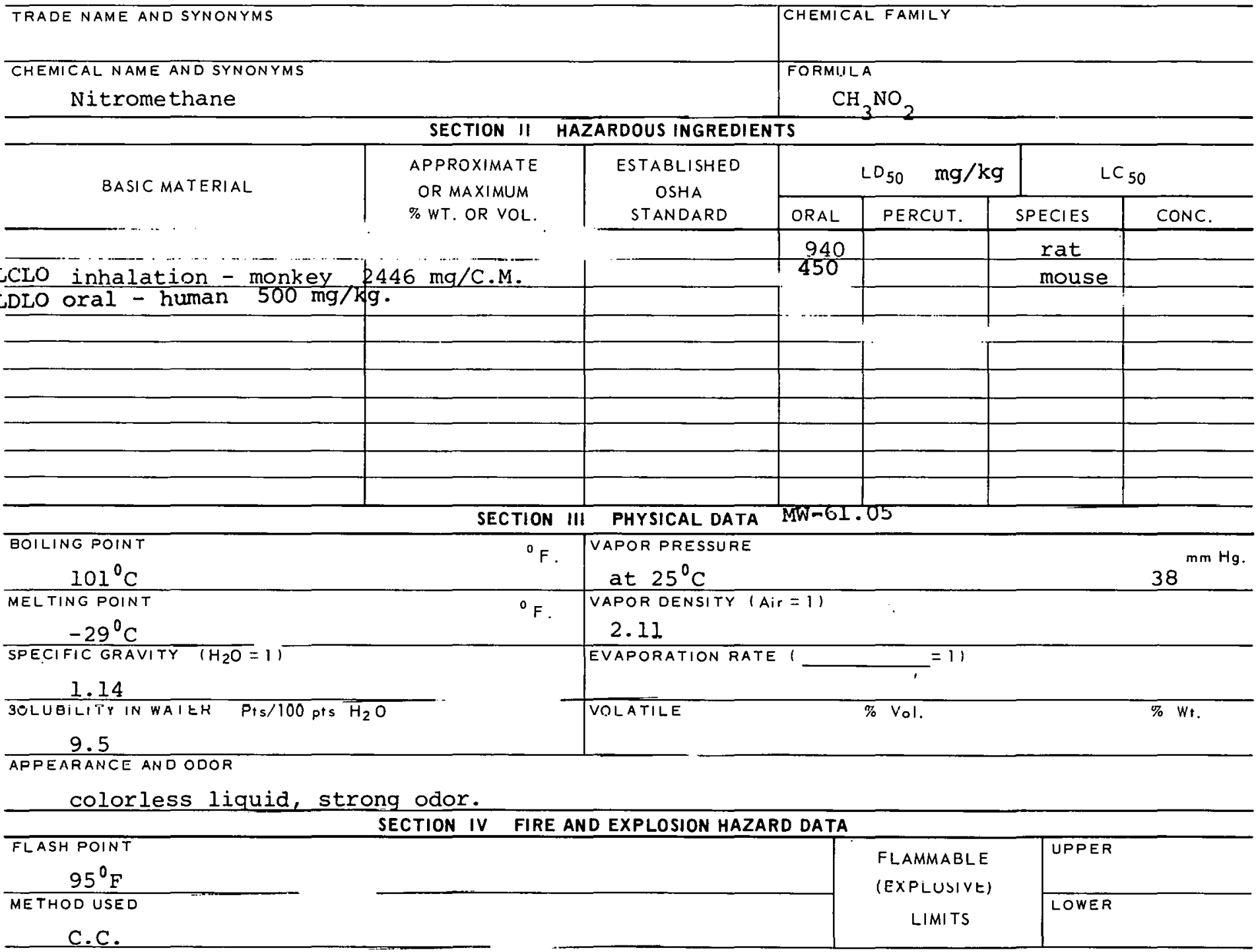

ExiINGUISHING MEDIA Alcohol foam, carbon dioxide, dry chemical. Water

may be ineffective.

SPECIAL FIRE FIGHTING PROCEDUHES

Wear self-contained breathing apparatus.

UNUSUAL FIRC AND CXFLOBMIN HAEARDS

Explosion hazard - moderate when exposed to heat or flame. 


\section{TOXIC LEVEL}

TIV - 100 PPM, $250 \mathrm{mg} / \mathrm{C} . \mathrm{M}$.

CARCINOGENIC

PRINCIPLE ROUTES OF ABSORBTION

SKIN AND EYE

Inhalation, ingestion

IRRITATION

REVELANT SYMPTOMS OF EXPOSURE Narcosis, irxitation of mucous membrane, central

nervous system excitation.

EFFECTS OF CHRONIC EXPOSURE

Liver and spleen damage.

EMERGENCY AND FIRST AID PROCEDURES If swallowed - induce vomiting, get medical aid, gastric

lavage; inhalation - artificial respiration, oxygen if needed; wash from skin \& eyes.

CONDITIONS CONTRIBUTING TO INSTABILITY

SECTION VI REACTIVITY DATA

CONDITIONS CONTRIBUTING TO HAZARDOUS POLYMERIZATION

INCOMPATABILITY (MATERIALS TO AVOID)

Avoid $\mathrm{Al} \mathrm{Cl}_{3}$ with organic matter, $\mathrm{Ca}(\mathrm{OH})_{2}, \mathrm{CaOCl}$, hydrocarbons, organic amines, HAZARDOUS DECOMPOSITION PRODUCTS

$\mathrm{NaOH}$ and $\mathrm{KOH}$

SECTION VII SPILL OR LEAK PROCEDURES

STEPS TO BE TAKEN IN CASE MATERIAL IS RELEASEU UR SPILLED

After covering spill with soda ash, spray with water and neutralize with $6 \mathrm{~N} \mathrm{HCl}$.

WASTE DISPOSAL METHOD

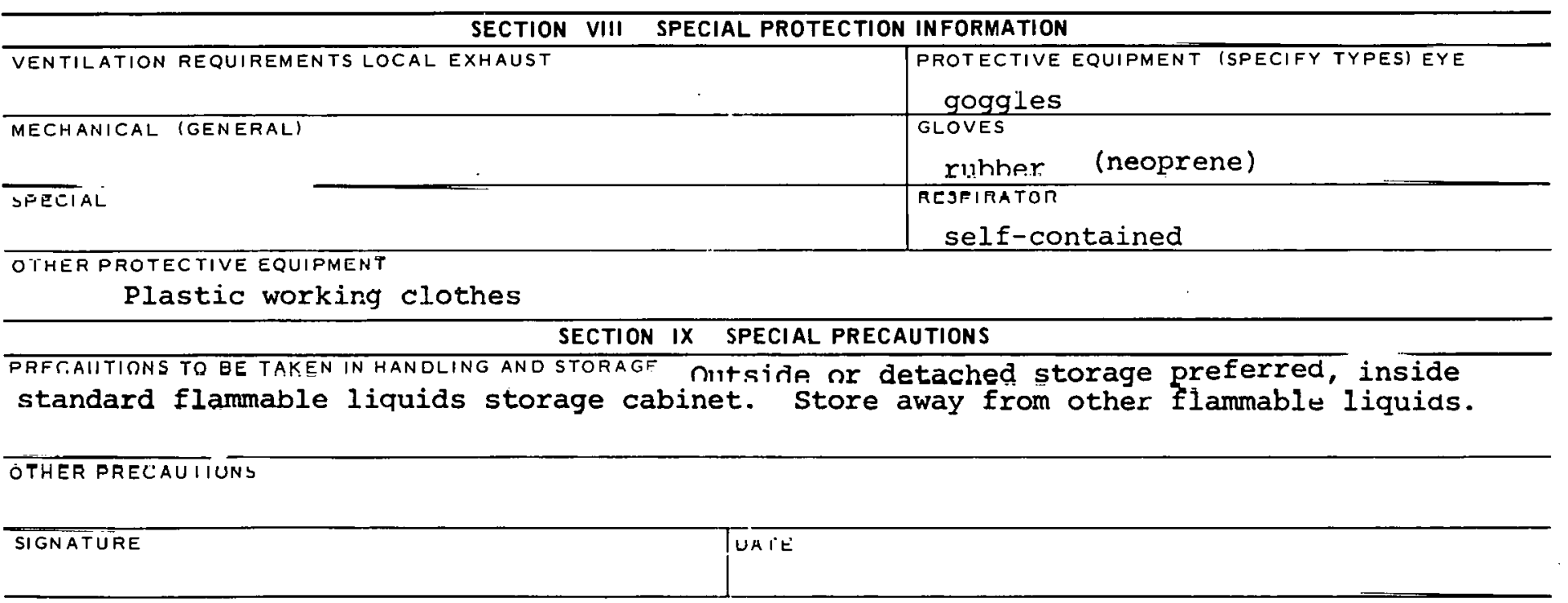

$316-9108 B$ 


\begin{tabular}{|l|l}
\hline \multicolumn{1}{|c|}{ PRODUCT DESIGNATION } \\
$\begin{array}{l}\text { 03-316-9608 } \\
\text { P-Nitromethylaniline }\end{array}$
\end{tabular}$\quad$ DATERIALSAFEY SAEST.

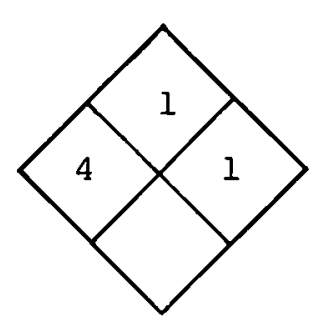

SECTION I SOURCE AND NOMENCLATURE

\begin{tabular}{l|l|l}
\hline MANUFACTURER'S NAME & EMERGENCY TELEPHONE NO. \\
\hline ADDRESS (NUMBER, STREET, CITY, STATE, ZIP CODE)
\end{tabular}

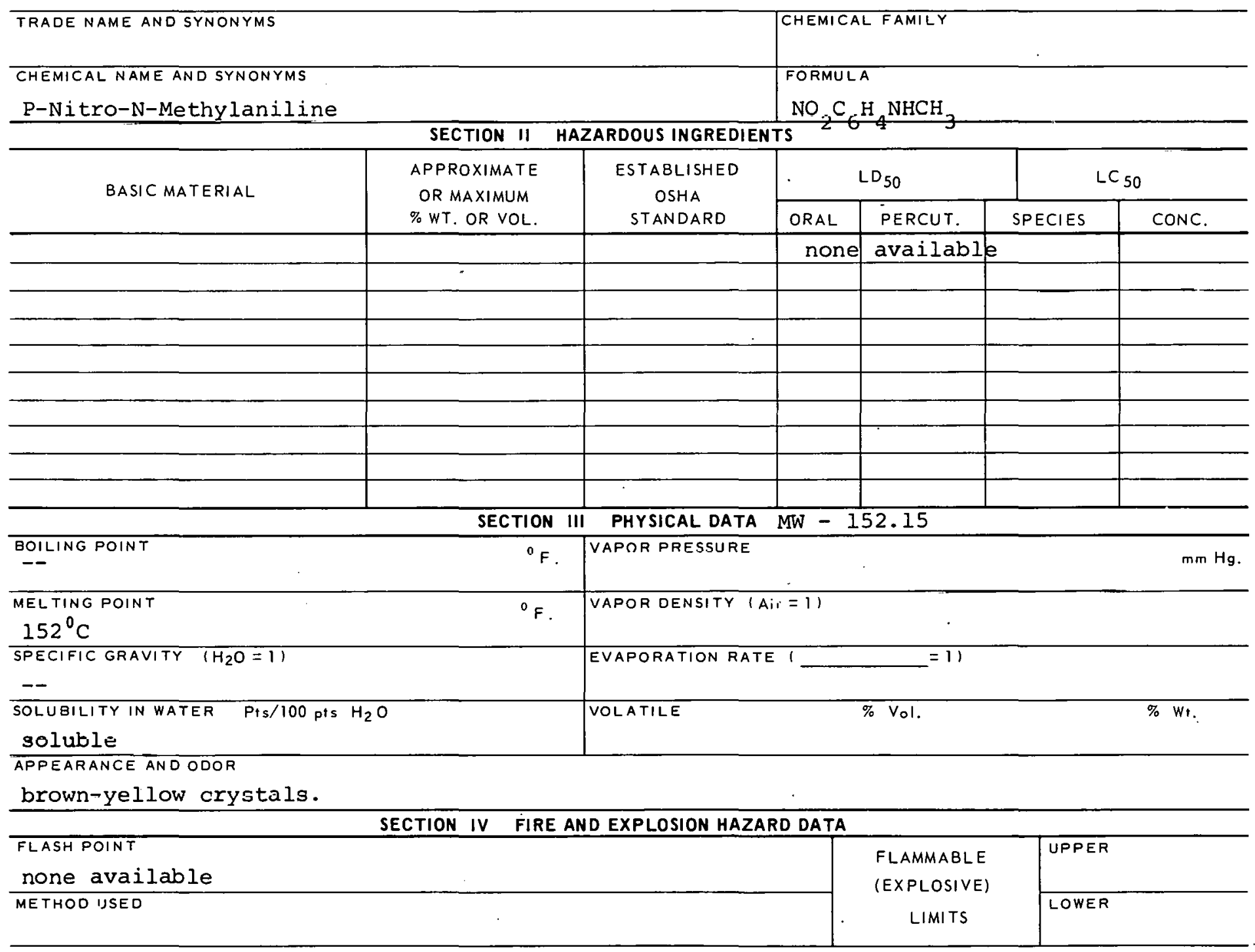

EXT!NGUISHING MEDIA

information nut available.

SPECIAL FIRE FIGHTING PROCEOURES

UNUSUAL FIRE AND EXPLOSION HAZARDS 


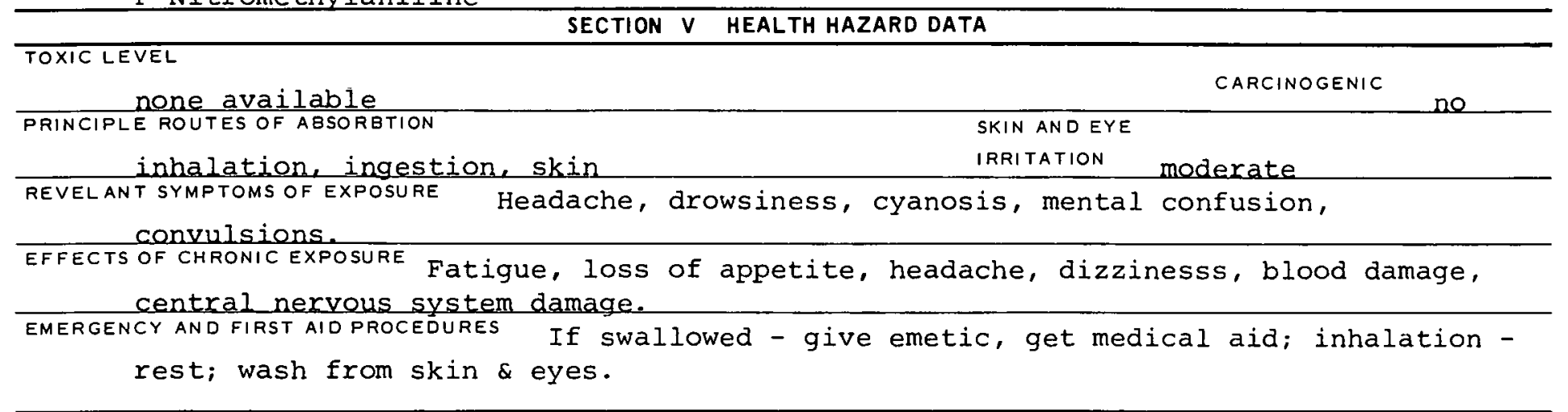

COMOITIONS CONTRIRIITING TO HAZARDOUS POLYMERIZATION

INCOMPATABILITY (MATERIALSTOAVOID)

HAZARDOUS DECOMPOSITION PRODUCTS

SECTION VII SPILL OR LEAK PROCEDURES

STEPS TO BE TAKEN IN CASE MA TERIAL IS RELEASED OR SPILLED

WASTE DISPOSAL METHOD

SECTION VIII SPECIAL PROTECTION INFORMATION

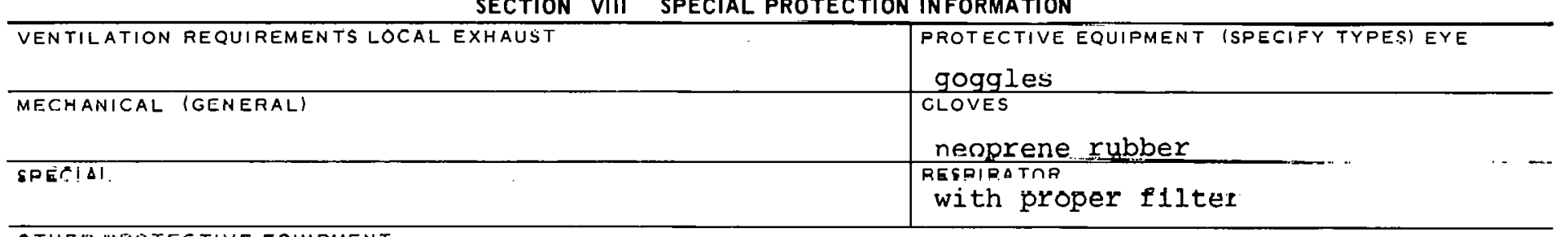

OTHER PROTECTIVE EQIIIPMENT

SECTION IX SPECIAL PRECAUTIONS

PRECAUTIONS TO BE TAKEN IN HANDLING AND STORAGE

OTHER TRECAUTIOHE

SIGNATURE

DATE

\begin{tabular}{l|l} 
SATE & ONATURE \\
\hline
\end{tabular}

$316-9608 B$ 


\begin{tabular}{|l|}
\hline \multicolumn{1}{|c|}{ PROdUCT DESIGNATION } \\
$03-317-1209$ \\
5 Nitro-1-Naphthylamine
\end{tabular}

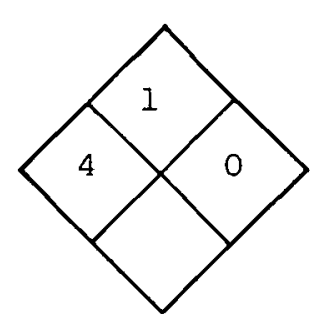

SECTION I SOURCE AND NOMENCLATURE

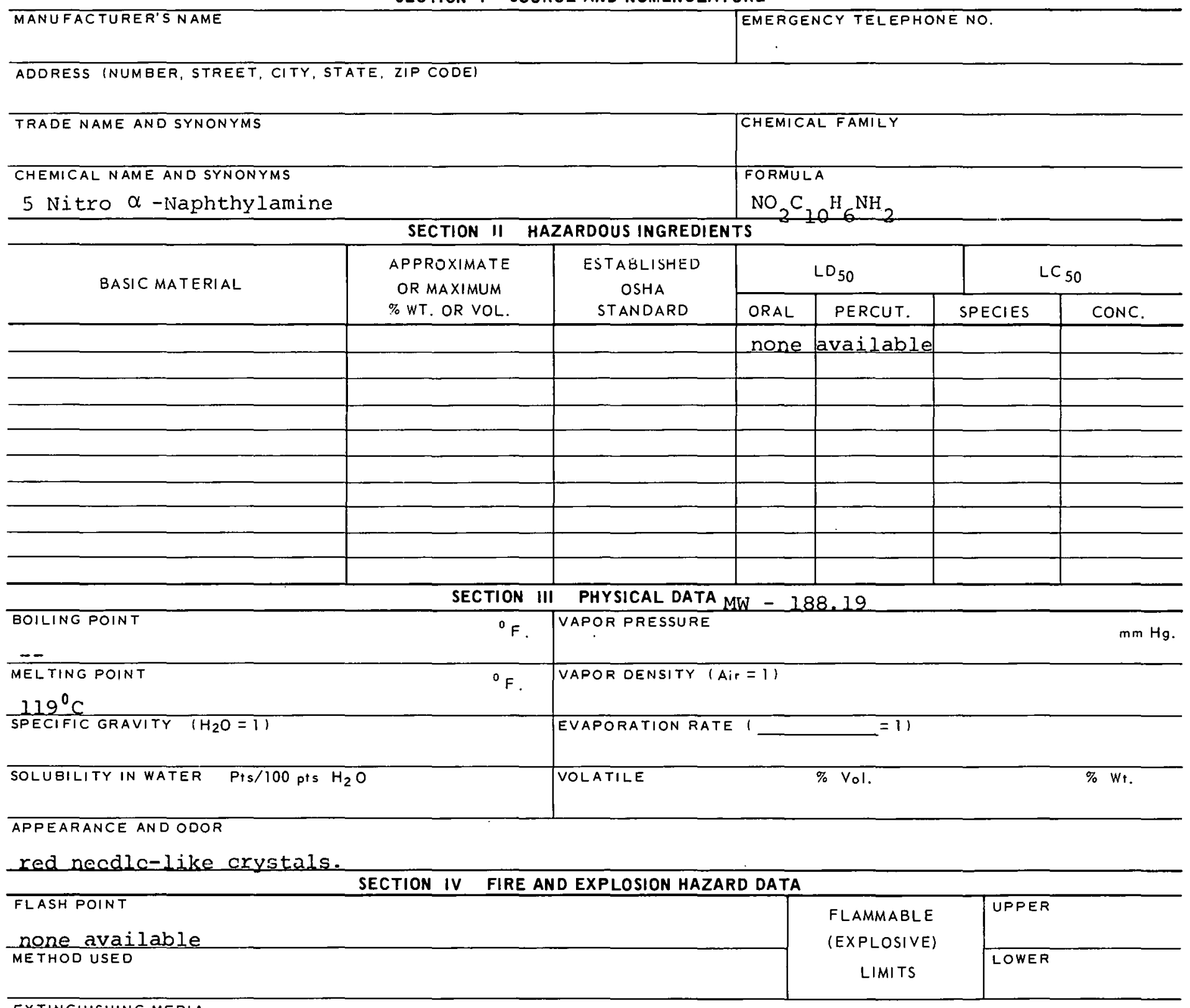

EXTINGUISHING MEDIA

information nol available.

SPECIAL FIRE FIGHTING PROCEDURES

UNUSUAL FIRE AND EXPLOSION HAZARDS 


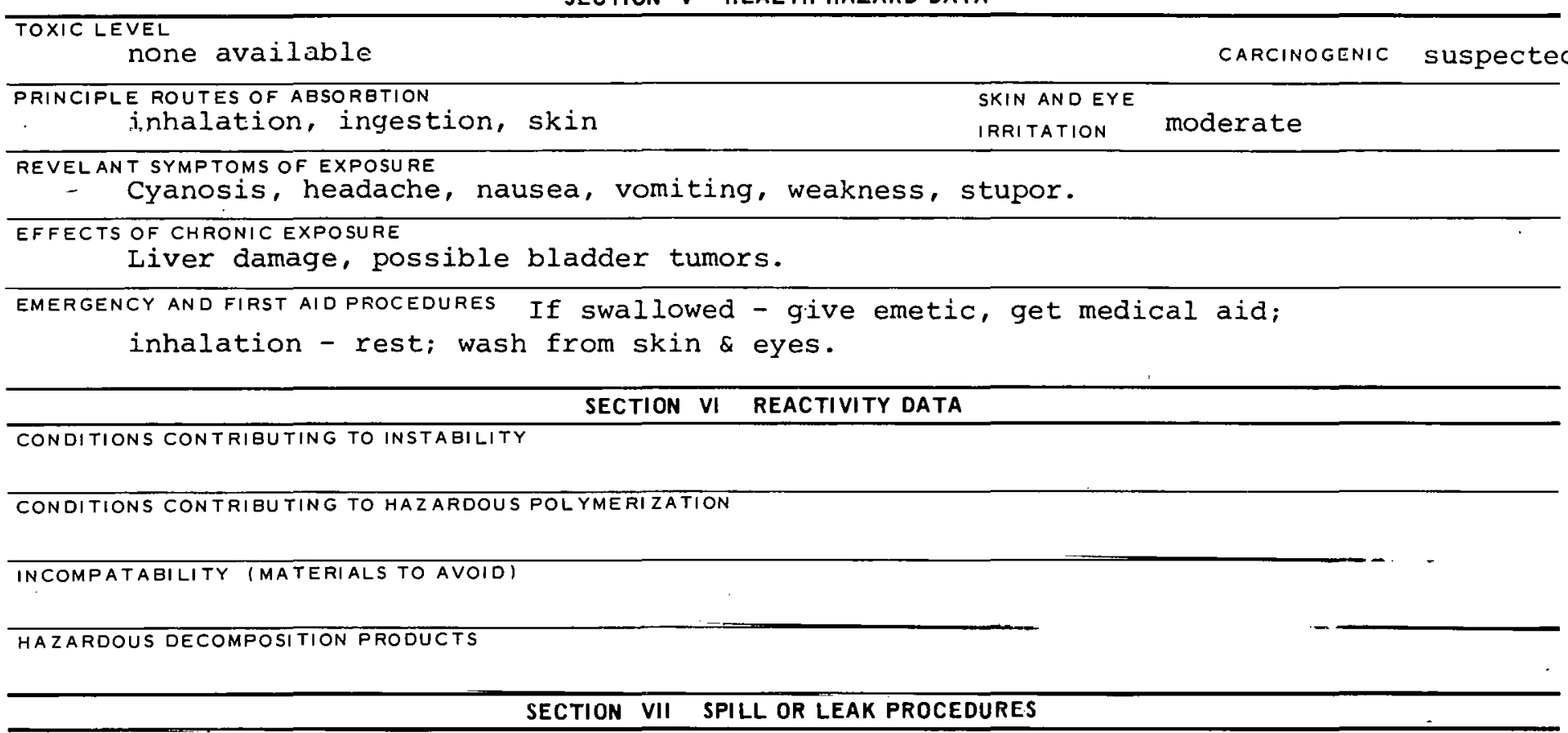
STEPS TO BE TAKEN IN CASE MATERIAL IS RELEASED OR SPILLED

WASTE DISPOSAL METHOD

SECTION VIII SPECIAL PROTECTION INFORMATION

\begin{tabular}{|c|c|}
\hline VENTILATION REQUIREMENTS LOCAL EXHAUST & $\begin{array}{l}\text { PROTECTIVE EQUIPMENT (SPECIFY TYPESI EYE } \\
\text { goggles }\end{array}$ \\
\hline MECHANICAL (GENERAL) & $\begin{array}{l}\text { GLOVES } \\
\text { rubber }\end{array}$ \\
\hline SPECIAL & $\begin{array}{l}\text { RESPIRATOR } \\
\text { with proper filter }\end{array}$ \\
\hline
\end{tabular}

OTHEH PROTECTIVE EQUIPMENT

SECTION IX SPECIAL PRECAUTIONS

PAFCAIITIONS TO BE TAKEN IN HANDLING AND STORAGE

OTHER PRECAUTIONS

SIGNATURE

DATE

317-1209B 
CAS : 000554847

\section{PRODUCT DESIGNATION}

03-317-3239

M-Nitrophenol
SM19250

MATERIAL SAFETY

DATA SHEET

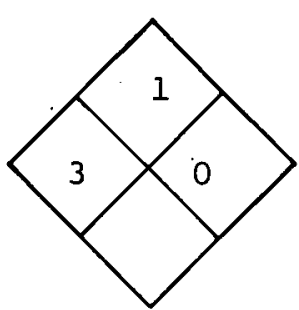

\section{SECTION I SOURCE AND NOMENCLATURE}

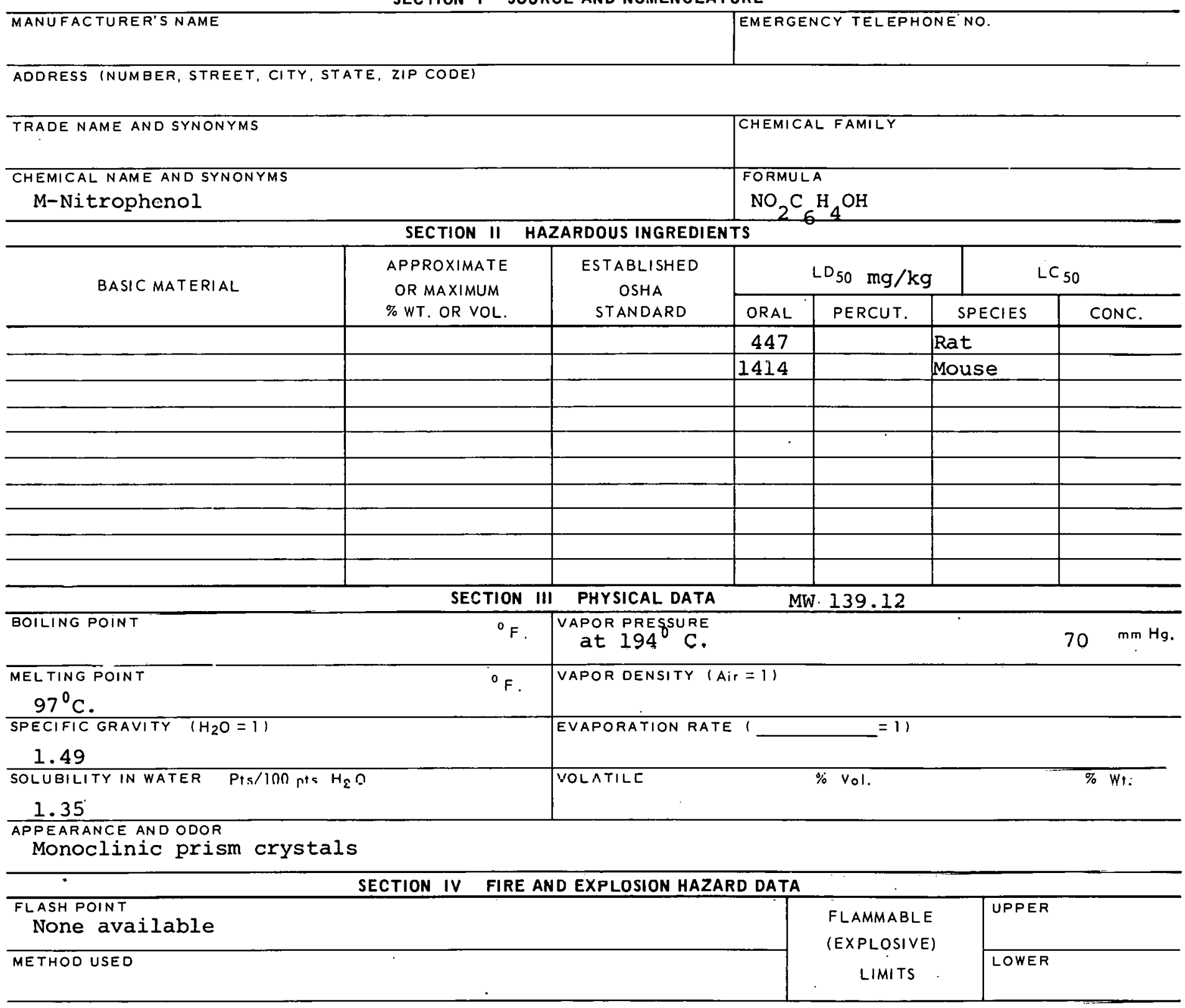

EXTINGUISHING MEDIA

Use water to cool down containers.

SPECIAL FIRE FIGHTING PROCEDURES

Wear full protective clothing, self-contained breathing apparatus.

UNUSUAL FIRE AND EXPLOSION HAZAROS

When heated to decomposition gives off toxic nitrogen oxide fumes. 
TOXIC LEVEL

None Available

PRINCIPLE ROUTES OF ABSORBTION

Inhalation, ingestion, skin
CARCINOGENIC No

SKIN AND EYE

IRRITATION
Moderate

REVELANT SYMPTOMS OF EXPOSURE

Eye and mucous membrane irritation, C.N.S. depressant.

EFFECTS OF CHRONIC EXPOSURE

Forms methemoglobin, dermatitis.

EMERGENCY AND FIRST AID PROCEDURES Inhalation - rest; wash from skin \& eyes. If swallowed remove by gastric lavage or emesis.

\begin{tabular}{|c|c|}
\hline SECTION VI & REACTIVITY DATA \\
\hline CONDITIONS CONTRIBUTING TO INSTABILITY & \\
\hline SOMIOITINNG RONTRIBUTING TO HAZARDOUS POLYMERIZATION & \\
\hline $\begin{array}{l}\text { INCOMPATAEILITY (MATERIALSTO. AVOID) } \\
\text { Keep from oxidizing materials. }\end{array}$ & \\
\hline
\end{tabular}

SECTION VII SPILL OR LEAK PROCEDURES

STEPS TO BE TAKEN IN CASE MATERIAL IS RELEASED OR SPILLED

WASTE DISPOSAL METHOD

SECTION VIII SPECIAL PROTECTION INFORMATION

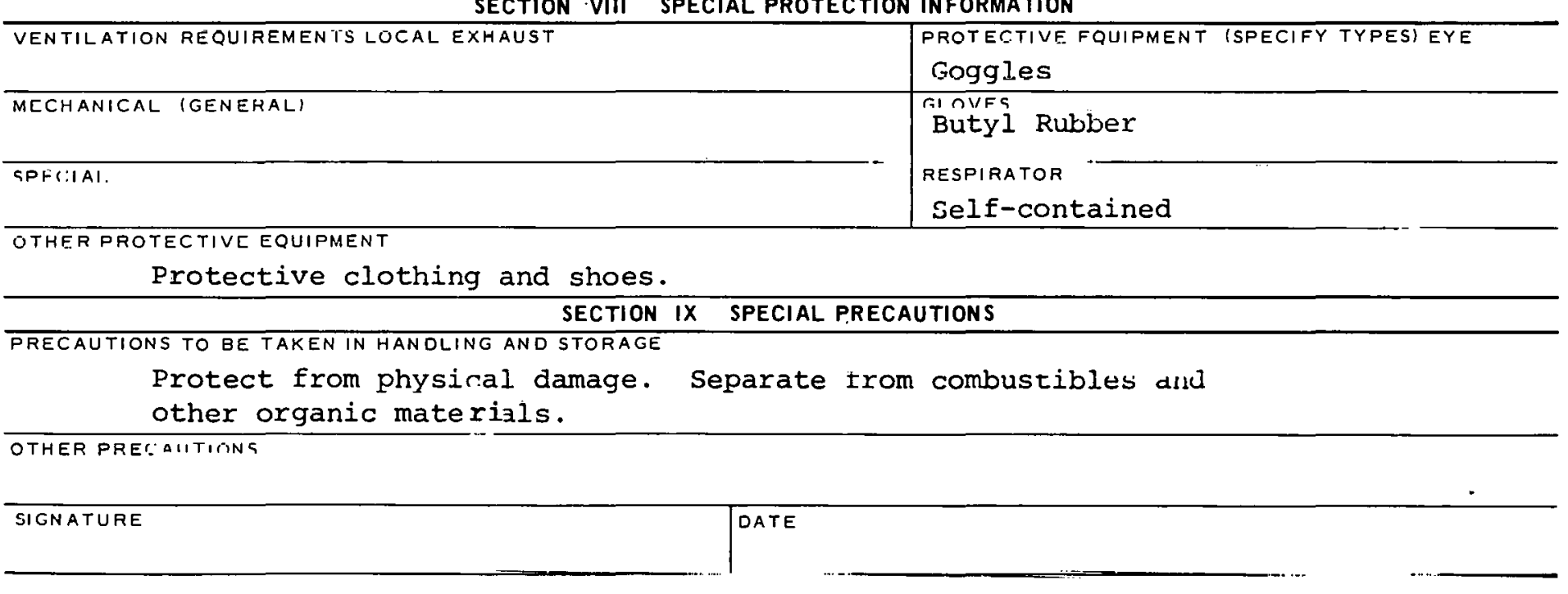

$317-3239 B$ 
CAS : 000100027

\begin{tabular}{|c|}
\hline PRODUCT DESIGNATION \\
03-317-3439 \\
P-Nitrophenol \\
\hline
\end{tabular}

MATERIAL SAFETY DATA SHEET

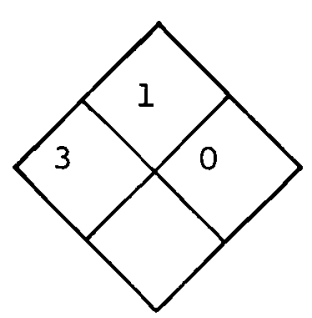

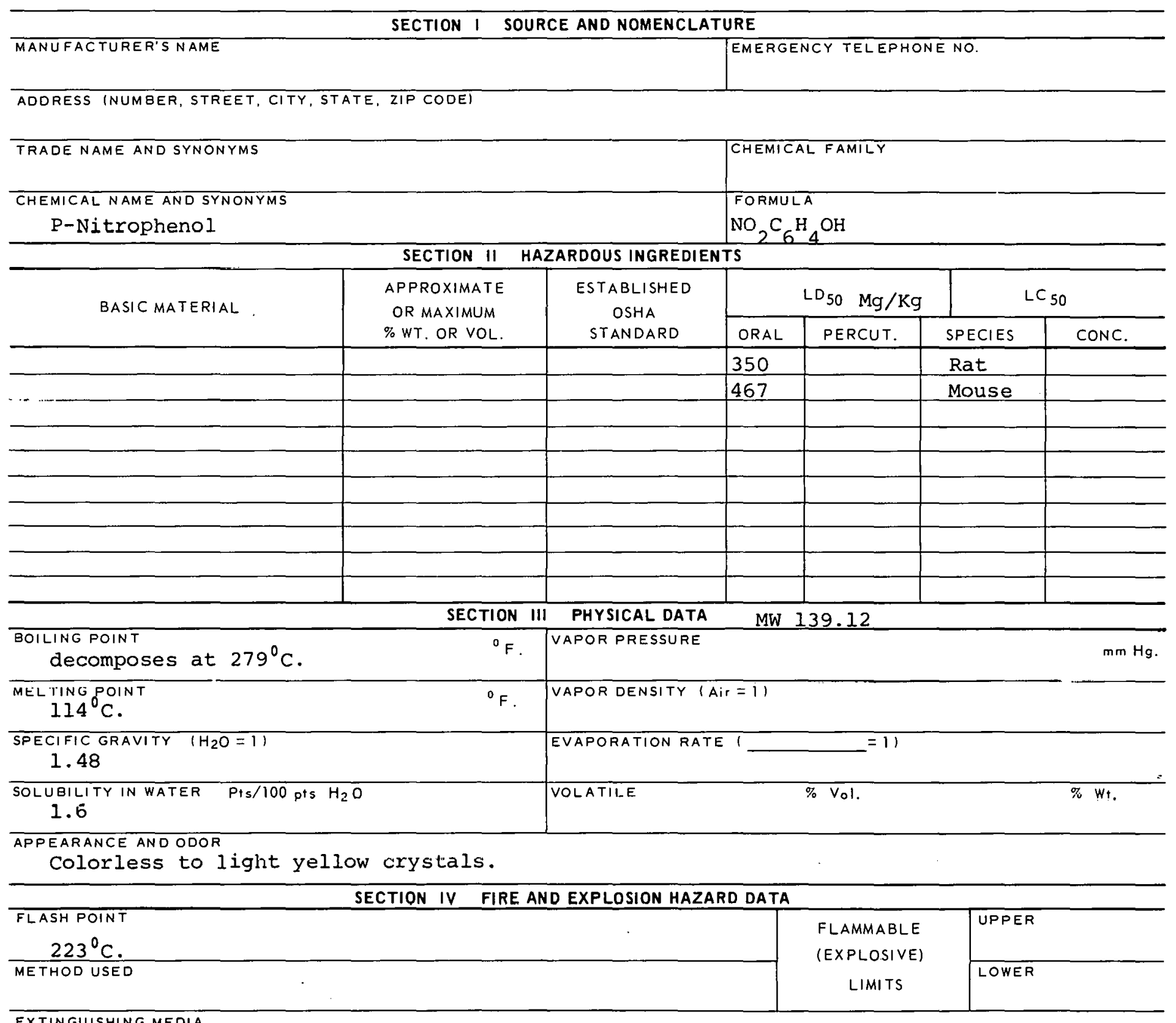

EXTINGUISHING MEDIA

Use water to wet down containers.

SPECIAL FIRE FIGHTING PROCEDURES

Wear full protective clothing, self-contained breathing apparatus.

UNUSUAL FIRE AND EXPLOSION HAZARDS

When heated to decomposition, gives off toxic nitruyen uxide funes. 


\begin{tabular}{ccc}
\hline & SECTION V HEALTH HAZARD DATA & \\
\hline TOXIC LEVEL & & CARCINOCENIC \\
None availlable & No \\
\hline $\begin{array}{c}\text { PRINCIPLE ROUTES OF ABSORBTION } \\
\text { Inhalation, ingestion, skin }\end{array}$ & SKIN ANDEYE \\
IRRITATION & Moderate \\
\hline
\end{tabular}

REVELANT SYMPTOMS OF EXPOSURE

Eye and mucous membrane irritation, CNS depressant.

EFFECTS OF CHRONIC EXPOSURE

Forms methemoglobin, dermatitis.

EMERGENCY AND FIRST AID PROCEDURES Inhalation remove by gastric lavage or emesis.

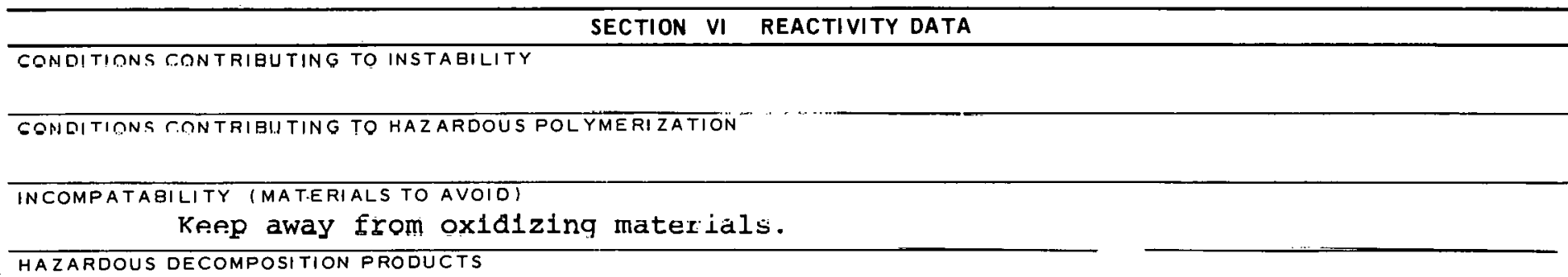

SECTION VII SPILL OR LEAK PROCEDURES

STEFS TO BE TAKEN IN CASF MATERIAL IS RELEASED OR SPILLED

WASTE DISPOSAL METHOD

\section{SECTION VIII SPECIAL PROTECTION INFORMATION}

\begin{tabular}{|c|c|c|}
\hline VFNTII ATIQN REQUIREMENTS LOCAL EXHAUST & & $\begin{array}{l}\text { PROTECTIVE EQUIPMENT (SPECIFY TYPES) EYE } \\
\text { GOgg LES }\end{array}$ \\
\hline MECHANICAL (GENERAL) & & $\begin{array}{l}\text { GLOVES } \\
\text { Rutyl Rubber }\end{array}$ \\
\hline SPECIAL & & $\begin{array}{l}\text { RESPIRATOOR } \\
\text { Self-contained }\end{array}$ \\
\hline \multicolumn{2}{|l|}{ Protective clothing } & \\
\hline \multicolumn{3}{|c|}{ SECTION IX SPECIAL PRECAUTIONS } \\
\hline \multicolumn{3}{|c|}{$\begin{array}{l}\text { PRECAUTIONS TO BE TAKEN IN HANDLING AND STORAGE } \\
\text { Keep from combustible materials. }\end{array}$} \\
\hline \multicolumn{3}{|l|}{ OTHER PRECAUTIONS } \\
\hline SIGNATURE & DATE & \\
\hline
\end{tabular}



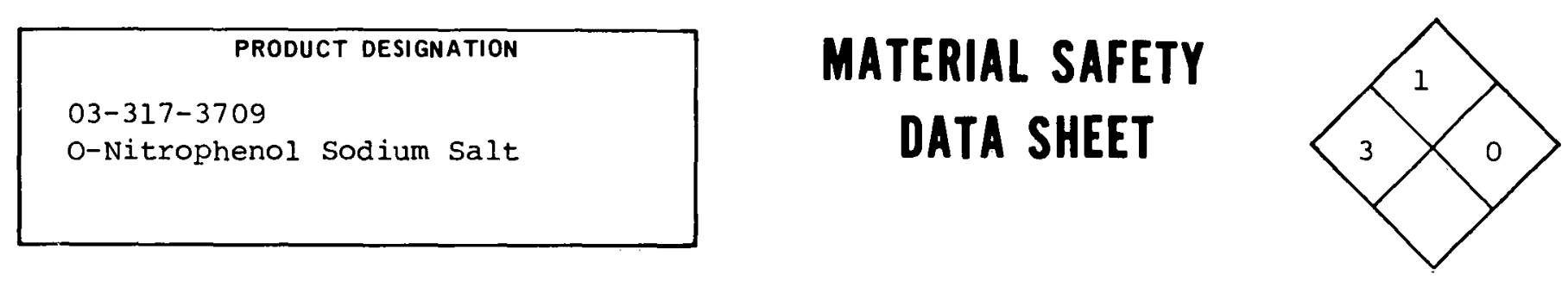

SECTION I SOURCE AND NOMENCLATURE

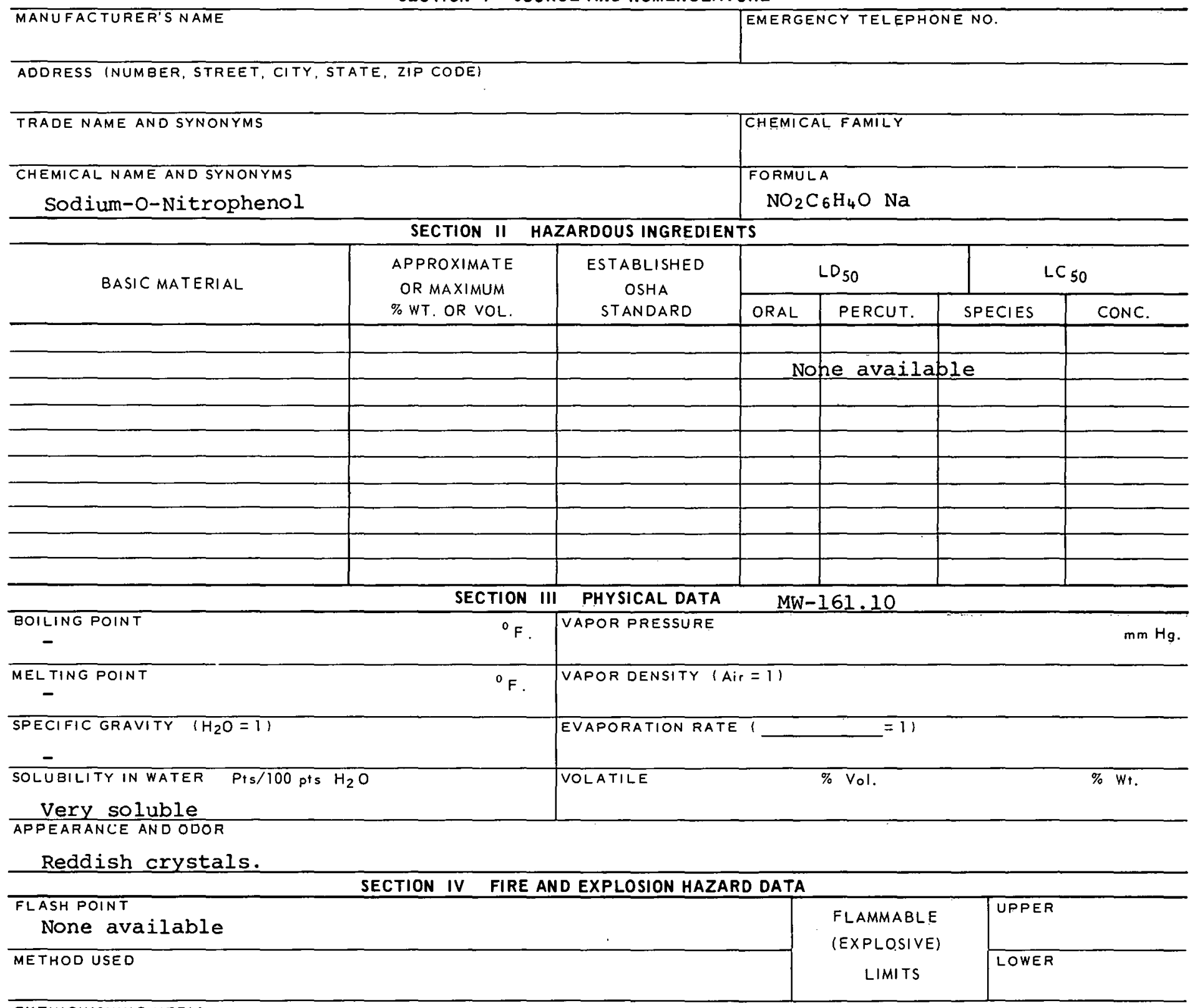

EXTINGUISHING MEDIA

Use water to wet down.containers.

SPECIAL FIRE FIGHTING PROCEDURES Wear full protective clothing; self-contained

breathing apparatus.

UNUSUAL FIRE AND EXPLOSION HAZAROS

oxide fumes.

When heated to decomposition give off toxic nitrogen 


\section{TOXIC LEVEL}

None available

PRINCIPLE ROUTES OF ABSORBTION

Inhalation, ingestion, skin REVELANT SYMPTOMS OF EXPOSURE

EFFECTS OF CHRONIC EXPOSURE

Forms methemoglobin, dermatitis

EMERGENCY AND FIRST AID PROCEDURES inhalation - rest; wash from eyes \& skin; if swallowed remove by gastric lavage or emesis.

\section{CONDITIONS CONTRIBUTING TO INSTABILITY \\ CONDITIONS CONTRIBUTING TO HAZARDOUS POL YMERIZATIUN \\ Keep from oxidizing materials.}

HAZAROOUS DECOMPOSITION PRODUCTS

SECTION VII SPILL OR LEAK PROCEDURES

STEPS TO BE TAKEN IN CASE MATERIAL IS RELEASED OR SPILLED

SECTION VIII SPECIAL PROTECTION INFORMATION

\begin{tabular}{l|l}
\multicolumn{1}{c}{ SECTION VIII SPECIAL PROTECTION INFORMATION } \\
\hline VENTILATION REQUIREMENTS LOCAL EXHAUST & $\begin{array}{c}\text { PROTECTIVE EQUIPMENT (SPECIFY TYPES) EYE } \\
\text { GOggles }\end{array}$ \\
\hline MECHANICAL (GENERAL) & $\begin{array}{c}\text { GLOVES } \\
\text { ButYl Rubber }\end{array}$ \\
\hline SPECIAL & RESPIRATOR \\
& Self-contained \\
\hline
\end{tabular}

OTHER PROTECTIVE EQUIPMENT

Protective clothing

SECTION IX SPECIAL PRECAUTIONS

PRECAUTIONS TO BE TAKEN IN HANDLING ANO STORAGE

Keep from combustible materials.

OIHEK RHELAUTIONS

SIGNATURE

$\overline{D A} \overline{T E}$

$317-3709 B$ 


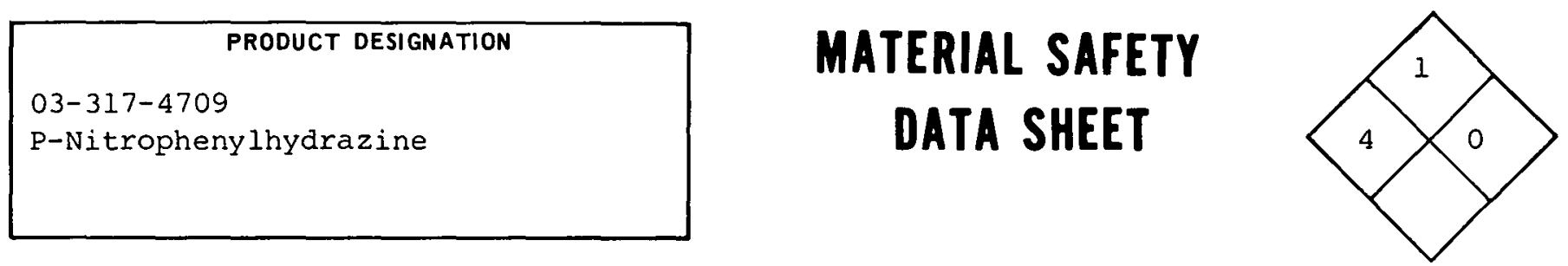

\section{SECTION I SOURCE AND NOMENCLATURE}

MANUFACTURER'S NAME
ADDRESS INUMBER, STREET, CITY,
TRADE NAME AND SYNONYMS
CHEMICAL NAME AND SYNONYMS
P-NitrOPhENYlhYdYaZine

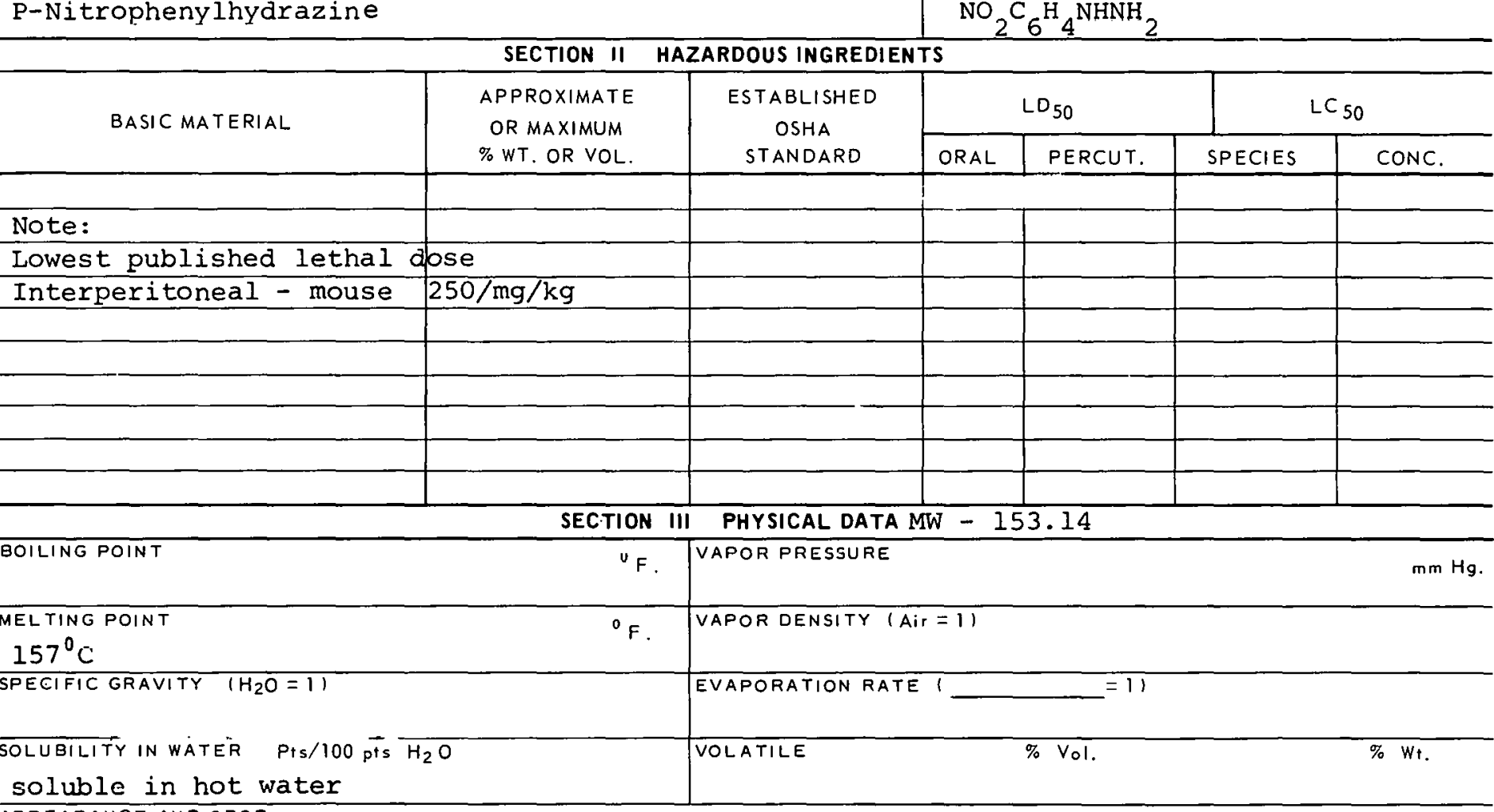

APPEARANCE AND ODOR

orange-red crystals

SECTION IV FIRE AND EXPLOSION HAZARD DATA

FLASH POINT

none available

METHOD USEO

\begin{tabular}{l|l} 
& CHEMICAL FAMILY \\
& $\begin{array}{l}\text { FORMULA } \\
\mathrm{NO}_{2} \mathrm{C}_{6} \mathrm{H}_{4} \mathrm{NHNH}_{2}\end{array}$ \\
\hline
\end{tabular}




\section{TOXIC LEVEL}

none available

CARCINOGENIC

PRINCIPLE ROUTES OF ABSORBTION

Inhalation, ingestion, skin

SKIN AND EYE

IRRITATION Severe

REVELANT SYMPTOMS OF EXPOSURE Irritation of skin, eyes and mucous membranes, gastroentric

disturbances, anemia.

EFFECTS OF CHRONIC EXPOSURE

Blood and liver damage, loss of appetite.

EMERGENCY AND FIRST AID PROCEDURES If swallowed - induce vomiting, get medical aid;

inhalation - rest; wash from skin \& eyes.

\section{SECTION VI REACTIVITY DATA}

CONDITIONS CONTRIBUTING TO INSTABILITY

CONDITIONS CONTRIBUTING TO HAZARDOUS POLYMERIZATION

-

INEUMPATABILIT' (MATERIAL3 TO AVOID.)

IIAZARDQUE DESOMROEITIOM PROQUETS

SECTION VII SPILL OR LEAK PROCEDURES

STEPS TO BE TAKEN IN CASE MATERIAL IS RELEASED OR SPILLED

SECTION VIII SPECIAL PROTECTION INFORMATION

\begin{tabular}{l|l}
\hline VENTILATION REQUIREMENTS LOCAL EXHAUST & PROTECTIVE EQUIPMENT (SPECIFY TYPES) EYE \\
\hline MECHANICAL IGENERAL) & GLOGglCE \\
\hline SPECIAL & Rubber \\
\hline
\end{tabular}

OTHER PROTECTIVE EQUIPMENT

SECTION IX SPECIAL PRECAUTIONS

PRECAUTIONS TO BE TAKEN IN HANDLING AND STORAGE

OTHER PRECAUTIONS

SIGNATURE

DATE

$317-4709 B$ 
P-Nitrophenylhydrazine

Hydroch loride

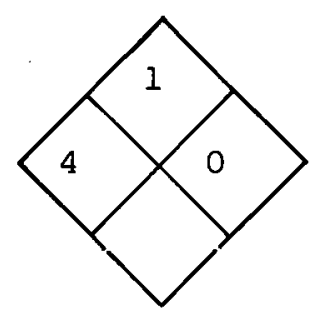

\section{SECTION I SOURCE AND NOMENCLATURE}

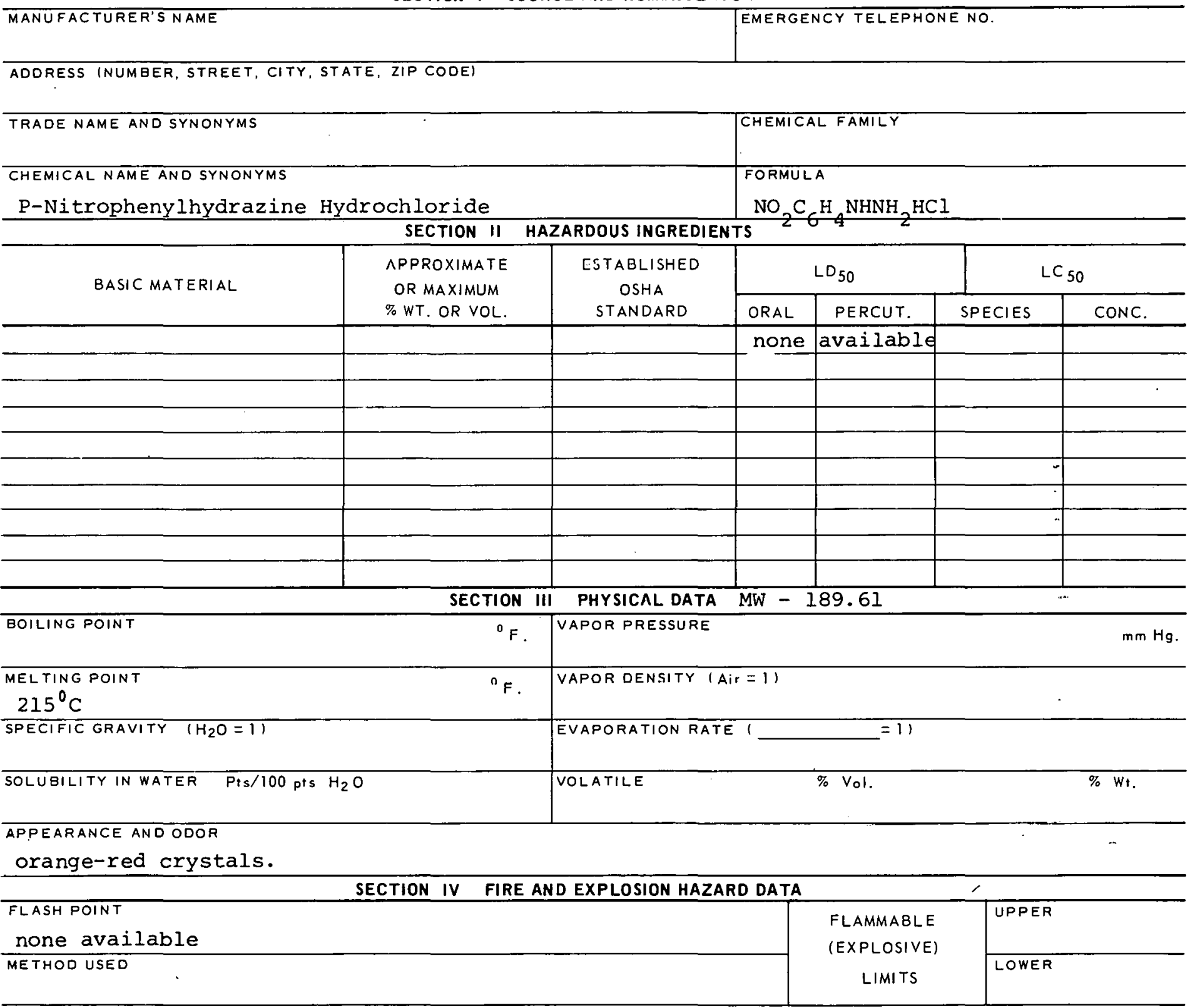

EXTINGUISHING MEDIA

information not available.

SPECIAL FIRE FIGHTING PROCEDURES

UNUSUAL FIRE AND EXPLOSION HAZARDS 


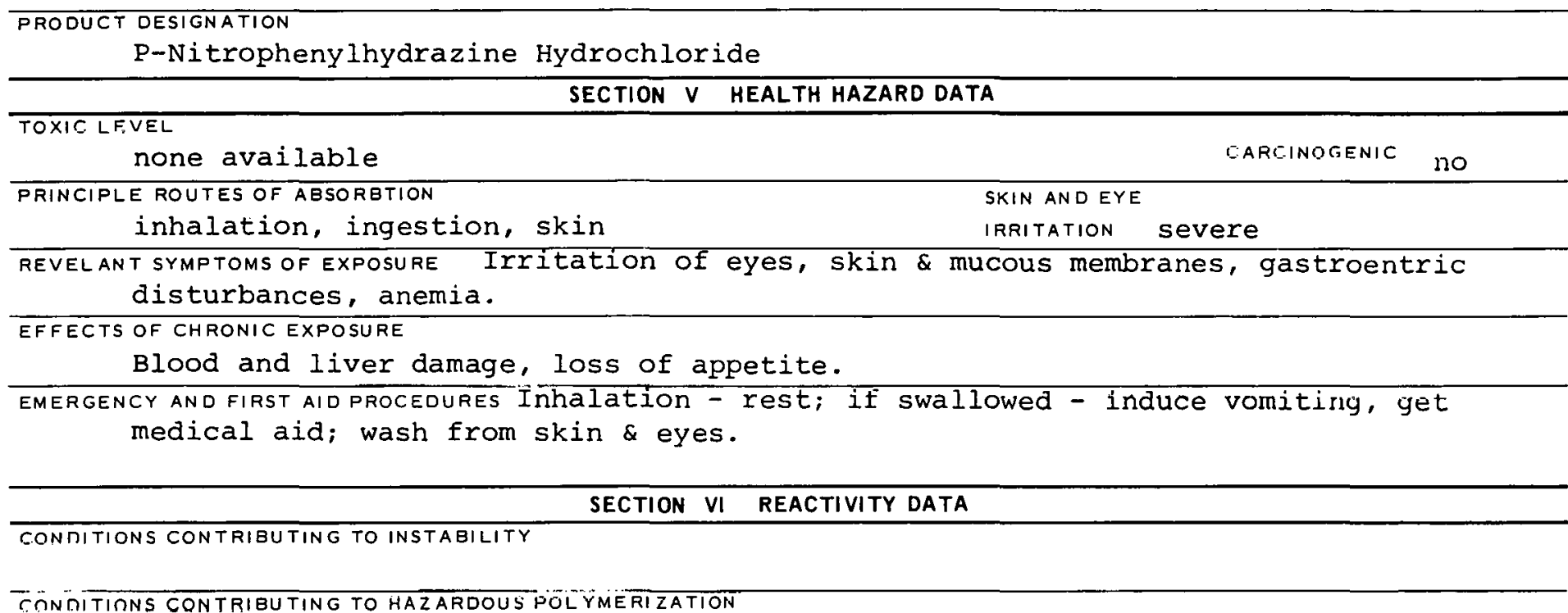

INCOMPATABILITY IMATERIALS TO AVOIDI
HAZARDOUS DECOMPOSITION PRODUCTS
STEPS TO BE TAKEN IN CASE MATERIAL IS RELEASED OR SPILLED
SECTION VII SPILL OR LEAK PROCEDURES

STEPS TO BE TAKEN IN CASE MATERIAL IS RELEASED OR SPILLED

WASTE DISPOSAL METHOD

SECTION VIII SPECIAL PROTECTION INFORMATION

\begin{tabular}{l|l|l}
\hline SECTION VIII SPECIAL PROTECTION INFORMATION \\
\hline VENTILATION REQUIREMENTS LOCAL EXHAUST & $\begin{array}{l}\text { PROTECTIVE EQUIPMENT (SPECIFY TYPES) EYE } \\
\text { Goggles }\end{array}$ \\
\hline MECHANICAL IGENERAL) & $\begin{array}{l}\text { GLOVES } \\
\text { Rubber } \\
\text { SPECIAL }\end{array}$ & $\begin{array}{l}\text { RESPIRATOR } \\
\text { Self-contained }\end{array}$ \\
\hline
\end{tabular}

ÖTHER PROTECTIVE EQUIPMENT

\section{SECTION IX SPECIAL PRECAUTIONS}

PRECAUTIONS TO BE TAKEN IN HANDLING AND STORAGE

OTHER PRECAUTIONS

SIGNATURE

DATE

$317-4909 B$ 


PRODUCT DESIGNATION
03-317-6209
1 -Nitropropane

\section{MATERIAL SAFETY DATA SHEET}

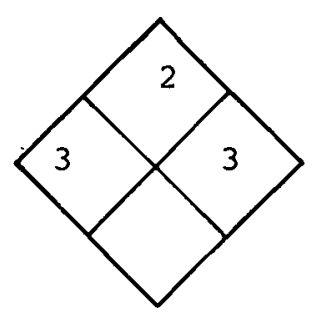

\section{SECTION I SOURCE AND NOMENCLATURE}

\begin{tabular}{l} 
MANU FACTURER'S NAME \\
ADDRESS INUMBER, STREET, CITY \\
TRADE NAME AND SYNONYMS \\
CHEMICAI.NAME AND SYNONYMS \\
I-NitrOPYOPANE \\
\hline
\end{tabular}

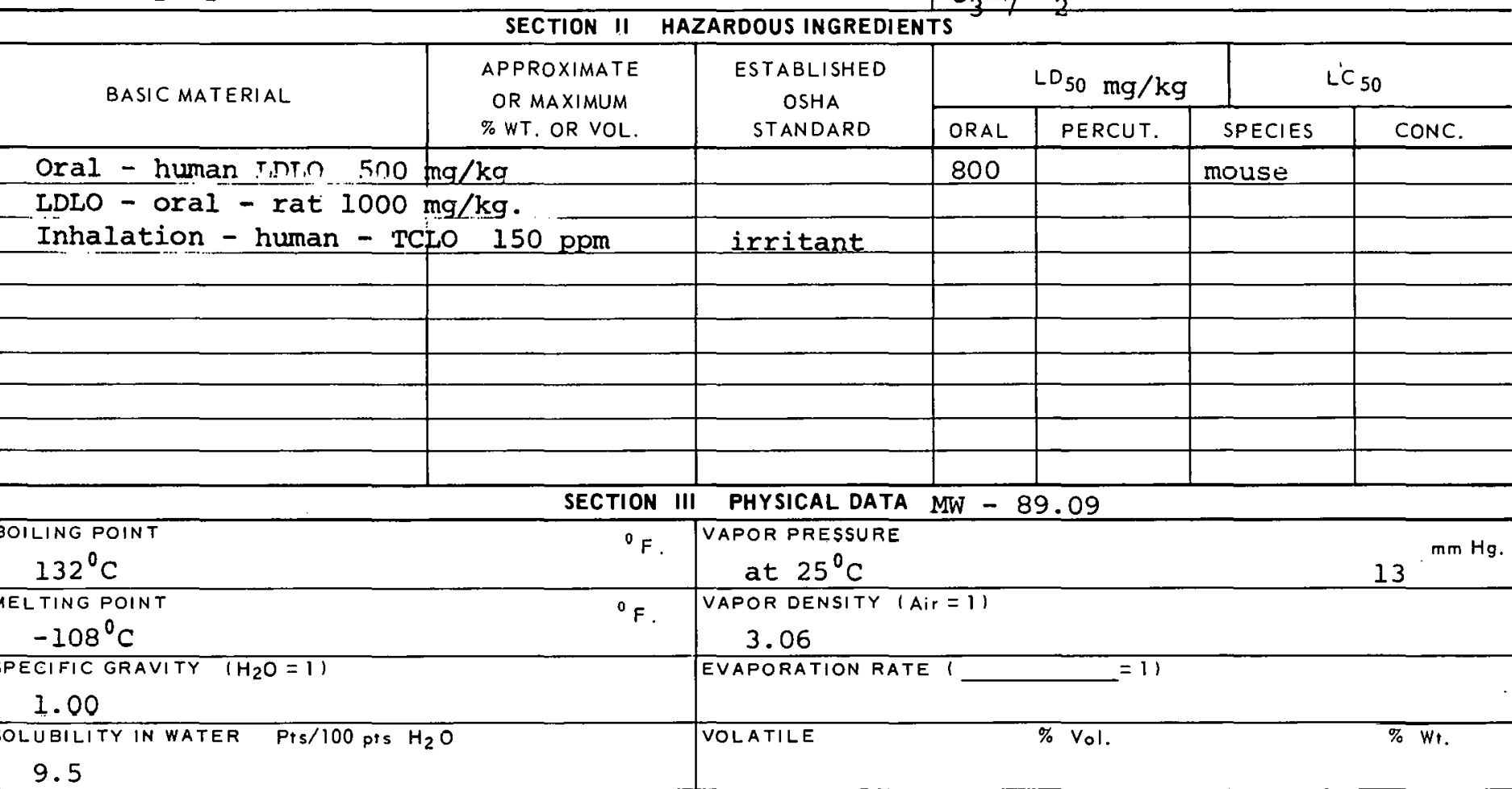

APPEARANCE AND ODOR

colorless liquid, with disagreeable odor.

\begin{tabular}{l|c|c}
\hline & SECTION IV FIRE AND EXPLOSION HAZARD DATA \\
\hline FLASH POINT & FLAMMABLE & UPPER \\
$120^{\circ} \mathrm{F}$ & (EXPLOSIVE) & LOWER \\
\hline METHOD USED & LIMITS & . \\
T.O.C. & & \\
\hline
\end{tabular}

EXTINGUISHING MEDIA Alcohol foam, carbon dioxide, dry chemical or

water spray.

SPECIAL FIRE FIGHTING PROCEDURES

Wear protective clothing and self-contained breathing apparatus.

UNIISUAL FIRE ANDEXPLOSION HAZAROS 
TOXIC LEVEL

$T L V$ - 25 PPM, $90 \mathrm{mg} / \mathrm{C.M}$. PRINCIPLE ROUTES OF ABSORBTION

Inhalation, ingestion

CARCINOGENIC

CARCINOGENIC nO
SKIN AND EYE

IRRITATION moderate

REVELANT SYMPTOMS OF EXPOSURE Headache, dizziness, nausea, vomiting, diarrhea, restlessness, muscular uncoordination.

EFFECTS OF CHRONIC EXPOSURE

Liver, kidney and heart damage.

EMERGENCY AND FIRST AID PROCEDURES If swallowed - induce vomiting, get medical aid,

gastric lavage; inhalation - artificial respiration, oxygen as needed, wash skin \& eyes.

SECTION VI REACTIVITY DATA

CONDITIONS CONTRIBUTING TO INSTABILITY

FINARITIRNS FONTRIRIITING TO HAZARDOUS POLYMERIZATION

INCOMPATABILITY (MATE RIALS TO AVOID)

Unstable with amines, acids, alkalis.

HAZARDOUS DECOMPOSITION PRODUCTS

SECTION VII SPILL OR LEAK PROCEDURES

STEPS TO BE TAKEN IN CASE MATERIAL IS RELEASED OR SPILLED

After mixing spill with soda ash, spray with water, neutralize with

WASTE OISPOSAL METHOD

SECTION VIII SPECIAL PROTECTION INFORMATION

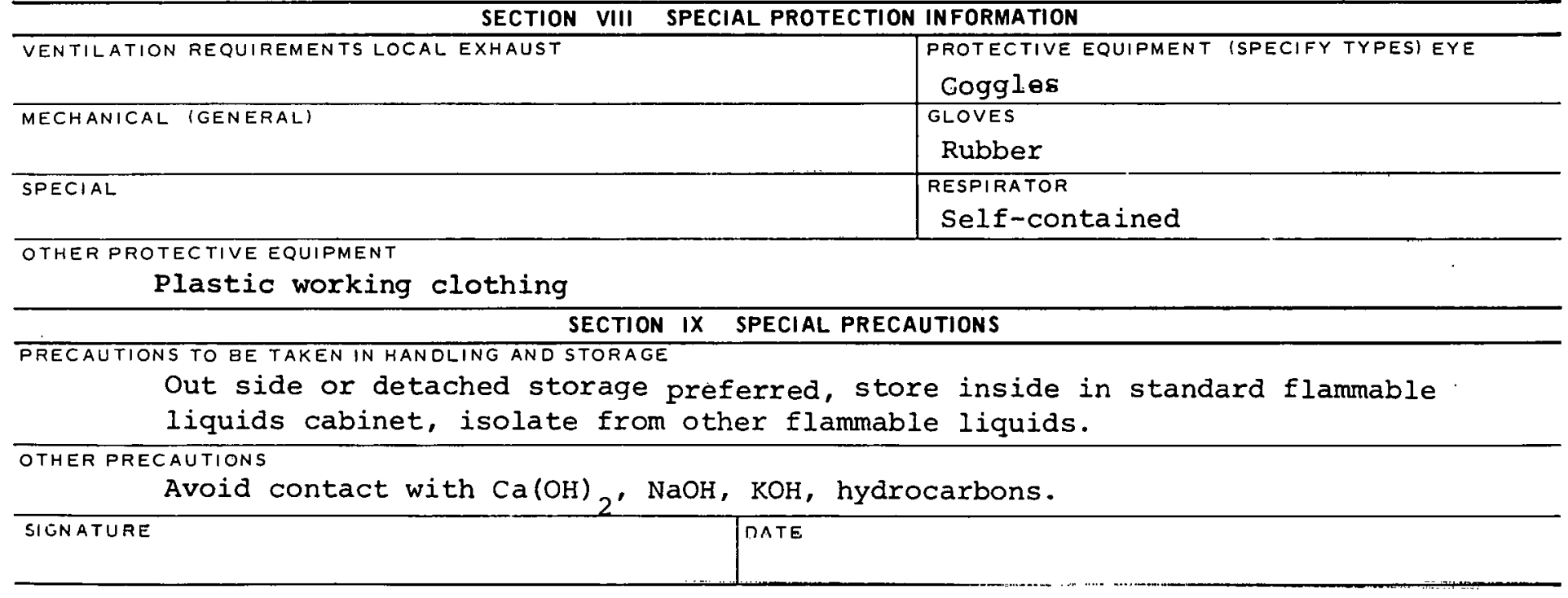


CAS : 000079469

\section{PRODUCT DESIGNATION}

$03-317-6309$

2-Nitropropane
MATERIAL SAFETY

DATA SHEET

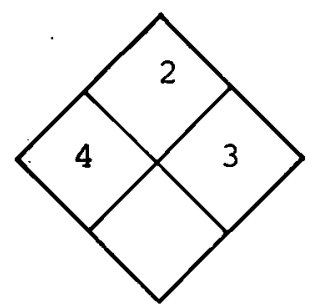

\section{SECTION I SOURCE AND NOMENCLATURE}

\section{MANUFACTURER'S NAME \\ TRADE NAME AND SYNONYMS \\ CHEMICAL NAME AND SYNONYMS \\ 2-Nitropropane}

ADDRESS (NUMBER, STREET, CITY, STATE, ZIP CODE)
BASIC MATERIAL

SECTION II HAZARDOUS INGREDIENTS

CHEMICAL FAMILY
FOAMULA
$\mathrm{C}_{3} \mathrm{H}_{7} \mathrm{NO}_{2}$

SECTION II HAZARDOUS INGREDIENTS

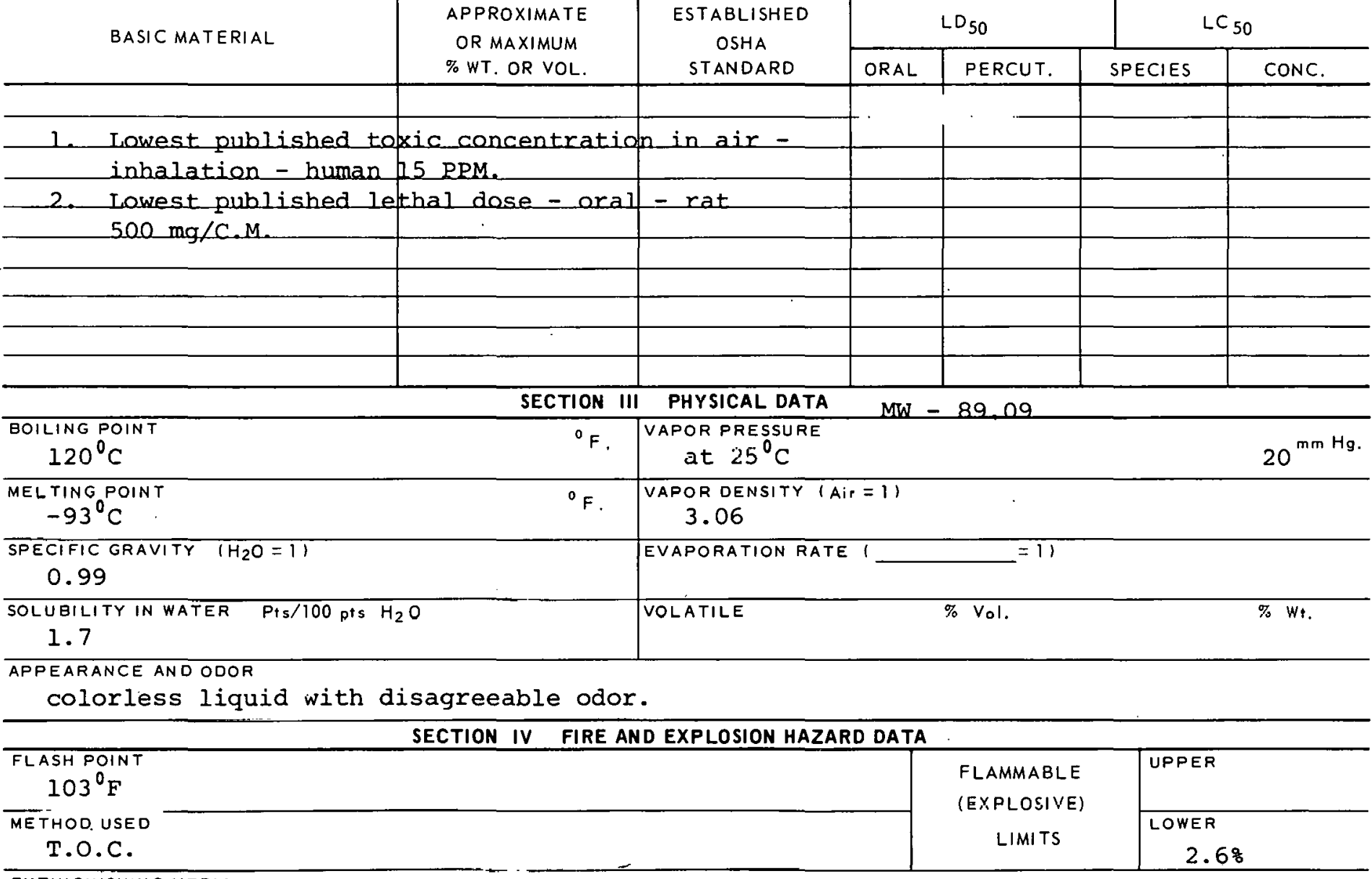

EXTINGUISHING MEDIA

Alcohol foam, carbon dioxide', dry chemical or water spray.

SPECIAL FIRE FIGHTING PROCEDURES

Wear protective clothing and self-contained breathing apparatus.

UNUSUAL FIRE AND E'XFLUUSIUN HALARUS 


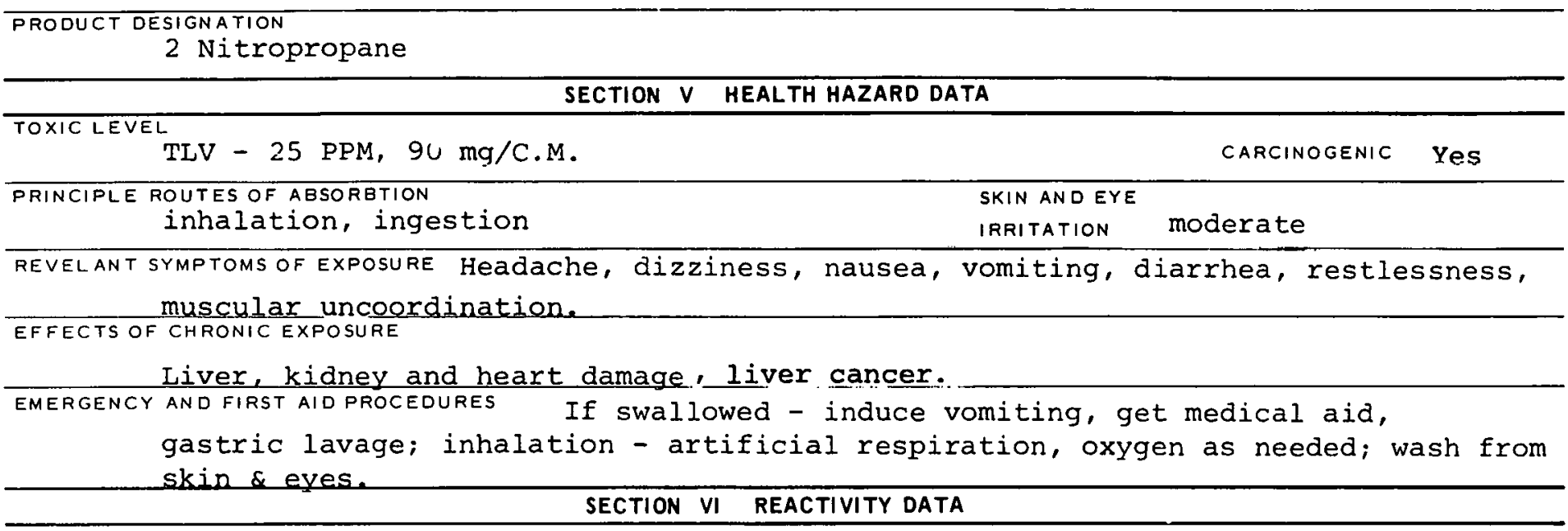

CONDITIONS CONTRIBUTING TO INSTABILITY

CONDITIQNS CONTRIBUTING TO HAZARDOUS POLYMERIZATION

INCOMPATABILITY (MATERIALS TO AVOID)

Unstable with amines, acids, alkalis.

HAZAROOUS DECOMPOSITION PRODUCTS

SECTION VII SPILL OR LEAK PROCEDURES

STEPS TO BE TAKEN IN CASE MATERIAL IS RELEASED OR SFILLED

After covering spill with soda ash, spray with water and neutralize with HCl.

WASTE.DISPOSAL METHOD

SECTION VIII SPECIAL PROTECTION INFORMATION

\section{SECTION IX SPECIAL PRECAUTIONS}

PRECAUTIONS TO BE TAKEN IN HANDLING AND STORAGE

Outside or detached storage preferred, store inside in standard flammable

liquids cabinet, isolate from other flammable liquids.

UTHER PREGAUTIONS

Avoid contact with $\mathrm{Ca}\left(\mathrm{OH}_{2}\right), \mathrm{NaOH}, \mathrm{KoH}$, hydrocarbon. 


\section{PRODUCT DESIGNATION}

03-317-7209

2-Nitroresorcinol
MATERIAL SAFETY

DATA SHEET

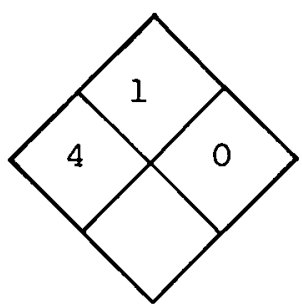

SECTION I SOURCE AND NOMENCLATURE

\begin{tabular}{|l|l|l}
\hline MANUFACTURER'S NAME & EMERGENCY TELEPHONE NO. \\
\hline
\end{tabular}

ADDRESS (NUMBER, STREET, CITY, STATE, ZIP CODE)

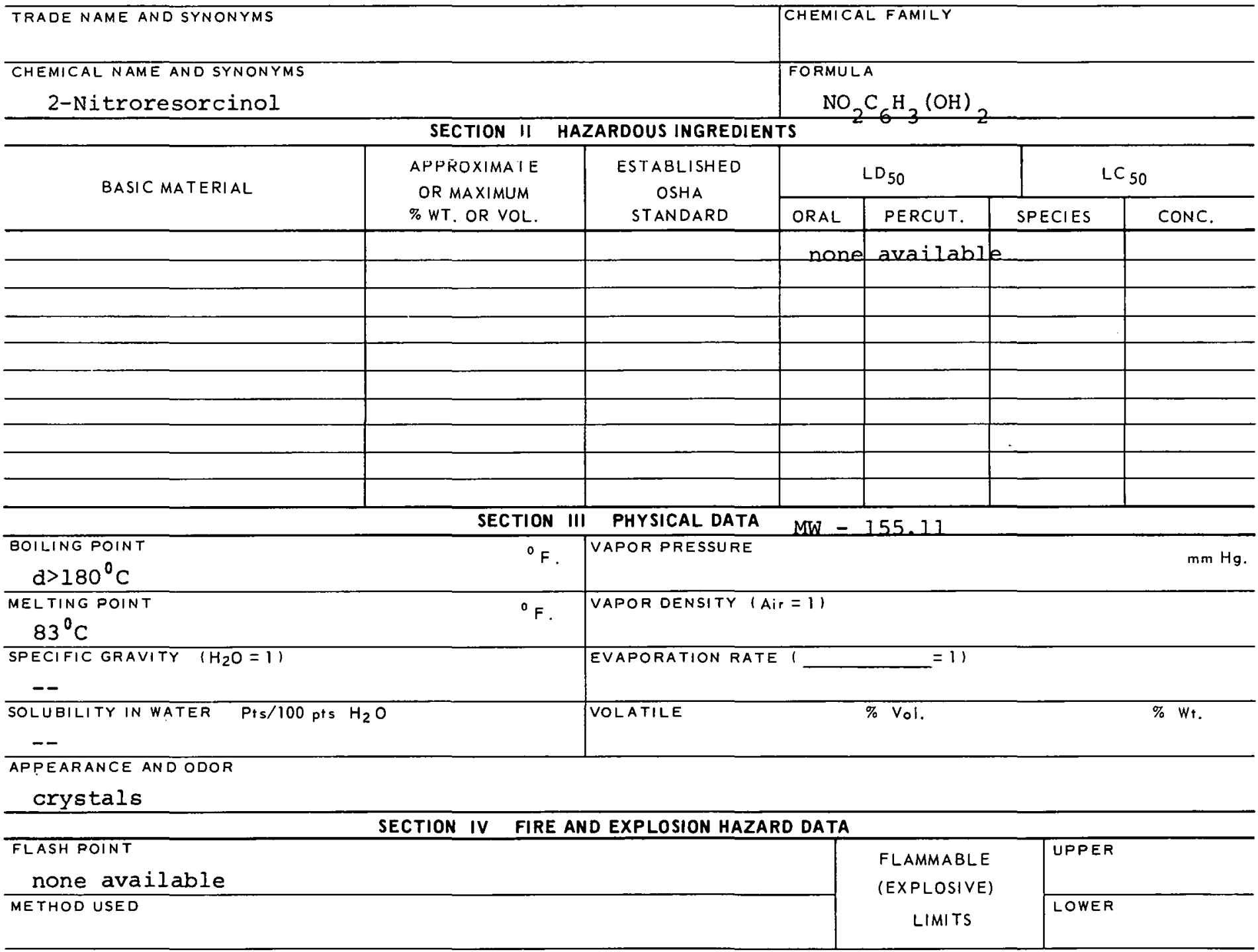

EXTINGUISHING MEDIA

information not available

SPECIAL FIRE FIGHTING PROCEDURES

UNUSUAL FIRE AND EXPLOSION HAZARDS 


\section{TOXIC LEVEL}

none available

PRINCIPLE ROUTES OF ABSORBTION

inhalation, ingestion, skin

CARCINOGENIC

SKIN AND EYE

IRRITATION moderate

REVELANT SYMPTOMS OF EXPOSURE Itching, dermatitis, restlessness, cyanosis, dizziness,

tremors, convulsion and death.

EFFECTS OF CHRONIC EXPOSURE

Liver, kidney and lung damage.

EMERGENCY AND FIRST AIO PROCEDURES If swallowed - induce vomiting, get medical aid;

inhalation - rest; wash skin \& eyes.

\section{SECTION VI REACTIVITY DATA}

CONDITIONS CONTRIBUTING TO INSTABILITY

CONDITIONS CONTRIBUTING TO HAZARDOUS POLYMERIZATION

INCOMPATABILITY (MATERIALS TO AVOID)

HAZARDOUS DECOMPOSITION PRODUCTS

SECTION VII SPILL OR LEAK PROCEDURES

STEPS TO BE TAKEN IN CASE MATERIAL IS RELEASED OR SPILLED

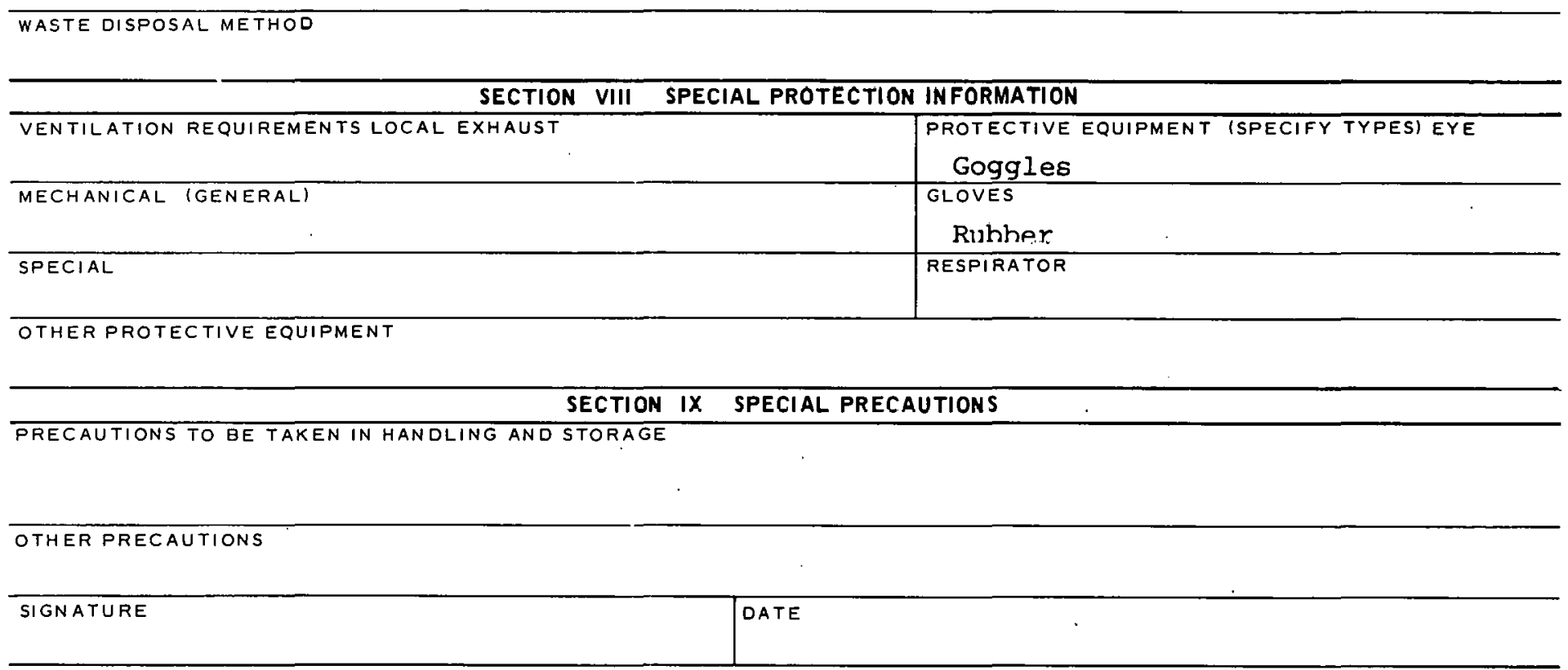




\begin{tabular}{|c|}
\hline \multicolumn{1}{|c|}{ PRODUCT DESIGNATION } \\
03-317-8009 \\
P-Nitrosodiethylaniline \\
\hline
\end{tabular}

\section{MATERIAL SAFETY DATA SHEET}

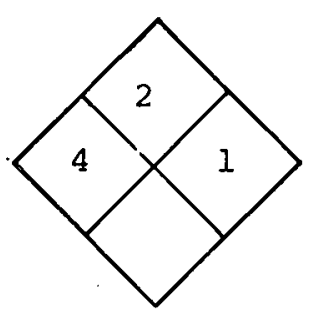

\section{SECTION I SOURCE AND NOMENCLATURE}

MANUFACTURER'S NAME
ADDRESS INUMBER, STREET, CITY, STATE,
TRADE NAME AND SYNONYMS
N, N-DiethYl-P-Nitrosoanilin
CHEMICAL NAME AND SYNONYMS
P-Nitrosodiethyaniline.

EMERGENCY TELEPHONE NO.

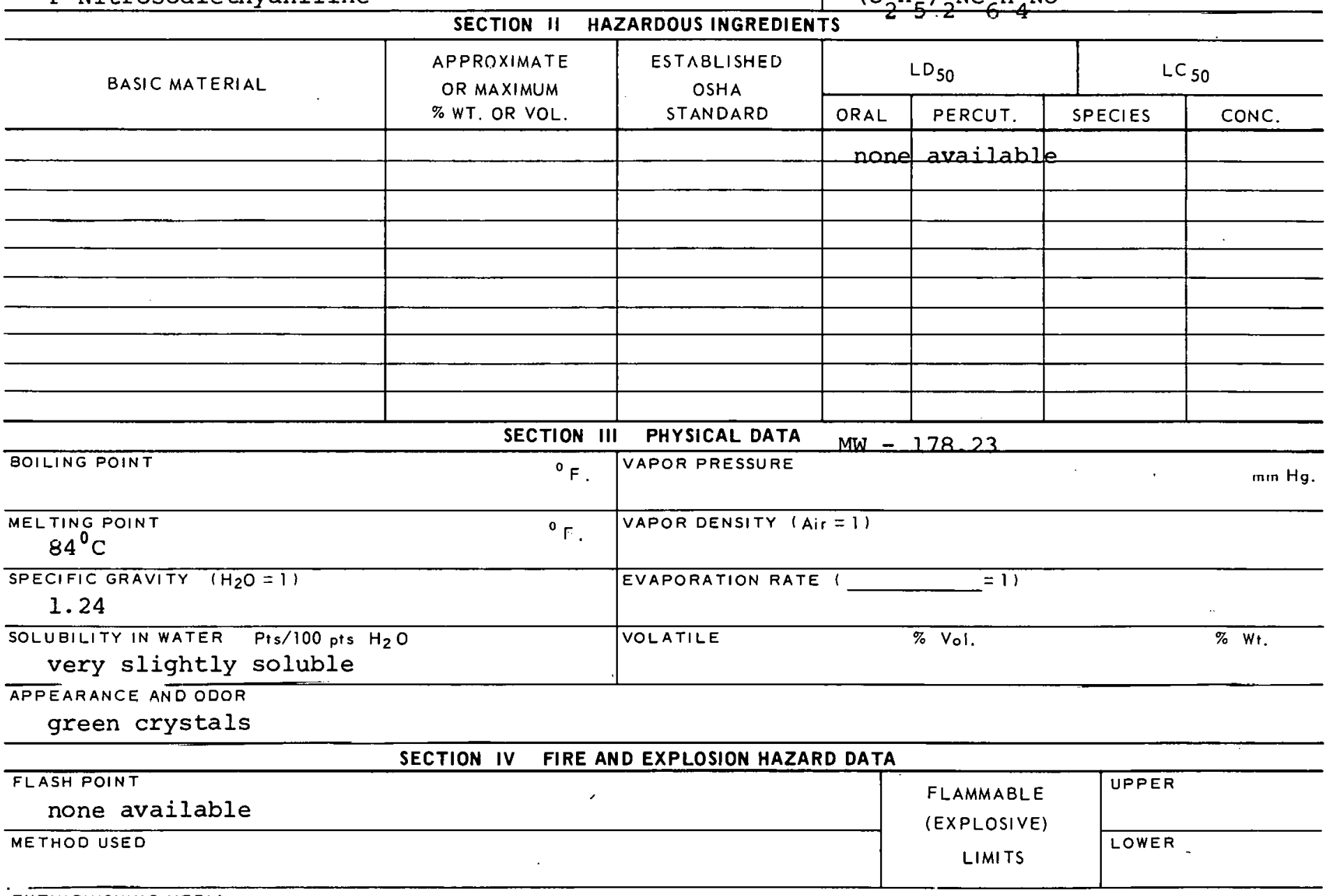

EXTINGUISHING MEDIA

Alcohol foam, water spray, carbon dioxide and dry chemical.

SPECIAL FIRE FIGHTING PROCEDURES

UNUSUAL FIRE AND EXPLOSION HAZARDS 


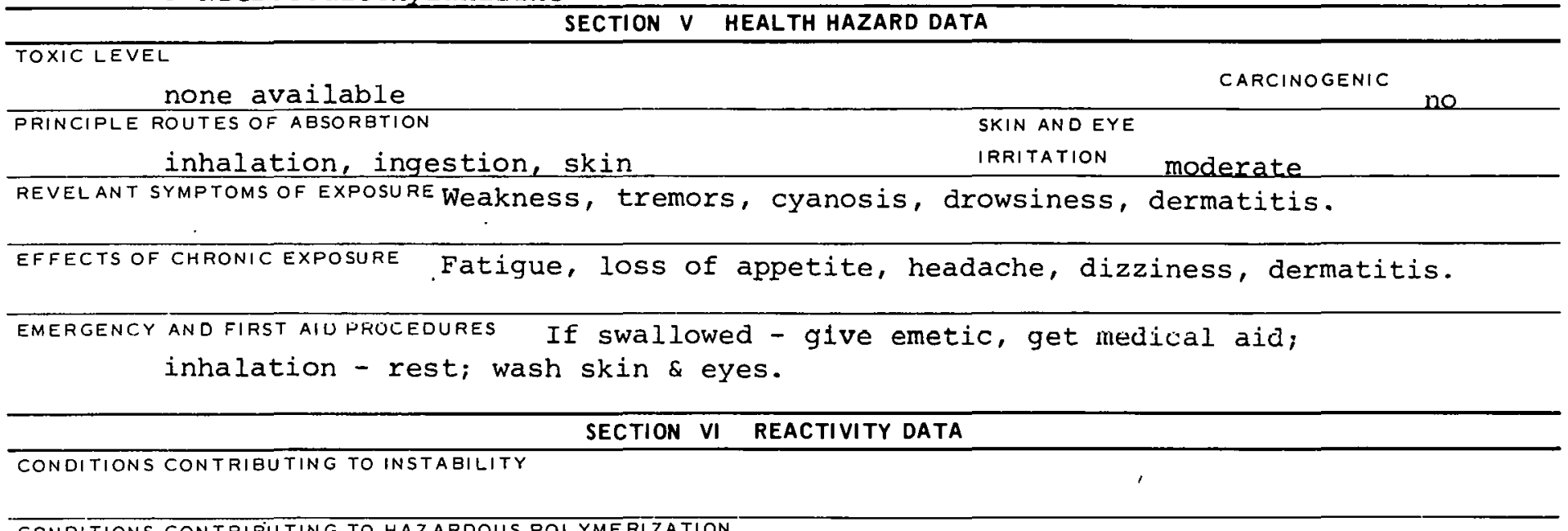

CONOITIOAS GOMTRIRIITING TR HAZARDOUS POLLYMERIZATION

INCOMPATABILITY (MATERIALS TO AVOID)

HAZARDOUS DECOMPOSITION PROUULTS

SECTION VII SPILL OR LEAK PROCEDURES

STEPS TO BE TAKEN IN CASE MATERIAL IS RELEASED OR SPILLED

\section{WASTE DISPOSAL METHOD}

\section{SECTION VIII SPECIAL PROTECTION INFORMATION}

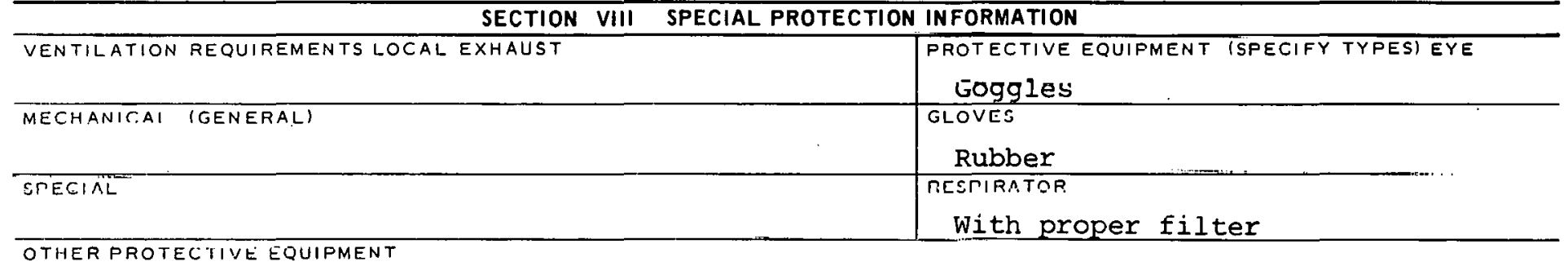




\section{PRODUCT DESIGNATION}

03-317-8209

P-Nitrosodimethylaniline
MATERIAL SAFETY DATA SHEET

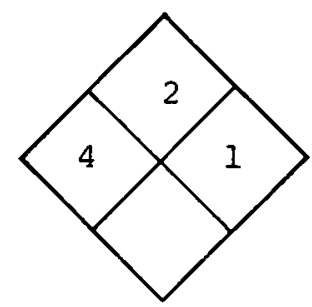

SECTION I SOURCE AND NOMENCLATURE

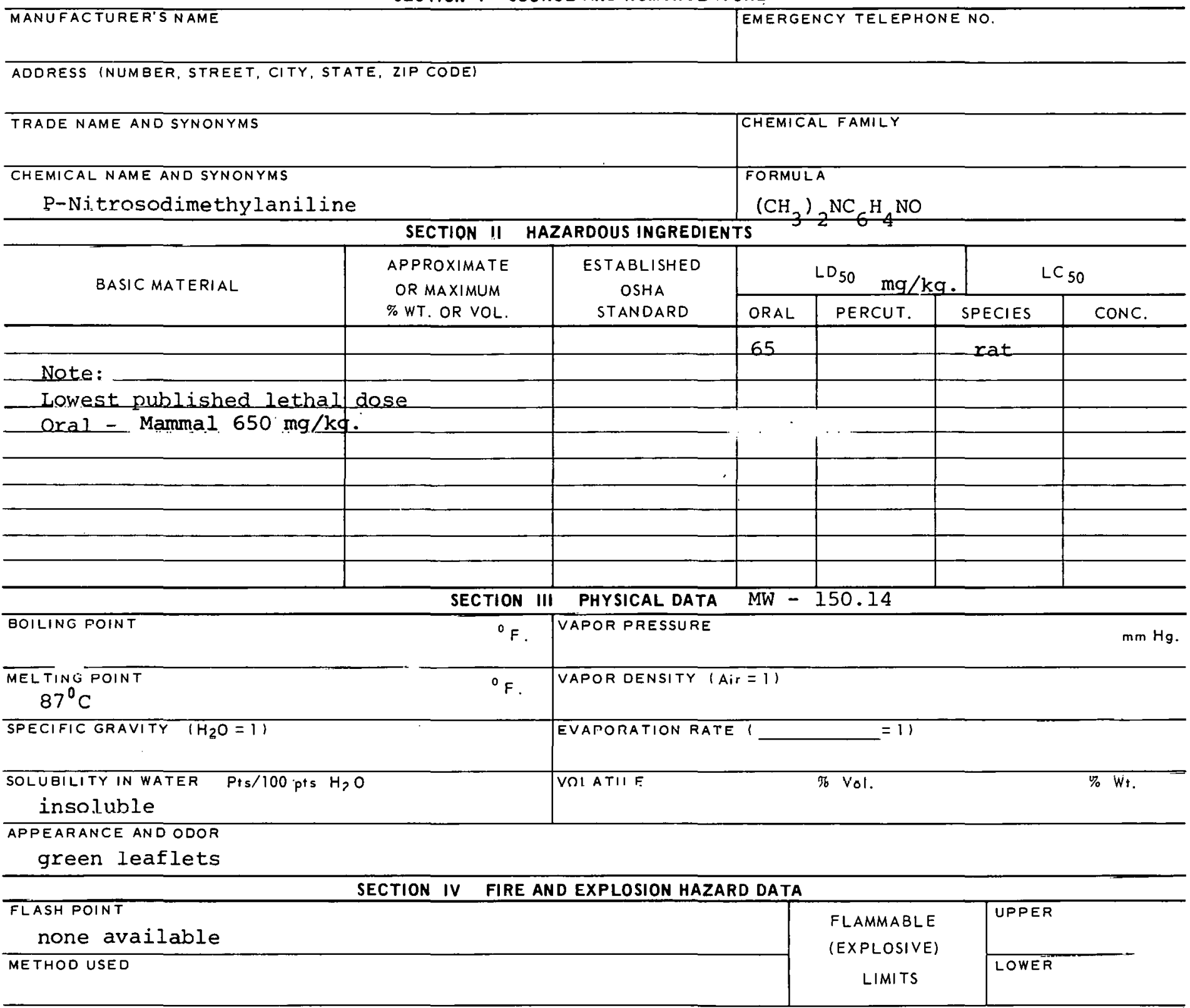

Extinguishing media Alcohol foam, dry cliemical, carbon dioxide and water spray.

SPECIAL FIRE FIGHTING PROCEDURES

UNUSUAL FIRE AND EXPLOSION HAZARDS 


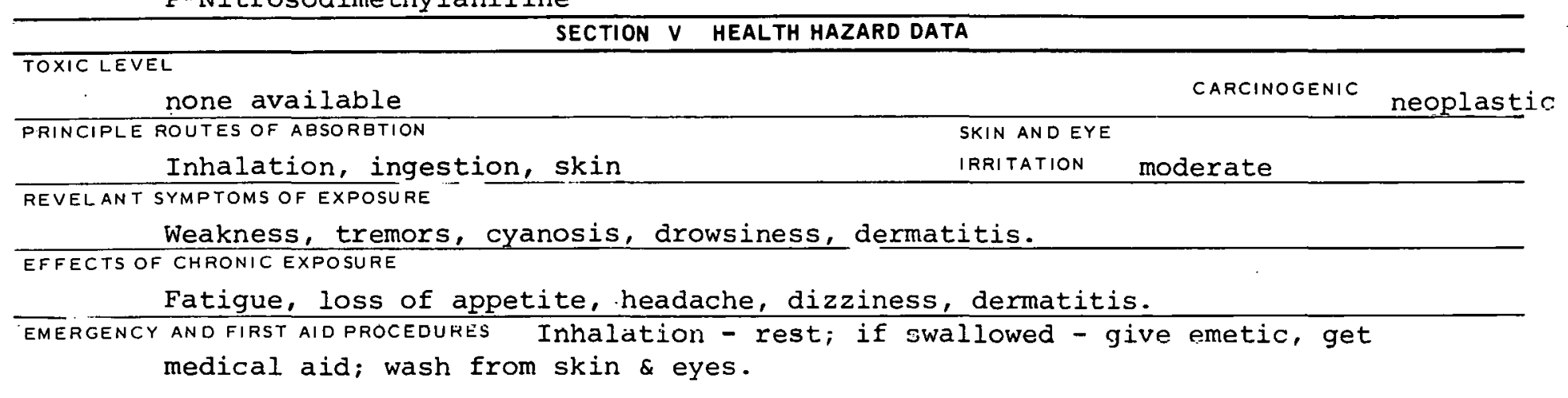

\section{SECTION VI REACTIVITY DATA}

CONDITIONS CONTRIBUTING TO INSTABILITY

CONDITIONS CONTRIBUTING TO HAZARDOUS POLYMERIZATION

INCOMPATABILITY (MATERIALS TO AVOIO)

HAZARDOUS DECOMPOSITION PRODUCTS

SECTION VII SPILL OR LEAK PROCEDURES

STEPS TO BE TAKEN IN CASE MATERIAL IS RELEASED OR SPILLED

WASTE DISPOSAL METHOD

SECTION VIII SPECIAL PROTECTION INFORMATION

\begin{tabular}{l|l} 
SECTION VIII SPECIAL PROTECTION INFORMATION \\
\hline MENTILATION REQUIREMENTS LOCAL EXHAUST & $\begin{array}{l}\text { PROTECTIVE EQUIPMENT ISPECIFY I'YPESI EYE } \\
\text { COggles }\end{array}$ \\
\hline SPELIAL & $\begin{array}{c}\text { GLOVES } \\
\text { Rubber }\end{array}$ \\
\hline
\end{tabular}

OTHER PRUTECTIVE EQUIPMENT

\section{SECTION IX SPECIAL PRECAUTIONS}

PRECAUTIONS TO RE TAKEN IN HANDLING AND STORAGE

OTHER PRECAUTTONS

SIGN ATURE

UATC

$317-8209 B$ 


\section{MATERIAL SAFETY DATA SHEET}

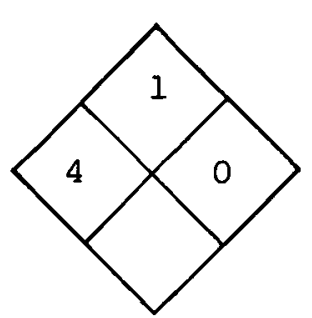

SECTION I SOURCE AND NOMENCLATURE

\section{MANUFACTURER'S NAME}

ADDRESS (NUMBER, STREET, CITY, STATE, ZIP CODE) \begin{tabular}{|l|l|} 
& CHEMICAL FAMILY \\
& $\begin{array}{c}\text { FORMULA } \\
\mathrm{HOC}_{2} \mathrm{OH}_{6} \mathrm{NO}\end{array}$ \\
\hline
\end{tabular}

SECTION II HAZARDOUS INGREDIENTS \begin{tabular}{l|l}
\hline APPROXIMATE & ESTABLISHED
\end{tabular} OR MAXIMUM OSHA $\%$ WT. OR VOL.

BASIC MATERIAL

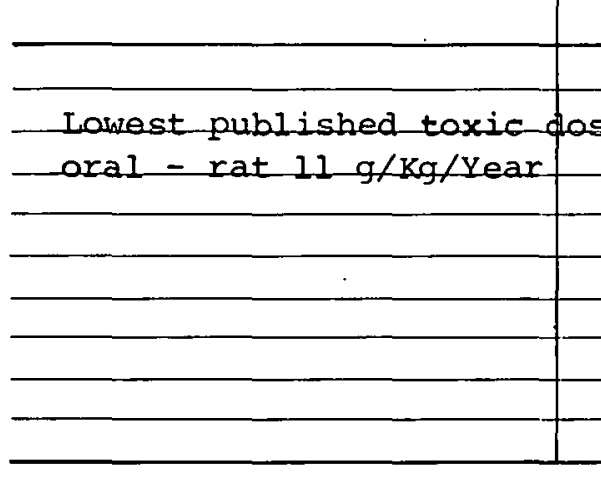

\section{BOILING POINT}

MELTING POINT

Decomp. $140^{\circ} \mathrm{C}$. SPECIFIC GRAVITY $\left(\mathrm{H}_{2} \mathrm{O}=1\right)$

SOLUBILITY IN WATER Pts/100 pis $\mathrm{H}_{2} \mathrm{O}$

Soluble in hot water APPEARANCE AND ODOR

Yellow needles

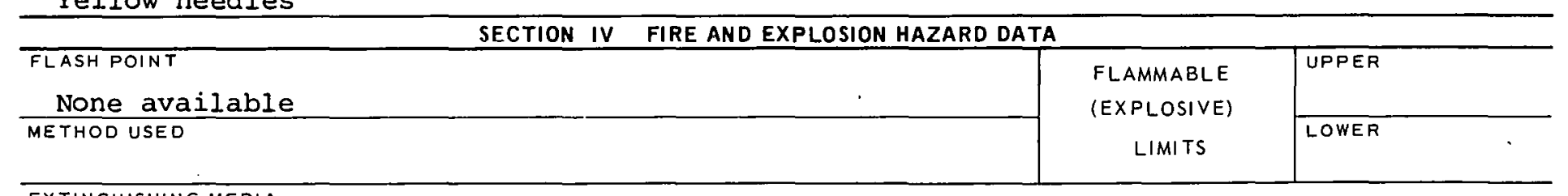

EXTINGUISHING MEDIA

Dry chemical, carbon dioxide, alcohol foam.

EMERGENCY TELEPHONE NO. 


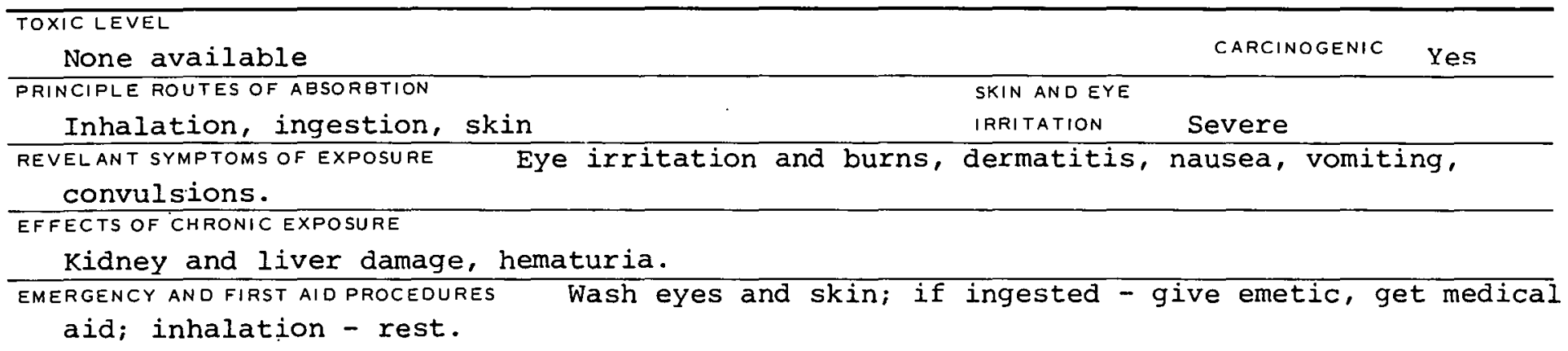

\section{SECTION VI REACTIVITY DATA}

CONDITIONS CONTRIBUTING TO INSTABILITY

GOMOITIQMS RONTRIRIITING TR HAZARDOUS POLYMERIZATION

INCOMPATABILITY (MATERIALS TO AVOID)

HAZARDOUS DECOMPOSITION HRUDUCTS

SECTION VII SPILL OR LEAK PROCEDURES

STEPS TO BE TAKEN IN CASE MATERIAL IS RELEASED OR SPILLED

WASTE DISPOSAL METHOD

SECTION VIII SPECIAL PROTECTION INFORMATION

\begin{tabular}{l|l}
\hline VENTILATION REQUIREMENTS LOCAL EXHAUS & $\begin{array}{c}\text { PROTECTIVE LQUITMENT (SPECIFY TYPES) EYE } \\
\text { GOgglES }\end{array}$ \\
\hline MECHANICAL IGENERAL) & $\begin{array}{c}\text { GLOVES } \\
\text { Rubber }\end{array}$ \\
\hline STECIAL & $\begin{array}{c}\text { RESPIRATOR } \\
\text { With particulatc tiltor }\end{array}$ \\
\hline
\end{tabular}

OTHER PROIEGTIVE EQUIPMENT

SECTION IX SPECIAL PRECAUTIONS

PRECAUTIONS TO BE TAKEN IN HANOLING ANO STORAGE

ITHER FRECAUTIOHS

SIGNATURE

DATE

$317-8809 B$ 


\section{PRODUCT DESIGNATION}

03-317-9409

Nitrosopiperidene
MATERIAL SAFETY

DATA SHEET

\section{SECTION I SOURCE AND NOMENCLATURE}

\section{MANUFACTURER'S NAME \\ 1 Nitrosopiperidene \\ CHEMICAL NAME AND SYNONYMS \\ Nitrosopiperidene}

ADDRESS (NUMBER, STREET, CITY, STATE, ZIP CODE)

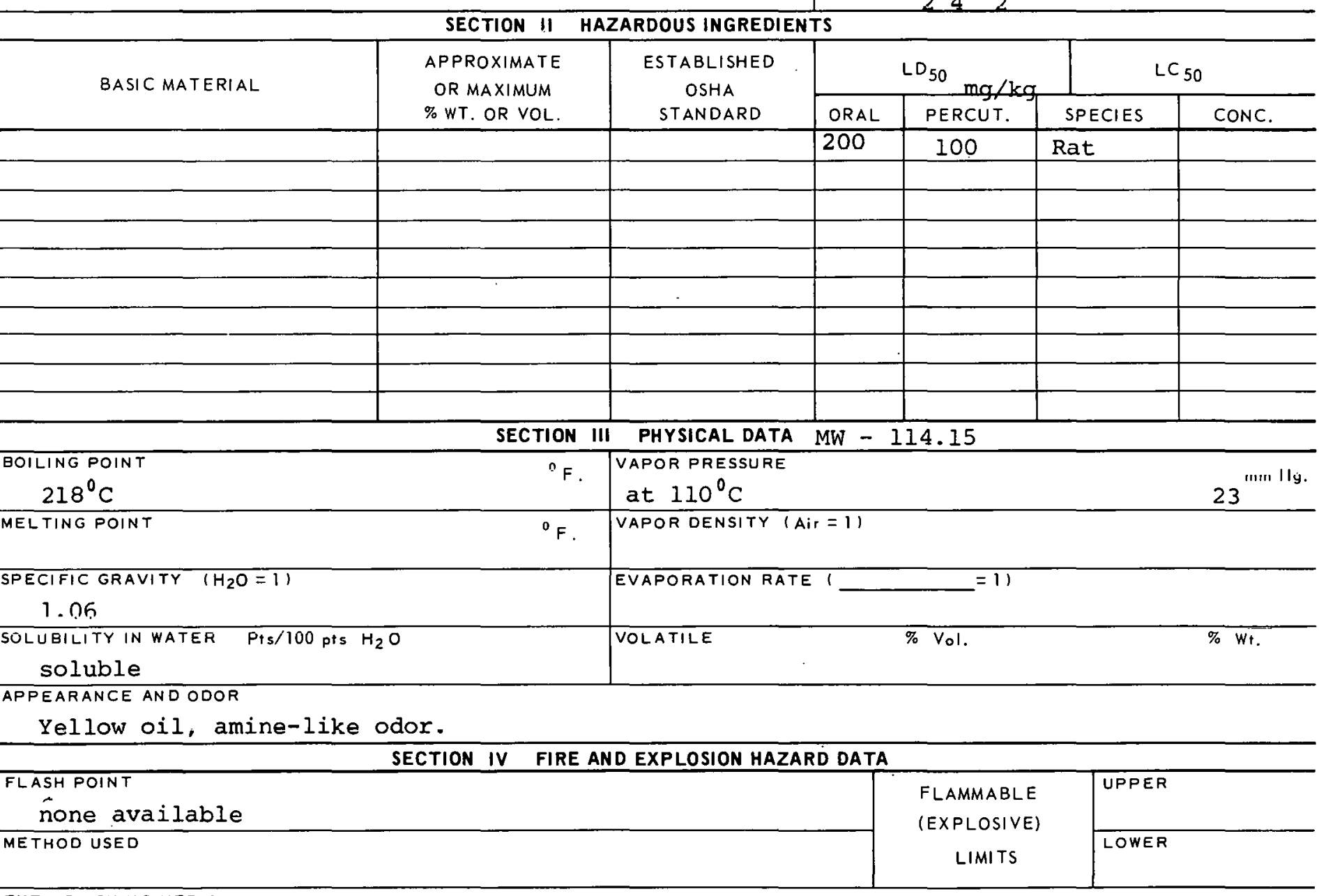

EXTINGUISHING MEDIA

foam, carbon dioxide, dry chemical.
CHEMICAL FAMILY

FORMULA

$\mathrm{NON}\left(\mathrm{CH}_{2}\right)_{4} \mathrm{CH}_{2}$ SECTION II HAZARDOUS INGREDIENTS
EMERGENCY TELEPHONE NO.

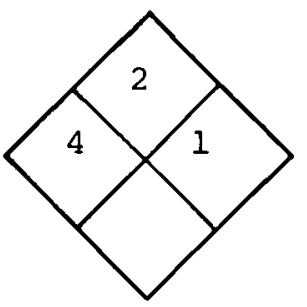




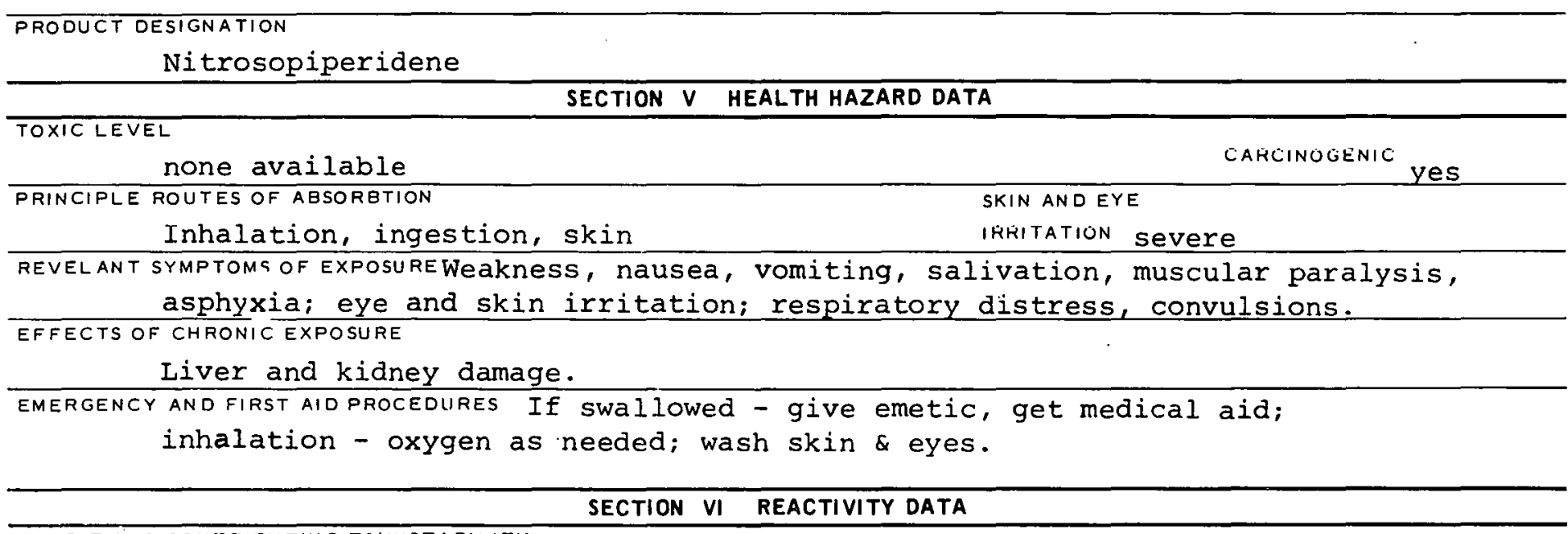

CONDITIONS CONTRIBUTING TOINSTABILITY

CONDITIONS CONTRIBU TING TO HAZARDOUS POLYMERIZATION

INCOMPATABILITY (MATERIALS TO AVOID)

HAZARDOUS DECOMPOSITION PRODUCTS

SECTION VII SPILL OR LEAK PROCEDURES

STEPS TO BE TAKEN IN CASE MATERIAL IS RELEASED OR SPILLED

WASTE DISPOSAL METHOD

SECTION VIII SPECIAL PROTECTION INFORMATION

\begin{tabular}{|c|c|}
\hline VENTILATION REQUIREMENTS LOCAL EXHAUST & $\begin{array}{l}\text { PROTECTIVE EQUIPMENT (SPECIFY TYPES) EYE } \\
\text { GOggleS }\end{array}$ \\
\hline MECHANICAL (GENERAL) & $\begin{array}{l}\text { GLOVES } \\
\text { Rubber }\end{array}$ \\
\hline SPECIAL & $\begin{array}{l}\text { FESPIRATOR } \\
\text { With proper filter }\end{array}$ \\
\hline
\end{tabular}

OTHER PROTECTIVE EQUIPMENT

\section{SECTION IX SPECIAL PRECAUTIONS}

PRECAUTIONS TO BE TAKEN IN HANDLING AND STORAGE

OTHER PRECAUTIONS

$\because$

SIGNATURE

DATE

$317-9409 B$ 


\section{PRODUCT DESIGNATION}

03-317-9609

Nitrosoresorcinol

Sodium Salt

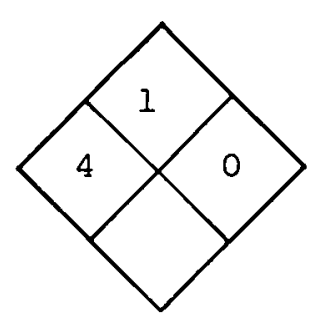

\section{SECTION I SOURCE AND NOMENCLATURE}

ADDRESS (NUMBER, STREET, CITY, STATE, ZIP CODE)

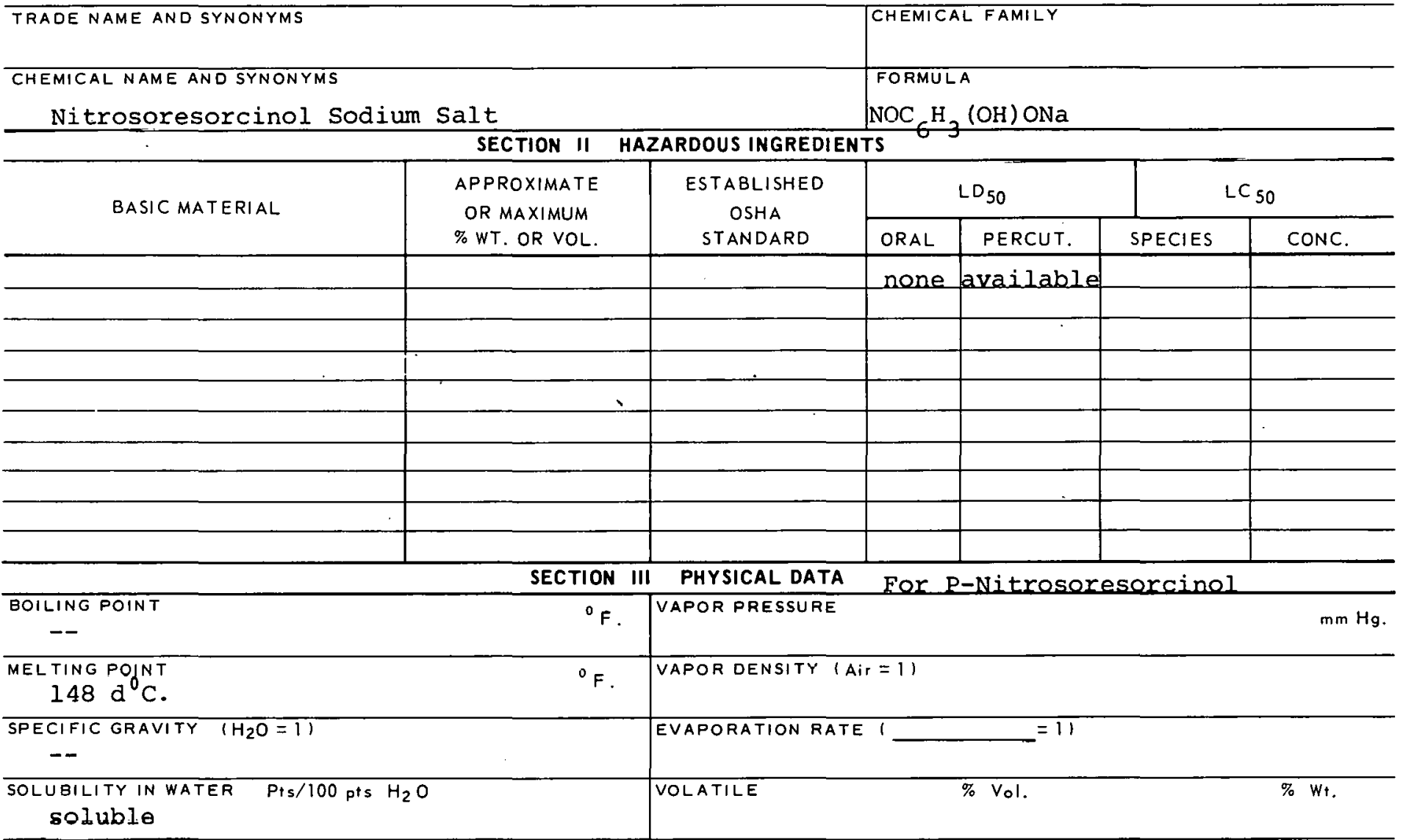

APPEARANCE AND ODOR yellow crystals

\begin{tabular}{l|l|l}
\hline \multicolumn{1}{c}{ SECTION IV FIRE AND EXPLOSION HAZARD DATA } \\
\hline $\begin{array}{l}\text { FLASH POINT } \\
\text { nOt aVailable }\end{array}$ & $\begin{array}{c}\text { FLAMMABLE } \\
\text { (EXPLOSIVE) } \\
\text { METHOD USED }\end{array}$ & UIMITS \\
\cline { 2 - 3 } & LOWER & \\
\hline
\end{tabular}

EXTINGUISHING MEDIA

information not available.

SPECIAL FIRE FIGHTING PROCEDURES

UNUSUAL FIRE AND EXPLOSION HAZARDS 


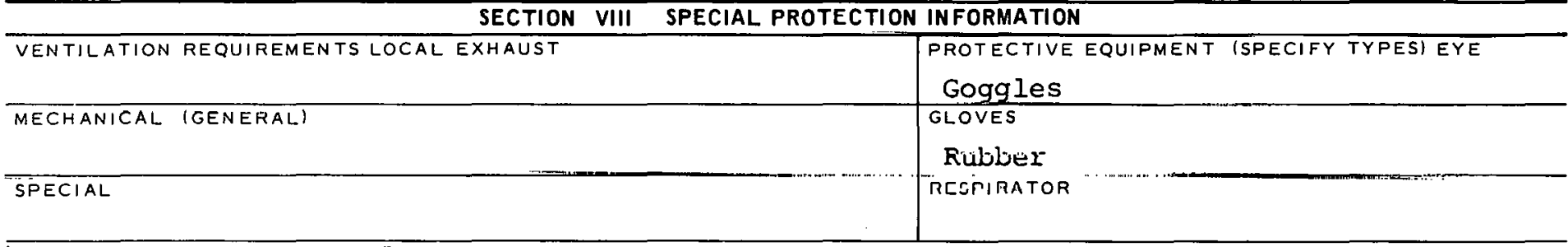

OTHER PROTECTIVE EQUIPMENT

\section{SECTION IX SPECIAL PRECAUTIONS}

PRECAUTIONS TO BE TAKEN IN HANDLING AND STORAGE

OTHER PRECAUTIONS

SIGNATURE

DATE

$317-9609 B$ 
CAS : 000099081

\section{PRODUCT DESIGNATION}

03-317-9709

$\mathrm{M}-\mathrm{Nitrotoluene}$
XT29750

MATERIAL SAFETY

DATA SHEET

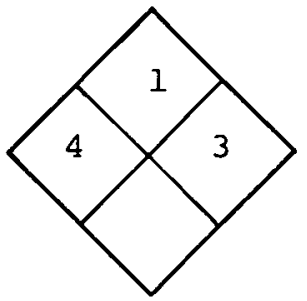

SECTION I SOURCE AND NOMENCLATURE

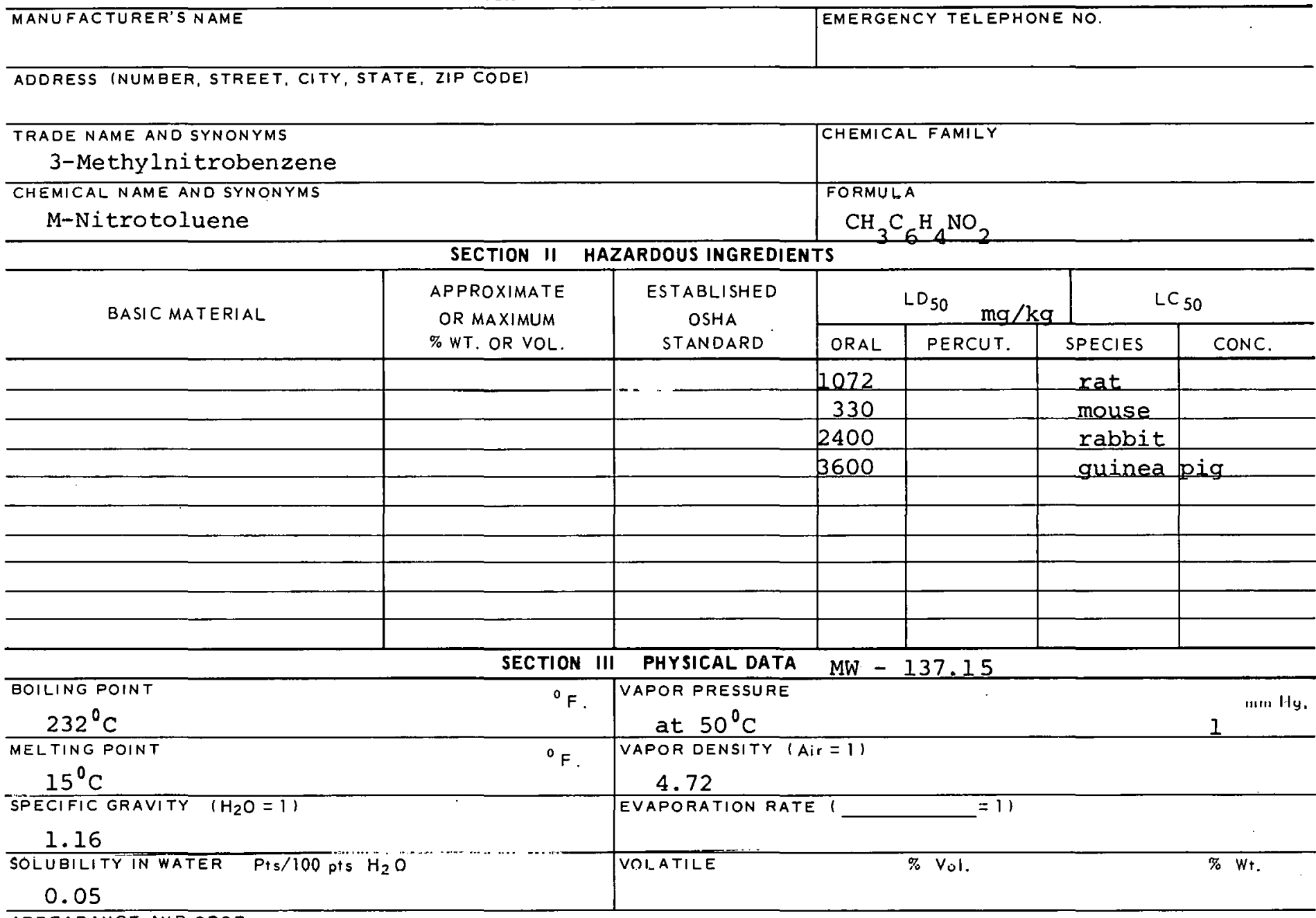

APPEARANCE AND ODOR

liquid

SECTION IV FIRE AND EXPLOSION HAZARD DATA

FLASH POINT

$233^{\circ} \mathrm{F}$

METHOD USED

C.C.

FLAMMABLE

([XPLOSIVE)

LIMITS
UPPER

LOWER

EXTINGUISHING MEDIA

Water, carbon dioxide, dry chemical.

SPECIAL FIRE FIGHTING PROCEDURES

UNUSUAL FIRE AND EXPLOSION HAZARDS 
TOXICLEVEL

$\mathrm{TLV}-5 \mathrm{PPM}, 30 \mathrm{mg} / \mathrm{CM}$

PRINCIPLE ROUTES OF ABSORBTION

Inhalation, inqestion, skin

CARCINOGENIC SKIN AND EYE no REVELANT SYMPTOMS OF EXPOSURE

Difficulty in breathing, cyanosis, vomiting, headache. EFFECTS OF CHRONIC EXPOSURE

Blood damage.

EMERGENCY AND FIRST AID PROCEDURES If swallowed - give emetic, get medical aid;

inhalation - rest; wash from skin \& eyes.

\section{SECTION VI REACTIVITY DATA}

CONDITIONS CONTRIBUTING TO INSTABILITY

CONDITIONS CONTRIBUTING TO HAZAROOUS POLYMERIZATION

INCOMPATABILITY (MATERIALS TO AVOID)

May explode when heated with sulfuric acid. HAZARDOUS DECOMPOSITION PRODUCTS

\section{SECTION VII SPILL OR LEAK PROCEDURES}

STEPSTO BE TAKEN IN CASE MATERIAL IS RELEASEO OR SPILLED

Add sodium bicarbonate or sand-soda àsh mixture (9/1). Transler intu cardboard WASTF DISPOSAL METHOD carton for aisposal.

\section{SECTION VIII SPECIAL PROTECTION INFORMATION}

\begin{tabular}{l|l}
\hline VENTILATION REQUIREMENTS LOCAL EXHAUST & $\begin{array}{l}\text { PROTECTIVE EQUIPMENT (SPECIFY TYPES) EYE } \\
\text { GoggleS }\end{array}$ \\
\hline MECHANICAL (GENERAL) & GLOVES \\
RublUeY (butyl) \\
\hline SPECIAL & RESPIRATOR \\
Self-contained
\end{tabular}

OTHER PROTECTIVE EQUIPMENT

\section{SECTION IX SPECIAL PRECAUTIONS}

PRECAUTIONS TO BE TAKEN IN HANDLING AND STORAGE

outside or detached storage preferred inside storage in standard flammable

Iiquids cabinet.

OTHER PRECAUTIONS 


\section{PRODUCT DESIGNATION}

03-317-9809

O-Nitrotoluene

\section{MATERIAL SAFETY \\ DATA SHEET}

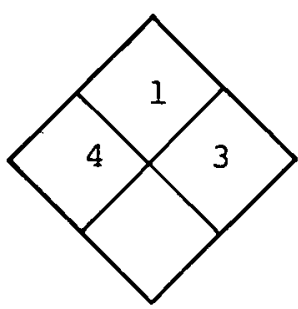

SECTION I SOURCE AND NOMENCLATURE

MANUFACTURER'S NAME

EMERGENCY TELEPHONE NO

ADDRESS (NUMBER, STREET, CITY, STATE, ZIP CODE)

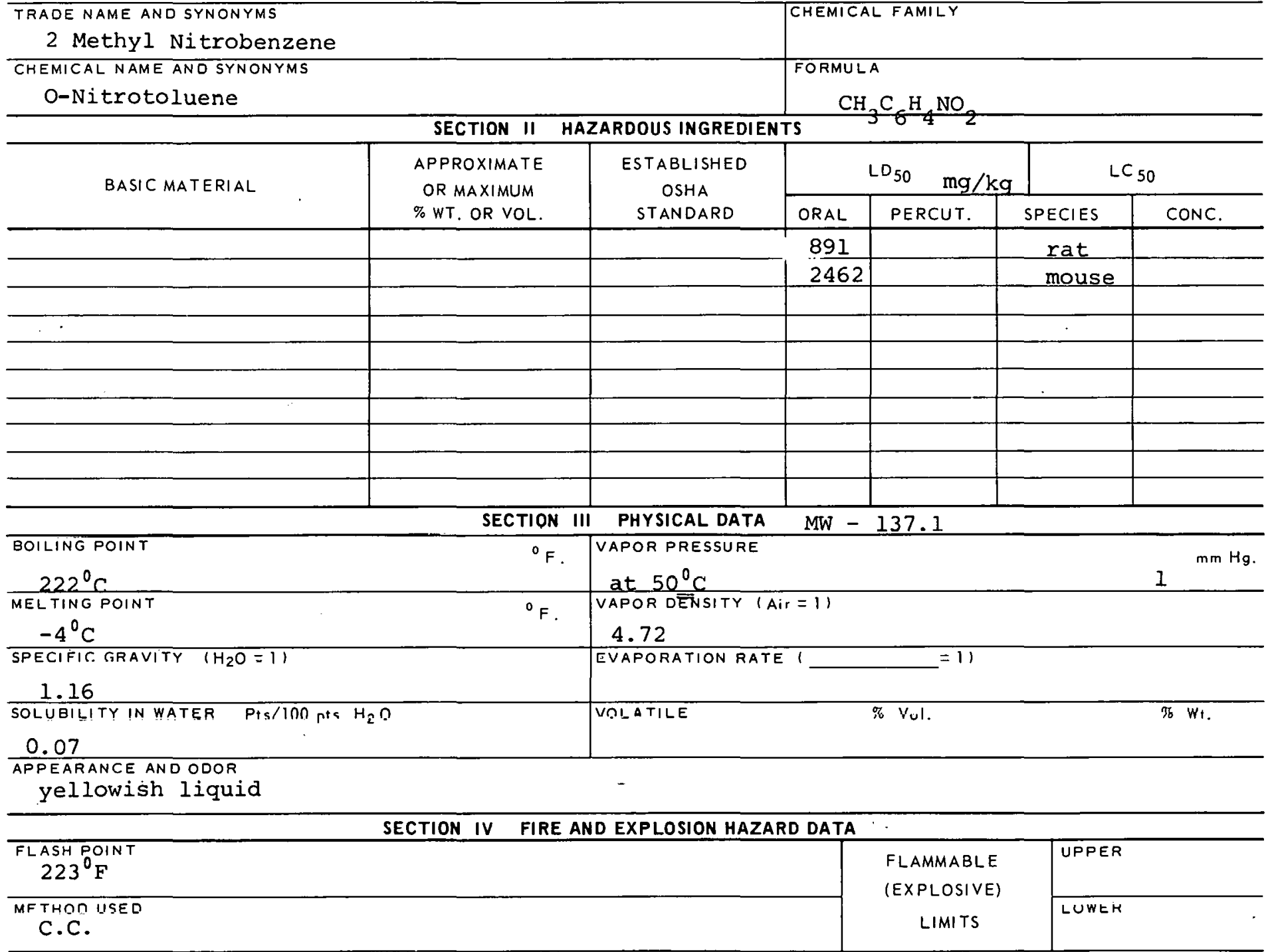

EXTINGUISHING MERIA

Water, carbon dioxide, dry chemical

SPECIAL FIRE FIGHTING PROCEDURES

UNUSUAL FIRE AND EXPLOSION HAZARDS 


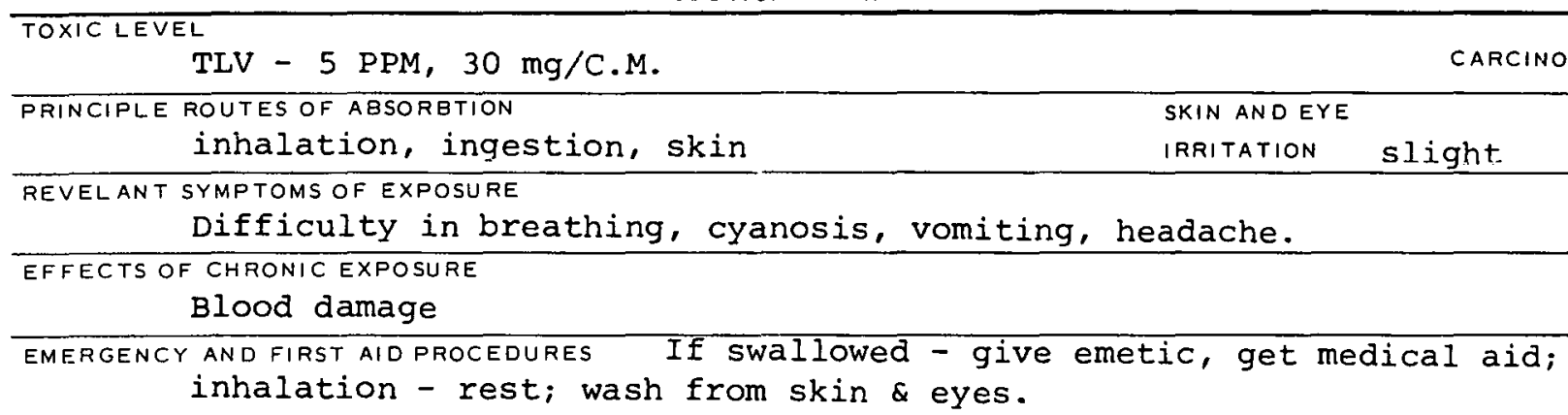




\section{PRODUCT DESIGNATION}

03-317-9909

P-Nitrotoluene
MATERIAL SAFETY DATA SHEET

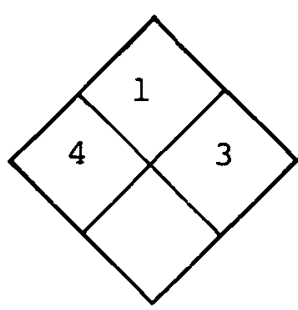

\section{SECTION I SOURCE AND NOMENCLATURE}

\section{MANUFACTURER'S NAME \\ TRADE NAME ANO SYNONYMS \\ 4 Methyl Nitrobenzene CHEMICAL NAME AND SYNONYMS \\ $\mathrm{P}-\mathrm{Nitrotoluene}$}

ADDRESS (NUMBER, STREET, CITY, STATE, ZIP CODE)

BASIC MATERIAL

\section{SECTION II HAZARDOUS INGREDIENTS}

$\mathrm{CH}_{3} \mathrm{C}_{6} \mathrm{H}_{4} \mathrm{NO}_{2}$

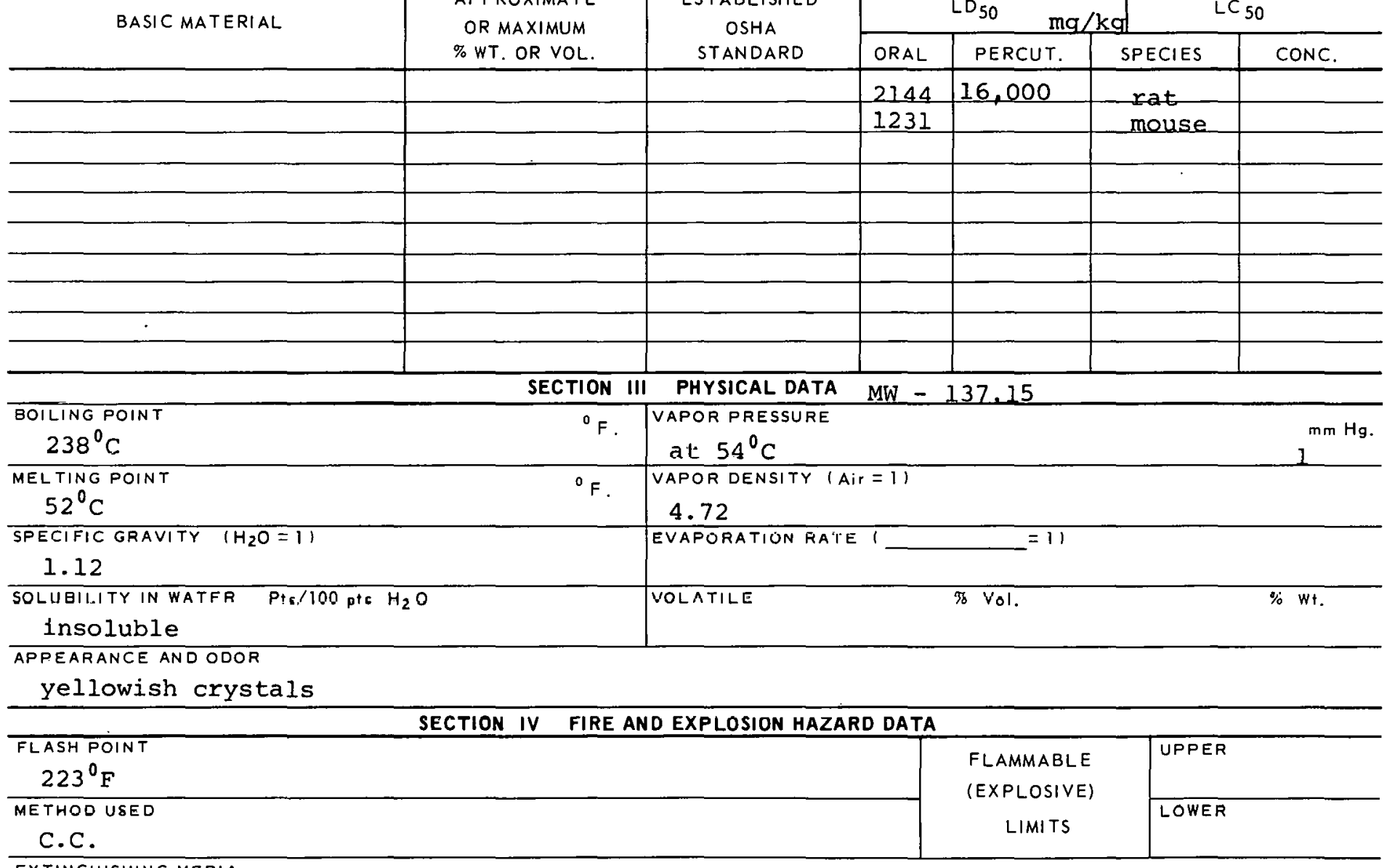

EXTINGIIISHING MEDIA

Water, carbon dioxide, dry chemicals.

SPECIAL FIRE FIGHTING PROCEDURES 
TOXIC LEVEL

TLV - 5 PPM, $30 \mathrm{mg} / \mathrm{C} . \mathrm{M}$.

GARCINOGENIC

PRINCIPLE ROUTES OF ABSORBTION

inhalation, ingestion, skin

SKIN AND EYE

IRRITATION Slight

REVELANT CVMOTOMSGFEXPOSURE

EFFECTS OF THRONIC EXPOSURE

Blood damage

EMERGENCY AND FIRST AID PROCEDURES If swallowed - give emetic, get medical aid;

inhalation - rest; wash from skin \& eyes.

\section{SECTION VI REACTIVITY DATA}

CONDITIONS CONTRIBUTING TO INSTABILITY

CONDITIONS CONTRIBU TING TO HAZARDQUS POLYMERIZATION

INCOMPATABILITY (MATERIALS TO AVOID)

May explode when heatec with sulfuric acid.

HAZARDOUS DECOMPOSIIIUN PRODUCTS

SECTION VII SPILL OR LEAK PROCEDURES

STEPS TO BE TAKEN IN CASE MATERIAL IS RELEASED OR SPILLED

Add (9:1) sand to soda ash to spill and transfer to cardboard container

tor dispósal.

WASTE DISPOSAL METHOD

SECTION VIII SPECIAL PROTECTION INFORMATION

\begin{tabular}{l|l}
\hline VENTILATION REQUIREMENTS LOCAL EXHAUST & $\begin{array}{l}\text { PHUTECTIVE EQUIPMENT (SPECIFY TYPES) EYE } \\
\text { GUYYlES }\end{array}$ \\
\hline MECHANICAL IGENERAL) & $\begin{array}{c}\text { GLOVES } \\
\text { Rubber (butYI) }\end{array}$ \\
\hline SPECIAL & $\begin{array}{l}\text { RESPIRATOR } \\
\text { Self-contained }\end{array}$ \\
\hline
\end{tabular}

OTHER PROTECTIVE EQUIPMENT

SECTION IX SPECIAL PRECAUTIONS

PRECAUTIONS TO BE TAKEN IN HANDLING AND STORAGE

Outside or detached storage preferred inside storage in standard flammable

liquids cabinet.

OTHER PRECAUTIONS

SIGNATURE

UAIE

317-9909B 


\begin{tabular}{|l|}
\hline \multicolumn{1}{|c|}{ PRODUCT DESIGNATION } \\
$\begin{array}{l}\text { M3-321-0301 } \\
\text { Oxaly1 Chloride }\end{array}$
\end{tabular}

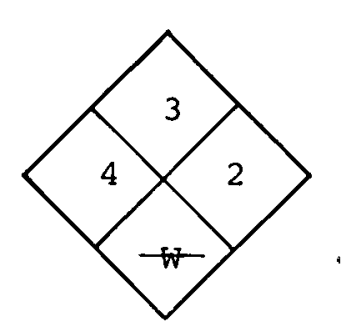

SECTION I SOURCE AND NOMENCLATURE

MANUFACTURER'SNAME

EMERGENCY TELEPHONE NO.

ADDRESS (NUMBER, STREET, CITY, STATE, ZIP CODE)

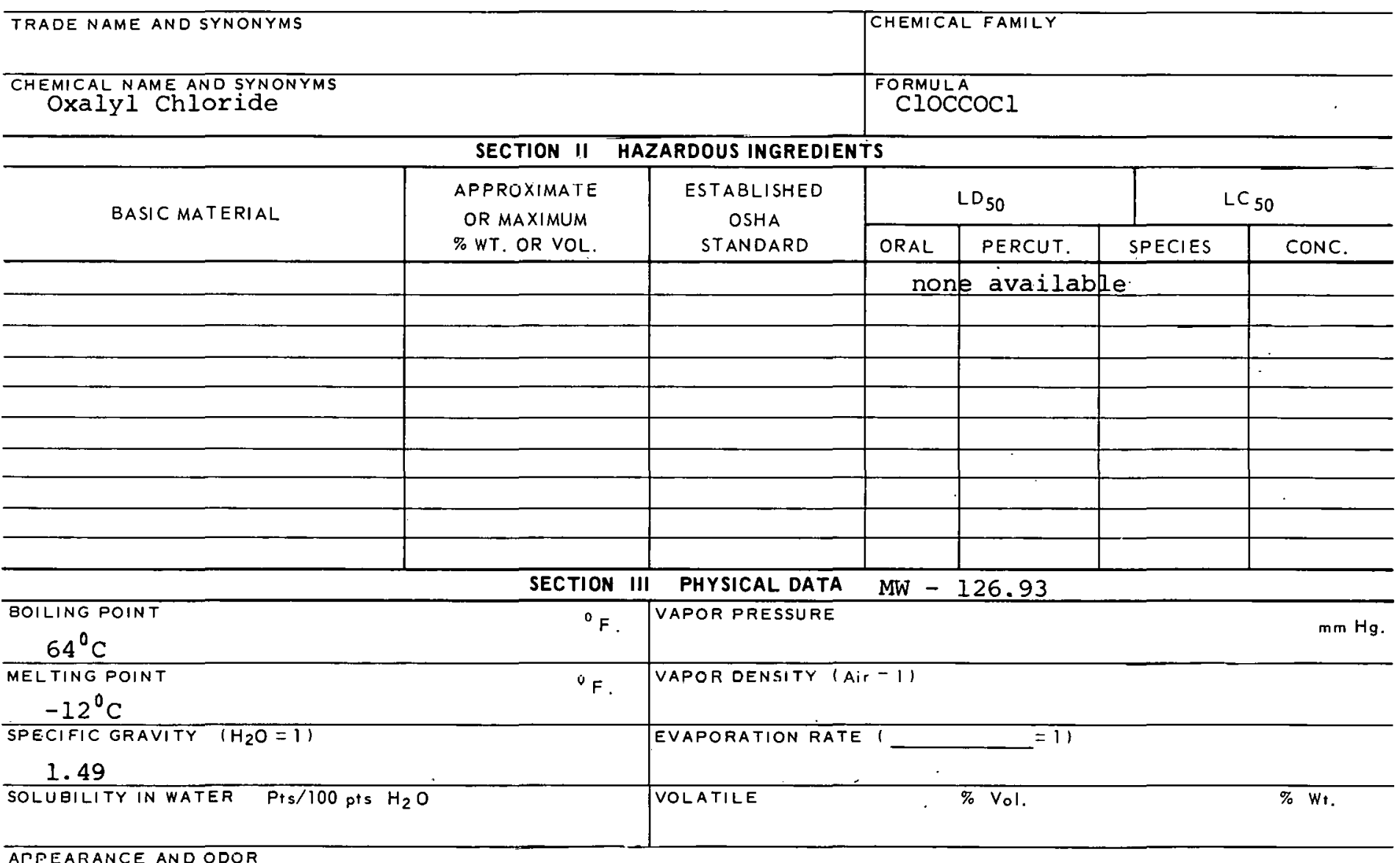

ATPEARANCE AND ODOR

SECTION IV FIRE AND EXPLOSION HAZARD DATA

\begin{tabular}{l|c|l}
\hline $\begin{array}{l}\text { FLASH POINT } \\
\text { nONe available }\end{array}$ & $\begin{array}{c}\text { FLAMMABLE } \\
\text { (EXPLOSIVE) }\end{array}$ \\
\hline METHOD USED & LIMITS & LOWER \\
\hline
\end{tabular}

EXTINGUISHING MEDIA

sarbon dioxidc, dry ehemical - avold water.

SPECIAL FIRE FIGHTING PROCEDURES

UNUSUAL FIRE AND EXPLOSION HAZARDS
(3) 1 (n.7n) 


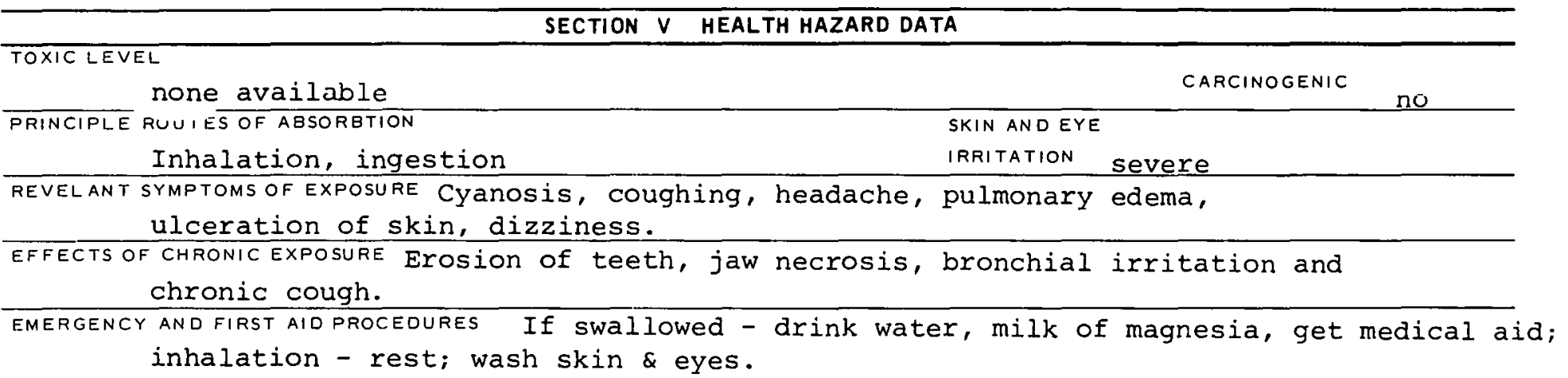

\section{SECTION VI REACTIVITY DATA}

CONDITIONS CONTRIBUTING TO INSTABILITY

CONQITIONS CONTRIBUTING TO HAZARDOUS POLYMERIZATION

INCOMPATABILITY IMATERIALSTOAVOIDI

water and alcohol.

HAZARDOUS DECOMFOSITION HKUUUL IS

SECTION VII SPILL OR LEAK PROCEDURES

STEPS TO BE TAKEN IN CASE MATERIAL IS RELEASED OR SPILLED

Cover spill with sodium bicarbonate, transfer to plastic bag for disposal.

WASTE UISHUSAL METHOD

SECTION VIII SPECIAL PROTECTION INFORMATION

\begin{tabular}{l|l}
\hline \multicolumn{1}{|c|}{ SECTION VIII SPECIALPROTECTION INFORMATION } \\
\hline MECHANIGAL IGENERAL) & $\begin{array}{l}\text { PROTECTIVE EQUIPMENT (SPECIFY TYPES) EYE } \\
\text { GOggles }\end{array}$ \\
\hline SHECIAL & $\begin{array}{l}\text { GLOVES } \\
\text { Rubber }\end{array}$ \\
\hline
\end{tabular}

OTIIER FROTECTIVE EQUIPMENT

Coveralls

SECTION IX SPECIAL PRECAUTIONS

PRECAUTIONS TO BE TAKEN IN HANDLING AND STORAGE

OTHER PRECAUTIONS

SIGNATURE

DATE

$321-0301 B$ 

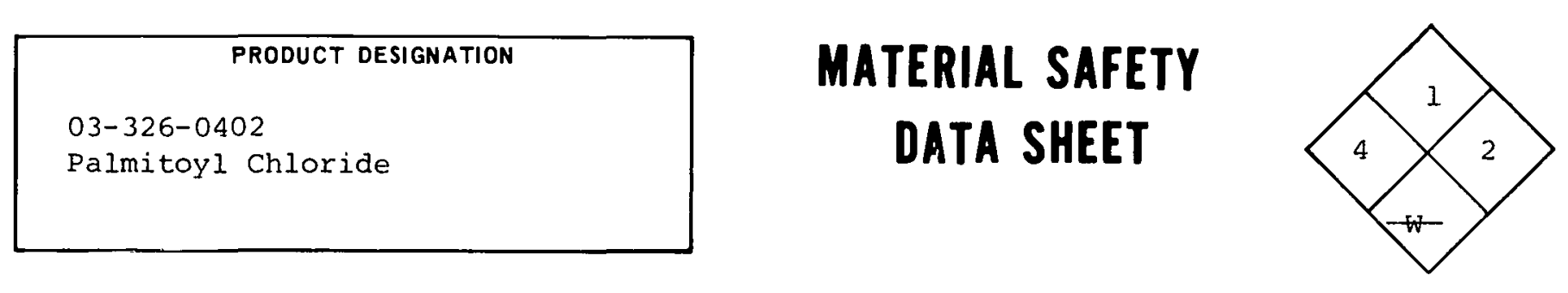

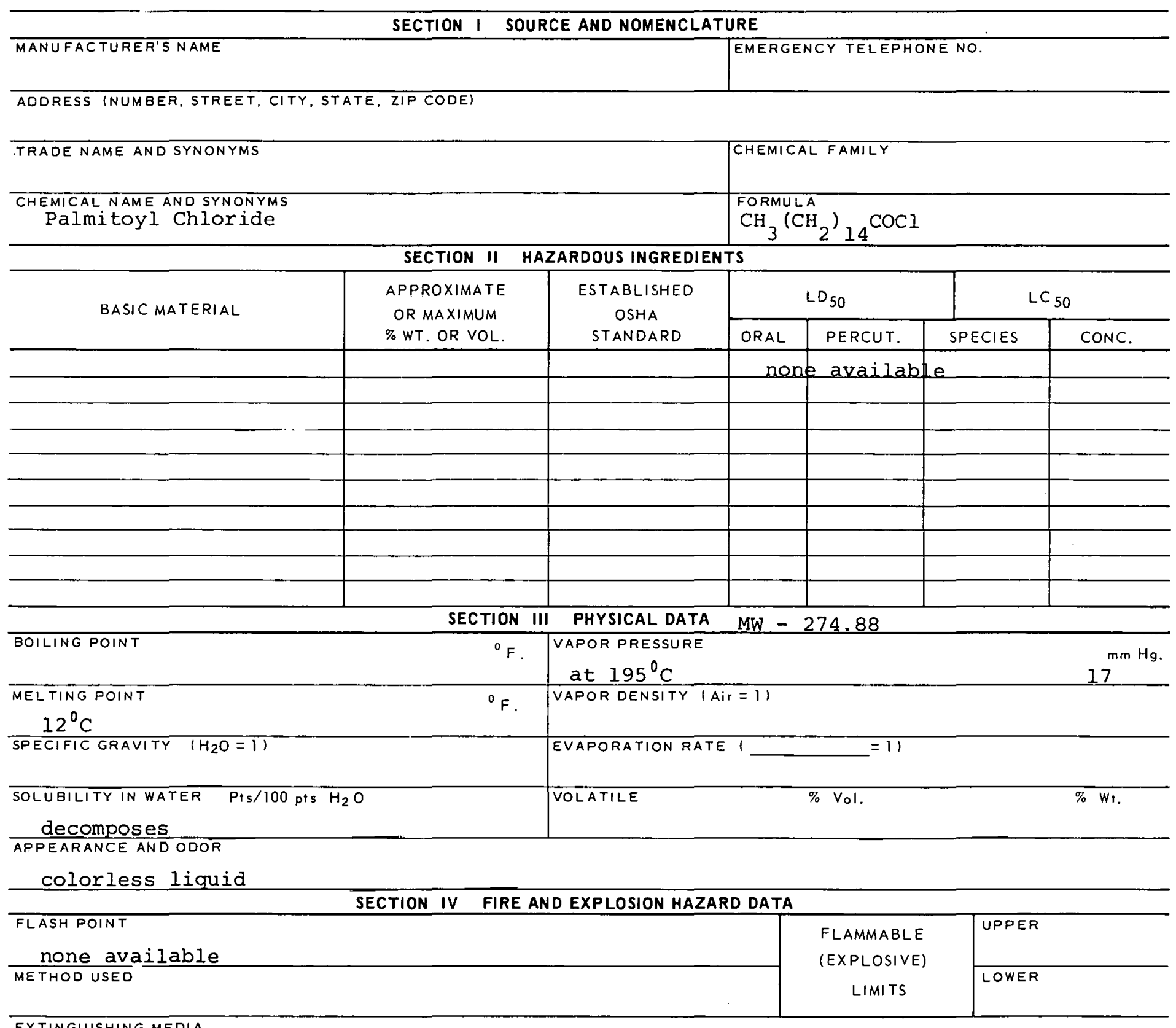

EXTINGUISHING MEDIA

Use carbon dioxide or dry chemical.

SPECIAL FIRE FIGHTING PROCEOURES

Wear full protective clothing.

UNUSUAL FIRE AND EXPLOSION HAZARDS 
TOXIC LEVEL

None available

PRINCIPLE ROUTES OF ABSORETION inhalation, ingestion
CARGINOGENIC

no SKIN AND EYE IRRITATION SEVERE

REVELANT SYMPTOMS OF EXPOSURE Cyanosis, coughing, headache, pulmonary edema, ulceration of skin, dizziness.

EFFECTS OF CHRONICEXPOSURE Erosion of teeth, jaw necrosis, bronchial irritation

and chronic cough.

EMERGENCY AND FIRST AID PROCEDURES Inhalation - rest, artificial respiration as needed; ingestion - get medical aid; wash from skin \& eyes.

\section{SECTION VI REACTIVITY DATA}

CONO!TINNS SONTRIBUTING TO INSTABILITY

COMDITIONG RINNTRIBUTING TO HAZARDOUS POLYMERIZATION

INCOMPATABILITY (MATERIALS TO AVOID)

Avoid contàct witls waler.

HAZARDOUS DECOMPOSITION FRODUCTS

Produces $\mathrm{HCl}$ on contact with water.

SECTION VII SPILL OR LEAK PROCEDURES

STEPS TO BE TAKEN IN CASE MATERIAL IS RELEASED OR SPILLED

WASTE DISPOSAL METHOD

SECTION VIII SPECIAL PROTECTION INFORMATION

\begin{tabular}{l|l}
\hline VFNTII ATION REQUIREMENTS LOCAL EXHAUST & $\begin{array}{c}\text { PROTECTIVE EQUIPMENT (SPECIFY TYRES) EYE } \\
\text { Safety gOggles }\end{array}$ \\
\hline MECHANICAL IGENERAL) & $\begin{array}{c}\text { GLOVES } \\
\text { Rubber }\end{array}$ \\
\hline SPECIAL & $\begin{array}{l}\text { HESHIRATUR } \\
\text { With proper. filter }\end{array}$ \\
\hline
\end{tabular}

OTHER PROTECTIVE EQUIPMENT

\section{SECTION IX SPECIAL PRECAUTIONS}

PRECAUTIONS TO BE TAKEN IN HANDLING AND STORAGE

Store in cool, dry area away from heat irside storage in standard flammable

liquids storage cabinet.

OTHER PRECAUTIONS

SIGNATURE

DATE

376-0402B 
03-326-3402

Pentamethylene Bromide

DATA SHEET

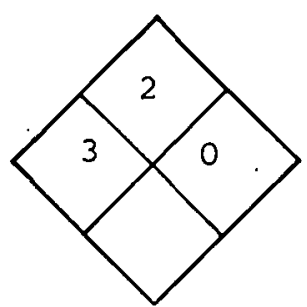

SECTION I SOURCE AND NOMENCLATURE

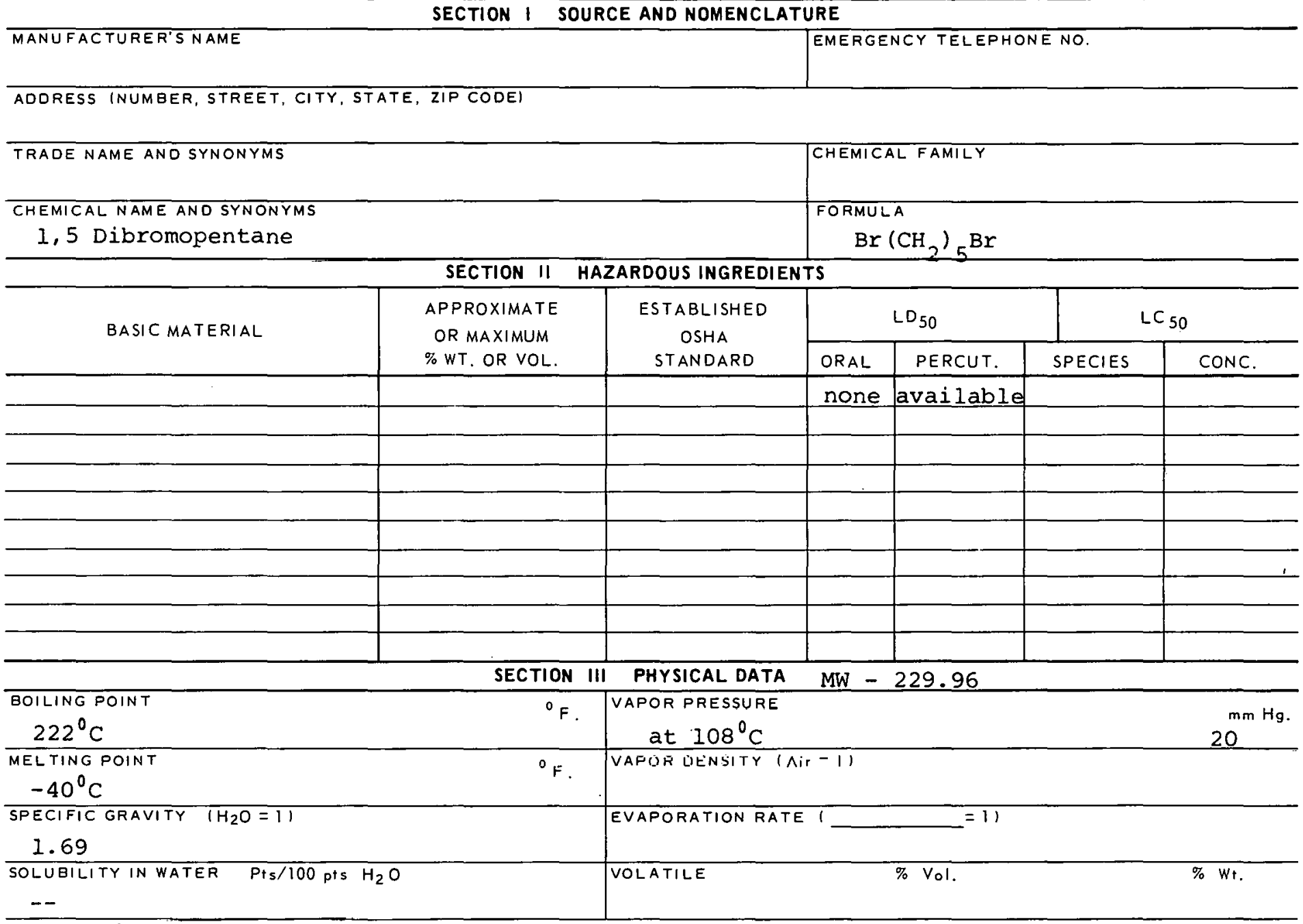

APPEARANCE AND ODOR

colorless liquid

\begin{tabular}{|c|c|c|c|c|}
\hline & SECTION IV & FIRE AND EXPLOSION HAZARD DATA & & \\
\hline $\begin{array}{l}\text { FLASH POINT } \\
\text { none available }\end{array}$ & & & \multirow{2}{*}{$\begin{array}{c}\text { FLAMMABLE } \\
\text { (EXPLOSIVE) } \\
\text { LIMITS }\end{array}$} & UPPER \\
\hline METHOD USED & & & & LOWER \\
\hline
\end{tabular}

EXTINGUISHING MEDIA

Water, foam, carbun dioxide and dry chemical.

SPECIAL FIRE FIGHTING PROCEDURES

Water may be ineffective.

UNUSUAL FIRE AND EXPLOSION HAZARDS 
PRODUCT DESIGNATION

Pentamethylene Bromide

SECTION $V$ HEALTH HAZARD DATA

TOXIC LEVEL

none

PRINCIPLE ROUTES OF ABSORBTION

inhalation, ingestion

CARCINOGENIC

no

REVELANT SYMPTOMS OF EXPOSURE

eye irritation, narcosis, drowsiness, headache, vomiting.

EFFECTS OF CHRONIC EXPOSURE dermatitis, liver \& kidney damage.

EMERGENCY AND FIRST AIDPROCEDURES Inhalation - rest; if swallowed - give emetic, get medical aid; wash from skin \& eyes.

SECTION VI REACTIVITY DATA

CONDITIONS CONTRIBUTING TO INSTABILITY

CONDITIONS CONTRIBUTING TO HAZARDOUS POLYMERIZATION

INCOMPATABILITY (MATERIALS TO AVOIU)

HAZAROOUS DECOMPOSITION PRODUCTS

SECTION VII SPILL OR LEAK PROCEDURES

STEPS TO BE TAKEN IN CASE MATERIAL IS RELEASED OR SPILLED

WASTE DISPOSAL METHOD

SECTION VIII SPECIAL PROTECTION INFORMATION

\begin{tabular}{l|l}
\hline VENTILATION REQUIREMENTS LOCAL EXHAUST & PROTECTIVE EQUIPMENT (SPECIFY TYPES) EYE
\end{tabular} Goggles

MECHANICAL (GENERAL)

GLOVES

Rubber

SPECIAL

OTHER PROTECTIVE EQUIPMENT

RESPIRATOR

With organic vapor resistant canister.

\section{SECTION IX SPECIAL PRECAUTIONS}

PRECAUTIONS TO BE TAKEN IN HANDLING AND STORAGE

OTHER PRECAUTIONS

SIGNATURE

DATE

$326-3402 B$ 


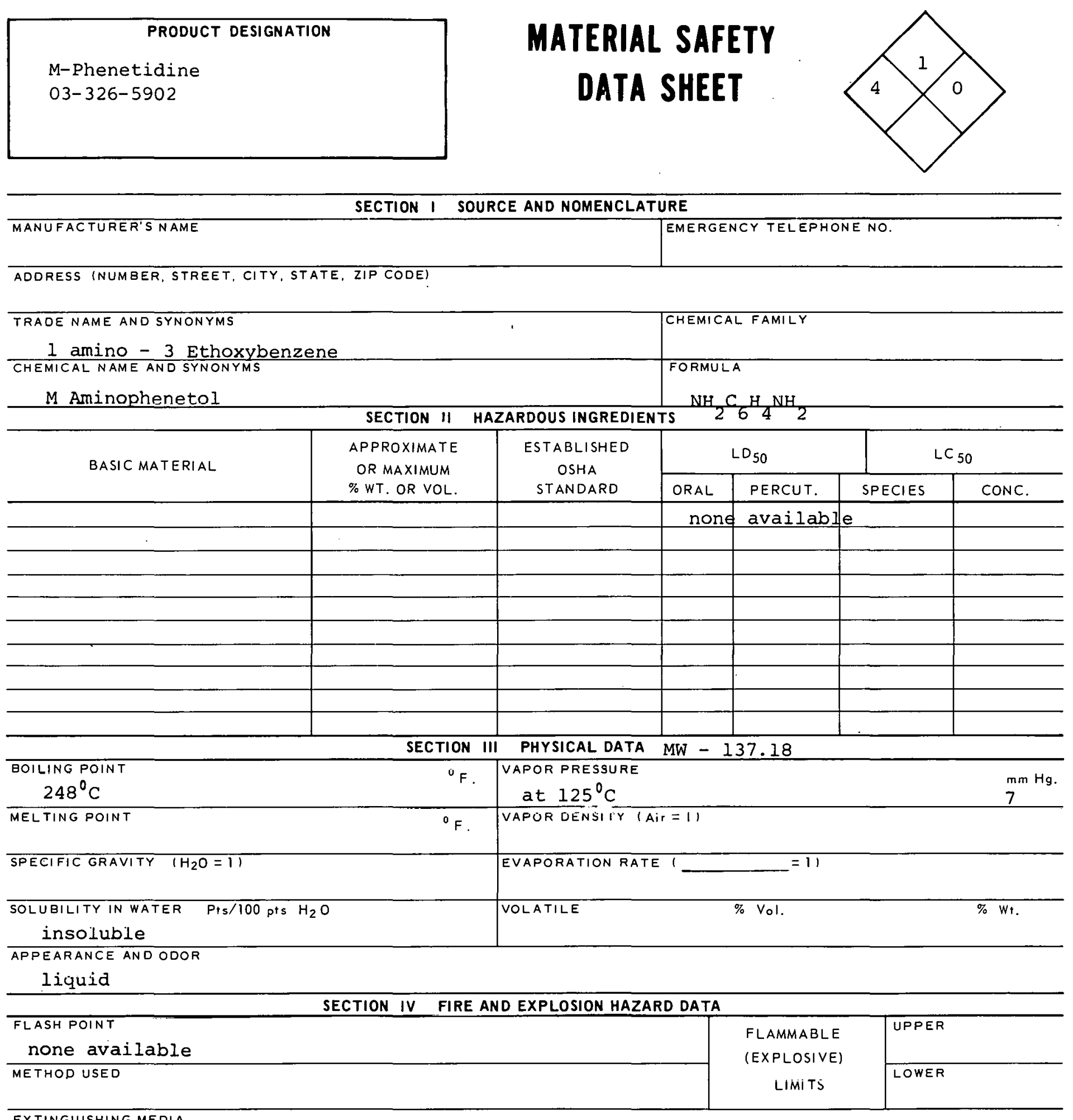

EXTINGUISHING MEDIA

carbon dioxide, dry chemical

SPECIAL FIRE FIGHTING PROCEDURES

water or foam may cause frothing.

UNUSUAL FIRE ANDEXPLOSION HAZARDS 


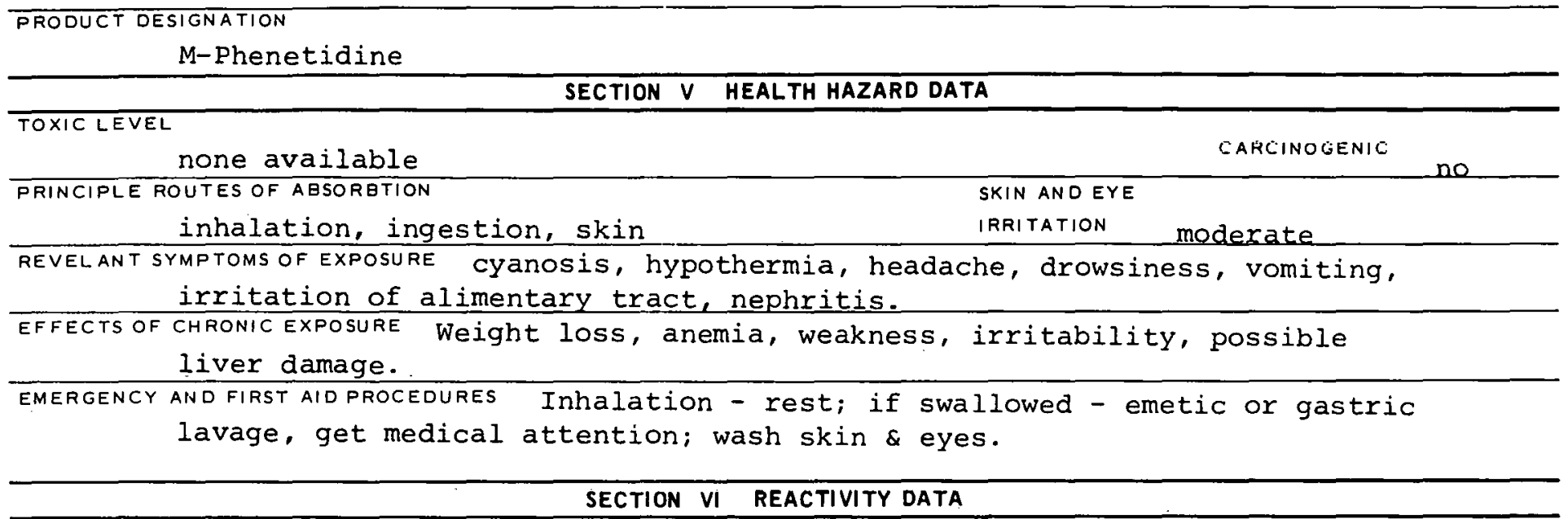

CONDITIONS CONTRIBUTING TO INSTABILITY

CONDITIONS CONTRIBUTING TO HAZARDOUS POLYMERIZ TTION

INCOMPATABILITY (MATERIALS TO AVOID)

HAZARDOUS DECOMPOSITION PRODUCTS

SECTION VII SPILL OR LEAK PROCEDURES

STEPS TO BE TAKEN IN CASE MATERIAL IS RELEASED OR SPILLED

Pour (9:1) sand to soda ash mixture on spill, put in cardboard carton

for disposal.

WASTE DISPOSAL METHOD

SECTION VIII SPECIAL PROTECTION INFORMATION

\begin{tabular}{l|l} 
SECTION VIII SPECIAL PROTECTION INFORMATION \\
\hline VENTILATION REQUIREMENTS LOCAL EXHAUST & $\begin{array}{l}\text { PROTECTIVE EQUIPMENT ISPECIFY TYPESI EYE } \\
\text { Goggles }\end{array}$ \\
\hline MECHANIGAL IGENERAL) & $\begin{array}{l}\text { GLOVES } \\
\text { Rubber } \quad \text { (butyl) }\end{array}$ \\
\hline STCCIAL & $\begin{array}{l}\text { RESPIRATOR } \\
\text { With proper filter }\end{array}$ \\
\hline
\end{tabular}

OTHER PROTLCTIVE EQUIFMENT

SECTION IX SPECIAL PRECAUTIONS

PRECAUTIONS TO BE TAKEN IN HANDLING AND STORAGE

store in dark, cool area.

UIHEH HRELAUTIUINS

SIGNATURE

DATE

$326-5902 B$ 


ProduCt DESIGNATION
03-326-6002
O-Phenetidine

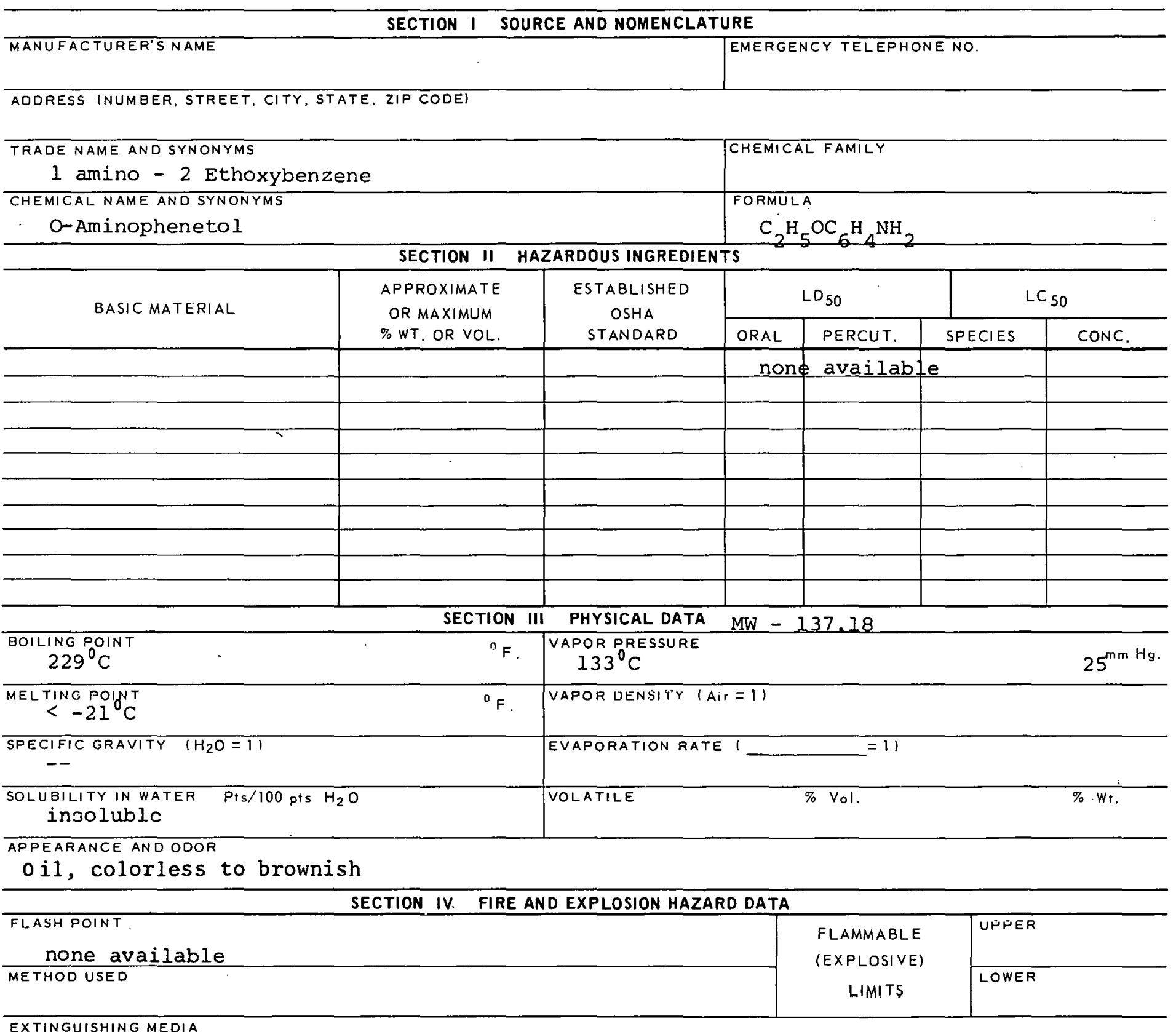

EXTINGUISHING MEDIA

carbon dioxide, dry chemical

SPECIAL FIRE FIGHTING PROCEDURES

water or foam may cause frothing.

UNUSUAL FIRE AND EXPLOSION HAZARDS 


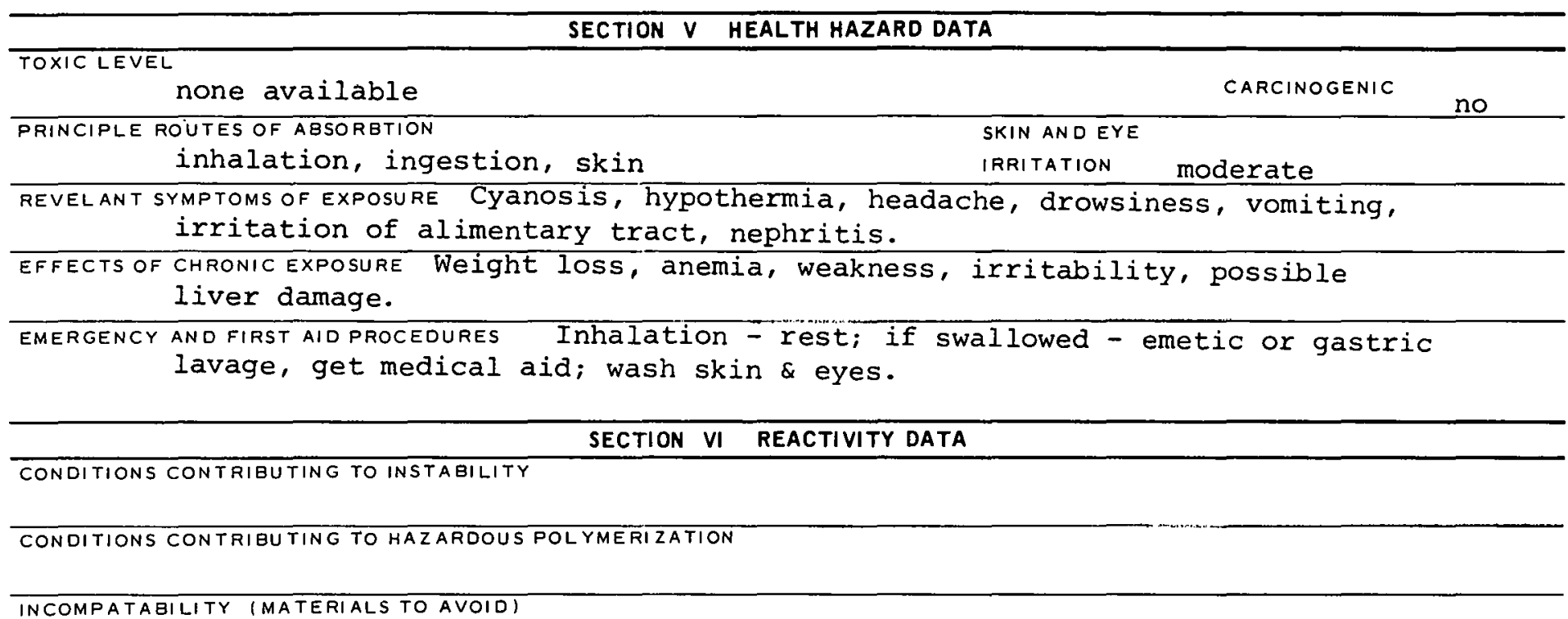

HAZAROOUS DECOMPOSITION PRODUCTS

SECTION VII SPILL OR LEAK PROCEDURES

STEPS TO $B E$ TAKEN IN CASE MATERIAL IS RELEASED OR SPILLED

Pour (9:I) sand to soda ash mixture on spill, put in cardboard carton for disposal.

WASTE DISPOSAL METHOD

\section{SECTION VIII SPECIAL PROTECTION INFORMATION}

\begin{tabular}{l|l}
\hline \multicolumn{1}{c}{ SECTION VIII SPECIAL PROTECTION INFORMATION } \\
\hline VENTILATION REQUIREMENTS LOCAL EXHAUST & $\begin{array}{c}\text { PROTECTIVE EQUIPMENT ISPECIFY TYPES) EYE } \\
\text { Goggles }\end{array}$ \\
\hline MECHANICAL (GENERAL) & $\begin{array}{c}\text { GLOVES } \\
\text { Rubber (butyl) }\end{array}$ \\
\hline SPECIAL & $\begin{array}{l}\text { HESPIRAIOR } \\
\text { With proper filter }\end{array}$ \\
\hline OTHER PROTECTIVE EQUIPMENT
\end{tabular}

OTHER PROTECTIVE EQUIPMENT

Plastic coveralls, self-contained breathing apparatus.

\section{SECTION IX SPECIAL PRECAUTIONS}

PRECAUTIONS TO BE TAKEN IN HANDLING AND STORAGE

Store in dark, cool area.

\section{OTHER PRECAUTIONS}


CAS : 000156434

\begin{tabular}{|c|}
\hline PRODUCT DESIGNATION \\
03-326-6102 \\
P-Phenetidine
\end{tabular}

SI64600

\section{MATERIAL SAFETY \\ DATA SHEET}

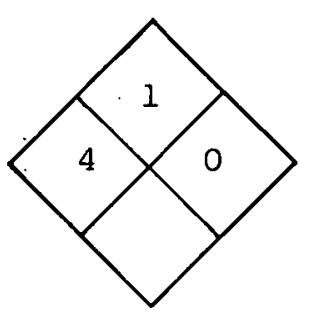

\section{SECTION I SOURCE AND NOMENCLATURE}

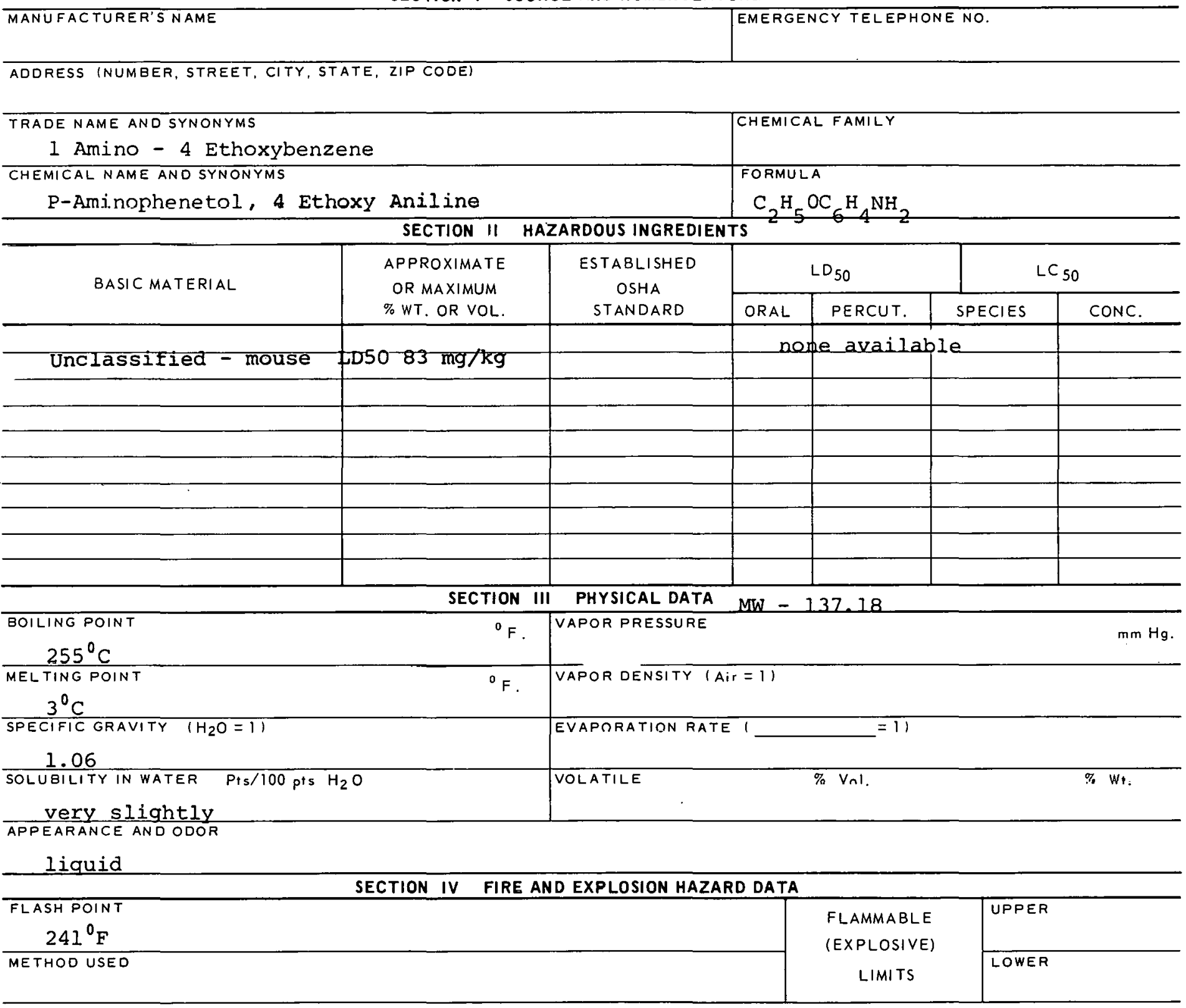

EXTINGUISHING MEDIA

carbon dioxide, dry chemical

SPECIAL FIRE FIGHTING PROCEOURES

water or foam may cause frothing.

UNUSUAL FIRE AND EXPLOSION HAZARDS 


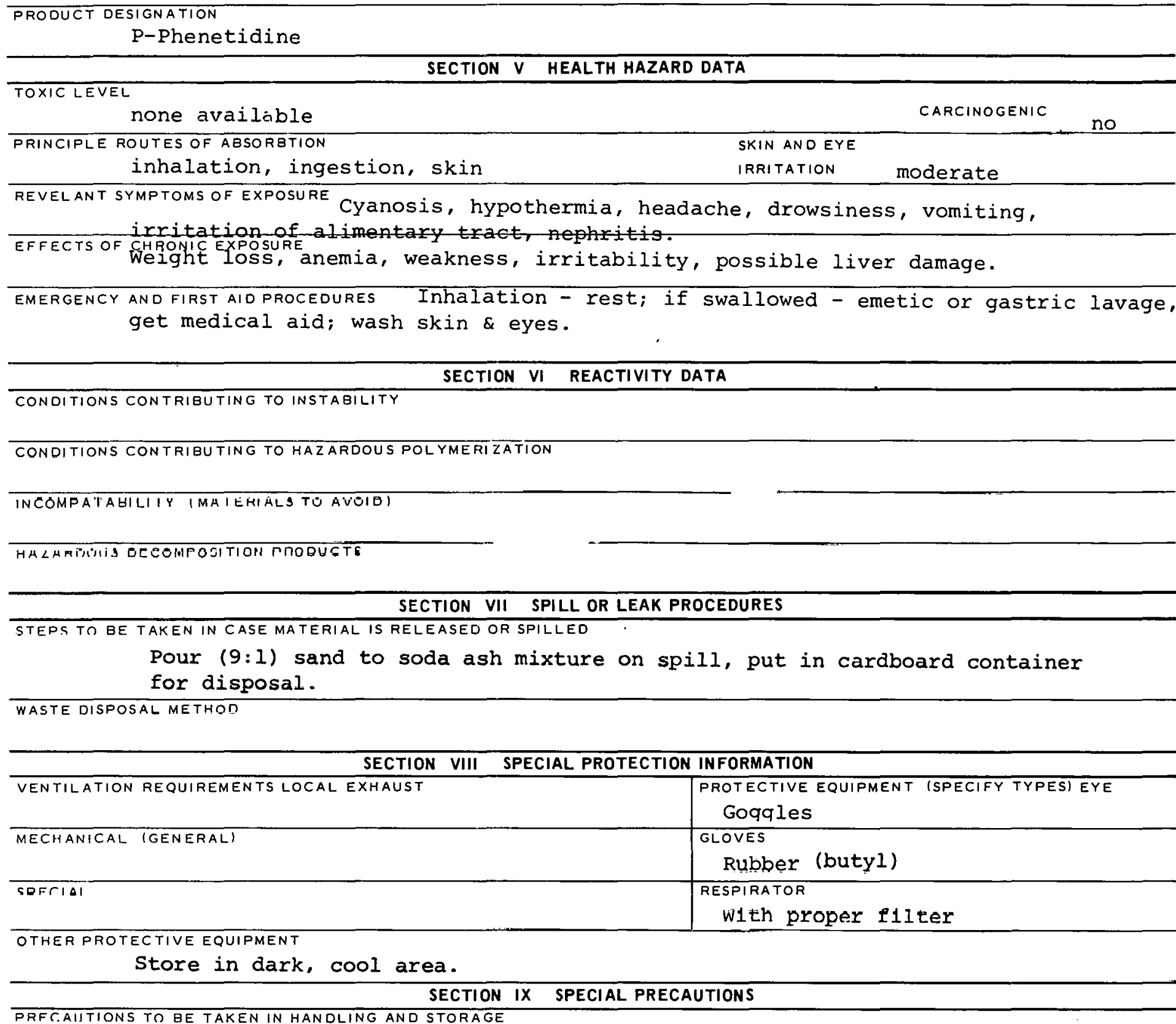

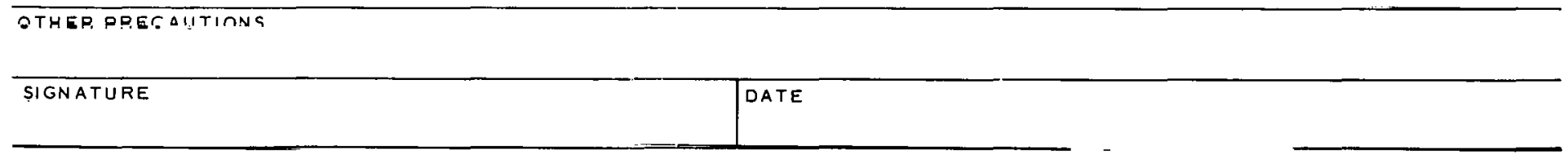


CAS : 000108952

SJ 33250

\section{PRODUCT DESIGNATION}

Phenol

03-326-6904

MATERIAL SAFETY

DATA SHEET

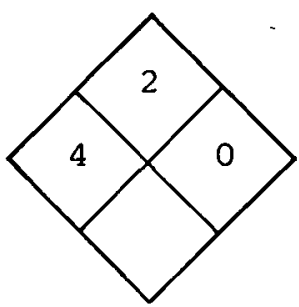

\section{SECTION I SOURCE AND NOMENCLATURE}

\section{MANUFACTURER'S NAME \\ TRADE NAME AND SYNONYMS \\ CHEMICAL NAME AND SYNONYMS \\ Carbolic Acid}

ADORESS (NUMBER, STREET, CITY, STATE, ZIP CODE)

EMERGENCY TELEPHONE NO.

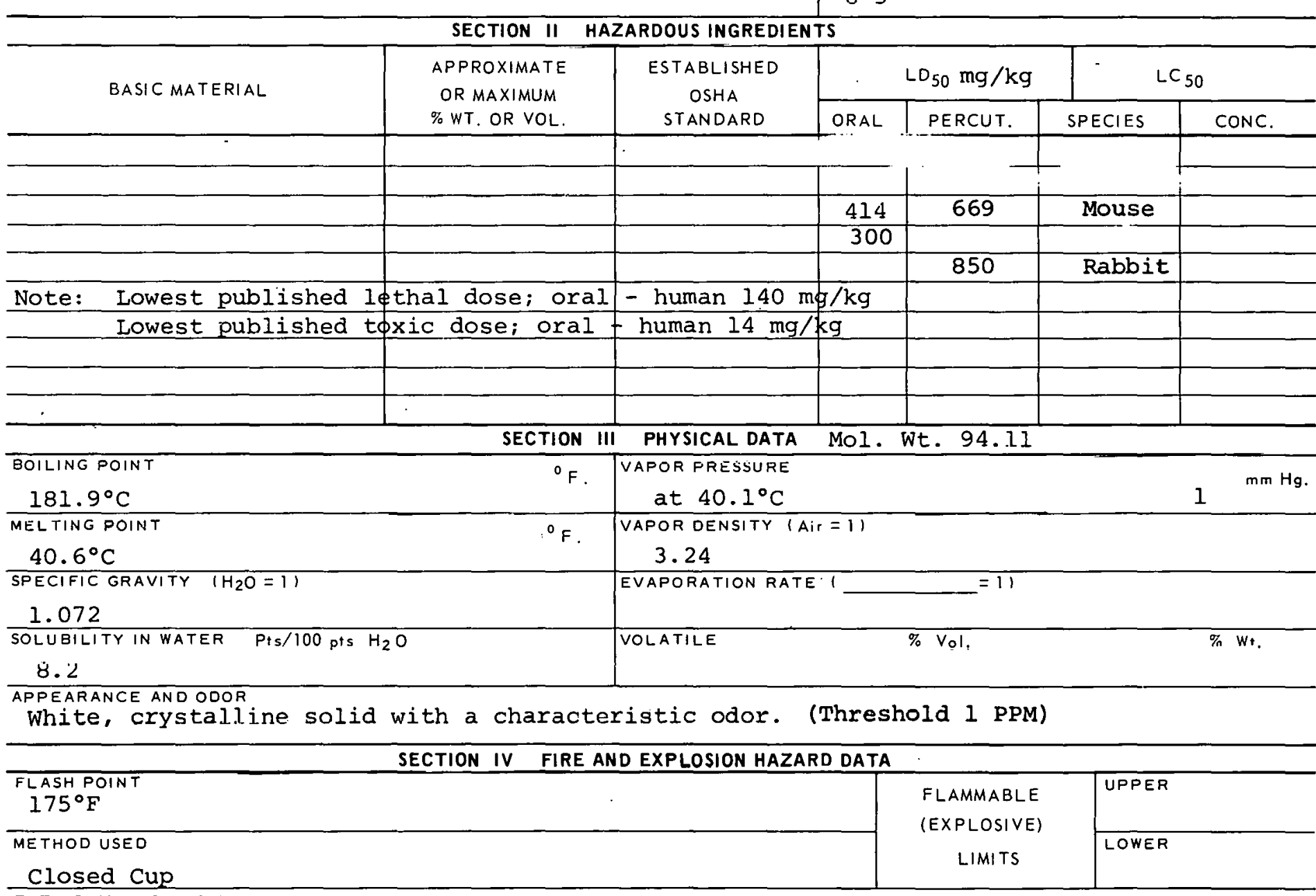

\begin{tabular}{l|l} 
& CHEMICAL FAMILY \\
& $\begin{array}{l}\text { FORMULA } \\
\mathrm{C}_{6} \mathrm{H}_{5} \mathrm{OH}\end{array}$ \\
\hline SECTION II HAZARDOUS INGREDIENTS
\end{tabular}




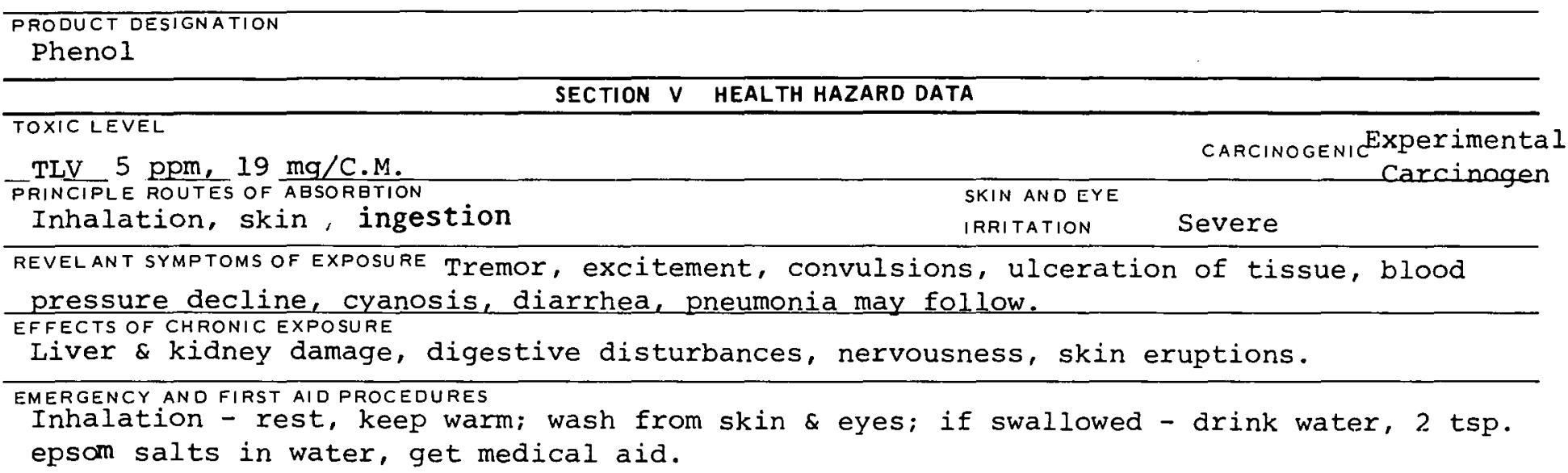

TOXIC LEVEL

TLV $5 \mathrm{ppm}, 19 \mathrm{mg} / \mathrm{C} . \mathrm{M}$. PRINCIPLE ROUTES OF ABSORBTION

Inhalation, skin, ingestion

SKIN AND EYE

CARCINOGENICXperimental IRRITATION Severe

REVELANT SYMPTOMS OF EXPOSURE Tremor, excitement, convulsions, ulceration of tissue, blood pressure decline, cyanosis, diarrhea, pneumonia may follow.

EFFECTS OF CHRONIC EXPOSURE

Liver \& kidney damage, digestive disturbances, nervousness, skin eruptions.

EMERGENCY ANO FIRST AID PROCEDURES

Inhalation - rest, keep warm; wash from skin \& eyes; if swallowed - drink water, 2 tsp. epsom salts in water, get medical aid.

\section{SECTION VI REACTIVITY DATA}

CONDITIONS CONTRIBUTING TO INSTABILITY

CONDITIONS CONTRIBUTING TO HAZARDOUS POLYMERIZATION

INCOMRATABILITY (MATERIALS TO AVOIO)

Calcium hypochlorite, butadiene and aluminum chloride and nitrobenzene

HAZARDQUS DECOMPOSITION PRODUCTS

SECTION VII SPILL OR LEAK PROCEDURES

STEPS TO BE TAKEN IN CASE MATERIAL IS RELEASED OR SPILLED

Flush with quantities of water (evacuate area) caustic soda solution used for neutralization.

WASTE DISPOSAL METHOD

SECTION VIII SPECIAL PROTECTION INFORMATION FUII-PIOLECLIOH

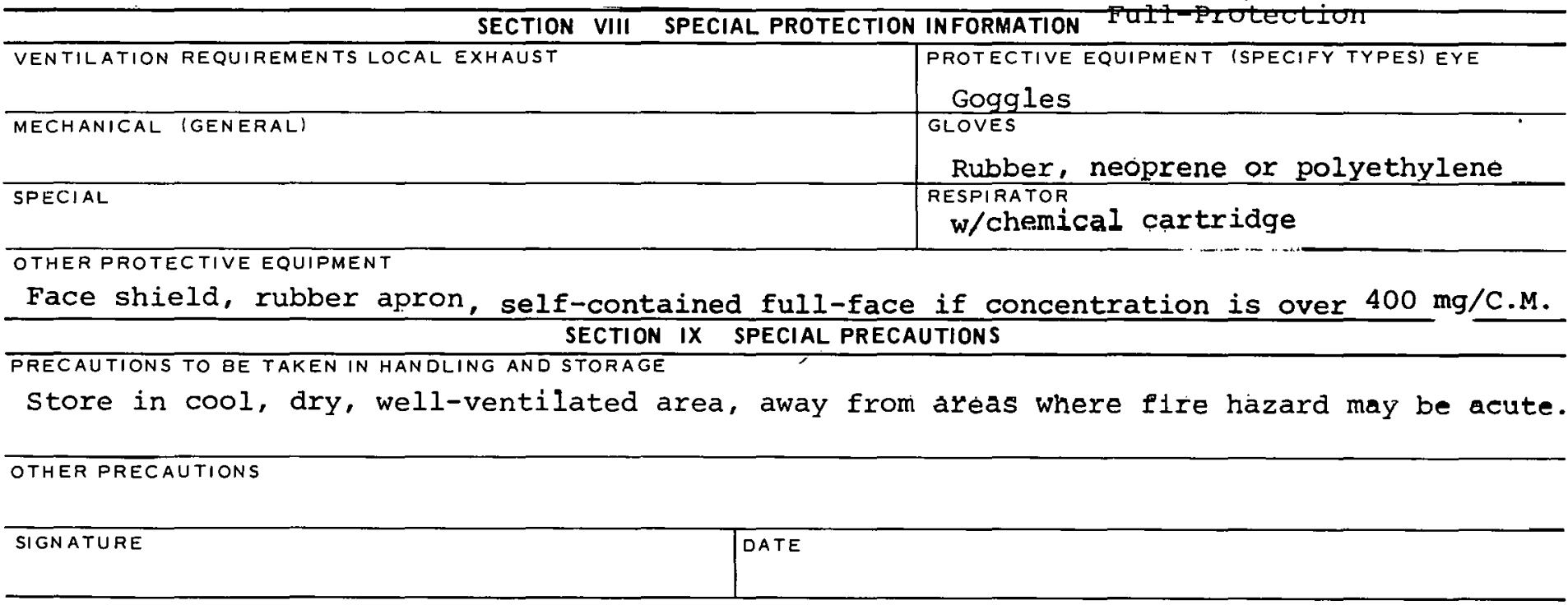



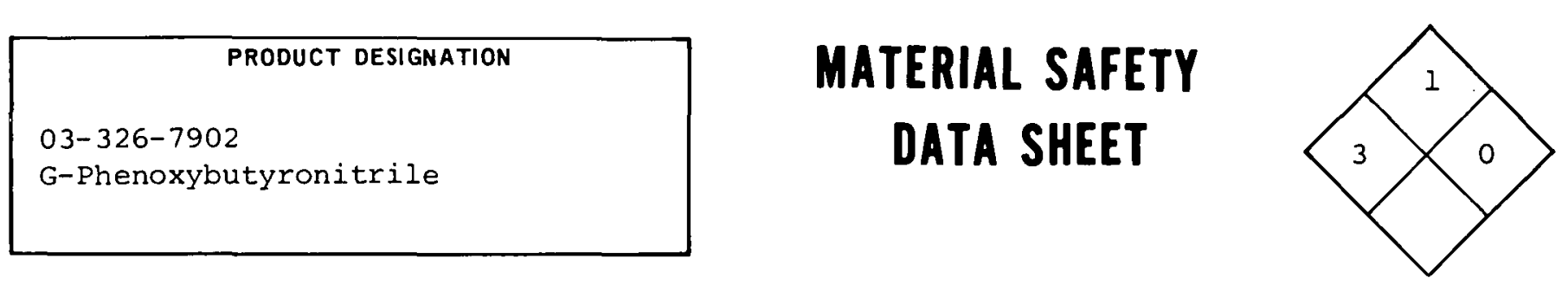

SECTION I SOURCE AND NOMENCLATURE

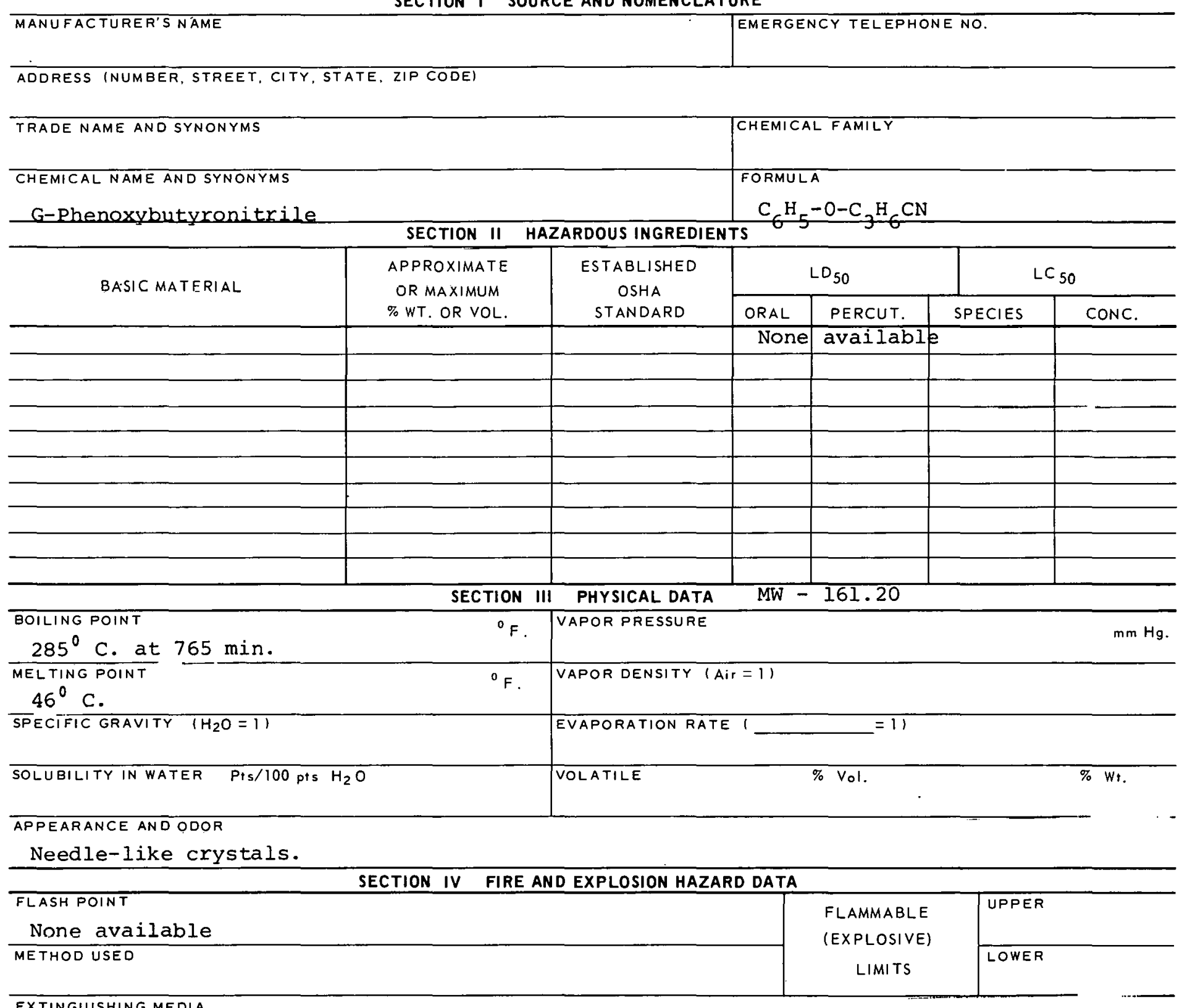

EXTINGUISHING MFDIA

Dry chemical, alcohol foam or carbon dioxide.

SPECIAL FIRE FIGHTING PROCEDURES

UNUSUAL FIRE ANDEXPLOSION HAZARDS 


\section{TOXIC LEVEL}

None available

PRINCIPLE ROUTES OF ABSORBTION

Inhalation, ingestion, skin SKIN AND EYE IRRITATION Moderate
CARCINOGENIC

REVELANT SYMPTOMS OF EXPOSURE Dizziness, rapid respiration, headache, drowsiness, drop in blood pressure and pulse, delayed symptoms. EFFECTS OF CHRONIC EXPOSURE

Eye irritation, loss of appetite, weight loss, mental deterioration. EMERGENCY AND FIRST AID PROCEDURES If swallowed - inhalation of amyl nitrite, induce vomiting or gastric lavage; wash skin and eyes; inhalation - rest.

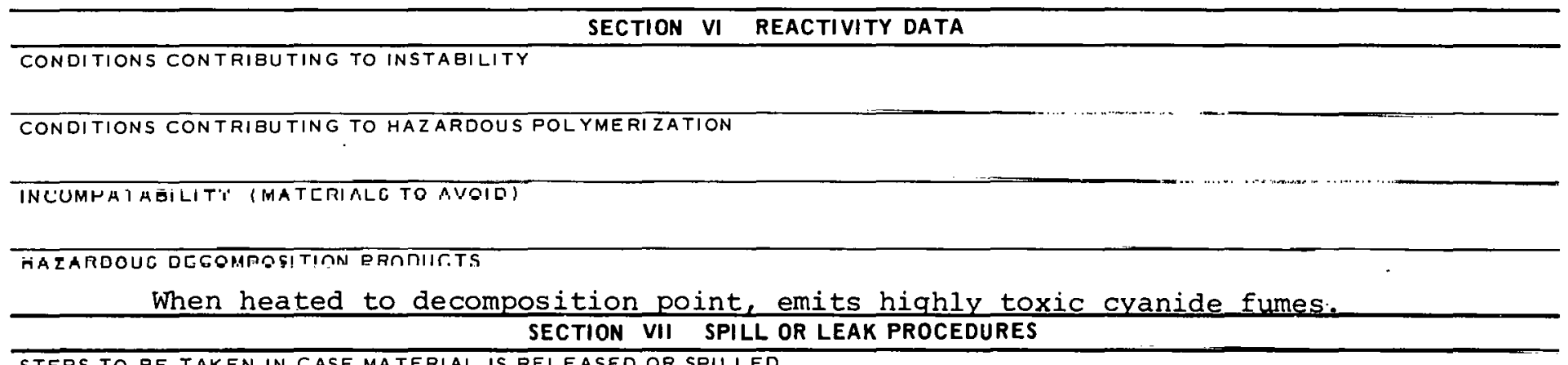
STEPS TO RF. TAKEN IN CASE MATERIAL IS RELEASED OR SPILLED

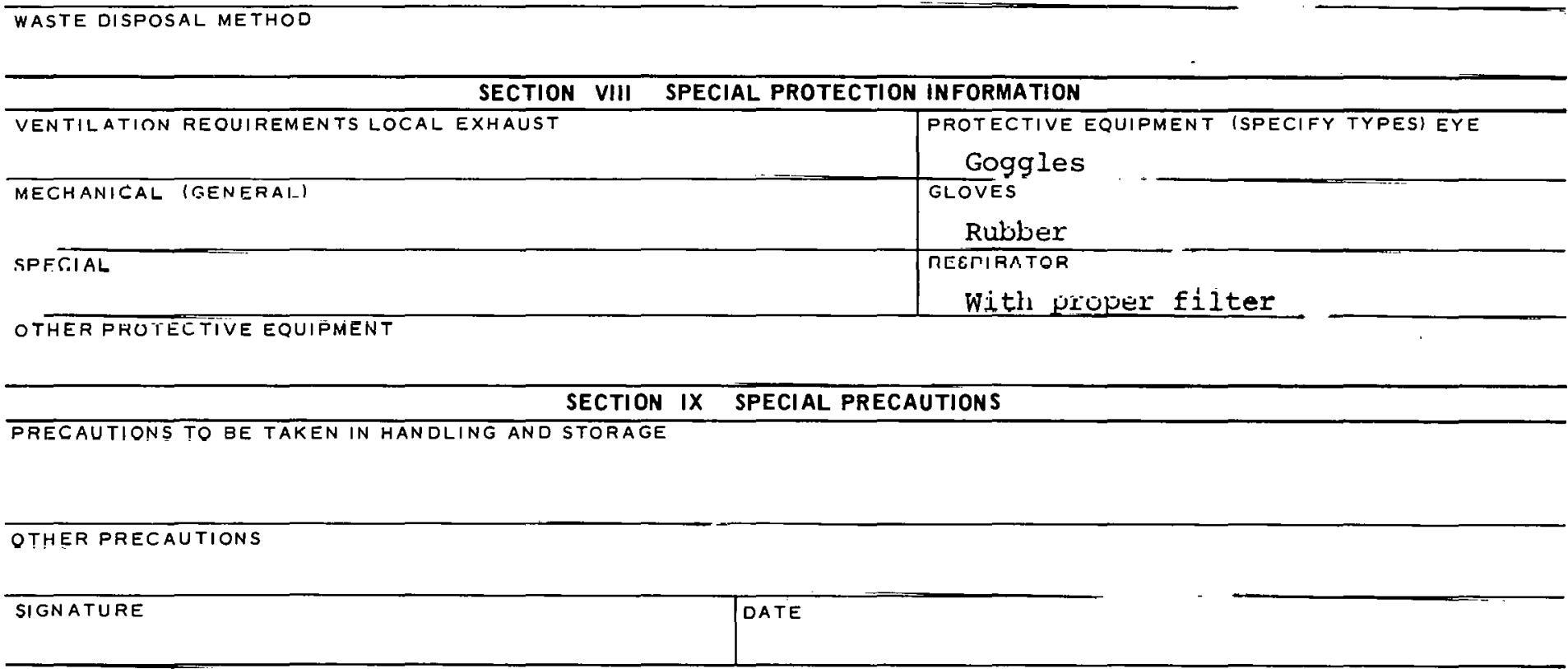




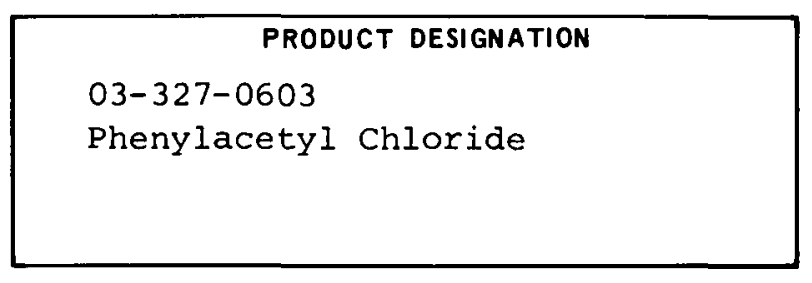

\section{MATERIAL SAFETY DATA SHEET}

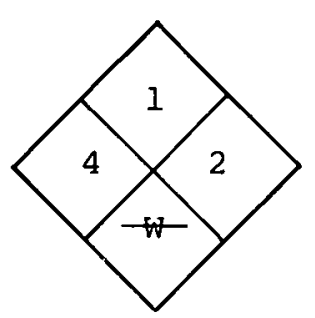

SECTION I SOURCE AND NOMENCLATURE

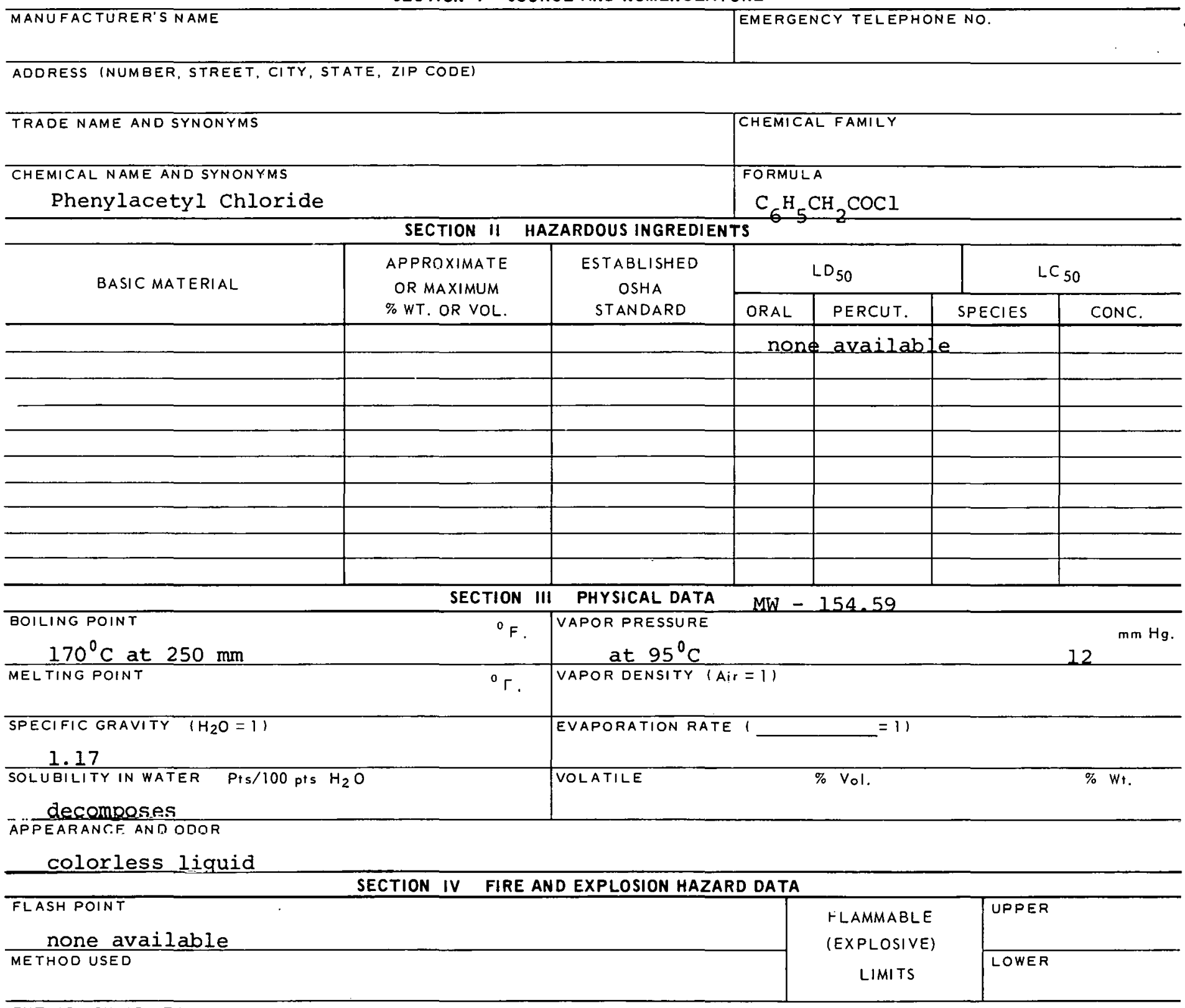

EXTINGUISHING MEDIA

carbon dinxide, dry chemical

SPECIAL FIRE FIGHTING PROCEDURES

wear full protective clothing.

UNUSHECESE WIThE WateELN HAZAROS 
Phenyl Acetyl Chloride

TOXIC LEVEL

none

CARCINOGENIC

PRINCIPLE ROUTES OF ABSORBTION

inhalation, ingestion

SKIN ANDEYE

IRRITATION

severe

REVELANT SYMPTOMS OF EXPOSURE Cyanosis, coughing, headache, pulmonary edema,

ulceration of skin, dizziness.

EFFECTS OF CHRONIC EXPOSURE Erosion of teeth, jaw necrosis, bronchial irritation and chronic cough.

EMERGENCY AND FIRST AIOPROCEDURES Inhalation - artificial respiration if necessary; ingestion drink water, milk of magnesia, get medical aid; wash from skin \& eyes.

SECTION VI REACTIVITY DATA

CONDITIONS CONTRIBUTING TO INSTABILITY

CONDITIONS CONTRIBU TING TO HAZARDOUS POLYMERIZATION

INCOMPAIABILIIY IMAIEHIRLS TU AUUIOI

Avoid water.

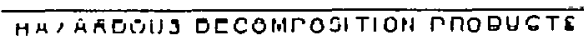

Produces $\mathrm{HCl}$ with water.

SECTION VII SPILL OR LEAK PROCEDURES

STEPS TO BE TAKEN IN CASE MATERIAL IS RELEASED OR SPILLED

WASTE DISPOSAL METHOD

SECTION VIII SPECIAL PROTECTION INFORMATION

\begin{tabular}{l|l|l}
\hline VENTILATION REQUIREMENTS LOCAL EXHAUST & PROTEC TIVE EQUIPMENT ISPECIFY TYPES\} EYE
\end{tabular}

MECHANICAL (GENERAL)

Goggles

GLOVES

Rubber

SPFC.IAL.

RESPIRATOR

With proper Eilter

OTHER PROTECTIVE EQUIPMENT

\section{SECTION IX SPECIAL PRECAUTIONS}

PRECAUTIONS TO BE TAKEN IN HANDLING AND STORAGE

Store in cool, dry well-ventilated room; inside storage in standard flammable liquids storage cabinet.

OTHER PRECAUTIOQNS 
CAS : 000108452

PRODUCT DESIGNATION

$03-327-2603$

M-Phenylenediamine

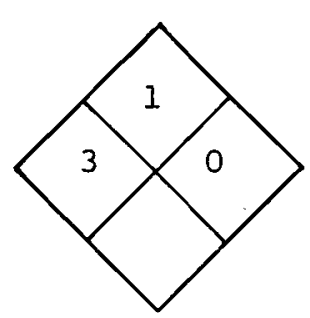

SECTION I SOURCE AND NOMENCLATURE

MANUFACTURER'S NAME

ADDRESS (NUMBER, STREET, CITY, STATE, ZIP CODE)

TRADE NAME AND SYNONYMS

3 Aminoaniline

CHEMICAL NAME AND SYNONYMS

1,3 Aminobenzene

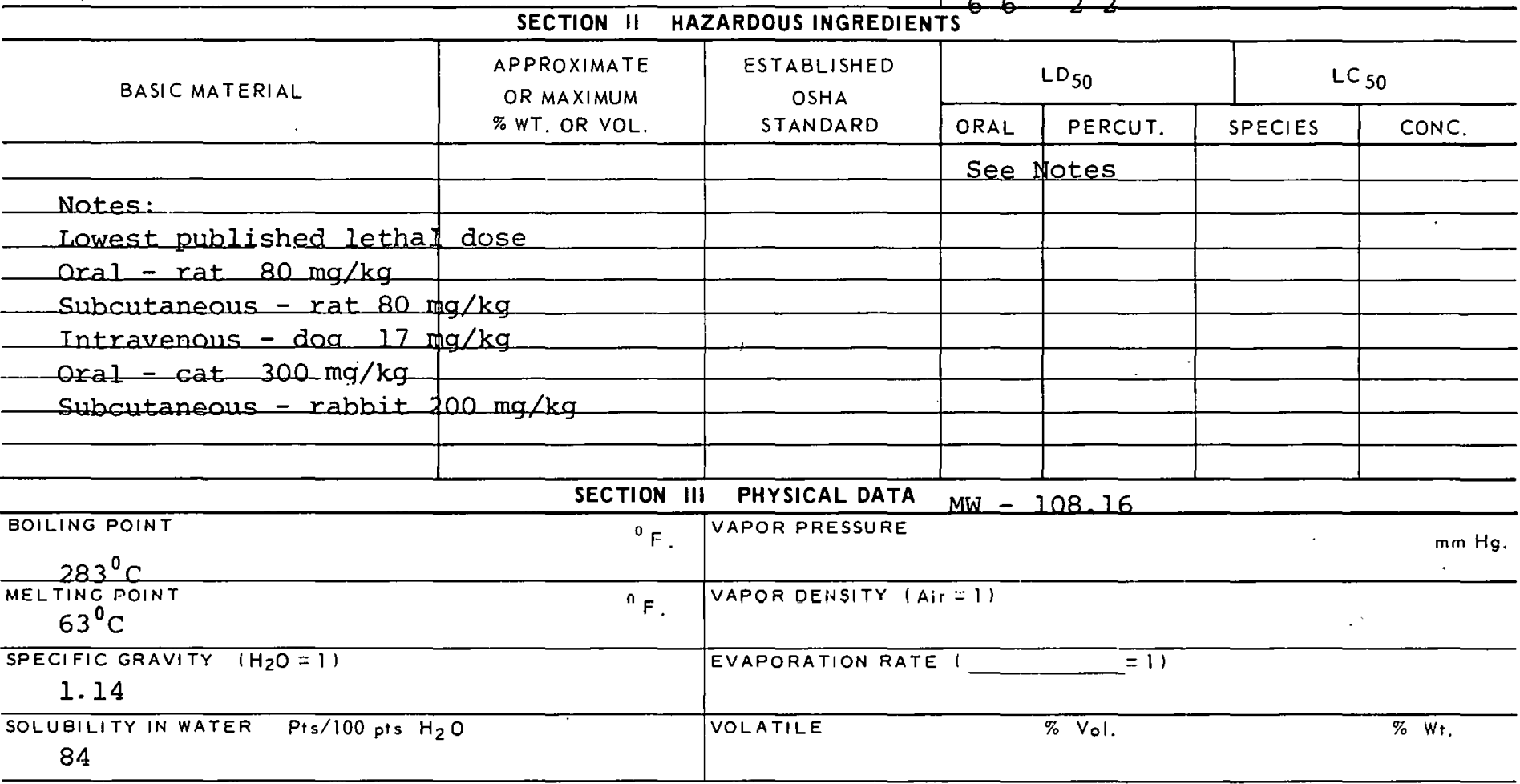

APPEARANCE AND ODOR

colorless crystal

SECTION IV FIRE AND EXPLOSION HAZARD DATA

FLASH FOINT
none available

METHOD USED

FLAMMABLE

(EXPLOSIVE)

LIMITS

EXTINGUISHING MEDIA

water, carbon dioxide, dry chemical.

SPECIAL FIRE FIGHTING PROCEDURES

UNUSUAL FIRE AND EXPLOSION HAZARDS 


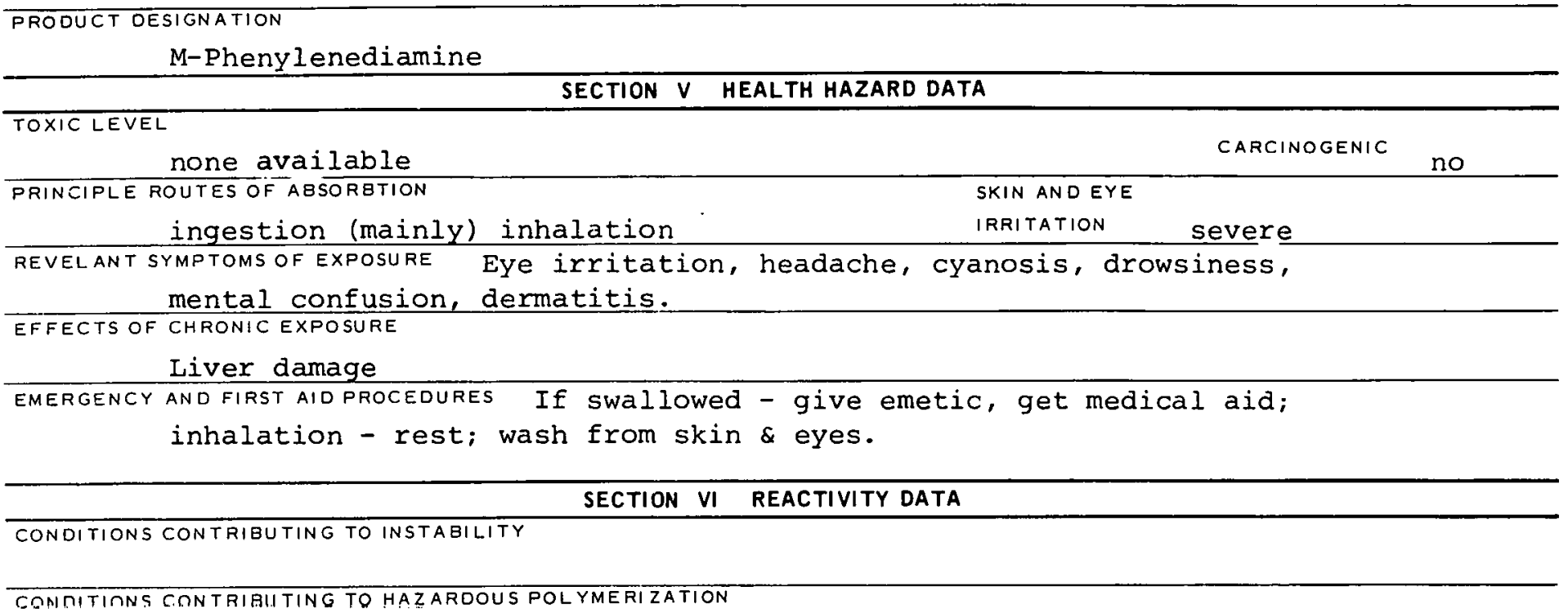

INCOMPATAEILITY (MATERIALS TO AVOIOI

HAZARDOUS DECOMPOSIIION PRODUCTS

SECTION VII SPILL OR LEAK PROCEDURES

STEPS TO BE TAKEN IN CASE MATERIAL IS RELEASED OR SPILLED

Cover with $(9: 1)$ sand to soda ash mixture; put in cardboard carton

for disposal.

WASTE DISPOSAL METHOD

SECTION VIII SPECIAL PROTECTION INFORMATION

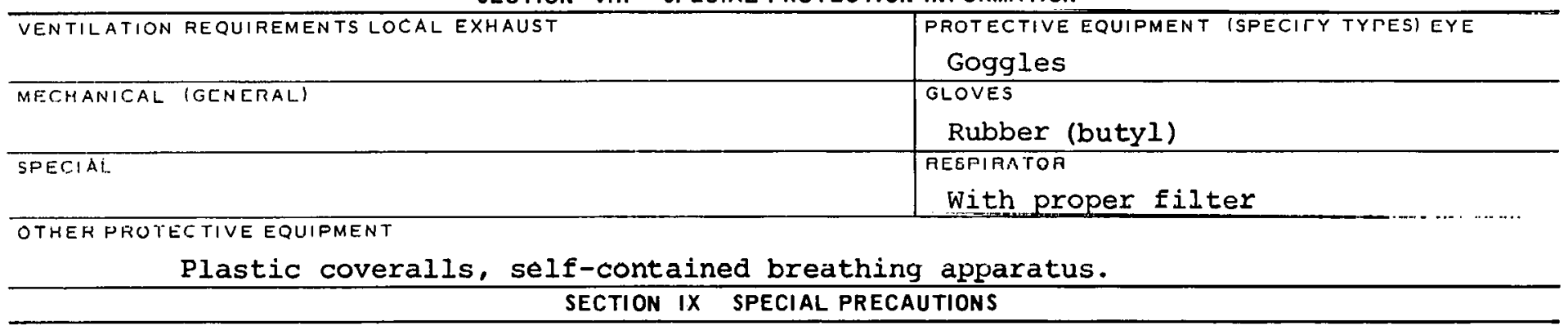

PRECAUTIONS TO BE TAKEN IN HANDLING AND STORAGE

OTHER PRECAUTIONS

SIGNAIUHE

பேंТอ

$327-6203 B$ 


\begin{tabular}{|l|}
\hline PRODUCT DESIGNATION \\
03-327-2813 \\
P-Phenylene Diamine \\
\end{tabular}

\section{MATERIAL SAFETY DATA SHEET}

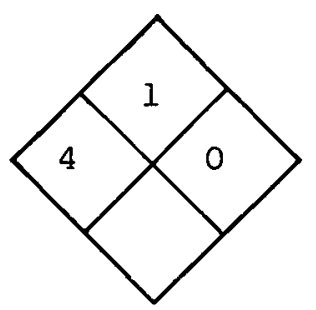

\section{SECTION I SOURCE AND NOMENCLATURE}

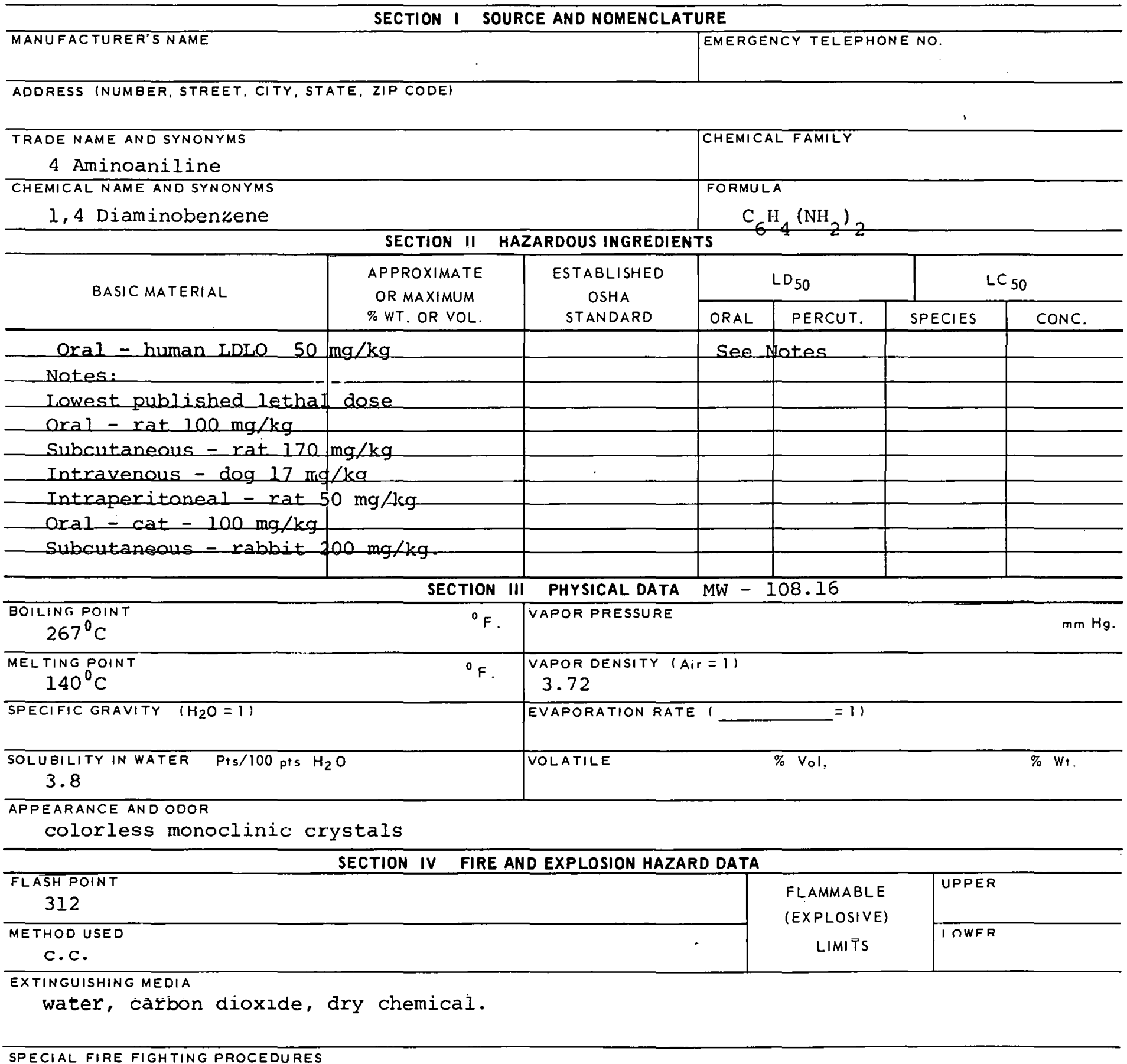

SPECIAL FIRE FIGHTING PROCEDURES

UNUSUAL FIRE AND EXPLOSION HAZARDS 
TOXIC LEVEL

$0.1 \mathrm{mg} / \mathrm{C} . \mathrm{M} .(\mathrm{skin})$

CARCINOGENIC no

PRINCIPLE ROUTES OF ABSORBTION

inhalation, ingestion, skin SKIN AND EYE

IRRITATION severe

REVELANT SYMPTOMS OF EXPOSURE

dermatitis, eye irritation.

EFFECTS OF CHRONIC EXPOSURE Allergenic bronchial asthma, dermatitis, liver damage.

EMERGENCY AND FIRST AID PROCEDURES If swallowed - give emetic, get medical aid;

inhalation - rest; wash from skin \& eyes.

SECTION VI REACTIVITY DATA

CONDITIONS CONTRIBUTING TO INSTABILITY

CONDITIONS CONTRIBUTING TO HAZARDOUS POLYMERIZ $\overline{\text { TION }}$

INCOMPATADILITY (AATEFIALE TO AVDIDI

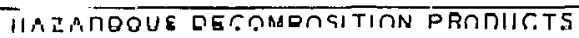

SECTION VII SPILL OR LEAK PROCEDURES

STFPS TO BE TAKEN IN CASE MATERIAL IS RELEASED OR SPILLED

Cover with $(9: 1)$ sand to soda ash mixture; put into cardboard carton

for disposal.

WASTE DISPOSAL METHOD

\section{SECTION VIII SPECIAL PROTECTION INFORMATION}

VFNTILATION REQUIREMENTS LOCAL EXHALST

ROTECTIVE EQUIPMENT (SPECIFY TYPES) EYE

MECHANICAL (GENERAL)

SPECIAL Goggles

GLOVES

Rubber (butyl)

REIFIRATOR

Will proper filter

OIHER NRUIEL IIVE EQUIPMENT

Plastic coveralls, self-contained breathing apparatus. SECTION IX SPECIAL PRECAUTIONS

PRECAUTIONS TO BE TAKEN IN HANDLING AND STORAGE

OTHER PRECAUTIONS

SIGNATURE

DATE

$327-2813 B$ 
03-327-2903

M-Phenylenediamine Dihydrochloride
MATERIAL SAFETY

DATA SHEET

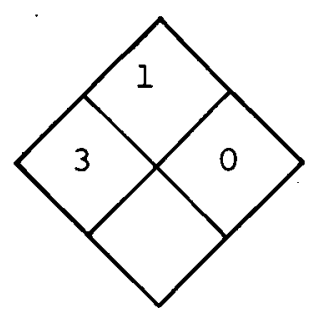

SECTION I SOURCE AND NOMENCLATURE

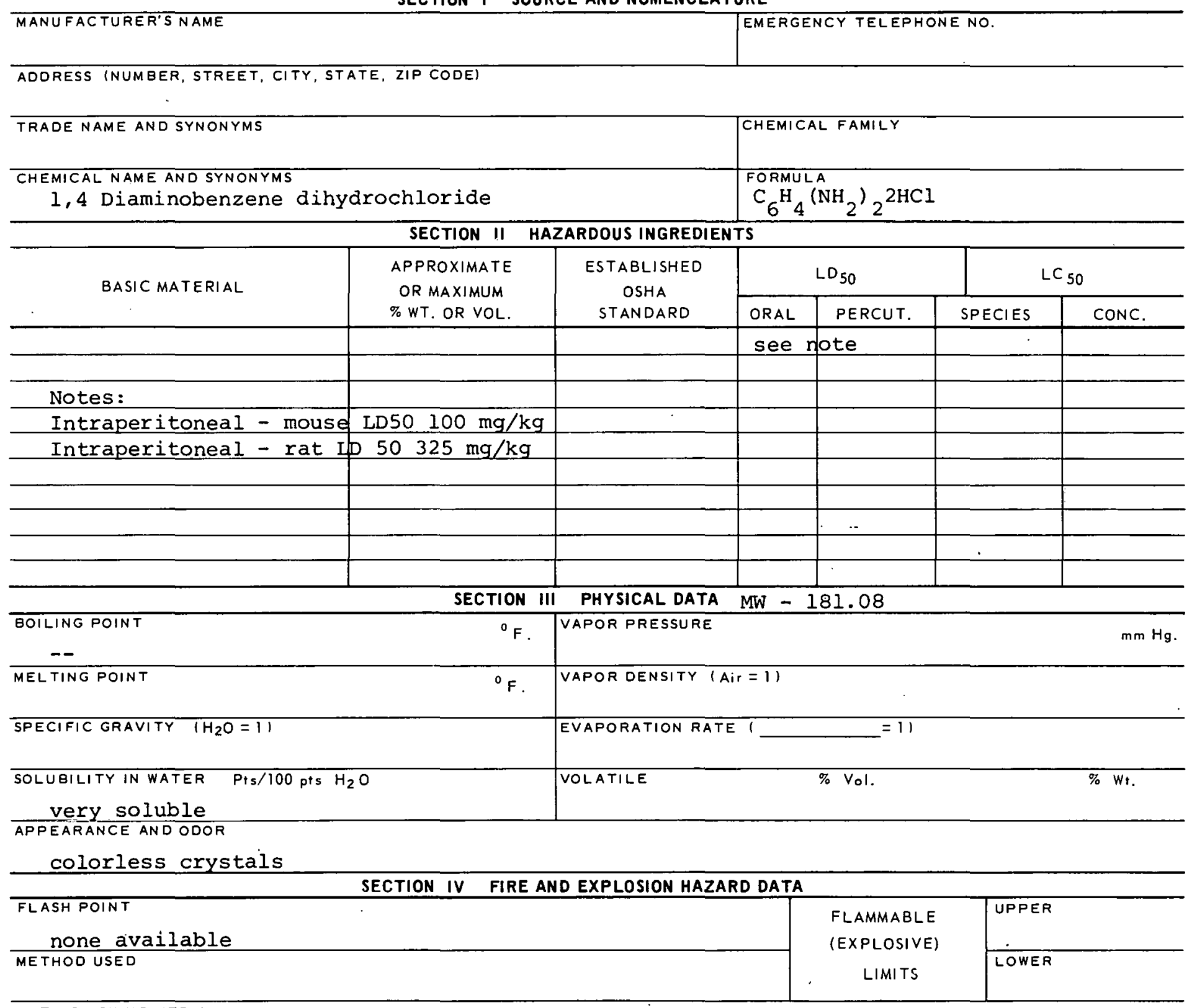

EXTINGUISHING MEDIA

water, carbon dioxide, dry chemical.

SPECIAL FIRE FIGHTING PROCEDURES

UNUSUAL FIRE AND EXPLOSION HAZARDS 
M-Phenylenediamine Dihydrochloride SECTION $V$ HEALTH HAZARD DATA

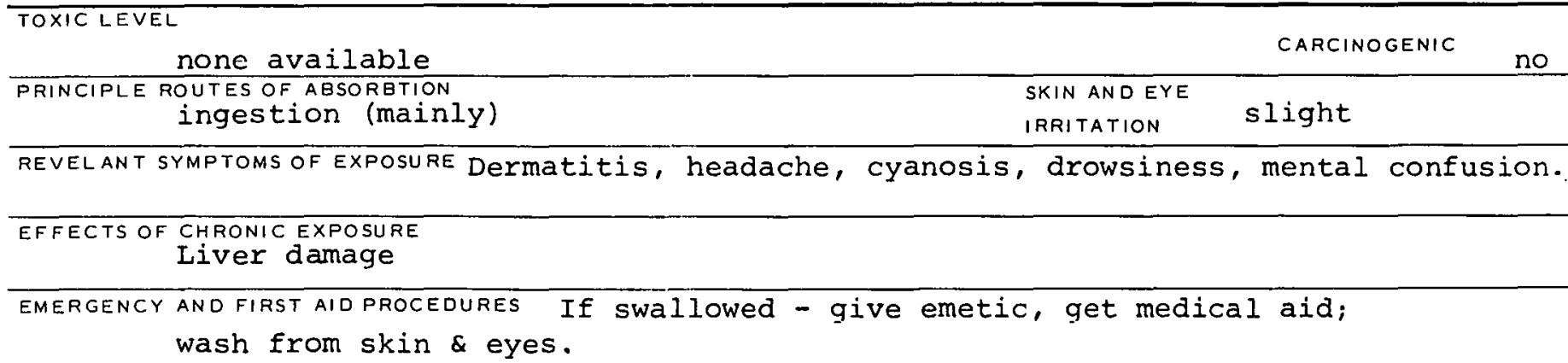

$\begin{aligned} \text { ingestion (mainly) } & \text { IRRITATION SIIght }\end{aligned}$

REVELANT SYMPTOMS OF EXPOSURE Dermatitis, headache, cyanosis, drowsiness, mental confusion.

\begin{tabular}{l|l}
\hline VENTILATION REQUIREMENTS LOCAL EXHAUST & $\begin{array}{l}\text { PROTECTIVE EQUIPMENT ISPECIFY TYPESI EYE } \\
\text { GOggles }\end{array}$ \\
\hline MECHANICAL IGENERALI & GLOVES \\
\hline SPECIAL & RESPIRATOR \\
\hline OTIIER PROTECTIVE CQUIPMENT
\end{tabular}

\section{SECTION IX SPECIAL PRECAUTIONS}

PRECAUTIONS TO BE TAKEN IN HANDLING AND STORAGE

\section{OTHER PRECAUTIONS}




\section{PRODUCT DESIGNATION}

03-327-3603

B-Phenylethyl Bromide
MATERIAL SAFETY

DATA SHEET

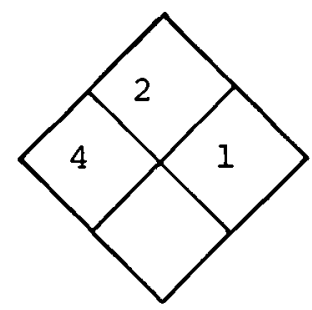

SECTION I SOURCE AND NOMENCLATURE

\section{MANUFACTURER'S NAME}

ADDRESS (NUMAER, STREET, CITY, STATE, ZIP CODE)
EMERGENCY TELEPHONE NO.

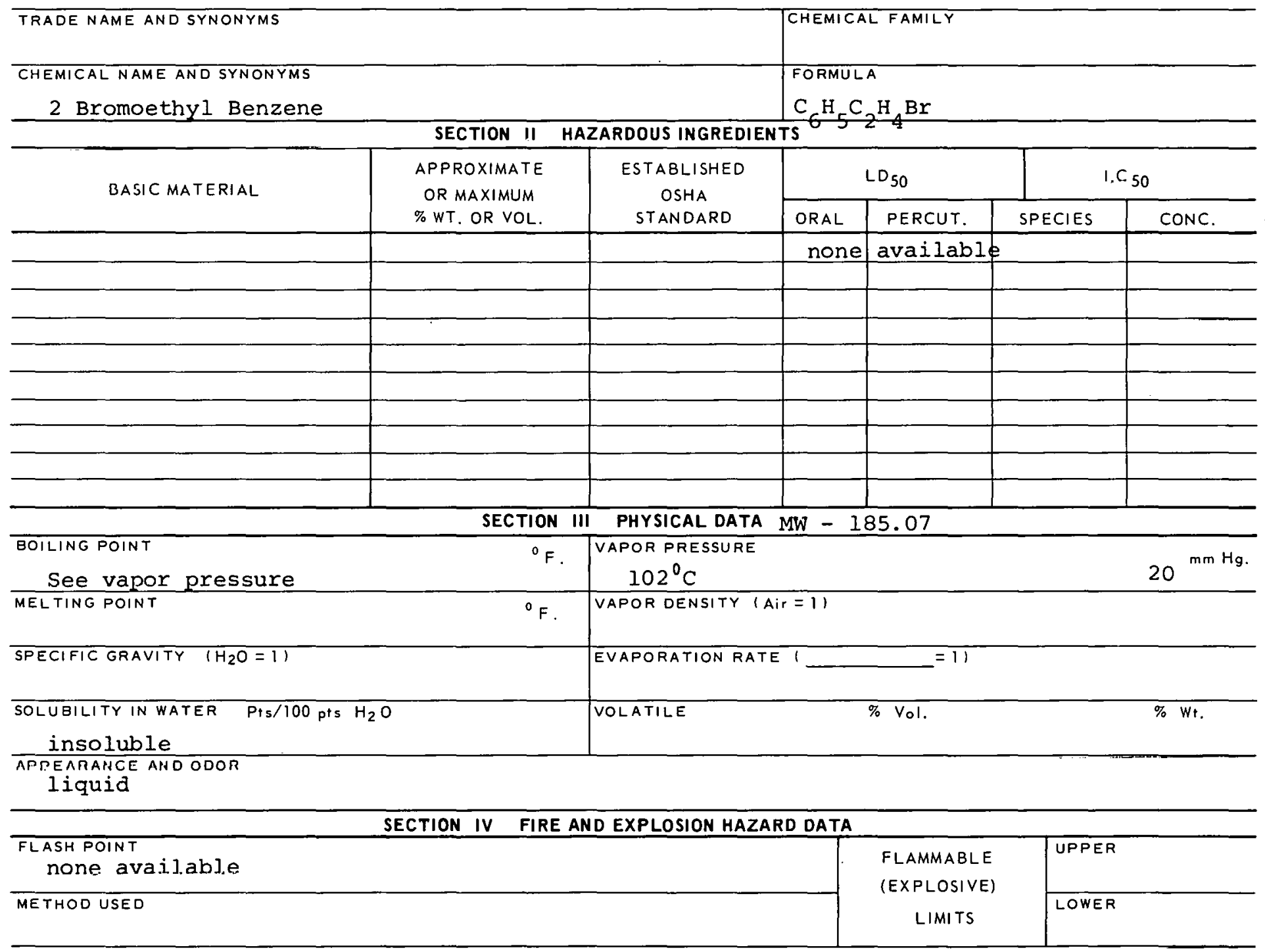

\section{EXTINGUISHING MEDIA}

water spray, dry chemical, carbon dioxide.

SPECIAL FIRE FIGHTING PROCEDURES 


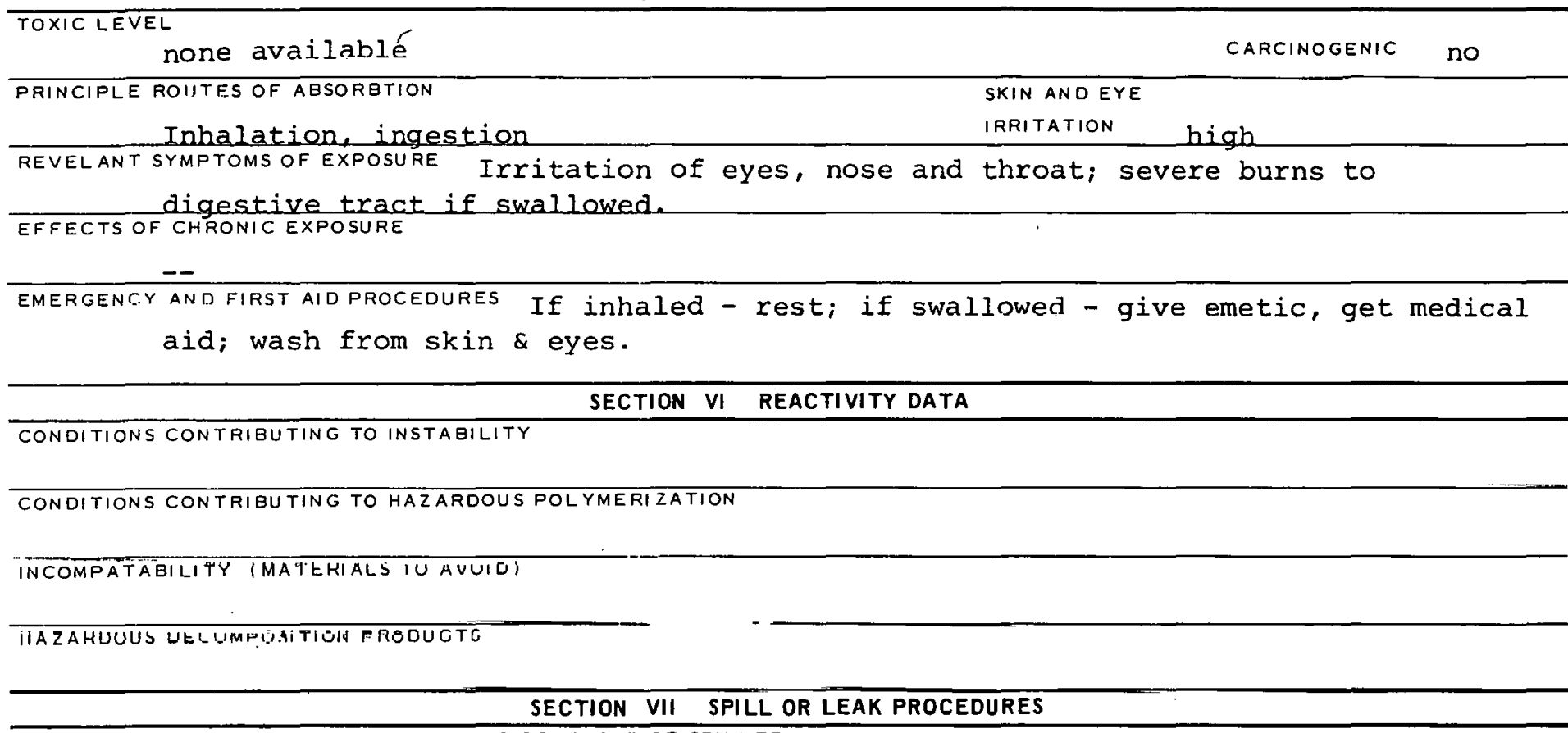

STEPS TO BE TAKEN IN CASE MATERIAL IS RELEASED OR SPILLED

WASTE DISPOSAL METHOD

SECTION VIII SPECIAL PROTECTION INFORMATION

\begin{tabular}{l|l}
\hline VENTILATION REQUIREMENTS LOCAL EXHAUST & $\begin{array}{c}\text { PROTECTIVE EQUIPMENT (SPECIFY TYPES) EYE } \\
\text { GOgGleS }\end{array}$ \\
\hline MECHANICAL IGENERAL) & $\begin{array}{c}\text { GLOVES } \\
\text { Rubber }\end{array}$ \\
\hline SREGIAL & $\begin{array}{r}\text { RESPIRATOR } \\
\text { Self-contained breathing apparatus }\end{array}$ \\
OTHER PROTECTIVE EQUIPMENT \\
\hline
\end{tabular}




PRODUCT DESIGNATION
$03-327-5103$
Phenylhydrazine

\section{MATERIAL SAFETY DATA SHEET}

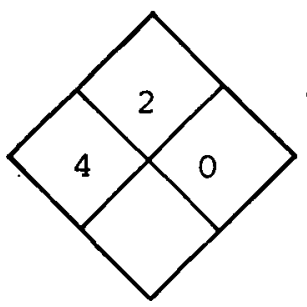

SECTION I SOURCE AND NOMENCLATURE

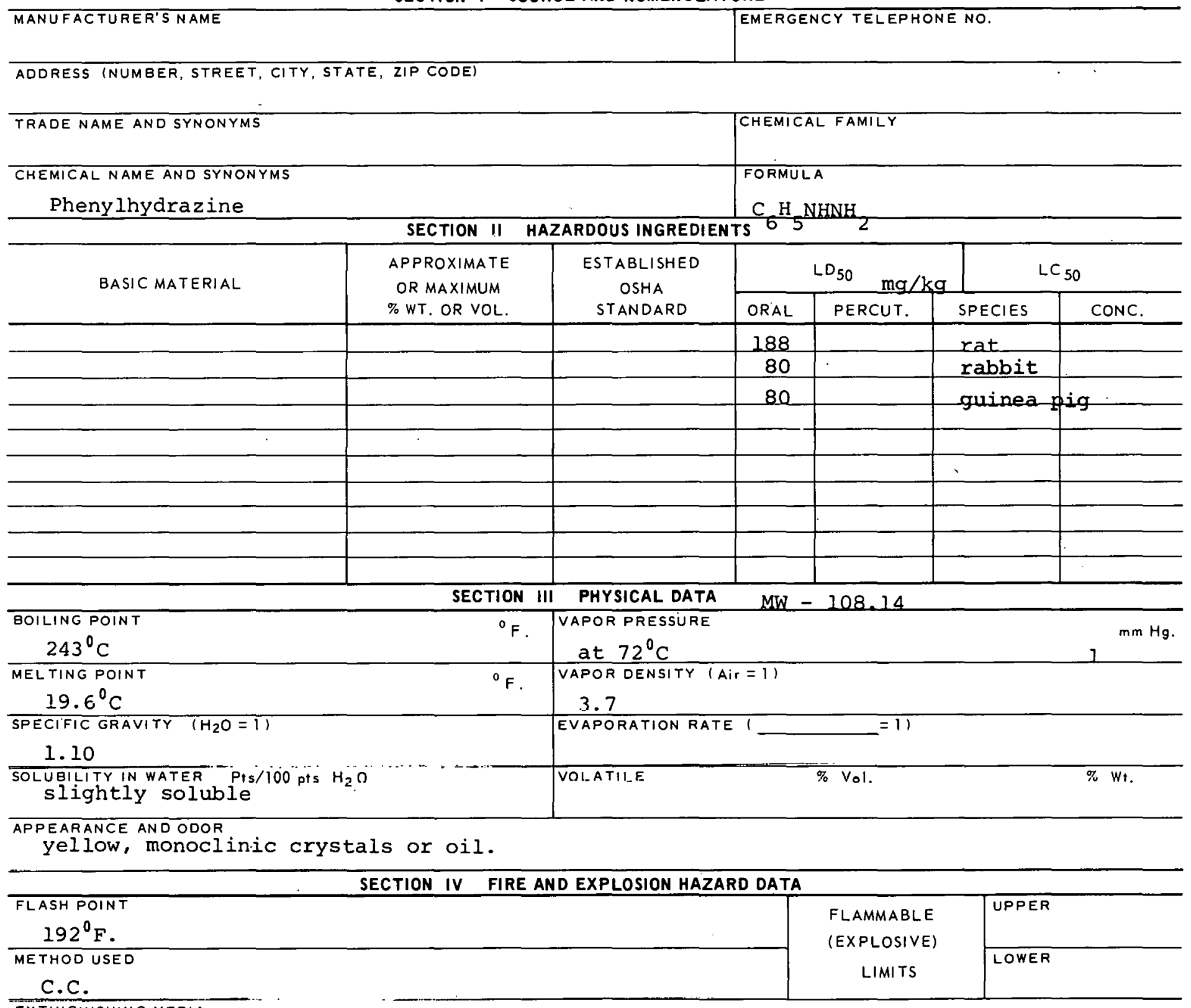

EXTINGUISHING MEDIA

Alcohol foam, water, carbon dioxide.

SPECIAL FIRE FIGHTING PROCEDURES

UNUSUAL FIRE ANU EXFLOSION HAZARDS

When heated to decomposition point, it gives off fumes of nitrogen compounds. 
TOXIC LEVEL

TLV 5 PPM, $22 \mathrm{mg} / \mathrm{C} . \mathrm{M}$.

PRINCIPLE ROUTES OF ABSORBTION

Inhalation, ingestion, skin

CARCINOGENIC

no

REVELANT SYMPTOMS OF EXPOSURE Irritation of skin, eyes and mucous membranes, gastroentric disturbances, anemia.

EFFECTS OF CHRONIC EXPOSURE

Blood and liver damage, loss of appetite.

EMERGENCY AND FIRST AID PROCEOURES Inhalation - rest; if swallowed - induce vomiting, get

medical aid; wash from skin \& eyes.

\begin{tabular}{|c|c|}
\hline & SECTION VI REACTIVITY DATA \\
\hline CONDITIONS CONTRIBUTING TO INSTABILI & \\
\hline CONDITIONS CONTRIBUTING TO HAZARDOU & YMERIZATION \\
\hline $\begin{array}{l}\text { INLUMHAIABILIT' IMATERIALSTO AVOID } \\
\text { AVoid lead dioxide. }\end{array}$ & \\
\hline
\end{tabular}

SECTION VII SPILL OR LEAK PROCEDURES

STEPS TO RE TAKEN IN CASE MATERIAL IS RELEASED OR SPILLED

Dilute with water, neutralize with sulfuric acid.

WASTE QISPOSAL METHOD

SECTION VIII SPECIAL PROTECTION INFORMATION

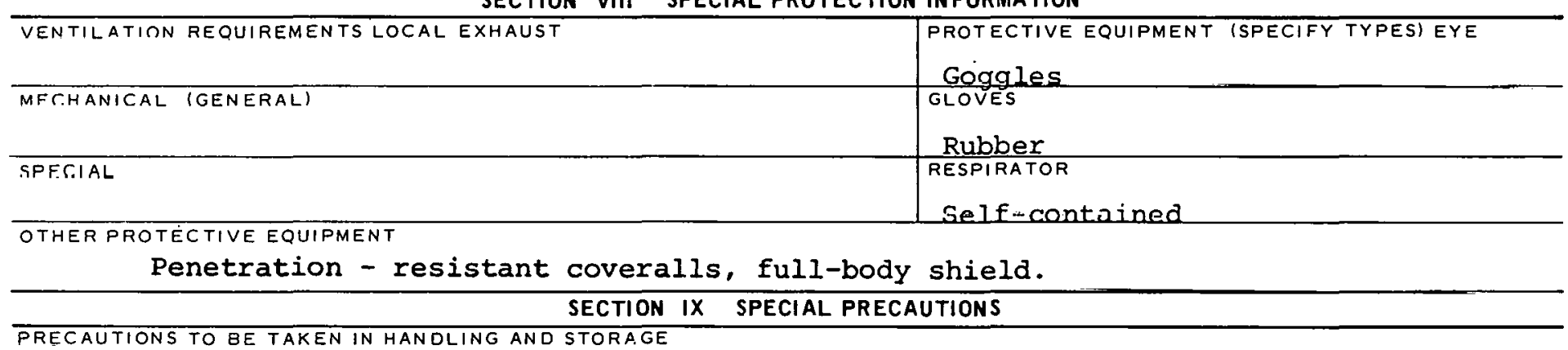

PRECAUTIONS TO BE TAKEN IN HANOLING AND STORAGE

Store in flammable liquids storage cabinet; detached storage preferred.

\section{OTHER PRECAUTIONS}




\begin{tabular}{|l} 
PRODUCT DESIGNATION \\
$03-327-5203$ \\
Phenylhydrazine Hydrochloride
\end{tabular}

\section{MATERIAL SAFETY DATA SHEET}

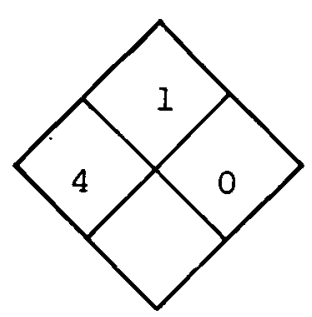

SECTION I SOURCE AND NOMENCLATURE

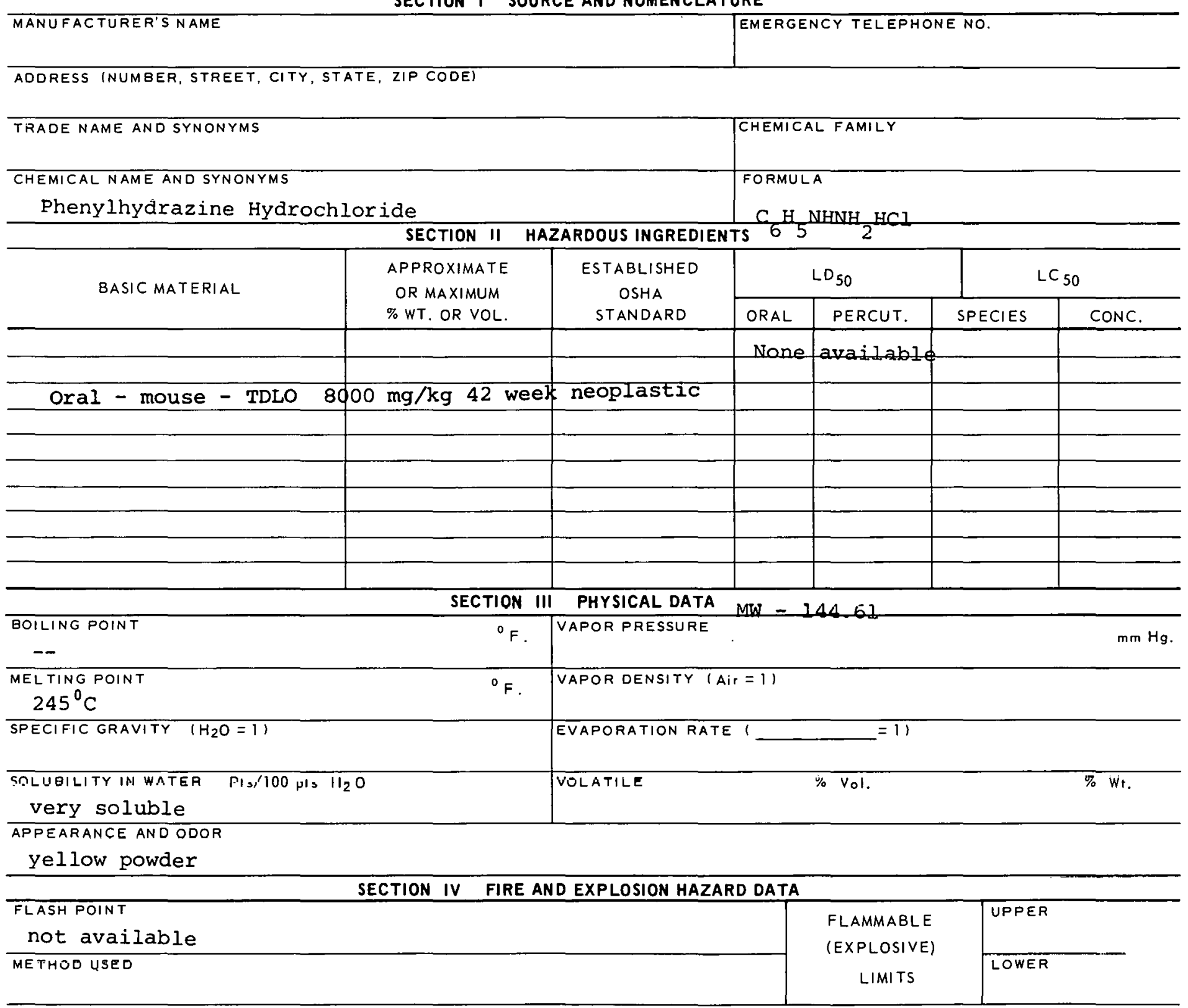

EXTIMCUISIIING MCDIA

alcohol foam, water, carbon dioxide

SPECIAL FIRE FIGHTING PROCEDURES

UNUSUAL FIRE ANDEXPI OSION HATARIS 
PRODUCT DESIGNATION

Phenylhydrazine Hydrochloride

SECTION $V$ HEALTH HAZARD DATA

TOXIC LEVEL

none available

CARCINOGENIC

neoplastic

PRINCIPLE ROUTES OF ABSORBTION

SKIN AND EYE

Inhalation of dust, ingestion, skin IRRITATION

severe

REVELANT SYMPTOMS OF EXPOSURE Irritation of skin, eyes and mucous membranes, gastroentric disturbances, anemia.

EFFECTS OF CHRONIC EXPOSURE

Blood and liver damage, loss of appetite.

EMERGENCY AND FIRST AIDPROCEDURES Inhalation - rest; if swallowed - induce vomiting, get medical aid; wash from skin \& eyes.

\section{SECTION VI REACTIVITY DATA}

CONOITIONS CONTRIBUTING TO INSTABILITY

CONDITIONS CONTRIBUTING TO HAZARDOUS POLYMERIZATION

INCOMPATABILITY (MATERIALS TO AVOID)

Avoid lead dioxide

HAZARDOUS OEECOMPOSITION PHUUUL I S

SECTION VII SPILL OR LEAK PROCEDURES

STEPS TO EE TAKEN IN CASE MATERIAL IS RELEASED OR SPILLED

WASTE DISPOSAL METHOD

SECTION VIII SPECIAL PROTECTION IN FORMATION

\begin{tabular}{l|l}
\hline VENTILATION REQUIREMENTS LOCAL EXHAUST & $\begin{array}{c}\text { PROTECTIVE EQUIPMENT (SPECIFY TYPES) EYE } \\
\text { GÜYYIES }\end{array}$ \\
\hline MECHANICAL (GENERAL) & $\begin{array}{c}\text { GLOVES } \\
\text { RUUbUE }\end{array}$ \\
\hline SHECIAL & RESPIRATOR \\
\hline
\end{tabular}

OTHER PROTECTIVE EQUIPMENT

SECTION IX SPECIAL PRECAUTIONS

PRECAUTIONS TO BE TAKEN IN HANDLING AND STORAGE

OTHER PRECAUTIONE

SIGNATURE

DATC

\begin{tabular}{l|l|l} 
DATE & \\
\hline
\end{tabular}

$327-5203 B$ 
CAS : 000103719

DAे 36750

PRODUCT DESIGNATION

MATERIAL SAFETY

$03-327-5603$

Phenyl Isocyanate

DATA SHEET

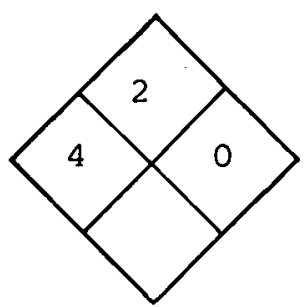

SECTION I SOURCE AND NOMENCLATURE

\section{MANUFACTURER'S NAME}

AODRESS (NUMBER, STREET, CITY, STATE, ZIP CODE)

\begin{tabular}{l|l} 
& CHEMICAL FAMILY \\
& FORMULA \\
& SECTION II HAZARDOUS INGREDIENTS 65
\end{tabular}

SECTION II HAZARDOUS INGREDIENTS 6 NCO

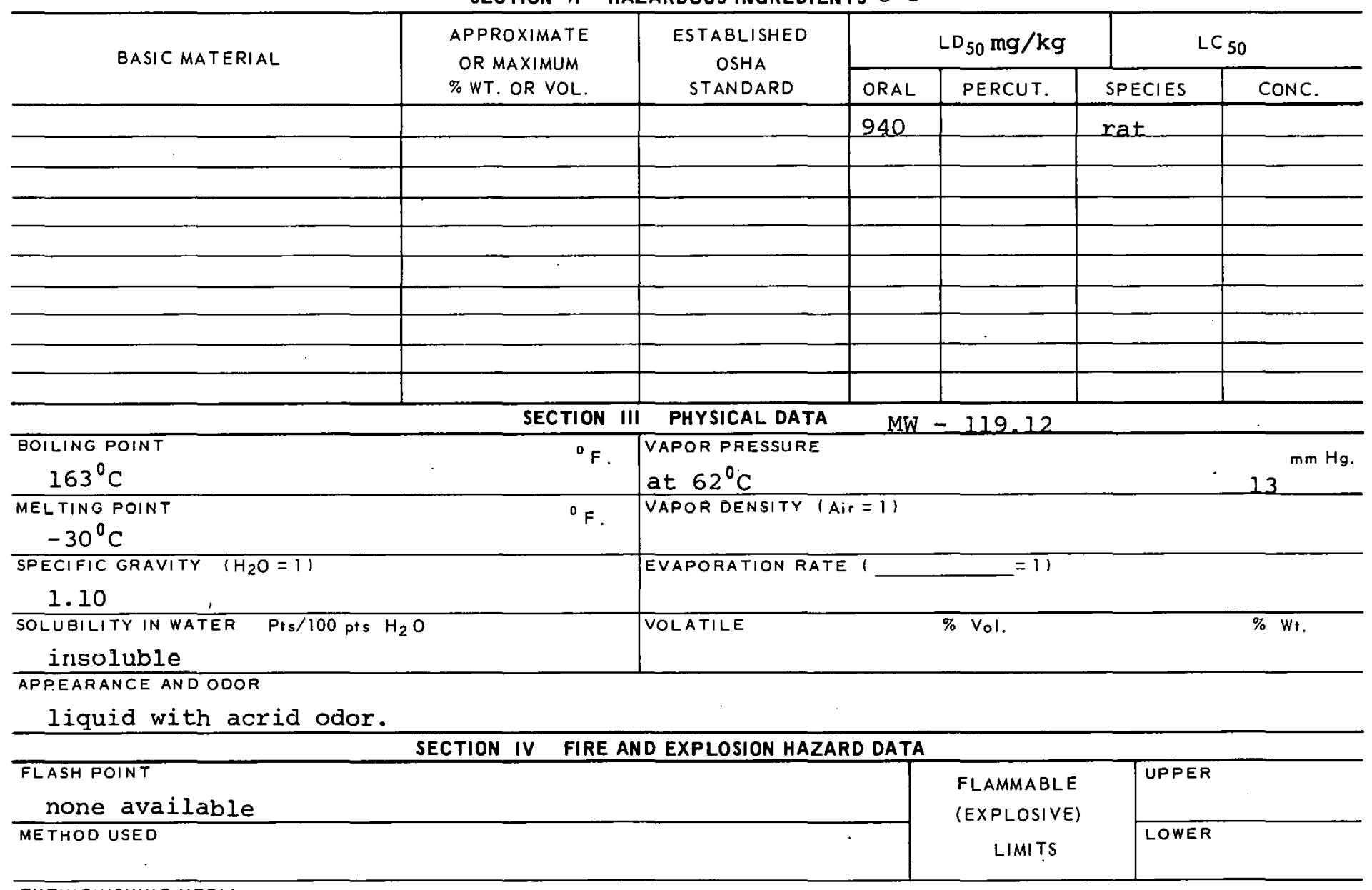

\section{EXTINGUISHING MEDIA}

Water, alcohol foam, carbon dioxide or dry chemical.

\section{SPECIAL FIRE FIGHTING PROCEDURES \\ Wear self-contained breathing apparatus and goggles.}

UNUSUAL FIRE AND EXPLOSION HAZARDS 
Phenyl Isocyanate

\section{SECTION V HEALTH HAZARD DATA}

\section{TOXIC LEVEL}

None available

PRINCIPLE ROUTES OF ABSORBTION

Inhalation, ingestion

CARCINOGENIC

SKIN ANDEYE

IRRITATION

Eye and mucous membrane irritation; skin irritation. Sensitizer

REVELANT SYMPTOMS OF EXPOSURE Eye and mucous membrane irritation; skin irritation. Sensitizer

EFFECTS OF CHRONIC EXPOSURE

Liver and kidney damage.

EMERGENCY AND FIRST AID PROCEDURES If swallowed - give emetic, medical aid, gastric lavage;

inhalation - rest, give oxygen as needed; wash from skin \& eyes.

SECTION VI REACTIVITY DATA

CONDITIONS CONTRIBUTING TO INSTABILITY

CONDITIONS CONTRIBU TING TO HAZARDOUS POLYMERIZATION

INCOMPATABILITY IMATERIALS TÓ AVOIDI

HAZARDOUS DECOMPOSITION PRODUCTS

SECTION VII SPILL OR LEAK PROCEDURES

STEPS TO BE TAKEN IN CASE MATERIAL IS RELEASED OR SPILLED

WASTE OISPOSAL METHOD

SECTION VIII SPECIAL PROTECTION INFORMATION

\begin{tabular}{l|l}
\hline \multicolumn{2}{c}{ SECTION VIII SPECIAL PROTECTION INFORMATION } \\
\hline VENTILATION REQUIREMENTS LOCAL EXHAUST & $\begin{array}{l}\text { PROTECTIVE EQUIPMENT (SPECIFY TYPES) EYE } \\
\text { COgglCO }\end{array}$ \\
\hline MEGHANICAL (GENERAI.) & GLOVES \\
\hline SHECIIAL & Rubber \\
& RESPIROTOR \\
\end{tabular}

OTHER FROTECTIVE EQUIPMENT

SECTION IX SPECIAL PRECAUTIONS

PRECAUTIONS TO BE TAKEN IN HANDLING AND STORAGE

OTHEH RRECAUTIONS

IIOAATURE

DATC

$327-5603 B$ 
CAS : 000103720

NX92750

\section{PRODUCT DESIGNATION}

03-327-6103

Phenyl Isothiocyanate

\section{MATERIAL SAFETY DATA SHEET}

\section{SECTION I SOURCE AND NOMENCLATURE}

MANUFACTURER'S NAME

ADDRESS INUMBER, STREET, CITY, STATE, ZIP CODE

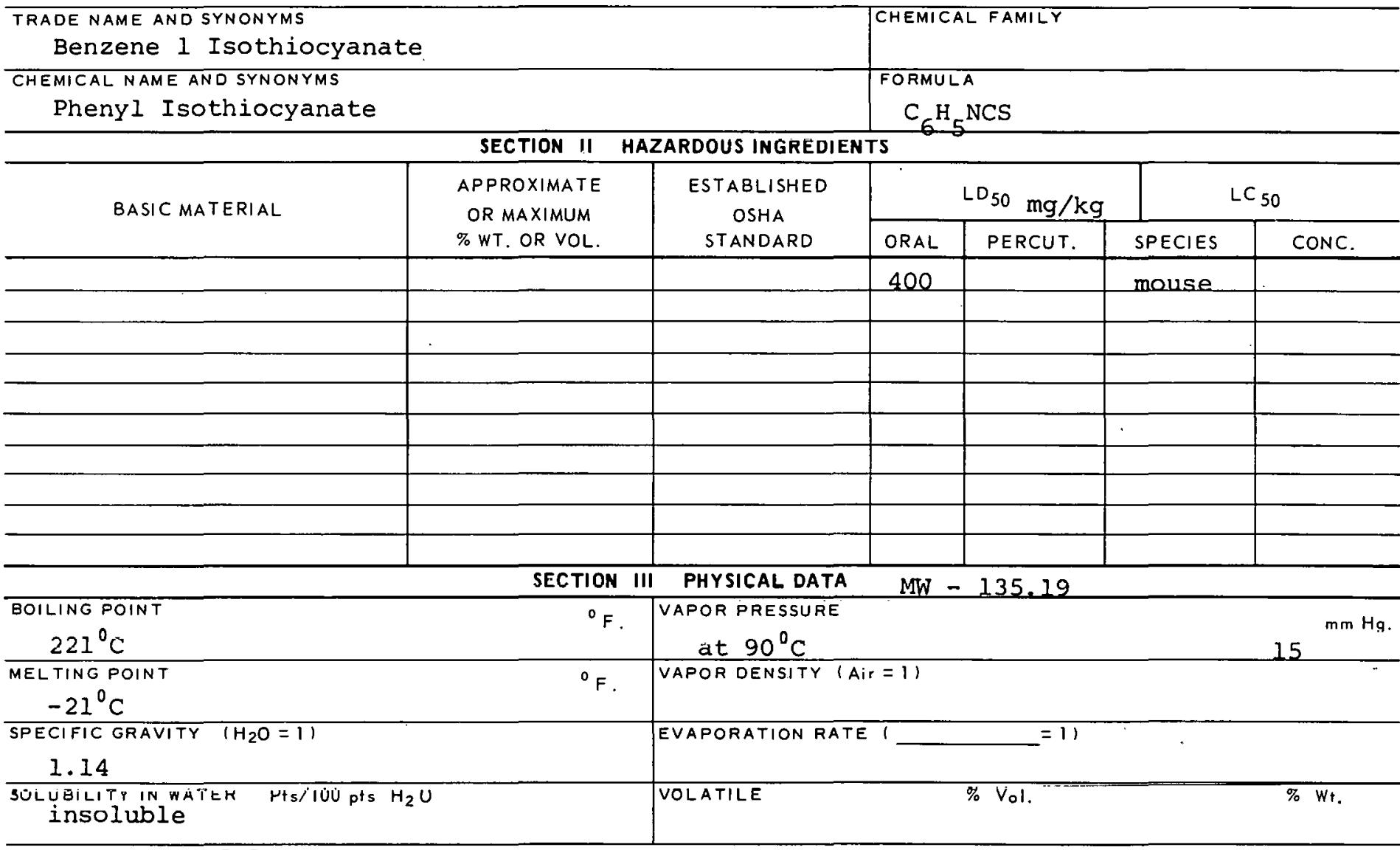

APFEARANCE AND ODOR

Pale yellow liquid

SECTION IV FIRE AND EXPLOSION HAZARD DATA

FLASH POINT

None available

METHOD USED

EXIINGUISHING MEOIA

Water, alcohol foam, carbon dioxide, or dry chemical.

FLAMMABLE

(EXPLOSIVE)

LIMITS
UPPER

LOWER

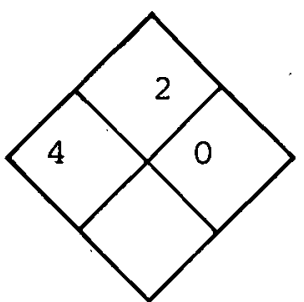

EMERGENCY TELEPHONE NO. 
Phenyl Isothiocyanate

\section{SECTION $V$ HEALTH HAZARD DATA}

TOXIC LEVEL

None available

CARCINOGENIC

PRINCIPLE ROUTES OF ABSORBTION SKIN AND EYE

Inhalation, ingestion

IRRITATION Severe

REVELANT SYMPTOMS OF EXPOSURE

Eye and mucous membrane irritation; skin irritation, sensitizer

EFFECTS OF CHRONIC EXPOSURE

Liver and kidney damage.

EMERGENCY AND FIRST AID PROCEDURES If swallowed - give emetic, medical aid, gastric lavage;

inhalation - rest, give oxygen as needed; wash from skin \& eyes.

SECTION VI REACTIVITY DATA

CONDITIONS CONTRIBUTING TO INSTABILITY

CONDITIONS CONTRIBUTING TO HAZARDOUS POLYMERIZATION

INCOMPATABILITY (MATERIALSTO AVQIO)

HAZARDOUS DECOMPOSITION PRODUCTS

SECTION VII SPILL OR LEAK PROCEDURES

STEPS TO BE TAKEN IN CASE MATERIAL IS RELEASEO OR SPILLED

WASTE DISPOSAL METHOD

SECTION VIII SPECIAL PROTECTION INFORMATION

\begin{tabular}{l|l}
\hline \multicolumn{1}{c}{ SECTION VIII SPECIAL PROTECTION INFORMATION } \\
\hline VENTILATION REQUIREMENTS LOCAL EXHAUST & $\begin{array}{l}\text { PROTECTIVE EQUIPMENT (SPECIFY TYPES) EYE } \\
\text { GOggles }\end{array}$ \\
\hline MECHANICAL IGENERAL; & $\begin{array}{l}\text { GLOVES } \\
\text { Rubber }\end{array}$ \\
\hline SPECIAL & $\begin{array}{l}\text { RESPIRATOR } \\
\text { Selfm COntaimed. }\end{array}$ \\
\hline
\end{tabular}

OIHER PROTECTIVE EQUIPMENT

\section{SECTION IX SPECIAL PRECAUTIONS}

PRECAUTIONS TO BE TAKEN IN HANDLING AND STORAGE

OTHER PRECAUTIONS

\begin{tabular}{l|l}
\hline SIGNATURE & DATE
\end{tabular}

$327-6103 B$ 


\begin{tabular}{|l|}
\hline \multicolumn{1}{|c|}{ PRODUCT DESIGNATION } \\
03-327-7603 \\
P-Phenylphenacyl Bromide
\end{tabular}

SECTION I SOURCE AND NOMENCLATURE

\begin{tabular}{l|l}
\hline MANUFACTURER'S NAME & EMERGENCY TELEPHONE NO. \\
\hline AODRESS (NUMBER, STREET, CITY, STATE, ZIP CODE)
\end{tabular}

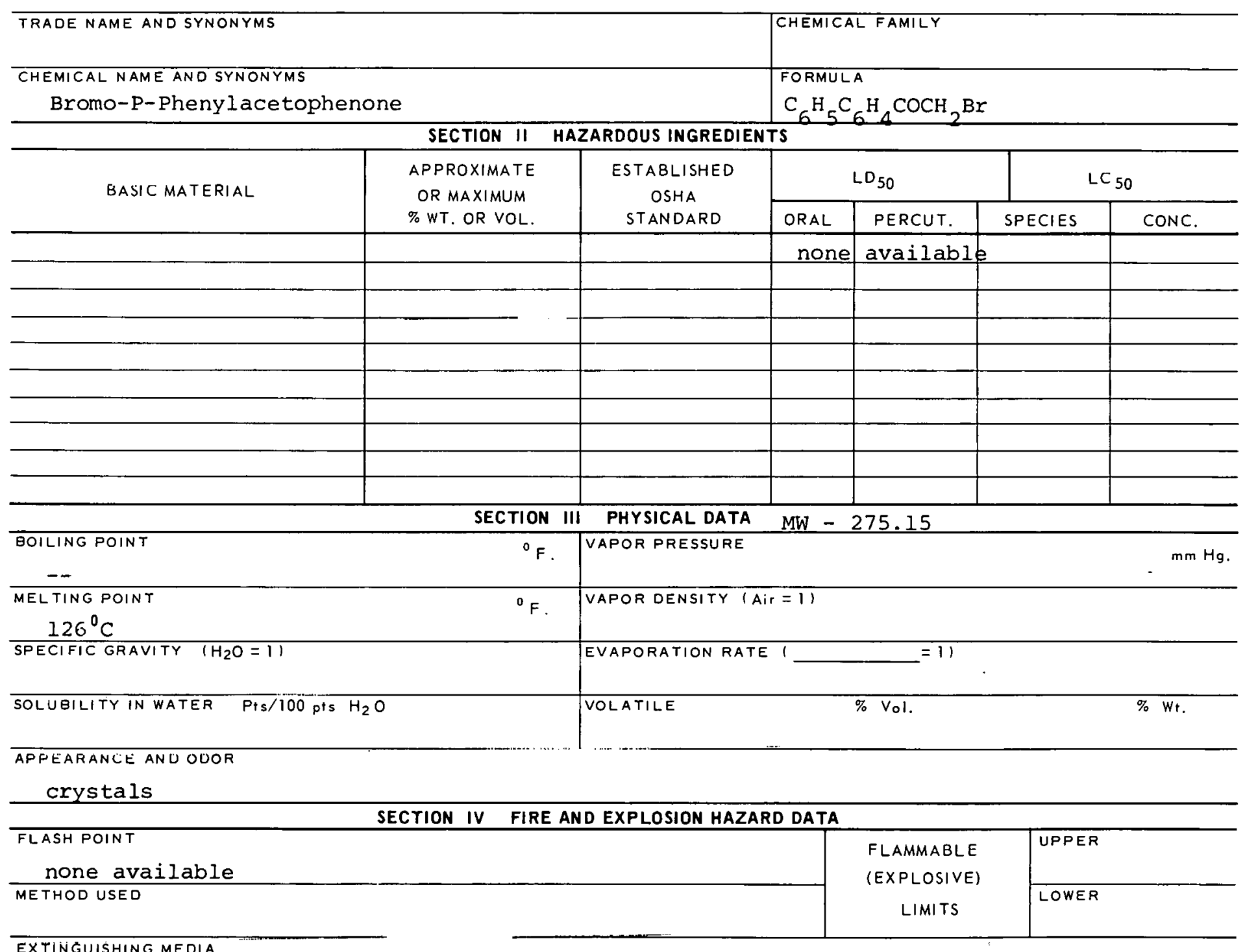

carbon dioxide, dry chemical.

SPECIAL FIRE FIGHTING PROCEDURES

UNUSUAL FIRE ANO EXPLOSION HAZAROS 
P Phenylphenacyl Bromide SECTION $V$ HEALTH HAZARD DATA

TOXIC LEVEL

none available

CARCINOGENIC

PRINCIPLE ROUUTES OF ABSORBTION inhalation, ingestion

SKIN AND EYE

IRRITATION

severe

REVELANT SYMPTOMS OF EXPOSURE

Eye irritation, corneal haziness and pain; burning of throat; skin irritation

EFFECTS OF CHRONIC EXPOSURE

$--$

EMERGENCY AND FIRST AID PROCEDURES

Inhalation - rest; if swallowed - wash mouth, give emetic, get medical aid; wash from skin \& eyes.

SECTION VI REACTIVITY DATA

CONDITIONS CONTRIBUTING TO INSTABILITY

CONDITIONS CONTRIBUTING TO HAZARDOUS POLYMERI ZATION

INLUMHATABILIT'Y (MATERIALS TO AVOID)

IIAZAROOUG BEGQMPOEITISM PORNIITA

SECTION VII SPILL OR LEAK PROCEDURES

STEPS TO BE TAKEN IN CASE MATERIAL IS RELEASED OR SPILLED

WASTE DISPOSAL METHOD

SECTION VIII SPECIAL PROTECTION INFORMATION

\begin{tabular}{l|l}
\hline VENTII.ATION REOUIREMENTS LOCAL EXHAUST & $\begin{array}{l}\text { PROTECTIVE EQUIPMENT (SPECIFY TYPES) EYE } \\
\text { Goggles }\end{array}$ \\
\hline MECHANICAL IGENERAL) & $\begin{array}{c}\text { GLOVES } \\
\text { Rubber }\end{array}$ \\
\hline SPECIAL & $\begin{array}{l}\text { RESPIRATOR } \\
\text { With proper filter }\end{array}$ \\
\hline
\end{tabular}

OTHER PROTECTIVE EQUIPMEN I

SECTION IX SPECIAL PRECAUTIONS

PRECAUTIONS TO BE TAKEN IN HANDLING AND STORAGE

OTHER PRECAUTIONS

SIGNATURE

DATE

$327-7603 B$ 


\begin{tabular}{|c|}
\hline \multicolumn{1}{|c|}{ PRODUCT DESIGNATION } \\
03-327-8103 \\
B-Phenylpropionyl Chloride \\
\hline
\end{tabular}

\section{MATERIAL SAFETY \\ DATA SHEET}

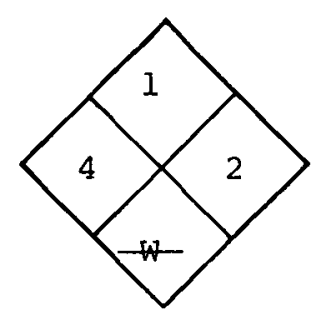

\section{SECTION I SOURCE AND NOMENCLATURE}

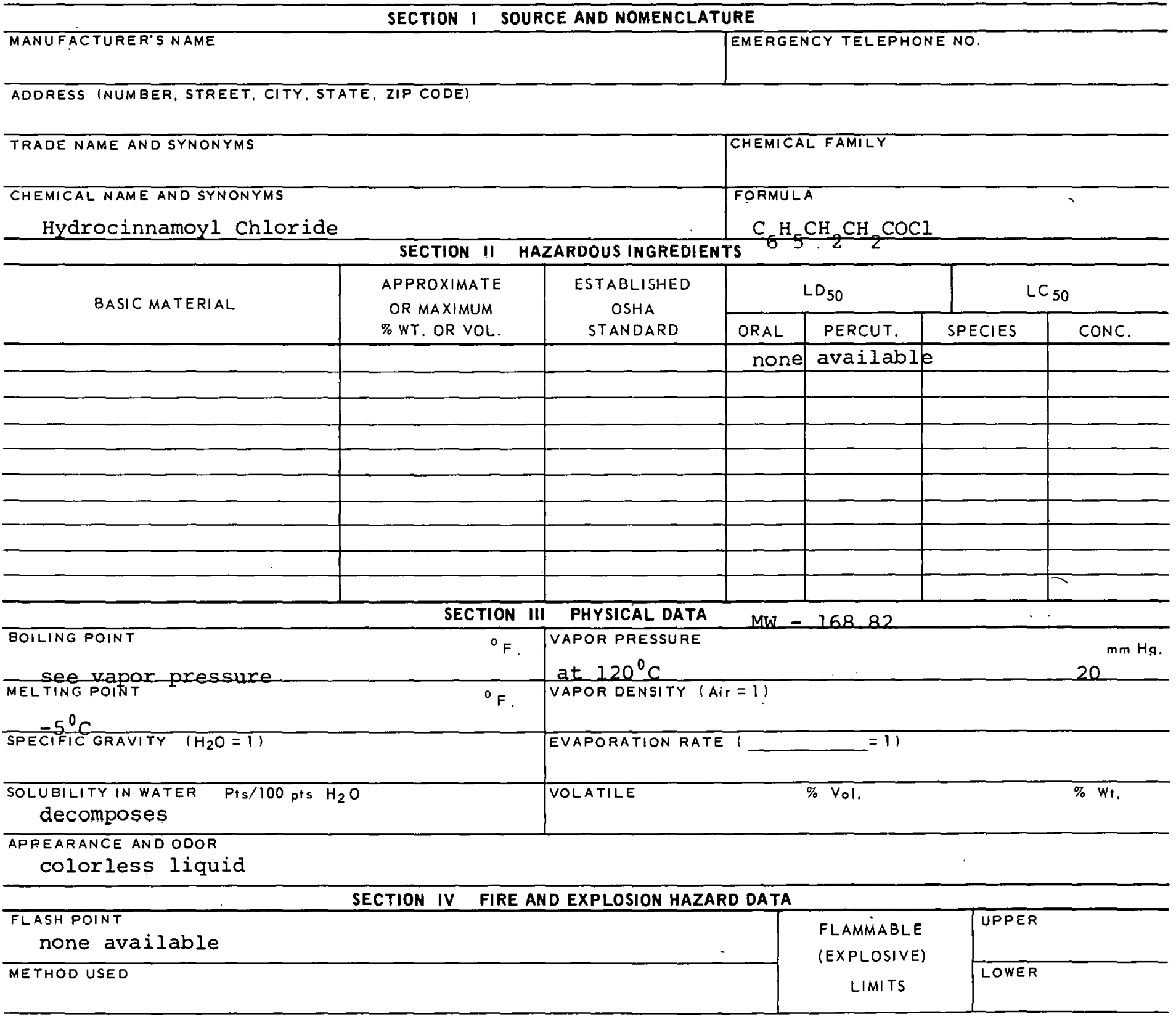

EXTINGUISHING MEOIA carbon dioxide, dry chemical.

SPECIAL FIRE FIGHTING PROCEDURES

Wear full protective clothing.

UNUSUAL FIRE AND EXPLOSION HAZAROS

Rcacts with watcr. 
TOXIC LEVEL

None available

CARCINOGENIC no

PRINCIPLE ROUTES OF ABSORBTION

Irhalatjon, ingestion

SKIN ANDEYE

IRRITATION

severe

REVELANT SYMPTOMS OF EXPosure Cyanosis, coughing, headache, pulmonary edema, ulceration of skin, dizziness.

EFFECTS OF CHRONIC EXPOSURE ErOsion of teeth, jaw necrosis, bronchial irritation and chronic cough.

EMERGENCY AND FIRST AID PROCEDURES Inhalation - artificial respiration if necessary; ingestion drink water, milk of magnesia, get medical aid; wash from skin \& eyes.

\section{SECTION VI REACTIVITY DATA}

CONDITIONS CONTRIBUTING TO INSTABILITY

CONDITIONS CONTRIBU TING TO HAZARDOUS POLYMERIZATION

INEUMPATABILITI (MATERIALS TU AVOIO)

Avoid water

IInZnRDOUG DEGQMnOEITIOH RחOQUGTE

Produces $\mathrm{HCl}$ with water

SECTION VII SPILL OR LEAK PROCEDURES

STEPS TO BE TAKEN IN CASE MATERIAL IS RELEASED OR SPILLED

WASTE DISPOSAL METHOD

SECTION VIII SPECIAL PROTECTION INFORMATION

\begin{tabular}{|c|c|}
\hline VENTILATION REQUIREMENTS LOCAL EXHAUST & PROTECTIVE EQUIPMENT (SPECIFY TYPES) EYE \\
\hline MECHANICAL (GENERAL) & Rubber \\
\hline SPECIAL & $\begin{array}{l}\text { RFGQIRATOR } \\
\text { With proper filter }\end{array}$ \\
\hline
\end{tabular}

OTHER PROTECTIVE EQUIPMENT

SECTION IX SPECIAL PRECAUTIONS

PRECAUTIONS TO BE TAKEN IN HANDLING AND STORAGE

Store in cool, dry, well-ventilated room; inside storage in standard flammable

liquids storage cabinet.

OTHER PRECAUTIONS

SIGNATURE

DATE

$327-8103 B$ 
CÁS:000103037

VT36750

\section{PRODUCT DESIGNATION}

03-328-0704

1-Phenylsemicarbazide

MATERIAL SAFETY

DATA SHEET

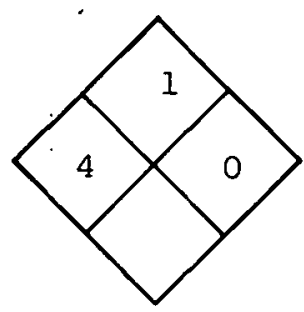

SECTION I SOURCE AND NOMENCLATURE

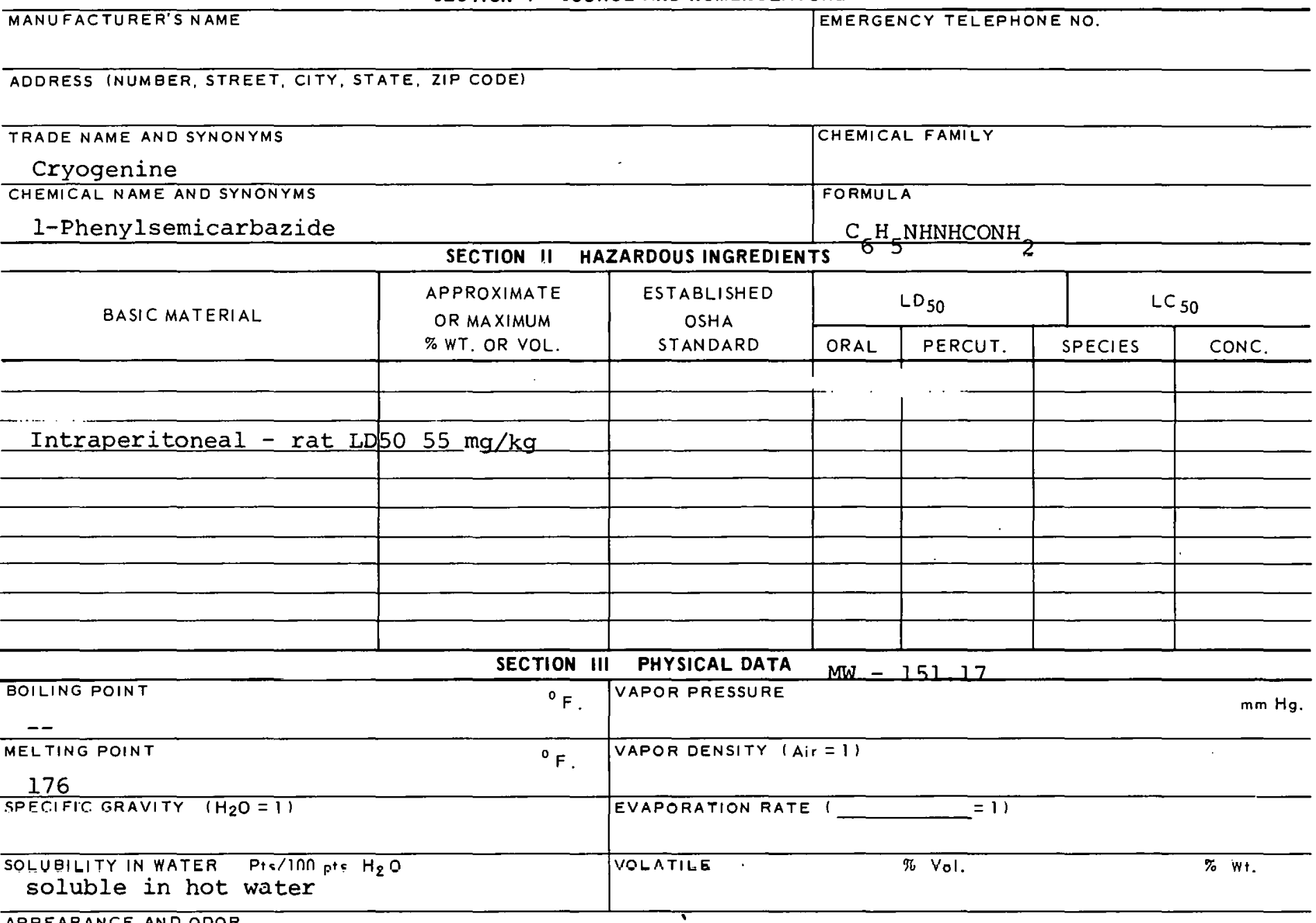

APPEARANCE AND ODOR

\section{SECTION IV FIRE AND EXPLOSION HAZARD DATA}

\begin{tabular}{|c|c|c|}
\hline $\begin{array}{l}\text { FLASH POINT } \\
\text { none available }\end{array}$ & \multirow{2}{*}{$\begin{array}{c}\text { FLAMMABLE } \\
\text { (EXPLOSIVE) } \\
\text { LIMITS }\end{array}$} & UPPER \\
\hline METHOD USED & & LOWER \\
\hline
\end{tabular}

EXTINGUISHING MEDIA

Alcohol foam, carbon dioxide, dry chemical.

SPECIAL FIRE FIGHTING PROCEOURES

UNUSUAL FIRE AND EXPLOSION HAZARDS 


\section{SECTION V HEALTH HAZARD DATA}

TOXIC LEVEL

none available

PRINCIPLE ROUTES OF ABSORBTION

inhalation, ingestion, skin

CARCINOGENIC nO SKIN AND EYE

IRRITATION SEvEre

REVELANT SYMPTOMS OF EXPOSURE Irritation of skin, eyes and mucous membranes, gastroentric disturbances, anemia.

EFFECTS OF CHRONIC EXPOSURE

Blood and liver damage, loss of appetite.

EMERGENCY AND FIRST AIO PROCEDURES Inhalation - rest; if swallowed - induce vomiting, get medical aid; wash from eyes and skin.

\section{SECTION VI REACTIVITY DATA}

CONDITIONS CONTRIBUTING TO INSTABILITY

CONDITIONS CONTRIBUTIN.G TO HAZARDOUS POLYMERIZATION

INCOMPATABILITY (MATERIALS TO AVOID)

HAZARDOUS DECOMPOSITION PROUUETS

SECTION VII SPILL OR LEAK PROCEDURES

STEPS TO BE TAKEN IN CASE MATERIAL IS RELEASED OR SPILLED

WASTE DISPOSAL METHOD

SECTION VIII SPECIAL PROTECTION INFORMATION

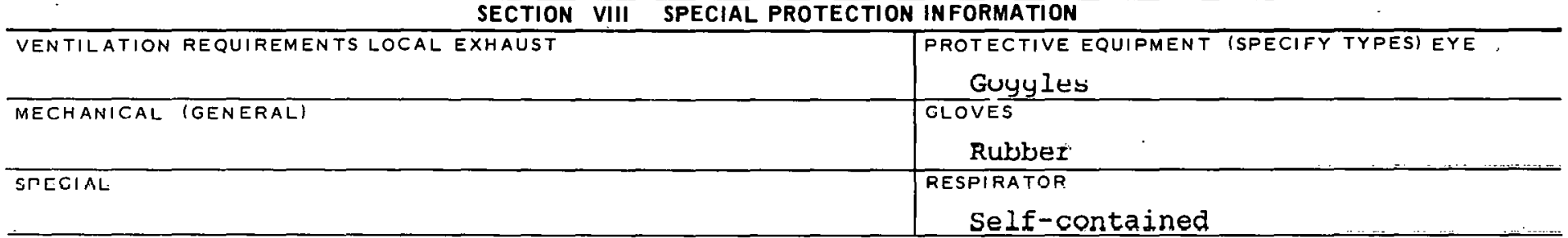

OTHER PROTECTIVE EQUIPMENT

\section{SECTION IX SPECIAL PRECAUTIONS}

PRECAUTIONS TO BE TAKEN IN HANDLING AND STORAGE

OTTHFR RRECAUTIONS

SI FNAATURE

DATE

$328-0704 B$ 


\section{PRODUCT DESIGNATION}

03-328-1704

4 Phenylthiosemicarbazide
MATERIAL SAFETY

DATA SHEET

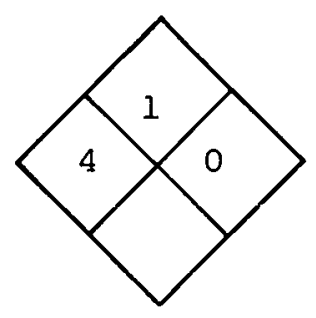

\section{SECTION I SOURCE AND NOMENCLATURE}

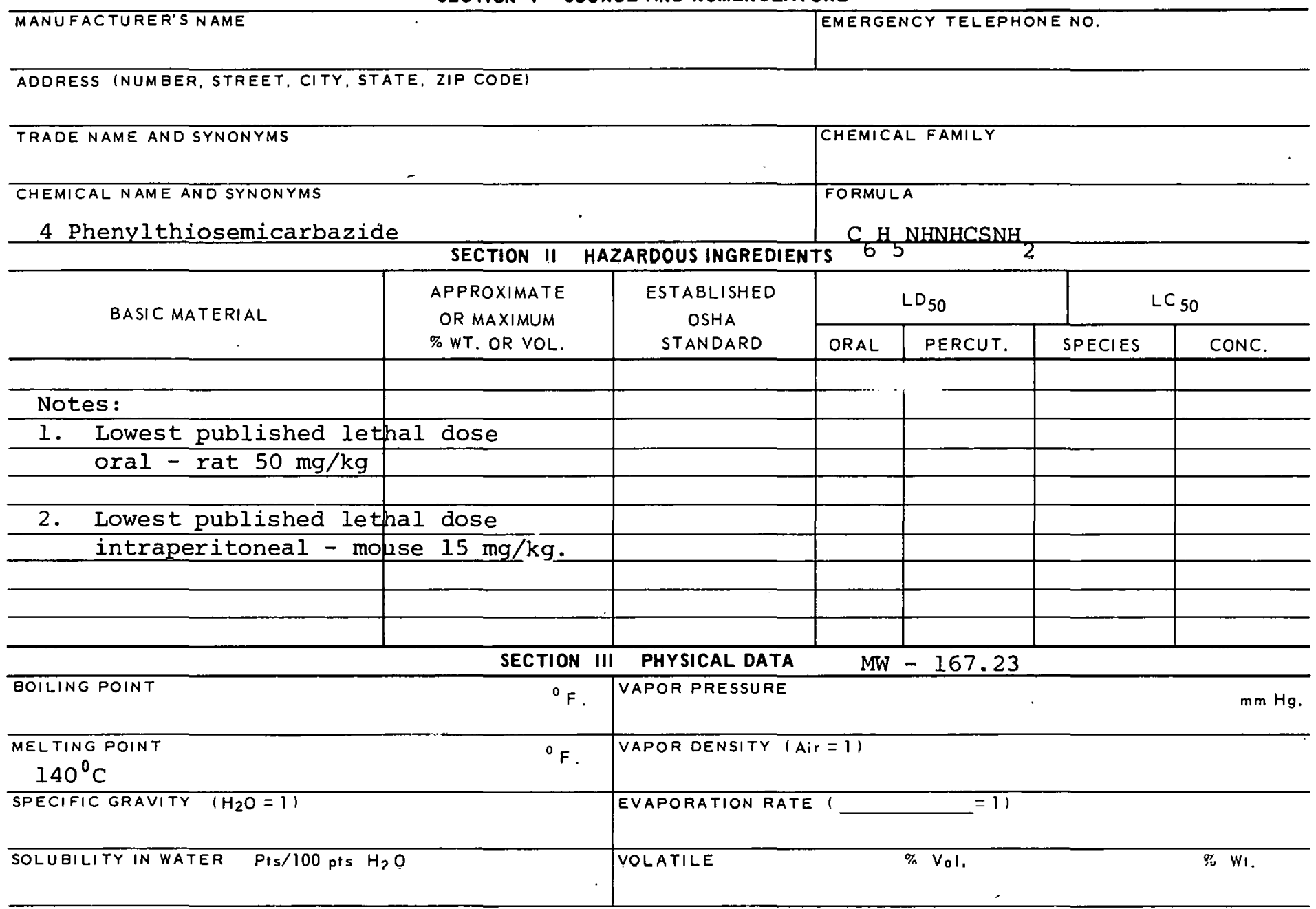

APPEARANCE AND ODOR

crystals

SECTION IV FIRE AND EXPLOSION HAZARD DATA

FLASH POINT

none available

METHOD USED

FLAMMABLE

(EXPLOSIVE)

LIMITS

UPPER

LOWER

EXTINGUISHING MEDIA

alcohol foam, dry chemical, carbon dioxide.

SPECIAL FIRE FIGHTING PROCEOURES

UNUSUAL FIRE AND EXPLOSION HAZARDS 
4 Phenylthiosemicarbazide SECTION $V$ HEALTH HAZARD DATA

TOXIC LEVEL

none available

PRINCIPLE ROUTES OF ABSORBTION

inhalation, ingestion, skin

CARCINOGENIC no

SKIN AND EYE

IRRITATION severe

REVELANT SYMPTOMS OF EXPOSURE Irritation of skin, eyes and mucous membranes, gastroentric disturbances, anemia.

EFFECTS OF CHRONIC EXPOSURE

Blood and liver damage, loss of appetite.

EMERGENCY AND FIRST AID PROCEDURES Inhalation - rest; if swallowed - induce vomiting, get medical aid; wash from skin \& eyes.

SECTION VI REACTIVITY DATA

CONDITIONS CONTRIBUTING TO INSTABILITY

CONDITIONS CONTRIBUTING TO HAZARDOUS POLYMERIZATION

INCOMPATABILITY (MATERIALS TO AVOID)

HAZARDOUS DECOMPOSITION PRODUCTS

SECTION VII SPILL OR LEAK PROCEDURES

STEPS TO BE TAKEN IN CASE MATERIAL IS RELEASED OR SPILLED

WASTE DISPOSAL METHOD

SECTION VIII SPECIAL PROTECTION INFORMATION

\begin{tabular}{|c|c|}
\hline VENTILATION REQUIREMENTS LOCAL EXHAUST & $\begin{array}{l}\text { PROTECTIVE EQUIPMENT (SPECIFY TYPES) EYE } \\
\text { GOggles }\end{array}$ \\
\hline MECHANICAL (GENERAL) & $\begin{array}{l}\text { FLöes } \\
\text { Rubber }\end{array}$ \\
\hline SPECIIAL & $\begin{array}{l}\text { REEPIRATQR } \\
\text { rigl f Eontoinot }\end{array}$ \\
\hline
\end{tabular}

OTHER PROTECTIVE EUUITMENT

SECTION IX SPECIAL PRECAUTIONS

PRECAUTIONS TO BE TAKEN IN HANDLING AND STORAGE

OTHER PRECAUTIONS 
CAS : 000103855

YU14000

\section{PRODUCT DESIGNATION}

03-328-2204

Phenyl thiourea

\section{MATERIAL SAFETY \\ DATA SHEET}

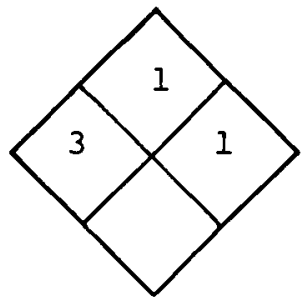

\section{SECTION I SOURCE AND NOMENCLATURE}

MANUFACTURER'S NAME
ADDRESS INUMBER, STREET, CITY
TRADE NAME AND SYNONYMS
CHEMICAL NAME AND SYNONYMS
PhENYlCarbamide

BASIC MATERIAL

SECTION II HAZARDOUS INGREDIENTS

EMERGENCY TELEPHONE NO.

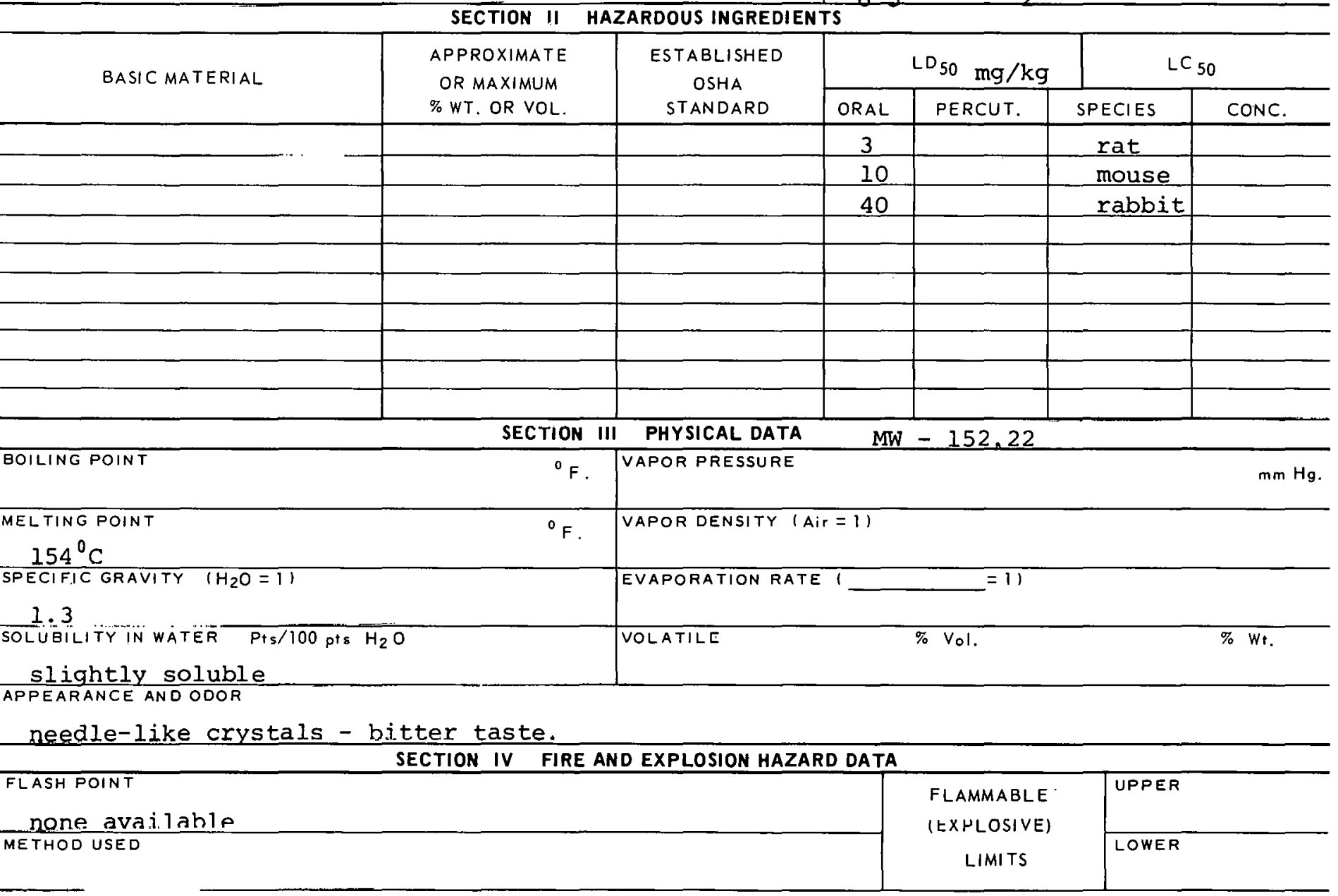

EXTINGUISHING MEDIA

carbon dioxide, water.

SPECIAL FIRE FIGHTING PROCEOURES

UNUSUAL FIRE AND EXPLLOSION HAZARDS

Burns to form $\mathrm{SO}_{2}$ tumes. 


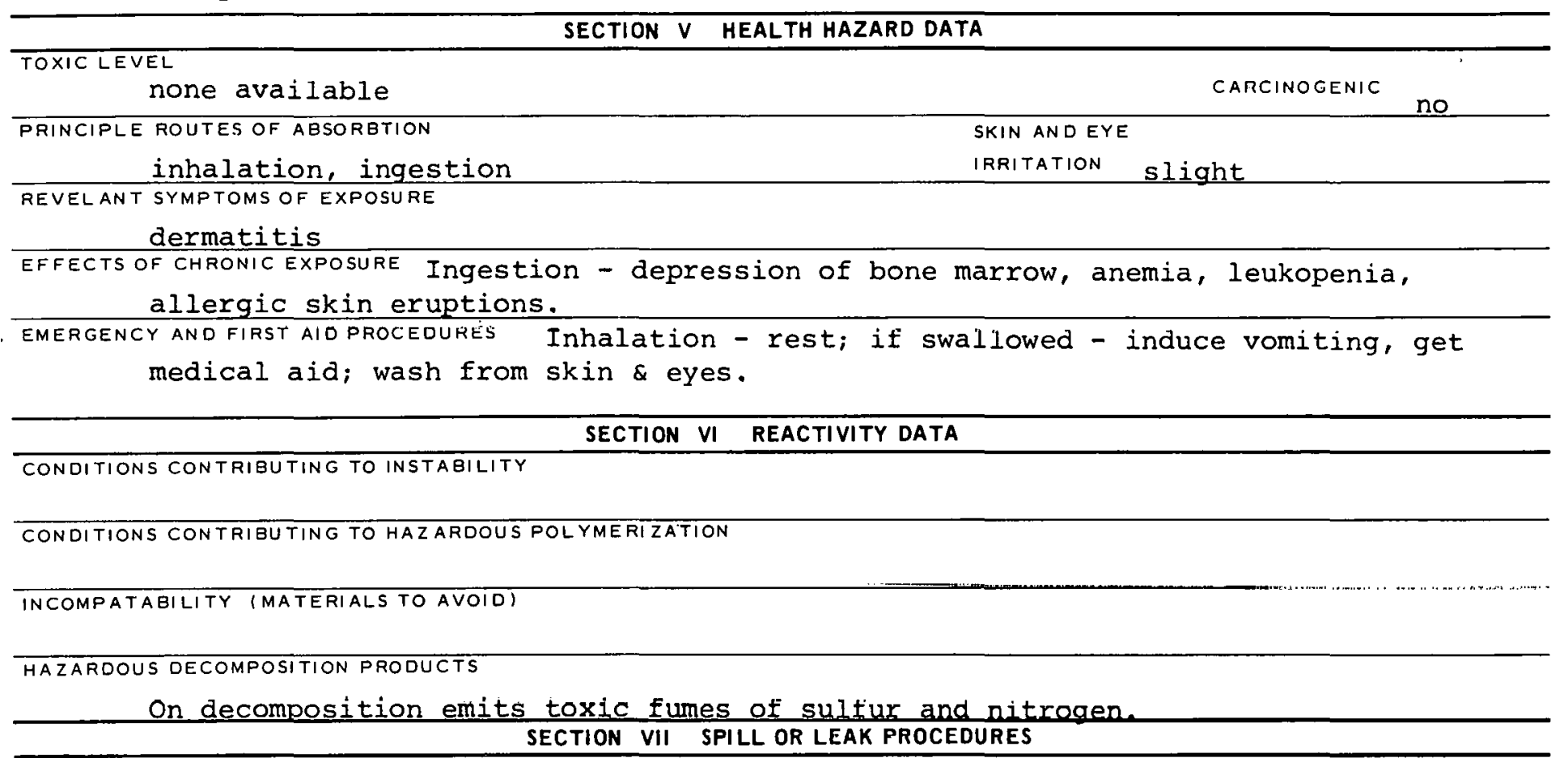

STEPS TO BE TAKEN IN CASE MATERIAL IS RELEASEU OH SFILLED

WASTE DISPOSAL METHOD

SECTION VIII SPECIAL PROTECTION INFORMATION 


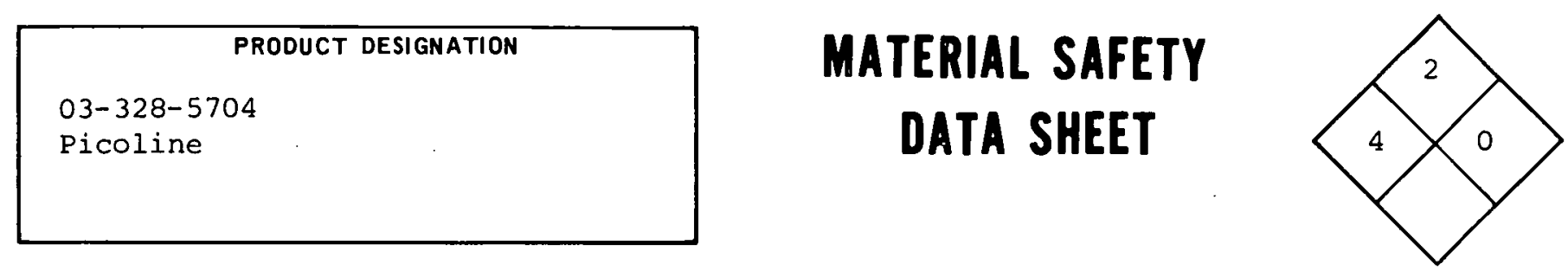

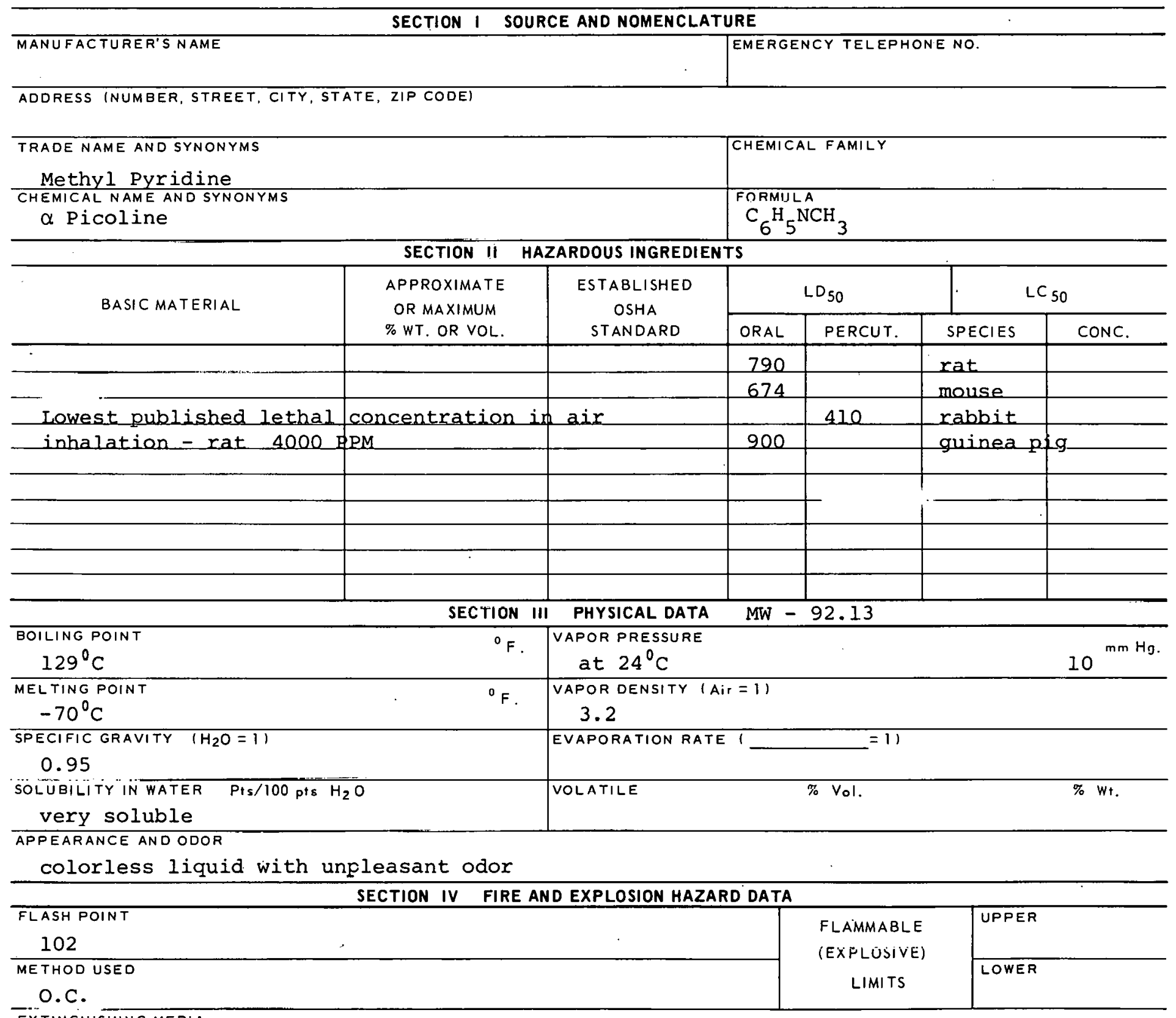

EXTINGUISHING MEDIA

Alcohol f.oam, carbon dioxide, dry chemical

SPECIAL FIRE FIGHTING PROCEDURES

Wear goggles and self-contained breathing apparatus.

UNUSUAL FIRE AND EXPLOSION HAZARDS 
TOXIC LEVEL

none available

CARCINOGENIC

PRINCIPLE ROUTES OF ABSORBTION

inhalation, ingestion, skin

SKIN AND EYE

REVELANT SYMPTOMS OF EXPOSURE

IRRITATION

Narcosis, headache, nausea, giddiness, vomiting

EFFECTS OF CHRONIC EXPOSURE OCCasional vomiting and diarrhea; weight loss and anemia; ocular and facial paralysis.

EMERGENCY AND FIRST AIO PROCEDURES If swallowed - induce vomiting, get medical aid; inhalation artificial respiration and oxygen as necessary; wash from skin \& eyes.

\section{SECTION VI REACTIVITY DATA}

CONDITIONS CONTRIBUTING TO INSTABILITY

CONRITIONS CONTRIBUTING TO HAZARDOUS POLYMERIZATION

INCOMPATARIIITY (MATERIALS TO AVOID)

May react with oxidizing materials.

HAZARDOUS DECOMPOSITION FROOUCTS

SECTION VII SPILL OR LEAK PROCEDURES

STEPS TO BE TAKEN IN CASE MATERIAL IS RELEASED OR SPILLED

SECTION VIII SPECIAL PROTECTION INFORMATION

\begin{tabular}{|c|c|}
\hline $\begin{array}{l}\text { VENTILATION KEQUIREMENTS LOCAL EXHAUST } \\
\text { USE in hoOd }\end{array}$ & $\begin{array}{l}\text { PROTECTIVE CQUIFMENT (SPECIFY TYPES) EYE } \\
\text { gOggles }\end{array}$ \\
\hline MECHANILAL U, GENERAI & $\begin{array}{l}\text { OLO'LS } \\
\text { rubber }\end{array}$ \\
\hline SトEEIAL & $\begin{array}{l}\text { RESPIPATOR } \\
\text { self-contained }\end{array}$ \\
\hline
\end{tabular}

OTHER PROTECTIVE EQUIPMENT

SECTION IX SPECIAL PRECAUTIONS

PRECAUTIONS TO BE TAKEN IN HANDLING AND STORAGE

Outside storage preferred, store inside in a flammable liquids storage cabinet.

OTHFR PRFTAIITIONS 


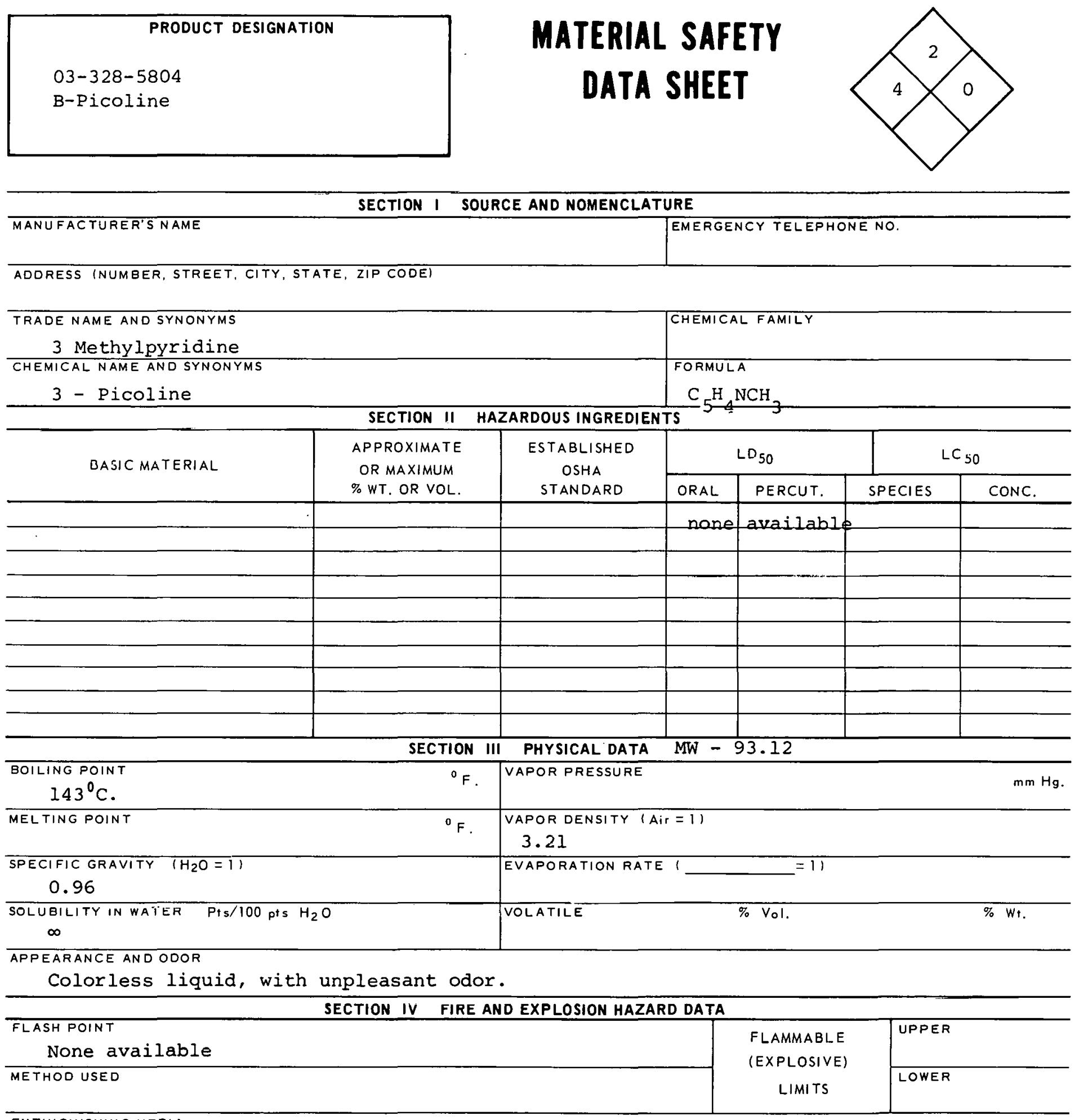

EXTINGUISHING MEDIA

Alcohol foam, carbon dioxide, dry chemical.

SPECIAL FIRE FIGHTING PROCEDURES

Wear goggles and self-contained breathing apparatus.

UNUSUAL FIRE AND EXPLOSION HAZARDS 


\section{TOXIC LEVEL}

Ione available

CARCINOGENIC no

PRINCIPLE ROUTES OF ABSORBTION

SKIN AND EYE

Inhalation, ingestion, skin IRRITATION

REVELANT SYMPTOMS OF EXPOSURE

Narcosis, headache, nausea, giddiness, vomiting.

EFFECTS OF CHRONIC EXPOSURE OCCasional vomiting and diarrhea; weight loss and anemia;

ocular and facial paralysis.

EMERGENCY AND FIRST AID PROCEOURES If swallowed - induce vomiting, get medical aid; inhalation -

artificial respixation and oxygen as needed; wash from skin \& eyes.

SECTION VI REACTIVITY DATA

CONOITIONS CONTRIBUTING TO INSTABILITY

CONDITIONS CONTRIBUTING TO HAZARDOUS POLYMERIZATION

INCOMPATABII-ITY (MATERIALS TO AVOID)

May react with oxidizing materials. HAZARDOUS DECOMPOSIIIUN IHULUCTS

SECTION VII SPILL OR LEAK PROCEDURES

STEPS TO BE TAKEN IN CASE MATERIAL IS RELEASED OR SPILLED

WASTE DISPOSAL METHOD

SECTION VIII SPECIAL PROTECTION INFORMATION

\begin{tabular}{l|l}
\hline $\begin{array}{l}\text { VENTILATION REQUIREMENTS LOCAL EXHAUST } \\
\text { USSEd In hOUd }\end{array}$ & $\begin{array}{l}\text { FROTECTIVE EQUIPMENT (SPECIFY TYPES) EYE } \\
\text { GOggles }\end{array}$ \\
\hline MECHANICAL (GENERAL) & GLOVES \\
Rubber \\
\hline SHELIML & RFGPIRATnR \\
& Self-Contained \\
\hline
\end{tabular}

OTHER PROTECTIVE EQUIPMENT

\section{SECTION IX SPECIAL PRECAUTIONS}

PRECAUTIONS TO BE TAKEN IN HANULING AND STORAGE

Outside storage preferred, store inside in a flammable liquids storage cabinet.

STHER FAECAUTIONG

SIGNATURE

DATE 
03-328-5904

G-Picoline

\section{SECTION I SOURCE AND NOMENCLATURE}

\section{ADORESS (NUMBER, STREET, CITY, STATE, ZIP CODE)}

TRADE NAME AND SYNONYMS

4 Methyl pyridine CHEMICAL NAME AND SYNONYMS

\section{Picoline}

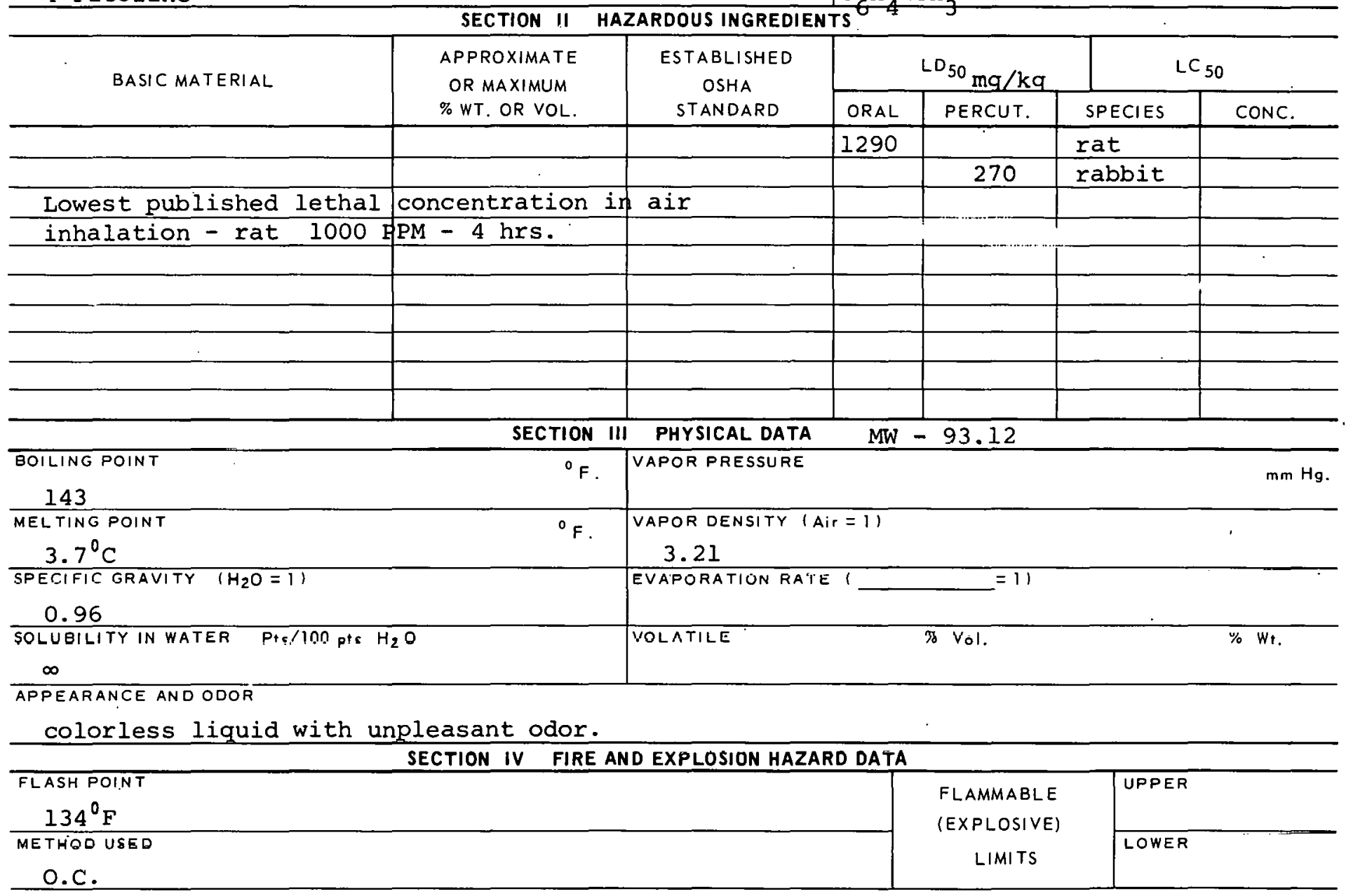

EXTINGUISHING MEDIA

Alcohol foam, carbon dioxide, dry chemical

SPECIAL FIRE FIGHTING PROCEOURES

Wear goggles and self-contained breathing apparatus.

UNUSUAL FIRE AND EXPLOSION HAZARDS 


\section{TOXIC LEVEL}

none available

PRINCIPLE ROUTES OF ABSORBTION

inhalation, ingestion, skin
CARCINOGENIC no

SKIN AND EYE

IRRITATION SEvere

REVELANT SYMPTOMS OF EXPOSURE

Narcosis, headache, nausea, giddiness, vomiting.

EFFECTS OF CHRONIC EXPOSURE OCCasional vomiting and diarrhea: weight loss and anemia; ocular and facial paralysis.

EMERGENCY AND FIRST AID PROCEDURES If swallowed - induce vomiting, get medical aid;

inhalation - artificial respiration and oxygen as needed; wash from skin \& eyes.

\section{SECTION VI REACTIVITY DATA}

CONDITIONS CONTRIBUTING TO INSTABILITY

CONDITIONS CONTRIBUTING TO HAZARDOUS POLYMERIZATION

INCOMPÁTABILITY (MATERIALS TO AVOIU)

May react with oxidizing materials.

HALAHUUUS LEGUMPUST THM FROEUETS

SECTION VII SPILL OR LEAK PROCEDURES

STEPS TO BE TAKEN IN CASE MATERIAL IS RELEASED OR SPILLED

WASTE DISPOSAL METHOO

SECTION VIII SPECIAL PROTECTION INFORMATION

\begin{tabular}{|c|c|}
\hline $\begin{array}{l}\text { VENTILATION REQUIREMENTS LOCAL EXHAUST } \\
\text { Use in hood }\end{array}$ & $\begin{array}{l}\text { PROTECTIVE EQUIPMENT (SPECIFY TYPES\} EYE } \\
\text { Goggles }\end{array}$ \\
\hline MCOHAMICAL IECMENAL) & $\begin{array}{l}\text { GLOVEC } \\
\text { Rubber }\end{array}$ \\
\hline SDFCIAI & $\begin{array}{l}\text { RFGPIRATOR } \\
\text { Selt-contained }\end{array}$ \\
\hline
\end{tabular}

OTHER PROTECTIVE EQUIPMENT

SECTION IX SPECIAL PRECAUTIONS

PRECAUTIONS TO BE TAKEN IN HANDLING AND STORAGE

Outside storage preferred, store inside in a flammable liquids storage cabinet.

OTHER PRECAUTIONS

SIGNATURE

NATF

$328-5904 \mathrm{~B}$ 


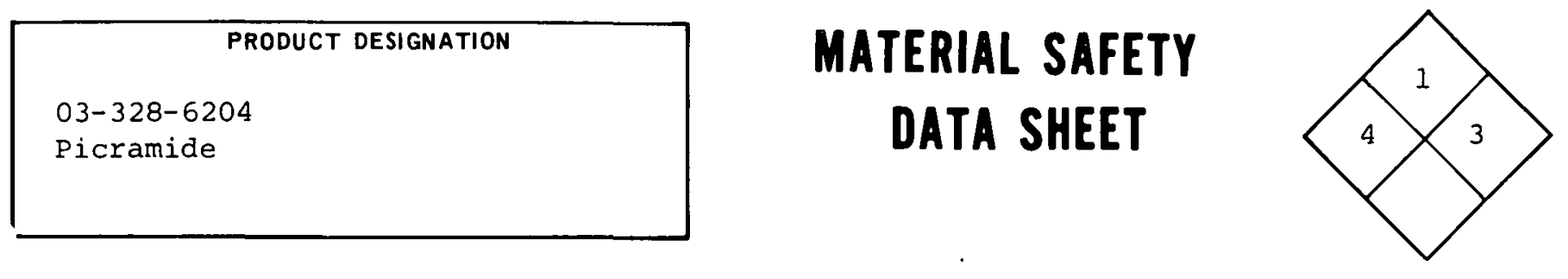

SECTION I SOURCE AND NOMENCLATURE

MANUFACTURER'S NAME

EMERGENCY TELEPHONE NO.

ADDRESS (NUMBER, STREET, CITY, STATE, ZIP CODE)

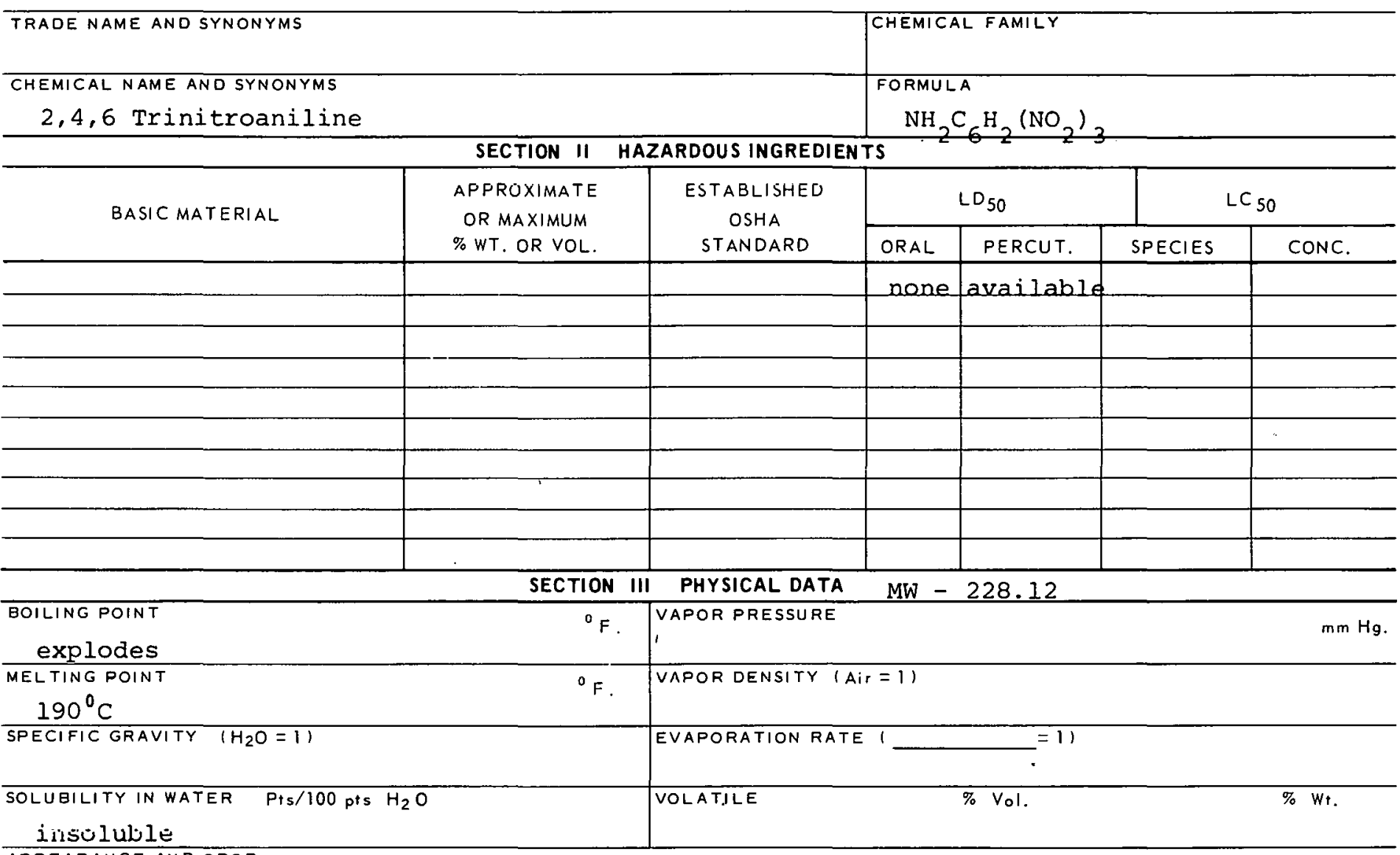

APPEARANCE AND ODOR

yellow monoclinic crystals

SECTION IV FIRE AND EXPLOSION HAZARD DATA

\begin{tabular}{l|c|l}
\hline \multicolumn{2}{c}{ SECTION IV FIRE AND EXPLOSION HAZARD DATA } \\
\hline FLASH POINT & FLAMMABLE & UFPER \\
nONe available & (EXPLOSIVE) & LIMITS \\
\hline METHOD USED & LOWER & \\
\hline
\end{tabular}

EXTINGUISHING MEDIA Explosive - do not fight fire except for unmanned

equipment - use water.

SPECIAL FIRE FIGHTING PROCEDURES

Wear full protective clothing for rescue operations.

UNUSUAL FIRE AND EXPLOSION HAZARDS

Severe exploeion hazard. 


\section{TOXIC LEVEL}

none available

CARCINOGENIC SKIN AND EYE

PRINCIPLE ROUTES OF ABSORBTION IRRITATION moderate

Inhalation of dust, ingestion, skin

REVELANT SYMPTOMS OF EXPOSURE Headache, drowsiness, weakness, cyanosis, respiratory

distress.

EFFECTS OF CHRONIC EXPOSURE

Weight loss, anemia, possible liver damage.

EMERGENCY AND FIRST AID PROCEDURES Inhalation - rest; if swallowed - give emetic, get medical aid; wash skin \& eyes.

\section{SECTION VI REACTIVITY DATA}

CONDITIONS CONTRIBUTING TO INSTABILITY

CONDITIONS CONTRIBUTING TO HAZARDOUS POLYMERIZATION

INCOMPATABILITY (MATERIALS TO AVOID)

Reacts with oxidizing materials.

HALARDOUS DECOMPOSITION PROOUC"'s

SECTION VII SPILL OR LEAK PROCEDURES

STEPS TO BE TAKEN IN CASE MATERIAL IS RELEASED OR SPILLED

WASTE DISPOSAL METHOD

SECTION VIII SPECIAL PROTECTION INFORMATION

\begin{tabular}{|c|c|}
\hline VENTILATION REQUIREMENTS LOCAL EXHAUST & $\begin{array}{l}\text { PROTECTIVE EQUIPMENT (SPECIFY TYPES) EYE } \\
\text { GOggleS }\end{array}$ \\
\hline MECHANICAL (GENERAL) & $\begin{array}{l}\text { GLOVES } \\
\text { Rubher }\end{array}$ \\
\hline \НE゙いA & $\begin{array}{l}\text { RESPIRATOA } \\
\text { With proper filter }\end{array}$ \\
\hline
\end{tabular}

OTHER PROTECTIVE EQUIPMENT

\section{SECTION IX SPECIAL PRECAUTIONS}

PRECAUTIONS TO BE TAKEN IN HANDLING AND STORAGE

store away from oxidizing materials and heat.

OTHER PRECAUTIONS

3IOHATURE

DATE

$328-6204 B$ 


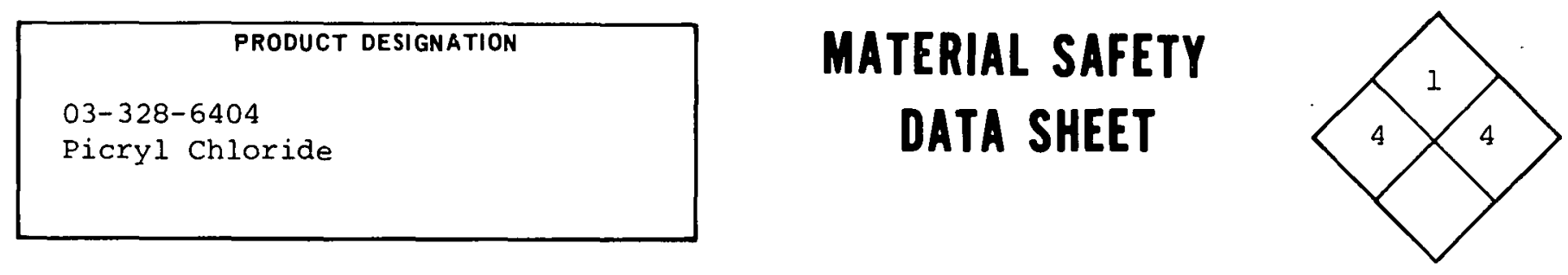

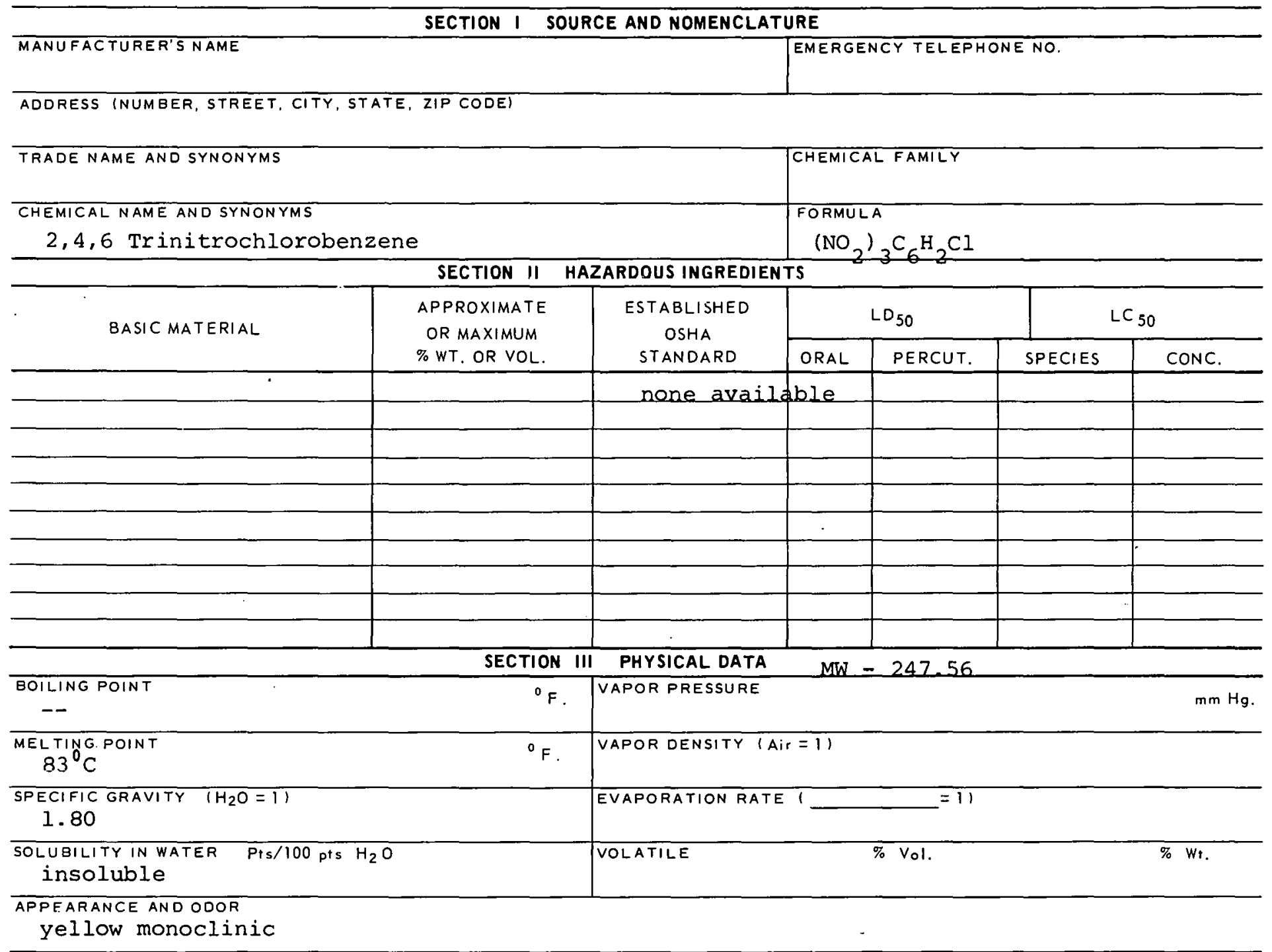

\begin{tabular}{|c|c|c|c|}
\hline & FIRE AND EXPLOSION HAZARD DATA & & \\
\hline $\begin{array}{l}\text { FLASH POINT } \\
\text { none available }\end{array}$ & & \multirow{2}{*}{$\begin{array}{c}\text { FLAMMABI,E } \\
\text { (EXPLOSIVE) } \\
\text { LIMITS }\end{array}$} & UPPER \\
\hline METHOD USED & & & LOWER \\
\hline
\end{tabular}

EXTINGUISHING MEDIA Explosive - do not fight fire except for unmanned equipment use water.

SPECIAL FIRE FIGHTING PROCEDURES

Wear full protective clothing for rescue operations.

UNUSUAL FIRE AND EXPLOSION HAZARDS

Severe explosion hazard. 
TOXIC LEVEL

none available

CARCINOGENIC

PRINCIPLE ROUTES OF ABSORBTION

SKIN AND EYE

inhalation, ingestion, skin

IRRITATION

REVELANT SYMPTOMS OF EXPOSURE

Dermatitis, irritation of respiratory and digestive systems. EFFECTS OF CHRONIC EXPOSURE

Dermatitis.

EMERGENCY AND FIRST AID PROCEDURES Inhalation - rest; if swallowed - give emetic, get medical aid; wash skin \& eyes.

\section{SECTION VI REACTIVITY DATA}

CONDITIONS CONTRIBUTING TO INSTABILITY

CONDITIONS CONTRIBU TING TO HAZARDOUS POLYMERIZATION

INCOMPATABILITY (MAIERIALS IU AVUIO)

Keep from oxidizing materials (explosive) inorganic nitrates, chlorates and HA LAHLUUS RPESUPOSTIOH RחOOUCTC ammointum nitrate.

\section{SECTION VII SPILL OR LEAK PROCEDURES}

STEPSTO BE TAKEN IN CASE MATERIAL IS RELEASED OR SPILLED

WASTE DISPOSAL METHOD

SECTION VIII SPECIAL PROTECTION INFORMATION

\begin{tabular}{l|l}
\hline VENTILATION REQUIREMENTS LOCAL EXHAUST & $\begin{array}{c}\text { PROTECTIVE EQUIPMENT (SPECIFY TYFESI EEYE } \\
\text { GOggles }\end{array}$ \\
\hline MECHANICAL IGENERAL) & GLOVES \\
Rubber
\end{tabular}

OTHER PROTECTIVE EQUIPMENT

\section{SECTION IX SPECIAL PRECAUTIONS}

PRECAUTIONS TO BE TAKEN IN HANDLING AND STORAGE

Keep from oxidizing materials, combustibles, and source of heat.

OTIIER PRECAUTIONS

SIGNATURE

INATF 
CAS : 000107120

\begin{tabular}{|l|}
\multicolumn{1}{|c|}{ PRODUCT DESIGNATION } \\
Propionitrile \\
o3-328-9504 \\
\hline
\end{tabular}

\section{UF96250 \\ MATERIAL SAFETY \\ DATA SHEET}

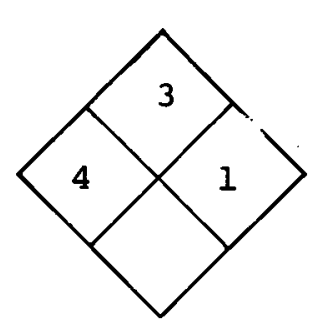

SECTION I SOURCE AND NOMENCLATURE

\begin{tabular}{l|l}
\hline MANUFACTURER'S NAME & EMERGENCY TELEPHONE NO. \\
\hline ADDRESS (NUMBER, STREET, CITY, STATE, ZIP CODE)
\end{tabular}

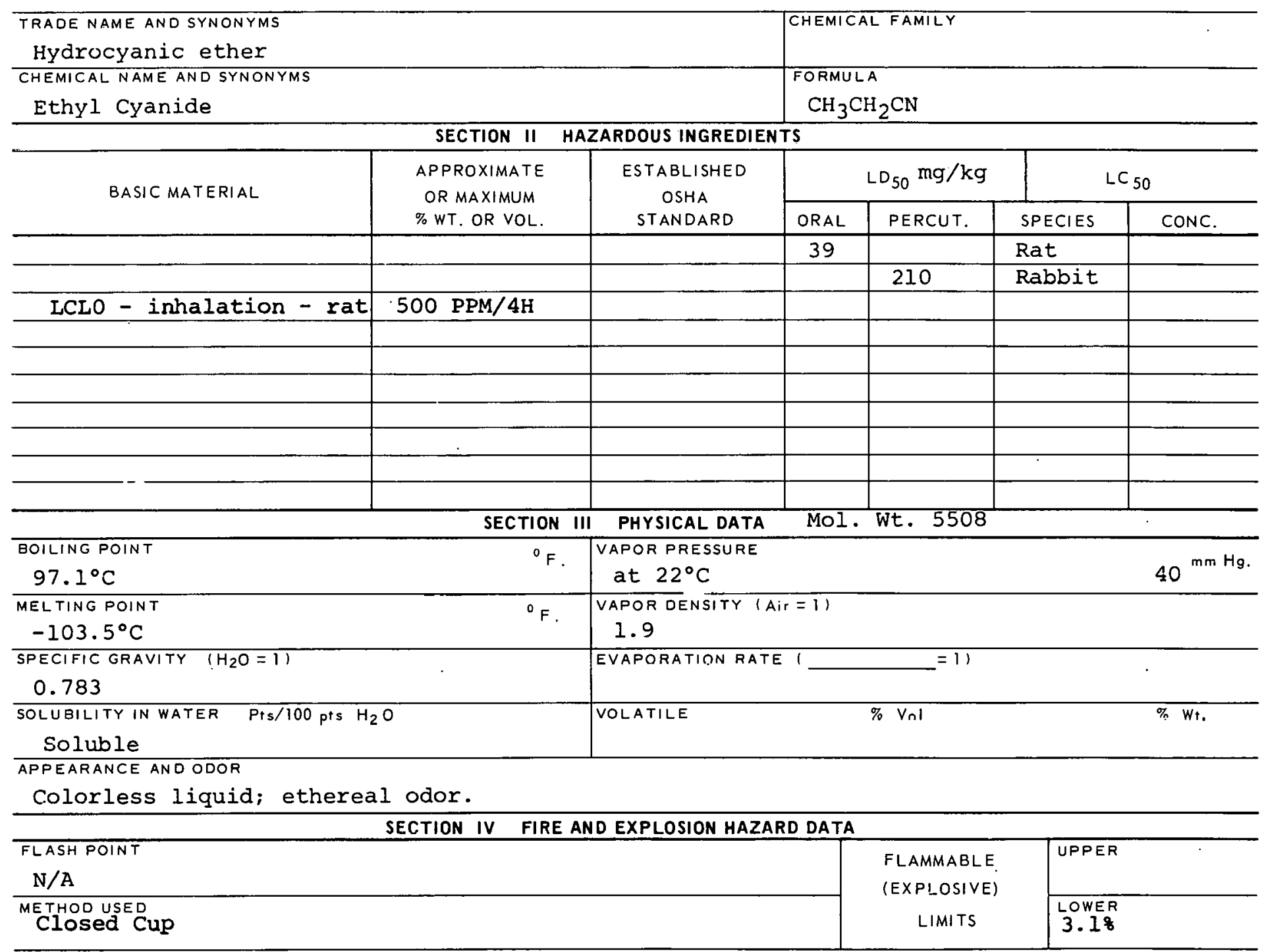

EXTINGUISHING MEDIA

Use dry chemicals, alcohol foam or carbon dioxide.

SPECIAL FIRE FIGHTING PROCEDURES

Wear protective clothing; use self-contained breathing apparatus.

UNUSUAL FIRE AND EXPLOSION HAZAROS 


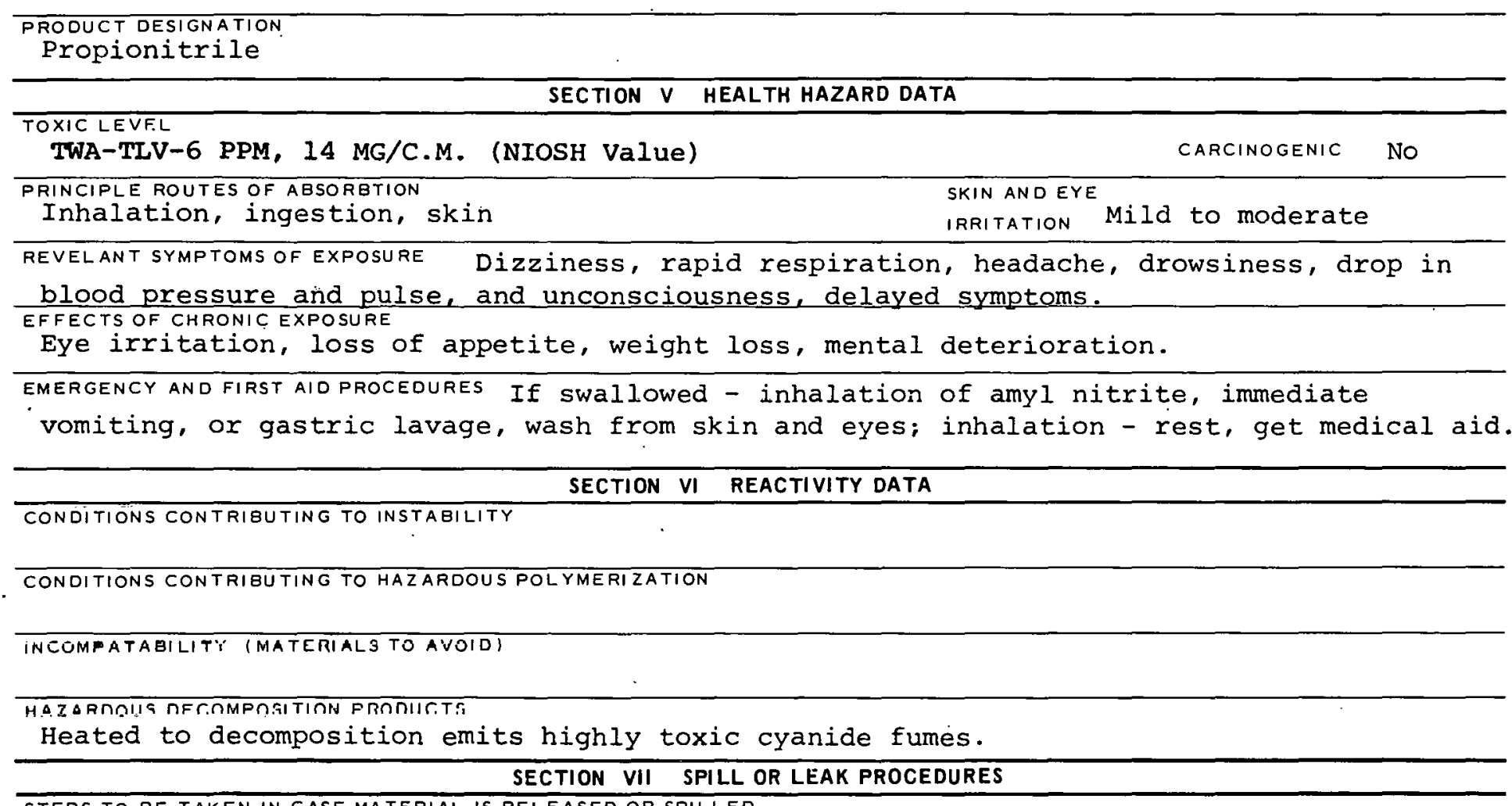

STEPS TO BE TAKEN IN CASE MATERIAL IS RELEASED OR SPILLED

Absorb with paper towels; evaporate in hood.

WASTE DISPOSAL METHOD

SECTION VIII SPECIAL PROTECTION INFORMATION

\begin{tabular}{l|l}
\multicolumn{1}{c}{ SECTION VIII SPECIAL PROTECTION INFORMATION } \\
\hline VENTILATION REQUIREMENTS LOCAL EXHAUST & $\begin{array}{l}\text { PROTECTIVE EOUIPMENT (SPECIFY TYPES) EYE } \\
\text { COgGlCC - SafCLY ClaCCCC }\end{array}$ \\
\hline MECHANICAL IGENERALI & $\begin{array}{c}\text { GLOVES } \\
\text { Rubbcr }\end{array}$ \\
\hline SPECIAL & $\begin{array}{c}\text { RESPIRATOR } \\
\text { w/proper filter }\end{array}$ \\
\hline OTHER PROTECTIVE EQUIPMENT
\end{tabular}

\section{Protective clothing}

SECTION IX SPECIAL PRECAUTIONS

PRECAUTIONS TO BE TAKEN IN HANDLING AND STORAGE

Store in flammable liquids storage cabinet.

OTHER PRECAUTIONS

SIGNATURE

DATE

$328-9504 \mathrm{~B}$ 


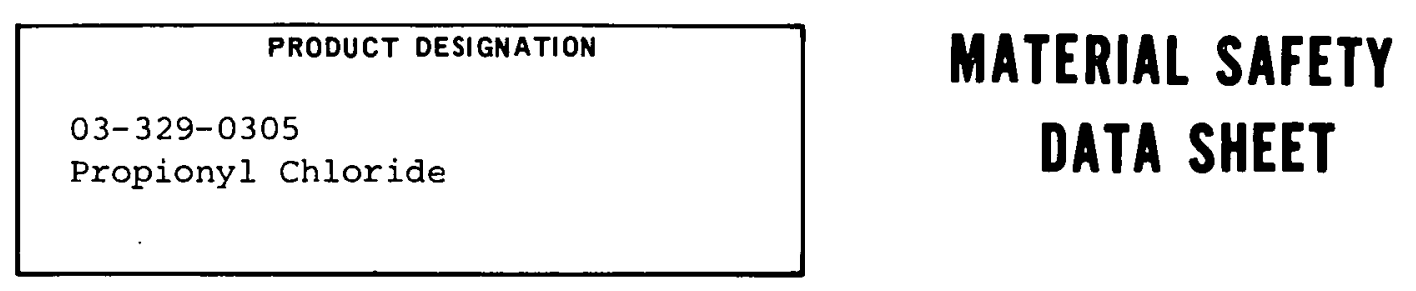

SECTION I SOURCE AND NOMENCLATURE

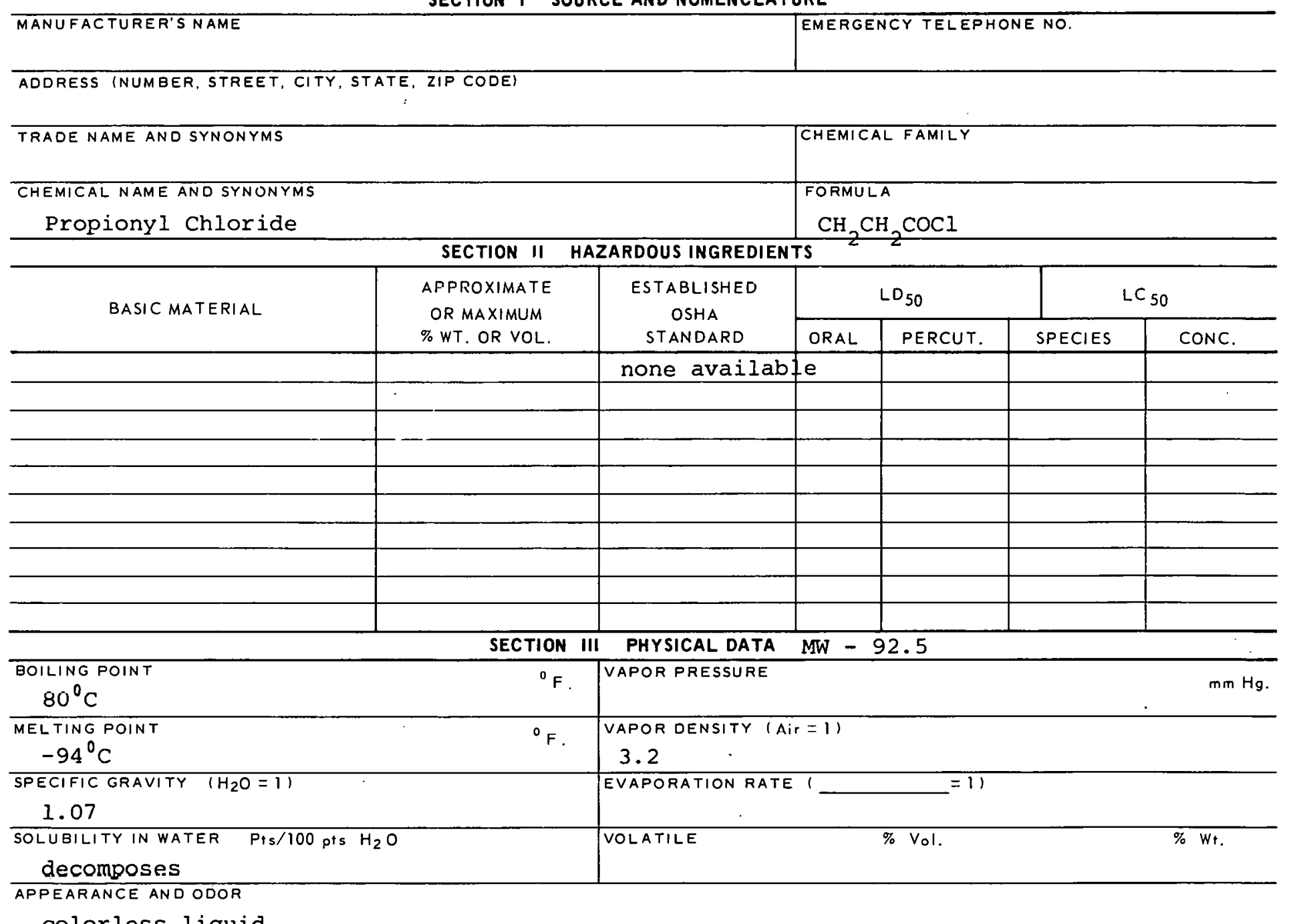

Colorless liquid

FLASH POINT

$54^{\circ} \mathrm{F}$

METHOD USED

EXTINGUISHING MEDIA

carbon dioxide, הry chemical.

SPECIAL FIRE FIGHTING PROCEDURES

UNUSUAL FIRE AND EXPLOSION HAZARDS

\section{FLAMMABLE}

(EXPLOSIVE)

LIMITS

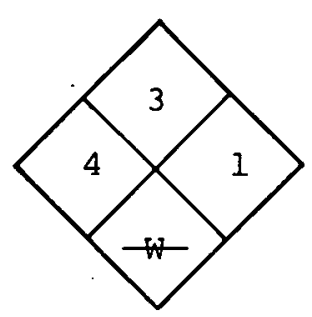




\section{TOXIC LEVEL}

none available

PRINCIPLE ROUTES OF ABSORBTION

inhalation, ingestion

CARCINOGENIC SKIN AND EYE IRRITATION

Cyanosis, coughing, headache, pulmonary edema, ulceration

of skin dizziness.

EFFECTS OF CHRONIC EXPOSURE Erosion of teeth, bronchial irritation and chronic cough.

EMERGENCY AND FIRST AID PROCEDURES Ingestion - drink water, milk of magnesia, get medical aid; inhalation - artificial respiration as needed; wash eyes and skin.

\section{SECTION VI REACTIVITY DATA}

CONDITIONS CONTRIBUTING TO INSTABILITY

CONDITIONS CONTRIBUTING TO HAZARDOUS POLYMERIZATION

INCOMPATABILITY (MATERIALS TOAVOID) May react violently with water, alcohol,

and dimethyl sulfoxide.

HAZARUOUS DECOMPOSITION PRODUCTS

SECTION VII SPILL OR LEAK PROCEDURES

STEPS TO BE TAKEN IN CASE MATERIAL IS RELEASED OR SPILLED

SECTION VIII SPECIAL PROTECTION INFORMATION

\begin{tabular}{l|l}
\hline VENTILATION REQUIREMENT'S LUCAL EXHAUST & $\begin{array}{l}\text { PROTECTIVE EQUIPMENT (STECIFY TYPESI EYE } \\
\text { GUYyleSS }\end{array}$ \\
\hline MECHANICAL IGENERAL) & $\begin{array}{l}\text { GLOVES } \\
\text { Rubber }\end{array}$ \\
\hline SPFLIIAL & REEFIRATOR \\
& Self-COntained \\
\hline
\end{tabular}

OTHER PROTECTIVE EQUIPMENT

SECTION IX SPECIAL PRECAUTIONS

PRECAUTIONS TO BE TAKEN IN HANDLING AND STORAGE

OTHER PRECAUTIONS

MIOINAIUKL

DATE

$329-0305 B$ 
CAS : 000108214

\section{PRODUCT DESIGNATION}

$03-329-1345$

03-329-1305

Iso-Propyl Acetate
AI49300

MATERIAL SAFETY

DATA SHEET

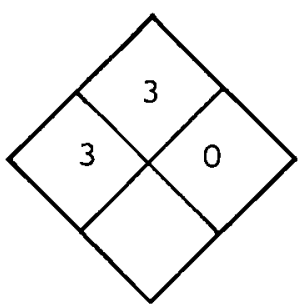

SECTION I SOURCE AND NOMENCLATURE

\begin{tabular}{l|l}
\hline MANUFACTURER'S NAME & EMERGENCY TELEPHONE NO. \\
\hline ADDRESS (NUMBER, STREET, CITY, STATE, ZIP CODE) &
\end{tabular}

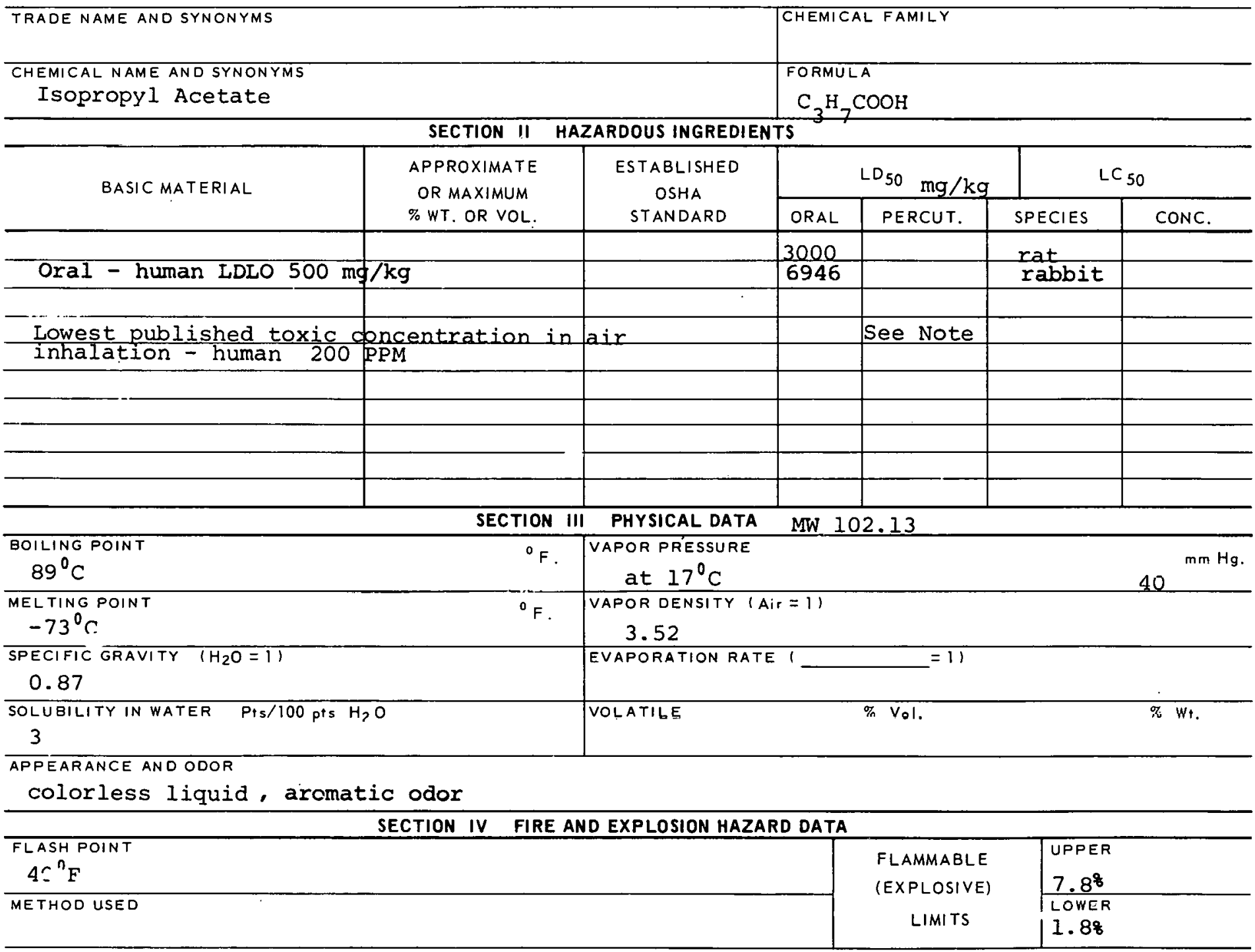

EXTINGUISHING MEDIA

Foam, carbon dioxide, dry chemical.

SPECIAL FIRE FIGHTING PROCEDURES

UNUSUAL FIRE AND EXPLOSION HAZARDS 
TOXIC LEVEL

TLV - 250 PPM, $950 \mathrm{mg} / \mathrm{C} . \mathrm{M}$.

CARCINOGENIC PRINCIPLE ROUTES OF ABSORBTION
Inhalation, ingestion

REVELANT SYMPTOMS OF EXPOSURE

Eye irritation, respiratory passage irritation

EFFECTS OF CHRONIC EXPOSURE

$--$

EMERGENCY AND FIRST AID PROCEDURES

If swallowed - give emetic, get medical aid; inhalation - rest; wash from skin \& eyes.

CONDITIONS CONTRIBUTING TO INSTABILITY

CONDITIONS CONTRIBUTING TO HAZARDOUS POLYMERIZATION

INCOMPATABILITY (MATERIALS TO AVOID)

HAZARDOUS DECOMPOSITION PRODUCTS

SECTION VII SPILL OR LEAK PROCEDURES

STEPS TO BE TAKEN IN CASE MATERIAL IS RELEASED OR SPILLED

Absorb on paper towel; evaporate in hood.

WASTE DISPOSAL METHOD

\section{SECTION VIII SPECIAL PROTECTION INFORMATION}

\begin{tabular}{l|l}
\hline VENTILATION REQUIREMENTS LOCAL EXHAUST & $\begin{array}{l}\text { PROTECTIVE EQUIPMENT (SPECIFY TYPES) EYE } \\
\text { Goggles }\end{array}$ \\
\hline MECHANICAL IGENERAL) & $\begin{array}{l}\text { GLOVES } \\
\text { Rubber gloves }\end{array}$ \\
\hline SFECIAL & $\begin{array}{l}\text { PESO!BATRR } \\
\text { With proper fj.lter }\end{array}$ \\
\hline
\end{tabular}

OTHER RROTECTIVE EQUIPMENT

SECTION IX SPECIAL PRECAUTIONS

PRECAUTIONS TO BE TAKEN IN HANDLING AND STORAGE

store in standard combustible liquids storage cabinet.

UIHER RRE: AUTIONS

SIGMATURE

DATE

$329-1305 B$ 
CAS - 000075310

PRODUCT DESIGNATION

Iso-propylamine

03-329-1805
NT84000

MATERIAL SAFETY

DATA SHEET

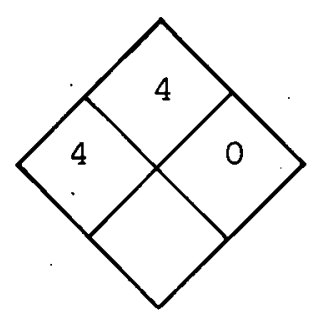

SECTION I SOURCE AND NOMENCLATURE

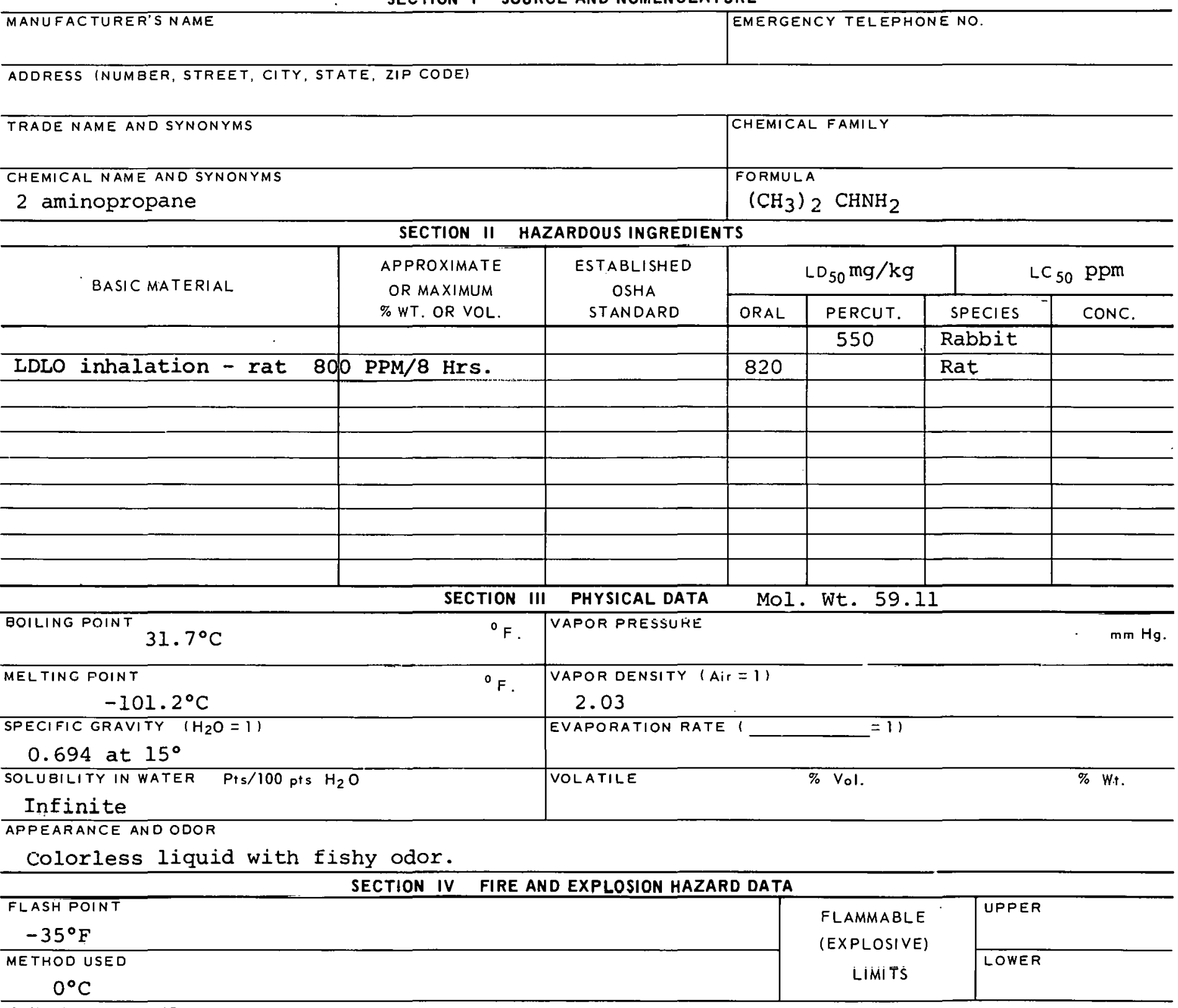

EXTINGUISHING MEDIA

Alcohol foam, $\mathrm{CO}_{2}$ or dry chemical. Water may be ineffective.

SPECIAL FIRE FIGHTING PROCEDURES

Wear full protective clothing.

UNUSUAL FIRE AND EXPLOSION HAZARDS 
TOXIC LEVEL

TLV $5 \mathrm{ppm}, 12 \mathrm{mg} / \mathrm{C} . \mathrm{M}$.

PRINCIPLE ROUTES OF ABSORBTION

CARCINOGENIC

Inhalation, ingestion

SKIN AND EYE

IRRITATION SEVERE

REVELANT SYMPTOMS OF EXPOSURE

Nose and throat irritation, lung edema, skin and eye burns.

EFFECTS OF CHRONIC EXPOSURE

EMERGENCY AND TIRST AID PROCEDURES

Inhalation - rest, keep warm, get medical aid; if swallowed - wash mouth, drink wator, get medical aid; wash from skin and eyes.

\begin{tabular}{|c|c|}
\hline \multicolumn{2}{|l|}{ SECTION VI REACTIVITY DATA } \\
\hline \multicolumn{2}{|l|}{ CONDITIONS CONTRIBUTING TO INSTABILITY } \\
\hline \multicolumn{2}{|l|}{ CONDITIONS CONTRIBUTING TO HAZARDOUS POLYMERIZATION } \\
\hline \multicolumn{2}{|l|}{ INCOMPATABILITY (MATERIALS TO AVOID) } \\
\hline \multicolumn{2}{|l|}{ HAZARDOUS DECOMPOSITION PRODUCTS } \\
\hline \multicolumn{2}{|c|}{ SECTION VII SPILL OR LEAK PROCEDURES } \\
\hline \multicolumn{2}{|l|}{ STEPS TO BE TAKEN IN CASE MATERIAL IS RELEASEO OR SPILLED } \\
\hline \multicolumn{2}{|c|}{ Cover with sodium bisulfate and sprinkle with water. Drain into sewer with much water. } \\
\hline \multicolumn{2}{|l|}{ WASTE DISPOSAL METHOD } \\
\hline \multicolumn{2}{|c|}{ SECTION VIII SPECIAL PROTECTION INFORMATION } \\
\hline \begin{tabular}{|l|} 
VENTILATION REQUIREMENTS LOCAL EXHAUST \\
\end{tabular} & $\begin{array}{l}\text { PROTECTIVE EQUIPMENT (SPECIFY TYPES) EYE } \\
\text { GOggICD }\end{array}$ \\
\hline \multirow[t]{2}{*}{ MECHANICAL (GENERAL) } & GLOVLS \\
\hline & Butyl rubber qloves \\
\hline SHEEIAL & $\begin{array}{l}\text { REEПIRATOR } \\
\text { w/proper filter }\end{array}$ \\
\hline
\end{tabular}

OTIIER FROTECTIVE EQUIPMENT

Face shield, rubber apron

SECTION IX SPECIAL PRECAUTIONS

PRECAUTIONS TO BE TAKEN IN HANDLING AND STORAGE

Store in flamable liquids storage cabinet.

OTHER PRECAUTIONS

BIGANTUAE

DATE

$329-1805 B$ 
CAS : 000107108

UH91000

\begin{tabular}{|c|}
\hline PRODUCT DESIGNATION \\
03-329-1905 \\
N-Propyiamine \\
\end{tabular}

MATERIAL SAFETY

DATA SHEET

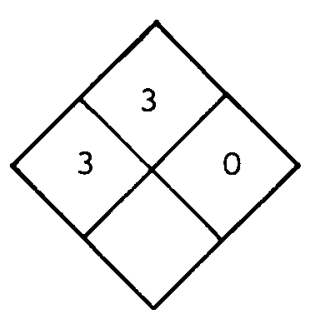

SECTION I SOURCE AND NOMENCLATURE

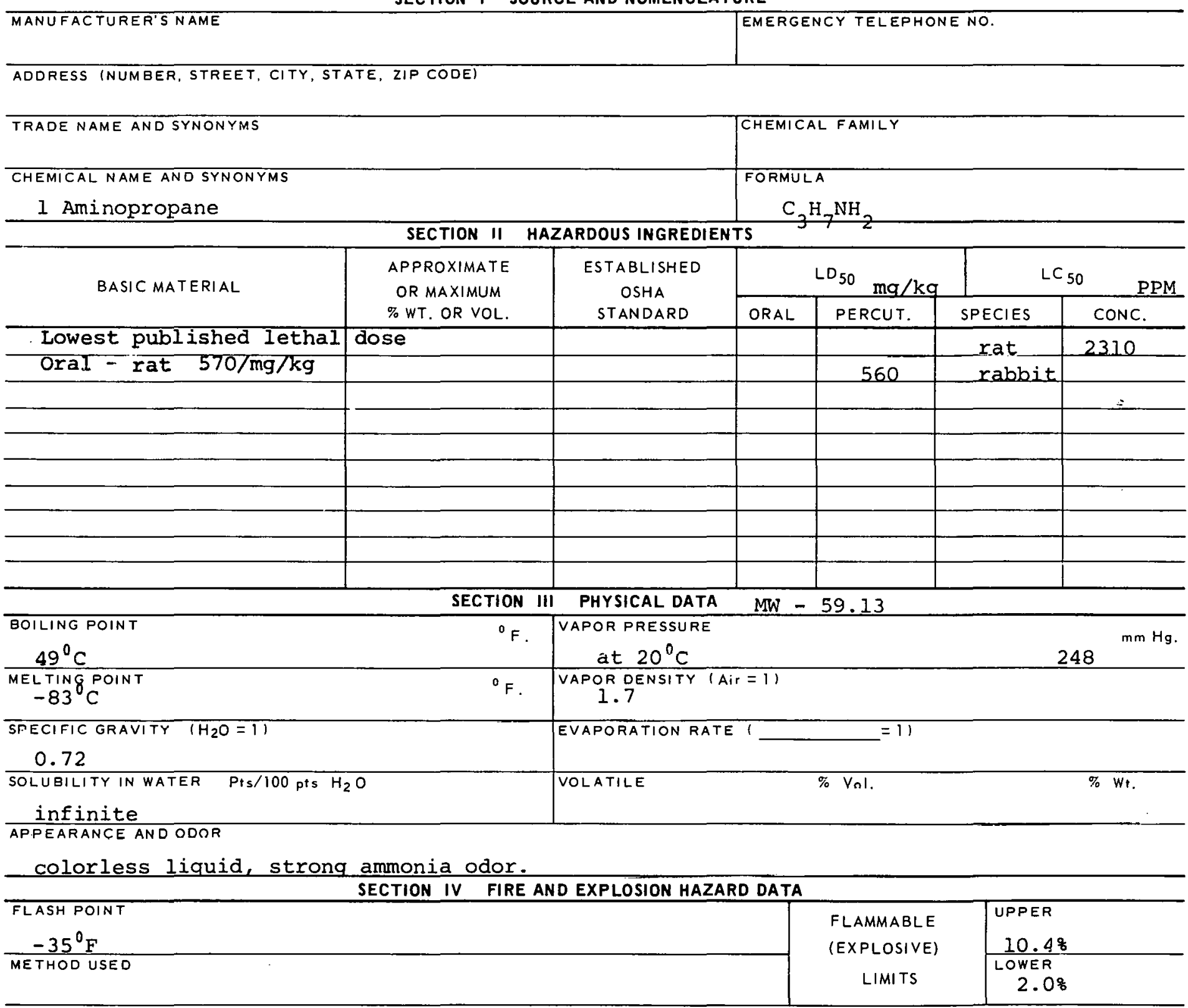

EXTINGUISHING MEOIA

carbon dioxide, dry chemical, alcohol foam. Water may be ineffective.

SPECIAL FIRE FIGHTING PROCEDURES

Wear full protective clothing.

UNUSUAL FIRE ANO EXPLOSION HATARTS 


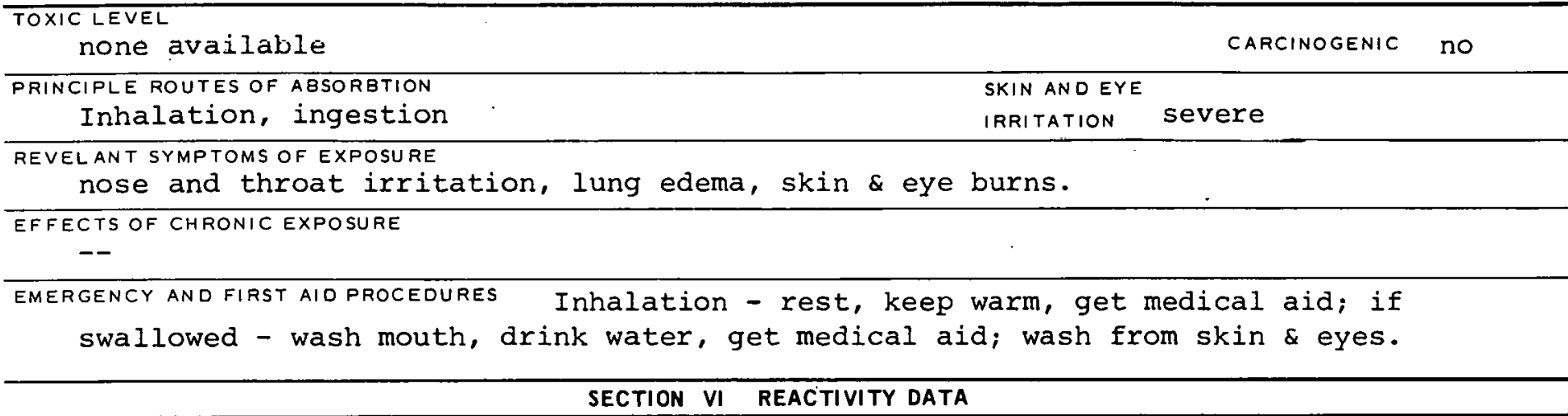

CONDITIONS CONTRIBUTING TO INSTABILITY

SOAOITIONG INNTRIRIITING TQ HAZARDOUS POLYMERIZATION

INCOMPATABILITY (MATERIALS TO AVOID)

HAZARDOUS DECOMPOSIIION PRODUCTS

SECTION VII SPILL OR LEAK PROCEDURES

STEPS TO BE TAKEN IN CASE MATERIAL IS RELEASED OR SPILLED

WASTE DISPOSAL METHOD

SECTION VIII SPECIAL PROTECTION INFORMATION

\begin{tabular}{l|l}
\hline VENTILATION REQUIREMENTS LOCAL EXHAUST & PROTECTIVE EQUIFMENT (SPECIFY TYPES) EYE
\end{tabular}

MESHANICAL (GENERAL) goggles

SPECINL GLOVES

AEERIRATOR

with proper filter

OTHER PROTECTIVE EQUIPMENT

Face shield, rubber aprón

SECTION IX SPECIAL PRECAUTIONS

PRECAUTIONS TO BE TAKEN IN HANDLING AND STORAGE

Protect against physical damage - outside or detached storage preferred. Inside storage

in a standard flammable liquids storage cabinet. OTHER PHECAUTIONS 


PRODUCT DESIGNATION
O3-329-2305
N-Propylaniline

\section{SECTION I SOURCE AND NOMENCLATURE}

MANUFACTURER'S NAME

ADDRESS (NUMBER, STREET, CITY, STATE, ZIP CODE)

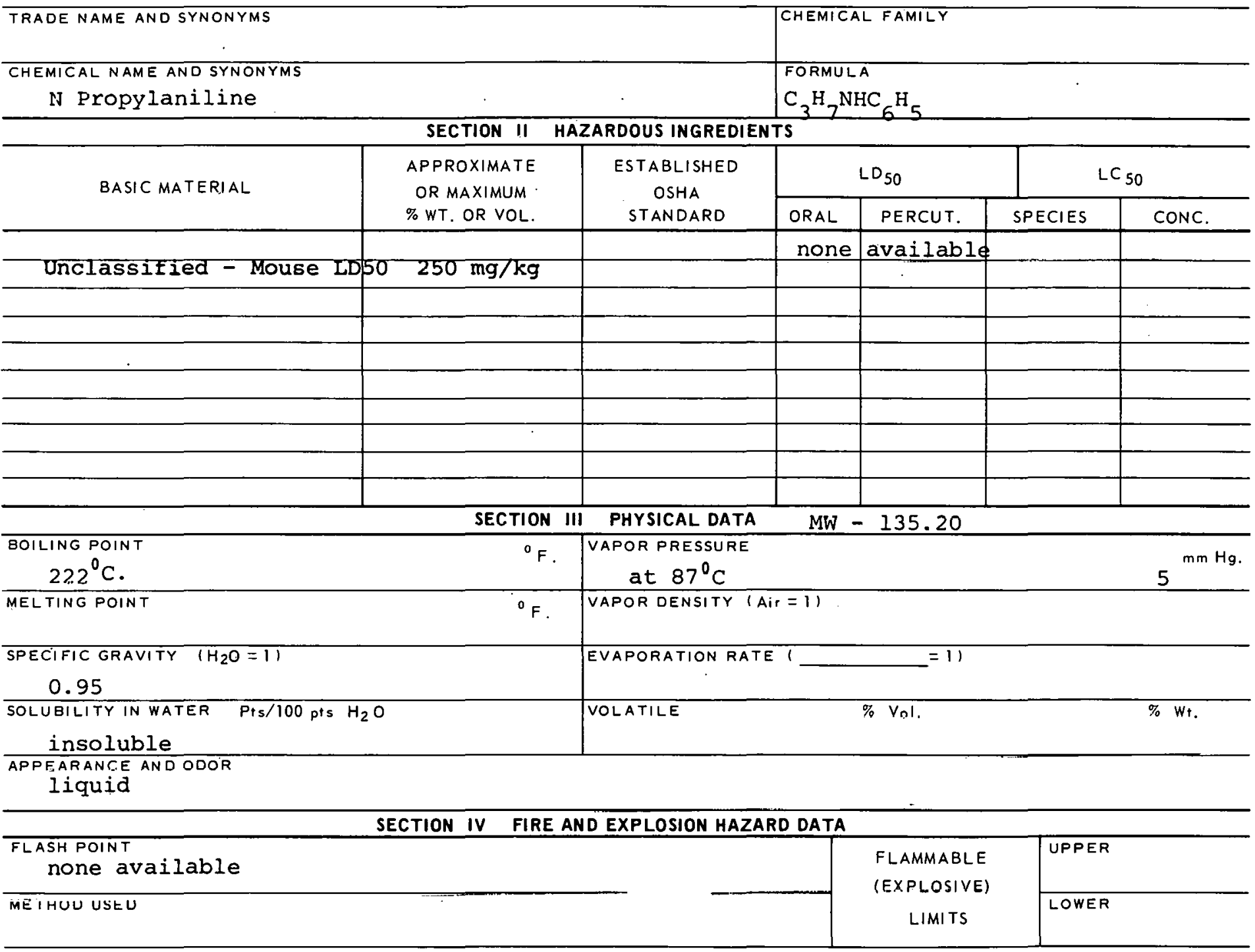

EXTINGUISHING MEDIA

Foam, carbon dioxide, dry chemical.

SPECIAL FIRE FIGHTING PROCEDURES

UNUSUAL. FIRF AN TQ EXPLOSION HAZARDS

When heated may decompose to give toxic aniline fumes. 


\section{TOXIC LEVEL}

none available

CARCINOGENIC

no

PRINCIPLE ROUTES OF ABSORETION

inhalation, ingestion, skin

SKIN AND EYE

IRRITATION moderate

REVELANT SYMPTOMS OF EXPOSURE Weakness, tremors, cyanosis, drowsiness, central nervous system depression.

EFFECTS OF CHRONIC EXPOSURE

Fatigue, loss of appetite, headache, dizziness.

EMERGENCY AND FIRST AID PROCEDURES Inhalation - rest; if swallowed - give emetic, get

medical aid; wash from skin \& eyes.

\section{SECTION VI REACTIVITY DATA}

CONDITIONS CONTRIBUTING TO INSTABILITY

CONDITIONS CONTRIBUTING TO HAZARDOUS POLYMERIZATION

INCOMPATABIL! TY (MATERIALS TO AVOID)

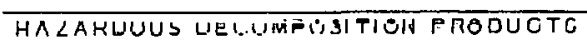

SECTION VII SPILL OR LEAK PROCEDURES

STEPS TO EE TAKEN IN CASE MATERIAL IS RELEASED OR SPILLED

WASTE DISPOSAL METHOD

SECTION VIII SPECIAL PROTECTION INFORMATION

\begin{tabular}{l|l}
\hline VENTILATION REQUIREMENTS LOCAL EXHAUST & $\begin{array}{l}\text { PROTECTIVE EQUIPMENT (SPECIFY TYPES) EYE } \\
\text { Goggles }\end{array}$ \\
\hline MECHANICAL IGENERAL) & $\begin{array}{l}\text { GLOVES } \\
\text { POlyVinYl alcohol }\end{array}$ \\
\hline SRECIAL & RESPIRATOR \\
& With proper filter \\
\hline
\end{tabular}

OTHER PROTECTIVE EQUIPMENT

SECTION IX SPECIAL PRECAUTIONS

PRECAUTIONS TO BE TAKEN IN HANDLING AND STORAGE

OTHER PRECAUTIONS

SIGNATURE

DATE

$329-2305 B$ 
CAS : 000103651

DA87500

PRODUCT DESIGNATION

03-329-3305

$\mathrm{N}$-Propylbenzene

MATERIAL SAFETY

DATA SHEET

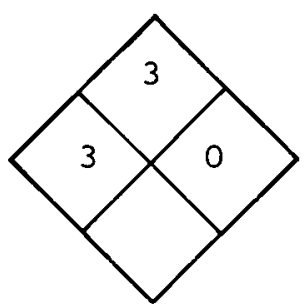

SECTION I SOURCE AND NOMENCLATURE

MANUFACTURER'S NAME

EMERGENCY TELEPHONE NO.

ADDRESS (NUMBER, STREET, CITY, STATE, ZIP CODE)

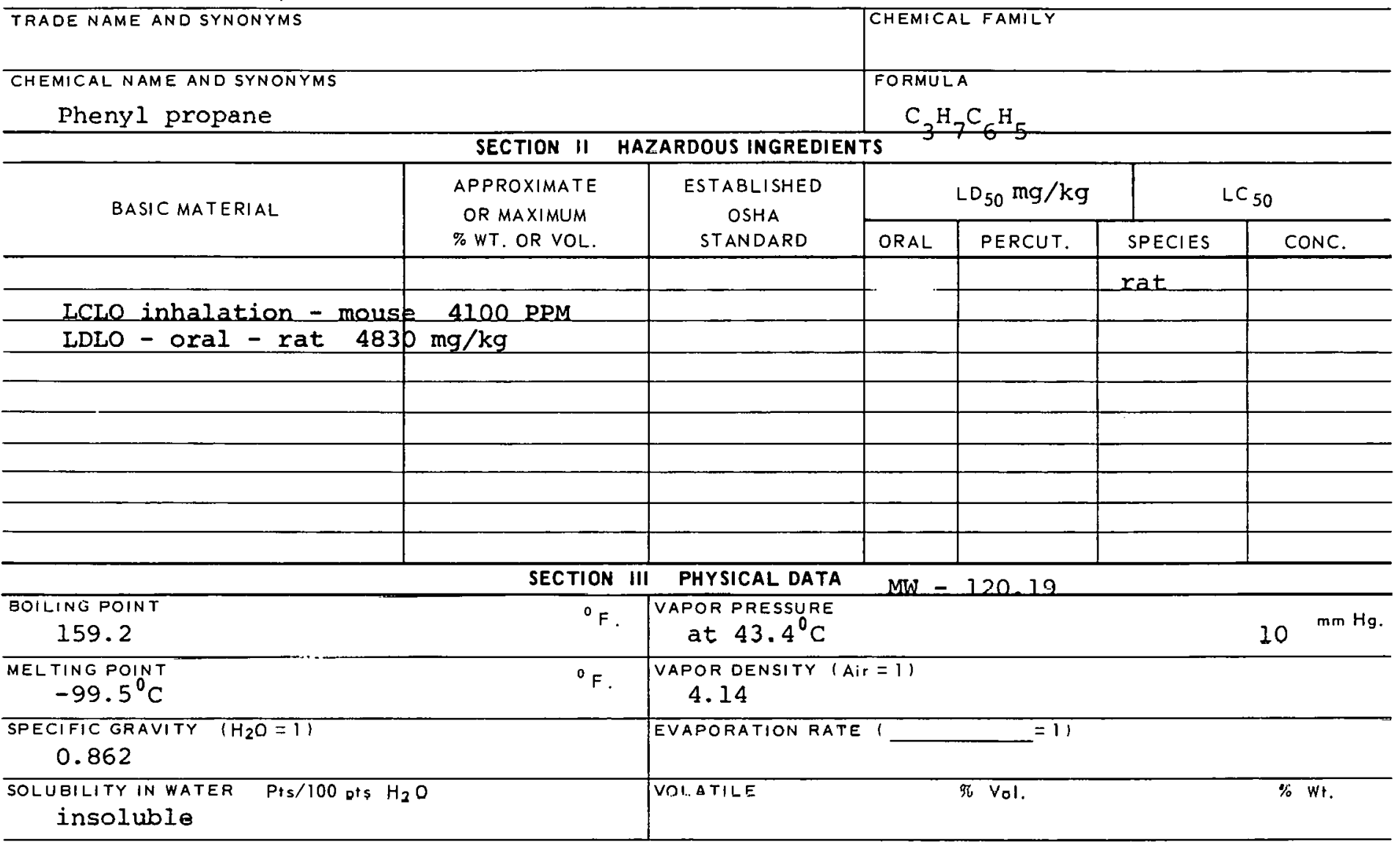

APPEARANCE AND ODOR

colorless liquid

SECTION IV FIRE AND EXPLOSION HAZARD DATA

\begin{tabular}{|c|c|c|}
\hline $\begin{array}{c}\text { FLASH POINT } \\
86^{\circ} \mathrm{F}\end{array}$ & \multirow{2}{*}{$\begin{array}{c}\text { FLAMMABLE } \\
\text { (EXPLOSIVE) } \\
\text { LIMITS }\end{array}$} & UPPER \\
\hline METHOD USED & & LOWER \\
\hline
\end{tabular}

EXTINGUISHING MEDIA

Water spray, carbon dioxide, dry chemical or foam.

SPECIAL FIRE FIGHTING PROCEDURES

Water may be ineffective. Wear goggles and self-contained breathing apparatus.

UNUSUAL FIRE AND EXPLOSION HAZARDS 


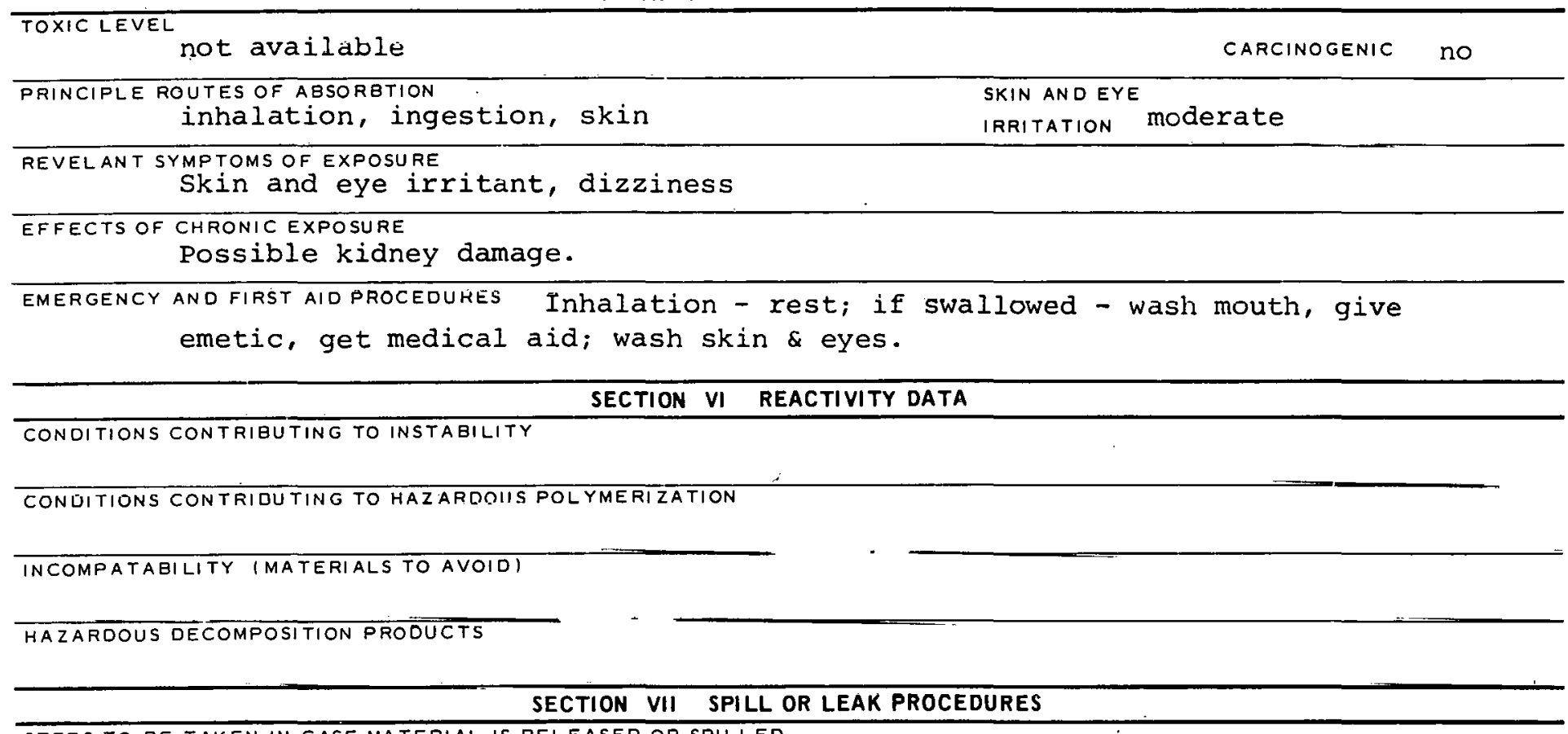
STEPS TO BE TAKEN IN CASE MATERIAL IS RELEASED OR SPILLED

WASTE DISPOSAL METHOD

SECTION VIII SPECIAL PROTECTION INFORMATION

\begin{tabular}{l|l}
\hline VENTILATION REQUIREMENTS LOCAL EXHAUST & $\begin{array}{l}\text { SROTECTIVE EQUIPMENT (SPECIFY TYPESIEYE } \\
\text { GOggleS }\end{array}$ \\
\hline MECHANICAL IGENERAL & $\begin{array}{l}\text { GLOVES } \\
\text { Ribber }\end{array}$ \\
\hline SPECIAL & $\begin{array}{l}\text { REEFIAATOR } \\
\text { Self-contained }\end{array}$ \\
\hline
\end{tabular}

OTHER PROTECTIVE EQUIPMENT 


\begin{tabular}{|l|}
\hline \multicolumn{1}{|c|}{ PRODUCT DESIGNATION } \\
$03-329-4405$ \\
N-Propyl Chlorocarbonate
\end{tabular}$\quad \begin{gathered}\text { MATERIAL SAFETY } \\
\end{gathered}$

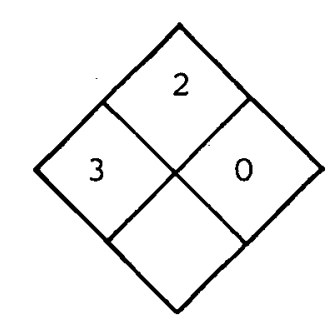

SECTION I SOURCE AND NOMENCLATURE

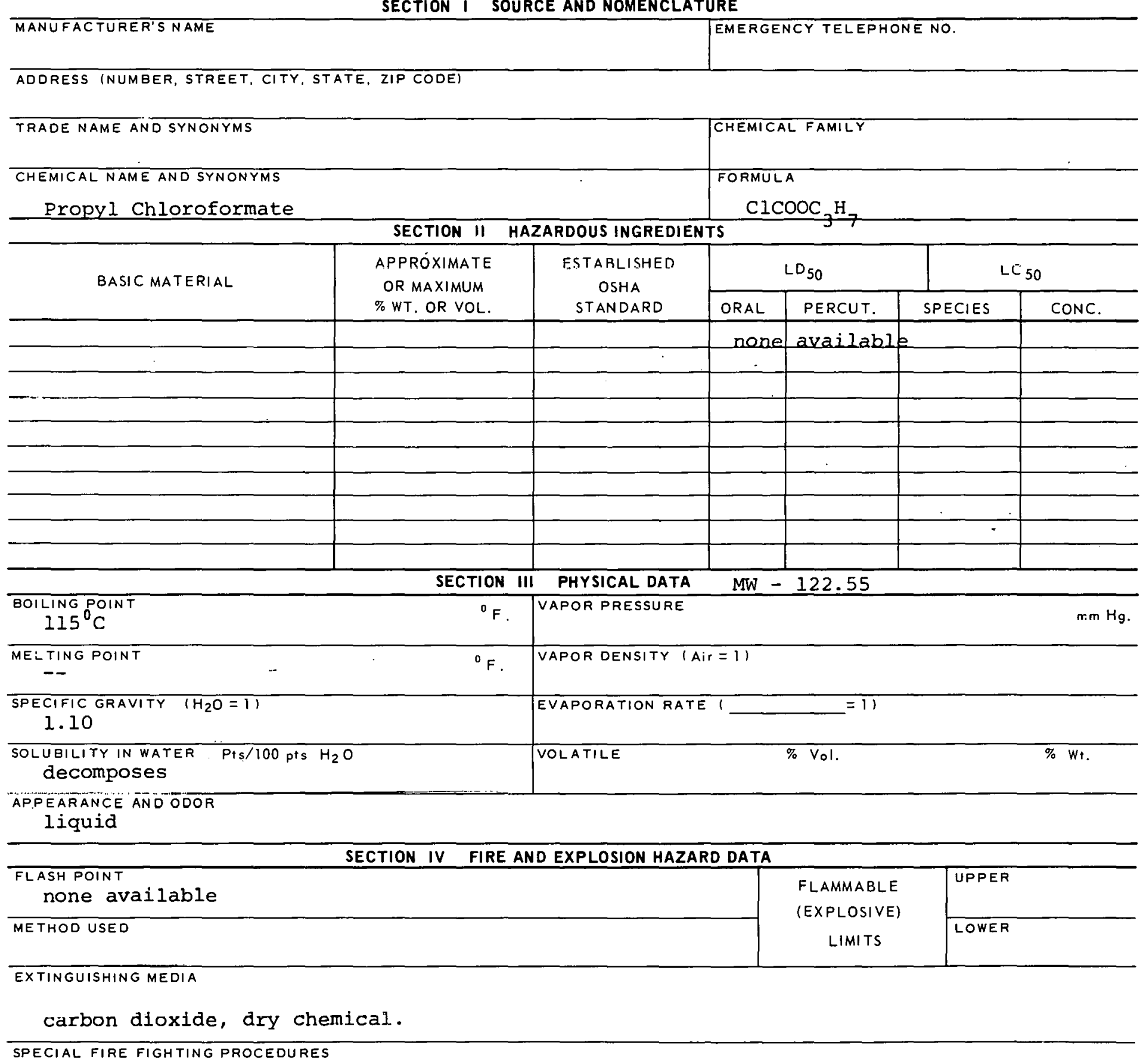




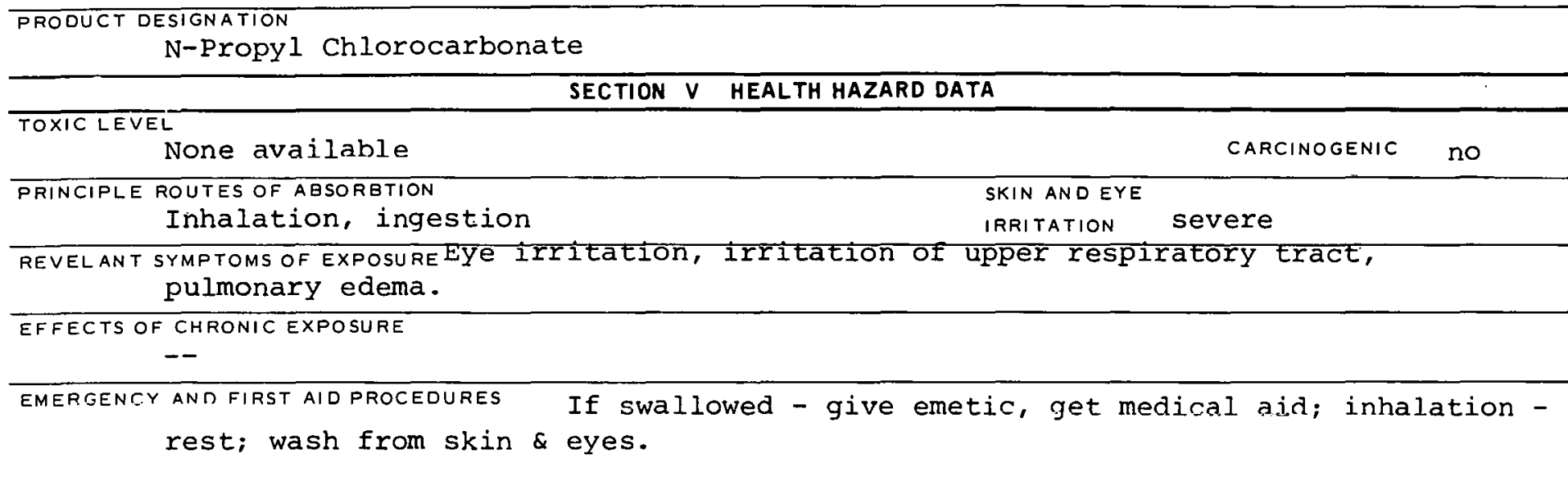

\section{SECTION VI REACTIVITY DATA}

CONDITIONS CONTRIBUTING TO INSTABILITY

CONDITIONS CONTRIBU TING TO HAZARDOUS POLYMERIZATION

INCOMPATABILITY (MATERIALS IUAVUIU)

IIAZARUUUS UELUMRUAT TIUIV PREBUCTS

SECTION VII SPILL OR LEAK PROCEDURES

STEPS TO BE TAKEN IN CASE MATERIAL IS RELEASED OR SPILLED

\section{WASTE DISPOSAL METHOD}

\section{SECTION VIII SPECIAL PROTECTION INFORMATION}

\begin{tabular}{|c|c|}
\hline VENTILATION REQUIREMENTS LOCAL EXHAIST & $\begin{array}{l}\text { PROTECTIVE EQUIPMENT (SPECIFY TYPES) EYE } \\
\text { Goggles }\end{array}$ \\
\hline MECIIANICAL (GENERAL) & $\begin{array}{l}\text { GLOVES } \\
\text { Rubber }\end{array}$ \\
\hline STECIAL & $\begin{array}{l}\text { RESPIRATOR } \\
\text { Self-contained }\end{array}$ \\
\hline
\end{tabular}

\section{SECTION IX SPECIAL PRECAUTIONS}

PRECAUTIONS TO BE TAKEN IN HANDLING AND STORAGE

\section{Keep away from water.}

OTHER TRECAUTIONS 
CAS : 000078751

\section{PRODUCT DESIGNATION}

03-329-4805

Propylene Bromide
TX85800

\section{MATERIAL SAFETY \\ DATA SHEET}

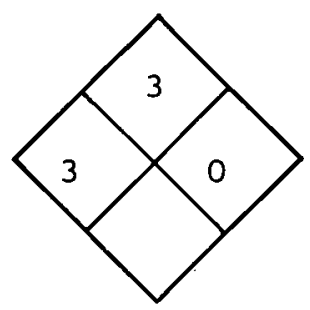

SECTION I SOURCE AND NOMENCLATURE

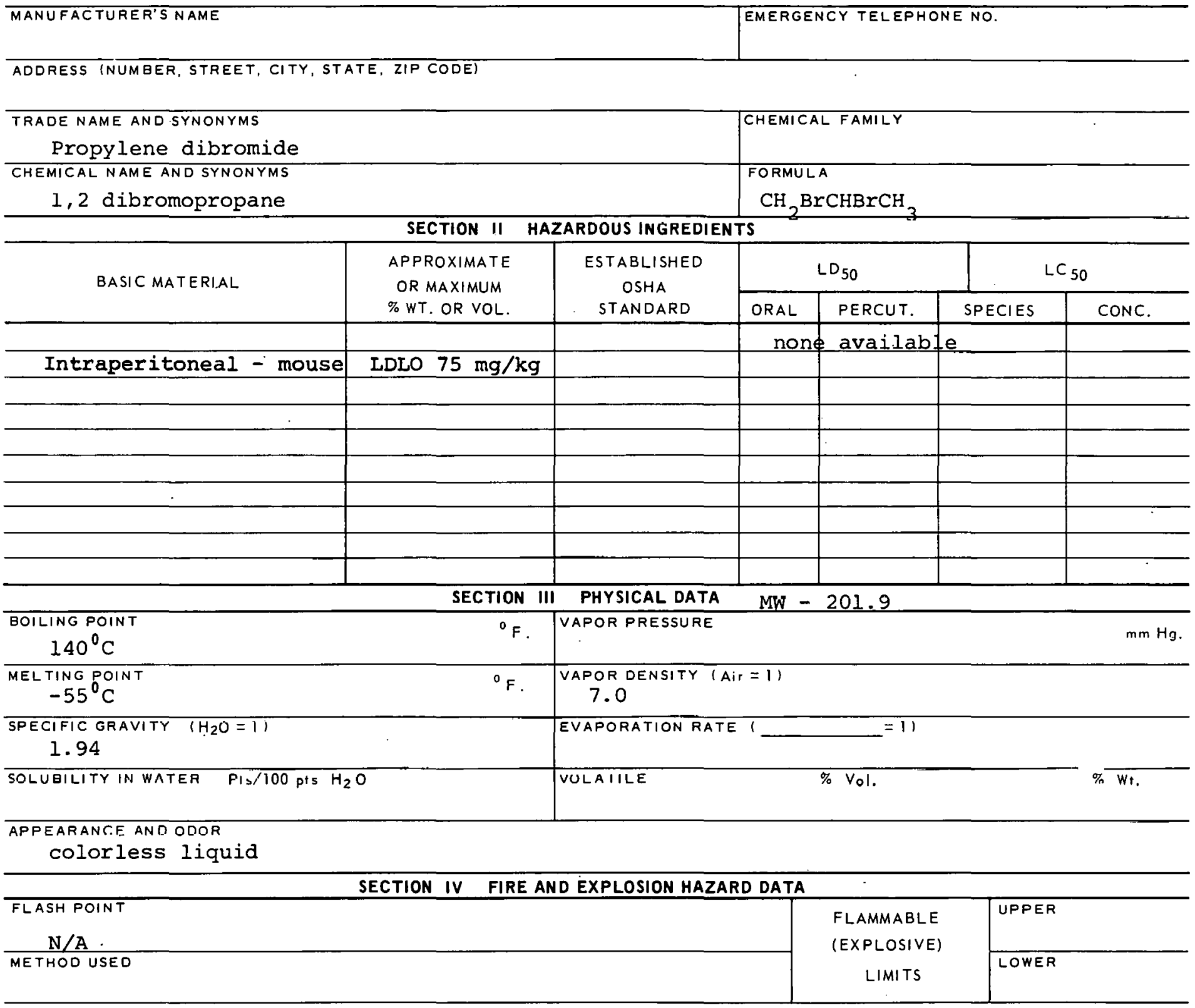

EXTINGUISHING MEDIA

Water, foam, carbon dioxide, dry chemical

SPECIAL FIRE FIGHTING PROCEDURES

Water may be ineffective.

UNUSUAL FIRF AN 
TOXIC LEVEL

not available

PRINCIPLE ROUTES OF ABSORBTION

Inhalation, ingestion

REVELANT SYMPTOMS OF EXPOSURE

Eye irritation, narcosis, drowsiness, headache, vomiting.

EFFECTS OF CHRONIC EYPOSURE

Dermatitis, liver and kidney damage.

EMERGENCY AND FIRST AID PROCEOURES Inhalation - rest; if swallowed - give emetic, get medical

aid; wash from skin \& eyes.

\section{SECTION VI REACTIVITY DATA}

CARCINOGENIC

\section{SKIN AND EYE}

IRRITATION no

medorate

CONDITIONS CONTRIBUTING TO INSTABILITY

TON IITINNS CONTRIBUTING TO HAZARDOUSS POLYMERIZATION

INCOMPATABILITY (MATERIALS TO AVOID)

HAZARDOUS DECUMPOSITION PRODUCTS

SECTION VII SPILL OR LEAK PROCEDURES

STEPS TO BE TAKEN IN CASE MATERIAL IS RELEASED OR SPILLED

Wear self-contained breathing apparatus.

WASTE DISPOSAL METHOD

\section{SECTION VIII SPECIAL PROTECTION INFORMATION}

VENTILATION REQUIREMENTS LOCAL EXHAUST

MECHANICAL (GENERAL)

SPECIAL

TROTECTIVE EQUIPMENT (SPECIFY TYPES) EYE

Goggles

GLOVES

Rulhher

OTHER PROTECTIVE EOUIPMENT

With organic vapor resistant canister

SECTION IX SPECIAL PRECAUTIONS

PRECAUTIONS TO BE TAKEN IN HANDLING AND STORAGE

OTHER FR[CAUITIOHS 


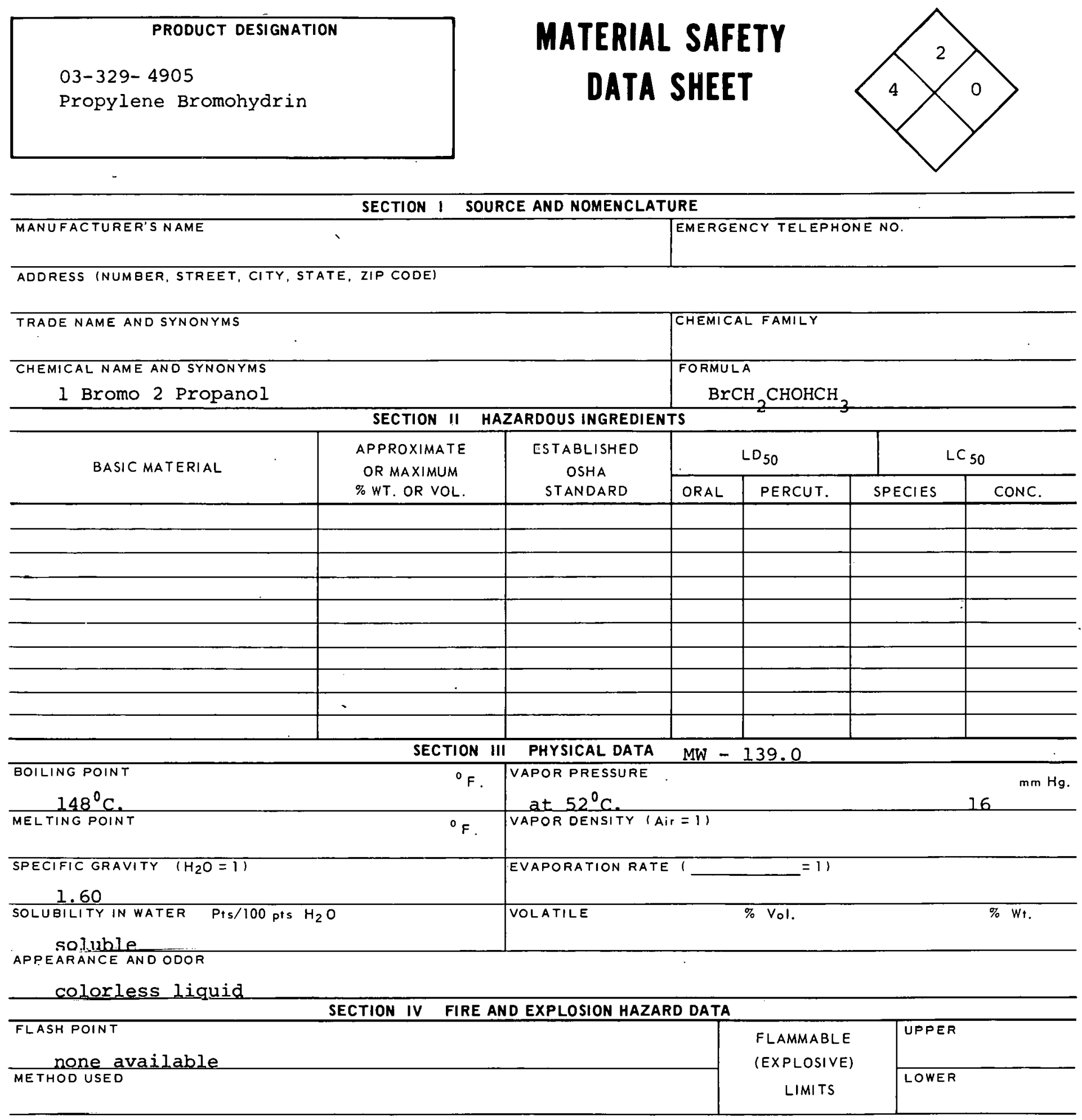

EXTINGUISHING MEDIA

al renhol foam, carbon dioxide, dry ehemical.

SPECIAL FIRE FIGHTING PROCEDURES

UNUSUAL FIRE AND EXPLOSION HAZARDS 
PRODUCT DESIGNATION

Propylene Bromohydrin

SECTION $V$ HEALTH HAZARD DATA

TOXIC LEVEL

None available

PRINCIPLE ROUTES OF ABSORBTION

Inhalation, ingestion, skin

CARCINOGENIC

SKIN AND EYE

IRRITATION

severe

REVELANT SYMPTOMS OF EXPOSURE EYe and nasal irritation, nausea, vomiting, giddiness,

dizziness, incoordination, visual disturbances, coma

EFFECTS OF CHRONIC EXPOSURE

EMERGENCY AND FIRST AID PROCEOURES Wash skin \& eyes; if swallowed - give emetic, get medical

aid; inhalation - rest, give oxygen as needed.

\section{SECTION VI REACTIVITY DATA}

CONDITIONS CONTRIBUTING TO INSTABILITY

CONDITIONS CONTRIBUTING TO HAZARDOUS POLYMERIZATION

INCOMPATABILITY (MAT́ERIALS TO AVOID)

HAZARDOUS DECOMPOSITION PRODUCTS

SECTION VII SPILL OR LEAK PROCEDURES

STEPS TO BE TAKEN IN CASE MATERIAL IS RELEASED OR SPILLED

WASTE DISPOSAL METHOD

SECTION VIII SPECIAL PROTECTION INFORMATION

\begin{tabular}{l|l}
\hline VENTILATION REQUIREMENTS LOCAL EXHAUST & $\begin{array}{c}\text { PROTECTIVE EQUIPMENT (SPECIFY TYPESIEYE } \\
\text { Goggles }\end{array}$ \\
\hline MECHANICAL IGENERAL) & $\begin{array}{c}\text { GLOVES } \\
\text { Ruhber. }\end{array}$ \\
3PECIAL & $\begin{array}{c}\text { RE3PIRAqOR } \\
\text { With proper filter }\end{array}$ \\
\hline OTHER PROTECTIVE EQUIPMENT &
\end{tabular}

OTMER PROTECTIVE EQUIPMENT

\section{SECTION IX SPECIAL PRECAUTIONS}

PRECAUTIONS TO BE TAKEN IN RANDLING AND STORAGE

OTHER HKECAUTIIUNS

SIGNATURE

DATE

$329-4905 B$ 
CAS : 000078875

\begin{tabular}{|l|}
\hline PRODUCT DESIGNATION \\
03-329-51C5 \\
Propylene Chloride \\
\end{tabular}

$\operatorname{TX} 96250$

MATERIAL SAFETY

DATA SHEET

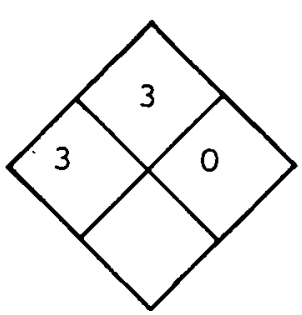

\section{SECTION I SOURCE AND NOMENCLATURE}

\section{MANUFACTURER'S NAME \\ TRADE NAME AND SYNONYMS \\ Propylene dichloride \\ CHEMICAL NAME AND SYNONYMS \\ 1,2 dichloropropane}

ADDRESS (NUMBER, STREET, CITY, STATE, ZIP CODE)

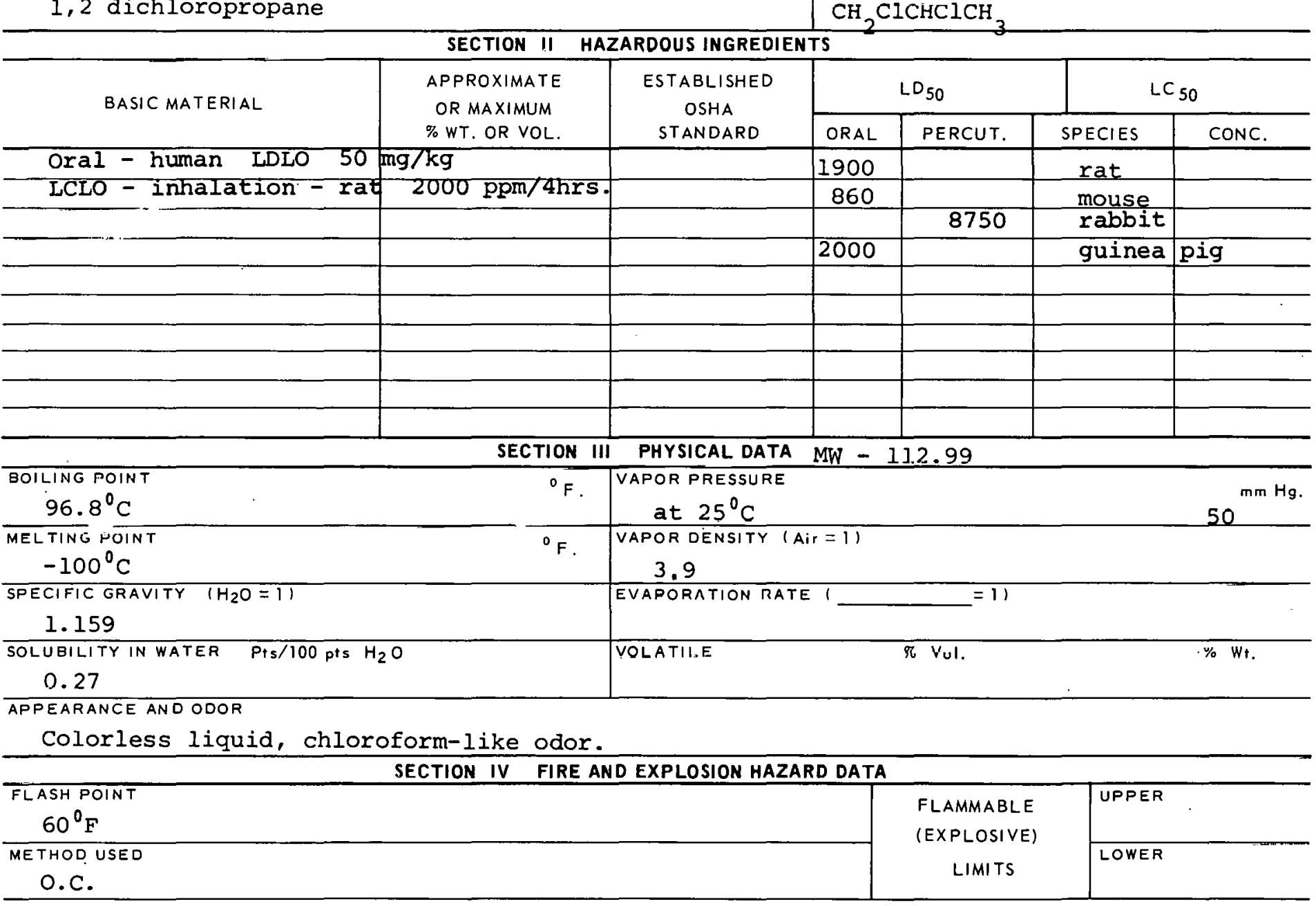

EXTINGUISHING MEDIA

Foam, carbon dioxide, dry chemical

SPECIAL FIRE FIGHTING PROCEDURES

Water may be ineffective.

EMERGENCY TELEPHONE NO.

FORMULA

$\mathrm{CH}_{2} \mathrm{ClCHClCH}_{3}$

\begin{tabular}{l|l} 
& CHEMICAL FAMILY \\
\hline SECTION II HAZARDOUS INGREDIENTS & $\begin{array}{l}\text { FORMULA } \\
\mathrm{CH}_{2} \mathrm{ClCHClCH}_{3}\end{array}$
\end{tabular}

\begin{tabular}{l|l} 
SEMERGENCY TELEPHONE NO. & ERTON \\
\hline
\end{tabular}


Propylene Chloride

\section{SECTION $V$ HEALTH HAZARD DATA}

TOXIC LEVEL

TLV $75 \mathrm{PPM}, 350 \mathrm{mg} / \mathrm{C} . \mathrm{M}$.

CARCINOGENIC

PRINCIPLE ROUTES OF ABSORBTION SKIN AND EYE

Inhalation, ingestion IRRITATION moderate

REVELANT SYMPTOMS OF EXPOSURE EYe irritation, narcosis, sleepiness, headache, vomiting.

EFFECTS OF CHRONIC EXPOSURE

Dermatitis, liver and kidney damage.

EMERGENCY AND FIRST AID PROCEDURES Inhalation - rest; if swallowed - give emetic, get

medical aid; wash from skin \& eyes.

\section{SECTION VI REACTIVITY DATA}

CONDITIONS CONTRIBUTING TO INSTABILITY

COINDITIOHE GOMTRIAITINS, TO HAZARDOUS POLYMERIZATION

INCOMPATABILITY (MATERIALS TO AVOID)

HAZARDOUS DECOMPOSITION PROUUCTS

SECTION VII SPILL OR LEAK PROCEDURES

STEPS TO BE TAKEN IN CASE MATERIAL IS RELEASED OR SPILLED

Wear self-contained breathing apparatus.

WASTE DISPOSAL METHOD

SECTION VIII SPECIAL PROTECTION INFORMATION

\begin{tabular}{l|l}
\hline VENTILATION REQUIREMENTS LOCAL EXHAUST & $\begin{array}{l}\text { PROTECTIVE EQUIPMENT (SPECIFY TYPESI EYE } \\
\text { GOGgles }\end{array}$ \\
\hline MECHANICAL (GENERAL) & $\begin{array}{l}\text { GLOVES } \\
\text { Rubber }\end{array}$ \\
SPECIAL & $\begin{array}{l}\text { RESPIPATOR } \\
\text { With organic vapor resistant canister }\end{array}$ \\
\hline
\end{tabular}

OTHER PROTECTIVE EQUIPMENT

SECTION IX SPECIAL PRECAUTIONS

PRECAUTIONS TO BE TAKEN IN HANDLING AND STORAGE

OTHER PREECAUTION'S

SIGNATURE

ONTE

$329-5105 B$ 
$C A-000127004$

\section{PRODUCT DESIGNATION}

03-329-5205

Propylene Chlorohydrin
MATERIAL SAFETY DATA SHEET

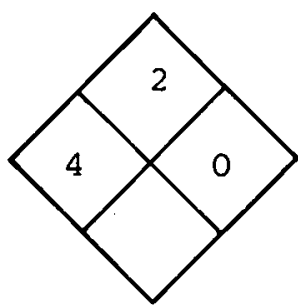

SECTION I SOURCE AND NOMENCLATURE

\section{MANUFACTURER'S NAME \\ TRADE NAME AND SYNONYMS \\ CHEMICAL NAME AND SYNONYMS \\ 1 Chloro 2 Propanol}

ADDRESS (NUMBER, STREET, CITY, STATE, ZIP CODE)
EMERGENCY TELEPHONE NO.
SECTION II HAZARDOUS INGREDIENTS

APPROXIMATE ESTABLISHED

OR MAXIMUM

$\%$ WT. OR VOL.
OSHA

STANDARD

\section{CHEMICAL FAMILY \\ FORMULA \\ $\mathrm{ClCH}_{2} \mathrm{CHOHCH}_{3}$}

\begin{tabular}{|l|c|c|c|c}
\multicolumn{3}{|c|}{ LD $_{50} \mathrm{mg} / \mathrm{kg}$} & \multicolumn{2}{c}{ LC $_{50}$} \\
\hline ORAL & PERCUT. & SPECIES & CONC. \\
\hline & 480 & rabbit & \\
\hline & & & \\
\hline & & & \\
\hline & & & & \\
\hline & & & \\
\hline & & & & \\
\hline & & & \\
\hline
\end{tabular}

PHYSICAL DATA

\begin{tabular}{l|l}
${ }^{\circ} \mathrm{F}$ & VAPOR PRESSURE
\end{tabular}

$\mathrm{mm} \mathrm{Hg}$.

$127^{\circ} \mathrm{C}$.

MELTING POINT

${ }^{\circ} \mathrm{F}$.

VAPOR DENSITY (Air $=1$ )

3.26

SPECIFIC GRAVITY $\left(\mathrm{H}_{2} \mathrm{O}=1\right)$

$$
1.10
$$

SOLUBILITY IN WATER Pis/100 pts $\mathrm{H}_{2} \mathrm{O}$

infinitely soluble

APPEARANCE AND ODOR

Colorless 1iquid, mild nonresidual odor.

EVAPORATION RATE ( $=11$

VOLATILE

$\% \cdot V_{n} l$

Wt:

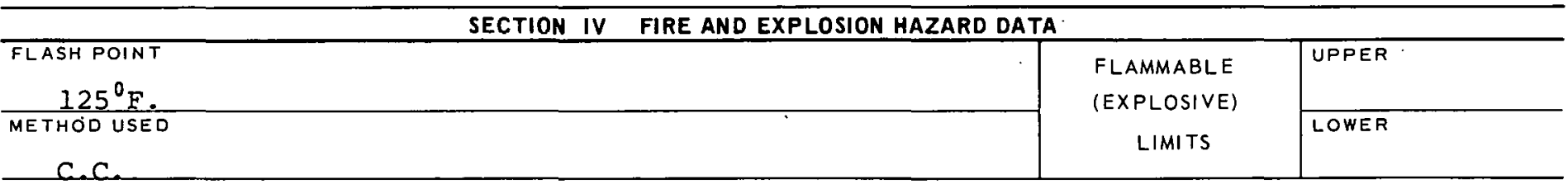

EXTINGUISHING MEDIA

Alcohol foam, carbon dioxide, dry chemical.

SPECIAL FIRE FIGHTING PROCEDURES

UNUSUAL FIRE AND EXPLOSION HAZARDS 
Propylene Chlorohydrin SECTION $V$ HEALTH HAZARD DATA

TOXIC LEVEL

None available

CARCINOGENIC

PRINCIPLE ROUTES OF ABSORETION

SKIN AND EYE

Inhalation, ingestion, skin

IRRITATION Severe

\section{REVELANT SYMPTOMS OF EXPOSURE \\ Eye and nasal irritation, nausea, vomiting, giddiness,}

dizziness, incoordination, visual disturbances, coma.

EFFECTS OF CHRONIC EXPOSURE

$--$

EMERGENCY AND FIRST AIO PROCEDURES Wash skin \& eyes; if swallowed - give emetic, get medical

aid; inhalation - rest, give oxygen as needed.

\begin{tabular}{l}
\hline SECTION VI REACTIVITY DATA \\
\hline CONDITIONS CONTRIBUTING TO INSTABILITY \\
\hline SONDITIONS CONTRIBUTING TO HAZARDOUS POLYMERIZATION \\
\hline INCOMPATABILITY (MATERIALS TO AVOIO) \\
HAZARDOUS DECOMPOSITION FRODUCTS \\
\hline STEPS TO BE TAKEN IN CASE MATERIAL IS RELEASED OR SPILLEO
\end{tabular}

WASTE DISPOSAL METHOD

\section{SECTION VIII SPECIAL PROTECTION INFORMATION}

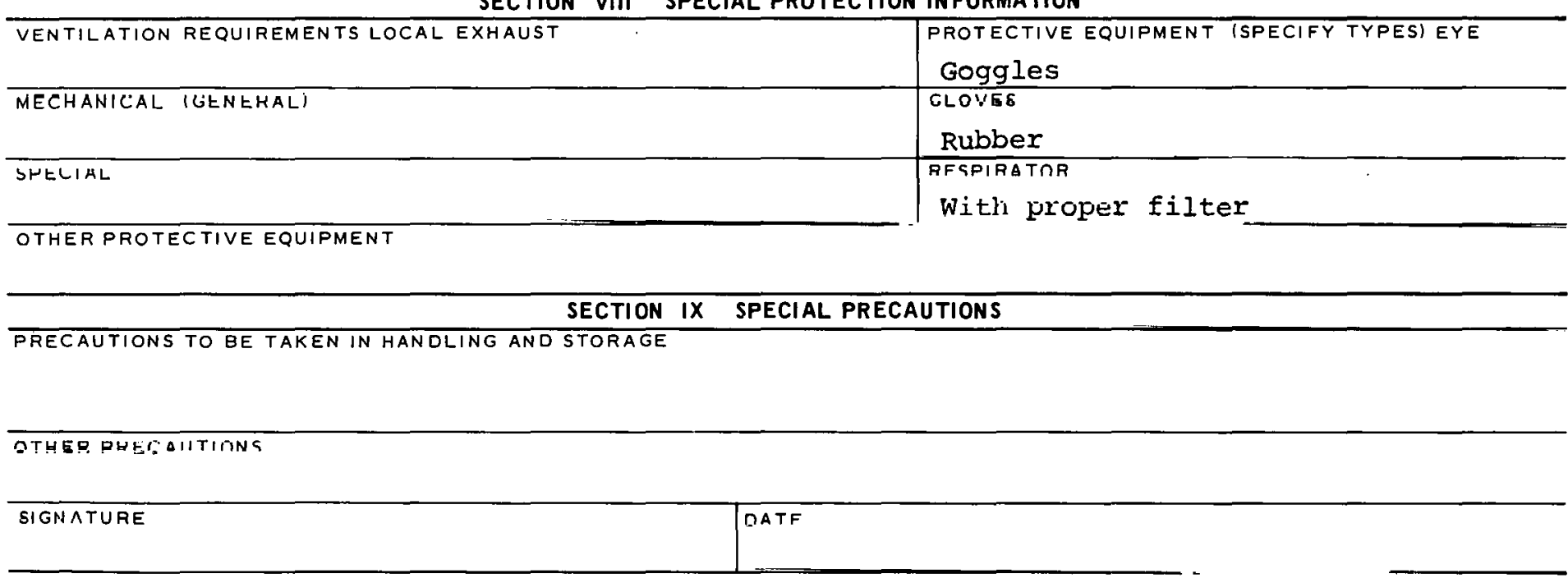


CAS : 000057556

TY20000

\section{PRODUCT DESIGNATION}

$03-329-5595$

03-329-5605

Propylene Glycol

MATERIAL SAFETY

DATA SHEET

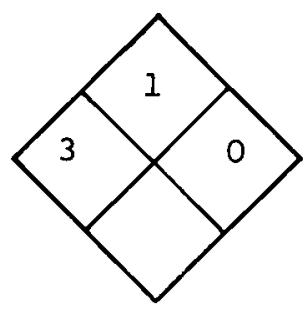

\section{SECTION I SOURCE AND NOMENCLATURE}

MANUFACTURER'S NAME

EMERGENCY TELEPHONE NO.

ADDRESS (NUMBER, STREET, CITY, STATE, ZIP CODE)

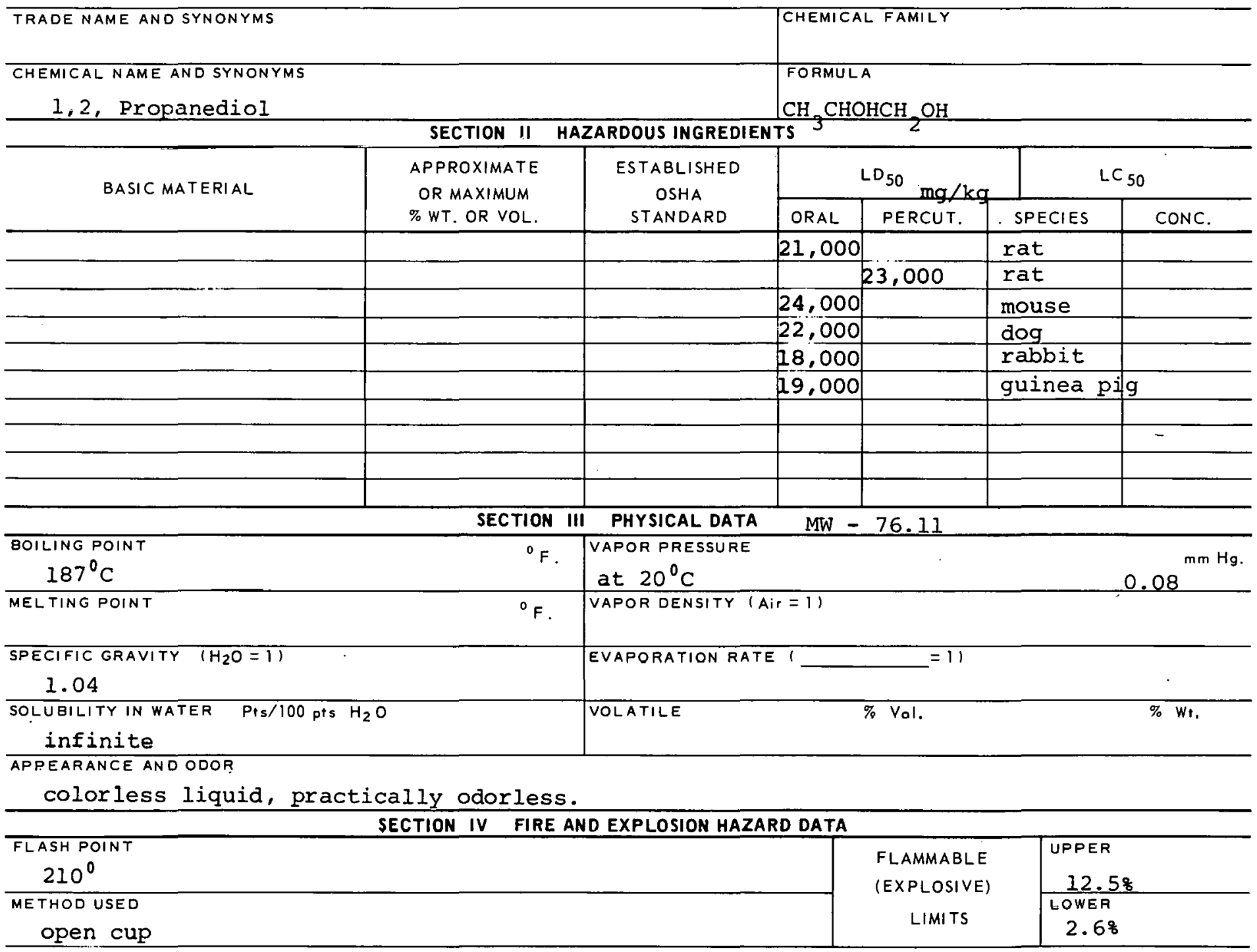

EXTINGUISHING MEDIA

Alcohol foam, carbon dioxide, dry chemical.

SPECIAL FIRE FIGHTING PROCEDURES

UNUSUAL FIRE ANDEXPLOSION HAZARDS 
TOXIC LEVEL

None available

PRINCIPLE ROUTES OF ABSORBTION

Inhalation, ingestion

CARCINOGENIC

SKIN AND EYE

IRRITATION Slight

REVELANT SYMPTOMS OF EXPOSURE Coldness, spastic contraction of muscles, loss of reflexes, coma, death; pulmonary edema, eye irritation.

EFFECTS OF CHRONIC EXPOSURE

Kidney damage, rapid weight loss, weakened reflexes.

EMERGENCY AND FIRST AID PROCEDURES If swallowed - give emetic, get medical aid;

inhalation - rest; wash from eyes.

\section{SECTION VI REACTIVITY DATA}

CONDITIONS CONTRIBUTING TO INSTABILITY

CINNITIONS CONTRIBUTING TO HAZARDOUS POLYMERIZATION

INCOMPATABILITY (MATERIALS TO AVOID)

HAZARDOUS DECOMPOSITION PRODUCTS

SECTION VII SPILL OR LEAK PROCEDURES

STEPS TO BE TAKEN IN CASE MATERIAL IS RELEASED OR SPILLED

\section{WASTE DISPOSAL METHOD}

\section{SECTION VIII SPECIAL PROTECTION INFORMATION}

VENTILATION REQUIREMENTS LOCAL EXHAUST
MECHANICAL (GENERAL)
SPFI:IAI.

PROTECTIVE EQUIPMENT (SPECIFY TYPES) EYE

Guggles

GLOVES

ПEEחIחATQA

OTHER PROTF,CTIVE EQUIPME'NT

SECTION IX SPECIAL PRECAUTIONS

PRECAUTIONS TO BE TAKEN IN HANDLING AND STORAGE

OTHER PRECAUTIONS

MGNATURE

DATC

$329-5605 B$ 


\begin{tabular}{|l} 
PRODUCT DESIGNATION \\
03-329-5805 \\
Propylene Oxide
\end{tabular}

\section{MATERIAL SAFETY DATA SHEET}

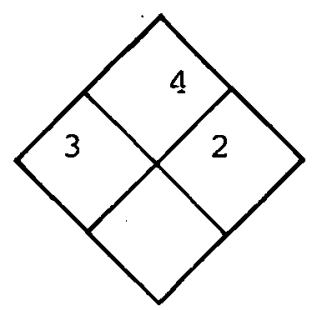

SECTION I SOURCE AND NOMENCLATURE

MANUFACTURER'S NAME
AODRESS INUMBER, STREET, CITY
TRADE NAME AND SYNONYMS
1,2 -EPOXYPYOPane
CHEMICAL NAME AND SYNONYMS
PYOPYlenE OXIde

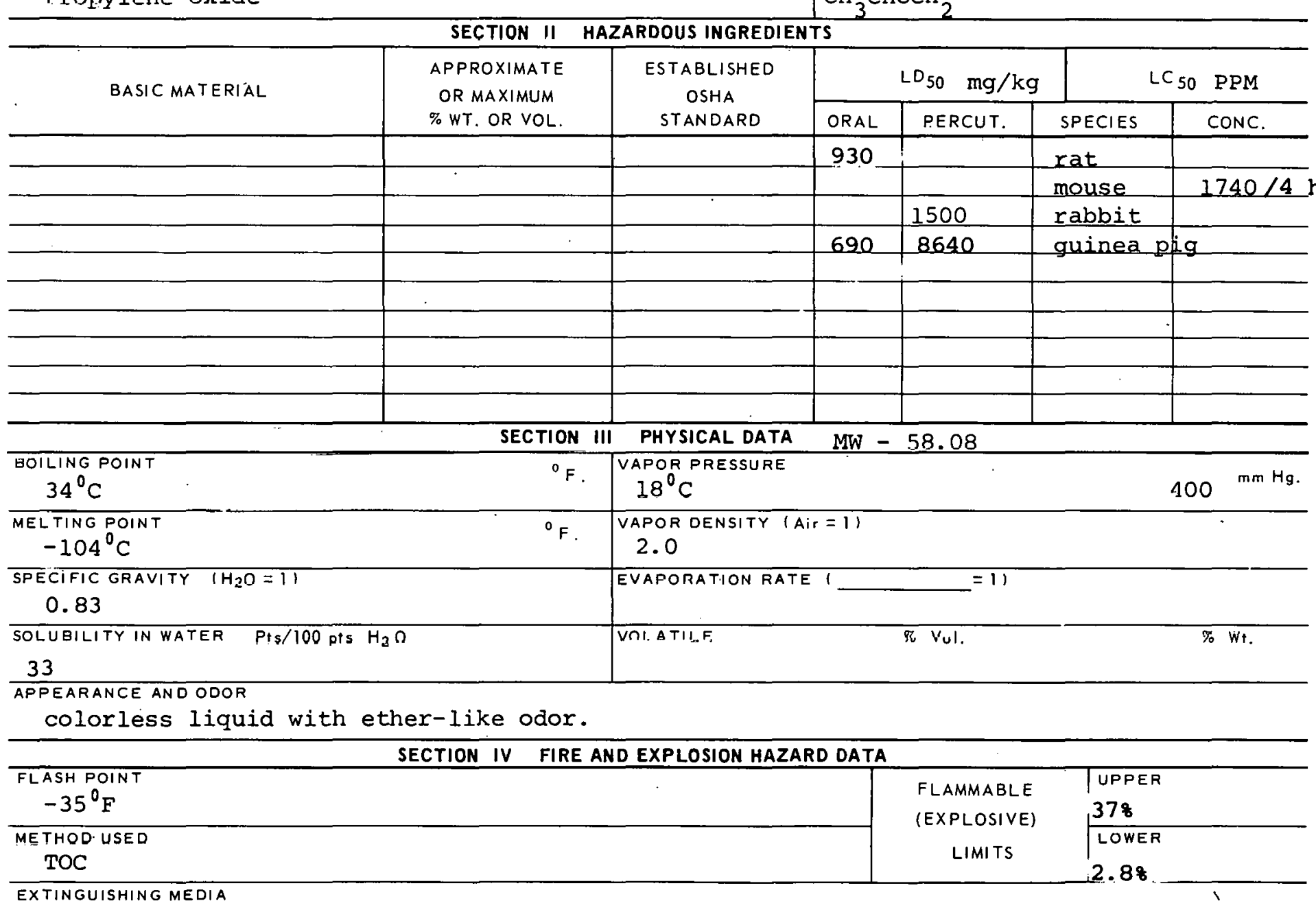

EXTINGUISHING MEDIA

Carbon dioxide, dry chemical, alcohol foam -

SPECIAL FIRE FIGHTING PROCEDURES

Wear self-contained breathing apparatus.

UNUSUAL FIRE AND EXPLOSION HAZARDS

May react vigorously with oxidizing materials. 
TOXIC LEVEL

TLV - 100 PPM, $240 \mathrm{mg} / \mathrm{C} . \mathrm{M}$.

CARCINOGENIC neOplastic

PRINCIPLE ROUTES OF ABSORBTION

Ingestion, inhalation SKIN AND EYE

IRRITATION severe

REVELANT SYMPTOMS OF EXPOSURE Irritation of eyes, upper respiratory tract and lungs; skin

irritation and corneal burns; periodic vomiting.

EFFECTS OF CHRONIC EXPOSURE

Lung damage.

EMERGENCY AND FIRST AID PROCEDURES If swallowed - get medical aid; inhalation - artificial

respiration if needed; wash from skin \& eyes.

\section{SECTION VI REACTIVITY DATA}

CONDITIONS CONTRIBUTING TO INSTABILITY

T.NNRITIONS CONTRIBUTING TO HAZARDOUS POLYMERIZATION

May polymerize with heat evolution in

contact with catalyst.

INCOMPATAB̈ILITY IMATERIALS TO AVUIUI Anhydrous chlorides of $\mathrm{Fe}, \mathrm{Sn}, \mathrm{Al}$; Peroxides of Fe

and $A_{1}$ alkali metal hydroxides.

HALAKUUUS UELUMHUSTIOUNAOEUCT3

SECTION VII SPILL OR LEAK PROCEDURES

STEPS TO BE TAKEN IN CASE MATERIAL IS RELEASED OR SPILLED

Absorb on paper towels; evaporate in hood.

WASTE DISPOSAL METHOD

SECTION VIII SPECIAL PROTECTION INFORMATION

\begin{tabular}{l|l}
\hline \multicolumn{1}{|c|}{ SECTION VIII SPECIALPROTECTION INFORMATION } \\
\hline MECHANILATION REQUIREMENTS LOCAL EXHAUST & $\begin{array}{c}\text { PROTECTIVE EQUIPMENT (SPECIFY TYPES) EYE } \\
\text { GOggleS }\end{array}$ \\
\hline SPES!A! & GLOVES \\
& Rubber \\
\hline
\end{tabular}

OTHER PROTECTIVE EOUIPMENT

Se] [-contained

SECTION IX SPECIAL PRECAUTIONS PRECAUTIONS TO BE TAKEN IN HANDLING AND STORAGE
Detached. Outside storage is preferred. Inside storage in standard flammable liquids
storage cabinet. Isolate from combustible materials.

OTHER PRECAUTIONS

SIGNATURE

DATE

$329-5805 B$ 


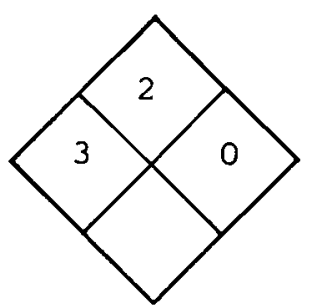

\section{SECTION I SOURCE AND NOMENCLATURE}

MANU FACTURER'S NAME
ADDRESS INUMEER, STREET, CI
TRADE NAME AND SYNONYMS
CHEMICAL NAME ANO SYNONYMS
2-IOdOPYOPane

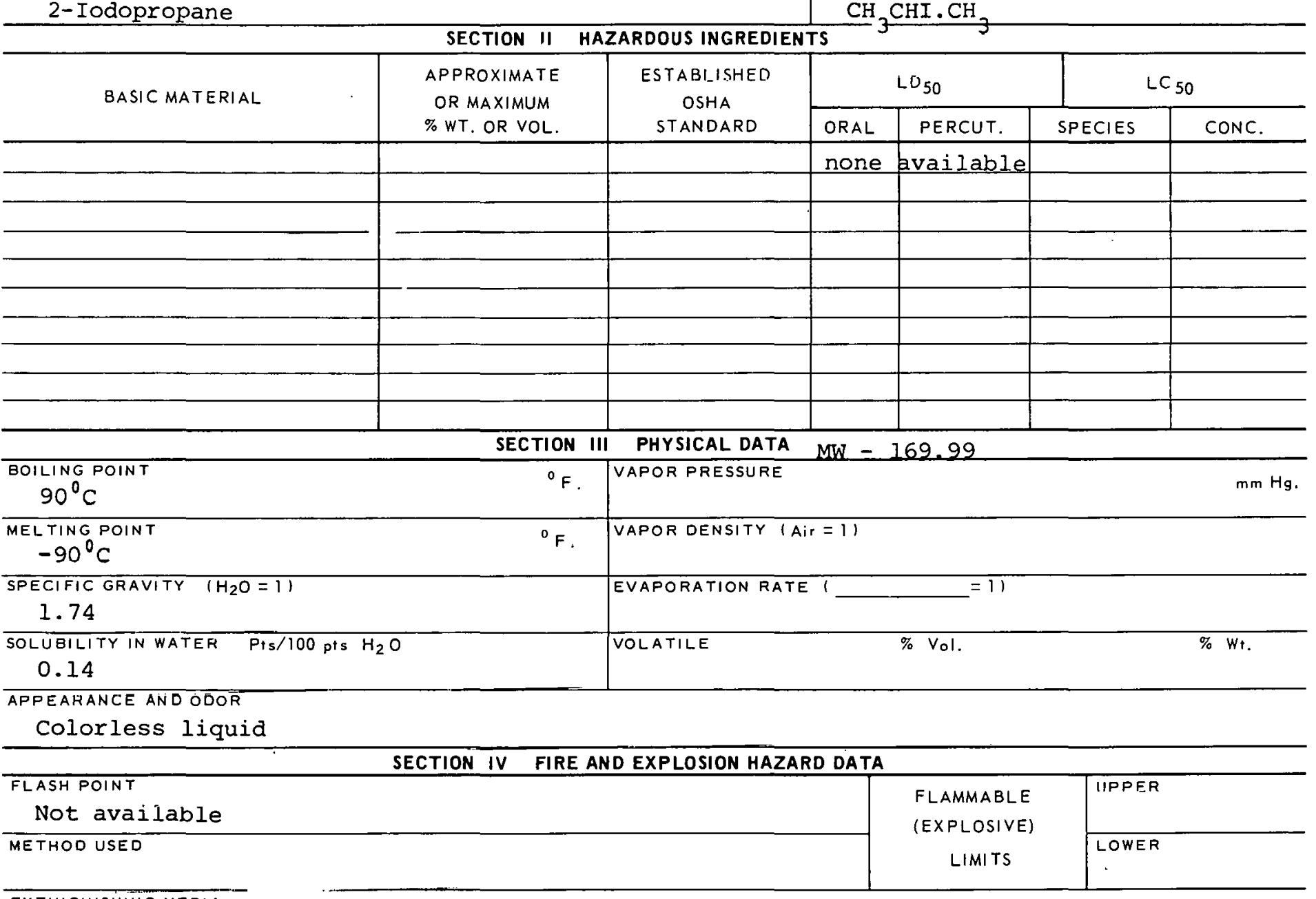

EXTINGUISHING MEDIA

Water, carbon dioxide, dry chemical.

SPECIAL FIRE FIGHTING PROCEDURES

--

UNUSUAL FIRE AND EXPLOSION HAZARDS

When heated to decomposition point emits toxic fumes of iodates. 


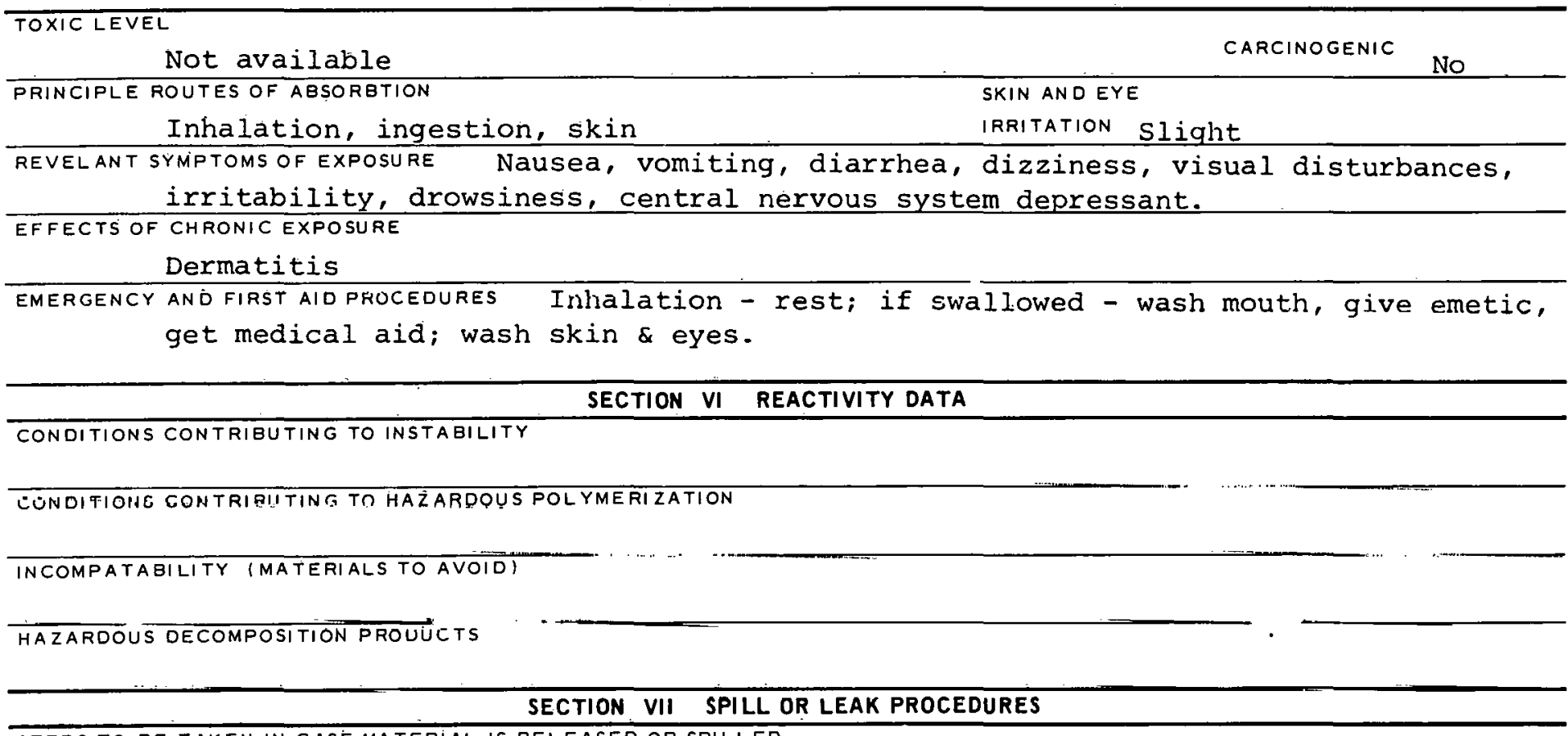

STEPS TO BE TAKEN IN CASE MATERIAL IS RELEASED OR SPILLED

WASTE DISPOSAL METHOD

SECTION VIII SPECIAL PROTECTION INFORMATION

\begin{tabular}{|c|c|c|}
\hline VENTILATION & REQUIREMENTS LOCAL EXHAUST & $\begin{array}{l}\text { PROTECTIVE EQUIPMENT (SPECIFY IYPES) EYE } \\
\text { SafCty gOgglCE }\end{array}$ \\
\hline MECHANICAL & (GENERAL) & $\begin{array}{l}\text { GLOVES } \\
\text { Rubber }\end{array}$ \\
\hline SPECIAL. & & $\begin{array}{l}\text { WFGPIRATRR } \\
\text { With proper filter }\end{array}$ \\
\hline
\end{tabular}

UTHER PROTECTIVE EQUUIPMENT

SECTION IX SPECIAL PRECAUTIONS

PREGAUTIONS TO $8 E$ TAKEN IN HANDLING AND STORAGE

OTHER PRECAUTIONS

SIGNATURE

DATE

329-6305B 
CAS : 000106514

\section{PRODUCT DESIGNATION}

03-341-1906

Quinone
DK26250

\section{MATERIAL SAFETY DATA SHEET}

\section{MANUFACTURER'S NAME \\ TRADE NAME AND SYNONYMS \\ Chinone \\ CHEMICAL NAME AND SYNONYMS \\ P-Benzoquinone}

ADDRESS (NUMBER, STREET, CITY, STATE, ZIP CODE)

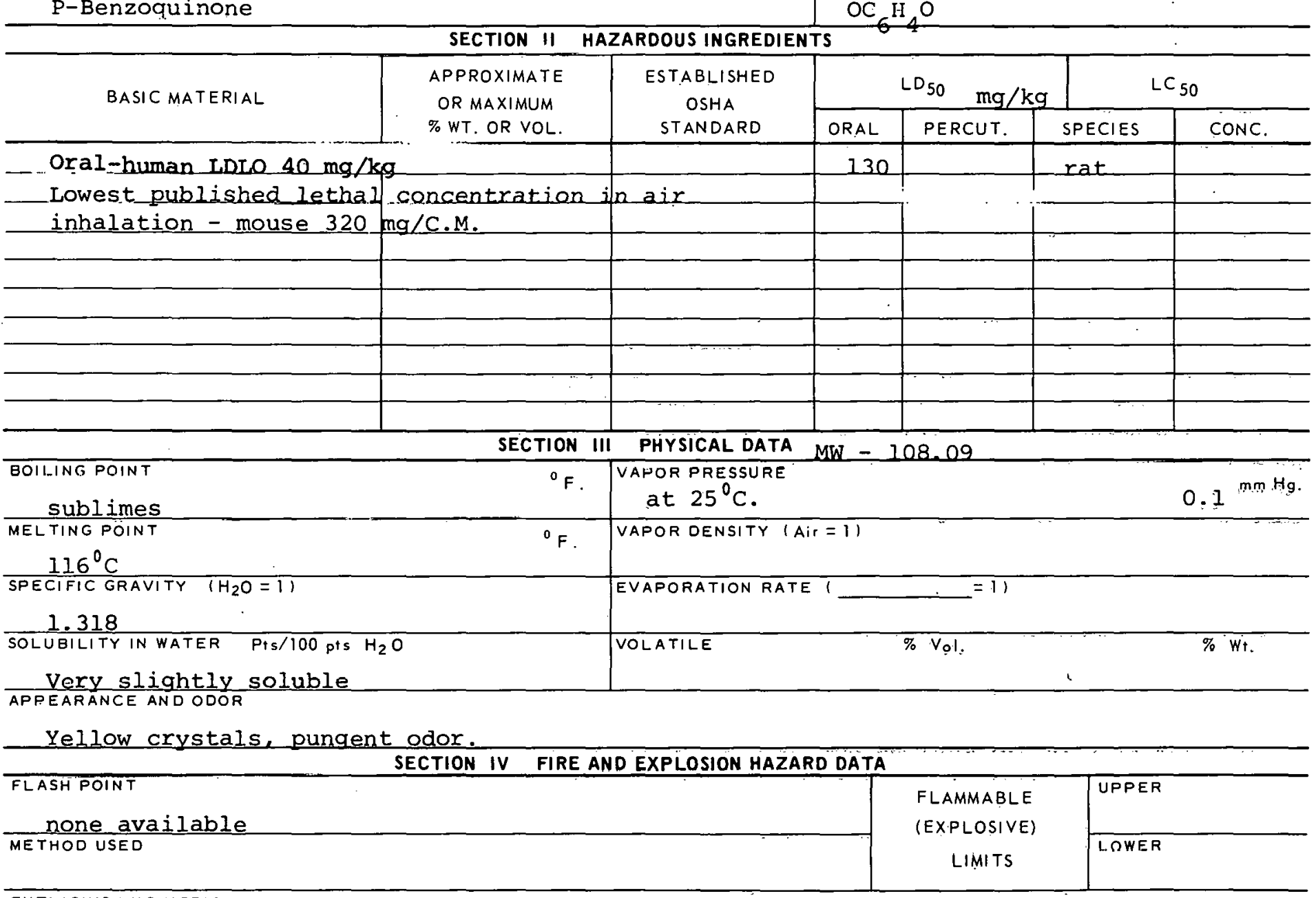

EXTINGUISHING MEDIA

Water, carbon dioxide, dry chemical.

EMERGENCY TELEPHONE NO.

CHEMICAL FAMILY

\section{FORMULA}

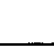

\section{SECTION I SOURCE AND NOMENCLATURE}

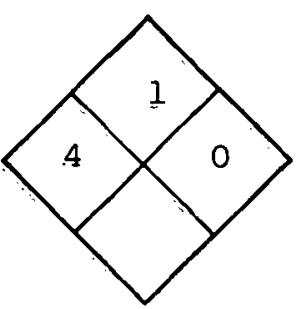




\section{SECTION V HEALTH HAZARD DATA}

TOXIC LEVEL

0.1 PPM, $0.4 \mathrm{mg} / \mathrm{C.M}$.

CARCINOGENIC neoplastic

PRINCIPLE ROUTES OF ABSORBTION

Inhalation, ingestion SKIN ANDEYE

IRRITATION

Severe

REVELANT SYMPTOMS OF EXPOSURE Irritation and damage to skin \& eyes, loss of vision, vomiting, collapse, coma.

EFFECTS OF CHRONIC EXPOSURE

Eye damage, discoloration, severe irritation, swelling.

EMERGENCY AND FIRST AID PROCEOURES If swallowed - give milk, emetic, get medical aid.

inhalation - rest; wash from skin \& eyes.

\section{SECTION VI REACTIVITY DATA}

CONDITIONS CONTRIBUTING TO INSTABILITY

CONDITIONS CONTRIBUTING TO HAZARDOUS POLYMERIZATION

INCOMPATABILITY IMATERIALS TO AVOIOI

HAZARDOUS DECOMPOSI TION PRODUCTS

SECTION VII SPILL OR LEAK PROCEDURES

STEPS TO BE TAKEN IN CASE MATERIAL IS RELEASED OR SPILLED

Collect with brush onto a paper, burn on iron pan in hood.

WASTE DISPOSAL METHOD

SECTION VIII SPECIAL PROTECTION INFORMATION

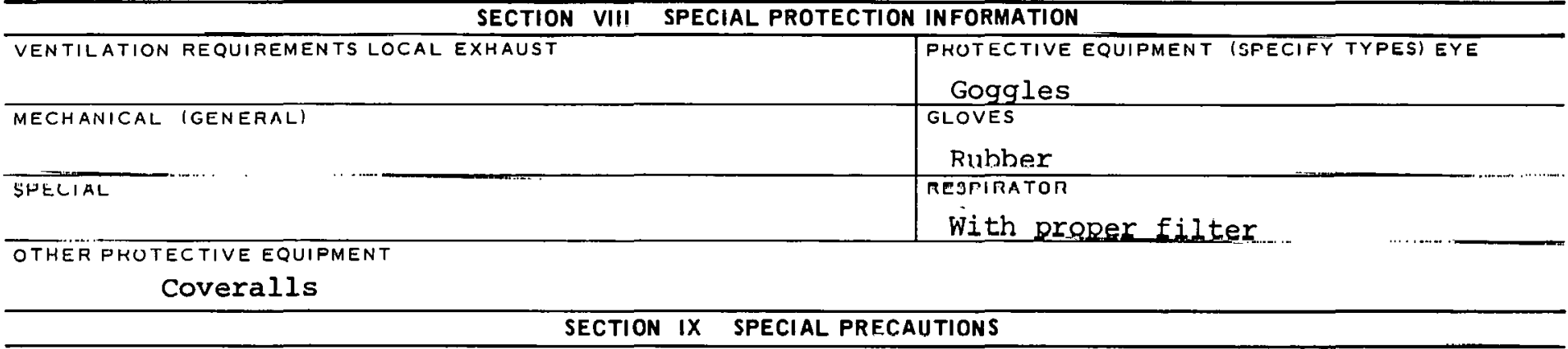

PRECAUTIONS TO BE TAKEN IN HANDLING AND STORAGE

OTHER PRECAUTIONS

SIGNATURE

DATE

34l-1906B 


\section{PRODUCT DESIGNATION}

03-348-0607

Resorcinol

MATERIAL SAFETY

DATA SHEET

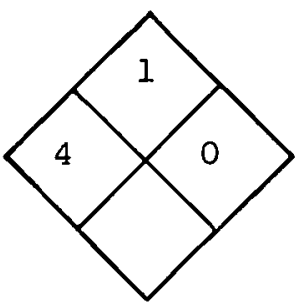

SECTION I SOURCE AND NOMENCLATURE

MANUFACTURER'S NAME

EMERGENCY TELEPHONE NO.

ADDRESS (NUMBER, STREET, CITY, STATE, ZIP CODE)

TRADE NAME AND SYNONYMS

m dihydroxybenzene

CHEMICAL NAME AND SYNONYMS

1,3 Benzene Diol

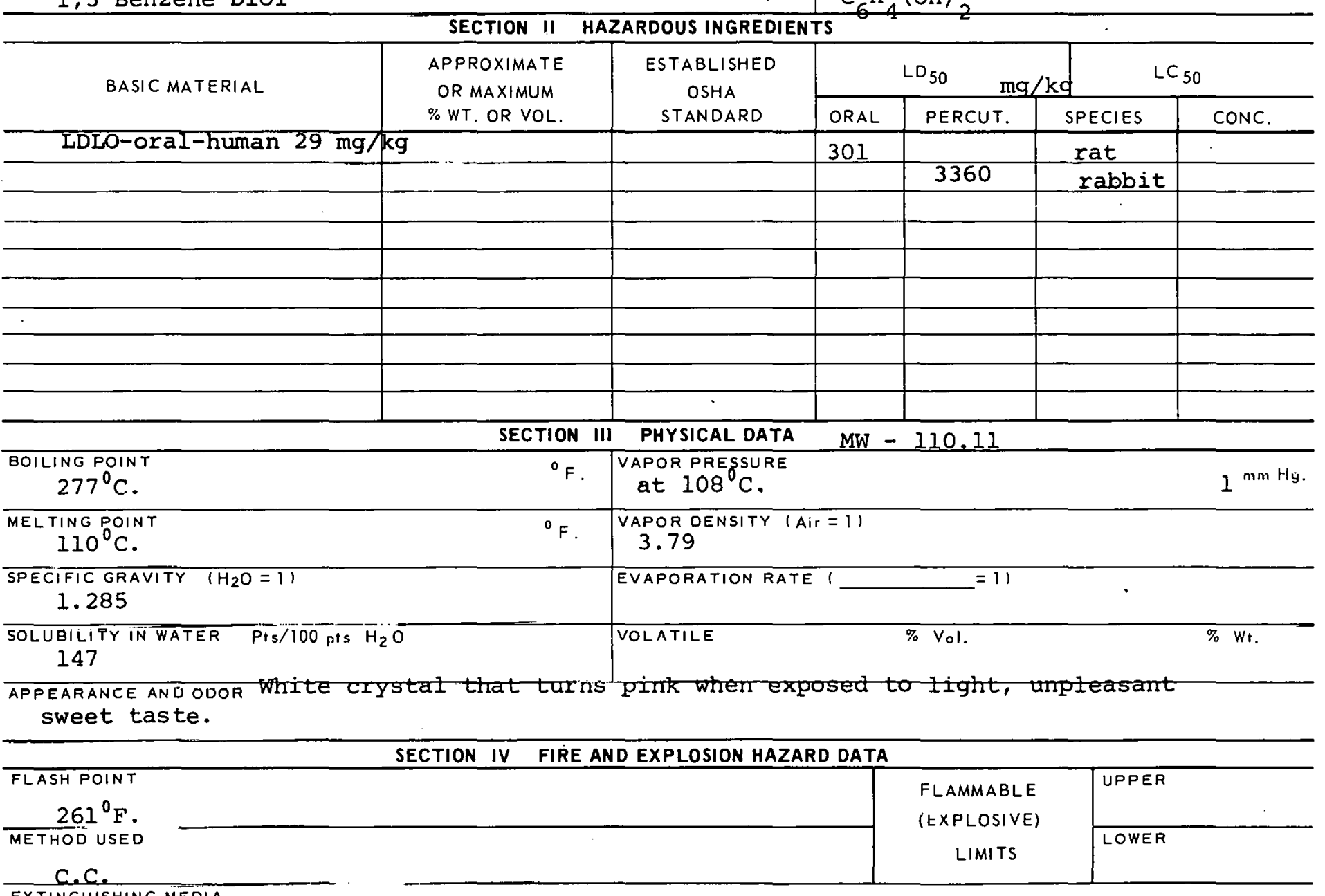

EXTINGUISHING MEDIA

Water, carbon dioxide, dry chemical.

SPECIAL FIRE FIGHTING PROCEDURES

UNU BUAL FIRE AND 
TOXICLEVEL

TLV - $10 \mathrm{PPM}, 45 \mathrm{mg} / \mathrm{C.M}$.

PRINCIPLE ROUTES OF ABSORBTION

Ingestion, skin, inhalation of dust.
CARCINOGENIC neoplastic SKIN AND EYE IRRITATION Moderate

REVELANT SYMPTOMS OF EXPOSURE HYperemia, itching, dermatitis, restlessness, cyanosis, dizziness; tremors, convulsions and death.

EFFECTS OF CHRONIC EXPOSURE

Possible liver, kidney and lung damage.

EMERGENCY AND FIRST AID PROCEDURES Inhalation - rest; if swallowed - induce vomiting, get medical aid; wash eye and skin.

\section{SECTION VI REACTIVITY DATA}

CONDITIONS CONTRIBUTING TO INSTABILITY

CONDITIONS CONTRIBUTING TO HAZARDOUS POLYMERIZATION

INCOMPATABILITY (MATERIALS TO AVOIO)

HAZARDOUS DECOMPOSITION PRODUL'TS

SECTION VII SPILL OR LEAK PROCEDURES

STEPS TO BE TAKEN IN CASE MATERIAL IS RELEASED OR SPILLED

Collect with brush onto a sheet of paper for disposal.

WASTE DISPOSAL METHOD

SECTION VIII SPECIAL PROTECTION INFORMATION

\begin{tabular}{l|l}
\hline VENTILATION REQUIREMENTS LOCAL EXHAUST & PROTECTIVE EQUIPMENT (SPECIFY TYPES) EYE
\end{tabular}

MECHANICAL. (GENERAL)

safecy goggles

GLOVES

Rubber

SPEGIAL

REGPIAATOR

OTHER PROTECTIVE EQUIPMENT

With chemical cartridge filler

SECTION IX SPECIAL PRECAUTIONS

PRECAUTIONS TO BE TAKEN IN HANDLING AND STORAGE

OTHER PRECAUTIUNS

SIÜNATURE

DATE

$348-0607 \mathrm{~B}$ 


\section{PRODUCT DESIGNATION}

03-357-1708

Semicarbazide Hydrochloride

MATERIAL SAFETY DATA SHEET

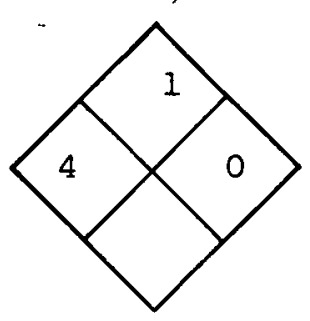

\section{SECTION I SOURCE AND NOMENCLATURE}

MANUFACTURER'S NAME

ADDRESS (NUMBER, STREET, CITY, STATE, ZIP CODE)

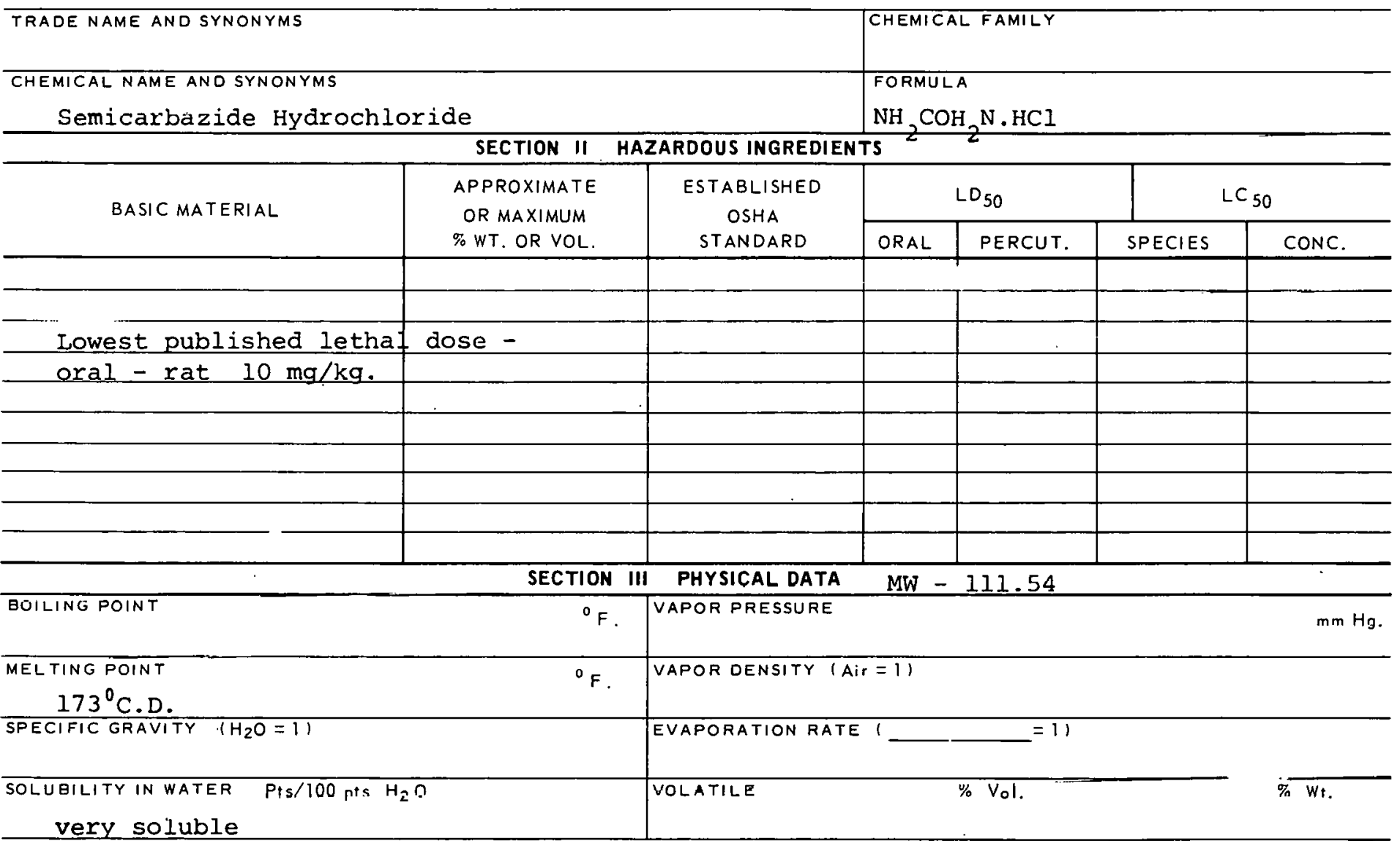
APPEARANCE AND ODOR

Crystals

SECTION IV FIRE AND EXPLOSION HAZARD DATA

\section{FLASH POINT \\ none available}

ME THOD USED

EXTINGUISHING MEDIA

Alcohol foam, carbon dioxide, dry chemical.
EMERGENCY TELEPHONE NO. 


\section{PRODUCT DESIGNATION \\ Semicarbazide Hydrochloride}

SECTION $V$ HEALTH HAZARD DATA

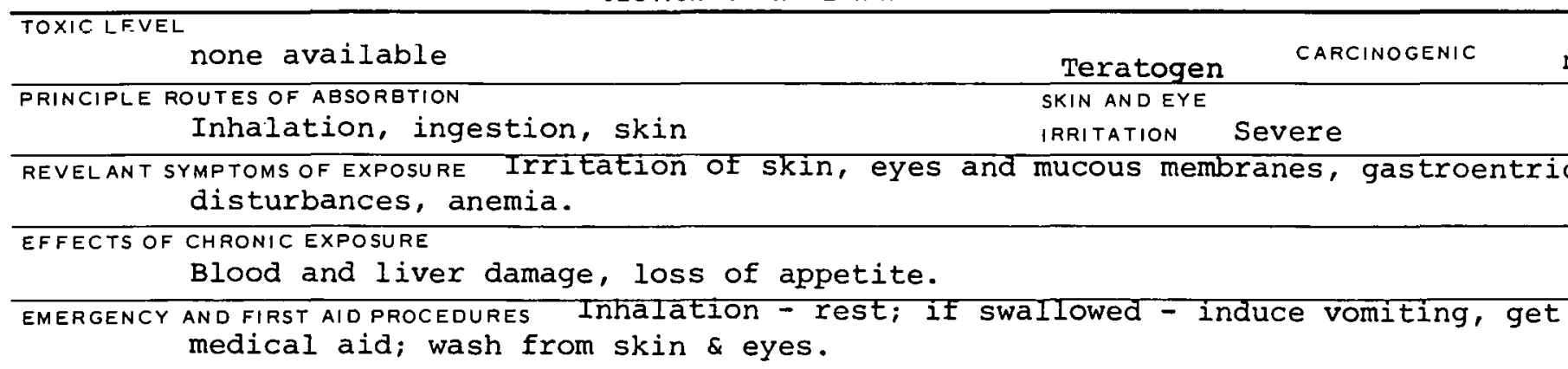

\section{SECTION VI REACTIVITY DATA}

CONDITIONS CONTRIBUTING TO INSTABILITY

TON IITIONS CONTRIBUTING TO HAZARDOUS POLYMERIZATION

INCOMPATABILITY (MATERIALS TO AVOID)

HAZARDOUS DECOMPOSITION PRODUCTS

SECTION VII SPILL OR LEAK PROCEDURES

STEPS TO BE TAKEN IN CASE MATERIAL IS RELEASED OR SPILLED

WASTE DISPOSAI_ METHOO

SECTION VIII SPECIAL PROTECTION INFORMATION

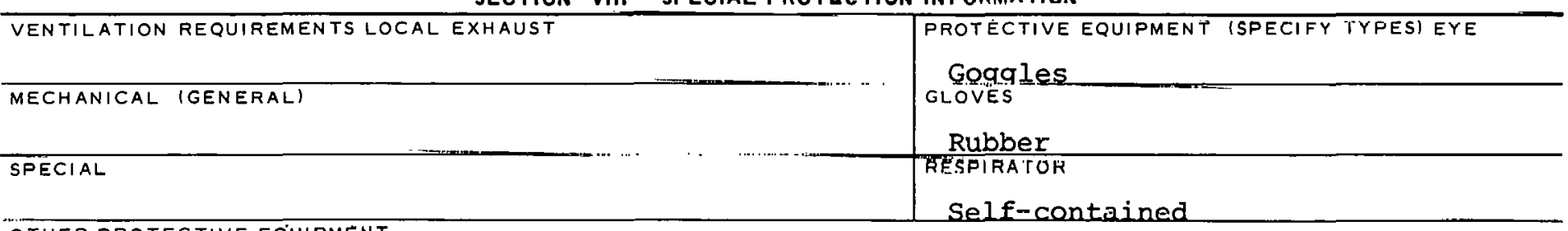

OTHER PROTECTIVE EQUIPMENT

SECTION IX SPECIAL PRECAUTIONS

PRECAUTIONS TO BE TAKEN IN HANDLING AND STORAGE

OTHER PRECAUTIONS

SIGNATURE

DATE

$357-1708 B$ 
CAS : 000060413

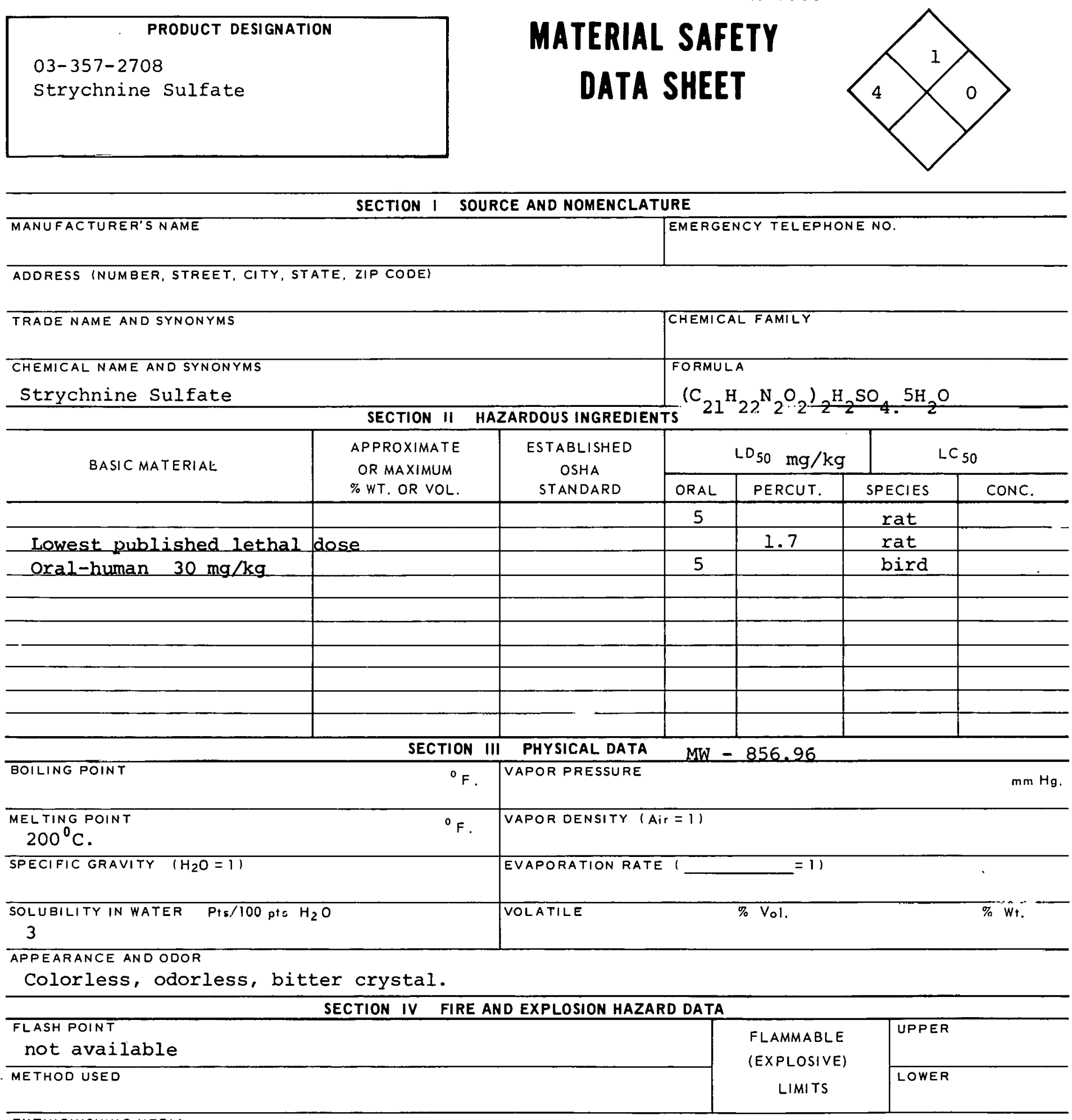

EXTINGUISHING MEDIA

Carbon dioxide, dry chemical.

SPECIAL FIRE FIGHTING PROCEDURES

UNUSUAL FIRE AND EXPLOSION HAZARDS 
TOXIC LEVEL

$0.15 \mathrm{mg} / \mathrm{C} . \mathrm{M}$.

PRINCIPLE ROUTES OF ABSORBTION Inhalation, ingestion
CARCINOGENIC NO

SKIN AND EYE

IRRITATION

slight

REVELANT SYMPTOMS OF EXPOSURE Increase in deep reflexes, stiffening of knees, extension spasms in arms and legs, death by respiratory failure.

EFFECTS OF CHRONIC EXPOSURE

$--$

EMERGENCY AND FIRST AID PROCEOURES If symptoms have begun, avold trying to make vOMIt; keep quiet, artificial respiration as needed.

SECTION VI REACTIVITY DATA

CONDITIONS CONTRIBUTING TO INSTABILITY

CONDITIONS CONTRIBUTING TO HAZARDOUS POLYMERIZATION

INCOMPATABILITY (MATERIALS TO AVOID)

HAZARDOUS DECOMPOSITION PRODUCTS

SECTION VII SPILL OR LEAK PROCEDURES

STEPS TO BE TAKEN IN CASE MATERIAL IS RELEASED OR SPILLED

WASTE DISPOSAL METHOD

SECTION VIII SPECIAL PROTECTION INFORMATION

\begin{tabular}{l|l}
\hline VENTILATION REQUIREMENTS LOCAL EXHAUST & PROTECTIVE EQUIPMENT (SPECIFY TYPES) EYE \\
\hline MECHANICAL (GENERAL) & GLOVES \\
\hline SPECIAL & RESPIRATOR \\
\hline
\end{tabular}

OTHER PROTECTIVE EQUIPMENT

SECTION IX SPECIAL PRECAUTIONS

PRFR AIITINNG TI RF TAKF.N IN HANDLING AND STORAGE

OTHER PRECAUTIONS

\begin{tabular}{l|l}
\hline SIGNATURE & UAIE
\end{tabular} 


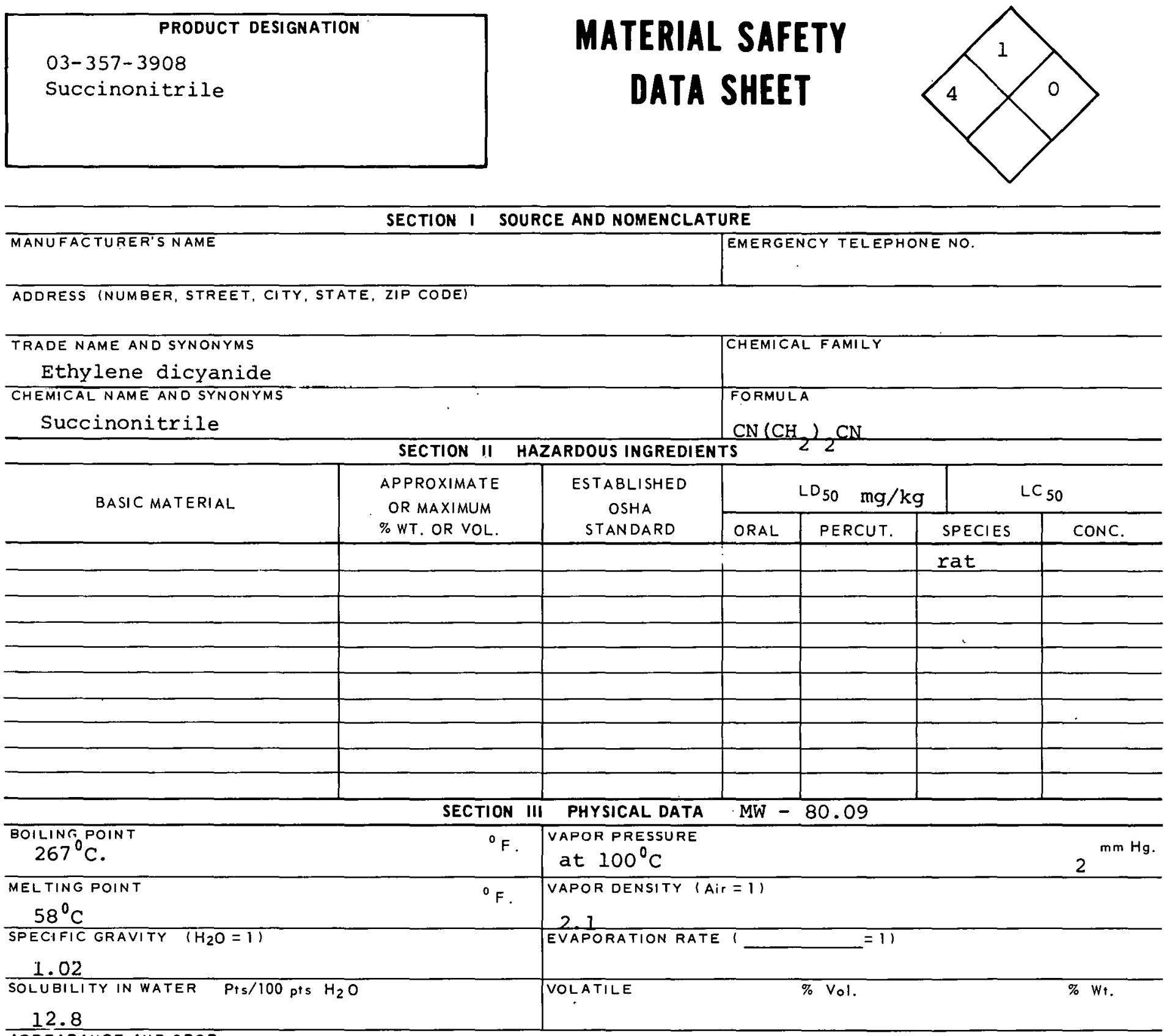

APPE ARANCE AND ODOR

Colorless, odorless, waxy material.

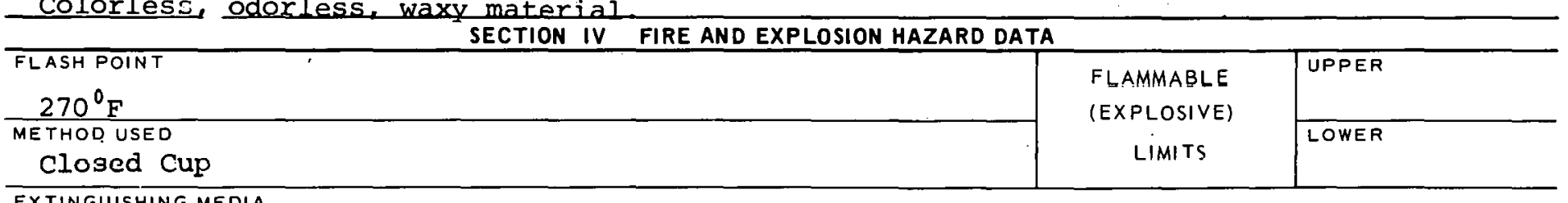

EXTINGUISHING MEDIA

Carbon dioxide, dry chemical - water or foam may cause frothing.

SPECIAL FIRE FIGHTING PROCEDURES

Wear self-contained breathing apparatus.

UNUSUAL FIRE AND EXPLOSION HAZARDS 


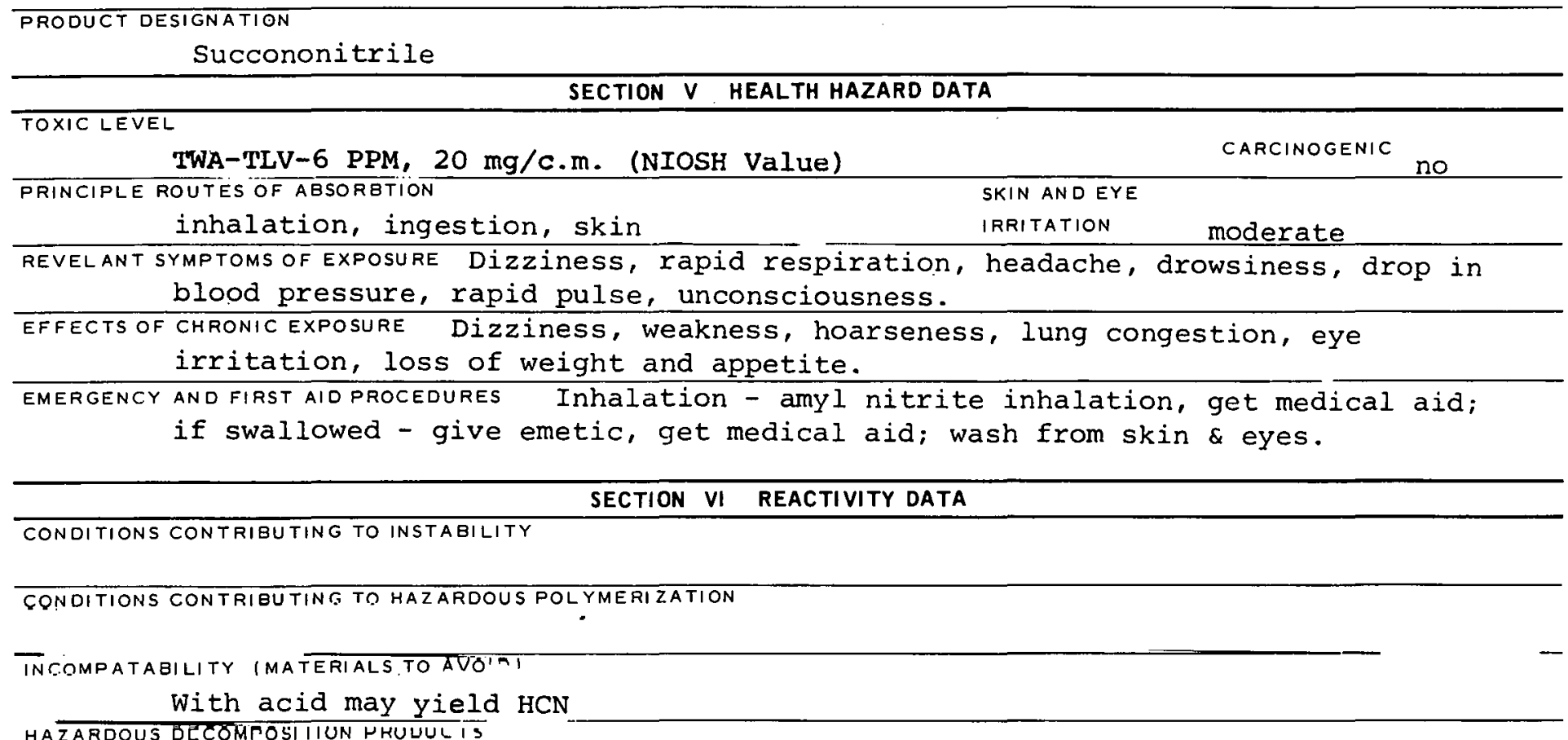

SECTION VII SPILL OR LEAK PROCEDURES

STEPS TO BE TAKEN IN CASE MATERIAL IS RELEASED OR SPILLED

WASTE DISPOSAL METHOD

SECTION VIII SPECIAL PROTECTION INFORMATION

\begin{tabular}{|c|c|}
\hline SECTION VIII & SPECIAL PROTECTION INFORMATION \\
\hline VENTILATION REQUIREMENTS LOCAL EXHAUST & \begin{tabular}{l|l} 
& PROTECTIVE EQUIPMENT (SPECIFY TYPES) EYE \\
& Goggles
\end{tabular} \\
\hline MECHANICAL (GENERAL) & $\begin{array}{l}\text { GI..OVES } \\
\text { Rubber }\end{array}$ \\
\hline SFECIAL & $\begin{array}{l}\text { RESPIRATOR } \\
\text { Self-contained }\end{array}$ \\
\hline
\end{tabular}

OTHER PROTECTIVE EQUIPMENT

SECTION IX SPECIAL PRECAUTIONS

PRECAUTIONS TO BE TAKEN IN HANDLING AND STORAGE

OTHER PRECALITTIONS

SIGNATURE

DATE

357-3908B 
C.AS : 00054.3204

PRODUCT DESIGNATION

03-357-4108

Succinyl Chloride
WH49000

MATERIAL SAFETY

DATA SHEET

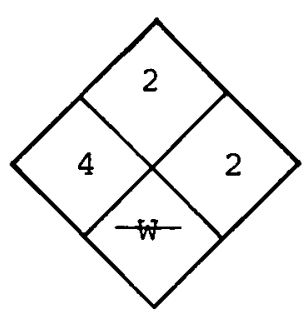

SECTION $\mid$ SOURCE AND NOMENCLATURE

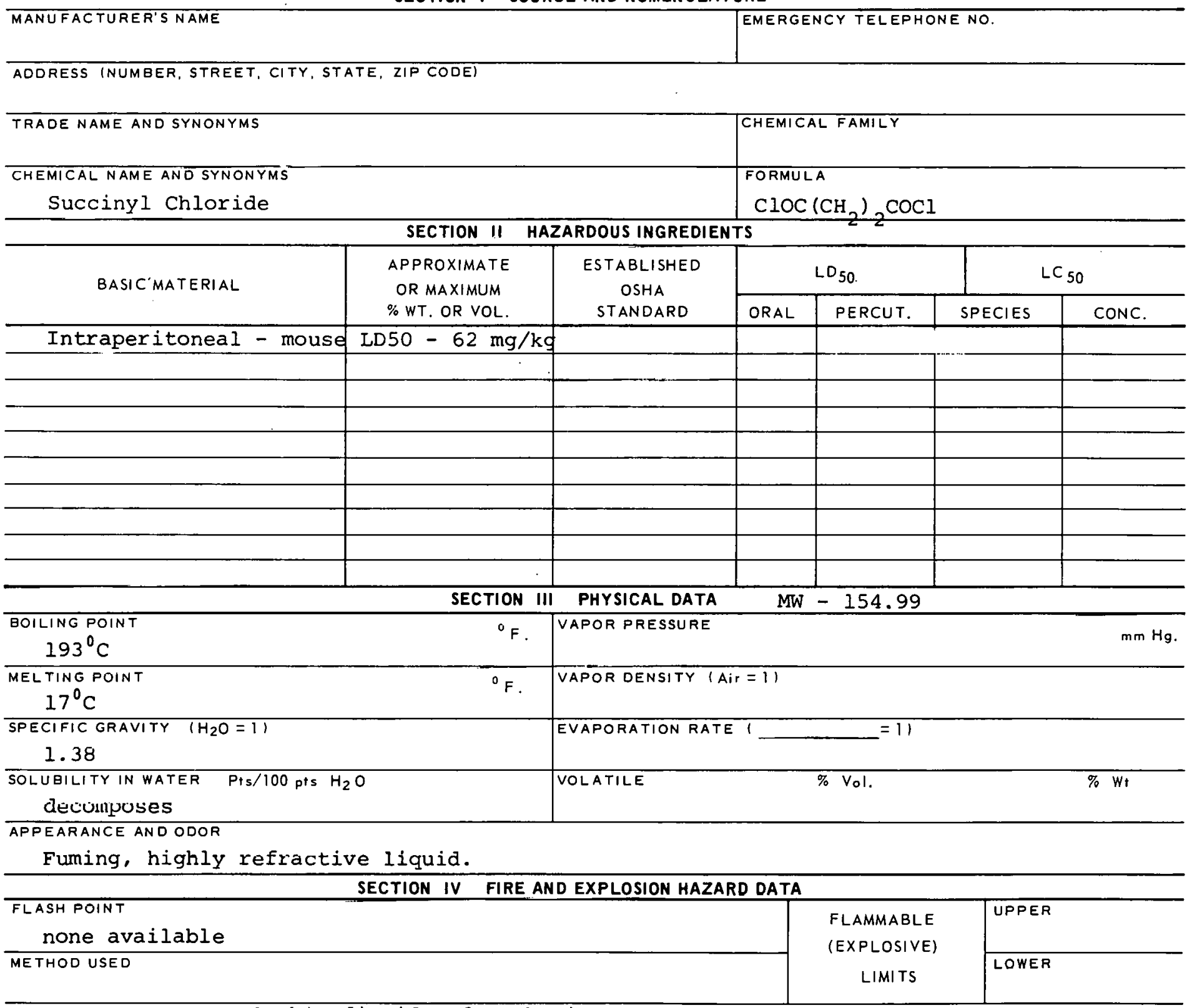

EXtinguishing media Carbón dioxide, dry chemical - do not use foam or water.

SPECIAL FIRE FIGHTING PROCEDURES

Wear full protective clothing.

UNUSUAL FIRE AND EXPLOSION HAZARDS

HCl is produced with water addition. 
Succinyl Chloride

SECTION $V$ HEALTH HAZARD DATA

\section{TOXIC LEVEL}

None available

CARCINOGENIC

PRINCIPLE ROUTES OF ABSORBTION

Inhalation, ingestion

SKIN AND EYE

IRRITATION SeVere

REVELANT SYMPTOMS OF EXPOSURE Cyanosis, headache, coughing, dizziness, weakness, pulmonary edema; on skin contact - severe pain, burns.

EFFECTS OF CHRONIC EXPOSURE Erosion of teeth, jaw necrosis, bronchial irritation and chronic cough.

EMERGENCY AND FIRST AID PROCEDURES Inhalation - artificial respiration as needed; if swallowed drink water, milk of magnesia, get medical aid; wash skin \& eyes.

\section{SECTION VI REACTIVITY DATA}

CONDITIONS CONTRIBUTING TO INSTABILITY

COHDITIDNS CONTRIRIITING TQ HAZARDOUS POLYMERIZATION

INCOMPATABILITY (MATERIALS TO AVOIDI

Ayuid water

HAZAROOUS DECOMPOSITION PRODUCT'S

produces $\mathrm{HCl}$.

SECTION VII SPILL OR LEAK PROCEDURES

STEPS TO BE TAKEN IN CASE MATERIAL IS RELEASED OR SPILLED

WASTE DISPOSAL METHOD

\section{SECTION VIII SPECIAL PROTECTION INFORMATION}

VENTILATION REQUIREMENTS LOCAL EXHAUST

ROTECTIVE EQUIPMENT (SPECIF'Y TYPES) EYE

Gougules

MESHANIC.AI (GENERAL)

GLOVES

SRECIAL

Rululis

RFSPIRATOR

self-cunlained

OTIIER PROTECTIVE EQUIPMENT

SECTION IX SPECIAL PRECAUTIONS

PRECAUTIONS TOBE TAKEN IN HANDLING AND STORAGE

Store in cool, dry well-ventilated plāce Inslde sturaye in rlammable liquias OTHER HHECAUTIONS

storage cabinet. 
CAS-000063741

PRODUCT DESIGNATION

03-357-5009

Sulfanilamide
W084000

MATERIAL SAFETY

DATA SHEET

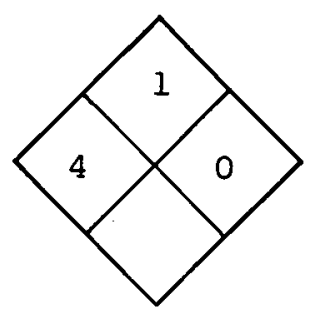

\section{SECTION I SOURCE AND NOMENCLATURE}

MANUFACTURER'S NAME

EMERGENCY TELEPHONE NO.

ADDRESS (NUMBER, STREET, CITY, STATE, ZIP CODE)

TRADE NAME AND SYNONYMS

P-Aminobenzenesulfonamide

CHEMICAL NAME AND SYNONYMS

Sulfanilamide

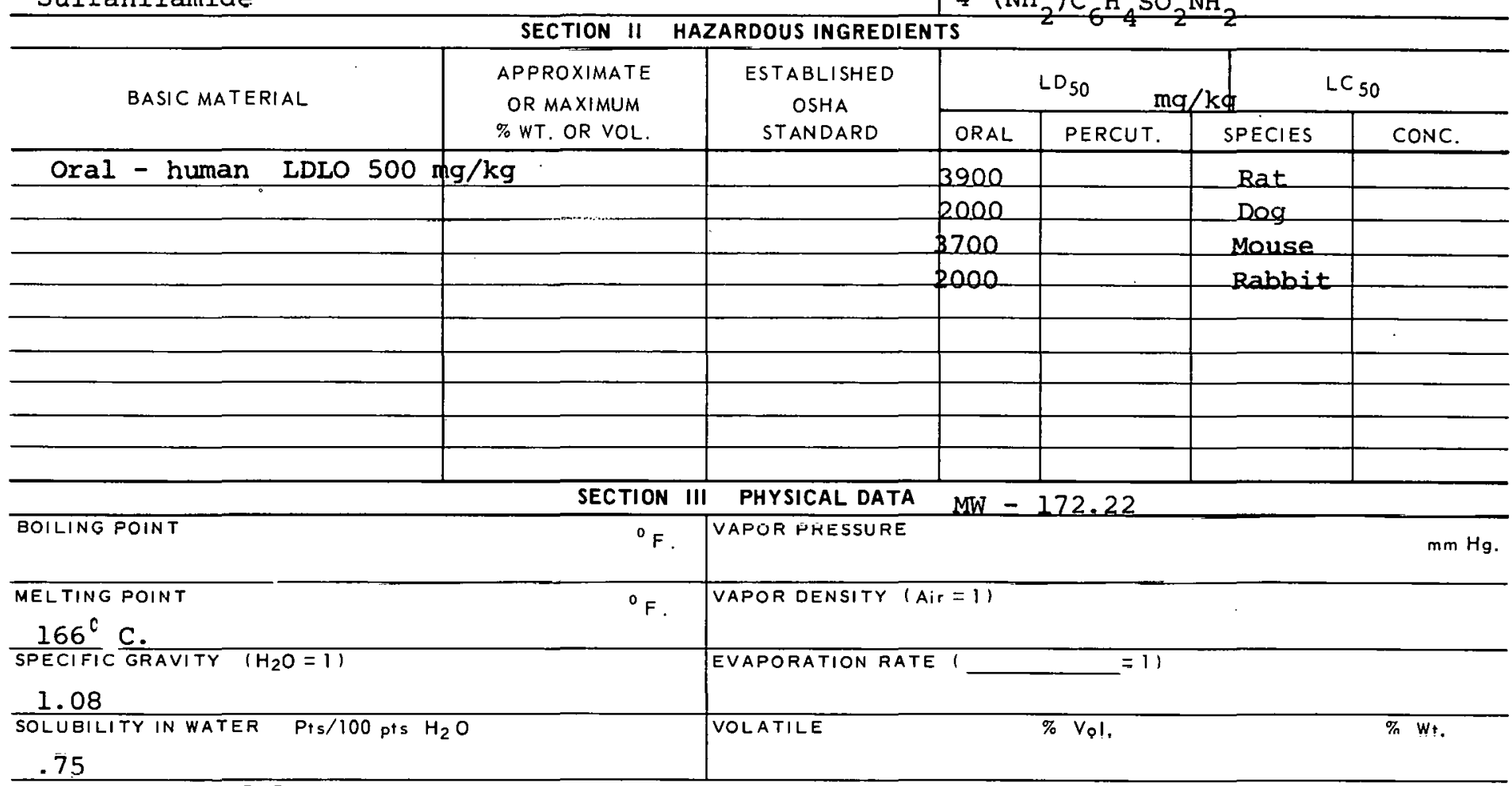

APPEARANCE AND ODOR

White to yellow powder.

SECTION IV FIRE AND EXPLOSION HAZARD DATA

FLASH POINT

None available

METHOD USED

FLAMMABLE

(EXPLOSIVE)

LIMITS
UPPER

LDWER

EXTINGUISHING MEDIA

Foam, carbon dioxide, dry chemical.

SPECIAL FIRE FIGHTING PROCEDURES

UNUSUAL FIRE AND EXPLOSION HAZARDS 
Sulfanilamide SECTION $V$ HEALTH HAZARD DATA

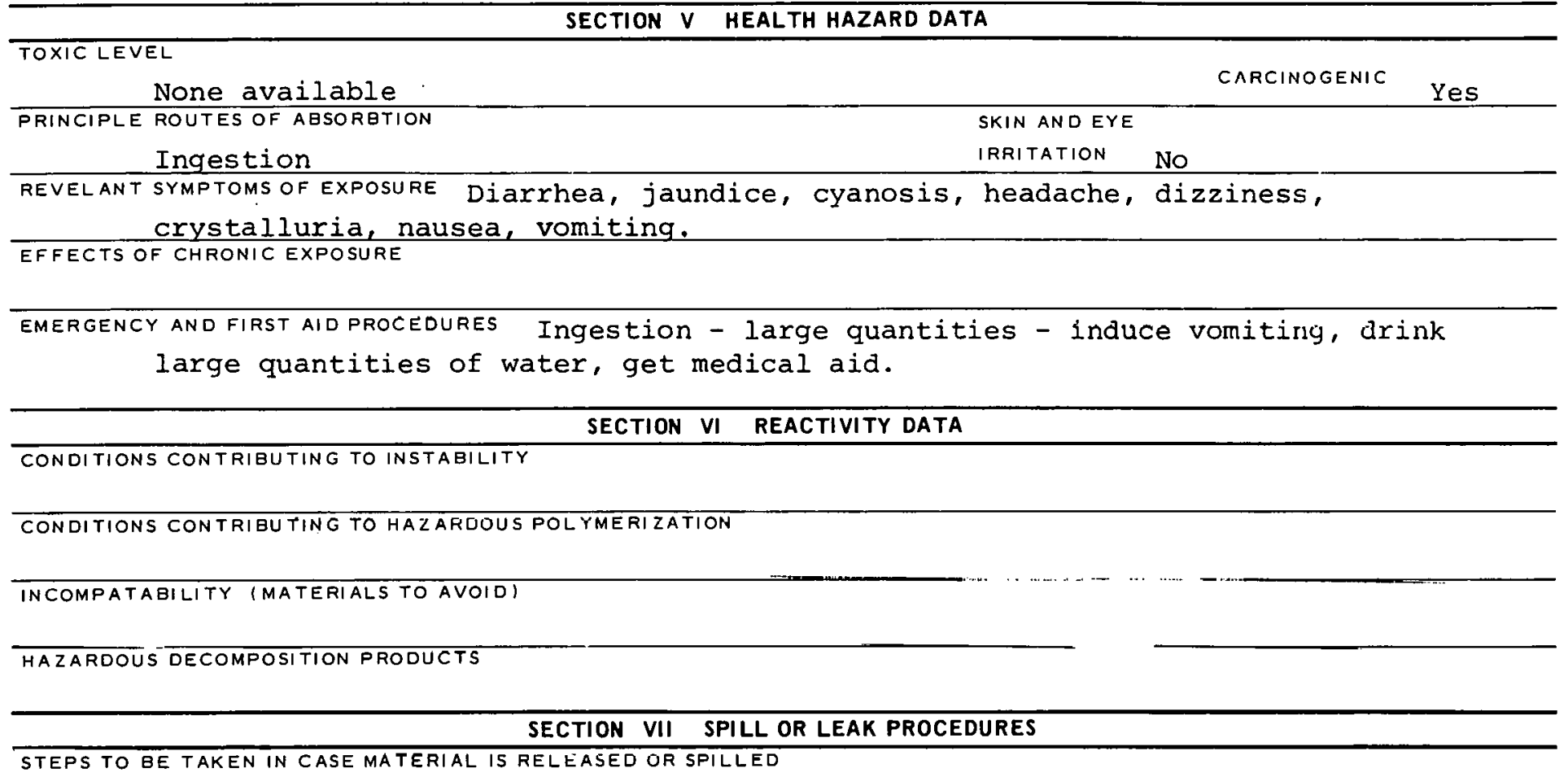
STEPS TO BE TAKEN IN CASE MATERIAL IS RELEASED OR SPILLED

WASTE DISPOSAL METHOD

\section{SECTION VIII SPECIAL PROTECTION INFORMATION}

\begin{tabular}{l|l}
\hline VENTILATION REQUIREMENTS LOCAL EXHAUST & PHUTECTIVE EQUIPMENT (SPECIFY TYPES) EYE
\end{tabular}

MECHANICAL (GENERAI.) GI.OVES

SPECIAL

REITIRATON

OTHER PROTECTIVE EQUIPMENI

SECTION IX SPECIAL PRECAUTIONS

PRECAUTIONS TO BE TAKEN IN HANDLING AND STORAGE

UIHEKHEE⿱ AUTIEIS 


\section{PRODUCT DESIGNATION}

Tetra bromoethane

(acetylene tetrabromide)

MATERIAL SAFETY

DATA SHEET

$03-365-0839$

\section{SECTION I SOURCE AND NOMENCLATURE}

\section{MANUFACTURER'S NAME}

EMERGENCY TELEPHONE NO.

ADORESS (NUMBER, STREET, CITY, STATE, ZIP CODE)

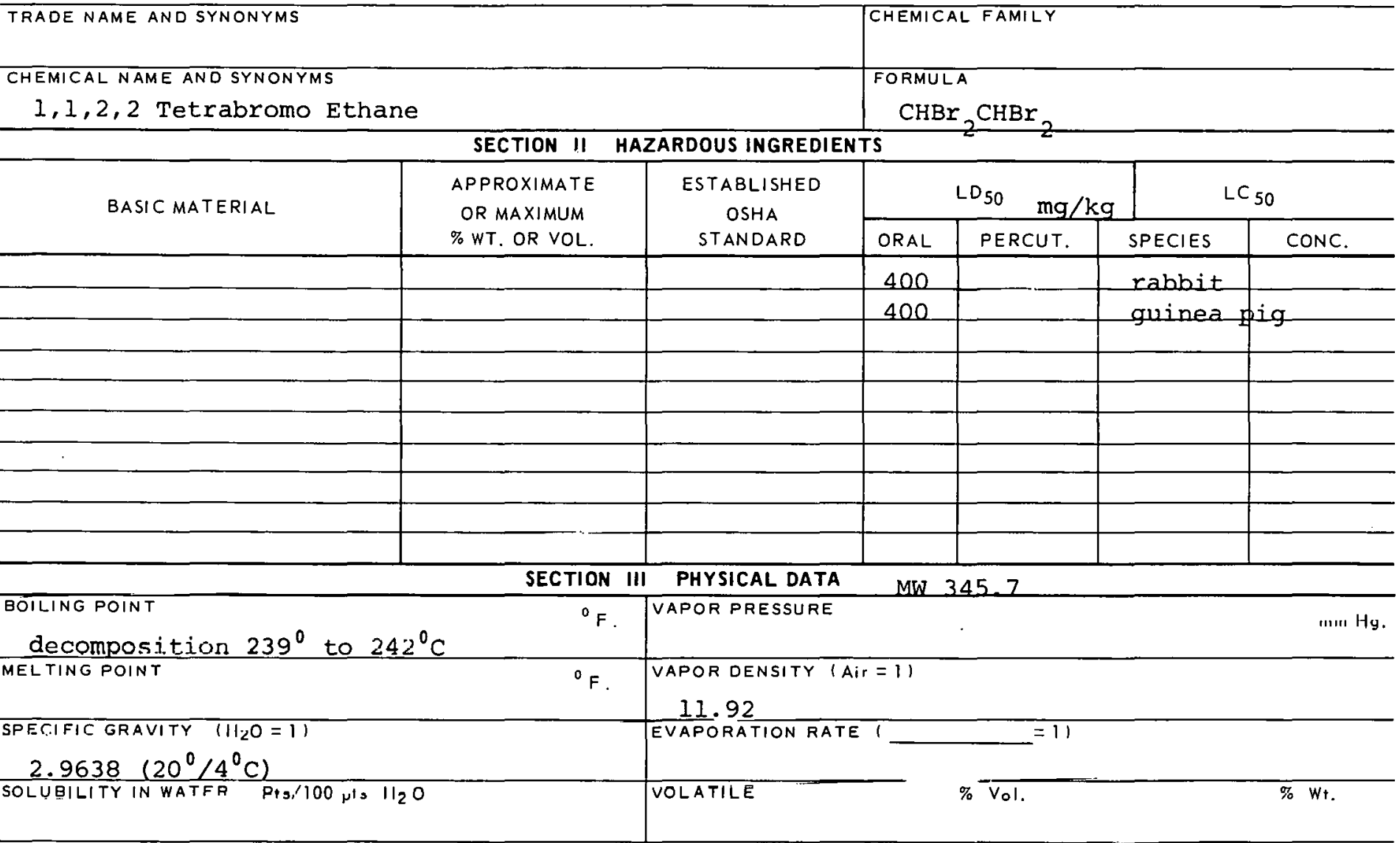

APPEARANCE ANO ODOR

Colorless to yellow Iiquid with chloroform-like odor. SECTION IV FIRE AND EXPLOSION HAZARD DATA

FLASH POINT

$-$

METHOD USED

EXTINGUISHING MEDIA

Use water for cooling containers.
FLAMMABLE

(EXPLOSIVE)

LIMITS

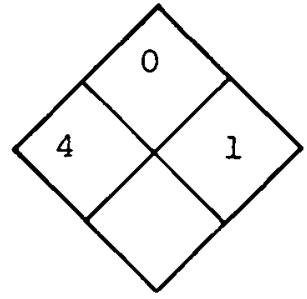




\section{TOXIC LEVEL}

TLV - 1 ppm, $14 \mathrm{mg}$.M.

\section{PRINCIPLE ROUTES OF ABSORBTION \\ Inhalation of vapor}

REVELANT SYMPTOMS OF EXPOSURE

Narcotic properties; is a c.n.s. and respiratory depressant;

drowsiness, etc.

Passibility of liver and kidey dame.

EFFECTS OF CHRONIC EXPOSURE

--

EMERGENCY AND FIRST AID PROCEDURES

rest and keep warm. Do not give stimulants.

\section{SECTION VI REACTIVITY DATA}

CONDITIONS CONTRIBUTING TO INSTABILITY

CONDITIONS CONTRIBUTING TO HAZARDOUS POLYMERIZATION

INCOMPATABILITY (MATERIALS TO AVOID)

HAZARDOUS DECOMPOSITION PRODUCTS

Toxic fumas of carboynl bromido.

SECTION VII SPILL OR LEAK PROCEDURES

STEPS TO BE TAKEN IN CASE MATERIAL IS RELEAASED OR SPILLED

WASTE DISPOSAL METHOD

SECTION VIII SPECIAL PROTECTION INFORMATION FUII protection

\begin{tabular}{l|l}
\hline VENTILATION REQUIREMENTS LOCAL EXHAUST & $\begin{array}{c}\text { FROTECTIVE EQUIPMENT (SPECIFY TYPESI EYE } \\
\text { GogqLES }\end{array}$ \\
\hline MECHANICAL IGENERAL) & $\begin{array}{c}\text { GLOVES } \\
\text { Kibber }\end{array}$ \\
\hline SHELIAL & REGFIRATON \\
W/Appropriate filter
\end{tabular}

OTHEH PROTECTIVE EQUIPMENT

(face shield), rubber apron.

SECTION IX SPECIAL PRECAUTIONS

PRECAUTIONS TOBE TAKEN IN HANDLING AND STORAGE

Be sure there is adequate ventilation.

OTHER PRECAUTIONS

SI GNATURE

JUATE

365-0839B 


\begin{tabular}{|c|}
\hline PRODUCT DESIGNATION \\
03-365-1809 \\
Tetrabromophthalic Anyhydride \\
\end{tabular}

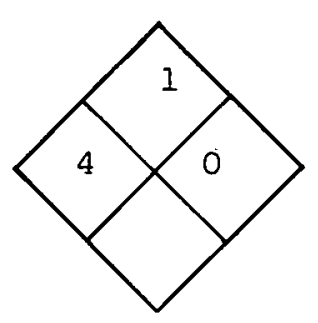

\section{SECTION I SOURCE. AND NOMENCLATURE}

EXTINGUISHING MEDIA

water, carbon dioxide, dry chemical.

SPECIAL FIRE FIGHTING PROCEOURES

UNUSUAL FIRE AND EXPLOSION HAZARDS 
Tetrabromophthalic Anhydride

SECTION $V$ HEALTH HAZARD DATA

TOXIC LEVEL

None available

PRINCIPLE ROUTES OF ABSORBTION

Inhalation (mainly)

REVELANT SYMPTOMS OF EXPOSURE eye irritation.

EFFECTS OF CHRONIC EXPOSURE Bronchitis, eye irritation, inflammation of respiratory tract; bloody nasal discharge. Sensitization

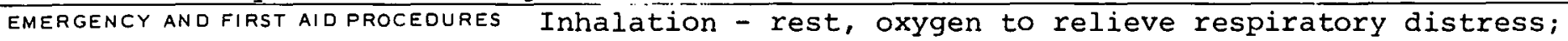
wash skin \& eyes; get medical aid.

\section{SECTION VI REACTIVITY DATA}

CONDITIONS CONTRIBUTING TO INSTABILITY

GOIDITIOAS TONTRIRIITING TO HAZARQQUS POLYMERIZATION

INCOMPATABILITY (MATERIALS TO AVOID)

HAZARDOUS DECOMPOSITION HRUDUCTS

SECTION VII SPILL OR LEAK PROCEDURES

STEPS TO BE TAKEN IN CASE MATERIAL IS RELEASED OR GPILLED

SECTION VIII SPECIAL PROTECTION INFORMATION

\begin{tabular}{l|l|l}
\hline VENTILATION REQUIREMENTS LOCAL EXHAUST & PROTECTIVE EQUIPMENT (SPECIFY TYPES) EYE
\end{tabular}

MECHANISA!. IGENERAL)

Salely yuyyles

STECINL

OTIIER RROTECTIVE EQUIFMENT

GLOVES

ПССПIRATQR

With proper filter

CARCINOGENIC nO

SKIN ANDEYE moderate

aermatitis, 


\begin{tabular}{|c|}
\hline PRODUCT DESIGNATION \\
$03-365-2309$ \\
Tetra-N-Butylammonium Iodide \\
\hline
\end{tabular}

\section{MATERIAL SAFETY DATA SHEET}

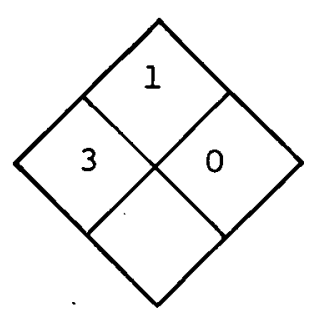

\section{SECTION I SOURCE AND NOMENCLATURE}

\begin{tabular}{l|l}
\hline MANUFACTURER'S NAME & EMERGENCY TELEPHONE NO. \\
\hline ADDRESS (NUMBER, STREET, CITY, STATE, ZIP CODE)
\end{tabular}

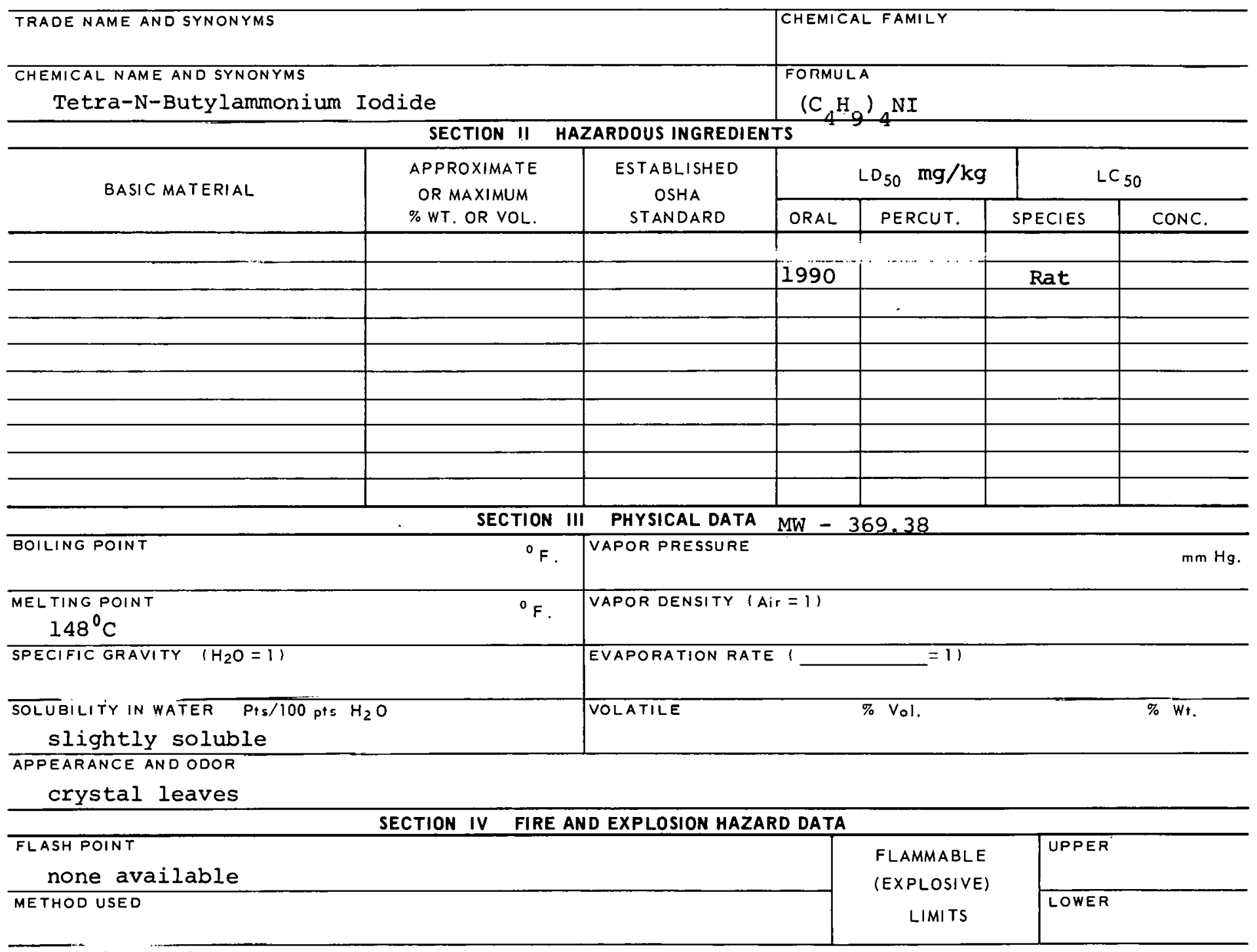

EXTINGUISHING MEDIA

Water, carbon dioxide, dry chemical.

SPECIAL FIRE FIGHTING PROCEDURES

UNUSUAL FIRE AND EXPLOSION HAZARDS 


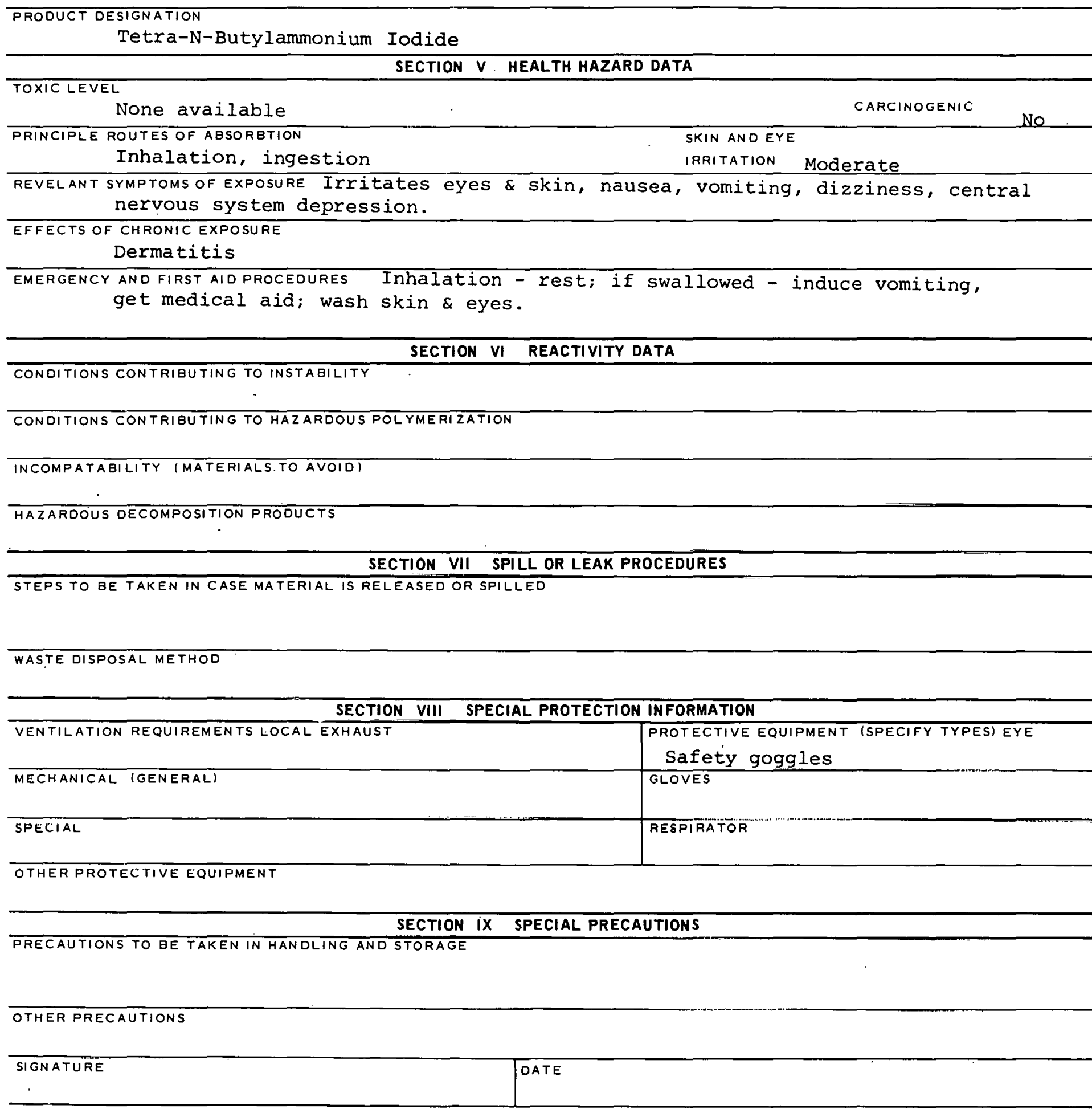




\section{PRODUCT DESIGNATION}

$1,1,2,2$-Tetrachloroethane 03-365-2809
MATERIAL SAFETY

DATA SHEET

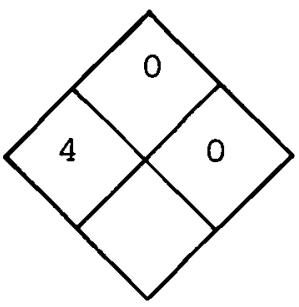

\section{SECTION I SOURCE AND NOMENCLATURE}

\begin{tabular}{l|l}
\hline MANUFACTURER'S NAME & EMERGENCY TELEPHONE NO. \\
\hline ADDRESS (NUMBER, STREET, CITY, STATE, ZIP CODE) &
\end{tabular}

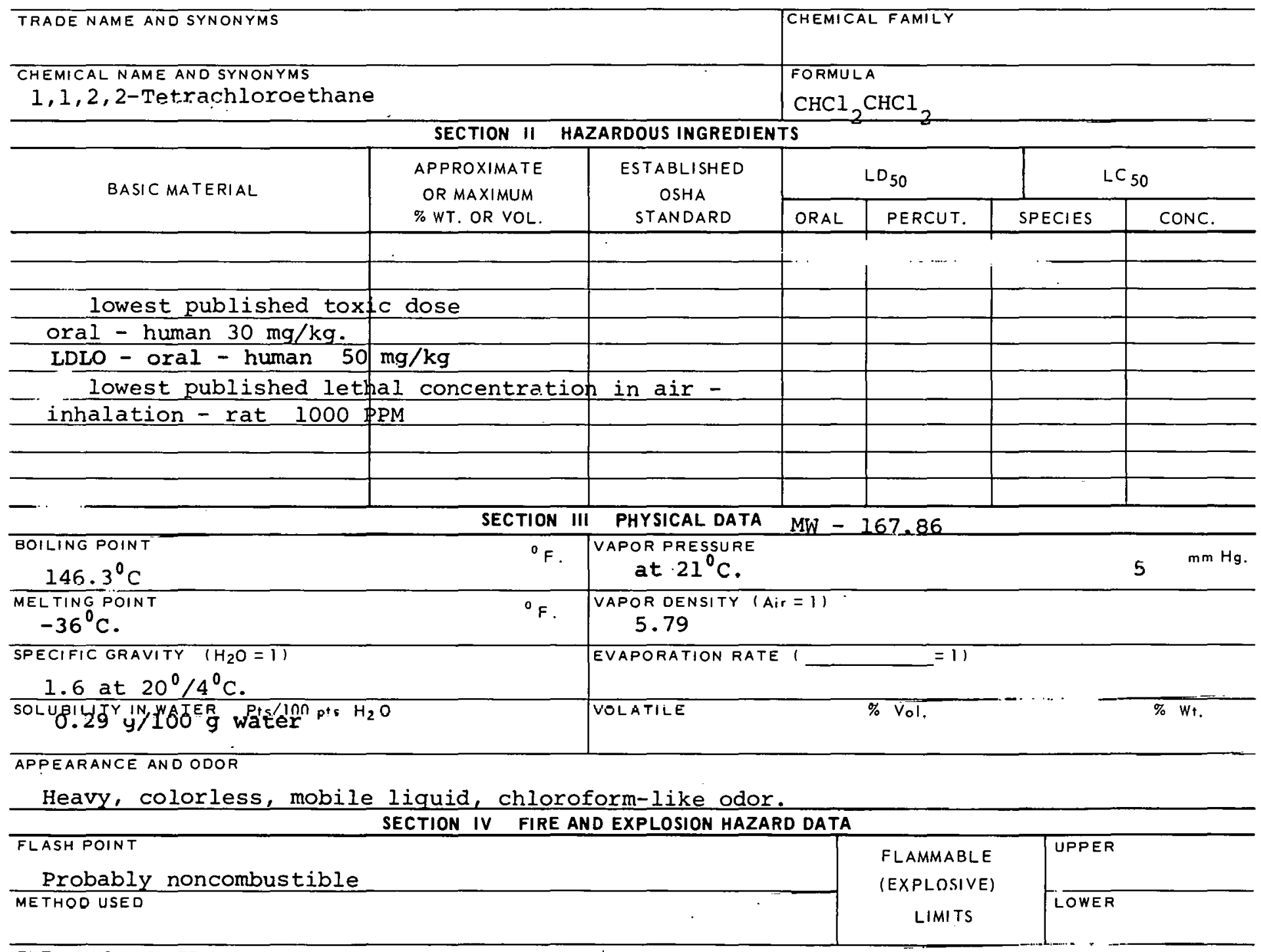

EXTINGUISHING MEDIA

SPECIAL FIRE FIGHTING PROCEDURES

Use water for cooling containers exposed by fire. Wear full protective clothing. UNUSUAL FIRE AND EXPLOSION HAZARDS

Upọn heating may decompose to produce flammable and toxic materials. 


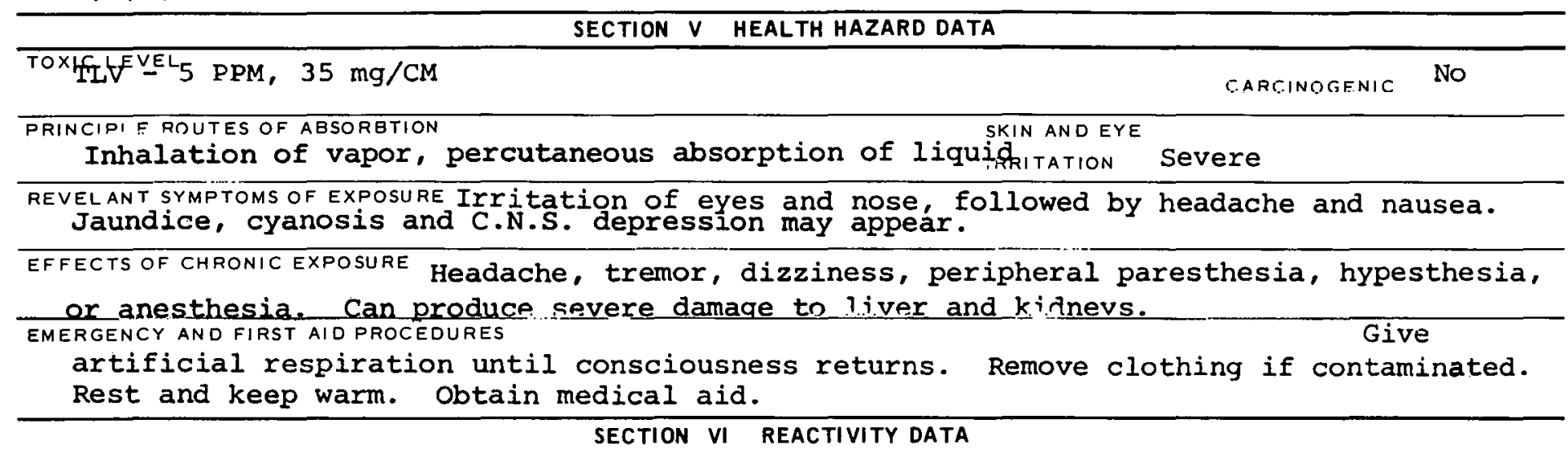

CONDITIONS CONTRIBUTING TO INSTABILITY

CONDITIONS CONTRIBUTING TO HAZARDOUS POLYMERIZATION

INCOMPATABILITY (MATERIALS TO AVOID) Nitrogen tetroxide, potassium, patassium hydroxide, sodium.

SECTION VII SPILL OR LEAK PROCEDURES

STEPS TO BE TAKEN IN CASE MATERIAL IS RELEASED OR SPILLED

Absorb spills on paper towels; evaporate in hood.

WASTE OISPOSAL METHOD

SECTION VIII SPECIAL PROTECTION INFORMATION

\begin{tabular}{l|l}
\hline \multicolumn{1}{c}{ SECTION VIII SPECIAL PROTECTION INFORMATION } \\
\hline $\begin{array}{l}\text { VENTILATION REQUIREMENTS LOCAL EXHAUST } \\
\text { MECHANICAL (GENERAL) }\end{array}$ & $\begin{array}{c}\text { PROTECTIVE EQUIPMENT (SPECIFY TYPES) EYE } \\
\text { Safety ylaSSES }\end{array}$ \\
\hline $\begin{array}{l}\text { SHECIAL } \\
\text { APION (solvent pront proof synthetic }\end{array}$ \\
\hline
\end{tabular}

OTHER PROTECTIVE EQUIPMENT

$>20000 \mathrm{ppm}$ self-contained breathing apparatus.

SECTION IX SPECIAL PRECAUTIONS

PRER DIITIONS TR RF TAKEN IN HANDLING AND STORAGE

store in dark, cool place. 


\begin{tabular}{|l|}
\hline PRODUCT DESIGNATION \\
03-365-3310 \\
03-365-3309 \\
Tetrachloroethylene \\
(Perchloroethylene) \\
\hline
\end{tabular}

\section{MATERIAL SAFETY DATA SHEET}

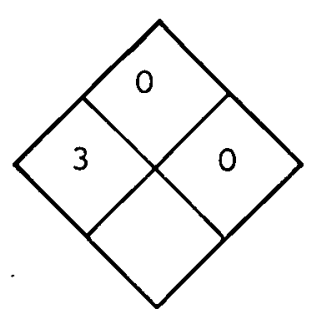

SECTION I SOURCE AND NOMENCLATURE ADDRESS (NUMBER, STREET, CITY, STATE, ZIP CODE)

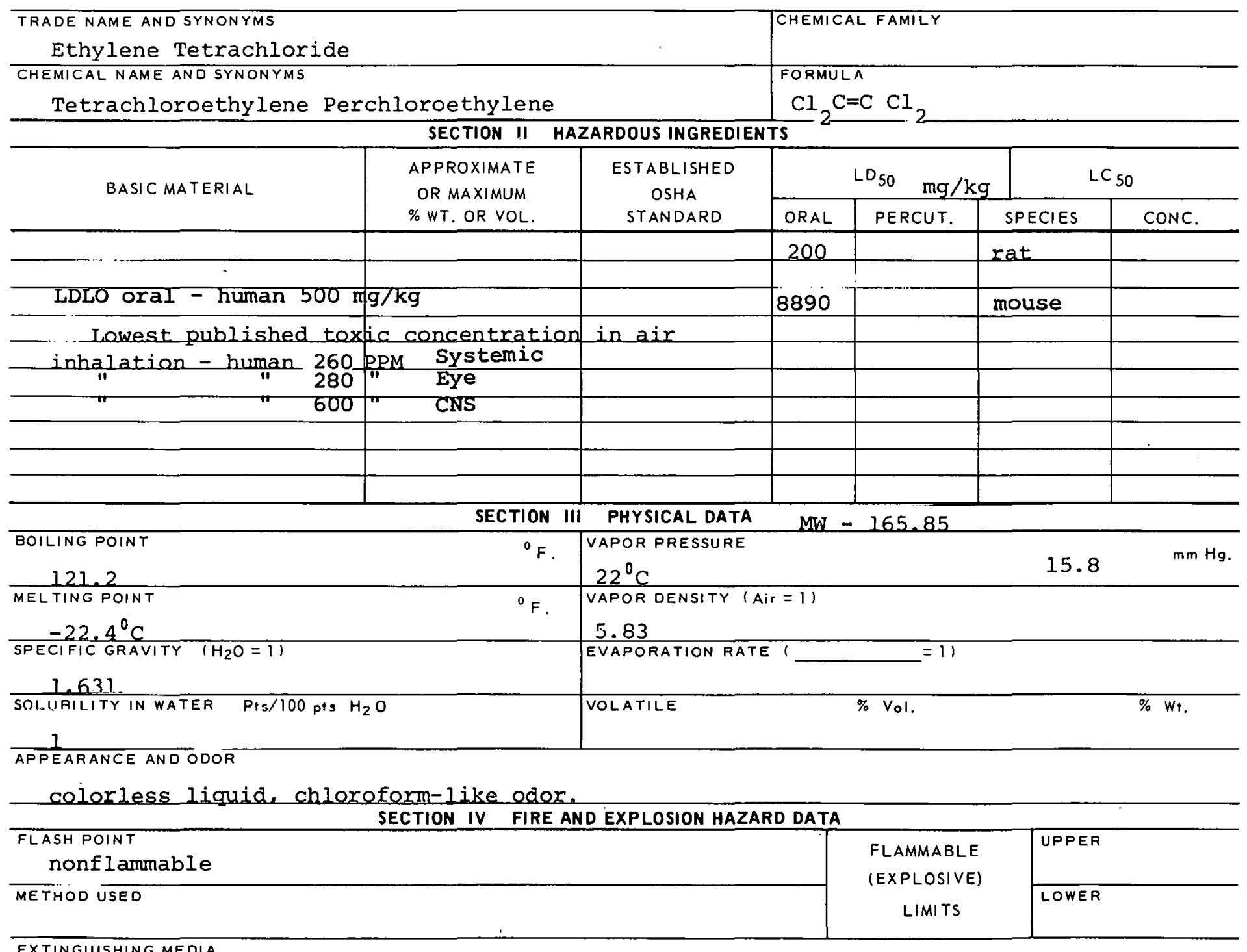

EXTINGUISHING MEDIA

water spray, $\mathrm{CO}_{2}$ or dry chemical -

SPECIAL FIRE FIGHTING PROCEDURES

Wear self-contained breathing apparatus.

UNUSUAL FIRE ANDEXPLOSION HAZARDS

When heated to decomposition point (above $140^{\circ} \mathrm{C}$ ) emits highly toxic fumes of chlorides. 


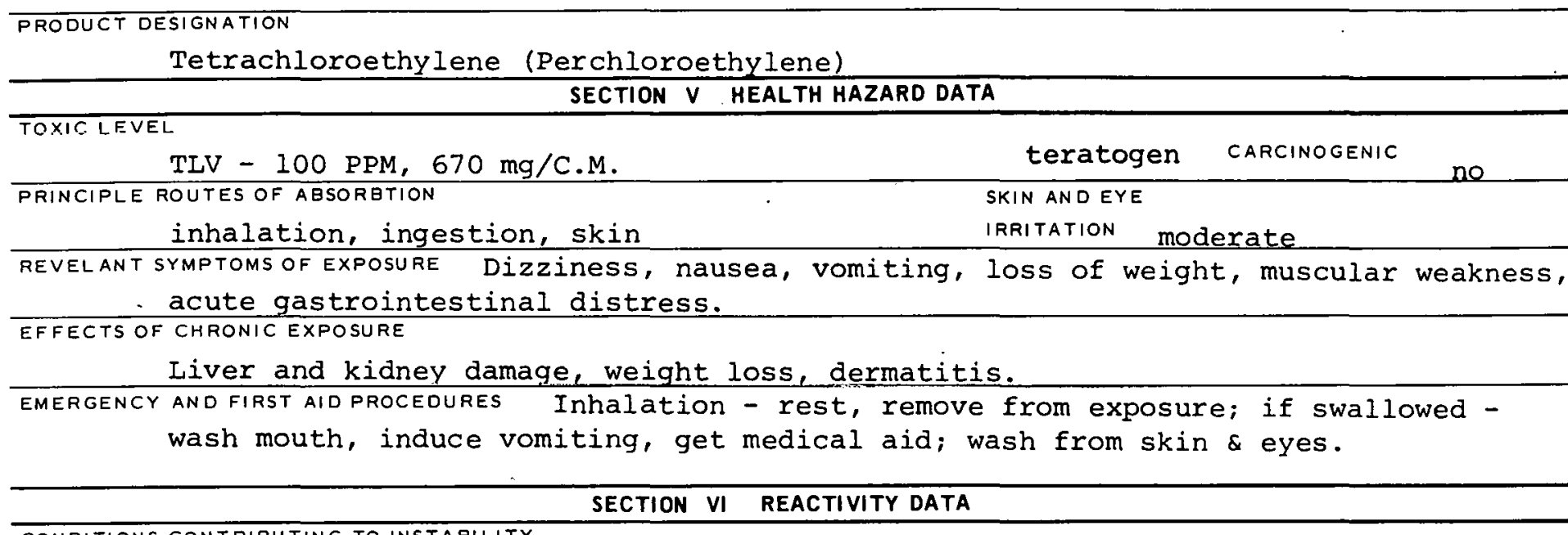

CONDITIONS CONTRIBUTING TO INSTABILITY

CONDITIONS CONTRIQUTING TO HAZARDOUS POLYMERIZATION

INCOMPATABILITY (MATERIALS TO AVOID)

HAZARDOUS DECOMPOSITION PRODUCTS

Upon decomposition, gives off toxic chloride fumes.

SECTION VII SPILL OR LEAK PROCEDURES

STEPS TO BE TAKEN IN CASE MATERIAL IS RELEASED OR SPILLED

Absorb on paper towels; evaporate in hood.

WASTE DISPOSAL METHOD

SECTION VIII SPECIAL PROTECTION INFORMATION

\begin{tabular}{l|l}
\hline \multicolumn{1}{c}{ SECTION VIII SPECIAL PROTECTION INFORMATION } \\
\hline $\begin{array}{l}\text { VENTILATION REQUIREMENTS LOCAL EXHAUST } \\
\text { In hOOd }\end{array}$ & $\begin{array}{c}\text { PROTECTIVE EQUIPMENT } \\
\text { GoggleS }\end{array}$ \\
\hline MECHANICAL (GENERAL) & $\begin{array}{c}\text { GLOVES } \\
\text { Rubber }\end{array}$ \\
\hline SPECIAL & $\begin{array}{c}\text { RESPIRATOR } \\
\text { With proper filter }\end{array}$ \\
\hline
\end{tabular}

OTHER PROTECTIVE EQUIPMENT

SECTION IX SPECIAL PRECAUTIONS

PRECAUTIONS TO BE TAKEN IN HANDLING AND STORAGE

Store in slosed container in cool place.

OTHER PRECAUTIONS

SIGNATURE

DATE

$365-3309 B$ 


\section{PRODUCT DESIGNATION}

03-365-3809

Tetrachlorohydroquinone

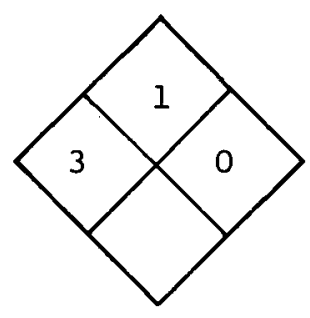

SECTION I SOURCE AND NOMENCLATURE

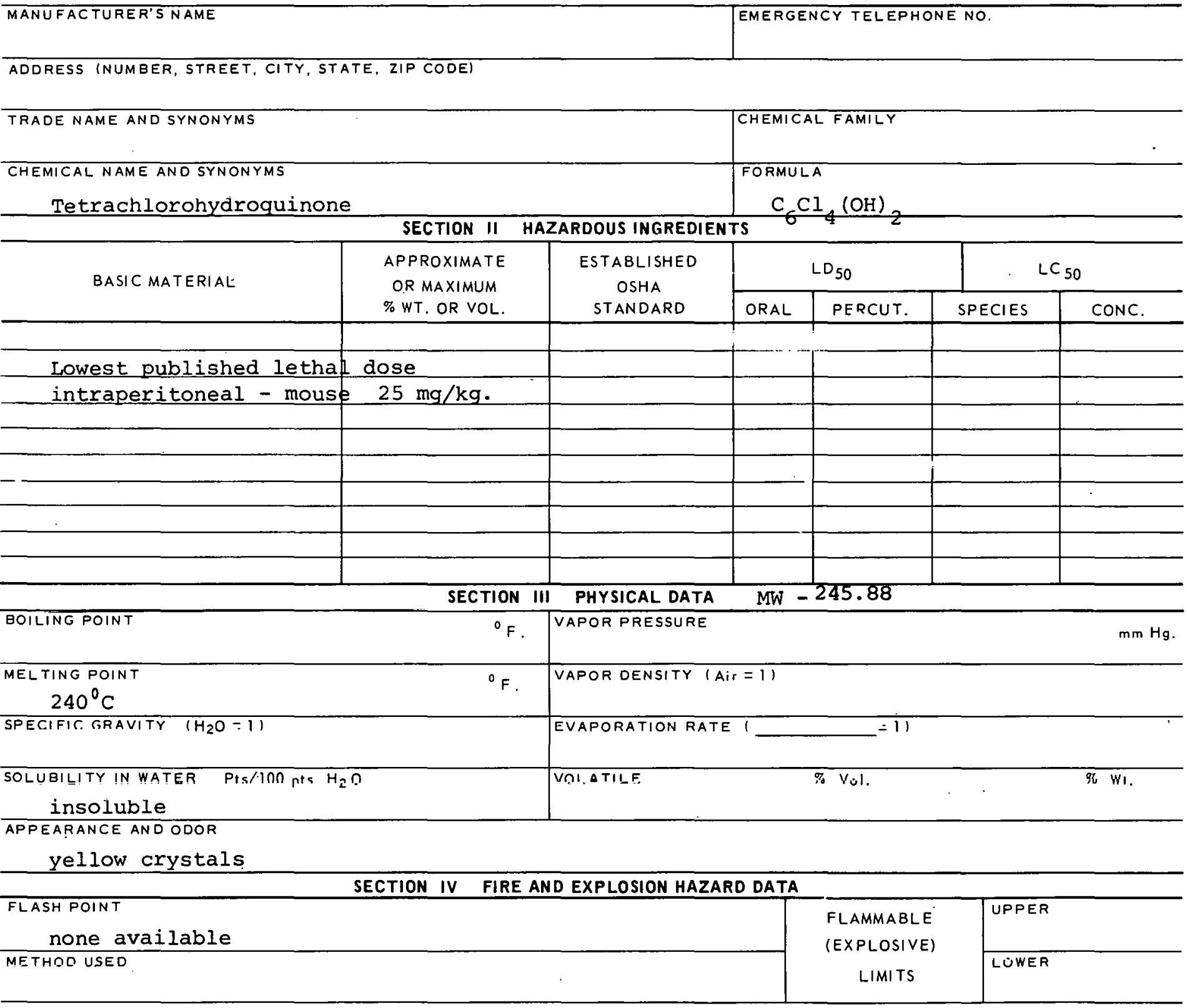

EXTINGUISHING MFOIA

Water, carbon dioxide, dry chemical.

SPECIAL FIRE FIGHTING PROCEDURES

UNUSUAL FIRE AND EXPLOSION HAZARDS 
Tetrachlorohydroquinone SECTION $V$ HEALTH HAZARD DATA

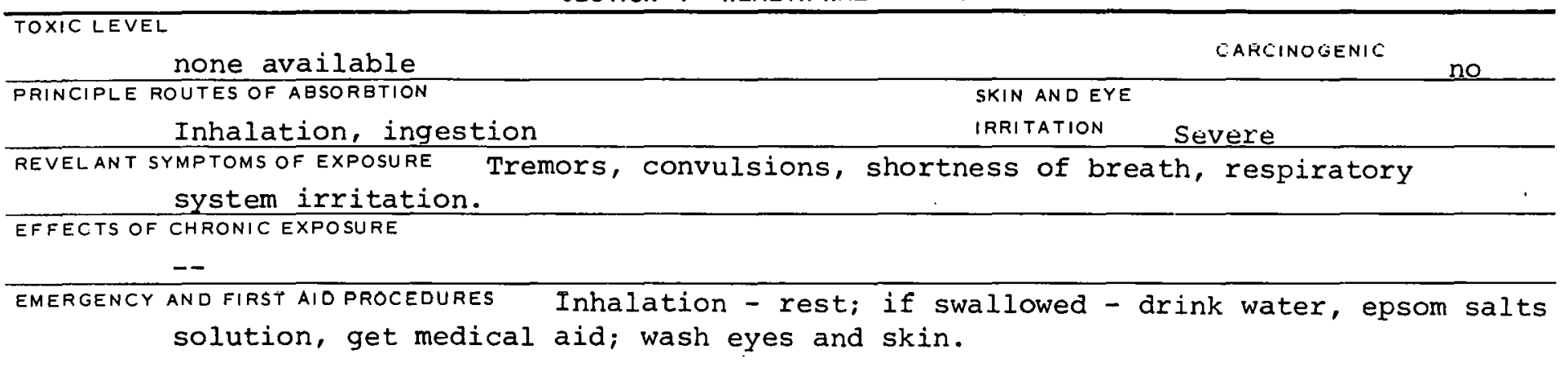

\section{SECTION VI REACTIVITY DATA}

CONDITIONS CONTRIBUTING TO INSTABILITY
CONDITIONS CONTRIBUTING TO HAZARDOUS POLYMERIZATION
INCOMPATABILITY (MATERIALS TO AVOID)
HAZARDOUS DECOMPOSITION PRODUCTS SECTION VII SPILL OR LEAK PROCEDURES

STEPS TO BE TAKEN IN CASE MATERIAL IS RELEASED OR SPILLËD

WASTE DISPOSAL METHOD

SECTION VIII SPECIAL PROTECTION INFORMATION

\begin{tabular}{l|l|l}
\hline VENTILATION REQUIREMENTS LOCAL EXHAUST & PROTECTIVE EQUIPMENT ISPECIFY TYPES) EYE
\end{tabular}

MECHANICAL (GENERAL)

Goggles

GLOVES

Rubber gloves

STECIAL

RESPIRATAR.

OTHER PROTCCTIVE EQUIFMENT

Self-contained

SECTION IX SPECIAL PRECAUTIONS

PRECAUTIONS TO BE TAKEN IN HANDLING AND STORAGE

TTHEK PHELAUTIUNS

SIGNATURE

ŌATE

$365-3809 B$ 


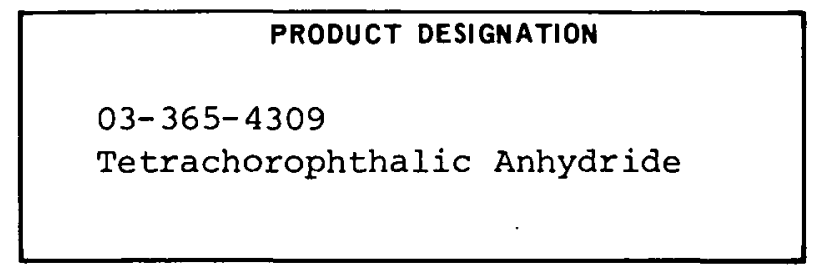

\section{MATERIAL SAFETY \\ DATA SHEET}

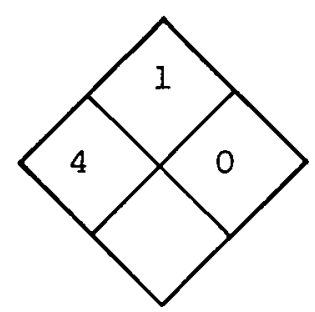

\section{SECTION I SOURCE AND NOMENCLATURE}

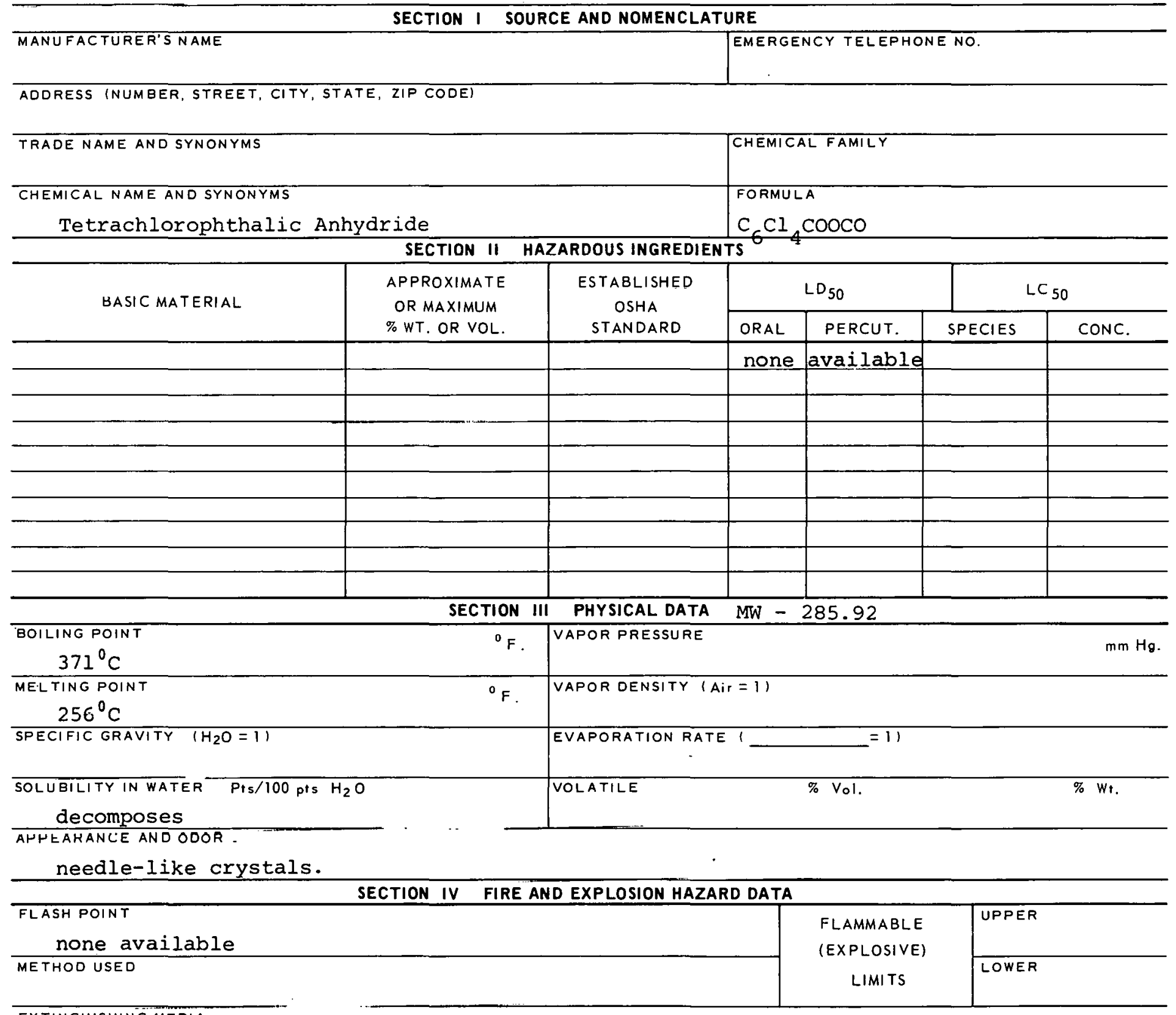

EXTINGUISHING MEDIA

Water, carbon dioxide, dry chemical.

SPECIAL FIRE FIGHTING PROCEDURES

UNUSUAL FIRE AND EXPLOSION HAZARDS 
Tetrachlorophthalic Anhydride

SECTION $V$ HEALTH HAZARD DATA

TOXIC LEVEL

none available

PRINCIPLE ROUTES OF ABSORBTION

Ingestion, inhalation (mainly)

CARCINOGENIC

no

SKIN AND EYE

IRRITATION Moderate

REVELANT SYMPTOMS OF EXPOSURE eye irritation.

Coughing, sneezing, respiratory irritation, dermatitis,

EFFECTS OF CHRONIC EXPOSURE

bloody nasal discharge.

Bronchitis, eye irritations, inflammation of respiratory tract;

AND FIRST AID PROCEDURES

Inhalation - rest, oxygen to relieve respiratory distress;

wash skin \& eyes; get medical aid.

SECTION VI REACTIVITY DATA

CONDITIONS CONTRIBUTING TO INSTABILITY

TONMITIONS CONTRIBUTING TO HAZARDOUS POLYMERIZATION

INCOMPATABILITY (MATERIALS TO AVOIO)

HAZARDOUS DECOMPOSITION PRODUCTS

SECTION VII SPILL OR LEAK PROCEDURES

STEPS TO BE TAKEN IN CASE MATERIAL IS RELEASED OR SPILLED

WASTE DISPOSAL METHOD

\section{SECTION VIII SPECIAL PROTECTION INFORMATION}

VENTILATION REQUIREMENTS LOCAL EXHAUST

INROTECTIVE EQUIPMENT (SPECIFY TYPES) EYE Safety goggles

MECHANICAL (GENERAL)

GLOVES

ŚDETIAI

DESPIBOTกR

OIHER PROTECTIVE EQUIPMENT

With proper filter

SECTION IX SPECIAL PRECAUTIONS

PRECAUTIONS TO BE TAKEN IN HANOLING AND STORAGE

OTHER TRECAUTIONG

SIGNATURE

DATE

$365-4309 B$ 


\begin{tabular}{|c|}
\hline PRODUCT DESIGNATION \\
$03-365-4809$ \\
$03-365-4819$ \\
Tetrahydrofuran
\end{tabular}

\section{MATERIAL SAFETY DATA SHEET}

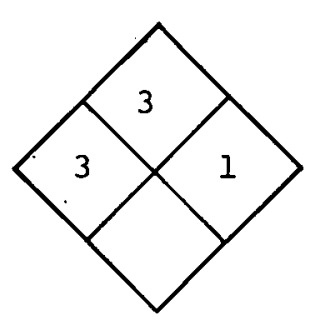

\section{SECTION I SOURCE AND NOMENCLATURE}

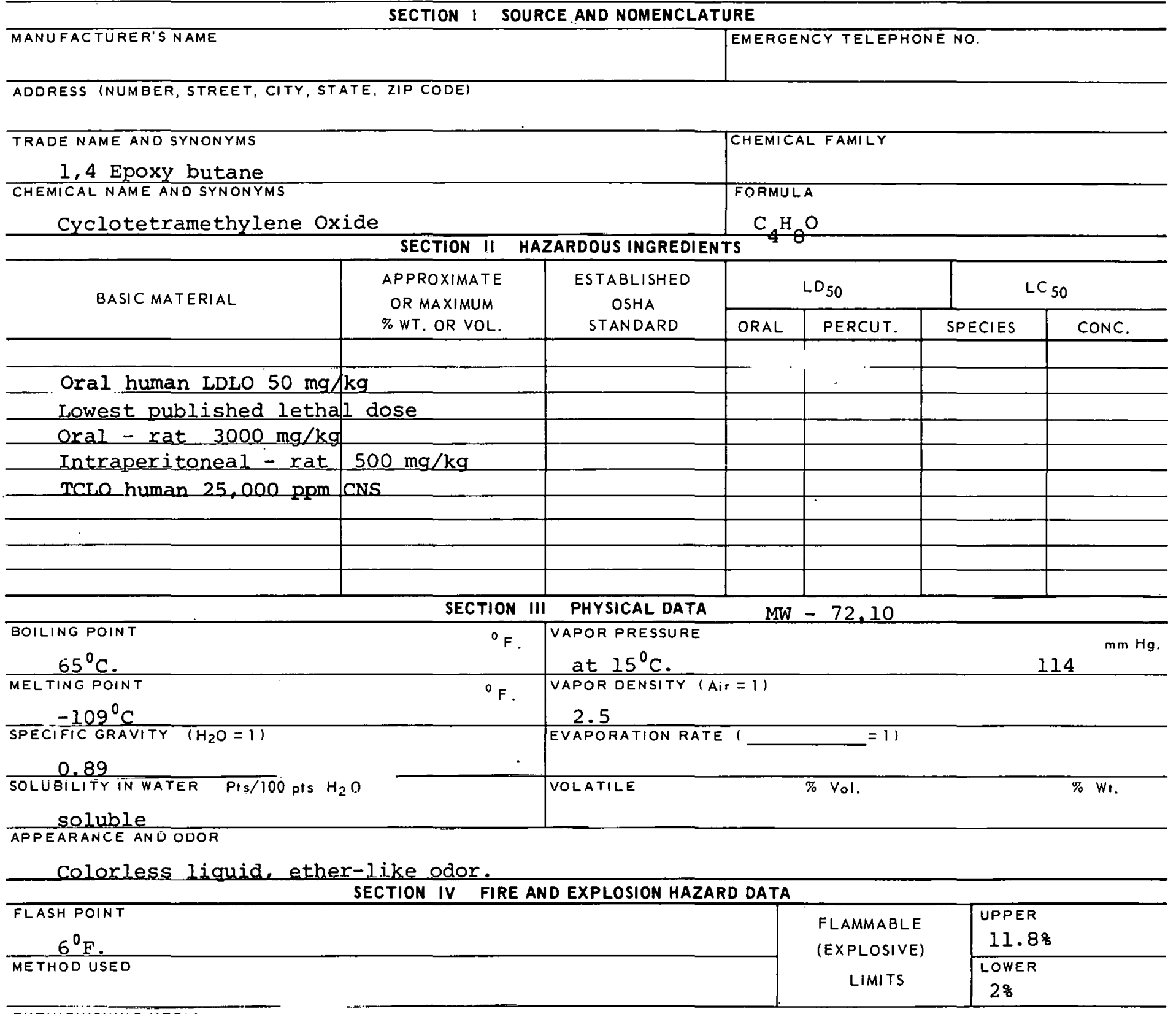

EXTINGUISHING MEDIA

Alcohol foam, carbon dioxide, dry chemical. Water may be ineffective. SPECIAL FIRE FIGHTING PROCEDURES

Wear goggles \& self-contained breathing apparatus. UNUSUAL FIRE ANÜEXHLOSION HAZAROS 
Tetrahydrofuran

\section{TOXIC LEVEL}

$200 \mathrm{PPM}, 590 \mathrm{mg} / \mathrm{C} . \mathrm{M}$.

PRINCIPLE ROUTES OF ABSORBTION

Ingestion, inhalation

SKIN AND EYE

IRRITATION Severe

REVELANT SYMPTOMS OF EXPOSURE

Eye and mucous membrane irritation, headache.

EFFECTS OF CHRONIC EXPOSURE

Liver and kidney damage - by ingestion.

EMERGENCY AND FIRST AID PROCEDURES If swallowed - give emetic, get medical aid;

inhalation - rest, oxygen as needed; wash from skin \& eyes.

CONDITIONS CONTRIBUTING TO INSTABILITY

CONDITIONS CONTRIBUTING TO HAZARDOUS POLYMERIZATION

INCOMPATABILITY (MATERIALS TO AVOID)

May form explosive mixture with sodium aluminum hydride.

HAZARDOUS DECOMPOSITION V'RUDUCTS

May form peroxide when exposed to light or air. SECTION VII SPILL OR LEAK PROCEDURES

STEPS TO $B E$ TAKEN IN CASE MATERIAL IS RELEASED OR SPILLED

Absorb on paper towels; evaporate in hood.

WASTE DISPOSAL METHOD

SECTION VIII SPECIAL PROTECTION INFORMATION

VENTILATION REQUIREMENTS LOCAL EXHAUST

MECHANILAL (GENERAL)

SPECIAL

OTHER PROTECTIVE EQUIPMENT

PROTECTIVE EQUIPMENT (SPECIFY TYPES) EYE Goggles

GLOVLC

Rubber

RESRIRATRR

With proper filter

\section{SECTION IX SPECIAL PRECAUTIONS}

PRECAUTIONS TO BE TAKEN IN HANDLING AND STORAGE

Store in cool, dark well-ventilated area. If inside, store in standard flammable liquids cabinet.

OTHFR PRFC.AUTIONS 


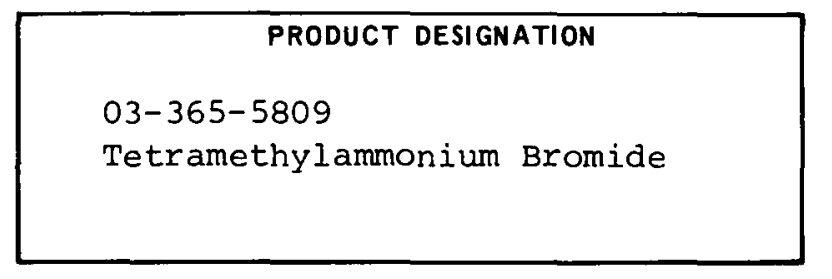

\section{MATERIAL SAFETY \\ DATA SHEET}

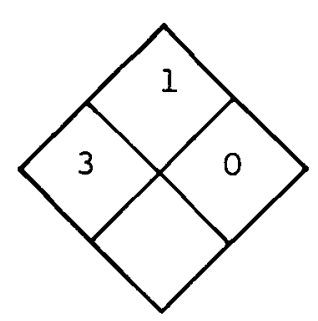

SECTION I SOURCE AND NOMENCLATURE

\begin{tabular}{l|l|l}
\hline MANUFACTURER'S NAME & EMERGENCY TELEPHONE NO. \\
\hline ADDRESS (NUMBER, STREET, CITY, STATE, ZIP CODE)
\end{tabular}

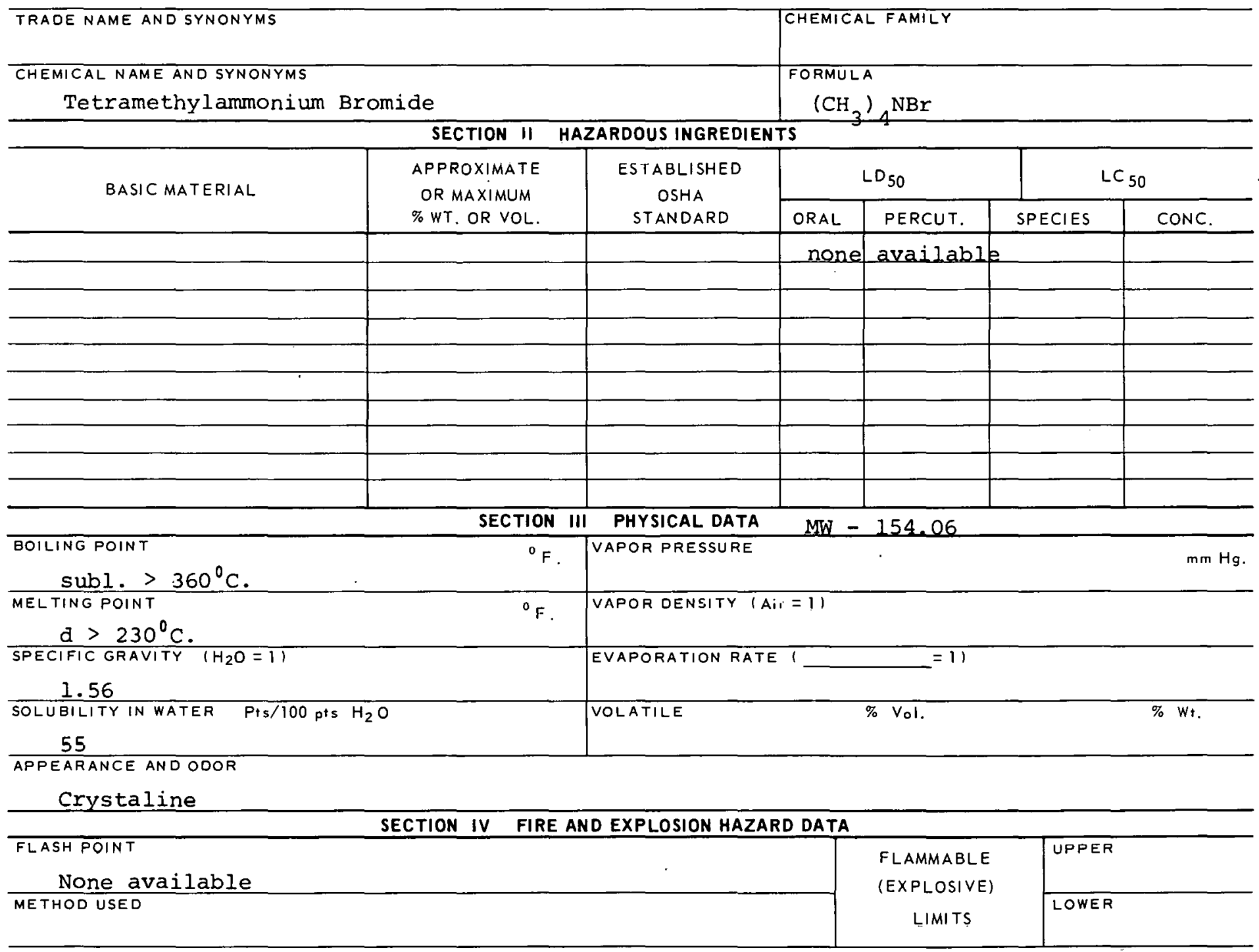

EXTINGUISHING MEDIA

Carbon dioxide, dry sliemical.

SPECIAL FIRE FISHTING PROTFIIIRFS

Wear full protective clothing.

UNUSUAL FIRE AND EXPLOSION HAZARDS 
Tetramethylammonium Bromide

\section{SECTION $V$ HEALTH HAZARD DATA}

TOXIC LEVEL

None available

PRINCIPLE ROUTES OF ABSORBTION

Inhalation, ingestion

CARCINOGENIC SKIN AND EYE IRRITATION Moderate

REVELANT SYMPTOMS OF EXPOSURE

Central nervous system depression, irritation of respiratory system. EFFECTS OF CHRONIC EXPOSURE

Possible liver and kidney damage.

EMERGENCY AND FIRST AID PROCEDURES Inhalation - rest; if swallowed - induce vomiting, get medical aid; wash from skin \& eyes.

\section{SECTION VI REACTIVITY DATA}

CONDITIONS CQONTRIBUTING TO INSTABILITY

T.ONDITIONS CONTRIBUTING TO HAZARDOUS POLYMERIZATION

INCOMPATABILITY (MATERIALS TO AVOIO)

HAZARDOUS DECOMPOSITION PRODUCTS

SECTION VII SPILL OR LEAK PROCEDURES

STEPS TO BE TAKEN IN CASE MATERIAL IS RELEASED OR SPILLED

WASTE DISPOSAL METHOD

SECTION VIII SPECIAL PROTECTION INFORMATION

\begin{tabular}{l|l|l}
\hline SECTION VIII SPECIAL PROTECTION INFORMATION \\
\hline VENTILATION REQUIREMENTS LOCAL EXHAUST & $\begin{array}{l}\text { PROTECTIVE EQUIPMENT (SPECIFY TYPES) EYE } \\
\text { GOggles }\end{array}$ \\
\hline MECHANICAL IGENERAL) & GLOVES \\
\hline SPECIAL & FEBPIRATOR \\
Self-contained breathing apparatus.
\end{tabular}

OTHER PROTECTIVE EQUIPMENT

SECTION IX SPECIAL PRECAUTIONS

PRECAUTIONS TO $8 E$ TAKEN IN HANDLING AND STORAGE

OTHER PRECAUTIONS

SIGNATURE

DATE

$365-5809 B$ 


\section{PRODUCT DESIGNATION}

03-365-5909

Tetramethylammonium Chloride
MATERIAL SAFETY

DATA SHEET

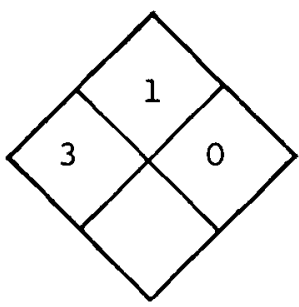

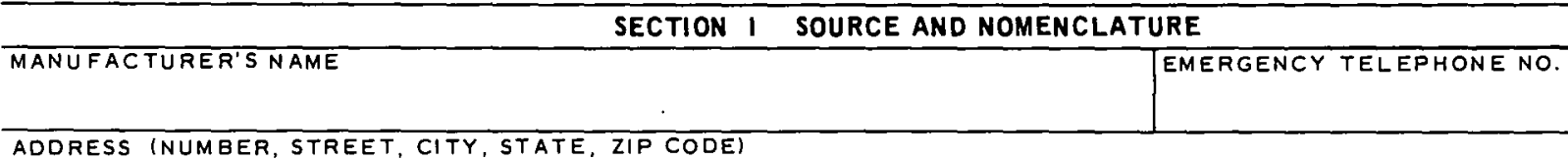

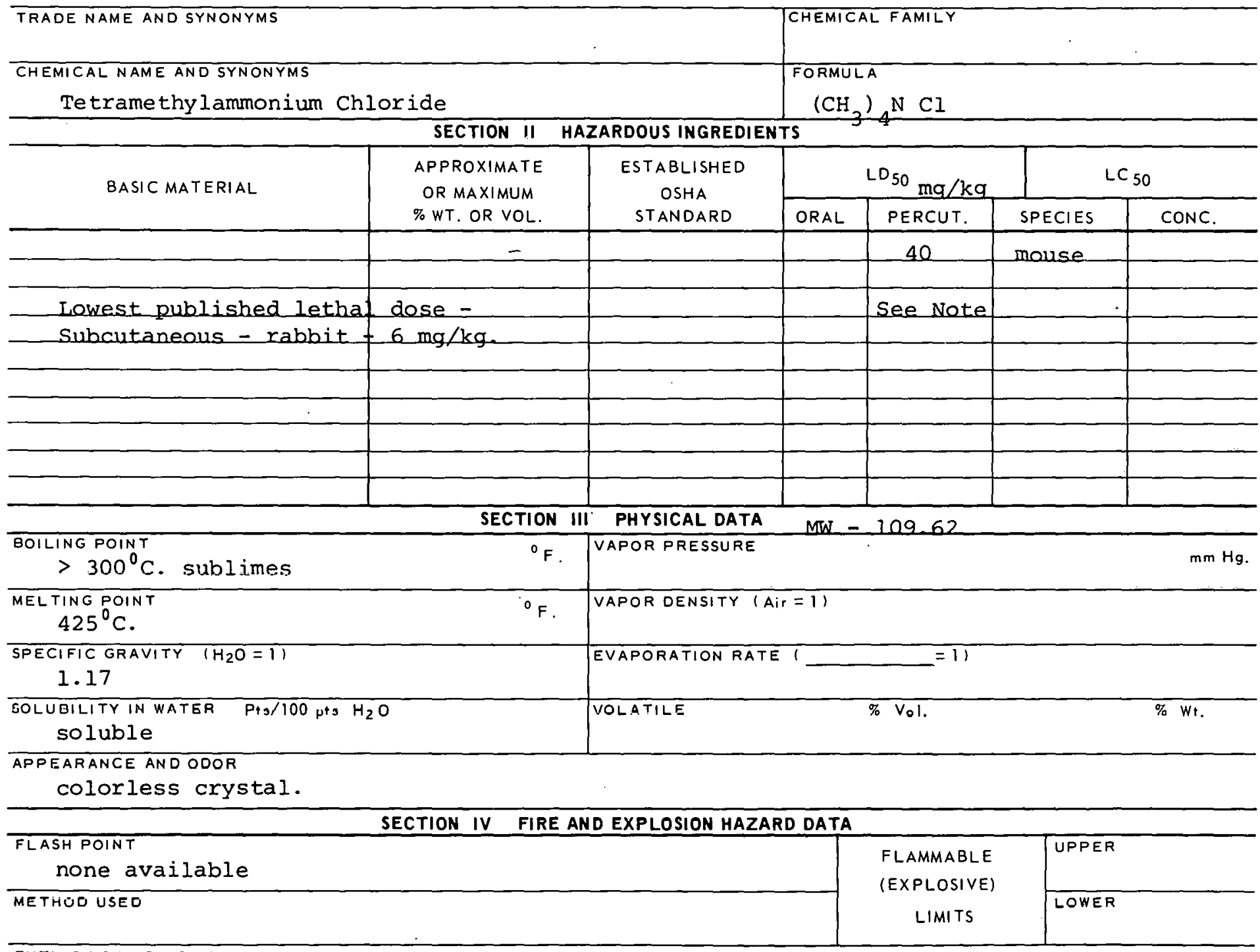

EXTINGUIGIIING MEDIA

Carbon dioxide, dry chemical.

SPECIAL FIRE FIGHTING PROCEDURES

Wear full protective clothing.

UNUSUAL FIRE AND EXPLOSION HAZARDS 


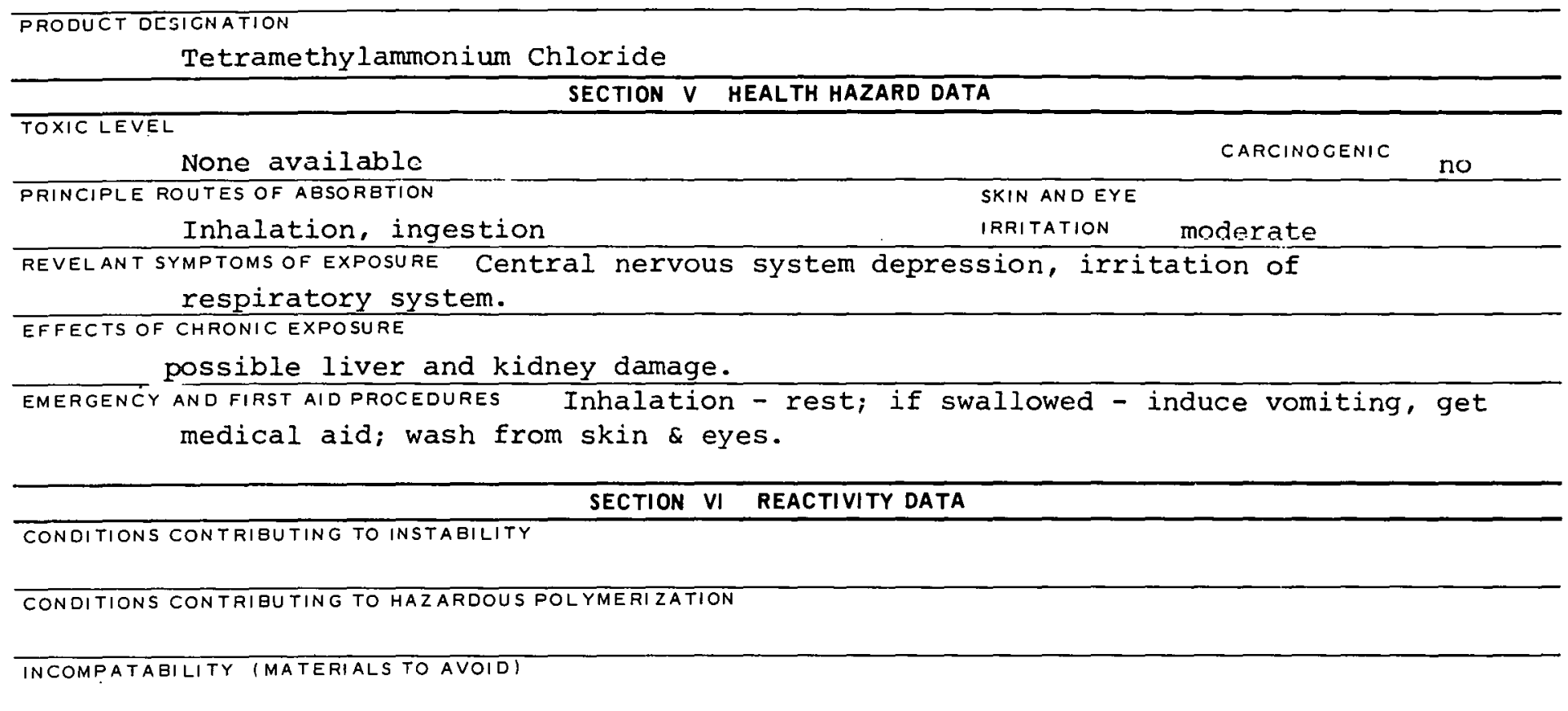

\section{HAZARDOUS DECOMPOSITION PRODUCTS}

SECTION VII SPILL OR LEAK PROCEDURES

STEPS TO BE TAKEN IN CASE MA TERIAL IS RELEASED OR SPILLED

WASTE DISPOSAL METHOD

\begin{tabular}{l|l}
\hline \multicolumn{1}{c}{ SECTION VIII SPECIAL PROTECTION INFORMATION } \\
\hline VENTILATION REQUIREMENTS LOCAL EXHAUST & $\begin{array}{c}\text { PROTECTIVE EQUIPMENT (SPECIFY TYPESIEYE } \\
\text { GOggles }\end{array}$ \\
\hline MECHANICAI. IGENERAL) & GI.OVES \\
\hline GPECIAL & $\begin{array}{c}\text { RE3PIRATOR } \\
\text { Self-contained }\end{array}$ \\
\hline
\end{tabular}

OTHER PROTECTIVE EQUIPMENT

SECTION IX SPECIAL PRECAUTIONS

PRECAUTIONS TO BE TAKEN IN HANDLING AND STORAGE

OTHER PRECAUTIONS

SIGNATURE

DATE

\begin{tabular}{l|l} 
DATEE \\
\hline
\end{tabular} 


\section{PRODUCT DESIGNATION}

03-365-6009

Tetramethylammonium Hydroxide
MATERIAL SAFETY

DATA SHEET

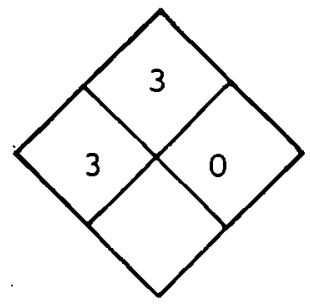

SECTION I SOURCE AND NOMENCLATURE

\begin{tabular}{l|l}
\hline MANUFAC TURER'S NAME & EMERGENCY TELEPHONE NO. \\
\hline ADDRESS (NUMBER, STREET, CITY, STATE, ZIP CODE)
\end{tabular}

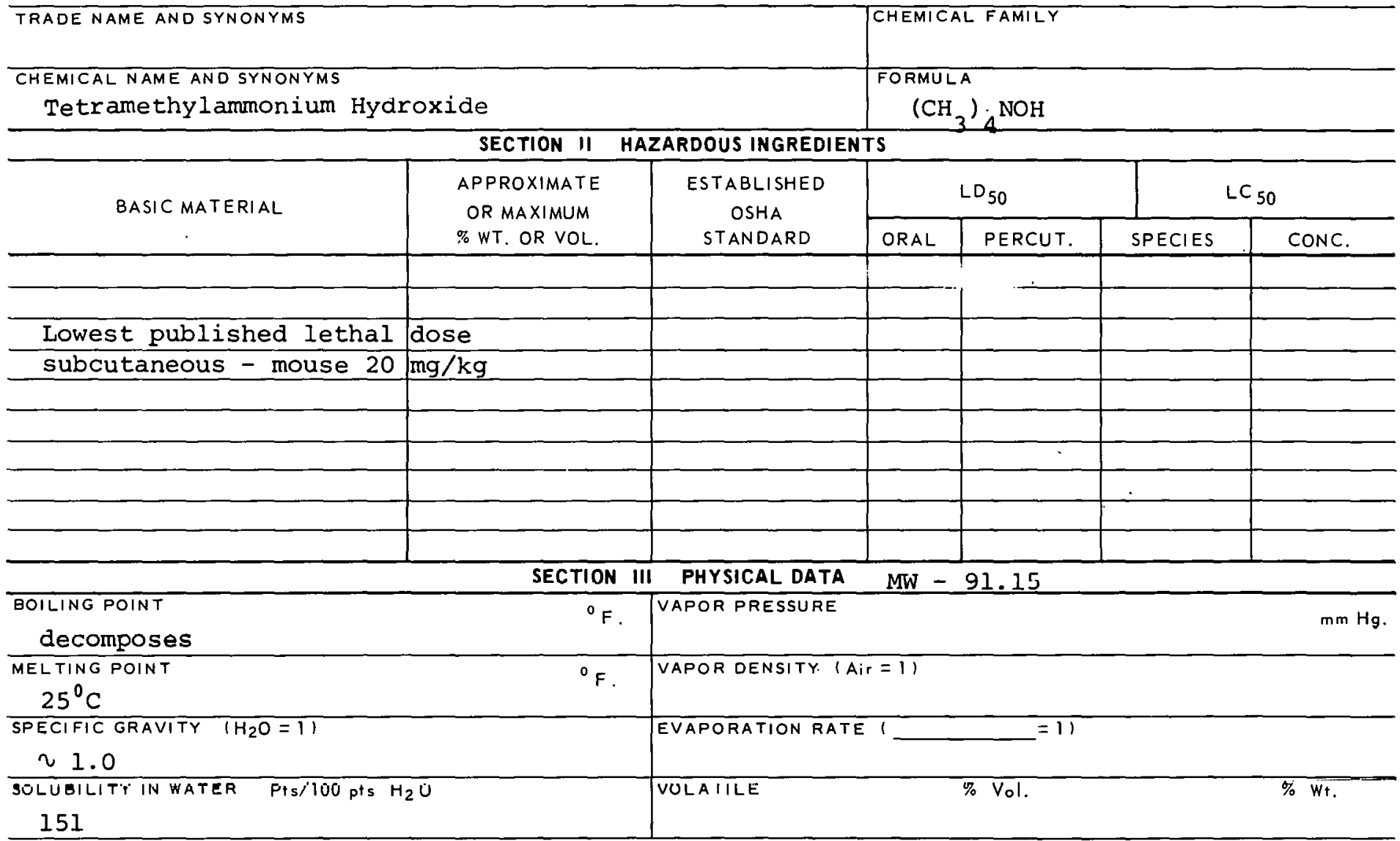
APPEARANCE AND ODOR

a liquid

\begin{tabular}{l|c|l}
\hline \multicolumn{3}{c}{ SECTION IV FIRE AND EXPLOSION HAZARD DATA } \\
\hline $\begin{array}{l}\text { FLASH POINT } \\
\text { none available }\end{array}$ & $\begin{array}{c}\text { FLAMMABLE } \\
\text { (EXPLOSIVE) } \\
\text { LIMITS }\end{array}$ & UPPER \\
\cline { 1 - 2 } & LOWER & \\
\hline
\end{tabular}

EXTINGUISHING MEDIA

Water, carbon dioxide, dry chemical.

SPECIAL FIRE FICHTINC PROCEDURES

Wear full protective clothing.

UNUSUAL FIRE AND EYPLOSION HAZAADE 


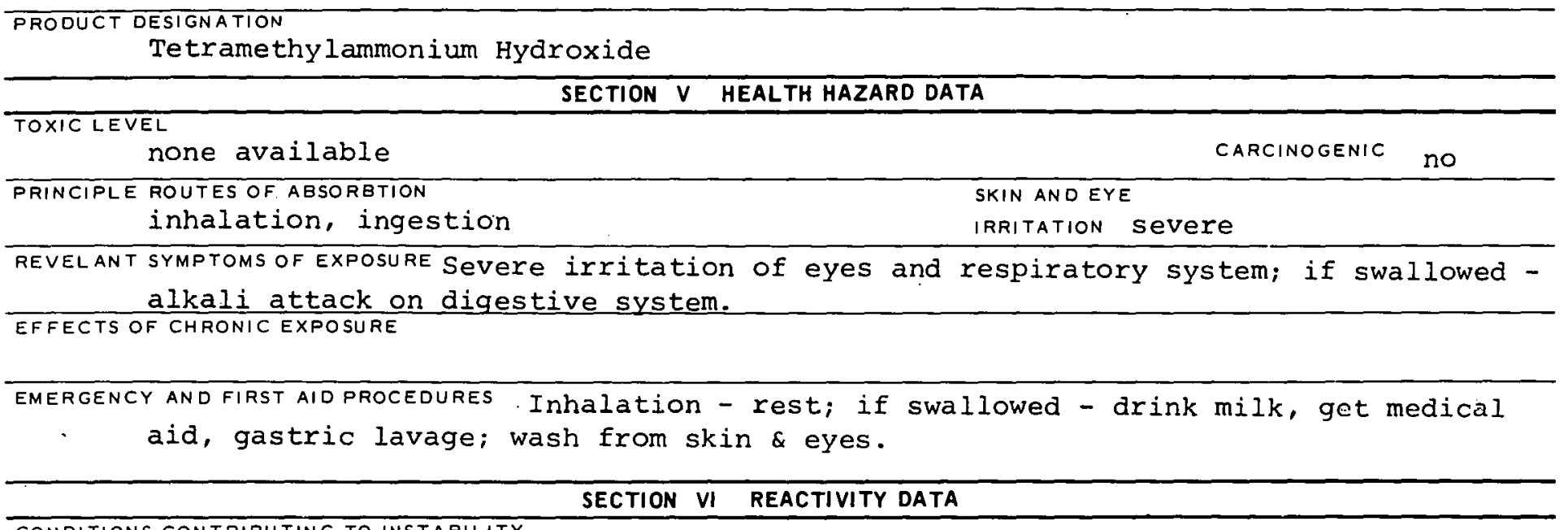

CONDITIONS CONTRIBUTING TO INSTABILITY

CONDITIONS CONTRIBUTING TO HAZARDOUS POLYMERIZATION

INCOMPATAGILITY (MATERIALS TO AVOID)

HAZARDOUS DECOMFOSITION FRODUCTS

SECTION VII SPILL OR LEAK PROCEDURES

STEPS TO BE TAKEN IN CASE MATERIAL IS RELEASED OR SPILLED

WASTE DISPOSAL METHOO

SECTION VIII SPECIAL PROTECTION INFORMATION

\begin{tabular}{l|l}
\hline VENTILATION REQUIREMENTS LOCAL EXHAUST & $\begin{array}{l}\text { PROTECTIVE EOUIPMENT (SPECIFY TYPES) EYE } \\
\text { GOgGIEC }\end{array}$ \\
\hline MECHANICAL IGENERALI & GLOVES \\
\hline SPECIAL & RESPIRATOR \\
& Self-contained \\
\hline
\end{tabular}

OTHER PROTECTIVE EQUIPMENT

Self-contained

SECTION IX SPECIAL PRECAUTIONS

PRECAUTIONS TO BE TAKEN IN HANDLING AND STORAGE

OTHER PRECAUTIONS

SIGNATURE

DATE

$365-6009 B$ 
CAS : 000075581

\section{PRODUCT DESIGNATION}

03-365-6109

Tetramethylammonium Iodide
PA10500

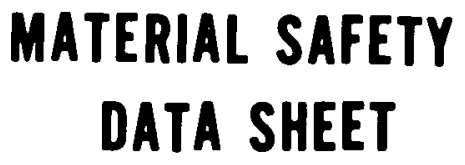

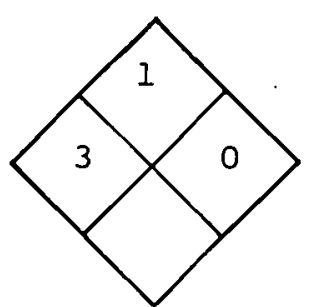

\section{SECTION I SOURCE AND NOMENCLATURE}

MANUFACTURER'S NAME

ADDRESS (NUMBER, STREET, CITY, STATE, ZIP CODE)

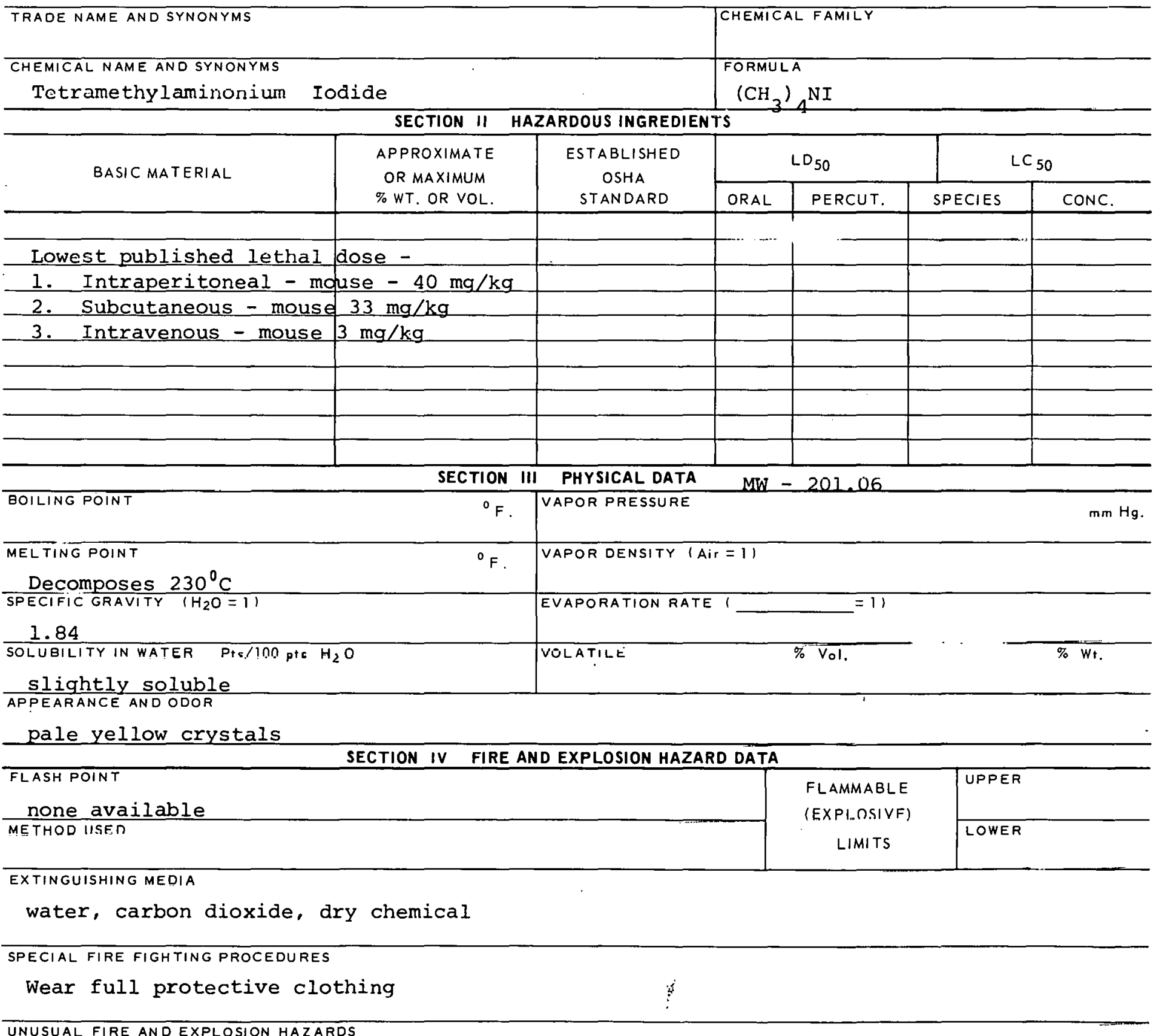

UNUSUAL FIRE AND EXPLOSION HAZARDS 
Tetramethylammonium Iodide

\section{SECTION $V$ HEALTH HAZARD DATA}

TOXIC LEVEL

none available

PRINCIPLE ROUTES OF ABSORBTION

inhalation, ingestion

CARCINOGENIC

SKIN AND EYE

IRRITATION moderate

REVELANT SYMPTOMS OF EXPOSURE Nausea, vomiting, diarrhea, slurred speech, visual

disturbances, drowsiness, coma.

EFFECTS OF CHRONIC EXPOSURE

Neurotoxic effects.

EMERGENCY AND FIRST AID PROCEDURES Tnhalation - rest; if swallowed - induce vomiting, get medical aid; wash from skin \& eyes.

\section{SECTION VI REACTIVITY DATA}

CONDITIONS CONTRIBUTING TO INSTABILITY

GONDITIONS CONTRIBUTING TO HAZARDOUS POLYMERIZATION

INCOMPATABILITY (MATERIALS TO AVOID)

HAZARDOUS DECOMPOSITION PRODUCTS

SECTION VII SPILL OR LEAK PROCEDURES

STEPS TO BE TAKEN IN CASE MATERIAL' IS RELEASED OR SPILLED

WASTE DISPUSAL METHOD

SECTION VIII SPECIAL PROTECTION INFORMATION

\begin{tabular}{l|l}
\hline VENTILATION REQUIREMENTS LOCAL EXHAUST & $\begin{array}{l}\text { PROTECTIVE EQUIPMENT (SPECIFY TYPES) EYE } \\
\text { GoggleS }\end{array}$ \\
\hline MECHANICAL IGENERAL) & GLOVES \\
\hline SPECIAL & $\begin{array}{l}\text { RESPIRATOR } \\
\text { With proper filter }\end{array}$ \\
\hline
\end{tabular}

OTHER PROTECTIVF, EQUIPMENT

SECTION IX SPECIAL PRECAUTIONS

PRECAUTIONS TO BE TAKEN IN HANDLING AND STORAGE

OTHER PRECAUTIONS

GIFNATURE

DATE

$365-6109 B$ 


\begin{tabular}{|c|}
\hline PRODUCT DESIGNATION \\
\hline
\end{tabular}

SECTION I SOURCE AND NOMENCLATURE

MANUFACTURER'S NAME
ADORESS INUMBER, STREET, CITY, STAT
TRADE NAME AND SYNONYMS
CHEMICAL NAME AND SYNONYMS
1,4 DiChlorobutane

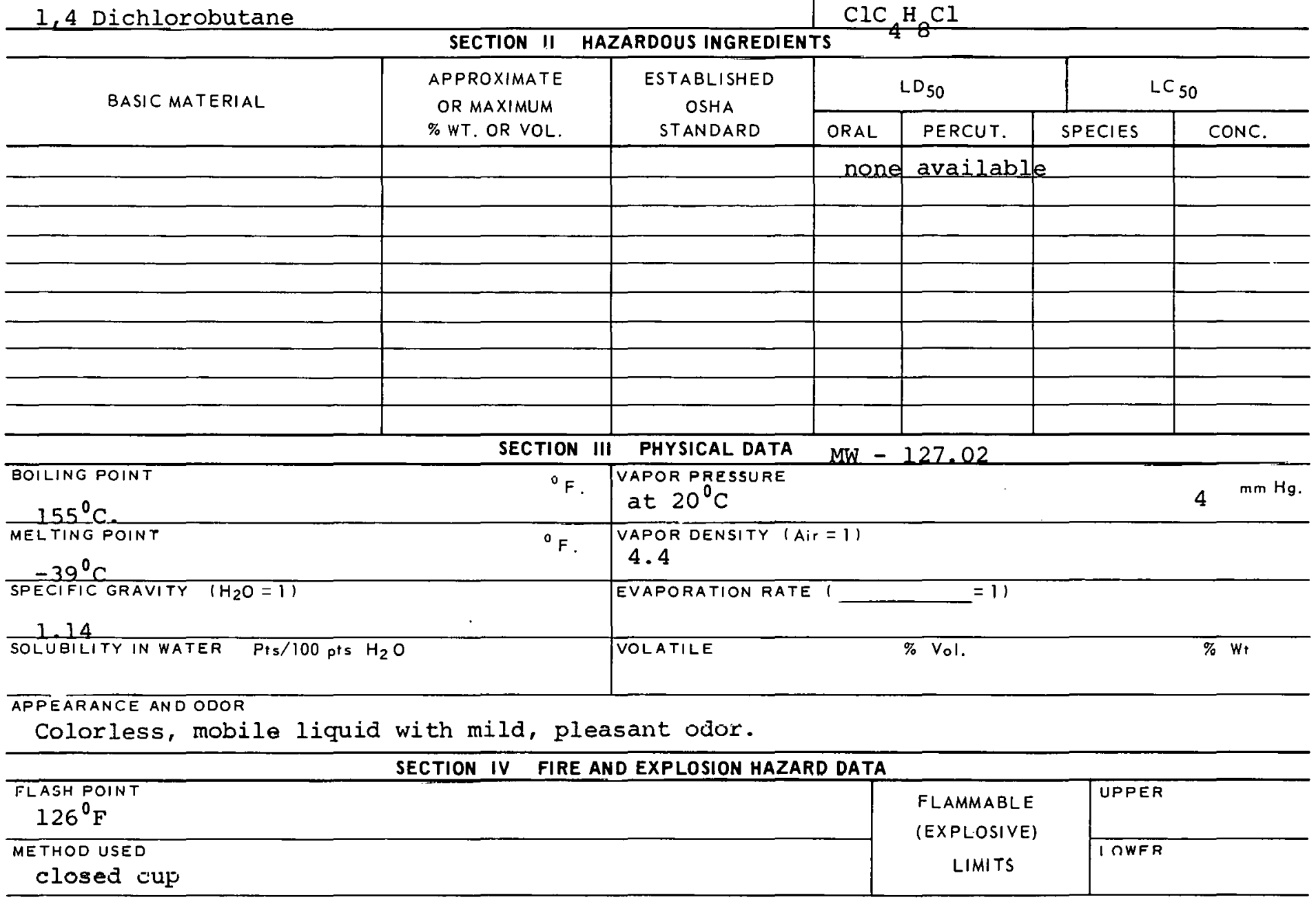

EXTINGUISHING MEDIA

carbon dioxide, ary chemical

SPECIAL FIRE FIGHTING PROCEDURES

Water may be ineffective.

UNUSUAL FIRE ANO EXPLOSION HAZARDS
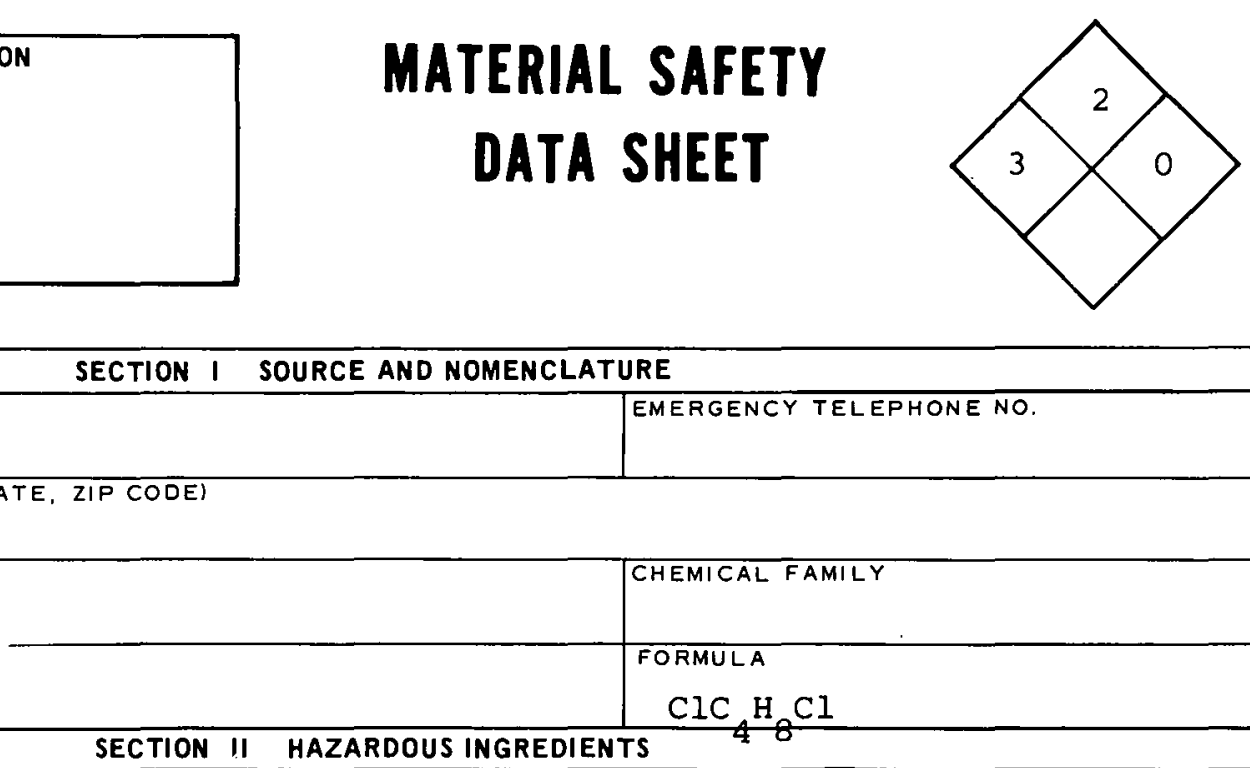

EMERGENCY TELEPHONE NO. 


\begin{tabular}{ccc}
\hline & SECTION $V$ HEALTH HAZARD DATA & \\
\hline $\begin{array}{c}\text { TOXIC LEVEL } \\
\text { none available }\end{array}$ & SKIN ANDEYE \\
\hline $\begin{array}{c}\text { PRINCIPLE ROUTES OF ABSORBTION } \\
\text { Inhalation, ingestion }\end{array}$ & IRRITATION moderate \\
\hline
\end{tabular}

REVELANT SYMPTOMS OF EXPOSURE

Eye irritation, headache, vomiting, drowsiness, narcosis.

EFFECTS OF CHRONIC EXPOSURE

Dermatitis, liver and kidney damage.

EMERGENCY AND FIRST AID PROCEDURES

Inhalation - rest; if swallowed - give emetic, get medical aid; wash from skin \& eyes.

SECTION VI REACTIVITY DATA

CONDITIONS RONTRIBUTING TO INSTABILITY

COHDITIONS IONTRIRIITING TQ HAZARDOUS POLYME RI ZATION

INCOMPATABILITY (MATERIALS TO AVOID)

HAZARDOUS DECOMPOSITION PRODUCTS

SECTION VII SPILL OR LEAK PROCEDURES

STEPS TO BE TAKEN IN CASE MATERIAL IS RELEASED OR SPILLED

Wear self-contained breathing apparatus.

WASTE DISFOSAL METHOO

SECTION VIII SPECIAL PROTECTION INFORMATION

\begin{tabular}{l|l}
\hline VENTII ATION REQUIREMENTS LOCAL EXHAUST & $\begin{array}{l}\text { PROTECTIVE EQUIPMENT (SPECIFY TYPES) EYE } \\
\text { GoggleS }\end{array}$ \\
\hline MECHANICAL IGENERAL) & $\begin{array}{l}\text { GLOVES } \\
\text { Rubber }\end{array}$ \\
\hline SPECIAL & $\begin{array}{l}\text { PESPIFATOR } \\
\text { With proper filter }\end{array}$ \\
\hline
\end{tabular}

OTHER PROTECTIVE EQUIPMENT

SECTION IX SPECIAL PRECAUTIONS

PRECAUTIONS TO BE TAKEN IN HANDLING AND STORAGE

OTHER PRECAUTIONS

SIGNATURE

\begin{tabular}{l} 
DATE \\
\hline
\end{tabular}

$365-7309 B$ 
CAS : 000062555

AC89250

\begin{tabular}{l|}
\multicolumn{1}{c}{ PRODUCT DESIGNATION } \\
$\begin{array}{l}\text { 03-365-8509 } \\
\text { Thioacetamide }\end{array}$ \\
MATERIAL SAFETY SHEET
\end{tabular}

SECTION I SOURCE AND NOMENCLATURE

MANUFACTURER'S NAME

ADORESS (NUMBER, STREET, CITY, STATE, ZIP CODE)

TRADE NAME AND SYNONYMS

\begin{tabular}{l|l} 
& CHEMICAL FAMILY \\
& $\begin{array}{l}\text { FORMULA } \\
\mathrm{CH}_{3} \mathrm{CSNH}_{2}\end{array}$ \\
\hline
\end{tabular}

Thioacetamide

EMERGENCY TELEPHONE NO.

CHEMICAL NAME AND SYNONYMS

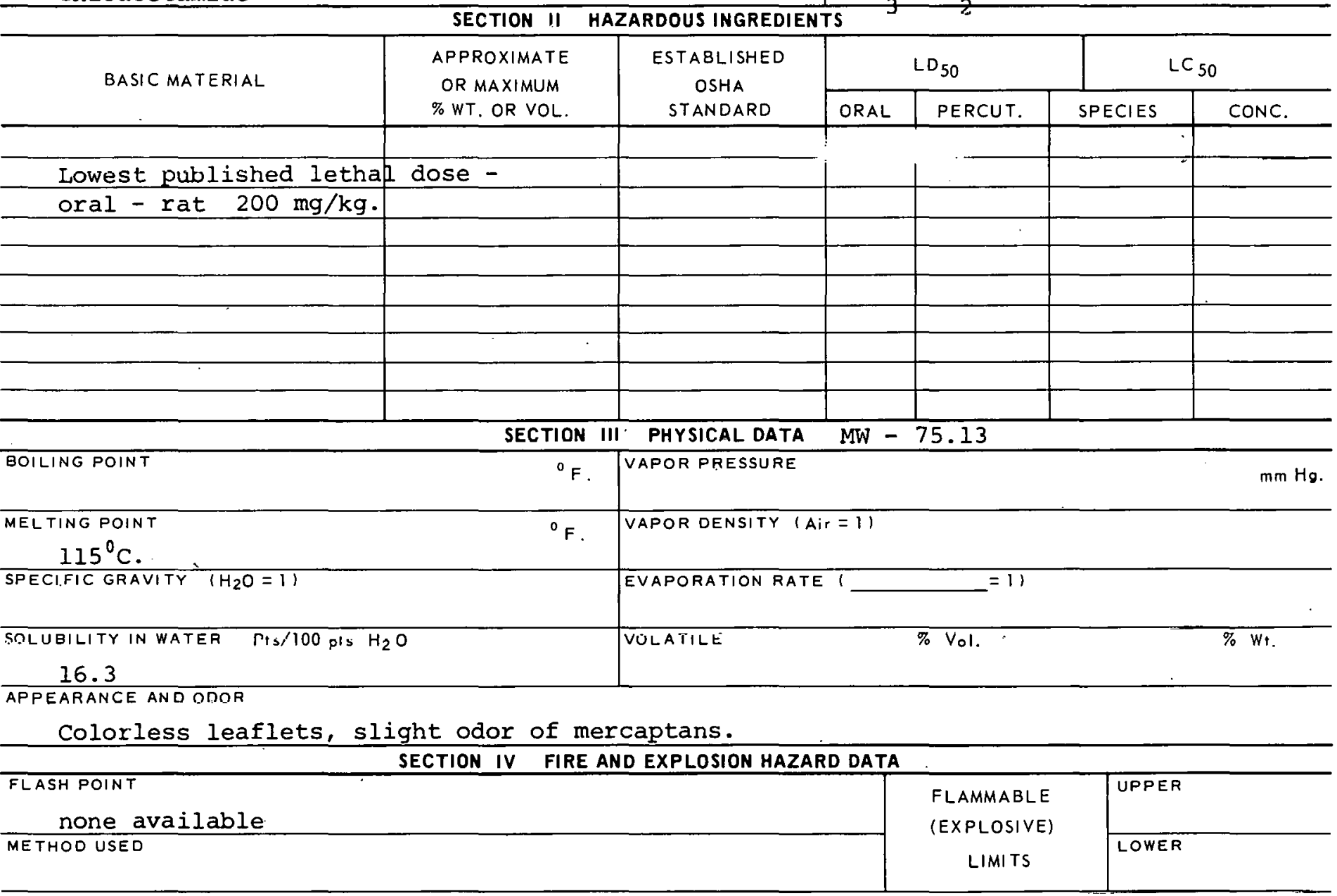

EXTINGUISHING MEDIA

Carbon dioxide, dry chemical.

SPECIAL FIRE FIGHTING PROCEDURES

UNUSUAL FIRE AND FXPI RSION HAZAROS 
Thioacetamide

SECTION V HEALTH HAZARD DATA

\section{TOXICLEVEL}

None available

PRINCIPLE ROUTES OF ABSORBTION

Inhalation, ingestion

CARCINOGENIC SKIN AND EYE

IRRITATION SIight

REVELANT SYMPTOMS OF EXPOSURE

Drowsiness, fatique, nausea, acidosis, skin eruptions.

EFFECTS OF CHRONIC EXPOSURE

Liver damage

EMERGENCY AND FIRST AID PROCEDURES

inhalation - rest.

If swallowed - induce vomiting, get medical aid;

SECTION VI REACTIVITY DATA

CONDITIONS CONTRIBUTING TO INSTABILITY

CON DITIONS CONTRIQUTING TO HAZARDOUS POLYMERIZATION

INCOMPATABILITY (MATERIALS TO AVOID)

HAZARDOUS DECOMPOSITION PRODUCTS

SECTION VII SPILL OR LEAK PROCEDURES

STEPS TO BE TAKF,N IN CASE MATERIAL IS RELEASED OR SPILLED

WASTE DISPOSAL METHOD

SECTION VIII SPECIAL PROTECTION INFORMATION

\begin{tabular}{l|l|l}
\hline \multicolumn{1}{c}{ SECTION VIII SPECIAL PROTECTION INFORMATION } \\
\hline VENTILATION REQUIREMENTS LOCAL EXHAUST & $\begin{array}{c}\text { PROTECTIVE EQUIPMENT (SPECIFY TYPES) EYE } \\
\text { Goggles }\end{array}$ \\
\hline MECHANICAL IGENERAL) & $\begin{array}{c}\text { RLOVES } \\
\text { Rubber }\end{array}$ \\
\hline SPECIAL & FEERIRATOR \\
\hline
\end{tabular}

OTHER PROTECTIVE EQUIPMENT

SECTION IX SPECIAL PRECAUTIONS

PRECAUTIONS TO BE TAKEN IN HANDLING AND STORAGE

OTHER PRECAUTIONS

SIGNATURE

DATE

$365-8509 B$ 
CAS : 007719097

XM51500

\begin{tabular}{l|}
\hline PRODUCT DESIGNATION \\
03-365-9309 \\
Thionyl Chloride
\end{tabular}

SECTION I SOURCE AND NOMENCLATURE

\section{TRADE NAME AND SYNONYMS \\ Sulfurous Oxychloride \\ CHEMICAL NAME AND SYNONYMS \\ Thionyl Chloride}

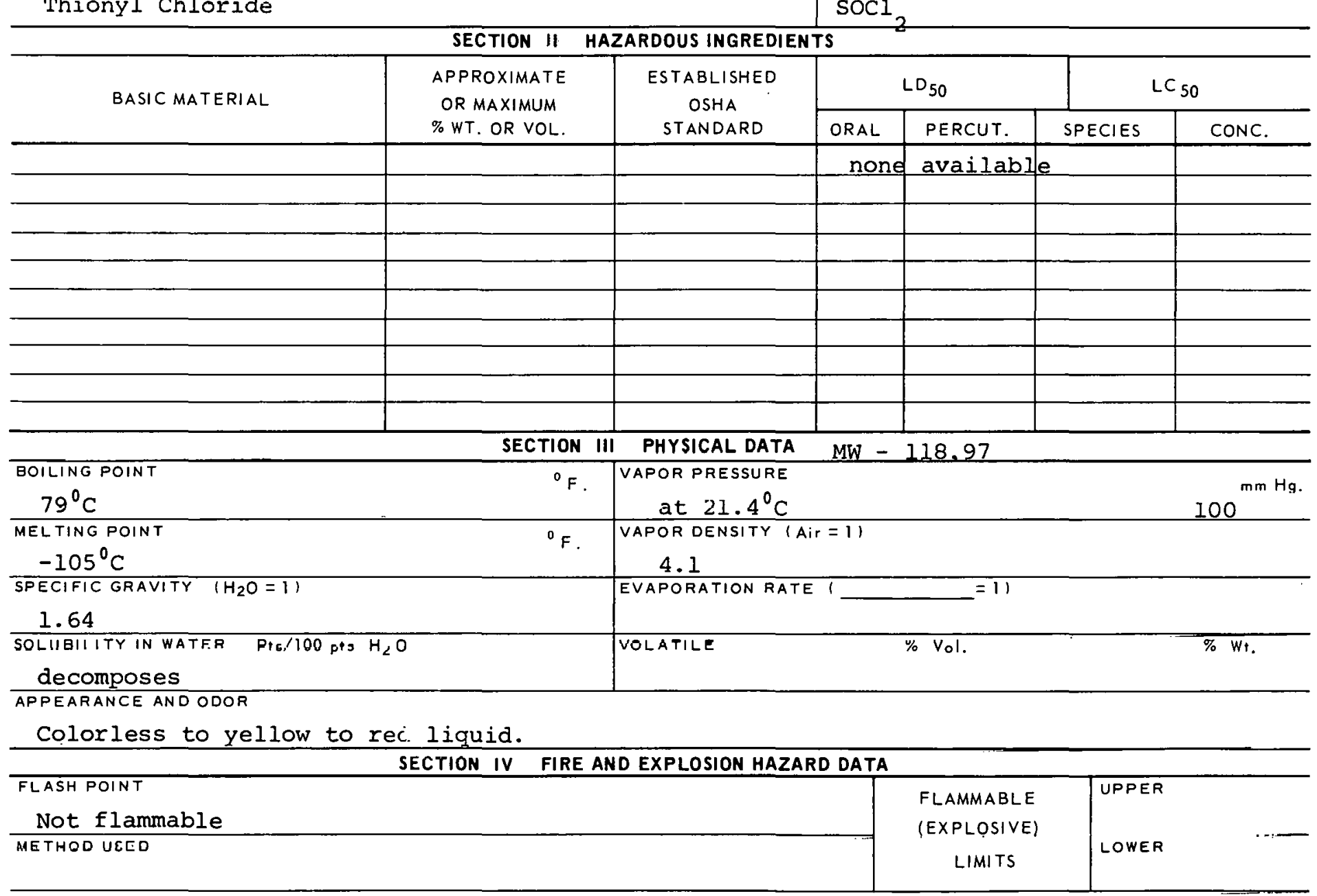

FXTINGIIISHING MEDIA

SPECIAL FIRE FIGHTING PROCEDURES

CHEMICAL FAMILY
FORMULA
SOCl $_{2}$

m H.

$$
\text { - }
$$

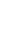


TOXIC LEVEL

none available

PRINCIPLE ROUTES OF ABSORBTION

inhalation, ingestion

CARCINOGENIC

\begin{tabular}{ll} 
CARCINOGENIC no \\
SKIN ANDEYE \\
IRRITATION \\
\hline
\end{tabular}

REVELANT SYMPTOMS OF EXPOSURE Irritation of skin, eyes, mucous membranes and respiratory tract.

EFFECTS OF CHRONIC EXPOSURE

$--$

EMERGENCY AND FIRST AID PROCEDURES Inhalation - rest; if swallowed - drink water, milk of magnesia, get medical aid; wash skin \& eyes.

\section{SECTION VI REACTIVITY DATA}

CONDITIONS CONTRIBUTING TO INSTABILITY

TONDITIONS CONTRIBUTING TO HAZ AROOUS POLYMERIZATION

INCOMPATABILITY (MATERIALS TO AVOID)

Watex

HAZARDOUS DECOMPOSITION PRODUCTS

Reacts with water to produce $\mathrm{HCl}$ and $\mathrm{SO}$.

SECTION VII SPILL OR LEAK PROCEDURES

STEPS TO EE TAKEN IN CASE MATERIAL IS RELEASED OR SPILLED

Cover with bicarbonate

WASTE DISPOSAL. METHOD

SECTION VIII SPECIAL PROTECTION INFORMATION

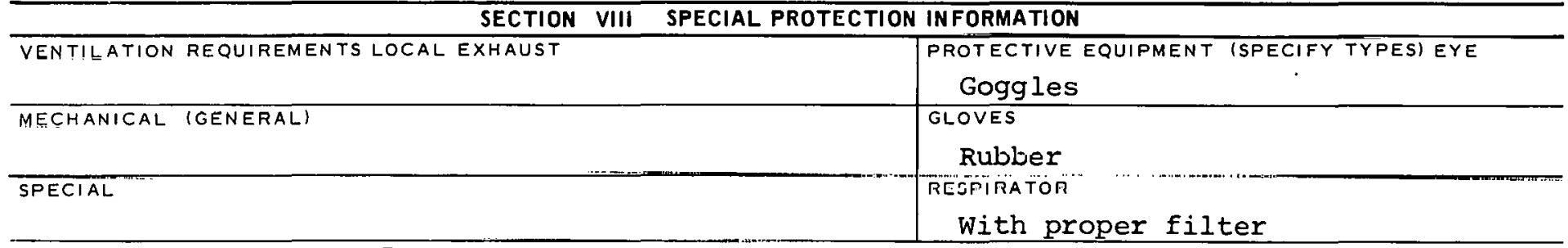

OTHER PROTECTIVE EQUIPMENT

Protective clothing

SECTION IX SPECIAL PRECAUTIONS

PRECAUTIONS TO BE TAKEN IN HANDLING AND STORAGE

QTHER PRECAUTIONS

SIGNATURE

OATE

.

$365-9309 B$ 


\section{PRODUCT DESIGNATION}

03-365-9709

Thiophenol
MATERIAL SAFETY DATA SHEET

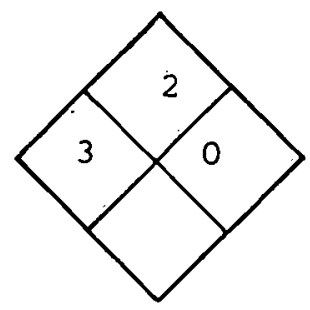

\section{SECTION I SOURCE AND NOMENCLATURE}

\section{MANUFACTURER'S NAME \\ ADDRESS (NUMBER, JTREET, CITY, STATE, ZIP CODE) \\ TRADE NAME AND SYNONYMS \\ Phenyl Mercaptain CHEMICAL NAME AND SYNONYMS \\ Thiophenol}

EMERGENCY TELEPHONE NO.

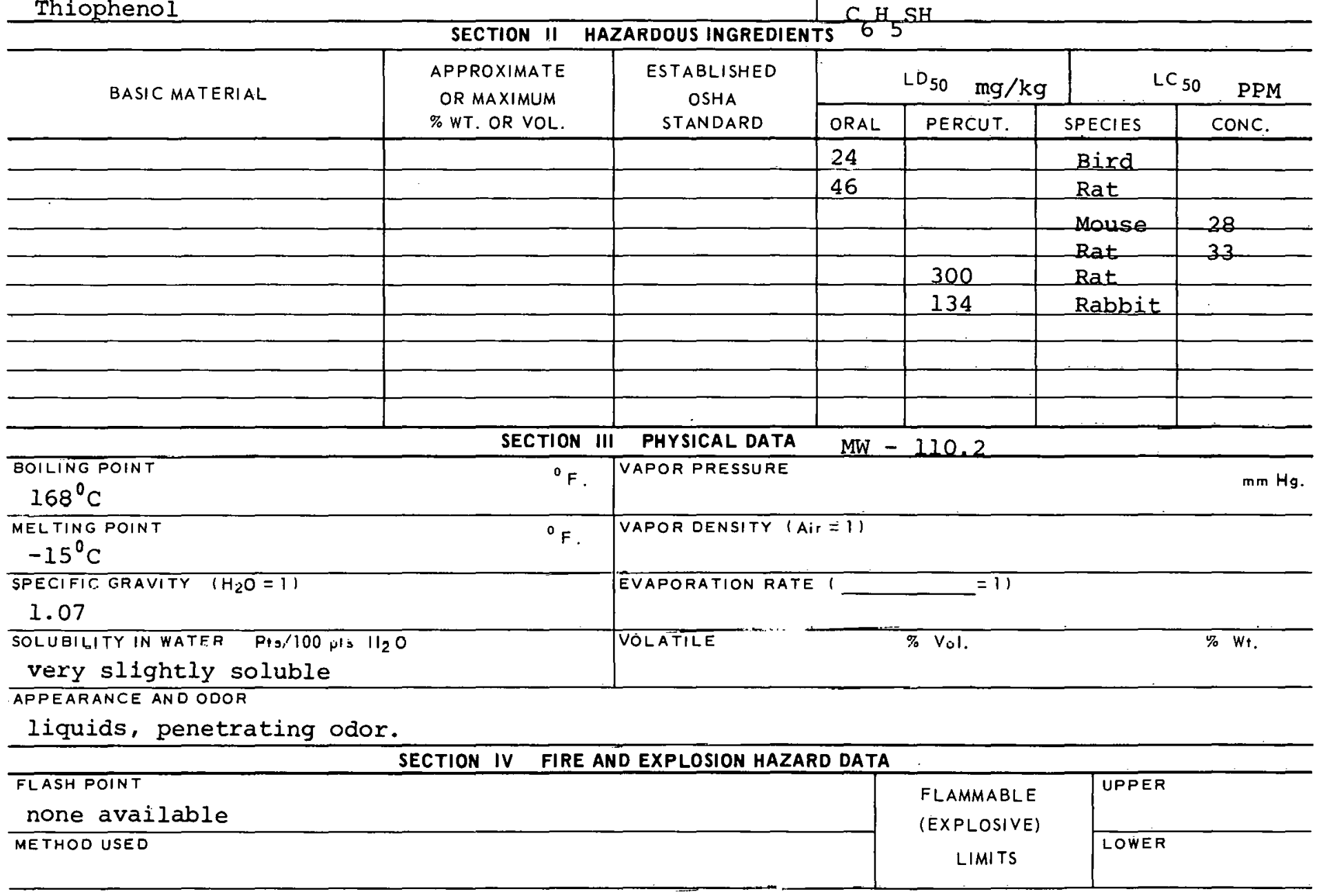

EXTINGUISHING MEDIA

carbon dioxide, dry chemical.

SPECIAL FIRE FIGHTING PROCEDURES

UNUSUAL FIRE AND EXPLOSION HAZARMS 
TOXICLEVEL

none available

PRINCIPLE ROUTES OF ABSORBTION inhalation, ingestion

REVELANT SYMPTOMS OF EXPOSURE Skin and mucous membrane irritation, headache, dizziness, dermatitis.

EFFECTS OF CHRONIC EXPOSURE

$--$

EMERGENCY ANO FIRST AID PROCEOURES Inhalation - artificial respiration as necessary; if swallowed - induce vomiting, get medical aid, gastric lavage; wash skin \& eyes.

SECTION VI REACTIVITY DATA

CONDITIONS CONTRIBUTING TO INSTABILITY

CONDITIONS CONTRIBUTING TO HAZARDOUS POLYMERIZATION

INCOMPATABILITY (MATERIALS TO AVOID)

HAZARDOUS DECOMPOSITION PRODUCTS

SECTION VII SPILL OR LEAK PREOCEDURES

STEPS TO BE TAKEN IN CASE MATERIAL IS RELEASED OR SPILLED

\section{WASTE DISPOSAL METHOD}

\section{SECTION VIII SPECIAL PROTECTION INFORMATION}

\begin{tabular}{l|l}
\hline VENTILATION REQUIREMENTS LOCAL EXHAUST & $\begin{array}{l}\text { PROTECTIVE EQUIFMENT (SPECIFY TYPES) FYYE } \\
\text { GUYYIES }\end{array}$ \\
\hline MECHANICAL IGENERAL) & GLOVES \\
& Rubber \\
\hline SHELIAL & nLCOIMATOA \\
& With proper filter
\end{tabular}

OTHER PROTECTIVE EQUIPMENT

SECTION IX SPECIAL PRECAUTIONS

PRFCAIITIONS TO BE TAKEN IN HANDLING AND STORAGE

OTIIER TRECAUIIUNS

SIGNATURE

DATL 
CAS : 000079196

VT42000

PRODUCT DESIGNATION

03-366-0401

Thiosemicarbazide

MATERIAL SAFETY

DATA SHEET

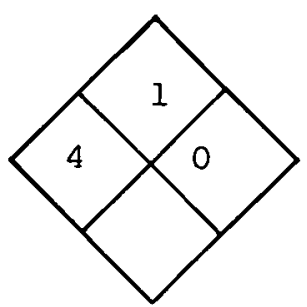

SECTION I SOURCE AND NOMENCLATURE

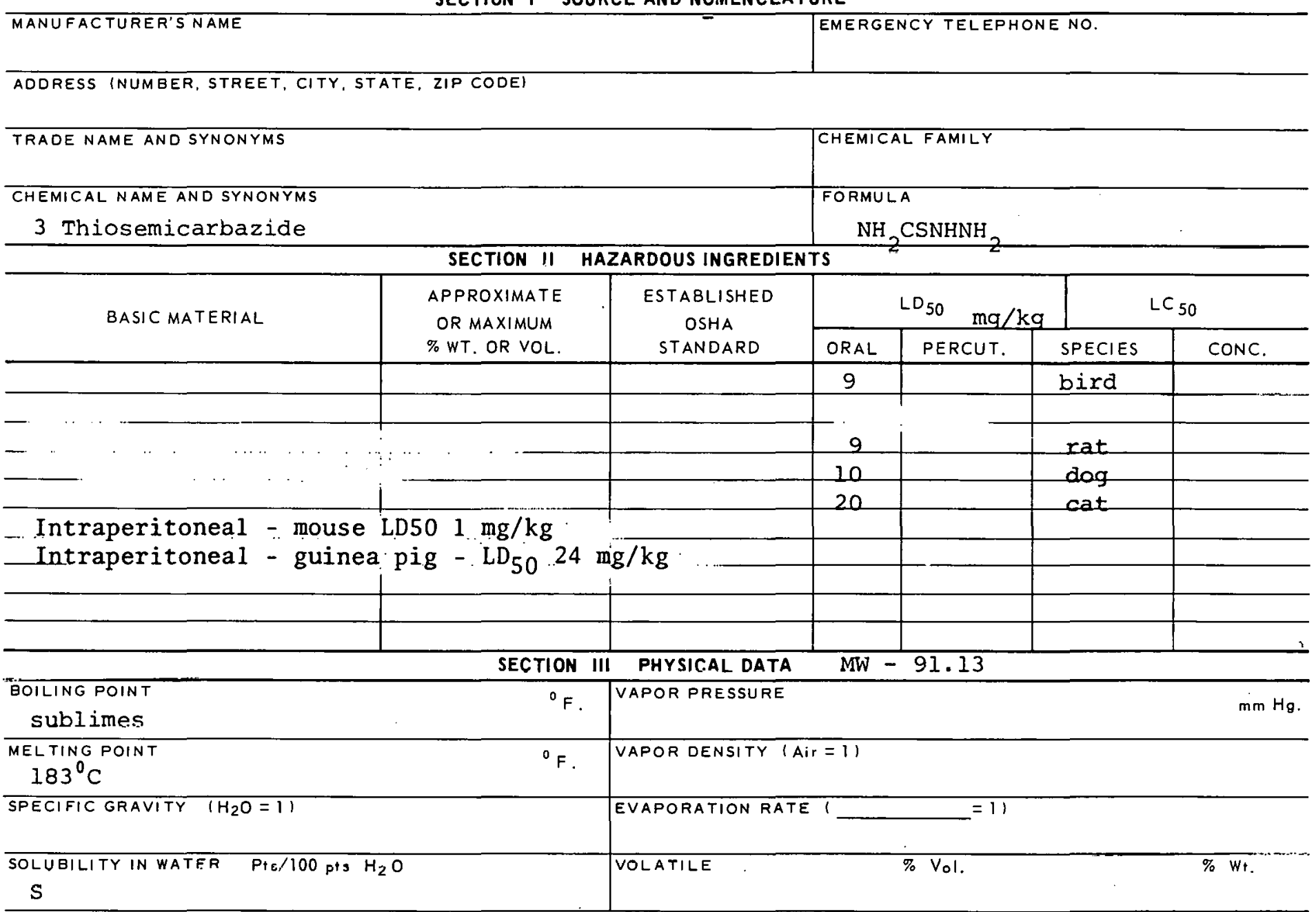

APPEARANCE AND ODOR

SECTION IV FIRE AND EXPLOSION HAZARD DATA

\begin{tabular}{|c|c|c|}
\hline $\begin{array}{l}\text { FLASH POINT } \\
\text { none available }\end{array}$ & \multirow{2}{*}{$\begin{array}{c}\text { FLAMMABLE } \\
\text { (EXPLOSIVF) } \\
\text { LIMITS }\end{array}$} & UPPER \\
\hline METHOD USED & & LOWER \\
\hline
\end{tabular}

EXTINGUISHING MEDIA

Alcohol foam, carbon dioxide, dry chemical.

SPECIAL FIRE FIGHTING PROCEDIIRES

UNUSUAL FIRE AND EXPLOSIQN HAZARDS 


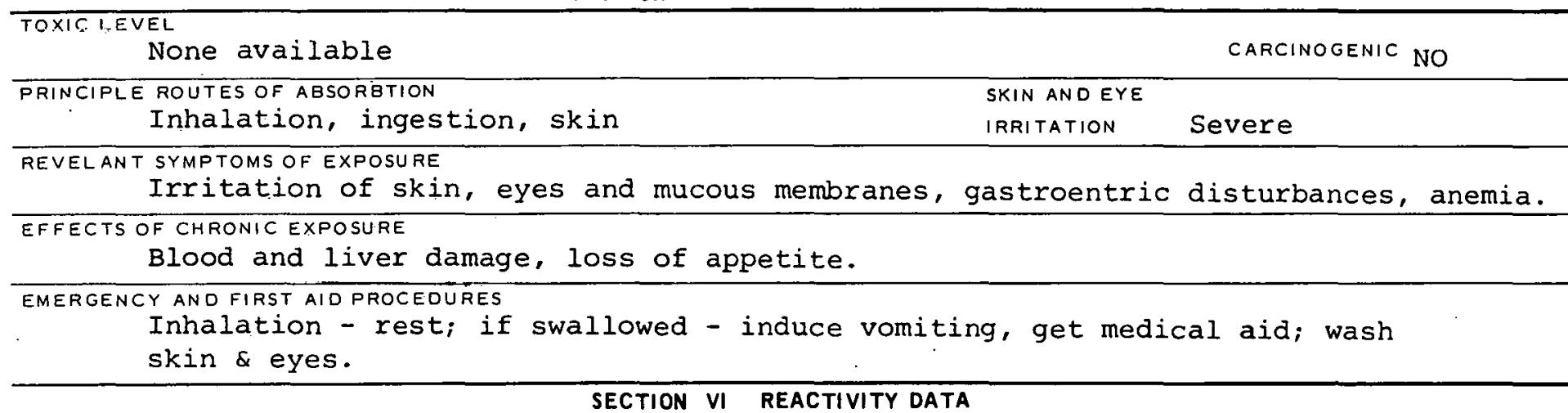

TINNITIONS CONTRIBUTING TO INSTABILITY

TONMITIONS CONTRIBUTING TO HAZARDOUS POLYMERIZATION

INCOMPATABILITY (MATERIALS TO AVOID)

HAZARDOUS DECOMPOSITION PRODUCTS

SECTION VII SPILL OR LEAK PROCEDURES

STEPS TO BE TAKEN IN CASE MATERIAL IS RELEASED OR SPILLED

WASTE DISPOSAL METHOO

SECTION VIII SPECIAL PROTECTION INFORMATION

\begin{tabular}{|c|c|}
\hline VENTILATION REQUIREMENTS LOCAL EXHAUST & $\begin{array}{l}\text { PROTECTIVE EQUIPMENT (SPECIFY TYPES) EYE } \\
\text { GOggles }\end{array}$ \\
\hline MECHAN!CAL (GENERAL) & Rubber \\
\hline$\overline{S P E C I A L}$ & $\begin{array}{l}\text { RESPIRATOR } \\
\text { Self-contained }\end{array}$ \\
\hline
\end{tabular}

OTHER PROTECTIVE EQUIPMENT

SECTION IX SPECIAL PRECAUTIONS

PRECAUTIONS TO BE TAKEN IN HANDLING AND STORAGE

OTHER PRECAUTIONS

SIGNATURE

-

DATE

$366-0401 B$ 
CAS : 000062566

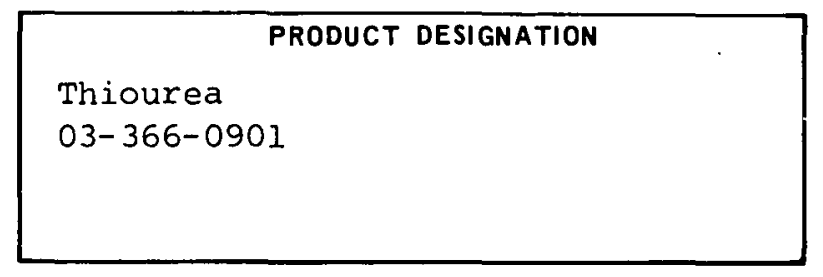

YU28000

\section{MATERIAL SAFETY \\ DATA SHEET}

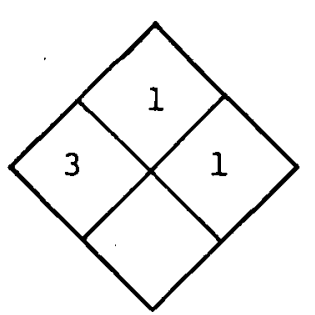

SECTION I SOURCE AND NOMENCLATURE

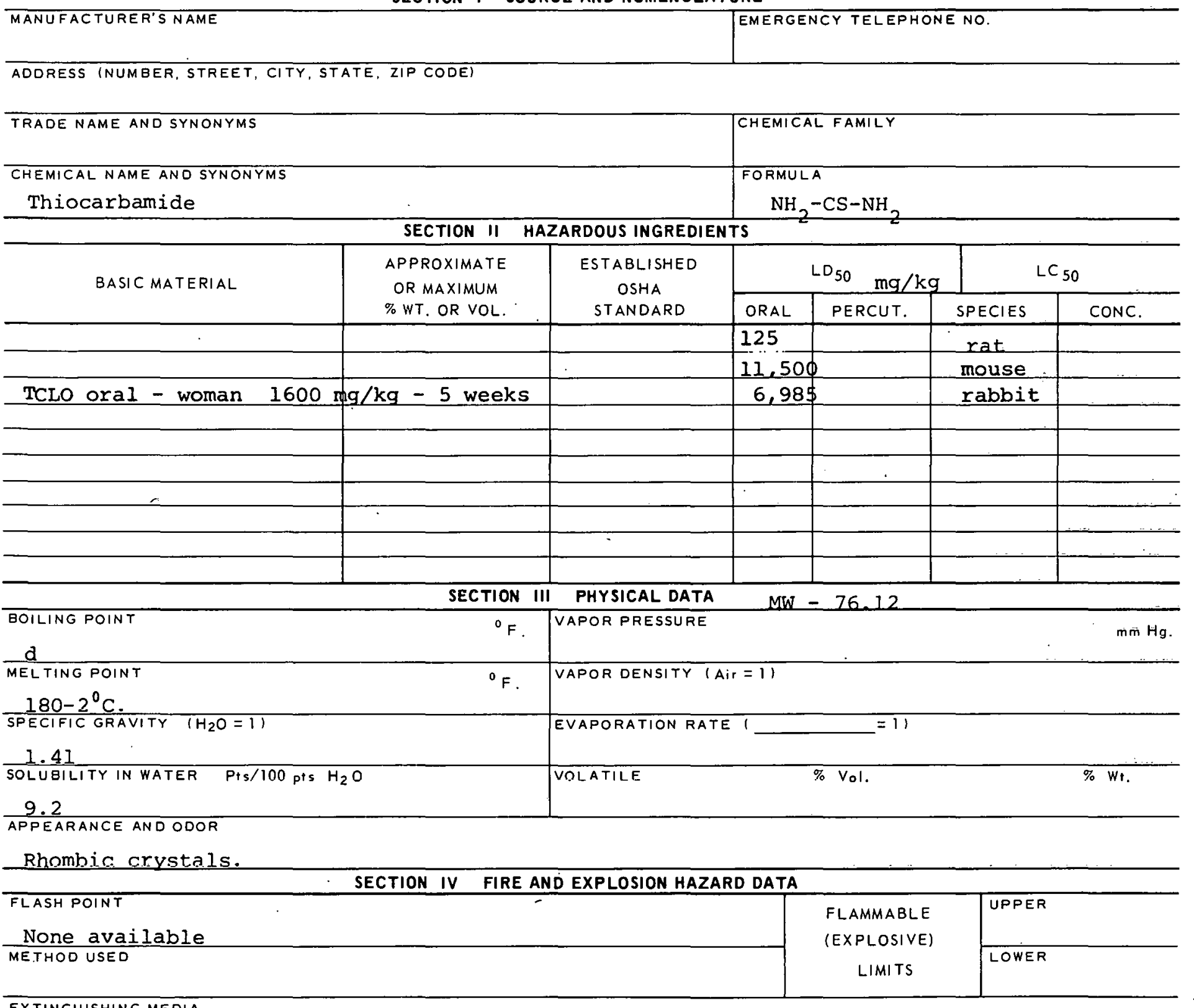

EXTINGUISHING MEDIA

Carbon dioxide, dry chemical.

SPECIAL FIRE FIGHTING PROCEDURES

UNUSUAL FIRE AND EXPLOSION HAZARDS

Burns to form $\mathrm{SO}_{2}$ fumes. 


\section{SECTION $V$ HEALTH HAZARD DATA}

TOXIC LEVE

None available

CARCINOGENIC

neoplastic

PRINCIPLE ROUTES OF ABSORBTION

SKIN AND EYE

Ingestion, inhalation of dust IRRITATION effects

REVELANT SYMPTOMS OF EXPOSURE

Dermatitis

EFFECTS OF CHRONIC EXPOSURE Ingestion - depression of bone marrow, anemia, leukopenia, allergic skin eruptions.

EMERGENCY AND FIRST AID PROCEOURES Inhalation - rest; if swallowed - induce vomiting, get medical aid; wash from skin \& eyes.

\section{SECTION VI REACTIVITY DATA}

CONDITIONS CONTRIBUTING TO INSTABILITY

TON MITIONS CON TRIBUTING TO HAZARDOUS POLYMERIZATION

INCOMPATABILITY (MATERIALS TO AVOID)

HAZARDOUS OECOMPOSITION PRODUCTS

Oxides of sulfur and nitrogen.

SECTION VII SPILL OR LEAK PROCEDURES

STEPS TO BE TAKEN IN CASE MATERIAL IS RELEASED OR SPILLED

WASTE DISPOSAL METHON

\section{SECTION VIII SPECIAL PROTECTION INFORMATION}

\begin{tabular}{|c|c|c|}
\hline VENTILATION REQUIREMENTS LOCAL EXHAUST & & $\begin{array}{l}\text { PROTECTIVE EQUIPMENT (SPECIFY TYPES) EYE } \\
\text { GOggIQS }\end{array}$ \\
\hline MECHANICAL (GENERAL) & & GLOVES \\
\hline SPECIAL & & RESPIRATOR \\
\hline \multicolumn{3}{|l|}{ OTHER PROTECTIVE EQUIPMENT } \\
\hline \multicolumn{3}{|c|}{ SECTION IX SPECIAL PRECAUTIONS } \\
\hline \multicolumn{3}{|c|}{ PRECAUTIONS TO BE TAKEN IN HANDLING AND STORAGE } \\
\hline \multicolumn{3}{|l|}{ OTHER PRECAUTIONS } \\
\hline SIGNATURE & DATE & \\
\hline
\end{tabular}



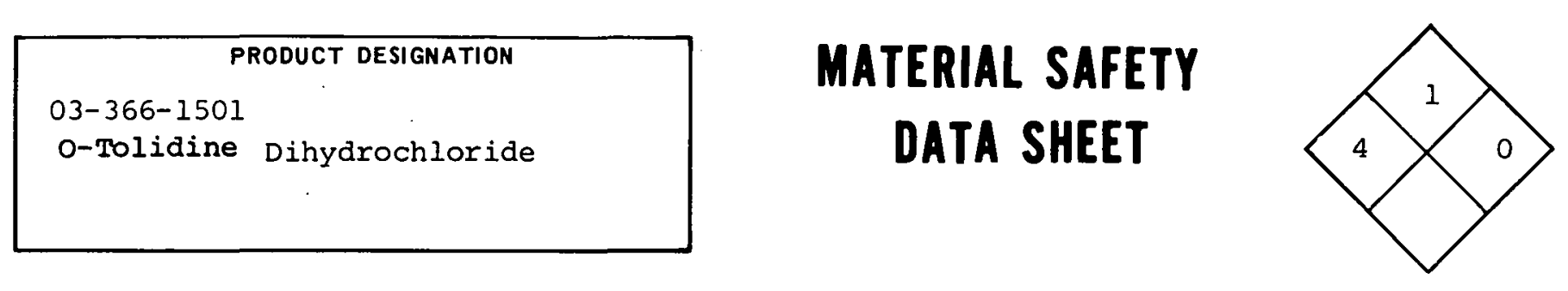

SECTION I SOURCE AND NOMENCLATURE

\begin{tabular}{l|l|l}
\hline MANU FAC TURER'S NAME & EMERGENCY TELEPHONE NO. \\
\hline ADORESS (NUMBER, STREET, CITY, STATE, ZIP CODE)
\end{tabular}

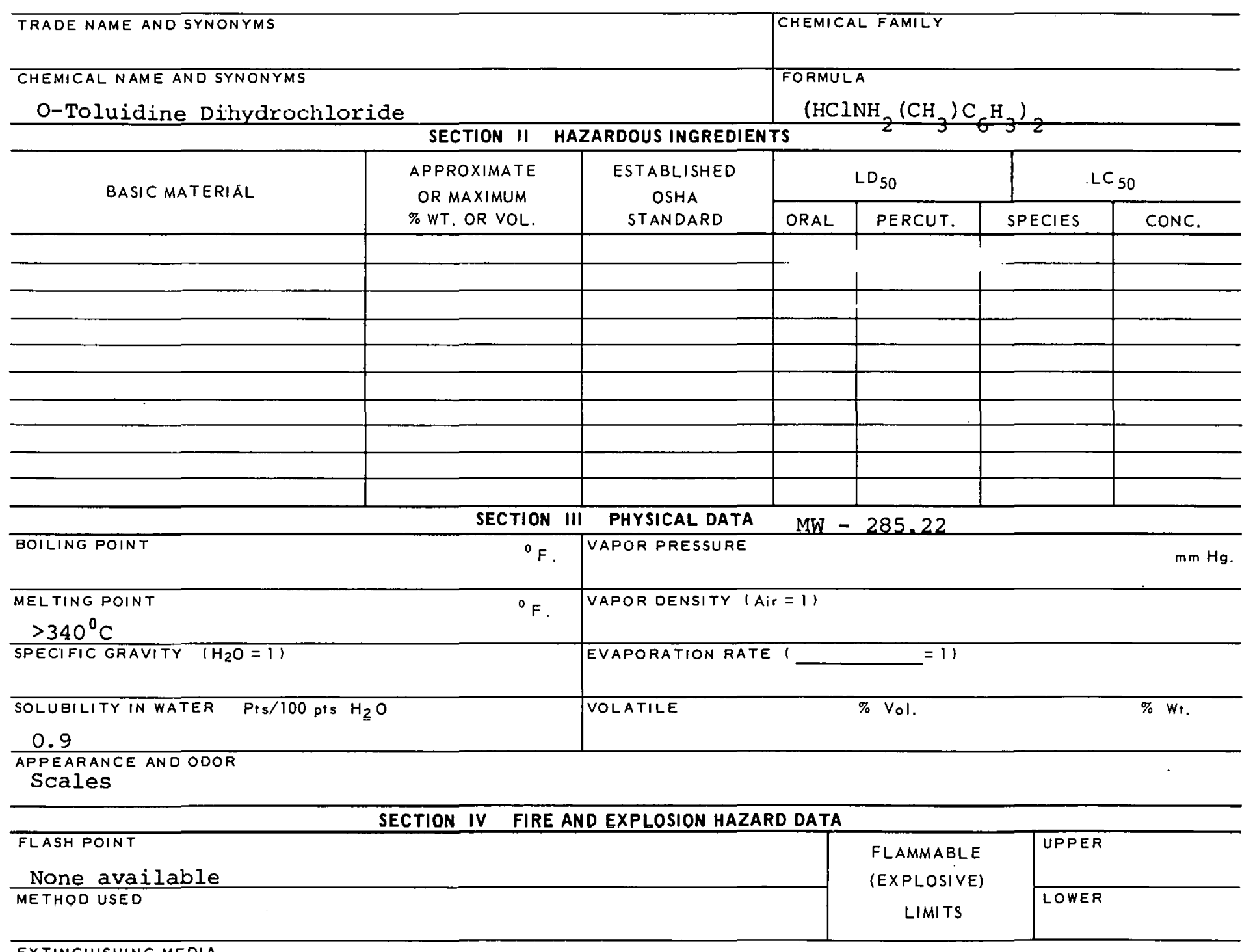

EXTINGUISHING MEOIA

Carbon dioxide, dry chemical.

SPECIAL FIRE FIGHTING PROCEDURES

Wear full protective clothing.

UNUSUAL FIRE ANDEXPLOSION HAZARDS 


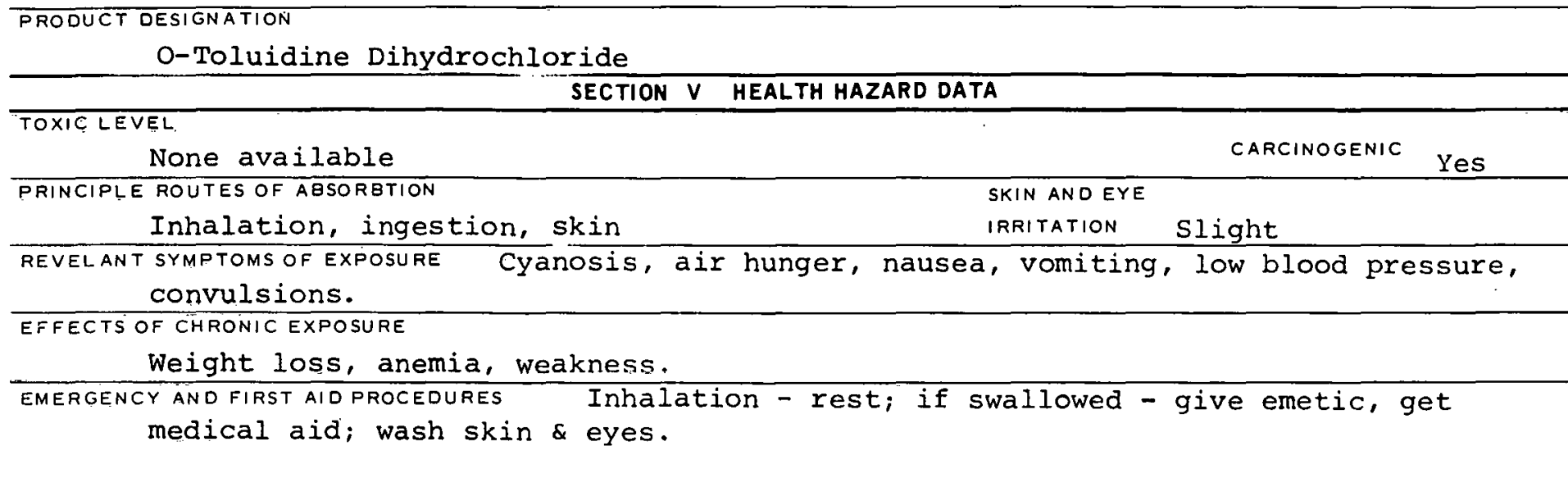

\begin{tabular}{l}
\hline SECTION VI REACTIVITY DATA \\
\hline CONDITIONS CONTRIBUTING TO INSTABILITY \\
CONOITIONS CONTRIBUTING TO HAZARDOUS POLYMERITATION \\
INCOMPATABILITY IMATERIALS TO AVOIO)
\end{tabular}


CAS : 000108883

\section{PRODUCT DESIGNATION}

To] uene

03-366-2401

03-366-2431

\section{MATERIAL SAFETY \\ DATA SHEET}

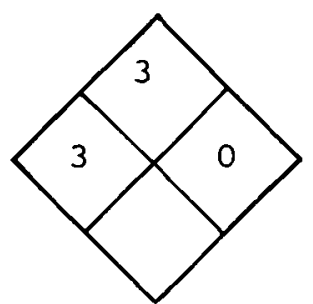

SECTION I SOURCE AND NOMENCLATURE

MANUFACTURER'S NAME

| EMERGENCY TELEPHONE NO.

ADDRESS (NUMBER, STREET, CITY, STATE, ZIP COOE)

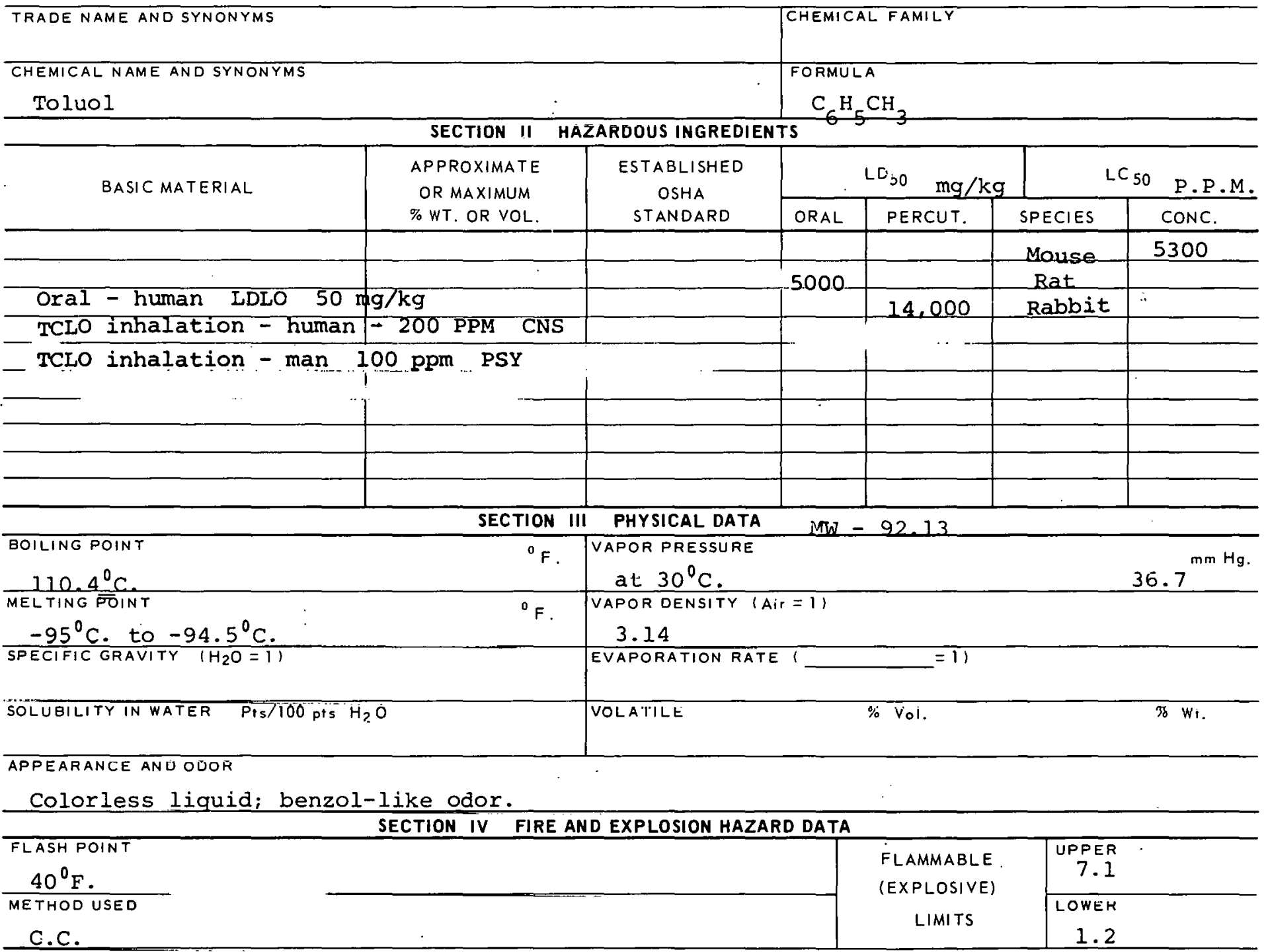

EXTINGUISHING MEDIA

Use carbon dioxide, dry chemical or foam. Water may be ineffective.

SPECIAL FIRE FIGHTING PROCEDURES

Wear goggles and self-contained breathing apparatus.

UNUSUAL FIRE ANDEXPLOBION HAZARDS

Vapors form explosive mixtures with air. 
TOXIC LEVEL TLV $100 \mathrm{PPM}, 375 \mathrm{mg} / \mathrm{C.M}$.

CARCINOGENIC

no

PRINCIPLE ROUTES OF ABSORBTION Inhalation of vapor, ingestion

SKIN ANDEYE

IRRITATION moderate

REVELANT SYMPTOMS OF EXPOSURE Dizziness, headache, nausea, vomiting, shallow, rapid respiration; paralysis, unconsciousness, convulsions, deleriums; tightness in chest.

EFFECTS OF CHRONIC EXPOSURE Headache, loss of appetite, drowsiness, nervousness, pallor, anemia, petechiae, and abnormal bleeding.

EMERGENCY AND FIRST AIO PROCEDURES Remove from exposure; give artificial respiration with oxygen, do not give epinephrine or ephedrine or related drugs. Obtain medical aid.

\begin{tabular}{|c|c|}
\hline SECTION VI & REACTIVITY DATA \\
\hline \multicolumn{2}{|l|}{ CONDITIONS CONTRIBUTING TO INSTABILITY } \\
\hline \multicolumn{2}{|l|}{ CONDITIONS CONTRIBU TING TO HAZARDOUS POLYMERIZATION } \\
\hline \multicolumn{2}{|c|}{$\begin{array}{l}\text { INCOMPATABILITY (MATERIALS TO AVOID) } \\
\text { Potentially hazardous reaction with mixture of nitric and sulfuric acids. }\end{array}$} \\
\hline \multicolumn{2}{|c|}{ HAZARDOUS DECOMPOSITION PRODUCTS } \\
\hline \multicolumn{2}{|c|}{ SECTION VII SPILL OR LEAK PROCEDURES } \\
\hline \multicolumn{2}{|c|}{$\begin{array}{l}\text { STEPS TO BE TAKEN IN CASE MATERIAL IS RELEASED OR SPILLED } \\
\text { Absorb on paper towels, evaporate in hood. }\end{array}$} \\
\hline \multicolumn{2}{|l|}{ WASTE DISPOSAL METHOD } \\
\hline \multirow{2}{*}{\multicolumn{2}{|c|}{\begin{tabular}{|c|c|} 
SECTION VIII SPECIAL PROTECTION INFORMATION \\
VENTILATION REQUIREMENTS LOCAL EXHAUST & $\begin{array}{l}\text { PROTECTIVE EQUIPMENT (SPECIFY TYPES) EYE } \\
\text { GOggleS }\end{array}$ \\
\end{tabular}}} \\
\hline & \\
\hline MECHANICAL (GENERAL) & 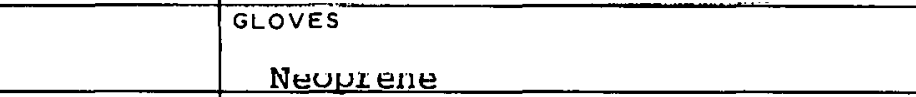 \\
\hline SPECIAL & $\begin{array}{l}\text { RESPIRATOR } \\
\text { W/appropriate filter }\end{array}$ \\
\hline $\begin{array}{l}\text { OTHER PROTECTIVE EQUIPMENT } \\
\qquad \text { In high concentrations positive pr } \\
\text { SECTION IX }\end{array}$ & essure air mask. \\
\hline \multicolumn{2}{|c|}{$\begin{array}{l}\text { PRECAUTIONS TO BE TAKEN IN HANDLING AND STORAGE } \\
\text { Protect against physical damage. Storage should be in a standard flammable } \\
\text { liquids storage room or cabinet. }\end{array}$} \\
\hline \multicolumn{2}{|l|}{ OTHER PRECAUTIONS. } \\
\hline SIGNATURE & DATE \\
\hline
\end{tabular}


03-366-3401

Toluhydroquinone
MATERIAL SAFETY DATA SHEET

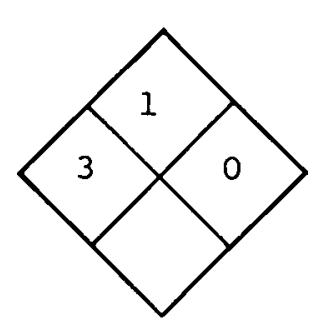

\section{SECTION I SOURCE AND NOMENCLATURE}

\section{MANUFACTURER'S NAME \\ TRADE NAME AND SYNONYMS \\ CHEMICAL NAME AND SYNONYMS \\ Tolyl Hydroquinone}

ADDRESS (NUMBER, STREET, CITY, STATE, ZIP CODE)

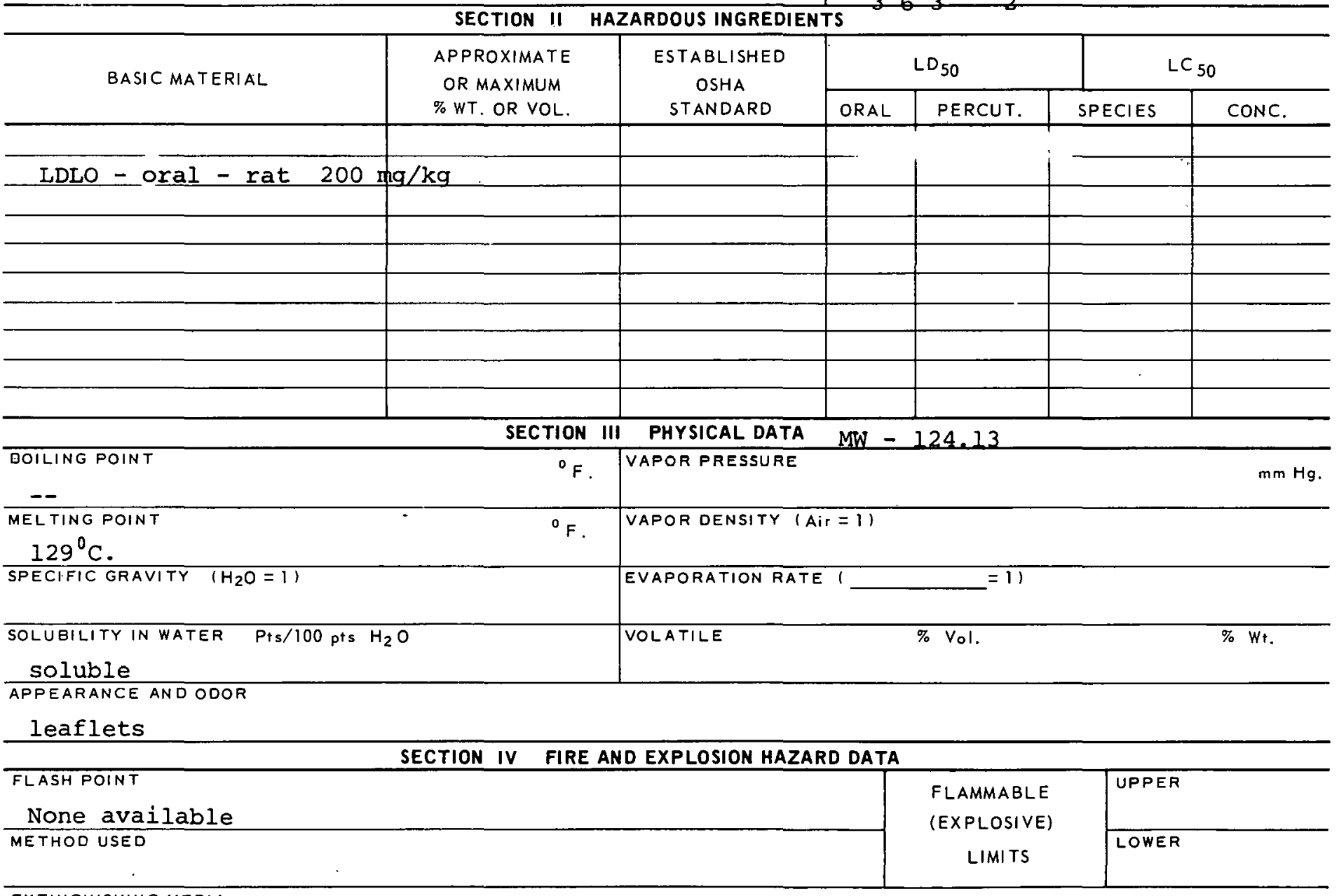

EXTINGUISHING MEDIA

Carbon dioxide, dry chemical, water.
EMERGENCY TELEPHONE NO.

CHEMICAL FAMILY

FORMULA

$\mathrm{CH}_{3} \mathrm{C}_{3} \mathrm{H}_{3}(\mathrm{OH})$

PECIAL FIRE FIGHTING PROCEDURES

UNUSUAL FIRE AND EXPLOSION HAZARDS 


\section{TOXIC LEVEL}

NONE aVailable

CARCINOGENIC

Inhalation, ingestion, skin

SKIN AND EYE

IRRITATION Severe

REVELANT SYMPTOMS OF EXPOSURE Nausea, dizziness, increased respiration, headache, cyanosis, delirium and collapse, depigmentation of eyes and skin.

EFFECTS OF CHRONIC EXPOSURE

Eye damage, dermatitis.

EMERGENCY AND FIRST AIDPROCEDURES Inhalation - rest; if swallowed - induce vomiting, gastric lavage, medical aid; wash from skin \& eyes.

\section{SECTION VI REACTIVITY DATA}

CONOITIONS CONTRIBUTING TO INSTABILITY

CONDITIONS CONTRIBUTING TO HAZARDOUS POLYMERIZATION

INCOMPATABILITY (MATERIALS TO AVOID)

HAZARDOUS DECOMPOSITION PRODUCTS

SECTION VII SPILL OR LEAK PROCEDURES

STEPS TO BE TAKEN IN CASE MATERIAL IS RELEASED OR SPILLED

WASTE DIGPOSAL METHOD

SECTION VIII SPECIAL PROTECTION INFORMATION

\begin{tabular}{l|l}
\hline VENTILATION REQUIREMENTS LOCAL EXHAUST & $\begin{array}{l}\text { PROTECTIVE EQUIPMENT (SPECIFY TYPES) EYE } \\
\text { EafEty gOggleS }\end{array}$ \\
\hline MECHANICAL IGENERALI & $\begin{array}{c}\text { GLOVES } \\
\text { Rubber }\end{array}$ \\
\hline SPECIAL & RESPIRATOR \\
\hline
\end{tabular}

OTHER PROTECTIVE EQUIPMENT

SECTION IX SPECIAL PRECAUTIONS

PRECAUTIONS TO BE TAKEN IN HANDLING AND STORAGE 
CAS : 000108441

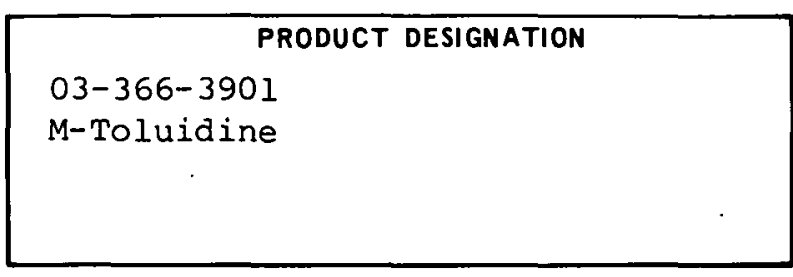

\section{SECTION I SOURCE AND NOMENCLATURE}

\section{MANUFACTURER'S NAME

TRADE NAME AND SYNONYMS
3-AminO TOluene
CHEMICAL NAME AND SYNONYMS
3-MethYl Aniline

MATERIAL SAFETY DATA SHEET

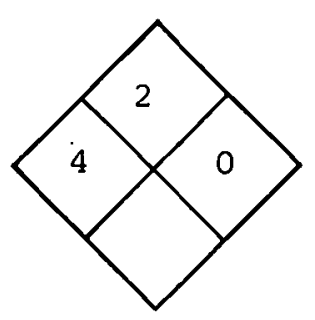

AODRESS (NUMBER, STREET, CITY, STATE, ZIP CODE)

BASIC MATERIAL SECTION II HAZARDOUS INGREDIENTS $\mathrm{CH}_{3} \mathrm{C}_{6} \mathrm{H}_{4} \mathrm{NH}_{2}$

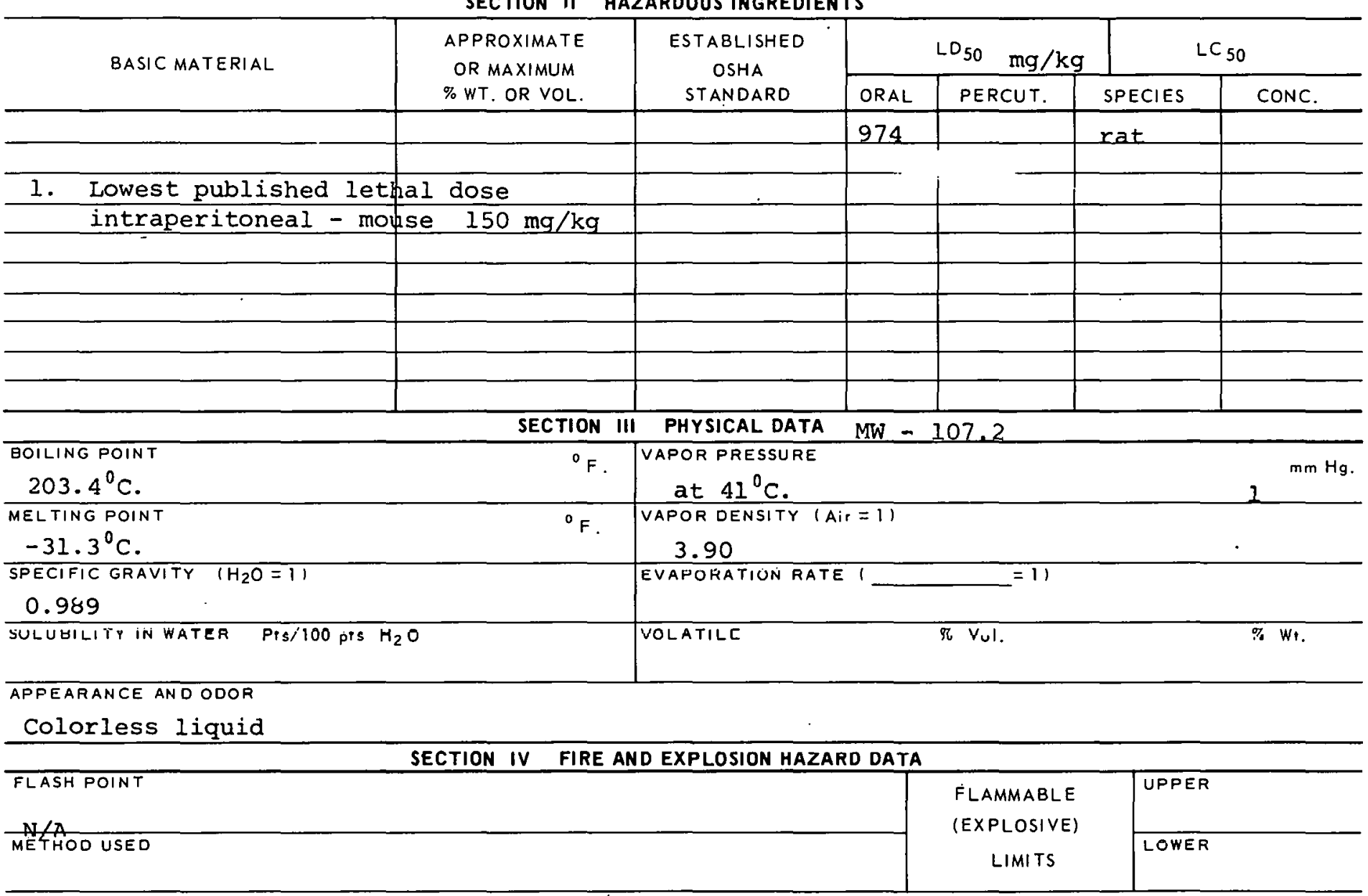

EXTINGUISHING MEOIA

Carbon dioxide, dry chemical, foam or water spray. SPECIAL FIRE FIGHTING PROCEDURES

Wear full protective clothing.

UHUSUAL FIRE AND EXPLOSION HAZAROS

When heated emits highly toxic fumes. 


\section{TOXIC LEVEL}

Not available

PRINCIPLE ROUTES OF ABSORBTION

Inhalation, ingestion, skin

REVELANT SYMPTOMS OF EXPOSURE Cyanosis, air hunger, nausea, vomiting, low blood pressure, convulsions.

\section{EFFECTS OF CHRONIC EXPOSURE}

Weight loss, anemia, weakness.

EMERGENCY AND FIRST AID PROCEDURES Inhalation - rest; if swallowed - qash mouth, give emetic, get medical aid; wash from skin \& eyes, skin may be washed with 5 acetic acid to aid removal.

CONDITIONS CONTRIBUTING TO INSTABILITY

CONDITIONS CONTRIBUTING TO HAZARDOUS POLYMERIZATION

May react vigorously with oxidizing materials.

INCOMPATABILITY (MATERIALS TO AVOID)

HAZARDOUS DECOMPOSITION PRODUCTS

SECTION VII SPILL OR LEAK PROCEDURES

STEPS TO EE TAKEN IN CASE MATERIAL IS RELEASED OR SPILLED

Cover with a 9:1 mixture of sand and soda ash, put in cardboard box for disposal.

\section{SECTION VIII SPECIAL PROTECTION IN FORMATION}

\begin{tabular}{|c|c|}
\hline VENTILATION REQUIREMENTS LOCAL EXHAUST & $\begin{array}{l}\text { PROTECTIVE EQUIPMENT (SPECIFY TYPES) EYE } \\
\text { Safety goggles }\end{array}$ \\
\hline MECHANICAL (GENERAL) & $\begin{array}{l}\text { GLOVES } \\
\text { Rubber }\end{array}$ \\
\hline SPECINL & RESTIRATOR \\
\hline
\end{tabular}

\section{SECTION IX SPECIAL PRECAUTIONS}

PRECAUTIONS TO BE TAKEN IN HANDLING AND STORAGE

Outside or detached storage is preferred; store in dry, well-ventilated, cool location away from fire hazards.

OTHEF HHECAUIIONS 
03-366-4001

PRODUCT DESIGNATION

o-Toluidine

MATERIAL SAFETY

DATA SHEET

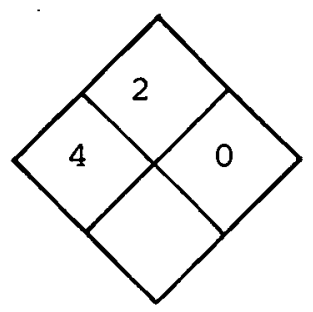

\section{SECTION I SOURCE AND NOMENCLATURE}

MANUFAC TURER'S NAME

|EMERGENCY TELEPHONE NO.

ADDRESS (NUMBER, STREET, CITY, STATE, ZIP CODE)

TRADE NAME AND SYNONYMS

2-Methyl Aniline

CHEMICAL NAME AND SYNONYMS

2 Amino Toluene

\begin{tabular}{l|l|l} 
& CHEMICAL FAMILY \\
& $\begin{array}{l}\text { FORMULA } \\
\mathrm{CH}_{3} \mathrm{C}_{6} \mathrm{H}_{4} \mathrm{NH}_{3}\end{array}$ \\
\hline
\end{tabular}

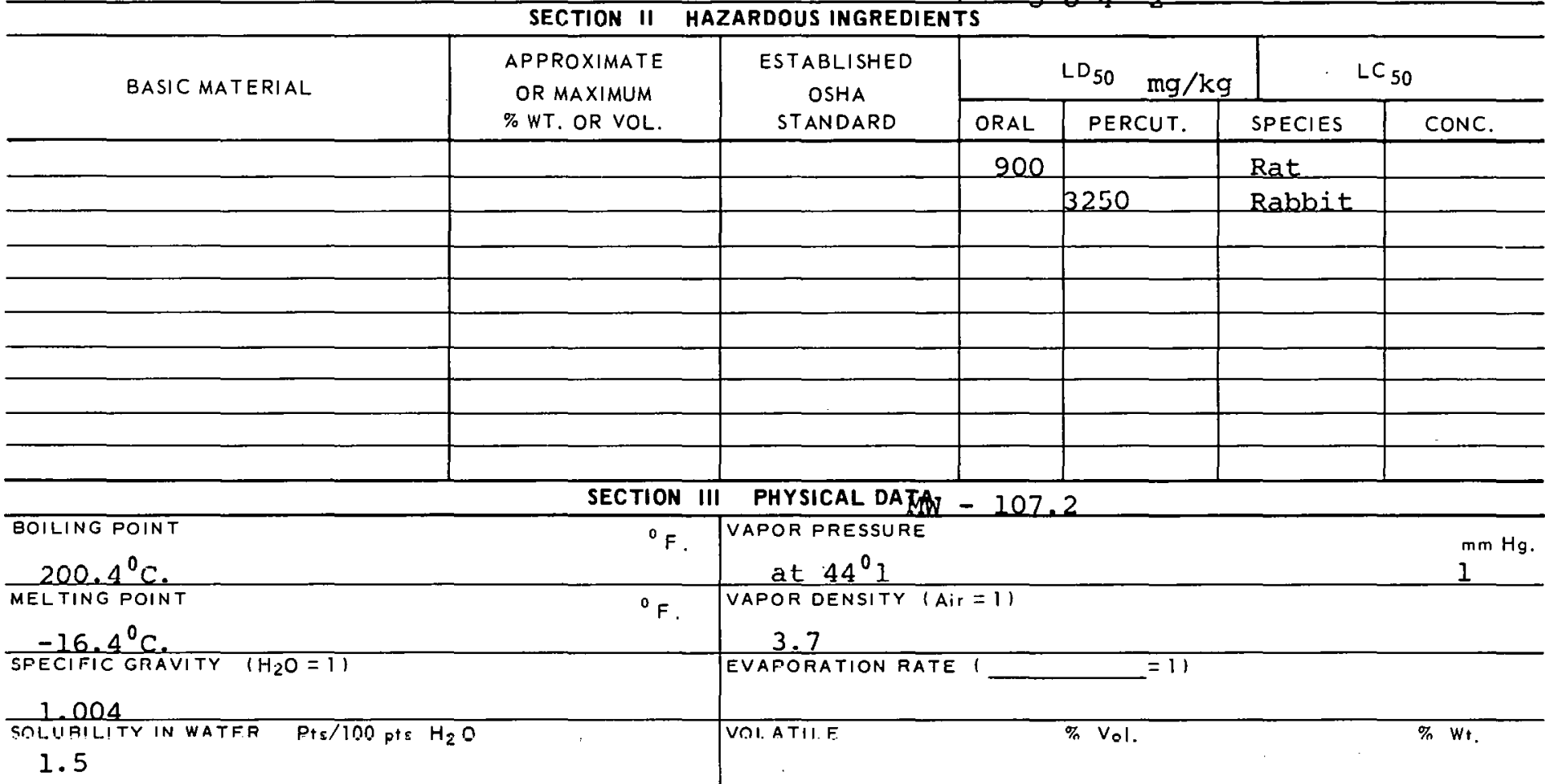

APPEARANCE AND ODOR

Colorless liquid

\begin{tabular}{|c|c|c|c|c|}
\hline & SECTION IV & FIRE AND EXPLOSION HAZARD DATA & & \\
\hline $\begin{array}{c}\text { FLASH POINT } \\
185^{\circ} \mathrm{F} .\end{array}$ & & & \multirow{2}{*}{$\begin{array}{c}\text { FLAMMABLE } \\
\text { (EXPLOSIVE) } \\
\text { LIMITS }\end{array}$} & UPPER \\
\hline $\begin{array}{l}\text { METHOD USED } \\
\text { C.C. }\end{array}$ & & & & LOWER \\
\hline
\end{tabular}

EXTINGUISHING MEDIA

Carbon dioxide, dry chemical, foam or water spray.

SPECIAL FIRE FIGHTING PROCEDURES

Wear full protective clothing.

UNUSUAL FIRE AND EXPLOSION HAZARDS

Emits toxic fumes when heated. 


\section{TOXIC LEVEL}

TLV - 5 PPM, $22 \mathrm{mg} / \mathrm{C} . \mathrm{M}$.

PRINCIPLE ROUTES OF ABSORBTION

Inhalation, ingestion, skin

\section{REVELANT SYMPTOMS OF EXPOSURE Cyanosis, air hunger, nausea, vomiting, low blood pressure,}

CARCINOGENIC

neoplastic convulsions.

EFFECTS OF CHRONIC EXPOSURE

Weight loss, anemia, weakness.

EMERGENCY AND FIRST AID PROCEDURES Inhalation - rest; if swallowed - wash mouth, give emetic, get medical aid; wash skin and eyes, skin may be washed with $5 \%$ acetic acid to aid removal.

SECTION VI REACTIVITY DATA

CONDITIONS CONTRIBUTING TO INSTABILITY

CONDITIONS CONTRIBUTING TO HAZARDOUS POLYMERIZATION

INCOMPATABILITY (MATERIALS TO AVOIO)

Can react with oxidizing materials. HAZARDOUS DECOMPOSITION PRODUCTS

SECTION VII SPILL OR LEAK PROCEDURES

STEPS TO BE TAKEN IN CASE MATERIAL IS RELEASED OR SPILLED

Add mixture $(9: I)$ sand to soda ash. Put into cardboard box for disposal.

WASTE DISPOSAL METHOD

SECTION VIII SPECIAL PROTECTION INFORMATION

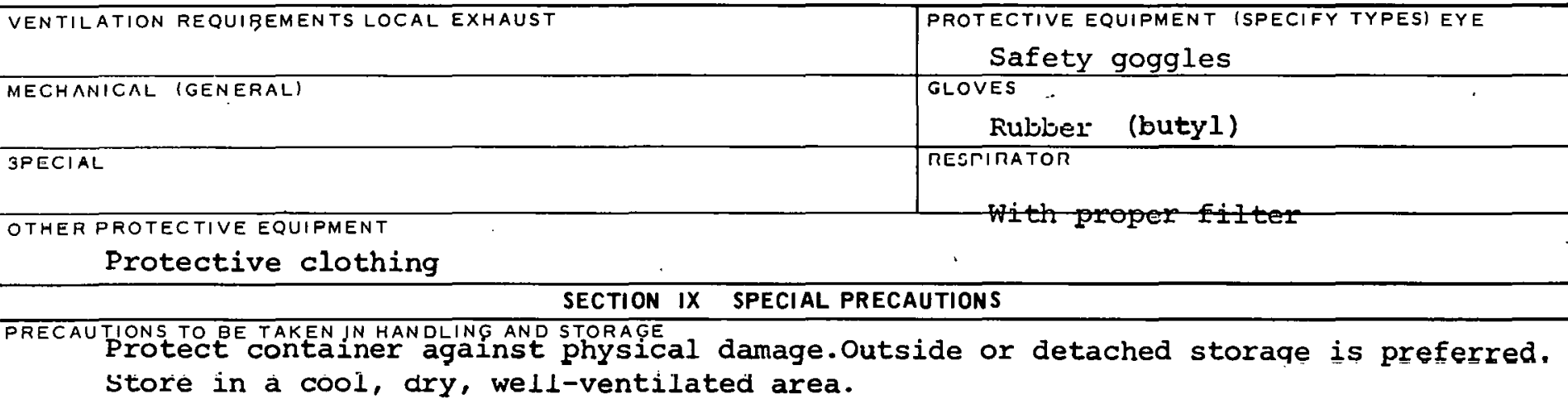

OTHER PHELAUIIUNS 
CAS : 000106490

XU31500

\begin{tabular}{|l|}
\hline PRODUCT DESIGNATION \\
O3-366-4101 \\
P-Toluidine
\end{tabular}

\section{MATERIAL SAFETY DATA SHEET}

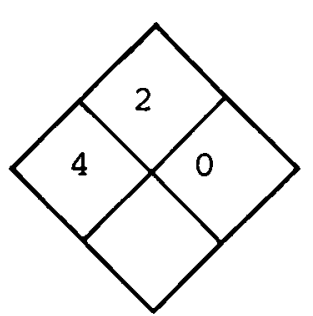

\section{SECTION I SOURCE AND NOMENCLATURE}

MANUFACTURER'SNAME

ADDRESS (NUMBER, STREET, CITY, STATE, ZIP CODE)

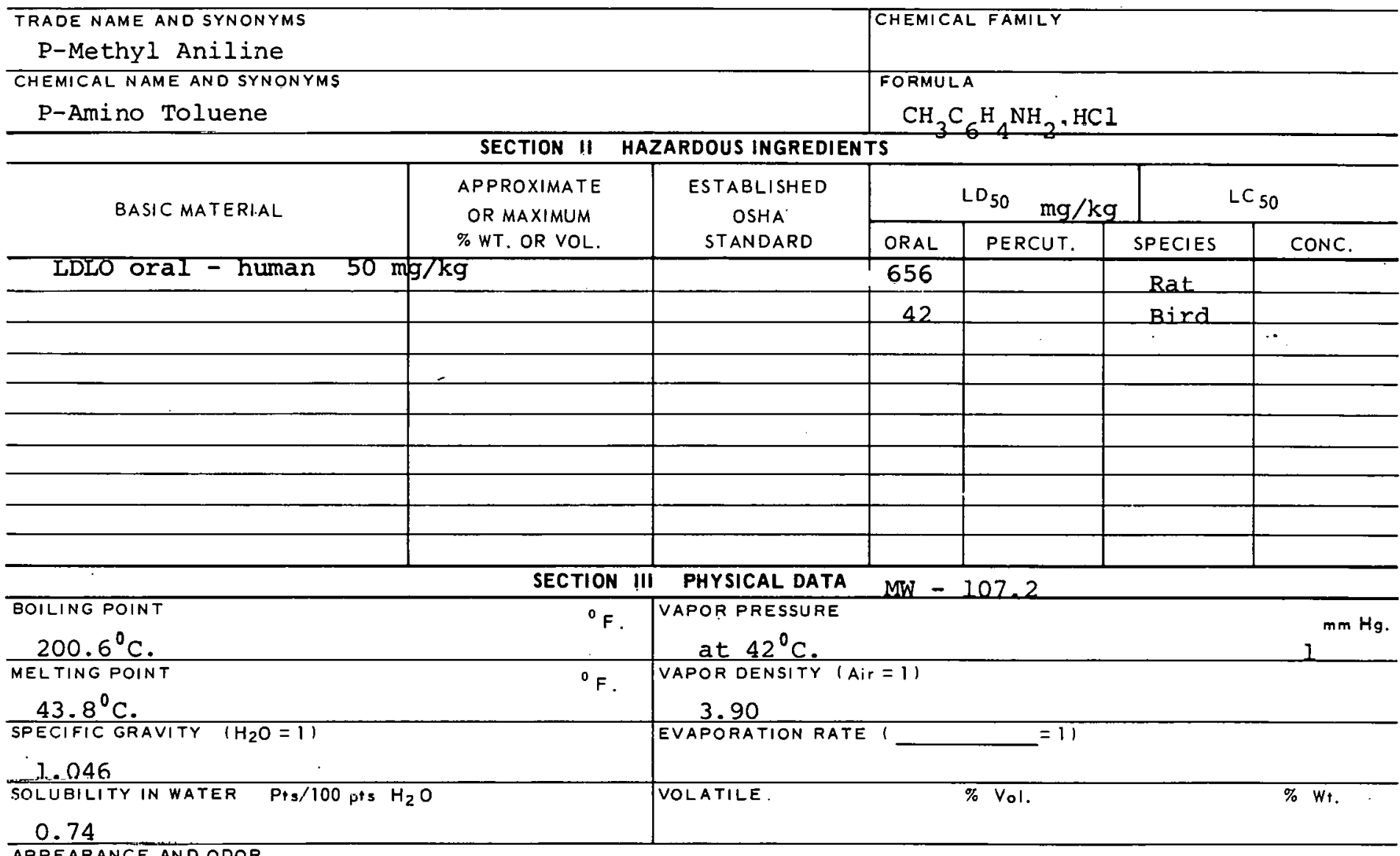

APPEARANCE AND ODOR

crystals

\begin{tabular}{|c|c|c|}
\hline & & \\
\hline $\begin{array}{c}\text { FLASH POINT } \\
188^{\circ} \mathrm{F} .\end{array}$ & \multirow{2}{*}{$\begin{array}{c}\text { FLAMMABLE } \\
\text { (EXPLOSIVE) } \\
\text { LIMITS }\end{array}$} & UPPER \\
\hline $\begin{array}{l}\text { METHOD USED } \\
\text { C.C. }\end{array}$ & & LOWER \\
\hline
\end{tabular}

EXTINGUISHING MEDIA

Carbon dioxide, dry chemical, foam and water spray.

SPECIAL FIRE FIGHTING PROCEDURES

Wear full protective clothing.

UNUSUAL FIRE AND EXPLOSION HATARDS

Emits toxic fumes when heated. 


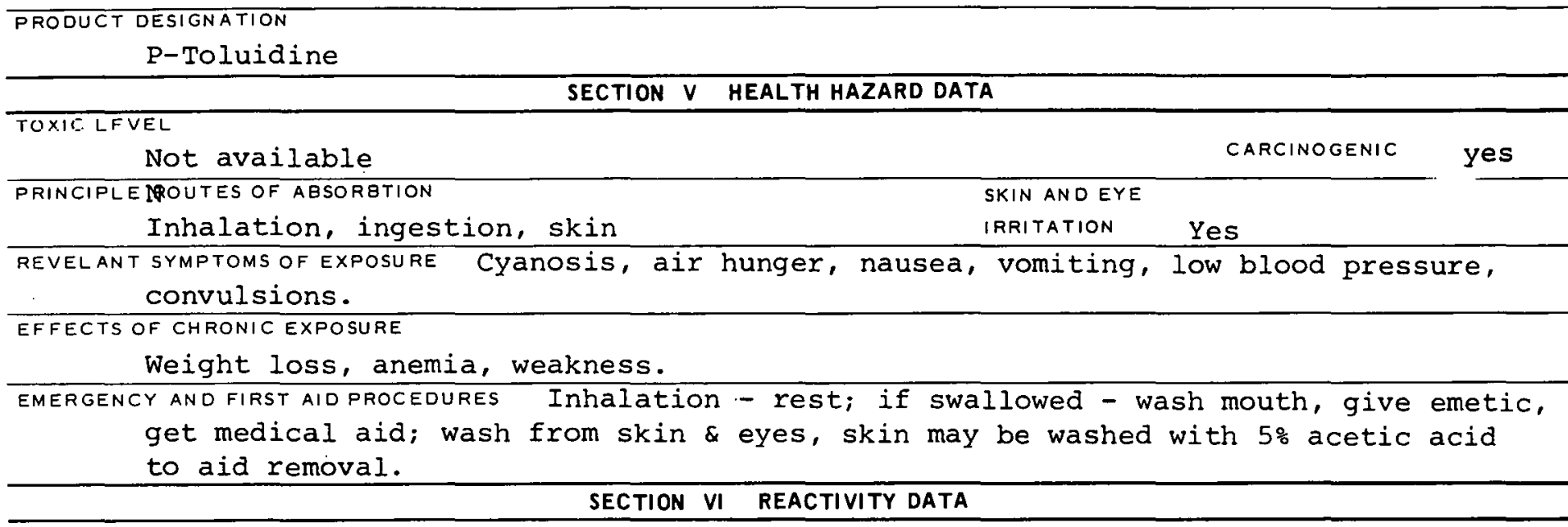

CONDITIONS CONTRIBUTING TO INSTABILITY

CONDITIONS CONTRIBUTING TO HAZARDOUS POLYMERIZATION

INCOMPATABILITY (MATERIALS TO AVOID)

May react vigorous $\perp y$ with oxidizing materials.

HAZARDOUS DECOMPOSITION PRODUCTS

SECTION VII SPILL OR LEAK PROCEDURES

STEPS TO BE TAKEN IN CASE MATERIAL IS RELEASED OR SPILLED

Cover with a $(9: 1)$ sand to soda ash mixture. Transfer to box for disposal.

WASTE UISPOSAL METHOD

\section{SECTION VIII SPECIAL PROTECTION INFORMATION}

VENTILATION REQUIREMENTS LOCAL EXHAUS

\section{SPECIAL PROTECTION INFORMATION}

EQUIPMENT (SPECIFY TYPES) EYE

MECHANICAL (GENERAL)

Safety goggles

GLOVES

Rubbex

SPECIAL

RESPIRATOR

With proper filter

OTHER PROTECTIVE EQUIPMENT

Protective clothing with self-contained breathing apparatus. SECTION IX SPECIAL PRECAUTIONS

PRECAUTIONS TO BE TAKEN IN HANDLING AND STORAGE

Protect container from physical damage,outside or detached storage preferred. Store

in cool, dry, well-ventilated area.

OTHER PRECAUTIONS 


\begin{tabular}{|c|}
\hline PRODUCT DESIGNATION \\
$03-366-4401$ \\
P-Toluidine Hydrochloride
\end{tabular} \\ MATERIAL SAFETY DATA SHEET}

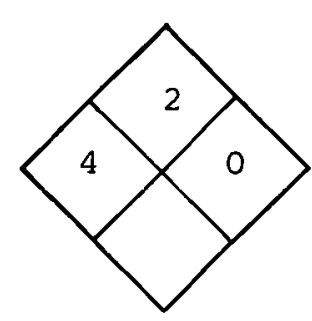

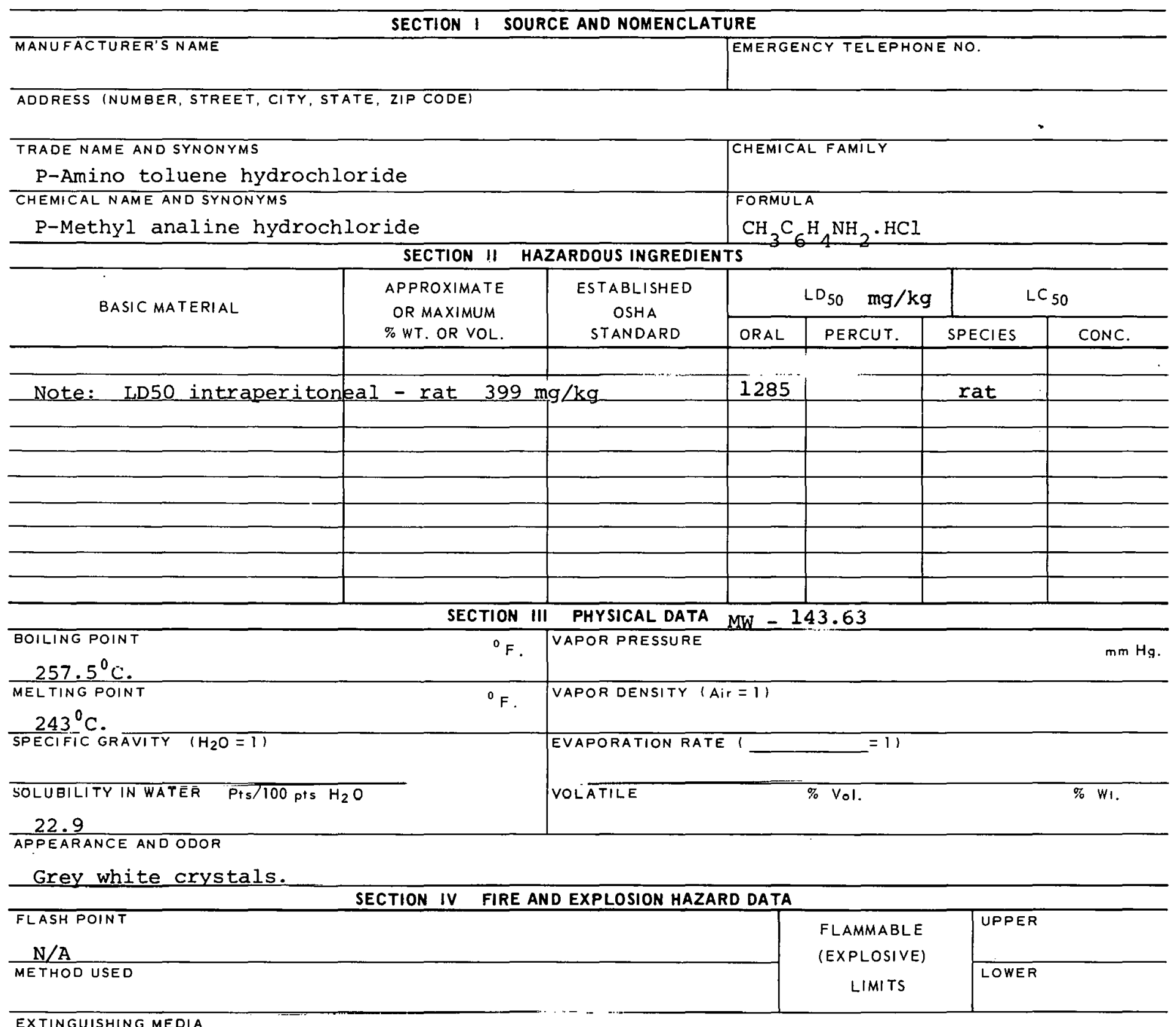

Carbon dioxide, dry chemical, foam or water spray.

SPECIAL FIRE FIGHTING PROCEOURES

Wear full protective clothing.

UNUSUAL TIRE AND EXPLOSION HAZARDS

Emits toxic fumes when heated. 
TOXIC.: I,EVEL

Not available

CARCINOGENIC YeS

PRINCIPLE ROUTES OF ABSORBTION

Inhalation, ingestion, skin

SKIN ANDEYE

IRRITATION SIIght

REVELANT SYMPTOMS OF EXPOSURE Cyanosis, air hunger, nausea, vomiting, low blood pressure, convulsions.

EFFECTS OF CHRONIC EXPOSURE

Weight loss, anemia, weakness.

EMERGENCY AND FIRST AIDPROCEDURES Inhalation - rest; if swallowed - wash mouth, give emetic, get medical aid; wash from skin \& eyes, skin may be washed with 5\% acetic acid to aid removal.

\section{SECTION VI REACTIVITY DATA}

CONDITIONS CONTRIBUTING TO INSTABILITY

CONMITINNS CONTRIBUTING TQ HAZARDOUS POLYMERIZATION

INCOMPATABILITY (MATERIALS TO AVOID)

May react vigorously with oxidizing materials. HAZARDOUS DECOMPOSITION PRODUCTS

SECTION VII SPILL OR LEAK PROCEDURES

STEPS TO BE TAKEN IN CASE MATERIAL IS RELEASED OR SPILLED

Cover with a $(9: 1)$ sand to soda ash mixture. Transfer to box for disposal.

WASTE DISPOSAL METHOD

SECTION VIII SPECIAL PROTECTION INFORMATION

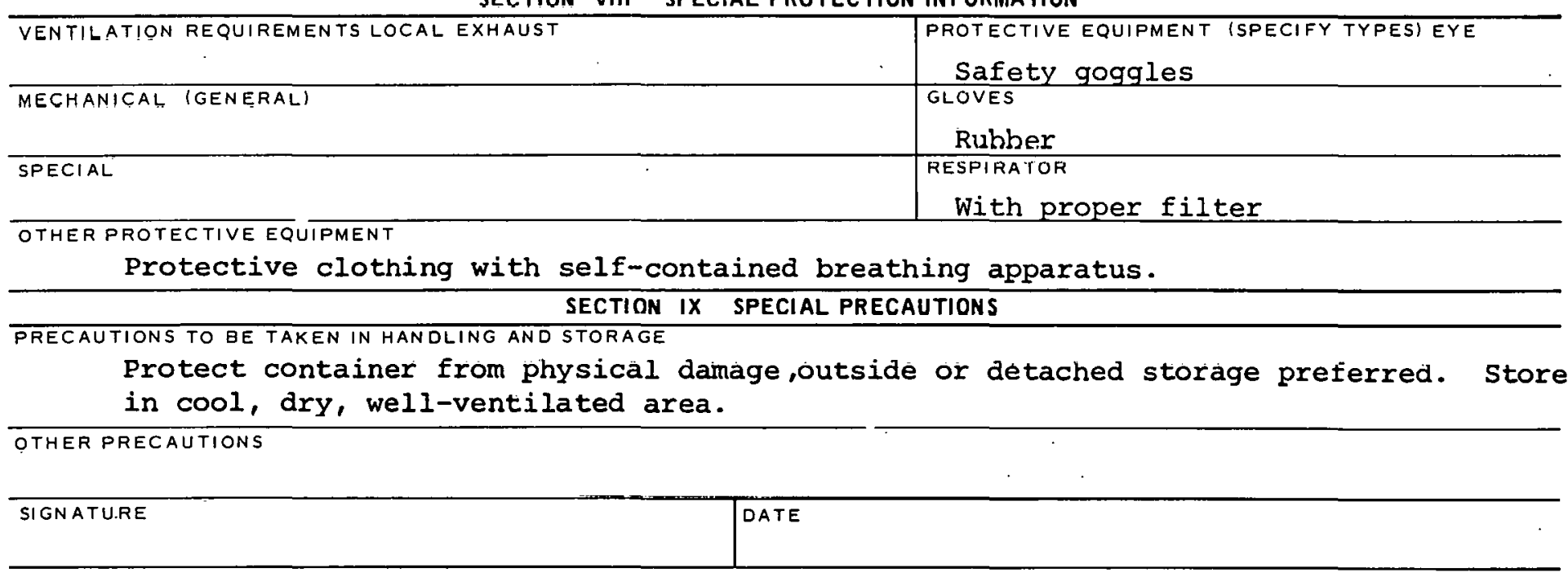


CAS: 000147820

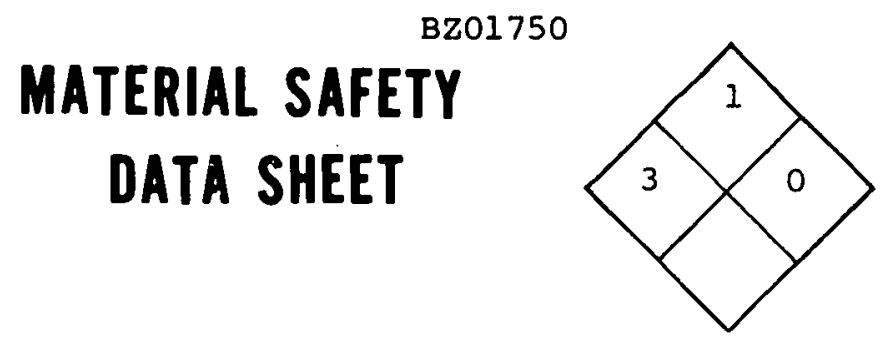

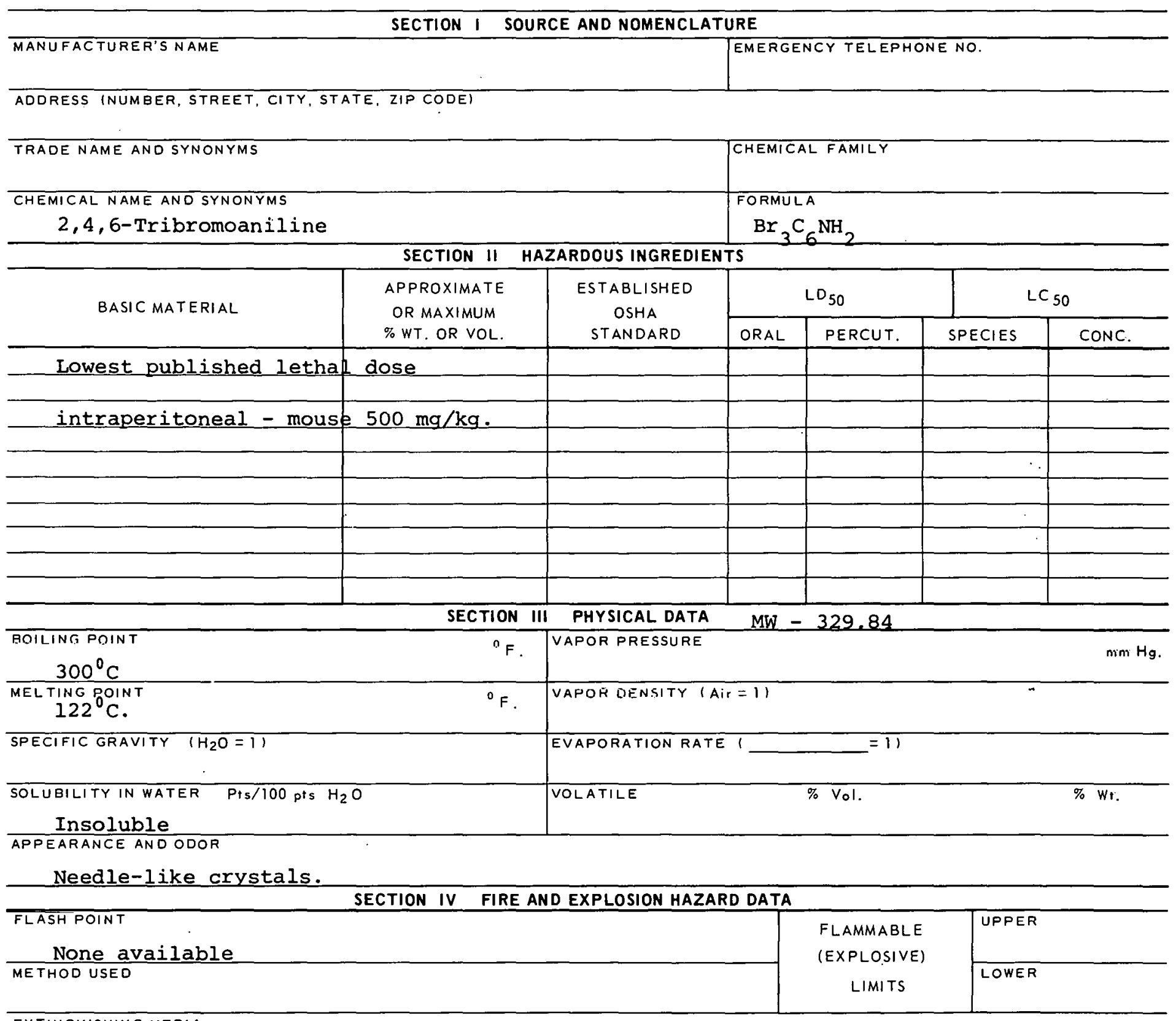

EXTINGUISHING MEDIA

Dry chemical or carbon dioxide. Water or foam may cause frothing.

SPECIAL FIRE FIGHTING PROCEDURES

UNUSUAL FIRE AND EXPLOSION HAZARDS 


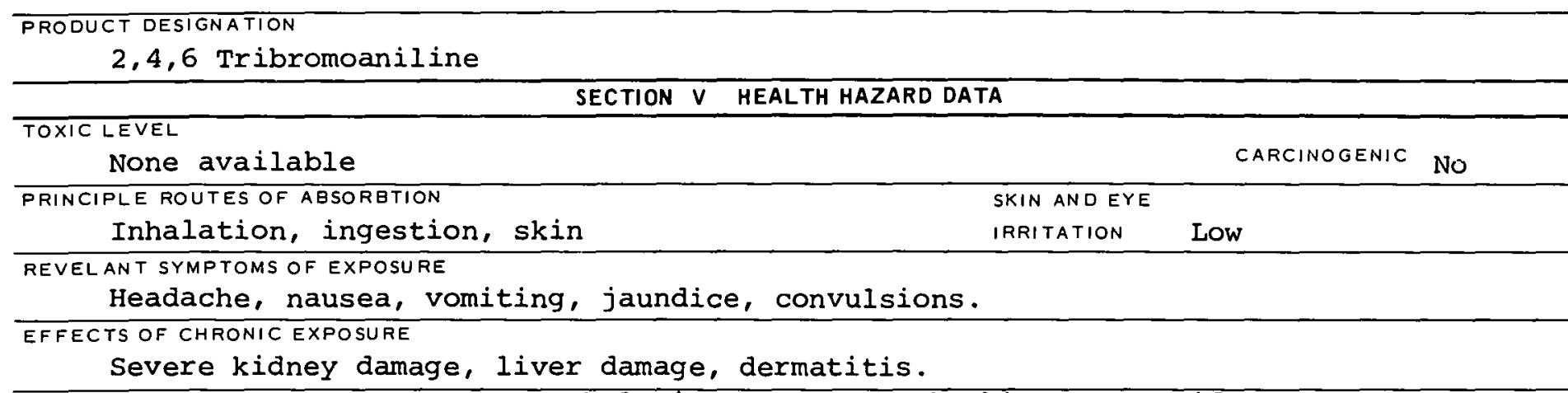

EMERGENCY AND FIRST AID PROCEDURES Inhalation - rest; wash skin \& eyes; if swallowed remove by gastric lavage; give oxygen as needed.

SECTION VI REACTIVITY DATA

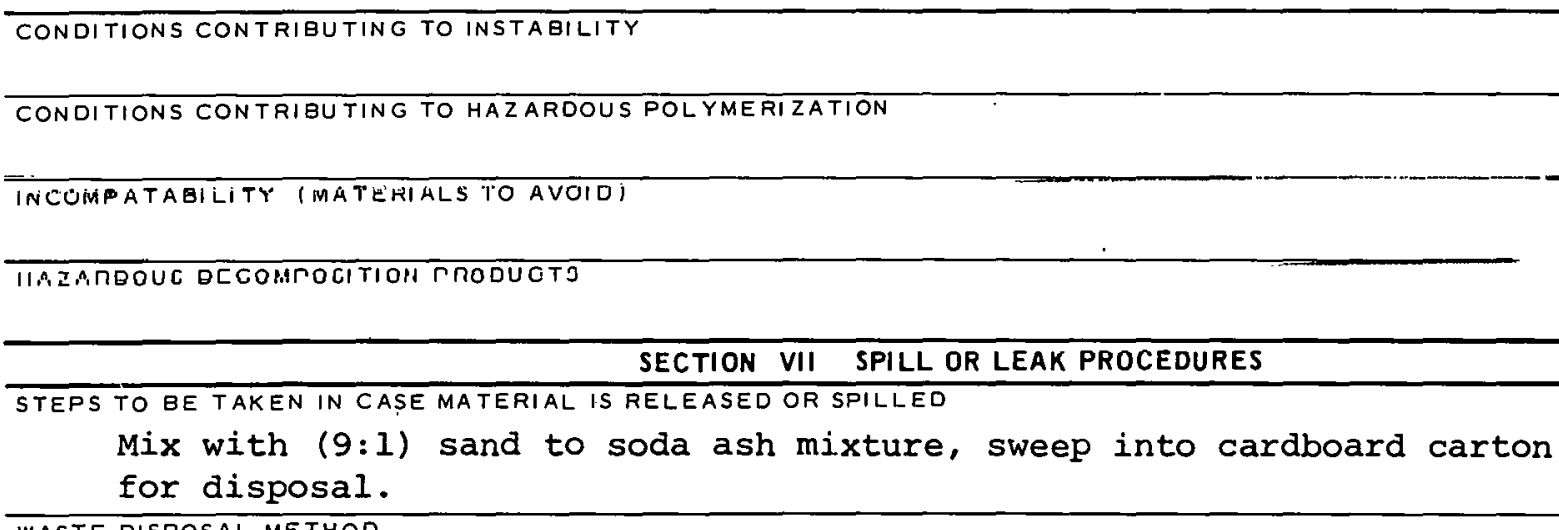

WASTE DISPOSAL METHOO

SECTION VIII SPECIAL PROTECTION INFORMATION

\begin{tabular}{|c|c|}
\hline VENTILATION REQUIREMENTS LOCAL EXHAUST & $\begin{array}{l}\text { PROTECTIVE EQUIPMENT (SPECIFY TYPES) EYE } \\
\text { GOggleS }\end{array}$ \\
\hline MECHANICAL (GENERAL) & $\begin{array}{l}\text { CLOVCS } \\
\text { Rubber }\end{array}$ \\
\hline$\overline{S P E C I A L}$ & $\begin{array}{l}\text { REERIRATOR } \\
\text { Self-contained }\end{array}$ \\
\hline
\end{tabular}

OTHER PROTECTIVE EQUIPMENT

Protective clothing

SECTION IX SPECIAL PRECAUTIONS

PRECAUTIONS TO BE TAKEN IN HANDLING AND STRRAGE
Protect container against physical damage, separate from oxidizing materials.

OTHER PRECAUTIONS

SIGNATURE

D^TF

$366-7401 B$ 


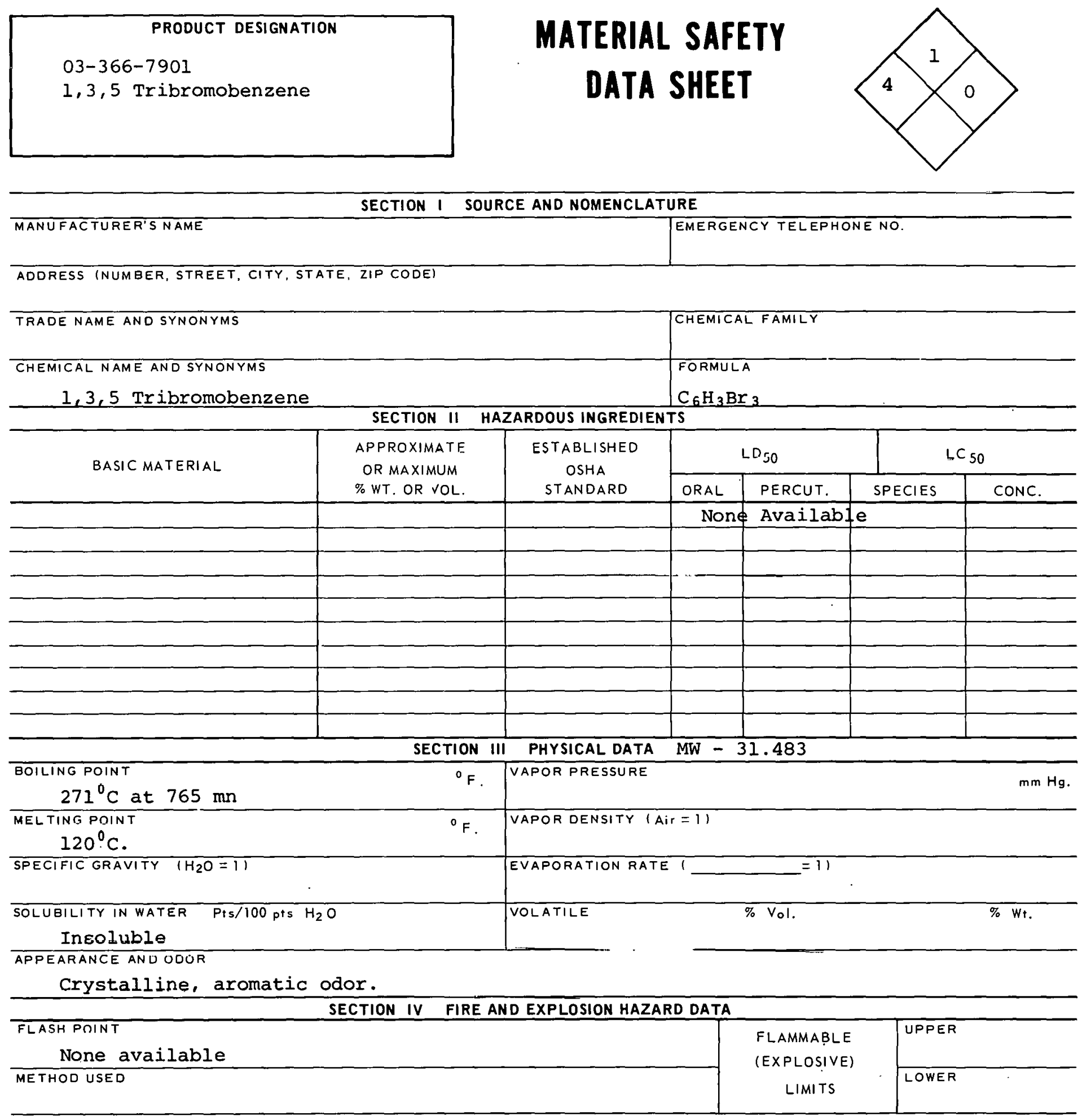

EXTINGUISHING MEDIA

Water may be used to blanket fire, alcohol foam, carbon dioxide. SPECIAL FIRE FIGHTING PROCEDURES

Self-contained mask.

UNUSUAL FIRE AND EXPLOSION HAZAROS 


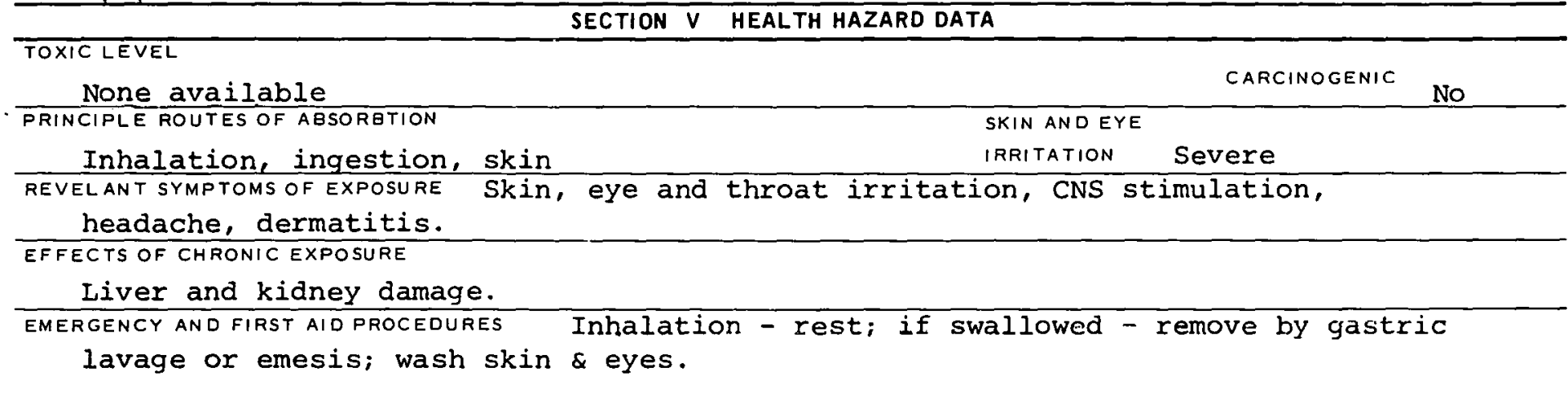

\begin{tabular}{ll}
\hline & SECTION VI REACTIVITY DATA \\
\hline CONDITIONS CONTRIBUTING TO INSTABILITY &
\end{tabular}

CONDITIONS CONTRIBUTING TO HAZARDOUS POLYMERIZATION

INCOMPATABILITY (MATERIALSTE AVOIOI

May react with oxidizing materials.

HAZARDOUS DECOMPOSITION PRODUCTS

SECTION VII SPILL OR LEAK PROCEDURES

STEPS TO BE TAKEN IN CASE MATERIAL IS RELEASED OR SPILLED

Absorb the spill with paper towels; evaporate in hood; then burn towels.

\section{WASTE DISPOSAL METHOD}

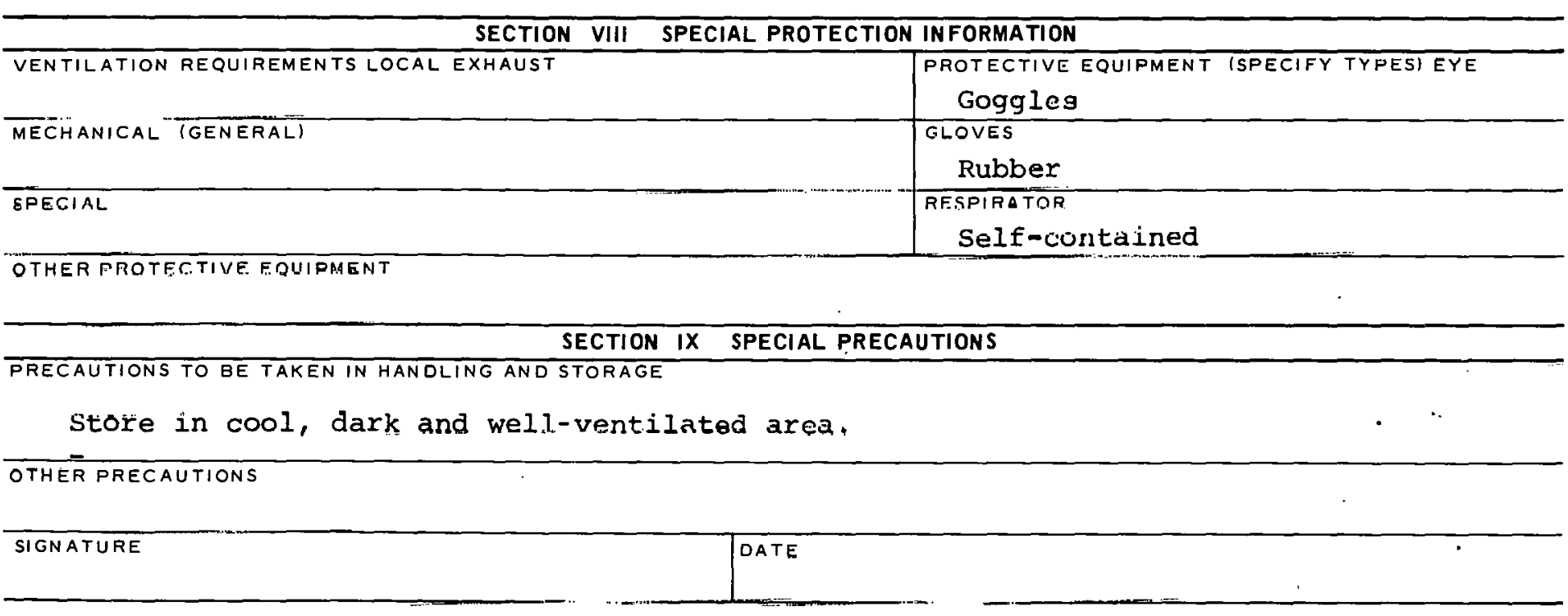




\begin{tabular}{|c|}
\hline PRODUCT DESIGNATION \\
$03-366-8401$ \\
$1,2,3$ Tribromopropane \\
\hline
\end{tabular}

\section{MATERIAL SAFETY \\ DATA SHEET}

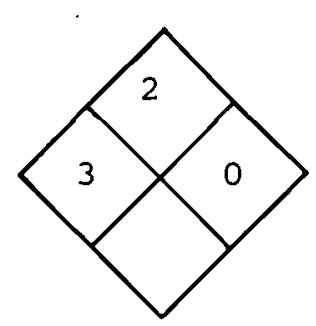

\section{SECTION I SOURCE AND NOMENCLATURE}

\section{MANUFACTURER'S NAME \\ TRADE NAME AND SYNONYMS \\ Glycerol Tribromohydrin CHEMICAL NAME AND SYNONYMS 1,2,3-Tribromopropane}

ADDRESS (NUMBER, STREET, CITY, STATE, ZIP CODE)

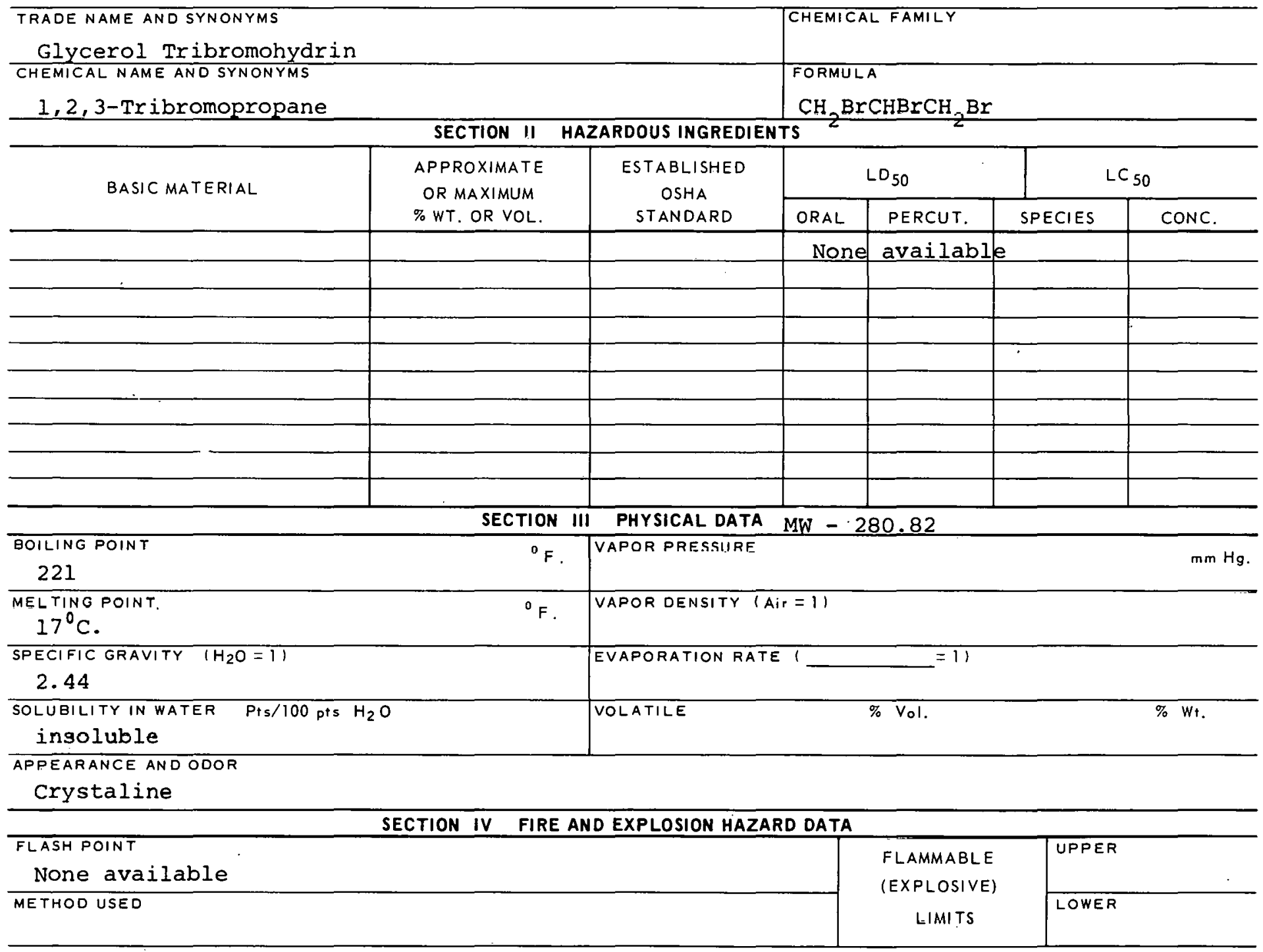

\section{EXTINGUISHING MEOIA}

Foam, carbon dioxide, dry chemical.

\section{SPECIAL FIRE FIGHTING PROCEDURES \\ Wear self-contained breathing apparatus.}

\section{FLAMMABLE}

LIANITS 
Tribromopropane SECTION $V$ HEALTH HAZARD DATA

TOXIC LEVEL

None available

CARCINOGENIC

PRINCIPLE ROUTES OF ABSORBTION

SKIN AND EYE

Inhalation ingestion skin IRRITATION

IRRITATION SEVEre

REVELANT SYMPTOMS OF EXPOSURE

lungs, narcosis.

Eye and throat irritation, edema and hemorrhaging in

EFFECTS OF CHRONIC EXPOSURE

Heart and liver damage.

EMERGENCY AND FIRST AIDPROCEDURES If swallowed - give emetic, get medical aid; inhalation artificial respiration if needed; wash from skin \& eyes.

SECTION VI REACTIVITY DATA

CONDITIONS CONTRIBUTING TO INSTABILITY

CONDITIONS CONTRIBUTING TO HAZAROOUS POLYMERI ZATION

INCOMPATABILITY IMATTERIALS TO AVOIDI

HAZARDOUS DECOMPOSITION PRODUCTS

SECTION VII SPILL OR LEAK PROCEDURES

STEPS TO BE TAKEN IN CASE MATERIAL IS RELEASED OR SPILLED

WASTE DISPOSAL METHOD

SECTION VIII SPECIAL PROTECTION INFORMATION

\begin{tabular}{|c|c|}
\hline VENTILATION REQUIREMENTS LOCAL EXHAUST & $\begin{array}{l}\text { PROTECTIVE EQUIPMENT (SPECIFY TYPES) EYE } \\
\text { GNGglPS. }\end{array}$ \\
\hline MECHANICAL (GENERAL) & $\begin{array}{l}\text { GLOVES } \\
\text { Rubber }\end{array}$ \\
\hline SPECIAL & $\begin{array}{l}\text { REGFIRATON } \\
\text { With proper filter }\end{array}$ \\
\hline
\end{tabular}

OTHER PROTECTIVE EQUIPMENT

SECTION IX SPECIAL PRECAUTIONS

PRECAUTIONS TO BE TAKEN IN HANDLING AND STORAGE

OTHER PRECAUTIONS

SIGNATURE

DATE

$366-8401 B$ 


\begin{tabular}{|c|}
\hline PRODUCT DESIGNATION \\
$03-366-9401$ \\
$2,4,6-$ Trichloroaniline \\
\hline
\end{tabular}

\section{MATERIAL SAFETY DATA SHEET}

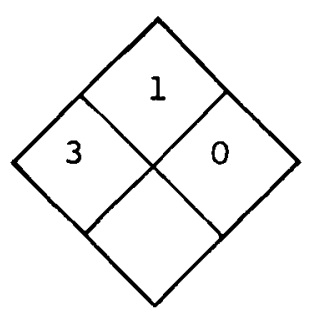

SECTION I SOURCE AND NOMENCLATURE

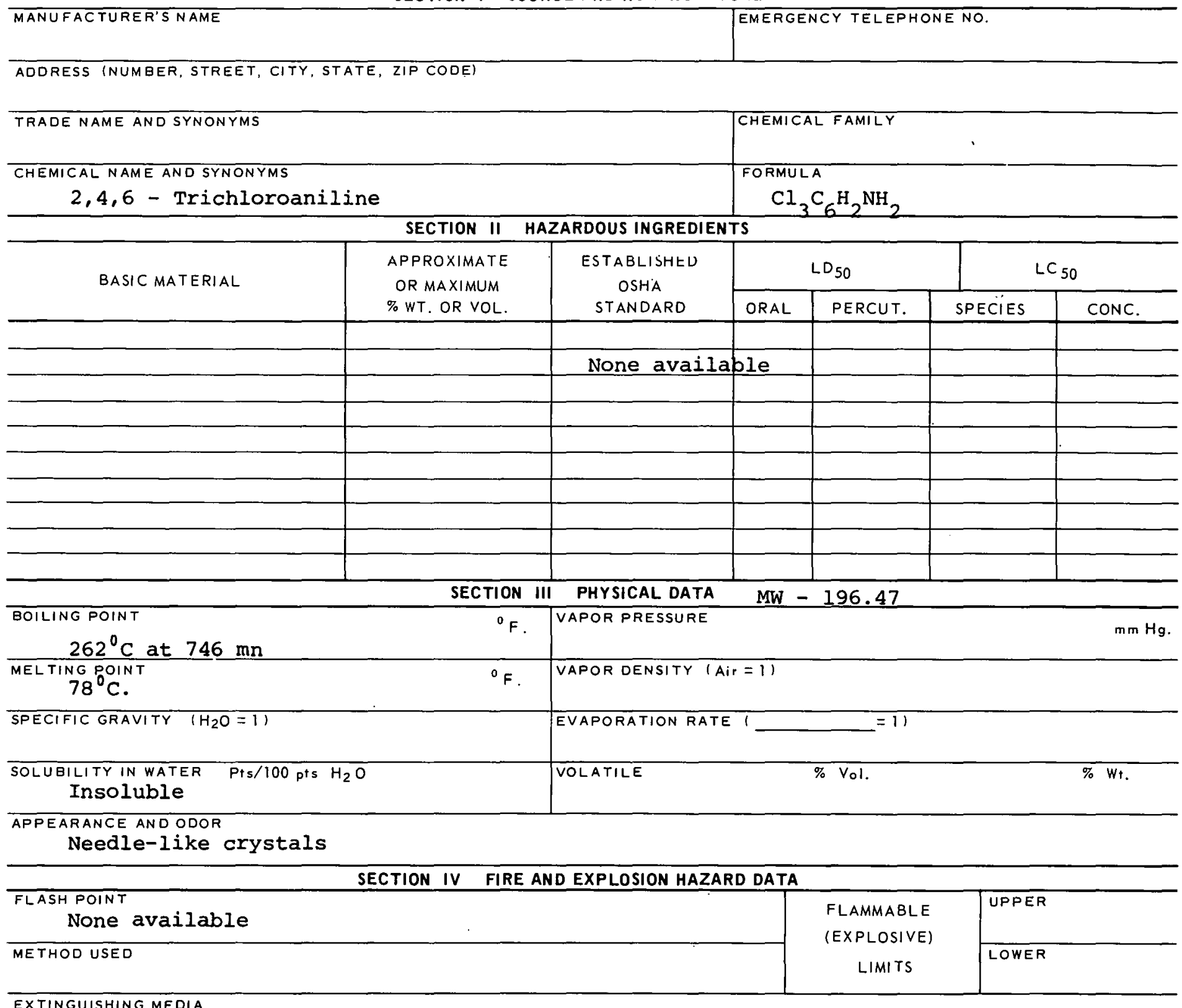

EXTINGUISHING MEDIA

Dry chcmical or earbon dioxide. Water ur fuam may cause frothing. SPECIAL FIRE FIGHTING PROCEDURES

UNUSUAL FIRE AND EXPLOSION HAZARDS 


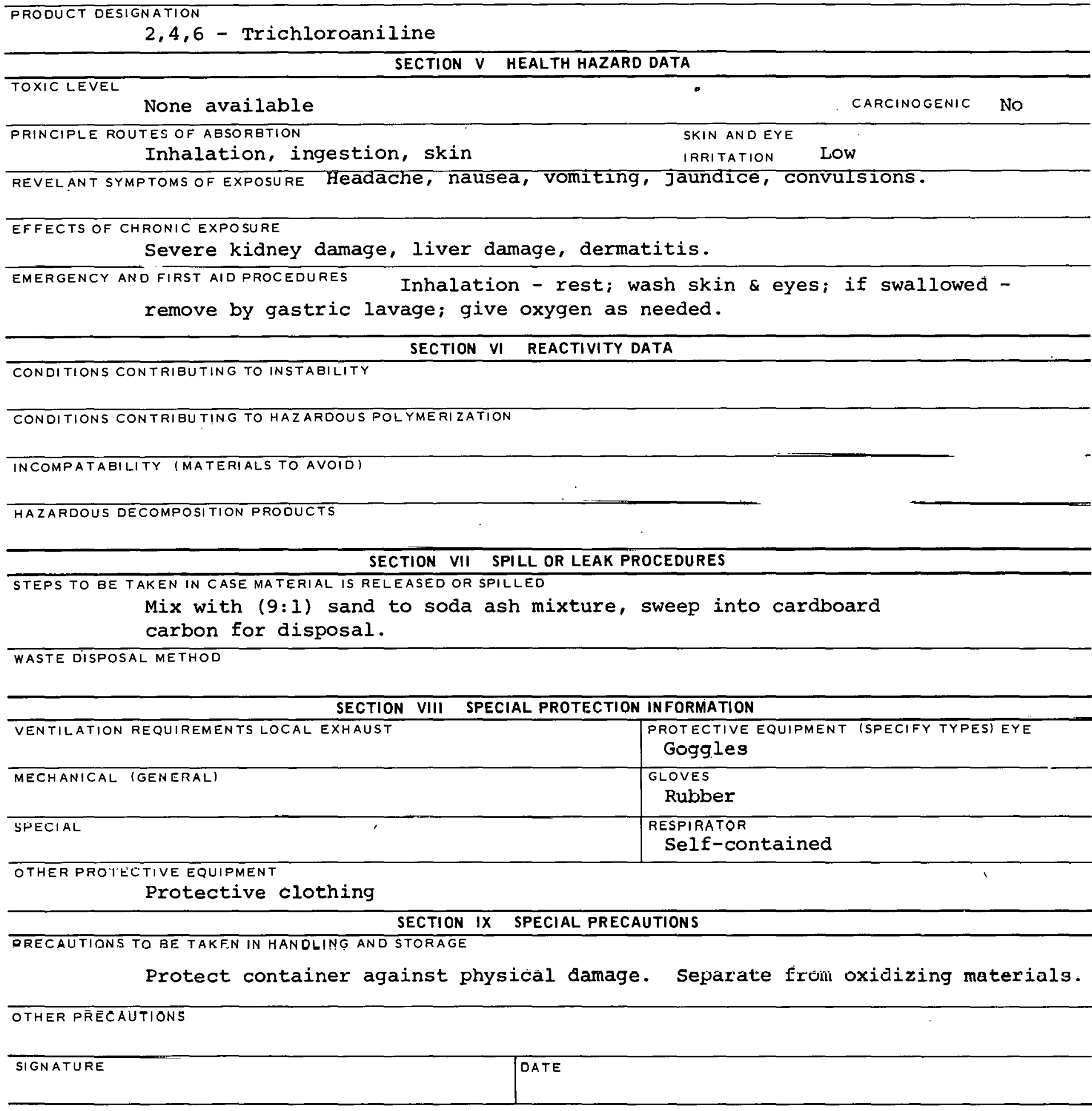




\section{PRODUCT DESIGNATION}

03-366-9611

1,2,4-Trichlorobenzene
MATERIAL SAFETY

DATA SHEET

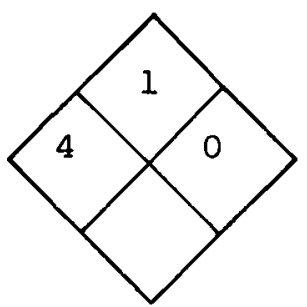

SECTION I SOURCE AND NOMENCLATURE

\begin{tabular}{l|l}
\hline MANUFACTURER'S NAME & EMERGENCY TELEPHONE NO. \\
\hline ADDRESS (NUMBER, STREET, CITY, STATE, ZIP CODE) &
\end{tabular}

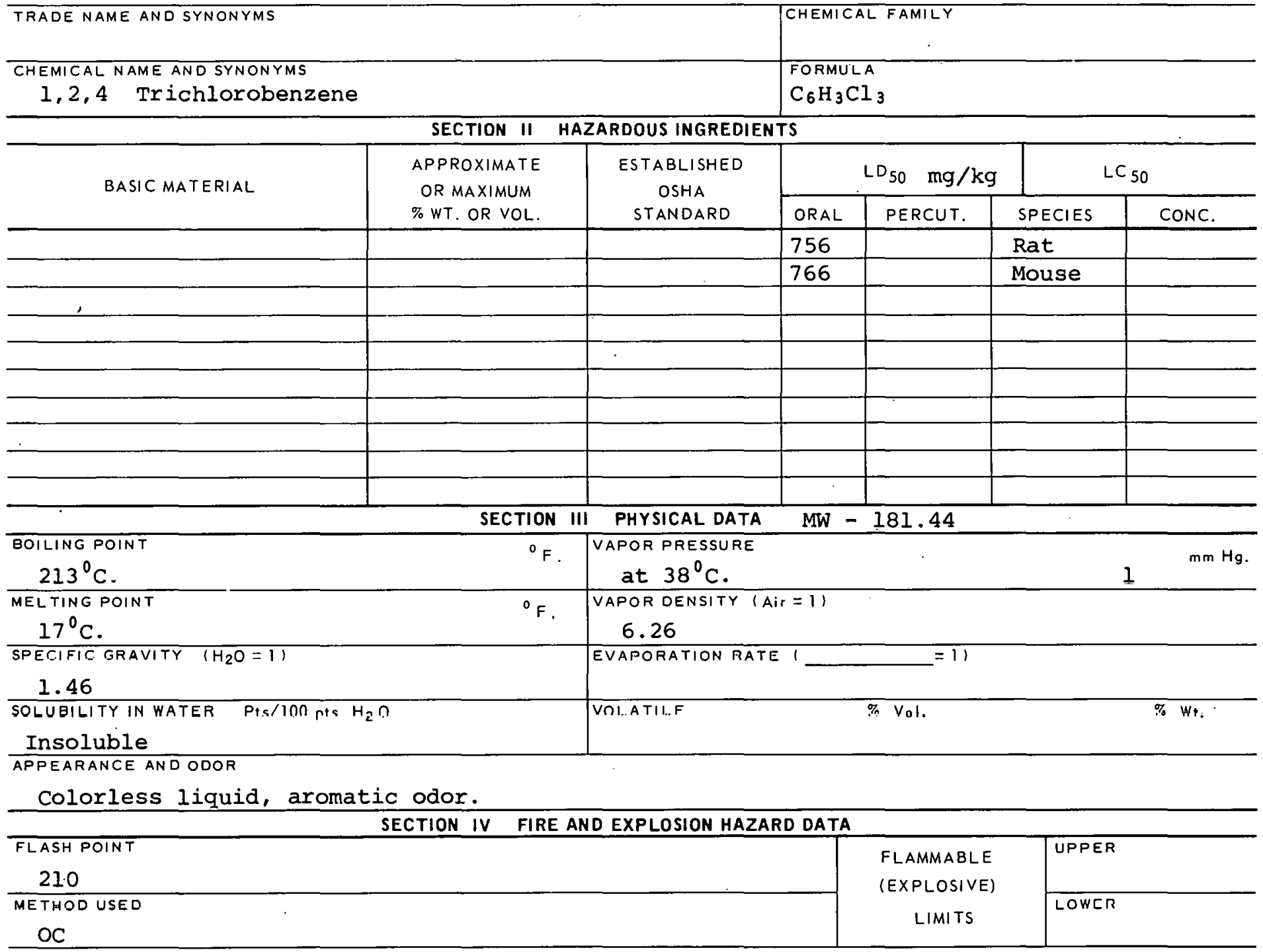

EXTINSIIISHINST MFRIA

Water may be used to blanket fire.

SPECIAL FIRE FIGHTING PROCEDURES

Self-contained mask.

UNUSUAL FIRE AND EXPLOSION HAZARDS 


\section{1,2,4 Trichlorobenzene}

\section{SECTION $V$ HEALTH HAZARD DATA}

\section{TOXIC LEVEL}

TLV-5 PPM, $40 \mathrm{mg} / \mathrm{C.M}$. ceiling value

PRINCIPLE ROUTES OF ABSORBTION

Inhalation, ingestion, skin

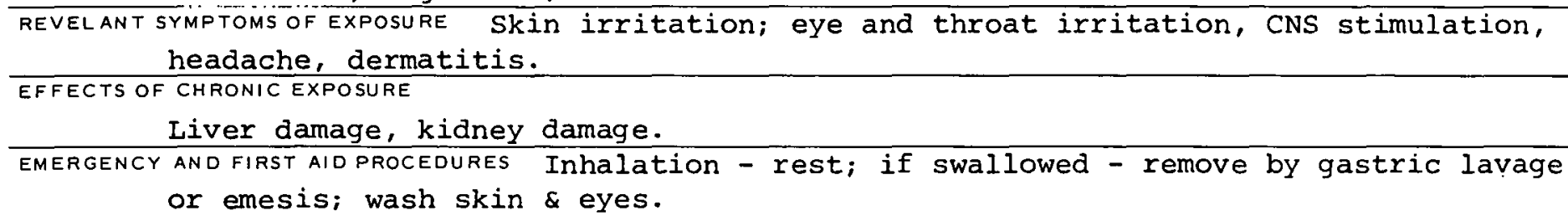

Liver damage, kidney damage.

EMERGENCY AND FIRST AID PROCEDURES Inhalation - rest; if swallowed - remove by gastric lavage or emesis; wash skin \& eyes.

SECTION VI REACTIVITY DATA

CONDITIONS CONTRIBUTING TO INSTABILITY
CONDITIONS CONTRIBUTING TO HAZARDOUS POLYMERIZATION
INCOMPATABILITY IMATERIALS TO AVOIOI
ReaCtS VIgOrOUSIY with OXIdizing materials.
HAZARDOUS DECOMPOSITION PRODUCTS

SECTION VII SPILL OR LEAK PROCEDURES

STEPS.TO BE TAKEN IN CASE MATERIAL IS RELEASED OR SPILLED

Absorb the spill with paper towels; evaporate in hood; then burn towels

WASTE DISPOSAL METHOD

SECTION VIII SPECIAL PROTECTION INFORMATION

\begin{tabular}{l|l} 
SECTION VIII SPECIAL PROTECTION INFORMATION \\
\hline VENTILATION REQUIREMENTS LOCAL EXHAUST & $\begin{array}{c}\text { PROTECTIVE EQUIPMENT (SPECIFY TYPES) EYE } \\
\text { GOggles }\end{array}$ \\
\hline MECHANICAL IGENERAL) & GI.OVES \\
Rubber
\end{tabular}

OTHER PROTECTIVE EQUIPMENT

\section{SECTION IX SPECIAL PRECAUTIONS}

PRECAUTIONS TO BE TAKEN IN HANDLING AND STORAGE

Store in well-ventilated, dark, cool area.

OTHER PRECAUTIONS

SIGNAIUKE 
CAS - 000079005

Cement T-339

1,1,2 Trichloroethane

03-367-0084

\section{MATERIAL SAFETY \\ DATA SHEET}

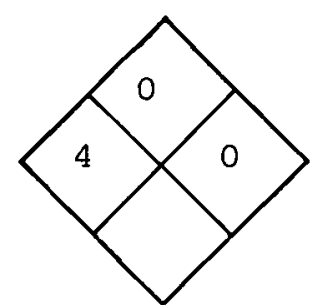

\section{SECTION I SOURCE AND NOMENCLATURE}

MANU FACTURER'S NAME

ADDRESS (NUMBER, STREET, CITY, STATE, ZIP CODE)

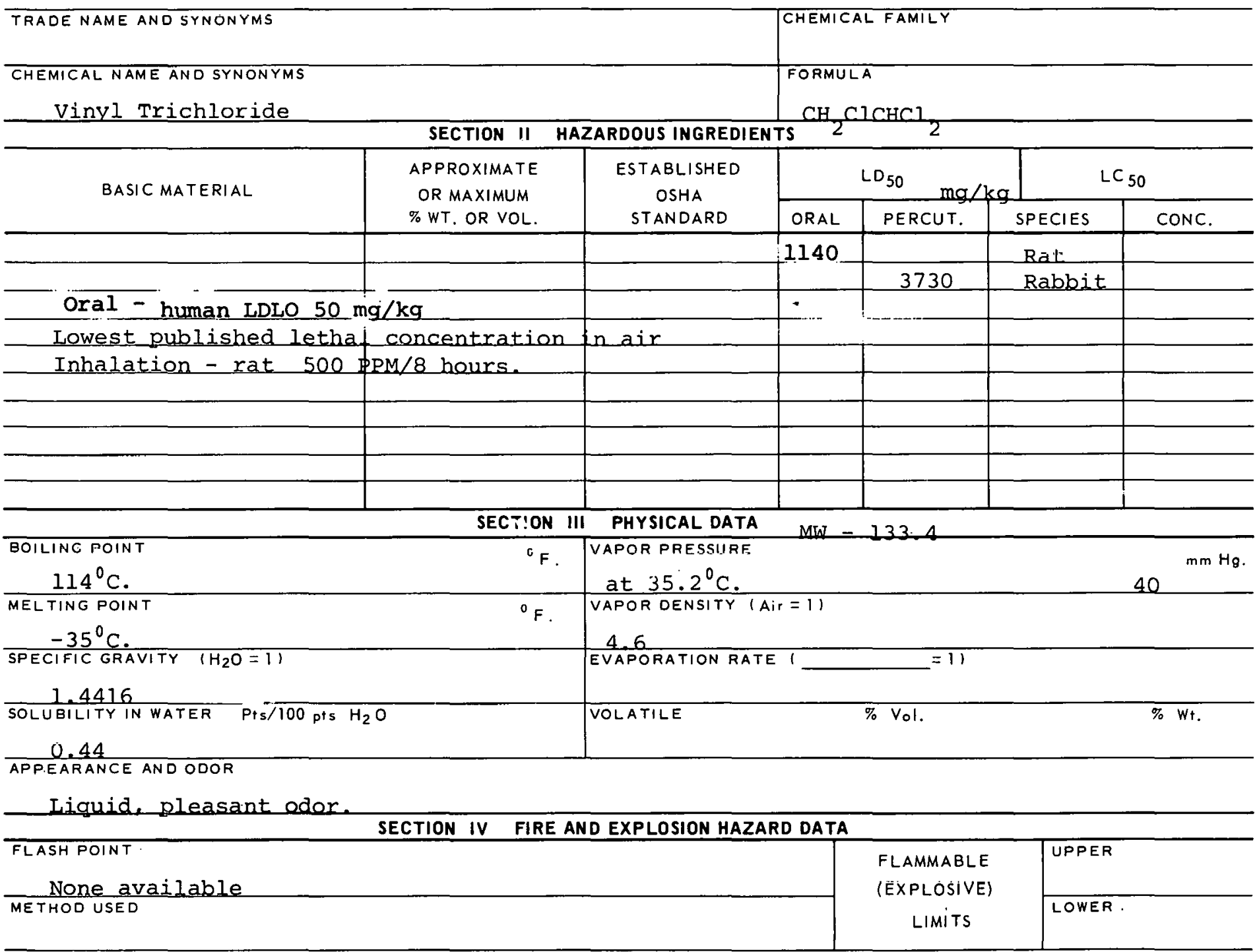

EXTINGUISHING MEDIA

Information not available.

SPECIAL FIRE FIGHTING PROCEDURES

UNUSUAL FIRE AND EXPLOSION HAZARDS 
Cement $\mathrm{T}-3391,1,2$ Trichloroethane

SECTION $V$ HEALTH HAZARD DATA

TOXIC LEVEL

$\mathrm{TLV}$ - $10 \mathrm{PPM}, 45 \mathrm{mg} / \mathrm{C} . \mathrm{M}$. PRINCIPLE ROUTES OF ABSORBTION

Inhalation or skin absorption.

CARCINOGENIC SKIN AND EYE

IRRITATION MOderate

REVELANT SYMPTOMSOF EXPOSURE Irritation of eyes and nose, headache, nausea, cyanosis and

C.N.S. depression.

EFFECTS OF CHRONIC EXPOSURE Headache, tremor, dizziness, peripheral parethesia, hypesthesia,

or anesthesia.

EMERGENCY AND FIRST AID PROCEDURES Artificial respiration, remove contaminated clothing. (Do

not give stimulants), 5\% glucose I.V. (to maintain blood pressure)

SECTION VI REACTIVITY DATA

CONDITIONS CONTRIBUTING TO INSTABILITY

CONDITIONS CONTRIBUTING TO HAZARDOUS POLYMERIZATION

INCOMPATABILITY (MATERIALS TO AVOID)

HAZARDOUS DECOMPOSITION PROOUC,TS

SECTION VII SPILL OR LEAK PROCEDURES

STEPS TO BE TAKEN IN CASE MATERIAL IS RELEASED OR SPILLED

.

WASTE DISPOSAL METHOD

SECTION VIII SPECIAL PROTECTION INFORMATION

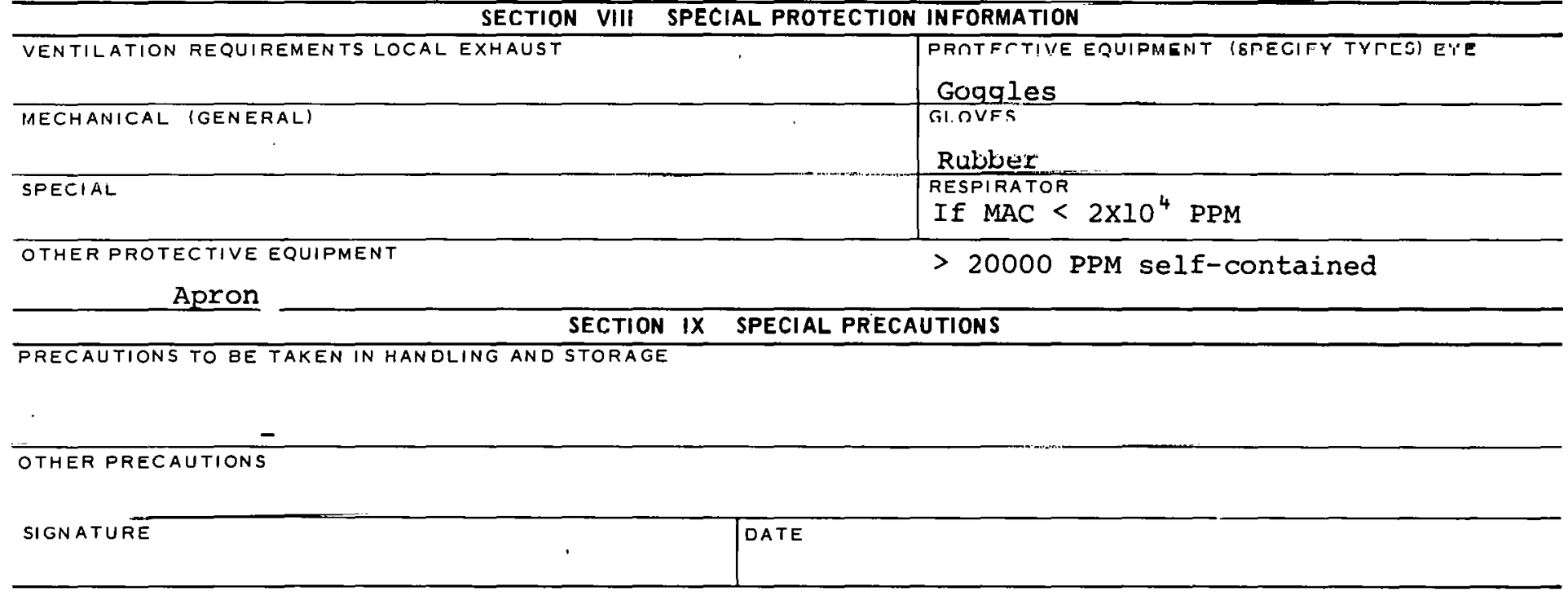

367-0084B 


\section{PRODUCT DESIGNATION}

Trichloroethylene

03-367-0102

$03-367-0118$

03-367-0143

\section{MATERIAL SAFETY \\ DATA SHEET}

\section{SECTION I SOURCE AND NOMENCLATURE}

MANUFACTURER'S NAME

ADORESS (NUMBER, STREET, CITY, STATE, ZIP CODE)

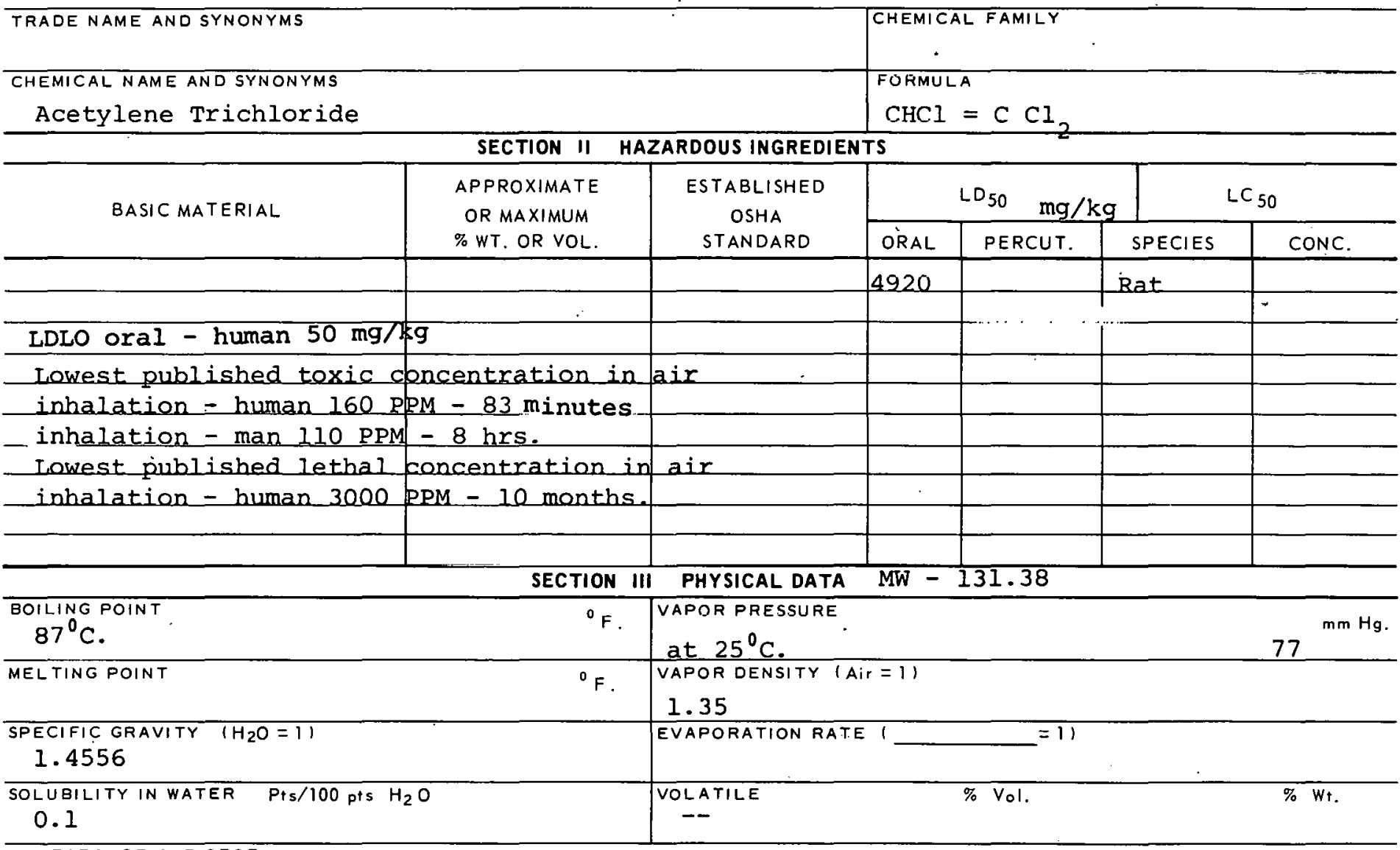

APPEARANCE AND ODOR

Colorless liquid with aromatic odor.

\begin{tabular}{l|c|l}
\hline \multicolumn{2}{c}{ SECTION IV FIRE AND EXPLOSION HAZARD DATA } & \\
\hline FLASH POINT & FLAMMABLE & UPPER \\
$90^{\circ} \mathrm{F}$. & (EXPLOSIVE) & LIMITS \\
\hline METHOD USED & LOWER & \\
\hline
\end{tabular}

EXTINGUISHING MEDIA

Alcohol foam, $\mathrm{CO}_{21}$

SPECIAL FIRE FIGHTING PROCEDURES

Wear self-contained gas mask.

UNUSUAL FIRE AND EXPLOSION HAZARDS

Reacts explosively with powdered aluminum. 


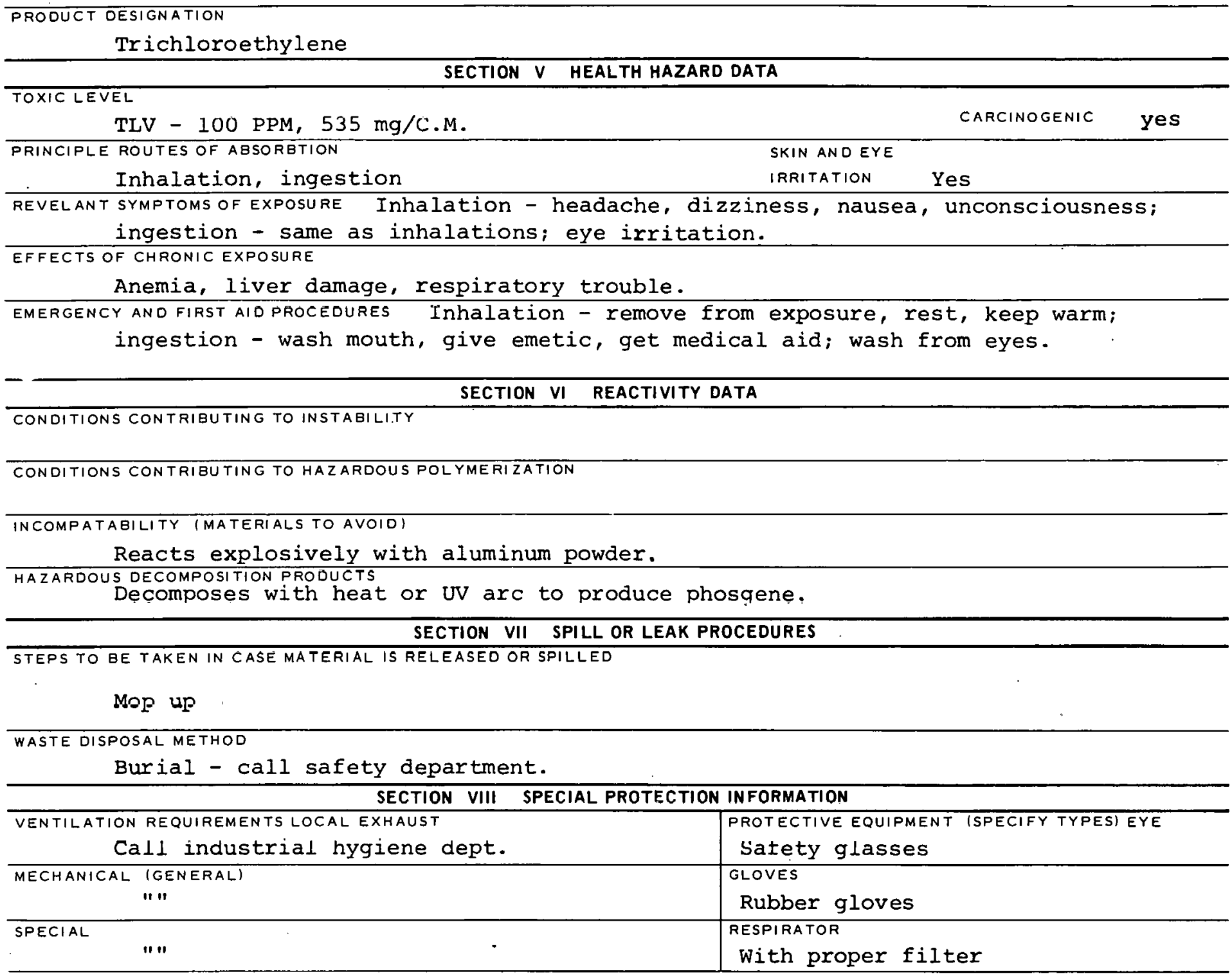

OTHER PROTECTIVE EQUIPMENT

\section{SECTION IX SPECIAL PRECAUTIONS}

PRECAUTIONS TO BE TAKEN IN HANDLING AND STORAGE

OTHER PRECAUTIONS

SIBNATURE

T̄ंTE

$367-0102 B$ 
PRODUCT DESIGNATION

03-367-0602

2, 4,6 Trichlorophenol

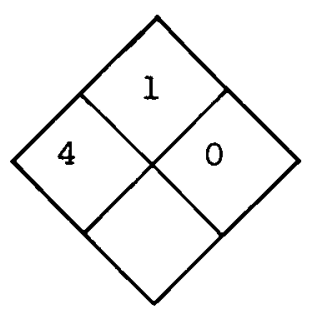

\section{SECTION I SOURCE AND NOMENCLATURE}

MANUFACTURER'S NAME
ADDRESS INUMBER, STREET, CITY,
TRADE NAME AND SYNONYMS
CHEMICAL NAME AND SYNONYMS
$2,4,6$ TriChlOYOPhENOI

2:4,6 Trichlorophenol

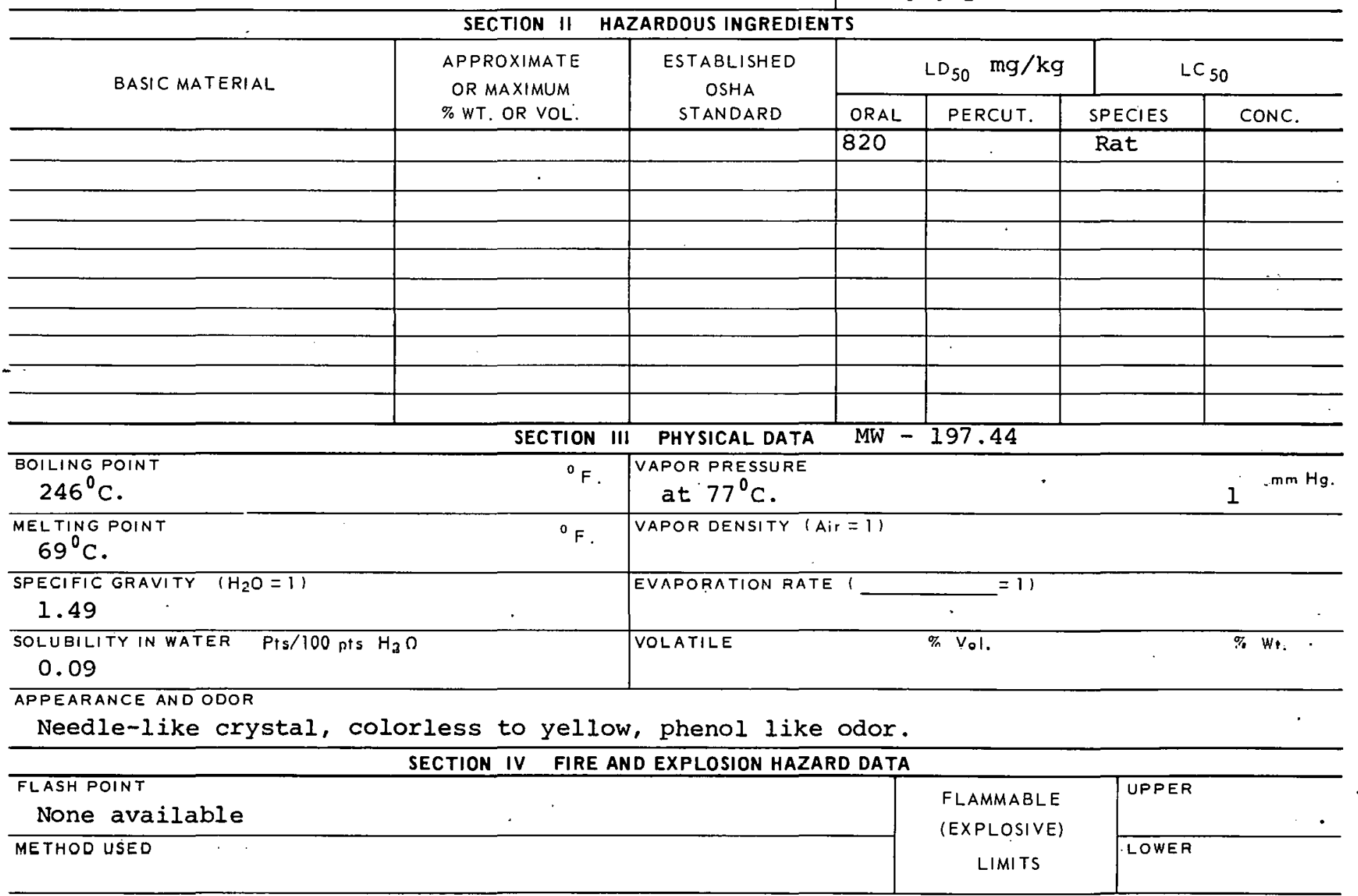

EXTINGUISHING MEDIA

Carbon dioxide, alcohol foam.

SPECIAL FIRE FIGHTING PROCEDURES

Self-contained breathing apparatus.
EMERGENCY TELEPHONE NO. 


\section{TOXIC LEVEL}

None available

PRINCIPLE ROUTES OF ABSORBTION

Inhalation, ingestion, skin

REVELANT SYMPTOMS OF EXPOSURE

irritation of digestive

EFFECTS OF CHRONIC EXPOSURE

- -

EMERGENCY AND FIRST AID PROCEDURES

get medical aid;

wash
Inhalation - rest; if swallowed - give epsom salts, SKIN AND EYE

IRRITATION SEvere

Irritation or severe burns on skin \& eyes; ingestion system.

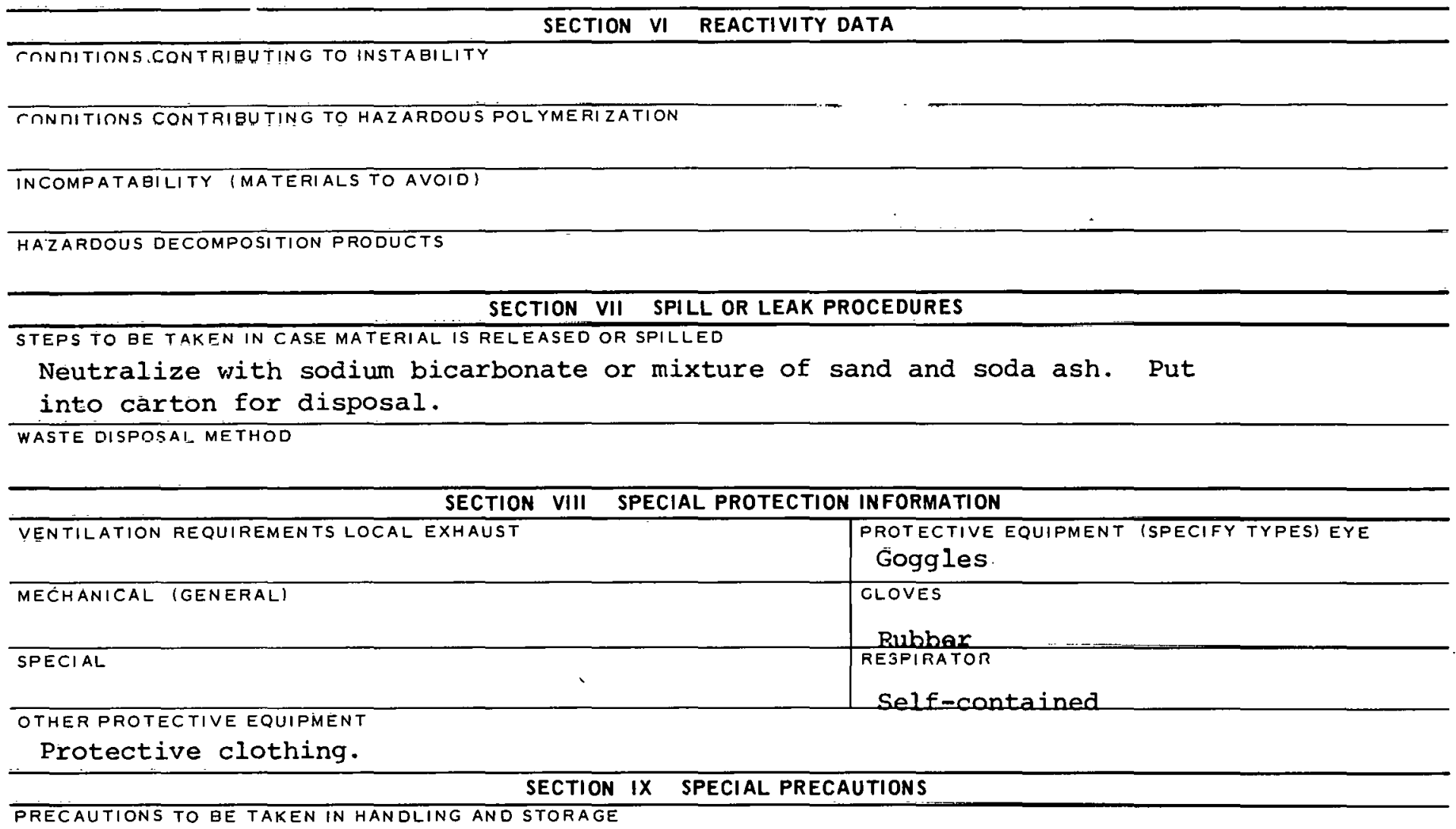

OTHER PRECAUTIONS 
CAS - 000096184

Tz92750

03-367-1102

PRODUCT DESIGNATION

1,2,3 Trichloropropane

MATERIAL SAFETY

DATA SHEET

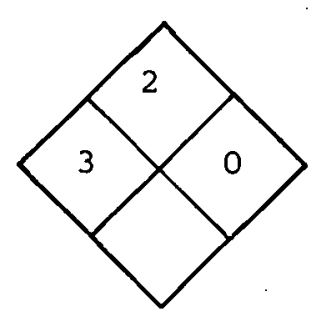

\section{SECTION I SOURCE AND NOMENCLATURE}

ADDRESS (NUMBER, STREET, CITY, STATE, ZIP CODE)

\section{TRADE NAME AND SYNONYMS}

Glycerol Trichlorohydrin

CHEMICAL NAME AND SYNONYMS

$1,2,3$ Trichloropropane

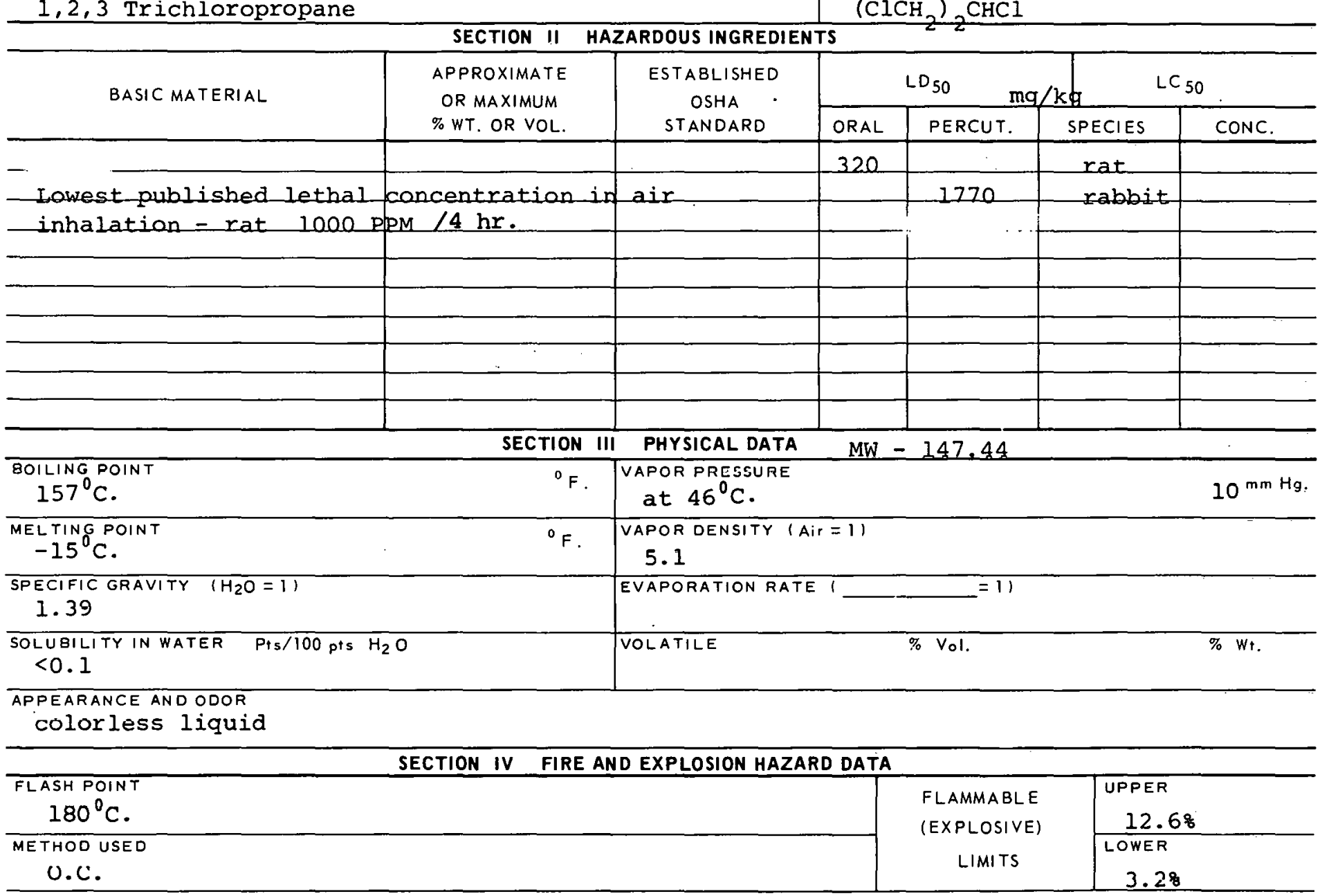

EXTINGUISHING MEDIA

Water may be used to blanket the fire, foam, carbon dioxide, dry chemical.

SPECIAL FIRE FIGHTING PROCEDURES

Wear self-contained breathing apparatus.

UNUSUAL FIRE AND EXPLOSION HAZARDS 


\section{SECTION $V$ HEALTH HAZARD DATA}

\section{TOXIC LEVEL}

50. PPM, $300 \mathrm{mg} / \mathrm{C} . \mathrm{M}$.

PRINCIPLE ROUTES OF ABSORBTION

CARCINOGENIC

\section{Inhalation, ingestion,skin \\ REVELANT SYMPTOMS OF EXPOSURE EYe and throat irritation, edema and hemorrhaging in}

$$
\text { lungs, narcosis. }
$$

EFFECTS OF CHRONIC EXPOSURE

Heart and liver damage

EMERGENCY AND FIRST AIO PROCEDURES If SWaIIOWed - gIVe emetic, get medicaI aId; lnhalation -

rest, artificial respiration if needed; wash from skin \& eyes.

\section{SECTION VI REACTIVITY DATA}

CONDITIONS CONTRIBUTING TO INSTABILITY
CONDITIONS CONTRIBUTING TO HAZARDOUS POLYMERI ZATION
INCOMPATABILITY IMATERIALS TO AVOIDI
HAZARDOUS DECOMPOSITION PRODUCTS
SECTION VII SPILL OR LEAK PROCEDURES

STEPS TO BE TAKEN IN CASE MATERIAL IS RELEASED OR SPILLED

WASTE DISPOSAL METHOD

SECTION VIII SPECIAL PROTECTION INFORMATION

\begin{tabular}{l|l}
\hline \multicolumn{1}{|c|}{ SECTION VIII SPECIAL PROTECTION INFORMATION } \\
\hline MECHANICAL (GENERAL) & $\begin{array}{c}\text { PROTECTIVE EQUIPMENT (SPECIFY TYPES) EYE } \\
\text { GOgGles }\end{array}$ \\
\hline SPEGIAL & $\begin{array}{c}\text { GLOVES } \\
\text { RUbber }\end{array}$ \\
\hline OTHER PROTECTIVE EQUIPMENT & RESPIRATOR \\
With proper filter
\end{tabular}

OTHER PROTECTIVE EQUIPMENT

\section{SECTION IX SPECIAL PRECAUTIONS}

PRECAUTIONS TO BE TAKEN IN HANDLING AND STORAGE

ETHER PRECAUTIUINS

SIGNATU R̄E

DATE

$367-1102 B$ 


\section{PRODUCT DESIGNATION}

03-367-2102

Triethylamine

MATERIAL SAFETY

DATA SHEET

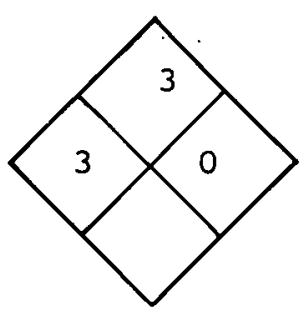

\section{SECTION I SOURCE AND NOMENCLATURE}

MANUFACTURER'S NAME
ADDRESS INUMBER, STREET, CITY
TRADE NAME AND SYNONYMS
CHEMICAL NAME AND SYNONYMS
Trie ThYlamine

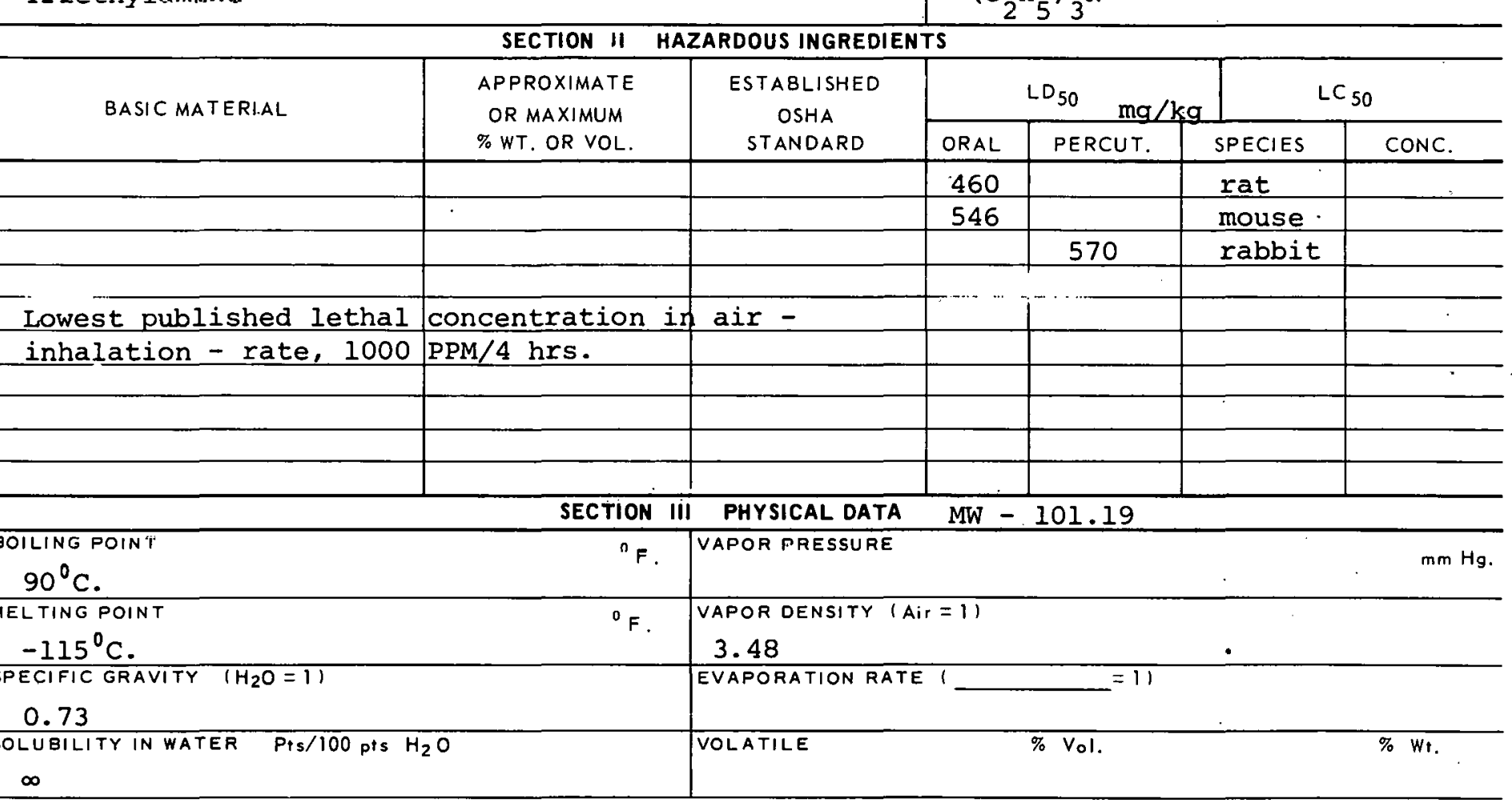

APPEARANCE AND ODOR

Colorless oil

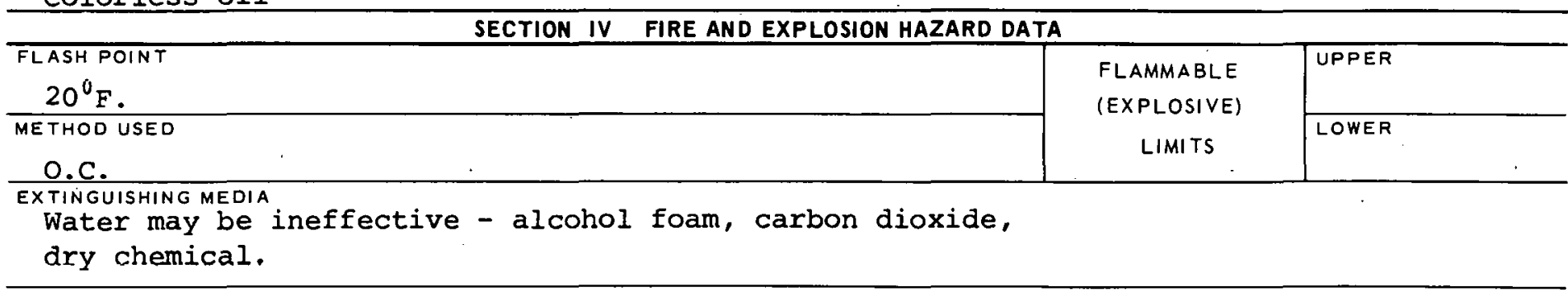

SPECIAL FIRE FIGHTING PROCEDURES

UNUSUAL FIRE AND EXPLOSION HAZARDS 
TOXICLEVEL

$25 \mathrm{PPM}, 100 \mathrm{mg} / \mathrm{C} . \mathrm{M}$.

PRINCIPLE ROUTES OF ABSORBTION

Ingestion, inhalation -

CARCINOGENIC SKIN AND EYE

IRRITATION

Severe

REVELANT SYMPTOMS OF EXPOSURE Eye and skin burns, eye irritation and corneal edema,

mucous membrane irritation.

EFFECTS OF CHRONIC EXPOSURE Lung irritation, liver and kidney damage, heart

damage and edema.

EMERGENCY AND FIRST AID PROCEDURES If swallowed - drink water, get medical aid;

inhalation - rest; wash eyes and skin.

\section{SECTION VI REACTIVITY DATA}

CONDITIONS CONTRIBUTING TO INSTABILITY

CONDITIONS CONTRIBUTING TO HAZARDOUS POLYMERIZATION

IN COMPATABILITY (MATERIALS TO AVOID)

Can react with oxidizing materials.

HAZARDOUS DECOMPOSITION PRODUCTS

SECTION VII SPILL OR LEAK PROCEDURES

STEPS TO BE TAKEN IN CASE MATERIAL IS REI,EASED OR SPILLED

WASTE DISPOSAL METHOD

SECTION VIII SPECIAL PROTECTION INFORMATION

\begin{tabular}{|c|c|}
\hline VENTILATION REQUIREMENTS LOCAL EXHAUST & 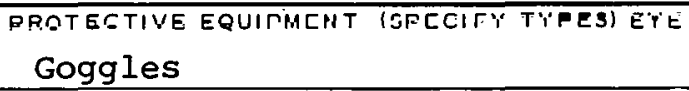 \\
\hline MECHANICAL (GENERAL) & $\begin{array}{l}\text { GLOVES } \\
\text { Rubber }\end{array}$ \\
\hline SPECIAL & $\begin{array}{l}\text { RESPIRATOR } \\
\text { With proper filter }\end{array}$ \\
\hline
\end{tabular}

OTHER PROTECTIVE EQUIPMENT

*

SECTION IX SPECIAL PRECAUTIONS

PRECAUTIONS TO BE TAKEN IN HANDLING AND STORAGE

OTHER PRECAUTIONS

\begin{tabular}{l|l|l}
\hline SIGNATURE & DATE \\
\hline
\end{tabular}

$367-2102 B$ 
CAS - 000075503

\begin{tabular}{|l|}
\hline PRODUCT DESIGNATION \\
$03-367-3102$ \\
Trimethylamine \\
\end{tabular}

\section{MATERIAL SAFETY DATA SHEET}

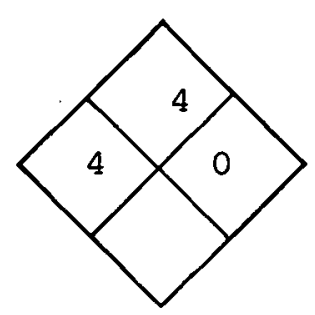

\section{SECTION I SOURCE AND NOMENCLATURE}

\begin{tabular}{l|l|l}
\hline MANUFACTURER'S NAME & SECTION I SOURCE AND NOMENCLATURE \\
\hline ADDRESS (NUMBER, STREET, CITY, STATE, ZIP CODE)
\end{tabular}

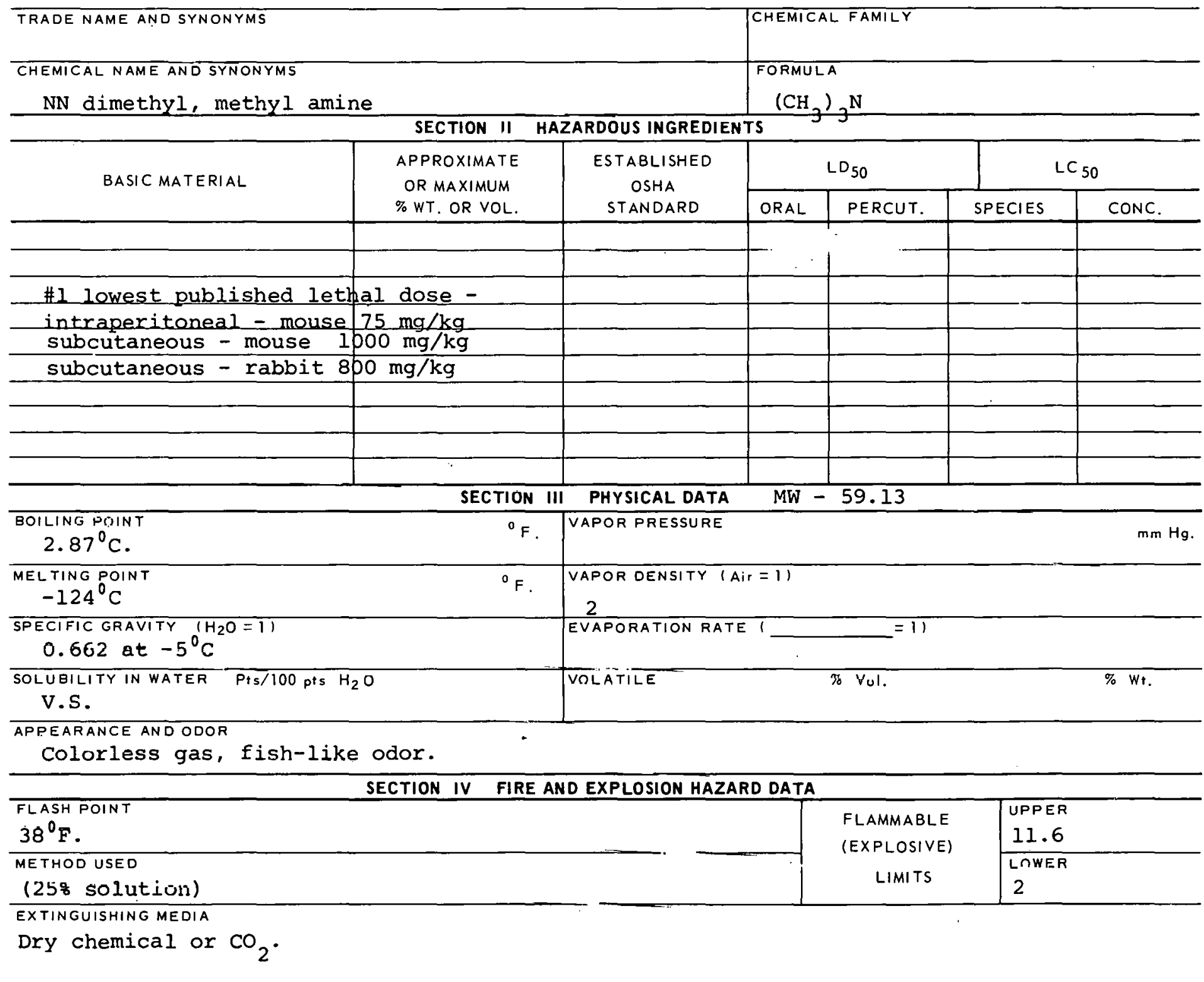

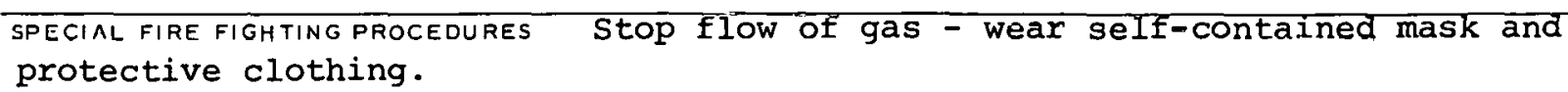




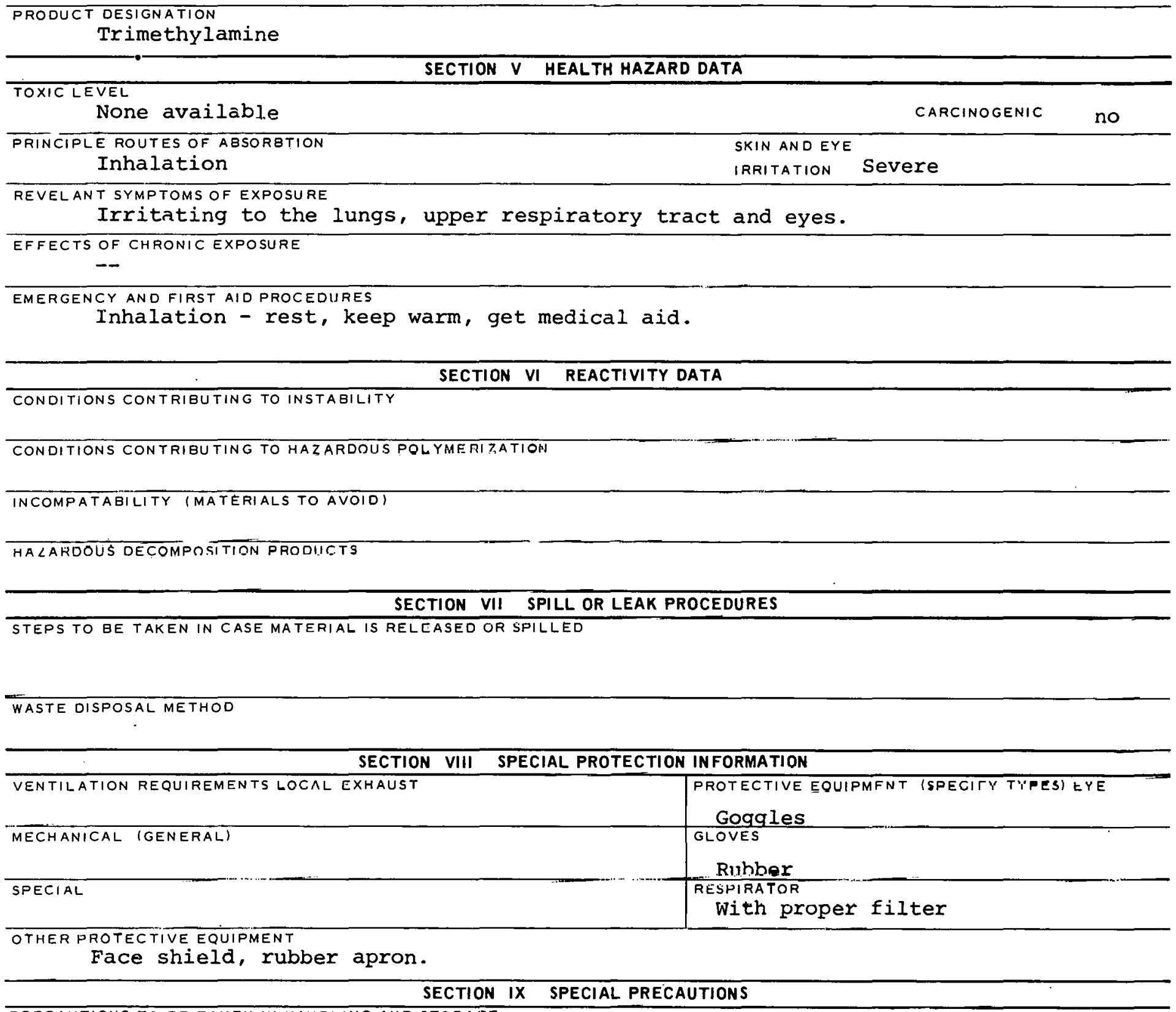

PRECAUTIONS TO BE TAKEN IN HANDLING AND STORAGE

OTHER PRECAUTIONS

SIGNATURE

D̄ATE

$367-3102 B$ 
PRODUCT DESIGNATION

03-367-3502

Trimethylene Bromide

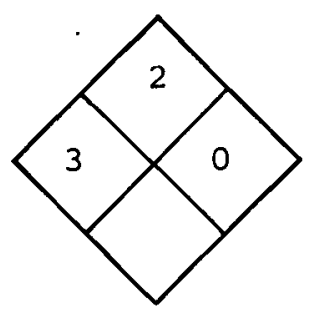

\section{SECTION I SOURCE AND NOMENCLATURE}

MANUFACTURER'S NAME

EMERGENCY TELEPHONE NO.

ADDRESS (NUMBER, STREET, CITY, STATE, ZIP CODE)

TRAOE NAME AND SYNONYMS

\begin{tabular}{|l|l|} 
& CHEMICAL FAMILY \\
& $\begin{array}{l}\text { FORMULA } \\
\mathrm{CH}_{2} \mathrm{BrCH}_{2} \mathrm{CH}_{2} \mathrm{Br}\end{array}$ \\
\hline SECTION II HAZARDOUS INGREDIENTS
\end{tabular}

\section{CHEMICAL NAME AND SYNONYMS}

1,3 - Dibromopropane

BASIC MATERIAL

Lowest published lethal
Intraperitoneal - mouse

APPROXIMATE

OR MAXIMUM

\% WT. OR VOL.

ANDARD

\section{BOILING POINT}

$167^{\circ} \mathrm{C}$.

MELTING POINT

$-36^{\circ} \mathrm{C}$.

SPECIFIC GRAVITY $\left(\mathrm{H}_{2} \mathrm{O}=1\right)$

1.97

SOLUBILITY IN WATER Pts/100 pts $\mathrm{H}_{2} \mathrm{O}$

0.02

APPEARANCE AND ODOR

Colorless liquid

\begin{tabular}{|c|c|c|c|c|}
\hline & SECTION IV & FIRE AND EXPLOSION HAZARD DATA & & \\
\hline $\begin{array}{l}\text { FLASH POINT } \\
\text { none available }\end{array}$ & 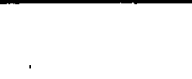 & & \multirow{2}{*}{$\begin{array}{c}\text { FLAMMABLE } \\
\text { (EXPLOSIVE) } \\
\text { LIMITS }\end{array}$} & UPPER \\
\hline METHOD USED & & & & LOWER \\
\hline
\end{tabular}

EXTINGUISHING MEDIA

Foam, carbon dioxide, dry chemical.

VOLATILE \% Vol. $\% W_{t}$.

SECTION III PHYSICAL DATA MW - 201.91

${ }^{\circ} \mathrm{F}$ VAPOR PRESSURE

SPECIAL FIRE FIGHTING PROCEDURES

UNUSUAL FIRE ANDEXPLOSION HAZARDS 
PRODUCT DESIGNATION

Trimethylene bromide

SECTION $V$ HEALTH HAZARD DATA

TOXIC LEVEL

none available

PRINCIPLE ROUTES OF ABSORBTION

inhalation, ingestion

CARCINOGENIC SKIN ANOEYE

IRRITATION no

REVELANT SYMPTOMS OF EXPOSURE EYe irritation, narcosis, sleepiness, headache,

vomiting.

EFFECTS OF CHRONIC EXPOSURE

Dermatitis, liver and kidney damage.

EMERGENCY AND FIRST AID PROCEDURES Inhalation - rest; if swallowed - give emetic, get medical aid; wash from skin and eyes.

\section{SECTION VI REACTIVITY DATA}

CONDITIONS CONTRIBUTING TO INSTABILITY

CONDITIONS CONTRIBUTING TO HAZARDOUS POLYMERIZATION

INCOMPATABILITY (MATERIALS TO AVOID)

HAZARDOUS DECOMPOSITION PROOUCTS

SECTION VII SPILL OR LEAK PROCEDURES

STEPS TO BE TAKEN IN CASE MATERIAL IS RELEASED OR SPILLED

WASTE DISPOSAL METHOD

SECTION VIII SPECIAL PROTECTION INFORMATION

\begin{tabular}{|c|c|}
\hline VENTILATION REQUIREMENTS LOCAL EXHAUST & $\begin{array}{l}\text { PROTECTIVE EOUIPMENT ISPFR.IFY TYPFSI EYE } \\
\text { GEŸت̆lES }\end{array}$ \\
\hline MECHANICAL (GENERAL) & $\begin{array}{l}\text { GLOVES } \\
\text { Rubber }\end{array}$ \\
\hline SPECIAL & $\begin{array}{l}\text { RESPIRATOR } \\
\text { With proper filter }\end{array}$ \\
\hline
\end{tabular}

OTHER PROTECTIVE EQUIPMENT

With proper filter

SECTION IX SPECIAL. PRECAUTIONS

PRECAUTIONS TO BE TAKEN IN HANOLING AND STORAGE

OTHER PRECAUTIONS

SIGNATURE

DATE

\begin{tabular}{l|l|l}
\hline & DATE \\
\hline
\end{tabular}

367-3502B 


\section{PRODUCT DESIGNATION}

03-367-3902

Trimethylene Glycol

\section{MATERIAL SAFETY \\ DATA SHEET}

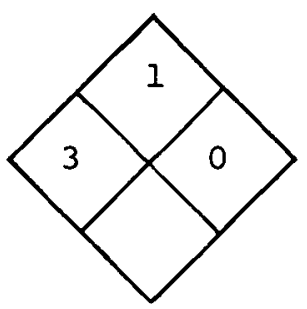

\section{SECTION I SOURCE AND NOMENCLATURE}

\section{MANUFACTURER'S NAME \\ TRADE NAME AND SYNONYMS \\ 1,3 dihydroxy propane \\ CHEMICAL NAME AND SYNONYMS \\ Trimethylene Glycol} ADDRESS (NUMBER, STREET, CITY, STATE, ZIP CODE)

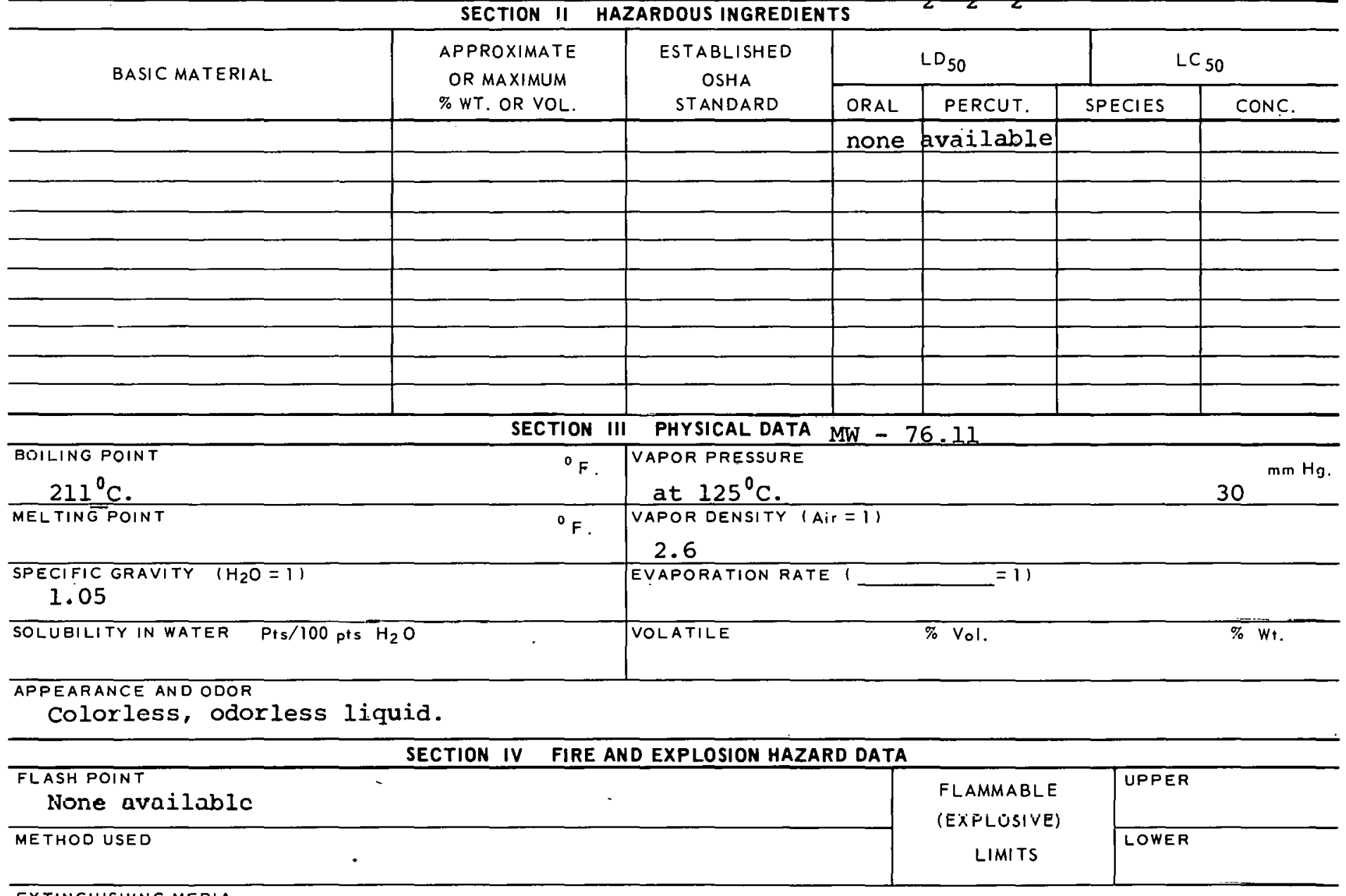

EXTINGUISHING MEDIA

Water, carbon dioxide, dry chemical.

SPECIAL FIRE FIGHTING PROCEDURES

UNUSUAL FIRE AND EXPLOSION HAZARDS 
Trimethylene Glycol

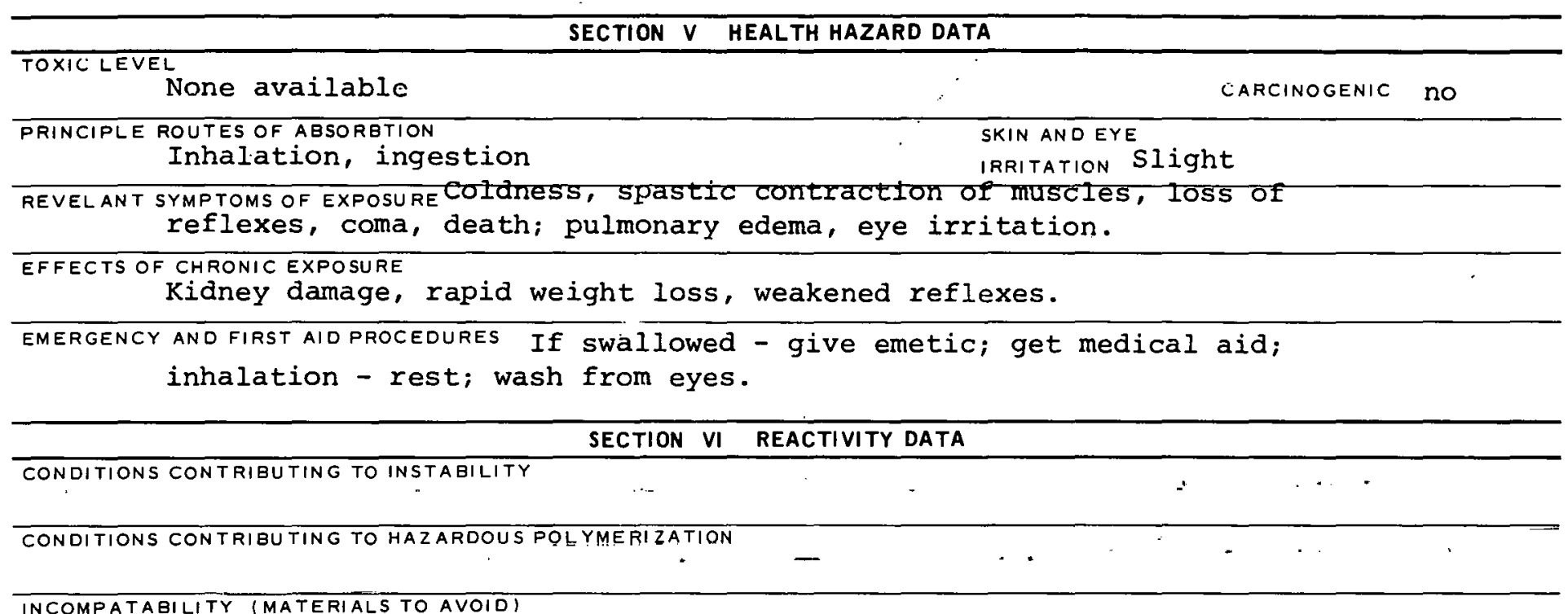

HAZARDOUS DECOMPOSITION PRODUCTS

SECTION VII SPILL OR LEAK PROCEDURES

STEPS TO BE TAKEN IN CASE MATERIAL IS RELEASED OR SPILLED

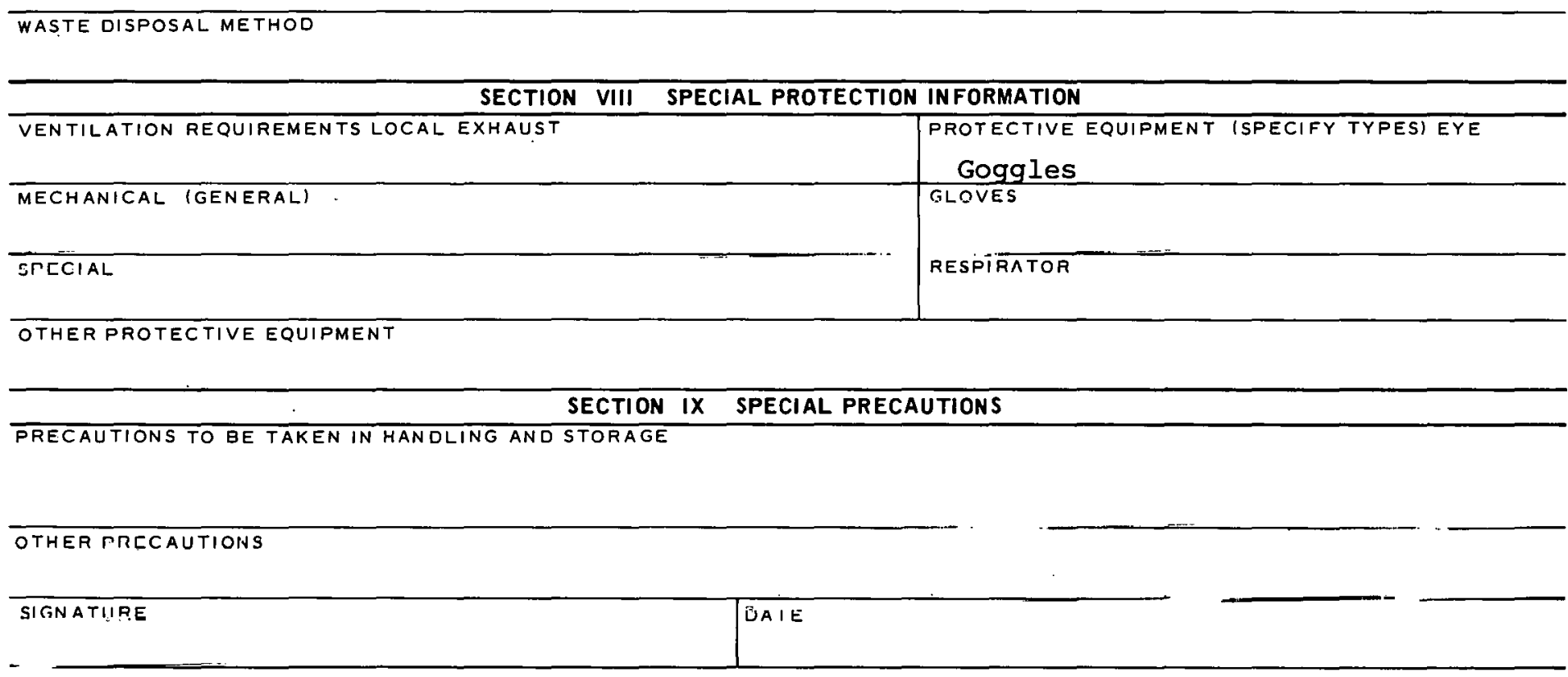




PRODUCT DESIGNATION
$03-367-5602$
$2,4,6-$ Trinitroresorcinol.

\section{MATERIAL SAFETY DATA SHEET}

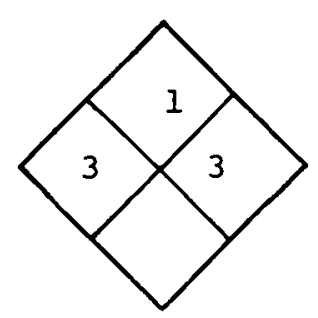

\section{SECTION I SOURCE AND NOMENCLATURE}

\section{MANUFACTURER'S NAME}

ADDRESS (NUMBER, STREET, CITY, STATE, ZIP CODE)

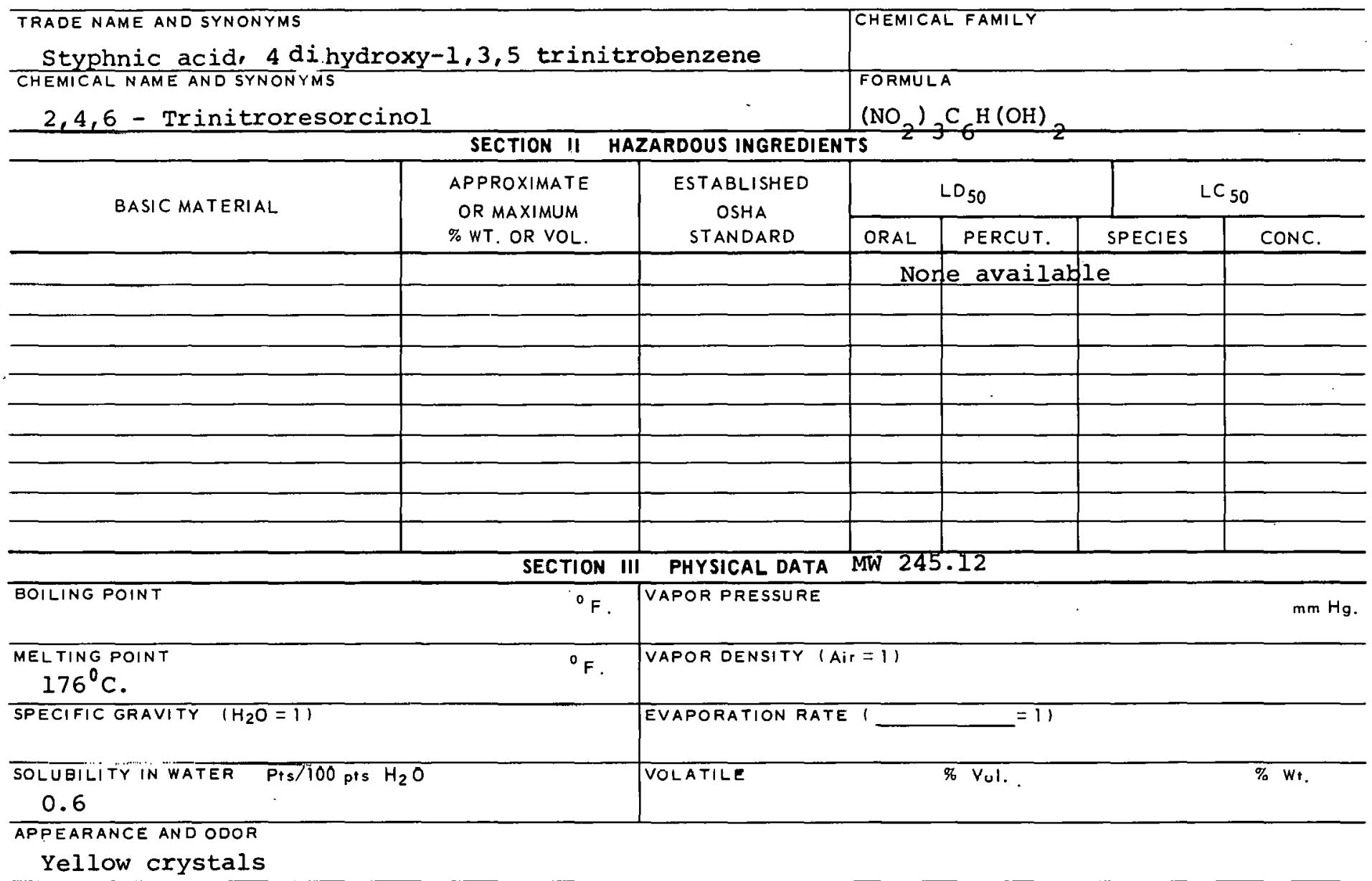

\begin{tabular}{|c|c|c|c|c|}
\hline & SECTION IV & FIRE AND EXPLOSION HAZARD DATA & & \\
\hline $\begin{array}{l}\text { FLASH POINT } \\
\text { None available }\end{array}$ & & & $\begin{array}{l}\text { FLAMMABLE } \\
\text { (EXPLOSIVE) }\end{array}$ & UPPER \\
\hline METHOD USED & & & & LOWER \\
\hline
\end{tabular}

EXTINGUISHING MEDIA Explosive - do not fiyhl fire except for unmanned equipment use water.

SPECIAL.FIRE FIGHTING PROCEDURES

Full protective clothing for rescue operations.

UNUSUAL FIRE ANU EXPLOSION HAZARDS

Severe explosion hazard. Dot class A explosive 


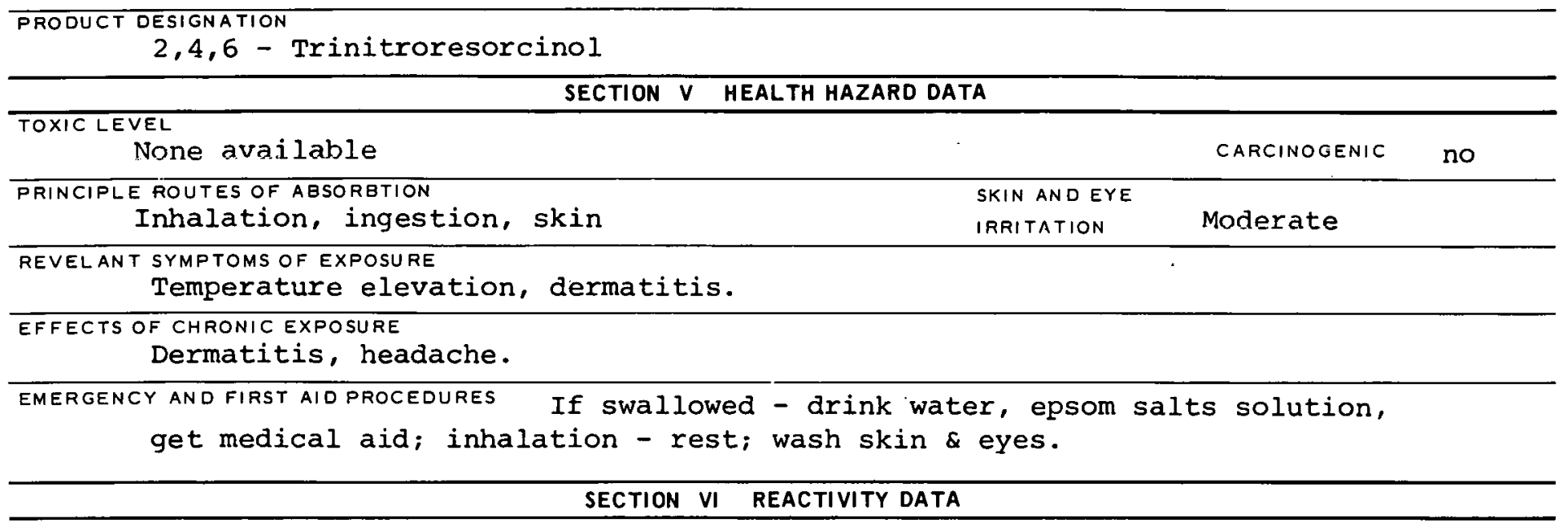

CONDITIONS CONTRIBUTING TO INSTABILITY

CONDITIONS CONTRIBUTING TO HAZARDOUS POLYMERIZATION

INCOMPATABILITY (MATERIALS TO AVOID) Explosive (keep from oxidizing materials) inorganic

nitrates and chlorates, ammonium nitrate.

HAZAROOUS DECOMPOSITION PRODUCTS

Oxides of nitregen.

SECTION VII SPILL OR LEAK PROCEDURES

STEPS TO BE TAKEN IN CASE MATERIAL IS RELEASED OR SPILLED

WASTE DISPOSAL METHOD

SECTION VIII SPECIAL PROTECTION INFORMATION

\begin{tabular}{|c|c|}
\hline SECTION VIII & SPECIAL PROTECTION INFORMATION \\
\hline VENTILATION REQUIREMENTS LOCAL EXHAUST & \begin{tabular}{|l|l} 
& $\begin{array}{l}\text { PROTECTIVE EQUIPMENT (SPECIFY TYPES) EYE } \\
\text { Goggles }\end{array}$ \\
\end{tabular} \\
\hline MECHANICAL (GENERAL) & $\begin{array}{l}\text { GI.OVES } \\
\text { Rubluer }\end{array}$ \\
\hline SRECIAL & $\begin{array}{l}\text { RESPIRATOR } \\
\text { Self-contained }\end{array}$ \\
\hline
\end{tabular}

OTHER PROTECTIVE EQUIPMENT

\section{SECTION IX SPECIAL PRECAUTIONS}

PRECAUTIONS TO BE TAKEN IN HANDLING AND STORAGE

Keep from oxidizing materials, combustibles and source of heat.

OIHEH PRECAUTIONS

SICNATURE

DATE

$367-5602 B$ 
CAS : 000102692

\begin{tabular}{|l} 
PRODUCT DESIGNATION \\
$03-367-8102$ \\
Tri-N-Propylamine
\end{tabular}

\section{MATERIAL SAFETY DATA SHEET}

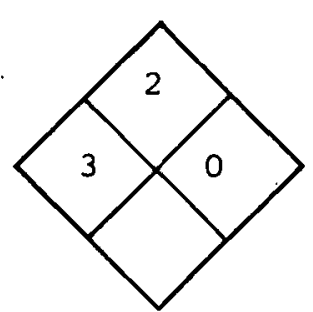

\section{SECTION I SOURCE AND NOMENCLATURE}

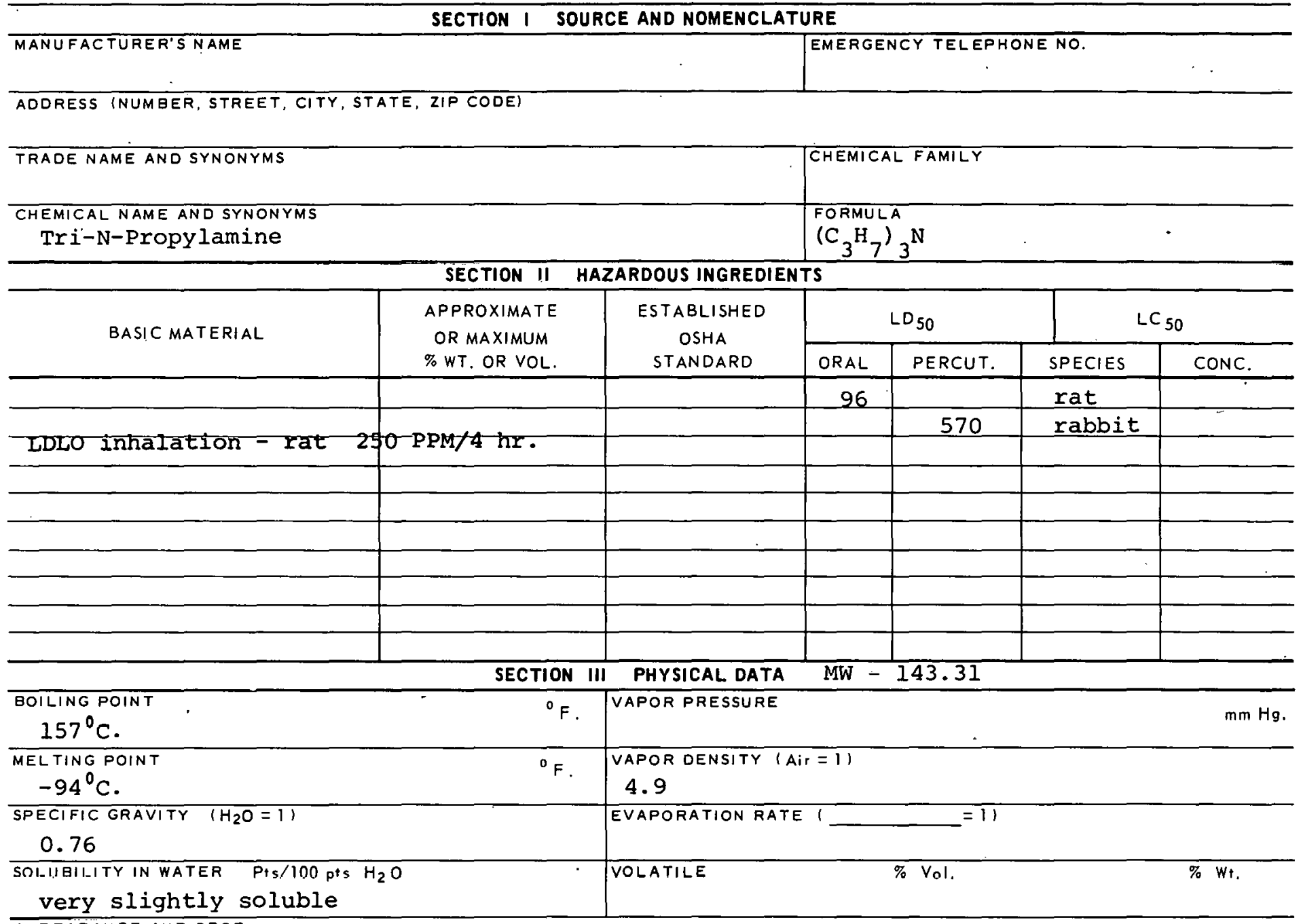
APPEARANCE ANDODOR

colorless liquid

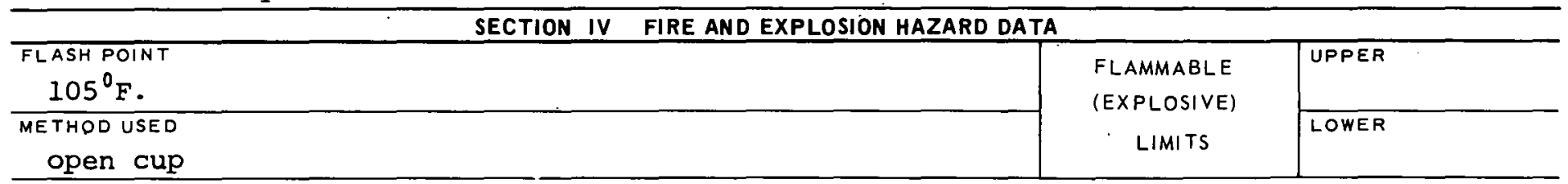

EXTINGUISHING MEDIA

Carbon dioxide, dry chemical

SPECIAL FIRE FIGHTING PROCEDURES

UNUSUAL FIRE AND EXPLOSION HAZARDS 


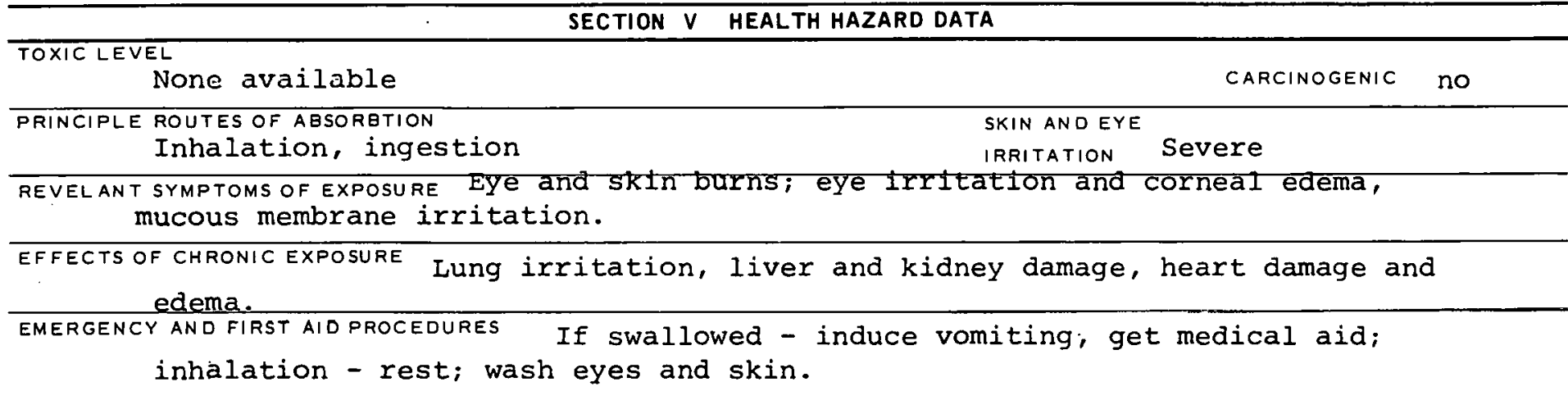

\section{SECTION VI REACTIVITY DATA}

CONDITIONS CONTRIBUTING TO INSTABILITY

CONDITIONS CONTRIBU TING TO HAZARDOUS POLYMERIZATION

INCOMPATABILITY (MATERIALS TO AVOID)

HAZARDOUS DECOMPOSITION PRODUCTS

SECTION VII SPILL OR LEAK PROCEDURES

STEPS TO BE TAKEN IN CASE MATERIAL IS RELEASEO OR SPILLED

WASTE DISPOSAL METHOO

SECTION VIII SPECIAL PROTECTION INFORMATION

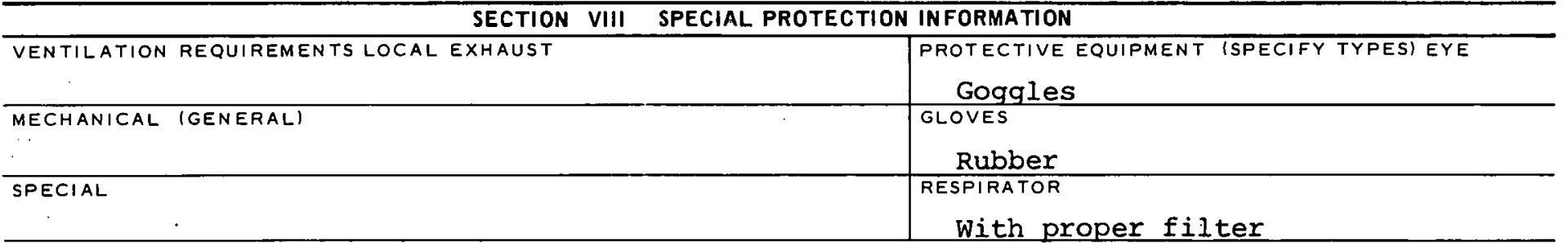

OTHER PROTECTIVE EQUIPMENT

\section{SECTION IX SPECIAL PRECAUTIONS}

PRECAUTIONS TO BE TAKEN IN HANDLING AND STORAGE

\section{OTHER PRECAUTIONS}

GIGATURE

DATE 


\begin{tabular}{|c|}
\hline PRODUCT DESIGNATION \\
03-367-8602 \\
Tribromoethylene
\end{tabular}

\section{MATERIAL SAFETY \\ DATA SHEET}

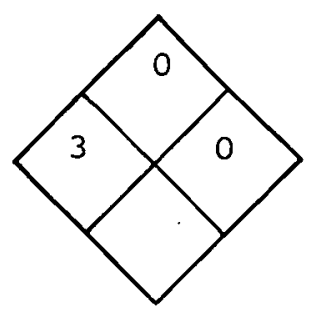

SECTION 1 SOURCE AND NOMENCLATURE

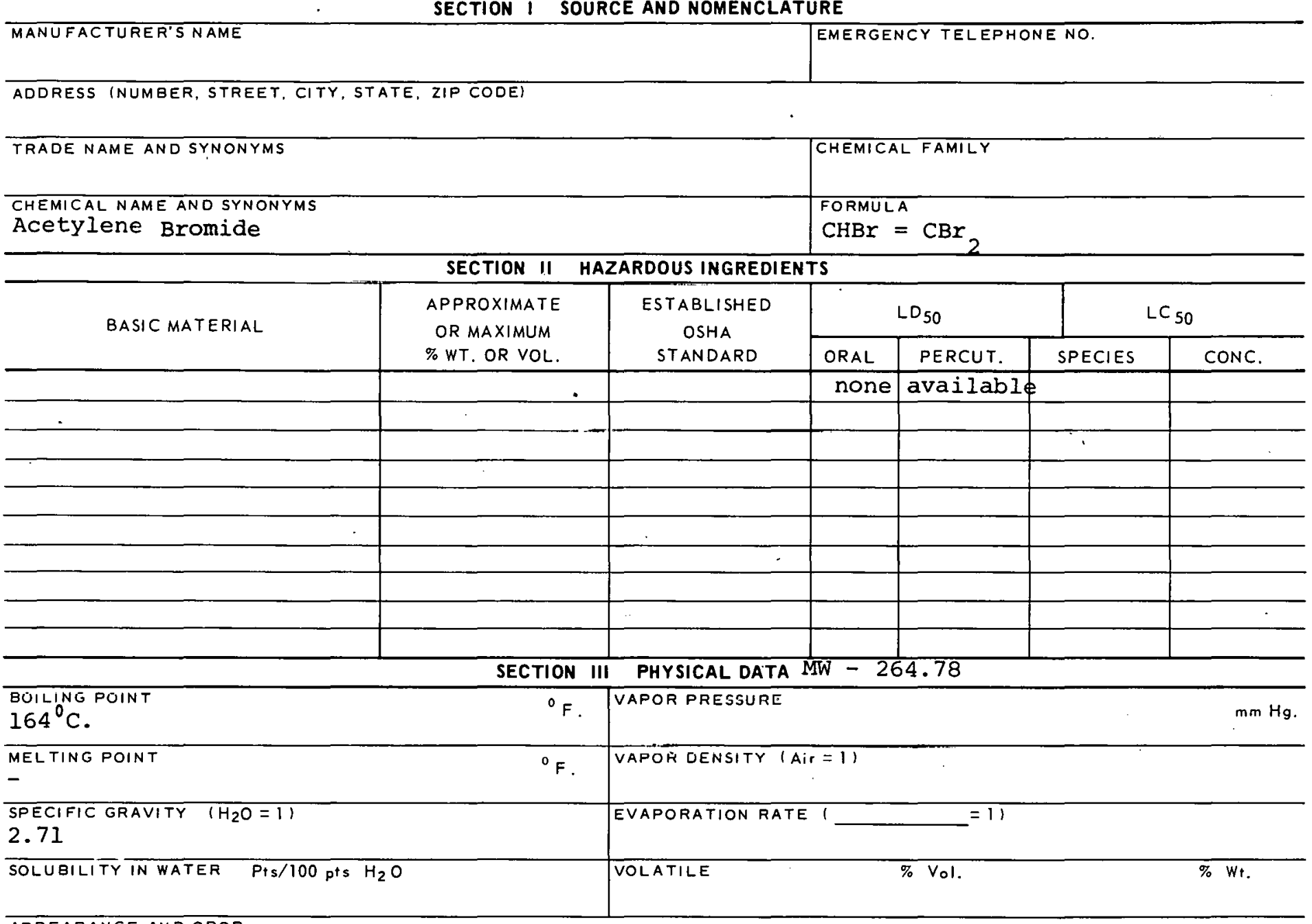

APPEARANCE AND ODOR

liquid

\section{SECTION IV FIRE AND EXPLOSION HAZARD DATA}

\begin{tabular}{l|c|c}
\hline FLASH POINT & FLAMMABLE \\
not flammable & UPPER \\
\hline METHOD USED & (EXPLOSIVE) \\
& LIMITS & LOWER \\
\hline
\end{tabular}

EXTINGUISHING MEDIA

SPECIAL FIRE FIGHTING PROCEDURES

UNUSUAL FIRE AND EXPLOSION HAZARDS 


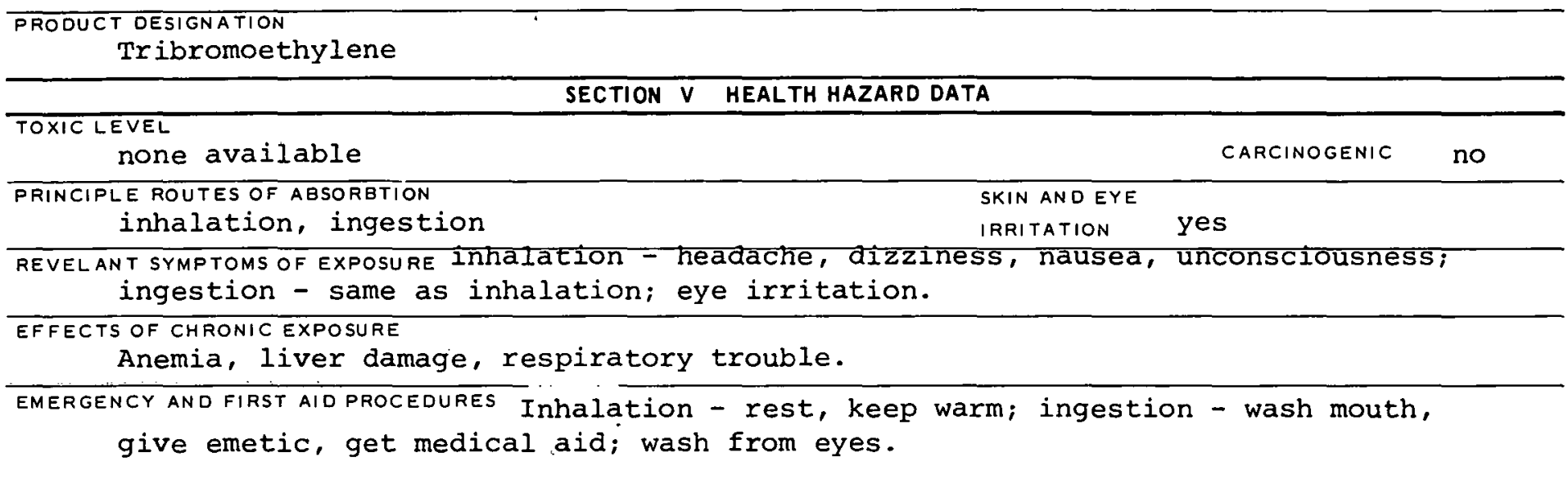

\section{SECTION VI REACTIVITY DATA}

CONDITIONS CONTRIBUTING TO INSTABILITY

CONDITIONS CONTRIQU TING TO HAZARDOUS POLYMEQRIZZTIOQN

INCOMPATABILITY (MATERIALS TO AVOID)

Reacts exploșively with aluminum powder.

HAZARDOUS DECOMPOSITION PRODUCTS

SECTION VII SPILL OR LEAK PROCEDURES

STEPS TO BE TAKEN IN CASE MATERIAL IS RELEASED OR SPILLED

Mop up

WASTE DISPOSAL METHOD

Burial - call safety department SECTION VIII SPECIAL PROTECTION INFORMATION

\begin{tabular}{|c|c|}
\hline VENTILATION REQUIREMENTS LOCAL EXHAUST & $\begin{array}{l}\text { PROTECTIVF FQIIIPMFNT ISPFCIFY TYPESI EYE } \\
\text { safety glasses }\end{array}$ \\
\hline MECHANICAL IGENERAL) & $\begin{array}{l}\text { GLOVES } \\
\text { rubber gloves }\end{array}$ \\
\hline SPECIAL & $\begin{array}{l}\text { RESPIRATOR } \\
\text { with proper filter }\end{array}$ \\
\hline
\end{tabular}

OTHER PROTECTIVE EQUIPMENT

SEC.TION IX SPECIAL PRECAUTIONS

PRECAUTIONS TO BE TAKEN IN HANDLING AND STORAGE

OTHER PRECAUTIONS

SIGNATURE

DATE

$367-8602 B$ 


PRODUCT DESIGNATION
O3-387-1425
Xylene

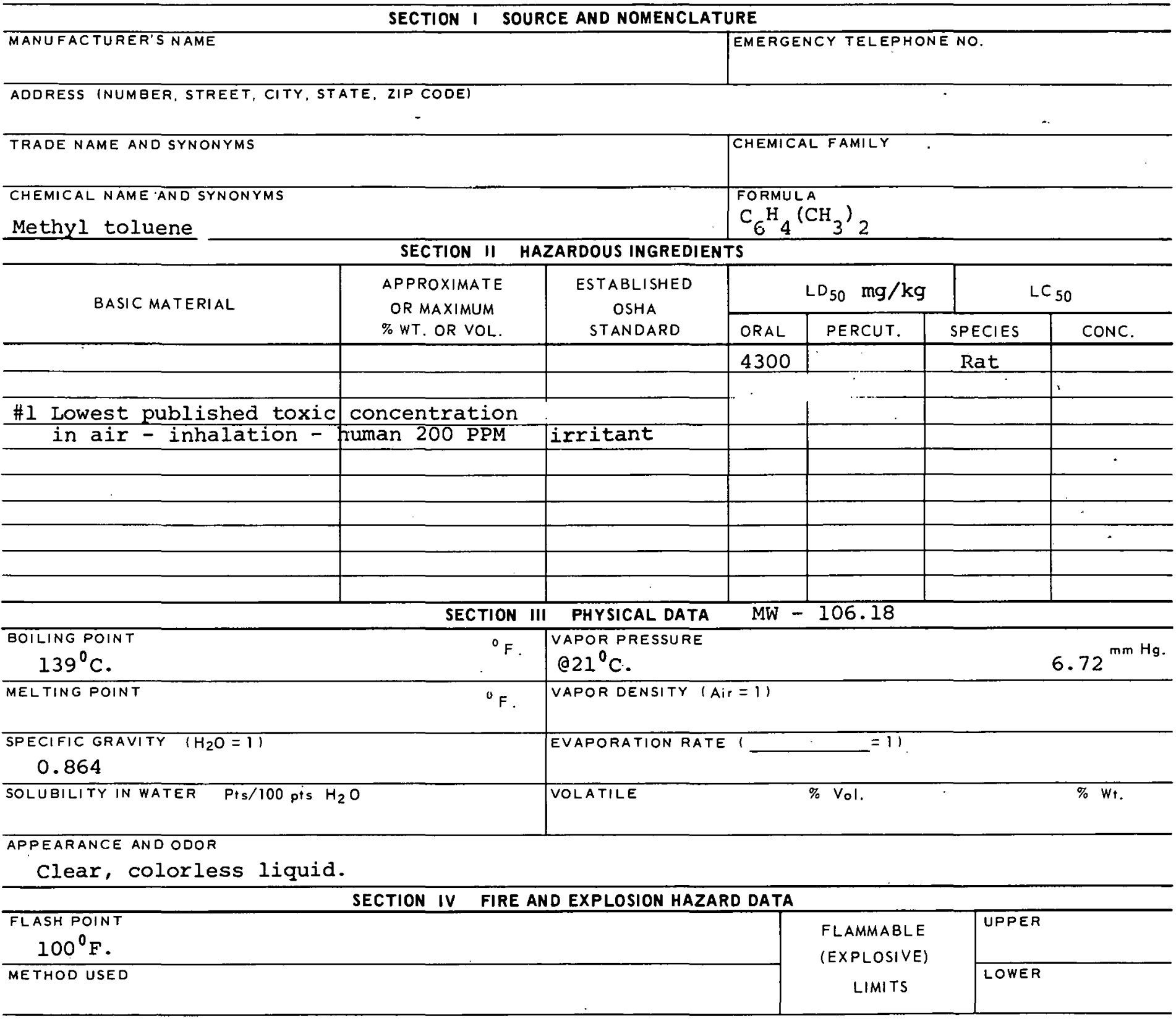

EXTINGUISHING MEDIA

Foam, carbon dioxide, dry chemical - water spray may be used to reduce rate of burning or cooling containers. SPECIAL FIRE FIGHTING PROCEDURES

Wear self-contained breathing apparatus.

UNUSUAL FIRE AND EXPLOSION HAZARDS 


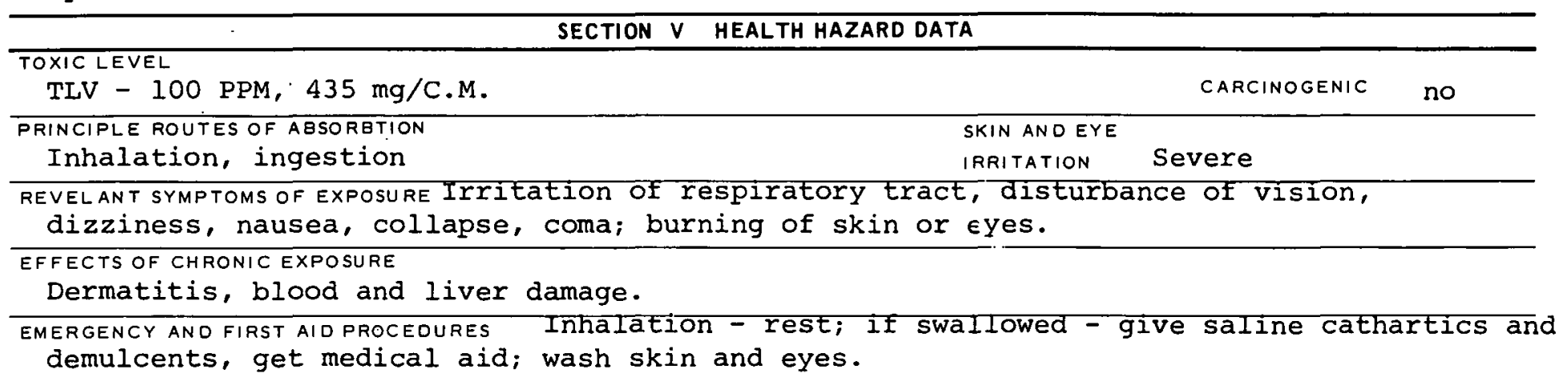

SECTION $V$ HEALTH HAZARD DATA

Inhalation, ingestion IRRITATION Severe


CAS - 000108383

ZE22750

\section{PRODUCT DESIGNATION}

M-Xylene

03-387-1505

MATERIAL SAFETY

DATA SHEET

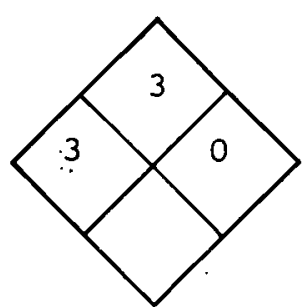

SECTION I SOURCE AND NOMENCLATURE

\begin{tabular}{l|l}
\hline MANUFACTURER'S NAME & EMERGENCY TELEPHONE NO: \\
\hline ADDRESS (NUMBER, STREET, CITY, STATE, ZIP CODE) & \\
\hline
\end{tabular}

TRADE NAME AND SYNONYMS
CHEMICAL, NAME AND SYNONYMS
M-MethYI TOluene

M-Methyl toluene

BASIC MATERIAL.

SECTION II HAZARDOUS INGREDIENTS

CHEMICAL FAMILY
FORMULA
$\mathrm{C}_{6} \mathrm{H}_{4}\left(\mathrm{CH}_{3}\right)_{2}$

\begin{tabular}{l|l} 
APPROXIMATE & ESTABLISHED
\end{tabular}

OR MAXIMUM

$\%$ WT. OR VOL.

OSHA

STANDARD

Lowest published lethal concentration in air -

inhalation - rat $8000 \mathrm{P} \$ \mathrm{M} / 4$ hours.

Lowest published lethal dose -

- intraperitoneal - rat $\$ 000 \mathrm{mg} / \mathrm{kg}$.

subcutaneous - rat $5000 \mathrm{mg} / \mathrm{kg}$

BOILING POINT

$139^{\circ} \mathrm{C}$.

MEL TING POINT

$-48^{\circ} \mathrm{C}$.

SPECIFIC GRAVITY $\left(\mathrm{H}_{2} \mathrm{O}=1\right)$

0.864

SOLUBILITY IN WATER Pts/100 pts $\mathrm{H}_{2} \mathrm{O}$

(2)

APPEARANCE AND ODOR

Clear, colorless liquid.

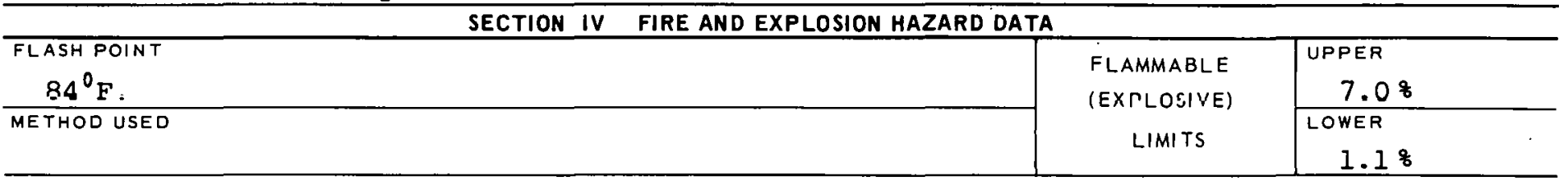

EXTINGUISHING MEDIA Foam, carbon dioxide, or dry chemical. Water spray may be used

to reduce rate of burning or to cool containers.

SPECIAL FIRE FIGHTING PROCEDURES

Wear self-contained breathing apparatus.

IUNIISUAL FIRE AND EXPLOSION HAZARDS 


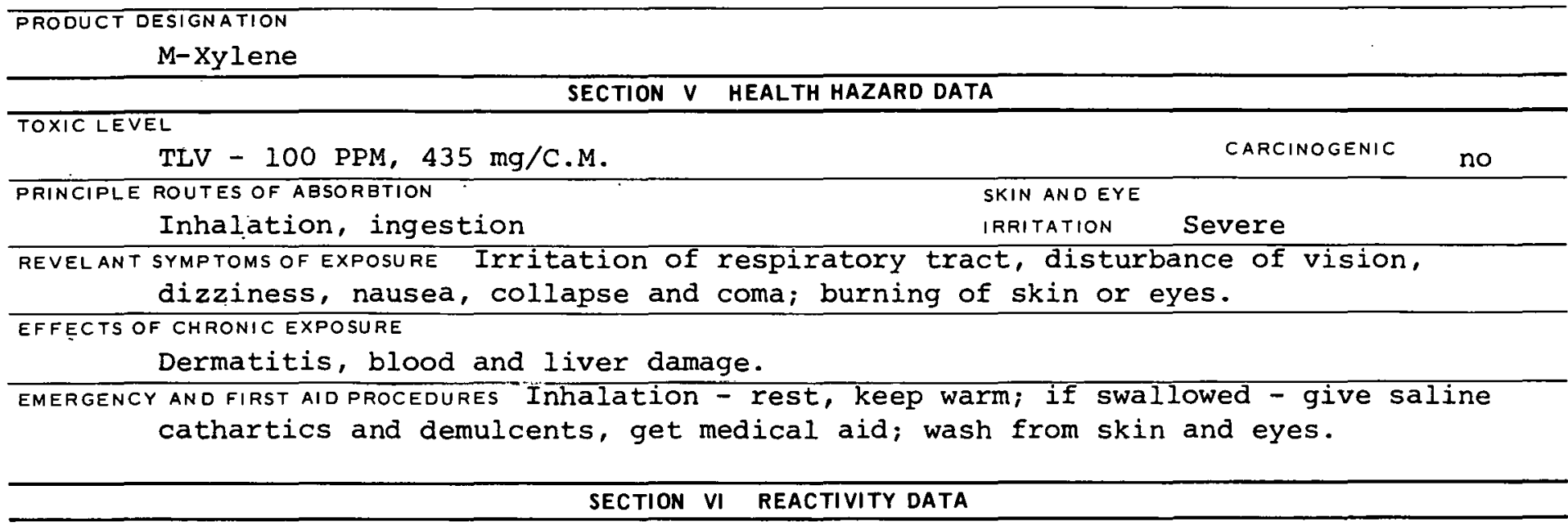

CONDITIONS CONTRIBUTING TO INSTABILITY

CONDITIONS CONTRIBUTING TO HAZARDOUS POLYMERIZATION

INCOMPATABILITY (MATERIALS TO AVOID)

HAZARDOUS DECOMPOSITION PRODUCTS

SECTION VII SPILL OR LEAK PROCEDURES

STEPS TO BE TAKEN IN CASE MATERIAL IS RELEASED OR SPILLED

WASTE DISPOSAL METHOD

SECTION VIII SPECIAL PROTECTION IN FORMATION

\begin{tabular}{l|l}
\hline $\begin{array}{l}\text { VENTILATION REQUIREMENTS LOCAL EXHAUST } \\
\text { ConSUlt Industrial HYgiene Dept. }\end{array}$ & $\begin{array}{l}\text { PROTECTIVE EQUIPMENT (SPECIFY TYPES) EYE } \\
\text { Safety glasSES }\end{array}$ \\
\hline MECHANICAL IGENERAL) & GLOVES \\
\hline SPEGIAL & $\begin{array}{l}\text { RESPIRATOR } \\
\text { Self-contained breathing apparatus. }\end{array}$ \\
\hline
\end{tabular}

OTHER PROTECTIVE EQUIPMENT

SECTION IX SPECIAL PRECAUTIONS

PRECAUTIONS TO BE TAKEN IN HANDLING AND STORAGE

OTHER PRECAUTIONS

SIGNATURE

DATE

$387-1505 B$ 


\begin{tabular}{l}
\multicolumn{1}{c|}{ PRODUCT DESIGNATION } \\
$03-387-1605$ \\
$0-X y$ lene
\end{tabular}

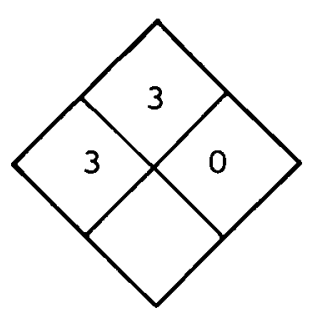

SECTION I SOURCE AND NOMENCLATURE

\begin{tabular}{ll}
\hline MANUFACTURER'S NAME &. EMERGENCY TELEPHONE NO. \\
\hline ADDRESS (NUMBER, STREET, CITY, STATE, ZIP CODE) & $\cdot$. \\
\hline
\end{tabular}

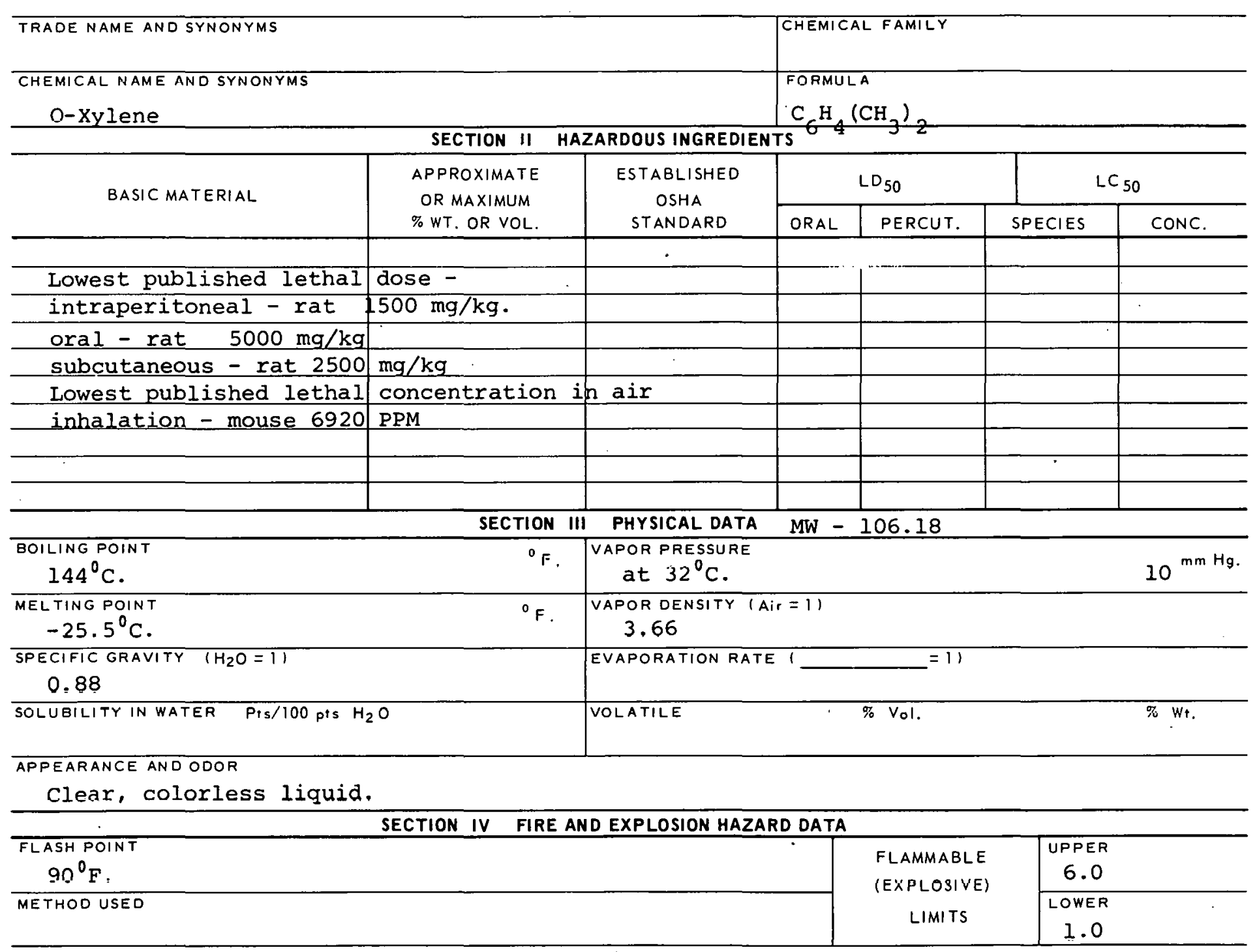

EXTINGUISHINGMEDIA ioxide or dry chemical. Water spray may be used to reduce

rate of burning or cooling containers.

SPECIAL FIRE FIGHTING PROCEDURES

Wear self-contained breathing apparatus.

UNUSUAL FIRE AND EXPLOSION HAZARDS 
TOXIC LEVEL

TLV - 100 PPM, $435 \mathrm{mg} / \mathrm{C} . \mathrm{M}$.

PRINCIPLE ROUTES OF ABSORBTION

Inhalation, ingestion
CARCINOGENIC nO

SKIN AND EYE

IRRITATION SEvere

REVELANT SYMPTOMS OF EXPOSURE Irritation of respiratory tract, disturbance of vision,

dizziness, nausea, collapse and coma; burning of skin or eyes.

EFFECTS OF CHRONIC EXPOSURE

Dermatitis, blood and liver damage.

EMERGENCY AND FIRST AID PROCEDURES Inhalation - rest; if swallowed - give saline cathartic

and demulcents, get medical aid; wash from skin \& eyes.

\section{SECTION VI REACTIVITY DATA}

CONDITIONS CONTRIBUTING TO INSTABILITY

CONDITIONS CONTRIBUTING TO HAZARDOUS POLYMERIZATION

INCOMPATABILITY (MATERIALS TO AVOID)

HAZARDOUS DECOMPOSITION PRODUCTS

SECTION VII SPILL OR LEAK PROCEDURES

STEPS TO BE TAKEN IN CASE MATERIAL IS RELEASED OR SPILLED

WASTE DISPOSAL METHOD

SECTION VIII SPECIAL PROTECTION INFORMATION

VEN TILATION REQUIREMENTS LOCAL EXHAUST

Consult Industrial Hygiene Dept.

MECHANICAL (GENERAL) PROTECTIVE EQUIPMENT (SPECIFY TYPES) EYE

Safety glasses GLOVES

SPECIAL

OTHER PROTECTIVE EQUIPMENT

PRECAUTIONS TO BE TAKEN IN HANDLING AND STORAGE

OTHER PRECAITIONS 
CAS - 000106423

ZE26250

PRODUCT DESIGNATION
$03-387-1705$
P-XYlene

MATERIAL SAFETY

DATA SHEET

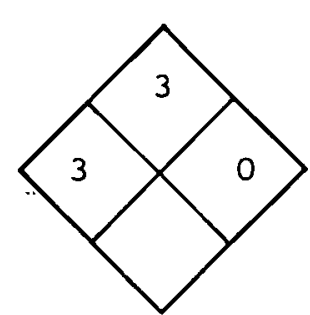

\section{SECTION I SOURCE AND NOMENCLATURE}

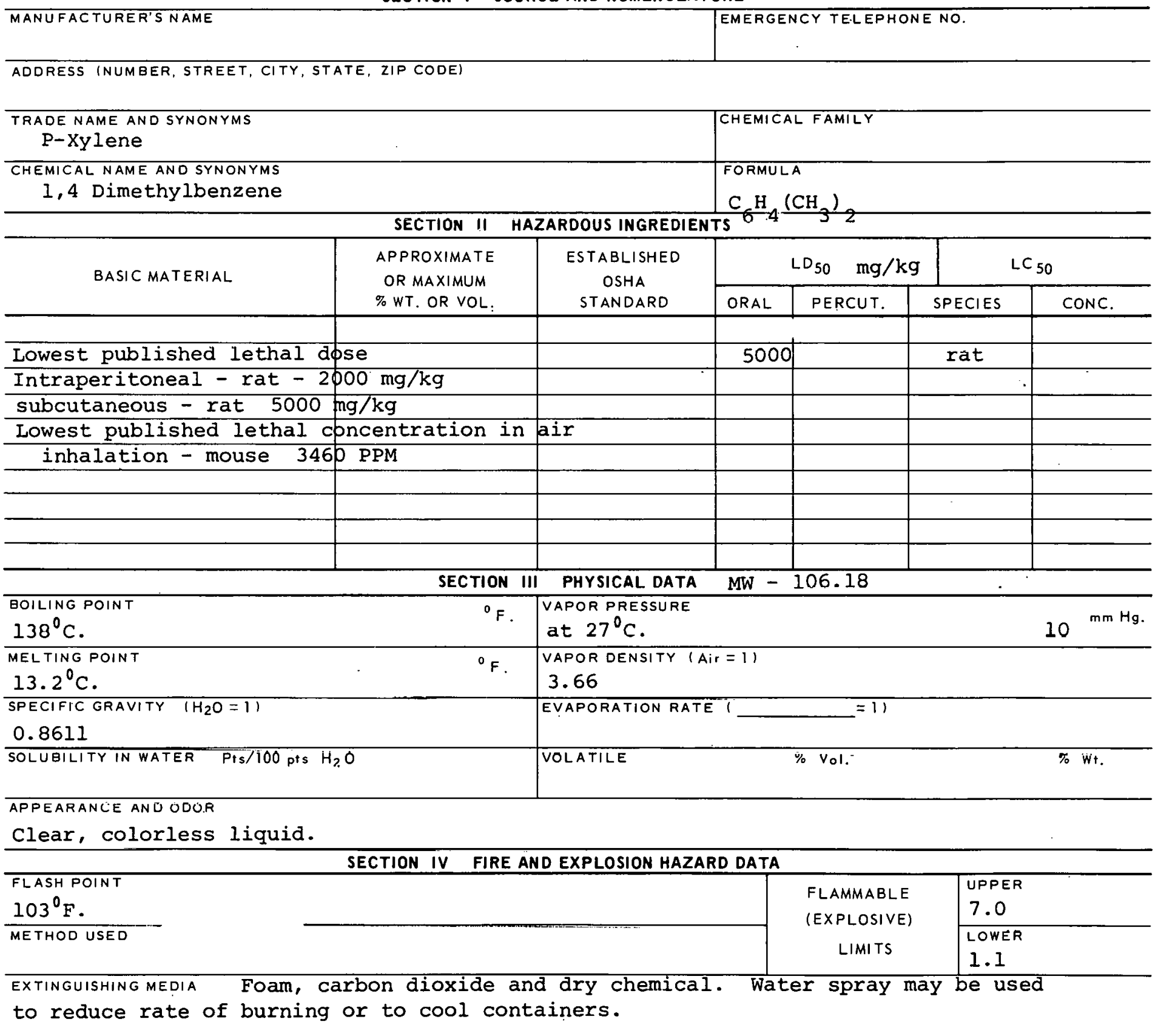

SPECIAL FIRE FIGHTING PROCEEUURES

Wear self-contained breathing apparatus.

UNUSUAL FIRE AND EXPLOSION HAZAROS 


\section{SECTION IX SPECIAL PRECAUTIONS}

PRECAUTIONS TO BE TAKEN IN HANDLING AND STORAGE 


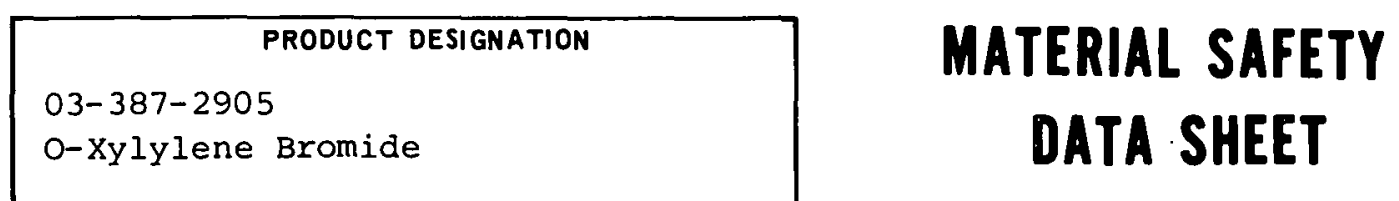

SECTION I SOURCE AND NOMENCLATURE

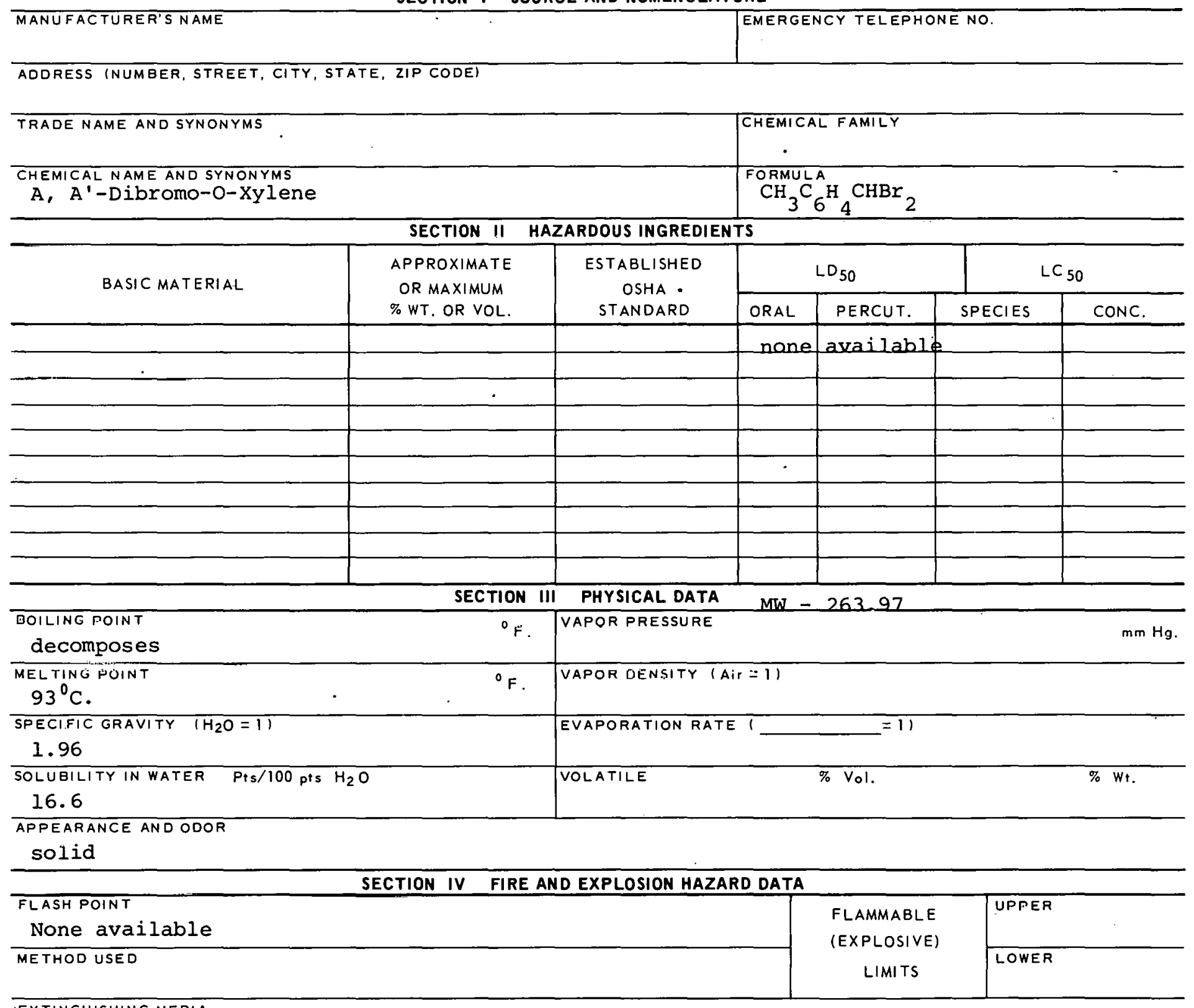

EXTINGUISHING MEDIA

Alcohol foam, carbon dloxide, dry chemical.

SPECIAL FIRE FIGHTING PROCEDURES

Self-contained breathing apparatus.

UNUSUAL FIRE AND EXPLOSION HAZARDS 


\begin{tabular}{|c|c|c|}
\hline SECTION V & HEALTH HAZARD DATA & \\
\hline $\begin{array}{l}\text { TOXIC LEVEL } \\
\text { None available }\end{array}$ & CARCINOGENIC & no \\
\hline $\begin{array}{l}\text { PRINCIPLE ROUTES OF ABSORBTION } \\
\text { Inhalation, ingestion }\end{array}$ & $\begin{array}{ll}\text { SKIN ANDEYE } & \\
\text { IRRITATION Severe }\end{array}$ & \\
\hline $\begin{array}{l}\text { REVELANT SYMPTOMS OF EXPOSURE EYe and respira } \\
\text { internal irritation of digestive }\end{array}$ & $\begin{array}{l}\text { tory system irritation, severe } \\
\text { tract. }\end{array}$ & \\
\hline $\begin{array}{c}\text { EFFECTS OF CHRONIC EXPOSURE } \\
--\end{array}$ & & \\
\hline
\end{tabular}

\begin{tabular}{l}
\hline CONDITIONS CONTRIBUTING TO INSTABILITY \\
\hline CONDITIONS CONTRIBUTING TO HAZARDOUS POLYYMERIZATION \\
\hline
\end{tabular}




\begin{tabular}{l} 
PRODUCT DESIGNATION \\
$03-387-3405$ \\
M-XYlyl Bromide \\
\hline
\end{tabular}

\section{MATERIAL SAFETY DATA SHEET}

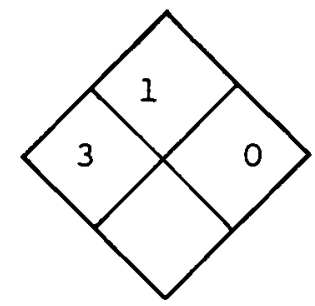

SECTION I SOURCE AND NOMENCLATURE

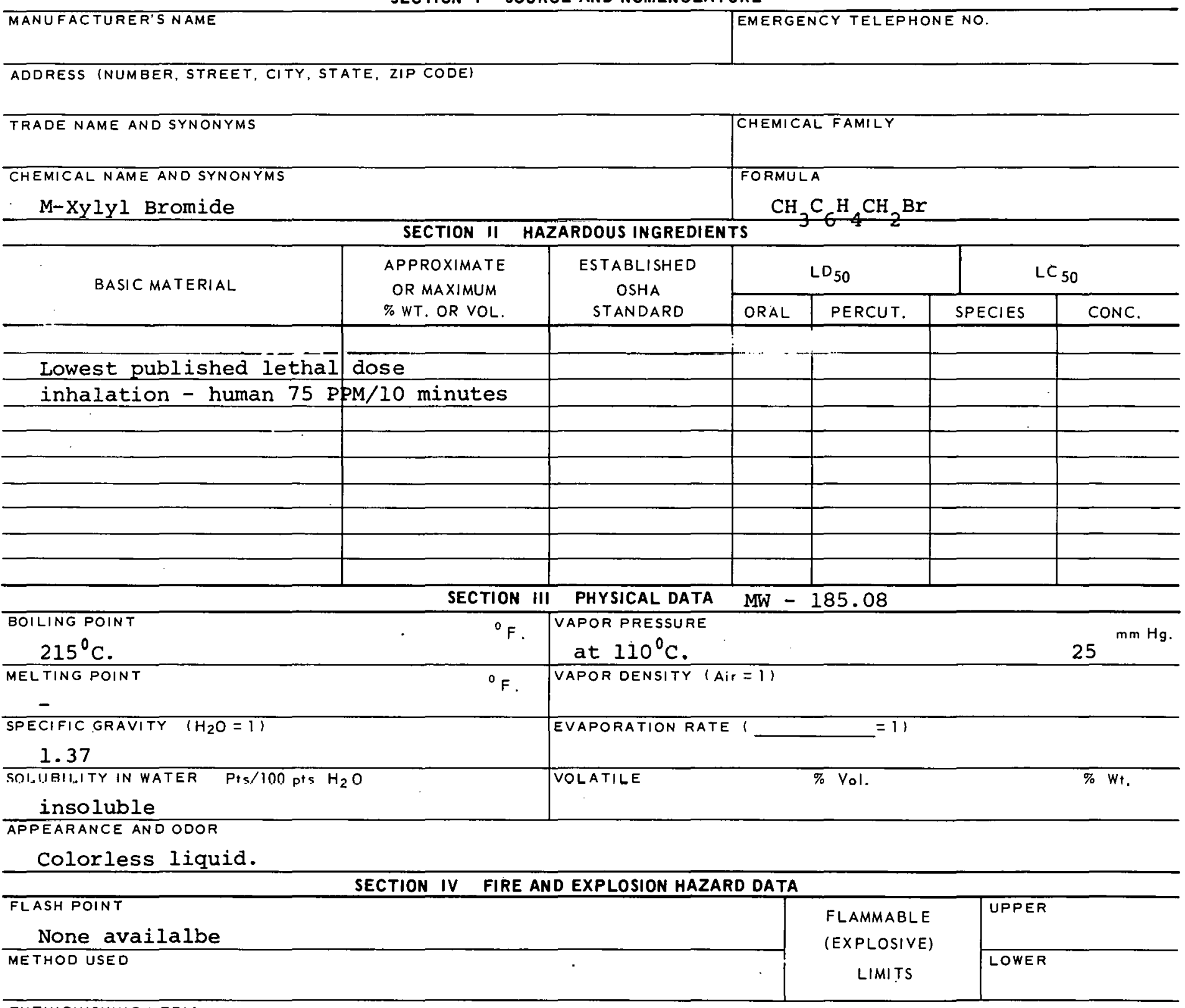

EXTINGUISHING MEDIA

Foam, carbon dioxide, dry chemical.

SPECIAL FIRE FIGHTING PROCEOURES

Self-contained breathing apparatus.

UNUSUAL FIRE AND EXPLOSION HAZARUS 


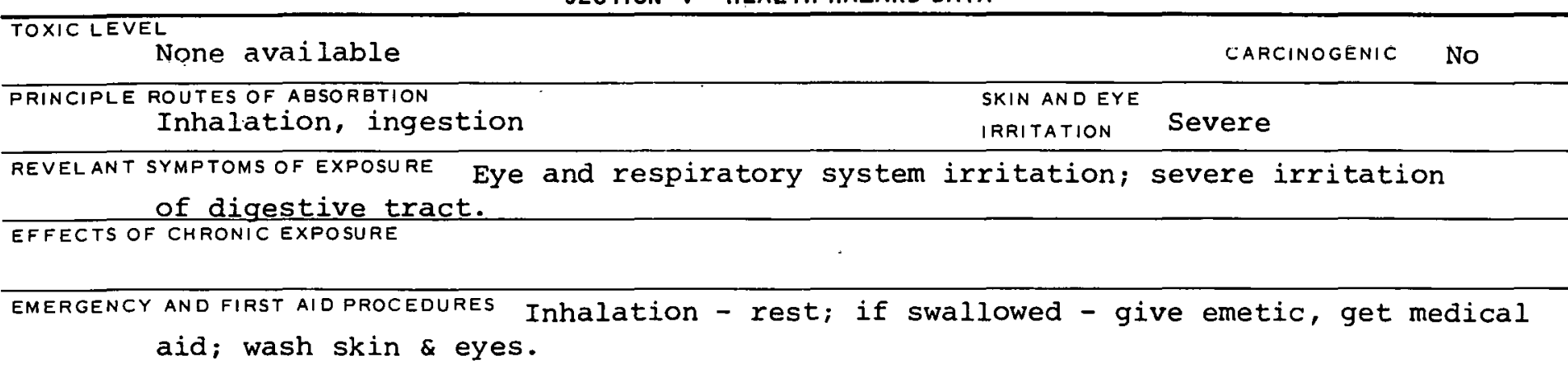

\section{SECTION VI - REACTIVITY DATA}

SECTION VI
CONDITIONS CONTRIBUTING TO INSTABILITY
CONOITIONS CONTRIBUTING TO HAZARDOUS POLYMERIZATION

INCOMPATABILITY (MATERIALS TO AVOID)

HAIARDOUS DECOMPUSITIUN PRUDUETS

SECTION VII SPILL OR LEAK PROCEDURES

STEPS TO BE TAKEN IN CASE MA TERIAL IS RELEASED OR SPILLED

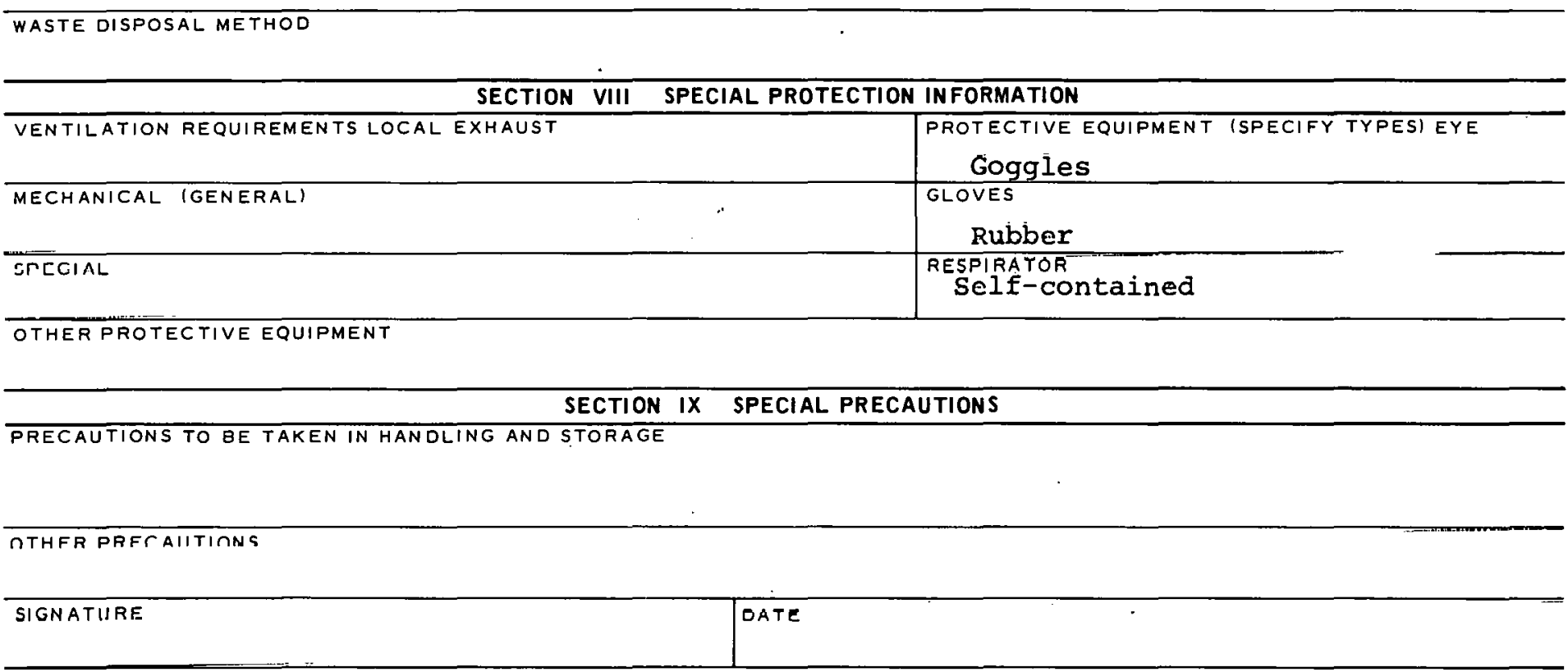




\begin{tabular}{|c|}
\hline PRODUCT DESIGNATION \\
$03-387-3505$ \\
$0-x y l y l$ bromide \\
\hline
\end{tabular}

\section{MATERIAL SAFETY DATA SHEET}

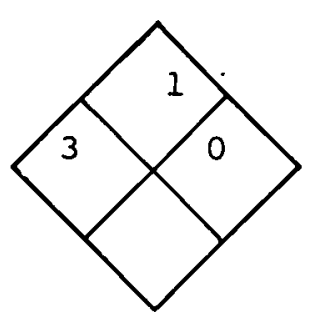

\section{SECTION I SOURCE AND NOMENCLATURE}

MANUFACTURER'S NAME

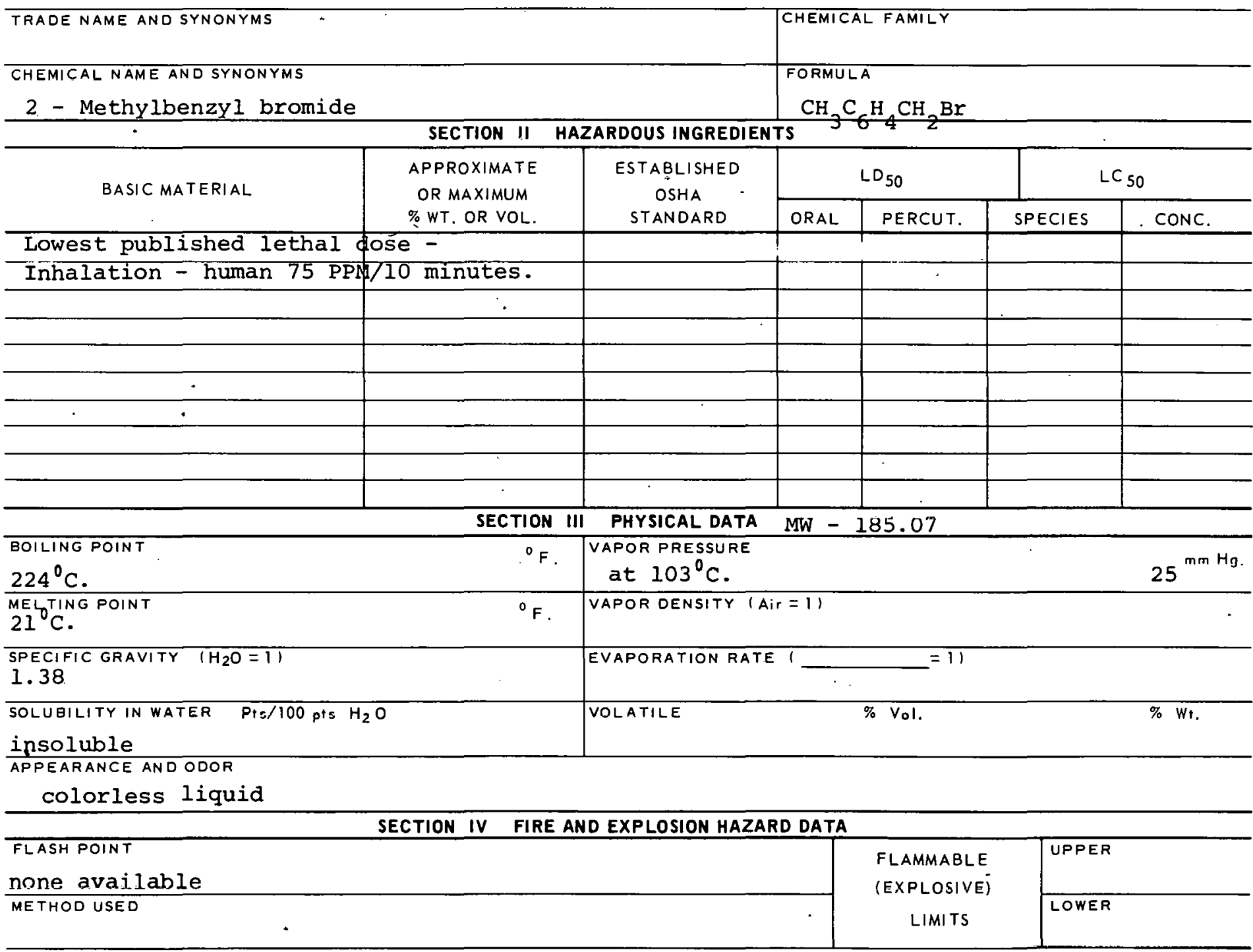

EXTINGUISHING MEDIA

Foam, carbon dioxide, ary chemical.

SPECIAL FIRE FIGHTING PROCEDURES

Self-contained breathing apparatus.

UNUSUAL FIRE AND EXPLOSION HAZARDS 


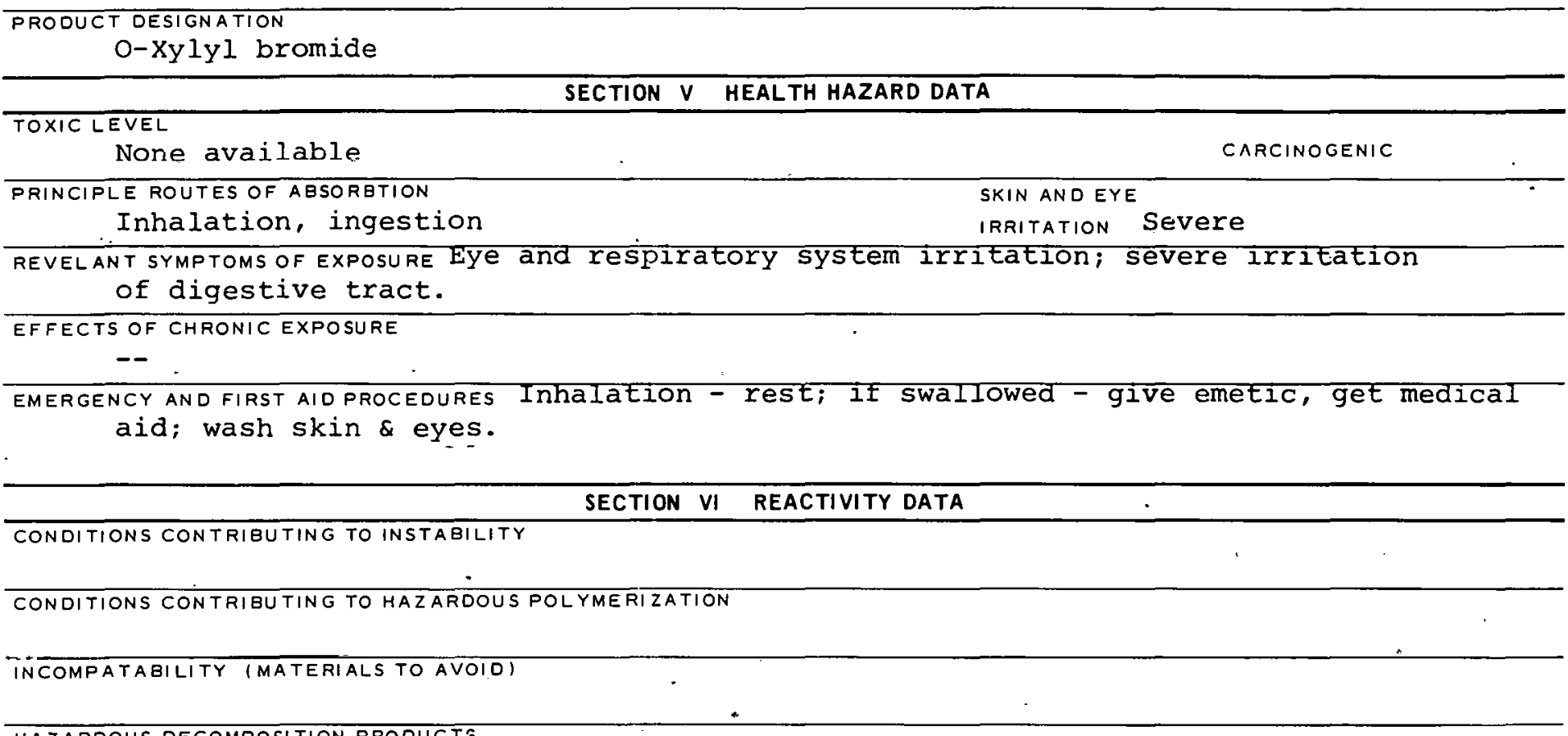

HAZARDOUS DECOMPOSITION PROOUCTS

SECTION VII SPILL OR LEAK PROCEDURES

STEPS TO BE TAKEN IN CASE MATERIAL IS RELEASED OR SPILLED

WASTE DISPOSAL METHOD

SECTION VIII SPECIAL PROTECTION INFORMATION

\begin{tabular}{l|l}
\hline VENTILATION REQUIREMENTS LOCAL EXHAUST & $\begin{array}{l}\text { PROTECTIVE EQUIPMENT (SPECIFY TYPES) EYE } \\
\text { GOGgles }\end{array}$ \\
\hline MECHANICAL IGENERAL) & $\begin{array}{c}\text { GLOVES } \\
\text { Rubber gloves }\end{array}$ \\
\hline SPECIAL & RESSPIRATOR \\
& Self-Contained \\
\hline
\end{tabular}

OTHER PROTECTIVE EQUIPMENT

SECTION IX SPECIAL PRECAUTIONS

PRECAUTIONS TO BE TAKEN IN HANDLING AND STORAGE

OTHER PRECAUTIOIN9

SIGNATIIRF

DATE

$387-3505 B$ 


\begin{tabular}{|c|}
\hline PRODUCT DESIGNATION .. \\
03-619-3032 \\
Camphor Gum \\
\hline
\end{tabular}

\section{MATERIAL SAFETY \\ DATA SHEET}

\section{SECTION I SOURCE AND NOMENCLATURE}

\begin{tabular}{l|l|l}
\hline & SECTION I SOURCE AND NOMENCLATURE \\
\hline MANUFACTURER'S NAME & EMERGENCY TELEPHONE NO. \\
\hline ADORESS INUMEER, STREET, CITY, STATE, ZIP CODE) &
\end{tabular}

TRADE NAME AND SYNONYMS -
2 CamphanONE
C.HEMICAL NAME AND SYNONYMS
CamphOY

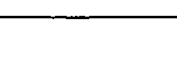

\begin{tabular}{l|l} 
& CHEMICAL FAMILY \\
\hline & $\begin{array}{l}\text { FORMULA } \\
C_{20}{ }_{2} \mathrm{H}_{2} \mathrm{O}\end{array}$ \\
\hline
\end{tabular}
SECTION II HAZARDOUS INGREDIENTS

\section{BASIC MATERIAL}

Oral - human IiDLo $50 \mathrm{mg} / \mathrm{kg}$

(1) Intraperitoneal - rat LD50 $900 \mathrm{mg} / \mathrm{kg}$

(2) Lowest published toxic dose

\begin{tabular}{|c|c|}
\hline skin - mouse $84 \mathrm{mg} / \mathrm{C} . \mathrm{M}$. & \\
\hline & \\
\hline & \\
\hline & \\
\hline & \\
\hline & \\
\hline
\end{tabular}

\begin{tabular}{c|c}
\hline APPROXIMATE & ESTABLISHED \\
OR MAXIMUM & OSHA \\
$\%$ WT. OR VOL. & STANDARD \\
\hline
\end{tabular}

\begin{tabular}{|c|c|c|c|c|}
\hline \multicolumn{2}{|c|}{ LD $_{50}$} & \multicolumn{2}{c}{ LC $_{50}$} \\
\cline { 2 - 5 } & ORAL & PERCUT. & SPECIES & CONC. \\
\hline
\end{tabular}

BOILING POINT

$204^{\circ} \mathrm{C}$.

MELTING POINT

$177^{\circ} \mathrm{C}$.

SPECIFIC GRAVITY $\left(\mathrm{H}_{2} \mathrm{O}=1\right)$

0.992

SOLUBILITY IN WATER Pts $/ 100$ pts $\mathrm{H}_{2} \mathrm{O}$

Insoluble

APPEARANCE AND ODOR White transparent crystaline mass, aromatic odor.

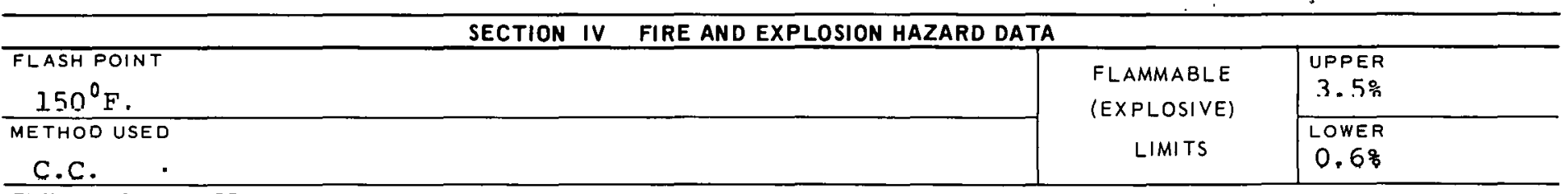

EXTINGUISHING MEDIA

Foam, carbon dioxide, dry chemical

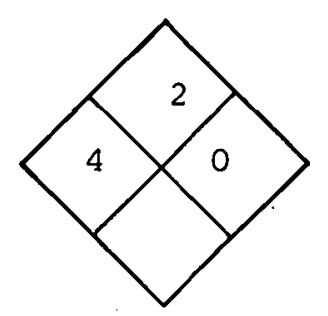


TOXIC LEVEL

TLV - 2 PPM, 12 mg/C.M.

CARCINOGENIC neoplastic

PRINCIPLE ROUTES OF ABSORBTION Inhalation, ingestion

SKIN AND EYE

IRRITATION Moderate

REVELANT SYMPTOMS OF EXPOSURE

Rigidity of jaw muscles, headache, convulsive seizures, coma.

EFFECTS OF CHRONIC EXPOSURE

$--$

EMERGENCY AND FIRST AID PROCEDURES If swallowed - medical aid, gastric lavage, cathartics, inhalation - rest; wash from eyes and skin.

\section{SECTION VI REACTIVITY DATA}

CONDITIONS CONTRIBUTING TO INSTABILITY

CONDITIONS CONTRIBU TING TO HAZARDOUS POLYMERIZATION

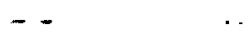

SECTION VII SPILL OR LEAK PROCEDURES

STEPS TO BE TAKEN IN CASE MATERIAL IS RELEASED OR SPILLED

WASTE DISPOSAL METHOD

SECTION VIII SPECIAL PROTECTION INFORMATION

\begin{tabular}{l|l}
\hline VENTILATION REQUIREMENTS LOCAL EXHAUST & PROTECTIVE EQUIPMENT (SPECIFY TYPES) EYE \\
\hline MECHANICAL IGENERAL) & GLOVES \\
\hline SPECIAL & $\begin{array}{c}\text { RESPIRATOR } \\
\text { With proper filter }\end{array}$ \\
\hline
\end{tabular}

OTHER PROTECTIVE EQUIPMENT

SECTION IX SPECIAL PRECAUTIONS

PRECAUTIONS TO BE TAKEN IN HANDLING AND STORAGE

\section{OTHER PKELAUIIUNS}




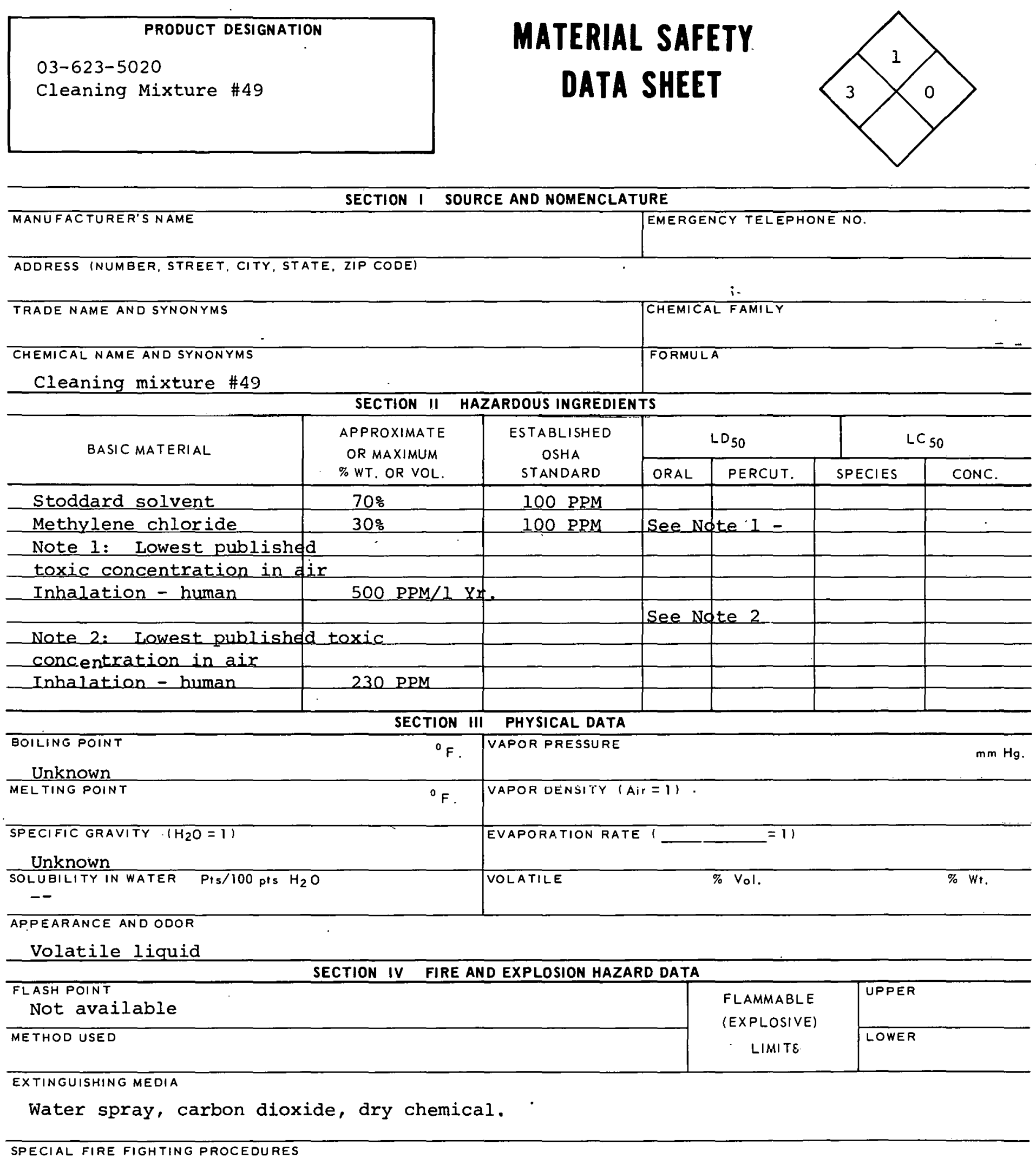

SPECIAL FIRE FIGHTING PROCEDURES

UNUSUAL FIRE AND EXPLOSION HAZARDS 
TOXICLEVEL

TLV - 100 PPM

CARCINOGENIC

PRINCIPLE ROUTES OF ABSORBTION SKIN AND EYE

Inhalation, ingestion, skin IRRITATION SEvere

REVELANT SYMPTOMS OF EXPOSURE Headache, giddiness, stupor, irxitability, fatigue, eye

and respiratory passage irritation.

EFFECTS OF CHRONIC EXPOSURE

Iiver and kidney damage.

EMERGENCY AND FIRST AID PROCEDURES If swallowed - give emetic, get medical aid; inhalation artificial respiration as needed; wash skin \& eyes.

SECTION VI REACTIVITY DATA

CONDITIONS CONTRIBUTING TO INSTABILITY

CONDITIONS CONTRIGUTING TO HAZARDOUS POLYMERIZATION

INCOMPATABILITY (MATERIALS TO AVOIO)

HAZARDOUS DECOMPOSITION PRODUCTS

SECTION VII SPILL OR LEAK PROCEDURES

STEPS TO BE TAKEN IN CASE MATERIAL IS RELEASED OR SPILLED

WASTE DISPOSAL METHOD

SECTION VIII SPECIAL PROTECTION INFORMATION

\begin{tabular}{l|l}
\hline VENTILATION REQUIREMENTS LOCAL EXHAUST & PROTECTIVE EQUIPMENT (SPECIFY TYPES) EYE
\end{tabular}

MECHANICAL (GENERAL)

Safety goggles

GLOVES

Rubber

RE3PIRATOR

SHECIAL

OTHER PROTECTIVE EQUIPMENT

Self-contained

SECTION IX SPECIAL PRECAUTIONS

PRECAUTIONS TO BE TAKEN IN HANDLING AND STORAGE

OTHER PRECAUTIONS

SIGNATURE

DATE

$.623-5020 B$ 


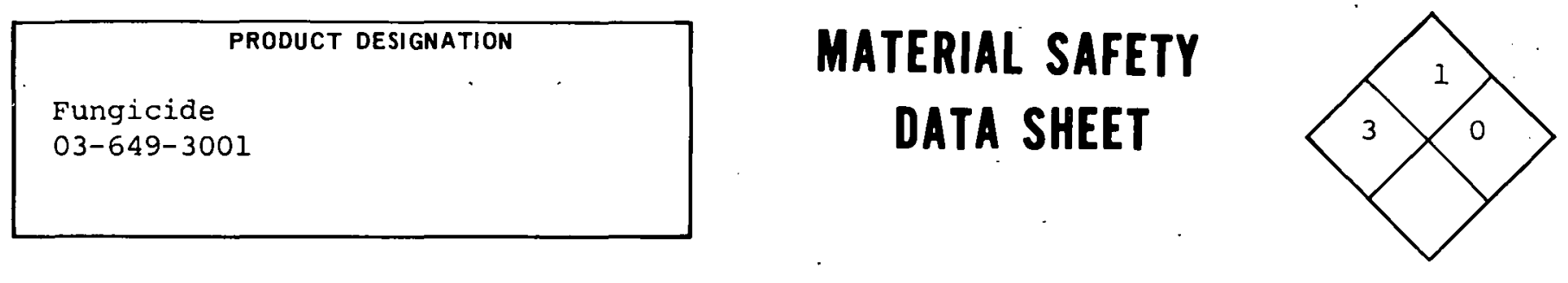

SECTION I SOURCE AND NOMENCLATURE

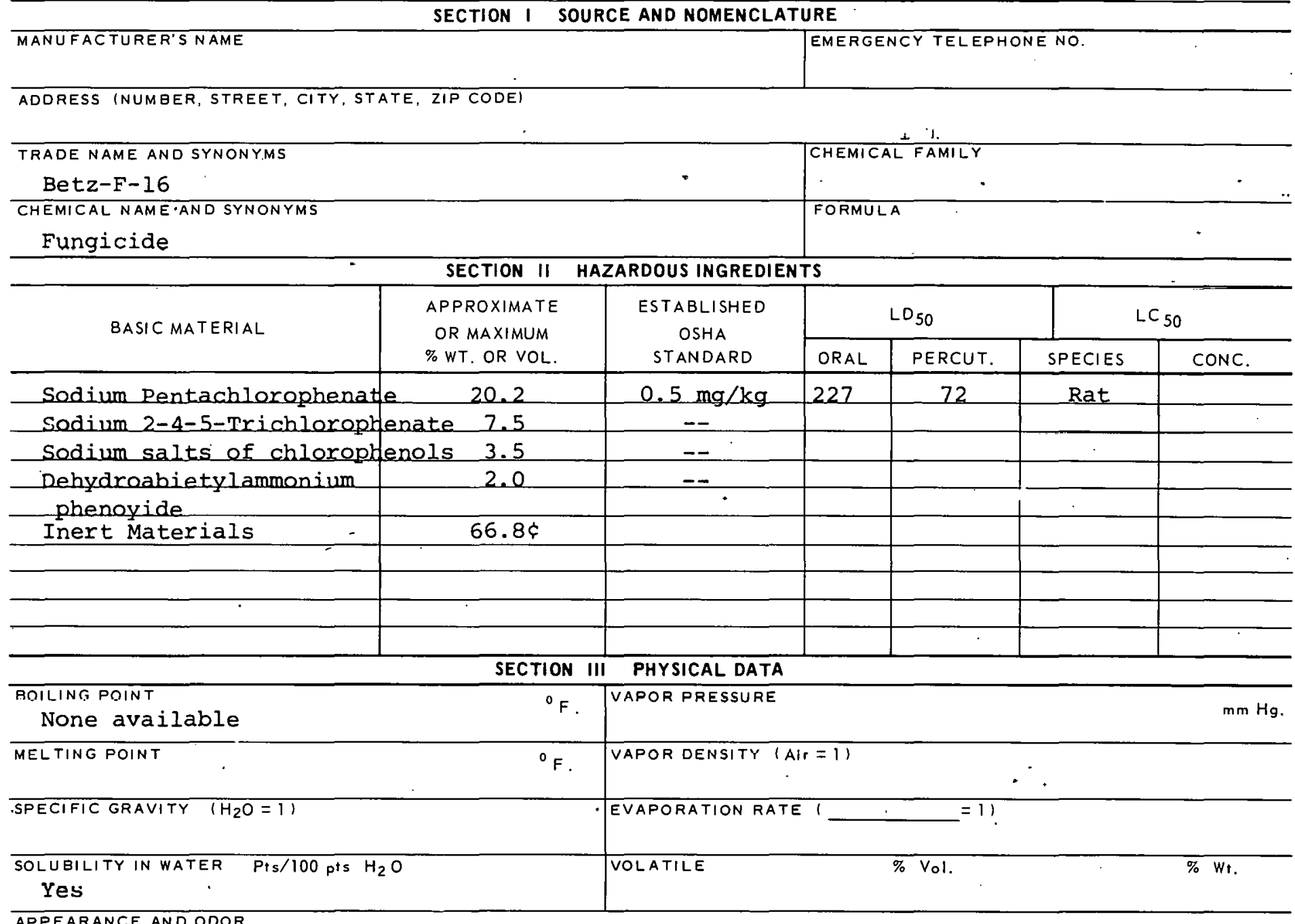

APPEARANCE AND ODOR.

Liquid

SECTION IV FIRE AND EXPLOSION HAZARD DATA

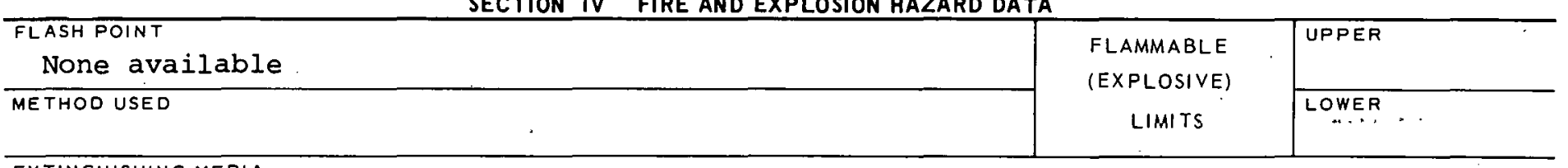

EXTINGUISHING MEDIA

SPECIAL FIRE FIGHTING PROCEDURES

UNUSUAL FIRE AND EXPLOSION HAZARDS

$U C N-11322$

(3) $10-74$ )

649-3001A 
TOXIC LEVEL

SECTION $V$ HEALTH HAZARD DATA

None available

CARCINOGENIC

no

PRINCIPLE ROUTES OF ABSORBTION

Inhalation, ingestion

SKIN AND EYE

IRRITATION severe

REVELANT SYMPTOMS OF EXPOSURE EYe and nose irritation, heart failure, respiratory problems, anesthesia, sweating, coma.

EFFECTS OF CHRONIC EXPOSURE

Dermatitis.

EMERGENCY AND FIRST AID PROCEDURES If swallowed - induce vomiting, get medical aid, gastric lavage, inhalation -. rest; wash from skin and eyes.

\section{SECTION VI REACTIVITY DATA}

CONDITIONS CONTRIBUTING TO INSTABILITY

CONDITIONS CONTRIBUTING TO HAZARDOUS POLYMERIZATION

INCOMPATABILITY (MATERIALS TO AVOID)

HAZARDOUS DECOMPOSITION PRODUCTS

SECTION VII SPILL OR LEAK PROCEDURES

STEPS TO BE TAKEN IN CASE MATERIAL IS RELEASED OR SPII, I, ED

WASTE DISPOSAL METHOD

SECTION VIII SPECIAL PROTECTION INFORMATION

\begin{tabular}{|c|c|}
\hline VENTILATION REQUIREMENTS LOCAL EXHAUST & $\begin{array}{l}\text { PROTECTIVE EOUIPMENT (SPECIFY TYPES) FYF } \\
\text { Goggles }\end{array}$ \\
\hline MECHANICAL (GENERAL) & $\begin{array}{l}\text { GLOVES } \\
\text { Rublier }\end{array}$ \\
\hline SPECIAL & $\begin{array}{l}\text { RESPIRATOR } \\
\text { With proper filter }\end{array}$ \\
\hline
\end{tabular}

Wear protective clothing.

SECTION IX SPECIAL PRECAUTIONS

PRECAUTIONS TO BE TAKEN IN HANDLING AND STORAGE

OTHER PRECAUTIONS

SIGNATURE

DATE

$649-3001 B$ 


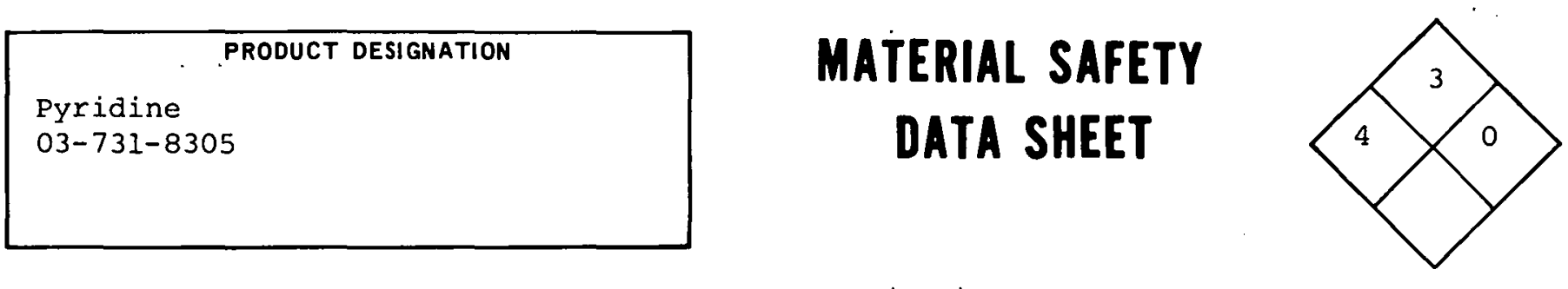

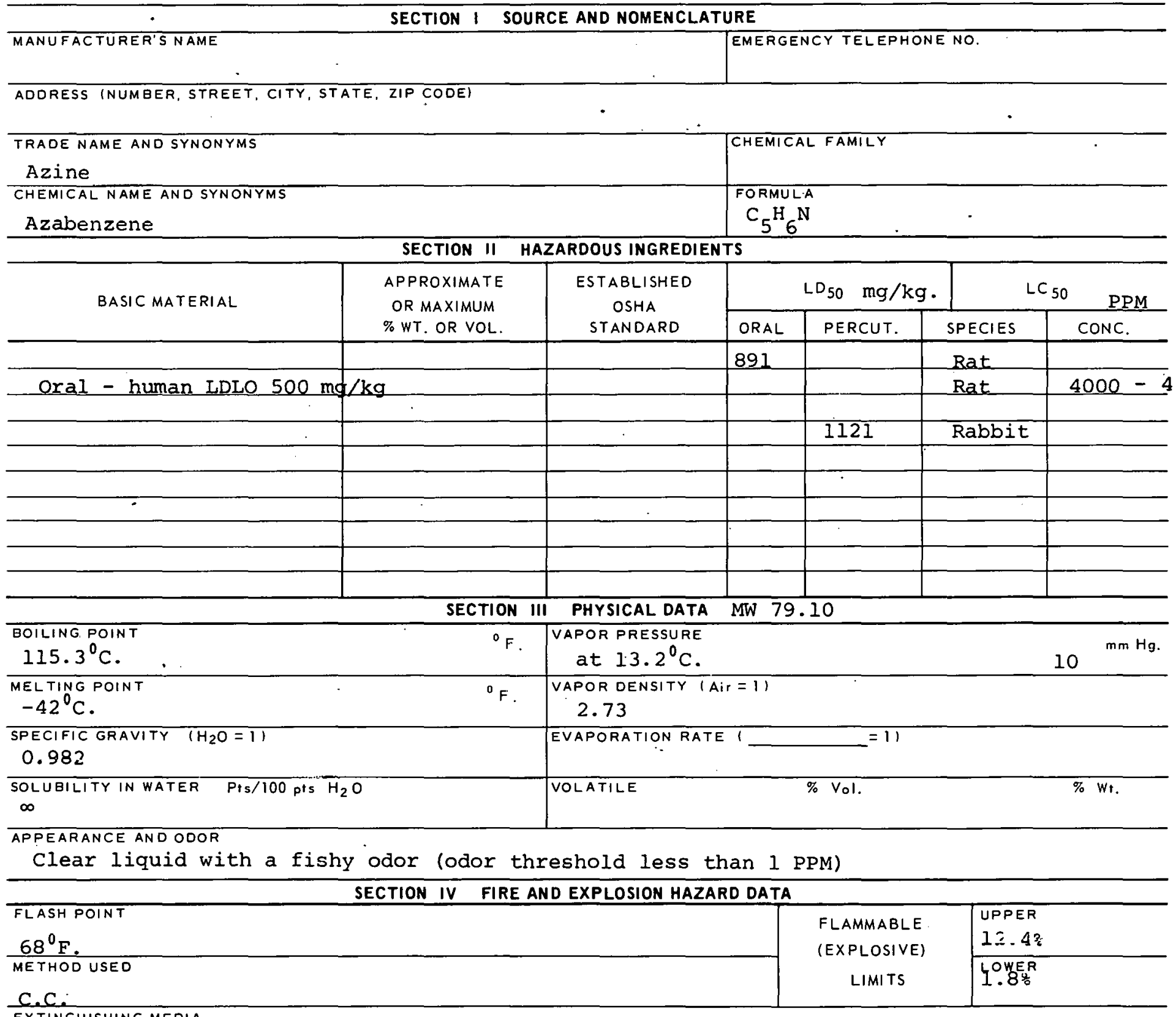

ExTingur.

EXTINGUISHING MEDIA

Use alcohol foam, carbon dioxide, or dry chemical. Water may be ineffective.

SPECIAL FIRE FIGHTING PROCEDURES

Wear goggles and self-contained breathing apparatus.

UNUSUAL FIRE AND EXPLOSION HAZARDS

Vapor may travel a considerable distance to a source of ignition and flashback. 


\section{TOXIC LEVEL \\ TLV - 5 PPM, $15 \mathrm{mg} / \mathrm{C.M}$.}

PRINCIPLE ROUTES OF ABSORBTION Inhalation, ingestion, skin
CARCINOGENIC

no SKIN AND EYE IRRITATION Severe

REVELANT SYMPTOMS OF EXPOSURE Narcosis, headache, nausea, giddiness, vomiting oral - liver and kidney damage, skin irritation.

EFFECTS OF CHRONIC EXPOSURE OCCasional vomiting and diarrhea; weight loss and anemia, ocular and facial paralysis.

EMERGENCY AND FIRST AID PROCEDURES InhaLaEION - rest, artifiCIaI respiration and oXYgen lf needed; ingestion - wash mouth, give emetic, get medical aid; wash skin and eyes thoroughly.

SECTION VI REACTIVITY DATA

CONDITIONS CONTRIBUTING TO INSTABILITY

CONDITIONS CONTRIBUTING TO HAZARDOUS POLYMERIZATION

INCOMPATABILITY (MATERIALS TO AVOID)

A $0.17 \%$ solution of pyridine in maleic anhydride at $185^{\circ} \mathrm{C}$. gives erothermir rernmpsition. HAZARDOUS DECOMPOSITION PRODUCTS

SECTION ViI SPILL OR LEATK PROCEDURES

STEPS TO BE TAKEN IN CASE MATERIAL IS RELEASED OR SPILLED

WASTE DISPOSAL METHOD

SECTION VIII SPECIAL PROTECTION INFORMATION

\begin{tabular}{l|l}
\hline VENTILATION REQUIREMENTS LOCAL EXHAUST & $\begin{array}{l}\text { PROTECTIVE EQUIPMENT (SPECIFY TYPES) EYE } \\
\text { GOggles }\end{array}$ \\
\hline MECHANICAL IGENERAL) & GLOVES \\
\hline SPECIAL & $\begin{array}{c}\text { RESPIRATOR } \\
\text { Self-contained breathing apparatus }\end{array}$ \\
\hline
\end{tabular}

OTMER PROTECTIVE EQUIPMENT

SECTION IX SPECIAL PRECAUTIONS

PRECAUTIONS TO BE TAKEN IN HANDLING AND STORAGE

OTHER PRECAUTIONS

SIGNATURE

DATE

$731-8305 B$ 


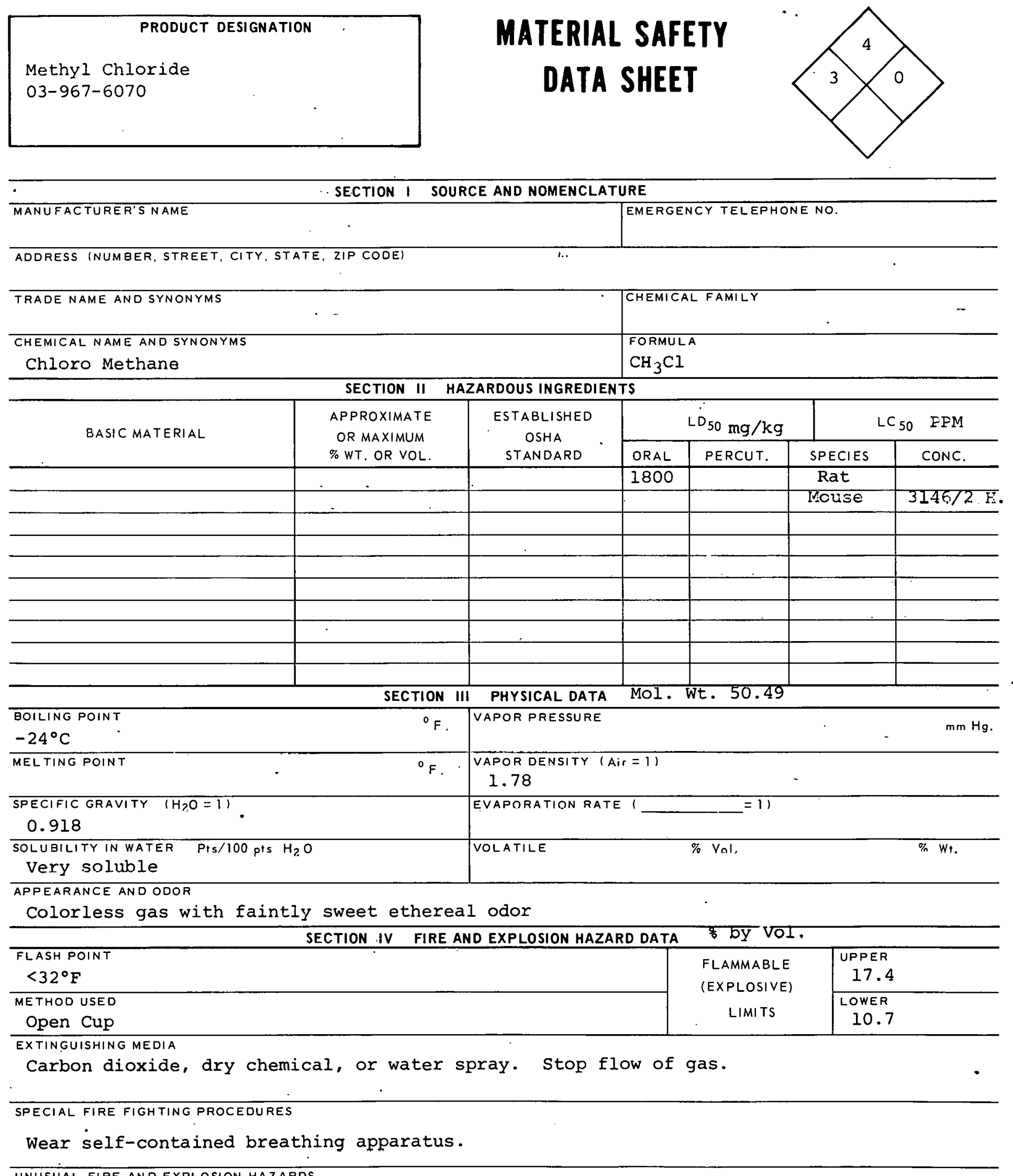
UNUSUAL FIRE AND EXPLOSION HAZARDS 
REVELANT SYMPTOMS OF EXPOSURE Narcosis, nausea, vomiting, blurred vision, drowsiness, confusion, coma, convulsions, pulmonary edema, bronchial pneumonia, frostbite.

EFFECTS OF CHRONIC EXPOSURE Blurring of vision, numbness of extremeties, confusion, hallucinations, fainting attacks, bronchospasms.

EMERGENCY AND FIRST AID PROCEDURES

Remove from exposure; antidote is dimercaprol; obtain medical aid.

\section{SECTION VI REACTIVITY DATA}

CONDITIONS CONTRIBUTING TO INSTABILITY

-

CONDITIONS CONTRIBU TING TO HAZARDOUS POLYMERIZATION

INCOMPATABILITY (MATERIALS TO AVOID)

Aluminum, magnesium, potassium, sodium.

HAZARDOUS UECUMFUSIIUN HHUÜUETS

SECTION VII SPILL OR LEAK PROCEDURES

STEPS TO BE TAKEN IN CASE MATERIAL IS RELEASED OR SPILLED

WASTE DISPOSAL METHOD

SECTION VIII SPECIAL PROTECTION INFORMATION

\begin{tabular}{|c|c|}
\hline $\begin{array}{l}\text { VENTILATION REQUIREMENTS LOCAL EXHAUST } \\
\text {. }\end{array}$ & $\begin{array}{l}\text { PROTECTIVE EQUIPMENT (SPECIFY TYPES) EYE } \\
\text { GNggllas }\end{array}$ \\
\hline MECHANICAL (GENERAL) & $\begin{array}{l}\text { GLOVES } \\
\text { Rubber }\end{array}$ \\
\hline 3FECIAL & $\begin{array}{l}\text { w/appropriate Eilter } \\
\text { WIITATRR }\end{array}$ \\
\hline
\end{tabular}

OTHER PROTECTIVE EQUIPMENT

Self-contained breathing apparatus.

SECTION IX SPECIAL PRECAUTIONS

PRECAUTIONS TO BE TAKEN IN HANDLING AND STORAGE

OTIIER PRESAUTIQHS

SIGNATURE

D.A.TE

SIONATURE 
4. APPENDIX 
CHEMICAL ABSTRACTS SERVICE REGISTRY NUMBERS

\section{Organic}

A,G-Dibromohydrin Glycerol

Heptoyl Chloride

Hexachloroethane

Hexane

Hydrazine Dihydrochloride

Hydrazine Hydrate

Hydrazine Sulfate

Hydroquinone

2 Hydroxy 1,4-Dimethylbenzene

4 Hydroxy-1,2-Dimethylbenzene

4 Hydroxy-1, 3-Dimethylbenzene

O-Hydroxydipheny 1

P-Hydroxydiphenyl

8-Hydroxyquinoline

Indene

P-Iodoaniline

Iodobenzene

o-Iodobenzoyl Chloride

2 Iodo-1, 3-Dimethylluenzene

2-Iodo-1,4-Dimethylbenzene

4-Iodo-1, 3-Dime thylbenzene

o-Iododiphenyl

P-Iododiphenyl

O-Iodophenol

M-Iodotoluene

P-Iodotoluene

Isophorone

2,4 Lutidine

Mesityl Oxide
CAS NO.

000096219

002528612

000067721

000110543

005341617

007803578

010034932

000123319

000095874

000095658

000105679

000090437

000092693

000148243

000095136

000540374

000591504

000609676

000608286

001122425

004214282

002113511

001591317

000533584

000625956

000624317

000078591.

000108474

0001.41797
Page No.

263-0606

267-1707

267-2407

267-3207

267-5507

267-5787

267-5947

267-6737

267-8207

267-8307

267-8407

267-8647

267-8677

267-9857

278-0308

278-1308

278-1808

278-2308

278-27กR

278-2808

278-2908

278-3308

278-3408

278-4308

278-4708

278-4908

278-5808

278-291.9

305-2601 
Mesitylene

Methyl Acetate

Methyl Acrylate

Methylamine

Methylamine Hydrochloride

Methyl N-Amyl Ketone

N-Methylaniline

Methyl Bromuacetate

Methyl B-Bromopropionate

Methyl $\perp$ so-buty $\perp$ Ketone

Methyl Carbonate

P-Methylcyclohexanol

M-Methylcyclohexanone

P-Methylcyclohexanone

Methyl A,B-Dibromopropionate

Methylene Bromide

Methylene chloride

Methylene Iodide

Methylethylamine Hydrochlorıde

Methylethylaniline

Methyl Ethyl Ketone

Methylhydrazine Sulfate

Methyl Iodide

A-Methylnaphthalene

B-Methylnaphthalene

4 Methyl-2-Pentanol

Methyl-P-Phenylenediamine

Dihydxochloride

A-Methyl-A-Phenylhydrazine

Metlıyl-Iso-Propylcarbinol

Methyl Iso-Propyl Ketone

Methyl Sulfate

Methyl Thiocyanate
000108678

000079209

000096333

000074895

000593511

000110430

000100618

000096322

003395913

UUULUYLUL

000616386

000589913

000591242

000589924

001729675

000074953

000075092

000075116

000624602

000589082

000078933

000302158

000074884

000090120

000091576

000108112

005395700

000618406

000590754

000563804

000077781

000556649
305-2701

305-5631

305-6101

305-7601

305-7701

305-9601

306-0202

$306-2702$

306-3702

306-4702

306-6202

307-0303

307-0703

307-0903

307-1303

307-2103

307-2203

307-2303

307-2803

307-3303

307-3843

307-5803

307-7803

308-0404

308-0474

308-4904

$308-5904$

308-6404

308-8404

308-8904

309-1605

309-2105 
Methyl-Iso-Thiocyanate

Methyl-M-Toluidine

Methyl-O-Toluidine

Methyl P Toluidine

Morpholine

Myristoyl Chloride

Amsco Diluent 125-82

Naphthalene

A-Naphthaleneacetonitrile

A-Naphthol

B-Naphthol

A-Naphthonitrile

A-Naphthylamine

B-Naphthylamine Hydrochloride

A-Naphthylhydrazine Hydrochloride

I-Naphthy I Isocyanate

B-Naphthyl Isocyanate

3 Nitro-4 Acetylaminotoluene

5 Nitro-2-Aminoanisole

5 Nitro-4-Amino-1, 3-Dimethyl Benzene

3-Nitro-4 Aminophenelole

3-Nitro-4-Aminotoluene

4-Nitro-2-Aminotoluene

M-Nitroaniline

o-Nitroaniline

P-Nitrobenzal Bromide

Nitrobenzene

P-Nitrobenzoyl Chloride

P-Nitrobenzyl Bromide

P-Nitrobenzyl Chloride

M-Nitrobromobenzene

O-Nitrobromobenzene
000556616

000696446

000611212

000623085

000110918

000112641

000091203

000132752

000090153

000135193

000086533

000134327

000612522

002243563

000086840

002243541

000612453

000099592

001635843

000616864

000089623

000099558

000099092

000088744

000619750

000098953

000122043

000100118

000100141

000585795

000577195
309-2205

309-3605

309-3705

309-3805

309-8205

309-9205

314-0286

314-0706

314-1206

314-2706

314-2806

314-3706

314-5306

314-5906

314-6306

314-6806

314-6906

315-0907

315-1907

315-2407

315-2907

315-3407

315-3507

315-3807

315-3907

315-5407

315-6857

316-0208

316-0608

316-0808

316-1108

316-1208 
000586787

000121733

000088733

000100005

000943157

005307142

000099569

口.2216173

000619318

000089587

000083410

000350469

000645001

000609734

000636986

000603714

บับับั75525

000100152

003272911

000534847

000100027

000824395

000100163

000636997

000108032

000079469

000601898

000120229

กกถ1 38896

000132536

000100754
316-1308

316-1708

316-1808

316-1908

316-2608

316-3108

316-3308

3163700

316-4108

316-4608

316-4808

316-7108

316-8108

316-8208

316-8308

316-8608

316-9108

$316-9608$

317-1209

$31 \%-323 y$

317-3439

31.7-3709

317-4709

317-4909

317-6200

317-6309

317-7209

317-8009

317-8309

317-8809

317-9409

317-9609 


M-Nitrotoluene
O-Nitrotoluene
P-Nitrotoluene
Oxalyl Chloride
Palmitoyl Chloride
Pentamethylene Bromide
M-Phenetidine
O-Phenetidine
P-Phenetidine
Phenol
G-Phenoxybutyronitrile
Phenylacetyl Chloride
M-Phenylenediamine
P-Phenylene Diamine
M-Phenylenediamine Dihydrochloride
B-Phenylethyl Bromide
Phenylhydrazine
Phenylhydrazine Hydrochloride
Phenyl Isocyanate
Phenyl Isothiocyanate
P-Phenylphenacyl Bromide
B-Phenylpropionyl Chloride
l-Phenylsemicarbazide
P Phenylthiosemicarbazide
Phenylthiourea
Picoline
B-Picoline
G-Picoline
Picramide
Propionyl Chloride
Pitrile
Phloride

000099081

000088722

000099990

000079378

000112674

000111240

000621330

000094702

000156434

000108952

002243438

000103800

000108452

000106503

000541695

000103639

000100630

000059881

000103719

000103720

000135739

000645454

039538937

005351699

000103855

000109068

000108996

000108894

000489985

000088880

000107120

000079038
31.7-9709

317-9809

317-9909

321-0301

326-0402

326-3402

326-5902

326-6002

326-6102

326-6904

326-7902

327-0603

327-2603

327-2813

327-2903

327-3603

327-5103

327-5203

327-5603

327-6103

327-7603

327-8103

328-0704

328-1704

328-2204

328-5704

328-5804

328-5904

328-6204

328-6404

328-9504

$329=0305$ 
A-1-6

Iso-Propyl Acetate

Iso-Propylamine

N-Propylamine

N-Propylaniline

$\mathrm{N}$-Propylbenzene

N-Propyl Chlorocarbonate

Fropylene Dromide

propylenè Bromohyarin

Propylene Chloride

Propylene Chlorohydrin

Propylene Glycol

Propylene Oxide

Isopropyl Iodide

Quinone

Resorcinol

Semicarbazide Hyảrochloride

Strychnine Sulfate

Succinonitrile

Succinyl Chloride

Sulfanilamide

Tetra Bromoethane

(Acetylene Tetrabromide)

Tetrabromophthalic Anyhydride

Tietra-N-Butylammonium Iodide

1, 1, 2,2-Tetrachloroethane

Tetrachloroethylene

(Perchloroethylene)

Tetrachlorohydroquinone

Tetrachlorophthalic Anhydride

Tetrahydrofuran

Tetramethylammonium Bromide

Tetramethylammonium chloride

Tetramethylammonium Hydroxide
000108214

000075310

000107108

000622800

000103651

000109615

000078751

019888738

UUUU78875

000127004

000057556

000075569

000075309

000106514

000108463

000563417

000060413

000110612

000543204

000063741

000079276

000632791

000311284

000079345

000127184

000087876

000117088

000109999

000064200

000075570

000075592
329-1305

329-1805

329-1905

329-2305

329-3305

329-4405

$339-1805$

$329-4905$

329-51ن்5

329-5205

329-5605

329-5805

329-6305

341-1906

348-0607

357-1708

357-2708

357-3908

$357-4108$

357-5009

365-0839

365-1809

365-2309

365-2809

365-3309

365-3809

365-4309

365-4809

365-5809

365-5909

365-6009 
Tetramethylammonium Iodide

Tetramethylene Chloride

Thioacetamide

Thionyl Chloride

Thiophenol

Thiosemicarbazide

Thiourea

o-Tolidine Dihydrochloride

Toluene

Toluhydroquinone

M-Toluidine

o-Toluidine

P-Toluidine

P-Toluidine Hydrochloride

2,4,6-Tribromoaniline

1,3,5 Tribromobenzene

1,2,3 Tribromopropane

2,4,6-Trichloroaniline

1,2,4-Trichlorobenzene

1,1,2 Trichloroethane

Trichloroethylene

2,4,6 Trichlorophenol

$1,2,3$ I'r1chloropropane

Triethylamine

Trimethylamine

Trimethylene Bromide

Trimethylene Glycol

2, 4,6-Trinitroresorcinol

Tri-N-propylamine

Tribomoethylene

Xylene

M-Xylene

o-Xylene
000075581

000110565

000062555

007719097

000108985

000079196

000062566

000612828

000108883

000095716

000108441

000095534

000106490

000540238

000147820

000626391

000096117

000634935

000120821

000079005

000079016

000088062

000096184

000121448

000075503

000109648

000057556

000082713

000102692

000598163

001330207

000108383

000095476
365-6109

365-7309

365-8509

365-9309

365-9709

366-0401

366-0901

366-1501

366-2401

366-3401

366-3901

366-4001

366-4101

366-4401

366-7401

366-7901

366-8401

366-9401

366-9611

367-0084

367-0102

367-0602

367-1102

367-2102

367-3102

367-3502

367-3902

367-5602

367-8102

367-8602

387-1425

387-1505

387-1605 
$A-1-8$

P-Xylene

000106423

387-1705

o-Xylylene Bromide

000091134

387-2905

M-Xylyl Bromide

000620133

387-3405

o-xylyl Bromide

000089929

387-3505

Materials

Campher Gum

000076222

$619-3032$

Cleaning Mixture \#49

623-5020

Funglicide

649-3001

Pyridine

000110861

731-8305

Organic Gases

Methyl Chloride

000074873

$967-6070$ 
ORNL/TM-6981/V3

INTERNAL DISTRIBUTION

1. Arakawa, E. T.

2. Atchley, R. L.

3. Auble, R. L.

4. Baes, C. F.

5. Baker, M. A.

6. Bandy, C. W.

7. Barclay, D. D.

8. Bel1, J. T.

9. Betz, N.

10. Beweley, W. D.

11. Binford, $F$. $T$.

12. Blevins, W. A.

13. Boarner, L. A.

14. Bolinsky, J.

15. Baunstein, J.

16. Breeding, S. W.

17. Brogan, D.

18. Brooksbank, R. E.

19. Brown, J. M.

20. Bryant, E. H.

21. Burch, W. D.

22. Busby, $W$.

23. Busing, W. R.

24. Cagle, C. D.

25. Cain, G. C.

26. Case, F. N.

27. Chandler, J. M. *

28. Chapman, G. T.

29. Clark, B. R.

30. Clark, R. L.

31. Cleland, J.W.

32. Clinton, S. D.

33. Coleman, C. F.

34. Collins, W. M.

35. Coltharp, H. G.

36. Colwell, B.

37. Conrad, M. C.

38. Corbett, B. L.

39. Corbin, L. T.

40. Cottrell, W. B.

41. Cox, J. A.

42. Cox, T. T.
43. Creswick, F. A.

44. Crowe11, R. A.

45. Crump, . B. F.

46. Datz, S.

47. DeBakker, R. J .

48. Demonbrun, J. R.

49. Dismuke, S. E.

50. Dixon, G. J.

51. Dobbs, N. A.

52. Doherty, D. G.

53. Dunning, D. E.

54. Ealy, J. A.

55. Edwards, R. S

56. Ehrlich, R. D.

57. E1rod, J. C.

58. Emerson, E. E.

59. Everett; W. S.

60. Fair, W. C.

61. Farnham, R. M.

62. Fields, J. A.

63. Fields, J. W.

64. Fontana, M. H.

65. Fortney, T. G.

66. Fox, C. I.

67. Garrett, A. S.

68. Garrett, W. R.

69. Gary, D. C.

70. Gilley, L. W.

71. Gilmore, R. D.

72. Glass. J.

73. Glass, R. W.

74. Graves, W. D.

75. Griest, W. H.

76. Groenier. W. S.

77. Guerin, M. R.

78. Hamrick, T. P.

79. Hartman, H. F.

80. Havmond, H. H.

81. Havnes, C. E.

82. Hicks, G. R.

83. Hightower, J.R.

84. Hoffman, H. W. 
85. Holbrook, J. L.

86. Holland, L. B.

87. Hood, W. B.

88. Hull, G. T.

89. Hurt, S. S.

90. Hutchins, J. D.

91. Ind. Hyg. Library (K-25)

92-102. Ind. Hyg. Dept. (ORNL)

103. Jackson, J. W.

104. Jamison, K. E.

105. Jenkins, L. H.

1nk. Tenkins, R. A

107. Jenness, R. G.

108. Jennings, R. M.

109. Johnson, C. E.

110. Johnston, R. L.

111. Jones, J. E., Jr.

112. Jones, J. R.

113. Jones, R. S.

114. Judish, T. P.

115. Kappelmann, F. A.

116. Keesee, H. F.

117-118. Ketchen, E. E.

119. King, E. M.

12U. King, L. J.

121. Klaus, H. V.

1.2.2. Knhisk, F. H,

1.7.5. kiwhler, W. C.

124. Lake, M. M.

125. Lampton, R. E.

126. Lance, E. D.

127. Langford, L.

128. Lasher, L. C.

129. Lees, E. M.

130. Leinart, R. L.

131. Leuzé, R. E.

132. Library (Paducah)

133. Lieberman, B.

134. Lines, R. L.

135. Livingston, $R$.

136. Lloyd, M. H.

137. Love, $\mathrm{G}$.

138. Lovelace, H. G.

139. Lyon, W. S.

140. MacPherson, R. E.

141. Macres, J .

142. Mailen, J. C.

143. Malinauskas, A. P.

144. Martin, J. R.
145. Massengill, J .

146. McCord, R. V.

147. McDougal, B. E.

148. McDowel1, J. W.

149. McNabb, J. G.

150. Mesmer, R. E.

151. Michel, J.W.

152. Milan,'D.

153. Millican, R.

154. Miller, J. M.

Ibb. Miller, 户. D.

156. Milder, W. H.

157. Mitchell, M.E.

158. Murray, J, A.

159. Nicol, R. G.

160. Noggle, T. S.

161. Oakes, T. W.

162. Ode11, T. T.

163. Oliphant, G. W.

164. Ottinger, C. L.

165. Parrott, J. R.

166. Parzyck, 0. C.

167. Perry, T.P.A.

168. Peterson, C. H.

169. Pickel, 'l'. W.

170. Pierce, E. E.

171. Pope, R, G.

172. Porter, W. E.

173. Posey, F. A.

174. Potcct, K. H.

175. Poutsma, M. L.

176. Pugh, L. P.

177. Pulley, H.

178. Ragland, W. R.

179. Rickard, C. R.

180. Rickard, R. R.

181. Rubbins, D.

182. Rohwer, F. S.

183. Ross, R. G.

184. Ruch, J. B.

185. Ryon, A. D.

186. Schaich, R. W.

187. Schmitt, C.

188. Scott, C. B.

189. Senkan, S. M.

190. Setaro, J. A.

191. Shanks, M. H.

192. Sherrod, J.

193. Shults, W. D. 
194. Skidmore, M. R.

195. Smith, G. P.

196. Snider, J. W.

197. Spiewak, I.

198. Stinton, L. H.

199. Stokely, J. R.

200. Swanks, J. H.

201. Taylor, W. C.

202. Testerman, G. E.

203. Thomas, I. L.

204. Thompson, W. E.

205. Tidweli, K. W.

207. Todd, W. D.

208. Tuck, H. H.

209. Tuholsky,'A. M.

210. Turok, C. W.

211. Vaughen, V.

212. Viar, C. L.

213. Vollman, C. L.

214. Walden, J. M.

215. Walls, A. A.

216. Ward, R. H.

217. Weeren, W. H.

218. White, J. E.

219. Whitman, G. D.

220. Whitson, W. R.

221. Wicker, C. D.

222. Wilson, G. R.

223. Wilson, M. A.

224. Winget, R. H.

225. Wolfenbarger, J. D.

226. Wood, R. F.

227. Wright, J. A.

228. Wright, J. R.

229. Young, F. W.

230. Young, G. B.

231. Youngblood, E. L.

232-233. Central Research Library

234. Document Reference Section

235. ORNL Patent Office

236-237. Laboratory Records

238. Laboratory Records (RC)

239-270. Industrial Hygiene Department (ORNL) 


\section{EXTERNAL DISTRIBUTION}

\section{1-297. Technical Information Center}

298. Office of Asst. Mgr. Energy Research \&

Development, Department of Energy, Oak Ridge Operations Office, Oak Ridge, TN

299. N. E. Boltọn, Union Carbide Corporation - NYO

300. Juhn Janous, 222 Rainbow B1vd. N. - Box 728, Niagara Fa11s, NY 14302 